\title{
DYNAMIC FUNCTIONING OF RESTING STATE NETWORKS IN PHYSIOLOGICAL AND PATHOLOGICAL CONDITIONS
}

\section{EDITED BY: Roberto Esposito, Filippo Cieri, Nicoletta Cera, Dante Mantini}

and Alessandra Griffa

PUBLISHED IN: Frontiers in Neuroscience, Frontiers in Neurology, Frontiers in Psychiatry and Frontiers in Human Neuroscience
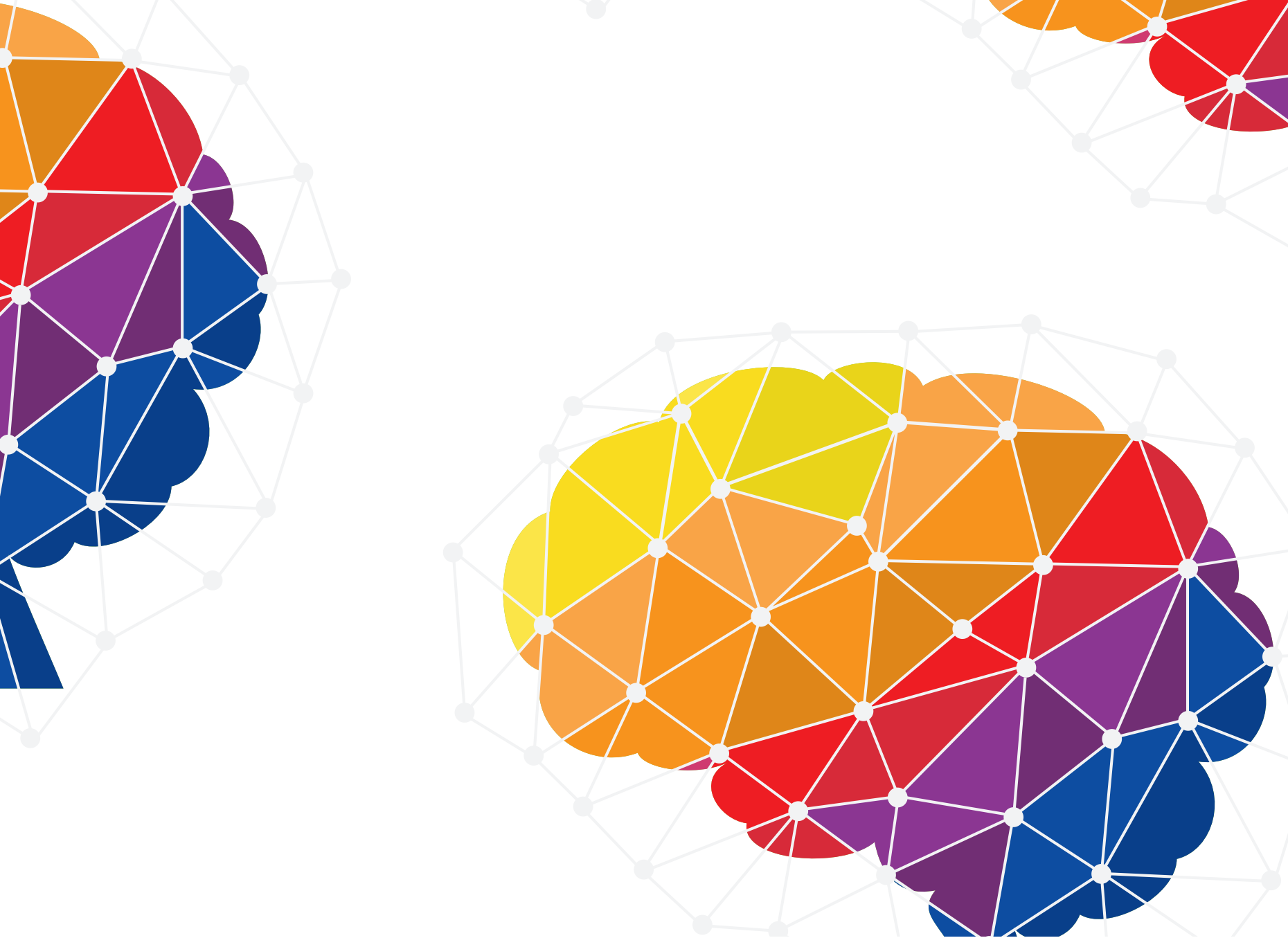


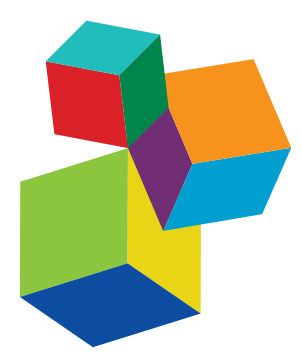

Frontiers eBook Copyright Statement

The copyright in the text of individual articles in this eBook is the property of their respective authors or their respective institutions or funders. The copyright in graphics and images within each article may be subject to copyright of other parties. In both cases this is subject to a license granted to Frontiers.

The compilation of articles constituting this eBook is the property of Frontiers.

Each article within this eBook, and the eBook itself, are published under the most recent version of the Creative Commons CC-BY licence.

The version current at the date of publication of this eBook is CC-BY 4.0. If the CC-BY licence is updated, the licence granted by Frontiers is automatically updated to the new version.

When exercising any right under the CC-BY licence, Frontiers must be attributed as the original publisher of the article or eBook, as applicable.

Authors have the responsibility of ensuring that any graphics or other materials which are the property of others may be included in the

CC-BY licence, but this should be checked before relying on the

CC-BY licence to reproduce those materials. Any copyright notices relating to those materials must be complied with.

Copyright and source acknowledgement notices may not be removed and must be displayed in any copy, derivative work or partial copy which includes the elements in question.

All copyright, and all rights therein, are protected by national and international copyright laws. The above represents a summary only.

For further information please read Frontiers' Conditions for Website

Use and Copyright Statement, and the applicable CC-BY licence.

ISSN 1664-8714

ISBN 978-2-88966-499-3

DOI 10.3389/978-2-88966-499-3

\section{About Frontiers}

Frontiers is more than just an open-access publisher of scholarly articles: it is a pioneering approach to the world of academia, radically improving the way scholarly research is managed. The grand vision of Frontiers is a world where all people have an equal opportunity to seek, share and generate knowledge. Frontiers provides immediate and permanent online open access to all its publications, but this alone is not enough to realize our grand goals.

\section{Frontiers Journal Series}

The Frontiers Journal Series is a multi-tier and interdisciplinary set of open-access, online journals, promising a paradigm shift from the current review, selection and dissemination processes in academic publishing. All Frontiers journals are driven by researchers for researchers; therefore, they constitute a service to the scholarly community. At the same time, the Frontiers Journal Series operates on a revolutionary invention, the tiered publishing system, initially addressing specific communities of scholars, and gradually climbing up to broader public understanding, thus serving the interests of the lay society, too.

\section{Dedication to Quality}

Each Frontiers article is a landmark of the highest quality, thanks to genuinely collaborative interactions between authors and review editors, who include some of the world's best academicians. Research must be certified by peers before entering a stream of knowledge that may eventually reach the public - and shape society; therefore, Frontiers only applies the most rigorous and unbiased reviews.

Frontiers revolutionizes research publishing by freely delivering the most outstanding research, evaluated with no bias from both the academic and social point of view. By applying the most advanced information technologies, Frontiers is catapulting scholarly publishing into a new generation.

\section{What are Frontiers Research Topics?}

Frontiers Research Topics are very popular trademarks of the Frontiers Journals Series: they are collections of at least ten articles, all centered on a particular subject. With their unique mix of varied contributions from Original Research to Review Articles, Frontiers Research Topics unify the most influential researchers, the latest key findings and historical advances in a hot research area! Find out more on how to host your own Frontiers Research Topic or contribute to one as an author by contacting the Frontiers Editorial Office: frontiersin.org/about/contact 


\section{DYNAMIC FUNCTIONING OF RESTING STATE NETWORKS IN PHYSIOLOGICAL AND PATHOLOGICAL CONDITIONS}

Topic Editors:

Roberto Esposito, ASUR Marche, Italy

Filippo Cieri, Cleveland Clinic, United States

Nicoletta Cera, University of Porto, Portugal

Dante Mantini, KU Leuven, Belgium

Alessandra Griffa, University of Geneva, Switzerland

Citation: Esposito, R., Cieri, F., Cera, N., Mantini, D., Griffa, A., eds. (2021). Dynamic Functioning of Resting State Networks in Physiological and Pathological Conditions. Lausanne: Frontiers Media SA. doi: 10.3389/978-2-88966-499-3 


\section{Table of Contents}

07 Editorial: Dynamic Functioning of Resting State Networks in Physiological and Pathological Conditions

Filippo Cieri, Nicoletta Cera, Alessandra Griffa, Dante Mantini and Roberto Esposito

13 Abnormal Topology of the Structural Connectome in the Limbic Cortico-Basal-Ganglia Circuit and Default-Mode Network Among Primary Insomnia Patients

Yunfan Wu, Mengchen Liu, Shaoqing Zeng, Xiaofen Ma, Jianhao Yan, Chulan Lin, Guang Xu, Guomin Li, Yi Yin, Shishun Fu, Kelei Hua, Chao Li, Tianyue Wang, Cheng Li and Guihua Jiang

25 Association Between Connectivity of Hippocampal Sub-Regions and Auditory Verbal Hallucinations in Schizophrenia

Lin Liu, Long-Biao Cui, Yi-Bin Xi, Xing-Rui Wang, Yu-Chen Liu, Zi-Liang Xu, Hua-Ning Wang, Hong Yin and Wei Qin

34 Correlation Between Hippocampus MRI Radiomic Features and Resting-State Intrahippocampal Functional Connectivity in Alzheimer's Disease

Qi Feng, Mei Wang, Qiaowei Song, Zhengwang Wu, Hongyang Jiang, Peipei Pang, Zhengluan Liao, Enyan Yu and Zhongxiang Ding

45 Effects of Second Language Learning on the Plastic Aging Brain: Functional Connectivity, Cognitive Decline, and Reorganization Giovanna Bubbico, Piero Chiacchiaretta, Matteo Parenti, Marcin di Marco, Valentina Panara, Gianna Sepede, Antonio Ferretti and Mauro Gianni Perrucci

58 Corrigendum: Effects of Second Language Learning on the Plastic Aging Brain: Functional Connectivity, Cognitive Decline, and Reorganization Giovanna Bubbico, Piero Chiacchiaretta, Matteo Parenti, Marcin di Marco, Valentina Panara, Gianna Sepede, Antonio Ferretti and Mauro Gianni Perrucci

60 Modeling the Switching Behavior of Functional Connectivity Microstates (FC $\mu$ states) as a Novel Biomarker for Mild Cognitive Impairment

Stavros I. Dimitriadis, María Eugenia López, Fernando Maestu and Ernesto Pereda

78 Tasked-Based Functional Brain Connectivity in Multisensory Control of Wrist Movement After Stroke

Benjamin T. Kalinosky, Kaleb Vinehout, Miguel R. Sotelo,

Allison S. Hyngstrom and Brian D. Schmit

95 Brain Molecular Connectivity in Neurodegenerative Diseases: Recent Advances and New Perspectives Using Positron Emission Tomography Arianna Sala and Daniela Perani 
Stefania Evangelisti, Francesca Pittau, Claudia Testa, Giovanni Rizzo, Laura Ludovica Gramegna, Lorenzo Ferri, Ana Coito, Pietro Cortelli, Giovanna Calandra-Buonaura, Fabio Bisquoli, Claudio Bianchini, David Neil Manners, Lia Talozzi, Caterina Tonon, Raffaele Lodi and Paolo Tinuper

120 Resting State Dynamic Functional Connectivity in Neurodegenerative Conditions: A Review of Magnetic Resonance Imaging Findings Massimo Filippi, Edoardo G. Spinelli, Camilla Cividini and Federica Agosta

128 The Principles of Electroconvulsive Therapy Based on Correlations of Schizophrenia and Epilepsy: A View From Brain Networks

Qi Li, Sha Liu, Meng Guo, Cheng-Xiang Yang and Yong Xu

136 Eigenvector Centrality Dynamics From Resting-State fMRI: Gender and Age Differences in Healthy Subjects

Alle Meije Wink

145 Characterizing Rapid Fluctuations of Resting State Functional Connectivity in Demyelinating, Neurodegenerative, and Psychiatric Conditions: From Static to Time-Varying Analysis

Paola Valsasina, Milagros Hidalgo de la Cruz, Massimo Filippi and Maria A. Rocca

174 Evaluation of Functional Connectivity in the Brain Using Positron Emission Tomography: A Mini-Review Tadashi Watabe and Jun Hatazawa

179 How Sensitive are Conventional MEG Functional Connectivity Metrics With Sliding Windows to Detect Genuine Fluctuations in Dynamic Functional Connectivity?

Lucrezia Liuzzi, Andrew J. Quinn, George C. O'Neill, Mark W. Woolrich, Matthew J. Brookes, Arjan Hillebrand and Prejaas Tewarie

195 EEG Resting-State Large-Scale Brain Network Dynamics are Related to Depressive Symptoms

Alena Damborská, Miralena I. Tomescu, Eliška Honzírková, Richard Barteček, Jana Hořínková, Sylvie Fedorová, Šimon Ondruš and Christoph M. Michel

205 Lower Functional Connectivity in Vestibular-Limbic Networks in Individuals With Subclinical Agoraphobia

Iole Indovina, Allegra Conti, Francesco Lacquaniti, Jeffrey P. Staab, Luca Passamonti and Nicola Toschi

218 Increased Nucleus Accumbens Connectivity in Resting-State Patients With Drug-Naive, First-Episode Somatization Disorder Yangpan Ou, Qinji Su, Feng Liu, Yudan Ding, Jindong Chen, Zhikun Zhang, Jingping Zhao and Wenbin Guo

225 Sex Moderates Amyloid and Apolipoprotein \&4 Effects on Default Mode Network Connectivity at Rest

Jessica Z. K. Caldwell, Xiaowei Zhuang, MacKenzie J. Leavitt, Sarah J. Banks, Jeffrey Cummings and Dietmar Cordes, for the Alzheimer's Disease Neuroimaging Initiative

234 Decreased Cross-Domain Mutual Information in Schizophrenia From Dynamic Connectivity States

Mustafa S. Salman, Victor M. Vergara, Eswar Damaraju and Vince D. Calhoun 
247 Early Pain Exposure Influences Functional Brain Connectivity in Very Preterm Neonates

Domenico Tortora, Mariasavina Severino, Carlo Di Biase, Maryia Malova, Alessandro Parodi, Diego Minghetti, Cristina Traggiai, Sara Uccella, Luca Boeri, Giovanni Morana, Andrea Rossi and Luca Antonio Ramenghi

258 Identification of the Early Stage of Alzheimer's Disease Using Structural $M R I$ and Resting-State $f M R I$

Seyed Hani Hojjati, Ata Ebrahimzadeh and Abbas Babajani-Feremi for the Alzheimer's Disease Neuroimaging Initiative

270 Structural and Functional Connectivity of the Anterior Cingulate Cortex in Patients With Borderline Personality Disorder

Xiaoxia Lei, Mingtian Zhong, Bowen Zhang, Huihui Yang, Wanrong Peng,

Qian Liu, Yu Zhang, Shuqiao Yao, Changlian Tan and Jinyao Yi

279 Dynamic Functional Connectivity Within the Fronto-Limbic Network Induced by Intermittent Theta-Burst Stimulation: A Pilot Study

Yingying Tang, Xiong Jiao, Junjie Wang, Tianyuan Zhu, Jie Zhou,

Zhenying Qian, Tianhong Zhang, Huiru Cui, Hui Li, Xiaochen Tang, Lihua Xu,

Ling Zhang, Yanyan Wei, Jianhua Sheng, Liu Liu and Jijun Wang

288 Altered Cingulate Cortex Functional Connectivity in Normal Aging and Mild Cognitive Impairment

Nicoletta Cera, Roberto Esposito, Filippo Cieri and Armando Tartaro

303 fMRI in Non-human Primate: A Review on Factors That Can Affect

Interpretation and Dynamic Causal Modeling Application

D. Blair Jovellar and Doris J. Doudet

316 Lessons From Astronomy and Biology for the Mind-Copernican

Revolution in Neuroscience

Georg Northoff

330 Neural Correlates of Facial Expression Recognition in Earthquake Witnesses

Francesca Pistoia, Massimiliano Conson, Mario Quarantelli,

Luca Panebianco, Antonio Carolei, Giuseppe Curcio, Simona Sacco,

Gennaro Saporito, Ernesto Di Cesare, Antonio Barile, Carlo Masciocchi and

Alessandra Splendiani

340 Brainstem Modulation of Large-Scale Intrinsic Cortical Activity Correlations

R. L. van den Brink, T. Pfeffer and T. H. Donner

358 Functional Connectivity Patterns and the Role of 5-HTTLPR

Polymorphism on Network Architecture in Female Patients With Anorexia Nervosa

Enrico Collantoni, Paolo Meneguzzo, Marco Solmi, Elena Tenconi, Renzo Manara and Angela Favaro

368 Atypical Temporal Dynamics of Resting State Shapes Stimulus-Evoked Activity in Depression-An EEG Study on Rest-Stimulus Interaction Annemnarie Wolff, Sara de la Salle, Alana Sorgini, Emma Lynn, Pierre Blier, Verner Knott and Georg Northoff 
384 Primary Open Angle Glaucoma is Associated With Functional Brain Network Reorganization

Silvia Minosse, Francesco Garaci, Alessio Martucci, Simona Lanzafame, Francesca Di Giuliano, Eliseo Picchi, Massimo Cesareo, Raffaele Mancino, Maria Guerrisi, Chiara Adriana Pistolese, Roberto Floris, Carlo Nucci and Nicola Toschi

395 The Impact of the Geometric Correction Scheme on MEG Functional Topology at Rest

Stefania Della Penna, Maurizio Corbetta, Vincent Wens and

Francesco de Pasquale

414 Multi-Level Clustering of Dynamic Directional Brain Network Patterns and Their Behavioral Relevance

Gopikrishna Deshpande and Hao Jia

436 Brain Resting-State Network Alterations Associated With Crohn's Disease Jennifer Kornelsen, Alyssia Wilson, Jennifer S. Labus, Kelcie Witges, Emeran A. Mayer and Charles N. Bernstein

445 Decreased Functional Connectivity Between the Left Amygdala and Frontal Regions Interferes With Reading, Emotional, and Executive Functions in Children With Reading Difficulties

Ohad Nachshon, Rola Farah and Tzipi Horowitz-Kraus

457 Abnormal Intrinsic Brain Activity and Neuroimaging-Based fMRI Classification in Patients With Herpes Zoster and Postherpetic Neuralgia Jiabin Huang, Yongxin Li, Huijun Xie, Shaomin Yang, Changyu Jiang, Wuping Sun, Disen Li, Yuliang Liao, Xiyuan Ba and Lizu Xiao

467 Associations Between Altered Cerebral Activity Patterns and Psychosocial Disorders in Patients With Psychogenic Erectile Dysfunction: A Mediation Analysis of fMRI

Tao Yin, Qi Liu, Ziyang Ma, Zhengjie Li, Ruirui Sun, Feiqiang Ren, Guangsen Li, Xiaopeng Huang, Degui Chang and Peihai Zhang 


\title{
Editorial: Dynamic Functioning of Resting State Networks in Physiological and Pathological Conditions
}

\author{
Filippo Cieri ${ }^{1}$, Nicoletta Cera ${ }^{2}$, Alessandra Griffa $^{3,4}$, Dante Mantini ${ }^{5,6}$ and \\ Roberto Esposito ${ }^{7,8 *}$
}

\begin{abstract}
${ }^{1}$ Department of Neurology, Cleveland Clinic Lou Ruvo Center for Brain Health, Las Vegas, NV, United States, ${ }^{2}$ Center for Psychology at University of Porto (CPUP), Faculty of Psychology and Educational Sciences, University of Porto, Porto, Portugal, ${ }^{3}$ Department of Clinical Neurosciences, Division of Neurology, Geneva University Hospitals and Faculty of Medicine, University of Geneva, Geneva, Switzerland, ${ }^{4}$ Institute of Bioengineering, Center of Neuroprosthetics, Ecole Polytechnique Fédérale De Lausanne (EPFL), Geneva, Switzerland, ${ }^{5}$ Research Center for Motor Control and Neuroplasticity, KU Leuven, Leuven, Belgium, ${ }^{6}$ Brain Imaging and Neural Dynamics Research Group, Istituto di Ricovero e Cura a Carattere Scientifico (IRCCS) San Camillo Hospital, Venice, Italy, ${ }^{7}$ Titano Diagnostic Clinic, Falciano, San Marino, ${ }^{8}$ Area Vasta 1, ASUR Marche, Pesaro, Italy
\end{abstract}

Keywords: default mode network, advanced neuroimaging, anticorrelations, dynamic brain activity, resting state networks (RSNs)

\section{Editorial on the Research Topic}

Dynamic Functioning of Resting State Networks in Physiological and Pathological Conditions

\section{OPEN ACCESS}

\author{
Edited and reviewed by: \\ Vince D. Calhoun, \\ Georgia State University, \\ United States \\ *Correspondence: \\ Roberto Esposito \\ resposito1979@gmail.com \\ Specialty section: \\ This article was submitted to \\ Brain Imaging Methods, \\ a section of the journal \\ Frontiers in Neuroscience
}

Received: 31 October 2020 Accepted: 20 November 2020 Published: 16 December 2020

Citation:

Cieri F, Cera N, Griffa A, Mantini D and Esposito R (2020) Editorial: Dynamic Functioning of Resting State Networks in Physiological and Pathological

Front. Neurosci. 14:624401 doi: 10.3389/fnins.2020.624401

\section{INTRODUCTION}

Modern neuroimaging techniques, such as Magnetic Resonance Imaging (MRI), allow for study of the brain from structural and functional perspectives. Particularly, resting state networks' (RSNs) connectivity explores the integration of activity across distant brain areas. In recent years a growing number of studies have shown that the resting state may provide a sensitive and valuable tool able to study cerebral functioning in normal and pathological conditions.

Cerebral activity recorded during cognitive tasks shows a baseline low frequency fluctuation at $0.01-0.1 \mathrm{~Hz}$ (Cordes et al., 2001). During rest, this baseline fluctuation organizes in a network of coordinated cerebral activity named the Default Network (DN), spanning the medial prefrontal, posterior cingulate, inferior parietal, and hippocampal cortices (Raichle et al., 2001; AndrewsHanna et al., 2014). In recent decades, this network has received great attention because it contains several regions that support cognitive functions and undergo critical changes upon aging, cognitive decline, and neuropsychiatric disorders. The DN has been associated with reflective activity and self-referential mental processes and has been extensively characterized in neurological, psychiatric, and psychotherapeutic contexts (Cieri and Esposito, 2018, 2019). To date, in addition to the DN, at least 10 resting state networks have been identified. Among these, the Dorsal Attention Network (DAN) comprises regions commonly activated in attention demanding tasks. The DN and DAN show a pattern of anticorrelated activity in both task and resting state studies, suggesting that they are intrinsically organized into anticorrelated networks (Esposito et al., 2017).

The overall goal of this Research Topic was to provide a comprehensive coverage of the latest advances in dynamic functioning of RSNs both in physiological and neuropsychiatric conditions. The studies have used different neuroimaging techniques to probe functional connectivity (FC) in the brain, including resting-state functional MRI (rs-fMRI), Electroencephalography (EEG), Magnetoencephalography (MEG), and Positron Emission Tomography (PET). 


\section{NEURODEGENERATIVE DISEASES}

Nine articles submitted to this Research Topic investigate FC changes occurring in physiological and pathological aging, with particular attention given to functional plasticity mechanisms and functional markers of prodromal dementia syndromes or conversion to dementia. The articles also highlight how the integration of multimodal functional imaging data (e.g., simultaneous EEG-fMRI recordings) and the analyses of dynamic functional connectivity (dFC) features, as well as subject-level molecular connectivity networks, may contribute to broadening our understanding of neurodegenerative disorders.

\section{Aging, Mild Cognitive Impairment, and Alzheimer's Disease}

Alzheimer's Disease (AD) is the most common form of dementia, and is becoming increasingly common in our increasingly older societies. $\mathrm{AD}$ is characterized by several brain changes including $\beta$-amyloid and tau proteins accumulation, synaptic dysfunctions, and brain atrophy, especially in the medial temporal lobe. In addition to these modifications, a loss of cognitive functions (mostly in the memory domain) and of independence in daily activities are observed. The transition from healthy aging to $\mathrm{AD}$ is usually not immediate and passes through different phases, such as Significant Memory Concerns (SMC) and Mild Cognitive Impairment (MCI). Functional imaging may play a crucial role in identifying early mechanisms predictive of dementia onset.

Hojjati et al. integrate rs-fMRI FC and structural MRI features to predict the conversion of $\mathrm{MCI}$ patients to $\mathrm{AD}$. By investigating $\mathrm{MCI}$ converter patients, MCI non-converter patients, $\mathrm{AD}$ patients, and healthy controls (HCs), the authors show the power of integrating multi-modal MRI data for the identification of early-stage AD.

Cera et al. compare FC patterns of the cingulate cortex between a sample of MCI patients and HCs with comparable levels of education. The authors explore RSNs activities, mapping the FC patterns of different subregions of the cingulate cortex. The cognitive decline observed in MCI patients relates to the global FC of the cingulate cortex, suggesting that the analysis of the cingulate cortex FC could be a helpful tool to better understand the brain mechanisms underlying MCI.

Dimitriadis et al. introduce a novel approach to identify MCI through MEG resting-state data, estimating a dFC graph using the imaginary part of phase lag value for both intra-frequency and cross-frequency couplings. This work shows how the adaptation of neuroinformatic tools combining advanced signal processing and network neuroscience elements can properly highlight the non-stationarity of time-resolved FC patterns, revealing a robust biomarker for MCI.

Bubbico et al. explore cerebral plasticity induced by learning a new language in elders, investigating how cognition together with functional brain organization can be improved late in life. This study analyzes the functional effects of a 4-month second language learning program in a group of HCs. After the program, in the intervention group, there are significant improvements in global cognition together with increased FC in the right frontal gyrus and left superior parietal lobule.
Caldwell et al. investigate how gender moderates typical biomarkers of $\mathrm{AD}$. The authors employed group independent component analysis (ICA) to analyze rs-fMRI data from the Alzheimer's Disease Neuroimaging Initiative dataset. Results suggest that stronger anterior/posterior DN connectivity may support verbal learning in women at risk for $\mathrm{AD}$ dementia.

Feng et al. investigate the correlation between hippocampal FC and MRI radiomic features in $\mathrm{AD}$. The $\mathrm{AD}$ group showed abnormalities of FC levels in the bilateral hippocampal functional network relating to hippocampal radiomic features.

\section{Parkinson's Disease}

Evangelisti et al. explore the effects of L-dopa administration in early-stage Parkinson's Disease (PD) patients on FC patterns revealed by simultaneous recording of fMRI and EEG data. This pilot study provides a first insight into the potentiality of simultaneous EEG-fMRI acquisitions in PD patients, showing for both techniques the analogous direction of increased FC after L-dopa intake, mainly involving motor, dorsal attention, and the DN.

\section{Neuroimaging of Neurodegenerative Diseases: Positron Emission Tomography and Magnetic Resonance Imaging}

Two interesting reviews were submitted about neurogenerative diseases providing an exhaustive overview of literature on rsfMRI and PET imaging.

Sala and Perani summarize available evidence in the field of PET molecular connectivity, offering an overview of how this approach may broaden our understanding of the pathogenesis of neurodegenerative diseases, over and above "traditional" structural/functional connectivity studies. The review gives focus to the available strategies to investigate molecular connectivity at the single-subject level, of potential relevance for both research and diagnostic purposes.

Filippi et al. summarize the main currently available approaches for $\mathrm{dFC}$ analysis and report the recent application of these methods for the assessment of the most common neurodegenerative conditions, including $\mathrm{AD}, \mathrm{PD}$, dementia with Lewy bodies, and frontotemporal dementia. The authors point out the key role of dFC analyses, highlighting both technical and clinical aspects.

\section{EMOTIONAL DISTURBANCES AND PSYCHIATRIC DISEASES}

One of the targets of neuroscience, and particularly of advanced neuroimaging techniques, is to identify specific biomarkers associated with neurological disorders. These biomarkers have induced a great progression in modern cognitive neuroscience but remain elusive in psychiatric disorders due to their clinical heterogeneity and comorbidities. A collection of thirteen studies of this Research Topic highlights the different functional changes occurring in a variety of psychiatric, emotional, learning, eating, and sleep disorders, possibly contributing to the identification of disease-specific biomarkers. Moreover, some of these studies 
show how new neuroimaging measures quantifying functional connectivity dynamics, rest-task transitions, or network information flow may provide a finer-grain characterization of brain functioning in psychiatric conditions. Finally, particular attention is given to the identification of brain functional features reflecting specific behavioral/cognitive/emotional traits (e.g., reading abilities) or clinical symptoms (e.g., verbal auditory hallucinations or depressive symptoms), tackling clinical heterogeneity and inter-individual variations in psychiatric conditions.

\section{Depression}

Damborská et al. explore whether resting-state EEG microstate temporal features can capture large-scale brain network dynamics relevant to depressive symptoms in patients with moderate to severe depression in bipolar affective disorder, depressive episode, and recurrent depressive disorder compared to HCs. Results suggest that the interindividual differences in resting-state microstate parameters could reflect altered largescale brain dynamics relevant to depressive symptomatology during depressive episodes.

Wolff et al. conduct a combined rest and task EEG study in acute depressed major depression disorder (MDD) patients, compared to HCs. Results show that resting-state dynamics are atypical in MDD and strongly shape subsequent stimulus-induced activity. The authors conclude that MDD is characterized by alterations of the rest-stimulus interaction.

\section{Schizophrenia}

Salman et al. employ a new information theoretic framework named dynamic functional domain connectivity (DFDC) to analyze resting-state $\mathrm{dFC}$ and the amount of information shared among functional domains in schizophrenia patients (SZ) and HCs. Functional domains are defined as subsets of functional networks, and their properties and interactions are quantified with entropy and mutual information measures. The authors show that the DFDC pairs tend to function in a more independent manner in SZ patients compared to HCs, suggesting higher uncertainty and randomness in SZ brain function.

Liu et al. explore hippocampal dysconnectivity in SZ patients and its correlation with auditory verbal hallucinations. This study suggests that locations in the hippocampus mediate the neural mechanism behind auditory verbal hallucinations in SZ.

Li et al. review functional network changes occurring in SZ and epilepsy to highlight possible similarities and differences. In light of the reviewed literature, the authors question whether electroconvulsive therapy (ECT), one of the oldest therapeutic modalities in psychiatric clinical practice, relies on antagonistic and/or affinitive mechanisms between these two disorders. The authors highlight how the study of RSNs, such as the default, salience, and dorsal attention networks, has provided a new perspective to understand the relationship between schizophrenia and epilepsy and has shown how ECT modifies the dynamics of these brain networks.

\section{Borderline Personality Disorder}

Emerging evidence supports the hypothesis that emotional dysregulation results from aberrant connectivity within the fronto-limbic neural networks in patients with borderline personality disorder (BPD). Despite its important role in emotional regulation, the anterior cingulate cortex (ACC) has not yet been fully explored in BPD patients. Using seed-based restingstate FC and probabilistic fiber tracking, Lei et al. explore the alterations of functional and structural connectivity of the ACC in young non-medicated BPD patients compared to HCs. The authors show that ACC structural and functional connectivity alteration underlie the deficient emotional regulation circuitry of BPD patients. Such alterations may be important biomarkers of BPD and point to potential BPD treatment targets.

\section{Phobia, Somatization Disorder, Psychogenic Erectile Dysfunction}

Indovina et al. test the hypotheses that individuals with agoraphobic symptoms have visual-vestibular network alterations similar to those of patients with persistent postural perceptual dizziness, and that these alterations are influenced by neuroticism and introversion. They find that the FC of two brain networks is lower in subjects with subclinical agoraphobia as compared to HCs. These networks integrate visual vestibular and emotional responses to guide movement in space.

$\mathrm{Ou}$ et al. investigate the seed-based nucleus accumbens (NAc) FC in first-episode, drug-naive patients with somatization disorder. This study reveals that patients have increased NAc connectivity within the frontal regions of the reward circuit. Increased left NAc-right gyrus rectus connectivity can be used as a potential marker to discriminate patients with somatization disorder from HCs.

Previous studies have illustrated neural changes in patients with psychogenic erectile dysfunction, while only a few works have focused on the neural underpinning of the psychosocial status in patients with this dysfunction. Yin et al. investigate associations among altered cerebral activity patterns, impaired erectile function, and disrupted psychosocial status in patients and HCs, pointing out the key role of psychosocial disorders with respect to the neural changes observed in psychogenic erectile dysfunction.

\section{Eating Disorders: Anorexia Nervosa}

Anorexia nervosa (AN) is a severe psychopathology characterized by intense fear of gaining weight, relentless pursuit of thinness, deep concerns about food, and a pervasive disturbance of body image. Nowadays, eating disorders are more widespread and characterized by diagnostic fluidity with other eating disorders, carry a high psychiatric comorbidity burden, and are associated with elevated suicide risk (Welch et al., 2016). FMRI studies try to shed light on the neurobiological underpinnings of these disorders (Esposito et al., 2018).

Collantoni et al. use a graph-theory approach to explore FC differences between AN patients and HCs, focusing on the effect of serotonin transporter (5-HTTLPR) genotype on regional and global network characteristics. AN patients display lower network clustering and altered hub distribution compared to 
HCs. Moreover, carriers of the short allele are characterized by lower small-world and modularity indexes in the patient group, while an opposite trend is present in HCs.

\section{Sleep Disorders: Primary Insomnia}

$\mathrm{Wu}$ et al. compare the network properties of the structural and functional connectomes derived from diffusion MRI and rs-fMRI data of primary insomnia patients and HCs. They investigate the relationship between abnormal network metrics and clinical characteristics, including disease duration, sleep quality, and anxiety and depression indexes. Patients show small-world architecture with lower global and local efficiencies compared to HCs and present five disrupted subnetworks in the limbic cortico-basal-ganglia circuit and left DN. These results suggest that abnormalities of brain network architecture may be closely linked to the clinical characteristics of primary insomnia.

\section{Post-traumatic Stress Disorder}

Major adverse events trigger different kinds of emotional dysfunctions or psychiatric disorders in the exposed subjects. Recent literature shows that exposure to natural disasters such as earthquakes can generate difficulties in identifying and describing feelings (alexitimia), correlated to the intensity of post-traumatic symptoms (Di Giacinto et al., 2015). Neuroimaging data show that trauma exposure is related to derangement of resting-state FC. Pistoia et al. investigate the neurofunctional changes related to the recognition of emotional faces in L'Aquila earthquake witnesses. The results show that, in earthquake-exposed subjects, there is a significant reduction in the correlation between the accuracy in recognizing facial expressions and the FC of the visual network and DN.

\section{Learning Disorder: Dyslexia}

Nachshon et al. compare functional and cognitive features of children with reading difficulties and age-matched typical readers. A negative correlation between reading, emotional, and executive abilities is found in both groups. Children with reading difficulties show significantly decreased emotional and executive abilities, altered network efficiency within the emotional network, and lower FC between the amygdala and frontal pole regions. Stronger FC between the amygdala, pre-central, and post-central gyri related to worse reading, emotional, and executive abilities in both typical readers and children with reading difficulties.

\section{OTHER CLINICAL CONDITIONS}

\section{Multiple Sclerosis}

In their review, Valsasina et al. describe the methods currently used to assess dFC from rs-fMRI data and summarize the main dFC findings in multiple sclerosis. An overview of the main results obtained in neurodegenerative and psychiatric conditions is also provided. The analysis of dynamic (or time-varying) FC contributes to providing significant information on intrinsic brain functional organization, both in healthy and diseased conditions, which complements data produced by static FC approaches. Time-varying FC seems to be an intrinsic property of the brain with a neural origin, although some open questions still remain about its correct interpretation.

\section{Chronic Inflammatory Bowel Disease: Crohn's Disease}

Kornelsen et al. investigate differences in brain structure and function in patients with Crohn's disease compared to HCs. Voxel-based morphometry analysis is performed to contrast Crohn's disease and HCs' structural images. ROI analyses are run to assess FC for RSN nodes. ICA identifies whole brain differences in FC associated with RSNs. In patients, changes of FC associated with sex are observed in both ROI and ICA analyses and suggest an influence of Crohn's disease on brain function.

\section{Eye Diseases: Glaucoma}

Minosse et al. explore a putative reorganization of functional brain networks in Glaucomatous patients and evaluate the potential of functional network measures as biomarkers of disease severity in terms of their relationship to clinical variables and select retinal layer thicknesses. The authors compare restingstate FC of glaucoma patients and HCs using disruption indices that measure the degree of overall reorganization of specific properties in the whole brain network. In Glaucoma, group-wise disruption indices are negative for all graph theoretical metrics. The disruption index of the clustering coefficient yielded the best discriminative power for differentiating patients from HCs. These results support a possible relationship between FC and disease severity in Glaucoma.

\section{Stroke}

Kalinosky et al. describe brain connectivity associated with multisensory integration during wrist control in stroke survivors, age-matched HCs, and healthy young adults. They use a novel fMRI task paradigm involving wrist movement developed to gain insight into the effects of multimodal (visual and auditory) sensory feedback on brain function in stroke participants. Results show that stroke participants have greater contralesional activation than HCs during the visual feedback condition and less ipsilesional activity than HCs during the auditory feedback condition. Connectivity analyses between the lesioned sensorimotor cortex and the contralesional cerebellum demonstrate decreased FC in stroke participants, positively correlated to manual dexterity. These results suggest that taskbased FC provides details on brain network reorganization in stroke survivors.

\section{Neonatal Pathology: Preterm Neonates}

Tortora et al. evaluate FC changes in preterm neonates that underwent invasive procedures during the postnatal period and correlate them with the neurodevelopmental outcome at 24 months. The authors investigate two groups of preterm neonates: subjected and non-subjected to painful invasive procedures during neonatal intensive care. The results show that early exposure to pain is associated with abnormal FC of developing networks involved in the modulation of noxious stimuli in preterm neonates, contributing to the neurodevelopmental consequence of preterm birth. 


\section{Neuropathic Pain: Post-herpetic Neuralgia}

Huang et al. compare patients with herpes zoster, individuals with post-herpetic neuralgia, and HCs, using fMRI to explore the effects of these diseases on brain activity and to detect the neural mechanisms of cognitive impairment in neuropathic pain patients. The results show that spontaneous brain activity is reduced in both patient groups compared to HCs. In particular, patients have decreased ALFF in the precuneus, posterior cingulate cortex, and middle temporal gyrus. The authors conclude that ALFF values in pain-related regions can be used as an fMRI-based biomarker for the classification of subjects with different pain conditions.

\section{BRAIN PHISIOLOGY AND METHODOLOGICAL APPROACHES}

Five articles of this Research Topics tackle more general aspects of FC assessment, such as the influence on FC measures of data processing and analysis steps (e.g., spatial leakage correction and sliding window approaches), of the uncertainty of oxygen consumption quantification, and of additional factors specific to the investigated species (human or non-human primates).

Della Penna et al. evaluate the impact of the geometric correction scheme (GCS) on MEG functional topology at rest. Source-projected MEG signals are affected by spatial leakage, leading to the estimation of spurious, blurred connections that may affect the topological properties of brain networks. To reduce leakage effects, several correction schemes have been proposed, including the GCS. The authors explore the impact of GCS correction on classical graph measures used to describe the architecture brain functional networks by comparing such measures between GCS-corrected and uncorrected MEG connectomes. The use of GCS considerably reorganizes the topology of connectivity, reducing withinhemisphere interactions mainly in the beta and gamma bands and increasing cross-hemisphere interactions mainly in the alpha and beta bands. Overall, the GCS leakage correction removes spurious local connections, but confirms the role of dynamic hub regions (specifically, the anterior and posterior cingulate cortices) in integrating information in the brain at rest.

Liuzzi et al. propose another interesting MEG study on FC, using multivariate autoregressive and neural mass models with a priori defined ground truths to systematically analyze the sensitivity of conventional metrics in combination with different window lengths to detect genuine fluctuations in connectivity for various underlying state durations. The authors show that fixed sliding window connectivity approaches can detect modulations of connectivity, but mostly if the underlying dynamics operate on moderate to slow timescales. In practice, this can be a drawback, as state durations can vary significantly in empirical data.

In their mini review Watabe and Hatazawa discuss the value of PET imaging to evaluate FC. Previous studies have assessed RSNs mainly based on spontaneous fluctuations in blood-oxygen-level-dependent (BOLD) fMRI signals. However, separation between regional increases in cerebral blood flow and oxygen consumption is theoretically difficult using BOLD-fMRI.
Such a separation can be achieved using quantitative $15 \mathrm{O}$-gas and water PET. In addition, 18F-FDG PET can be used to investigate FC based on changes in glucose metabolism, which reflects local brain activity. Previous studies have highlighted the feasibility and clinical usefulness of 18F-FDG-PET for the analysis of RSNs, and recent studies have utilized simultaneous PET/fMRI recordings for such analyses. PET and fMRI provide different types of information and integrating these modalities may help elucidate the pathological mechanisms underlying certain brain diseases and in characterizing individual patients.

Jovellar and Doudet discuss anatomical and functional differences between human brains and non-human primate brains that can affect pre-processing and analysis of fMRI data, anesthetic effects on BOLD signal and FC, and factors that can affect dynamic causal modeling application in fMRI. There are established preprocessing methods that prepare human fMRI data for subsequent analyses, such as dynamic causal modeling to infer effective connectivity; however, these are not optimized for non-human primate fMRI image analysis. The majority of fMRI imaging in non-human primates is done under anesthesia, which can decrease BOLD signal-to-noise ratio and spontaneous fluctuations. While dynamic causal modeling is a tool that can reliably ascertain directed causal influence (effective connectivity) and address enduring questions on fMRI hemodynamic responses, many uncertainties remain.

Complementarily, van Den Brink et al. review recent works investigating how neuromodulatory systems shape correlations of large-scale cortical activity fluctuations. They discuss functional studies in the human, monkey, and rodent brain and provide a structured but selective overview of these works, distilling a number of emerging principles. The authors underline that efforts to chart the effect of specific neuromodulators and, in particular, of specific receptors, on intrinsic correlations may help in identifying shared or antagonistic principles between different neuromodulatory systems. Such principles can inform models of healthy brain function and may provide an important reference for understanding cortical dynamic alterations observed in neurological and psychiatric disorders, potentially paving the way for mechanistically inspired biomarkers and individualized treatments.

Two studies explore new methodological approaches to characterize brain network centrality dynamics and dynamic effective connectivity.

Wink explores the use of centrality dynamics extracted from eigenvector centrality mapping of fMRI data for measuring group-differences in imaging studies. The analyses on the OpenNeuro dataset show that centrality dynamics can be used to identify age and gender between-group differences, and that age and gender distributions need to be considered in functional imaging studies.

Deshpande and Jia employ a dynamic multivariate autoregressive model to estimate dynamic effective connectivity (DEC), a method first validated on simulated data and then applied to real rs-fMRI data. The authors perform a dynamic clustering (adaptive evolutionary clustering) of DEC matrices across multiple levels -spatial locations, time, and subjectswhich highlights a small number of directional brain network 
configurations akin to brain microstates, alternating over time in a quasi-stable manner. The dominant DEC networks involved spatially distributed brain regions mainly pertaining to memory, emotion, and executive and language functions. Finally, the authors use a larger cohort of rs-fMRI and behavioral data from the Human Connectome Project to show that metrics derived from DEC analysis can explain larger variance in 70 behavioral scores compared to static effective connectivity measures.

One study illustrates how FC analyses can be used to assess in real time the impact of external interventions on brain function. Tang et al. conducted a multi-session analysis combing transcranial magnetic stimulation (TMS) and fMRI experiments to explore the spatiotemporal effects of TMS within the fronto-hippocampal network. Ten healthy volunteers are modulated by intermittent theta-burst stimulation at a precise site within the left dorsolateral prefrontal cortex, navigated by individual structural MRI images. The findings suggest that the intermittent theta-burst stimulation effect dynamically changed over time, from local neural activations at the

\section{REFERENCES}

Andrews-Hanna, J. R., Smallwood, J., and Spreng, R. N. (2014). The default network and self-generated thought: component processes, dynamic control, and clinical relevance. Ann. N. Y. Acad. Sci. 1316, 29-52. doi: $10.1111 /$ nyas. 12360

Cieri, F., and Esposito, R. (2018). Neuroaging through the lens of the resting state networks. Biomed. Res. Int. 2018:5080981. doi: 10.1155/2018/5080981

Cieri, F., and Esposito, R. (2019). Psychoanalysis and neuroscience: the bridge between mind and brain. Front. Psychol. 10:1983. doi: 10.3389/fpsyg.2019.01983

Cordes, D., Haughton, V. M., Arfanakis, K., Carew, J. D., Turski, P. A., Moritz, C. H., et al. (2001). Frequencies contributing to functional connectivity in the cerebral cortex in "resting-state" data. AJNR Am. J. Neuroradiol. 22, 1326-1333.

Di Giacinto, A., Lai, C., Cieri, F., Cinosi, E., Massaro, G., Angelini, V., et al. (2015). Difficulty describing feelings and post-traumatic symptoms after a collective trauma in survivors of L'Aquila earthquake. J. Ment. Health. 24, 150-154. doi: 10.3109/09638237.2015.1019055

Esposito, R., Cieri, F., Chiacchiaretta, P., Cera, N., Lauriola, M., Di Giannantonio, M., et al. (2017). Modifications in resting state functional anticorrelation between default mode network and dorsal attention network: comparison among young adults, healthy elders and mild cognitive impairment patients. Brain Imaging Behav. 12, 127-141. doi: 10.1007/s11682-017-9686-y stimulated site to its connected remote regions within the frontohippocampal network.

Finally, in his hypothesis and theory contribution Northoff reflects on the relationship between neuronal activity, worldbrain interactions, and mental features. Northoff proposes a change in our methodological strategy to approach the brain, from a pre-Copernican vantage point from within brain to a postCopernican vantage point from beyond brain. This change would allow neuroscience to take into view what happens beyond the brain itself, e.g., the world, and how that shapes the brain and its neural activity, e.g., world-brain relation. This view converges with the free energy principle proposed by Karl Friston.

\section{AUTHOR CONTRIBUTIONS}

RE and FC conceived the topic. RE, FC, DM, AG, and NC managed and checked the review processes. RE and FC drafted the editorial and worked on the revisions with DM, AG, and NC. All authors contributed to the article and approved the submitted version.

Esposito, R., Cieri, F., di Giannantonio, M., and Tartaro, A. (2018). The role of body image and self-perception in anorexia nervosa: the neuroimaging perspective. J. Neuropsychol. 12, 41-52. doi: 10.1111/jnp. 12106

Raichle, M. E., MacLeod, A. M., Snyder, A. Z., Powers, W. J., Gusnard, D. A., and Shulman, G. L. (2001). A default mode of brain function. Proc. Natl. Acad. Sci. U.S.A. 98, 676-682. doi: 10.1073/pnas.98.2.676

Welch, E., Jangmo, A., Thornton, L. M., Norring, C., von Hausswolff-Juhlin, Y., Herman, B. K., et al. (2016). Treatment-seeking patients with binge-eating disorder in the Swedish national registers: clinical course and psychiatric comorbidity. BMC Psychiatry 16:163. doi: 10.1186/s12888-016-0840-7

Conflict of Interest: The authors declare that the research was conducted in the absence of any commercial or financial relationships that could be construed as a potential conflict of interest.

Copyright (c) 2020 Cieri, Cera, Griffa, Mantini and Esposito. This is an open-access article distributed under the terms of the Creative Commons Attribution License (CC $B Y)$. The use, distribution or reproduction in other forums is permitted, provided the original author(s) and the copyright owner(s) are credited and that the original publication in this journal is cited, in accordance with accepted academic practice. No use, distribution or reproduction is permitted which does not comply with these terms. 


\section{OPEN ACCESS}

Edited by:

Roberto Esposito,

A.O. Ospedali Riuniti Marche Nord,

Italy

Reviewed by:

Alexander Nikolaevich

Savostyanov,

State Scientific-Research Institute of Physiology \& Basic Medicine,

Russia

Sheng Zhang,

Yale University, United States

*Correspondence:

Guihua Jiang

jiangguihua1970@163.com

${ }^{\dagger}$ These authors have contributed equally to this work

Specialty section: This article was submitted to Brain Imaging Methods, a section of the journal

Frontiers in Neuroscience

Received: 12 September 2018 Accepted: 05 November 2018

Published: 23 November 2018

Citation:

Wu Y, Liu M, Zeng S, Ma X, Yan J, Lin C, Xu G, Li G, Yin Y, Fu S, Hua K, Li C, Wang T, LiC and Jiang G (2018) Abnormal Topology of the Structural

Connectome in the Limbic

Cortico-Basal-Ganglia Circuit and Default-Mode Network Among

Primary Insomnia Patients.

Front. Neurosci. 12:860

doi: 10.3389/fnins.2018.00860

\section{Abnormal Topology of the Structural Connectome in the Limbic Cortico-Basal-Ganglia Circuit and Default-Mode Network Among Primary Insomnia Patients}

Yunfan $\mathrm{Wu}^{1,2+}$, Mengchen Liu',2t, Shaoqing Zeng', Xiaofen $\mathrm{Ma}^{2}$, Jianhao Yan'2, Chulan Lin'2, Guang Xu', Guomin Li', Yi Yin', Shishun Fu', Kelei Hua', Chao Li², Tianyue Wang ${ }^{2}$, Cheng $L^{2}{ }^{2}$ and Guihua Jiang ${ }^{2,1 *}$

${ }^{1}$ The Second School of Clinical Medicine, Southern Medical University, Guangzhou, China, ${ }^{2}$ Department of Medical Imaging, Guangdong Second Provincial General Hospital, Guangzhou, China

Purpose: Primary insomnia (PI) is the second most common mental disorder. However, the topologic alterations in structural brain connectome in patients with PI remain largely unknown.

Methods: A total of $44 \mathrm{PI}$ patients and 46 age-, gender-, and education level matched healthy control $(\mathrm{HC})$ participants were recruited in this study. Diffusion tensor imaging (DTI) and resting state MRI were used to construct structural connectome for each participant, and the network parameters were employed by nonparametric permutations to evaluate the significant differences between the two groups. Relationships between abnormal network metrics and clinical characteristics, including the disease duration, the Pittsburgh Sleep Quality Index (PSQI), the Insomnia Severity Index (ISI), the Self-Rating Anxiety Scale (SAS), and the Self-Rating Depression Scale (SDS), were investigated with Spearman's correlation analysis in PI patients.

Results: PI patients demonstrated small-world architecture with lower global $(P=0.005)$ and local $(P=0.035)$ efficiencies compared with the $\mathrm{HC}$ group. The unique hub nodal properties in $\mathrm{PI}$ patients were mainly in the right limbic cortico-basal-ganglia circuit. Five disrupted subnetworks in PI patients were observed in the limbic corticobasal-ganglia circuit and left default-mode networks (DMN) $(P<0.05$, NBS corrected). Moreover, most unique hub nodal properties in the right limbic cortico-basal-ganglia circuit were significantly correlated with disease duration, and clinical characteristics (SAS, SDS, ISI scores) in PI processing.

Conclusion: These findings suggested the abnormal anatomical network architecture may be closely linked to clinical characteristics in PI. The study provided novel insights into the neural substrates underlying symptoms and neurophysiologic mechanisms of PI.

Keywords: primary insomnia, diffusion tensor imaging, human connectome, fMRI, limbic system

Abbreviations: DTI, diffusion tensor imaging; FA, fractional anisotropy; FN, streamline number; HC, healthy control; HCs, healthy control subjects; PI, primary insomnia; PIs, primary insomnia patients; WM, white matter. 


\section{INTRODUCTION}

Primary insomnia is the second most common mental disorder (Wittchen et al., 2011). PI is characterized by difficulty in initiating and maintaining sleep and early morning awakening for at least 3 months (American Psychiatric Association, 2013). Insomnia profoundly affects health and aging (Musiek and Holtzman, 2016) and is a significant risk factor for the development of other medical or psychiatric diseases. Although previous studies (Riemann et al., 2009, 2010, 2015; Levenson et al., 2015) have reported that heritability, polygenic vulnerability, specific cellular mechanisms and hyperarousal are involved in the pathophysiology of insomnia, the underlying neural substrate symptoms and pathological mechanisms of PI are not fully elucidated (Levenson et al., 2015; Morin et al., 2015).

Rapid development of neuroimaging technologies has provided diverse tools to non-invasively assess abnormal brain activity and anomalous structure for exploring the pathophysiology of insomnia. Two positron emission tomography studies (Nofzinger et al., 2004, 2006) have demonstrated that compared with healthy control participants (HCs), 18-fluorodeoxyglucose metabolism was lower in the prefrontal cortex while awake, and higher in the thalamus (THA), anterior cingulate gyrus, temporal region and pontine tegmentum during non-rapid eye movement sleep in PI patients (PIs). Wang T. et al. (2016) found that regional homogeneity values were higher in the left insula (INS.L), right anterior cingulate gyrus (ACG.R), left insula, bilateral precentral gyrus and left cuneus (CUN.L) in PIs than in HCs. They further used seed-based functional connectivity (FC) analysis and reported that the increased FC between left insular connectivity with many brain regions was related to emotional scores in PIs, primarily in the bilateral anterior cingulate cortex, bilateral thalamus, right fusiform, and middle temporal gyrus (Wang et al., 2017). A surface-based approach reported (Joo et al., 2014) that hippocampal volume decreased in PIs compared with that in HCs. However, a voxel-based morphometric (VBM) study (Li M. et al., 2018) found that gray matter volumes increased in the hippocampus (HIP) and decreased in the dorsolateral prefrontal and middle cingulate cortices compared with the HC group. These results were inconsistent and were mainly focused on single or few seed regions. Recently, many researchers have supported the presence of complex and advanced neural networks in different human brain regions. Brain hub regions particularly play important roles in information integration and are susceptible to attack during disease progression (Crossley et al., 2014). Thus, we speculated that brain network analysis may be more suitable to elucidate the pathophysiological mechanisms of PI.

Diffusion tensor imaging tractography can identify changes in the WM microstructure, cerebral anatomical connections and neural circuits in vivo. Using DTI, Spiegelhalder et al. (2014) demonstrated reduced integrity of WM tracts in the anterior internal capsule, indicating that disturbed frontosubcortical connectivity is a cause or consequence of PI. Similarly, Li et al. (2016) found that the integrity of the right lateralized WM was disrupted in PIs on DTI and tract-based spatial statistics, which involved the right internal capsule, right corona radiate, right superior longitudinal fasciculus, corpus callosum body and right thalamus (THA.R). These two studies demonstrated that DTI could detect changes and provide objective evidence to enhance our understanding of the underlying neurobiological mechanisms of PI. However, these studies focused on the reduced integrity of WM tracts, especially with regard to the internal capsule, and the results revealed no interconnections between abnormal WM tracts and cortical regions. Currently, anatomical networks constructed using DTI tractography successfully identify neuropathological changes in diverse diseases, such as Alzheimer's disease (AD) (Shu et al., 2018), schizophrenia (van den Heuvel et al., 2013), post-traumatic stress disorder (Suo et al., 2017), and other such diseases. Lu et al. (2017a) already demonstrated disrupted structural connectivity in healthy adults with insomnia. However, topological alterations in the anatomical network, including the hub regions, remain largely unknown in PIs.

Considering these findings, we hypothesized that the PIrelated brain WM network exhibits some abnormal network properties, especially in the hub regions, which are more closely related to the severity of insomnia or emotional disorders. We thus aimed to investigate the anatomical brain networks in PI patients, using DTI and graph theory analysis.

\section{MATERIALS AND METHODS}

\section{Subjects}

At the beginning, this prospective study recruited 100 righthanded individuals between April 2010 and April 2016. Adequate images of subjects could not be obtained because of severely head movement or brain lesions as detected by MRI. In the final analyses, the study included 44 PI patients and 46 age-, gender-, and education level matched subjects. This study was approved by the Ethics Committee of the Guangdong Second Provincial General Hospital. All participants signed informed consent forms to participate in this study.

The diagnosis of PI was performed by two neurologists with 15 years of experience. PI patients were enrolled in this study according to the following criteria: (a) patients were diagnosed and confirmed as PI based on the criteria of Diagnostic and Statistical Manual of Mental Disorders, version 5 (DSM-V) (American Psychiatric Association, 2013); (b) patients with a selfcomplaint of difficulty falling asleep, maintaining sleep or early awakening for at least 3 months; (c) patients with no other sleep disorders, such as hypersomnia, parasomnia, obstructive sleep apnea, or sleep-related movement disorder; (d) patients with no serious organic diseases or no severe mental diseases, such as brain stroke, depression (SDS $<70$ ), and anxiety (SAS < 70); (e) all participants were right-handed according to the Edinburgh handedness inventory; (f) all subjects were aged 18-60 years. And HC subjects have good sleep quality and the Insomnia Severity Index (ISI) score $<7$ or the Pittsburgh Sleep Quality Index (PSQI) score $<7$, who were enrolled from the local community.

All participants were excluded according to the following criteria: (a) pregnant, nursing or menstruating females; (b) 
subjects who had an abnormal signal as verified by conventional T1- or T2-weighted fluid-attenuated inversion recovery MR imaging; (c) patients who had severe brain lesions as detected

TABLE 1 | All cortical and subcortical regions with abbreviations and full name in the AAL-90 templates defined in our study.

\begin{tabular}{|c|c|c|}
\hline Index & Regions abbreviations & Brain regions (full name) \\
\hline 1 & PreCG & Precentral gyrus \\
\hline 2 & SFGdor & Superior frontal gyrus, dorsolateral \\
\hline 3 & ORBsup & Superior frontal gyrus, orbital part \\
\hline 4 & MFG & Middle frontal gyrus \\
\hline 5 & ORBmid & Middle frontal gyrus, orbital part \\
\hline 6 & IFGoperc & Inferior frontal gyrus, opercular part \\
\hline 7 & IFGtriang & Inferior frontal gyrus, triangular part \\
\hline 8 & ORBinf & Inferior frontal gyrus, orbital part \\
\hline 9 & ROL & Rolandic operculum \\
\hline 10 & SMA & Supplementary motor area \\
\hline 11 & OLF & Olfactory cortex \\
\hline 12 & SFGmed & Superior frontal gyrus, medial \\
\hline 13 & ORBsupmed & Superior frontal gyrus, medial orbital \\
\hline 14 & REC & Gyrus rectus \\
\hline 15 & INS & Insula \\
\hline 16 & $A C G$ & Anterior cingulate and paracingulate gyrus \\
\hline 17 & DCG & Median cingulate and paracingulate gyrus \\
\hline 18 & PCG & Posterior cingulate gyrus \\
\hline 19 & HIP & Hippocampus \\
\hline 20 & PHG & Parahippocampal gyrus \\
\hline 21 & AMYG & Amygdala \\
\hline 22 & CAL & Calcarine fissure and surrounding cortex \\
\hline 23 & CUN & Cuneus \\
\hline 24 & LING & Lingual gyrus \\
\hline 25 & SOG & Superior occipital gyrus \\
\hline 26 & MOG & Middle occipital gyrus \\
\hline 27 & $I O G$ & Inferior occipital gyrus \\
\hline 28 & $\mathrm{FFG}$ & Fusiform gyrus \\
\hline 29 & PoCG & Postcentral gyrus \\
\hline 30 & SPG & Superior parietal gyrus \\
\hline 31 & $\mathrm{IPL}$ & $\begin{array}{l}\text { Inferior parietal, but supramarginal and } \\
\text { angular gyrus }\end{array}$ \\
\hline 32 & SMG & Supramarginal gyrus \\
\hline 33 & ANG & Angular gyrus \\
\hline 34 & PCUN & Precuneus \\
\hline 35 & PCL & Paracentral lobule \\
\hline 36 & CAU & Caudate nucleus \\
\hline 37 & PUT & Lenticular nucleus, putamen \\
\hline 38 & PAL & Lenticular nucleus, pallidum \\
\hline 39 & THA & Thalamus \\
\hline 40 & HES & Heschl gyrus \\
\hline 41 & STG & Superior temporal gyrus \\
\hline 42 & TPOsup & Temporal pole: superior temporal gyrus \\
\hline 43 & MTG & Middle temporal gyrus \\
\hline 44 & TPOmid & Temporal pole: middle temporal gyrus \\
\hline 45 & ITG & Inferior temporal gyrus \\
\hline
\end{tabular}

Due to space limitations, the detailed descriptions (including $L$, left; $R$, right) could be found at http://neuro.imm.dtu.dk/wiki/Automated_Anatomical_Labeling. by MR; and (d) the subjects who had head motion of more than $1.5 \mathrm{~mm}$ or $1.5^{\circ}$ during MR imaging.

\section{Assessment of Sleep Situation and Mental Status}

Each participant was asked to complete the PSQI, ISI, SAS, and SDS to determine the scores for estimating sleep quality and mental status prior to MRI.

\section{MR Data Acquisition}

MR images of all PIs and healthy control participants (HCs) were obtained on a Philips 1.5T MRI system (Achieva Nova-Dual; Best, Netherlands) at the Department of Medical Imaging, Guangdong Second Provincial General Hospital. Each participant was placed in the supine position with eyes closed and the head snugly restricted by a belt and foam pads. T1WI were acquired with the following parameters: TR, $25 \mathrm{~ms}$; TE, $4 \mathrm{~ms}$; matrix, $256 \times 256$; FOV of $230 \mathrm{~mm} \times 230 \mathrm{~mm}$; a flip angle of $30^{\circ}$; section thickness, $1 \mathrm{~mm}$; 160 transverse sections without gap covering the whole brain. DTI images were collected using an echo planar imaging sequence with the following parameters: TR, 10,700 ms; TE,

TABLE 2 | The definitions of global and nodal topological properties in the study.

\begin{tabular}{|c|c|}
\hline \multicolumn{2}{|l|}{ Global network properties } \\
\hline Small-world coefficient (sigma, $\sigma$ ) & $\begin{array}{l}\text { Sigma }=\text { lambda/gamma, a real network would } \\
\text { be considered small world if } \gamma>1 \text { and } \lambda \approx 1 \text {, } \\
\text { or } \sigma=\lambda / \gamma>1\end{array}$ \\
\hline Clustering coefficient (Cp) & $\begin{array}{l}\mathrm{Cp} \text { is the average clustering coefficient over all } \\
\text { nodes, which measures by calculating the } \\
\text { fraction of the node's neighbors that are also } \\
\text { neighbors of each other. }\end{array}$ \\
\hline Characteristic path length (Lp) & $\begin{array}{l}\text { Lp is the average distance of the shortest path } \\
\text { between every pair of nodes in all nodes, which } \\
\text { indicates the efficiency of information } \\
\text { transferred on a network. }\end{array}$ \\
\hline Normalized Cp (gamma, $\gamma$ ) & $\begin{array}{l}\gamma=C_{\text {preal }} / \mathrm{C}_{\text {prand }}, \mathrm{C}_{\text {prand }} \text { is the mean clustering } \\
\text { coefficient of } 1,000 \text { matched random networks. }\end{array}$ \\
\hline Normalized Lp (lambda, $\lambda$ ) & $\begin{array}{l}\lambda=L_{\text {preal }} / L_{\text {prand }}, L_{\text {prand }} \text { is the mean shortest } \\
\text { path length of } 1,000 \text { matched random networks }\end{array}$ \\
\hline Global efficiency (Eg) & $\begin{array}{l}\text { Eg is defined as the mean value of shortest } \\
\text { path length between all pairs of nodes in the } \\
\text { network. It is a measure of functional } \\
\text { integration. }\end{array}$ \\
\hline Local efficiency (Eloc) & $\begin{array}{l}\text { Eloc is defined as the inverse of the average } \\
\text { shortest path connecting all neighbors of a } \\
\text { node. It is a measure of functional segregation. }\end{array}$ \\
\hline
\end{tabular}

Nodal network properties Nodal efficiency $(\mathrm{Ne})$

Betweenness centrality $(\mathrm{Bc})$

$\mathrm{Ne}$ is defined as the efficient between a node and all other nodes in the network, which evaluate the capacity of a given node for information communication.

$\mathrm{Bc}$ is defined as the fraction of shortest paths passing through a node, which evaluate the contribution of a node on the communication for other nodes.

Degree centrality (Dc)

Dc is defined as the number of edges that a node shares with other nodes in the network, which is a measure of node importance in the network. 
TABLE 3 | Demographics and clinical characteristics of all participants.

\begin{tabular}{lccc}
\hline Characteristic & PI participants $(\boldsymbol{n}=\mathbf{4 4})$ & HC participants $(\boldsymbol{n}=\mathbf{4 6})$ & $\boldsymbol{P}$-value \\
\hline Age (years) & $40.59 \pm 11.51$ & $39.29 \pm 9.25$ & 0.458 \\
Sex & $20 / 24$ & $17 / 29$ & 0.233 \\
(male/female) & & & \\
Duration of & $8.12 \pm 3.36$ & $7.45 \pm 5.21$ & 0.372 \\
education & & & \\
(yeas) & & & \\
PSQI & $16.49 \pm 3.66$ & $2.28 \pm 2.46$ & $<0.001$ \\
ISI & $20.84 \pm 3.67$ & $2.35 \pm 2.63$ & $<0.001$ \\
SAS & $47.43 \pm 9.52$ & $5.28 \pm 10.43$ & $<0.001$ \\
SDS & $52.03 \pm 9.77$ & $6.25 \pm 11.50$ & $<0.001$
\end{tabular}

- Unless otherwise noted, data are expressed as mean \pm SD. PSQI, Pittsburgh Sleep Quality Index; ISI, Insomnia Severity Index; SAS, Self-Rating Anxiety Scale; SDS = Self-Rating Depression Scale; PI, primary insomnia; HC, healthy control.

$80 \mathrm{~ms}$; FOV of $256 \mathrm{~mm} \times 256 \mathrm{~mm}$; matrix size of $128 \times 128$; a flip angle of $90^{\circ}$; section thickness, $2 \mathrm{~mm}$; b-value, $1000 \mathrm{~s} / \mathrm{mm}^{2}$ together with an acquisition without diffusion weighting (bvalue $=0$ ); 75 transverse sections without gap covering the whole cerebellum. All images were reviewed and verified by two radiologists with more than 10 years of experience.

\section{Data Preprocessing and DTI Network Construction}

According to Cui et al. (2013), we preprocessed and analyzed all raw DTI and T1 data with the Diffusion Toolkit 0.6.4 in PANDA software (Cui et al., 2013). Because images of the cerebellum were incomplete, a WM deterministic fiber tracking approach using by PANDA software was used to construct a weighted network including 90 nodes without the cerebellum for each subject, which were defined by the Automated Anatomic Labeling (AAL90) template (Tzourio-Mazoyer et al., 2002). The abbreviations and full names of the AAL90 template are listed in
Table 1. It was terminated if the WM deterministic fiber tracking with a turned angle greater than $45^{\circ}$ or a voxel with a FA less than 0.2 by Continuous Tracking (FACT) algorithm (Chen et al., 2013). In lined with several previous brain DTI network studies (Lo et al., 2010; Wang X. N. et al., 2016; Lu et al., 2017a), we defined the weight of each effective edge between two nodes ( $i$ and $j$ ) of the WM structure as the product of FA and the fiber number (FN) along the fiber bundles, and normalized by the average volume of the two connecting regions (wi $\mathrm{j}=\mathrm{FN}^{*}$ FA/volume). As previous studies (Chen et al., 2013; Wang X. N. et al., 2016), the threshold value for the FN between two regions was defined as 3. Therefore, a weighted matrix of $90 \times 90$ WM structural network was constructed for each subject. After PANDA generated registration images for quality inspection, these images of each subject were carefully checked to ensure registration and segmentation quality by a radiologist with 15 years of experience.

\section{Network Analysis Small-World Properties}

Network analyses were performed with the GRETNA toolbox 2.0.0 release $\left(\right.$ GRETNA $^{1}$ ) (Wang et al., 2015). All global and nodal metrics were defined by Rubinov and Sporns (2010), which including following: small-world coefficient $(\sigma)$, clustering coefficient (Cp), characteristic path length (Lp), normalized Cp $(\gamma)$, normalized Lp $(\lambda)$, global efficiency (Eg), local efficiency (Eloc), nodal efficiency ( $\mathrm{Ne}$ ), betweenness centrality $(\mathrm{Bc})$, and degree centrality (Dc) (the definitions of all network metrics are listed in Table 2). We used the sparsity threshold range of 0.05-0.23 with an interval of 0.01 to discriminate the betweengroup difference with 1,000 matched random networks. The sparsity threshold was selected based on the minimum threshold as determined by the average degree of all network nodes at each

${ }^{1}$ http://www.nitrc.org/projects/gretna
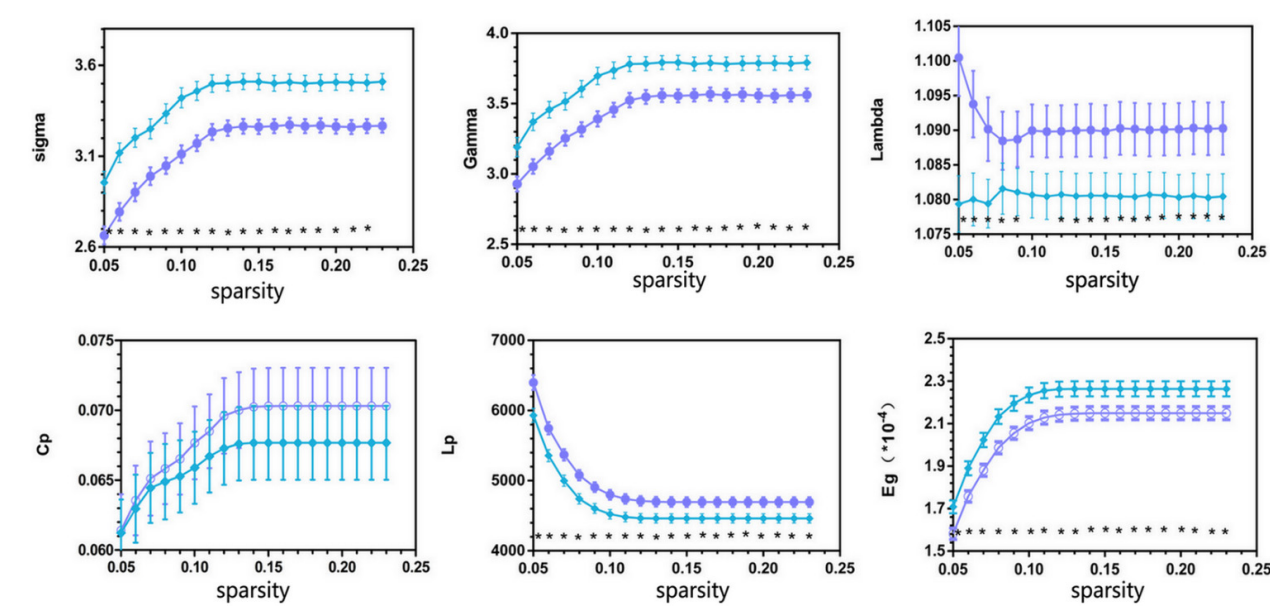
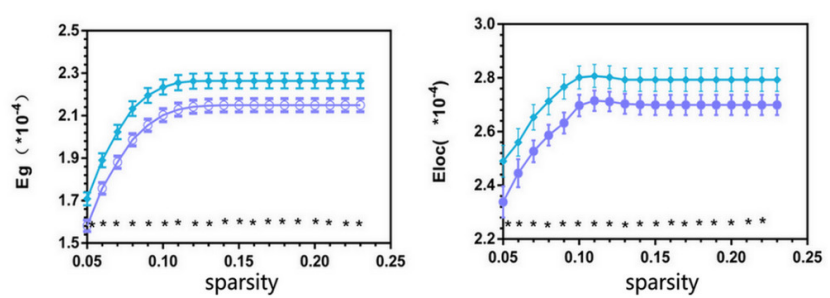

FIGURE 1 | Overall sparsity of group comparison of global network topological properties (Sigma, Gamma, Lambda, Cp, Lp, Eg, and Eloc) of AAL-90 structural connectivity network between the PI and $\mathrm{HC}$ groups. The stars indicate the significantly statistical difference between two groups (10,000 permutations, $p<0.05$, FDR corrected). The vertical bar indicates the standard deviation across subjects. For the abbreviations of network metrics, see Table 2; PI, primary insomnia; HC, healthy control. 


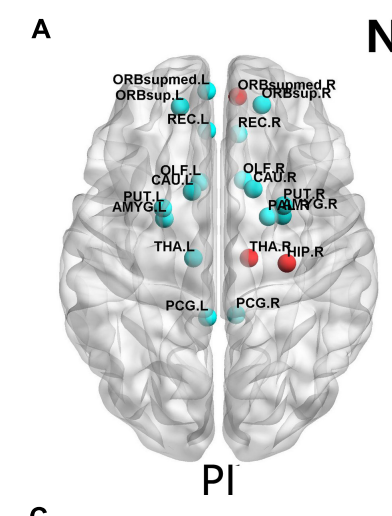

$\mathrm{Ne}$

C
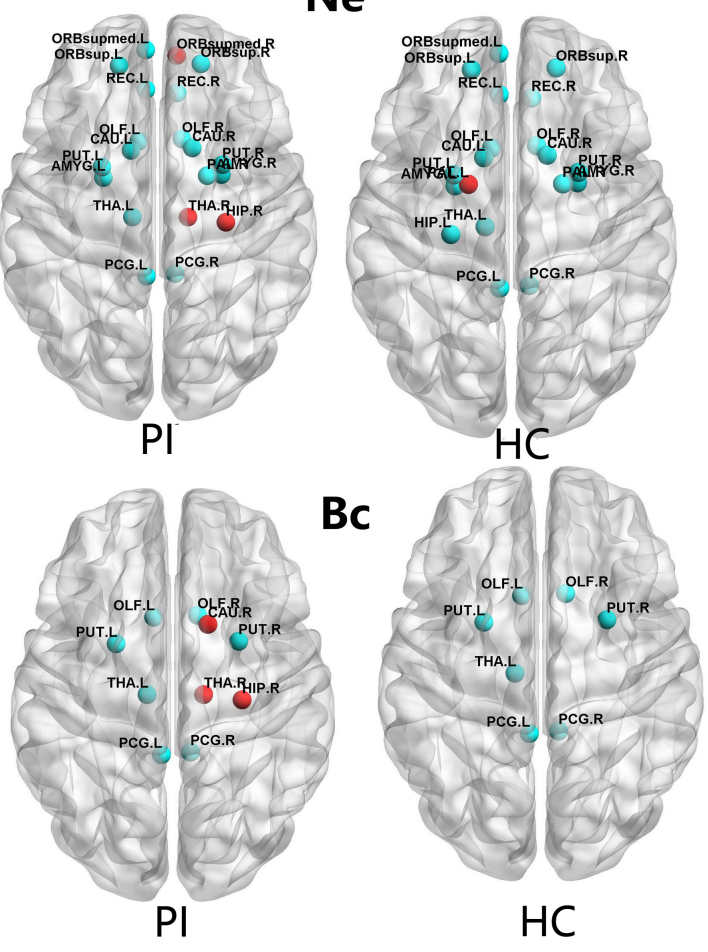

B

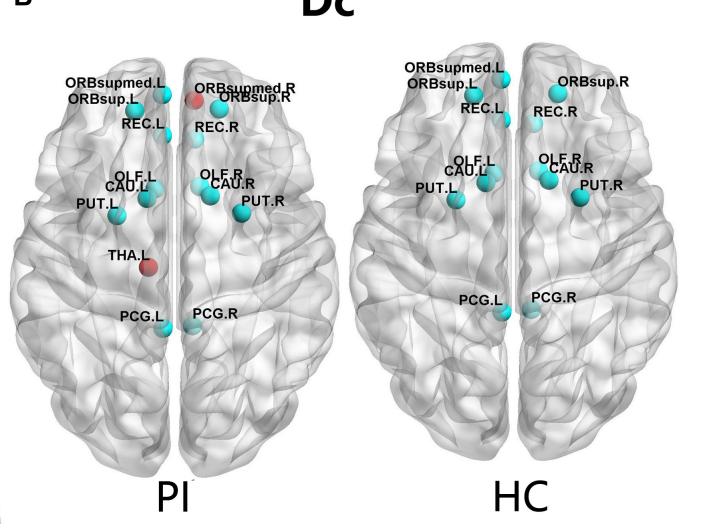

FIGURE 2 | The distribution of the hub regions, left $(\mathrm{L})$ and right $(\mathrm{R})$. The hub regions were identified of three type nodal properties (Ne, Dc, and Bc) at least one standard deviation (SD) above the mean nodal properties across all brain nodes in each group. Nodes with the red color presented unique nodal properties in PI patients compared with HC subjects. Nodes with the cyan point color presented the same hub nodes in both groups. The nodal efficiency was computed in the WM connections with a density of 15\%. (A) Shows nodal properties in Ne. (B) Shows nodal properties in Dc. (C) Shows nodal properties in Bc. Ne, nodal efficiency; Bc, betweenness centrality; Dc, degree centrality. For the abbreviations of the brain nodes, see Table 1; L, left; R, right; PI, primary insomnia; HC, healthy control.

threshold which should be larger than $\log (N)(N=90, N$ means the total number of nodes), and the max threshold as determined by the sigma of all individual networks must be larger than 1.1 to ensure compliance with the small world structure and was in line with previous studies (Tzourio-Mazoyer et al., 2002; Shu et al., 2018).

\section{Hub Distribution}

Hub nodes were defined with nodal properties ( $\mathrm{Ne}, \mathrm{Dc}, \mathrm{Bc}$ ) at least one standard deviation (SD) above the mean nodal properties across all regions in each group. Meanwhile, we further compared between-hemisphere differences in three nodal properties (FDR corrected, $P<0.05$ ) in the PI and HC groups.

\section{PI-Related Subnetwork Analysis}

According to the detailed descriptions in (Zalesky et al., 2010) study, network-based statistic (NBS) connectomes were employed to determinate PI-related subnetwork by the NBS toolkit (version 1.2) $\left(\mathrm{NBS}^{2}\right)$. 10,000 non-parametric permutation tests and NBS corrected $(P<0.01)$ were performed to estimate the significance of each component in identifying the connected subnetworks.

\footnotetext{
${ }^{2}$ https://www.nitrc.org/projects/nbs
}

\section{Statistical Analysis}

SPSS 16.0 software (SPSS Inc., Chicago, IL, United States) was used to compare demographic and clinical characteristics. We used the Shapiro-Wilk test to test the normality of all data. We evaluated the differences of education time, age, PSQI, ISI, SAS, and SDS scores in PIs and HCs by the MannWhitney $U$ test. A $\chi^{2}$ test was used to compare the qualitative variables of gender. In MATLAB software, 10,000 non-parametric permutation tests (Nichols and Holmes, 2002) were employed to assess between-group differences in global and regional network metrics after adjusted age, gender, and education levels as covariates. Briefly, all differences were randomly divided into two groups, and the same primary threshold $(P<0.05)$ was set to compare by recalculating the mean differences between the two randomized groups (10,000 permutations). For comparisons of global and nodal metrics, Benjamin-Hochberg false discovery rate (FDR) correction (Benjamini and Hochberg, 1995) was performed to address the multiple comparisons at a significance level of 0.05 ( $p<0.05)$. After between-group differences of network metrics were identified in the topological properties and nodal metrics, Spearman's correlation was performed by SPSS 16.0 software to assess the associations of these nodal metrics with clinical scores (SAS, SDS, ISI, PSQI scores and disease duration) in PI patients, removing age, gender, and education 
A

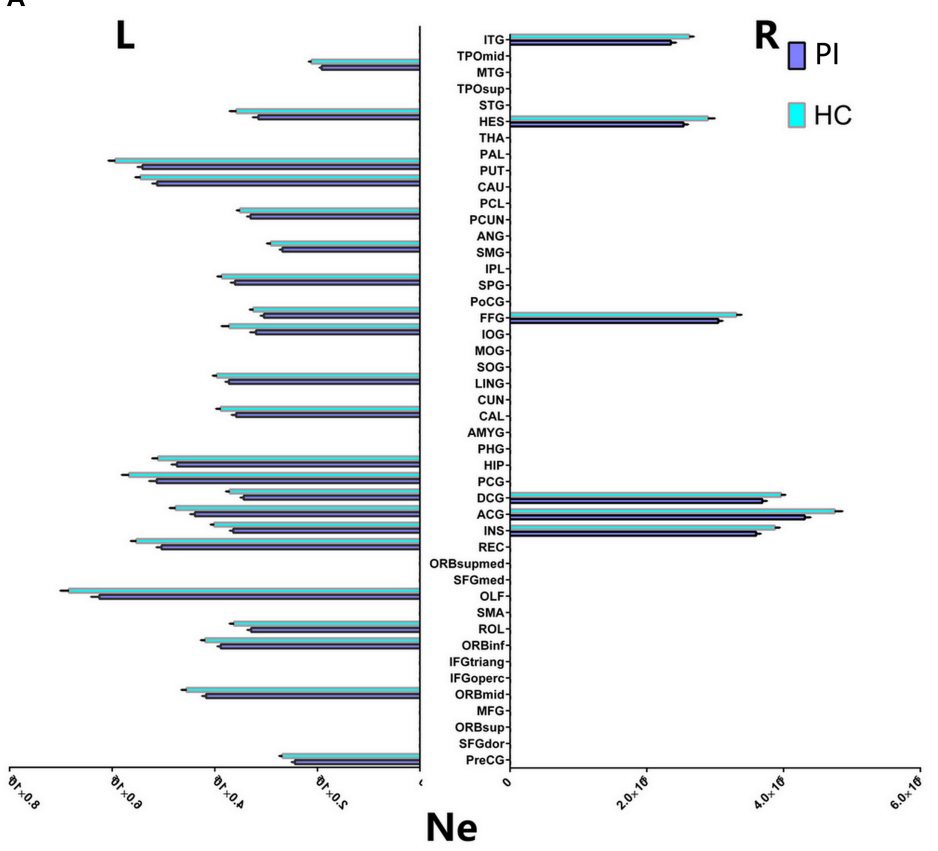

B

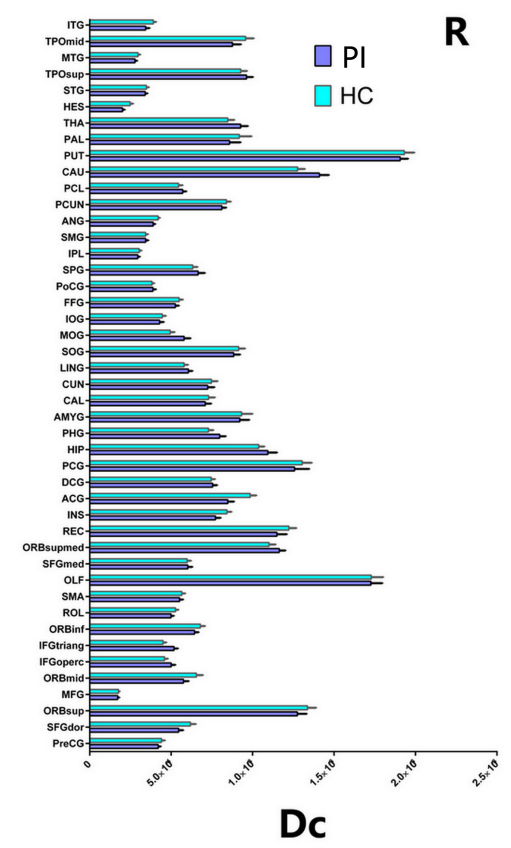

FIGURE 3 | Cortical regions with hemispheric asymmetry in node properties. (A) Bars and error bars represent the significant asymmetric effect of Ne (6 right nodes and 22 left nodes) in both hemispheres in structural connectome ( $p<0.05$, FDR corrected). (B) Bars and error bars represent significant differences of Dc only in right hemispheres in structural connectome. Ne, nodal efficiency; Dc, degree centrality; L, left; R, right; PI, primary insomnia; HC, healthy control.

levels as covariates. All differences of network properties were statistically analyzed by SPSS 16.0 software, MATLAB 2016 software (Matlab, MathWorks, United States), and GRETNA 2.0.0 release software (Gretna, Beijing Normal University, China).

\section{Reproducibility Analysis}

To evaluate the reliability and reproducibility of the research results, we repeated network analysis with different thresholds $(\mathrm{FN} \geq 1,2,3,5$, and 10) in AAL-90 templates and a threshold $(\mathrm{FN}=1)$ in AAL-1024 as previous studies (Bai et al., 2012; Chen et al., 2013). This finding can be replicated with different thresholds and parcellation schemes over the sparisity threshold range of $0.05-0.23$ with an interval of 0.01 .

\section{RESULTS}

\section{Demographic and Clinical Characteristics}

No significant differences were found in age, gender, and education between the PI and HC groups $(P>0.05$; Table 3$)$. The 44 PI patients (20 males, mean age: $42.4 \pm 12.65$ years and 24 females, mean age: $39.83 \pm 11.04$ years) and 46 HCs (17 males, mean age: $38.88 \pm 7.26$ years and 29 females, mean age: $39.41 \pm 9.81$ years) showed significant differences in PSQI, ISI, SAS, and SDS scores $(P<0.05$, Table 3).

\section{Global Topological Organization of Structural Connectome}

Both PIs and HCs showed characteristic small-world topology in the brain structural connectome across all selected thresholds $(\gamma>1, \lambda \approx 1$, and $\sigma>1)$ compared with 1,000 matched random networks. Compared with HCs, PIs exhibited significantly lower $\sigma(P<0.001), \gamma(P<0.001), \operatorname{Eg}(P=0.005)$, and Eloc $(P=0.035)$, higher $\lambda(P=0.027)$ and $\operatorname{Lp}(P=0.004)$ (Figure 1).

\section{Different Hub Regions of Regional Topological Organization of Structural Connectome in Two Groups}

The PI patients and HCs had similar hub regions as shown in Figure 2 by cyan color. In nodal efficiency (Ne), PI patients presented twenty regions acting as hubs, while HCs presented nineteen regions (Figure 2, cyan point). Five unique regions of nodal properties ( $\mathrm{Ne}, \mathrm{Dc}$, and $\mathrm{Bc}$ ) in PIs were located in ORBsupmed.R, HIP.R, bilateral THA and CAU.R compared with HCs (Figure 2). PAL.L was the only unique $\mathrm{Ne}$ region in $\mathrm{HC}$ group. More specifically, three different regions were located in the right orbital part of the medial superior frontal gyrus (ORBsupmed.R), HIP.R and right thalamus (THA.R) in PI patients (Figure 2A, red point). The left lenticular nucleus and pallidum (PAL.L) was the only unique $\mathrm{Ne}$ region in $\mathrm{HC}$ group (Figure $\mathbf{2 A}$, red point). In the degree centrality (Dc), fifteen hub regions were commonly identified in each group. Two unique regions in PI patients were found, locating in the ORBsupmed.R and THA.L (Figure 2B, red point). Meanwhile, PI 


\section{A negative (each independent negative subnetwork)}

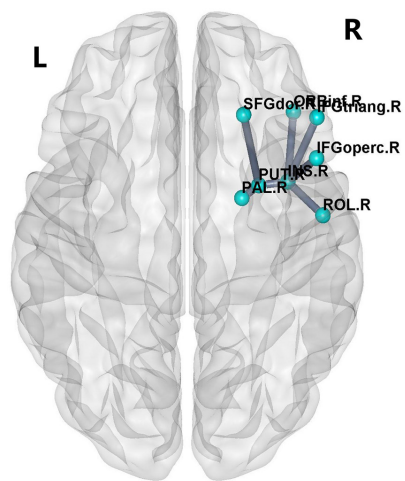

(1)

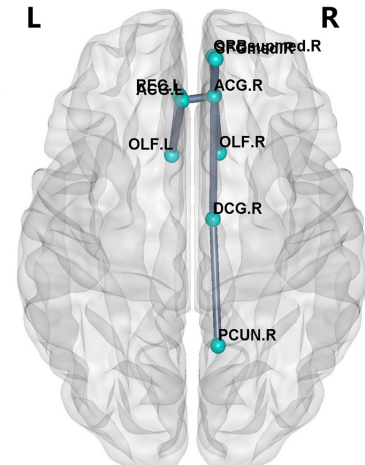

(2)

$B$ negative (full negative subnetworks)
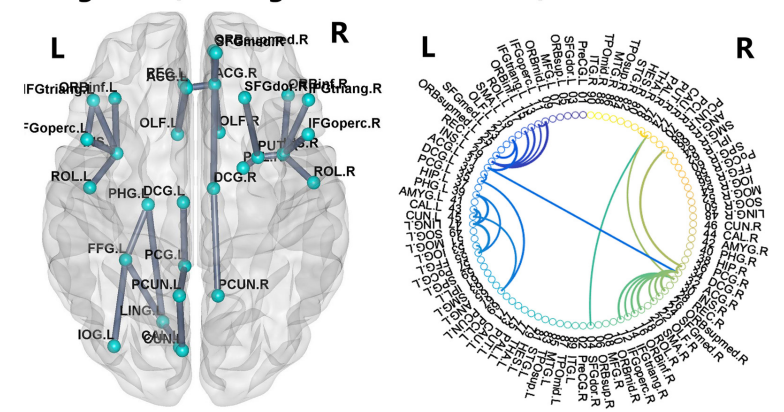

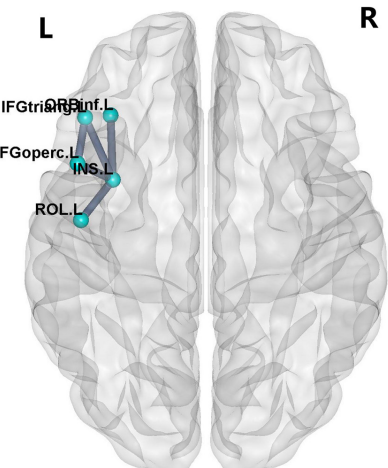

(3)

C a positive subnetwork

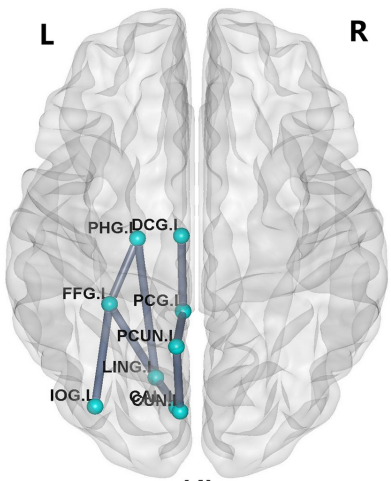

(4)

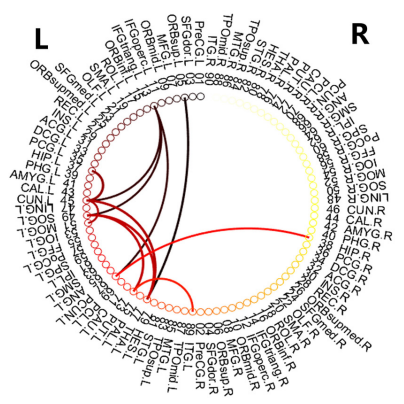

FIGURE 4 | The network-based statistic (NBS) shows the disrupted structural connection in the insomnia participants compared with the HCs. (A,B) Four structural subnetworks with decrease connections were separated $(P<0.01$, NBS corrected). (C) One subnetwork with increase connections was uniformly significantly increased $(P<0.001$, NBS corrected). For the abbreviations of the brain nodes, see Table 1; L, left; $R$, right.

patients presented three different brain nodes in the betweenness centrality $(\mathrm{Bc})$ compared with $\mathrm{HCs}$, including right caudate nucleus (THA.R), HIP.R and caudate nucleus (CAU.R) (left of Figure 2C, red point). These common hub regions shared by both patients and HCs were often bilateral brain regions, including orbital part of superior frontal gyrus (ORBsup), CAU, olfactory cortex (OLF), rectus gyrus (REC), posterior cingulate gyrus (PCG), and lenticular nucleus and putamen (PUT), which were found in more than two types of hub regions. Above all, five different hub regions were identified between the two groups with four regions in the right brain structural connectome except THA.L. There was a interesting asymmetric effect of the nodal properties ( $\mathrm{Ne}, \mathrm{Dc}$, and $\mathrm{Bc}$ ) between the $\mathrm{PI}$ and $\mathrm{HC}$ groups $(P<0.05,10,000$ non-parametric permutation tests) (Figure 3).

\section{Different Nodal Properties of Both Hemispheres in Structural Connectome}

Our study further suggested that the nodal properties showed significant hemispheric effect $(\mathrm{Ne}, \mathrm{Dc}$, and $\mathrm{Bc}$ ) between the PI and HC groups $(P<0.05,10,000$ non-parametric permutation tests). All patients with $\mathrm{Ne}$ showed lower $\mathrm{Ne}$ in PI patients compared with HC group, including all unique hub nodes in PI patients. In comparison with the $\mathrm{HC}$ group, $\mathrm{Ne}$ in $\mathrm{PI}$ patients showed a significant asymmetric effect (6 right nodes and 22 left nodes) in both the hemispheres in structural connectome ( $P<0.05$, Benjamin-Hochberg FDR correction, FDR for short). Specifically, REC.L, INS.L, and PUT.L showed lower Ne in the PI left hemisphere $(P<0.001$, FDR corrected, Figure 3$)$. Similarly, the Dc of all right brain regions showed significant differences between the two groups ( $P<0.05$, no FDR corrected, Figure 3 ), including all right unique hub nodes in PI patients. But the Dc of left hemisphere and the $\mathrm{Bc}$ of both hemispheres showed no significant differences when compared between PI patients and the HCs. For the abbreviations of the brain nodes, these can see Table 1 of the main manuscript.

\section{PI-Related Subnetwork Connectivity}

For the PI group, five subnetworks were revealed in the WM connectome (details listed in Figure 4 and Table 4) that were related to the limbic cortico-basal-ganglia circuit and defaultmode networks (prefrontal cortex, CUN.L, left superior occipital gyrus, left middle occipital gyrus and left precuneus). Four decreased structural subnetworks were separated and mainly distributed in the bilateral prefrontal cortex, left occipital and temporal cortex, and many middle line regions of the brain $(P<0.01$, NBS corrected; Figure 4). Most regions of the uniquely increased subnetworks were located in the left hemisphere, 
TABLE 4 | Regions of four insomnia-related subnetworks.

\begin{tabular}{|c|c|c|}
\hline Index & Region 1 abbreviations & Region 2 abbreviations \\
\hline \multicolumn{3}{|c|}{ Regions of decrease insomnia-related subnetwork 1} \\
\hline$(12,30)$ & IFGoperc.R & INS.R \\
\hline$(14,30)$ & IFGtriang.R & INS.R \\
\hline$(16,30)$ & ORBinf.R & INS.R \\
\hline$(18,30)$ & ROL.R & INS.R \\
\hline$(4,74)$ & SFGdor.R & PUT.R \\
\hline$(30,74)$ & INS.R & PUT.R \\
\hline$(74,76)$ & PUT.R & PAL.R \\
\hline \multicolumn{3}{|c|}{ Regions of decrease insomnia-related subnetwork 2} \\
\hline$(21,27)$ & OLF.L & REC.L \\
\hline$(21,31)$ & OLF.L & ACG.L \\
\hline$(22,32)$ & OLF.R & ACG.R \\
\hline$(24,32)$ & SFGmed.R & ACG.R \\
\hline$(26,32)$ & ORBsupmed.R & ACG.R \\
\hline$(31,32)$ & ACG.L & ACG.R \\
\hline$(32,34)$ & ACG.R & DCG.R \\
\hline$(34,68)$ & DCG.R & PCUN.R \\
\hline \multicolumn{3}{|c|}{ Regions of decrease insomnia-related subnetwork 3} \\
\hline$(11,13)$ & IFGoperc.L & IFGtriang.L \\
\hline$(11,29)$ & IFGoperc.L & INS.L \\
\hline$(13,29)$ & IFGtriang.L & INS.L \\
\hline$(15,29)$ & ORBinf.L & INS.L \\
\hline$(17,29)$ & ROL.L & INS.L \\
\hline \multicolumn{3}{|c|}{ Regions of decrease insomnia-related subnetwork 4} \\
\hline$(33,35)$ & DCG.L & PCG.L \\
\hline$(43,45)$ & CAL.L & CUN.L \\
\hline$(39,47)$ & PHG.L & LING.L \\
\hline$(43,47)$ & CAL.L & LING.L \\
\hline$(39,55)$ & PHG.L & FFG.L \\
\hline$(47,55)$ & LING.L & FFG.L \\
\hline$(53,55)$ & IOG.L & FFG.L \\
\hline$(35,67)$ & PCG.L & PCUN.L \\
\hline$(45,67)$ & CUN.L & PCUN.L \\
\hline \multicolumn{3}{|c|}{ Regions of increase insomnia-related subnetwork 5} \\
\hline$(13,45)$ & IFGtriang.L & CUN.L \\
\hline$(37,45)$ & HIP.L & CUN.L \\
\hline$(13,49)$ & IFGtriang.L & SOG.L \\
\hline$(45,51)$ & CUN.L & MOG.L \\
\hline$(13,67)$ & IFGtriang.L & PCUN.L \\
\hline$(38,67)$ & HIP.R & PCUN.L \\
\hline$(49,73)$ & SOG.L & PUT.L \\
\hline$(5,77)$ & ORBsup.L & THA.L \\
\hline$(45,77)$ & CUN.L & THA.L \\
\hline$(49,77)$ & SOG.L & THA.L \\
\hline$(73,89)$ & PUT.L & ITG.L \\
\hline
\end{tabular}

but with right hippocampus (HIP.R, $P<0.01$, NBS corrected; Figure 4C). Moreover, our study showed the frontal cortex had lower the connection strength with the bilateral INS, ACG, PUT.R and increased the connection strength with THA.L, CUN.L, left precuneus (PCUN.L), and left superior occipital gyrus (SOG.L).

\section{Relationship Between Unique Nodal Properties of Hub Regions and Clinical Characteristics in PI Patients}

We further assessed the associations of hub nodal properties $(\mathrm{Ne}$, $\mathrm{Dc}$, and $\mathrm{Bc}$ ) with clinical characteristics in PI patients (Figure 5 and Table 5). There were significant positive correlations between SAS scores with unique hub nodal properties in PI patients $(\mathrm{Ne}$ of THA.L, $r=0.495, P=0.001$; Ne of ORBsupmed.R, $r=0.332$, $P=0.028$; Ne of HIP.R, $r=0.332, P=0.028$; Bc of CAU.R, $r=0.319, P=0.035)$. Meanwhile, significant positive correlations between SDS scores and Ne of THA.L $(r=0.438, P=0.003)$ were observed. In addition, a significant negative correlation between Dc of ORBsupmed.R with ISI scores $(r=-0.336, P=0.026)$ was observed. Moreover, significant positive correlations between disease duration and unique hub nodal properties of PI patients (Dc of ORBsupmed.R, $r=0.308, P=0.042$; Dc of HIP.R, $r=0.350$, $P=0.020$; Bc of HIP.R, $r=0.328, P=0.030$ ) were observed. However, no significant correlation between unique hub nodal values and PSQI scores was found in PI patients.

\section{DISCUSSION}

We found that PIs had small-world architectures with lower global and local efficiencies than HCs. Asymmetric changes in unique hub nodes and five disrupted subnetworks of the PI WM network suggested that topological alterations were mostly involved in the limbic cortico-basal-ganglia circuit (Haynes et al., 2018) and default-mode networks (DMN) (Van Calster et al., 2017). Additionally, there were significant correlations between clinical characteristics (SAS, SDS, ISI scores and disease duration) and unique hub nodal properties in PIs.

In our study, anatomical topology analysis revealed that the two study groups had characteristic small-world organization, which was consistent with the finding of previous studies ( $\mathrm{Lu}$ et al., 2017b; Ma et al., 2018). Eg and Eloc were used as measures of functional integration and segregation, respectively (Rubinov and Sporns, 2010). Our finding implied neuroinformation transfer disruption in PI. A previous study (Crossley et al., 2014) suggested hub nodes may serve as important centers of information integration and segregation and may be targets of priority attack in diseases. Therefore, we speculated that these unique hub nodes may be affected in PI.

Here, abnormal unique hub nodes were indeed observed, including the prefrontal cortex, right HIP, right caudate nucleus (CAU.R) and THA.R in PIs compared with those in HCs. These hub nodes all are part of the limbic cortico-basal-ganglia circuit. Abnormal prefrontal cortexes in PIs have been reported by many previous studies; for example, Li S. et al. (2018) demonstrated that functional connections decreased in the right fronto-parietal network regions, including the superior frontal gyrus, which are linked to working memory and attention. Using EEG, Muzur et al. (2002) demonstrated that prefrontal cortex influences executive functions in PIs. Furthermore, wholebrain VBM studies demonstrated that orbitofrontal gray matter volume (Altena et al., 2010) and density (Stoffers et al., 2012) 

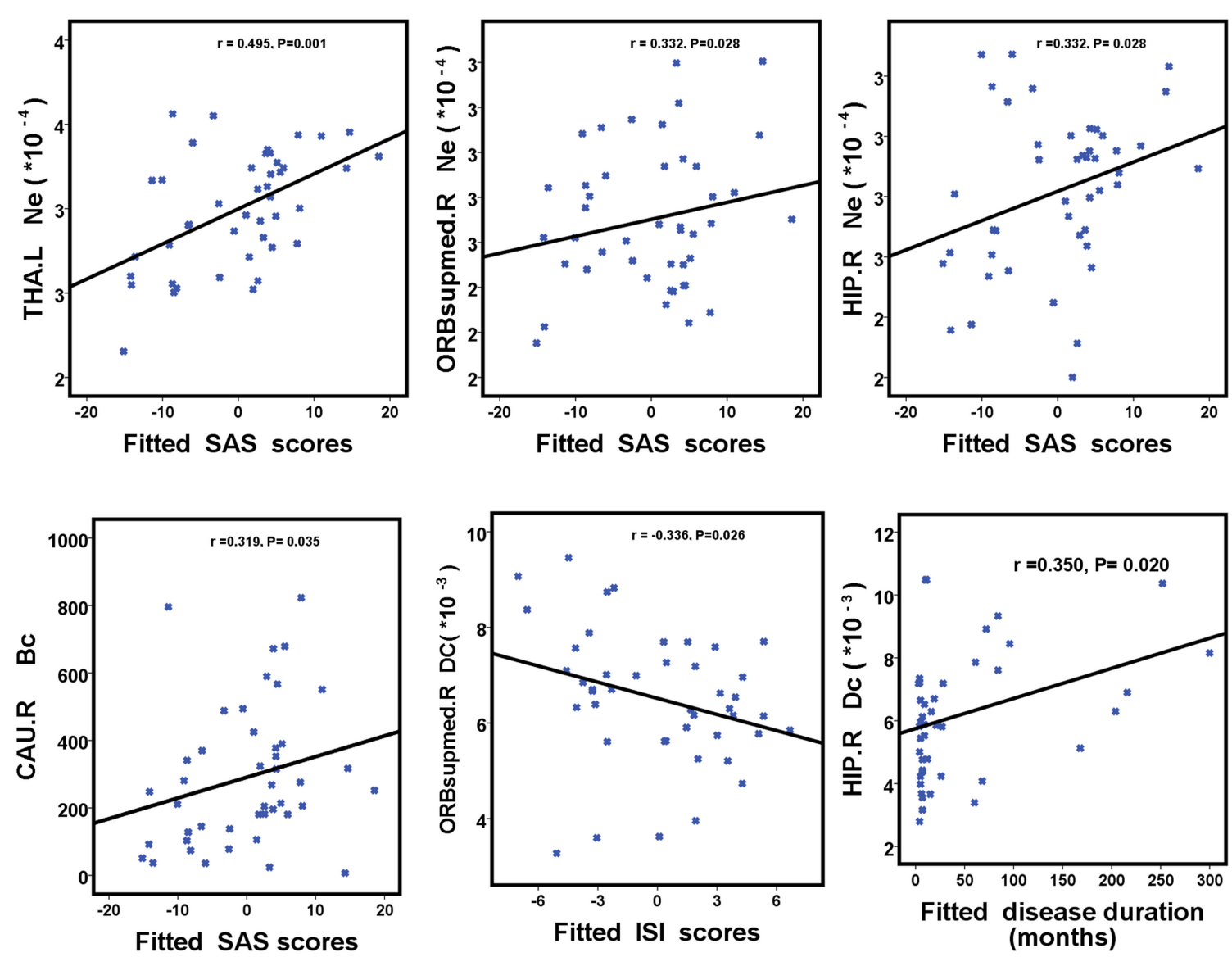

FIGURE 5 | Scatterplots show the relationships between hub nodal properties and clinical characteristics in participants with PI. The fitted values indicate the residuals of PSQI, ISI, SAS, and SDS scores after removing the effects of age, sex, and years of education. PI, primary insomnia; HC, healthy control; SAS, the Self-Rating Anxiety Scale; SDS, Self-Rating Depression Scale; PSQI, Pittsburgh Sleep Quality Index; ISI, Insomnia Severity Index.

were associated with PI complaints. de Vivo et al. (2016) used an adolescent mouse model to investigate the ultrastructure of the frontal cortex that can predict and identify the effects of sleep and sleep loss. We found a negative correlation between the Dc of ORBsupmed.R and ISI scores in structural

TABLE 5 | Relationship between unique nodal properties of hub regions and clinical characteristics in PI patients.

\begin{tabular}{lclrl}
\hline Brain hub region & Nodal properties & Clinical characteristics & $\boldsymbol{r}$ & $\boldsymbol{P}$-value \\
\hline ORBsupmed.R & $\mathrm{Ne}$ & SAS scores & 0.332 & 0.028 \\
& $\mathrm{Dc}$ & ISI scores & -0.336 & 0.026 \\
HIP.R & $\mathrm{Dc}$ & Disease duration & 0.308 & 0.042 \\
& $\mathrm{Ne}$ & SAS scores & 0.332 & 0.028 \\
& $\mathrm{Dc}$ & Disease duration & 0.350 & 0.020 \\
THA.L & $\mathrm{Bc}$ & Disease duration & 0.328 & 0.030 \\
& $\mathrm{Ne}$ & SAS scores & 0.495 & 0.001 \\
CAU.R & $\mathrm{Ne}$ & SDS scores & 0.438 & 0.003 \\
& $\mathrm{Bc}$ & SAS scores & 0.319 & 0.035
\end{tabular}

PIQS, Pittsburgh Sleep Quality Index; ISI, Insomnia Severity Index; SAS, Self-Rating Anxiety Scale; SDS, Self-Rating Depression Scale; PI, primary insomnia. networks among PIs. Our study, as well as these previous studies, suggested that the prefrontal cortex may be associated with abnormal function or structure in PI. HIP is associated with information consolidation and working memory in sleeprelated emotional processing (Murkar and De Koninck, 2018). Despite the inconsistent HP volume results of previous studies (Joo et al., 2014; Li M. et al., 2018), recent animal model studies (Lopez-Virgen et al., 2015; de Vivo et al., 2016; Wadhwa et al., 2017) provided compelling evidence that changes in neuronal ultrastructure in HIP are associated with sleep loss or deprivation. We speculated that these inconsistencies in neuroimaging findings with regard to PI are associated with clinical heterogeneity and use of various techniques and designs for the assessment of substructures in HIP. Our results provide further evidence to support the relation between HIP and PI severity, because PIs were susceptible to negative emotions and had consistent consolidation of negative or dreadful information. CAU is associated with executive dysfunction and control of the sleep-wake behavior, which promotes frequent sleep-wake transitions (Qiu et al., 2010; Stoffers et al., 2014). THA can regulate autonomic and endocrine activation and is associated with a hyperarousal state in PI (Lugaresi, 1992). We speculated 
that abnormalities in the hub nodes of THA and CAU may be associated with these difficulties in maintaining sleep and early morning awakening owing to frequent sleep-wake transitions in PI processing. Meanwhile, in the disrupted subnetworks, we found decreased connections of the frontal cortex with the bilateral INS, ACG, and PUT.R and increased connections of the frontal cortex with THA.L, CUN.L, PCUN.L and left superior occipital gyrus (SOG.L). Our results further showed the presence of complex and neuroinformation interaction in these hub regions. PIs often have a high comorbidity incidence associated with PI and anxiety/depression. Poor PI quality and cognitive emotional hyperarousal predisposition might trigger and maintain a negative cascade (Watling et al., 2017). Therefore, we speculated that these hub nodes may be associated with abnormal emotional reactivity, such as depression and anxiety in PI, which was supported by significant correlations of emotional scores (SAS and SDS) with the nodal properties of the ORBsupmed.R, CAU.R and THA.R in PIs. Our findings demonstrate that changes in the hub topological properties of the limbic cortico-basal-ganglia circuit may be related to the underlying symptoms of PI, especially in sleep-dependent emotional processing.

Meanwhile, some hub regions and PI-related subnetworks were related to DMN. A DMN is an essential network in the human brain and plays important roles in memory, dreaming, auditory/visual processing, self-awareness and selfprocessing operations (Domhoff and Fox, 2015; Zuo et al., 2016). Malfunctioning of the DMN may lead to sustained sleep difficulties and sleep architecture disturbances in PIs, particularly, decrease in connectivity between the DMN and HIP can increase sleep depth (Regen et al., 2016). Our NBS results revealed that PIs exhibited increased connectivity in the left hemisphere between many regions in the DMN with right hippocampus, which further demonstrated that the DMN plays an important role in PI processing.

Additionally, we interestingly found asymmetric distribution of these abnormal hub nodes in PIs, which may be due to have several reasons. First, the result might be associated with anatomical and functional lateralization in the two hemispheres. The left hemisphere of right-handed individuals plays a leading role in language, auditory and visual processing ( $\mathrm{Li}$ et al., 2014; Shu et al., 2015), while the right hemisphere plays an important role in spatial attention, emotion and memory ( $\mathrm{Li}$ et al., 2014). Over the last 3 years, however, studies have shown that the abnormal asymmetric topological properties of both hemispheres are associated with neurophysiological mechanisms in some diseases, such as AD (Yang et al., 2017), schizophrenia (Sun et al., 2017), and autism spectrum disorder (Sun et al., 2017). PIs often have high reactivity with regard to the functions of emotion and memory, which are closely related to the right hemisphere. Our findings showed that most abnormal hub nodes were located in the right limbic cortico-basal -ganglia circuit and were related to patient symptoms (clinical scores and disease duration). PIs also have heightened sensitivity, dreaminess and self-awareness (Domhoff and Fox, 2015; Zuo et al., 2016), and this is closely associated with the left hemisphere. This study also showed increased connections of DMN in the left hemisphere on NBS analysis. Therefore, we speculated that the asymmetric topology in structural networks provides novel insights into the neural substrates underlying patient symptoms, which requires further investigation. Second, asymmetric changes in nodal properties and disrupted subnetworks in both hemispheres might lead to the asymmetric distribution of these unique hub nodes. The observed rightward asymmetric hub nodes were attributed to changes in the complex topological properties of the left hemisphere in PIs, which is consistent with the findings in a previous AD study (Yang et al., 2017). Third, the definition approach for the WM network had a great effect on the constructed networks and results. Li S. et al. (2018) performed DTI and suggested disruption in the integrity of the right lateralized WM in PIs.

\section{Limitations}

The present study has some limitations. First, cognitive functions were not evaluated in this study, which limited the evaluation of the potential impact of cognitive function in PIs. Second, the sample size of PIs was relatively small. Third, the study was limited by the hardware used. We constructed WM networks according to DTI data using the deterministic fiber-tracking algorithm, which has been often used for DTI data with 'not-so-good' quality. However, deterministic tractography has some deficiencies in estimating the crossing fibers. Further investigations involving probabilistic fiber-tracking algorithms and 3T MR scanners are required.

\section{CONCLUSION}

Using DTI and graph theory analysis, we demonstrated abnormal hub nodal properties and subnetworks involving the limbic cortico-basal-ganglia circuit and DMN in PIs. Moreover, the altered network architecture may be related to the neural substrates underlying patient symptoms and the neurophysiologic mechanisms involved in PI.

\section{AUTHOR CONTRIBUTIONS}

YW and GJ conceived and designed the experiments. YW, ML, GX, GL, YY, SF, and KH acquired the data. SZ, XM, JY, ClL, GX, CoL, and TW performed the clinical data, which YW, CgL, and ML analyzed. YW and GJ wrote the article, which all authors reviewed and approved for submission.

\section{FUNDING}

This study has received funding by the National Natural Science Foundation of China (Grant Nos. 81471639 and 81771807), the Natural Science Foundation of Guangdong (Grant No. 2015A030313723), and the Science and Technology Planning Project of Guangdong (Grant Nos. 201607010056, 2016A020215125, and 2017A020215077). 


\section{REFERENCES}

Altena, E., Vrenken, H., Van Der Werf, Y. D., van den Heuvel, O. A., and Van Someren, E. J. (2010). Reduced orbitofrontal and parietal gray matter in chronic insomnia: a voxel-based morphometric study. Biol. Psychiatry 67, 182-185. doi: 10.1016/j.biopsych.2009.08.003

American Psychiatric Association (2013). Diagnostic and Statistical Manual of Mental Disorders, 5th Edn. Virginia : American Psychiatric Association. doi: 10.1176/appi.books.9780890425596

Bai, F., Shu, N., Yuan, Y., Shi, Y., Yu, H., Wu, D., et al. (2012). Topologically convergent and divergent structural connectivity patterns between patients with remitted geriatric depression and amnestic mild cognitive impairment. J. Neurosci. 32, 4307-4318. doi: 10.1523/JNEUROSCI.5061-11.2012

Benjamini, Y., and Hochberg, Y. (1995). Controlling the false discovery rate: a practical and powerful approach to multiple testing. J. R. Stat. Soc. B 57, 289-300.

Chen, Z., Liu, M., Gross, D. W., and Beaulieu, C. (2013). Graph theoretical analysis of developmental patterns of the white matter network. Front. Hum. Neurosci. 7:716. doi: 10.3389/fnhum.2013.00716

Crossley, N. A., Mechelli, A., Scott, J., Carletti, F., Fox, P. T., McGuire, P., et al. (2014). The hubs of the human connectome are generally implicated in the anatomy of brain disorders. Brain 137, 2382-2395. doi: 10.1093/brain/aw u132

Cui, Z., Zhong, S., Xu, P., He, Y., and Gong, G. (2013). PANDA: a pipeline toolbox for analyzing brain diffusion images. Front. Hum. Neurosci. 7:42. doi: 10.3389/ fnhum.2013.00042

de Vivo, L., Nelson, A. B., Bellesi, M., Noguti, J., Tononi, G., and Cirelli, C. (2016). Loss of sleep affects the ultrastructure of pyramidal neurons in the adolescent mouse frontal cortex. Sleep 39, 861-874. doi: 10.5665/sleep.5644

Domhoff, G. W., and Fox, K. C. (2015). Dreaming and the default network: a review, synthesis, and counterintuitive research proposal. Conscious. Cogn. 33, 342-353. doi: 10.1016/j.concog.2015.01.019

Haynes, W. I. A., Clair, A. H., Fernandez-Vidal, S., Gholipour, B., Morgieve, M., and Mallet, L. (2018). Altered anatomical connections of associative and limbic cortico-basal-ganglia circuits in obsessive-compulsive disorder. Eur. Psychiatry 51, 1-8. doi: 10.1016/j.eurpsy.2018.01.005

Joo, E. Y., Kim, H., Suh, S., and Hong, S. B. (2014). Hippocampal substructural vulnerability to sleep disturbance and cognitive impairment in patients with chronic primary insomnia: magnetic resonance imaging morphometry. Sleep 37, 1189-1198. doi: 10.5665/sleep.3836

Levenson, J. C., Kay, D. B., and Buysse, D. J. (2015). The pathophysiology of insomnia. Chest 147, 1179-1192. doi: 10.1378/chest.14-1617

Li, M., Chen, H., Wang, J., Liu, F., Long, Z., Wang, Y., et al. (2014). Handednessand hemisphere-related differences in small-world brain networks: a diffusion tensor imaging tractography study. Brain Connect. 4, 145-156. doi: 10.1089/ brain.2013.0211

Li, M., Yan, J., Li, S., Wang, T., Wen, H., Yin, Y., et al. (2018). Altered gray matter volume in primary insomnia patients: a DARTEL-VBM study. Brain Imaging Behav. doi: 10.1007/s11682-018-9844-x [Epub ahead of print].

Li, S., Tian, J., Li, M., Wang, T., Lin, C., Yin, Y., et al. (2018). Altered resting state connectivity in right side frontoparietal network in primary insomnia patients. Eur. Radiol. 28, 664-672. doi: 10.1007/s00330-017-5012-8

Li, S., Tian, J., Bauer, A., Huang, R., Wen, H., Li, M., et al. (2016). Reduced integrity of right lateralised white matter in patients with primary insomnia: a diffusion-tensor imaging study. Radiology 280, 520-528. doi: 10.1148/radiol. 2016152038

Lo, C. Y., Wang, P. N., Chou, K. H., Wang, J., He, Y., and Lin, C. P. (2010). Diffusion tensor tractography reveals abnormal topological organization in structural cortical networks in Alzheimer's disease. J. Neurosci. 30, 1687616885. doi: 10.1523/JNEUROSCI.4136-10.2010

Lopez-Virgen, V., Zarate-Lopez, D., Adirsch, F. L., Collas-Aguilar, J., and Gonzalez-Perez, O. (2015). Effects of sleep deprivation in hippocampal neurogenesis. Gac. Med. Mex. 151, 99-104.

Lu, F. M., Dai, J., Couto, T. A., Liu, C. H., Chen, H., Lu, S. L., et al. (2017a). Diffusion tensor imaging tractography reveals disrupted white matter structural connectivity network in healthy adults with insomnia symptoms. Front. Hum. Neurosci. 11:583. doi: 10.3389/fnhum.2017.00583
Lu, F. M., Liu, C. H., Lu, S. L., Tang, L. R., Tie, C. L., Zhang, J., et al. (2017b). Disrupted topology of frontostriatal circuits is linked to the severity of insomnia. Front. Neurosci. 11:214. doi: 10.3389/fnins.2017.00214

Lugaresi, E. (1992). The thalamus and insomnia. Neurology 42, 28-33.

Ma, X., Jiang, G., Fu, S., Fang, J., Wu, Y., Liu, M., et al. (2018). Enhanced network efficiency of functional brain networks in primary insomnia patients. Front. Psychiatry 9:46. doi: 10.3389/fpsyt.2018.00046

Morin, C. M., Drake, C. L., Harvey, A. G., Krystal, A. D., Manber, R., Riemann, D., et al. (2015). Insomnia disorder. Nat. Rev. Dis. Primers 1:15026. doi: 10.1038/ nrdp. 2015.26

Murkar, A. L. A., and De Koninck, J. (2018). Consolidative mechanisms of emotional processing in REM sleep and PTSD. Sleep Med. Rev. 41, 173-184. doi: 10.1016/j.smrv.2018.03.001

Musiek, E. S., and Holtzman, D. M. (2016). Mechanisms linking circadian clocks, sleep, and neurodegeneration. Science 354, 1004-1008. doi: 10.1126/science. aah4968

Muzur, A., Pace-Schott, E. F., and Hobson, J. A. (2002). The prefrontal cortex in sleep. Trends Cogn. Sci. 6, 475-481. doi: 10.1016/S1364-6613(02)01992-7

Nichols, T. E., and Holmes, A. P. (2002). Nonparametric permutation tests for functional neuroimaging: a primer with examples. Hum. Brain Mapp. 15, 1-25. doi: 10.1002/hbm.1058

Nofzinger, E. A., Buysse, D. J., Germain, A., Price, J. C., Miewald, J. M., and Kupfer, D. J. (2004). Functional neuroimaging evidence for hyperarousal in insomnia. Am. J. Psychiatry 161, 2126-2128. doi: 10.1176/appi.ajp.161.11.2126

Nofzinger, E. A., Nissen, C., Germain, A., Moul, D., Hall, M., Price, J. C., et al. (2006). Regional cerebral metabolic correlates of WASO during NREM sleep in insomnia. J. Clin. Sleep Med. 2, 316-322.

Qiu, M. H., Vetrivelan, R., Fuller, P. M., and Lu, J. (2010). Basal-ganglia control of sleep-wake behavior and cortical activation. Eur. J. Neurosci. 31, 499-507. doi: 10.1111/j.1460-9568.2009.07062.x

Regen, W., Kyle, S. D., Nissen, C., Feige, B., Baglioni, C., Hennig, J., et al. (2016). Objective sleep disturbances are associated with greater waking resting-state connectivity between the retrosplenial cortex/ hippocampus and various nodes of the default mode network. J. Psychiatry Neurosci. 41, 295-303. doi: 10.1503/ jpn.140290

Riemann, D., Kloepfer, C., and Berger, M. (2009). Functional and structural brain alterations in insomnia: implications for pathophysiology. Eur. J. Neurosci. 29, 1754-1760. doi: 10.1111/j.1460-9568.2009.06721.x

Riemann, D., Nissen, C., Palagini, L., Otte, A., Perlis, M. L., and Spiegelhalder, K. (2015). The neurobiology, investigation, and treatment of chronic insomnia. Lancet Neurol. 14, 547-558. doi: 10.1016/S1474-4422(15)00 021-6

Riemann, D., Spiegelhalder, K., Feige, B., Voderholzer, U., Berger, M., Perlis, M., et al. (2010). The hyperarousal model of insomnia: a review of the concept and its evidence. Sleep Med. Rev. 14, 19-31. doi: 10.1016/j.smrv.2009.04.002

Rubinov, M., and Sporns, O. (2010). Complex network measures of brain connectivity: uses and interpretations. Neuroimage 52, 1059-1069. doi: 10.1016/ j.neuroimage.2009.10.003

Shu, N., Liu, Y., Duan, Y., and Li, K. (2015). Hemispheric asymmetry of human brain anatomical network revealed by diffusion tensor tractography. Biomed. Res. Int. 2015:908917. doi: 10.1155/2015/908917

Shu, N., Wang, X., Bi, Q., Zhao, T., and Han, Y. (2018). Disrupted topologic efficiency of white matter structural connectome in individuals with subjective cognitive decline. Radiology 286, 229-238. doi: 10.1148/radiol.201716 2696

Spiegelhalder, K., Regen, W., Prem, M., Baglioni, C., Nissen, C., Feige, B., et al. (2014). Reduced anterior internal capsule white matter integrity in primary insomnia. Hum. Brain Mapp. 35, 3431-3438. doi: 10.1002/hbm.22412

Stoffers, D., Altena, E., van der Werf, Y. D., Sanz-Arigita, E. J., Voorn, T. A., Astill, R. G., et al. (2014). The caudate: a key node in the neuronal network imbalance of insomnia? Brain 137, 610-620. doi: 10.1093/brain/awt329

Stoffers, D., Moens, S., Benjamins, J., van Tol, M. J., Penninx, B. W., Veltman, D. J., et al. (2012). Orbitofrontal gray matter relates to early morning awakening: a neural correlate of insomnia complaints? Front. Neurol. 3:105. doi: 10.3389/ fneur.2012.00105

Sun, Y., Chen, Y., Collinson, S. L., Bezerianos, A., and Sim, K. (2017). Reduced hemispheric asymmetry of brain anatomical networks is linked to 
schizophrenia: a connectome study. Cereb. Cortex 27, 602-615. doi: 10.1093/ cercor/bhv255

Suo, X., Lei, D., Chen, F., Wu, M., Li, L., Sun, L., et al. (2017). Anatomic insights into disrupted small-world networks in pediatric posttraumatic stress disorder. Radiology 282, 826-834. doi: 10.1148/radiol.2016160907

Tzourio-Mazoyer, N., Landeau, B., Papathanassiou, D., Crivello, F., Etard, O., Delcroix, N., et al. (2002). Automated anatomical labeling of activations in SPM using a macroscopic anatomical parcellation of the MNI MRI single-subject brain. Neuroimage 15, 273-289. doi: 10.1006/nimg.2001. 0978

Van Calster, L., D’Argembeau, A., Salmon, E., Peters, F., and Majerus, S. (2017). Fluctuations of attentional networks and default mode network during the resting state reflect variations in cognitive states: evidence from a novel restingstate experience sampling method. J. Cogn. Neurosci. 29, 95-113. doi: 10.1162/ jocn_a_01025

van den Heuvel, M. P., Sporns, O., Collin, G., Scheewe, T., Mandl, R. C., Cahn, W., et al. (2013). Abnormal rich club organization and functional brain dynamics in schizophrenia. JAMA Psychiatry 70, 783-792. doi: 10.1001/jamapsychiatry. 2013.1328

Wadhwa, M., Prabhakar, A., Ray, K., Roy, K., Kumari, P., Jha, P. K., et al. (2017). Inhibiting the microglia activation improves the spatial memory and adult neurogenesis in rat hippocampus during $48 \mathrm{~h}$ of sleep deprivation. J. Neuroinflammation 14:222. doi: 10.1186/s12974-017-0998-Z

Wang, J., Wang, X., Xia, M., Liao, X., Evans, A., and He, Y. (2015). GRETNA: a graph theoretical network analysis toolbox for imaging connectomics. Front. Hum. Neurosci. 9:386. doi: 10.3389/fnhum.2015.00386

Wang, T., Li, S., Jiang, G., Lin, C., Li, M., Ma, X., et al. (2016). Regional homogeneity changes in patients with primary insomnia. Eur. Radiol. 26, 1292-1300. doi: 10.1007/s00330-015-3960-4

Wang, X. N., Zeng, Y., Chen, G. Q., Zhang, Y. H., Li, X. Y., Hao, X. Y., et al. (2016). Abnormal organization of white matter networks in patients with subjective cognitive decline and mild cognitive impairment. Oncotarget 7, 48953-48962. doi: 10.18632/oncotarget.10601
Wang, T., Yan, J., Li, S., Zhan, W., Ma, X., Xia, L., et al. (2017). Increased insular connectivity with emotional regions in primary insomnia patients: a resting-state fMRI study. Eur. Radiol. 27, 3703-3709. doi: 10.1007/s00330-016-4 $680-0$

Watling, J., Pawlik, B., Scott, K., Booth, S., and Short, M. A. (2017). Sleep loss and affective functioning: more than just mood. Behav. Sleep Med. 15, 394-409. doi: $10.1080 / 15402002.2016 .1141770$

Wittchen, H. U., Jacobi, F., Rehm, J., Gustavsson, A., Svensson, M., Jonsson, B., et al. (2011). The size and burden of mental disorders and other disorders of the brain in Europe 2010. Eur. Neuropsychopharmacol. 21, 655-679. doi: 10.1016/j.euroneuro.2011.07.018

Yang, C., Zhong, S., Zhou, X., Wei, L., Wang, L., and Nie, S. (2017). The abnormality of topological asymmetry between hemispheric brain white matter networks in Alzheimer's disease and mild cognitive impairment. Front. Aging Neurosci. 9:261. doi: 10.3389/fnagi.2017.00261

Zalesky, A., Fornito, A., and Bullmore, E. T. (2010). Network-based statistic: identifying differences in brain networks. Neuroimage 53, 1197-1207. doi: 10.1016/j.neuroimage.2010.06.041

Zuo, N., Song, M., Fan, L., Eickhoff, S. B., and Jiang, T. (2016). Different interaction modes for the default mode network revealed by resting state functional magnetic resonance imaging. Eur. J. Neurosci. 43, 78-88. doi: 10.1111/ejn.13112

Conflict of Interest Statement: The authors declare that the research was conducted in the absence of any commercial or financial relationships that could be construed as a potential conflict of interest.

Copyright (C) $2018 \mathrm{Wu}$, Liu, Zeng, Ma, Yan, Lin, Xu, Li, Yin, Fu, Hua, Li, Wang, Li and Jiang. This is an open-access article distributed under the terms of the Creative Commons Attribution License (CC BY). The use, distribution or reproduction in other forums is permitted, provided the original author(s) and the copyright owner(s) are credited and that the original publication in this journal is cited, in accordance with accepted academic practice. No use, distribution or reproduction is permitted which does not comply with these terms. 
OPEN ACCESS

Edited by:

Filippo Cieri,

Cleveland Clinic, United States

Reviewed by:

Yue Cui,

Chinese Academy of Sciences, China

Elisabetta C. del Re,

Harvard Medical School,

United States

*Correspondence:

Long-Biao Cui

Ibcui@fmmu.edu.cn

Hong Yin

yinhong@fmmu.edu.cn

Wei Qin

wqin@xidian.edu.cn

${ }^{\dagger}$ These authors have contributed equally to this work

Specialty section: This article was submitted to Brain Imaging Methods, a section of the journal Frontiers in Neuroscience

Received: 22 November 2018 Accepted: 12 April 2019

Published: 03 May 2019

Citation: Liu L, Cui L-B, Xi Y-B, Wang $X-R$, Liu Y-C, Xu Z-L, Wang H-N, Yin H and Qin W (2019) Association Between

Connectivity of Hippocampal Sub-Regions and Auditory Verbal Hallucinations in Schizophrenia. Front. Neurosci. 13:424. doi: 10.3389/fnins.2019.00424

\section{Association Between Connectivity of Hippocampal Sub-Regions and Auditory Verbal Hallucinations in Schizophrenia}

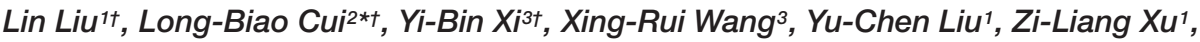 \\ Hua-Ning Wang ${ }^{4}$, Hong Yin ${ }^{3 *}$ and Wei Qin ${ }^{1 *}$ \\ ${ }^{1}$ School of Life Sciences and Technology, Xidian University, Xi'an, China, ${ }^{2}$ Department of Clinical Psychology, School \\ of Medical Psychology, Fourth Military Medical University, Xi'an, China, ${ }^{3}$ Department of Radiology, Xijing Hospital, Fourth \\ Military Medical University, Xi'an, China, ${ }^{4}$ Department of Psychiatry, Xijing Hospital, Fourth Military Medical University, \\ X'an, China
}

Background: Hippocampal dysconnectivity has been detected in schizophrenia patients with auditory verbal hallucinations (AVHs). Neuroanatomical evidence has indicated distinct sub-regions in the hippocampus, but which sub-regions within the hippocampus may emerge dysfunction in the brain network, and the relationship between connection strength and the severity of this debilitating disorder have yet to be revealed. Masked independent component analysis (mICA), i.e., ICA restricted to a defined region of interest, can provide insight into observing local functional connectivity in a particular brain region. We aim to map out the sub-regions in the hippocampus with dysconnectivity linked to AVHs in schizophrenia.

Methods: In this functional magnetic resonance imaging study of schizophrenia patients with $(n=57)$ and without $(n=83)$ AVHs, and 71 healthy controls, we first examined hippocampal connectivity using $\mathrm{mICA}$, and then the correlation between connection metric and clinical severity was generated.

Results: As compared with patients without AVHs, mICA showed a group of hyper-connections for the left middle part, as well as another group of hypo-connections for the bilateral antero-lateral and right antero-medial parts in patients with AVHs. Connectivity was linked to the clinical symptoms scores in the sample of patients with AVHs.

Conclusion: These findings demonstrate that the left middle part is more densely connected, but the bilateral antero-lateral and right antero-medial parts are more sparsely connected in schizophrenia patients with AVHs. The findings in the present study show proof of precious location in the hippocampus mediating the neural mechanism behind AVHs in schizophrenia.

Keywords: auditory verbal hallucinations, schizophrenia, masked ICA, hippocampus, sub-regions, functional connectivity 


\section{INTRODUCTION}

Hippocampal abnormalities have been implicated in the pathophysiology of hallucinations in schizophrenia (Behrendt, 2010, 2016). Converging lines of evidence point to an association between the hippocampus and auditory verbal hallucinations (AVHs) in schizophrenia (Jardri et al., 2011; Alderson-Day et al., 2016). Structural findings include a positive correlation between AVHs severity and hippocampal volume principally affecting the right side (Modinos et al., 2009) and a reduction in the total gray matter volume in schizophrenia with AVHs (Maller et al., 2012). Functional abnormalities have been reported in a variety of studies. There is evidence of reduced connectivity between the left hippocampus and superior temporal gyrus and increased connectivity between the left hippocampus/fusiform gyrus and thalamus in patients with AVHs (Sommer et al., 2012; Clos et al., 2014). Disrupted directed thalamic-auditory cortical-hippocampal connectivity has been found to be involved in AVHs in schizophrenia (Li et al., 2017). Studies comparing auditory and visual hallucinations continue to elucidate the contribution of hippocampal connectivity to schizophrenia. In patients with auditory and visual hallucinations, the hippocampus is hyperconnected to the medial prefrontal cortex and caudate, and there is higher white matter connectivity between the hippocampus and visual cortex (Amad et al., 2014), although no difference was detected in hippocampal connectivity between auditory hallucinations and auditory and visual hallucinations (Ford et al., 2015). Moreover, left hippocampal amplitudes of low-frequency fluctuations (ALFF) across all of the cases were related to reported hallucination severity in both auditory and visual domains (Hare et al., 2017). Patients who were experiencing hallucinations (24 out 25 patients had auditory hallucinations) were linked to effective connectivity from the hippocampus to the salience network (Lefebvre et al., 2016). Particularly, integrating ALFF and functional network connectivity, hippocampal ALFF was positively associated with functional network connectivity between the primary auditory cortex and the salience network in patients reporting auditory hallucinations (Hare et al., 2018). From the neuroanatomical perspective, the hippocampus consists of the dentate gyrus, hippocampus proper, and subiculum (Fogwe and Mesfin, 2018). However, it remains unclear which part in the hippocampus with dysconnectivity is linked to this core psychopathology of schizophrenia.

On structural MRI, the hippocampus is segmented into six sub-regions (Dalton et al., 2017). Despite a harmonized protocol for manual segmentation of hippocampal and parahippocampal sub-regions developed by the Hippocampal Subfields Group (Wisse et al., 2017), hippocampal sub-region segmentation is evolving and plays a limited role in fMRI research. To this end, masked independent component analysis (mICA), i.e., ICA restricted to a defined region of interest, is a high-resolution functional parcellation technique and provides insight into observing local functional connectivity in a particular brain region (Blessing et al., 2016; Moher Alsady et al., 2016). Using mICA, subdivisions of the caudate showing reduced cortical functional connectivity have been established in patients with traumatic brain injury (De Simoni et al., 2018).

Given such a background, we aimed to map out the sub-regions with dysconnectivity in the hippocampus associated with AVHs in schizophrenia. We hypothesized that dysconnectivity can be harnessed via mICA to aid in identifying symptom-specific pathophysiology of schizophrenia.

\section{MATERIALS AND METHODS}

The workflow of this research is presented in Figure $\mathbf{1 .}$

\section{Participants}

Two independent datasets were recruited in this study (Cui et al., 2017c, 2018). Dataset 1 included 74 patients (33 with AVHs, 43 without AVHs) with schizophrenia and 35 healthy controls, which were collected from May 2011 to September 2013 (Cui et al., 2017c). The structural clinical interview for Diagnostic and Statistical Manual of Mental Disorders, Fourth Edition, Text Revision (DSM-IV-TR) was used, and consensus diagnoses were made using all of the available information. Each patient was assessed by using the Positive and Negative Syndrome Scale (PANSS) at the time of imaging. Dataset 2 included 64 patients (24 with AVHs, 40 without AVHs) with schizophrenia and 36 healthy controls, a partial sample of the dataset investigated in Cui et al. (2018). Patients were diagnosed according to DSM, Fifth Edition (DSM-5) from April 2015 to December 2017, with no more than two weeks of cumulative exposure to antipsychotics. We have previously described the criteria for AVHs and Non-AVHs (Chang et al., 2015; Cui et al., 2016, 2017a,b, 2018). This study was approved by the local ethics committee. All participants (or their parents for those under age of 18 years) gave written informed consent after a full description of the aims and design of the study. Table 1 provides further details of the two patient populations.

\section{Image Acquisition}

High-resolution structural imaging and resting-state functional data were acquired on a Siemens $3.0 \mathrm{~T}$ scanner for dataset 1 and a GE $3.0 \mathrm{~T}$ scanner for dataset 2 using protocols published previously (Cui et al., 2019). More details are shown in Supplementary Table 1. Those participants whose head motion exceeded more than $2.5 \mathrm{~mm}$ or $3.0^{\circ}$ during resting-state functional MRI were detected and removed using our own Matlab scripts.

\section{Data Preprocessing}

Functional imaging data were processed using tools from CONN toolbox and FSL 5.0.11 (Oxford Centre for Functional MRI of the Brain, Oxford, United Kingdom ${ }^{1}$ ), mICA $^{2}$ and scripts written in Matlab (MathWorks, Natick, MA, United States). Anatomical images were segmented using FEAT of FSL into gray matter, white matter and cerebrospinal fluid (CSF) using

\footnotetext{
${ }^{1}$ http://www.fmrib.ox.ac.uk/fsl/

${ }^{2}$ https://www.nitrc.org/projects/mica
} 


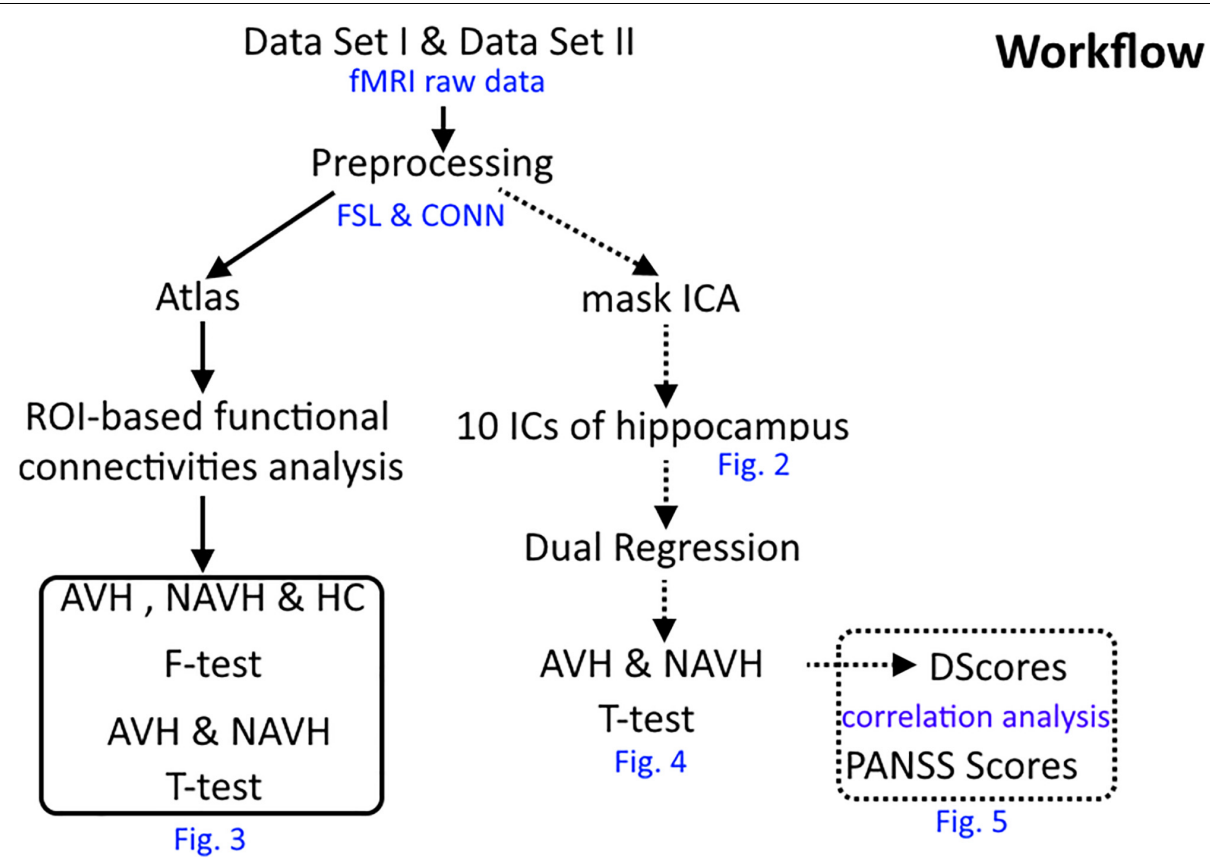

FIGURE 1 | The workflow of this research.

TABLE 1 | Clinical and demographical data.

\begin{tabular}{|c|c|c|c|c|c|c|c|c|}
\hline \multirow[b]{2}{*}{ Characteristic } & \multicolumn{4}{|c|}{ Dataset 1} & \multicolumn{4}{|c|}{ Dataset 2} \\
\hline & $\begin{array}{c}\text { AVHs } \\
(n=33)\end{array}$ & $\begin{array}{c}\text { Non-AVHs } \\
(n=43)\end{array}$ & $P$ values & $\begin{array}{c}\text { HCs } \\
(n=35)\end{array}$ & $\begin{array}{c}\text { AVHs } \\
(n=24)\end{array}$ & $\begin{array}{c}\text { Non-AVHs } \\
(n=40)\end{array}$ & $P$ values & $\begin{array}{c}\text { HCs } \\
(n=36)\end{array}$ \\
\hline Age (y) & $24.7 \pm 6.6$ & $25.5 \pm 5.6$ & 0.53 & $27.1 \pm 7.3$ & $21.6 \pm 5.5$ & $23.7 \pm 7.3$ & 0.49 & $29.3 \pm 9.1$ \\
\hline Gender (M/F) & $17 / 16$ & $26 / 17$ & 0.24 & $22 / 13$ & $12 / 12$ & $27 / 13$ & 0.08 & $14 / 22$ \\
\hline Education level (y) & $13.0 \pm 2.0$ & $13.3 \pm 1.8$ & 0.41 & $13.6 \pm 3.5$ & $12.4 \pm 2.9$ & $12.3 \pm 2.9$ & 0.92 & $15.0 \pm 4.1$ \\
\hline Duration of illness (mon) & $23.0 \pm 26.7$ & $20.4 \pm 29.2$ & 0.80 & NA & $8.2 \pm 13.5$ & $15.4 \pm 20.6$ & 0.12 & NA \\
\hline \multicolumn{9}{|l|}{ PANSS score } \\
\hline Total score & $100.2 \pm 18.5$ & $91.1 \pm 15.1$ & 0.06 & NA & $85.2 \pm 16.7$ & $85.0 \pm 15.1$ & 0.52 & NA \\
\hline Positive score & $27.5 \pm 7.8$ & $21.2 \pm 5.3$ & 0.33 & NA & $23.5 \pm 5.3$ & $20.8 \pm 5.8$ & 0.80 & NA \\
\hline Negative score & $25.5 \pm 7.1$ & $21.2 \pm 7.8$ & 0.15 & NA & $17.1 \pm 7.0$ & $20.9 \pm 7.7$ & 0.44 & NA \\
\hline General psychopathology score & $47.2 \pm 9.5$ & $48.7 \pm 8.6$ & 0.65 & NA & $43.6 \pm 8.8$ & $43.3 \pm 8.3$ & 0.51 & NA \\
\hline
\end{tabular}

HCs, healthy controls; PANSS, Positive and Negative Syndrome Scale.

FEAT. Preprocessed functional data consisted of 240 time points, and due to a decrease in the magnetic field effect the first 10 volumes were removed. Preprocessing included realignment of echo-planar images to remove the effects of motion (using Motion Correction FMRIB's linear image registration tool, MCFLIRT), brain extraction (using Brain Extraction Tool, BET), spatial smoothing with a $6 \mathrm{~mm}$ full width at half maximum Gaussian kernel, and high-pass temporal filtering by using a cutoff frequency of $150 \mathrm{sec}(\sim 0.007 \mathrm{~Hz})$. The Motion Correction FMRIB's non-linear image registration tool (FNIRT) was used to register functional MR imaging volumes into standard montreal neurological institute (MNI) space templates (step1: individual functional space to individual structural space; step2: individual structural space to MNI standard space), and post-registration images were re-sampled to $2 \mathrm{~mm}$ for further analysis (Cui et al., 2017b, 2018). Furthermore, the factors of sex and age of all subjects were regressed using the FSL toolbox. In order to avoid the impact of the two different datasets in imaging, we compared the data preprocessing from the two datasets and did not find any significant differences (Supplementary Figure 1 and Supplementary Table 2).

\section{mICA: Based on Hippocampus}

Further analysis after preprocessing was implemented in mICA tools (see footnote 2) for both patients and healthy controls. Based on Blessing et al.'s (2016) research, left and right hippocampi in the Harvard-Oxford subcortical structural atlas were selected as the seed ROIs, and dimensionality of 10 was selected as the number of components (Figure 2; Blessing et al., 
2016; Moher Alsady et al., 2016). In order to verify the stability of the ingredients, the 10 independent components (ICs) were identified in all subjects of two data sets separately (GE data set and Siemens data set) as suggested by Blessing et al. (2016) and then we compared the spatial correlation of each of the 10 ICs (Supplementary Figure 1 and Supplementary Table 2). The 10 hippocampus components generated by the combined data set were selected as the ICs for further analysis.

\section{Functional Connectivity Analyses Based on 10 Hippocampus Components}

Multivariate functional connectivity between 10 components and the whole brain was assessed via a modified dual regression approach. Data analysis was implemented in FSL tools (see footnote 1) according to the method of Filippini et al. (2009).
Briefly, in the first step of dual regression, the concatenated multiple functional MR imaging data sets were decomposed by using ICA, in which 10 hippocampus components were applied to detect large-scale patterns of functional connectivity in subjects. Second, the dual-regression approach was used to identify subject-specific temporal dynamics and associated spatial maps within each subject's functional MR imaging data set. This involved using the spatial maps of the group ICA in a linear model fit (spatial regression) against the separate functional MR imaging data sets, resulting in matrices that describe the temporal dynamics of each component and subject. Then, subject-specific spatial maps were estimated by using these time-course matrices in a linear model fit (temporal regression) against the associated functional MR imaging data sets. Third, the different component maps were collected across subjects

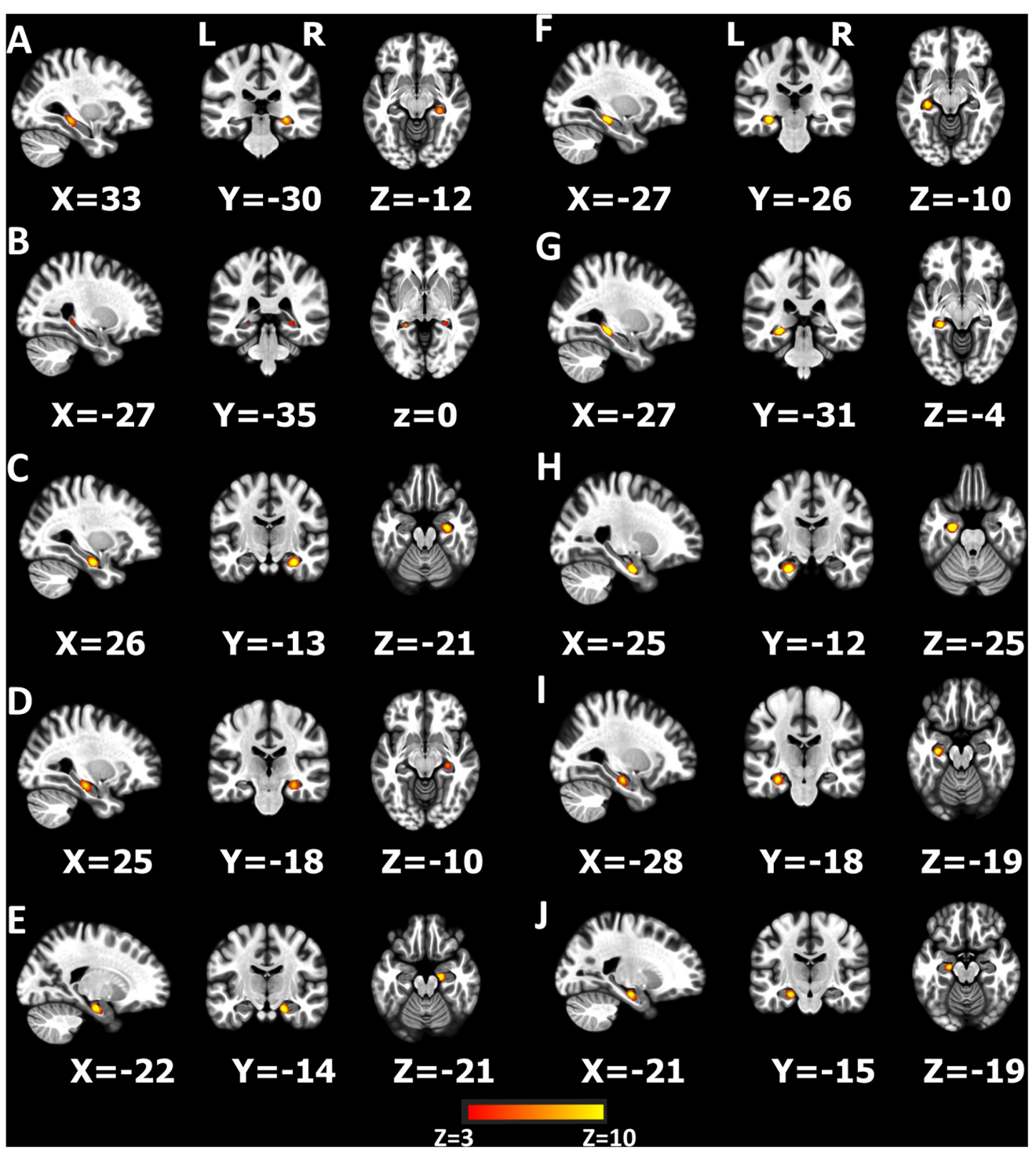

FIGURE 2 | Ten components produced by independent component analysis (ICA) restricted within the hippocampus (A-J). The $z$-scores $>3$ are colored in the figure. 
into single four-dimensional files (one per original ICA map, with the fourth dimension being subject identification) and them tested voxel-wise for statistically significant differences between groups by using non-parametric permutation testing (5000 permutations). The maps were thresholded by using an alternative hypothesis test based on a mixed Gaussian and gamma distribution model in previous work and by controlling the local false discovery rate at $P$ less than 0.05 (Cui et al., 2017b).

\section{ROI-Based Functional Connectivity of the Hippocampus}

After preprocessing, ROI-based correlational analyses were performed using the Functional Connectivity (CONN) toolbox ${ }^{3}$ between the hippocampus and whole brain ROIs. Briefly, (1) after preprocessing, the confounding effects of white matter, CSF, realignment and scrubbing were removed using linear regression. Then, (2) linear detrending. (3) first-level analysis, left- and right- hippocampi were selected as the seed ROIs to analyze the functional connectivity between the seed ROIs and all brain ROIs. (4) second-level analysis. Any differences between seed

${ }^{3} \mathrm{http}: / /$ web.mit.edu/swg/software.htm
ROIs and all brain ROIs for the group comparison among AVHs, N-AVHs, and HCs using a general linear model (GLM) and differences between AVHs and Non-AVHs were calculated (Cui et al., 2018).

\section{Statistical Analysis}

The difference scores (removed the value $>\mid$ Mean $\pm 3 \mathrm{SD} \mid$ ) of significant different regions (difference scores, dscore $=$ $\left|\sum_{R=1}^{\mathrm{k}} \sum_{\mathrm{i}=1}^{\mathrm{n}} S_{\mathrm{i}, \mathrm{R}}\right|, R$, the difference regions of each hippocampus component; $k$, the number of regions; $S$, the scores of difference regions; and $i$, the number of voxels of each significant different region) were calculated for AVHs and Non-AVHs patients. The correlation between PANSS scores and difference scores was calculated for patients.

\section{RESULTS}

\section{Clinical Characteristics}

Table 1 shows the full description of demographic and clinical characteristics of patients and healthy controls. No significant difference was found in age, gender, education, and handedness among the patient groups.

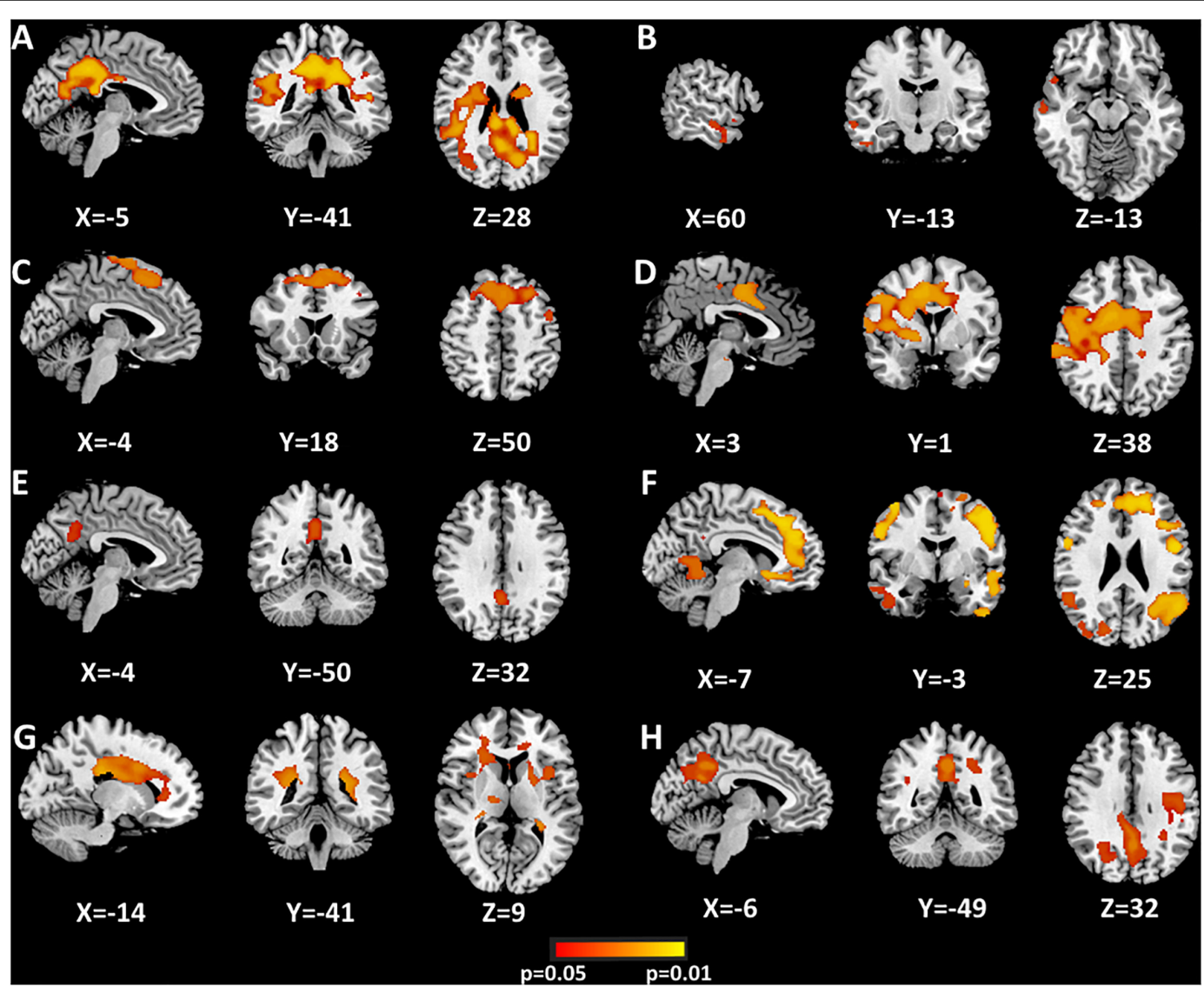

FIGURE 3 | Significant differences of hippocampus components between AVHs and Non-AVHs using dual regression (A-H). Correction via Bonferroni and the areas where $P<0.05$ are shown in the figure. The color bar indicates the $P$-value. 


\section{mICA Findings-Based Connectivity}

Figure 3, Table 2, and Supplementary Table 3 summarize the regions where hippocampal components-seeded connections were altered between AVHs and Non-AVHs. Supplementary Figure 2 and Table 3 show the mean connection strength in each group. In contrast to patients without AVHs, patients with AVHs had increased connection strength in the left middle part and decreased connection strength in the bilateral antero-lateral and right antero-medial parts.

\section{ROI-Based Connectivity}

The bilateral hippocampi were utilized as seeds for the ROI-based connectivity analysis. A few regions had altered functional connectivity with the seed areas (Figure 4 and Supplementary Table 4). Altered connectivity was seen between the right hippocampus and bilateral superior temporal gyrus among the three groups. No significant difference was found between patients with and without AVHs.

\section{Clinical Correlates}

Positive correlation was found between difference scores of functional connectivity and PANSS total scores in patients with AVHs $(r=0.67, P<0.001)$, suggesting a significant effect of AVHs on clinical severity (Figure 5). There was no clear association between connectivity metrics and PANSS total scores in patients without AVHs $(r=0.18, P=0.08)$. We also added supplementary

TABLE 2 | The differences areas of mICA.

\begin{tabular}{lccccc}
\hline Position & $\begin{array}{c}\text { Number of } \\
\text { voxels }\end{array}$ & \multicolumn{2}{c}{ Peak MNI coordinate } & $\begin{array}{c}\text { Peak } \\
\text { intensity }\end{array}$ \\
\cline { 3 - 5 } & & $\mathbf{x}$ & $\mathbf{y}$ & $\mathbf{z}$ & \\
\hline Anterior.L & 6578 & -26 & -54 & 28 & 0.99 \\
Anterior.R & 362 & 52 & -14 & -32 & 0.966 \\
Antero-medial.L & 5751 & 0 & 18 & 50 & 0.976 \\
Antero-medial.R & 8944 & 40 & -18 & -18 & 0.986 \\
Antero-lateral.L & 593 & 4 & -58 & 24 & 0.966 \\
Antero-lateral.R & 32601 & -40 & 32 & -20 & 0.992 \\
Middle.L & 2743 & -16 & -26 & 26 & 0.984 \\
Middle.R & 3512 & 2 & -56 & 24 & 0.974
\end{tabular}

analysis to calculate the correlation between PANSS positive, negative, general psychopathology, and supplemental scores and neuroimaging measures (Supplementary Table 5).

\section{DISCUSSION}

Identifying the disrupted hippocampal structure and function in schizophrenia at a sub-large-scale level has a critical role in fundamental research. In this study, combining neuroimaging and mICA analysis, we reported and presented a full view of hippocampal regional connectivity pattern for AVHs in schizophrenia, including bilateral anterior, antero-medial, antero-lateral, middle, and posterior parts.

The present study extends the previous findings (Sommer et al., 2012; Clos et al., 2014; Li et al., 2017) by providing a hippocampal sub-region-level pattern of AVHs-specific disrupted connectivity in schizophrenia via mICA. The unique advantage of mICA is separating the hippocampus into several subregions for the connectivity analysis. We explored the subregional aspects of hippocampal connectivity in schizophrenia patients with AVHs, which allows and ensures displaying dysfunctional brain regions involving AVHs in schizophrenia. We found patients with AVHs with increased connection strength in the left middle part and decreased connection strength in the bilateral antero-lateral and right antero-medial parts compared with patients without AVHs, indicating disrupted sensory representation or memory underlying hallucinations in this disorder.

In our study, another result is that patients in this cohort showed right hippocampus-seeded dysconnectivity with bilateral superior temporal gyri, which is in line with a previous report (Sommer et al., 2012). With the exception of the superior temporal gyrus, which proved to be a reasonable AVHs-related brain area (Orlov et al., 2018; Spray et al., 2018), we uncovered a much more complicated network for AVHs in schizophrenia by means of mICA. This finding is suggestive of aberrant patterns of multiple brain areas in schizophrenia patients suffering from AVHs, and has made an unexpected discovery of AVH proneness. ROI-based connectivity analysis of the whole hippocampus could

TABLE 3 | Mean connection strength for each group.

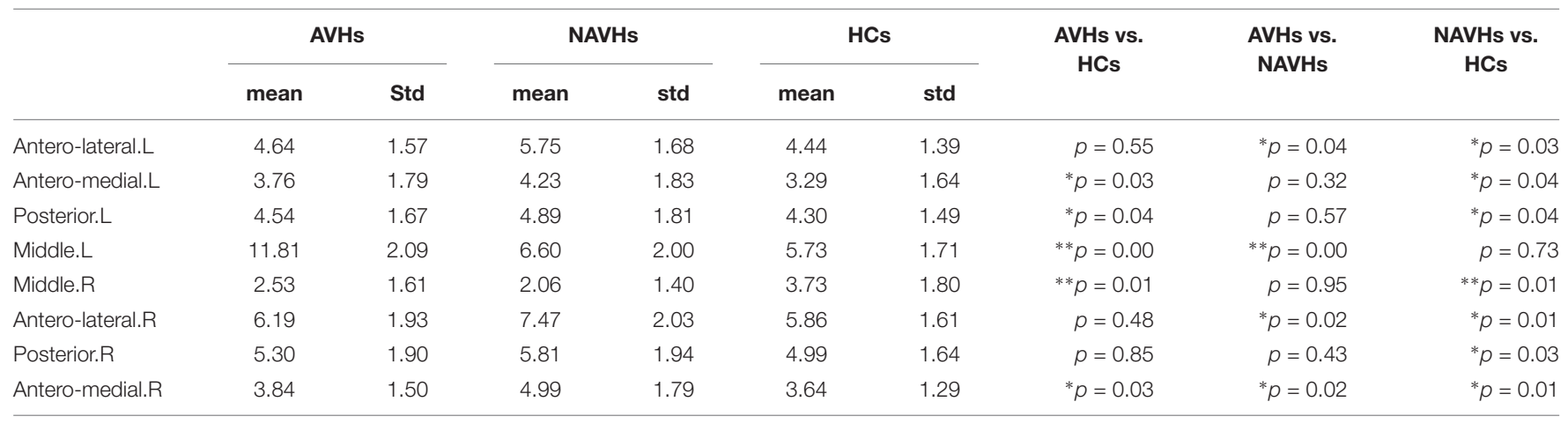

${ }^{*} p<0.05 ; * * p<0.01$. 


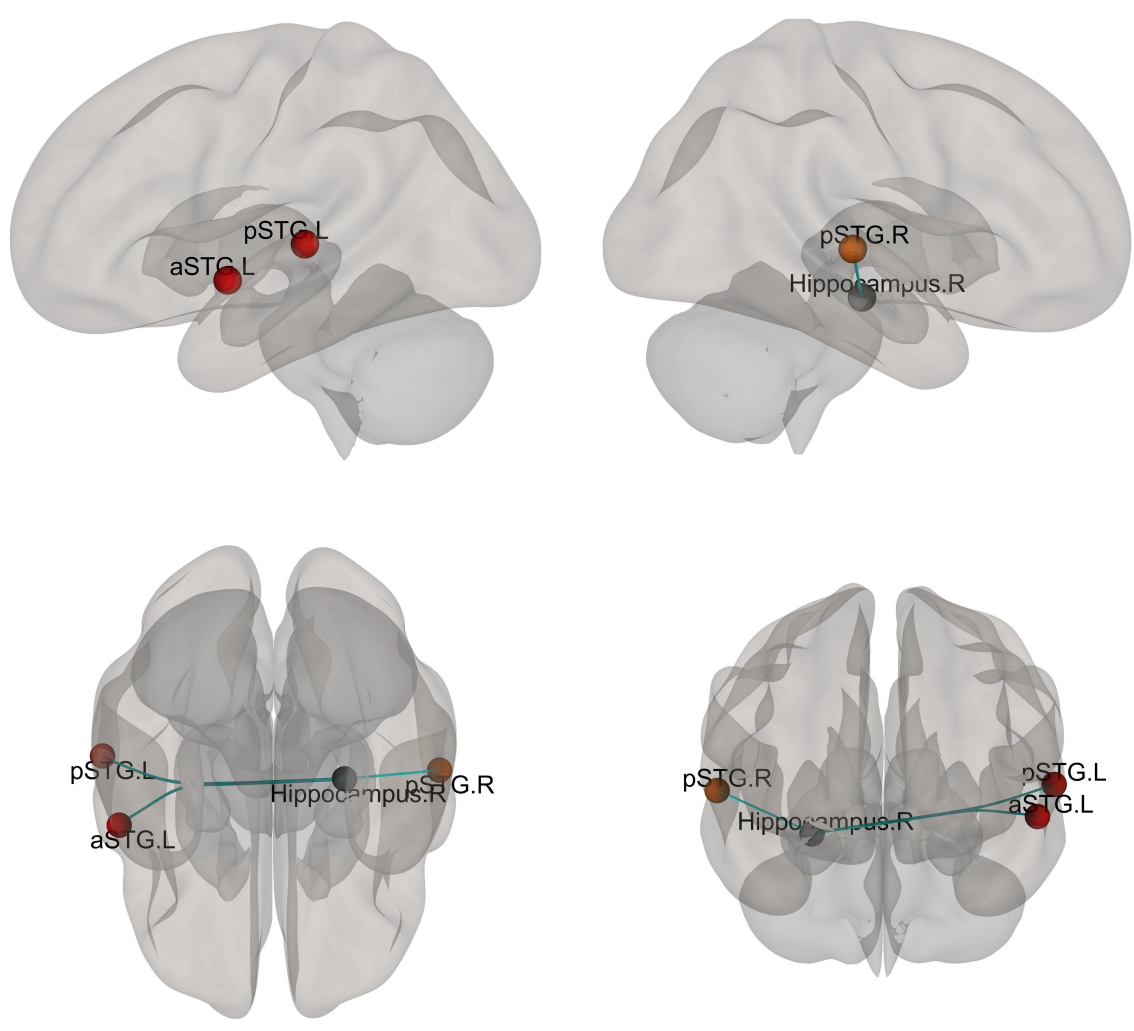

FIGURE 4 | Different functional connectivity between whole hippocampus and ROls of the brain mask. F-tests were calculated and the functional connectivity with a significant difference after FDR correction is shown. The color of the ball indicated the relationship between mask ROI (Hippocampus) and the other ROls (Gray ball: the mask ROI, the warm color indicated that the connection increased).

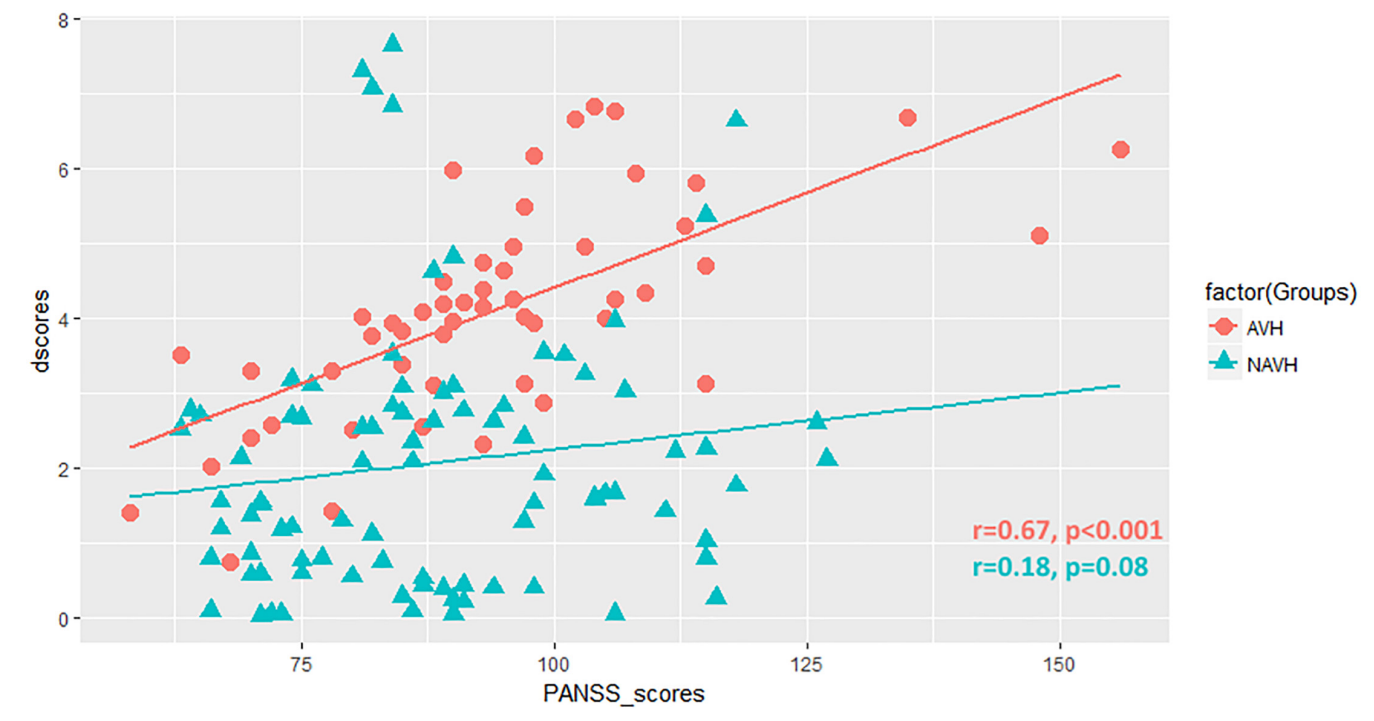

FIGURE 5 | Scatter plot showing the correlation between difference scores (dscores) and PANSS total scores (PANSS_scores) in patients.

be an impediment to detecting some subtle alterations for functional neuroimaging. On the contrary, mICA might be an alternative approach to explore much more changes missed by ROI-based analysis.
Affected memory to some extent is known to exist in cognitive symptoms of schizophrenia (Ricarte et al., 2017). The implications of hallucinatory experience as aberrant event memory formation for the pathophysiology of schizophrenia 
have been established (Behrendt, 2016). A sizable number of studies have implicated hippocampal association with AVHs in schizophrenia. A recently proposed model for AVHs in schizophrenia is that "the aberrant neuromodulation within RSNs involving sensory information filtering, auditory processing/language processing, and memory retrieving might implicate a contribution to this distressing symptom" [see Figure $\mathbf{5}$ in Cui et al. (2017b) for review]. While it remains to be determined, neural mechanisms underlying disrupted connectivity of hippocampal sub-regions, the evidence from this study could support such a model involving memory retrieval.

Several aspects of the current study deserve further comments. With the mICA Toolbox, ICA dimensionality was set to a value of 10 for the hippocampus in our study, but this value was 12 in a previous study (Moher Alsady et al., 2016). The number of components varies from data to data in the ICA decomposition. In addition to 10 hippocampal components, we performed 8, 12, and 15 components, revealing consistent results (for details see Supplementary Materials). The neurobiological underpinnings behind findings of this study are currently not known. Schizophrenia-patient-derivedCA3 neurons from human pluripotent stem cells have been efficiently generated and enable modeling of deficit hippocampal connectivity in vitro (Sarkar et al., 2018). What should be done in the future includes linking the network of hippocampal sub-regions in relation to AVHs in schizophrenia using fMRI and cell physiology.

In summary, our study shows that schizophrenia patients with AVHs exhibit disrupted connectivity of hippocampal sub-regions, which represents a core symptom-specific pathophysiology of schizophrenia. Mapping out the sub-regions with dysconnectivity in the hippocampus associated with AVHs in schizophrenia may help elucidate neurobiological substrates of this disorder.

\section{ETHICS STATEMENT}

This study was carried out in accordance with the recommendations of the Institutional Ethics Committee, First Hospital of the Fourth Military Medical University with written informed consent from all subjects. All subjects gave written informed consent in accordance with the Declaration of Helsinki. The protocol was approved by the Institutional

\section{REFERENCES}

Alderson-Day, B., Diederen, K., Fernyhough, C., Ford, J. M., Horga, G., Margulies, D. S., et al. (2016). Auditory hallucinations and the brain's resting-state networks: findings and methodological observations. Schizophr. Bull. 42, 1110-1123. doi: 10.1093/schbul/sbw078

Amad, A., Cachia, A., Gorwood, P., Pins, D., Delmaire, C., Rolland, B., et al. (2014). The multimodal connectivity of the hippocampal complex in auditory and visual hallucinations. Mol. Psychiatry 19, 184-191. doi: 10.1038/mp.2012.181

Behrendt, R. P. (2010). Contribution of hippocampal region CA3 to consciousness and schizophrenic hallucinations. Neurosci. Biobehav. Rev. 34, 1121-1136. doi: 10.1016/j.neubiorev.2009.12.009
Ethics Committee, First Hospital of the Fourth Military Medical University.

\section{AUTHOR CONTRIBUTIONS}

Y-BX, HY, and WQ were responsible for the entire study. All authors contributed to the study concepts and study design, data acquisition, data analysis and interpretation, manuscript drafting, manuscript revision for important intellectual content, approval of the final version of the manuscript, and agreed to ensure that any questions related to the work are appropriately resolved. L-BC, X-RW, Y-BX, and HY contributed to the literature research. LL, L-BC, X-RW, H-NW, and HY performed the clinical studies. L-BC, Y-BX, X-RW, and HY performed the experimental studies. LL, L-BC, Y-CL, Z-LX, HY, and WQ contributed to the statistical analysis. LL, L-BC, HY, and WQ were responsible for the manuscript revision.

\section{FUNDING}

This study was supported by the NSFC under Grant 81671651 and Key Research and Development Program of Shaanxi Province Grant 2017ZDXM-SF-048 to HY, the NSFC grant 81801675 and Wisdom in Creation to L-BC, the National Basic Research Program of China Grants 2014CB543203 and 2015CB856403, the Science and Technology Projects of Xi'an, China under Grant 201809170CX11JC12, and the NSFC under Grants 81771918,81471811 , and 81471738 to WQ.

\section{ACKNOWLEDGMENTS}

The authors acknowledge their patients and patients' family, and thank Prof. Florian Beissner from Somatosensory and Autonomic Therapy Research, Institute for Neuroradiology, Hannover Medical School, Germany and Dr. Karen M. von Deneen from Xidian University for their kind help.

\section{SUPPLEMENTARY MATERIAL}

The Supplementary Material for this article can be found online at: https://www.frontiersin.org/articles/10.3389/fnins. 2019.00424/full\#supplementary-material

Behrendt, R. P. (2016). Hallucinatory experience as aberrant event memory formation: implications for the pathophysiology of schizophrenia. Prog. Neuropsychopharmacol. Biol. Psychiatry 71, 203-209. doi: 10.1016/j.pnpbp. 2016.07.009

Blessing, E. M., Beissner, F., Schumann, A., Brunner, F., and Bar, K. J. (2016). A data-driven approach to mapping cortical and subcortical intrinsic functional connectivity along the longitudinal hippocampal axis. Hum. Brain Mapp. 37, 462-476. doi: 10.1002/hbm.23042

Chang, X., Xi, Y. B., Cui, L. B., Wang, H. N., Sun, J. B., Zhu, Y. Q., et al. (2015). Distinct inter-hemispheric dysconnectivity in schizophrenia patients with and without auditory verbal hallucinations. Sci. Rep. 5:11218. doi: 10.1038/ srep 11218 
Clos, M., Diederen, K. M., Meijering, A. L., Sommer, I. E., and Eickhoff, S. B. (2014). Aberrant connectivity of areas for decoding degraded speech in patients with auditory verbal hallucinations. Brain Struct. Funct. 219, 581-594. doi: 10.1007/s00429-013-0519-5

Cui, L. B., Cai, M., Wang, X. R., Zhu, Y. Q., Wang, L. X., Xi, Y. B., et al. (2019). Prediction of early response to overall treatment for schizophrenia: a functional magnetic resonance imaging study. Brain Behav. 9:e01211. doi: 10.1002/brb3. 1211

Cui, L. B., Chen, G., Xu, Z. L., Liu, L., Wang, H. N., Guo, L., et al. (2017a). Cerebral blood flow and its connectivity features of auditory verbal hallucinations in schizophrenia: a perfusion study. Psychiatry Res. 260, 53-61. doi: 10.1016/j. pscychresns.2016.12.006

Cui, L. B., Liu, L., Guo, F., Chen, Y. C., Chen, G., Xi, M., et al. (2017b). Disturbed brain activity in resting-state networks of patients with firstepisode schizophrenia with auditory verbal hallucinations: a cross-sectional functional mr imaging study. Radiology 283, 810-819. doi: 10.1148/radiol.20161 60938

Cui, L. B., Wang, L. X., Tian, P., Wang, H. N., Cai, M., Guo, F., et al. $(2017 \mathrm{c})$. Aberrant perfusion and its connectivity within default mode network of first-episode drug-naive schizophrenia patients and their unaffected first-degree relatives. Sci. Rep. 7:16201. doi: 10.1038/s41598-017-14 343-7

Cui, L. B., Liu, K., Li, C., Wang, L. X., Guo, F., Tian, P., et al. (2016). Putamenrelated regional and network functional deficits in first-episode schizophrenia with auditory verbal hallucinations. Schizophr. Res. 173, 13-22. doi: 10.1016/j. schres.2016.02.039

Cui, L. B., Liu, L., Wang, H. N., Wang, L. X., Guo, F., Xi, Y. B., et al. (2018). Disease definition for schizophrenia by functional connectivity using radiomics strategy. Schizophr. Bull. 44, 1053-1059. doi: 10.1093/schbul/sby007

Dalton, M. A., Zeidman, P., Barry, D. N., Williams, E., and Maguire, E. A. (2017). Segmenting subregions of the human hippocampus on structural magnetic resonance image scans: an illustrated tutorial. Brain Neurosci. Adv. 1:2398212817701448. doi: 10.1177/2398212817701448

De Simoni, S., Jenkins, P. O., Bourke, N. J., Fleminger, J. J., Hellyer, P. J., Jolly, A. E., et al. (2018). Altered caudate connectivity is associated with executive dysfunction after traumatic brain injury. Brain 141, 148-164. doi: 10.1093/ brain/awx309

Filippini, N., MacIntosh, B. J., Hough, M. G., Goodwin, G. M., Frisoni, G. B., Smith, S. M., et al. (2009). Distinct patterns of brain activity in young carriers of the APOE-epsilon4 allele. Proc. Natl. Acad. Sci U.S.A. 106, 7209-7214. doi: 10.1073/pnas.0811879106

Fogwe, L. A., and Mesfin, F. B. (2018). Neuroanatomy, Hippocampus. Treasure Island, FL: StatPearls Publishing.

Ford, J. M., Palzes, V. A., Roach, B. J., Potkin, S. G., van Erp, T. G., Turner, J. A., et al. (2015). Visual hallucinations are associated with hyperconnectivity between the amygdala and visual cortex in people with a diagnosis of schizophrenia. Schizophr. Bull. 41, 223-232. doi: 10.1093/schbul/sbu031

Hare, S. M., Ford, J. M., Ahmadi, A., Damaraju, E., Belger, A., Bustillo, J., et al. (2017). Modality-dependent impact of hallucinations on low-frequency fluctuations in schizophrenia. Schizophr. Bull. 43, 389-396. doi: 10.1093/schbul/ sbw093

Hare, S. M., Law, A. S., Ford, J. M., Mathalon, D. H., Ahmadi, A., Damaraju, E., et al. (2018). Disrupted network cross talk, hippocampal dysfunction and hallucinations in schizophrenia. Schizophr. Res. 199, 226-234. doi: 10.1016/j. schres.2018.03.004
Jardri, R., Pouchet, A., Pins, D., and Thomas, P. (2011). Cortical activations during auditory verbal hallucinations in schizophrenia: a coordinate-based metaanalysis. Am. J. Psychiatry 168, 73-81. doi: 10.1176/appi.ajp.2010.09101522

Lefebvre, S., Demeulemeester, M., Leroy, A., Delmaire, C., Lopes, R., Pins, D., et al. (2016). Network dynamics during the different stages of hallucinations in schizophrenia. Hum. Brain Mapp. 37, 2571-2586. doi: 10.1002/hbm.23197

Li, B., Cui, L. B., Xi, Y. B., Friston, K. J., Guo, F., Wang, H. N., et al. (2017). Abnormal effective connectivity in the brain is involved in auditory verbal hallucinations in schizophrenia. Neurosci. Bull. 33, 281-291. doi: 10.1007/ s12264-017-0101-x

Maller, J. J., Daskalakis, Z. J., Thomson, R. H., Daigle, M., Barr, M. S., and Fitzgerald, P. B. (2012). Hippocampal volumetrics in treatment-resistant depression and schizophrenia: the devil's in de-tail. Hippocampus 22, 9-16. doi: 10.1002/hipo.20873

Modinos, G., Vercammen, A., Mechelli, A., Knegtering, H., McGuire, P. K., and Aleman, A. (2009). Structural covariance in the hallucinating brain: a voxel-based morphometry study. J. Psychiatry Neurosci. 34, 465-469.

Moher Alsady, T., Blessing, E. M., and Beissner, F. (2016). MICA-A toolbox for masked independent component analysis of fMRI data. Hum. Brain Mapp. 37, 3544-3556. doi: 10.1002/hbm.23258

Orlov, N. D., Giampietro, V., O’Daly, O., Lam, S. L., Barker, G. J., Rubia, K., et al. (2018). Real-time fMRI neurofeedback to down-regulate superior temporal gyrus activity in patients with schizophrenia and auditory hallucinations: a proof-of-concept study. Transl. Psychiatry 8:46. doi: 10.1038/s41398-0170067-5

Ricarte, J. J., Ros, L., Latorre, J. M., and Watkins, E. (2017). Mapping autobiographical memory in schizophrenia: clinical implications. Clin. Psychol. Rev. 51, 96-108. doi: 10.1016/j.cpr.2016.11.004

Sarkar, A., Mei, A., Paquola, A. C. M., Stern, S., Bardy, C., Klug, J. R., et al. (2018). Efficient generation of CA3 neurons from human pluripotent stem cells enables modeling of hippocampal connectivity in vitro. Cell Stem Cell 22, 684-697.e9. doi: 10.1016/j.stem.2018.04.009

Sommer, I. E., Clos, M., Meijering, A. L., Diederen, K. M., and Eickhoff, S. B. (2012). Resting state functional connectivity in patients with chronic hallucinations. PLoS One 7:e43516. doi: 10.1371/journal.pone.0043516

Spray, A., Beer, A. L., Bentall, R. P., Sluming, V., and Meyer, G. (2018). Microstructure of the superior temporal gyrus and hallucination proneness - a multi-compartment diffusion imaging study. Neuroimage Clin. 20, 1-6. doi: 10.1016/j.nicl.2018.06.027

Wisse, L. E. M., Daugherty, A. M., Olsen, R. K., Berron, D., Carr, V. A., Stark, C. E. L., et al. (2017). A harmonized segmentation protocol for hippocampal and parahippocampal subregions: why do we need one and what are the key goals? Hippocampus 27, 3-11. doi: 10.1002/hipo.22671

Conflict of Interest Statement: The authors declare that the research was conducted in the absence of any commercial or financial relationships that could be construed as a potential conflict of interest.

Copyright (c) $2019 \mathrm{Liu}, \mathrm{Cui}, \mathrm{Xi}$, Wang, Liu, Xu, Wang, Yin and Qin. This is an open-access article distributed under the terms of the Creative Commons Attribution License (CC BY). The use, distribution or reproduction in other forums is permitted, provided the original author(s) and the copyright owner(s) are credited and that the original publication in this journal is cited, in accordance with accepted academic practice. No use, distribution or reproduction is permitted which does not comply with these terms. 
OPEN ACCESS

Edited by:

Filippo Cieri,

Cleveland Clinic, United States

Reviewed by:

Roser Sala-Llonch,

University of Barcelona, Spain

Paola Valsasina,

San Raffaele Scientific Institute

(IRCCS), Italy

*Correspondence:

Zhongxiang Ding

hangzhoudzx73@126.com

Specialty section:

This article was submitted to

Brain Imaging Methods,

a section of the journal

Frontiers in Neuroscience

Received: 29 January 2019 Accepted: 15 April 2019

Published: 07 May 2019

Citation:

Feng $Q$, Wang $M$, Song $Q, W u Z$, Jiang $H$, Pang P, Liao Z, Yu E and

Ding Z (2019) Correlation Between

Hippocampus MRI Radiomic Features and Resting-State Intrahippocampal

Functional Connectivity in Alzheimer's

Disease. Front. Neurosci. 13:435.

doi: 10.3389/fnins.2019.00435

\section{Correlation Between Hippocampus MRI Radiomic Features and Resting-State Intrahippocampal Functional Connectivity in Alzheimer's Disease}

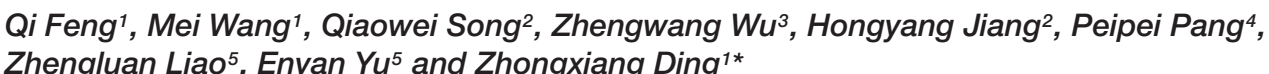

1 Department of Radiology, Affiliated Hangzhou First People's Hospital, Zhejiang University School of Medicine, Hangzhou, China, ${ }^{2}$ Department of Radiology, Zhejiang Provincial People's Hospital, Hangzhou Medical College, Hangzhou, China, ${ }^{3}$ Department of Radiology and BRIC, The University of North Carolina at Chapel Hill, Chapel Hill, NC, United States, ${ }^{4}$ GE Healthcare Life Sciences, Hangzhou, China, ${ }^{5}$ Department of Psychiatry, Zhejiang Provincial People's Hospital, Hangzhou Medical College, Hangzhou, China

Alzheimer's disease (AD) is a neurodegenerative disease with main symptoms of chronic primary memory loss and cognitive impairment. The study aim was to investigate the correlation between intrahippocampal functional connectivity (FC) and MRI radiomic features in AD. A total of $67 \mathrm{AD}$ patients and 44 normal controls (NCs) were enrolled in this study. Using the seed-based method of resting-state functional MRI (rs-fMRI), the whole-brain FC with bilateral hippocampus as seed was performed, and the FC values were extracted from the bilateral hippocampus. We observed that $A D$ patients demonstrated disruptive FC in some brain regions in the left hippocampal functional network, including right gyrus rectus, right anterior cingulate and paracingulate gyri, bilateral precuneus, bilateral angular gyrus, and bilateral middle occipital gyrus. In addition, decreased FC was detected in some brain regions in the right hippocampal functional network, including bilateral anterior cingulate and paracingulate gyri, right dorsolateral superior frontal gyrus, and right precentral gyrus. Bilateral hippocampal radiomics features were calculated and selected using the A.K. software. Finally, Pearson's correlation analyses were conducted between these selected features and the bilateral hippocampal FC values. The results suggested that two gray level runlength matrix (RLM) radiomic features and one gray level co-occurrence matrix (GLCM) radiomic feature weakly associated with $\mathrm{FC}$ values in the left hippocampus. However, there were no significant correlations between radiomic features and FC values in the right hippocampus. These findings present that the AD group showed abnormalities in the bilateral hippocampal functional network. This is a prospective study that revealed the weak correlation between the MRI radiomic features and the intrahippocampal FC in AD patients.

Keywords: Alzheimer's disease, resting-state functional magnetic resonance imaging, functional connectivity, hippocampus, radiomics 


\section{INTRODUCTION}

Alzheimer's disease $(\mathrm{AD})$ is a neurodegenerative disease with main symptoms of chronic primary memory loss and cognitive impairment. $\mathrm{AD}$ has become a public health problem due to its hidden onset, high incidence, and lack of effective drug treatment. The exact cause of AD is still unclear. It is believed some may be related to heredity, neurotransmitter changes, virus infection, immune dysfunction, and free radical damage. Nowadays, the clinical diagnosis of $\mathrm{AD}$ is based on cognitive measures and one or more biomarkers including structural MRI, PET, and cerebrospinal fluid analysis of amyloid $\beta$ or tau proteins (Bruno et al., 2007). Researchers are investigating non-invasive neuroimaging biomarkers for the early diagnosis of AD (Claudia et al., 2010; Yong et al., 2014).

Cell degeneration in the hippocampus plays an important role in the onset of $\mathrm{AD}$. It proved to be the cause of $\mathrm{AD}$ that amyloid- $\beta$ plaques and Tau proteins are selectively deposited in the special cortex of the hippocampus in AD patients (Ball, 1997; Guzman et al., 2013). These special cortexes are the main pathways that connect the hippocampus to the other cortexes of the brain. A large number of magnetic resonance imaging (MRI) studies have revealed atrophy and some other changes in microstructure in the hippocampus in $\mathrm{AD}$ dementia (De et al., 2015; Mak et al., 2017).

MRI is an important technique for radiomics. MR images can acquire numerous sequences and do not receive a radiation dose. MRI shows both the structural change and the functional dynamic change. Several MRI techniques have been applied in $\mathrm{AD}$ research, including voxel-based morphometry (Whitwell et al., 2007), diffusion tensor imaging (DTI) (Denise et al., 2004), resting-state functional MRI (rs-fMRI; Wang et al., 2011), as well as radiomics analysis (Qi et al., 2018). Radiomics is a new frontier subject based on quantitative imaging, feature calculation, feature selection, and model construction. It uses a large number of automated feature extraction algorithms to transform the original image data into first-order or higherorder data, and then analyzes the deep relationship between the data to further improve the accuracy of clinical diagnosis and prognostic value. Radiomics has strong power for radiotherapy, chemotherapy, and immunotherapy evaluation (Horvat et al., 2018; Sun et al., 2018; Wang et al., 2018), cancer patients survival prediction (Kickingereder et al., 2016), molecular subtyping of tumor (Lu et al., 2018), and cancer recurrence prediction (Li H. et al., 2017). Nowadays, radiomics is also applied to non-tumor diseases, such as attention deficit hyperactivity disorder (Port, 2018) and autism spectrum disorder (Chaddad et al., 2017a). In this context, we select the high-resolution T1-weighted MR images for hippocampal microstructural radiomics analysis.

Rs-fMRI is a functional MRI technique that based on blood oxygen level dependent (BOLD), and has emerged as one of the most important techniques for analysis of human brain function. Functional connectivity (FC) is the indirect reflection of synaptic connections. Rs-fMRI FC analysis is increasingly used to detect brain network changes in AD. There are two main methods used in rs-fMRI FC analysis. One is the "seed-based" approach, and the other is independent component analysis (ICA). In seed-based analysis, first, you need to set up a region of interest (ROI), then select BOLD signal fluctuations from the ROI, and associate them with BOLD signal fluctuations for all other voxels in the brain (Sheline and Raichle, 2013). The default mode network (DMN) is most commonly shown to be active when a person is not focused on the outside world and the brain is at wakeful rest, such as during daydreaming and mind-wandering. It is highly correlated with cognitive function (Menon, 2011). FC altered in DMN becomes a potential non-invasive biomarker in diagnosis of $\mathrm{AD}$ and amnestic mild cognitive impairment (aMCI) (Damoiseaux et al., 2012; Toussaint et al., 2014; Yu et al., 2016; Alderson et al., 2017). Functional activity in the hippocampus is the hot topic of $\mathrm{AD}$ research. One study found decreased synchrony of low-frequency fluctuations within the hippocampus in AD patients (Shi-Jiang et al., 2002). An ICA study of fMRI showed that the hippocampal activity diminished in $\mathrm{AD}$ (Wu et al., 2011). Intrinsic connectivity altered in hippocampal functional networks has been observed in aMCI patients (De et al., 2017). However, most of the rs-fMRI studies were focused on the $\mathrm{DMN}$ and some local brain regions; little is known regarding the hippocampus functional network.

Many studies investigated the altered structure or function in the hippocampus in AD patients. Nevertheless, little is known regarding the relationship between hippocampal microstructure and $\mathrm{FC}$ in $\mathrm{AD}$. The hypothesis of the present study is that the local structural change in hippocampus is correlated to the functional changes of the brain in AD patients. The aim of the study was to investigate the correlation between hippocampal MRI radiomic features and intrahippocampal FC in $\mathrm{AD}$, and to explore noninvasive imaging biomarkers for early diagnosis of $\mathrm{AD}$.

\section{MATERIALS AND METHODS}

\section{Study Cohort}

There were $82 \mathrm{AD}$ patients and 50 normal controls (NCs) recruited initially. AD patients were enrolled prospectively in the study at the Zhejiang Provincial People's Hospital from September 2016 to June 2018. The NCs were volunteers collected from the health promotion center of the hospital. All subjects were right-handed and provided written informed consent.

TABLE 1 | Demographics and cognitive characteristics of the participants.

\begin{tabular}{|c|c|c|c|c|}
\hline & AD patients & NC group & Statistic & $P$-value \\
\hline Sample size & 67 & 44 & NA & NA \\
\hline $\begin{array}{l}\text { Age (years, } \\
\text { mean } \pm S D \text { ) }\end{array}$ & $68.75 \pm 11.69$ & $65.48 \pm 9.69$ & 1.54 & 0.13 \\
\hline $\begin{array}{l}\text { Gender } \\
\text { (male/female) }\end{array}$ & $29: 38$ & $20: 24$ & $0.05^{*}$ & $0.82^{*}$ \\
\hline $\begin{array}{l}\text { Education } \\
\text { (years, } \\
\text { mean } \pm \mathrm{SD} \text { ) }\end{array}$ & $6.10 \pm 3.78$ & $7.11 \pm 3.36$ & -1.44 & 0.15 \\
\hline MMSE & $17.16 \pm 5.54$ & $29.09 \pm 0.77$ & -14.17 & $<0.01$ \\
\hline
\end{tabular}

$S D$, standard deviation; MMSE, Mini-Mental State Examination. AD, Alzheimer's disease; NC, normal control. Statistics were calculated with t-tests, unless otherwise indicated. * $x^{2}$-test was used. 


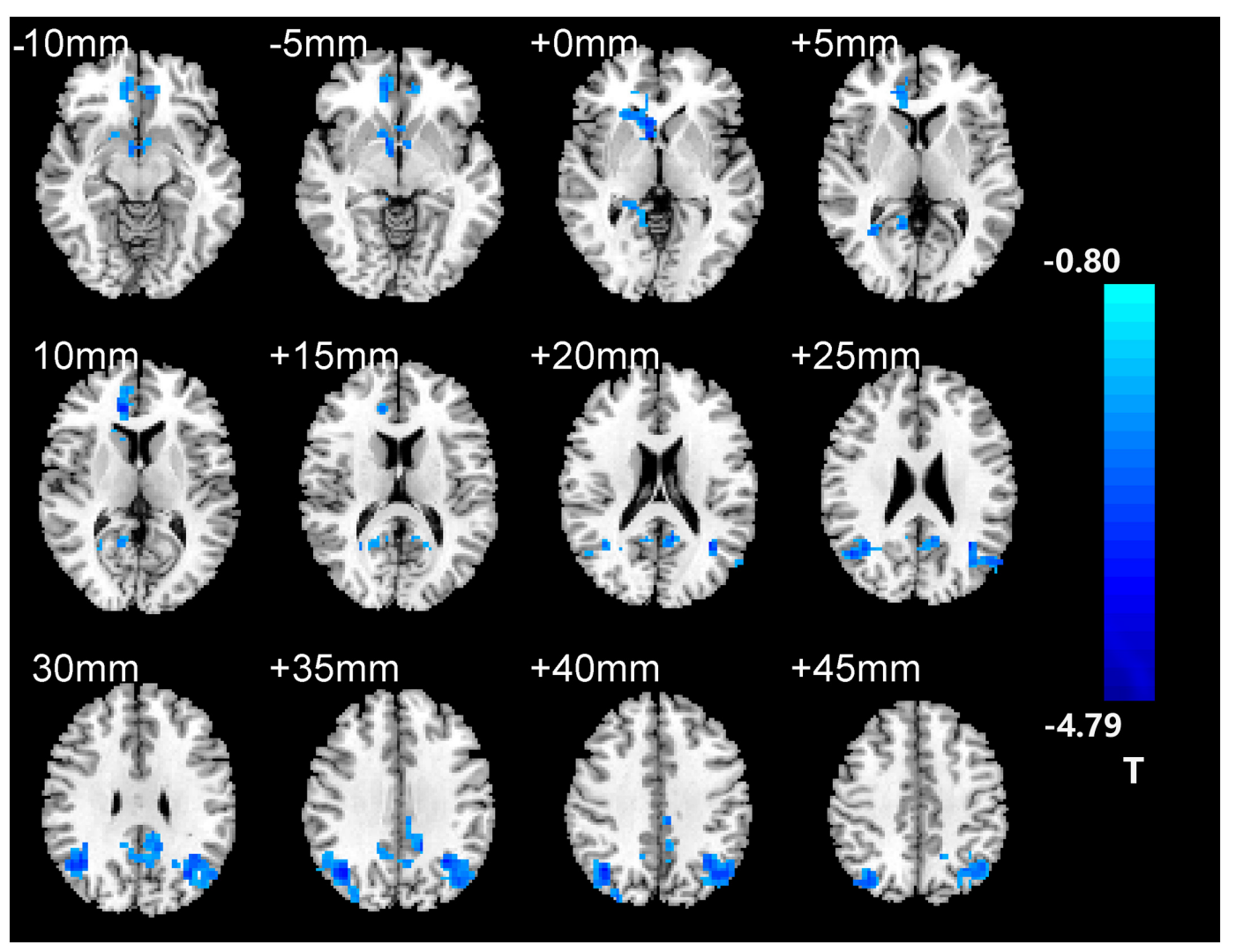

FIGURE 1 | The left hippocampus seed-based functional connectivity (FC) maps in the Alzheimer's disease (AD) patients compared with normal control (NC) subjects.

This study was approved by the Ethics Committee of Zhejiang Provincial People's Hospital (No. 2012KY002) and had been carried out in accordance with the Declaration of Helsinki.

All subjects underwent medical history collection, laboratory examination, neuropsychological test, physical examination, and conventional brain MRI scans. The neuropsychological tests included the Mini-Mental State Examination (MMSE) and Montreal Cognitive Scale (MoCA). AD patients were diagnosed in terms of the revised NINCDS-ADRDA (National Institute of Neurological and Communicative Disorders and Stroke and the Alzheimer's Disease and Related Disorders Association) criteria (Amp, 2011) with MMSE score $\leq 24$ and MoCA score $\leq 26$. The inclusion criteria for NC subjects were as follows: (1) no neurological deficits, such as hearing or vision loss; (2) no neurological or mental diseases, such as stroke, epilepsy, or depression; (3) no evidence of infarction, hemorrhage, or tumor on routine MRI; and (4) MMSE score $\geq 28$. For both AD and NC groups, the exclusion criteria were (1) stroke; (2) brain trauma; (3) brain tumors, Parkinson's disease, epilepsy, and other neurological diseases that cause memory disorders; (4) serious anemia, hypertension, diabetes, and other systemic diseases; (5) history of mental illness; and (6) signal abnormalities in the medial temporal lobe caused by infectious or vascular factors on MRI FLAIR or T2 images (Dubois et al., 2007). There were 73 AD patients and $45 \mathrm{NC}$ subjects who completed all the required MR sequences successfully.

TABLE 2 | Regions showing altered FC for AD patients and NC subjects with the left hippocampus as seed.

\begin{tabular}{|c|c|c|c|c|}
\hline Anatomical region & BA & MNI coordinates $(x, y, z)$ & Cluster size (voxels) & $T$-value \\
\hline REC.R, ACG.R, ORBmid.R, CAU.R & $11 / 10 / 32 / 25$ & $-6, \quad 39,-15$ & 344 & -3.458 \\
\hline PCUN.L, PCUN.R, DCG.L & $31 / 7$ & $-15,-48,33$ & 264 & -3.762 \\
\hline ANG.R, MOG.R & $39 / 19 / 7$ & $39,-66,39$ & 280 & -3.600 \\
\hline ANG.L, MOG.L, IPL.L & $39 / 40 / 19$ & $-33,-60,33$ & 337 & -3.492 \\
\hline
\end{tabular}

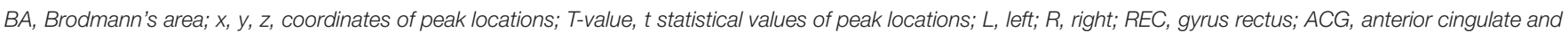

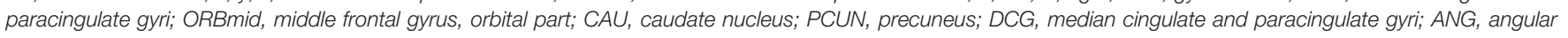
gyrus; MOG, middle occipital gyrus; IPL, inferior parietal but supramarginal and angular gyri. 


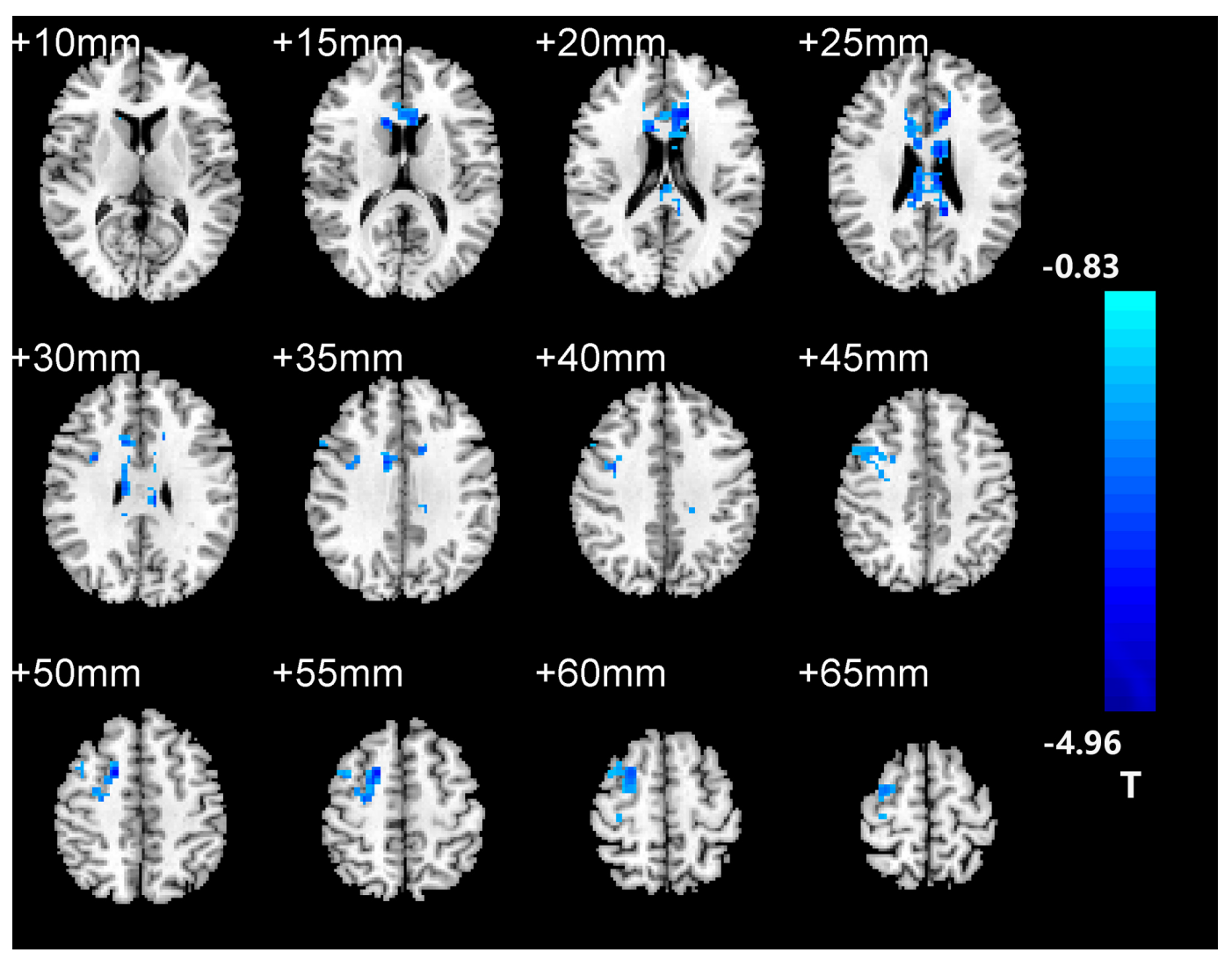

FIGURE 2 | The right hippocampus seed-based FC maps in the AD patients compared with NC subjects.

\section{MRI Acquisition}

Each subject underwent both structural and fMRI examinations on a 3.0-T MR scanner (Discovery MR750; GE Healthcare, Waukesha, WI, United States). The structural MRIs were acquired using a high-resolution three-dimensional T1-weighted magnetization-prepared rapid gradient echo (MPRAGE) sagittal sequence with scanning parameters of repetition time $(\mathrm{TR})=6.7 \mathrm{~ms}$, echo time $(\mathrm{TE})=2.9 \mathrm{~ms}$, inversion time $(\mathrm{TI})=450 \mathrm{~ms}$, slice thickness $/$ gap $=1 / 0 \mathrm{~mm}, \mathrm{FOV}=256 \times 256$ $\mathrm{mm}^{2}$, flip angle $=12^{\circ}$, matrix $=256 \times 256$; there were 192 sagittal slices collected from each subject. The rs-fMRI images were acquired using an echo-planar imaging (EPI) sequence with scanning parameters of $\mathrm{TR}=2,000 \mathrm{~ms}, \mathrm{TE}=30 \mathrm{~ms}$, slice thickness $/$ gap $=3.2 / 0 \mathrm{~mm}, \mathrm{FOV}=220 \times 220 \mathrm{~mm}^{2}$, and flip angle $=90^{\circ}$. Each rs-fMRI sequence contained 210 time points and each time point contained 44 slices. During rs-fMRI, all subjects were instructed to keep still and keep their eyes closed, but to not fall asleep.

\section{Preprocessing of Resting-State Functional MRI}

The image analysis of rs-fMRI after acquisition consists of preprocessing and FC analysis. The preprocessing was performed using the Data Processing Assistant for rs-fMRI (DPARSF ${ }^{1}$ ). The preprocessing steps were as follows:

(1) removing the first 10 time points (considering the time when the magnetic field of the machine was stable and the time when the subject adapted to the environment);

(2) slice timing correction and head motion correction;

\footnotetext{
${ }^{1}$ http://rfmri.org/DPARSF
}

TABLE 3 | Regions showing altered FC for AD patients and NC subjects with the right hippocampus as seed.

\begin{tabular}{|c|c|c|c|c|}
\hline Anatomical region & BA & MNI coordinates $(x, y, z)$ & Cluster size (voxels) & $T$-value \\
\hline ACG.L, ACG.R, DCG.R & $24 / 32 / 23$ & $-9,-21,27$ & 429 & -4.703 \\
\hline SFGdor.R, PreCG.R, MFG.R & $6 / 8$ & $18,6,51$ & 276 & -3.793 \\
\hline
\end{tabular}



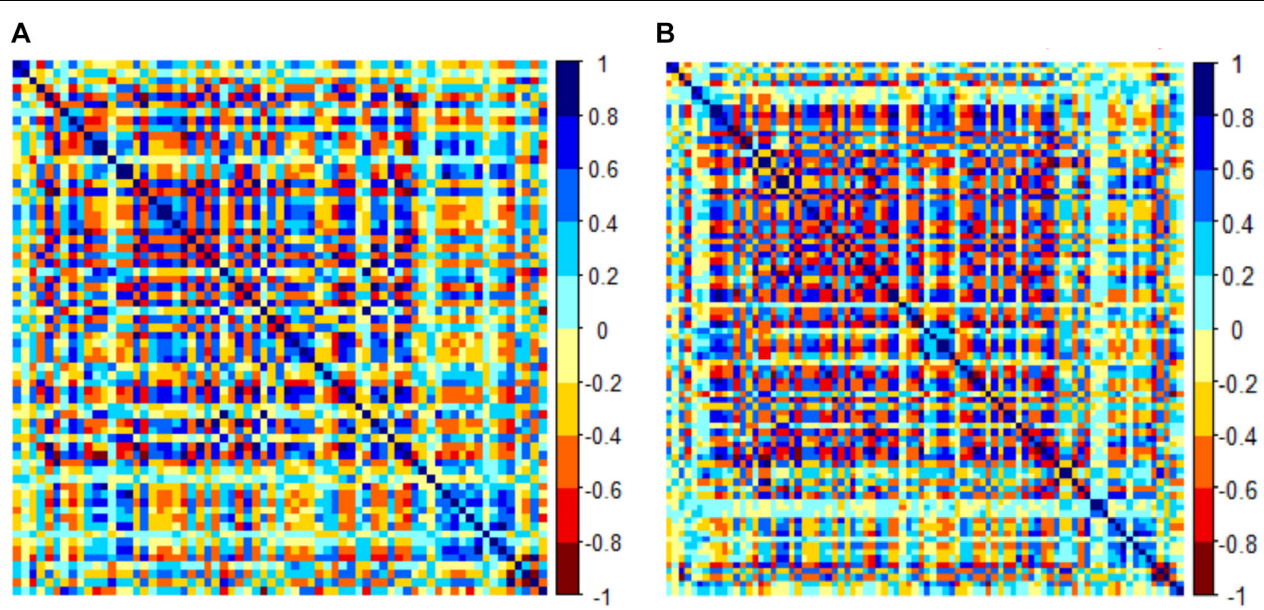

FIGURE 3 | Correlation analysis graph of the left hippocampus (A). Correlation analysis graph of the right hippocampus (B). Each grid represents the feature that enters the correlation analysis after the first step of dimensionality reduction.

(3) normalization to the Montreal Neurological Institute $(\mathrm{MNI})$ space and resampling (with voxels of $3 \times 3 \times 3 \mathrm{~mm}$ );

(4) spatial smoothing using a 4-mm isotropic Gaussian kernel; removing the linear trend; bandpass filtering (0.01$0.08 \mathrm{~Hz}$ ); and

(5) regression of covariates, including the six head motion parameters, the white matter, and cerebrospinal fluid signal.

\section{Segmentation of Hippocampus}

The 3D T1-weighted MPRAGE images were used in the hippocampus segmentation using an efficient learning-based deformable model (Wu et al., 2017). A joint classification and regression model was established to predict the location of the hippocampus. In the training stage, the extracted features were used to train the structured random forest classifier, and in the testing stage, the extracted features were input into the classifier in order to predict the segmentation of each hippocampus, and the prediction segmentation is iteratively improved through the training model. Finally, the hippocampal shape model gradually deformed to the target image to adapt the target hippocampus. We used this method to segment the right and left hippocampus, respectively. The segmentation results had been checked by a senior neuroradiologist, and the data with poor segmentation quality were resegmented.

\section{Resting-State Functional Connectivity Analysis}

The FC analysis of the bilateral hippocampus network was performed using REST plus $\mathrm{V} 1.2^{2}$. We took the averaged time courses of all voxels in ROI (above segmented left and right hippocampus as defined) as the reference sequence. And Pearson's correlation was conducted between the BOLD time course within the ROI and each voxel in the brain. Then, ROI-wise whole-brain FC maps were obtained. Fisher's

${ }^{2}$ http://restfmri.net/forum/RESTplusV1.2 $r$-to- $z$ transformation was used to convert FC maps into normalized zFC maps for subsequent statistical analyses. Onesample $t$-test was conducted in the $\mathrm{AD}$ and $\mathrm{NC}$ groups to make a mask using Gaussian random field (GFR) correction. Two-sample $t$-test was then performed using the above mask between the AD and NC groups. Then, GFR correction was performed (voxel-level $P<0.05$, cluster-level $P<0.05$, twotailed). Finally, the brain areas of significant differences were obtained. Furthermore, in order to quantify the FC values of the bilateral hippocampus in the brain network for next correlation analysis, the mean $Z$-value within the bilateral hippocampus was calculated using the above segmented left and right hippocampus images as ROI definition. The mean $Z$-value represents the average FC value within the hippocampus. These steps were performed in the left and right hippocampus data, respectively.

\section{MRI Radiomic Analysis}

Artificial Intelligence Kit (A.K) is a commercially available software developed by GE Healthcare Institute of Precision Medicine. It performs data loading, segmentation, feature calculation, feature selection, and model establishment of radiomics.

First, we loaded the original 3D T1-weighted images and ROI images into the A.K. software. Then, image features were calculated including Formfactor, Histogram, Haralick, gray level co-occurrence matrix (GLCM), and gray level runlength matrix (RLM). Formfactor features use mathematical methods to characterize the shape of the lesion, and describe the shape and compactness of the lesion. Histogram features calculate the gray intensity information of the lesion and describe the overall distribution of gray level information. GLCM obtains the co-occurrence matrix by counting the probability of the occurrence of pixel pairs in different directions and displacement vectors. It describes the complexity of the lesion, the level variation, and the degree of texture 

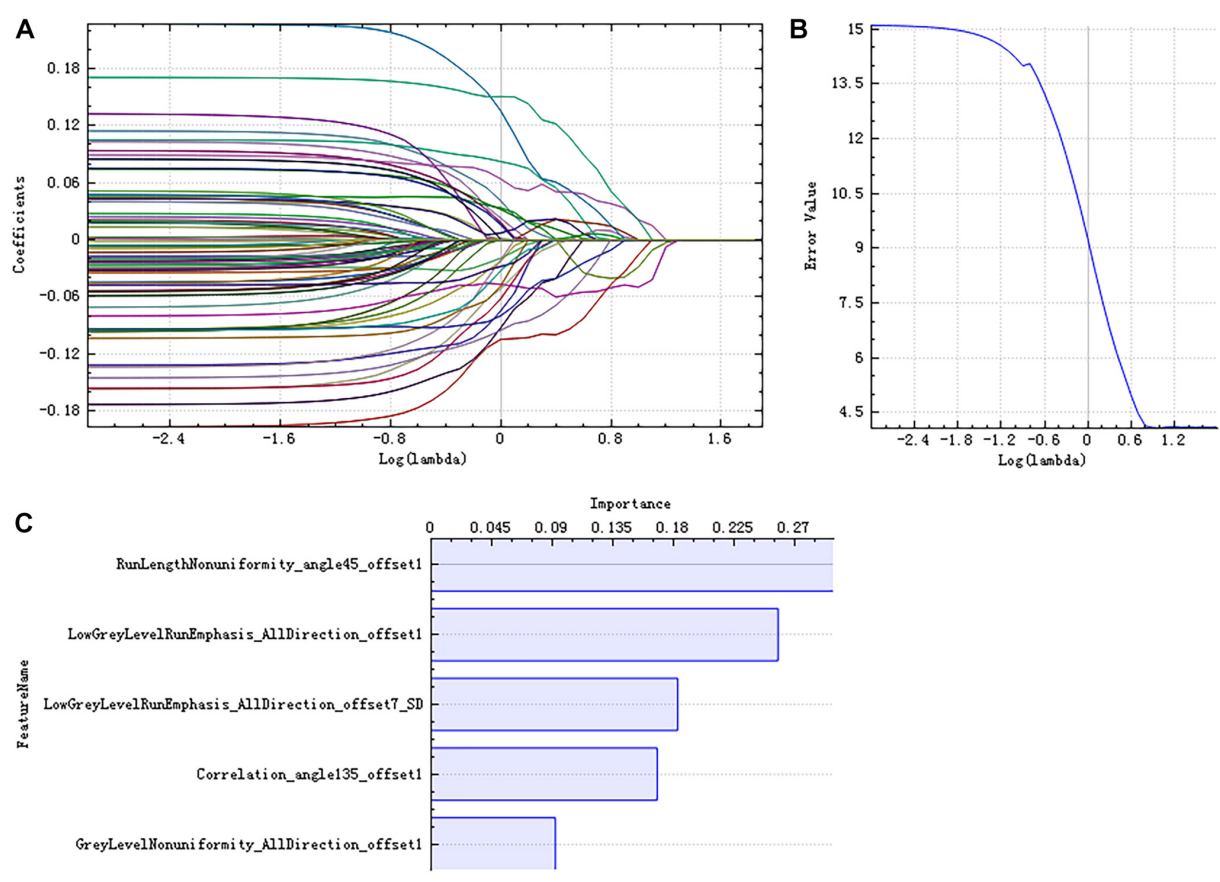

FIGURE 4 | Least absolute shrinkage and selection operator (LASSO) dimensionality reduction of the left hippocampus. Error-lambda graph (A); coefficients-lambda graph (B). We chose $\lambda$ according to the lowest error rate. Importance of the selected features (C).

thickness (Seongin et al., 2011). Haralick also based on the co-occurrence matrix to extract the corresponding features, but it has directional invariance. RLM obtains the length matrix by calculating the probability of the pixels appearing repeatedly in succession with different directions and displacement vectors. It also describes the complexity of the lesion, the level variation and the degree of texture thickness (Galloway, 1975). We chose "1, 4, and 7" for displacement vectors.

After feature calculation, we added labels " 0 " and " 1 " for each subject data representing $\mathrm{NC}$ and $\mathrm{AD}$. The preprocessing for feature selection included replaced the abnormal value with the mean; set the training set proportion and testing set proportion to 0.7 and 0.3 , respectively, and eliminate the unit restriction for each feature column through normalization. The feature selection steps were as follows. Step 1: T-test and rank sum test were used to identify the features with significant differences between the two groups $(P<0.05)$. Step 2: The correlation analysis was used to reduce the dimension. The filter threshold was set to 0.9 , and the Spearman rank correlation coefficient was selected. Correlation analysis was performed between any two feature columns; one of the two highly correlated features was removed when the correlation coefficient was greater than 0.9. Step 3: The least absolute shrinkage and selection operator (LASSO) regression model was applied to reduce the dimension using 10-fold cross-validation. This method is applicable to the regression analysis of high-dimensional data. We performed feature selection on the left and right hippocampus data, respectively.

\section{Statistical Analysis}

Demographic, neuropsychological comparison, RS FC comparison of the two groups, and correlation analysis between the bilateral hippocampus radiomic features and FC were performed using SPSS version 22.0. All statistical methods about radiomic analysis were performed using the A.K. software.

\section{RESULTS}

\section{Comparison of Demographic and Neuropsychological Performance}

Among the remaining $73 \mathrm{AD}$ patients and $45 \mathrm{NC}$ subjects, $67 \mathrm{AD}$ patients and $44 \mathrm{NC}$ subjects were finally collected for analysis, who had head motion $<3.0 \mathrm{~mm}$ translation and $3.0^{\circ}$ rotation in any direction during preprocessing of rs-fMRI. Table 1 showed the statistical analysis results of demographics and neuropsychological performance. There was no significant difference between $\mathrm{AD}$ and $\mathrm{NC}$ subjects in demographics $(P>0.05)$. However, there were statistically significant differences in MMSE performance between the two groups $(P<0.05)$.

\section{Functional Connectivity Analysis}

After two-sample $t$-tests, RS FC had no significant difference between $\mathrm{AD}$ and $\mathrm{NC}$ within the left hippocampal mask $(t=0.34, P>0.05)$ and the right hippocampal mask $(t=-1.01, P>0.05)$. In addition, we observed that FC 

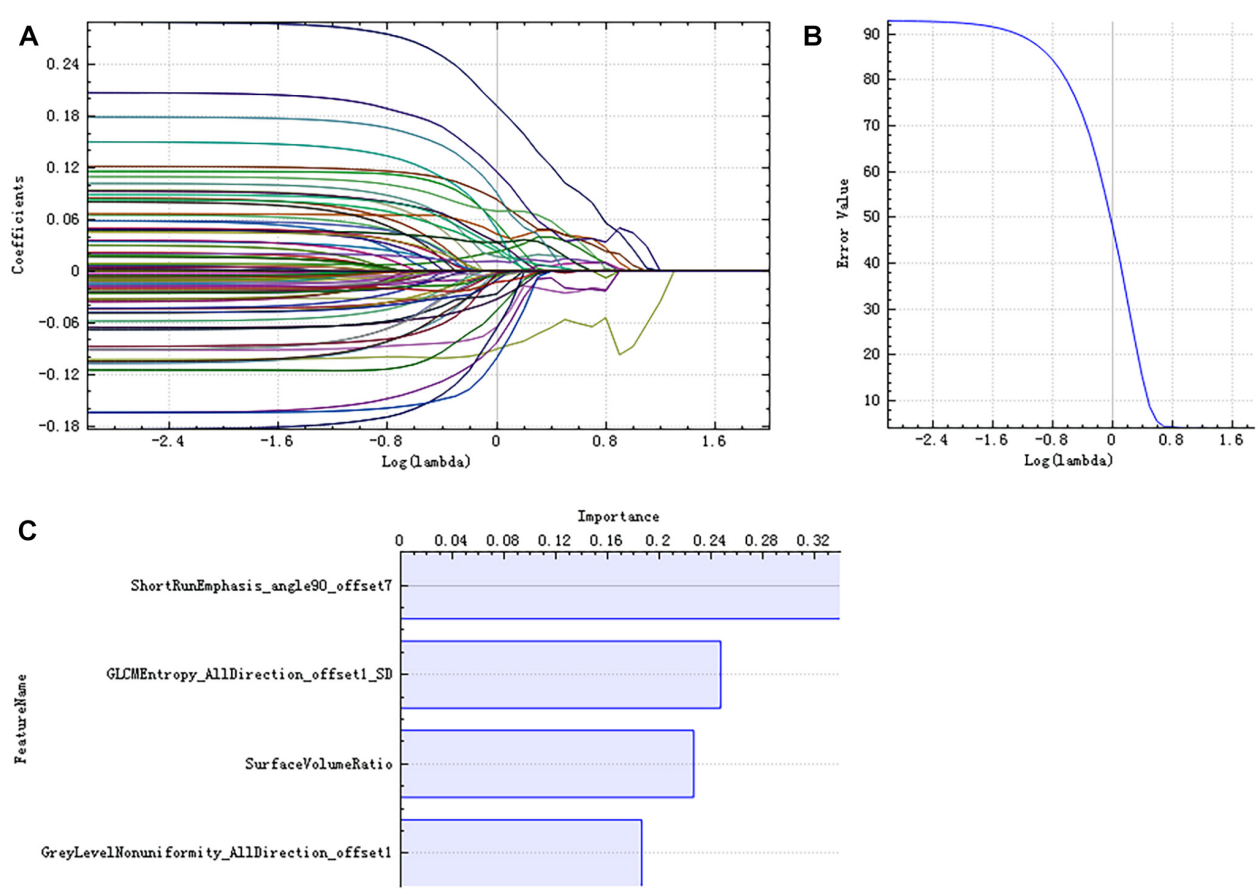

FIGURE 5 | LASSO dimensionality reduction of the right hippocampus. Error-lambda graph (A); coefficients-lambda graph (B). We chose $\lambda$ according to the lowest error rate. Importance of the selected features (C).

A

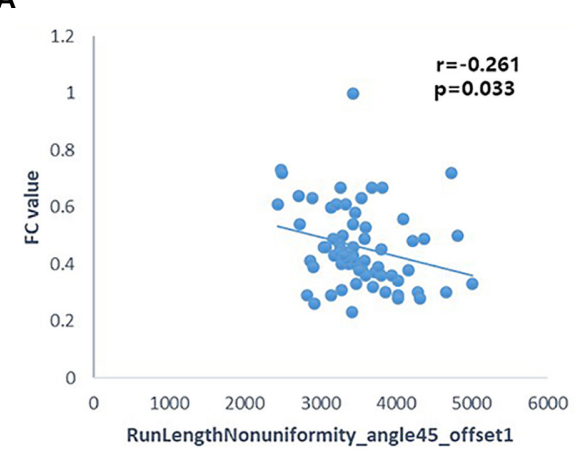

C

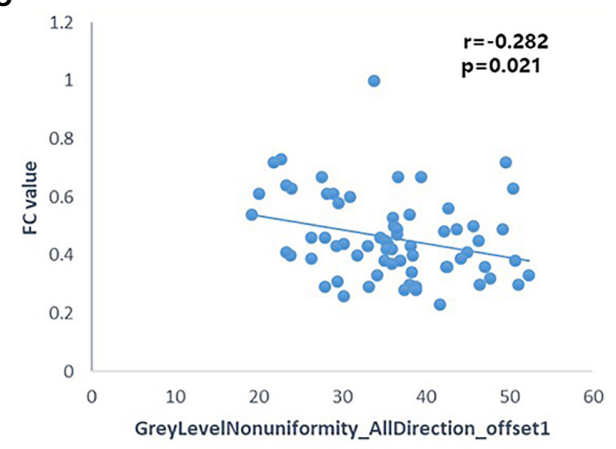

\section{B}

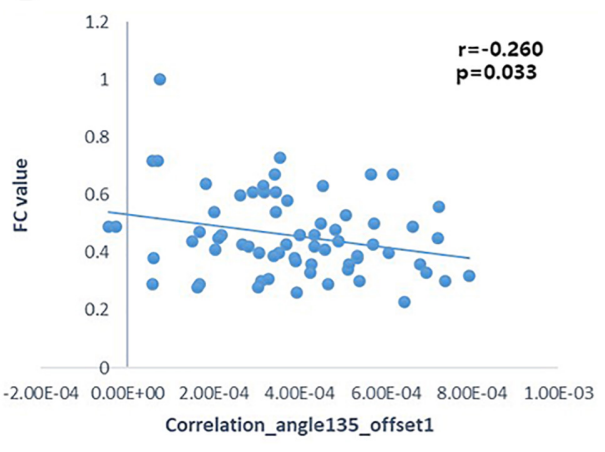

FIGURE 6 | Pearson correlation analysis between some radiomic features and FC of the left hippocampus $(p<0.05)$. Pearson's correlation analysis between "RunLengthNonuniformity_angle45_offset1" and FC value (A); Pearson's correlation analysis between "Correlation_angle135_offset1" and FC value (B); Pearson's correlation analysis between "GreyLevelNonuniformity_AllDirection_offset1" and FC value (C). 
in some brain regions was disrupted in the left hippocampal functional network in the $\mathrm{AD}$ patient group; these regions are right gyrus rectus, right anterior cingulate and paracingulate gyri, right orbital part of middle frontal gyrus, right caudate nucleus, left and right precuneus, left median cingulate and paracingulate gyri, left and right angular gyrus, left and right middle occipital gyrus, and inferior parietal but supramarginal and angular gyri (Figure $\mathbf{1}$ and Table 2). We also observed some brain regions with decreased FC in the right hippocampal functional network, including left and right anterior cingulate and paracingulate gyri, right median cingulate and paracingulate gyri, right dorsolateral superior frontal gyrus, right precentral gyrus, and right middle frontal gyrus (Figure 2 and Table 3 ).

\section{Radiomic Analysis}

There were 385 features extracted in the bilateral hippocampus after feature calculation. For left and right hippocampus, after $T$-test and rank sum test, the remaining feature numbers were 196 and 215. After correlation analysis, the remaining feature numbers were reduced to 70 and 81 (Figures 3A,B). Finally, using the LASSO regression model, five and four features were selected (Figures 4A-C, 5A-C).

\section{Correlations Between Radiomic Features and Functional Connectivity}

Pearson's correlation analysis suggested that there were three radiomic features (RunLengthNonuniformity_angle45_offset1,

TABLE 4 | Correlation between the left hippocampal radiomic features and functional connectivity values.

\begin{tabular}{lcc}
\hline & $\begin{array}{c}\text { Type of } \\
\text { parameters }\end{array}$ & $\begin{array}{c}\text { r, AD patients } \\
(\boldsymbol{n}=\mathbf{6 7})\end{array}$ \\
\hline RunLengthNonuniformity_angle45_offset1 & RLM & $-0.261^{*}$ \\
LowGreyLevelRunEmphasis_AllDirection_offset1 & RLM & 0.222 \\
LowGreyLevelRunEmphasis_AllDirection_offset7_SD & RLM & -0.008 \\
Correlation_angle135_offset1 & GLCM & $-0.260^{*}$ \\
GreyLevelNonuniformity_AllDirection_offset1 & RLM & $-0.282^{*}$ \\
\hline
\end{tabular}

Pearson correlation analyses were conducted to calculate the correlations between these selected features and the left hippocampal FC values. ${ }^{*} P<0.05$. RLM, runlength matrix; GLCM, gray level co-occurrence matrix.

TABLE 5 | Correlation between the right hippocampal radiomic features and functional connectivity values.

\begin{tabular}{lcc}
\hline & $\begin{array}{c}\text { Type of } \\
\text { parameters }\end{array}$ & $\begin{array}{c}\text { r, AD patients } \\
(\boldsymbol{n}=\mathbf{6 7})\end{array}$ \\
\hline ShortRunEmphasis_angle90_offset7 & RLM & 0.203 \\
GLCMEntropy_AllDirection_offset1_SD & GLCM & 0.104 \\
SurfaceVolumeRatio & Formfactor & 0.174 \\
GreyLevelNonuniformity_AllDirection_offset1 & RLM & -0.044
\end{tabular}

Pearson correlation analyses were conducted to calculate the correlations between these selected features and the right hippocampal FC values.
Correlation_angle135_offset1, GreyLevelNonuniformity_AllDirection_offset1) associated with FC values in the left hippocampus $(P<0.05)$; the correlation coefficient values were -0.261 , -0.260 , and -0.282 , respectively (Figure 6 and Table 4). However, there were no significant correlations between selected radiomic features and FC values in the right hippocampus (Table 5).

\section{DISCUSSION}

Our study indicated that the AD group showed abnormalities in the left and right hippocampal functional network compared with the NC group. Meanwhile, the present study selected the closely related radiomic features of the bilateral hippocampus. Pearson correlation analysis suggested weak relationship between some radiomic features and FC values in the left hippocampus. It's a prospective paper to study the correlation between the hippocampal radiomic features and fMRI characteristics in AD.

In addition to hippocampal structural studies, some rsfMRI studies suggest that hippocampal functional characteristics changed in the AD or aMCI stage. For example, Sorg et al. (2007) found that only selected brain areas such as the hippocampus showed reduced activities in MCI patients. Some $\mathrm{AD}$ and $\mathrm{MCI}$ studies indicated the destruction of functional connections between the hippocampus and the PCC, medial prefrontal cortex, inferior parietal lobule, and other brain areas (Wang et al., 2006a; Dennis and Thompson, 2014; Krajcovicova et al., 2014). One study showed alterations of three hippocampal subfield functional networks in aMCI patients (De et al., 2017). Our results have demonstrated diminished FC in the right gyrus rectus, right anterior cingulate and paracingulate gyri, bilateral precuneus, bilateral angular gyrus, and bilateral middle occipital gyrus of the left hippocampus functional networks. We also observed some brain regions of decreased FC in the right hippocampal functional network, including bilateral anterior cingulate and paracingulate gyri, right dorsolateral superior frontal gyrus, and right precentral gyrus. Some of these brain regions are major components of the DMN. Most seed-based and ICA studies have shown that functional connections of DMN and other brain networks are reduced in AD or MCI (Greicius et al., 2004; Christian et al., 2007). We observed no increase or decrease of FC in PCC; maybe there are both disruption and compensation effects in the AD stage. However, many studies have reported areas of aberrant increased connectivity in $\mathrm{AD}$ (Damoiseaux et al., 2012; Simona et al., 2015; Li M. et al., 2017). An ICA study by Damoiseaux et al. (2012) showed that the FC of posterior DMN began to decrease in the early stage of $\mathrm{AD}$ patients, while the connection increased within the anterior and ventral DMN. As the disease progressed, all Internet connections decreased. We observed most of the regions of decreased FC in the bilateral hippocampal functional networks, probably because patients with severe $\mathrm{AD}$ were in the majority of our AD subjects. Moreover, the disruption patterns of the left and right hippocampal networks are 
different in $\mathrm{AD}$ and aMCI patients (Wang et al., 2006b; Xie et al., 2013). Our result was consistent with these recent discoveries.

Radiomics analysis has been applied to some neuropsychiatric diseases. A radiomics study about autism spectrum disorder found significant differences in the texture features in the right hippocampus, corpus callosum, cerebellar white matter, and left choroid plexus between patients and controls (Chaddad et al., 2017b). Some texture analysis studies have found that there are texture differences in hippocampus, corpus callosum, and thalamus between AD patients and NCs (Li et al., 2010; Oliveira et al., 2011). The nine radiomic features selected in this study reflect the differences in image gray value distribution, texture characteristics, spatial heterogeneity, and other microstructural information in AD patients. Among the five selected features of the left hippocampus, "RunLengthNonuniformity_angle45_ offset1," "LowGreyLevelRunEmphasis_AllDirection_offset1," "LowGreyLevel RunEmphasis_AllDirection_offset7_SD," and "GreyLevelNonuniformity_AllDirection_offset1" are RLM parameters. The higher the value of "RunLengthNonuniformity" or "GreyLevelNonuniformity" is, the more heterogeneous the lesion is. "LowGreyLevelRunEmphasis" describes the overall brightness of the lesion; the higher the value, the darker the lesion. "Correlation_angle135_offset1" is one of the GLCM parameters. "Correlation" describes the similarity of the gray levels in adjacent pixels and displays the correlation between a pixel and its neighbors across the image. Among the four selected features of the right hippocampus, "ShortRunEmphasis_angle90_offset7" and "GreyLevelNonuniformity_AllDirection_offset1" are RLM parameters. "ShortRunEmphasis" describes the degree of difference in gray value between adjacent pixels of the lesion; the higher the value is, the more complex and heterogeneous the lesion is. "GLCMEntropy_AllDirection_offset1_SD" is a GLCM parameter. "Entropy" describes the complexity of the co-occurrence matrix; the larger the value is, the more complex the co-occurrence matrix will be. "SurfaceVolumeRatio" is a Formfactor parameter; it describes the three-dimensional size and shape of the hippocampus. If the edge irregularity of three-dimensional lesions is large, the ratio is also large, indicating greater heterogeneity. These features extracted from the hippocampus structure reflect high-order imaging patterns and heterogeneity characteristics of microstructure in hippocampus in $\mathrm{AD}$ patients.

Although there was no significant difference in hippocampal FC between the two groups, there were significant differences in hippocampal radiomic features. In addition, our study showed weak and negative correlation between the intrahippocampal FC and the radiomic features. It suggests that the changes of hippocampal microstructure appeared before the changes of hippocampal function in $\mathrm{AD}$ patients. MR volumetry and DTI studies indicated the decreased volumes and increased mean diffusivity of the hippocampus in AD patients (Palesi et al., 2011; Brueggen et al., 2015). Structural MRI studies have shown that microstructural abnormalities of the hippocampus can be the neuroimaging biomarkers of early cognitive impairment. In future studies, we will pay more attention to the study of hippocampal microstructure to provide imaging basis for the early diagnosis of $\mathrm{AD}$. The occurrence and development of $\mathrm{AD}$ is a complex process, and we can obtain more structural and functional information by using a variety of MRI techniques in future studies.

However, there were several limitations in our study. Firstly, a complete 1:1 match in age and sex ratio had not been achieved. Secondly, although patients with MCI were excluded, the severity of the disease in $\mathrm{AD}$ patients was not distinguished in the study, and these subjects were not followed up. Mild, moderate, and severe $\mathrm{AD}$ patients were all included in the $\mathrm{AD}$ group. Lastly, we did not correct the between-group comparison of RS FC adjusting for hippocampal volume. It may influence the results to a certain degree.

In summary, this study observed that there are decreased activity in hippocampus functional network in AD patients. It also indicates that the closely related hippocampal radiomic features can be neuroimaging biomarkers for the diagnosis of AD. Moreover, we explored the correlations between the MRI radiomic features and intrahippocampal $\mathrm{FC}$ in $\mathrm{AD}$ patients. It provides a very important reference for further understanding the pathogenesis of AD.

\section{ETHICS STATEMENT}

This study was carried out in accordance with the recommendations of "Ethics Committee of Zhejiang Provincial People's Hospital (No. 2012KY002)" with written informed consent from all subjects. All subjects gave written informed consent in accordance with the Declaration of Helsinki. The protocol was approved by the "Ethics Committee of Zhejiang Provincial People's Hospital.”

\section{AUTHOR CONTRIBUTIONS}

$\mathrm{ZD}$, EY, and QF designed the experiments. QF, MW, QS, and $\mathrm{HJ}$ performed the experiments and analyzed the data. ZW segmented the MR images. ZD, QF, PP, and $\mathrm{ZL}$ interpreted the results and drafted the manuscript. All the authors read and approved the final version of the manuscript.

\section{FUNDING}

This study was funded by the National Natural Science Foundation of China (No. 81871337).

\section{ACKNOWLEDGMENTS}

We thank Prof. Wenli Cai for his thoughtful comments and assistance with English editing. 


\section{REFERENCES}

Alderson, T., Kehoe, E., Maguire, L., Farrell, D., Lawlor, B., Kenny, R. A., et al. (2017). Disrupted thalamus white matter anatomy and posterior default mode network effective connectivity in amnestic mild cognitive impairment. Front. Aging Neurosci. 9:370. doi: 10.3389/fnagi.2017.00370

Amp, A. S. (2011). The diagnosis of mild cognitive impairment due to Alzheimer's disease: recommendations from the national institute on aging-Alzheimer's association workgroups on diagnostic guidelines for Alzheimer's disease. Alzheimers Dement. 7, 270-279.

Ball, M. J. (1997). Frequency of stages of Alzheimer-related lesions in different age categories: concurrences and cautions. Neurobiol. Aging 18, 375-376. doi: 10.1016/s0197-4580(97)00052-3

Brueggen, K., Dyrba, M., Barkhof, F., Hausner, L., Filippi, M., Nestor, P. J., et al. (2015). Basal forebrain and hippocampus as predictors of conversion to Alzheimer's disease in patients with mild cognitive impairment-a multicenter DTI and volumetry study. Alzheimers Dement. J. Alzheimers Assoc. 11, 72-72. doi: $10.3233 / J A D-150063$

Bruno, D., Feldman, H. H., Claudia, J., Dekosky, S. T., Pascale, B. G., Jeffrey, C., et al. (2007). Research criteria for the diagnosis of Alzheimer's disease: revising the NINCDS-ADRDA criteria. Lancet Neurol. 6, 734-746. doi: 10.1016/s14744422(07)70178-3

Chaddad, A., Desrosiers, C., Hassan, L., and Tanougast, C. (2017a). Hippocampus and amygdala radiomic biomarkers for the study of autism spectrum disorder. BMC Neurosci. 18:52. doi: 10.1186/s12868-017-0373-0

Chaddad, A., Desrosiers, C., and Toews, M. (2017b). Multi-scale radiomic analysis of sub-cortical regions in MRI related to autism, gender and age. Sci. Rep. 7:45639. doi: 10.1038/srep45639

Christian, S., Valentin, R., Mark, M., Calhoun, V. D., Tom, E., Leonhard, L. E., et al. (2007). Selective changes of resting-state networks in individuals at risk for Alzheimer's disease. Proc. Natl. Acad. Sci. U.S.A. 104, 18760-18765. doi: 10.1073/pnas.0708803104

Claudia, P., Teipel, S. J., Annahita, O., Christian, B. H., Thomas, M., Janaina, M. M., et al. (2010). Automated detection of brain atrophy patterns based on MRI for the prediction of Alzheimer's disease. Neuroimage 50, 162-174. doi: 10.1016/j.neuroimage.2009.11.046

Damoiseaux, J. S., Prater, K. E., Miller, B. L., and Greicius, M. D. (2012). Functional connectivity tracks clinical deterioration in Alzheimer's disease. Neurobiol. Aging 33:828.e19-30

De, F. R., La, J. R., and Chételat, G. (2015). Structural imaging of hippocampal subfields in healthy aging and Alzheimer's disease. Neuroscience 309, 29-50. doi: 10.1016/j.neuroscience.2015.08.033

De, F. R., Mutlu, J., Bejanin, A., Gonneaud, J., Landeau, B., Tomadesso, C., et al. (2017). Intrinsic connectivity of hippocampal subfields in normal elderly and mild cognitive impairment patients. Hum. Brain Mapp. 38, 4922-4932. doi: 10.1002/hbm. 23704

Denise, H., Buckner, R. L., Shimony, J. S., Williams, L. E., Erbil, A., Conturo, T. E., et al. (2004). Differential vulnerability of anterior white matter in nondemented aging with minimal acceleration in dementia of the Alzheimer type: evidence from diffusion tensor imaging. Cereb. Cortex 14, 410-423. doi: 10.1093/cercor/ bhh003

Dennis, E. L., and Thompson, P. M. (2014). Functional brain connectivity using fMRI in aging and Alzheimer's disease. Neuropsychol. Rev. 24, 49-62. doi: 10.1007/s11065-014-9249-6

Dubois, B., Feldman, H. H., Jacova, C., Dekosky, S. T., Barberger-Gateau, P., Cummings, J., et al. (2007). Research criteria for the diagnosis of Alzheimer's disease: revising the NINCDS-ADRDA criteria. Lancet Neurol. 6, 734-746. doi: 10.1016/s1474-4422(07)70178-3

Galloway, M. M. (1975). Texture analysis using gray level run lengths. Comput. Graphics Image Proc. 4, 172-179. doi: 10.1016/s0146-664x(75)80008-6

Greicius, M. D., Gaurav, S., Reiss, A. L., and Vinod, M. (2004). Default-mode network activity distinguishes Alzheimer's disease from healthy aging: evidence from functional MRI. Proc. Natl. Acad. Sci. U.S.A. 101, 4637-4642. doi: 10. 1073/pnas.0308627101

Guzman, V. A., Carmichael, O. T., Schwarz, C., Tosto, G., Zimmerman, M. E., and Brickman, A. M. (2013). White matter hyperintensities and amyloid are independently associated with entorhinal cortex volume among individuals with mild cognitive impairment. Alzheimers Dement. J. Alzheimers Assoc. 9, S124-S131. doi: 10.1016/j.jalz.2012.11.009

Horvat, N., Veeraraghavan, H., Khan, M., Blazic, I., Zheng, J., Capanu, M., et al. (2018). MR imaging of rectal cancer: radiomics analysis to assess treatment response after neoadjuvant therapy. Radiology 287:172300. doi: 10.1148/radiol. 2018172300

Kickingereder, P., Burth, S., Wick, A., Götz, M., Eidel, O., Schlemmer, H. P., et al. (2016). Radiomic profiling of glioblastoma: identifying an imaging predictor of patient survival with improved performance over established clinical and radiologic risk models. Radiology 280, 880-889. doi: 10.1148/radiol. 2016160845

Krajcovicova, L., Marecek, R., Mikl, M., and Rektorova, I. (2014). Disruption of resting functional connectivity in Alzheimer's patients and at-risk subjects. Curr. Neurol. Neurosci. Rep. 14:491. doi: 10.1007/s11910-014-0491-3

Li, X., Xia, H., Zhou, Z., and Tong, L. (2010). “3D texture analysis of hippocampus based on MR images in patients with Alzheimer disease and mild cognitive impairment," in Proceedings of the 2010 3rd International Conference on Biomedical Engineering and Informatics, (Yantai: IEEE).

Li, H., Zhu, Y., Burnside, E. S., Drukker, K., Hoadley, K. A., Fan, C., et al. (2017). MR imaging radiomics signatures for predicting the risk of breast cancer recurrence as given by research versions of mammaprint, oncotype DX, and PAM50 gene assays. Radiology 281:152110.

Li, M., Zheng, G., Zheng, Y., Xiong, Z., Xia, R., Zhou, W., et al. (2017). Alterations in resting-state functional connectivity of the default mode network in amnestic mild cognitive impairment: an fMRI study. BMC Med. Imaging 17:48. doi: 10.1186/s12880-017-0221-9.

Lu, C. F., Hsu, F. T., Hsieh, K. L., Kao, Y. J., Cheng, S. J., Hsu, J. B., et al. (2018). Machine learning-based radiomics for molecular subtyping of gliomas. Clin. Cancer Res. Clincanres. 24, 4429-4436. doi: 10.1158/1078-0432.CCR-173445

Mak, E., Gabel, S., Su, L., Williams, G. B., Arnold, R., Passamonti, L., et al. (2017). Multi-modal MRI investigation of volumetric and microstructural changes in the hippocampus and its subfields in mild cognitive impairment, Alzheimer's disease, and dementia with Lewy bodies. Int. Psychogeriatr. 29, 545-555. doi: $10.1017 /$ S1041610216002143

Menon, V. (2011). Large-scale brain networks and psychopathology: a unifying triple network model. Trends Cognit. Sci. 15, 483-506. doi: 10.1016/j.tics.2011. 08.003

Oliveira, M. S., De Balthazar, M. L. F., D’Abreu, A., Yasuda, C. L., Damasceno, B. P., et al. (2011). MR imaging texture analysis of the corpus callosum and thalamus in amnestic mild cognitive impairment and mild Alzheimer disease. Ajnr. Am. J. Neuroradiol. 32, 60-66. doi: 10.3174/ajnr.A2232

Palesi, F., Vitali, P., Chiarati, P., Castellazzi, G., Caverzasi, E., Pichiecchio, A., et al. (2011). DTI and MR volumetry of hippocampus-PC/PCC circuit: in search of early micro- and macrostructural signs of Alzheimer's disease. Neurol. Res. Int. 2012:517876. doi: 10.1155/2012/517876

Port, J. D. (2018). Diagnosis of attention deficit hyperactivity disorder by using MR imaging and radiomics: a potential tool for clinicians. Radiology 287:631-632. doi: 10.1148/radiol.2018172804

Qi, F., Yuanjun, C., Zhengluan, L., Hongyang, J., Dewang, M., Mei, W., et al. (2018). Corpus callosum radiomics-based classification model in Alzheimer's disease: a Case-control study. Front. Neurol. 9:618. doi: 10.3389/fneur.2018.00618

Seongjin, P., Bohyoung, K., Jeongjin, L., Mo, G. J., and Yeong-Gil, S. (2011). GGO nodule volume-preserving nonrigid lung registration using GLCM texture analysis. IEEE Trans. Biomed. Eng. 58, 2885-2894. doi: 10.1109/TBME.2011. 2162330

Sheline, Y. I., and Raichle, M. E. (2013). Resting state functional connectivity in preclinical Alzheimer's disease. Biol. Psychiatry 74, 340-347. doi: 10.1093/ cercor/bhu259

Shi-Jiang, L., Zhu, L., Gaohong, W., Mei-Jie, Z., Malgorzata, F., and Antuono, P. G. (2002). Alzheimer disease: evaluation of a functional MR imaging index as a marker. Radiology 225, 253-259. doi: 10.1148/radiol.2251011301

Simona, G., Annalena, V., Fabio, S., Fernando, C., Fabrizio, F., Massimo, M., et al. (2015). Increased functional connectivity in the default mode network in mild cognitive impairment: a maladaptive compensatory mechanism associated with poor semantic memory performance. J. Alzheimers Dis. 45, 457-470. doi: 10.3233/JAD- 142547 
Sorg, C., Riedl, V., Mühlau, M., Calhoun, V. D., Eichele, T., Läer, L., et al. (2007). Selective changes of resting-state networks in individuals at risk for Alzheimer's disease. Proc. Natl. Acad. Sci. U.S.A. 104, 18760-18765. doi: 10.1073/pnas. 0708803104

Sun, R., Limkin, E. J., Vakalopoulou, M., Dercle, L., Champiat, S., Han, S. R., et al. (2018). A radiomics approach to assess tumour-infiltrating CD8 cells and response to anti-PD-1 or anti-PD-L1 immunotherapy: an imaging biomarker, retrospective multicohort study. Lancet Oncol. 19, 1180-1191. doi: 10.1016/ S1470-2045(18)30413-3

Toussaint, P. J., Maiz, S., Coynel, D., Doyon, J., Messé, A., Souza, L. C. D., et al. (2014). Characteristics of the default mode functional connectivity in normal ageing and Alzheimer's disease using resting state fMRI with a combined approach of entropy-based and graph theoretical measurements. Neuroimage 101, 778-786. doi: 10.1016/j.neuroimage.2014.08.003

Wang, G., He, L., Yuan, C., Huang, Y., Liu, Z., and Liang, C. (2018). Pretreatment MR imaging radiomics signatures for response prediction to induction chemotherapy in patients with nasopharyngeal carcinoma. Eur. J. Radiol. 98, 100-106. doi: 10.1016/j.ejrad.2017.11.007

Wang, L., Zang, Y., He, Y., Liang, M., Zhang, X., Tian, L., et al. (2006a). Changes in hippocampal connectivity in the early stages of Alzheimer's disease: evidence from resting state fMRI. Neuroimage 31, 496-504. doi: 10.1016/j.neuroimage. 2005.12.033

Wang, L., Zang, Y., Liang, M., Zhang, X., Tian, L., and Jiang, T. (2006b). Changes in hippocampal connectivity in the early stages of Alzheimer's disease: evidence from resting state fMRI. Neuroimage 31, 496-504. doi: 10.1016/j.neuroimage. 2005.12.033

Wang, Z., Yan, C., Zhao, C., Qi, Z., Zhou, W., Lu, J., et al. (2011). Spatial patterns of intrinsic brain activity in mild cognitive impairment and Alzheimer's disease: a resting-state functional MRI study. Hum. Brain Mapp. 32, 1720-1740. doi: 10.1002/hbm. 21140

Whitwell, J. L., Przybelski, S. A., Weigand, S. D., Knopman, D. S., Boeve, B. F., Petersen, R. C., et al. (2007). 3D maps from multiple MRI illustrate changing atrophy patterns as subjects progress from mild cognitive impairment to
Alzheimer's disease. Brain J. Neurol. 130(Pt 7), 1777-1786. doi: 10.1093/brain/ awm 112

Wu, X., Li, R., Fleisher, A. S., Reiman, E. M., Guan, X., Zhang, Y., et al. (2011). Altered default mode network connectivity in Alzheimer's disease-a resting functional MRI and Bayesian network study. Hum. Brain Mapp. 32, 1868-1881. doi: 10.1002/hbm. 21153

Wu, Z., Gao, Y., Shi, F., Ma, G., Jewells, V., and Shen, D. (2017). Segmenting hippocampal subfields from 3T MRI with multi-modality images. Med. Image Anal. 43, 10-22. doi: 10.1016/j.media.2017.09.006

Xie, C., Li, W., Chen, G., Ward, B. D., Franczak, M. B., Jones, J. L., et al. (2013). Late-life depression, mild cognitive impairment and hippocampal functional network architecture. Neuroimage Clin. 3, 311-320. doi: 10.1016/j.nicl.2013. 09.002

Yong, L., Chunshui, Y., Xinqing, Z., Jieqiong, L., Yunyun, D., Alexander-Bloch, A. F., et al. (2014). Impaired long distance functional connectivity and weighted network architecture in Alzheimer's disease. Cereb. Cortex 24, 1422-1435. doi: 10.1093/cercor/bhs410

Yu, E., Liao, Z., Mao, D., Zhang, Q., Ji, G., Li, Y., et al. (2016). Directed functional connectivity of posterior cingulate cortex and whole brain in Alzheimer's disease and mild cognitive impairment. Curr. Alzheimer Res. 14, 628-635. doi: $10.2174 / 1567205013666161201201000$

Conflict of Interest Statement: The authors declare that the research was conducted in the absence of any commercial or financial relationships that could be construed as a potential conflict of interest.

Copyright (c) 2019 Feng, Wang, Song, Wu, Jiang, Pang, Liao, Yu and Ding. This is an open-access article distributed under the terms of the Creative Commons Attribution License (CC BY). The use, distribution or reproduction in other forums is permitted, provided the original author(s) and the copyright owner(s) are credited and that the original publication in this journal is cited, in accordance with accepted academic practice. No use, distribution or reproduction is permitted which does not comply with these terms. 


\section{OPEN ACCESS}

Edited by: Alessandra Griffa, VU University Amsterdam,

Netherlands

Reviewed by:

John George Grundy, lowa State University, United States

Jiu Chen,

Nanjing Medical University, China

*Correspondence: Mauro Gianni Perrucci maurogianni.perrucci@unich.it; gianni.perrucci@gmail.com

Specialty section:

This article was submitted to Brain Imaging Methods,

a section of the journal Frontiers in Neuroscience

Received: 06 November 2018 Accepted: 12 April 2019 Published: 15 May 2019

Citation:

Bubbico G, Chiacchiaretta $P$ Parenti M, di Marco M, Panara V, Sepede G, Ferretti $A$ and Perrucci MG (2019) Effects of Second Language Learning on the Plastic Aging Brain: Functional Connectivity, Cognitive

Decline, and Reorganization.

Front. Neurosci. 13:423

doi: 10.3389/fnins.2019.00423

\section{Effects of Second Language Learning on the Plastic Aging Brain: Functional Connectivity, Cognitive Decline, and Reorganization}

\author{
Giovanna Bubbico 1,2, Piero Chiacchiaretta 1,2, Matteo Parenti'3, Marcin di Marco ${ }^{3}$, \\ Valentina Panara ${ }^{1,4}$, Gianna Sepede ${ }^{1,5,6}$, Antonio Ferretti ${ }^{1,2}$ and Mauro Gianni Perrucci, ${ }^{1,2 *}$ \\ ${ }^{1}$ Department of Neuroscience, Imaging and Clinical Sciences, "G. d'Annunzio" University of Chieti-Pescara, Chieti, Italy, \\ ${ }^{2}$ Institute for Advanced Biomedical Technologies, "G. d'Annunzio" University of Chieti-Pescara, Chieti, Italy, ${ }^{3}$ Department \\ of Medicine and Science of Aging, "G. d'Annunzio" University of Chieti-Pescara, Chieti, Italy, ${ }^{4}$ Section of Diagnostic Imaging \\ and Therapy, Radiology Division, Department of Neuroscience and Imaging, "SS Annunziata" Hospital, "G. D'Annunzio" \\ University, Chieti, Italy, ${ }^{5}$ Department of Basic Medical Sciences, Neurosciences and Sense Organs, University "A. Moro" \\ Bari, Chieti, Italy, ${ }^{6}$ National Health Trust, Department of Mental Health, Chieti, Italy
}

Learning a new language requires the use of extensive neural networks and can represent a powerful tool to reorganize brain neuroplasticity. In this study, we analyze how a 4 months long second language learning program (16, 2 h sessions) can lead to functional changes in the brain of healthy elderly individuals. A large number of studies point out a decline of brain-skills with age; here it is analyzed how cognition together with functional brain organization can be improved later in life. Twentysix older adults (59-79 years old) were enrolled in the present study. A complete neuropsychological examination was administered before and after the intervention to measure global cognition levels, short- and long-term memory, attention, language access and executive functions. At the end of the program, in the intervention group, the results showed a significant improvement in global cognition together with an increased functional connectivity in the right inferior frontal gyrus (rIFG), right superior frontal gyrus (rSFG) and left superior parietal lobule (ISPL). These findings can be added to the current neurobiological breakthroughs of reshaping brain networks with a short language learning practice in healthy elderly subjects. Therefore, learning a foreign-language may represent a potentially helpful cognitive intervention for promoting healthy aging.

Keywords: aging, brain plasticity, second language learning, cognitive decline, resting state, functional connectivity

\section{INTRODUCTION}

Economic and social implications of pathological aging are dramatically growing (Winblad et al., 2016). Aging is the major risk factor for neurodegenerative diseases and dementia (Niccoli and Partridge, 2012; Alzheimer's Disease International, 2018). In our society, healthy aging is an objective to be achieved in order to prevent dementia in epidemic proportions. Simple and affordable solutions should be investigated for reducing risks linked to aging, both for the well-being of the individual and caregivers (Lwi et al., 2017). In the course of lifetime, lifestyle factors, such as 
education, hypertension, diet and depression represent modifiable variables which dramatically impact the risk of pathological aging (Granzotto and Zatta, 2011, 2014; Isopi et al., 2015; Martin Prince et al., 2015; Davies, 2017; Frankish and Horton, 2017; Frisoni et al., 2017; Prince, 2017). It has been shown that older adults can benefit from cognitive and physical interventions (Greenwood and Parasuraman, 2003; Coffman et al., 2014; Ferrucci and Priori, 2014; Luber and Lisanby, 2014; Strenziok et al., 2014). Cognitive and aerobic trainings emerged as potent modulators of cognitive decline (Kivipelto et al., 2013; Ngandu et al., 2015; Firth et al., 2018). Clinical aspects can be supervised and treated with lifestyle factors such as physical exercise or cognitive stimulation. Social stimulation and nutritional components, together with the learning of new cognitive tasks, even late in life, can make the person more autonomous in daily routines and less dependent on caregivers (Granzotto and Zatta, 2011, 2014; Hughes et al., 2012; Isopi et al., 2015; Tan et al., 2016).

Dementia has a multifactorial etiology (Iqbal and GrundkeIqbal, 2010; Alkadhi and Eriksen, 2011). Recent studies showed how cognitive and brain reserve can prevent detrimental brain aging. Cognitive activities in lifetime boost brain resilience against aging and neurodegenerative disease, this process is known as cognitive reserve (CR) (Schweizer et al., 2012).

In addition, evidence indicates that early bilingualism has defensive effects on our aging brain (Iqbal and Grundke-Iqbal, 2010; Alkadhi and Eriksen, 2011; Boissy et al., 2011; Hughes et al., 2012; Schweizer et al., 2012; Abutalebi and Weekes, 2014; Isopi et al., 2015; Perani and Abutalebi, 2015; Bialystok et al., 2016; Perani et al., 2017). However, it is still debated whether language learning in older monolingual individuals can bring neuroplastic changes on the brain, since life-long bilingual older adults show increased functional connectivity compared to monolingual individuals (Grundy et al., 2017), the investigation focused on the reorganization of distributed brain networks after learning a second language. Few studies have examined differences in language experiences in older adults, especially of a language learning experience later in life (Grady et al., 2015).

Recent works of Grundy et al. (2017), Anderson et al. (2018), DeLuca et al., 2019, and Rosselli et al. (2019) analyzed brain and cognitive modifications effects of bilingualism in young and old adults.

Learning a foreign language could improve cognitive plasticity as this learning task requires the recruitment of extensive neural networks and stimulates different cognitive abilities such as working memory, inductive reasoning, sound discrimination, speech segmentation, task switching, rule learning, and semantic memory (Ware et al., 2017).

Therefore, tests showed that learning a second language during adulthood may exert neuroprotective effects, promote strengthening of brain networks, and improve cognitive reserve (Stern, 2001). Since pharmacological tools with a longterm efficacy to prevent or delay dementia are still missing (Extance, 2010; Collis and Waterfield, 2015; Selkoe and Hardy, 2016), simple and affordable non-pharmacological solutions should be improved in order to train our brain before neurodegenerative condition.
Many studies have investigated the neuroprotective effects of bilingualism in different settings (Gold et al., 2013; Green and Abutalebi, 2013; Gold, 2015; Houtzager et al., 2017), however, it is still largely unknown whether a late intervention is, similarly, effective in monolingual elderly healthy individuals. In addition, functional and structural changes occurring in the brain and underpinning the protective effect of bilingualism have been only partially investigated.

To fill the gap on the effects of late second language learning on brain connectivity, tests concerned the effect of a 4 months intervention focused on learning a second language.

The stimulation of language skills can indirectly stimulate different cognitive abilities (Kowoll et al., 2016; Schroeder and Marian, 2017) and indirectly, eventually counteract detrimental brain aging boosting cognitive abilities. A controlled intervention study, in which 14 healthy Italian-speaking adults were subjected to a 4 months English course, was performed. The objectives of the study were the effects on cognitive status, which were assessed with a comprehensive neuropsychological battery and brain functional connectivity, which was measured by restingstate functional magnetic resonance imaging (rs-fMRI). Subjects underwent rs-fMRI and neuropsychological assessment before and after the language course; the results were compared with those of a control group of monolingual Italian-speaking elderly subjects who did not change their daily habits during the period of the study.

\section{MATERIALS AND METHODS}

\section{Study Description}

The Ethic Committee of University "G. d'Annunzio" of Chieti approved all procedures and all experiments were performed in accordance with the relevant guidelines and regulations. Thirty participants were recruited from the local community and randomly assigned to one of two groups (1:1) after giving informed written consent. One group was involved in a second language learning course training program, in this case an English course for beginners, which lasted 16 weeks with 120 min of training per week. In the current intervention, each training week consisted of a $1 \mathrm{~h}$ and a half classroom session, interspersed with 15 min break and half an hour of homework exercises. The intervention consisted of group lessons with a native teacher. Throughout the intervention, the participants worked on improving their English skills. They acquired basic vocabulary and grammar skills, so they could start communicating in English in everyday social situations. They also learned about British and American English traditions, customs and culture. Participants further developed their speaking and writing skills. They worked on team projects, which provided ample opportunities to practice oral and written communication in English. Participants focused on developing their grammar and vocabulary in areas such as: traveling, shopping and family. All participants were assessed qualitatively by the native teacher at the beginning and at the end of the course.

The control group also completed pre- and post-tests but did not engage in training. In addition, all participants completed a 
neuropsychological battery prior to and following the training period. Control participants received each month a telephone call to make sure they did not change their lifestyle over the 4 months of the study. According to prior cognitive training research, the battery of tasks measured multiple cognitive abilities, including measures of executive functions, working memory, episodic memory and fluid intelligence. In addition to neuropsychological examination, participants underwent an rs-fMRI acquisition pre- and post-training; the same procedure was applied for the control group.

\section{Participants}

Among the enrolled 30 participants, 26 finished the study (12 in the control group and 14 in the intervention group). Two did not accept to be re-tested at the post-training condition phase, one did not attend enough lessons and another one did not meet inclusion criteria for a re-test. The enrolled participants were right-handed subjects of both genders, aged between 59 and 79 years old. Exclusion criteria were a suspected of cognitive decline after the neuropsychological assessment as well as disorders affecting safe engagement in the intervention (i.e., depression, symptomatic cardiovascular disease, severe self-reported loss of vision, hearing, or communicative ability and coincident participation in another intervention trial, together with any contraindication to MRI scanning, including metal implants and claustrophobia). Smokers and drug abuse subjects were excluded. Participants were asked to refrain from caffeine and alcohol for $24 \mathrm{~h}$ prior to the fMRI experimental session to control for external confounders. We randomly assigned participants into the two groups (1:1). Mastery of English proficiency was determined by the teacher during the first meeting through informal conversation and questions concerning the participants' previous experience with English. All participants were identified as beginner. It is common that Italian elderly people did not have English language lessons during their education. Table $\mathbf{1}$ describes participant characteristics.

\section{Conditions}

Participants in the intervention group ( 12 female, 2 male; average age $=69.5$ ) underwent a learning program located in the Abruzzo region. Classes were held by a native English teacher. Participants in the control group ( 7 female, 5 male; average age $=65.66$ ) did not change their daily routine during the 4 months. Subjects were called monthly; an informal interview was used to ask for changes in lifestyle. All participants completed the same pre- and postcognitive evaluation as well as the rs-fMRI acquisition (for study design see Figure 1).

TABLE 1 | Participants demographics.

\begin{tabular}{lccc}
\hline & $\begin{array}{c}\text { Control group } \\
(\boldsymbol{n}=\mathbf{1 2})\end{array}$ & $\begin{array}{c}\text { Intervention group } \\
(\boldsymbol{n}=\mathbf{1 4})\end{array}$ & ANOVA one-way \\
\hline Age (mean, $S D)$ & $65.7(3.7)$ & $69.5(5.3)$ & $F=4.42, p=0.04$ \\
Education & $13(2.7)$ & $9.6(2.9)$ & $F=8.43, p=0.008$ \\
Sex & $5 \mathrm{M} / 7 \mathrm{~F}(Y .=41.7)$ & $2 \mathrm{M} / 12 \mathrm{~F}(\mathrm{Y} .=14.3)$ & $\begin{array}{l}X^{2} \text { fisher corrected } \\
X^{2}=2.46, p=0.19\end{array}$
\end{tabular}

\section{Behavioral Assessment}

A comprehensive neuropsychological assessment to investigate different cognitive abilities was performed at baseline and after the 4 months intervention. All subjects included in the study, of both control and intervention groups, completed the tests for cognitive domains scores. The battery included measures of: global cognition with a Mini Mental State Examination (MMSE) test (Measso et al., 1993); attention, in the present case sustained spatial attention evaluated by Trial Making Test (TMT) A; divided spatial attention evaluated by with TMT B; cognitive flexibility TMT AB (Giovagnoli et al., 1996); phonological lexicon access with a Verbal Fluency Test (FAS), which is also a measure of the executive functioning domain (Novelli et al., 1986; Bianchi and Dai Prà, 2008); short and long term episodic memory with Babcock Memory test (Carlesimo et al., 1996) and executive functions with the Frontal Assessment Battery (FAB) test (Lezak and Lezak, 2004; Appollonio et al., 2005). This procedure was repeated at the post-test phase and these scores were analyzed using Statistical 8 and Statistical Package for Social Sciences (SPSS, Inc, Chicago), version 15.0.T.

\section{Behavioral Analysis}

Arithmetic mean and standard deviation, as well as median, percentage and range were used to report the general characteristics of the study population and controlled between group using $t$-test or Chi-square statistic (Table 1). To compare the intervention group and the control group at enrollment, general linear model statistical test was performed. The analyzed outcomes were the pre- and post-differences in performance. To indicate statistical difference, two-tailed $P$-value of less than 0.05 was considered. The baseline cognition was included as a covariate. The significance threshold was further adjusted for multiple comparisons using Bonferroni's correction. This data analysis was carried out using the software Statistica 8.

\section{Imaging Procedure}

Images were acquired with a Philips Achieva 3 Tesla scanner (Philips Medical Systems, Best, Netherlands) using a wholebody radiofrequency coil for signal excitation and an 8-channel phased-array head coil for signal reception. A high-resolution structural volume was first acquired using a 3D fast field echo T1-weighted sequence (sagittal, matrix $240 \times 240$, FOV $=256 \mathrm{~mm}$, slice thickness $=1 \mathrm{~mm}$, no gap, in-plane voxel size $=1 \times 1 \mathrm{~mm}$, flip angle $=8^{\circ}$, TR $=8.2 \mathrm{~ms}$ and $\mathrm{TE}=4 \mathrm{~ms}$ ). Afterward, the data from Blood Oxygen Level Dependent (BOLD) fMRI were obtained using a gradient-echo T2*-weighted echo-planar (EPI) sequence with the following parameters: matrix $64 \times 64$, voxel size $3.6 \mathrm{~mm} \times 3.6 \mathrm{~mm} \times 5 \mathrm{~mm}$, SENSE 1.8, TE $=30 \mathrm{~ms}, \mathrm{TR}=1.1 \mathrm{~s}$. Three runs were acquired, with 300 volumes per run. During fMRI, cardiac (ppu) and respiratory (belt) data were also acquired. Physiological signals were recorded using a pulse oximeter placed on a finger of the left hand and a pneumatic belt strapped around the upper abdomen. Cardiac and respiratory data were both sampled at $100 \mathrm{~Hz}$ and stored by the scanner software in a file for each run. 


\section{Enrollement phase: 30 healthy elderly \\ Neuropsychological Battery and fMRI \\ Randomization}

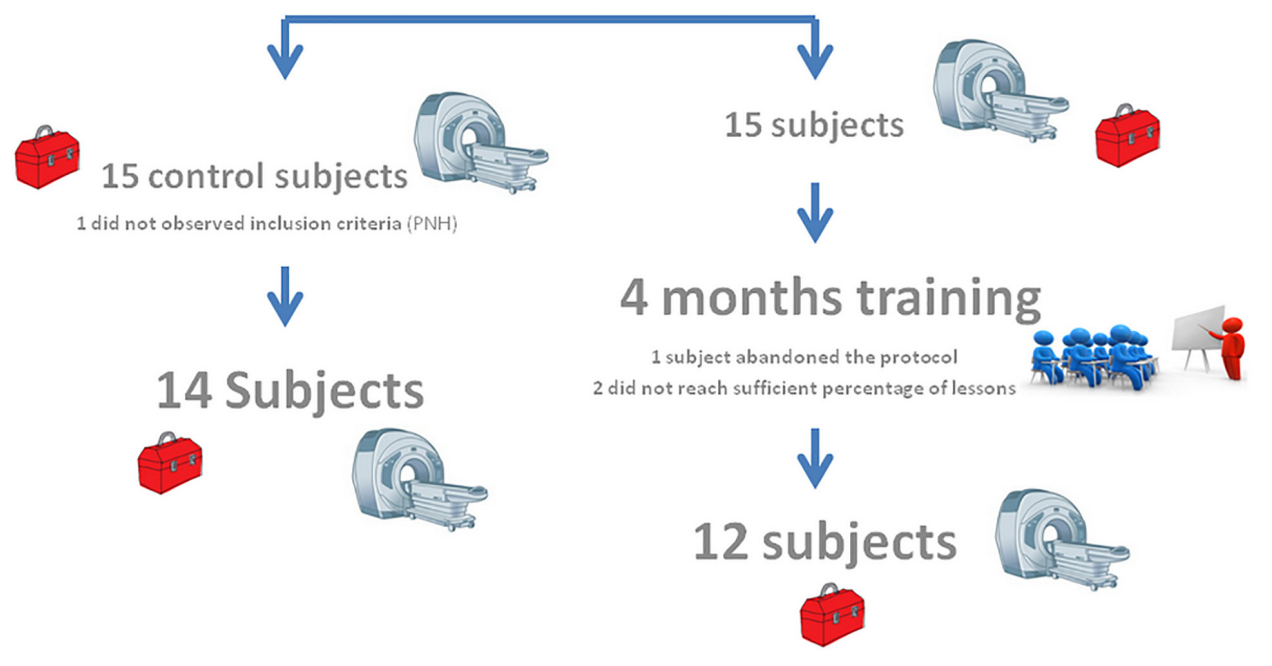

FIGURE 1 | Study design. The pictogram illustrates the study design paradigm.

\section{fMRI Data Pre-processing}

AFNI Software was used to perform the analysis of fMRI data ${ }^{1}$. To allow T1 balancing equilibration of the MR signals, the first five volumes of each functional run were discarded. First, despiking (AFNI's "3d Despike") was performed to remove transient signal spikes from the EPI time series, followed by RETROICOR (Glover et al., 2000) to remove signal fluctuations related to cardiac and respiratory cycles and slice scan time correction. Motion correction was performed using rigid body registration of EPI images to the sixth volume of the first run. To remove further physiological and hardware related confounds, ANATICOR (Jo et al., 2010) was employed for additional pre-processing. A global nuisance regressor was obtained extracting the EPI average time course within the ventricle mask and local nuisance regressors were obtained calculating for each gray matter voxel the average signal time course for all white matter voxels within a $3 \mathrm{~cm}$ radius (Jo et al., 2010). AFNI’s @ANATICOR was used to remove nuisance regressors and the six regressors derived from motion parameters from the EPI timeseries of each run. Structural scans segmentation done by FreeSurfer ${ }^{2}$ permitted to obtain individual masks of large ventricles and white matter. Then we performed a co-registration to EPI using an affine transformation.

Finally, preprocessed functional scans were normalized to the MNI space, spatial smoothing ( $6 \mathrm{~mm}$ FWHM), and bandpass filtering $(0.01-0.1 \mathrm{~Hz})$ were performed. The framewise displacement $(\mathrm{FD})$ and the root average square value of the differentiated BOLD timeseries were calculated (DVARS) within

${ }^{1}$ afni.nimh.nih.gov/afni

${ }^{2}$ http://surfer.nmr.mgh.harvard.ed a whole brain spatial mask. Quality control measures to inspect between-groups differences of motion effects, which could potentially not be calculated, were added in the special registration and regression of motion parameters accounted by spatial registration and regression of motion parameters (Power et al., 2012, 2014).

\section{Functional Connectivity Analysis}

First of all, seed-based resting state connectivity maps were created for individual subjects calculating the Pearson correlation coefficient ( $r$-value) between the Posterior Cingulate Cortex (PCC) of the Default Mode Network (DMN) time series and the time series at each voxel. The PCC time series was derived by averaging the time courses of voxels inside a sphere with a $6 \mathrm{~mm}$ radius (Table 2). Individual correlation maps were converted using the $\mathrm{z}$-Fisher transformation $(\mathrm{z}=$ atanh $(\mathrm{r})$, where $\mathrm{r}$ is the correlation coefficient) to approach a normal distribution before calculating the random effect group analysis.

A one-sample $t$-test was performed on the z-Fisher maps to obtain group statistical functional connectivity maps, separately for the control and the intervention groups. These group statistical maps were thresholded at $p<0.05$ corrected for multiple comparisons using False Discovery Rate (FDR) and were utilized to visually inspect the level of connectivity for the two groups. Then, to quantify statistically significant differences across groups and time, a number of spherical nodes $(6 \mathrm{~mm}$ of radius) for each region, which is known to have a correlation, were defined using independent coordinates from the literature (see Table 2). The examined nodes have been chosen to be correlated, that is they increase their activity simultaneously, 
TABLE 2 | Principal brain networks investigated in MNI coordinates.

\begin{tabular}{rrrcll}
\hline $\boldsymbol{x}$ & $\boldsymbol{y}$ & $\boldsymbol{z}$ & Seed & \multicolumn{1}{c}{ Brain region } & $\begin{array}{c}\text { Brain } \\
\text { network }\end{array}$ \\
\hline 0 & -51 & 29 & PCC & Posterior cingulate cortex & DMN \\
-32 & 16 & -8 & LAIFO & Anterior insula frontal operculum & SLN \\
27 & 3 & 57 & FEF & Frontal eye field & DAN \\
-56 & -44 & 22 & LTPJ & Temporal parietal junction & LAN \\
1 & -87 & -2 & LG & Lingual gyrus & VIS \\
-51 & -18 & 7 & LSTG & Superior temporal gyrus & AUD \\
-52 & -9 & 31 & PCG L & Pre-central gyrus & MOT \\
-51 & -54 & 37 & LIFC & Bilateral parietal cortex & FPCN \\
-13 & -14 & 4 & IPG & Inferior parietal gyrus & CEN \\
\hline
\end{tabular}

or anti-correlated, i.e., they decrease their activity with the PCC (Esposito et al., 2018).

This procedure was utilized in order to avoid circularity problems in the analysis (Kriegeskorte et al., 2009). Individual connectivity values were extracted from these regions of interest (ROI) and compared across groups and conditions using a repeated measure analysis based on multivariate modeling (MVM) approach as implemented in R Software (Chen et al., 2014). A multivariate, seed-based approach was employed to assess functional connectivity in brain networks simultaneously by including a seed for the DMN (the posterior cingulate cortex, or PCC). This seed-approach is useful for distinguishing network activity between groups of participants, and for distinguishing connectivity patterns that differ across brain regions (Campbell et al., 2013). Although there are conflicting opinions (Mineroff et al., 2018; DeLuca et al., 2019), multifaceted cognitive abilities (and language is one of those) depend on multiple mental processes that engage different large-scale functional networks including parietal, frontal and temporal cortical regions of the brain. In particular, executive processes involved in second language learning (including cognitive control, semantic processing, and working memory) are supported by DMN, executive control network (ECN) and language network (Varela et al., 2001; Woolgar et al., 2017). Since the DMN has been shown to be involved in age related changes, which are reflected in both within and between network connectivity modifications (Wu J.-T. et al., 2011; Esposito et al., 2018), the PCC (which is considered the main hub of the DMN) was chosen as a seed region for our analysis.

In contrast, choosing a seed region of the language network could have limited the investigation of potential plasticity effects due to the present training in regions not strictly linked to linguistic aspects.

Furthermore, semantic/conceptual processing engages regions of both DMN and language network (Chen et al., 2006; Toro et al., 2008; Uddin et al., 2009).

Data were analyzed with a linear mixed effects model in R3, which estimates both parameters using Maximum Likelihood Estimation and effects using specific contrast matrices. The fixed factors were defined as the group (control versus intervention) and time (T0 versus T1), and the subject of either group was entered as a random factor. By considering the nine ROIs, the number of statistical tests which were performed were 18 comparisons. To prevent Type I error, contrasts were both assessed at $p<0.05$ corrected for Bonferroni multiple comparisons.

\section{fMRI Data Analysis and Cognition}

Pearson's correlation analysis was performed in each group separately to examine the association between cognition (MMSE corrected score for age and education) and functional variables (connectivity signal variation in regions showing between-group effects). Sex and educational level were also included as a co-variate. Statistical Package for Social Sciences (SPSS, Inc, Chicago), version 15.0.T was used for the purpose. Statistical significance for correlation analysis was set at $p<0.05$, corrected for multiple comparisons using Bonferroni correction.

\section{RESULTS}

Results of the study indicate that a 4 months second language learning intervention improves global cognitive performances and reorganizes functional connectivity.

\section{Cognitive Performances}

Control and intervention subjects were evaluated at the baseline phase (T0) and at the end of the 4 months (T1) period for their neuropsychological abilities. Four subjects were excluded, one did not observe inclusion criteria [had periventricular nodular heterotopia $(\mathrm{PNH})$ ], two did not accept to be retested at posttraining condition, and one did not attend a sufficient percentage of lessons. We observed slight differences between group in terms of age (Control group Mean: 65.7, SD 3.7; Intervention group: Mean 69.5, SD 5.3; One-Way ANOVA $F=4.42, p=0.046$ ) and education (Controls: Mean 13.0, SD 3.0; Intervention group Mean 9.6 SD 2.9; One-Way ANOVA $F=8.43, p=0.0008$ ). A detailed description of statistical analysis results can be seen in Table 1. The normality of the distribution was controlled by Kolmogorov-Smirnov test (Ksd $d=0.11, p>0.20$ ). Statistically significant differences in MMSE score were found within and between the two groups at both T0 and T1 $(p=0.009)$. In more details, the two groups significantly differ at T0, with the control group performing better than the intervention group (29.35 versus 27.23, Duncan post hoc $p=0.001$ ); on the contrary, the between group difference disappeared at T1 (28.28 versus 27.81 , Duncan post hoc $p=0.42$ ). In fact, only the control group significantly decreased its performances over time (29.35 versus 28.28 Duncan post hoc $p=0.017$ ), whereas the intervention group remained stable (27.23 versus 27.81) (see Table 3 and Figure 2). Since language is a task that involves many cognitive abilities, several cognitive domains were investigated for this purpose, but all these domains were involved in the aging-related cognitive decline. Therefore, the performance in prose memory was then studied using the prose memory test (Babcock story version A) (Horner et al., 2002), a test that investigates short-term and long-term memory. The performance of both groups in their attention skills was evaluated by using TMT (Giovagnoli et al., 
TABLE 3 | MMSE values; Group 0 is referred to Control group while Group 1 is referred to Intervention group.

\begin{tabular}{|c|c|c|c|c|c|}
\hline \multicolumn{6}{|c|}{ MMSE score } \\
\hline & \multicolumn{2}{|c|}{ Group 0} & \multicolumn{2}{|c|}{ Group 1} & \\
\hline & Mean & $S D$ & Mean & $S D$ & \\
\hline TO & 29.35 & 1.18 & 27.23 & 1.72 & \\
\hline \multirow[t]{2}{*}{ T1 } & 28.28 & 1.76 & 27.81 & 1.01 & \\
\hline & $\boldsymbol{F}$ & $D F$ & $P$ & \multicolumn{2}{|r|}{ Duncan post hoc effect } \\
\hline Within effect (Time) & 0.68 & 1.24 & 0.419 & 0.549 & - \\
\hline Between effect (Group) & 6.88 & 1.24 & 0.015 & 0.015 & Group 0 > Group 1 \\
\hline Quadratic interaction & 7.98 & 1.24 & 0.009 & 0.017 & Group 0 T0 > Group 0 T1 \\
\hline \multicolumn{6}{|l|}{ (Group × Time) } \\
\hline & & & & 0.17 & Group $1 \mathrm{TO}=$ Group $1 \mathrm{~T} 1$ \\
\hline & & & & 0.001 & Group 0 TO > Group 1 TO \\
\hline & & & & 0.422 & Group 0 T1 = Group 1 T1 \\
\hline
\end{tabular}

1996), a test that analyzes visual attention and task switching. The results of different subsets, $\mathrm{A}$ and $\mathrm{B}$ (Test-A: sustained attention; Test B: divided attention; and Test B-A: task coordination and set-shifting) were also analyzed.

Moreover, Frontal Assessment Battery (FAB) (Dubois et al., 2000; Cyarto et al., 2010) test and Verbal Fluency (FAS) (Tombaugh et al., 1999; Costa et al., 2014) test were performed: they are two tests that are employed to evaluate the functioning of frontal lobes (FAB) as well as attention or lexical production (FAS) (see Table 4 ).

Finally, the subject autonomy for daily and instrumental activities (ADL, IADL) (Song et al., 2014) was investigated. No statistically significant differences were found within and between the groups for these tests (see Supplementary Figure 1).

\section{Brain Functional Connectivity}

Performing MVM FMRI interaction analysis between baseline (T0) and after 4 months (T1) in both groups (ANCOVA interaction, $p=0.001$, FDR corrected), significant connectivity changes in specific areas of language network (LAN) and control executive network (CEN) were found (Figure 3). All FMRI data were controlled for age and education, entering these variables as co-variates. Significant $(p<0.001)$ longitudinal increases were found in the intervention group in the LAN for the strength of functional connectivity in the right inferior frontal gyrus (rIFG) (MNI 35.5, 27.5, -11.5) and in the right superior frontal gyrus (rSFG) (MNI 14.5, 54.5, 30.5) regions ( $t$-test between $\mathrm{T} 0$ and $\mathrm{T} 1, p=0.001, t=3.703$, Figures 4, 5). Moreover, CEN network revealed a change in the strength of functional connectivity in the trained group in the superior parietal lobule (SPL), (MNI - 24.5 - 56.5 60.5), (SPL; $t$-test between $\mathrm{T} 0$ and $\mathrm{T} 1$ in the training group, $p=0.001$; $t=3.703$, Figure 6).

Analysis of the other Resting State Networks (RSNs, Table 2) did not show significant modifications.

Finally, a Pearson Correlation analysis between significant neuropsychological results and fMRI data was performed.

TABLE 4 | Neuropsychological performances.

\begin{tabular}{lccccc}
\hline \multicolumn{7}{c}{$\begin{array}{c}\text { Intervention T0 } \\
\text { Mean/SD }\end{array}$} & $\begin{array}{c}\text { Intervention T1 } \\
\text { Mean/SD }\end{array}$ & $\begin{array}{c}\text { Controls T0 } \\
\text { Mean/SD }\end{array}$ & $\begin{array}{c}\text { Controls T1 } \\
\text { Mean/SD }\end{array}$ & p \\
\hline Global cognition & & & & \\
MMSE & $27.23 / 1.66$ & $27.81 / 0.97$ & $29.35 / 1.13$ & $28.28 / 1.68$ & $0.009^{*}$ \\
Speed attention & & & & \\
TMT A $\quad 33.78 / 27.74$ & $26.90 / 16.40$ & $62.91 / 42.11$ & $49.91 / 23.18$ & 0.61 \\
TMT B $\quad 58.35 / 68.16$ & $55.85 / 60.99$ & $83.25 / 38.39$ & $72.66 / 28.80$ & 0.62 \\
TMT AB $\quad 18.60 / 31.69$ & $25.95 / 54.41$ & $20.83 / 61.50$ & $29.08 / 11.67$ & 0.96 \\
Immediate and delayed verbal memory & & & \\
IR & $6.75 / 1.95$ & $7.67 / 0.98$ & $6.37 / 1.50$ & $7.08 / 1.47$ & 0.79 \\
DR & $6.73 / 1.50$ & $7.64 / 1.08$ & $6.36 / 1.43$ & $6.83 / 1.34$ & 0.55 \\
Language & & & & & \\
FAS & $36.5 / 6.86$ & $37.42 / 8.53$ & $34.42 / 9.46$ & $35.3 / 9.13$ & 0.98 \\
Executive functions & & & & \\
FAB & $17.10 / 1.32$ & $17.14 / 1.47$ & $17.1 / 1.2$ & $17.04 / 1.53$ & 0.90
\end{tabular}

Means and standard deviation. *Indicates $p<0.05$.
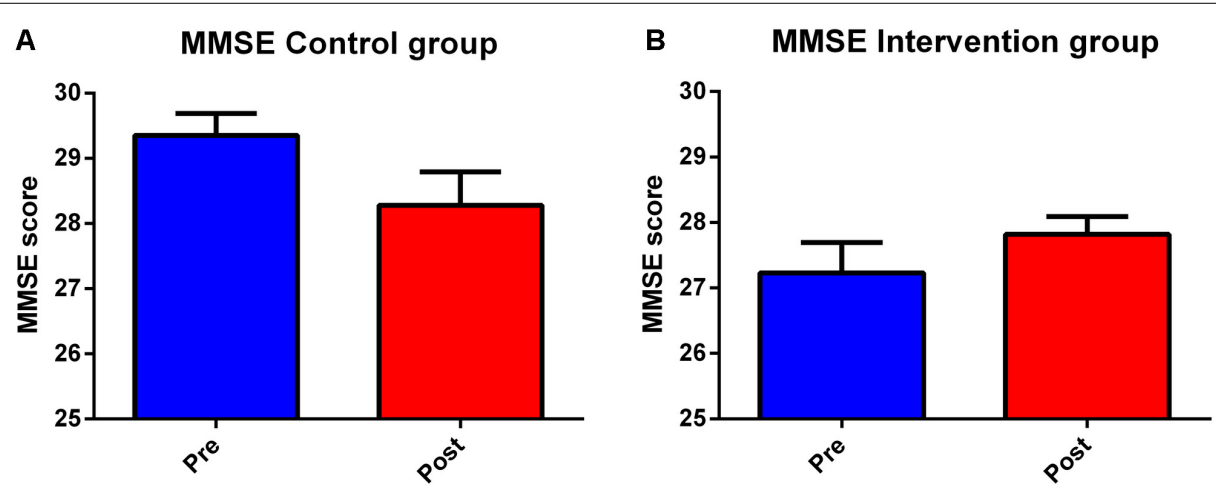

FIGURE 2 | Second language learning positively affects global cognition performances. Histograms depict results of neuropsychological evaluation in control (A) and intervention (B) groups at the beginning of the study (Pre) and after 4 months (Post). Graphs show results, expressed as means of MMSE and SEM. The trained group shows, after 4 months (T1), a statistically significant improvement compared to control group. 

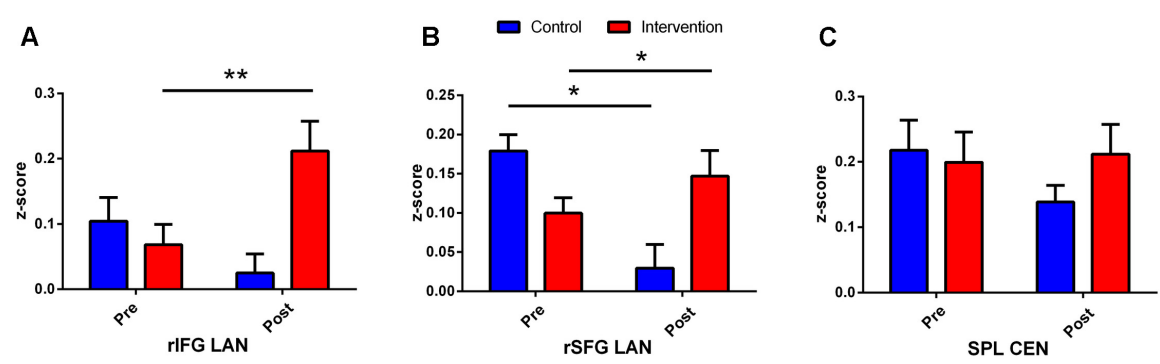

FIGURE 3 | Second language learning improves neuronal connectivity: effects in the Language Network (LAN) and in the Central Executive Network (CEN). The graphics depict the connectivity values from rIFG (A), rSFG (B), and SPL (C) extrapolated from control and intervention group difference (T1-T0). *Indicates $p<0.05$ and $* *$ indicates $p<0.01$.

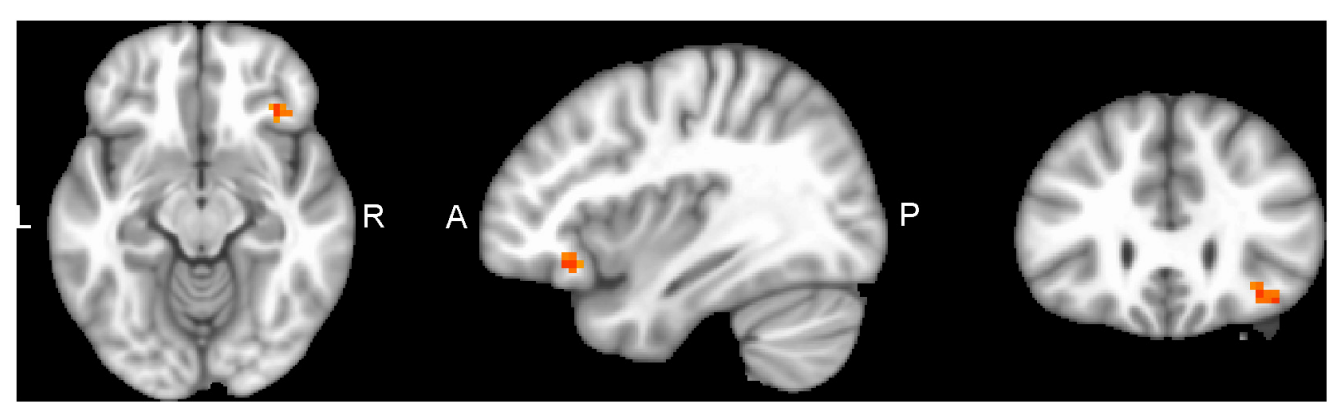

FIGURE 4 | rIFG of LAN network connectivity, non-radiological system L = L. Results from the right inferior frontal gyrus (rIFG, MNI 35.5 27.5 - 11.5) from multivariate modeling (MVM) approach correlation analysis is displayed. BOLD connectivity showed a greater increase in the rIFG from pre- to post-training $(p<0.05$, FDR corrected).

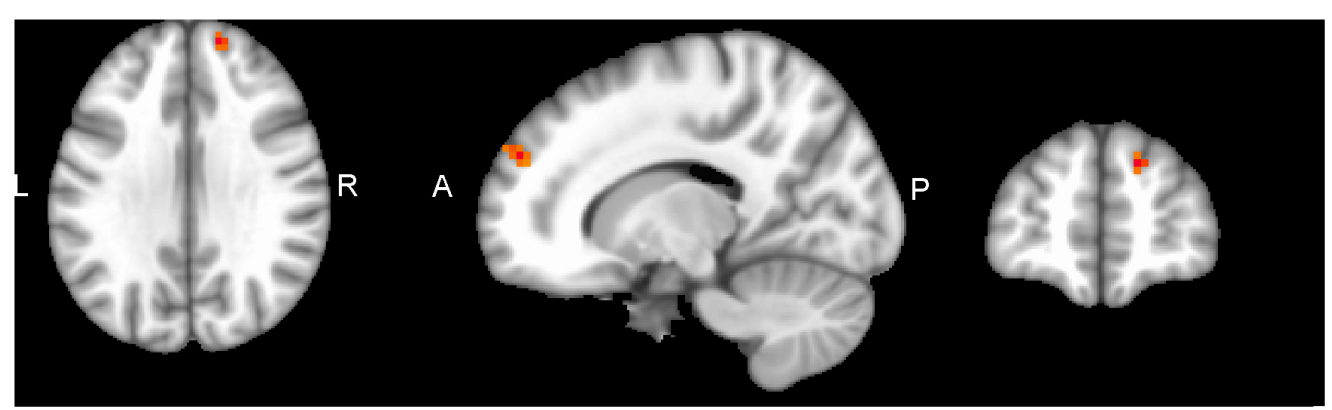

FIGURE 5 | rSFG of LAN network connectivity, non-radiological system L = L. Results from the right superior frontal gyrus (rSFG MNI 14.5 54.5 30.5) from multivariate modeling (MVM) approach correlation analysis is displayed. Connectivity values showed a greater increase in the rSFG from pre- to post-training ( $p<0.05$, FDR corrected).

The relationships between longitudinal functional connectivity changes and global cognition changes were assessed by extracting connectivity values from the mean values of the voxels in the cluster showing a group $\times$ time interaction effect on connectivity.

For the trained group, a positive significant correlation between the strength of functional connectivity in $\mathrm{rSFG}$ and the global cognition abilities (Pearson $r=0.4255, p=0.03$ ) was found.

Changes in connectivity values correlated positively with changes in MMSE score, and all this supported the hypothesis that all the observed changes in the treated group were driven by the language learning course exposure and not by the 4 months time interval. A detailed description of correlation analysis is shown in Figure 7.

To consider the possible confounding effect of differences in MMSE between groups at baseline phase, the MMSE at T0 was entered as a co-variate in the general linear model analysis. Also in this case, results remained significant in the $\operatorname{rIFG}[p=0.02$, $F(1,23)=5.68]$ and in the $\operatorname{rSFG}[p=0.04, F(1,23)=4.70]$ instead SPL showed only a statistical trend $[p=0.08, F(1,23)=3.13]$.

Moreover, to verify if the MMSE differences were linked to connectivity changes, GLM between $\Delta$ (T1 minus T0) 


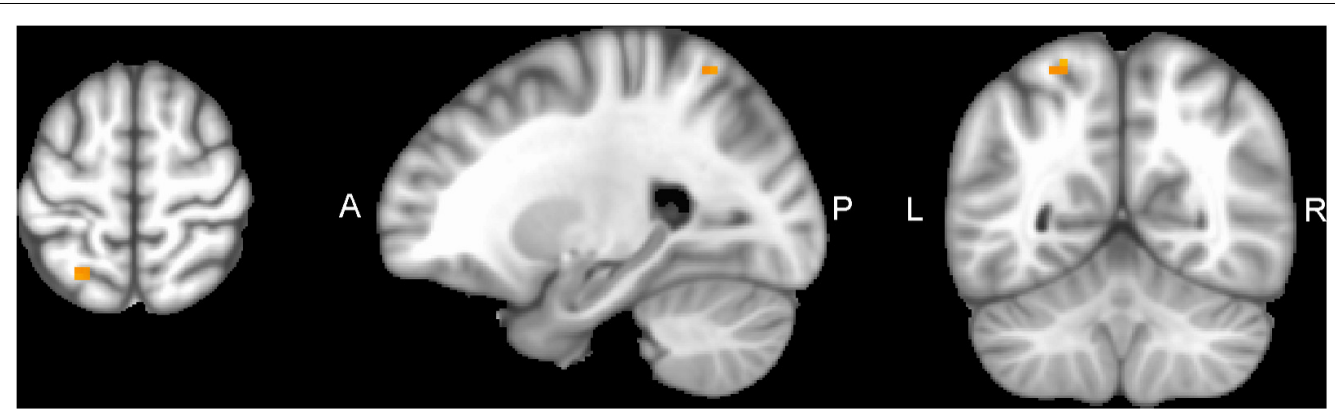

FIGURE 6 I SPL of CEN network connectivity, non-radiological system L = L. Results from the superior parietal lobule (SPL MNI $-24.5-56.560 .5)$ from multivariate modeling (MVM) approach correlation analysis are displayed. Connectivity values showed a greater increase in the SPL from pre- to post-training $(p<0.05$, FDR corrected).

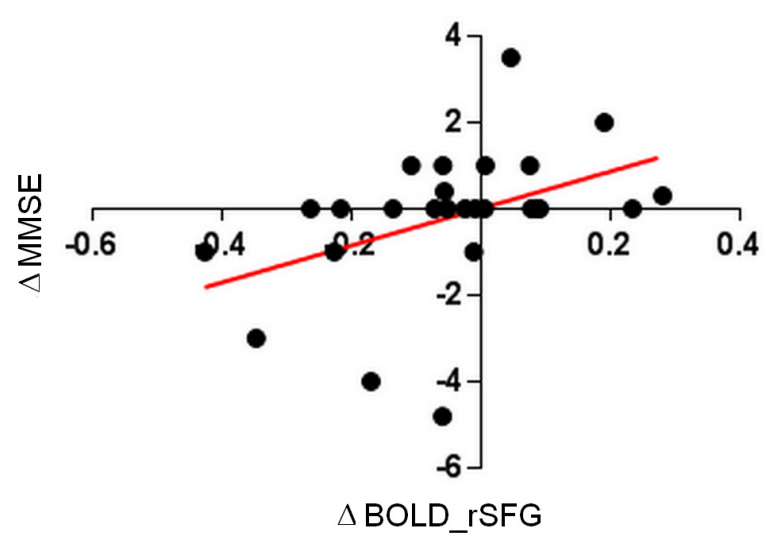

FIGURE 7 | Pearson correlation of global cognition differences between T1 -TO (DMMSE) and connectivity differences values for right superior frontal gyrus ( $\triangle \mathrm{rSFG})$.

connectivity values for LAN and CEN clusters and the $\Delta$ MMSE was performed.

Results were significant for $\mathrm{rSFG}[p=0.012, F(1,23)=7.35]$ and for $\operatorname{rIFG}[p=0.013, F(1,23)=7.08]$; SPL showed, however, a trend $[p=0.08, F(1,23)=3.13]$ Yates corrected Chi-square. (Figure 3)

Only in the intervention group there was a significant change in both functional and behavioral measures after intervention, thus supporting the initial hypothesis that language learning training can improve cognition in healthy elderly subjects.

The present data were also controlled for age and educational level, in this case results were significant for $\operatorname{rIFG}[p=0.024$, $F(1,21)=5.89]$, instead rSFG showed, however, a trend $[p=0.07, F(1,21)=3.59]$ and SPL was not significant for this condition $[p=0.39, F(1,21)=0.73]$.

\section{DISCUSSION}

The present study aimed at investigating neuroplastic-related effects of second language learning in terms of cognitive and brain networks functional connectivity changes. For this purpose, two groups of healthy elderly undergoing 4 months of second language learning course were tested. Resting-state fMRI was employed to observe effects on brain functional connectivity and cognition. Short-term longitudinal changes in functional connectivity together with an improvement in global cognition were also observed. Three new findings can be detected through the present study: an increase in global cognition scores in the intervention group (1), a significant short-term increase in functional connectivity in language network and CEN. The present work showed a significant longitudinal increase in the right inferior frontal gyrus and in the rSFG regions. Analysis of the CEN revealed a change in the strength of functional connectivity in the superior parietal lobule (2). Rate of change in global cognition was positively correlated with functional connectivity improvements, suggesting a relationship between behavioral change and functional alterations (3). There are several studies showing an improvement in cognition after physical or cognitive training or combined (Colcombe et al., 2006; Voss et al., 2010; Erickson et al., 2011; Kivipelto et al., 2013; Carlson et al., 2015; Ngandu et al., 2015). As far as one can know, this is the first study demonstrating an impact of short-term second language learning on cognition along with functional connectivity of language and control network in aging. Studies report brain connectivity changes in response to cognitive learning, motor training or both (Pieramico et al., 2012; Antonenko and Flöel, 2014). Nevertheless, as far as the authors' know, functional neuroplastic effects of a short language learning intervention in healthy elderly individuals, have not been analyzed with functional magnetic resonance (fMRI) methods (Antoniou et al., 2013).

The present results showed increased functional connectivity in the language network, in particular in right inferior frontal gyrus and rSFG, together with the left parietal lobule of the control network. These regions are critical for different processes. The right inferior frontal gyrus, which is involved in inhibition and attentional control, also known as Brodmann Area 44, has been implicated in go/no go tasks (Hampshire et al., 2010), more specifically it runs impulse control through inhibition. This kind of process is needed in the switching between two languages in bilingual people, and it has been shown that it can protect brain in dementia condition (Luk et al., 2011b; Green and Abutalebi, 
2013; Costa and Sebastián-Gallés, 2014; Rossi and Diaz, 2016). The rSFG is involved in control of impulsive response, a hallmark of cognitive control (Hu et al., 2016). The superior frontal gyrus (SFG) is located at the superior part of the prefrontal cortex and is involved in a variety of functions, it has also been parcellated in subregions which are: anteromedial (SFGam), dorsolateral (SFGdl), and posterior (SFGp) subregions which were divided according to the diffusion tensor tractography (Li et al., 2013; Hsiao et al., 2015). The SFGam is anatomically connected with the anterior and mid-cingulate cortices, which are critical nodes of the cognitive control network and the default mode network. The SFGdl was connected with the middle and inferior frontal gyri, which are involved in the executive network. The SFGp was connected with the precentral gyrus, caudate, thalamus, and frontal operculum, which are nodes of the motor control network. Resting-state functional connectivity analysis further revealed that the SFGam is mainly correlated with the cognitive control network and the DMN; the SFGdl was correlated with the cognitive execution network and the DMN; and the SFGp was correlated with the sensorimotor-related brain regions. The LPL is involved in retrieval of learnt facts and these are also involved in conceptual decisions on object names (Cappelletti et al., 2010). The present results are in line with and corroborate previous studies indicating the role of those brain regions in control processes. Also in bilingual or multilingual people, between-language competition requires neuronal effort to suppress activation of the non-target language (Marian et al., 2014, 2017). This neural effort is supposed to be involved in protecting against pathological aging and in the dementia delay (Abutalebi and Weekes, 2014; Alladi et al., 2014; Bak et al., 2014; Bialystok et al., 2016).

It has already been discussed that functional brain connectivity may be sensitive to disease-specific network changes in neurodegenerative diseases (Pievani et al., 2011). Assessed with resting-state connectivity, fMRI has shown distinct patterns of network disruption across the major neurodegenerative diseases.

Different works show how default mode network undergoes functional anomalies in Alzheimer Disease (AD) (Wu X. et al., 2011) or functional changes in the salience network in frontotemporal dementia (Filippi et al., 2017).

Properties of brain networks in healthy controls, compared to patients with behavioral variant of frontotemporal dementia (bvFTD) and patients with early-onset Alzheimer disease (EOAD) has been observed with graph analysis, a method for the analysis and representation of complex networks. In those studies, DMN is crucially impaired in AD, whereas ECN, dorsolateral prefrontal attention network, and semantic appraisal network are impaired in bvFTD. It can be hypothesized that the observed changes in LAN and CON in healthy aging can indirectly modify the functional connectivity of DMN together with other main brain networks. The brain is intrinsically organized into dynamic, correlated and anticorrelated functional networks, second language learning can longitudinally preserve principal network from deterioration (Fox et al., 2005). Moreover, plastic changes in the language network are, in the present work, lateralized to right hemisphere. Language functions are normally lateralized to the left hemisphere for right handed individuals. However, plasticity changes in the right hemisphere were observed. This could be expected in the case of second language acquisition whose process showed in adults right hemisphere involvement as well (Briganti et al., 2012; Plante et al., 2015). Moreover, other studies on bilingualism have provided evidence of reduced left lateralization (i.e., greater right hemisphere participation) for verbal tasks performed using the second language rather than the first, suggesting that the right hemisphere plays a role in the early stages of both child and adult language acquisition (Oxford and Ehrman, 1995).

This result can be explained with recent findings indicating that learning a second language triggers the recruitment of contralateral brain areas. With diffusion tensor imaging (DTI) measuring resting-state functional connectivity in monolingual and bilingual older adults, Luk et al. (2011a,b), showed higher white matter integrity in bilingual older adults, primarily in the corpus callosum connecting the two hemispheres but also extending to bilateral superior longitudinal fasciculi, right inferior frontal-occipital fasciculus and uncinate fasciculus. Luk and colleagues, with a resting-state functional connectivity analysis, showed that, while both monolinguals and bilinguals had correlating brain activity with contralateral regions at rest, bilinguals had increased anterior-posterior connectivity.

The present work shows that the approach of characterizing the brain as a network using rs-fMRI and MVM analysis can provide new insights into how language learning affects brain function and functional connectivity in aging.

Several protocols have been used with the purpose of maintaining healthy cognitive functions (Cotlarciuc et al., 2016; Brem and Sensi, 2018). In a recent study, Ware et al. (Ware et al., 2017) have shown that computer-assisted learning of a second language leads to social and motivational benefits, although they did not investigate different cognitive domains. In the present study, subjects were tested with a comprehensive battery for cognitive abilities as well as for changes in brain functional connectivity. Brain plasticity and potential reorganization against behavioral and functional brain deterioration were investigated. These results are in line with studies showing changes in brain reserve and metabolic connectivity in bilinguals ( $\mathrm{Li}$ et al., 2017; Perani et al., 2017). The results of the analysis indicate beneficial effects of second language learning late in life on global cognition. Despite starting from a lower global cognitive level, the intervention group reached a higher global cognition improvement (MMSE T0 MEAN $=27.23, S D=1.66$; T1 MEAN = $27.81, S D=0.97)$, in comparison to the control group (T0 MEAN = 29.35, $S D=1.13$; T1 MEAN = 28.28, $S D=1.68$ ).

Learning a new language, also late in life, is a big cognitive challenge (Blumenfeld et al., 2017). The present work supports the idea that the aging brain is a dynamic set of biological features that can plastically reorganize against pathological decline. Achieving positive results is possible thanks to a reorganization of a set of brain mechanisms, including adult neurogenesis, synaptic changes, dendritic branching, axon sprouting or changes in the number and morphology of glia cells, for both astrocytes and microglia (Wenger et al., 2017). 
In the field of non-pharmacological stimulation against pathological aging, the present results suggest that rs-fMRI can be used to detect connectivity changes after a period of 4 months, demonstrating sensitivity of BOLD signal as an imaging biomarker for functional connectivity short-term changes in aging. Thanks to the adaptive and plastic structure of our brain, even late in the elderly, the brain is able to respond dynamically to cognitive challenges. It remains to be investigated whether such brain changes will be maintained over time.

However, some technical limitation must be included. The size of the present cohort is relatively small, and the training effects of the intervention group were compared with those of a passive, but not an active, control group.

Collectively, our findings show that just 4 months of learning a second language leads to functional reorganization processes in the mature human brain together with an improvement in global cognition. These findings crucially complement current neural concepts of neuroplasticity in aging brain, a condition that can delay any pathological cognitive process and dementia. The current study gives a contribution in the field of brain training. The concept of brain and cognitive reserve, that is the brain resilience capacity (Stern, 2012), become a resource that could be one shock-absorber for pathological aging and can be increased even later in life.

The present study confirms the ability-capability of the aging brain in reorganizing neural networks maintaining and even improve mental functioning despite aging.

\section{CONCLUSION}

In conclusion, the present results demonstrate that longitudinal changes in functional connectivity and in cognition can be detected over an interval of merely 4 months in middle-aged and older adults. Furthermore, connectivity changes differed between the control and the intervention groups, suggesting a positive impact of second language learning on short-term functional connectivity trajectories.

Change in global cognition performances addressed by the present intervention, correlated positively with the rate of change in the bold signal. This can support rs-fMRI as a behaviorally relevant imaging biomarker.

Aging is paralleled by an increase in deterioration of cognitive abilities, however, with a simple, short and economic training, robust effects in terms of brain connectivity, global cognition functioning, and brain plasticity can be provided. Challenging aging with a learning stimulation can be a powerful tool to reorganize neuronal networks and cognitive behavior with the involvement of boosted neuronal activity.

Brain dynamism of the aging system can be more consistent and can bring global improvement taking advantage of mechanisms like cognitive reserve and functional plasticity (Greenwood and Parasuraman, 2003).

A simple and short cognitive intervention can be designed to improve cognition supported by the reorganization of functional brain circuitry and the increase in neuronal structures. A picture of a static decline with aging can be easily improved with a dynamic way of life, by means of stimulation to continuous learning of new knowledge and with healthy and dynamic lifestyles. These results should consider that a second language learning program, even late in life, can be considered a non-pharmacological treatment able to counteract cognitive aging along with the onset of dementia. Learning a second language is a powerful tool that can be part of a healthy lifestyle program that can preserve brain plasticity in aging individuals. Further studies are needed to explore whether these improvements are long-lasting or are reverted at the end of the training period.

\section{ETHICS STATEMENT}

This study was performed in accordance with the recommendations of the Declaration of Helsinki. All subjects signed written informed consent. The protocol was approved by the Ethics Committee of G. d'Annunzio University of Chieti, Italy.

\section{AUTHOR CONTRIBUTIONS}

GB conceived the idea, designed the research and wrote the manuscript. GB, MdM, MP, AF, and MGP supervised the experiments, exported the data, and reviewed the manuscript. PC and GS ran the statistical analysis. MP and MGP supervised the research and reviewed the manuscript for intellectual content. VP did the neuroradiological examinations. All authors approved the final version.

\section{FUNDING}

This study was supported by a grant from University G. d'Annunzio (FFABR - Funding Fund for Basic Research Activities) to MGP.

\section{ACKNOWLEDGMENTS}

GB would like to thank Dr. A. Granzotto for plotting and Dr. Errico Di Fabio for language revision. D. Petrucci and D. Calvo Garcia for precious support during subject's acquisition. We are grateful to all study participants for their time and effort. Moreover, we thank Prof. N. Ciacio and Prof. F. D'Ettorre for performing language lessons at the C. d'Ascanio College of Montesilvano. Thanks also to G. Tini and the Mazzaferro University of Third Age of Montesilvano for subject recruitment and language lesson organization. GB would like to thank Oelilag di Otesor and Attollap di Alletidnacasac for valuable advice and support.

\section{SUPPLEMENTARY MATERIAL}

The Supplementary Material for this article can be found online at: https://www.frontiersin.org/articles/10.3389/fnins.2019. 00423/full\#supplementary-material 
FIGURE S1 | Second language learning affects cognitive performances. Histograms depict measures of neuropsychological evaluation in control and intervention groups at the beginning of the study (Pre) and after 4 months (Post). Graphs show results, expressed as means and SEM for the performance of both groups in their sustained attention skills using TMT_A test for controls (A) and interventions (B), divided attention (TMT_B) for controls (C), and interventions (D);

\section{REFERENCES}

Abutalebi, J., and Weekes, B. S. (2014). The cognitive neurology of bilingualism in the age of globalization. Behav. Neurol. 2014, 1-3. doi: 10.1155/2014/536727

Alkadhi, K., and Eriksen, J. (2011). The complex and multifactorial nature of Alzheimer's disease. Curr. Neuropharmacol. 9:586. doi: 10.2174/157015911798 376235

Alladi, S., Bak, T. H., Duggirala, V., Surampudi, B., Shailaja, M., Shukla, A. K., et al. (2014). Bilingualism delays age at onset of dementia, indipendent of education and immigration status. Neurology 82:1936. doi: 10.1212/01.WNL.0000437347. 92583.fc

Alzheimer's Disease International (2018). World Alzheimer Report 2018. Available at: https://www.alz.co.uk/research/world-report-2018 (accessed April 10, 2019).

Anderson, J. A. E., Grundy, J. G., De Frutos, J., Barker, R. M., Grady, C., and Bialystok, E. (2018). Effects of bilingualism on white matter integrity in older adults. Neuroimage 167, 143-150. doi: 10.1016/j.neuroimage.2017.11.038

Antonenko, D., and Flöel, A. (2014). Healthy aging by staying selectively connected: a mini-review. Gerontology 60, 3-9. doi: 10.1159/000354376

Antoniou, M., Gunasekera, G. M., and Wong, P. C. M. (2013). Foreign language training as cognitive therapy for age-related cognitive decline: a hypothesis for future research. Neurosci. Biobehav. Rev. 37, 2689-2698. doi: 10.1016/j. neubiorev.2013.09.004

Appollonio, I., Leone, M., Isella, V., Piamarta, F., Consoli, T., Villa, M. L., et al. (2005). The frontal assessment battery (FAB): normative values in an Italian population sample. Neurol. Sci. 26, 108-116. doi: 10.1007/s10072-005-0443-4

Bak, T. H., Nissan, J. J., Allerhand, M. M., and Deary, I. J. (2014). Does bilingualism influence cognitive aging? Ann. Neurol. 75, 959-963. doi: 10.1002/ana.24158

Bialystok, E. (2017). The bilingual adaptation: how minds accommodate experience. Psychol. Bull. 143, 233-262. doi: 10.1037/bul0000099

Bialystok, E., Abutalebi, J., Bak, T. H., Burke, D. M., and Kroll, J. F. (2016). Aging in two languages: implications for public health. Ageing Res. Rev. 27, 56-60. doi: 10.1016/j.arr.2016.03.003

Bianchi, A., and Dai Prà, M. (2008). Twenty years after spinnler and tognoni: new instruments in the Italian neuropsychologist's toolbox. Neurol. Sci. 29, 209-217. doi: 10.1007/s10072-008-0970-x

Blumenfeld, H. K., Quinzon, S. J. R., Alsol, C., and Riera, S. A. (2017). Predictors of successful learning in multilingual older adults acquiring a majority language. Front. Commun. 2:23. doi: 10.3389/FCOMM.2017.00023

Boissy, P., Brière, S., Corriveau, H., Grant, A., Lauria, M., and Michaud, F. (2011). "Usability testing of a mobile robotic system for in-home telerehabilitation," in Proceedings of the 2011 Annual International Conference of the IEEE Engineering in Medicine and Biology Society, (Boston, MA: IEEE), 1839-1842.

Brem, A.-K., and Sensi, S. L. (2018). Towards combinatorial approaches for preserving cognitive fitness in aging. Trends Neurosci. 41, 885-897. doi: 10. 1016/J.TINS.2018.09.009

Briganti, C., Sestieri, C., Mattei, P. A., Esposito, R., Galzio, R. J., Tartaro, A., et al. (2012). Reorganization of functional connectivity of the language network in patients with brain gliomas. Am. J. Neuroradiol. 33, 1983-1990. doi: 10.3174/ ajnr.A3064

Campbell, K. L., Grigg, O., Saverino, C., Churchill, N., and Grady, C. L. (2013). Age differences in the intrinsic functional connectivity of default network subsystems. Front. Aging Neurosci. 5:73. doi: 10.3389/fnagi.2013.00073

Cappelletti, M., Lee, H. L., Freeman, E. D., and Price, C. J. (2010). The role of right and left parietal lobes in the conceptual processing of numbers. J. Cogn. Neurosci. 22, 331-346. doi: 10.1162/jocn.2009.21246

Carlesimo, G. A., Caltagirone, C., Gainotti, G., Facida, L., Gallassi, R., Lorusso, S., et al. (1996). The mental deterioration battery: normative data, diagnostic reliability and qualitative analyses of cognitive impairment. Eur. Neurol. 36, 378-384. doi: 10.1159/000117297 task coordination and set-shifting (TMT_BA) for controls (E) and interventions (F). Global prose memory test (Babcock story, version A) was also performed, with immediate recall $(\mathrm{IR}) \mathbf{( I , J )}$ and delayed recall (DR) subtest $\mathbf{( M , N )}$. Scores for functioning of frontal lobes (FAB) are represented for controls (K) and intervention group (L). Differences for scores for lexical production (FAS) are also

displayed $(\mathbf{G}, \mathbf{H})$, together with $A D L(\mathbf{O}, \mathbf{P})$ and $I A D L$ scales $\mathbf{( Q , R )}$.

Carlson, M. C., Kuo, J. H., Chuang, Y.-F., Varma, V. R., Harris, G., Albert, M. S. et al. (2015). Impact of the baltimore experience corps trial on cortical and hippocampal volumes. Alzheimer's Dem. 11, 1340-1348. doi: 10.1016/j.jalz. 2014.12.005

Chen, E., West, W. C., Waters, G., and Caplan, D. (2006). Determinants of bold signal correlates of processing object-extracted relative clauses. Cortex 42 , 591-604. doi: 10.1016/S0010-9452(08)70397-6

Chen, G., Adleman, N. E., Saad, Z. S., Leibenluft, E., and Cox, R. W. (2014). Applications of multivariate modeling to neuroimaging group analysis: a comprehensive alternative to univariate general linear model. Neuroimage 99, 571-588. doi: 10.1016/j.neuroimage.2014.06.027

Coffman, B. A., Clark, V. P., and Parasuraman, R. (2014). Battery powered thought: enhancement of attention, learning, and memory in healthy adults using transcranial direct current stimulation. Neuroimage 85, 895-908. doi: 10.1016/j.neuroimage.2013.07.083

Colcombe, S. J., Erickson, K. I., Scalf, P. E., Kim, J. S., Prakash, R., McAuley, E., et al. (2006). Aerobic exercise training increases brain volume in aging humans. J. Gerontol. A Biol. Sci. Med. Sci. 61, 1166-1170. doi: 10.1093/gerona/61.11.1166

Collis, D., and Waterfield, J. (2015). The understanding of pain by older adults who consider themselves to have aged successfully. Musculoskelet. Care 13, 19-30. doi: 10.1002/msc.1083

Costa, A., Bagoj, E., Monaco, M., Zabberoni, S., De Rosa, S., Papantonio, A. M., et al. (2014). Standardization and normative data obtained in the Italian population for a new verbal fluency instrument, the phonemic/semantic alternate fluency test. Neurol. Sci. 35, 365-372. doi: 10.1007/s10072-013-1520-8

Costa, A., and Sebastián-Gallés, N. (2014). How does the bilingual experience sculpt the brain? Nat. Rev. Neurosci. 15, 336-345. doi: 10.1038/nrn3709

Cotlarciuc, I., Marjot, T., Khan, M. S., Hiltunen, S., Haapaniemi, E., Metso, T. M., et al. (2016). Towards the genetic basis of cerebral venous thrombosisthe BEAST consortium: a study protocol. BMJ Open 6:e012351. doi: 10.1136/ bmjopen-2016-012351

Cyarto, E. V., Cox, K. L., Almeida, O. P., Flicker, L., Ames, D., Byrne, G., et al. (2010). The fitness for the ageing brain study II (FABS II): protocol for a randomized controlled clinical trial evaluating the effect of physical activity on cognitive function in patients with Alzheimer's disease. Trials 11:120. doi: 10.1186/1745-6215-11-120

Davies, R. (2017). Gill livingston: transforming dementia prevention and care. Lancet 390:2619. doi: 10.1016/S0140-6736(17)31913-X

DeLuca, V., Rothman, J., Bialystok, E., and Pliatsikas, C. (2019). Redefining bilingualism as a spectrum of experiences that differentially affects brain structure and function. Proc. Natl. Acad. Sci. U.S.A. 116, 7565-7574. doi: 10. 1073/PNAS.1811513116

Dubois, B., Slachevsky, A., Litvan, I., and Pillon, B. (2000). The FAB: a frontal assessment battery at bedside. Neurology 55, 1621-1626. doi: 10.1212/wnl.55. 11.1621

Erickson, K. I., Voss, M. W., Prakash, R. S., Basak, C., Szabo, A., Chaddock, L., et al. (2011). Exercise training increases size of hippocampus and improves memory. Proc. Natl. Acad. Sci. U.S.A. 108, 3017-3022. doi: 10.1073/pnas.1015950108

Esposito, R., Cieri, F., Chiacchiaretta, P., Cera, N., Lauriola, M., Di Giannantonio, M., et al. (2018). Modifications in resting state functional anticorrelation between default mode network and dorsal attention network: comparison among young adults, healthy elders and mild cognitive impairment patients. Brain Imaging Behav. 12, 127-141. doi: 10.1007/s11682-0179686-y

Extance, A. (2010). Alzheimer's failure raises questions about diseasemodifying strategies. Nat. Rev. Drug Discov. 9, 749-751. doi: 10.1038/nrd 3288

Ferrucci, R., and Priori, A. (2014). Transcranial cerebellar direct current stimulation (tcDCS): motor control, cognition, learning and emotions. Neuroimage 85, 918-923. doi: 10.1016/J.NEUROIMAGE.2013.04.122 
Filippi, M., Basaia, S., Canu, E., Imperiale, F., Meani, A., Caso, F., et al. (2017). Brain network connectivity differs in early-onset neurodegenerative dementia. Neurology 89, 1764-1772. doi: 10.1212/WNL.0000000000004577

Firth, J., Stubbs, B., Vancampfort, D., Schuch, F., Lagopoulos, J., Rosenbaum, S., et al. (2018). Effect of aerobic exercise on hippocampal volume in humans: a systematic review and meta-analysis. Neuroimage 166, 230-238. doi: 10.1016/ J.NEUROIMAGE.2017.11.007

Fox, M. D., Snyder, A. Z., Vincent, J. L., Corbetta, M., Van Essen, D. C., and Raichle, M. E. (2005). The human brain is intrinsically organized into dynamic, anticorrelated functional networks. Proc. Natl. Acad. Sci. U.S.A. 102, 9673-9678. doi: 10.1073/pnas.0504136102

Frankish, H., and Horton, R. (2017). Prevention and management of dementia: a priority for public health. Lancet 390, 2614-2615. doi: 10.1016/S01406736(17)31756-7

Frisoni, G. B., Boccardi, M., Barkhof, F., Blennow, K., Cappa, S., Chiotis, K., et al. (2017). Strategic roadmap for an early diagnosis of Alzheimer's disease based on biomarkers. Lancet Neurol. 16, 661-676. doi: 10.1016/S1474-4422(17)30159-X

Giovagnoli, A. R., Del Pesce, M., Mascheroni, S., Simoncelli, M., Laiacona, M., and Capitani, E. (1996). Trail making test: normative values from 287 normal adult controls. Ital. J. Neurol. Sci. 17, 305-309. doi: 10.1007/bf01997792

Glover, G. H., Li, T. Q., and Ress, D. (2000). Image-based method for retrospective correction of physiological motion effects in fMRI: RETROICOR. Magn. Reson. Med. 44, 162-167. doi: 10.1002/1522-2594(200007)44:1<162::aid-mrm23>3.3. co;2-5

Gold, B. T. (2015). Lifelong bilingualism and neural reserve against Alzheimer's disease: a review of findings and potential mechanisms. Behav. Brain Res. 281, 9-15. doi: 10.1016/j.bbr.2014.12.006

Gold, B. T., Johnson, N. F., and Powell, D. K. (2013). Lifelong bilingualism contributes to cognitive reserve against white matter integrity declines in aging. Neuropsychologia 51, 2841-2846. doi: 10.1016/j.neuropsychologia.2013. 09.037

Grady, C. L., Luk, G., Craik, F. I. M., and Bialystok, E. (2015). Brain network activity in monolingual and bilingual older adults. Neuropsychologia 66, 170-181. doi: 10.1016/j.neuropsychologia.2014.10.042

Granzotto, A., and Zatta, P. (2011). Resveratrol acts not through anti-aggregative pathways but mainly via its scavenging properties against $A \beta$ and $A \beta$-metal complexes toxicity. PLoS One 6:e21565. doi: 10.1371/journal.pone.0021565

Granzotto, A., and Zatta, P. (2014). Resveratrol and Alzheimer's disease: message in a bottle on red wine and cognition. Front. Aging Neurosci. 6:95. doi: 10.3389/ fnagi.2014.00095

Green, D. W., and Abutalebi, J. (2013). Language control in bilinguals: the adaptive control hypothesis. J. Cogn. Psychol. 25, 515-530. doi: 10.1080/20445911.2013. 796377

Greenwood, P. M., and Parasuraman, R. (2003). Normal genetic variation, cognition, and aging. Behav. Cogn. Neurosci. Rev. 2, 278-306. doi: 10.1177/ 1534582303260641

Grundy, J. G., Anderson, J. A., and Bialystok, E. (2017). Neural correlates of cognitive processing in monolinguals and bilinguals. Ann. N.Y. Acad. Sci. 1396, 183-201. doi: 10.1111/nyas.13333

Hampshire, A., Chamberlain, S. R., Monti, M. M., Duncan, J., and Owen, A. M. (2010). The role of the right inferior frontal gyrus: inhibition and attentional control. Neuroimage 50, 1313-1319. doi: 10.1016/j.neuroimage.2009.12.109

Horner, M. D., Teichner, G., Kortte, K. B., and Harvey, R. T. (2002). Construct validity of the babcock story recall test. Appl. Neuropsychol. 9, 114-116. doi: 10.1207/S15324826AN0902_7

Houtzager, N., Lowie, W., Sprenger, S., and De bot, K. (2017). A bilingual advantage in task switching? Age-related differences between German monolinguals and Dutch-Frisian bilinguals. Biling. Lang. Cogn. 20, 69-79. doi: 10.1017/ S1366728915000498

Hsiao, F. J., Yu, H. Y., Chen, W. T., Kwan, S. Y., Chen, C., Yen, D. J., et al. (2015). Increased intrinsic connectivity of the default mode network in temporal lobe epilepsy: evidence from resting-state MEG recordings. PLoS One 10:e128787. doi: 10.1371 /journal.pone.0128787

Hu, S., Ide, J. S., Zhang, S., and Li, C.-S. R. (2016). The Right superior frontal gyrus and individual variation in proactive control of impulsive response. J. Neurosci. 36, 12688-12696. doi: 10.1523/JNEUROSCI.1175-16.2016

Hughes, T. F., Chang, C.-C. H., Bilt, J., Vander Snitz, B. E., and Ganguli, M. (2012). Mild cognitive deficits and everyday functioning among older adults in the community: the monongahela-youghiogheny healthy aging team study. Am. J. Geriatr. Psychiatry 20, 836-844. doi: 10.1097/JGP.0b013e3182423961

Iqbal, K., and Grundke-Iqbal, I. (2010). Alzheimer's disease, a multifactorial disorder seeking multitherapies. Alzheimer's Dem. 6, 420-424. doi: 10.1016/j. jalz.2010.04.006

Isopi, E., Granzotto, A., Corona, C., Bomba, M., Ciavardelli, D., Curcio, M., et al. (2015). Pyruvate prevents the development of age-dependent cognitive deficits in a mouse model of Alzheimer's disease without reducing amyloid and tau pathology. Neurobiol. Dis. 81, 214-224. doi: 10.1016/j.nbd.2014.11.013

Jo, H. J., Saad, Z. S., Simmons, W. K., Milbury, L. A., and Cox, R. W. (2010). Mapping sources of correlation in resting state FMRI, with artifact detection and removal. Neuroimage 52, 571-582. doi: 10.1016/j.neuroimage.2010. 04.246

Kivipelto, M., Solomon, A., Ahtiluoto, S., Ngandu, T., Lehtisalo, J., Antikainen, R., et al. (2013). The finnish geriatric intervention study to prevent cognitive impairment and disability (FINGER): study design and progress. Alzheimer's Dem. 9, 657-665. doi: 10.1016/j.jalz.2012.09.012

Kowoll, M. E., Degen, C., Gorenc, L., Küntzelmann, A., Fellhauer, I., Giesel, F., et al. (2016). Bilingualism as a contributor to cognitive reserve? evidence from cerebral glucose metabolism in mild cognitive impairment and alzheimer's disease. Front. psychiatry 7:62. doi: 10.3389/fpsyt.2016.00062

Kriegeskorte, N., Simmons, W. K., Bellgowan, P. S. F., and Baker, C. I. (2009). Circular analysis in systems neuroscience: the dangers of double dipping. Nat. Neurosci. 12, 535-540. doi: 10.1038/nn.2303

Lezak, M. D., and Lezak, M. D. (2004). Neuropsychological Assessment. New York, NY: Oxford University Press.

Li, L., Abutalebi, J., Emmorey, K., Gong, G., Yan, X., Feng, X., et al. (2017). How bilingualism protects the brain from aging: insights from bimodal bilinguals. Hum. Brain Mapp. 38, 4109-4124. doi: 10.1002/hbm.23652

Li, W., Qin, W., Liu, H., Fan, L., Wang, J., Jiang, T., et al. (2013). Subregions of the human superior frontal gyrus and their connections. Neuroimage 78, 46-58. doi: 10.1016/j.neuroimage.2013.04.011

Luber, B., and Lisanby, S. H. (2014). Enhancement of human cognitive performance using transcranial magnetic stimulation (TMS). Neuroimage 85, 961-970. doi: 10.1016/J.NEUROIMAGE.2013.06.007

Luk, G., Bialystok, E., Craik, F. I. M., and Grady, C. L. (2011a). Lifelong bilingualism maintains white matter integrity in older adults. J. Neurosci. 31, 16808-16813. doi: 10.1523/JNEUROSCI.4563-11.2011

Luk, G., Green, D. W., Abutalebi, J., and Grady, C. (2011b). Cognitive control for language switching in bilinguals: a quantitative meta-analysis of functional neuroimaging studies. Lang. Cogn. Process. 27, 1479-1488. doi: 10.1080/ 01690965.2011.613209

Lwi, S. J., Ford, B. Q., Casey, J. J., Miller, B. L., and Levenson, R. W. (2017). Poor caregiver mental health predicts mortality of patients with neurodegenerative disease. Proc. Natl. Acad. Sci. U.S.A. 114, 7319-7324. doi: 10.1073/pnas. 1701597114

Marian, V., Bartolotti, J., Rochanavibhata, S., Bradley, K., and Hernandez, A. E. (2017). Bilingual cortical control of between- and within-language competition. Sci. Rep. 7:11763. doi: 10.1038/s41598-017-12116-w

Marian, V., Chabal, S., Bartolotti, J., Bradley, K., and Hernandez, A. E. (2014). Differential recruitment of executive control regions during phonological competition in monolinguals and bilinguals. Brain Lang. 139, 108-117. doi: 10.1016/j.bandl.2014.10.005

Martin Prince, A., Wimo, A., Guerchet, M., Gemma-Claire Ali, M., Wu, Y.-T., Prina, M., et al. (2015). World Alzheimer Report 2015 The Global Impact of Dementia An AnAlysIs of prevAlence, IncIDence, cosT AnD TrenDs. Available at: http://www.worldalzreport2015.org/downloads/world-alzheimerreport-2015.pdf (accessed August 30, 2017).

Measso, G., Cavarzeran, F., Zappalà, G., Lebowitz, B. D., Crook, T. H., Pirozzolo, F. J., et al. (1993). The mini-mental state examination: normative study of an Italian random sample. Dev. Neuropsychol. 9, 77-85. doi: 10.1080/ 87565649109540545

Mineroff, Z., Blank, I. A., Mahowald, K., and Fedorenko, E. (2018). A robust dissociation among the language, multiple demand, and default mode networks: evidence from inter-region correlations in effect size. Neuropsychologia 119, 501-511. doi: 10.1016/J.NEUROPSYCHOLOGIA.2018.09.011

Ngandu, T., Lehtisalo, J., Solomon, A., Levälahti, E., Ahtiluoto, S., Antikainen, R., et al. (2015). A 2 year multidomain intervention of diet, exercise, cognitive 
training, and vascular risk monitoring versus control to prevent cognitive decline in at-risk elderly people (FINGER): a randomised controlled trial. Lancet 385, 2255-2263. doi: 10.1016/S0140-6736(15)60461-5

Niccoli, T., and Partridge, L. (2012). Ageing as a risk factor for disease. Curr. Biol. 22, R741-R752. doi: 10.1016/J.CUB.2012.07.024

Novelli, G. P. C., Papagno, E., Capitani, M., Laiacona, G., and Vallar, S. F. C. (1986). Tre test clinici di ricerca e produzione lessicale. Taratura su soggetti normali. Arch. Psicol. Neurol. Psichiatr. 47, 477-506.

Oxford, R. L., and Ehrman, M. E. (1995). Adults' language learning strategies in an intensive foreign language program in the United States. System 23, 359-386. doi: 10.1016/0346-251X(95)00023-D

Perani, D., and Abutalebi, J. (2015). Bilingualism, dementia, cognitive and neural reserve. Curr. Opin. Neurol. 28, 618-625. doi: 10.1097/WCO.000000000000 0267

Perani, D., Farsad, M., Ballarini, T., Lubian, F., Malpetti, M., Fracchetti, A., et al. (2017). The impact of bilingualism on brain reserve and metabolic connectivity in Alzheimer's dementia. Proc. Natl. Acad. Sci.U.S.A. 114, 1690-1695. doi: 10. 1073/pnas.1610909114

Pieramico, V., Esposito, R., Sensi, F., Cilli, F., Mantini, D., Mattei, P. A., et al. (2012). Combination training in aging individuals modifies functional connectivity and cognition, and is potentially affected by dopamine-related genes. PLoS One 7:e43901. doi: 10.1371/journal.pone.0043901

Pievani, M., de Haan, W., Wu, T., Seeley, W. W., and Frisoni, G. B. (2011). Functional network disruption in the degenerative dementias. Lancet Neurol. 10, 829-843. doi: 10.1016/S1474-4422(11)70158-2

Plante, E., Almryde, K., Patterson, D. K., Vance, C. J., and Asbjørnsen, A. E. (2015). Language lateralization shifts with learning by adults. Laterality 20, 306-325. doi: 10.1080/1357650X.2014.963597

Power, J. D., Barnes, K. A., Snyder, A. Z., Schlaggar, B. L., and Petersen, S. E. (2012). Spurious but systematic correlations in functional connectivity MRI networks arise from subject motion. Neuroimage 59, 2142-2154. doi: 10.1016/j. neuroimage.2011.10.018

Power, J. D., Mitra, A., Laumann, T. O., Snyder, A. Z., Schlaggar, B. L., and Petersen, S. E. (2014). Methods to detect, characterize, and remove motion artifact in resting state fMRI. Neuroimage 84, 320-341. doi: 10.1016/j.neuroimage.2013. 08.048

Prince, M. (2017). Progress on dementia-leaving no one behind. Lancet 390, E51-E53. doi: 10.1016/S0140-6736(17)31757-9

Rosselli, M., Loewenstein, D. A., Curiel, R. E., Penate, A., Torres, V. L., Lang, M., et al. (2019). Effects of bilingualism on verbal and nonverbal memory measures in mild cognitive impairment. J. Int. Neuropsychol. Soc. 25, 15-28. doi: 10.1017/ S135561771800070X

Rossi, E., and Diaz, M. T. (2016). How aging and bilingualism influence language processing: theoretical and neural models. Linguist. Approaches Biling. 6, 9-42. doi: 10.1075/lab.14029.ros

Schroeder, S. R., and Marian, V. (2017). Cognitive consequences of trilingualism. Int. J. Biling. 21, 754-773. doi: 10.1177/1367006916637288

Schweizer, T. A., Ware, J., Fischer, C. E., Craik, F. I. M., and Bialystok, E. (2012). Bilingualism as a contributor to cognitive reserve: evidence from brain atrophy in Alzheimer's disease. Cortex 48, 991-996. doi: 10.1016/j.cortex.2011.04.009

Selkoe, D. J., and Hardy, J. (2016). The amyloid hypothesis of Alzheimer's disease at 25 years. EMBO Mol. Med. 8, 595-608. doi: 10.15252/emmm. 201606210

Song, H. J., Meade, K., Akobundu, U., and Sahyoun, N. R. (2014). Depression as a correlate of functional status of community-dwelling older adults: utilizing a short-version of 5-item geriatric depression scale as a screening tool. J. Nutr. Health Aging 18, 765-770. doi: 10.1007/s12603-014-0452-1

Stern, Y. (2001). What is Cognitive Reserve? Theory and Research Application of the Reserve Concept. Available at: http://www.cumc.columbia.edu/dept/sergievsky/ pdfs/CogResTheory.pdf (accessed August 30, 2017).

Stern, Y. (2012). Cognitive reserve in ageing and Alzheimer's disease. Lancet. Neurol. 11, 1006-1012. doi: 10.1016/S1474-4422(12)70191-6
Strenziok, M., Clarke, E., Cisler, D. S., Thompson, J. C., and Greenwood, P. M. (2014). Neurocognitive enhancement in older adults: comparison of three cognitive training tasks to test a hypothesis of training transfer in brain connectivity. Neuroimage 85, 1027-1039. doi: 10.1016/J.NEUROIMAGE.2013. 07.069

Tan, Z. S., Spartano, N. L., Beiser, A. S., DeCarli, C., Auerbach, S. H., Vasan, R. S., et al. (2016). Physical activity, brain volume, and dementia risk: the framingham study. J. Gerontol. Ser. A Biol. Sci. Med. Sci. 72:glw130. doi: 10.1093/gerona/ glw 130

Tombaugh, T. N., Kozak, J., and Rees, L. (1999). Normative data stratified by age and education for two measures of verbal fluency: FAS and animal naming. Arch. Clin. Neuropsychol. 14, 167-177. doi: 10.1016/S0887-6177(97)00 095-4

Toro, R., Fox, P. T., and Paus, T. (2008). Functional coactivation map of the human brain. Cereb. Cortex 18, 2553-2559. doi: 10.1093/cercor/ bhn014

Uddin, L. Q., Clare Kelly, A. M., Biswal, B. B., Xavier Castellanos, F., and Milham, M. P. (2009). Functional connectivity of default mode network components: correlation, anticorrelation, and causality. Hum. Brain Mapp. 30, 625-637. doi: 10.1002/hbm.20531

Varela, F., Lachaux, J.-P., Rodriguez, E., and Martinerie, J. (2001). The brainweb: phase synchronization and large-scale integration. Nat. Rev. Neurosci. 2, 229239. doi: $10.1038 / 35067550$

Voss, M. W., Prakash, R. S., Erickson, K. I., Basak, C., Chaddock, L., Kim, J. S., et al. (2010). Plasticity of brain networks in a randomized intervention trial of exercise training in older adults. Front. Aging Neurosci. 2:32. doi: 10.3389/fnagi. 2010.00032

Ware, C., Damnee, S., Djabelkhir, L., Cristancho, V., Wu, Y. H., Benovici, J., et al. (2017). Maintaining cognitive functioning in healthy seniors with a technologybased foreign language program: a pilot feasibility study. Front. Aging Neurosci. 9:42. doi: 10.3389/fnagi.2017.00042

Wenger, E., Brozzoli, C., Lindenberger, U., and Lövdén, M. (2017). Expansion and renormalization of human brain structure during skill acquisition. Trends Cogn. Sci. 21, 930-939. doi: 10.1016/j.tics.2017.09.008

Winblad, B., Amouyel, P., Andrieu, S., Ballard, C., Brayne, C., Brodaty, H., et al. (2016). Defeating Alzheimer's disease and other dementias: a priority for European science and society. Lancet. Neurol. 15, 455-532. doi: 10.1016/S14744422(16)00062-4

Woolgar, A., Duncan, J., and Fedorenko, E. (2017). Fluid intelligence is supported by the multiple-demand system not the language system. Nat. Hum. Behav. 2, 200-204. doi: 10.1038/s41562-017-0282-3

Wu, J.-T., Wu, H.-Z., Yan, C.-G., Chen, W.-X., Zhang, H.-Y., He, Y., et al. (2011). Aging-related changes in the default mode network and its anti-correlated networks: a resting-state fMRI study. Neurosci. Lett. 504, 62-67. doi: 10.1016/j. neulet.2011.08.059

Wu, X., Li, R., Fleisher, A. S., Reiman, E. M., Guan, X., Zhang, Y., et al. (2011). Altered default mode network connectivity in Alzheimer's disease-a resting functional MRI and Bayesian network study. Hum. Brain Mapp. 32, 1868-1881. doi: $10.1002 / \mathrm{hbm} .21153$

Conflict of Interest Statement: The authors declare that the research was conducted in the absence of any commercial or financial relationships that could be construed as a potential conflict of interest.

Copyright (c) 2019 Bubbico, Chiacchiaretta, Parenti, di Marco, Panara, Sepede, Ferretti and Perrucci. This is an open-access article distributed under the terms of the Creative Commons Attribution License (CC BY). The use, distribution or reproduction in other forums is permitted, provided the original author(s) and the copyright owner(s) are credited and that the original publication in this journal is cited, in accordance with accepted academic practice. No use, distribution or reproduction is permitted which does not comply with these terms. 


\section{OPEN ACCESS}

Edited and reviewed by:

Alessandra Griffa,

Vrije Universiteit

Amsterdam, Netherlands

${ }^{*}$ Correspondence:

Mauro Gianni Perrucci

maurogianni.perrucci@unich.it;

gianni.perrucci@gmail.com

Specialty section

This article was submitted to

Brain Imaging Methods,

a section of the journal

Frontiers in Neuroscience

Received: 05 December 2019

Accepted: 28 January 2020

Published: 19 February 2020

Citation:

Bubbico G, Chiacchiaretta $P$

Parenti M, di Marco M, Panara V, Sepede G, Ferretti $A$ and Perrucci MG

(2020) Corrigendum: Effects of

Second Language Learning on the

Plastic Aging Brain: Functional

Connectivity, Cognitive Decline, and

Reorganization.

Front. Neurosci. 14:108

doi: 10.3389/fnins.2020.00108

\section{Corrigendum: Effects of Second Language Learning on the Plastic Aging Brain: Functional Connectivity, Cognitive Decline, and Reorganization}

\author{
Giovanna Bubbico ${ }^{1,2}$, Piero Chiacchiaretta ${ }^{1,2}$, Matteo Parenti $^{3}$, Marcin di Marco ${ }^{3}$, \\ Valentina Panara ${ }^{1,4}$, Gianna Sepede ${ }^{1,5,6}$, Antonio Ferretti ${ }^{1,2}$ and Mauro Gianni Perrucci ${ }^{1,2 *}$ \\ 'Department of Neuroscience, Imaging and Clinical Sciences, "G. d'Annunzio" University of Chieti-Pescara, Chieti, Italy, \\ ${ }^{2}$ Institute for Advanced Biomedical Technologies, "G. d'Annunzio" University of Chieti-Pescara, Chieti, Italy, ${ }^{3}$ Department of \\ Medicine and Science of Aging, "G. d'Annunzio" University of Chieti-Pescara, Chieti, Italy, ${ }^{4}$ Section of Diagnostic Imaging \\ and Therapy, Radiology Division, Department of Neuroscience and Imaging, "SS Annunziata" Hospital, "G. D'Annunzio" \\ University, Chieti, Italy, ${ }^{5}$ Department of Basic Medical Sciences, Neurosciences and Sense Organs, University "A. Moro" Bari, \\ Chieti, Italy, ${ }^{6}$ National Health Trust, Department of Mental Health, Chieti, Italy
}

Keywords: aging, brain plasticity, second language learning, cognitive decline, resting state, functional connectivity

\section{A Corrigendum on}

Effects of Second Language Learning on the Plastic Aging Brain: Functional Connectivity, Cognitive Decline, and Reorganization

by Bubbico, G., Chiacchiaretta, P., Parenti, M., di Marco, M., Panara, V., Sepede, G., et al. (2019). Front. Neurosci. 13:423. doi: 10.3389/fnins.2019.00423

In the original article, the statistically significant differences in the MMSE scores between the two groups were incorrect. A correction has been made to the Results, subsection Cognitive Performances, paragraph one:

Control and intervention subjects were evaluated at the baseline phase (T0) and at the end of the 4 months (T1) period for their neuropsychological abilities. Four subjects were excluded, one did not observe inclusion criteria [had periventricular nodular heterotopia (PNH)], two did not accept to be retested at post-training condition, and one did not attend a sufficient percentage of lessons. We observed slight differences between group in terms of age (Control group Mean: 65.7, SD 3.7; Intervention group: Mean 69.5, SD 5.3; One-Way ANOVA $F=4.42, p=0.046$ ) and education (Controls: Mean 13.0, SD 3.0; Intervention group Mean 9.6 SD 2.9; One-Way ANOVA $F=8.43, p=0.0008$ ). A detailed description of statistical analysis results can be seen in Table 1. The normality of the distribution was controlled by Kolmogorov-Smirnov test (Ksd $d=0.11$, $p>0.20$ ). Statistically significant differences in MMSE score were found within and between the two groups at both T0 and T1 $(p=0.009)$. In more details, the two groups significantly differ at T0, with the control group performing better than the intervention group (29.35 versus 27.23 , Duncan post hoc $p=0.001)$; on the contrary, the between group difference disappeared at T1 (28.28 versus 27.81 , Duncan post hoc $p=0.42$ ). In fact, only the control group significantly decreased its 
performances over time (29.35 versus 28.28 Duncan post hoc $p=0.017)$, whereas the intervention group remained stable (27.23 versus 27.81) (see Table 3 and Figure 2).

The authors apologize for this error and state that this does not change the scientific conclusions of the article in any way. The original article has been updated.
Copyright (c) 2020 Bubbico, Chiacchiaretta, Parenti, di Marco, Panara, Sepede, Ferretti and Perrucci. This is an open-access article distributed under the terms of the Creative Commons Attribution License (CC BY). The use, distribution or reproduction in other forums is permitted, provided the original author(s) and the copyright owner(s) are credited and that the original publication in this journal is cited, in accordance with accepted academic practice. No use, distribution or reproduction is permitted which does not comply with these terms. 
OPEN ACCESS

Edited by:

Filippo Cieri,

Cleveland Clinic, United States

Reviewed by:

Marios Antonakakis,

University of Münster, Germany

Han Zhang,

The University of North Carolina at Chapel Hill, United States

${ }^{*}$ Correspondence:

Stavros I. Dimitriadis stidimitriadis@gmail.com DimitriadisS@cardiff.ac.uk

Specialty section: This article was submitted to Brain Imaging Methods, a section of the journal Frontiers in Neuroscience

Received: 14 February 2019 Accepted: 09 May 2019

Published: 11 June 2019

Citation:

Dimitriadis SI, López ME, Maestu F and Pereda E (2019)

Modeling the Switching Behavior of Functional Connectivity Microstates (FC $\mu$ states) as a Novel Biomarker for Mild Cognitive Impairment.

Front. Neurosci. 13:542. doi: 10.3389/fnins.2019.00542

\section{Modeling the Switching Behavior of Functional Connectivity Microstates (FC $\mu$ states) as a Novel Biomarker for Mild Cognitive Impairment}

\author{
Stavros I. Dimitriadis ${ }^{1,2,3,4,5,6 *}$, María Eugenia López ${ }^{7,8,9}$, Fernando Maestu ${ }^{7,8,9}$ and \\ Ernesto Pereda 8,10
}

\begin{abstract}
${ }^{1}$ Cardiff University Brain Research Imaging Centre, School of Psychology, Cardiff University, Cardiff, United Kingdom, ${ }^{2}$ Neuroinformatics Group, Cardiff University Brain Research Imaging Centre, School of Psychology, Cardiff University, Cardiff, United Kingdom, ${ }^{3}$ Division of Psychological Medicine and Clinical Neurosciences, School of Medicine, Cardiff University, Cardiff, United Kingdom, ${ }^{4}$ School of Psychology, Cardiff University, Cardiff, United Kingdom, ${ }^{5}$ Neuroscience and Mental Health Research Institute, School of Medicine, Cardiff University, Cardiff, United Kingdom, ${ }^{6}$ MRC Centre for Neuropsychiatric Genetics and Genomics, School of Medicine, Cardiff University, Cardiff, United Kingdom, ${ }^{7}$ Department of Experimental Psychology, Cognitive Processes and Speech Therapy, Universidad Complutense de Madrid, Madrid, Spain, ${ }^{8}$ Laboratory of Cognitive and Computational Neuroscience, Center for Biomedical Technology, Universidad Complutense de Madrid - Universidad Politécnica de Madrid, Madrid, Spain, ${ }^{9}$ Networking Research Center on Bioengineering, Biomaterials and Nanomedicine (CIBER-BBN), Madrid, Spain, ${ }^{10}$ Electrical Engineering and Bioengineering Group, Department of Industrial Engineering and Institute of Biomedical Technology, Universidad de La Laguna, Tenerife, Spain
\end{abstract}

The need for designing and validating novel biomarkers for the detection of mild cognitive impairment $(\mathrm{MCl})$ is evident. $\mathrm{MCl}$ patients have a high risk of developing Alzheimer's disease (AD), and for that reason the introduction of novel and reliable biomarkers is of significant clinical importance. Motivated by recent findings on the rich information of dynamic functional connectivity graphs (DFCGs) about brain (dys) function, we introduced a novel approach of identifying $\mathrm{MCl}$ based on magnetoencephalographic (MEG) resting state recordings. The activity of different brain rhythms $\{\delta, \theta, \alpha 1, \alpha 2, \beta 1, \beta 2, \gamma 1, \gamma 2\}$ was first beamformed with linear constrained minimum norm variance in the MEG data to determine 90 anatomical regions of interest (ROIs). A DFCG was then estimated using the imaginary part of phase lag value (iPLV) for both intra-frequency coupling (8) and cross-frequency coupling pairs (28). We analyzed DFCG profiles of neuromagnetic resting state recordings of $18 \mathrm{MCl}$ patients and 22 healthy controls. We followed our model of identifying the dominant intrinsic coupling mode (DICM) across MEG sources and temporal segments, which further leads to the construction of an integrated DFCG (iDFCG). We then filtered statistically and topologically every snapshot of the iDFCG with data-driven approaches. An estimation of the normalized Laplacian transformation for every temporal segment of the iDFCG and the related eigenvalues created a 2D map based on the network metric time series of the eigenvalues (NMTS ${ }^{\text {eigs }}$ ). The NMTS ${ }^{\text {eigs }}$ preserves the non-stationarity of the fluctuated synchronizability of iDCFG for each subject. Employing the initial set of 20 healthy elders and $20 \mathrm{MCl}$ patients, as training set, we built an overcomplete dictionary set of network 
microstates (n $\mu$ states). Afterward, we tested the whole procedure in an extra blind set of 20 subjects for external validation. We succeeded in gaining a high classification accuracy on the blind dataset (85\%), which further supports the proposed Markovian modeling of the evolution of brain states. The adaptation of appropriate neuroinformatic tools that combine advanced signal processing and network neuroscience tools could properly manipulate the non-stationarity of time-resolved FC patterns revealing a robust biomarker for $\mathrm{MCl}$.

Keywords: magnetoencephalography, mild cognitive impairment, dynamic functional connectivity, resting state, brain states, chronnectome analysis, symbolic dynamics, connectomic biomarker

\section{INTRODUCTION}

The major cause of clinical dementia in the elderly is that of Alzheimer's type (DAT; Qiu et al., 2009), which is mainly characterized by loss of synapses, the accumulation of the Beta amyloid protein $(\mathrm{A} \beta)$ and the phosphorylation of the Tau protein. Due to the progressive loss of synapses, which alters the efficient communication within and between various brain subsystems, the DAT may be considered a disconnection syndrome (Delbeuck et al., 2003). The pathological changes of DAT start decades before the first clinical symptoms appear, thus it is important to design proper analytic pathways for analyzing neuroimaging datasets via, e.g., the notion of brain connectivity, which allows the early detecting of such changes (Gómez et al., 2009a; Stam et al., 2009; Maestú et al., 2015). It is extremely important to Alzheimer's disease (AD) research to identify early on preclinical and prodromal $\mathrm{AD}$ as it can assist clinical trials and targeted interventions (Livingston et al., 2017).

Mild cognitive impairment (MCI) is considered to be an intermediate clinical stage between the normal cognitive decline and DAT (Petersen and Negash, 2008). The main parts of the affected brain during the MCI, apart from those involved in action and thought, are those related to memory. For that reason, MCI patients face memory problems on a higher level compared to normal aged population but with no prevalent characteristic symptomatology of dementia-like reasoning or impaired judgment (Petersen et al., 2009). MCI is a heterogeneous state with different subtypes, which complicates in many cases the prediction of DAT (Portet et al., 2006). Additionally, it is also difficult to accurately discriminate symptomatic predementia (MCI) from healthy aging or dementia (DAT) (Petersen and Negash, 2008). Despite these difficulties to achieve an early diagnosis, an accurate identification of $\mathrm{MCI}$ should be attempted. Early diagnosis of MCI, even in the absence of a healing strategy, is significant for both pharmacological and non-pharmacological interventions. For that reason, new tools based on neuroimaging approaches are needed to increase sensitivity in the detection of MCI.

Analysis of magnetoencephalographic (MEG) recordings untangled the association between neural oscillations, functional connectivity assessment and neurophysiological activity (Brookes et al., 2011). Altered frequency-dependent functional patterns have been linked to the progression of cognitive decline (Poza et al., 2007). Alternative scenarios of analyzing MEG recordings include single channel analysis, e.g., power analysis, functional connectivity, and brain network analysis in resting state and also in task-based experiments (for a review, see Mandal et al., 2018). Analysis of single channel recordings is a less complex approach that identified aberrant oscillations in $\mathrm{AD}$ primarily in the left temporal-parietal-occipital brain areas (Gómez et al., 2009b). Functional connectivity (FC) and effective connectivity (EC) analysis revealed a loss of connectivity in $\mathrm{AD}$ compared to healthy control (HC) subjects found mostly in higher frequency bands (Gómez et al., 2017) while multiplex network analysis of MEG study in AD identified affected regions of the hippocampus, posterior default mode network (DMN) and occipital areas (Yu et al., 2017). However, the current clinical literature is limited and no strong conclusion can be drawn.

A recent multicenter MEG study addressed this issue using FC analysis (Maestú et al., 2015). It revealed hypersynchronization in MCI as the most discriminative feature of brain connectivity, mainly over the fronto-parietal and inter-hemispheric links. This pattern was stable across the five different neuroimaging centers that participated in the study (Accuracy $\sim=80 \%$ ), which might thereby be considered as a preclinical connectomic biomarker for MCI/DAT. Previous MEG studies based on connectivity analysis described a less organized functional brain network, a hypersynchrony in the fronto-parietal network in MCI subjects (Bajo et al., 2010; Buldú et al., 2011), while patients with DAT demonstrated a less synchronized brain network accompanied with cognitive decline (Stam et al., 2009). This hypersynchronization might be a compensatory mechanism but it cannot be adaptive since the patient's network is closer to a random network compared to healthy elderly controls (Buldú et al., 2011). In a recent MEG study comparing progressive $\mathrm{MCI}$ and stable $\mathrm{MCI}$, authors described hypersynchronization in the $\alpha$ band between the anterior cingulate and posterior brain areas in the progressive MCI group (López et al., 2014).

Spontaneous fluctuations of functional MRI (fMRI) blood-oxygen-level-dependent (BOLD) signals are temporally coherent between distinct spatial brain areas and not random. Biswal et al. (1995) demonstrated that fluctuations from motor areas were correlated even in the absence of a motor task. FC based on BOLD signal is modulated by cognitive and affective states (Richiardi et al., 2011; Shirer et al., 2012), by learning (Bassett et al., 2011), and also spontaneously (Kitzbichler et al., 2009; Britz et al., 2010; Chang and Glover, 2010). 
When non-stationarity is taken into account and a dynamic functional connectivity (DFC) approach is adopted for studying FC patterns even in the absence of a task (resting state), more sophisticated algorithmic analyses should be used. In this line, two studies have recently been published simultaneously that presented a data-driven methodology. In the first one, Allen et al. (2014) proposed a method based on $k$-means clustering, aimed at detecting distinct "FC states" in the resting brain. These authors clearly showed differences from the stationary static functional brain networks. The second study proposed a data-driven method focused on extracting, out of hundreds of functional connectivity graphs (FCGs) in a multi-trial experimental paradigm, distinct brain states called functional connectivity microstates (FC $\mu$ states; Dimitriadis et al., 2013a). Both approaches revealed the need of dynamic FC to explore brain dynamics via the notion of brain connectivity, as it is clear that brain FC "hops" from one state to another (FC $\mu$ state) leading to a Markovian chain with characteristic favored transitions between distinct pairs of FC $\mu$ states (Dimitriadis et al., 2010b, 2013a,b; Allen et al., 2014).

In the last years, an increasing amount of human brain research based on functional imaging methods (electro-encephalography: EEG/magnetoencephalography: MEG/functional Magnetic Resonance Imaging: fMRI) has adopted a dynamic approach for exploring how brain connectivity fluctuates during resting-state and tasks alike (Laufs et al., 2003; Mantini et al., 2007; Dimitriadis et al., 2009, 2010b, 2012b, 2013a,b, 2015a,b,c,d, 2016a,b; Chang and Glover, 2010; Bassett et al., 2011; Handwerker et al., 2012; Ioannides et al., 2012; Hutchison et al., 2013; Liu and Duyn, 2013; Allen et al., 2014; Braun et al., 2014; Mylonas et al., 2015; Toppi et al., 2015; Yang and Lin, 2015; Calhoun and Adali, 2016). The aforementioned studies have demonstrated the superiority of DFC as compared to a static connectivity analysis.

In parallel, the concept of cross-frequency coupling (CFC) is gaining attention lately in the neuroscience community, as evinced by the increasing number of papers published with the incorporation of this type of interaction in the analysis (van Wijk and Fitzgerald, 2014; Dimitriadis et al., 2015a,c, 2016a,b; Florin and Baillet, 2015; Antonakakis et al., 2016a,b; Tewarie et al., 2016). Specifically, intrinsic coupling modes and especially CFC bias the task-related response and are sensitive to various brain diseases and disorders such as DAT, Parkinson, etc. (see, e.g., Engel et al., 2013 for a review). More recent studies have shown that the dynamics of spontaneously generated neural activity can be informative regarding the functional organization of large-scale brain networks (Fox et al., 2005; He et al., 2008; Hipp et al., 2012), revealing intrinsically generated "coupling modes" at multiple spatial and temporal scales (Deco and Corbetta, 2011; Engel et al., 2013).

Based on the aforementioned methodological evidence in microscale, it is significant to explore the repertoire of intra- and cross frequency interactions across brain rhythms and brain areas under the same integrated graph model (Dimitriadis et al., 2016a, 2017b, 2018a; Antonakakis et al., 2017).

In a previous study, we demonstrated how to design a connectomic biomarker for MCI based on source-reconstructed
MEG activity via static brain network analysis (Dimitriadis et al., 2018a). Here, we extended this work by proposing a scheme to design a dynamic connectomic biomarker under the framework of DFC analysis. Additionally, the proposed scheme will be validated in a second blind dataset (SID did not know anything about the labels).

To this aim, we analyzed the MEG activity of healthy controls and MCI patients at resting-state (eyes-open) via DFC analysis. Based on a previous approach (Dimitriadis et al., 2016a, 2017b), we detected the dominant type of interaction per pair of MEG sources and temporal segment (Dimitriadis, 2018). This approach produced a subject-specific dynamic functional connectivity graph (DFCG). This approach created a 2D matrix of size sources $\times$ temporal segments that described the evolution of the eigenvalues across experimental time. Afterward, we used neural gas to design overcomplete dictionaries for sparse representation of NMTS eigen independently for the two groups (Dimitriadis et al., 2013a). Then, we validated the whole approach in a blind dataset to quantify the generalization of the proposed method.

In the Section "Materials and Methods," we described the data acquisition, preprocessing steps, information about the datasets and the proposed methodological scheme. The Section "Results" is devoted to describing the results-including the prototypical network FC $\mu$ states, the accuracy of prediction in a blind dataset and network-based information of brain states. Finally, the Section "Discussion" includes the discussion of the current research results with future extensions.

\section{MATERIALS AND METHODS}

\section{Subjects and Ethics Statement}

The training dataset includes 18 right-handed individuals with MCI (71.89 \pm 4.51 years of age), and 22 age- and gendermatched neurologically intact controls $(70.91 \pm 3.85$ years of age) were also recorded. Table 1 summarizes their demographic characteristics. All participants were recruited from the Neurological Unit of the "The Hospital Universitario San Carlos," Madrid, Spain. They were right-handed (Oldfield, 1971) and native Spanish speakers. We used also a set of 20 subjects of unknown label (blind author SD) for further validation of the proposed dynamic connectomic biomarker (DCB). Table 2 summarizes the mean and standard deviation of the demographic characteristics of controls and MCI subjects from the blind dataset. Including the blind subjects, the total sample consisted of $29 \mathrm{MCI}$ and 31 controls. At the beginning, we used $18 / 22$ subjects for $\mathrm{MCI} /$ control group, correspondingly to train the algorithm and we kept 20 (nine control subjects and $11 \mathrm{MCI}$ ) for blind classification.

To explore their cognitive and functional status, all participants were screened by means of a variety of standardized diagnostic instruments and underwent an extensive cognitive assessment, as described in López et al. (2016).

Mild cognitive impairment diagnosis was established according to the National Institute on Aging-Alzheimer Association (NIA-AA) criteria (Albert et al., 2011), with all of them being categorized as "MCI due to $\mathrm{AD}$ 
TABLE 1 | Mean \pm standard deviation of the demographic characteristics of controls and MCls.

\begin{tabular}{|c|c|c|c|c|c|c|}
\hline & Age & Gender (M/F) & Educational level & MMSE & LH ICV & RH ICV \\
\hline Control $(n=22)$ & $70.91 \pm 3.853$ & $9 / 13$ & $3.50 \pm 1.225$ & $29.32 \pm 0.646$ & $0.0025 \pm 0.0003$ & $0.0025 \pm 0.0003$ \\
\hline $\mathrm{MCl}(n=18)$ & $71.89 \pm 4.510$ & $7 / 11$ & $2.71 \pm 1.359$ & $27.24 \pm 1.954$ & $0.0022 \pm 0.0005$ & $0.0021 \pm 0.0005$ \\
\hline
\end{tabular}

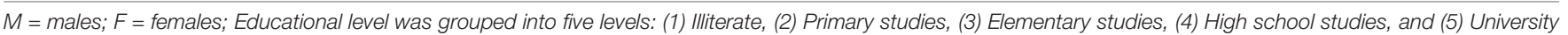

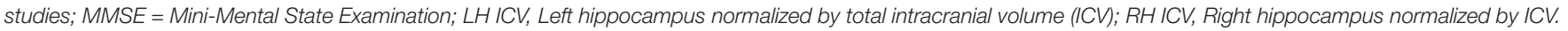

TABLE 2 | Mean \pm standard deviation of the demographic characteristics of the blind sample of controls and MCls.

\begin{tabular}{|c|c|c|c|c|c|c|}
\hline & Age & Gender (M/F) & Educational level & MMSE & LH ICV & RH ICV \\
\hline Control $(n=9)$ & $70.22 \pm 3.8333$ & $1 / 8$ & $3.44 \pm 1.333$ & $29.33 \pm 0.707$ & $0.0026 \pm 0.0005$ & $0.0027 \pm 0.0004$ \\
\hline $\mathrm{MCl}(n=11)$ & $73.45 \pm 3.297$ & $7 / 4$ & $3.91 \pm 1.221$ & $26.90 \pm 2.132$ & $0.0018 \pm 0.0004$ & $0.0021 \pm 0.0004$ \\
\hline
\end{tabular}

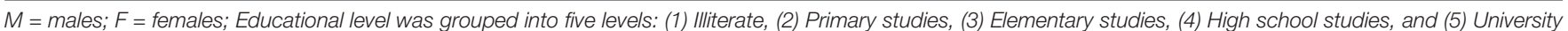

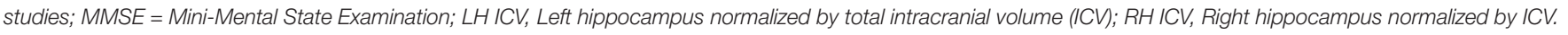

intermediate likelihood." They met the clinical criteria and also presented hippocampal atrophy, which was measured by magnetic resonance (MRI). According to their cognitive profile, they were also classified as amnestic subtype (Petersen et al., 2001).

The whole sample was free of significant medical, neurological and/or psychiatric diseases (other than MCI). Exclusion criteria included: a modified Hachinski Ischemic score $\geq 4$ (Rosen et al., 1980); a geriatric depression scale short-form score $\geq 5$ (Yesavage et al., 1983); a T2-weighted MRI within 12 months before MEG screening with indication of infection, infarction or focal lesions (rated by two independent experienced radiologists, Bai et al., 2012); and other possible causes of cognitive decline such as $\mathrm{B}_{12}$ deficit, diabetes mellitus, thyroid problems, syphilis or human immunodeficiency virus (HIV). Finally, those participants with medical treatment that could affect MEG activity (e.g., cholinesterase inhibitors) were required to interrupt it $48 \mathrm{~h}$ before the MEG recordings.

The present study was approved by the local ethics committee and all subjects signed an informed consent prior to their MEG recording.

\section{MRI Acquisition and Hippocampal Volumes}

Three-dimensional T1-weighted anatomical brain magnetic MRI scans were collected with a General Electric 1.5 TMRI scanner, using a high resolution antenna and a homogenization PURE filter (Fast Spoiled Gradient Echo (FSPGR) sequence with parameters: TR/TE/TI $=11.2 / 4.2 / 450 \mathrm{~ms}$; flip angle $12^{\circ} ; 1 \mathrm{~mm}$ slice thickness, a $256 \times 256$ matrix and FOV $25 \mathrm{~cm}$ ). Freesurfer software (version 5.1.0.; Fischl et al., 2002) was used to obtain the hippocampal volumes, which were normalized with the overall intracranial volume (ICV) of each subject.

\section{MEG Acquisition and Preprocessing}

4 min of eyes-open resting state data were recorded while the participants were seated in a 306-channel (one magnetometer and two orthogonal planar gradiometers per recording site, sampling frequency of $1 \mathrm{kHz}$ ) Vectorview system (Elekta Neuromag) placed in a magnetically shielded room (VacuumSchmelze $\mathrm{GmbH}$, Hanau, Germany) at the "Laboratory of Cognitive and Computational Neuroscience" (Madrid, Spain). Subjects had to fix their gaze at a cross, which was projected in a screen. The position of the head relative to the sensor array was monitored by four head position indicator (HPI) coils attached to the scalp (two on the mastoids and two on the forehead). These four coils along with the head shape of each subject (referenced to three anatomical fiducials: nasion and left-right preauricular points) were acquired by using a three-dimensional Fastrak Polhemus system. Vertical ocular movements were monitored by two bipolar electrodes, which were placed above and below the left eye, and a third one on the earlobe, for electrical grounding.

Four HPI coils were placed in the head of the subject, two in the forehead and two in the mastoids, for an online estimate of the head position. The HPI coils were fed during the whole acquisition, allowing for offline estimation of the head position.

Maxfilter software (version 2.2 Elekta Neuromag) was used to remove the external noise from the MEG data using the temporal extension of signal space separation (tSSS) with movement compensation (correlation threshold $=0.9 \mathrm{~m}$ time window $=10 \mathrm{~s}$ ) (Taulu and Simola, 2006). This algorithm removes the signals, whose origin is estimated outside the MEG helmet, while keeping intact the signals coming from inside the head. In addition, the continuous HPI acquisition, combined with the tSSS algorithm, allowed continuous movement compensation. As a result, the signal used in the next steps came from a set of virtual sensors whose position remained static in respect to the head of the subject. Recordings from those subjects whose movement along the recording was larger than $25 \mathrm{~mm}$ were discarded, following the recommendations of the manufacturer.

\section{Source Reconstruction}

We generated a volumetric grid for the MNI template by adopting a homogenous separation of $1 \mathrm{~cm}$ in each direction, with one source placed in $(0,0,0)$ in MNI coordinates. The 
whole procedure resulted in a source model with 2,459 sources inside the brain surface where each one consisted of three perpendicular dipoles. Every source was then labeled using the automated anatomical labeling (AAL) atlas (Tzourio-Mazoyer et al., 2002). We finally considered 1,467 cortical sources. The computed grid was then transformed to subject specific space employing the original T1 image. The realignment of the grid and brain surface was realized manually to the Neuromag coordinate system following the three fiducials and the head shape guides. Employing a realistically shaped head, we estimated a lead field (Nolte, 2003). We source reconstructed frequency-dependent brain activity using a Linearly Constrained Minimum Variance (LCMV) beamformer (Van Veen et al., 1997). We ran the LCMV beamformer independently for the following eight frequency bands: $\delta(1-4 \mathrm{~Hz}), \theta(4-8 \mathrm{~Hz}), \alpha_{1}(8-10 \mathrm{~Hz}), \alpha_{2}(10-13 \mathrm{~Hz}), \beta_{1}$ $(13-20 \mathrm{~Hz}), \beta_{2}(20-30 \mathrm{~Hz}), \gamma_{1}(30-49 \mathrm{~Hz})$, and $\gamma_{2}(51-90 \mathrm{~Hz})$. The resulting spatial filters were projected over the maximal radial direction, getting only one spatial filter per source. "Radial direction" means the direction of the segment connecting the dipole location to the center of the sphere best approximating the brain surface. Radial dipoles in a spherical conductor do not produce a magnetic field outside of the conductor (Sarvas, 1987), so this projection avoids the creation of undetectable sources among the target dipoles. Finally, we represented every brain area region of interest according to the AAL atlas by one source-space time series per frequency band using two alternative solutions: (1) the PCA of all the sources in the area or (2) the source closest to the centroid of the area (CENT).

Figure 1 illustrates the source-localization procedure and the different frequency-dependent representative Virtual Sensor time series for the two ROI representation schemes, PCA and the CENT.

\section{Dynamic Functional Connectivity Graphs (DFCGs)}

\section{Construction of the Integrated DFCGs}

The DCFG analysis was restricted to the 90 ROIs of the AAL atlas. Adopting a common sliding window of width equal to $1 \mathrm{~s}$ to get at least 1 cycle of $\delta$ activity and a moving step of $50 \mathrm{~ms}$, we estimated the dynamic networks for both intra-frequency (8 frequency bands) and inter-frequency coupling modes $\left(8^{*} 7 / 2=28\right.$ cross-frequency pairs) using the following formula of the imaginary part of phase locking value (iPLV).

$$
i P L V=\frac{1}{T}^{*}\left|\operatorname{Im}\left(\sum_{t=1}^{T} e^{i\left(\varphi_{i}(t)-\varphi_{j}(t)\right)}\right)\right|,
$$

where $\phi(t)$ is the phase of the signal in the corresponding frequency band (intra-frequency modes) and between frequencies (CFCs). For further details regarding phase-to-amplitudeCFC, see Dimitriadis et al. (2015a) and the Section "Construction of the Integrated Dynamic Functional Connectivity Graph" in Supplementary Material.

This procedure, whose implementation details can be found elsewhere (Dimitriadis et al., 2010b, 2015a, 2016a, 2017a,b, 2018b), resulted in a four-dimensional tensor of size [coupling modes $\times$ temporal segments $\times$ ROIs $\times$ ROIs] or $[36 \times 2,401 \times 90 \times 90]$ time-varying PAC graphs per participant ( $\left.{ }^{\mathrm{TV}} \mathrm{PAC}\right)$. Following proper surrogate analysis and a framework which have been presented in a previous study (Dimitriadis et al., 2018b), we defined the dominant intrinsic coupling mode (DICM) per pair of sources and across temporal segments. This procedure generates two three-dimensional tensors of size [temporal segments $\times$ ROIs $\times$ ROIs]. The first one keeps the functional coupling strength (iPLV) across anatomical space and time, while the second tabulates the DICM using an index for every possible case : $\{1$ for $\delta, 2$ for $\theta, 3$ for $\alpha_{1}, \ldots, 8$ for $\gamma_{2}, 9$ for $\delta-\theta, \ldots, 36$ for $\left.\gamma_{1}-\gamma_{2}\right\}$. The following section describes briefly the surrogate analysis appropriate for reducing pitfalls in CFC analysis and also to define the DICM.

\section{Statistical Filtering Scheme}

First, we must identify true CFC interactions that are not driven by the changes in signal power. Secondly, following a proper surrogate analysis our DICM model can detect the DICM between every pair of sources and $\boldsymbol{\delta}$

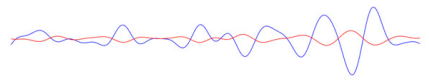

$\theta$

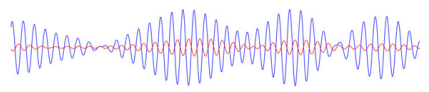

$\alpha_{1}$

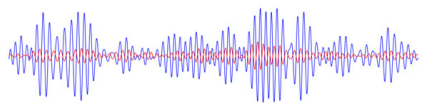

$\boldsymbol{\alpha}_{2}$

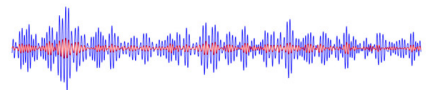

$\beta_{1}$
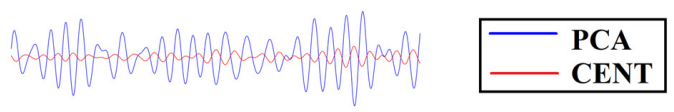

$\boldsymbol{\beta}_{2}$

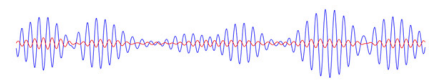

$\gamma_{1}$

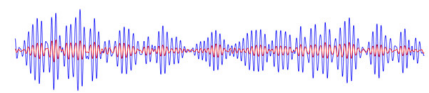

$\gamma_{2}$

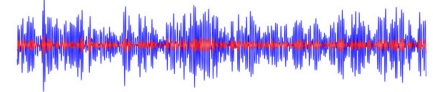

FIGURE 1 | ROI Virtual Sensor representation of left precentral gyrus magnetoencephalographic activity from the first healthy control subject. Virtual sensor time series with blue and red color represent brain activity for (A) PCA and (B) CENT time series, respectively. 
at every temporal segment. The whole procedure of analysis is described elsewhere in detail in Dimitriadis et al. (2016a), Dimitriadis and Salis (2017), and Dimitriadis (2018) and also in the Section "From Prominent Intrinsic Coupling Modes to Dominant Intrinsic Coupling Modes" in Supplementary Material.

Figure 2 illustrates the whole procedure of the DICM model for the first two temporal segments of resting-state activity of the first healthy control subject.

Figure 3A demonstrates the first 10 snapshots of the DFCG from the first healthy control subject.

\section{Topological Filtering Scheme Based on OMSTs}

Apart from surrogate analysis, which is a statistical filtering procedure of the functional couplings within an FCG akin to a regularization to sparsify the $4 \mathrm{D}$ array described above, we adopted a topological filtering to further enhance the network topology and the most significant interactions. To this aim, we applied a novel data-driven thresholding scheme, proposed by our group and termed Orthogonal Minimal Spanning Trees (OMSTs; Dimitriadis et al., 2017a,b), to each FCG derived from each subject and temporal segment independently.

Figure 3B demonstrates the temporal evolution of the topologically filtered dFCG for the first 10 temporal segments.

\section{Graph Signal Processing}

After extracting the most significant connections in DCFGs from each individual, we transformed every snapshot of the DFCG into the graph Laplacian variant called the normalized Laplacian matrix. With A being the functional connectivity graph and $\mathrm{D}$ being the degree matrix containing the degree of every node in the main diagonal, graph Laplacian $\mathrm{L}$ can be defined as $\mathrm{L}=\mathrm{D}-\mathrm{A}$. The normalized graph Laplacian is defined as $\mathrm{L}_{\text {sym }}=\mathrm{D}^{-1 / 2} \mathrm{LD}^{-1 / 2}$ (Shuman et al., 2013). We estimated the sorted eigenvalues of the Lsym for every snapshot of DFCG resulting in a twodimensional matrix of size [source $(90) \times$ temporal segments (2.401)] per subject. These two-dimensional matrices were concatenated separately for the healthy control and disease group of the training set. Practically, the concatenation was performance was performed along the temporal direction.

Figure 3C shows the temporal evolution of the normalized Laplacian transformation of the dFCG for the first 10 temporal segments while Figure 3D is dedicated to the temporal evolution of the eigenvalues.

\section{A Vector-Quantization (VQ) Modeling of Group NMTSeigen}

This subsection describes briefly our symbolization scheme, presented in greater details elsewhere (Dimitriadis et al., 2011, 2012a, 2013a,b). The group-specific NMTS ${ }^{\text {eigen }}$ patterns can

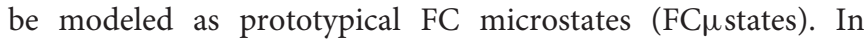
our previous studies, we demonstrated a better modeling of DFCG based on vector quantization approach (Dimitriadis et al., 2013a, 2017b, 2018b). A codebook of $k$ prototypical
FC states (i.e., functional connectivity microstates-FC $\mu$ states) was first designed by applying the neural-gas algorithm (Dimitriadis et al., 2013a). This algorithm is an artificial neural network model, which converges efficiently to a small number $k$ of codebook vectors, using a stochastic gradient descent procedure with a soft-max adaptation rule that minimizes the average distortion error (Martinetz et al., 1993). A neural-gas algorithm has been applied independently to each group by concatenating the $2 \mathrm{D}$ matrix of size $[2.401 \times 90]$ that describes the fluctuation of Laplacian eigenvalues.

The outcome of the neural-gas algorithm over NMTS eigen is the construction of a symbolic sequence of group-specific prototypical FCustates, one per subject. An example of such a symbolic time series (STS) is a Markovian chain with three FC $\mu$ states: $\{1,2,3,2,1,3,2 . \ldots\}$ where each integer defines a unique brain state (FCHstates) assigned to every quasi-static temporal segment.

\section{External Validation in a Blind Dataset}

We designed a novel approach for classifying a blind subject. We reconstructed the subject-specific NMTS eigen with both HC-based prototypical FC $\mu$ states and MCI-based prototypical FC $\mu$ states. Specifically, for every temporal segment expressed via a vector of 90 eigenvalues we estimated which of the prototypical FCustates is much closer, employing Euclidean distance for an appropriate criterion. Under this scheme, we rebuilt the original NMTS eigen twice, once using prototypical FC $\mu$ states of $\mathrm{HC}$ and once using prototypical FC $\mu$ states of MCI. Then, we estimated the reconstruction mean squared error between the original NMTSeigen and the two rebuilt NMTS ${ }^{\text {eigen }}$ based on prototypical FC $\mu$ states. Finally, we assigned the test sample to the class with the lowest reconstruction error (see Figure 6).

\section{Markov Chain Modeling for Synchro State Transitions}

The temporal sequence of spontaneous activity can be modeled as a Markovian process, which predicts the probabilities of several discrete states recurring or switching among themselves at different time points analyzing time-pointbased brain activity (Van de Ville et al., 2010; Gärtner et al., 2015). Several studies have investigated transition probabilities between phase-synchronized states on a subsecond temporal scale, untangling the Markovian property and the switching behavior of finite network-level brain states (Dimitriadis et al., 2013c, 2015b; Baker et al., 2014; Jamal et al., 2014).

\section{Markovian Process of Time-Sequential FC $\mu$ states}

A Markov model describes the underlying dynamical nature of a system that follows a chain of linked states, where the appearance of a state at any given instant depends only on the preceding ones (Gagniuc, 2017). In the Markov chain modeling for synchrostate transitions during the deductive reasoning and task-free processes, the first order transition matrices were estimated in a probabilistic framework. According 


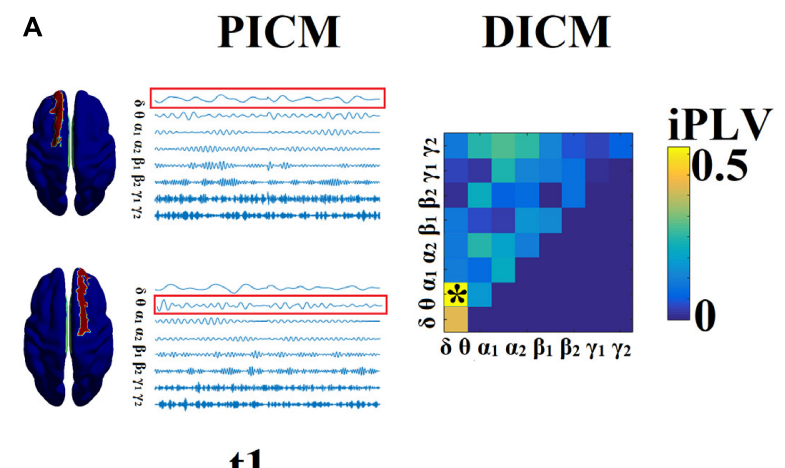

t1
PICM

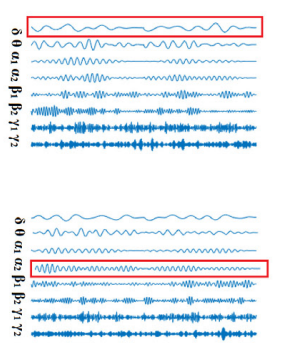

$\mathbf{t} 2$

B

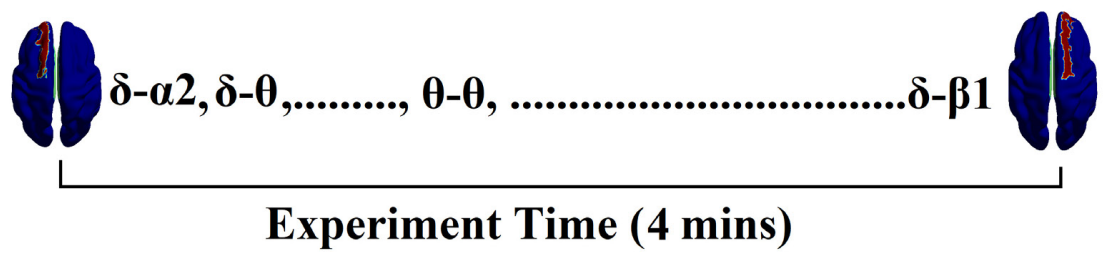

C
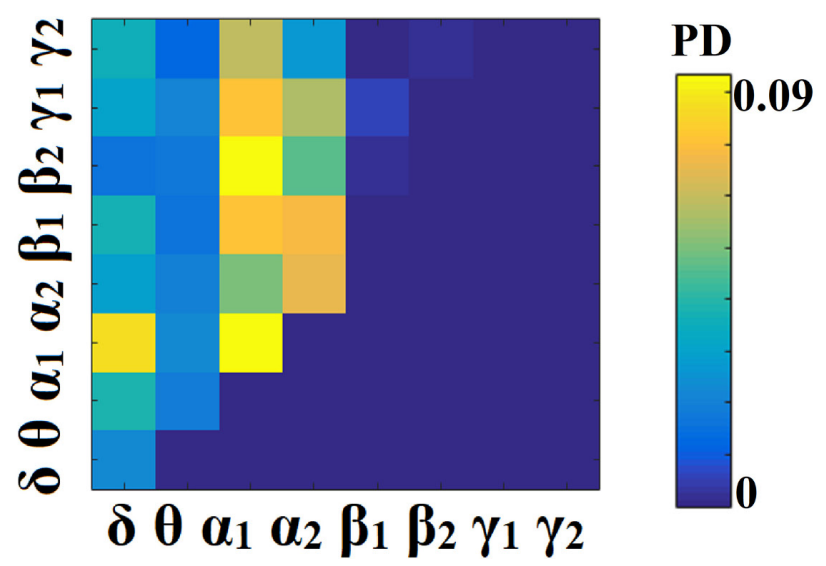

FIGURE 2 | Determining DICM. An example for AU task derived from the first trial of the first subject. (A) Schematic illustration of our approach employed to identify the DICM between two sources (Left superior frontal gyrus, Right superior frontal gyrus) for two consecutive sliding time windows ( $t_{1}, t_{2}$ ) during the first $4 \mathrm{~s}$ of resting-state activity from the first healthy control subject. In this example, the functional synchronization between band-passed signals from the two sources was estimated by imaginary Phase Locking (iPLV). In this manner iPLV was computed between the two sources either for same-frequency oscillations (e.g., $\delta$ to $\delta . . .$, $\gamma_{2}-\gamma_{2} ; 8$ intra-frequency couplings) or between different frequencies (e.g., $\delta$ to $\theta, \delta$ to $\alpha_{1} \ldots, \gamma_{1}-\gamma_{2} ; 28$ cross-frequency pairs). The sum of $8+28=36$ refers to Potential Intrinsic Coupling Modes (PICM), which are tabulated in a matrix format. In the main diagonal, we inserted the intra-frequency couplings while in the off-diagonal the cross-frequency pairs were inserted. Statistical filtering, using surrogate data for reference, was employed to assess whether each iPLV value was significantly different from chance. During $t_{1}$ the DICM reflected significant phase locking between $\alpha_{1}$ and $\alpha_{2}$ oscillations (indicated by red rectangles) in the oscillation list and $a * *$ "in the comodulogram. The DICM remains stable also for the $t_{2}$ between $\alpha_{1}$ and $\alpha_{2}$ oscillations whereas during $t_{3}$ the dominant interaction was detected between $\theta$ and $\alpha_{2}$ oscillations. (B) Burst of DICM between Left and Right superior frontal gyrus. This packing can be thought to associate the "letters" contained in the DICM series to form a neural "word," a possible integration of many DICMs. From this burst of DICM, we can quantify the probability distribution (PD) of DICM across experimental time (see C). (C) Tabular representation of the probability distribution (PD) of DICM for left and right superior frontal gyrus across the experimental time shown in B. This matrix is called a comodulogram and keeps the information of PD from the 36 possible coupling modes. In the main diagonal the PD of the 8 possible intra-frequency coupling can be seen while in the off-diagonal are the 28 possible cross-frequency pairs. PICM, Prominent Intrinsic Coupling Modes; DICM, Dominant Intrinsic Coupling Modes; PLV, imaginary part of Phase Locking Value; PD, probability distribution.

to discrete-time Markov chain theory (Jarvis and Shier, 1999), a finite number $\left(S_{1}, S_{2} \ldots, S_{m}\right)$ of inferred states that evolve in discrete time with a time-homogeneous transition structure can be mathematically represented by either its transition probability matrix or its directed graph (digraph). Here, the inferred states refer to the prototypical FC $\mu$ states. A feasible transition is one 

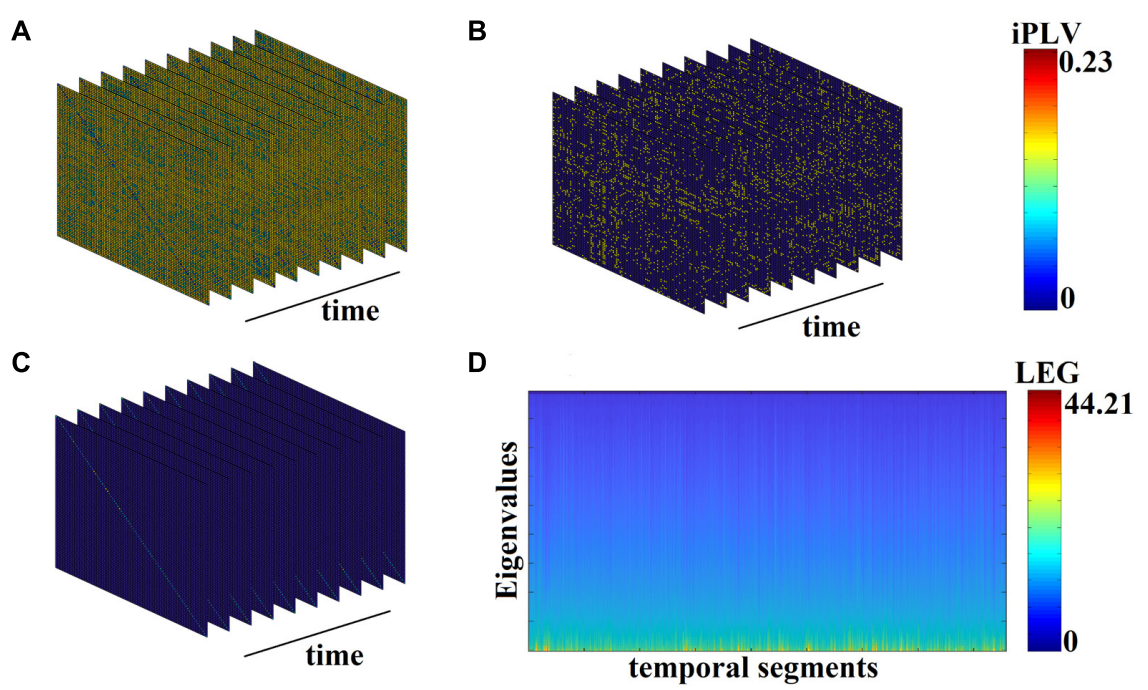

FIGURE 3 | From DFCG to the temporal evolution of Laplacian eigenvalues (LEG; from the first healthy control subject). (A) The first 10 snapshots of DFCG. (B) The quasi-static FCGs shown in A were topologically filtered with OMST. (C) The normalized Laplacian transformation of the topologically filtered FCGs shown in B. (D) Temporal evolution of the Laplacian eigenvalues for the 2,401 temporal segments.

whose occurring probability is greater than zero. The probability of transition from node (state) $i$ to node $j$ is defined as

$$
P_{i j}=\frac{N_{i j}}{\sum_{i j} N_{i j}}, i=1,2, \ldots, n \quad j=1,2, \ldots, m,
$$

where Nij is the number of transitions from node $i$ to node $j$. Obviously, the sum of the transition probabilities along each row of the transition matrix $P$ equals one. The complete digraph for a finite-state Markov process has edges of transition probabilities between every node $i$ and every other node $j$. Here, nodes refer to FCustates in the Markov chain. In the digraphs created in this study, $P_{i j}$ survives a $p$-value derived from 10,000 shuffledsurrogates of the original STS.

\section{Temporal Measurements of an $\mathrm{FC} \mu$ state Symbolic Sequence}

For further summarizing inter-FC $\mu$ state transition patterns, relevant temporal measurements were obtained and analyzed from the Markov chain structures of the subject-specific FC $\mu$ state sequence, including: (1) fractional occupancy for each class of

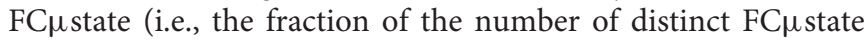
of a given class occurring within 2,401 temporal segments), (2) dwell time for each FC $\mu$ state which gives the average time the brain spends within a specific FC $\mu$ state in consecutive temporal segments, (3) transition probabilities (TP) of a given FCustate to any other functional connectivity state, (4) the complexity index (CI) that quantifies the richness of the spectrum of code words formed up to a length based on the symbolic time series (Dimitriadis, 2018), and (5) the flexibility index (FI) that quantifies the transition of the brain states (FC $\mu$ states) between consecutive temporal segments.

\section{Assessing the Statistically Significant Level of the Symbolic-Based Estimates}

To assess the statistically significant level of the aforementioned four estimates (excluding CI), we shuffled the group symbolic time series 10,000 times and re-estimated the surrogate-based $p$-values for every estimate per subject. CI is normalized by default with surrogates.

\section{Linking MMSE With Chronnectomics}

To investigate the possible relation between MMSE and the chronnectomics derived by the FC $\mu$ state symbolic sequence (see section "Temporal Measurements of an FC $\mu$ state Symbolic Sequence"), we used the canonical correlation analysis (CCA) approach to see whether MMSE correlates with seven chronnectomic variables. In our analyses, the significance of the correlation was estimated using Bartlett's approximate chi-squared statistic as implemented in MATLAB.

\section{Algorithms and MATLAB Code}

All the algorithmic steps of constructing the DCFGs were implemented on inhouse software written in MATLAB, freely available from the first author's website. LCMV beamformer was programmed under Fieldtrip's environment (Oostenveld et al., 2011).

\section{RESULTS}

\section{Group Prototypical FC $\mu$ states}

Figure 4 illustrates the prototypical group-specific FC $\mu$ states for each group by assigning the $90 \mathrm{AAL}$ brain areas to five well-known brain networks. The size and color of every circle decode the mean degree within every brain network while the color of each connection defines the mean functional strength 


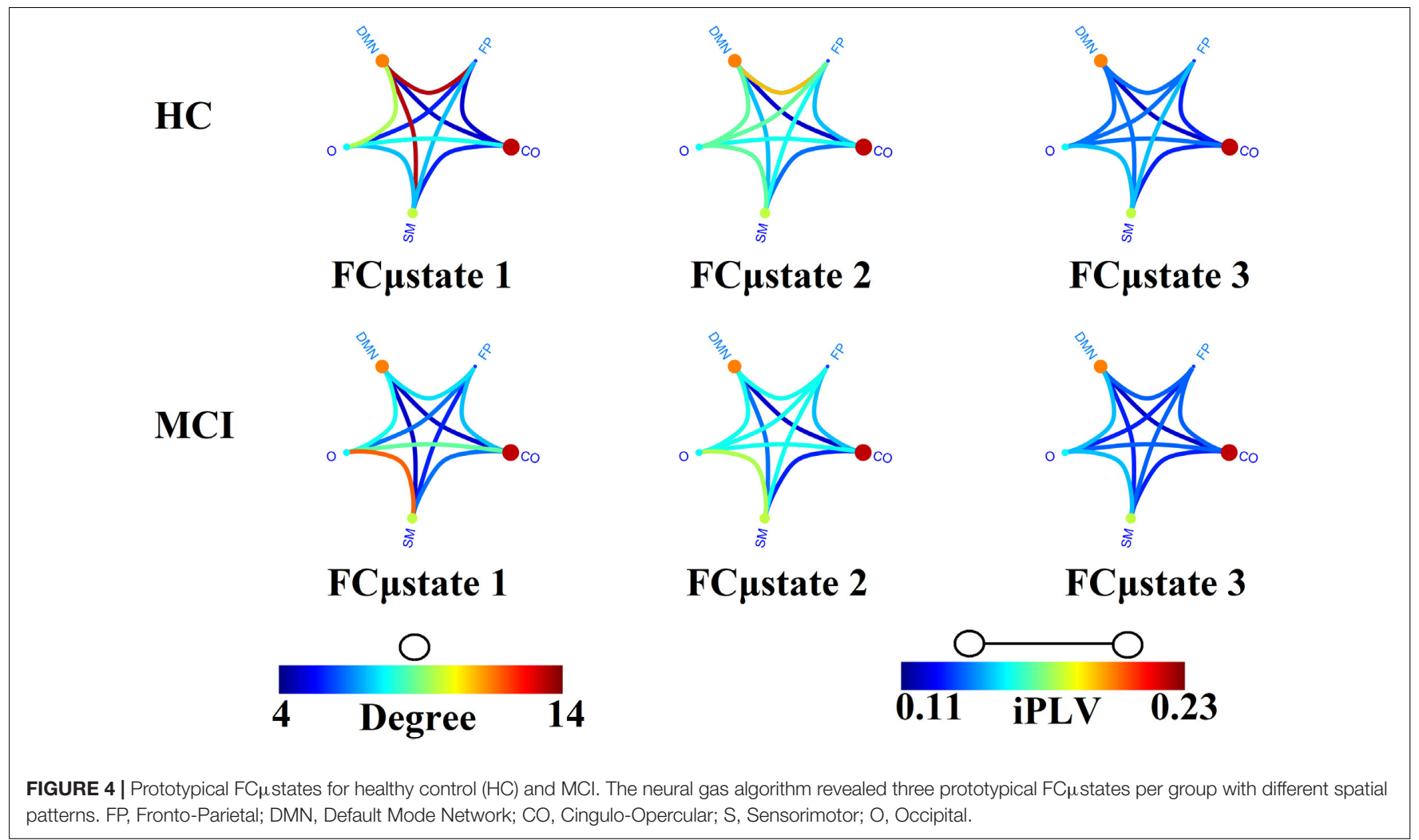

between every pair of brain networks. FC $\mu$ states can be described based on the most connected brain networks focusing on their degree. The most connected brain networks are the DMN and CO. Following a statistical test by comparing the functional coupling strength between FPN and DMN independently for every FC $\mu$ state, we found significant higher values for FC $\mu$ states 1 and 3 for HC compared to MCI ( $p=0.00045$ for FC $\mu$ state 1 and $p=0.000012$ for FC $\mu$ states 3, Wilcoxon Rank Sum Test).

Figure 5 demonstrates the dynamic reconfiguration of prototypical FCustates for the first subject of both groups for the 1 st $\mathrm{min}$.

\section{Classification of Blind Samples via Representations With Prototypical Net $\mu$ states $^{\text {eigen }}$}

Each test sample with an unknown label was classified to one of the two classes using as a criterion the minimization of the reconstruction error. The minimum reconstruction error denotes the class label of the sample. In our study, we used 20 samples with a distribution of 11 MCIs and 9 controls with $85 \%$ accuracy for CENT (17 out of 20) and 70\% for the PCA representation scheme (14 out of 20). SID received the blind dataset from MEL, who evaluated the outcome of this research. Figure 6 illustrates the methodological approach. Figure $\mathbf{6 A}$ refers to the temporal resolution of the Laplacian eigenvalues of a blind $\mathrm{HC}$ subject while Figures 6B,C the reconstruction of Figure 6A matrix employing the Prototypical Net $\mu$ states ${ }^{\text {eigen }}$ related to $\mathrm{HC}$ and MCI, correspondingly. Based on the reconstruction error between the original matrix (Figure 6A) and the two reconstructed matrixes (Figures 6B,C), a decision regarding the label of the blind subject was taken based on the lowest reconstruction error (Figure 6D).

\section{Group-Differences of Temporal Measurements Derived From FC $\mu$ state Symbolic Sequence}

FI, OT, and DT were significantly higher than the surrogates based values derived from the shuffled symbolic time series $(p<0.001)$. We detected significant higher FI and CI for HC compared to MCI applying a Wilcoxon Rank-Sum test (Figure 7, $p$-value $<0.00000001)$. Summarizing the results from OT and DT, HC subjects spent significantly higher time compared to MCI to first and third FC $\mu$ state while MCI spent significantly more time to the second FC $\mu$ state Figure 8, $p$-value $<0.00000001$ ).

\section{Modeling Dynamic Reconfiguration of Functional Connectivity Graphs as a Markovian Chain}

The outcome of the VQ modeling of NMTSeigen is the

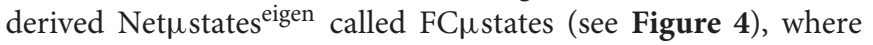
its evolution is described via a symbolic time series, a Markovian chain. Figure 9 illustrates a well-known scheme of the group-averaged transition probabilities (TP) between the three FC $\mu$ states for both groups. Our analysis revealed significant group differences in terms of TP, while the TPs were significantly different compared to the surrogates' symbolic time series. 

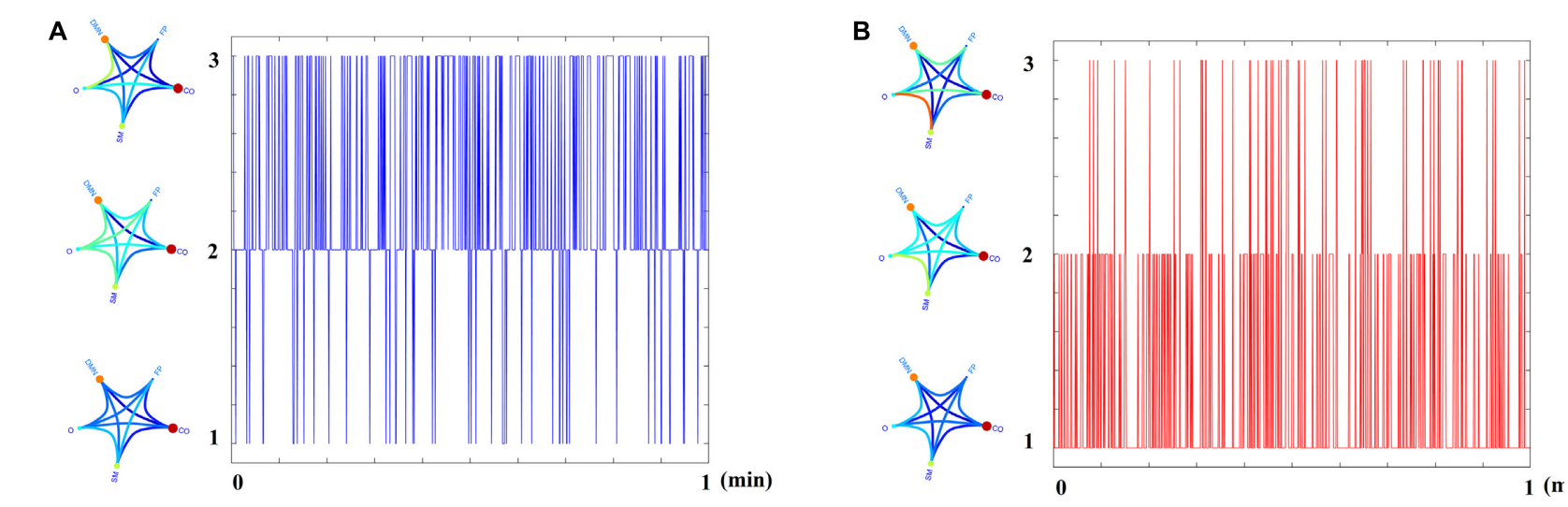

FIGURE 5 | Temporal Evolution of Prototypical FC $\mu$ states for the first subject of (A) HC and (B) MCl group for the 1 st min of resting state.

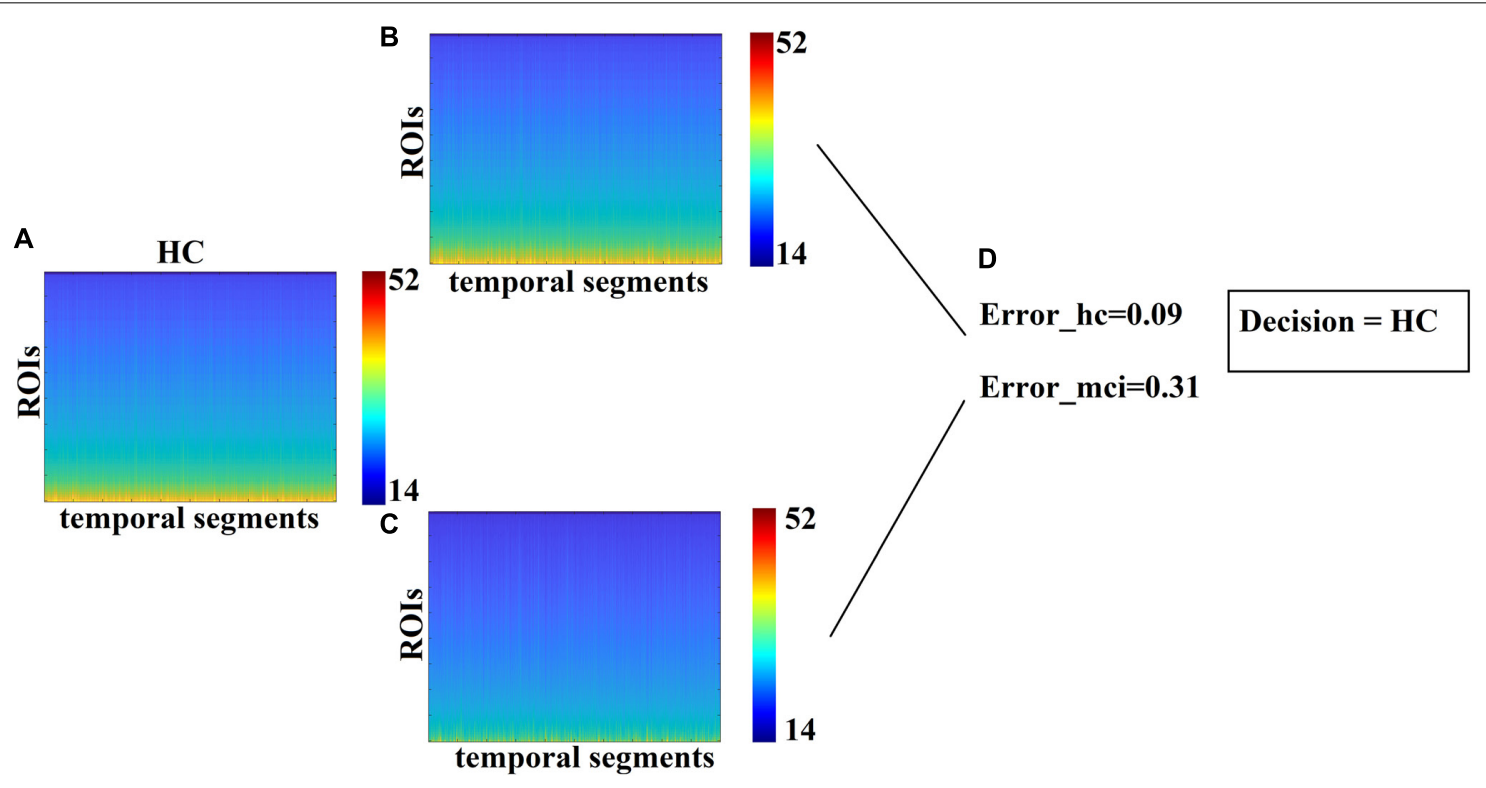

FIGURE 6 | Classification of blind subjects via prototypical Net $\mu$ states ${ }^{\text {eigen }}$. (A) Evolution of eigenvalues for the test sample. (B,C) Reconstruction of the temporal evolution of eigenvalues of the train sample with both group-specific prototypical Net $\mu$ states ${ }^{\text {eigen }}$. (D) Estimation of the reconstruction error between the original temporal evolution of eigenvalues in $\mathbf{A}$. and the two prototypical-based shown in $\mathbf{B}$. The decision of the subject's label was taken via the lower reconstruction error.

Self-loops defined the "staying" TP of brain dynamics to the same brain state.

The symbolic time series illustrated in Figure 5 is a Markovian chain of order 1 and it is shown schematically with a diagram of three nodes defining the three FCustates (Figures 4, 9) while the arrows from one state to the other show the TP. Our results revealed significant group differences between every possible brain state transition (Wilcoxon Rank-Sum test, $p<0.0001 / 9)$.

\section{Comodulograms of Dominant Intrinsic Coupling Modes (DICM)}

Probability distributions (PD) of prominent intrinsic coupling modes across all sources pairs and time windows were summarized for each group in the form of an $8 \times 8$ matrix. The horizontal axis refers to the phase modulating frequency $(\mathrm{Hz})$ while the $y$-axis refers to the amplitude modulated frequency $(\mathrm{Hz})$. The main diagonal of the comodulograms keeps the PD of intra-frequency phase-to-phase coupling. Group-averaged comodulograms in Figure $\mathbf{1 0}$ demonstrate the empirical PD of DICM revealing a significant role of $\alpha_{1}$ as phase modulator of the whole studying spectrum up to high-gamma $\left(\gamma_{2}\right)$ activity, which covers almost $50 \%$ of pairwise source connections and time windows. No significant trend was detected regarding the PD of each pair of frequencies between the two groups $(p<0.05$, Wilcoxon rank-sum test, Bonferroni corrected). Moreover, no significant difference was found regarding the PD of the groups for every possible pair of sources $(p<0.05$, Wilcoxon rank-sum test, 


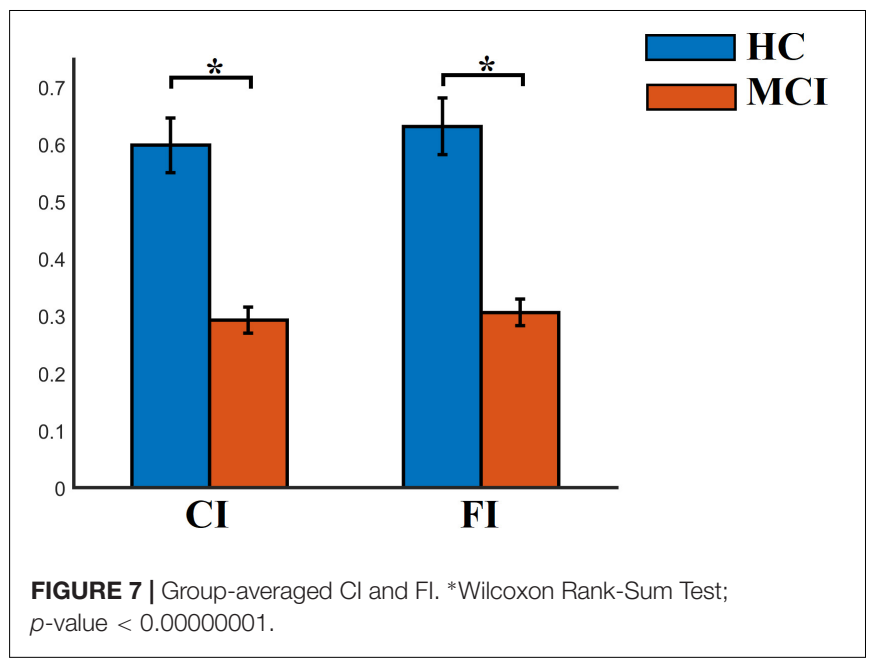

Bonferroni corrected). Finally, transition dynamics of DICM between consecutive time windows at every source pair did not uncover any group difference (for further details, see Dimitriadis et al., 2016a).

\section{Correlation of MMSE With Chronnectomics}

Figure 11 demonstrates the outcome of CCA analysis between chronnectomics and the well-known MMSE. The Chi-square was 26.95 and the related $p$-value $=0.00033886$. $x$-axis refers to the canonical variable scores of the chronnectomics, where the DT of the three NMTS ${ }^{\text {eigen }}$ contributes most to the maximization of their canonical correlation with MMSE. OC_2 did not associate with the CCA mode of MMSE variability. The 1st canonical component is:
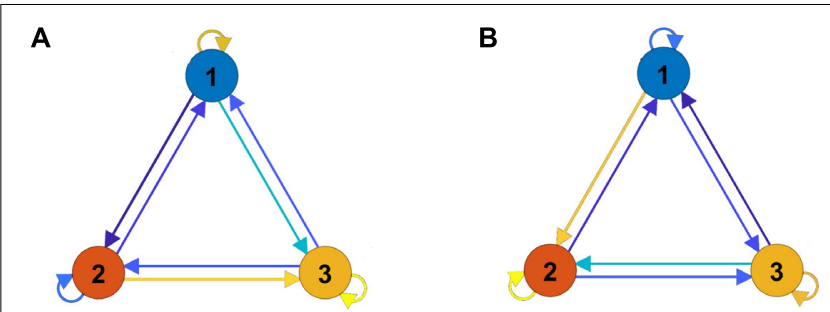

\section{TP

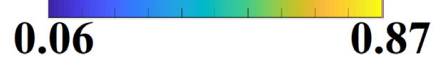

FIGURE 9 | A finite-state diagram showing group-averaged transition probability matrix (TP) of the symbolic time series, which describes the temporal evolution of the brain, states (FC $\mu$ state). (A) For healthy control and (B) forMCl.

$$
\begin{aligned}
C C_{1}= & 0.11^{*} F I+0.02^{*} O C_{1}+0.02^{*} O C_{3} \pm 0.0056^{*} C I+ \\
& 3.38^{*} D T_{1}+5.76^{*} D T_{2}+2.65^{*} D T_{3}
\end{aligned}
$$

and the second is:

$\mathrm{CC} 2=0.59^{*} \mathrm{MMSE}$.

\section{DISCUSSION}

We have demonstrated here a novel framework for designing a proper DCB for the detection of MCI subjects from spontaneous neuromagnetic activity. The whole approach exhibits novel, datadriven, algorithmic steps that can be summarized as follows:
A

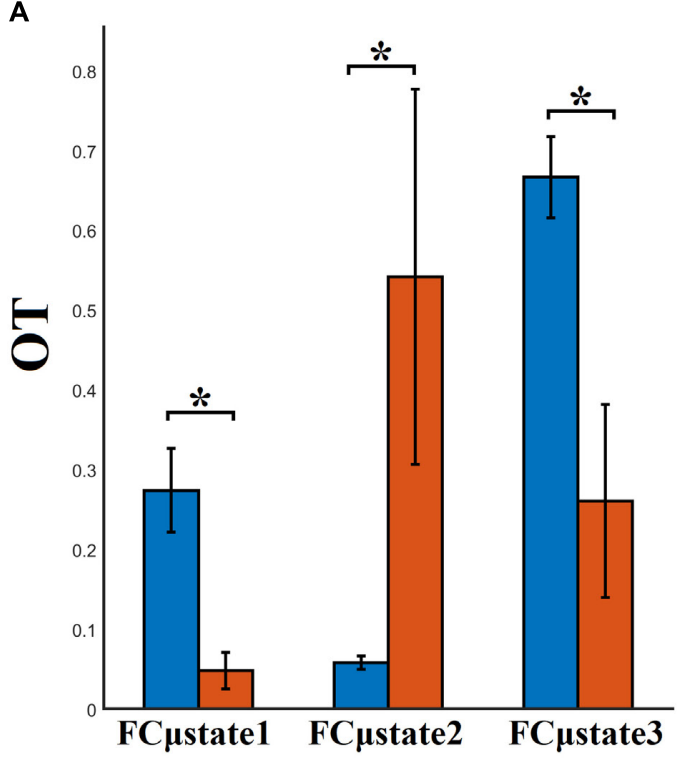

B

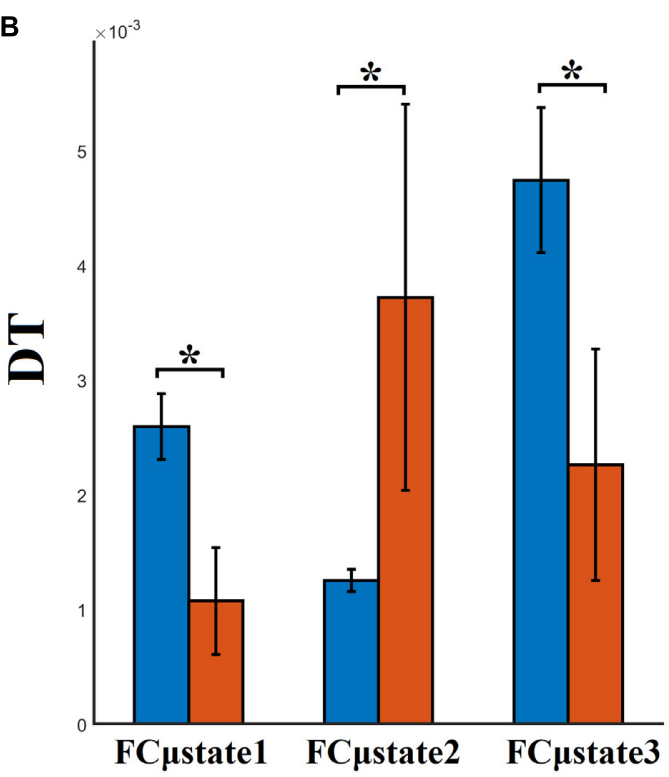

$\square$ HC $\square \mathrm{MCI}$

FIGURE 8 | Group-averaged (A) OT and (B) DT per FC $\mu$ state. *Wilcoxon Rank-Sum Test; $p$-value $<0.00000001$. 
- The construction of a IDFCG that incorporates dominant types of interactions, either intra- (e.g., $\theta-\theta$ ) or inter-frequency [phase-to-amplitude coupling (PAC) (e.g., $\theta-\gamma)]$ coupling.

- The application of a new thresholding scheme termed OMSTs as a topological filtering applied to DFCG to extract a "true" network topology.

- The VQ modeling of network metric time series (NMTS) based on nodal Laplacian eigenvalues for prototyping the spatiotemporal dynamics of both control and MCI subjects.

- Modeling of the switching behavior of brain states as a Markovian chain

- The validation of the whole approach to a second blind dataset achieving an 85\% classification accuracy for the CENT ROI representation scheme compared to $70 \%$ for the PCA scheme

- Regions of interest representation scheme matters on the designing of connectomic biomarker in general and also for MCI

- Canonical correlation analysis between chronnectomics and MMSE revealed that the DT of brain states associates strongly with the CCA mode of MMSE variability.

We proved that the VQ modeling of NMTS eigen is an effective approach to extract an overcomplete dictionary for the representation of DFC that can accurately classify subjects as either control or MCI based on their resting state MEG activity. Adopting a static network analysis, the classification accuracy was 12 out of 20, demonstrating the need of a DFC approach for studying resting brain dynamics (Dimitriadis et al., 2010b, 2012a,c, 2013a,b, 2015a,b, 2016a, 2017a,b, 2018a,b; Allen et al., 2014; Damaraju et al., 2014; Kopell et al., 2014).
The capture of time-varying coupling between variables is a topic that has been heavily studied in other fields and in communications for signal processing in particular. However, the specific application to whole-brain functional connectivity is relatively new (Sakoglu et al., 2010; Dimitriadis et al., 2013a; Calhoun et al., 2014), and its application to brain-imaging data poses particular challenges, which are the topic of active current research. One important challenge is how to best identify relevant features from the high-dimensional brain imaging data. The main algorithms used for manipulating functional brain network dynamics in fMRI are group ICA (Calhoun and Adali, 2012) or spatial-constrained ICA (Lin et al., 2013) and tensor decompositions (Acar and Yener, 2009). To characterize the dynamics of time-varying connectivity brain patterns, the basic approach is the metastate analysis based on the sliding window or more adaptive approach (Dimitriadis et al., 2013a, 2015d; Damaraju et al., 2014; Nomi et al., 2016). From the dynamic connectivity patterns, FC $\mu$ states are extracted that are "quasi-stable" distinct brain states. Then, the state vectors can be modeled via a Markovian chain (Dimitriadis et al., 2013a, 2015b; Calhoun et al., 2014; Damaraju et al., 2014).

Cross-frequency coupling mechanisms support the brain interactions across space over multiple temporal scales (Canolty and Knight, 2010; Fell and Axmacher, 2011). Computational models have explored the theoretical advantages of the existence of cross frequency coupling (Lisman and Idiart, 1995; Neymotin et al., 2011). These models untangled the major mechanisms of the importance of CFC, which may serve as the brain's neural syntax. Segmentation of spike trains into cell clusters ("letters") and sequences of assemblies (neural "words") are supported by the existing syntactic rules (Buzsaki, 2010).

In the present study, we demonstrated a methodology whose main scope is to provide a framework for modeling DFCG into a repertoire of distinct "quasi-static" brain states called

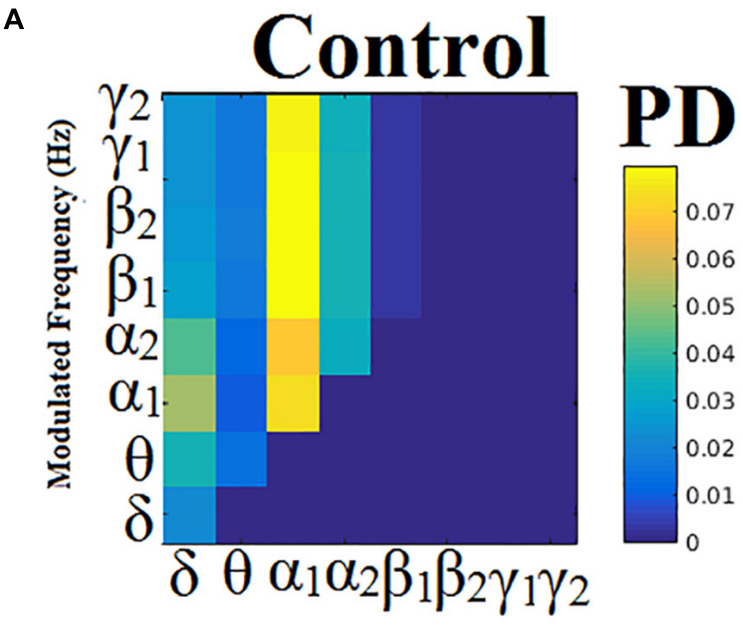

Modulating Frequency $(\mathrm{Hz})$

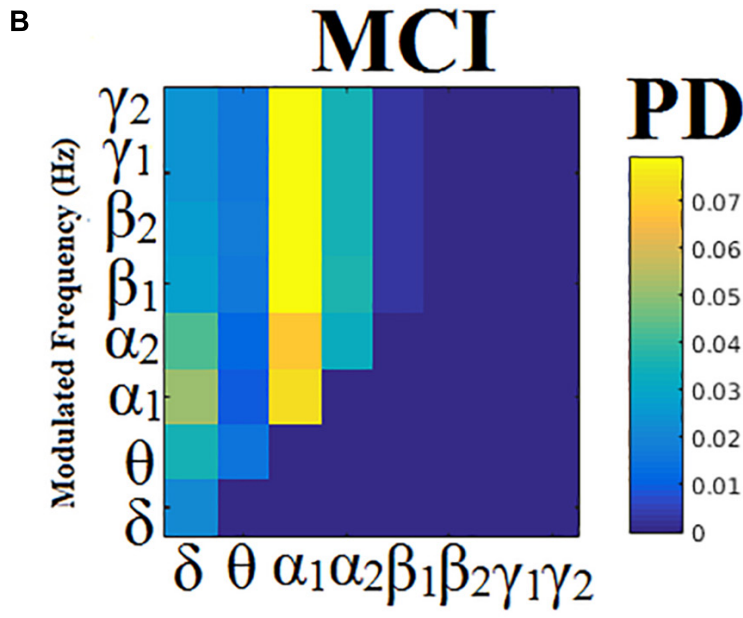

Modulating Frequency $(\mathrm{Hz})$

FIGURE 10 | Group-averaged empirical Probability Distribution values of DICMs for MCI (B) compared to control group (A). 


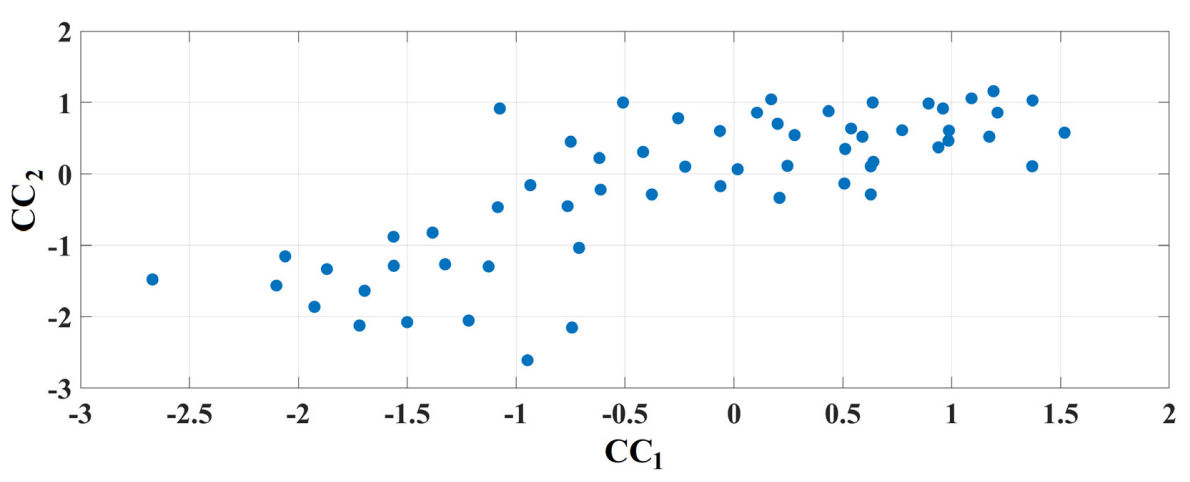

FIGURE 11 | Canonical Correlation Analysis of Chronnectomics and MMSE. Figure plots the canonical variable scores referring to the two sets.

FC $\mu$ states. Here, we modeled the NMTS derived from the DFC patterns expressed via the Laplacian eigenvalues (Dimitriadis et al., 2015d, 2017b, 2018b). After extracting the virtual source time series, we followed an algorithmic approach with the main aim of minimizing the effect of a priori selection of variables that can minimize the reproducibility of the results. The main steps of the proposed methodology are: (1) the construction of one integrated DFC per subject-which incorporates the DICM per each pair of brain areas and at every temporal segment, (2) the application of a data-driven topological filtering scheme to reveal the backbone of the network topology at every temporal segment, (3) the estimation of Laplacian eigenvalues to extract the so-called NMTSeigen (Dimitriadis et al., 2010a, 2015d, 2017b, 2018b), (4) the modeling of these NMTS ${ }^{\text {eigen }}$ via a vector-quantization approach, and (5) the validation of the whole approach to a second blind dataset.

The analysis of the spatiotemporal evolution of Laplacian eigenvalues during the training phase revealed three prototypical brain states (FC $\mu$ states). For a better illustration of the FCGs linked to the prototypical eigenvalues, we assigned the $90 \mathrm{AAL}$ brain areas to five well-known brain networks. In Figure 4, we mapped the average functional strength between ROIs belonging to every pair of brain networks while the size and color of every node define the within-brain network degree. The most connected brain networks in FC $\mu$ states are the DMN and CO. CO plays a key role in working memory mechanisms (Wallis et al., 2015) while cognitive complaints related to $\mathrm{AD}$ are linked to alterations of resting-state brain networks and mostly FPN and DMN (Contreras et al., 2017). The functional coupling strength between FPN and DMN was significantly higher for $\mathrm{HC}$ compared to MCI for FCustates 1 and 3 (Figure 4). The functional strength between FPN-DMN was positively correlated with a better episodic memory performance (Contreras et al., 2017).

Well-known and novel chronnectomics were estimated from the Markovian (symbolic) Chain that describes the evolution of brain states. We detected significantly higher flexibility and complexity for HC as compared to MCI described from FI and CI, correspondingly (Figure 7). A summarization derived from OT and DT revealed a significant trend: HC subjects spent significantly more time compared to MCI in FC $\mu$ states 1 and 3 while MCI spent significantly more time in the second FC $\mu$ state (Figure 8). Following a CCA analysis between the extracted chronnectomics and the MMSE score, we found a significant contribution of the DT for the three NMTS ${ }^{\text {eigen }}$. OC related to the 2nd NMTS eigen did not associate with the CCA mode of MMSE variability (Figure 11).

In the era of data sharing and aggregating large datasets from different research groups worldwide who contribute to large consortiums, it is important to test the reproducibility of the proposed biomarkers (Abraham et al., 2017). Our study is a first step in this direction to diminish the effect of any arbitrary selection of algorithmic steps up to the extraction of biomarkers. The next step is to extend the analysis in larger populations from different sites and MEG scanners. A recent study showed that 70 percent of the preclinical research from academic labs could not be replicated (Collins and Tabak, 2014). Abraham's work is one of the very first neuroimaging studies that lays the ground for the reliability and reproducibility of biomarkers extracted from neuroimaging data.

There is a large body of research based on different imaging methods covering various temporal and spatial scales that documents the association of electrophysiological rhythms with distinct cognitive processes within narrowly or broadly anatomical areas (for review, see Engel et al., 2001; Buszaky, 2006; Siegel et al., 2012; Başar and Güntekin, 2013). For example, low-frequency $\delta$ rhythms $(1-4 \mathrm{~Hz})$ are known to coordinate large portions of the brain (Fujisawa and Buzsaki, 2011; Nacher et al., 2013) while $\gamma$ oscillations play a dominant role in stimulus processing and detection is shown to be locally anatomically constrained (Engel et al., 2001). Recently, an extension of Brodmann's areas was suggested in order to associate distinct anatomical areas with preferable connectivity estimators and cognitive functions in both normal and brain disease/disorder populations as an initial step toward summarizing the large body of current brain connectivity research (Başar and Düzgün, 2016).

In the last few years, an increasing number of studies appeared studying CFC at resting state (Antonakakis et al., 2016a), during cognitive tasks (Dimitriadis et al., 2015a,c, 2016a,b) 
and in various brain diseases and disorders such as mild traumatic brain injury (Antonakakis et al., 2016a), amnestic MCI (Dimitriadis et al., 2015a), dyslexia (Dimitriadis et al., 2016a), schizophrenia (Kirihara et al., 2012), etc. It has been suggested that CFC is the key mechanism for the integration of information between anatomical distribution subsystems that function on a dominant frequency (Canolty and Knight, 2010; Jirsa and Muller, 2013; Florin and Baillet, 2015). However, only a few MEG studies have explored CFC at resting state (Antonakakis et al., 2015, 2016a,b; Florin and Baillet, 2015) and especially in a more dynamic fashion (Dimitriadis et al., 2015a, 2016a; Antonakakis et al., 2016b).

MEG source connectivity is at a mature level compared to a decade ago (Ioannides et al., 2012), and it is an active research area aimed at improving many aspects of "true" brain connectivity (Schoffelen and Gross, 2009; Colclough et al., 2016). The most significant issue is the parcellation of the cerebral cortex. In many cases, the AAL template (90 ROIs) is feasible for the detection of those changes induced by a specific task or obtained after comparing different groups. But in others such as the design of a reliable connectomic biomarker, there is a need to oversample more than 90 areas. The FC, which is directly linked to functional parcellation of the cerebral cortex, is an active area, which will further improve both the interpretation and the predictive power of source connectivity of many brain diseases such as MCI. The solution of a functional parcellation template for MEG source connectivity will improve the classification performance on the source level with the additional advantage, compared to sensor level, of facilitating the anatomical interpretation of the results.

Adopting the same framework and including also stable and progressive MCI groups, we will attempt to connect DCB with neuropsychological measures and cognitive scores (Cuesta et al., 2014, 2015). It is evident that a multifactorial model that includes cognition, neuropsychological measures and anatomical information can reliably predict the conversion from MCI to DAT, while genetic variation of risk genes like the APOE-e4 allele or cognitive reserve might play a secondary role (López et al., 2016).

Going one step further from our previous studies demonstrating the significance of a DCB (Dimitriadis et al., 2013b, 2015b), where we used network microstates extracted from DFCG patterns, in the present study we introduced a modeling approach of NMTS eigen estimated over DFCGs that preserve the dominant type of coupling (intra- or inter-frequency intrinsic coupling mode). Our study demonstrates the effectiveness of the data-driven analytic pipeline tailored to DFCG to the correct classification of a blind dataset based on control and MCI subjects compared to a static connectivity approach. Given these outcomes, the need is evident over the next years to adopt data-driven techniques that will not introduce bias, subjectivity and assumptions in neuroimaging datasets and also to improve the reproducibility of the outcome in large databases.

In magnetoencephalography (MEG) the conventional approach to source reconstruction is to solve the underdetermined inverse problem independently over time and space. Different algorithms have been proposed so far with alternative regularization procedures of space and time as with a Gaussian random field model (Solin et al., 2016).

Commonly used techniques include the minimum-norm estimate (MNE) (Hämäläinen and Ilmoniemi, 1994) and Linearly Constrained Minimum-Variance (LCMV) beamformer (Van Veen et al., 1997). It is in the right direction to compare the consistency of the outcome of the current study with alternative inverse solution algorithms to measure their consistency and sensitivity to the design of connectomic biomarkers tailored to MCI.

\section{CONCLUSION}

In this study, we presented a novel DCM for the prediction of MCI from an age-matched control group validated over a blind dataset. The novelties of the proposed analytic scheme are the incorporation in the DFCGs of the DICM (DICM, either intra- or inter-frequency coupling based on PAC), the adaptation of a novel data-driven thresholding scheme based on OMSTs, the estimation of Laplacian eigenvalues across time and the extraction of prototypical network microstates (FC $\mu$ states) for both the control and MCI group.

It is important for the near future to work in source space on MCI subjects that convert to AD after a following up study to further validate the proposed scheme as a potential tool of clinical importance. It would also be interesting to explore how the Apoe-e 4 allele can induce changes to the DFC of spontaneous activity. Moreover, multimodal neuroimaging biomarkers is a novel trend that will further be validated (Jack et al., 2016).

\section{DATA AVAILABILITY}

All datasets generated for this study are included in the manuscript and/or the Supplementary Files.

\section{AUTHOR CONTRIBUTIONS}

SD conceptualized the research analysis, methods, and design, data analysis, and drafting the manuscript. ML acquired the data. ML, FM, and EP criticized the revision of the manuscript. All authors read and approved the final version of the manuscript.

\section{FUNDING}

This study was supported by three projects from the Spanish Ministry of Economy and Competitiveness (PSI2009-14415-C0301, PSI2012-38375-C03-01, and TEC2016-80063-C3-2-R) and the National Centre for Mental Health (NCMH) at Cardiff University. SD was supported by MRC grant MR/K004360/1 (Behavioural and Neurophysiological Effects of Schizophrenia Risk Genes: A Multi-locus, Pathway Based Approach). SD is also supported by a MARIE-CURIE COFUND EU-UK Research Fellowship. 


\section{ACKNOWLEDGMENTS}

We would like to acknowledge RCUK of Cardiff University and the Welcome Trust for covering the publication fee. We would also like to acknowledge "Madrid Neurocenter".

\section{REFERENCES}

Abraham, A., Milham, M., Craddock, R. C., Samaras, D., Thirion, B., and Varoquaux, G. (2017). Deriving reproducible biomarkers from multi-site resting-state data: an Autism-based example. Neuroimage 147, 736-745. doi: 10.1016/j.neuroimage.2016.10.045

Acar, E., and Yener, B. (2009). Unsupervised multiway data analysis: a literature survey. IEEE Trans. Knowledge Data Eng. 21, 6-20. doi: 10.1109/tkde.2008.112

Albert, M. S., DeKosky, S. T., Dickson, D., Dubois, B., Feldman, H. H., Fox, N. C., et al. (2011). The diagnosis of mild cognitive impairment due to Alzheimer's disease: recommendations from the National Institute on AgingAlzheimer's Association workgroups on diagnostic guidelines for Alzheimer's disease. Alzheimers Dement. 7, 270-279.

Allen, E. A., Damaraju, E., Plis, S. M., Erhardt, E. B., Eichele, T., and Calhoun, V. D. (2014). Tracking whole-brain connectivity dynamics in the resting state. Cereb. Cortex 24, 663-676. doi: 10.1093/cercor/bhs352

Antonakakis, M., Dimitriadis, S. I., Zervakis, M., Micheloyannis, S., Rezaie, R., Babajani-Feremi, A., et al. (2016a). Altered cross-frequency coupling in restingstate MEG after mild traumatic brain injury. Int. J. Psychophysiol. 102, 1-11. doi: 10.1016/j.ijpsycho.2016.02.002

Antonakakis, M., Dimitriadis, S. I., Zervakis, M., Zouridakis, G., and Papanicolaou, A. C. (2016b). "Mining cross-frequency coupling microstates from resting state MEG: an application to mild traumatic brain injury," in Proceedings of the 38th Annual International Conference of the IEEE Engineering in Medicine and Biology Society, Orlando, FL. doi: 10.1016/j.ijpsycho.2016.02.002

Antonakakis, M., Dimitriadis, S. I., Zervakis, M., Papanicolaou, A. C., and Zouridakis, G. (2017). Altered rich-club and frequency-dependent subnetwork organization in mild traumatic brain injury: a MEG resting-state study. Front. Hum. Neurosci. 11:416. doi: 10.3389/fnhum.2017.00416

Antonakakis, M., Dimitriadis, S. I., Zervakis, M., Rezaie, R., Babajani-Feremi, A., Micheloyannis, S., et al. (2015). "Uncovering the brain model of MEG brain networks from cross-frequency coupling estimates via an attacking strategy," in Proceedings of the 37th Annual International Conference of the IEEE Engineering in Medicine and Biology Society, Milano.

Bai, F., Shu, N., Yuan, Y., Shi, Y., Yu, H., Wu, D., et al. (2012). Topologically convergent and divergent structural connectivity patterns between patients with remitted geriatric depression and amnestic mild cognitive impairment. J. Neurosci. 32, 4307-4318. doi: 10.1523/JNEUROSCI.5061-11.2012

Bajo, R., Maestú, F., Nevado, A., Sancho, M., Gutiérrez, R., Campo, P., et al. (2010). Functional connectivity in mild cognitive impairment during a memory task: implications for the disconnection hypothesis. J. Alzheimers Dis. 22, 183-193. doi: 10.3233/JAD-2010-100177

Baker, A. P., Brookes, M. J., Rezek, I. A., Smith, S. M., Behrens, T., Probert Smith, P. J., et al. (2014). Fast transient networks in spontaneous human brain activity. eLife 3:e01867. doi: 10.7554/eLife.01867

Başar, E., and Düzgün, A. (2016). The CLAIR model: extension of Brodmann's areas based on brain oscillations and connectivity. Int. J. Psychophysiol. 103, 185-198. doi: 10.1016/j.ijpsycho.2015.02.018

Başar, E., and Güntekin, B. (2013). Review of delta, theta, alpha, beta and gamma response oscillations in neuropsychiatric disorders. Suppl. Clin. Neurophysiol. 62, 303-341. doi: 10.1016/b978-0-7020-5307-8.00019-3

Bassett, D., Wymbs, N., Porter, M., Mucha, P., Carlson, J., and Grafton, S. (2011). Dynamic reconfiguration of human brain networks during learning. Proc. Natl. Acad. Sci. U.S.A. 108, 7641-7646. doi: 10.1073/pnas.1018985108

Biswal, B., Yetkin, F. Z., Haughton, V. M., and Hyde, J. S. (1995). Functional connectivity in the motor cortex of resting human brain using echo-planar MRI. Magn. Reson. Med. 34, 537-554.

Braun, U., Muldoon, S. F., and Bassett, D. S. (eds). (2014). "On human brain networks in health and disease," in eLS, (Chichester: Wiley), 1-9. doi: 10.1002/ 9780470015902.a0025783

\section{SUPPLEMENTARY MATERIAL}

The Supplementary Material for this article can be found online at: https://www.frontiersin.org/articles/10.3389/fnins. 2019.00542/full\#supplementary-material

Britz, J. D., Van De Ville, C. M., and Michel, C. M. (2010). BOLD correlates of EEG topography reveal rapid resting-state network dynamics. Neuroimage 52, 1162-1170. doi: 10.1016/j.neuroimage.2010.02.052

Brookes, M. J., Hale, J. R., Zumer, J. M., Stevenson, C. M., Francis, S. T., Barnes, G. R., et al. (2011). Measuring functional connectivity using MEG: methodology and comparison with fcMRI. Neuroimage 56, 1082-1104. doi: 10.1016/j.neuroimage.2011.02.054

Buldú, J. M., Bajo, R., Maestú, F., Castellanos, N., Leyva, I., Gil, P., et al. (2011). Reorganization of functional networks in mild cognitive impairment. PLoS One 6:e19584. doi: 10.1371/journal.pone.0019584

Buszaky, G. (2006). Rhythms of the Brain. New York, NY: Oxford University Press, 488.

Buzsaki, G. (2010). Neural syntax: cell assemblies, synapsembles, and readers. Neuron 68, 362-385. doi: 10.1016/j.neuron.2010.09.023

Calhoun, V. D., and Adali, T. (2012). Multi-subject independent component analysis of fMRI: a decade of intrinsic networks, default mode, and neurodiagnostic discovery. IEEE Rev. Biomed. Eng. 5, 60-73. doi: 10.1109/ rbme.2012.2211076

Calhoun, V. D., and Adali, T. (2016). Time-varying brain connectivity in fMRI data: whole-brain data-driven approaches for capturing and characterizing dynamic states. IEEE Signal Process. Mag. 33, 52-66. doi: 10.1109/MSP.2015. 2478915

Calhoun, V. D., Miller, R., Pearlson, G., and Adali, T. (2014). The chronnectome: time-varying connectivity networks as the next frontier in fMRI data discovery. Neuron 84, 262-274. doi: 10.1016/j.neuron.2014.10.015

Canolty, R. T., and Knight, R. T. (2010). The functional role of cross-frequency coupling. Trends Cogn. Sci. 14, 506-515. doi: 10.1016/j.tics.2010.09.001

Chang, C., and Glover, G. (2010). Time-frequency dynamics of resting-state brain connectivity measured with fMRI. Neuroimage 50, 81-98. doi: 10.1016/j. neuroimage.2009.12.011

Colclough, G. L., Woolrich, M. W., Tewarie, P. K., Brookes, M. J., Quinn, A. J., and Smith, S. M. (2016). How reliable are MEG resting-state connectivity metrics? Neuroimage [Epub ahead of print].

Collins, F. S., and Tabak, L. A. (2014). Policy: NIH plans to enhance reproducibility. Nature 505, 612-613. doi: 10.1038/505612a

Contreras, J. A., Goni, J., Risacher, S. L., Amico, E., Yoder, K., Dzemidzic, M., et al. (2017). Cognitive complaints in older adults at risk for Alzheimer's disease are associated with altered resting-state networks. Alzheimers Dement. 6, 40-49. doi: 10.1016/j.dadm.2016.12.004

Cuesta, P., Barabash, A., Aurtenetxe, S., Garcés, P., López, M. E., Bajo, R., et al. (2014). Source analysis of spontaneous magnetoencephalograpic activity in healthy aging and mild cognitive impairment: influence of apolipoprotein $\mathrm{E}$ polymorphism. J. Alzheimers Dis. 43, 259-273. doi: 10.3233/JAD-140633

Cuesta, P., Garcés, P., Castellanos, N. P., López, M. E., Aurtenetxe, S., Bajo, R., et al. (2015). Influence of the APOE $\varepsilon 4$ allele and mild cognitive impairment diagnosis in the disruption of the MEG resting state functional connectivity in sources space. J. Alzheimers Dis. 44, 493-505. doi: 10.3233/jad- 141872

Damaraju, E., Allen, E., Belger, A., Ford, J., McEwen, S., Mathalon, D., et al. (2014). Dynamic functional connectivity analysis reveals transient states of dysconnectivity in schizophrenia. Neuroimage Clin. 5, 298-308. doi: 10.1016/ j.nicl.2014.07.003

Deco, G., and Corbetta, M. (2011). The dynamical balance of the brain at rest. Neuroscientist 17, 107-123. doi: 10.1177/1073858409354384

Delbeuck, X., Van der Linden, M., and Collette, F. (2003). Alzheimer's disease as a disconnection syndrome? Neuropsychol. Rev. 13, 79-92.

Dimitriadis, S. I. (2018). Complexity of brain activity and connectivity in functional neuroimaging. J. Neurosci. Res. 96, 1741-1757. doi: 10.1002/jnr.24316

Dimitriadis, S. I., Laskaris, N. A., Del Rio-Portilla, Y., and Koudounis, G. C. (2009). Characterizing dynamic functional connectivity across sleep stages from EEG. Brain Topogr. 22, 119-133. doi: 10.1007/s10548-008-0071-4 
Dimitriadis, S. I., Laskaris, N. A., Simos, P. G., Micheloyannis, S., Fletcher, J. M., Rezaie, R., et al. (2013a). Altered temporal correlations in resting-state connectivity fluctuations in children with reading difficulties detected via MEG. Neuroimage 83, 307-331. doi: 10.1016/j.neuroimage.2013. 06.036

Dimitriadis, S. I., Laskaris, N. A., and Tzelepi, A. (2013b). On the quantization of time-varying phase synchrony patterns into distinct functional connectivity microstates (FC $\mu$ states) in a multi-trial visual ERP paradigm. Brain Topogr. 26, 397-409. doi: 10.1007/s10548-013-0276-z

Dimitriadis, S. I., Sun, Y., Kwok, K., Laskaris, N. A., Thakor, N., and Bezerianos, A. (2013c). "A tensorial approach to access cognitive workload related to mental arithmetic from EEG functional connectivity estimates," in Proceedings of the Annual International Conference of the IEEE Engineering in Medicine and Biology Society, Minneapolis, MN, 2940-2953.

Dimitriadis, S. I., Laskaris, N. A., Tsirka, V., Erimaki, S., Vourkas, M., Sifis, M., et al. (2011). A novel symbolization scheme for multichannel recordings with emphasis on phase information and its application to differentiate EEG activity from different mental tasks. Cogn. Neurodyn. 6, 107-113. doi: 10.1007/s11571011-9186-5

Dimitriadis, S. I., Kanatsouli, K., Laskaris, N. A., Tsirka, V., Vourkas, M., and Micheloyannis, S. (2012a). Surface EEG shows that functional segregation via phase coupling contributes to the neural substrate of mental calculations. Brain Cogn. 80, 45-52. doi: 10.1016/j.bandc.2012.04.001

Dimitriadis, S. I., Laskaris, N. A., Tsirka, V., Vourkas, M., and Micheloyannis, S. (2012b). An EEG study of brain connectivity dynamics at the resting state. Nonlinear Dynamics Psychol. Life Sci. 16, 5-22.

Dimitriadis, S. I., Laskaris, N. A., Tzelepi, A., and Economou, G. (2012c). Analyzing functional brain connectivity by means of commute times: a new approach and its application to track event-related dynamics. IEEE Trans. Biomed. Eng. 59, 1302-1309. doi: 10.1109/TBME.2012.2186568

Dimitriadis, S. I., Laskaris, N. A., Tsirka, V., Vourkas, M., and Micheloyannis, S. (2010a). What does delta band tell us about cognitive Processes: a mental calculation study? Neurosci. Lett. 483, 11-15. doi: 10.1016/j.neulet.2010. 07.034

Dimitriadis, S. I., Laskaris, N. A., Tsirka, V., Vourkas, M., Micheloyannis, S., and Fotopoulos, S. (2010b). Tracking brain dynamics via time-dependent network analysis. J. Neurosci. Methods 193, 145-155. doi: 10.1016/j.jneumeth.2010. 08.027

Dimitriadis, S. I., López, M. E., Bruña, R., Cuesta, P., Marcos, A., Maestú, F., et al. (2018a). How to build a functional connectomic biomarker for mild cognitive impairment from source reconstructed MEG resting-state activity: the combination of ROI representation and connectivity estimator matters. Front. Neurosci. 12:306. doi: 10.3389/fnins.2018.00306

Dimitriadis, S. I., Routley, B., Linden, D. E., and Singh, K. D. (2018b). Reliability of static and dynamic network metrics in the resting-state: a MEG-beamformed connectivity analysis. Front. Neurosci. 12:506. doi: 10.3389/fnins.2018.00506

Dimitriadis, S. I., Antonakakis, M., Simos, P., Fletcher, J. M., and Papanicolaou, A. (2017a). Data-driven topological filtering based on orthogonal minimal spanning trees: application to multi-group MEG resting-state connectivity. Brain Connect. 7, 661-670. doi: 10.1089/brain.2017.0512

Dimitriadis, S. I., Salis, C., Tarnanas, I., and Linden, D. (2017b). Topological filtering of dynamic functional brain networks unfolds informative chronnectomics: a novel data-driven thresholding scheme based on orthogonal minimal spanning trees (OMSTs). Front. Neuroinform. 11:28. doi: 10.3389/fninf.2017.00028

Dimitriadis, S. I., and Salis, C. I. (2017). Mining time-resolved functional brain graphs to an EEG-based chronnectomic brain aged index (CBAI). Front. Hum. Neurosci. 11:423. doi: 10.3389/fnhum.2017.00423

Dimitriadis, S. I., Laskaris, N. A., Simos, P. G., Fletcher, J. M., and Papanicolaou, A. C. (2016a). Greater repertoire and temporal variability of cross-frequency coupling (CFC) modes in resting-state neuromagnetic recordings among children with reading difficulties. Front. Hum. Neurosci. 10:163. doi: 10.3389/ fnhum.2016.00163

Dimitriadis, S. I., Sun, Y., Laskaris, N., Thakor, N., and Bezerianos, A. (2016b). Revealing cross-frequency causal interactions during a mental arithmetic task through symbolic transfer entropy: a novel vector-quantization approach. IEEE Trans. Neural Syst. Rehabil. Eng. 24, 1017-1028. doi: 10.1109/tnsre.2016. 2516107
Dimitriadis, S. I., Laskaris, N. A., Bitzidou, M. P., Tarnanas, I., and Tsolaki, M. N. (2015a). A novel biomarker of amnestic MCI based on dynamic cross-frequency coupling patterns during cognitive brain responses. Front. Neurosci. 9:350. doi: $10.3389 /$ fnins.2015.00350

Dimitriadis, S. I., Laskaris, N. A., and Micheloyannis, S. (2015b). Transition dynamics of EEG-based Network Microstates unmask developmental and task differences during mental arithmetic and resting wakefulness. Cogn. Neurodyn. 9, 371-387. doi: 10.1007/s11571-015-9330-8

Dimitriadis, S. I., Sun, Y., Kwok, K., Laskaris, N. A., Thakor, N., and Bezerianos, A. (2015c). Cognitive workload assessment based on the tensorial treatment of EEG estimates of cross-frequency phase interactions. Ann. Biomed. Eng. 43, 977-989. doi: 10.1007/s10439-014-1143-0

Dimitriadis, S. I., Zouridakis, G., Rezaie, R., Babajani-Feremi, A., and Papanicolaou, A. C. (2015d). Functional connectivity changes detected with magnetoencephalography after mild traumatic brain injury. Neuroimage Clin. 9, 519-531. doi: 10.1016/j.nicl.2015.09.011

Engel, A. K., Fries, P., and Singer, W. (2001). Dynamic predictions: oscillations and synchrony in top-down processing. Nat. Rev. Neurosci. 2, 704-716. doi: 10.1038/35094565

Engel, A. K., Gerloff, C., Hilgetag, C. C., and Nolte, G. (2013). Intrinsic coupling modes: multiscale interactions in ongoing brain activity. Neuron $80,867-886$. doi: 10.1016/j.neuron.2013.09.038

Fell, J., and Axmacher, N. (2011). The role of phase synchronization in memory processes. Nature Rev. Neurosci. 12, 105-118. doi: 10.1038/nrn2979

Fischl, B., Salat, D. H., Busa, E., Albert, M., Dieterich, M., Haselgrove, C., et al. (2002). Whole brain segmentation. Neuron 33, 341-355.

Florin, E., and Baillet, S. (2015). The brain's resting-state activity is shaped by synchronized cross-frequency coupling of neural oscillations. Neuroimage 111, 26-35. doi: 10.1016/j.neuroimage.2015.01.054

Fox, M. D., Snyder, A. Z., Vincent, J. L., Corbetta, M., Van Essen, D. C., and Raichle, M. E. (2005). The human brain is intrinsically organized into dynamic, anticorrelated functional networks. Proc. Natl. Acad. Sci. U.S.A. 102, 9673-9678. doi: 10.1073/pnas.0504136102

Fujisawa, S., and Buzsaki, G. (2011). A $4 \mathrm{~Hz}$ oscillation adaptively synchronizes prefrontal, VTA, and hippocampal activities. Neuron 72, 153-165. doi: 10.1016/ j.neuron.2011.08.018

Gagniuc, P. A. (2017). Markov Chains: From Theory to Implementation and Experimentation. Hoboken, NJ: John Wiley \& Sons.

Gärtner, M., Brodbeck, V., Laufs, H., and Schneider, G. (2015). A stochastic model for EEG microstates Equence analysis. Neuroimage 104, 199-208. doi: 10.1016/ j.neuroimage.2014.10.014

Gómez, C., Hornero, R., Abásolo, D., Fernández, A., and Escudero, J. (2009a). Analysis of MEG background activity in Alzheimer's disease using nonlinear methods and ANFIS. Ann. Biomed. Eng. 37, 586-594. doi: 10.1007/s10439-0089633-6

Gómez, C., Stam, C. J., Hornero, R., Fernández, A., and Maestú, F. (2009b). Disturbed beta band functional connectivity in patients with mild cognitive impairment: an MEG study. IEEE Trans. Biomed. Eng. 56, 1683-1690. doi: 10.1109/TBME.2009.2018454

Gómez, C., Juan-Cruz, C., Poza, J., Ruiz-Gómez, S. J., Gomez-Pilar, J., Núñez, P., et al. (2017). Alterations of effective connectivity patterns in mild cognitive impairment: an meg study. J. Alzheimers Dis. 65, 843-854. doi: 10.3233/JAD170475

Hämäläinen, M. S., and Ilmoniemi, R. J. (1994). Interpreting magnetic fields of the brain: minimum norm estimates. Med. Biol. Eng. Comput. 32, 35-42. doi: 10.1007/bf02512476

Handwerker, D. A., Roopchansingh, V., Gonzalez-Castillo, J., and Bandettini, P. A. (2012). Periodic changes in fMRI connectivity. Neuroimage 63, 1712-1719. doi: 10.1016/j.neuroimage.2012.06.078

He, B. J., Snyder, A. Z., Zempel, J. M., Smyth, M. D., and Raichle, M. E. (2008). Electrophysiological correlates of the brain's intrinsic large-scale functional architecture. Proc. Natl. Acad. Sci. U.S.A. 105, 16039-16044. doi: 10.1073/pnas. 0807010105

Hipp, J. F., Hawellek, D. J., Corbetta, M., Siegel, M., and Engel, A. K. (2012). Large-scale cortical correlation structure of spontaneous oscillatory activity. Nat. Neurosci. 15, 884-890. doi: 10.1038/nn.3101

Hutchison, R. M., Womelsdorf, T., Allen, E. A., Bandettini, P. A., Calhoun, V. D., Corbetta, M., et al. (2013). Dynamic functional connectivity: promise, issues, 
and interpretations. Neuroimage 80, 360-378. doi: 10.1016/j.neuroimage.2013. 05.079

Ioannides, A. A., Dimitriadis, S. I., Saridis, G. A., Voultsidou, M., Poghosyan, V., Liu, L., et al. (2012). Source space analysis of event-related dynamic reorganization of brain networks. Comput. Math. Methods Med. 2012:452503. doi: $10.1155 / 2012 / 452503$

Jack, C. R., Bennett, D. A., Blennow, K., Carrillo, M. C., Feldman, H. H., Frisoni, G. B., et al. (2016). A/T/N: an unbiased descriptive classification scheme for Alzheimer disease biomarkers. Neurology 87, 539-547. doi: 10.1212/WNL. 0000000000002923

Jamal, W., Das, S., Oprescu, I. A., Maharatna, K., Apicella, F., and Sicca, F. (2014). Classification of autism spectrum disorder using supervised learning of brain connectivity measures extracted from synchrostates. J. Neural Eng. 11:046019. doi: 10.1088/1741-2560/11/4/046019

Jarvis, J. P., and Shier, D. R. (1999). "Graph-theoretic analysis of finite Markov chains," in Applied Mathematical Modeling: A Multidisciplinary Approach, eds D. R. Shier and K. T. Wallenius (Boca Raton, FL: CRC Press), 85.

Jirsa, V., and Muller, V. (2013). Cross-frequency coupling in real and virtual brain networks. Front. Comput. Neurosci. 7:78. doi: 10.3389/fncom.2013.00078

Kirihara, K., Rissling, A. J., Swerdlow, N. R., Braff, D. L., and Light, G. A. (2012). Hierarchical organization of gamma and theta oscillatory dynamics in schizophrenia. Biol. Psychiatry 71, 873-880. doi: 10.1016/j.biopsych.2012. 01.016

Kitzbichler, M. G., Smith, M. L., Christensen, S. R., and Bullmore, E. (2009). Broadband criticality of human brain network synchronization. PLoS Comput. Biol. 5:e1000314. doi: 10.1371/journal.pcbi.1000314

Kopell, N. J., Gritton, H. J., Whittington, M. A., and Kramer, M. A. (2014). Beyond the connectome: the dynome. Neuron 83, 1319-1328. doi: 10.1016/j.neuron. 2014.08.016

Laufs, H., Krakow, K., Sterzer, P., Eger, E., Beyerle, A., Salek-Haddadi, A., et al. (2003). Electroencephalographic signatures of attentional and cognitive default modes in spontaneous brain activity fluctuations at rest. Proc. Natl. Acad. Sci. U.S.A. 100, 11053-11058. doi: 10.1073/pnas.1831638100

Lin, Q. H., Liu, J., Zheng, Y. R., Liang, H., and Calhoun, V. D. (2013). Semiblind spatial ICA of fMRI using spatial constraints. Hum. Brain Mapp. 31, 1076-1088. doi: 10.1002/hbm.20919

Lisman, J. E., and Idiart, M. A. (1995). Storage of $7+/-2$ short-term memories in oscillatory subcycles. Science $267,1512-1515$. doi: 10.1126/science.7878473

Liu, X., and Duyn, J. H. (2013). Time-varying functional network information extracted from brief instances of spontaneous brain activity. Proc. Natl. Acad. Sci. U.S.A. 110, 4392-4397. doi: 10.1073/pnas. 1216856110

Livingston, G., Sommerlad, A., Orgeta, V., Costafreda, S. G., Huntley, J., Ames, D., et al. (2017). Dementia prevention, intervention, and care. Lancet doi: 10.1016/S0140-6736(17)31363-6 [Epub ahead of print].

López, M. E., Bruña, R., Aurtenetxe, S., Pineda-Pardo, J. A., Marcos, A., Arrazola, J., et al. (2014). Alpha-band hypersynchronization in progressive mild cognitive impairment: a magnetoencephalography study. J. Neurosci. 34, 14551-14559. doi: 10.1523/JNEUROSCI.0964-14.2014

López, M. E., Turrero, A., Cuesta, P., López-Sanz, D., Bruña, R., Marcos, A., et al. (2016). Searching for primary predictors of conversion from mild cognitive impairment to Alzheimer's disease: a multivariate follow-up study. J. Alzheimers Dis. 52, 133-143. doi: 10.3233/JAD-151034

Maestú, F., Peña, J. M., Garcés, P., González, S., Bajo, R., Anto Bagic, A., et al. (2015). A multicenter study of the early detection of synaptic dysfunction in mild cognitive impairment using magnetoencephalography-derived functional connectivity. Neuroimage Clin. 9, 103-109. doi: 10.1016/j.nicl.2015. 07.011

Mandal, P. K., Banerjee, A., Tripathi, M., and Sharma, A. (2018). A comprehensive review of magnetoencephalography (MEG) studies for brain functionality in healthy aging and Alzheimer's disease (AD). Front. Comput. Neurosci. 12:60. doi: 10.3389/fncom.2018.00060

Mantini, D., Perrucci, M. G., Del Gratta, C., Romani, G. L., and Corbetta, M. (2007). Electrophysiological signatures of resting state networks in the human brain. Proc. Natl. Acad. Sci. U.S.A. 104, 13170-13175. doi: 10.1073/pnas.0700668104

Martinetz, T. M., Berkovich, S. G., and Schulten, K. J. (1993). 'Neural-gas' network for vector quantization and its application to time-series prediction. IEEE Trans. Neural Netw. 4, 558-569. doi: 10.1109/72.238311
Mylonas, D. S., Siettos, C. I., Evdokimidis, I., Papanicolaou, A. C., and Smyrnis, N. (2015). Modular patterns of phase desynchronization networks during a simple visuomotor task. Brain Topogr. 29, 118-129. doi: 10.1007/s10548-015-0451-5

Nacher, V., Ledberg, A., Deco, G., and Romo, R. (2013). Coherent delta-band oscillations between cortical areas correlate with decision making. Proc. Natl. Acad. Sci. U.S.A. 110, 15085-15090. doi: 10.1073/pnas.1314681110

Neymotin, S. A., Lazarewicz, M. T., Sherif, M., Contreras, D., Finkel, L. H., and Lytton, W. W. (2011). Ketamine disrupts $\theta$ modulation of $\gamma$ in a computer model of hippocampus. J. Neurosci. 31, 11733-11743. doi: 10.1523/ JNEUROSCI.0501-11.2011

Nolte, G. (2003). The magnetic lead field theorem in the quasi-static approximation and its use for magnetoencephalography forward calculation in realistic volume conductors. Phys. Med. Biol. 48, 3637-3652. doi: 10.1088/0031-9155/48/22/002

Nomi, J. S., Vij, S. G., Dajani, D. R., Steimke, D. R., Damaraju, E., Rachakonda, S., et al. (2016). Chronnectomic patterns and neural flexibility underlie executive function. Neuroimage 147, 861-871. doi: 10.1016/j.neuroimage.2016.10.026

Oldfield, R. C. (1971). The assessment and analysis of handedness: the Edinburgh inventory. Neuropsychologia 9, 97-113. doi: 10.1016/0028-3932(71)90067-4

Oostenveld, R., Fries, P., Maris, E., and Schoffelen, J. M. (2011). FieldTrip: open source software for advanced analysis of MEG, EEG, and invasive electrophysiological data. Comput. Intell. Neurosci. 2011:156869. doi: 10.1155/ 2011/156869

Petersen, R. C., Doody, R., Kurz, A., Mohs, R. C., Morris, J. C., Rabins, P. V., et al. (2001). Current concepts in mild cognitive impairment. Arch. Neurol. 58, 1985-1992.

Petersen, R. C., and Negash, S. (2008). Mild cognitive impairment: an overview. CNS Spectr. 13, 45-53. doi: 10.1017/s1092852900016151

Petersen, R. C., Roberts, R. O., Knopman, D. S., Boeve, B. F., Geda, Y. E., Ivnik, R. J., et al. (2009). Mild cognitive impairment: ten years later. Arch. Neurol. 66, 1447-1455. doi: 10.1001/archneurol.2009.266

Portet, F., Ousset, P. J., Visser, P. J., Frisoni, G. B., Nobili, F., Scheltens, P., et al. (2006). Mild cognitive impairment (MCI) in medical practice: a critical review of the concept and new diagnostic procedure. Report of the MCI working group of the European consortium on Alzheimer's disease. J. Neurol. Neurosurg. Psychiatry 77, 714-718. doi: 10.1136/jnnp.2005.085332

Poza, J., Hornero, R., Abásolo, D., Fernández, A., and García, M. (2007). Extraction of spectral based measures from MEG background oscillations in Alzheimer's disease. Med. Eng. Phys. 29, 1073-1083. doi: 10.1016/j.medengphy.2006.11.006

Qiu, C., Kivipelto, M., and von Strauss, E. (2009). Epidemiology of Alzheimer's disease: occurrence, determinants, and strategies toward intervention. Dial. Clin. Neurosci. 11, 111-128.

Richiardi, J., Eryilmaz, H., Schwartz, S., Vuilleumier, P., and Van De Ville, D. (2011). Decoding brain states from fMRI connectivity graphs. Neuroimage 56, 616-626. doi: 10.1016/j.neuroimage.2010.05.081

Rosen, W. G., Terry, R. D., Fuld, P. A., Katzman, R., and Peck, A. (1980). Pathological verification of ischemic score in differentiation of dementias. Ann. Neurol. 7, 486-488. doi: 10.1002/ana.410070516

Sakoglu, U., Pearlson, G. D., Kiehl, K. A., Wang, Y. M., Michael, A. M., and Calhoun, V. D. (2010). A method for evaluating dynamic functional network connectivity and task-modulation: application to schizophrenia. Magn. Reson. Mater. Phys. 23, 351-366. doi: 10.1007/s10334-010-0197-8

Sarvas, J. (1987). Basic mathematical and electromagnetic concepts of the biomagnetic inverse problem. Phys. Med. Biol. 32, 11-22. doi: 10.1088/00319155/32/1/004

Schoffelen, J. M., and Gross, J. (2009). Source connectivity analysis with MEG and EEG. Hum. Brain Mapp. 30, 1857-1865. doi: 10.1002/hbm.20745

Shirer, W. R., Ryali, S., Rykhlevskaia, E., Menon, V., and Greicius, M. D. (2012). Decoding subject-driven cognitive states with whole-brain connectivity patterns. Cereb. Cortex 22, 158-165. doi: 10.1093/cercor/ bhr099

Shuman, D. I., Narang, S. K., Frossard, P., Ortega, A., and Vandergheynst, P. (2013). The emerging field of signal processing on graphs: extending high-dimensional data analysis to networks and other irregular domains. IEEE Signal Process. Mag. 30, 83-98. doi: 10.1109/msp.2012.2235192

Siegel, M., Donner, T. H., and Engel, A. K. (2012). Spectral fingerprints of largescale neuronal interactions. Nat. Rev. Neurosci. 13, 121-134. doi: 10.1038/ nrn3137 
Solin, A., Jylänki, P., Kauramäki, J., Heskes, T., van Gerven, M. A. J., and Särkkä, S. (2016). Regularizing solutions to the MEG inverse problem using space-time separable covariance functions. arXiv [preprint]. arXiv:1604.04931

Stam, C. J., de Haan, W., Daffertshofer, A., Jones, B. F., Manshanden, I., van Cappellen van Walsum, A. M., et al. (2009). Graph theoretical analysis of magnetoencephalographic functional connectivity in Alzheimer's disease. Brain 132, 213-224. doi: 10.1093/brain/awn262

Taulu, S., and Simola, J. (2006). Spatiotemporal signal space separation method for rejecting nearby interference in MEG measurements. Phys. Med. Biol. 51, 1759-1768. doi: 10.1088/0031-9155/51/7/008

Tewarie, P., Hillebrand, A., van Dijk, B. W., Stam, C. J., O’Neill, G. C., Van Mieghem, P., et al. (2016). Integrating cross-frequency and within band functional networks in resting-state MEG: a multi-layer network approach. Neuroimage 142, 324-336. doi: 10.1016/j.neuroimage.2016.07.057

Toppi, J., Astolfi, L., Poudel, G. R., Innes, C. R., Babiloni, F., and Jones, R. D. (2015). Time-varying effective connectivity of the cortical neuroelectric activity associated with behavioural microsleeps. Neuroimage 124, 421-432. doi: 10. 1016/j.neuroimage.2015.08.059

Tzourio-Mazoyer, N., Landeau, B., Papathanassiou, D., Crivello, F., Etard, O., Delcroix, N., et al. (2002). Automated anatomical labeling of activations in SPM using a macroscopic anatomical parcellation of the MNI MRI single-subject brain. NeuroImage 15, 273-289. doi: 10.1006/nimg.2001. 0978

Van de Ville, D., Britz, J., and Michel, C. M. (2010). EEG microstate sequences in healthy humans at rest reveal scale-free dynamics. Proc. Natl. Acad. Sci. U.S.A. 107, 18179-18184. doi: 10.1073/pnas.1007841107

Van Veen, B. D., van Dronglen, W., Yuchtman, M., and Suzuki, A. (1997). Localization of brain electric activity via linearly constrained minimum variance spatial filtering. IEEE Trans. Biomed. Eng. 44, 867-880. doi: 10.1109/ 10.623056 van Wijk, B. C., and Fitzgerald, T. H. (2014). Thalamo-cortical cross-frequency coupling detected with MEG. Front. Hum. Neurosci. 8:187. doi: 10.3389/fnhum. 2014.00187

Wallis, G., Stokes, M., Cousijn, H., Woolrich, M., and Nobre, A. C. (2015). Frontoparietal and cingulo-opercular networks play dissociable roles in control of working memory. J. Cogn. Neurosci. 27, 2019-2034. doi: 10.1162/jocn_a_ 00838

Yang, C. Y., and Lin, C. P. (2015). Time-varying network measures in resting and task states using graph theoretical analysis. Brain Topogr. 28, 529-540. doi: 10.1007/s10548-015-0432-8

Yesavage, J. A., Brink, T. L., Rose, T. L., Lum, O., Huang, V., Adey, M., et al. (1983). Development and validation of a geriatric depression screening scale: a preliminary report. J. Psychiatr. Res. 17, 37-49. doi: 10.1016/0022-3956(82) 90033-4

Yu, M., Engels, M. M. A., Hillebrand, A., van Straaten, E. C. W., Gouw, A. A., Teunissen, C., et al. (2017). Selective impairment of hippocampus and posterior hub areas in Alzheimer's disease: an MEG-based multiplex network study. Brain 140, 1466-1485. doi: 10.1093/brain/aw $\mathrm{x} 050$

Conflict of Interest Statement: The authors declare that the research was conducted in the absence of any commercial or financial relationships that could be construed as a potential conflict of interest.

Copyright ( 2019 Dimitriadis, López, Maestu and Pereda. This is an open-access article distributed under the terms of the Creative Commons Attribution License (CC BY). The use, distribution or reproduction in other forums is permitted, provided the original author(s) and the copyright owner(s) are credited and that the original publication in this journal is cited, in accordance with accepted academic practice. No use, distribution or reproduction is permitted which does not comply with these terms. 
OPEN ACCESS

Edited by:

Nicoletta Cera

University of Porto, Portugal

Reviewed by:

Edward Ofori

Arizona State University, United States

Soha Saleh,

Kessler Foundation, United States

${ }^{*}$ Correspondence:

Brian D. Schmit

brian.schmit@marquette.edu

Specialty section:

This article was submitted to Applied Neuroimaging,

a section of the journal

Frontiers in Neurology

Received: 12 March 2019

Accepted: 23 May 2019

Published: 13 June 2019

Citation:

Kalinosky BT, Vinehout K, Sotelo MR, Hyngstrom AS and Schmit BD (2019)

Tasked-Based Functional Brain Connectivity in Multisensory Control of Wrist Movement After Stroke.

Front. Neurol. 10:609. doi: 10.3389/fneur.2019.00609

\section{Tasked-Based Functional Brain Connectivity in Multisensory Control of Wrist Movement After Stroke}

\author{
Benjamin T. Kalinosky ${ }^{1}$, Kaleb Vinehout ${ }^{1}$, Miguel R. Sotelo ${ }^{1}$, Allison S. Hyngstrom ${ }^{2}$ and \\ Brian D. Schmit ${ }^{1 *}$
}

${ }^{1}$ Integrative Neural Engineering and Rehabilitation Laboratory, Department of Biomedical Engineering, Marquette University and the Medical College of Wisconsin, Milwaukee, WI, United States, ${ }^{2}$ Integrative Neural Engineering and Rehabilitation Laboratory, Department of Physical Therapy, Marquette University, Milwaukee, WI, United States

In this study we documented brain connectivity associated with multisensory integration during wrist control in healthy young adults, aged matched controls and stroke survivors. A novel functional MRI task paradigm involving wrist movement was developed to gain insight into the effects of multimodal sensory feedback on brain functional networks in stroke participants. This paradigm consisted of an intermittent position search task using the wrist during fMRI signal acquisition with visual and auditory feedback of proximity to a target position. We enrolled 12 young adults, 10 participants with chronic post-stroke hemiparesis, and nine age-matched controls. Activation maps were obtained, and functional connectivity networks were calculated using an independent component analysis (ICA) approach. Task-based networks were identified using activation maps, and nodes were obtained from the ICA components. These nodes were subsequently used for connectivity analyses. Stroke participants demonstrated significantly greater contralesional activation than controls during the visual feedback condition and less ipsilesional activity than controls during the auditory feedback condition. The sensorimotor component obtained from the ICA differed between rest and task for control and stroke participants: task-related lateralization to the contralateral cortex was observed in controls, but not in stroke participants. Connectivity analyses between the lesioned sensorimotor cortex and the contralesional cerebellum demonstrated decreased functional connectivity in stroke participants ( $p<0.005)$, which was positively correlated the Box and Blocks arm function test $\left(r^{2}=0.59\right)$. These results suggest that task-based functional connectivity provides detail on changes in brain networks in stroke survivors. The data also highlight the importance of cerebellar connections for recovery of arm function after stroke.

Keywords: stroke, functional connectivity, upper limb, sensory integration, task based approach

\section{INTRODUCTION}

In this study, we used functional magnetic resonance imaging (fMRI) and a novel task paradigm to investigate the effects of multimodal sensory feedback on detection of brain functional networks after stroke. In prior studies, the primary motor cortical regions and their pathways have been a major focus in investigating the functional effects of stroke lesions on the brain (1-4). However, 
brain lesions might have an even stronger impact on integrative networks that process multisensory inputs and plan movements in a functional context. Lesions affecting sensorimotor integrative networks of the brain are likely to play a critical role in recovery, and damage to these networks could lead to chronic impairment, as they are important to motor learning and recovery (2, 4-9). Although current measures of functional connectivity characterize communication between brain motor regions, the changes in connectivity of the sensorimotor association areas have been largely unexplored in stroke survivors. In order to characterize the function of sensorimotor networks in stroke survivors, we measured brain connectivity and activation with functional magnetic resonance imaging (fMRI) during rest and during tasks that invoked key features of sensorimotor and multisensory integration.

fMRI has been used to characterize differences in brain activation patterns in stroke survivors and to document cortical plasticity with natural recovery or following targeted therapeutic interventions. In general, increased intensity and spread of brain activation after stroke have been associated with decreased functional outcomes. During finger movement, stroke survivors have increased cortical activation with a broader spatial extent in the ipsilesional hemisphere as well as contralesional activity that is absent in controls (10). Increases in cortical activity distant from primary motor areas impacted by a stroke lesion are considered evidence for cortical reorganization (11). These plastic changes might be compensatory or conversely, they could be maladaptive for functional recovery. During recovery, the intensity, and spread of brain activation associated with hand grip decreases in lower functioning stroke survivors (4), suggesting that simple task-related brain activation volumes are inversely correlated with functional recovery. In contrast, decreased activity in cortical areas have been documented in stroke survivors using the relatively more complex task of bilateral pedaling during fMRI (12), possibly due to a greater reliance on integrative sensorimotor regions that might become dysfunctional after stroke. The recent emergence of brain functional connectivity analyses $(13,14)$ offers the opportunity to interpret changes in task-related brain activity in the context of brain networks, potentially offering insight into the mechanisms underlying changes in brain function after stroke.

Functional connectivity analyses also provide evidence of changes in brain function after stroke. Functional connectivity MRI (fcMRI) $(13,15)$ infers coactivation of one or more cortical areas by their correlated fMRI signal over time. This analysis can be used to identify functional networks using fMRI signals obtained at rest (13) or during a task (15). For this analysis, nodes can be defined using predetermined anatomical regions of interest or identified by measuring regional homogeneity of voxel-wise intrinsic functional connectivity (16). Alternatively, functional connectivity can be decomposed into a set of spatiotemporal networks using an independent component analysis $(17,18)$; an independent component consists of a $3 \mathrm{D}$ volume that provides each voxel's contribution to a network and a BOLD time-course that is shared by all voxels within that network. In resting-state fMRI, one of the most consistent findings in stroke participants is decreased functional connectivity between the ipsilesional and contralesional sensorimotor cortices (19-21). While resting state functional connectivity and task-based connectivity share correspondence, they also have many notable differences (2224). In healthy individuals, the spatial extent of nodes determined by an independent component analysis is similar across task and resting state paradigms (25). In contrast, task-based connectivity exhibits "local specialization" with increased connectivity of long-distance connections compared to resting state analyses $(26,27)$. This means that areas engaged in a given task have increased local connectivity in the task-specific area (i.e., local specialization) and increased global connectivity (long distance connections) between the different areas engaged with that task. For a multimodal sensorimotor task, we expect increased local specialization within the primary sensorimotor region and increased long-distance communication between the ipsilesional sensorimotor cortices and contralesional cerebellum, similar to activation results reported during complex motor tasks (28).

Functional connectivity analyses are particularly effective in quantifying functionally relevant changes in brain networks after stroke and during recovery (29). It has been suggested that network integrity and reorganization is critical for functional recovery after stroke (30). Each cortical region can actively participate in multiple functional networks, allowing the brain to reorganize after damage to a particular node. Network plasticity has been documented in the motor network of people with stroke (31) and the integrity of contralesional parietofrontal and sensorimotor cortical networks has been associated with lower motor impairment after stroke (6). These findings suggest that resting state and task-based connectivity of sensorimotor integration areas predict motor function, and plasticity of these networks provide mechanisms for restoring motor function.

In order to identify changes in sensorimotor networks in chronic stroke survivors, we calculated the functional connectivity of the brain using resting state and task-based MRI, with a unique sensorimotor task that employs sensorimotor and multisensory integration. The task was specifically designed to engage integrative sensorimotor networks during controlled wrist movement. We then compared the changes in these networks to a clinical measure of upper limb function. We hypothesized that only during the task, functional connectivity between brain networks associated with sensorimotor integration would be reduced in stroke survivors, and that the reduction would be correlated to arm function.

\section{METHODS}

\section{Data Collection}

\section{Participant Recruitment and Clinical Testing}

Twelve young adults (four female, $25.2 \pm 2.4$ years), 10 individuals with chronic post-stroke hemiparesis (four female, age $66.7 \pm 7.94$ years, at least 1.1 years since stroke), and nine age-matched control participants (five female, age $64.2 \pm 7.73$ years) participated in this study. The young adults were included as additional data to validate the new task paradigm in its ability to show areas of the brain involved in performing the task. Each participant provided informed written consent to the 
experimental protocol, which was approved by the Institutional Review Boards at Marquette University and the Medical College of Wisconsin. Inclusion criteria included a history of an ischemic cortical or subcortical stroke that occurred no $<6$ months prior to recruitment. Participants with no ability to perform supination, pronation, ulnar deviation, or radial deviation of the wrist were excluded. Controls without history of stroke or other neurological impairments were age-matched (within 3 years) and gendered-matched to the stroke participants. Each stroke participant completed the upper extremity (UE) portion of the Fugl-Meyer Assessment (32) for a maximum possible score of 126. Participants also completed the Box and Blocks Test of Manual Dexterity (33), the Wolf Motor Function Test (34) for upper extremity motor ability (maximum score of 75), and the Modified Ashworth Scale (35). These clinical measurements were correlated with measured brain activity during the task and the functional connectivity during resting state and during the task. The stroke participant clinical scores and lesion locations are shown in Table 1, and Figure S1 shows the lesion distribution; although there is little lesion overlap, the lesion distribution shows damage in the sensorimotor pathways.

\section{Experimental Paradigm}

Our sensorimotor integration experiment was designed with cues and feedback that contrasted the effects of auditory and visual sensation. We introduced a task paradigm for studying the role of sensory integration in complex movement. Chronic stroke participants have more difficulty coordinating sensorimotor behavior, especially in tasks with higher complexity (36). Our task required the participant to produce movement while integrating multiple sensory modalities.

\section{Motion recording and audiovisual feedback}

Every participant completed two sessions on separate days no more than 2 weeks apart. Participants were trained to perform a wrist-movement task during the first session. The second session used the same wrist movement task and consisted of a second training period $1 \mathrm{~h}$ before the task-based fMRI session. The experimental apparatus is shown in Figure 1 (left). The forearm of the impaired limb was fixed on a small ramp to allow for radial and ulnar deviation of the wrist. Both the elbow and the forearm were secured on the ramp to ensure that the task was controlled by wrist movement only. The hand gripped the end of a ShapeTape device (Measurand Inc., Canada), an array of 16 optical fiber sensor pairs that provide 3D Euclidean coordinates along the sensor region. Each sensor pair also provided a $3 \mathrm{D}$ rotation matrix expressed in quaternion form. Visual feedback was presented on a computer monitor, and speakers provided auditory feedback. Motion data were recorded every $24 \mathrm{~ms}$, or $41.67 \mathrm{~Hz}$.

Shown in Figure 1 (left), two sensor pairs at the end of the ShapeTape were used to calculate a $3 \mathrm{D}$ ray with angle $\boldsymbol{\theta}=\left\{\theta_{x}, \theta_{z}\right\}$ relative to the horizontal $(\mathrm{x}-\mathrm{z})$ plane, which was used to define the orientation of the wrist. Effectively, pronation/supination was mapped to an angle within the $\mathrm{x}-\mathrm{y}$ plane $\theta_{z}$, and radial and ulnar deviation was mapped to an angle in the $z-y$ plane. Letting $\mathbf{p}_{n}$ and $\mathbf{p}_{n-1}$ be the $3 \mathrm{D}$ coordinates of the last two sensors, an orientation

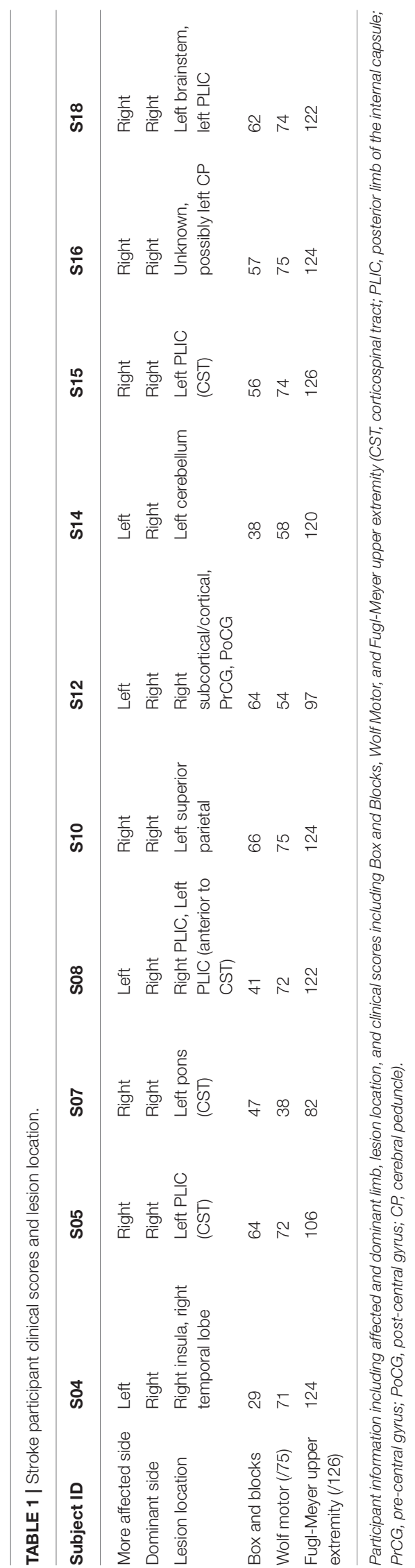



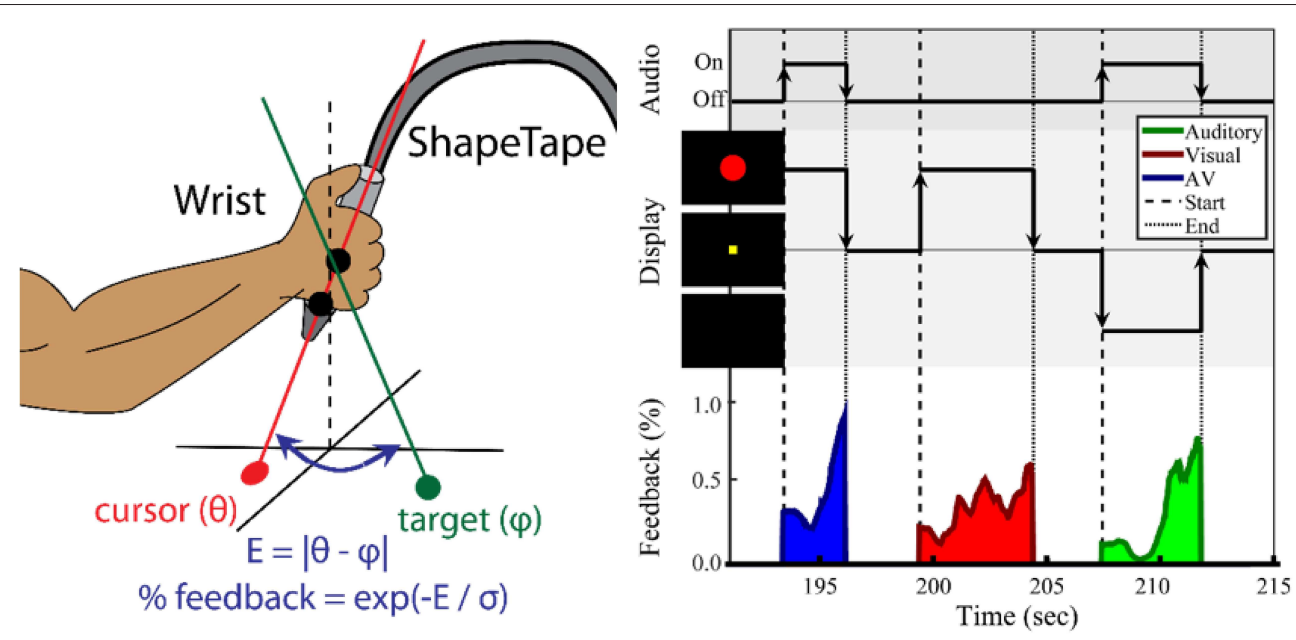

FIGURE 1 | Task paradigm for wrist movement and sensorimotor integration. (Left) Illustration of the ShapeTape apparatus. The forearm position was fixed. (Right) Experimental design of the multisensory search task. During each trial, the participant maximized sensory feedback using wrist joint angles to minimize error to a target angle. The participant was instructed to move the wrist to maximize the circle diameter and/or a sound volume. Auditory, visual, or audiovisual feedback were presented at the start and during each trial. After reaching the target, the participant fixated on a yellow square during a $2-4 \mathrm{~s}$ intertrial period. The average trial duration was $5 \mathrm{~s}$.

vector $\boldsymbol{v}$ was calculated as:

$$
\boldsymbol{v}=\frac{\mathbf{p}_{n}-\mathbf{p}_{n-1}}{\left\|\mathbf{p}_{n}-\mathbf{p}_{n-1}\right\|}
$$

The wrist orientation was estimated as $\theta_{x}=\left(v_{X} / v_{Y}\right)$, and $\theta_{Z}=\left(v_{Z} / v_{Y}\right)$. For each search task, a target angle, $\boldsymbol{\varphi}$, was created. The error to $\varphi$ during the search task was calculated as $E=\sqrt{\left(\theta_{X}-\varphi_{X}\right)^{2}+\left(\theta_{Z}-\varphi_{Z}\right)^{2}}$. $E$ was then used to provide feedback to the participant related to wrist proximity to the target. The feedback intensity, $w$, was calculated as $w=$ $\exp (-E / \sigma)$, with the sensitivity parameter $\sigma$ fixed at 0.1 radians for all trials. Intensity $w$ was used to modulate visual and auditory feedback stimuli. Auditory feedback was a $440 \mathrm{~Hz}$ tone and its volume was modulated linearly by $w$. Visual feedback was presented as a solid red circle at the center of a black screen. The circle diameter was modulated linearly by $w$ from 15 to 160 pixels or $0.38-4.06 \mathrm{~cm}$. The screen was placed $\sim 2$ feet away from the eyes. The resulting aperture angle was effectively varied 0.179-1.91 degrees.

\section{Experimental design}

As shown in Figure 1 (right), a search-based wrist movement task was designed to invoke brain networks involved in sensorimotor and multisensory integration. The first session began with up to five familiarization trials, in which the participant used the wrist to move a white cursor to a yellow square target at the center of the screen. These trials were also used to verify the participant's range of motion. Once comfortable reaching the square, the participant was then informed that the square and cursor would not be visible. At the start of each trial, one of three types of sensory feedback was presented. For both the training session and the fMRI session, a series of two search-task runs ("search") (6 min each) and one sensory-motor ("SM") only run (6 min) were conducted.

The "search" task run consisted of a series of trials, each trial including visual, auditory, or combined audiovisual feedback. In the visual-only feedback condition, a solid red circle appeared and grew larger as error decreased. Once the participant reached the target, the solid red circle was changed into an outline and then disappeared. During auditory-only trials, the participant searched for the target with feedback provided by the tone volume. Upon reaching the target, the tone was altered to a fixed pitch of $880 \mathrm{~Hz}$, giving a "beep" sound, and turned off. In audiovisual feedback trials, the red circle and auditory tone were mapped independently to the $\mathrm{x}$-coordinate and $\mathrm{y}$-coordinate errors. The goal was to maximize both feedback intensities. After the target was reached, the participant fixated at a yellow square at the center of the screen for an inter-trial period with a normal random duration of $2.5 \pm 0.5 \mathrm{~s}$. Participants were notified that the trial would end automatically after an unspecified time if they failed to reach the target. A maximum trial duration of $15 \mathrm{~s}$ was used for all experiments.

A control task ("SM") involving isolated sensory and motor tasks was conducted after the two search-based task runs. In the motor task, the words "Keep Moving" appeared on the screen, and the participant was told to move the wrist randomly in a similar pattern as during the search task. The participant was instructed to stop moving once the words disappeared. The message "Relax" was displayed on the screen for $2 \mathrm{~s}$ prior to the sensory only trials. For this condition, the participant was warned that there would be times during which the red circle and sound would appear and change outside of the participant's control. The participant was trained to not move and just watch and listen. The last instruction to the participant was "If you see the words "Keep moving" then move. If you do not see the words "Keep moving," 
then stay still no matter what happens." Throughout this final run, the experiment would alternate between motor-only and sensory-only conditions every $12 \pm 2.0 \mathrm{~s}$.

\section{MRI Scans}

Every participant was screened for MRI safety before entering the magnetic environment. An axial T1-weighted anatomical image was acquired using a fast spoiled gradient recalled (SPGR) pulse sequence, with TE: $3.2 \mathrm{~ms}$, TR: $8.16 \mathrm{~ms}$, flip angle: $12 \mathrm{deg}$, prep time: 450, bandwidth: 22.73, FOV: $240 \mathrm{~mm}, 1561 \mathrm{~mm}$ slices, matrix size: $256 \times 240$. For functional MRI, a sagittal view gradient-echo echo-planar sequence was acquired with TE: $25 \mathrm{~ms}$, TR: 2,000 ms, flip angle: $77 \mathrm{deg}$, FOV: $240 \times 240 \mathrm{~mm}$, 41 slices with $3.5 \mathrm{~mm}$ thickness. Four $6 \mathrm{~min}$ fMRI scans were performed, one for resting state, two for the search task, and one for the sensory motor only task.

\section{MRI Experimental Setup}

As the participant lay supine, the forearm was elevated with foam and fixed in place with sandbags. The ShapeTape was placed into the participant's impaired hand, or right hand in healthy adults. Visual feedback was projected to a visor attached to the head coil, and earbuds were placed into the ears to provide auditory feedback. The MRI scan session consisted of one resting-state run followed by three task-based runs. During the 6 min resting-state run, each participant was asked to close their eyes and stay alert. After the resting-state scan, the ShapeTape was placed into the participant's hand. If the participant had difficulty gripping the device, then a surgical wrap was used to keep the hand closed. The participant completed three 6-min runs of the same experiment conducted for the first session, including two search-based and one sensory-motor only.

\section{Image Registration and Lesion Side Normalization}

Intersubject and intermodality image registration was completed in both healthy adults and stroke participants using fully automated techniques. Each participant's anatomical T1weighted MRI volume was registered to a 152-brain MNI space using a 12-parameter affine registration, and then non-linear image registration was performed using Maxwell's demons algorithm (37). Local histogram matching was performed prior to deformable image registration in order to mitigate errors caused by lesion contrasts (38). In brief, each voxel in the subject's T1-weighted image was assigned a value from 0 to 1 based on its percentile for a $5 \times 5 \times 5$ voxel neighborhood centered at that voxel. The resulting deformed T1-weighted image was histogram-matched to the MNI template, where the local percentile of the subject's T1-weighted image was matched to the same percentile of the MNI template. Finally, 5 iterations of the demons algorithm were performed at the full $1 \mathrm{~mm}$ resolution to align the edges of the images.

The images of all stroke participants that completed the experiment with the left arm were flipped over the sagittal plane so that all lesions were on the left side of MNI space. One of these participants had a lesion within the left cerebellum, which was flipped to the right side. The flipping placed all strokes outside the cerebellum on the left side of MNI space.

\section{fMRI Data Processing General Linear Model for the Search Task}

Task-based functional MRI analysis was performed with AFNI (Analysis of Functional NeuroImages, RRID:SCR_005927, afni.nimh.nih.gov/afni). Data were temporally resampled in order to correct for non-uniform slice acquisition timing within each volume. BOLD signal changes related to head translation and rotation were corrected by affine coregistration between volumes using AFNI's 3 dvolreg function. The data were highpass filtered above $0.01 \mathrm{~Hz}$. The motion parameters included roll, yaw, pitch, and $\mathrm{x}, \mathrm{y}, \mathrm{z}$ translations, and were treated as coregressors for all subsequent analyses.

\section{General Linear Model and Cortical Activation Maps}

We were interested in stroke-related differences in cortical activity involved in sensorimotor integration during movement. In addition, we are interested in the young and age-matched control differences during this task. As previously shown in Figure 1, at the start of the trial the sensory feedback was at its lowest value. On the contrary, the level of wrist motion (not displayed in the figure) was greatest at the start of the search. As the participant closed in on the target, sensory feedback increased. In this latter phase of the task, finer wrist movements were required. Thus, wrist movement was greatest at the start of each trial, and sensory feedback was greatest at the end. In order to identify the brain activity associated with movement, wrist motion was estimated as the absolute change in $\theta$ with time. However, as mentioned above, the wrist motion parameters throughout time were used as co-regressors to help control for the variation in movement during the earlier and later stages of the task.

The sensory feedback and wrist movement signals, both produced with our in-house software from the ShapeTape data, were median filtered with a window of $2 \mathrm{~s}$. Using the "waver" function in AFNI, the signals were then convolved with a doublegamma variate hemodynamic response function to produce modeled BOLD responses and resampled to the fMRI temporal resolution of $0.5 \mathrm{~Hz}$. This method was repeated for the auditory, visual, and audiovisual feedback conditions to produce three movement regressors $\left(A_{M}, V_{M}, A V_{M}\right)$ and three sensory feedback regressors $\left(A_{S}, V_{S}, A V_{S}\right)$. Using the $3 \mathrm{dDeconvolve}$ program in AFNI, a multilinear regression was performed for each voxel, with the six task-related regressors and six head motion parameters (three rotation, three translation) contributing to the BOLD signal. The marginal $t$-value for the beta coefficient of each task-related regressor was resampled into $1 \mathrm{~mm}$ MNI space. These six cortical activation maps were calculated for every stroke participant and age-matched control.

\section{Within Network Functional Connectivity}

We were interested in the within network and between network functional connectivity. For the within network functional connectivity, functional connectivity MRI analysis was performed with Multivariate Exploratory Linear 
Decomposition into Independent Components (MELODIC) Version 3.14 available with the FMRIB's Software Library (FSL, RRID:SCR_002823, www.fmrib.ox.ac.uk/fsl). All runs and the stroke and aged matched control participants were time concatenated for a single 75-run (19 participants and 4 runs) group ICA. The sensory-motor only task run of stroke participant S04 was not included in the analysis since data collection was not complete. The data were high-pass filtered with a cutoff frequency of $0.01 \mathrm{~Hz}$ (39). The first five TRs were discarded to exclude signal drifts due to system ramp-up. This left each run with 175 volumes over $350 \mathrm{~s}$. The functional image volumes were motion corrected using the MCFLIRT implementation (40). Slice-time correction was applied using linear interpolation. Skull-stripping was automatically performed with the brain extraction tool (BET) (41), and the data were spatially smoothed with a $5 \mathrm{~mm}$ full-width half-max Gaussian kernel. The skull stripped images were visually inspected to ensure the quality of the skull stripping. The resulting brain mask was used to exclude non-brain voxels from the remaining analysis. All participants were spatially normalized to an anatomical MNI standard template using a 12-parameter affine registration implemented in FLIRT (42). The voxel BOLD times series were demeaned, variance normalized, and whitened.

The number of independent components were estimated using a Bayesian approach described by Minka et al. (18). Once the independent components for the combined stroke and age matched control data were calculated, a dual regression (43) was used to estimate individual spatial maps and time courses for each participant and session. Components with vertical stripes in the axial view were associated with motion and excluded from further analysis. Voxel-based general linear modeling was performed between stroke and age-matched controls, and between rest and task conditions for each independent component. The resulting maps of $t$ values were used as representations of the contributions of each condition to the component of interest. The term "within network functional connectivity" was used to refer to this relationship between the group/task and the network component.

\section{Task-Based Network Identification}

Overall relationships between resting-state networks and the task conditions were estimated with temporal correlation. The BOLD response to each task condition was modeled by convolving the stimulus presentation time signal with a double-gamma hemodynamic response function. The modeled time courses were concatenated across all 19 participants and four runs using the same arrangement used for the time-concatenated group ICA. The relationship between an independent component and the experimental variable was estimated by correlating the spatial component's data time course to the modeled BOLD response of the experimental variable. Since the movement-only and sensory-only conditions were presented in regular $15 \mathrm{~s}$ intervals, their modeled time courses were used to identify the functional brain network associated with the task. Shown in Figure S2, one particular task-related independent component $\left(\mathrm{SM}_{\mathrm{L}}\right)$ had a high correlation $(r>0.9)$ with the movement-only condition. This component, shown in Figure $\mathbf{S 2}$ below, will be referred to as the active sensorimotor network.
TABLE 2 | Locations used for between network seed-based FC analysis.

\begin{tabular}{|c|c|c|c|c|}
\hline Network & Region & $\mathbf{x}$ & $y$ & $\mathbf{z}$ \\
\hline DMN & Precuneus & 0 & -55 & 26 \\
\hline DMN & IPL left & 48 & -63 & 30 \\
\hline DMN & IPL right & -40 & -59 & 30 \\
\hline PF left & aPFC left & 44 & -55 & 42 \\
\hline PF left & IPL left & 48 & 33 & 18 \\
\hline PF right & aPFC right & -28 & -63 & 38 \\
\hline PF right & IPL right & -44 & 17 & 22 \\
\hline SM left & M1 left & 36 & -19 & 62 \\
\hline SM left & Cbl ant right & -24 & -51 & -30 \\
\hline SM left & Cbl post right & -24 & -55 & -58 \\
\hline SM left & M1 right & -36 & -19 & 62 \\
\hline SM left & Cbl ant left & 24 & -51 & -30 \\
\hline SM left & Cbl post left & 24 & -55 & -58 \\
\hline SM right & S1 right & 40 & -31 & 42 \\
\hline SM right & S1 left & -40 & -31 & 42 \\
\hline Cbl & Cbl left & 24 & -67 & -38 \\
\hline Cbl & Cbl right & -24 & -67 & -38 \\
\hline Vis & V1 left & -20 & -91 & -6 \\
\hline Vis & V1 right & 20 & -91 & -6 \\
\hline Visual Ext. & V5 right & 44 & -59 & -18 \\
\hline Visual Ext. & V5 left & -40 & -67 & -10 \\
\hline Aud left & A1 left & 64 & -7 & -2 \\
\hline Aud right & A1 right & -56 & -11 & 2 \\
\hline Thal & Thal right & -24 & 9 & -6 \\
\hline Thal & Thal left & 28 & 13 & -6 \\
\hline
\end{tabular}

Table of point locations used for seed-based functional connectivity analysis. The network name (i.e., independent component), anatomical region, and MNI coordinates are provided for each seed. Abbreviations: (DMN, default-mode network; PF left, left parieto-frontal network; PF right, right parietofrontal network; SM left, left sensorimotor network; Cbl, Cerebellum network; Vis, primary visual network; Visual Ext., extrastriate visual network; Aud left, left primary auditory network; Aud right, right primary auditory network; Thal, thalamus network). Region abbreviations: (IPL, inferior parietal lobule; aPFC, anterior prefrontal cortex; M1, primary motor cortex; S1, primary sensory cortex; V1, primary visual cortex; A1, primary auditory cortex; V5, middle temporal visual area).

\section{Between-Network Functional Connectivity Analysis}

In order to assess the between-network functional connectivity, group ICA network maps were thresholded at a $\mathrm{z}$-score of 30 , and local maxima of clusters $>2 \mathrm{~cm}^{3}$ were treated as nodes for a subsequent seed-based functional connectivity analysis. The z-score threshold and cluster size threshold were chosen heuristically such that only one or two clusters remained for each network. The voxel with the maximum z-score was used from each cluster for seed-based analysis. Shown in Table 2, a total of 27 local maxima were extracted from the left and right sensorimotor networks, the left and right parietofrontal control networks, the default-mode network, the bilateral cerebellar network, the bilateral extrastriate visual network, the primary visual network, the left and right auditory networks, and the bilateral thalamic network. Since the task-fMRI data were also included in the ICA, the left sensorimotor network map contained three clusters. These included the left precentral gyrus, and two clusters within the right cerebellum. The inclusion 
of task-positive BOLD data also caused the right sensorimotor network's local maximum to occur within the postcentral gyrus. Since the contralesional sensorimotor network has been shown to be involved in motor plasticity after stroke, the nodes from left and right sensorimotor networks were reflected over the mid-sagittal plane to produce 4 additional nodes. Independent components related to motion or cardiac artifact were regressed out of the raw BOLD data. The six motion regressors that were calculated by the MCFLIRT function prior to the group ICA were also used to clean the original BOLD data. The temporal correlation coefficient was calculated from the cleaned BOLD time courses of each pair of seed points.

\section{Statistical Analyses \\ Voxel-Based Analysis of BOLD Activation Maps and Spatial Network Maps}

All spatial map group comparisons were performed in MNI space and corrected for multiple comparisons using FDR correction. Group differences in functional connectivity maps were calculated in a $4 \mathrm{~mm}$ resolution MNI space as output by MELODIC. In our AFNI pipeline, we upsampled the BOLD activation maps into $1 \mathrm{~mm}$ MNI space before group comparison. Three BOLD activation maps from AFNI and four spatial network maps from the FSL dual regression analysis were compared between groups and conditions by using voxelbased Student's $t$-tests. Since we were focused on sensorimotor function in this study, only the spatial maps of networks included in Table 2 were analyzed. Voxel-level Student's $t$-tests were performed to test contrasts between stroke and control groups. In the functional network maps, additional paired $t$-tests were performed to determine within-group contrasts between resting-state and search. Spatial clusters of significantly different voxels $(p<0.01)$ were identified. In order to account for multiple comparisons, a corrected alpha value of 0.05 was used to remove clusters less than a threshold size determined by 3 dClustSim tool in AFNI. 3dClustSim, which corrects for multiple comparisons using FDR correction, was applied to both $4 \mathrm{~mm}$ and $1 \mathrm{~mm}$ MNI space templates to estimate cluster size thresholds for FDR correction. The cluster size threshold was 359 voxels for the $1 \mathrm{~mm}$ BOLD contrasts from AFNI and 8 voxels for the $4 \mathrm{~mm}$ functional connectivity maps from MELODIC. Multiple comparisons correction was further applied for the number of contrasts performed (3 activation maps and 20 functional networks). For between-network connectivity, a Student's $t$-test with was performed between stroke participants and age-matched controls for the edge strength between each pair of seed points. Multiple comparisons FWE correction was applied for the number of pairwise $t$-tests, which was $\left(\mathrm{n}^{*}(\mathrm{n}\right.$ $1) / 2)=\left(27^{*} 26 / 2\right)=702$.

\section{Correlational Analysis With Clinical Functional Scores} We hypothesized that stroke-related differences in task-related BOLD activation and functional network spatial maps would be correlated with the clinical evaluations of sensorimotor impairment/function. A linear regression analysis was performed with each BOLD activation contrast and functional network maps as a predictor of the Box and Blocks Score for the impaired arm. No linear regressions were performed with our activation and connectivity measures and the Fugl-Meyer or the Wolf Motor scores because there was a ceiling effect in our stroke participants with the Fugl-Meyer upper extremity (Table 1), and the Wolf Motor correlated with the Fugl-Meyer $(\mathrm{R}=0.87)$. The independent components that were compared between groups included the left and right sensorimotor networks. The $p$-values were multiplied by the number of analyzed networks to correct for multiple comparisons.

\section{RESULTS}

\section{The Search Task Produced Cortical Activation Patterns Within Motor and Multisensory Integration Areas}

Figure 2 demonstrates that in young healthy individuals and in the age-matched control group, our search task successfully produced unique cortical activation patterns for different sensory feedback conditions. Activity is reported where the group mean is significantly positive ( $t>2.79, p<0.01$, corrected). Search task-related activation common to all conditions was detected in contralateral sensorimotor cortex, bilateral premotor cortex, bilateral somatosensory association cortex, and bilateral anterior cerebellum. Purely visual or auditory activity was found in the primary visual and auditory cortices. An inferior-to-superior spatial gradient in overlapping activation maps were seen along the bilateral occipital surface. The superior occipital gyrus activity was exclusive to the unimodal auditory feedback condition. The middle occipital gyrus responded to the auditory and audiovisual conditions.

In young adults, there was unique activation during the audiovisual feedback condition within the bilateral dorsolateral prefrontal cortex and bilateral posterior parietal cortex, corresponding to the anterior and posterior multimodal association areas. BOLD activation in the control group that was age-matched to the stroke survivors was similar with the young healthy adults. However, this group did not have unique activation within the prefrontal and posterior parietal areas. Figure S3 illiterates stroke activity and the difference between stroke and control.

\section{During Sensory-Guided Movement, BOLD Activation in Stroke Survivors Depends on Sensory Feedback Modality}

As demonstrated in Figure 3 and Table 3, the BOLD activation in stroke survivors was dependent on the modality of sensory feedback. First, in the visual search condition, stroke survivors had similar activation to the age-matched controls within the active contralateral sensorimotor cortex. There was an increased activation in the contralesional prefrontal, posterior parietal, and sensorimotor cortices ( $p<0.01$, corrected). Increased activation was also observed within the ipsilesional prefrontal cortex ( $p<0.01$, corrected). Second, overall BOLD activity in stroke participants was lower than controls during the auditory feedback condition. Thirdly, the task-related BOLD activity in stroke survivors during the audiovisual search condition was significantly different from the age-matched controls in the contralesional inferior occipital gyrus and the posterior 


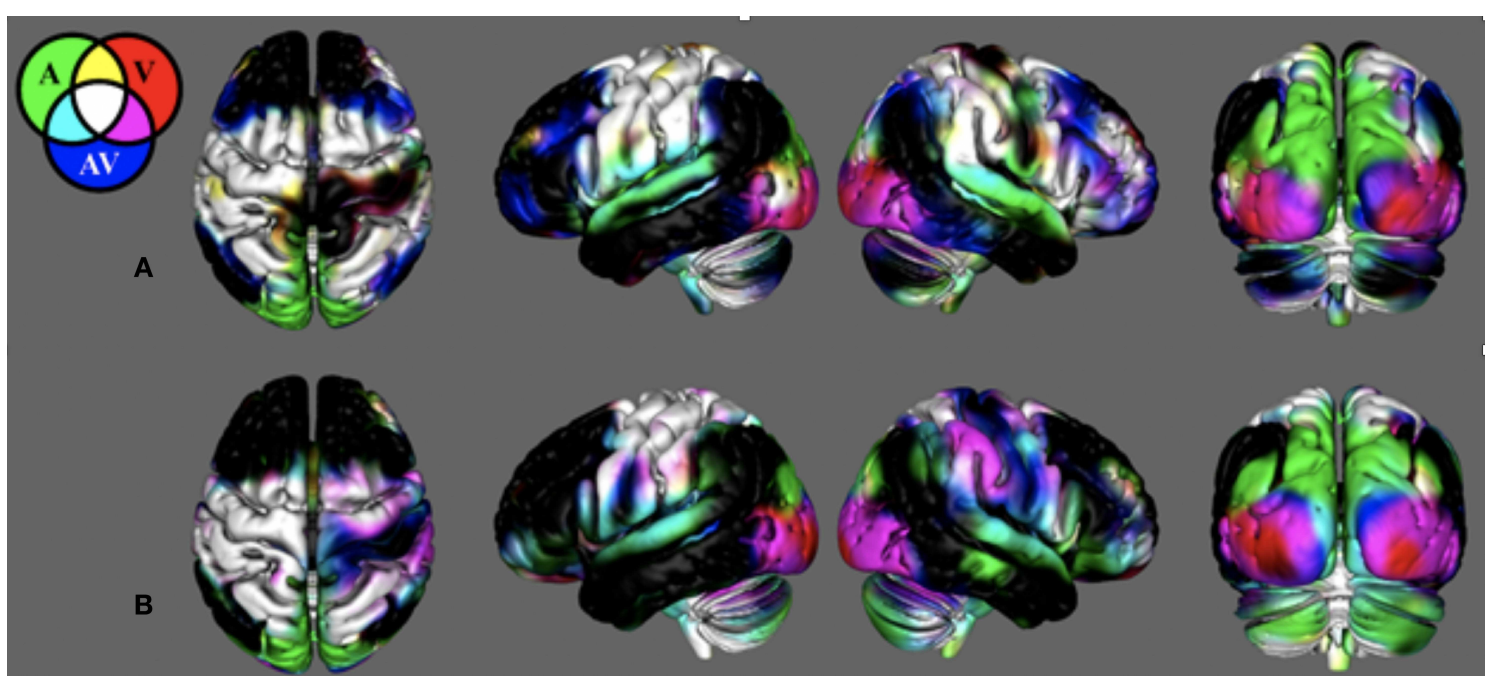

FIGURE 2 | Search-task BOLD activation maps in healthy individuals. These activations were produced from the novel search task paradigm. Mean BOLD activation for the auditory $\left(A_{M}\right)$, visual $\left(V_{M}\right)$, and audiovisual $\left(A V_{M}\right)$ task conditions in $(\mathbf{A})$ the young healthy adult group and (B) the control group age-matched to the stroke survivors. Note the BOLD contrasts are shown for the movement regressors.

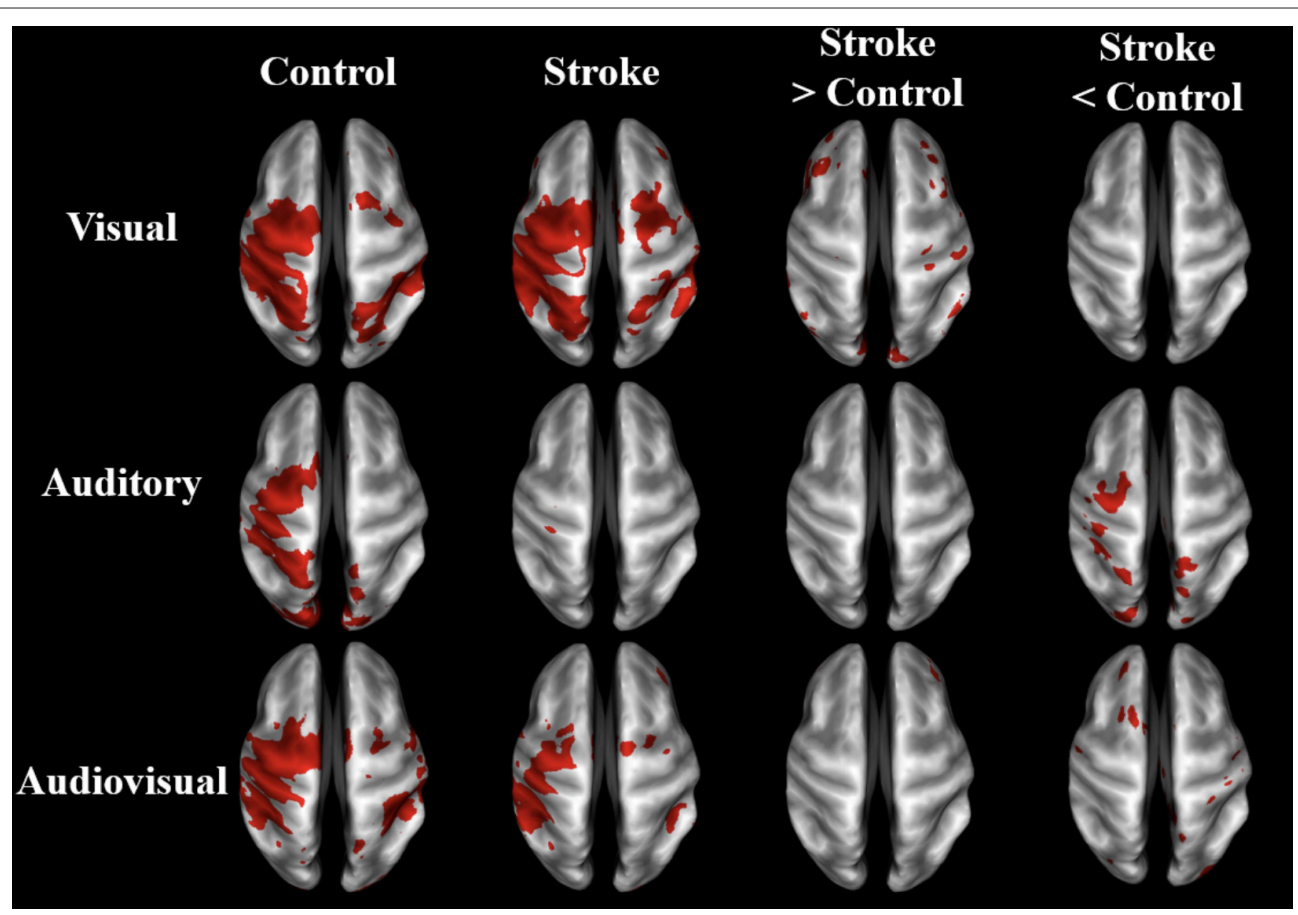

FIGURE 3 | BOLD activation maps of stroke vs. controls. BOLD activation in visual, auditory, and audiovisual sensory guided movement. Group-averaged activity and significant differences $(p<0.01$, un-corrected) are shown in stroke survivors $(n=10)$ and controls $(n=9)$ on inflated brain surfaces. The three sensory feedback conditions had similar activation in the contralateral sensorimotor cortex, bilateral premotor cortex, and bilateral somatosensory association cortex. Stroke participants had greater contralesional activation than controls during the visual condition and less ipsilesional activity during the auditory condition. The contralateral (ipsilesional) side is depicted on the left, and the ipsilateral (contralesional) side is depicted on the right.

thalamic radiation. During the visual and the audiovisual search, contralesional regions positively correlated with the Box and Blocks score, with the exception of the superior temporal gyrus
(Table 3). In the visual search task, the contralesional middle occipital gyrus, and superior temporal gyrus correlated with the Box and Blocks score; similarly, the inferior occipital and 
TABLE 3 | Localized group differences in BOLD activation.

\begin{tabular}{|c|c|c|c|c|c|c|c|c|c|c|c|}
\hline Condition & ROI & $\mathbf{x}$ & $y$ & $\mathbf{z}$ & nVox & $\mathbf{t}$ & & $p(t)$ & corr B\&B & & p (slope) \\
\hline Visual & MOG_R & 46 & -73 & 2 & 850 & -3.16 & $t t$ & 0.00636 & 0.716 & $t$ & 0.03610 \\
\hline \multirow[t]{2}{*}{ Search } & STG_R & 68 & -17 & 2 & 655 & 3.49 & $t t$ & 0.00321 & -0.765 & $t$ & 0.01972 \\
\hline & SPG_R & 31 & -62 & 63 & 35 & -2.00 & & 0.06249 & 0.851 & $t+$ & 0.00389 \\
\hline Visual & Cbl_R & 10 & -46 & -59 & 12 & -2.35 & t & 0.03182 & 0.697 & t & 0.04527 \\
\hline Target & $A G \_L$ & -40 & -75 & 49 & 96 & 2.34 & t & 0.03275 & -0.845 & $t+$ & 0.00471 \\
\hline Auditory & LG_L & -19 & -82 & -9 & 3616 & -3.65 & $t t$ & 0.00221 & 0.844 & $t t$ & 0.00481 \\
\hline \multirow[t]{5}{*}{ Search } & LG_R & 11 & -80 & -1 & 1234 & -3.79 & $t t$ & 0.00167 & 0.698 & $t$ & 0.04486 \\
\hline & SPG_L & -27 & -55 & 67 & 80 & -2.73 & $t$ & 0.01503 & 0.761 & $t$ & 0.02112 \\
\hline & MCP_L & -12 & -22 & -33 & 50 & -3.15 & $t+$ & 0.00651 & 0.672 & & 0.05666 \\
\hline & STG_R & 30 & 24 & -34 & 12 & -2.00 & & 0.06236 & 0.742 & + & 0.02676 \\
\hline & SPG_L & -15 & -63 & 71 & 12 & -4.27 & $t+$ & 0.00062 & 0.655 & & 0.06525 \\
\hline Auditory & STWM_L & -52 & -21 & -1 & 6027 & -3.77 & $t t$ & 0.00174 & 0.887 & $t t$ & 0.00154 \\
\hline \multirow[t]{8}{*}{ Target } & Cu_L & -4 & -96 & 6 & 963 & -2.58 & $t$ & 0.02122 & 0.916 & $t t$ & 0.00049 \\
\hline & PoCWM_R & 27 & -35 & 74 & 528 & -3.58 & $t t$ & 0.00265 & 0.837 & $t t$ & 0.00566 \\
\hline & Cbl_R & 8 & -82 & -47 & 296 & -5.21 & $t+$ & 0.00009 & 0.698 & $t$ & 0.04485 \\
\hline & IFG_R & 35 & 17 & 14 & 166 & -3.77 & $t+$ & 0.00173 & 0.632 & & 0.08274 \\
\hline & PrCG_L & -58 & -1 & 24 & 86 & -2.44 & t & 0.02772 & 0.848 & $t+$ & 0.00427 \\
\hline & PrCG_L & -3 & -30 & 74 & 37 & -3.24 & tt & 0.00546 & 0.885 & tt & 0.00162 \\
\hline & STG_R & 70 & -22 & -2 & 35 & -3.63 & tt & 0.00235 & 0.590 & & 0.11181 \\
\hline & MCP_R & 21 & -64 & -35 & 34 & -3.79 & tt & 0.00168 & 0.485 & & 0.21533 \\
\hline Audiovisual & IOG_R & 39 & -77 & 3 & 643 & -4.46 & $t t$ & 0.00041 & 0.740 & $t$ & 0.02721 \\
\hline Search & PTR_R & 36 & -61 & 0 & 484 & -3.89 & $t t$ & 0.00138 & 0.807 & $t$ & 0.01023 \\
\hline Audiovisual & MTWM_L & -49 & -49 & 1 & 70 & 4.77 & $t+$ & 0.00022 & -0.602 & & 0.10391 \\
\hline \multirow[t]{5}{*}{ Target } & Cbl_L & -31 & -88 & -29 & 52 & -1.54 & & 0.14873 & 0.910 & $t+$ & 0.00067 \\
\hline & ITG_L & -63 & -53 & -18 & 40 & -3.55 & $t+$ & 0.00282 & 0.712 & $t$ & 0.03807 \\
\hline & STG_L & -47 & -41 & 5 & 32 & 2.93 & $t$ & 0.01033 & -0.692 & $t$ & 0.04777 \\
\hline & PoCWM_L & -39 & -27 & 36 & 25 & 2.46 & $t$ & 0.02672 & -0.752 & $t$ & 0.02369 \\
\hline & SPG_L & -31 & -49 & 34 & 12 & 2.48 & $t$ & 0.02587 & -0.743 & $t$ & 0.02650 \\
\hline
\end{tabular}

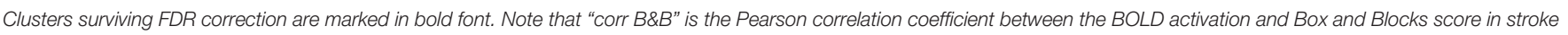

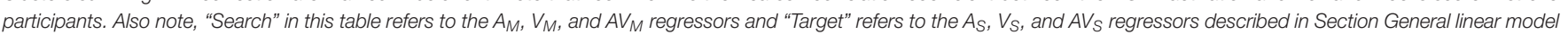

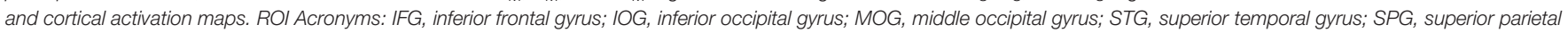

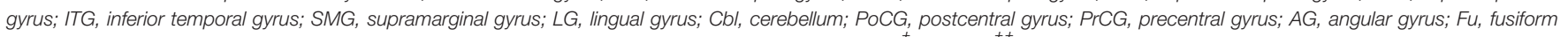
gyrus; MTG, middle temporal gyrus; MCP, middle cerebellar peduncle; PTR, posterior thalamic radiation. ${ }^{\dagger} p<0.05,{ }^{\dagger+} p<0.01$.

the posterior thalamic radiation correlated with the Box and Blocks score during the audiovisual task. As mentioned, the audio search task had decreased BOLD activity in the stroke group as compared to the controls, and these bilateral decreases were positively correlated with the Box and Blocks score.

\section{Stroke Participants Have Increased Contralesional Within-Network Functional Connectivity During the "Search" Task}

Within-network functional connectivity information provided by the independent component analysis is shown in Figure 4; Table 4. Figure 4A presents the network that had the highest temporal correlation with the recorded movement. This network included the contralateral sensorimotor cortex, bilateral premotor cortex, and ipsilateral cerebellum, which had the highest temporal correlation with the modeled BOLD response
( $\mathrm{R}=0.716$ across all participants). During both rest and the search task, this movement-related network was similar between stroke participants and age-matched controls. In controls, the participation of sensorimotor cortices became lateralized during the "search" task. Both hemispheres contributed to this shift in laterality, with greater positivity in contralateral sensorimotor areas and negative coefficients in the ipsilateral sensorimotor cortex. There was also an increased bilateral contribution from the supplementary motor area. These taskrelated network changes were not seen in stroke participants. Rather, contralateral somatosensory cortex and ipsilateral SMA and ventromedial premotor cortex increased in network participation. Thus, stroke participants had greater withinnetwork participation in the ipsilateral hemisphere during the search task. This result also held after subtracting the resting-state network values. 


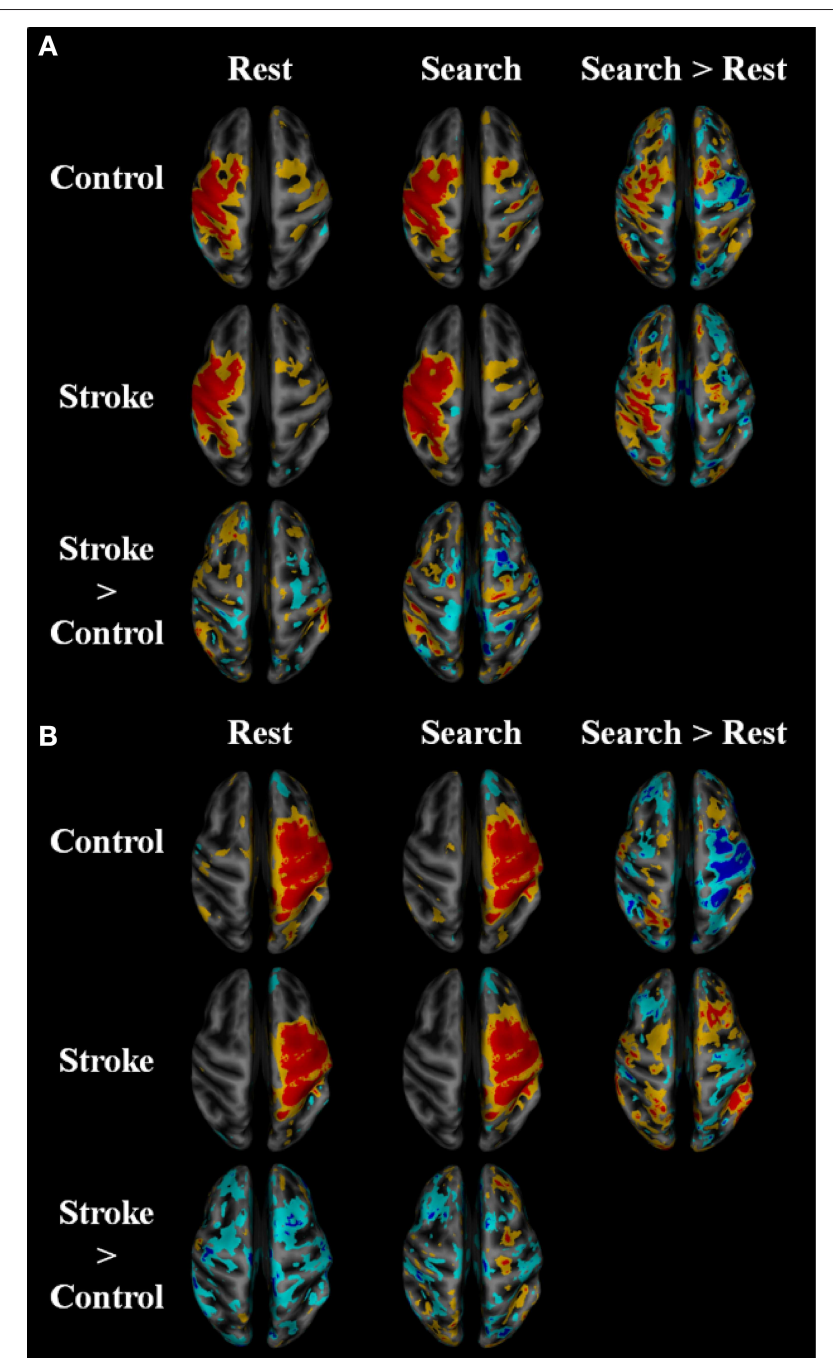

FIGURE 4 | Within network functional connectivity: stroke vs. control functional network maps. Independent component spatial maps for the ipsilesional/contralateral (A) and contralesional/ipsilateral (B) sensorimotor networks. Stroke and control $t$-value group averages and differences for rest and task conditions are overlaid on an inflated cortical map surface. Group averages with a mean normalized component intensity above three are colored red. Difference maps show clusters $>11$ cubic millimeters after thresholding at $p<0.01$. The ipsilesional side is depicted on the left, contralesional on the right. The colors represent $p$-values corresponding to the $t$-test run after the individual ICA maps were created with dual regression. Yellow and cyan are $p<0.05$, where red and blue are $p<0.01$. Blue/cyan represent significantly lower voxels and red/yellow represent significantly higher voxels for the test indicated.

\section{Stroke Survivors Have Decreased Between-Network Interhemispheric Connectivity and Increased Functional Connectivity to Visual Areas}

Between-network functional connectivity at rest and during the search task are shown in Figure 5. The resting-state functional connectivity between left and right sensorimotor networks was lower $(p<0.05$, corrected) in the stroke group. During both the task and resting state, functional connectivity in stroke participants showed an increase in connectivity between the sensorimotor areas and the visual areas in the occipital lobe as shown in Figure 5A. During the "search" task the left and right parietofrontal networks demonstrated lower between-network functional connectivity, both between the parietofrontal areas and the rest of the networks. Connections to the cerebellum (Figure 5D) were also lower in participants with stroke.

\section{Decreased Task-Based Functional Connectivity With the Cerebellum During Sensorimotor Integration Correlates With Motor Impairment After Stroke}

Figure 6 shows that with the "search" task, decreases in network functional connectivity of the cerebellum and visual association areas were associated with Box and Blocks score in individuals with stroke. In stroke participants, the contralesional cerebellum had decreased functional connectivity with the active sensorimotor cortex as previously mentioned (Figure 5; $p<0.005$, corrected). As shown in Figure 7, this cortico-cerebellar connectivity was significantly and positively correlated with Box and Blocks score $\left(r^{2}=\right.$ 0.64). Also, the connectivity of right and left extrastriate cortical regions (V5) measured during the search task were significantly and positively correlated with Box and Blocks score $\left(r^{2}=0.82\right)$. There were no significant correlations between resting state functional connectivity and the Box and Blocks score.

\section{DISCUSSION}

This study provides several novel findings that advance our understanding of network function of the brain after stroke. Consistent with our hypothesis, we found that the search task activated different parts of the brain dependent on visual, auditory, or audiovisual cues. For instance, in order to complete the visual search task control participants used greater contralateral activation, while stroke participants used greater ipsilateral activation. The connectivity analysis of the search task revealed more stroke-related deficits as compared to resting state. Of particular interest, we found a stroke-related increase in functional connectivity between the sensorimotor and visual areas only during the search task (not resting state), suggesting stroke survivors might rely more on visual feedback for motor control. The dependence of hand function on cerebellar and sensorimotor connectivity was only detected during the search task, indicative of the importance of performing task-based functional connectivity.

\section{Multisensory Control in Wrist Movement Evokes Activation in Bilateral Motor and Association Areas}

The activation patterns seen in the neurologically intact control group revealed that wrist movement during single 
TABLE 4 | Localized group differences in network spatial maps.

\begin{tabular}{|c|c|c|c|c|c|c|c|c|c|c|c|c|}
\hline Run & IC & ROI & $\mathbf{x}$ & $y$ & $\mathbf{z}$ & nVox & $t$ & & $p(t)$ & corr B\&B & & p (slope) \\
\hline \multirow[t]{3}{*}{ Rest } & Right PF & ITG_R & 44 & -1 & -44 & 13 & -3.42 & tt & 0.00360 & 0.928 & tt & 0.00028 \\
\hline & DAN/CblL & SMG_R & 68 & -33 & 20 & 11 & 3.72 & $t+$ & 0.00188 & -0.794 & $t$ & 0.01276 \\
\hline & & & & & & & & & & & tt & \\
\hline \multirow[t]{7}{*}{ Search } & V1 Medial & LWM_R & 12 & -81 & 0 & 32 & -3.18 & $t+$ & 0.00617 & 0.853 & tt & 0.00377 \\
\hline & Left SM & Cbl_R & 44 & -65 & -40 & 54 & 3.71 & $t+$ & 0.00192 & -0.915 & tt & 0.00049 \\
\hline & Left SM & Cbl_R & 32 & -53 & -24 & 39 & -3.26 & $t+$ & 0.00515 & 0.883 & tt & 0.00171 \\
\hline & Left SM & PoCWM_L & -20 & -33 & 40 & 35 & 3.52 & $t+$ & 0.00301 & -0.857 & $t+$ & 0.00347 \\
\hline & Left SM & PrCG_R & 36 & -5 & 60 & 17 & -4.43 & $t+$ & 0.00043 & 0.899 & tt & 0.00097 \\
\hline & Right PF & AWM_R & 36 & -53 & 32 & 11 & 2.99 & $t+$ & 0.00900 & -0.732 & $t$ & 0.02957 \\
\hline & & & & & & & & & & & tt & \\
\hline \multirow[t]{5}{*}{ S xor M } & Right Insula & Fu_R & 28 & -81 & -8 & 12 & 2.64 & t & 0.01955 & -0.953 & tt & 0.00020 \\
\hline & DMNmpf & MTG_L & -60 & -21 & -16 & 11 & -4.28 & $t+$ & 0.00069 & 0.892 & tt & 0.00305 \\
\hline & Left SM & Cbl_R & 50 & -61 & -44 & 22 & 3.12 & tt & 0.00720 & -0.965 & tt & 0.00007 \\
\hline & Left SM & Cbl_R & 36 & -53 & -28 & 17 & -3.01 & $t+$ & 0.00903 & 0.810 & t & 0.01711 \\
\hline & Central M1 & Cbl_R & 36 & -77 & -40 & 11 & -3.81 & tt & 0.00177 & 0.913 & $t+$ & 0.00152 \\
\hline
\end{tabular}

${ }^{\dagger} p<0.05,{ }^{+\dagger} p<0.01$.

MNI spatial coordinates are provided for clusters with significant group differences between stroke participants and age-matched controls.

ROI Acronyms: ITG, inferior temporal gyrus; SMG, supramarginal gyrus; LWM, lingual gyrus; Cbl, cerebellum; PoCWM, postcentral gyrus; PrCG, precentral gyrus; AWM, angular gyrus; Fu, fusiform gyrus; MTG, middle temporal gyrus.

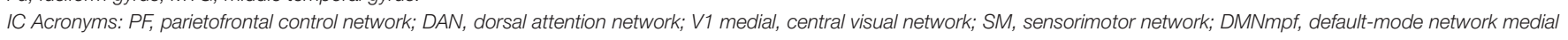
prefrontal node.

and multisensory search tasks elicits activation patterns mainly in the contralateral sensorimotor, and the bilateral premotor and somatosensory association cortices (Figure 2). As expected, the visual feedback task produced additional activation in the occipital lobe (44-46), while the auditory feedback produced activation in the temporal and superior occipital gyrus (4749). Interestingly, the combined audiovisual condition in young adults also recruited areas in the dorsolateral prefrontal cortex and the bilateral posterior parietal cortex. Similarly, the withinnetwork and between-network functional connectivity also suggests that the complex search task used in the current study connects motor and association areas bilaterally, which contrasts to the lateralized contralateral activation seen in finger tapping fMRI protocols $(50,51)$. This is notable in Figure 4A, where the independent component network with the highest temporal correlation with the recorded movement during the search tasks included the contralateral sensorimotor cortex, the bilateral premotor cortex, and the ipsilateral cerebellum (not visible in figure). Our study shows that a search task paradigm reveals the effects of multimodal sensory feedback on brain functional networks in neurologically intact participants, providing evidence that single-joint movement during multisensory control recruits areas of the brain beyond contralateral motor regions.

Brain activation was sensitive to the search task fMRI paradigm in stroke participants. As seen in Figure 3, stroke participants' task-related activation was dependent on the type of sensory feedback. Stroke participants had increased activation in the bilateral prefrontal cortex, and contralesional posterior parietal and sensorimotor cortices during the visual search condition. During auditory feedback, stroke participants had reduced ipsilesional activity. In the combined audiovisual search task, activation of the inferior occipital and the posterior temporal gyri was significantly reduced in stroke, and activation of these regions positively correlated with the Box and Blocks score (Table 3). These results suggest that task-related activation is sensory-dependent, and that multisensory integration is impacted by stroke (52).

Functional connectivity was also dependent on the search task in the current study. The within-network functional connectivity results suggest that stroke participants engage bilateral motor regions to a higher extent to complete the search task. Figure 4 shows that the functional connectivity within the ipsilateral sensorimotor network was greater during the search task as compared to rest in stroke participants. Whereas, the sensorimotor cortices in the neurologically intact group became more lateralized toward the contralateral hemisphere, the stroke group had greater within network participation in the ipsilateral hemisphere during the search task, suggesting that stroke participants engage bilateral motor networks during a search task and wrist movement. This observation is consistent with prior reports on brain activation $(10,53,54)$.

\section{Task-Based Functional Connectivity Provides Unique Information About Sensorimotor Integration and Motor Control After Stroke}

Consistent with our hypothesis, the search task revealed "local specialization" as evidenced by increased withinnetwork functional connectivity, and greater between-network connectivity after stroke. The control group saw an increase in 


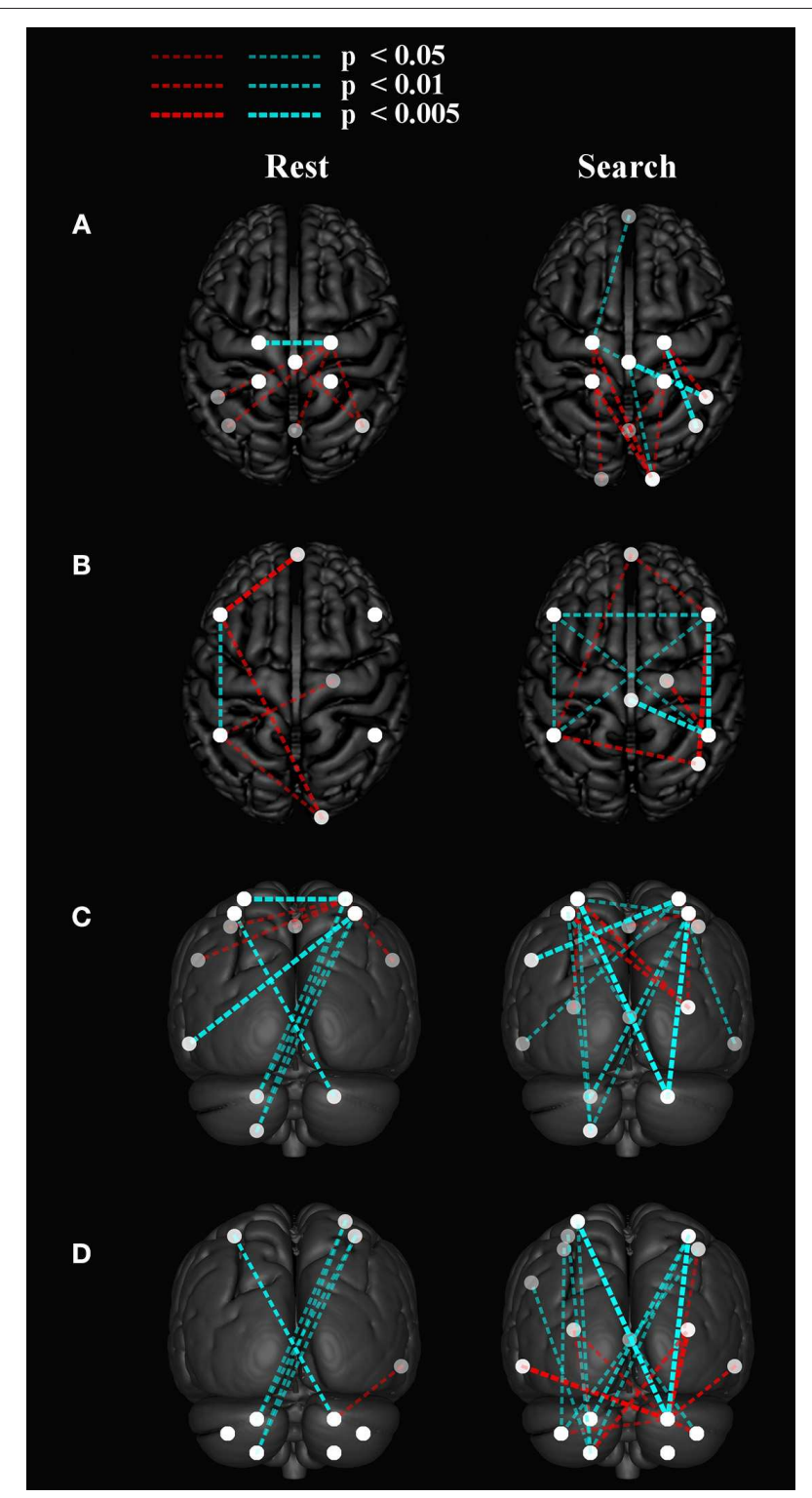

FIGURE 5 | Group differences in between network functional connectivity. An inflated pial surface of a template brain is shown with color-coded independent components and functional network graph overlay. The ipsilesional side is depicted on the left, contralesional on the right. Stroke-related increased connections are in red and decreases are in cyan. The overhead view in (A) shows the connectivity to the left and right sensorimotor network nodes and in (B) presents connectivity to the bilateral parietofrontal networks. (C) The posterior views show connectivity differences to four (C) sensorimotor network nodes, and (D) eight cerebellar nodes. Note that all nodes are within brain tissue, although some of the network edges are difficult to visualize.

within-network connectivity in the contralateral sensorimotor areas and bilateral SMA (Figure 4A). As shown in Figure 4B, cortical areas within the ipsilateral sensorimotor network were lower during the search task in control participants, further suggesting task-related inhibition of the ipsilateral cortex in healthy individuals and lateralization toward the contralateral hemisphere (22). The increase in bilateral SMA contributions

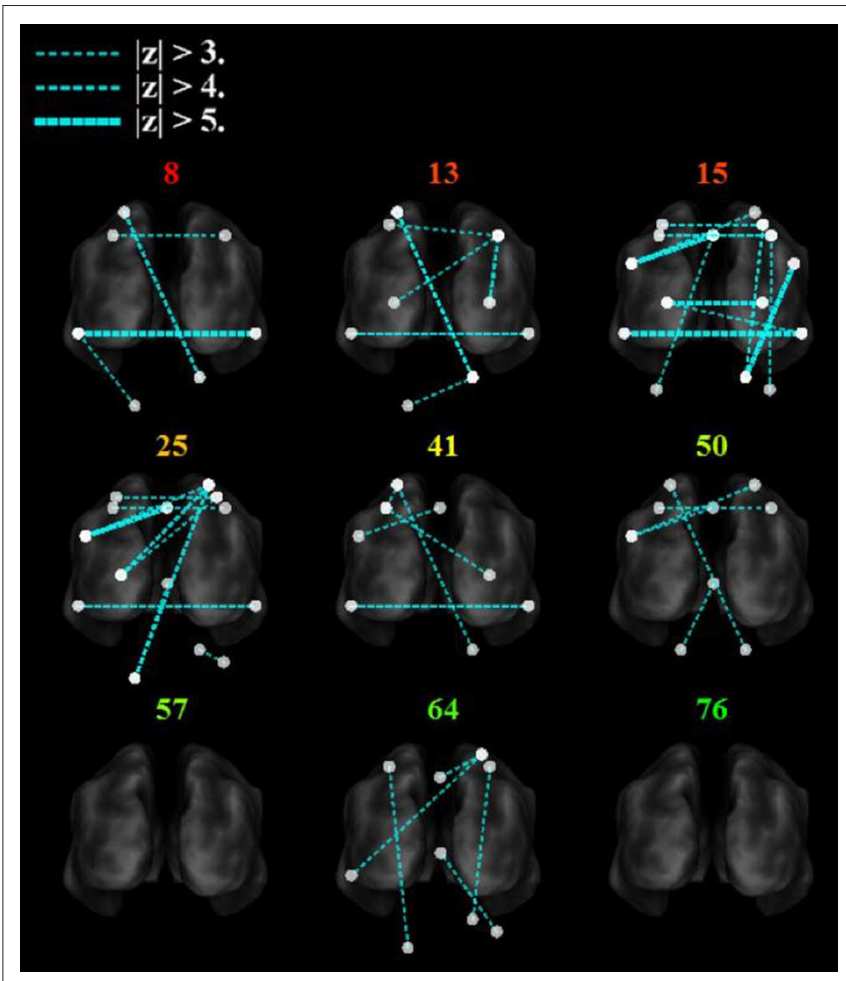

FIGURE 6 | Functional trends in seed-based functional connectivity. Differences in functional connectivity in individual stroke survivors during the search task vary with Box and Blocks Score (colored number indicated on top of each brain). Note that the $z$-score is calculated as the difference in connectivity strength between the age-matched controls and the individual stroke participant. The ipsilesional side is depicted on the left, contralesional on the right. Note that all nodes are within brain tissue, although some areas in the cerebellum are difficult to visualize with this slice.

to the sensorimotor network with the task might be due to the strong reciprocal connections between the left and right SM (55). In contrast, the stroke group saw increased within network connectivity in the ipsilateral SMA and ventromedial premotor areas during the search task (Figure 4B). Due to lesion location in the contralateral hemisphere, stroke participants were unlikely to be able to increase contralateral cortical participation in the SM network with the task. Thus, the stroke group appeared to produce local reorganization in the ipsilateral side.

The task-based fMRI revealed stroke-related network connectivity changes that positively correlated with motor impairment (Figure 7). The resting-state functional connectivity between left and right sensorimotor networks was lower $(p<$ 0.05 ) in the stroke group, which is consistent with other reports of decreased interhemispheric connectivity after stroke (21); however, these changes were not correlated with the box and blocks scores (Figure 7A). During the "search" task, reduced functional connectivity between the left and right V5 was strongly correlated with the box and blocks score in stroke individuals (Figure 7D). Additionally, the functional connectivity between the middle temporal gyrus and parietofrontal nodes showed 

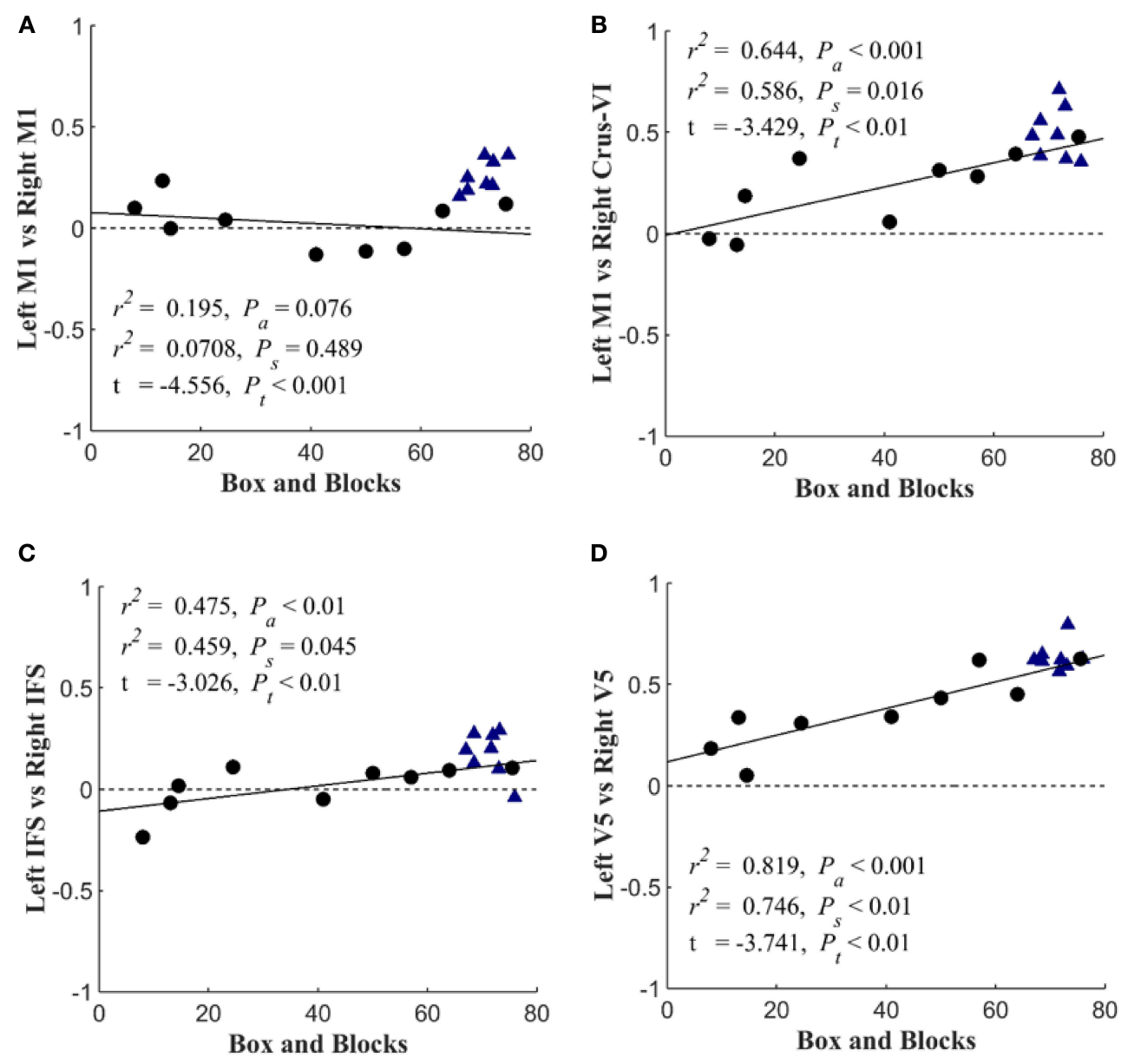

FIGURE 7 | Scatterplots of seed-based connectivity during "search" task vs. motor function. Stroke participants are shown with black dots and controls are shown with blue triangles. The dependent variable in each plot is the partial correlation between two specified seed points. Linear regressions were performed within stroke participants and repeated across all participants (assuming that controls scored a 76). The first $r^{2}$ and $\mathrm{Pa}_{\mathrm{a}}$ are the coefficient of variation and slope's $p$-value for the regression analysis across all participants. The second $r^{2}$ and $\mathrm{P}_{\mathrm{S}}$ are associated with the analysis that included only stroke survivors. Group differences are reported by $t$-value and corresponding $P_{\mathrm{t}}$-value. The figure shows the Box and blocks correlation with (A) Left M1 \& Right M1 connectivity, (B) Left M1 \& Right Crus-V1 connectivity, (C) Left IFS \& Right IFS connectivity, and (D) Left V5 \& Right V5 connectivity.

stroke-related increases (Figure 5). The middle temporal gyrus has been shown to play a role in processing motion-related visual information (56).

\section{Clinical Correlations During the Search Task Reveal Sensorimotor Integration Deficits in Stroke}

A unique finding in this study is a stroke-related increase in functional connectivity between the sensorimotor and visual areas during a search task, suggesting that stroke survivors may rely on visual feedback for motor control. During the auditory feedback conditions, stroke participant's auditory cortex was less active (Table 3; Figure 3). In contrast, ipsilesional cortical activity was more widespread in stroke participants than controls during the visuomotor task. While auditory feedback has been shown to improve weight bearing and gait characteristics in stroke survivors (57), individuals with stroke have been shown to rely on visual feedback for posturing (58). Additionally, visual feedback during grip-force control increases activation in the visual cortex, premotor cortex, supplementary motor areas, and the ipsilateral cerebellum in stroke participants (59), and limb apraxia has been associated with a deficit in visuo-motor integration due to lesions in the fronto-parietal motor network (60). The findings suggest that visual information during a complex motor task is an important source of feedback in stroke survivors and highlights the importance of measuring brain activation and calculating between-network connectivity during task-state fMRI.

Although the results showed an effect during visual feedback that was not present during the auditory condition, it would be overreaching to conclude that stroke survivors are more sensitive to visual over auditory feedback during fine-motor control. We do not know the visual vs. auditory sensitivity in each subject, which is information that would require a psychophysical exam. 
Although we did not record the $\mathrm{dB}$ gain of the maximum auditory feedback, a reasonable volume was used during the experiment. The volume was set such that the subject reported that the auditory feedback could be heard.

In addition to identifying stroke participant's dependence on visual feedback for motor control, this work emphasized the importance of cerebellar functional connectivity after stroke, specifically during sensorimotor integration. As indicated in Figure 5, the bilateral cerebellum exhibited stroke-related decreases in functional connectivity during both at rest and during the search task. Additionally, the connectivity between the ipsilateral cerebellar crus VI and the contralateral M1 was positively correlated with the box and blocks score when tested using the search task (Figure 7B). Involvement of the cerebellum in different networks involving movement and multisensory integration give it a critical role in brain plasticity after stroke (61). The motor cortex and cerebellum together have been shown to be involved in plasticity during motor training and in sensorimotor integration (62). Past imaging studies have shown that the connectivity between the cerebellum and parietofrontal areas are related to post-stroke motor function $(63,64)$.

\section{Study Limitations}

This study is limited by its small sample size for each group, especially for the age matched control and stroke participants. Head motion is a potential confounding factor in any study using functional activity or connectivity MRI, especially those involving task-based paradigms and patient populations. Indeed, head motion has been found in past studies to be greater in patients than in controls, and also increases with age (65). Van Dijk et al. found that although most variability in functional connectivity is not associated with head movement, there is significantly reduced functional coupling between the parietofrontal and default-mode network nodes in young adults. Furthermore, greater mean head motion leads to increased local functional coupling and interhemispheric connectivity between sensorimotor areas. Stroke participants in this study had significantly greater mean head motion than controls $(p<0.05)$ at rest and during the search task. Thus, our findings that stroke survivors had decreased between-network connectivity to the bilateral parietofrontal networks and increased within-network connectivity contralesionally could be in part due to increased head motion. However, we observed decreased interhemispheric connectivity, opposite to what was observed by Van Dijk et al. Since head motion was regressed out of the original data before our seed-based analysis, we do not believe that it was the prime contributor to our findings.

Changes in vasculature after stroke can lead to differences in neurovascular coupling near the lesion, which may influence correlations of these voxels with distant areas. Differences in brain structure can have an impact on functional connectivity metrics due to changes in the partial volume of gray matter (66). Using simulations, Dukart and Bertolino showed that between-group differences in brain structure leads to significant differences in functional connectivity between the groups. Due to the large variability in lesion location in this study, partial volume was not expected to have a significant impact on functional connectivity results.
Non-stroke related lateralization of cortical activation and functional connectivity is a potential confounding factor in this study. Studies of healthy adults have shown significant lateralization of resting-state functional connectivity (67). Nielson et al. showed that there are 20 "lateralization hubs" that have the most lateralized functional connectivity. Some of these hubs included the dorsolateral prefrontal cortex, supplementary motor area, premotor cortex, Broca's area, insula, and junctions between the parietal, occipital, and temporal lobes. Many of these regions are unimodal and multimodal sensory association areas. Handedness of our participants and the procedures of flipping the brain over the mid-sagittal plane to place all lesions on the same side of the brain could have had an impact on the results.

Spatial group differences in BOLD contrasts and functional connectivity could have been affected by group differences in the level of head motion during the task. The fsl_motion_outliers program in the FMRIB Software Library was used to estimate mean frame-wise displacement (FD) and mean square of successive differences (DVARS) (68) from the BOLD data during the search task runs of each subject. Shown in Figure S4, both metrics of head motion were significantly different $(p$ $<0.01)$ between groups, but neither DVARS $(\mathrm{r} 2=0.02)$ nor FD $(\mathrm{r} 2=0.08)$ were correlated with Box and Blocks score. Although head motion regressors were included in all fMRI analyses in this study, greater motion in stroke subjects may have reduced the peak values of the functional connectivity maps.

Differences between study groups in range of wrist movement, and task performance could also impact the interpretation of our imaging findings. We report the number of completed search-task trials as a rough estimate of performance in Figure S5a. On average, stroke subjects completed 49.4 trials, which was significantly $(p<0.01)>61.1$ trials completed in age-matched controls. However, the number of completed trials did not correlate $(\mathrm{r} 2=0.04)$ with the Box and Blocks score. Shown in Figures S5a-c, the number of search trials (Figure S5a) and the average error accumulated (Figures S5b,c) was not correlated with the box and blocks scores. Shown in Figures S5d,e, the range of wrist motion was not significantly different between groups nor correlated with Box and Blocks score in stroke subjects.

\section{CONCLUSION}

In conclusion, our novel functional MRI task paradigm involving wrist movement and multisensory feedback revealed changes in BOLD activation and functional connectivity after stroke, suggesting that task-based fMRI can highlight alterations in brain functional networks after stroke. We documented widespread bilateral activation in neurologically intact participants during an audiovisual search task, indicating that multisensory wrist control recruits primary motor and association areas. In contrast, stroke participant's activation patterns were task dependent; visual feedback produced increased contralesional activation compared to controls, while auditory feedback resulted in decreased activation ipsilesionally. Additionally, our within-network functional connectivity 
analysis detected a task-related lateralization to contralateral sensorimotor regions in control participants that was not found in stroke. Lastly, between-network functional connectivity during the search task revealed decreased connectivity between the ipsilesional sensorimotor cortex and the contralesional cerebellum, and between interhemispheric V5 regions, which both correlated with box and blocks scores of hand function. These results suggest that task-based functional connectivity provides detail on changes in brain networks in stroke survivors. The data also highlight the importance of cerebellar connections for recovery of arm function after stroke. Future work will investigate the role of structural connectivity in these mechanistic changes between sensorimotor and sensory integration networks.

\section{DATA AVAILABILITY}

The raw data supporting the conclusions of this manuscript will be made available by the authors, without undue reservation, to any qualified researcher.

\section{ETHICS STATEMENT}

This study was carried out in accordance with the recommendations of the Institutional Review Boards of the Medical College of Wisconsin and Marquette University with written informed consent from all subjects. All subjects gave written informed consent in accordance with the Declaration of Helsinki. The protocol was approved by the Institutional Review Board of the Medical College of Wisconsin.

\section{AUTHOR CONTRIBUTIONS}

BK involved in conception and design of work, software development, subject recruitment, data acquisition, image processing, results interpretation, manuscript drafting, and editing. KV and MS involved in results interpretation, manuscript drafting, and editing. $\mathrm{AH}$ involved in conception and design of work, clinical measures, results interpretation. BS involved in conception and design of work, results interpretation, manuscript drafting, and editing.

\section{FUNDING}

This work was supported by the Marquette University Strategic Innovation Fund and the Ralph and Marion C. Falk Medical Research Trust. The contents of this article were developed under a grant from the National Institute on Disability, Independent Living, and Rehabilitation Research (NIDILRR grant number

\section{REFERENCES}

1. Cheng L, Wu Z, Fu Y, Miao F, Sun J, Tong S. Reorganization of functional brain networks during the recovery of stroke: A functional MRI study. Conf Proc IEEE Eng Med Biol Soc. (2012) 2012:4132-5. doi: 10.1109/EMBC.2012.6346876
90RE5006, formerly NIDRR H133E100007). NIDILRR is a Center within the Administration for Community Living (ACL), Department of Health and Human Services (HHS). The contents of this article do not necessarily represent the policy of NIDILRR, ACL, HHS, and you should not assume endorsement by the Federal Government.

\section{ACKNOWLEDGMENTS}

We would like to thank the Center for Imaging Research at the Medical College of Wisconsin for assistance with data collection. This work is part of Dr. Kalinosky's dissertation at Marquette University.

\section{SUPPLEMENTARY MATERIAL}

The Supplementary Material for this article can be found online at: https://www.frontiersin.org/articles/10.3389/fneur. 2019.00609/full\#supplementary-material

Figure S1 | Stroke lesion distribution. Spatial distribution of lesioned voxels in the stroke subject cohort overlaid on eight slices in MNI space. Note that three stroke subjects' images have been flipped over the $x$-axis so that lesions are on the left and cerebellar lesions on the right. The lesions share limited overlap, with three lesions overlapping in the posterior limb of the internal capsule and two lesions overlapping in the superior temporal gyrus and sensorimotor cortex. Regions limited to a single lesion include the cerebellum, brainstem, and insula, and frontal gray and white matter.

Figure S2 | Overlay of task-based network time-course and GLM model. A comparison of the active sensorimotor network time-course (blue) and the modeled BOLD response to the motor-only condition (dotted black line) for a single control participant.

Figure S3 | BOLD Activation Maps for the search task. Respectively, the visual, auditory, and audiovisual feedback conditions are mapped to red, green, and blue. Cortical surface overlay with auditory, visual, and audiovisual brain activation regressors mapped to red, green, and blue by t-value. Coefficients are shown for (a) young adults, (b) age-matched controls, (c) stroke subjects, differences between groups are shown for Stroke > Controls (d) and Controls > Stroke (e), where $t>2.3$.

Figure S4 | Head motion in stroke subjects and age-matched controls. Comparison of head motion across all search task trials in stroke subjects (filled circles) and age-matched controls (triangles). (a) Mean framewise displacement (FD) and (b) mean square of successive differences (DVARS) (68) are plotted against Box and Blocks score. Age-matched controls were given an artificial score of 76 for visualization purposes only. Correlations were only performed on stroke subjects.

Figure S5 | Wrist range of motion and task performance in stroke subjects and age-matched controls. Comparison of stroke subjects (filled circles) and age-matched controls (triangles) in range of wrist motion and number of completed search task trials. (a) Number of search task trials, (b) Average $\theta_{x}$ error, (c) and $\theta_{y}$ error in radians, (d) angular range of $\theta_{x}$ dominantly controlled by supination/pronation, (e) angular range of $\theta$ y associated with radial/ulnar deviation, plotted against Box, and Blocks score. Age-matched controls were given an artificial score of 76 for visualization purposes only. Correlations were only performed on stroke subjects.
2. Sharma N, Baron J-C, Rowe JB. Motor imagery after stroke: relating outcome to motor network connectivity. Ann Neurol. (2009) 66:604-16. doi: 10.1002/ana.21810

3. Wang L, Yu C, Chen H, Qin W, He Y, Fan F, et al. Dynamic functional reorganization of the motor execution network after stroke. Brain. (2010) 133:1224-38. doi: 10.1093/brain/awq043 
4. Ward NS, Brown MM, Thompson AJ, Frackowiak RSJ. Neural correlates of motor recovery after stroke: a longitudinal fMRI study. Brain. (2003) 126:2476-96. doi: 10.1093/brain/awg245

5. Bosnell RA, Kincses T, Stagg CJ, Tomassini V, Kischka U, Jbabdi S, et al. Motor practice promotes increased activity in brain regions structurally disconnected after subcortical stroke. Neurorehabil Neural Repair. (2011) 25:607-16. doi: 10.1177/1545968311405675

6. Buch ER, Modir Shanechi A, Fourkas AD, Weber C, Birbaumer N, Cohen LG. Parietofrontal integrity determines neural modulation associated with grasping imagery after stroke. Brain. (2012) 135:596-614. doi: 10.1093/brain/awr331

7. Evans AC. Networks of anatomical covariance. Neuroimage. (2013) 80:489504. doi: 10.1016/j.neuroimage.2013.05.054

8. Lotze M, Beutling W, Loibl M, Domin M, Platz T, Schminke U, et al. Contralesional motor cortex activation depends on ipsilesional corticospinal tract integrity in well-recovered subcortical stroke patients. Neurorehabil Neural Repair. (2012) 26:594-603. doi: 10.1177/1545968311427706

9. Murphy TH, Corbett D. Plasticity during stroke recovery: from synapse to behaviour. Nat Rev Neurosci. (2009) 10:861-72. doi: 10.1038/nrn2735

10. Cramer SC, Nelles G, Benson RR, Kaplan JD, Parker RA, Kwong KK, et al. A functional MRI study of subjects recovered from hemiparetic stroke. Stroke. (1997) 28:2518-27. doi: 10.1161/01.STR.28.12.2518

11. Grefkes C, Fink GR. Reorganization of cerebral networks after stroke: new insights from neuroimaging with connectivity approaches. Brain. (2011) 134:1264-76. doi: 10.1093/brain/awr033

12. Promjunyakul N, Schmit BD, Schindler-Ivens SM. A novel fMRI paradigm suggests that pedaling-related brain activation is altered after stroke. Front Hum Neurosci. (2015) 9:324. doi: 10.3389/fnhum.2015.00324

13. Biswal B, Yetkin FZ, Haughton VM, Hyde JS. Functional connectivity in the motor cortex of resting human brain using echo-planar MRI. Magn Reson Med. (1995) 34:537-41. doi: 10.1002/mrm.1910340409

14. Van den Heuvel MP, Hulshoff Pol HE. Exploring the brain network: a review on resting-state fMRI functional connectivity. Eur Neuropsychopharmacol. (2010) 20:519-34. doi: 10.1016/j.euroneuro.2010.03.008

15. Friston KJ. Functional and effective connectivity in neuroimaging: a synthesis. Hum Brain Mapp. (1994) 2:56-78. doi: 10.1002/hbm.460020107

16. Yeo BTT, Krienen FM, Sepulcre J, Sabuncu MR, Lashkari D, Hollinshead $\mathrm{M}$, et al. The organization of the human cerebral cortex estimated by intrinsic functional connectivity. J Neurophysiol. (2011) 106:1125-65. doi: 10.1152/jn.00338.2011

17. Beckmann CF, Smith SM. Probabilistic independent component analysis for functional magnetic resonance imaging. IEEE Trans Med Imaging. (2004) 23:137-52. doi: 10.1109/TMI.2003.822821

18. Minka T. (2000). Automatic choice of dimensionality for PCA. In: Advances in Neural Information Processing Systems. 1-16. Available online at: https:// papers.nips.cc/paper/1853-automatic-choice-of-dimensionality-for-pca.pdf

19. Rehme AK, Grefkes C. Cerebral network disorders after stroke: evidence from imaging-based connectivity analyses of active and resting brain states in humans. J Physiol. (2013) 591:17-31. doi: 10.1113/jphysiol.2012.243469

20. Grefkes C, Eickhoff SB, Nowak DA, Dafotakis M, Fink GR. Dynamic intra-and interhemispheric interactions during unilateral and bilateral hand movements assessed with fMRI and DCM. Neuroimage. (2008) 41:1382-94. doi: 10.1016/j.neuroimage.2008.03.048

21. Carter AR, Astafiev SV, Lang CE, Connor LT, Rengachary J, Strube MJ, et al. Resting interhemispheric functional magnetic resonance imaging connectivity predicts performance after stroke. Ann Neurol. (2010) 67:365-75. doi: 10.1002/ana.21905

22. Cole MW, Bassett DS, Power JD, Braver TS, Petersen SE. Intrinsic and taskevoked network architectures of the human brain. Neuron. (2014) 83:238-51. doi: 10.1016/j.neuron.2014.05.014

23. Kaufmann T, Alnæs D, Brandt CL, Doan NT, Kauppi K, Bettella F, et al. Task modulations and clinical manifestations in the brain functional connectome in 1615 fMRI datasets. Neuroimage. (2017) 147:243-52. doi: 10.1016/j.neuroimage.2016.11.073

24. Barch DM, Burgess GC, Harms MP, Petersen SE, Schlaggar BL, Corbetta $\mathrm{M}$, et al. Function in the human connectome: task-fMRI and individual differences in behavior. Neuroimage. (2013) 80:169-89. doi: 10.1016/j.neuroimage.2013.05.033
25. Smith SM, Fox PT, Miller KL, Glahn DC, Fox PM, Mackay CE, et al. Correspondence of the brain's functional architecture during activation and rest. Proc Natl Acad Sci USA. (2009) 106:13040-5. doi: 10.1073/pnas.0905267106

26. Di X, Gohel S, Kim EH, Biswal BB. Task vs. rest-different network configurations between the coactivation and the resting-state brain networks. Front Hum Neurosci. (2013) 7:493. doi: 10.3389/fnhum.2013.00493

27. Gonzalez-Castillo J, Bandettini PA. Task-based dynamic functional connectivity: recent findings and open questions. Neuroimage. (2018) 180:526-33. doi: 10.1016/j.neuroimage.2017.08.006

28. Alahmadi AAS, Samson RS, Gasston D, Pardini M, Friston KJ, D'Angelo E, et al. Complex motor task associated with non-linear BOLD responses in cerebro-cortical areas and cerebellum. Brain Struct Funct. (2016) 221:2443-58. doi: 10.1007/s00429-015-1048-1

29. Westlake KP, Nagarajan SS. Functional connectivity in relation to motor performance and recovery after stroke. Front Syst Neurosci. (2011) 5:8. doi: 10.3389/fnsys.2011.00008

30. Grefkes C, Fink GR. Connectivity-based approaches in stroke and recovery of function. Lancet Neurol. (2014) 13:206-16. doi: 10.1016/S1474-4422(13)70264-3

31. Jiang L, Xu H, Yu C. Brain connectivity plasticity in the motor network after ischemic stroke. Neural Plast. (2013) 2013:924192. doi: 10.1155/2013/924192

32. Fugl-Meyer AR. The post-stroke hemiplegic patient. Scand J Rehab Med. (1975) 7:13-31.

33. Mathiowetz V, Volland G, Kashman N, Weber K. Adult norms for the Box and Block Test of manual dexterity. Am J Occup Ther. (1985) 39:386-91. doi: 10.5014/ajot.39.6.386

34. Wolf SL, Catlin PA, Ellis M, Archer AL, Morgan B, Piacentino A. Assessing Wolf motor function test as outcome measure for research in patients after stroke. Stroke. (2001) 32:1635-9. doi: 10.1161/01.STR.32.7.1635

35. Bohannon RW, Smith MB. Interrater reliability of a modified Ashworth scale of muscle spasticity. Phys Ther. (1987) 67:206-7. doi: 10.1093/ptj/67.2.206

36. Hollands KL, Pelton TA, Tyson SF, Hollands MA, van Vliet PM. Interventions for coordination of walking following stroke: systematic review. Gait Posture. (2012) 35:349-59. doi: 10.1016/j.gaitpost.2011.10.355

37. Thirion J-P. Image matching as a diffusion process: an analogy with Maxwell's demons. Med Image Anal. (1998) 2:243-60. doi: 10.1016/S1361-8415(98)80022-4

38. Kalinosky BT. Structural-Functional Brain Connectivity Underlying Integrative Sensorimotor Function After Stroke (dissertation thesis). Milwaukee, WI: Marquette University (2016).

39. Marchini JL, Ripley BD. A new statistical approach to detecting significant activation in functional MRI. Neuroimage. (2000) 12:366-80. doi: 10.1006/nimg.2000.0628

40. Jenkinson M, Bannister P, Brady M, Smith S. Improved optimization for the robust and accurate linear registration and motion correction of brain images. Neuroimage. (2002) 17:825-41. doi: 10.1006/nimg.2002.1132

41. Smith SM. Fast robust automated brain extraction. Hum Brain Mapp. (2002) 17:143-55. doi: 10.1002/hbm.10062

42. Jenkinson $M$, Smith S. A global optimisation method for robust affine registration of brain images. Med Image Anal. (2001) 5:143-56. doi: 10.1016/S1361-8415(01)00036-6

43. Beckmann CF, Mackay CE, Filippini N, Smith SM. Group comparison of resting-state FMRI data using multi-subject ICA and dual regression. Neuroimage. (2009) 47:S148. doi: 10.1016/S1053-8119(09)71511-3

44. DeYoe EA, Raut RV. Visual mapping using blood oxygen level dependent functional magnetic resonance imaging. Neuroimaging Clin N Am. (2014) 24:573-84. doi: 10.1016/j.nic.2014.08.001

45. Engel SA, Glover GH, Wandell BA. Retinotopic organization in human visual cortex and the spatial precision of functional MRI. Cereb Cortex. (1997) 7:181-92. doi: 10.1093/cercor/7.2.181

46. Wandell BA, Winawer J. Imaging retinotopic maps in the human brain. Vision Res. (2011) 51:718-37. doi: 10.1016/j.visres.2010.08.004

47. Cavaliere C, Longarzo M, Orsini M, Aiello M, Grossi D. Fronto-temporal circuits in musical hallucinations: a PET-MR case study. Front Hum Neurosci. (2018) 12:385. doi: 10.3389/fnhum.2018.00385

48. Green B, Jääskeläinen IP, Sams M, Rauschecker JP. Distinct brain areas process novel and repeating tone sequences. Brain Lang. (2018) 187:104-14. 
49. Tsai C-G, Li C-W. Increased activation in the left ventrolateral prefrontal cortex and temporal pole during tonality change in music. Neurosci Lett. (2019) 696:162-7. doi: 10.1016/j.neulet.2018.12.019

50. Olman CA, Pickett KA, Schallmo M-P, Kimberley TJ. Selective BOLD responses to individual finger movement measured with fMRI at 3T. Hum Brain Mapp. (2012) 33:1594-606. doi: 10.1002/ hbm. 21310

51. Yoo S-S, Wei X, Dickey CC, Guttmann CRG, Panych LP. Long-term reproducibility analysis of fMRI using hand motor task. Int $J$ Neurosci. (2005) 115:55-77. doi: 10.1080/00207450490512650

52. Hanna-Pladdy B, Choi H, Herman B, Haffey S. Audiovisual lexical retrieval deficits following left hemisphere stroke. Brain Sci. (2018) 8:206. doi: 10.3390/brainsci8120206

53. Weiller C, Ramsay SC, Wise RJ, Friston KJ, Frackowiak RS. Individual patterns of functional reorganization in the human cerebral cortex after capsular infarction. Ann Neurol. (1993) 33:181-9. doi: 10.1002/ana.410330208

54. Cramer SC, Finklestein SP, Schaechter JD, Bush G, Rosen BR. Activation of distinct motor cortex regions during ipsilateral and contralateral finger movements. J Neurophysiol. (1999) 81:383-7. doi: 10.1152/jn.1999.81.1.383

55. Nguyen VT, Breakspear M, Cunnington R. Reciprocal interactions of the SMA and cingulate cortex sustain premovement activity for voluntary actions. $J$ Neurosci. (2014) 34:16397-407. doi: 10.1523/JNEUROSCI.2571-14.2014

56. Lui LL, Rosa MGP. Structure and function of the middle temporal visual area (MT) in the marmoset: comparisons with the macaque monkey. Neurosci Res. (2015) 93:62-71. doi: 10.1016/j.neures.2014.09.012

57. Cha Y-J, Kim J-D, Choi Y-R, Kim N-H, Son S-M. Effects of gait training with auditory feedback on walking and balancing ability in adults after hemiplegic stroke. Int J Rehabil Res. (2018) 41:239-43. doi: 10.1097/MRR.0000000000000295

58. Bonan IV, Colle FM, Guichard JP, Vicaut E, Eisenfisz M, Tran Ba Huy $\mathrm{P}$, et al. Reliance on visual information after stroke. Part I: balance on dynamic posturography. Arch Phys Med Rehabil. (2004) 85:268-73. doi: 10.1016/j.apmr.2003.06.017

59. Archer DB, Kang N, Misra G, Marble S, Patten C, Coombes SA. Visual feedback alters force control and functional activity in the visuomotor network after stroke. NeuroImage Clin. (2018) 17:505-17. doi: 10.1016/j.nicl.2017.11.012

60. Nobusako S, Ishibashi R, Takamura Y, Oda E, Tanigashira Y, Kouno M, et al. Distortion of visuo-motor temporal integration in apraxia: evidence from delayed visual feedback detection tasks and voxel-based lesion-symptom mapping. Front Neurol. (2018) 9:709. doi: 10.3389/fneur.2018.00709
61. Proville RD, Spolidoro M, Guyon N, Dugu,é GP, Selimi F, Isope P, et al. Cerebellum involvement in cortical sensorimotor circuits for the control of voluntary movements. Nat Neurosci. (2014) 17:1233-9. doi: 10.1038/ nn.3773

62. Baarbé J, Yielder P, Daligadu J, Behbahani H, Haavik H, Murphy B. A novel protocol to investigate motor training-induced plasticity and sensorimotor integration in the cerebellum and motor cortex. J Neurophysiol. (2014) 111:715-21. doi: 10.1152/jn.00661.2013

63. Kalinosky BT, Barillas RB, Schmit BD. Structurofunctional resting-state networks correlate with motor function in chronic stroke. Neuroimage. (2017) 16:610-23. doi: 10.1016/j.nicl.2017.07.002

64. Schulz R, Koch P, Zimerman M, Wessel M, Bönstrup M, Thomalla G, et al. Parietofrontal motor pathways and their association with motor function after stroke. Brain. (2015) 138:1949-60. doi: 10.1093/brain/awv100

65. Van Dijk KRA, Sabuncu MR, Buckner RL. The influence of head motion on intrinsic functional connectivity MRI. Neuroimage. (2012) 59:431-8. doi: 10.1016/j.neuroimage.2011.07.044

66. Dukart J, Bertolino A. When structure affects function - the need for partial volume effect correction in functional and resting state magnetic resonance imaging studies. PLoS ONE. (2014) 9:e114227. doi: 10.1371/journal.pone.0114227

67. Nielsen JA, Zielinski BA, Ferguson MA, Lainhart JE, Anderson JS. An evaluation of the left-brain vs. right-brain hypothesis with resting state functional connectivity magnetic resonance imaging. PLoS ONE. (2013) 8:71275. doi: 10.1371/journal.pone.0071275

68. Power JD, Barnes KA, Snyder AZ, Schlaggar BL, Petersen SE. Spurious but systematic correlations in functional connectivity MRI networks arise from subject motion. Neuroimage. (2012) 59:2142-54. doi: 10.1016/j.neuroimage.2011.10.018

Conflict of Interest Statement: The authors declare that the research was conducted in the absence of any commercial or financial relationships that could be construed as a potential conflict of interest.

Copyright (c) 2019 Kalinosky, Vinehout, Sotelo, Hyngstrom and Schmit. This is an open-access article distributed under the terms of the Creative Commons Attribution License (CC BY). The use, distribution or reproduction in other forums is permitted, provided the original author(s) and the copyright owner(s) are credited and that the original publication in this journal is cited, in accordance with accepted academic practice. No use, distribution or reproduction is permitted which does not comply with these terms. 


\section{OPEN ACCESS}

Edited by:

Roberto Esposito, Azienda Ospedaliera Ospedali Riuniti

Marche Nord, Italy

Reviewed by:

Stephen Cunnane,

Université de Sherbrooke, Canada

Konstantinos Chiotis,

Karolinska Institutet (KI), Sweden

*Correspondence:

Daniela Perani

perani.daniela@hsr.it

Specialty section:

This article was submitted to

Brain Imaging Methods,

a section of the journal

Frontiers in Neuroscience

Received: 28 February 2019

Accepted: 29 May 2019

Published: 14 June 2019

Citation:

Sala A and Perani D (2019) Brain

Molecular Connectivity

in Neurodegenerative Diseases:

Recent Advances and New

Perspectives Using Positron Emission

Tomography.

Front. Neurosci. 13:617.

doi: 10.3389/fnins.2019.00617

\section{Brain Molecular Connectivity in Neurodegenerative Diseases: Recent Advances and New Perspectives Using Positron Emission Tomography}

\author{
Arianna Sala ${ }^{1,2}$ and Daniela Perani ${ }^{2,3,4 *}$ \\ ${ }^{1}$ Faculty of Medicine and Surgery, Vita-Salute San Raffaele University, Milan, Italy, ${ }^{2}$ Division of Neuroscience, Faculty of \\ Psychology, San Raffaele Scientific Institute (IRCCS), Milan, Italy, ${ }^{3}$ Faculty of Psychology, Vita-Salute San Raffaele University, \\ Milan, Italy, ${ }^{4}$ Nuclear Medicine Unit, Faculty of Psychology, San Raffaele Hospital (IRCCS), Milan, Italy
}

Positron emission tomography (PET) represents a unique molecular tool to get in vivo access to a wide spectrum of biological and neuropathological processes, of crucial relevance for neurodegenerative conditions. Although most PET findings are based on massive univariate approaches, in the last decade the increasing interest in multivariate methods has paved the way to the assessment of unexplored cerebral features, spanning from resting state brain networks to whole-brain connectome properties. Currently, the combination of molecular neuroimaging techniques with multivariate connectivity methods represents one of the most powerful, yet still emerging, approach to achieve novel insights into the pathophysiology of neurodegenerative diseases. In this review, we will summarize the available evidence in the field of PET molecular connectivity, with the aim to provide an overview of how these studies may increase the understanding of the pathogenesis of neurodegenerative diseases, over and above "traditional" structural/functional connectivity studies. Considering the available evidence, a major focus will be represented by molecular connectivity studies using [18F]FDG-PET, today applied in the major neuropathological spectra, from amyloidopathies and tauopathies to synucleinopathies and beyond. Pioneering studies using PET tracers targeting brain neuropathology and neurotransmission systems for connectivity studies will be discussed, their strengths and limitations highlighted with reference to both applied methodology and results interpretation. The most common methods for molecular connectivity assessment will be reviewed, with particular emphasis on the available strategies to investigate molecular connectivity at the singlesubject level, of potential relevance for not only research but also diagnostic purposes. Finally, we will highlight possible future perspectives in the field, with reference in particular to newly available PET tracers, which will expand the application of molecular connectivity to new, exciting, unforeseen possibilities.

Keywords: amyloid PET, brain networks, connectivity, FDG-PET, multivariate analysis, neurodegenerative diseases, neurotransmission, tau PET 


\section{INTRODUCTION}

During the last decades, positron emission tomography (PET) has established itself as a relevant tool, in providing in vivo biomarkers for neurodegenerative diseases associated with cognitive decline and dementia, and playing a leading role in the diagnostic work-up of these conditions (Albert et al., 2011; Gorno-Tempini et al., 2011; McKhann et al., 2011; Rascovsky et al., 2011; Sperling et al., 2011; Armstrong et al., 2013; Dubois et al., 2014; McKeith et al., 2017). PET represents a unique tool to in vivo measure different molecular processes that are key to the pathophysiology of neurodegenerative conditions (cf. Iaccarino et al., 2017b). Together with well-established tracers, such as $[18 \mathrm{~F}]$ FDG, measuring cellular glucose metabolism, new tracers have and are being developed, providing access to a widespread set of biological and pathological processes, from neurotransmission to amyloid and tau pathology.

Recently, the field of neurodegenerative diseases has witnessed a paradigmatic shift, with the research focus shifting from evaluating the effect of underlying pathology on local neuronal function to assessing the long-distance effects of brain pathology on interconnected neural systems (Fornito and Bullmore, 2015). Pathophysiological models of neurodegeneration now take into account brain inter-regional anatomical and functional networks, considered as relevant targets of pathology, on the one hand (Palop et al., 2006), and as key players in pathology spreading, on the other hand (Seeley, 2017).

The knowledge on functional and structural brain networks and connectivity is increased rapidly, with a plethora of studies focusing on magnetic resonance imaging (MRI), as a widely available and cost-effective in vivo tool (see for review Fornito and Bullmore, 2015; Fornito et al., 2015).

Information on molecular brain networks and connectivity, as assessed by PET, is still scarce, with the few studies mostly focusing on [18F]FDG-PET metabolic connectivity. Here we review the most recent advances in this emerging field. Following a brief introduction on the available PET tracers and on the theoretical and methodological framework of brain connectivity, we will review available molecular connectivity studies using PET, particularly in combination with [18F]FDG tracer, as a functional measure of brain metabolism. Pioneering studies assessing molecular connectivity with tracers for neurotransmission and brain pathology will also be discussed. Finally, methodological advances and future directions in the field will be reviewed.

\section{PET: RELEVANT TRACERS FOR NEURODEGENERATIVE DISEASES}

Positron emission tomography studies of neurodegenerative diseases have greatly contributed to the research in clinical neuroscience (Jagust, 2018), by providing access to a series of molecular measures impossible to obtain in vivo with other neuroimaging techniques (Iaccarino et al., 2017b). PET is increasingly showing its potential in supporting clinical diagnosis of neurodegenerative conditions, also in the early, if not preclinical, disease phases, by allowing the detection of subtle pathological and functional neural changes even before clinical symptoms become manifest (Albert et al., 2011; Sperling et al., 2011).

Traditionally, the focus of brain PET studies has been on brain metabolism, as accurately measured by [18F]FDGPET. It is well-established that [18F]FDG-PET signal, reflecting both oxidative metabolism in neurons and aerobic glycolysis in astrocytes, is strictly coupled to synaptic function (Stoessl, 2017) and dysfunction. Since synaptic dysfunction can arise from several neuropathological events, among which altered intracellular signaling cascades and mitochondria bioenergetics, impaired neurotransmitter release, accumulation of neurotoxic protein species, and long-distance disconnections (Perani, 2014), [18F]FDG-PET can be considered as a "funnel" biomarker, able to capture all the different pathological events that produce a perturbation in glucose metabolism. Decades of research have shown that specific patterns of hypometabolism can be consistently detected in the major neurodegenerative conditions, from Alzheimer's disease to dementia with Lewy bodies to the different syndromes of the frontotemporal dementia spectrum (cf. Perani et al., 2014; Cerami et al., 2015, 2016; Caminiti et al., 2019).

Positron emission tomography can also be used to measure receptor density (both at pre- and post-synaptic level) and transporter binding in neurotransmission systems. PET tracers have been developed for measuring the integrity of several brain neurotransmission systems, from the aminenergic to the $\mu$-opioid systems. Although the major application of tracers for neurotransmission is in the field of psychiatric disorders, some of these tracers have been used to measure neurochemical alterations in neurodegenerative diseases. Among the most used tracers, [11C]MP4A - and analogous tracers for cholinergic presynaptic function - have shown reduced acetylcholinesterase activity in the cortex, hippocampus, and amygdala in Alzheimer's disease (Herholz et al., 2004; Marcone et al., 2012), and even more severe reductions in dementia with Lewy bodies and Parkinson's disease with dementia (Klein et al., 2010). In parallel, PET studies targeting the dopaminergic system have allowed to consistently show, in vivo, reduced dopaminergic transporter activity - a transmembrane protein regulating extracellular levels of dopamine - in Parkinson's disease and atypical Parkinsonian conditions (Varrone and Halldin, 2010; Caminiti et al., 2017b). Still, the use of these tracers, usually carbon-labeled and thus requiring the presence of a cyclotron, on-site, is limited to research studies, with more restricted applications in daily clinical practice.

More recently, efforts in tracer development have focused on targeting brain aggregates of pathological proteins, with successful validation of tracers for amyloid and (partially) tau pathology, representing the key pathological aggregates of Alzheimer's disease (Jack et al., 2018). The binding properties of currently available amyloid tracers have been well-characterized, with tracers binding selectively and with high affinity to the $\beta$-sheet structure of fibrillary amyloid plaques, with low affinity to diffuse plaques and showing no affinity for other amyloid isoforms, such as protofibrils or oligomers (Fodero-Tavoletti et al., 2012; Ni et al., 2013; Sabri et al., 2015). The availability of these in vivo 
markers for amyloid pathology has greatly improved the design of clinical trials for Alzheimer's disease, advancing strategies for patients' selection and allowing in vivo evaluation of target engagement (Vandenberghe et al., 2013). As for tau tracers, available data derive mainly from first-generation tau tracers, binding with high affinity to tau neurofibrillary tangles (FoderoTavoletti et al., 2011; Xia C.F. et al., 2013; Hashimoto et al., 2014). Preliminary studies have shown that tau PET imaging could be a valuable tool for the in vivo staging of Alzheimer's disease pathology progression (Schöll et al., 2016; Schwarz et al., 2016). Still, major areas of concern remain, regarding the type of tau pathology being targeted, i.e., 3-repeat vs. 4-repeat tau isoforms, and presence of non-specific binding in the striatum and choroid plexus, and the off-target binding to neuromelanin and monoamine oxidase (MAO-A/B) (Saint-Aubert et al., 2017; Lemoine et al., 2018).

\section{THE BRAIN AS A NETWORK}

Although most imaging findings are based on massive univariate approaches, the increasing interest in multivariate methods has paved the way to the assessment of unexplored cerebral features, spanning from resting state brain networks to whole-brain connectome properties. The advantage of using multivariate methods is that they allow to assess variations in the relationship between brain regions, over and above local regional changes, measureable with univariate methods (Clark and Stoessl, 1986). The great majority of multivariate findings derive from structural and functional MRI (fMRI) studies, respectively, providing information on brain axonal pathways, and on the correlation of blood-oxygen-level-dependent (BOLD)-signal time course across brain regions. Still, the first seminal studies assessing covariations in brain function were performed, already in the 1980s, using brain metabolic data derived from [18F]FDG-PET (Horwitz et al., 1984, 1987). From the 1990s, the popularity of multivariate approaches steeply increased, following the development of fMRI, and the observation that spontaneous activity in the primary motor cortex correlates with the activity of a widespread, spatially distributed, network of brain regions (Biswal et al., 1995). Later, based on [18F]FDG-PET evidence of coherent metabolic decreases during cognitive tasks vs. resting condition, it was hypothesized that different sets of brain regions organize into different brain networks (Raichle et al., 2001). Subsequent fMRI studies confirmed that other large-scale networks, whose regions show coherent patterns of dynamic activity, exist in the "resting brain" (Greicius et al., 2003) and that brain spontaneous activity can essentially be decomposed in a series of internally coherent large-scale functional brain networks (Beckmann et al., 2005). From the 2000s, building on this evidence, and borrowing methodological tools from the field of graph theory, a new theoretical framework was proposed, under the name of "connectomics" (Sporns et al., 2005). This framework, also known as the new "systems biology of the brain," uses graph theory indices to investigate the properties of the brain functional and structural architecture, on the assumption that a comprehensive characterization of the brain as a network is necessary to understand brain function (and dysfunction) (Sporns et al., 2005). In recent years, these methodological advances have been further extended to PET data. An excellent review of the analysis methods cited in this paragraph, and their adaptation to PET imaging data, is also available (Yakushev et al., 2017).

In the study of neurodegenerative diseases, the relevance of modeling the brain as a system of interconnected regions spans from two foundational hypotheses, one conceptualizing brain networks as passive targets of brain pathology (Palop et al., 2006) and the other as active players in the spreading of pathology (Seeley, 2017).

In the first "passive" conceptualization, brain networks are deemed relevant targets of brain pathology, dynamically altered by plasticity mechanisms that arise from pathological processes (Palop et al., 2006). It is assumed that pathological processes not only alter activity of isolated regions, but also produce distributed effects on brain networks, by prompting a reorganization of regional interconnections through induction of dedifferentiation and compensation processes (Fornito et al., 2015). A decade of evidence indeed suggests that the effects of molecular pathological alterations underlying neurodegeneration invariantly pass through large-scale brain networks, as a class-wide phenomena affecting each neurodegenerative disease (Seeley et al., 2009). The impairment of large-scale brain networks represents the endpoint of a chain reaction, where the perturbation of molecular processes at the microscale level propagates through the mesoscale to eventually affect the macroscale level. At the microscale level, the abnormal protein assemblies, that are the very basis of the neurodegenerative process alter, for example, receptor expression, neurotransmitters release, and synaptic plasticity, producing synaptic dysfunction and failure (Bellucci et al., 2015). In the long term, synaptic impairment alters neuronal functioning by affecting activitydependent signaling and gene expression, also producing distributed effects, at the mesoscale, on local neuronal circuits (Palop et al., 2006). Dysfunction in specific brain circuits eventually reverberates onto distant brain regions, resulting in disintegration of large-scale brain networks (Palop et al., 2006).

The idea of an "active" role for neuron-to-neuron interconnections in the spread of pathology stems from the observation that stereotypical patterns of pathology spreading are detectable in every neurodegenerative disease (see Brettschneider et al., 2015). Sequential stages of pathology spreading have been identified from post-mortem data, suggesting that propagation of pathology follows highly specific topographies (Braak and Braak, 1991; Braak et al., 2003, 2006; Brettschneider et al., 2013). Specifically, it was shown that tau spreads from the locus coeruleus to the transentorhinal cortex to cortical areas (Braak and Braak, 1991; Braak et al., 2006); amyloid plaques, from the neocortex to subcortical and brainstem regions (Braak and Braak, 1991); Lewy bodies (composed of immunoreactive $\alpha$-synuclein), from the olfactory bulbar/dorsal motor nucleus of the vagus nerve through the basal forebrain to the neocortex (Braak et al., 2003); TAR DNA-binding protein 43 (TDP-43) pathology, from the agranular motor cortex to brainstem motor nuclei and spinal cord, eventually reaching the neocortex in later 
disease stages (Brettschneider et al., 2013). This post-mortem evidence is complemented by recent in vivo and in vitro studies, demonstrating that pathological proteins, similarly to prions, spread trans-synaptically along neuronal interconnections (Dujardin et al., 2014; Song et al., 2014; Narasimhan et al., 2017). Notably, injection of pathological proteins triggers protein spreading to spatially remote but anatomically connected brain regions, and the pattern of spreading depends only on the site of injection and on the neural connectome at that specific site of injection, and not on the type of protein strain (Narasimhan et al., 2017). Brain networks are thus active players in pathology spreading, setting the topographical constraints according to which pathology can propagate from its initial site of aggregation (Zhou et al., 2013). Available in vivo studies support this view, as spreading of both pathology and neurodegeneration map onto functional and structural brain networks (Seeley et al., 2009; Schmidt et al., 2016; Franzmeier et al., 2019). In this framework, brain connectomics is a powerful tool to investigate and predict the pattern of long-distance pathology spreading, as pathology spreading is strictly dependent on the topology of the underlying brain connectome (Fornito et al., 2015).

Although both the "active" and "passive" conceptualizations of brain networks are backed up by solid evidence, it stands to reason that their relevance might change along the time course of the disease. We can hypothesize that, at the very beginning of the neurodegenerative processes, the brain connectome would indeed act as an hard-wired "roadmap," determining the pattern of pathology spreading (Zhou et al., 2013). Early on, pathological changes would affect connectome functional and structural properties, disrupting the "healthy" neuronal pathways and brain networks (Prescott et al., 2014). At this stage, pathology-related alterations of the brain connectome would progressively superimpose on the "original" connectome, dynamically interacting with the pre-morbid brain architecture, to determine subsequent spreading of the disease.

\section{PET MOLECULAR CONNECTIVITY}

Molecular evidence on brain networks and connectivity pathways, obtained from PET imaging data, is now becoming increasingly available. The first PET connectivity studies, tracing back to the 1980s, took into account [18F]FDGPET data to investigate metabolic molecular connectivity, defined as the association between inter-regional metabolic demands on the assumption that regions with similar metabolic demands are functionally associated (Horwitz et al., 1984). These pioneering studies remained "isolated experiments," and were not replicated until the last decade, when the number of molecular metabolic connectivity studies steeply increased, in a "renaissance" (Yakushev et al., 2017) mainly driven by the renewed interest in the newborn field of connectomics. Together with [18F]FDG-PET metabolic connectivity studies, the application of connectivity approaches has now been extended to other PET targets, including neurotransmission systems. So far, molecular connectivity approaches have demonstrated novel network-level alterations in a wide range of neurodegenerative disorders. Crucially, the combination of connectivity approaches with PET molecular data provides extremely specific results on the underlying target, thus overcoming the "lack of specificity" typical of functional connectivity, as estimated from fMRI (Hahn et al., 2018). It is expected that molecular connectivity will greatly broaden the field of connectomics, providing an integrated, network-oriented, and biologically rooted, perspective, leading to a deeper understanding of the complexity of the brain architecture (Veronese et al., 2019).

\section{Basic Principles and Methods in PET Molecular Connectivity}

Different analytical approaches have been implemented for molecular connectivity modeling (Yakushev et al., 2017). Three main analytical approaches are commonly used to estimate PET molecular connectivity:

(i) Seed correlation or interregional correlation analysis (IRCA): this voxel-wise approach relies on the selection of a region of interest (ROI), or seed, from which the average value of tracer uptake is extracted (Figure 1A). The correlation between average uptake in the seed and uptake in each voxel in the rest of the brain is then tested (Lee et al., 2008), to obtain an estimation of the connectivity profile, or connectivity map, of the seed of interest. This method yields a certain flexibility, as the researcher can select the ROI, or seed, in either a data-driven fashion (e.g., Morbelli et al., 2013; Iaccarino et al., 2018) or based on an a priori hypothesis (e.g., Ballarini et al., 2016; Malpetti et al., 2018). In the former case, the seed is derived directly from data analysis, usually by inputting, as seed, the cluster derived from a first round of univariate analyses (e.g., Morbelli et al., 2013; Iaccarino et al., 2018). In the latter case, this method has been commonly used to estimate large-scale brain networks in [18F]FDGPET molecular connectivity studies (e.g., Ballarini et al., 2016; Malpetti et al., 2018). Resulting networks have similar topographies to the ones obtained with resting state-fMRI (Passow et al., 2015).

(ii) Independent component analysis (ICA): this voxel-wise approach is based on the multivariate decomposition of PET signal across the brain (Di et al., 2012), under the assumption that PET signal can be described as a mixture of statistically independent components (Pagani et al., 2017). This approach allows identification of highly coherent brain networks in a data-driven fashion, without requiring any a priori selection of specific ROI (Figure 1B). Still, researcher's intervention is needed to set the number of components to be extracted, and to select components with pathophysiological/anatomo-functional meaning, while discarding unimportant components of pure statistical noise. Although ICA represents the election method for connectivity analysis with fMRI data, contrasting results have emerged on its application to 


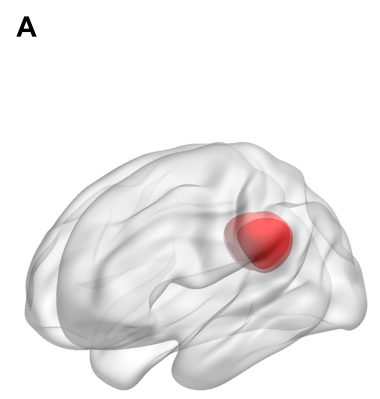

SEED SELECTION

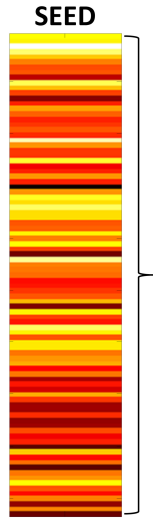

EXTRACTION OF AVERAGE TRACER UPTAKE
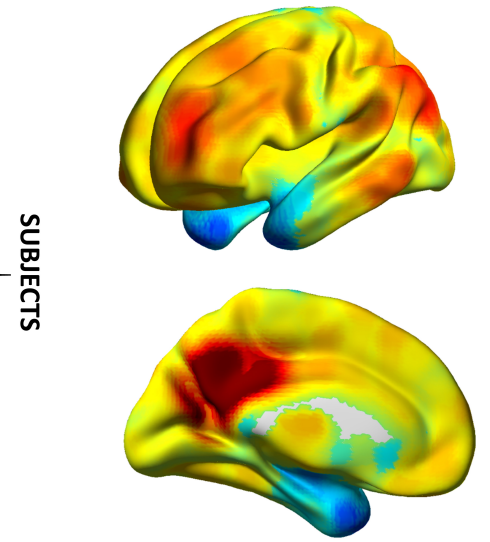

ESTIMATION OF VOXELWISE CORRELATIONS
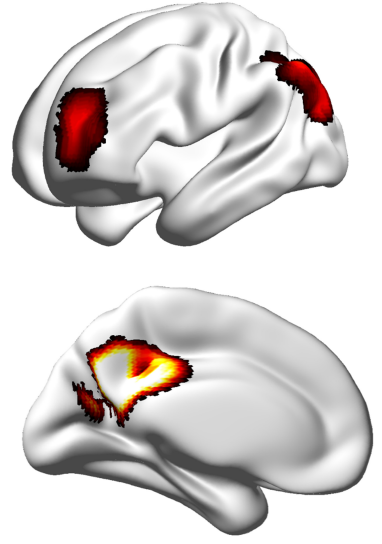

THRESHOLDING OF THE CONNECTIVITY MAP

B

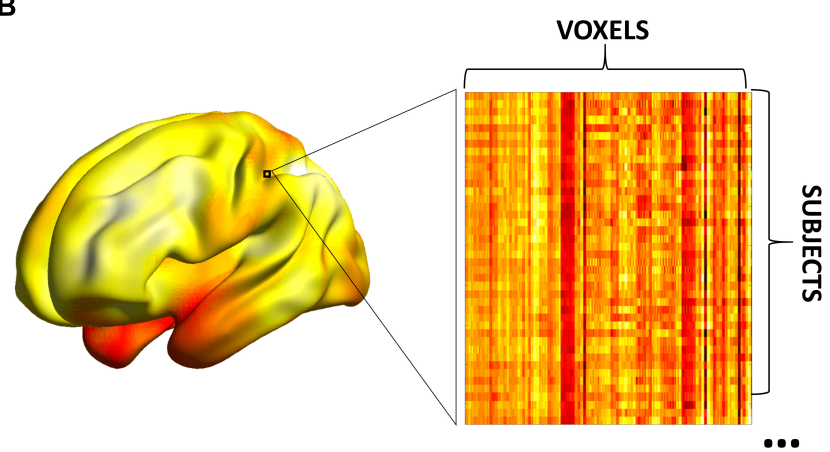

PET IMAGE

C

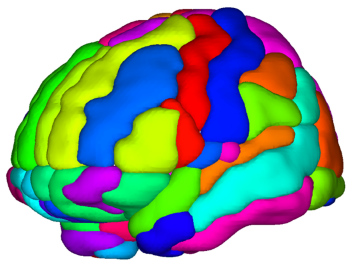

ROIS SELECTION
EXTRACTION OF VOXEL-WISE TRACER UPTAKE

ROIs

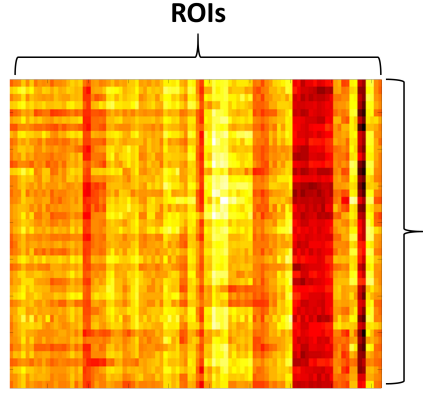

EXTRACTION OF AVERAGE TRACER UPTAKE

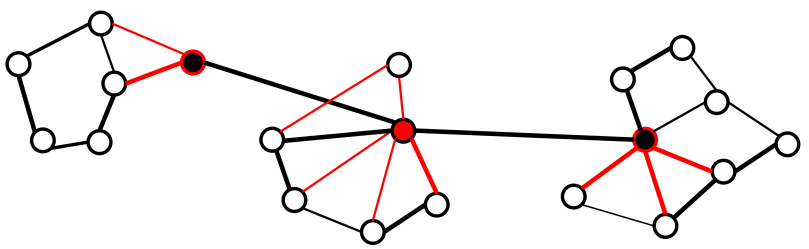

COMPUTATION OF GRAPH THEORY INDICES
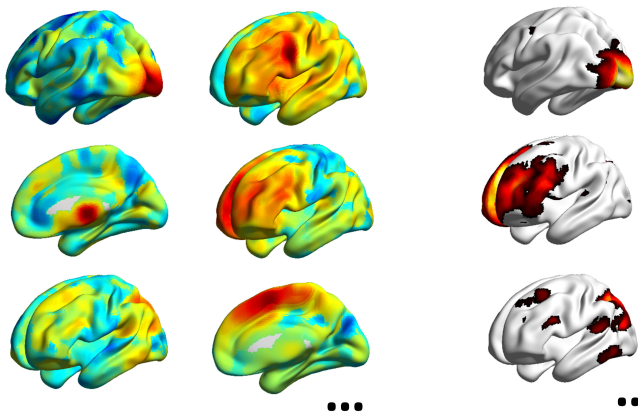

SIGNAL DECOMPOSITION INTO DIFFERENT INDEPENDENT COMPONENTS

SELECTION AND THRESHOLDING OF INDEPENDENT COMPONENTS

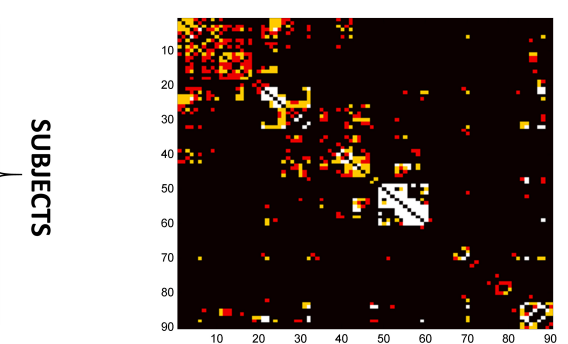

ESTIMATION AND THRESHOLDING OF THE CONNECTIVITY MATRIX
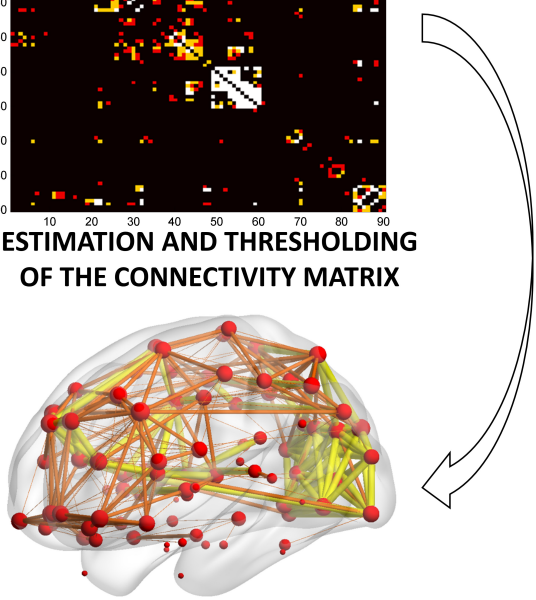

NETWORK TRANSLATION 
FIGURE 1 | Schematic representation of the most common analytical approaches for molecular connectivity modeling. (A) Seed correlation analysis: molecular connectivity estimation is performed from a specific seed of interest, selected by the researcher. Here, the seed corresponds to a cluster encompassing the precuneus/posterior cingulate cortex; the resulting connectivity map corresponds to the default mode network. (B) Independent component analysis: whole-brain tracer uptake signal is decomposed into multiple statistically independent components, at voxel-wise level. The number of extracted components is set by the researcher. Here, $N=20$ components are extracted (only six are shown for visualization purposes). By comparing the topography of the identified components with known anatomo-functional networks, the researcher can then select the components of interest. Here, three selected components are shown for visualization purposes, corresponding to the primary visual network, executive network, and default mode network. (C) Partial correlation analysis: molecular connectivity estimation is performed from ROls selected by the researcher. Here, the ROls comprehensively cover the whole brain. If the number of subject is smaller than the number of ROls, sparse inverse covariance estimation (SICE) is used to estimate a connectivity matrix. This is then translated into a network, where nodes are represented by ROls and edges by molecular connections. Here, a weighted connectivity matrix and weighted networks are shown, with edges computed at three different network densities. A wide array of graph theory metrics can then be estimated. BrainNet Viewer (http://www.nitrc.org/projects/bnv/) was used for rendering (Xia M. et al., 2013). ROls, regions of interest.

[18F]FDG-PET data for large-scale network estimation (Di et al., 2012; Savio et al., 2017).

(iii) Partial correlation analysis: this ROI-based approach allows to compute a comprehensive "connectivity matrix" following selection of a series of target regions, either based on a specific a priori hypothesis, i.e., ROIs belonging to a specific anatomo-functional system of interest or in a data-drive fashion, i.e., ROIs covering the whole brain (Figure 1C). This approach allows to estimate the degree of linear association between each couple of selected ROIs, after factoring out the contribution of all remaining ROIs to the target association. As such, partial correlation analysis overcomes the limitations of simple correlation analysis, that, by capturing pairwise information only, cannot characterize the effects of multiple brain regions interacting together (Huang et al., 2010). This method has subsequently been refined into a more advanced approach, known as sparse inverse covariance estimation (SICE) (Huang et al., 2010). The advantage of SICE is that it allows to estimate molecular connectivity even when the number of subjects included in the analysis is smaller than the number of ROIs, a relatively frequent scenario in PET studies (Huang et al., 2010). This is crucial particularly in connectivity studies for connectome assessment, where an elevated number of ROIs, covering the whole-brain, are selected. Once the whole-brain connectivity matrix is estimated through SICE, graph theory indices can be eventually computed, molecular hubs and modules identified, and changes in nodal and global network properties assessed [see Bullmore and Sporns (2009) for a review on graph theory indices].

Although these approaches are methodologically quite different, they all rely on the assessment of regional co-variation in PET tracer uptake across subjects to estimate molecular connectivity (Figure 1). This is quite different from fMRI studies, where the availability of a time series for each subject allows to estimate functional connectivity based on regional co-variation of BOLD signal, through time, within the same subject. Since a times series is not available in PET studies, i.e., parametric PET images are inherently "static," molecular connectivity studies always rely on the identification of patterns of inter-subject co-variation of regional tracer uptake. This will be further detailed in the last paragraph of the present review.

Independent of the analytical method used to estimate molecular connectivity, and the resulting outcome, i.e., seedbased maps of connectivity, mutually independent networks or connectivity matrices, results of molecular connectivity studies are usually translated into a common lexicon, with reference to decreased or increased connectivity. This is usually achieved through statistical comparison of connectivity metrics (e.g., network topography, connectivity stregth, and number of significant connections) between the target population and a reference group of healthy controls. At a basic level, interpretation of decreased and increased connectivity is quite straightforward, i.e., decreased (or increased) connectivity of a region to another indicates that the former region has a tracer uptake that is less (or more) associated with tracer uptake in the latter. At a more informative level, however, interpretation of connectivity changes becomes non-trivial and varies greatly depending on the type of PET tracer being used. In [18F]FDG-PET studies, similarly to fMRI studies, connectivity changes are usually interpreted in terms of function: connectivity decreases indicate functional disconnection between regions, while connectivity increases indicate increased functional coupling between regions. Interpreting the significance of connectivity increases is particularly non-trivial (Pievani et al., 2014): when increased connectivity affects metabolically preserved brain regions, it might be indicative of a "beneficial" compensatory process, with recruitment of brain regions that are still functional; when increased connectivity affects metabolically impaired brain regions, it might be indicative of a common underlying pathological process, conjunctly affecting metabolism of multiple brain regions in a similar fashion. For tracers targeting pathology, molecular connectivity increases can be expected with progressive pathology spreading. For tracers targeting neurotransmission, interpretation strictly depends on the specific neurotransmission system and target being studied: connectivity decreases might be indicative, for example, of selective denervation from the neurotransmitter nuclei projecting to the target regions being evaluated. Of note, changes in molecular connectivity can, but do not necessarily, reflect changes in anatomical connections between regions. Although anatomical disconnection between regions would likely result in changes in molecular connectivity, changes in molecular connectivity can be expected also without structural 
disconnection. Accordingly, and similarly to fMRI functional connectivity (Smith et al., 2013), molecular connections reflect both direct or indirect (polysynaptic) connections between regions, i.e., regions can be functionally connected, with or without a direct anatomical connection.

\section{Molecular Connectivity Studies Targeting Brain Metabolism}

The investigation of brain metabolic connectivity has its foundation in the principle that regions whose metabolism is associated are functionally interconnected (Horwitz et al., 1984). This assumption stems from a pioneering study, demonstrating that metabolic connectivity results are largely consistent with known anatomo-functional data (Horwitz et al., 1984). Among the different declinations of molecular connectivity, metabolic connectivity theoretically represents the one most closely akin to functional connectivity, as assessed by fMRI. Available evidence suggests a close link between resting-state functional brain connectivity, as measured by resting-state fMRI, and glucose consumption, as measured by [18F]FDG-PET (Riedl et al., 2014; Passow et al., 2015). In a recent cross-modal study, it was shown that network properties of the brain metabolic covariance network indeed resemble that of the fMRI network, retaining the small-worldness property typical of functionally relevant organizations, as opposed to the randomness typical of structural organizations (Di et al., 2017). Direct comparison of the topography of brain networks as identified by [18F]FDGPET vs. resting-state fMRI provided, however, contrasting results. Passow et al. (2015) demonstrated remarkably overlapping patterns of functional and metabolic connectivity seeding from the posterior cingulate gyrus, clearly corresponding to the topography of the default mode network (Passow et al., 2015). Savio et al. (2017), using simultaneously acquired [18F]FDGPET and resting-state fMRI data, expanded these findings, reporting again substantial overlap within the major large-scale brain networks (Savio et al., 2017). While these results seem to point at a common underlying neural substrate for functional and metabolic connectivity (Passow et al., 2015; Savio et al., 2017), Di and colleagues (2012) reported mostly dissimilar findings, especially pertaining the default mode network (Di et al., 2012). It must be noted that, while both [18F]FDGPET and fMRI signals represent proxies of synaptic function, they record extremely different aspects of neural activity. fMRI BOLD signal measures changes in the relative levels of oxy- and deoxy-hemoglobin, and is coupled to neural activity through the hemodynamic response, a neurovascular mechanism aimed at increasing blood flow to cover the energy demands of local brain activity (Bullmore and Sporns, 2009). BOLD signal is dependent on a combination of oxidative metabolism, blood flow, and blood volume (Gauthier and Fan, 2019), and is also affected by vessels size (Liu, 2013). fMRI is thus inherently more dependent of neuro-vascular coupling, and less directly linked to synaptic function, as compared to [18F]FDG-PET (cf. Yakushev et al., 2017). [18F]FDG-PET signal measures glucose consumption and is coupled to neuronal activity through a specific biochemical pathway, where excitatory glutamate release in the synaptic cleft elicits activation of the sodium/potassium pump, stimulating glucose consumption via aerobic glycolysis (Stoessl, 2017). Notably, energy consumption represents a proxy for directional signaling, as increases in local metabolism are indicative of increased afferent neuronal activity (cf. Riedl et al., 2016). Interestingly, this observation can be exploited to estimate effective brain connectivity with a completely data-driven approach, by combining undirected signaling pathways estimated from fMRI, with the information on directionality derived from [18F]FDG-PET data (Riedl et al., 2016).

In the following sections, we review the available metabolic connectivity findings in the main neurodegenerative disease spectra.

Amyloidopathies/Tauopathies - Among metabolic connectivity studies, Alzheimer's disease represents definitely the nosographic entity that has received the greatest attention. The first study of metabolic connectivity in neurodegenerative conditions, in the 1980s, was indeed performed on a small sample of patients with Alzheimer's disease dementia (Horwitz et al., 1987). Subsequent studies have consistently shown reduced metabolic connectivity seeding from the posterior cingulate gyrus/precuneus (Morbelli et al., 2012; Ballarini et al., 2016; Herholz et al., 2017), crucially representing the main hub of the default mode network. This finding was replicated in both late- and early onset Alzheimer's disease, with involvement of additional brain networks in the latter group (Ballarini et al., 2016). It remains to be determined whether the pattern of metabolic connectivity alterations differs among atypical presentations of Alzheimer's disease. To this regard, preliminary data suggest that, in atypical variants, specific alterations in metabolic connectivity might co-exist with the typical default mode network dysfunction (Herholz et al., 2017). Notably, crosssectional studies suggest that metabolic connectivity deficits become increasingly more pronounced as disease progresses, with a gradual disintegration of the default mode network from prodromal to overt disease phases (Pagani et al., 2017). Interestingly, the majority of these studies reported reduced metabolic connectivity in both hypo-metabolic and metabolically preserved brain regions, at consistence with the view that connectivity alterations can exceed local metabolic deficits (Clark and Stoessl, 1986). This is also in accordance with recent pathophysiological models of neurodegenerative diseases, suggesting that connectivity alterations can spread at longdistance, affecting brain regions that would be otherwise spared by pathology (Warren et al., 2013). Together with decreases metabolic connectivity, increased network connectivity has also been reported in Alzheimer's disease, in association to reserve proxies such as education (Morbelli et al., 2013; Malpetti et al., 2017) and bilingualism (Perani et al., 2017). It has been suggested that these results might be indicative of a common compensatory mechanism, according to which lifelong protective factors promote stronger integration of large-scale brain networks, in spite of more severe hypometabolism (Yakushev et al., 2017). Of note, in a recent report in Alzheimer's disease, we found that -in females only - high body mass index levels determine decreased connectivity in the very same brain networks involved in the above-mentioned compensatory mechanisms 

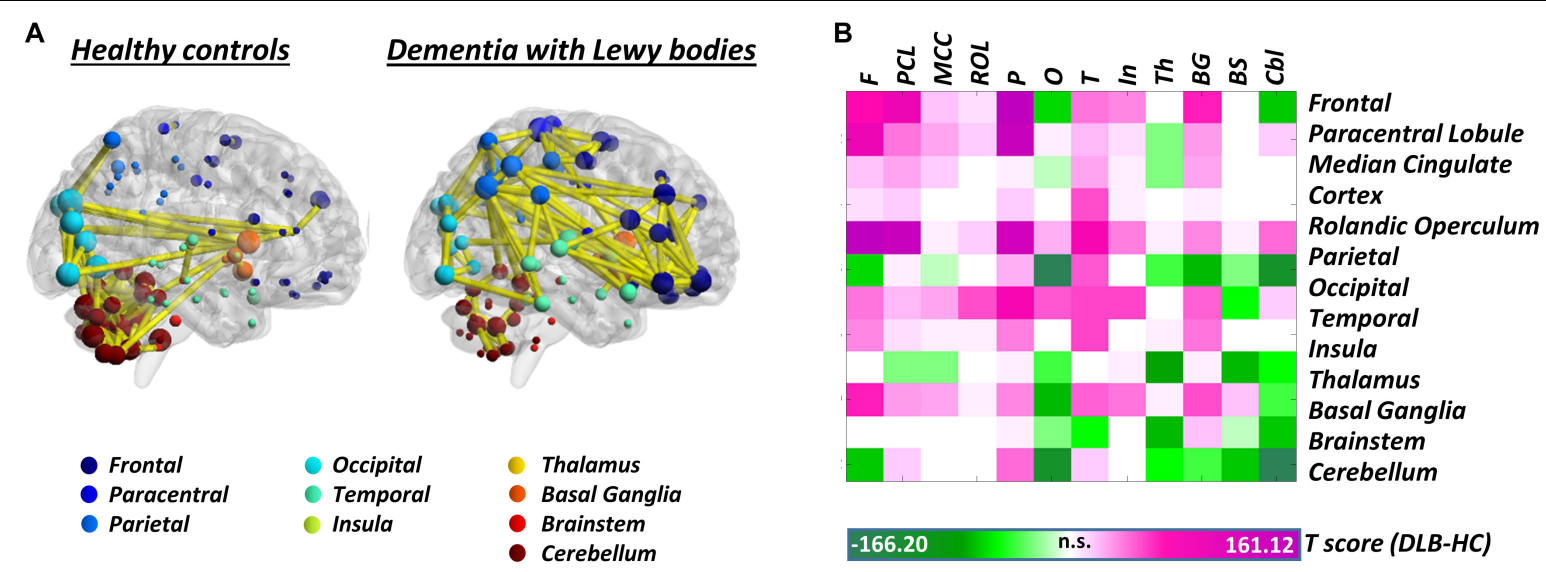

FIGURE 2 | Whole-brain metabolic connectome in Dementia with Lewy bodies. (A) Brain connectivity graphs in healthy controls and patients with dementia with Lewy bodies. A global connectivity reconfiguration is evident in patients with dementia with Lewy bodies, with metabolic connectivity decreases mainly affecting occipital cortex, thalamus, and cerebellum. Only the strongest connections (density $\approx 3 \%$ ) are shown (yellow edges). The size of each node depends on the node total number of connections, whereas the color indicates its anatomical localization. (B) The $T$-score matrix reports $T$-test statistics, derived from the direct comparison of the number of metabolic connections (within and between each macroarea) between patients and healthy controls, following a bootstrapping procedure. Connectivity decreases are indicated by negative T-scores (green); connectivity increases by positive $T$-scores (violet). BrainNet Viewer (http://www.nitrc.org/projects/bnv/) was used for rendering (Xia M. et al., 2013). Modified from Caminiti et al. (2017c). F, frontal; PCL, paracentral lobule; MCC, median cingulate cortex; ROL, rolandic operculum; P, parietal; O, occipital; T, temporal; In, insula; Th, thalamus; BG, basal ganglia; BS, brainstem; Cbl, cerebellum.

(Malpetti et al., 2018), suggesting a multi-factorial modulation of brain connectivity in Alzheimer's disease.

As for primary tauopathies, to the best of our knowledge, only one metabolic connectivity study is available. In this study, Titov et al. (2017) reported that pathological metabolic connections tend to cluster in the frontal and temporal lobes of subjects with the behavioral variant of frontotemporal dementia (Titov et al., 2017).

Although patterns of focal metabolic alterations have been relatively well-characterized in the other primary tauopathies, namely corticobasal degeneration and progressive supranuclear palsy (e.g., Niethammer et al., 2014; Caminiti et al., 2017a), a network-level characterization of these diseases is still lacking.

Synucleinopathies - In the spectrum of synucleinopathies, metabolic connectivity studies have focused mainly on Parkinson's disease. Spetsieris et al. (2015) reported a perturbation of metabolic connectivity in the default mode network in late Parkinson's disease, associated with subsequent development of cognitive dysfunction (Spetsieris et al., 2015). In a more recent study, we replicated these findings, and additionally showed that connectivity alterations, in Parkinson's disease, go well beyond the default mode network, with perturbations of frontal connectivity in virtually each large-scale brain network (Sala et al., 2017). In the same study, we also assessed whole-brain connectome alterations, reporting connectivity decreases, locally and at long-distance, in the frontolateral cortex, opposed to connectivity increases, of possible compensatory significance, in occipital regions. This pattern of connectivity impairment was antithetical to the one observed in dementia with Lewy bodies, characterized by local and long-distance occipital connectivity decreases, and frontal connectivity increases (Caminiti et al., 2017c; Figure 2). Still, the two synucleinopathies showed a common backbone of connectivity alterations, involving cerebellum and mesencephalic-pontine regions, notably representing very early sites of $\alpha$-synuclein aggregation into Lewy bodies, as the pathological hallmark of both diseases (Caminiti et al., 2017c; Sala et al., 2017). To the best of our knowledge, no metabolic connectivity studies are available in multiple system atrophy.

Other - Little is known on network-level molecular alterations in neurodegenerative diseases underlid by other pathological proteins, such as TDP-43 and huntingtin proteins. To this regard, the only available study using IRCA in the TDP-43 spectrum, specifically in amyotrophic lateral sclerosis, reported a positive association between metabolism in the midbrain and white matter in the corticospinal tract (Pagani et al., 2014). Still, results deriving from group-level metabolic connectivity analysis in amyotrophic lateral sclerosis should be interpreted with caution, as they might be affected by the intrinsic metabolic heterogeneity that characterizes this condition (Matías-Guiu et al., 2016; Sala et al., 2019). Further reflection on group inhomogeneity and molecular connectivity is reported in the last section of this review.

\section{Molecular Connectivity Studies Targeting Brain Neurotransmission Systems}

Positron emission tomography studies targeting brain neurotransmission systems make up a significant proportion of molecular connectivity evidence. So far, the majority of these studies have focused on assessing network-level properties of neurotransmission systems in healthy controls and their alterations in neuropsychiatric disorders, with only a few studies available in neurodegenerative conditions. These studies are based on the assumption that cell firing and neurotransmitter release from a given neurotransmitter nucleus would affect 
the expression of tracer binding sites in the nucleus' target regions (Hahn et al., 2018), and would do so in a similar way for targets innervated by the same nucleus. The first attempt to apply multivariate methods to tracers for neurotransmission was carried out by Cervenka et al. (2010), assessing patterns of correlations between striatal and extrastriatal dopaminergic D2-receptor in 16 control subjects. They found that the pattern of correlations of the radioligands [11C]raclopride and $[11 \mathrm{C}]$ FLB457 was consistent with the known biochemical architecture of the dopaminergic systems, reflecting the segregation between nigrostriatal, mesolimbic, and mesocortical pathways (Cervenka et al., 2010). Additional studies followed, extending the investigation of molecular connectivity to the serotoninergic (e.g., Hahn et al., 2014; Tuominen et al., 2014; Pillai et al., 2018) and $\mu$-opioid systems (Tuominen et al., 2014). During the last years, these studies have progressively moved from "simple" seed-based correlative approaches, to the implementation of methods borrowed from functional/structural connectomics, adopting graph theory measures to estimate neurotransmission systems' organization properties. From the theoretical standpoint, these studies have progressively situated their results in the framework of connectomics, enriching "system level" knowledge with "molecular-level" information (see Tuominen et al., 2014). Altogether, this evidence shows that PET can reliably reconstruct brain connectivity patterns within and between neurotransmitter systems, providing in vivo access to the biochemical architecture of the brain.

To the best of our knowledge, only one study has measured molecular connectivity alterations of neurotransmission systems in neurodegenerative diseases. In this study, Caminiti et al. (2017b) investigated the nigrostriatal and mesolimbic dopaminergic pathways in a series of Parkinson's disease patients, reporting a severe reduction in dopaminergic connectivity between substantia nigra and putamen, a portion of the striatum known to be early affected by nigral denervation (cf. Caminiti et al., 2017b). The mesolimbic network was also affected, with loss of connectivity between homotopic regions, but no change in connectivity between the ventrotegmental area and its subcortical targets. These results support the view of Parkinson's disease as a disconnection syndrome, with axonal damage representing an early occurrence in the degeneration of the nigrostriatal system (Caminiti et al., 2017b; Fazio et al., 2018). Other studies, based, however, on single photon emission computed tomography (SPECT) radioligands for dopaminergic transporter imaging also adopted a similar approach (Premi et al., 2016, 2017). These studies showed abnormal patterns of subcortico-cortical molecular connectivity in Parkinson's disease (Premi et al., 2016, 2017), with additional putaminal-cingulate disconnection specifically associated with the presence of impulse control disorder (Premi et al., 2016).

It must be mentioned that a few PET studies adopted an alternative strategy, mapping changes in neurotransmission pathways via estimation of molecular metabolic connectivity in selected brain regions (see Figure 3 for an example). This approach is based on the assumption that energy consumption is influenced by multiple pathological events, notably including altered neurotransmission (Perani, 2014) and builds on the evidence of a significant coupling between neurotransmission impairment and integrity of metabolic networks (Holtbernd et al., 2015). Using this approach, it was shown that metabolic connectivity alterations can be detected within the nigrostriatal dopaminergic and mesocorticolimbic system, in both Parkinson's disease (Sala et al., 2017) and dementia with Lewy bodies (Caminiti et al., 2017c), with reconfigurations more prominent in the latter. Using a similar rationale, Verger et al. (2018) reported a disruption of metabolic connectivity in regions belonging to the mesocorticolimbic system, specifically in Parkinson's disease patients presenting with impulse control disorder (Verger et al., 2018). Further application of this method extends to the cholinergic pathways, where derangement was reported running along cholinergic projections from the basal forebrain and brainstem nuclei, in dementia with Lewy bodies (Caminiti et al., 2017c; Figure 3).

\section{Molecular Connectivity Studies Targeting Brain Pathology}

The most recent application of brain molecular connectivity involves the use of PET tracers for brain amyloidosis and tau pathology. Contrarily to molecular connectivity studies targeting metabolism and neurotransmission systems, where the focus is on assessing the effects of pathology on the energetic and biochemical architecture of the brain, this branch of molecular connectivity clearly builds upon the hypothesis of brain networks as active players in the spreading of pathology (see above, paragraph: "The brain as a network"). Since pathology spreading maps onto the underlying structural brain networks, superimposing on the brain connectome, pathology spreading might show, per se, "network" properties, and thus be modeled with network-level approaches. Accordingly, recent studies have started to make reference to the "amyloid network" and "tau network” (Sepulcre et al., 2013; Hoenig et al., 2018). Quite a few molecular connectivity studies using established PET tracers for amyloidosis are available, while molecular connectivity studies with tau tracers are emerging.

As a side note, it must be underlined that although the models of connectivity-based pathology spreading hold true for tau pathology and amyloid pathology in the form of oligomers (Ahmed et al., 2014; Domert et al., 2014), some evidence suggests that spreading of extracellular fibrillary amyloid plaques, targeted by currently available PET tracers, might be led by spatial proximity and not by brain connectivity (Mezias and Raj, 2017). Still, this finding remains controversial.

Amyloidosis - In the first study targeting the "amyloid network," Sepulcre et al. (2013) demonstrated that it is possible to use molecular connectivity data to derive information on putative mechanisms of amyloid spreading (Sepulcre et al., 2013). They showed that "hubs" in the amyloid network act as seeding nodes from where amyloid accumulation spread to more peripheral regions (Sepulcre et al., 2013). The hubs that they identified, including the medial temporal lobe and orbitofrontal cortex, are partially consistent with the early regions of amyloid accumulation, as defined by post-mortem studies (Braak and Braak, 1991; Thal et al., 2002). Braak and Braak (1991) 


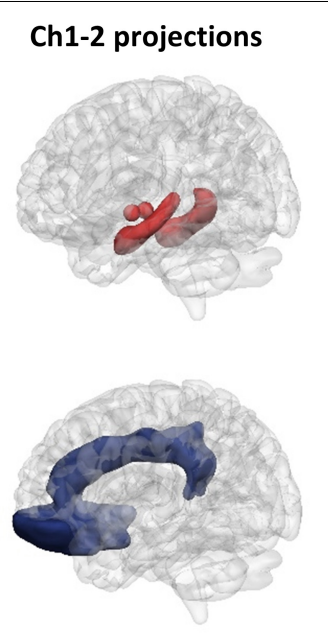

Ch4 medial projections

\section{Ch3 projections}
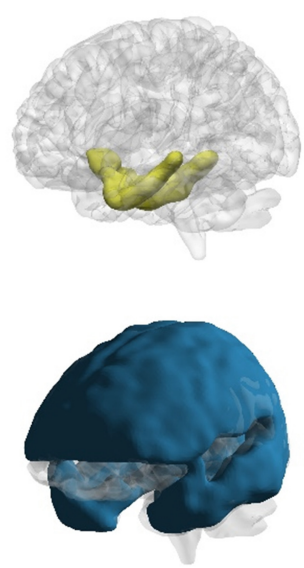

Ch4 lateral projections capsular subdivision

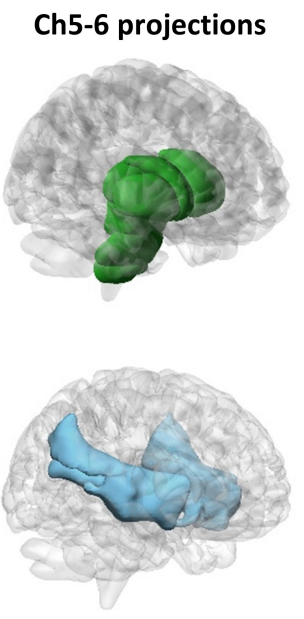

Ch4 lateral projections perisylvian subdivision

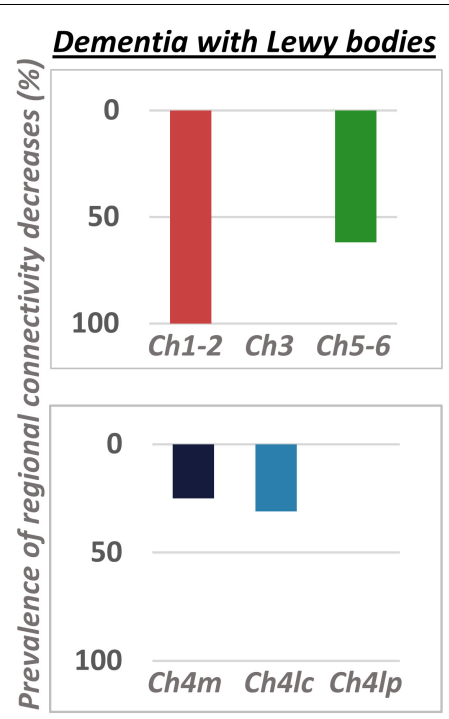

FIGURE 3 | Metabolic connectivity targeting cholinergic pathways. Left panel: selection of ROls for assessment of metabolic connectivity within the major cholinergic pathways. The first row shows regions supplied by basal forebrain (Ch1-2; Ch3) and brainstem (Ch5-6) nuclei. The second row shows the three cholinergic pathways projecting from the nucleus basalis of Meynert (Ch4). Right panel: histograms show the prevalence of regional connectivity decreases in dementia with Lewy bodies, in each cholinergic pathway. Prevalence of metabolic connectivity decreases in each pathway was computed as the number of regions, within the pathway, presenting with significantly decreased metabolic connectivity, divided by the total number of regions belonging to that pathway. Only connections within each pathway (i.e., between regions innervated by the same cholinergic nucleus) were taken into account to compute the prevalence of connectivity decreases within each pathway. Prevalence of metabolic connectivity decreases was higher in regions supplied by Ch1-Ch2 nuclei (100\% of regions presenting with significantly decreased metabolic connectivity within this pathway) and Ch5-Ch6 nuclei, with additional involvement of the medial and lateral projection (capsular subdivision) of the nucleus basalis of Meynert (Ch4). BrainNet Viewer (http://www.nitrc.org/projects/bnv/) was used for rendering (Xia M. et al., 2013). Modified from Caminiti et al. (2017c). Ch4m, Ch4 medial projections; Ch4lc, Ch4 lateral projections, capsular subdivision; Ch4lp, Ch4 lateral projections, perisylvian subdivision.

described the presence of amyloid pathology in basal portions of the isocortex (including orbitofrontal cortex), together with mild involvement of medial temporal lobe structures, already in Stage A (Braak and Braak, 1991). Thal et al. (2002) reported involvement of the neocortex first (including orbitofrontal cortex) (Phase 1), followed by medial temporal lobe structures already in Phase 2 (Thal et al., 2002). Although presence of amyloid pathology in the medial temporal lobe is described as an early (Stage A according to Braak's staging) or relatively early (Phase 2 according to Thal's phases) event, it is worth noting that medial temporal amyloid burden remains mild even in the latest stages of amyloid accumulation (Braak and Braak, 1991). Due to the mismatch between timing and severity of amyloid pathology, univariate approaches based on severity of amyloid pathology only would necessarily not be able to identify the same amyloid hubs as those reported by Sepulcre et al. (2013) using multivariate connectivity techniques. Interestingly, a recent study has shown that the localization of pathological hubs in the amyloid network might not be invariant, but differ across different variant of the Alzheimer's disease spectrum (Leyton et al., 2015). This result contrast with the well-established homogeneity of amyloid burden reported across different conditions (Iaccarino et al., 2017a). Further studies on large population of patients are necessary to better define these aspects.

Other recent studies have compared nodal and global properties of the amyloid network in healthy controls, subjects with mild cognitive impairment and patients with Alzheimer's disease dementia (Jiang et al., 2015; Son et al., 2015; Duan et al., 2017). These studies have however reported contrasting results, with respect to both the direction and the localization of the amyloid network's alterations, possibly due to differences in the proportion of amyloid positive/negative cases in the tested cohorts (Duan et al., 2017). To this regard, a recent study adopted a radically different approach, classifying subjects not on the basis of their clinical status, but on a multi-modal biomarker-based amyloid staging, allowing classification of subjects into "negative," "early," and "late" amyloid accumulators (Pereira et al., 2017). This elegant study has shown that the amyloid network is characterized by a "community" of strongly interconnected regions, notably partly overlapping with the default mode network, invariantly detectable across all amyloid stages (Pereira et al., 2017). Interestingly, as amyloid accumulation becomes more severe, this community progressively expands to include additional neocortical regions (Pereira et al., 2017), suggesting that changes in network topology reflect amyloid pathology progression and spreading.

Tau pathology - To the best of our knowledge, only one study has investigated molecular connectivity using tau tracers. In this extremely recent study, Franzmeier et al. (2019) reported that tau covariance is linearly associated with functional connectivity, independently of spatial proximity (Franzmeier et al., 2019). These results provide in vivo support to the 
view that patterns of tau spreading map onto the underlying connectome, and that tau spread preferentially across connected (but not necessarily adjacent) brain regions (Franzmeier et al., 2019). Notably, these results were confirmed not only in Alzheimer's disease, but also in normal aging and cerebrovascular cognitive impairment, suggesting a strong coupling between tau propagation and functional connectivity, independent of amyloidosis (Franzmeier et al., 2019). Still, these findings need to be interpreted with caution, as significant off-target binding has been reported for the $[18 \mathrm{~F}] \mathrm{AV}-1451$ tau tracer used in this study (cf. Lemoine et al., 2018).

a-Synuclein pathology - Currently, there is no validated tracer to in vivo measure $\alpha$-synuclein pathology. Several compounds have been investigated, but subsequently discarded as they did not show sufficient in vivo binding or acceptable selectivity for the target (Jovalekic et al., 2017; Mathis et al., 2017). To remedy the lack of information on molecular connectivity alterations within the $\alpha$-synuclein network, we used an alternative approach based on [18F]FDG-PET, following the same rationale explained above. We mapped [18F]FDG-PET metabolic connectivity in a series of a priori selected brain regions, as derived from currently available neuropathological models of $\alpha$-synuclein staging (Braak et al., 2003). Using this approach, we showed that metabolic connectivity alterations map onto the underlying pathology, with the most severe alterations involving regions early affected by $\alpha$-synuclein pathology, in both Parkinson's disease (Sala et al., 2017) and dementia with Lewy bodies (Caminiti et al., 2017c). In spite of the relative lack of specificity of the [18F]FDG-PET signal, these results show that $\alpha$-synuclein pathology leaves a unique imprint on metabolic connectivity.

\section{Limitations and Future Directions in Molecular Connectivity - Getting at the Single-Subject Level?}

Although compelling, the net majority of molecular connectivity results are based on group-level analyses, due to the inherently "static" nature of PET images - containing either tracer uptake values averaged over a certain time window or parametric values derived from the tracer's dynamics. As a consequence, quantified PET images do not possess the temporal "dynamic" component that is typical, for example, of fMRI data (Yakushev et al., 2017). This limitation makes a within-subject "fMRI-like" analysis of PET images impossible. For this reason, molecular connectivity analysis is necessarily performed at group level. It follows that the reliability of molecular connectivity findings depends on the definition of a relatively homogeneous cohort, on the one hand, and on the normalization of between-subject differences, e.g., due to variability in image acquisitions, on the other hand (cf. Veronese et al., 2019). Currently, little is known on the effect of sample heterogeneity on molecular connectivity results, and, more in general, on test-retest reliability and reproducibility of molecular connectivity findings. Encouragingly, a very recent validation study has shown good reproducibility of molecular connectivity results, obtained with an ROI-based correlative approach and graph theory, and confirmed using three different tracers, suggesting a general applicability within typical experimental settings (Veronese et al., 2019). More studies are urgently needed to confirm and extend these findings using other analytical methods and other tracers, and address the effect of other experimental variables, e.g., sample size, on molecular connectivity results.

Together with validation studies, derivation of individual metrics to quantify molecular connectivity alterations at the single-subject level represents a top priority in the field of neurodegenerative diseases. Availability of single-subject metrics would allow to test the value of molecular connectivity as a biomarker of diagnostic and prognostic interest, and to perform correlative analysis, e.g., to investigate the association between individual connectivity metrics and other imaging parameters, clinical symptoms/neuropsychological deficits or, eventually, as complementary outcome measures to evaluate the effects of new emerging treatments (e.g., Fortier et al., 2019). Although the estimation of molecular connectivity patterns necessarily requires - as a first step - a grouplevel analysis, different approaches have been developed to subsequently derive information at the single-subject level. So far, it has been shown that single-subjects connectivity metrics can be estimated following group-level molecular connectivity analysis, from: (i) independent connectivity analysis, followed by computation of the loading coefficient (Savio et al., 2017); (ii) partial correlation analysis, followed by a bootstrapping procedure that allows to obtain a distribution of connectivity parameters, still at group-level, but that can nevertheless be used for correlative analysis (Franzmeier et al., 2019); and (iii) SICE, using the equation of the multivariate Gaussian distribution (Titov et al., 2017). Finally, a radically different approach has been proposed, involving the use of dynamic PET data, traditionally used only to estimate the final parametric "static" PET image (Passow et al., 2015; Tomasi et al., 2017). This approach takes full advantage of the dynamic nature of PET data, using the temporal fluctuations in tracer uptake to estimate within-subject "temporal" molecular connectivity, adopting an analytical pipeline similar to the one used in resting-state fMRI analysis (Passow et al., 2015; Tomasi et al., 2017). This allows to obtain a "direct" estimation of the patterns of connectivity alterations at single-subject level, similarly to fMRI data. Still, it has been noted that results obtained with this approach, based on estimation of molecular connectivity using tracer dynamics, might lack biological specificity (Veronese et al., 2019), in particular for [18F]FDG tracer. In general, tracer dynamics are dependent not only on tracer's specific binding, but also on its nonspecific binding and its delivery properties (Veronese et al., 2019), making the interpretation of results obtained with such approach less straightforward, as compared to "traditional" approaches based on parametric static PET images. In addition, the use of individual frames of PET acquisitions might harbor other limitations; most importantly, the necessarily shorter duration of individual frames would yield lower signal-tonoise ratio compared to static images derived from the whole acquisition time. 


\section{CONCLUSION}

Molecular connectivity represents a powerful tool to investigate the pathophysiology of neurodegenerative diseases, providing in vivo access to a potentially endless series of biological processes, from cellular metabolism, to neurotransmission, to aggregation of pathological proteins. The potential of molecular connectivity is still emerging, and might possibly be endless, as new areas of application would arise in parallel with the validation of new tracers for new biological targets. A recent development is related to the possibility to assess not only brain glucose metabolism, as measured by [18F]FDG-PET, but also brain ketone metabolism, using [11C]AcAc-PET (Courchesne-Loyer et al., 2017). Although contribution of ketones to brain energy requirements is scarce under standard conditions $(<5 \%)$, recent evidence suggests that ketone metabolism might hold relevance in both aging and Alzheimer's disease (Croteau et al., 2018). Combining [18F]FDG and [11C]AcAc tracers would allow to test for similarities and differences between glucosebased and ketone-based brain metabolic networks. Another interesting development would concern the possibility to assess molecular connectivity using tracers for synaptic activity, such as the newly developed [11C]UCB-J tracer (Finnema et al., 2016). This would give us a direct access to molecular networks of "pure" neural activity, without

\section{REFERENCES}

Ahmed, Z., Cooper, J., Murray, T. K., Garn, K., McNaughton, S. A., Clarke, H., et al. (2014). A novel in vivo model of tau propagation with rapid and progressive neurofibrillary tangle pathology: the pattern of spread is determined by connectivity, not proximity. Acta Neuropathol. 127, 667-683. doi: 10.1007/ s00401-014-1254-6

Albert, M. S., DeKosky, S. T., Dickson, D., Dubois, B., Feldman, H. H., Fox, N. C., et al. (2011). The diagnosis of mild cognitive impairment due to Alzheimer's disease: recommendations from the National Institute on Aging and Alzheimer's Association workgroup. Alzheimers Dement. 7, 270-279. doi: 10.1016/j.jalz.2011.03.008

Armstrong, M. J., Litvan, I., Lang, A. E., Bak, T. H., Bhatia, K. P., Borroni, B., et al. (2013). Criteria for the diagnosis of corticobasal degeneration. Neurology 80, 496-503. doi: 10.1212/WNL.0b013e31827f ofd 1

Ballarini, T., Iaccarino, L., Magnani, G., Ayakta, N., Miller, B. L., Jagust, W. J., et al. (2016). Neuropsychiatric Subsyndromes and Brain Metabolic Network Dysfunctions in Early Onset Alzheimer's Disease. Hum. Brain Mapp. 37, 42344247. doi: 10.1002/hbm.23305

Beckmann, C. F., DeLuca, M., Devlin, J. T., and Smith, S. M. (2005). Investigations into resting-state connectivity using independent component analysis. Philos. Trans. R. Soc. Lond. Ser. B Biol. Sci. 360, 1001-1013. doi: 10.1098/rstb.2005.1634

Bellucci, A., Mercuri, N. B., Venneri, A., Faustini, G., Longhena, F., Pizzi, M., et al. (2015). Parkinson's disease: from synaptic loss to connectome dysfunction. Neuropathol. Appl. Neurobiol. 42, 77-94. doi: 10.1111/nan.1 2297

Biswal, B., Yetkin, F. Z., Haughton, V. M., and Hyde, J. S. (1995). Functional connectivity in the motor cortex of resting. Magn. Reson. Med. 34, 537-541.

Braak, H., Alafuzoff, I., Arzberger, T., Kretzschmar, H., and Tredici, K. (2006). Staging of Alzheimer disease-associated neurofibrillary pathology using paraffin sections and immunocytochemistry. Acta Neuropathol. 112, 389-404. doi: 10. 1007/s00401-006-0127-z

Braak, H., and Braak, E. (1991). Neuropathological stageing of Alzheimer-related changes. Acta Neuropathol. 82, 239-259. doi: 10.1007/BF00308809 the mediation of BOLD signal or metabolic processes (Heurling et al., 2017).

Thus, the progressive implementation of molecular connectivity techniques, with possibly unlimited applications brought by the development of new PET tracers, will allow unique breakthroughs in our understanding of neurodegenerative mechanisms. Once approaches to estimate single-subject brain molecular connectivity will become well-established, brain connectivity signatures might hold promises to be validated as biomarkers for diagnostic and prognostic use, or, eventually, as complementary outcome measures to evaluate the effects of new emerging treatments. Hopefully, molecular connectivity studies "will gain momentum, and deservedly so!" (Yakushev et al., 2017).

\section{AUTHOR CONTRIBUTIONS}

AS performed the PubMed search and wrote the review. DP wrote the review and critically revised the article for intellectual content.

\section{ACKNOWLEDGMENTS}

We thank Dr. Silvia Paola Caminiti and Prof. Marco Tettamanti for their invaluable insights and discussions.

Braak, H., Del Tredici, K., Rüb, U., de Vos, R. A., Jansen Steur, E. N., and Braak, E. (2003). Staging of brain pathology related to sporadic Parkinson's disease. Neurobiol. Aging 24, 197-211.

Brettschneider, J., Del Tredici, K., Lee, V. M.-Y., and Trojanowski, J. Q. (2015). Spreading of pathology in neurodegenerative diseases: a focus on human studies. Nat. Rev. Neurosci. 16, 109-120. doi: 10.1038/nrn3887

Brettschneider, J., Del Tredici, K., Toledo, J. B., Robinson, J. L., Irwin, D. J., Grossman, M., et al. (2013). Stages of pTDP-43 pathology in amyotrophic lateral sclerosis. Ann. Neurol. 74, 20-38. doi: 10.1002/ana.23937

Bullmore, E., and Sporns, O. (2009). Complex brain networks: graph theoretical analysis of structural and functional systems. Nat. Rev. Neurosci. 10, 186-198. doi: $10.1038 / \mathrm{nrn} 2575$

Caminiti, S. P., Alongi, P., Majno, L., Volontè, M. A., Cerami, C., Gianolli, L., et al. (2017a). Evaluation of an optimized [18F]fluoro-deoxy-glucose positron emission tomography voxel-wise method to early support differential diagnosis in atypical Parkinsonian disorders. Eur. J. Neurol. 24, 687-e26. doi: 10.1111/ene. 13269

Caminiti, S. P., Presotto, L., Baroncini, D., Garibotto, V., Moresco, R. M., Gianolli, L., et al. (2017b). Axonal damage and loss of connectivity in nigrostriatal and mesolimbic dopamine pathways in early Parkinson's disease. Neuroimage Clin. 14, 734-740. doi: 10.1016/j.nicl.2017.03.011

Caminiti, S. P., Sala, A., Iaccarino, L., Beretta, L., Pilotto, A., Gianolli, L., et al. (2019). Brain glucose metabolism in Lewy body dementia: implications for diagnostic criteria. Alzheimers. Res. Ther. 11:20.

Caminiti, S. P., Tettamanti, M., Sala, A., Presotto, L., Iannaccone, S., Cappa, S. F., et al. (2017c). Metabolic connectomics targeting brain pathology in dementia with Lewy bodies. J. Cereb. Blood Flow Metab. 37, 1311-1325. doi: 10.1177/ $0271678 X 16654497$

Cerami, C., Crespi, C., Della Rosa, P. A., Dodich, A., Marcone, A., Magnani, G., et al. (2015). Brain changes within the visuo-spatial attentional network in posterior cortical atrophy. J. Alzheimers Dis. 43, 385-395. doi: 10.3233/JAD-141275

Cerami, C., Dodich, A., Lettieri, G., Iannaccone, S., Magnani, G., Marcone, A., et al. (2016). Different FDG-PET metabolic patterns at single-subject level in the behavioral variant of fronto-temporal dementia. Cortex 83, 101-112. doi: 10.1016/j.cortex.2016.07.008 
Cervenka, S., Varrone, A., Fransén, E., Halldin, C., and Farde, L. (2010). PET studies of D2-receptor binding in striatal and extrastriatal brain regions: Biochemical support in vivo for separate dopaminergic systems in humans. Synapse 64, 478-485. doi: 10.1002/syn.20765

Clark, C. M., and Stoessl, A. J. (1986). Glucose use correlations: a matter of inference. J. Cereb. Blood Flow Metab. 6, 511-512. doi: 10.1038/jcbfm.1986.87

Courchesne-Loyer, A., Croteau, E., Castellano, C. A., St-Pierre, V., Hennebelle, M., and Cunnane, S. C. (2017). Inverse relationship between brain glucose and ketone metabolism in adults during short-term moderate dietary ketosis: A dual tracer quantitative positron emission tomography study. J. Cereb. Blood Flow Metab. 37, 2485-2493. doi: 10.1177/0271678X16669366

Croteau, E., Castellano, C. A., Fortier, M., Bocti, C., Fulop, T., Paquet, N., et al. (2018). A cross-sectional comparison of brain glucose and ketone metabolism in cognitively healthy older adults, mild cognitive impairment and early Alzheimer's disease. Exp. Gerontol. 107, 18-26. doi: 10.1016/j.exger.2017.07.004

Di, X., Biswal, B. B., and Alzheimer's Disease Neuroimaging Initiative (2012). Metabolic Brain Covariant Networks as Revealed by FDG-PET with Reference to Resting-State fMRI Networks. Brain Connect. 2, 275-283. doi: 10.1089/brain. 2012.0086

Di, X., Gohel, S., Thielcke, A., Wehrl, H. F., and Biswal, B. B. (2017). Do all roads lead to Rome? A comparison of brain networks derived from inter-subject volumetric and metabolic covariance and moment-to-moment hemodynamic correlations in old individuals. Brain Struct. Funct. 222, 3833-3845.

Domert, J., Rao, S. B., Agholme, L., Brorsson, A.-C., Marcusson, J., Hallbeck, M., et al. (2014). Spreading of amyloid- $\beta$ peptides via neuritic cell-to-cell transfer is dependent on insufficient cellular clearance. Neurobiol. Dis. 65, 82-92. doi: 10.1016/j.nbd.2013.12.019

Duan, H., Jiang, J., Xu, J., Zhou, H., Huang, Z., Yu, Z., et al. (2017). Differences in $\mathrm{A} \beta$ brain networks in Alzheimer's disease and healthy controls. Brain Res. 1655, 77-89. doi: 10.1016/j.brainres.2016.11.019

Dubois, B., Feldman, H. H., Jacova, C., Hampel, H., Molinuevo, J. L., Blennow, K., et al. (2014). Advancing research diagnostic criteria for Alzheimer's disease: The IWG-2 criteria. Lancet Neurol. 13, 614-629.

Dujardin, S., Lécolle, K., Caillierez, R., Bégard, S., Zommer, N., Lachaud, C., et al. (2014). Neuron-to-neuron wild-type Tau protein transfer through a transsynaptic mechanism: Relevance to sporadic tauopathies. Acta Neuropathol. Commun. 2:14. doi: 10.1186/2051-5960-2-14

Fazio, P., Svenningsson, P., Cselényi, Z., Halldin, C., Farde, L., and Varrone, A. (2018). Nigrostriatal dopamine transporter availability in early Parkinson's disease. Mov. Disord. 33, 592-599. doi: 10.1002/mds.27316

Finnema, S. J., Nabulsi, N. B., Eid, T., Detyniecki, K., Lin, S. F., Chen, M.-K., et al. (2016). Imaging synaptic density in the living human brain. Sci. Transl. Med. 8:348ra96. doi: 10.1126/scitranslmed.aaf6667

Fodero-Tavoletti, M. T., Brockschnieder, D., Villemagne, V. L., Martin, L., Connor, A. R., Thiele, A., et al. (2012). In vitro characterization of [18F]-florbetaben, an A $\beta$ imaging radiotracer. Nucl. Med. Biol. 39, 1042-1048. doi: 10.1016/j. nucmedbio.2012.03.001

Fodero-Tavoletti, M. T., Okamura, N., Furumoto, S., Mulligan, R. S., Connor, A. R., McLean, C. A., et al. (2011). 18F-THK523: A novel in vivo tau imaging ligand for Alzheimer's disease. Brain 134, 1089-1100. doi: 10.1093/brain/awr038

Fornito, A., and Bullmore, E. T. (2015). Connectomics: a new paradigm for understanding brain disease. Eur. Neuropsychopharmacol. 25, 733-748. doi: 10.1016/j.euroneuro.2014.02.011

Fornito, A., Zalesky, A., and Breakspear, M. (2015). The connectomics of brain disorders. Nat. Rev. Neurosci. 16, 159-172. doi: 10.1038/nrn3901

Fortier, M., Castellano, C.-A., Croteau, E., Langlois, F., Bocti, C., St-Pierre, V., et al. (2019). A ketogenic drink improves brain energy and some measures of cognition in mild cognitive impairment. Alzheimers Dement. 15, 625-634. doi: 10.1016/j.jalz.2018.12.017

Franzmeier, N., Rubinski, A., Neitzel, J., Kim, Y., Damm, A., Na, D. L., et al. (2019). Functional connectivity associated with tau levels in ageing, Alzheimer's, and small vessel disease. Brain 142, 1093-1107. doi: 10.1093/brain/awz026

Gauthier, C. J., and Fan, A. P. (2019). BOLD signal physiology: models and applications. Neuroimage. 187, 116-127.

Gorno-Tempini, M. L., Hillis, A. E., Weintraub, S., Kertesz, A., Mendez, M., Cappa, S. F., et al. (2011). Classification of primary progressive aphasia and its variants. Neurology 76, 1006-1014. doi: 10.1212/WNL.0b013e318211 $03 \mathrm{e} 6$
Greicius, M. D., Krasnow, B., Reiss, A. L., and Menon, V. (2003). Functional connectivity in the resting brain: a network analysis of the default mode hypothesis. Proc. Natl. Acad. Sci. U.S.A. 100, 253-258. doi: 10.1073/pnas. 0135058100

Hahn, A., Haeusler, D., Kraus, C., Höflich, A. S., Kranz, G. S., Baldinger, P., et al. (2014). Attenuated serotonin transporter association between dorsal raphe and ventral striatum in major depression. Hum. Brain Mapp. 35, 3857-3866. doi: $10.1002 / \mathrm{hbm} .22442$

Hahn, A., Lanzenberger, R., and Kasper, S. (2018). Making sense of connectivity. Int. J. Neuropsychopharmacol. 22, 194-207. doi: 10.1093/ijnp/pyy100

Hashimoto, H., Kawamura, K., Igarashi, N., Takei, M., Fujishiro, T., Aihara, Y., et al. (2014). Radiosynthesis, photoisomerization, biodistribution, and metabolite analysis of 11C-PBB3 as a clinically useful PET probe for imaging of tau pathology. J. Nucleic Med. 55, 1532-1538. doi: 10.2967/jnumed.114.139550

Herholz, K., Haense, C., Gerhard, A., Jones, M., Anton-Rodriguez, J., Segobin, S., et al. (2017). Metabolic regional and network changes in Alzheimer's disease subtypes. J. Cereb. Blood Flow Metab. 38, 1796-1806. doi: 10.1177/ 0271678X17718436

Herholz, K., Weisenbach, S., Zündorf, G., Lenz, O., Schröder, H., Bauer, B., et al. (2004). In vivo study of acetylcholine esterase in basal forebrain, amygdala, and cortex in mild to moderate Alzheimer disease. Neuroimage 21, 136-143. doi: 10.1016/j.neuroimage.2003.09.042

Heurling, K., Leuzy, A., Jonasson, M., Frick, A., Zimmer, E. R., Nordberg, A., et al. (2017). Quantitative positron emission tomography in brain research. Brain Res. 1670, 220-234. doi: 10.1016/j.brainres.2017.06.022

Hoenig, M. C., Bischof, G. N., Seemiller, J., Hammes, J., Kukolja, J., Onur, ÖA., et al. (2018). Networks of tau distribution in Alzheimer's disease. Brain 141, 568-581. doi: 10.1093/brain/awx353

Holtbernd, F., Ma, Y., Peng, S., Schwartz, F., Timmermann, L., Kracht, L., et al. (2015). Dopaminergic correlates of metabolic network activity in Parkinson's disease. Hum. Brain Mapp. 36, 3575-3585. doi: 10.1002/hbm.22863

Horwitz, B., Duara, R., and Rapoport, S. I. (1984). Intercorrelations of glucose metabolic rates between brain regions: application to healthy males in a state of reduced sensory input. J. Cereb. Blood Flow Metab. 4, 484-499. doi: 10.1038/ jcbfm.1984.73

Horwitz, B., Grady, C. L., Schlageter, N. L., Duara, R., and Rapoport, S. I. (1987). Intercorrelations of regional cerebral glucose metabolic rates in Alzheimer's disease. Brain Res. 407, 294-306.

Huang, S., Li, J., Sun, L., Ye, J., Fleisher, A., Wu, T., et al. (2010). Learning brain connectivity of Alzheimer's disease by sparse inverse covariance estimation. Neuroimage 50, 935-949. doi: 10.1016/j.neuroimage.2009.12.120

Iaccarino, L., Chiotis, K., Alongi, P., Almkvist, O., Wall, A., Cerami, C., et al. (2017a). A Cross-Validation of FDG- and Amyloid-PET Biomarkers in Mild Cognitive Impairment for the Risk Prediction to Dementia due to Alzheimer's Disease in a Clinical Setting. J. Alzheimers Dis. 59, 603-614. doi: 10.3233/JAD170158

Iaccarino, L., Sala, A., Caminiti, S. P., and Perani, D. (2017b). The emerging role of PET imaging in dementia. F1000Res. 6:1830. doi: 10.12688/f1000research. 11603.1

Iaccarino, L., Sala, A., Caminiti, S. P., Santangelo, R., Iannaccone, S., Magnani, G., et al. (2018). The brain metabolic signature of visual hallucinations in dementia with Lewy bodies. Cortex 108, 13-24. doi: 10.1016/j.cortex.2018.06.014

Jack, C. R., Bennett, D. A., Blennow, K., Carrillo, M. C., Dunn, B., Haeberlein, S. B., et al. (2018). NIA-AA Research Framework: toward a biological definition of Alzheimer's disease. Alzheimers Dement. 14, 535-562. doi: 10.1016/j.jalz.2018. 02.018

Jagust, W. (2018). Imaging the evolution and pathophysiology of Alzheimer disease. Nat. Rev. Neurosci 11, 687-700. doi: 10.1038/s41583-018-0067-3

Jiang, J., Duan, H., Huang, Z., and Yu, Z. (2015). Study of amyloid- $\beta$ peptide functional brain networks in AD, MCI and HC1. Biomed. Mater. Eng. 26, S2197-S2205. doi: 10.3233/BME-151525

Jovalekic, A., Koglin, N., Mueller, A., and Stephens, A. W. (2017). New protein deposition tracers in the pipeline. EJNMMI Radiopharm. Chem. 1:11. doi: 10. 1186/s41181-016-0015-3

Klein, J. C., Eggers, C., Kalbe, E., Weisenbach, S., Hohmann, C., Vollmar, S., et al. (2010). Neurotransmitter changes in dementia with Lewy bodies and Parkinson disease dementia in vivo. Neurology 74, 885-892. doi: 10.1212/WNL. ob013e3181d55f61 
Lee, D. S., Kang, H., Kim, H., Park, H., Oh, J. S., Lee, J. S., et al. (2008). Metabolic connectivity by interregional correlation analysis using statistical parametric mapping (SPM) and FDG brain PET; methodological development and patterns of metabolic connectivity in adults. Eur. J. Nucl. Med. Mol. Imaging 35, 1681-1691. doi: 10.1007/s00259-008-0808-z

Lemoine, L., Leuzy, A., Chiotis, K., Rodriguez-Vieitez, E., and Nordberg, A. (2018). Tau positron emission tomography imaging in tauopathies: The added hurdle of off-target binding. Alzheimers Dement. Diagn. Assess. Dis. Monit. 10, 232-236. doi: 10.1016/j.dadm.2018.01.007

Leyton, C. E., Cassidy, B., Villemagne, V. L., Jones, G., Kwok, J. B., Rowe, C. C., et al. (2015). Divergent Network Patterns of Amyloid- $\beta$ Deposition in Logopenic and Amnestic Alzheimer's Disease Presentations. Biol. Psychiatry Cogn. Neurosci. Neuroimaging 1, 24-31. doi: 10.1016/j.bpsc.2015.09.004

Liu, T. T. (2013). Neurovascular factors in resting-state functional MRI. Neuroimage 15, 339-348. doi: 10.1016/j.neuroimage.2013.04.071

Malpetti, M., Ballarini, T., Presotto, L., Garibotto, V., Tettamanti, M., and Perani, D. (2017). Gender differences in healthy aging and Alzheimer's Dementia: A18F-FDG-PET study of brain and cognitive reserve. Hum. Brain Mapp. 38, 4212-4227. doi: 10.1002/hbm.23659

Malpetti, M., Sala, A., Vanoli, E. G., Gianolli, L., Luzi, L., and Perani, D. (2018). Unfavourable gender effect of high body mass index on brain metabolism and connectivity. Sci. Rep. 8:12584. doi: 10.1038/s41598-018-30883-y

Marcone, A., Garibotto, V., Moresco, R. M., Florea, I., Panzacchi, A., Carpinelli, A., et al. (2012). [11C]-MP4A PET cholinergic measurements in amnestic mild cognitive impairment, probable Alzheimer's disease, and dementia with Lewy bodies: a Bayesian method and voxel-based analysis. J. Alzheimers Dis. 31, 387-399.

Mathis, C. A., Lopresti, B. J., Ikonomovic, M. D., and Klunk, W. E. (2017). Smallmolecule PET Tracers for Imaging Proteinopathies. Semin. Nucleic Med. 47, 553-575. doi: 10.1053/j.semnuclmed.2017.06.003

Matías-Guiu, J. A., Pytel, V., Cabrera-Martín, M. N., Galán, L., Valles-Salgado, M., Guerrero, A., et al. (2016). Amyloid- and FDG-PET imaging in amyotrophic lateral sclerosis. Eur. J. Nucl. Med. Mol. Imaging 43, 2050-2060. doi: 10.1007/ s00259-016-3434-1

McKeith, I., Boeve, B. F., Dickson, D. W., Halliday, G., Taylor, J. P., Weintraub, D., et al. (2017). Diagnosis and management of dementia with Lewy bodies. Neurology 65, 1863-1872. doi: 10.1212/01.wnl.0000224698. 67660.45

McKhann, G. M., Knopman, D. S., Chertkow, H., Hyman, B. T., Jack, C. R. Jr., Kawas, C. H., et al. (2011). The diagnosis of dementia due to Alzheimer's disease: recommendations from the National Institute on Aging-Alzheimer's Association workgroups on diagnostic guidelines for Alzheimer's disease. Alzheimers Dement. 7, 263-269.

Mezias, C., and Raj, A. (2017). Analysis of Amyloid- $\beta$ pathology spread in mouse models suggests spread is driven by spatial proximity, not connectivity. Front. Neurol. 18:653. doi: 10.3389/fneur.2017.00653

Morbelli, S., Drzezga, A., Perneczky, R., Frisoni, G. B., Caroli, A., van Berckel, B. N. M., et al. (2012). Resting metabolic connectivity in prodromal Alzheimer's disease. A European Alzheimer Disease Consortium (EADC) project. Neurobiol. Aging 33, 2533-2550. doi: 10.1016/j.neurobiolaging.2012. 01.005

Morbelli, S., Perneczky, R., Drzezga, A., Frisoni, G. B., Caroli, A., van Berckel, B. N. M., et al. (2013). Metabolic networks underlying cognitive reserve in prodromal Alzheimer disease: a European Alzheimer disease consortium project. J. Nucleic Med. 54, 894-902. doi: 10.2967/jnumed.112.113928

Narasimhan, S., Guo, J., Changolkar, L., Stieber, A., McBride, J., Silva, L., et al. (2017). Pathological Tau Strains from Human Brains Recapitulate the Diversity of Tauopathies in Nontransgenic Mouse Brain. J. Neurosci. 37, 11406-11423. doi: 10.1523/jneurosci.1230-17.2017

Ni, R., Gillberg, P.-G., Bergfors, A., Marutle, A., and Nordberg, A. (2013). Amyloid tracers detect multiple binding sites in Alzheimer's disease brain tissue. Brain 136, 2217-2227. doi: 10.1093/brain/awt142

Niethammer, M., Tang, C. C., Feigin, A., Allen, P. J., Heinen, L., Hellwig, S., et al. (2014). A disease-specific metabolic brain network associated with corticobasal degeneration. Brain 137, 3036-3046. doi: 10.1093/brain/awu256

Pagani, M., Chiò, A., Valentini, M. C., Öberg, J., Nobili, F., Calvo, A., et al. (2014). Functional pattern of brain FDG-PET in amyotrophic lateral sclerosis. Neurology 83, 1067-1074. doi: 10.1212/WNL.0000000000000792
Pagani, M. M., Giuliani, A., Öberg, J., De Carli, F., Morbelli, S., Girtler, N., et al. (2017). Progressive disgregation of brain networking from normal aging to Alzhimer's Disease. Independent Component Analysis on FDG-PET data. J. Nucleic Med. 58, 1132-1139. doi: 10.2967/jnumed.116.184309

Palop, J. J., Chin, J., and Mucke, L. (2006). A network dysfunction perspective on neurodegenerative diseases. Nature 443, 768-773. doi: 10.1038/nature05289

Passow, S., Specht, K., Adamsen, T. C., Biermann, M., Brekke, N., Craven, A. R., et al. (2015). Default-mode network functional connectivity is closely related to metabolic activity. Hum. Brain Mapp. 36, 2027-2038. doi: 10.1002/hbm.22753

Perani, D. (2014). FDG-PET and amyloid-PET imaging: the diverging paths. Curr. Opin. Neurol. 27, 405-413. doi: 10.1097/WCO.0000000000000109

Perani, D., Della Rosa, P. A., Cerami, C., Gallivanone, F., Fallanca, F., Vanoli, E. G., et al. (2014). Validation of an optimized SPM procedure for FDG-PET in dementia diagnosis in a clinical setting. Neuroimage Clin. 6, 445-454. doi: 10.1016/j.nicl.2014.10.009

Perani, D., Farsad, M., Ballarini, T., Lubian, F., Malpetti, M., and Fracchetti, A. (2017). The impact of bilingualism on brain reserve and metabolic connectivity in Alzheimer's s dementia. Proc. Natl. Acad. Sci. U.S.A. 114, 1690-1695. doi: 10.1073/pnas.1610909114

Pereira, J. B., van Westen, D., Volpe, G., Strandberg, T. O., Westman, E., Palmqvist, S., et al. (2017). Amyloid Network Topology Characterizes the Progression of Alzheimer's Disease During the Predementia Stages. Cereb. Cortex 28, 340-349. doi: 10.1093/cercor/bhx294

Pievani, M., Filippini, N., van den Heuvel, M. P., Cappa, S. F., and Frisoni, G. B. (2014). Brain connectivity in neurodegenerative diseases [mdash] from phenotype to proteinopathy. Nat. Rev. Neurol. 10, 620-633. doi: 10.1038/ nrneurol.2014.178

Pillai, R. L. I., Zhang, M., Yang, J., Mann, J. J., Oquendo, M. A., Parsey, R. V., et al. (2018). Molecular connectivity disruptions in males with major depressive disorder. J. Cereb. Blood Flow Metab. 9:271678X18764053. doi: 10. 1177/0271678X18764053

Premi, E., Calhoun, V. D., Garibotto, V., Turrone, R., Alberici, A., Cottini, E., et al. (2017). Source-Based Morphometry Multivariate Approach to Analyze [123I]FP-CIT SPECT Imaging. Mol. Imaging Biol. 19, 772-778.

Premi, E., Pilotto, A., Garibotto, V., Bigni, B., Turrone, R., Alberici, A., et al. (2016). Impulse control disorder in PD: a lateralized monoaminergic frontostriatal disconnection syndrome? Parkinsonism Relat. Disord. 30, 62-66. doi: 10.1016/ j.parkreldis.2016.05.028

Prescott, J. W., Guidon, A., Doraiswamy, P. M., Roy Choudhury, K., Liu, C., and Petrella, J. R. (2014). The Alzheimer structural connectome: changes in cortical network topology with increased amyloid plaque burden. Radiology 273, 175-185. doi: 10.1148/radiol.14132593

Raichle, M. E., MacLeod, A. M., Snyder, A. Z., Powers, W. J., Gusnard, D. A., and Shulman, G. L. (2001). A default mode of brain function. Proc. Natl. Acad. Sci. U.S.A. $98,676-682$.

Rascovsky, K., Hodges, J. R., Knopman, D., Mendez, M. F., Kramer, J. H., Neuhaus, J., et al. (2011). Sensitivity of revised diagnostic criteria for the behavioural variant of frontotemporal dementia. Brain 134, 2456-2477. doi: 10.1093/brain/ awr179

Riedl, V., Bienkowska, K., Strobel, C., Tahmasian, M., Grimmer, T., Förster, S., et al. (2014). Local activity determines functional connectivity in the resting human brain: a simultaneous FDG-PET/fMRI study. J. Neurosci. 34, 6260-6266. doi: 10.1523/JNEUROSCI.0492-14.2014

Riedl, V., Utz, L., Castrillón, G., Grimmer, T., Rauschecker, J. P., Ploner, M., et al. (2016). Metabolic connectivity mapping reveals effective connectivity in the resting human brain. Proc. Natl. Acad. Sci. U.S.A. 113, 428-433. doi: 10.1073/ pnas. 1513752113

Sabri, O., Sabbagh, M. N., Seibyl, J., Barthel, H., Akatsu, H., Ouchi, Y., et al. (2015). Florbetaben PET imaging to detect amyloid beta plaques in Alzheimer's disease: Phase 3 study. Alzheimers Dement. 11, 964-974. doi: 10.1016/j.jalz.2015.02.004

Saint-Aubert, L., Lemoine, L., Chiotis, K., Leuzy, A., Rodriguez-Vieitez, E., and Nordberg, A. (2017). Tau PET imaging: present and future directions. Mol. Neurodegener. 12:19. doi: 10.1186/s13024-017-0162-3

Sala, A., Caminiti, S. P., Presotto, L., Premi, E., Pilotto, A., Turrone, R., et al. (2017). Altered brain metabolic connectivity at multiscale level in early Parkinson's disease. Sci. Rep. 7:4256. doi: 10.1038/s41598-017-04102-z

Sala, A., Iaccarino, L., Fania, P., Vanoli, E. G., Fallanca, F., Pagnini, C., et al. (2019). Testing the diagnostic accuracy of [18F]FDG-PET in discriminating spinal- and 
bulbar-onset amyotrophic lateral sclerosis. Eur. J. Nucl. Med. Mol. Imaging 46, 1117-1131. doi: 10.1007/s00259-018-4246-2

Savio, A., Fünger, S., Tahmasian, M., Rachakonda, S., Manoliu, A., Sorg, C., et al. (2017). Resting state networks as simultaneously measured with fMRI and PET. J. Nucleic Med. 58, 1314-1317. doi: 10.2967/jnumed.116.185835

Schmidt, R., de Reus, M. A., Scholtens, L. H., van den Berg, L. H., and van den Heuvel, M. P. (2016). Simulating disease propagation across white matter connectome reveals anatomical substrate for neuropathology staging in amyotrophic lateral sclerosis. Neuroimage 1, 762-769. doi: 10.1016/j. neuroimage.2015.04.005

Schöll, M., Lockhart, S. N., Schonhaut, D. R., O’Neil, J. P., Janabi, M., Ossenkoppele, R., et al. (2016). PET imaging of tau deposition in the aging human brain. Neuron 89, 971-982. doi: 10.1016/j.neuron.2016.01.028

Schwarz, A. J., Yu, P., Miller, B. B., Shcherbinin, S., Dickson, J., Navitsky, M., et al. (2016). Regional profiles of the candidate tau PET ligand 18F-AV-1451 recapitulate key features of Braak histopathological stages. Brain 139, 15391550. doi: 10.1093/brain/aww023

Seeley, W. W. (2017). Mapping neurodegenerative disease onset and progression. Cold Spring Harb. Perspect. Biol. 9:a023622. doi: 10.1101/cshperspect.a023622

Seeley, W. W., Crawford, R. K., Zhou, J., Miller, B. L., and Greicius, M. D. (2009). Neurodegenerative diseases target large-scale human brain networks. Neuron 62, 42-52. doi: 10.1016/j.neuron.2009.03.024

Sepulcre, J., Sabuncu, M. R., Becker, A., Sperling, R., and Johnson, K. A. (2013). In vivo characterization of the early states of the amyloid-beta network. Brain 136, 2239-2252. doi: 10.1093/brain/awt146

Smith, S. M., Vidaurre, D., Beckmann, C. F., Glasser, M. F., Jenkinson, M., Miller, K. L., et al. (2013). Functional connectomics from resting-state fMRI. Trends Cogn. Sci. 17, 666-682. doi: 10.1016/j.tics.2013.09.016

Son, S. J., Kim, J., Seo, J., Lee, J. M., and Park, H. (2015). Connectivity analysis of normal and mild cognitive impairment patients based on FDG and PiB-PET images. Neurosci. Res. 98, 50-58. doi: 10.1016/j.neures.2015.04.002

Song, H. L., Shim, S., Kim, D. H., Won, S. H., Joo, S., Kim, S., et al. (2014). $\beta$-Amyloid is transmitted via neuronal connections along axonal membranes. Ann. Neurol. 75, 88-97. doi: 10.1002/ana.24029

Sperling, R. A., Aisen, P. S., Beckett, L. A., Bennett, D. A., Craft, S., Fagan, A. M., et al. (2011). Toward defining the preclinical stages of Alzheimer's disease: recommendations from the National Institute on Aging-Alzheimer's Association workgroups on diagnostic guidelines for Alzheimer's disease. Alzheimers Dement. 7, 280-292. doi: 10.1016/j.jalz.2011.03.003

Spetsieris, P. G., Ko, J. H., Tang, C. C., Nazem, A., Sako, W., Peng, S., et al. (2015). Metabolic resting-state brain networks in health and disease. Proc. Natl. Acad. Sci. U.S.A. 112, 2563-2568. doi: 10.1073/pnas.1411011112

Sporns, O., Tononi, G., and Kötter, R. (2005). The human connectome: a structural description of the human brain. PLoS Comput. Biol. 1:e42. doi: 10.1371/journal. pcbi.0010042

Stoessl, A. J. (2017). Glucose utilization: still in the synapse. Nat. Neurosci. 20, 382-384. doi: 10.1038/nn.4513

Thal, D. R., Rüb, U., Orantes, M., and Braak, H. (2002). Phases of A beta-deposition in the human brain and its relevance for the development of AD. Neurology 58, 1791-1800. doi: 10.1212/WNL.58.12. 1791
Titov, D., Diehl-schmid, J., Shi, K., Perneczky, R., Zou, N., Grimmer, T., et al. (2017). Metabolic connectivity for differential diagnosis of dementing disorders. J. Cereb. Blood Flow Metab. 37, 252-262. doi: 10.1177/0271678X15622465

Tomasi, D. G., Shokri-Kojori, E., Wiers, C. E., Kim, S. W., Demiral, Ş. B., Cabrera, E. A., et al. (2017). Dynamic brain glucose metabolism identifies anti-correlated cortical-cerebellar networks at rest. J. Cereb. Blood Flow Metab. 37, 3659-3670. doi: 10.1177/0271678X17708692

Tuominen, L., Nummenmaa, L., Keltikangas-Järvinen, L., Raitakari, O., and Hietala, J. (2014). Mapping neurotransmitter networks with PET: An example on serotonin and opioid systems. Hum. Brain Mapp. 35, 1875-1884. doi: 10. 1002/hbm.22298

Vandenberghe, R., Adamczuk, K., Dupont, P., Van Laere, K., and Chételat, G. (2013). Amyloid PET in clinical practice: Its place in the multidimensional space of Alzheimer's disease. Neuroimage Clin. 6, 497-511. doi: 10.1016/j.nicl.2013. 03.014

Varrone, A., and Halldin, C. (2010). Molecular imaging of the dopamine transporter. J. Nucleic Med. 51, 1331-1334. doi: 10.2967/jnumed.109.065656

Verger, A., Klesse, E., Chawki, M. B., Witjas, T., Azulay, J. P., Eusebio, A., et al. (2018). Brain PET substrate of impulse control disorders in Parkinson's disease: a metabolic connectivity study. Hum. Brain Mapp. 39, 3178-3186. doi: 10.1002/ hbm. 24068

Veronese, M., Moro, L., Arcolin, M., Dipasquale, O., Rizzo, G., Expert, P., et al. (2019). Covariance statistics and network analysis of brain PET imaging studies. Sci. Rep 9:2496. doi: 10.1038/s41598-019-39005-8

Warren, J. D., Rohrer, J. D., Schott, J. M., Fox, N. C., Hardy, J., and Rossor, M. N. (2013). Molecular nexopathies: A new paradigm of neurodegenerative disease. Trends Neurosci. 36, 561-569. doi: 10.1016/j.tins.2013.06.007

Xia, C. F., Arteaga, J., Chen, G., Gangadharmath, U., Gomez, L. F., Kasi, D., et al. (2013). [18F]T807, a novel tau positron emission tomography imaging agent for Alzheimer's disease. Alzheimers Dement. 9, 666-676. doi: 10.1016/j.jalz.2012. 11.008

Xia, M., Wang, J., and He, Y. (2013). BrainNet Viewer: a network visualization tool for human brain connectomics. PLoS One 8:e68910. doi: 10.1371/journal.pone. 0068910

Yakushev, I., Drzezga, A., and Habeck, C. (2017). Metabolic connectivity: methods and applications. Curr. Opin. Neurol. 30, 677-685. doi: 10.1097/WCO. 0000000000000494

Zhou, J., Gennatas, E. D., Kramer, J. H., Miller, B. L., and Seeley, W. W. (2013). Predicting regional neurodegeneration from the healthy brain functional connectome. Neuron 73, 1216-1227. doi: 10.1016/j.neuron.2012.03.004

Conflict of Interest Statement: The authors declare that the research was conducted in the absence of any commercial or financial relationships that could be construed as a potential conflict of interest.

Copyright (c) 2019 Sala and Perani. This is an open-access article distributed under the terms of the Creative Commons Attribution License (CC BY). The use, distribution or reproduction in other forums is permitted, provided the original author(s) and the copyright owner(s) are credited and that the original publication in this journal is cited, in accordance with accepted academic practice. No use, distribution or reproduction is permitted which does not comply with these terms. 


\section{OPEN ACCESS}

Edited by:

Roberto Esposito,

Azienda Ospedaliera Ospedali Riuniti

Marche Nord, Italy

Reviewed by:

Bei Wang,

East China University of Science and Technology, China

Chunyan Luo,

Sichuan University, China

*Correspondence:

Caterina Tonon

caterina.tonon@unibo.it

tThese authors have contributed equally to this work

Specialty section:

This article was submitted to Brain Imaging Methods, a section of the journal Frontiers in Neuroscience

Received: 27 March 2019 Accepted: 28 May 2019

Published: 14 June 2019

Citation:

Evangelisti S, Pittau F, Testa C, Rizzo G, Gramegna LL, Ferri L, Coito A, Cortelli $P$,

Calandra-Buonaura G, Bisquoli F, Bianchini C, Manners DN, Talozzi L,

Tonon C, Lodi R and Tinuper P (2019)

L-Dopa Modulation of Brain Connectivity in Parkinson's Disease

Patients: A Pilot EEG-fMRI Study. Front. Neurosci. 13:611. doi: 10.3389/fnins.2019.00611

\section{L-Dopa Modulation of Brain Connectivity in Parkinson's Disease Patients: A Pilot EEG-fMRI Study}

\author{
Stefania Evangelisti ${ }^{1}$, Francesca Pittau ${ }^{2}$, Claudia Testa ${ }^{3}$, Giovanni Rizzo 4,5, \\ Laura Ludovica Gramegna ${ }^{1,5}$, Lorenzo Ferri ${ }^{4}$, Ana Coito ${ }^{6}$, Pietro Cortelli4,5, \\ Giovanna Calandra-Buonaura ${ }^{4,5}$, Fabio Bisquoli4,5, Claudio Bianchini ${ }^{1}$, \\ David Neil Manners ${ }^{1}$, Lia Talozzi ${ }^{1}$, Caterina Tonon ${ }^{1,5 *}$, Raffaele Lodi ${ }^{1,5+}$ and \\ Paolo Tinuper ${ }^{4,5+}$
}

\begin{abstract}
${ }^{1}$ Functional MR Unit, Department of Biomedical and NeuroMotor Sciences, University of Bologna, Bologna, Italy, ${ }^{2}$ EEG and Epilepsy Unit, Geneva University Hospitals, Geneva, Switzerland, ${ }^{3}$ Department of Physics and Astronomy, University of Bologna, Bologna, Italy, ${ }^{4}$ Department of Biomedical and NeuroMotor Sciences, University of Bologna, Bologna, Italy, ${ }^{5}$ IRCCS Istituto delle Scienze Neurologiche di Bologna, Bologna, Italy, ${ }^{6}$ Functional Brain Mapping Lab, Department of Fundamental Neurosciences, University of Geneva, Geneva, Switzerland
\end{abstract}

Studies of functional neurosurgery and electroencephalography in Parkinson's disease have demonstrated abnormally synchronous activity between basal ganglia and motor cortex. Functional neuroimaging studies investigated brain dysfunction during motor task or resting state and primarily have shown altered patterns of activation and connectivity for motor areas. L-dopa administration relatively normalized these functional alterations. The aim of this pilot study was to examine the effects of L-dopa administration on functional connectivity in early-stage PD, as revealed by simultaneous recording of functional magnetic resonance imaging (fMRI) and electroencephalographic (EEG) data. Six patients with diagnosis of probable PD underwent EEG-fMRI acquisitions (1.5 T MR scanner and 64-channel cap) before and immediately after the intake of L-dopa. Regions of interest in the primary motor and sensorimotor regions were used for resting state $\mathrm{fMRI}$ analysis. From the EEG data, weighted partial directed coherence was computed in the inverse space after the removal of gradient and cardioballistic artifacts. fMRI results showed that the intake of L-dopa increased functional connectivity within the sensorimotor network, and between motor areas and both attention and default mode networks. EEG connectivity among regions of the motor network did not change significantly, while regions of the default mode network showed a strong tendency to increase their outflow toward the rest of the brain. This pilot study provided a first insight into the potentiality of simultaneous EEG-fMRI acquisitions in PD patients, showing for both techniques the analogous direction of increased connectivity after L-dopa intake, mainly involving motor, dorsal attention and default mode networks.

Keywords: Parkinson's disease, EEG-fMRI, functional connectivity, L-dopa, pilot study 


\section{INTRODUCTION}

Alterations in the temporal pattern of neuronal discharge have been found to be associated with parkinsonian symptoms (Marsden and Obeso, 1994; Obeso et al., 1997), but the central mechanisms underlying motor deficits in Parkinson's Disease (PD) is still unclear (Brown and Marsden, 1998; Jankovic, 2008).

Findings based on functional neurosurgery in PD patients suggest the existence of excessive neuronal synchronization mainly in the subthalamic nucleus (STN) and globus pallidus (Levy et al., 2002b; Williams et al., 2002; Brown, 2003), in particular within the beta band (13-30 Hz). It has been demonstrated that treatment with L-dopa not only reduces this synchronization, but it is also associated with a stronger synchronization within the gamma band (30-100 Hz) (Brown et al., 2001; Levy et al., 2002a,b; Priori et al., 2002; Williams et al., 2002; Brown, 2003). Moreover, abnormal synchronized activity in the basal ganglia may be coupled to activity in the motor cortex: oscillatory synchronization within and between cortical areas is increasingly recognized as a key mechanism in motor organization (Ohara et al., 2001; Serrien and Brown, 2003) and over the last few decades, electroencephalographic (EEG) studies have shown a high incidence of background and focal intermittent EEG slowing in PD (Yeager et al., 1966; McPherson, 1970). The correlation between motor disability and slowing of the background EEG suggests that this effect may be related to failure of nigrostriatal modulation of basal ganglia inputs to the cortex (Neufeld et al., 1988). In PD patients, relevant changes may also occur in the pattern of synchronization across distributed areas of the cortex (cortico-cortical coherence) (Silberstein et al., 2005; Hirschmann et al., 2011, 2013). This finding is especially significant in the beta and gamma bands, given the importance of cortico-cortical coherence at these frequencies in motor organization and the evidence that basal ganglia activity is preferentially synchronized in these bands. In particular, it has been shown that, when exploring resting state scalp EEG, there is a correlation between EEG-EEG coherence over the $10-35 \mathrm{~Hz}$ range and the severity of parkinsonism, and that the reduction of cortical coupling correlates with L-dopa therapy, STN stimulation and with the consequent clinical improvement (Silberstein et al., 2005). The frequency-specific functional connectivity between basal ganglia and cortex was investigated in PD patients also using simultaneous magnetoencephalography (MEG) and local field potentials recordings (Hirschmann et al., 2011, 2013). Coherent activity was observed in the beta range in the ipsilateral sensorimotor and premotor cortices, as well as in the alpha range in the ipsilateral temporal lobe, and, besides, it was found that beta coherence between primary motor cortex (M1) and STN was suppressed after the administration of L-dopa.

Brain magnetic resonance techniques are valuable methods to explore the pathophysiological bases of PD for both structural and functional aspects, and might provide new potential biomarkers for the in vivo differential diagnosis with atypical parkinsonism (Bajaj et al., 2017; Heim et al., 2017; Zanigni et al., 2017; Morisi et al., 2018). In particular, functional neuroimaging techniques have been mostly applied to give further insights into the pathophysiology of motor dysfunction in PD and to explore brain dysfunction underlying core motor symptoms such as bradykinesia and rigidity by using specific motor tasks during the MRI acquisitions (Rizzo et al., 2012). Overall, these studies mainly reported altered functional activation in supplementary motor area (SMA), pre-SMA, M1, premotor cortex, prefrontal cortex, parietal cortex, cingulum, basal ganglia, thalamus and cerebellum. Some discrepancies in the findings could be related to the stage of disease and/or to differences between the motor fMRI paradigms. L-dopa administration relatively normalized the functional activation patterns (Haslinger et al., 2001; Buhmann et al., 2003; Wu and Hallett, 2005). More recently, resting state fMRI has been used to investigate brain functional connectivity in PD patients. Typically, such patients show lower functional connectivity, compared to healthy subjects, for M1, SMA, dorsolateral prefrontal cortex, temporal lobe, putamen, substantia nigra, striatum, STN, thalamus, cerebellum and the default mode network, and alterations in connectivity were likewise appreciably normalized by L-dopa administration (Wu et al., 2009, 2011, 2012; Sharman et al., 2013). The differences among the results reported by resting state fMRI studies might depend, in addition to clinical samples and methodological differences, on resting state being an uncontrolled condition, with a risk of unstable wakefulness (Tagliazucchi and Laufs, 2014).

The combined acquisition of EEG and fMRI was originally developed with a clinical interest to investigate the neuronal activity and the hemodynamic response simultaneously to try to detect and localize epileptic discharges (Rosenkranz and Lemieux, 2010; Huster et al., 2012; Vitali et al., 2015). In the recent years, however, EEG-fMRI became a promising techniques also in experimental neuroscience, in psychiatric and behavioral disorders related to mesocorticolimbic dopamine pathway dysfunction, such as attention-deficit hyperactivity disorder or schizophrenia, in narcolepsy, and in neurodegenerative disorders, such as Alzheimer's disease (Boecker et al., 2014; Lei et al., 2014; Schneider et al., 2015; Drissi et al., 2016; Brueggen et al., 2017; Syed Nasser et al., 2019).

Given the evidence that both fMRI and EEG independently provided important insights into PD pathophysiology and specific functional alterations, the aim of this exploratory pilot study was to investigate the effect of acute L-dopa administration on functional connectivity in early-stage PD patients as revealed by simultaneous recording of hemodynamic (fMRI) and electrical (EEG) activity. The combined use of EEG and fMRI techniques guaranteed a controlled resting state acquisition and might provide a more comprehensive perspective on functional connectivity changes in PD: the simultaneous acquisition of these techniques gives the possibility to have both high temporal resolution EEG electrical measure and fMRI whole-brain neurometabolic evaluation with good spatial resolution at the same time and in the same group of patients.

\section{MATERIALS AND METHODS}

\section{Subjects}

Eight PD patients were recruited at the Movement Disorders outpatients center, IRCCS Istituto delle Scienze Neurologiche 
di Bologna, DIBINEM, University of Bologna, Italy. Inclusion criteria were: diagnosis of probable idiopathic PD, according to Gelb's criteria (Gelb et al., 1999); ongoing L-dopa therapy at stable dosage in at least two previous months (other drug therapies, such as dopaminergic agents, MAO or COMT inhibitors, were stopped during the week before the EEG-fMRI session); L-dopa efficacy documented by kinetic-dynamic monitoring within 1 year from the session. Exclusion criteria were: cognitive impairment and/or inability to provide informed consent; MR contraindications; neuroimaging findings inconsistent with PD diagnosis, severe involuntary movements; antidepressant or other therapies acting on central nervous system.

The same neurologist (GR) performed neurological evaluation of all patients using the Unified Parkinson's Disease Rating Scale (UPDRS) - III testing and the Hoehn and Yahr scale (HY) (Hoehn and Yahr, 1967).

This study was carried out in accordance with the Declaration of Helsinki. The protocol was approved by the Local Public Health Service (AUSL) of Bologna Ethics Committee and all participants provided written informed consent. No healthy controls were included, as the primary goal of the study was to compare connectivity patterns before and after the administration of L-dopa in PD patients.

\section{EEG-fMRI Acquisitions}

Each participant was instructed to go to bed at his or her preferred time on the evening before the examination, to fast after midnight, and not to take the first daily dose of L-dopa tablet $(100 \mathrm{mg})$ in the morning. All acquisitions were performed between 8 am and $1 \mathrm{pm}$. Patients first underwent UPDRSIII and HY testing before taking L-dopa (i.e., in the OFF state), then the MR compatible EEG cap was set-up. This was followed by the positioning in the MR scanner and the OFF state acquisition. Immediately afterward, L-dopa was administered and patients underwent UPDRS-III retesting every $10 \mathrm{~min}$ in order to identify the ON state (i.e., under L-dopa effect). Within 15 min following the onset of the effect of the medication, patients were repositioned in the MR scanner and the ON state EEG-fMRI was acquired. Scalp EEG was recorded with an MRcompatible 64-channel cap (Brain Vision), according to the 10-20 International System (reference: central top electrode, near $\mathrm{Cz}$ ). The recording resolution was $0.1 \mu \mathrm{V}$ and the sampling frequency was $5 \mathrm{kHz}$. The ECG trace was continuously recorded at the same sampling frequency by means of a specific ECG cable.

Brain MRI acquisitions were performed using a $1.5 \mathrm{~T}$ scanner (GE Medical Systems Signa HDx 15) equipped with an 8channel phased array brain coil. Participants were instructed to stay during the scan awake, relaxed and motionless with their eyes closed, and to avoid goal-directed attention. Simultaneously to the EEG recordings, two resting state runs were acquired during the $\mathrm{OFF}$ and the $\mathrm{ON}$ conditions $(13 \mathrm{~min}$ for each condition) with a pure axial GE-EPI sequence (Gradient EchoEcho Planar Imaging, TR/TE $=3000 / 40 \mathrm{~ms}$, flip angle $=90^{\circ}$, $\mathrm{FOV}=24 \mathrm{~cm}$, voxel $=1.875 \times 1.875 \times 4 \mathrm{~mm}$ ); for each run the first five volumes were discarded. A high-resolution volumetric sequence was acquired at the end of the session (T1-weighted
Fast SPoiled Gradient, TR/TE/TI $=12 / 5 / 600 \mathrm{~ms}, \mathrm{FOV}=25.6 \mathrm{~cm}$, voxel $=1 \mathrm{~mm}^{3}$ ).

\section{Data Analysis}

Subjects' UPDRS rating was compared between OFF and ON states with the Wilcoxon test.

\section{EEG}

Electroencephalographic signal preprocessing was performed with Brain Vision Analyzer 2.0 software. This included gradient artifact off-line correction and EEG signal filtering (Allen et al., 2000). A 50- $\mathrm{Hz}$ low-pass filter was also applied to remove the remaining artifact. The ballistocardiogram and eye-movements and blinking artifacts were removed by Independent Components Analysis (ICA) (Bénar et al., 2003). Finally, signals were down-sampled to $250 \mathrm{~Hz}$ and examined by visual inspection to remove sections containing muscular artifacts and sleep patterns. In both conditions, the artifact-free sections were concatenated.

To perform EEG-directed connectivity analysis, a previously published approach (Coito et al., 2015, 2016) was used, and is summarized below. For regional electrical source imaging (ESI), the forward model consisted of a simplified realistic head model [Locally Spherical Model with Anatomical Constraints, LSMAC (Birot et al., 2014)]. Based on the MNI template, about 5000 solution points were equally distributed within gray matter, which represented the solution space. A linear distributed inverse solution with biophysical constraints was then used to calculate the 3D current density distribution [Local AutoRegressive Averages, LAURA (Grave de Peralta Menendez et al., 2004)]. The brain was then parcellated into 82 regions of interest (ROIs) using the AAL atlas, and the source activity of the solution point closest to the geometric center of each ROI was considered as the representative source activity of the ROI. To account for the changing tridimensional orientation of the source dipoles, these were projected at each time point on the predominant dipole direction of each ROI to obtain scalar values of the current density. Directed functional connectivity was determined with a Granger-causality measure: the time-varying weighted Partial Directed Coherence (wPDC) (Astolfi et al., 2008; Van Mierlo et al., 2011; Plomp et al., 2014). This gave a 4-dimensional matrix $(82$ ROIs $\times 82$ ROIs $\times$ time $\times$ frequency) that was then reduced to the theta, alpha and beta frequency bands and averaged over time. Specifically, The time-frequency distribution of the power spectral density (PSD) was calculated using the S-transform (ST). To determine the PSD for each voxel in the inverse space, ST was computed for each scalp electrode, and source estimation was then applied to this frequency-domain complex data. The mean PSD for each patient was computed and normalized (0-1) across regions, time, and frequencies $(1-100 \mathrm{~Hz})$ by subtracting the minimum power and dividing by the range. The PDC (partial directed coherence) was analyzed for each frequency band. Time-varying PDC estimates directed interactions between pairs of signals in the time and frequency domain using adaptive multivariate autoregressive models (AMVAR). We estimated the time-varying AMVAR parameters by means of a recursive least squares (RLS) algorithm (Astolfi et al., 2008). PDC values were 
scaled, in the same way as the ST, and multiplied by the spectral power (weighted PDC, wPDC) (Van Mierlo et al., 2011; Plomp et al., 2014). The summed outflow for a given ROI and time point was defined as the sum of wPDC values from that ROI to all others ROIs at that time point. For each time point, the summed outflow for a given ROI and time point was then computed, as the sum of WPDC values from that ROI to all others. For each patient, the summed outflow of each region at each time point of the OFF segments was compared to each time point of the ON segments with a non-parametric test (MannWhitney-Wilcoxon, $p<0.05$ ). As 82 different regions are tested at each band, a Bonferroni multiple comparisons correction is considered. EEG connectivity analysis and ESI were carried out using the software Cartool ${ }^{1}$ and Matlab 2012b (MathWorks Inc.).

\section{fMRI}

Functional magnetic resonance imaging data processing and analysis were mainly performed using FSL (version 4.1.4) (Jenkinson et al., 2012). Functional connectivity was investigated with a seed-based approach, using four distinct ROIs: right and left supplementary motor area (R-SMA, L-SMA), and right and left precentral gyri (R-PG, L-PG). Seeds were drawn in the MNI space as spheres of radius $5 \mathrm{~mm}$ centered at the following coordinates: R-SMA $(6,0,54)$, L-SMA $(-6,0,54), \mathrm{R}-\mathrm{PG}(44,-8$, $38)$, and L-PG $(-44,-8,38)$.

The measured ECG trace was used to remove cardiac physiological noise from fMRI data by means of a retrospective method [RETROICOR, RETROspective Image-based CORrection (Glover et al., 2000)]. We used the RETROICOR implementation in AFNI [version AFNI_2008_07_18_1710), that follows the original method described by Glover et al. (2000)]. The input data were raw fMRI data with only volume registration performed. Since respiration could not be monitored during the acquisitions due to technical limitations, breathing noise was estimated with PESTICA [Physiologic EStimation by Temporal ICA (Beall and Lowe, 2007)] from the data themselves. The respiration timecourses obtained in this way were then included in RETROICOR correction as well.

For two patients (Table 1, subjects 2 and 5) the disease symptoms were on the left at onset, corresponding to the right brain hemisphere, so we flipped MR functional and structural images in the right/left direction, in order to have all patients' predominantly affected hemisphere on the left side of the brain. Structural images were then registered to a symmetrical version of MNI template created by copying, flipping along the $\mathrm{x}$ axis and averaging the original and the mirrored version of the template, similarly to the procedure used by Kwak et al. (2012).

Functional MR data preprocessing also included high-pass filtering (cut-off $=100 \mathrm{~s}$ ), motion correction (motion parameters were then added as confounding variables to the model), slice timing correction, brain extraction and spatial smoothing (Gaussian filter, FWHM $=6 \mathrm{~mm}$ ). Functional data were aligned to structural images using a linear registration (flirt) and structural images were non-linearly aligned to standard MNI space (fnirt), so that, combining the two steps, functional images could be

${ }^{1}$ https://sites.google.com/site/cartoolcommunity/home registered to MNI as well. Once ROIs were aligned to fMRI space, their voxel time series were averaged. The first-level of the analysis was based on a general linear model, constructed with the time series of each seed used separately as a regressor. Clusters were determined in Z-statistic images $(|Z| \geq 2.3)$ and the family wise-error was controlled with a cluster significance threshold of $p=0.05$. A second level within-subject fixed-effects analysis was then performed, in order to combine the results of the two OFF resting state runs and the two ON runs, giving each subject's mean response for each of the two conditions. Finally, the thirdlevel analysis was a mixed-effects group statistics: a two-sample paired $t$-test was performed to compare the connectivity with each seed before and after L-dopa. Clusters were determined with a threshold of $Z=2.3$ then family wise-error was controlled with a cluster significance threshold of $p=0.05$. We did not explore whether the variations of brain connectivity correlated with the disease severity because of the small cohort and the homogeneity of the UPDRS motor score in the OFF state.

\section{RESULTS}

Two out of eight patients were unable to complete the EEG-fMRI acquisition protocol. Demographic and clinical data are reported in Table 1.

Patients' motor performances as assessed by the UPDRS were significantly ameliorated when on medication than when off medication (UPDRS score reduction after L-dopa: 49\%, $p<0.05$ ).

\section{fMRI}

A significantly higher connectivity with all four seeds was found for the contrast ON vs. OFF (Figure 1 and Table 2). Brain areas showing increased connectivity with both L-PG and R-PG after L-dopa intake were right angular gyrus, middle cingulate gyrus, posterior cingulate gyrus, bilateral middle frontal gyrus, superior frontal gyrus, superior occipital cortex, superior parietal gyrus, postcentral gyrus, precentral gyrus, precuneus and SMA, while left middle and posterior cingulate gyri showed increased connectivity only with L-PG.

As for the SMA, L-SMA showed increased connectivity with right angular gyrus, posterior cingulate gyrus, left superior occipital cortex, bilateral middle cingulate gyrus, middle and superior frontal gyri, superior parietal gyrus, postcentral gyrus, precentral gyrus, precuneus and SMA, while R-SMA had an increased connectivity only with right middle and superior frontal giri and right precentral gyrus. The group analysis showed no significant variations in connectivity for the contrast OFF vs. ON.

\section{EEG}

When evaluating the connections among the four seeds of the motor system (R-PG, L-PG, R-SMA, and L-SMA) we did not find any significant difference in PDC in ON vs. OFF $(p>0.05)$ for all the studied frequencies (alpha, beta, and theta). Although not significant, a trend toward a decreased FC in ON vs. OFF among the different structures of the motor system was observed for each frequency. 
TABLE 1 | Demographic and clinical data of the patients at the time of the MR session.

\begin{tabular}{|c|c|c|c|c|c|c|c|c|}
\hline $\mathbf{N}$ & 1 & 2 & 3 & 4 & 5 & 6 & Mean & $(S D)$ \\
\hline Age (yrs) & 46 & 58 & 52 & 46 & 48 & 57 & 51.2 & $(5.4)$ \\
\hline Sex & $\mathrm{F}$ & $\mathrm{M}$ & $\mathrm{F}$ & $\mathrm{M}$ & $M$ & $\mathrm{M}$ & - & - \\
\hline Age at onset (yrs) & 60 & 55 & 48 & 41 & 54 & 51 & 51.5 & $(6.5)$ \\
\hline Side of onset & $\mathrm{R}$ & $L$ & $\mathrm{R}$ & $\mathrm{R}$ & $L$ & $\mathrm{R}$ & - & - \\
\hline Disease duration (yrs) & 6 & 3 & 4 & 5 & 4 & 6 & 4.7 & $(1.2)$ \\
\hline HY stage & 2 & 2 & 2 & 2 & 2 & 2 & 2 & - \\
\hline UPDRS III - OFF & 19 & 17 & 18 & 17 & 19 & 15 & 17.5 & $(1.5)$ \\
\hline UPDRS III - ON & 9 & 6 & 10 & 8 & 10 & 10 & 8.8 & (1.6) \\
\hline UPDRS III reduction (\%) & 53 & 65 & 44 & 53 & 48 & 33 & $49.3^{*}$ & $(10.7)$ \\
\hline
\end{tabular}

Yrs, years; HY, Hoehn and Yahr staging of severity of Parkinson disease; UPDRS, unified Parkinson's Disease Rating Scale reported as score; *significant ( $p$ < 0.05).
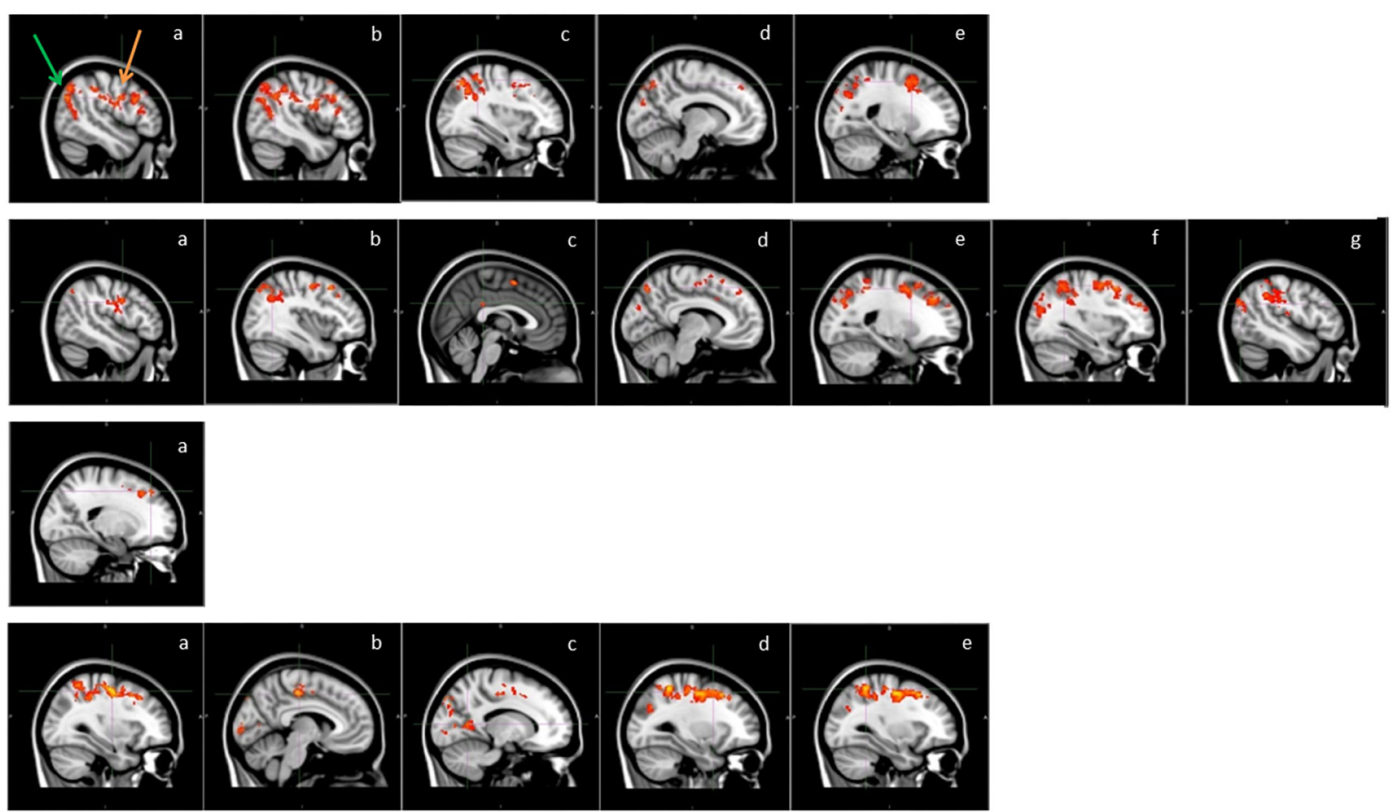

FIGURE 1 | Functional connectivity in the group analysis of ON state vs. OFF state; images show sagittal views of the main areas with significant clusters of voxels, superimposed on the MNI template; box R-PG: connectivity with seed in right precentral gyrus (a: occipital cortex -green arrow-, motor areas -orange arrow-, b: angular gyrus, c: superior parietal lobe, d: precuneus, e: superior frontal gyrus); box L-PG: connectivity with seed in left precentral gyrus (a: motor areas, b: superior frontal gyrus, c: superior parietal lobe, d: angular gyrus, e: precuneus, f: cingulum, g: occipital cortex); box R-SMA: connectivity with seed in right SMA (a: frontal pole - superior frontal gyrus); box L-SMA: connectivity with seed in left SMA (a,b: motor areas, c: precuneus, d: middle/superior frontal gyrus, e: superior parietal lobe).

Similarly, no significant differences for ON vs. OFF were observed when evaluating whether the motor system was changing its outflow (in term of summed outflow from the four ROIs) toward the remaining regions of the brain. In this case as well, we can observe a tendency toward a decreased summed outflow from the motor system, especially concerning the alpha and the theta bands, after L-dopa intake.

As for the PDC from each ROI of the whole brain, we observed a strong tendency $(p<0.06)$ toward a change in the summed outflow between ON and OFF conditions mainly in the following structures: posterior cingulate, left amygdala, left hippocampus, right anterior cingulate and right lingual gyrus in the alpha band; left amygdala, bilateral hippocampi and right anterior cingulate in the beta band; posterior cingulate, left amygdala, bilateral hippocampi and right anterior cingulate in the theta band (Supplementary Figures 1-3).

Although this difference was not statistically significant, an interesting common behavior is that in the OFF state, the strongest connections originated from the posterior regions of the default mode network (DMN), while in ON they came from the anterior cingulate, part of the anterior portion of the DMN (Figure 2). Specifically, in the alpha band the main driver of connection is the posterior cingulate in the OFF state and the anterior cingulate in the $\mathrm{ON}$ state; in the beta band the main driver of connections is the right hippocampus in the OFF state and the anterior cingulate in the ON state; in the theta band the main driver of connections is the right hippocampus in the OFF state and the anterior cingulate in the ON state. 


\section{DISCUSSION}

This exploratory pilot study is the first that describes the potentialities of resting state functional connectivity measured simultaneously with EEG and fMRI in early-stage PD patients to describe acute L-dopa effect.

Resting state fMRI results showed, after the administration of L-dopa, an increased bilateral connectivity of PG and SMA with cortical areas that belong to three resting state brain networks: sensorimotor, DMN and dorsal-attention network (Figure 1 and Table 2). The sensorimotor network deals with the integration of sensitive and motor stimuli and its characteristic spatial pattern includes pre- and post-central gyri, extending from the superior bank of the sylvian fissure to the medial wall of the interhemispheric fissure and the SMA (Damoiseaux et al., 2006).

Our findings of increased connectivity within the sensorimotor system after the intake of L-dopa are in line with previous results (Esposito et al., 2013) that showed enhanced function connectivity within the SMA in drugnaïve PD patients after acute L-dopa administration. Effects of L-dopa administration on resting connectivity were also investigated in patients with advanced $\mathrm{PD}$, and a higher resting state functional connectivity was found between striatum and prefrontal cortex, and a lower connectivity between pallidum, STN, and supplementary and primary motor cortices (Akram et al., 2017). Compared to previous studies (Wu et al., 2012;
Esposito et al., 2013; Akram et al., 2017) we detected limited variations of functional connectivity between the motor cortex and deep brain structures: this result may be related to differences in clinical samples and methodological approaches.

The DMN is typically constituted by ventro-medial prefrontal cortex, cingulate cortex, inferior parietal lobule, lateral temporal cortex, dorsal medial prefrontal cortex and hippocampus, and it is particularly active during rest condition while it deactivates when specific goal-directed behavior is needed (Damoiseaux et al., 2006). For PD patients, a stronger connection of DMN with PG and SMA after the administration of L-dopa may contribute to an increased readiness to plan and perform movements. In line with this hypothesis, Krajcovicova et al. (2012) found no differences in DMN integrity between PD patients on dopaminergic medication and healthy controls, suggesting that dopaminergic therapy may have specific effects on restoring default mode functional integrity. It has also been shown (Van Eimeren et al., 2009) that in PD patients, deficits in executive tasks such as planning and set-shifting were associated with less deactivation of posterior cingulate cortex and precuneus. On the other hand, a resting state fMRI study in cognitively unimpaired PD patients (Tessitore et al., 2012b) showed lower DMN functional connectivity in patients compared to healthy controls, and this impairment was uncorrelated with the L-dopa dosage.

The dorsal attention system is crucial in voluntary orientation and attention and it mainly involves the intraparietal sulcus,

TABLE 2 | Brain areas showing a significant variation in fMRI connectivity with the four seeds (R-PG, L-PG, R-SMA, and L-SMA) when comparing the ON and the OFF state.

\begin{tabular}{|c|c|c|c|c|c|c|c|c|c|}
\hline \multirow[t]{2}{*}{ BRAIN AREAS } & \multirow[t]{2}{*}{ BRODMANN AREAS } & \multicolumn{2}{|c|}{$\mathbf{R}-\mathbf{P G}$} & \multicolumn{2}{|c|}{ L-PG } & \multicolumn{2}{|c|}{ R-SMA } & \multicolumn{2}{|c|}{ L-SMA } \\
\hline & & VOXELS & Z MAX & VOXELS & Z MAX & VOXELS & $Z$ MAX & VOXELS & Z MAX \\
\hline Angular gyrus $L$ & $39,19,22$ & 72 & 2.83 & 33 & 2.86 & 0 & 0 & 0 & 0 \\
\hline Angular gyrus $\mathrm{R}$ & $39,19,22$ & 685 & 3.29 & 195 & 2.97 & 0 & 0 & 51 & 2.80 \\
\hline Middle Cingulate L & $31,32,23,24$ & 0 & 0.00 & 7 & 2.50 & 0 & 0 & 78 & 3.19 \\
\hline Middle Cingulate R & $31,32,23,24$ & 13 & 2.57 & 3 & 2.46 & 0 & 0 & 31 & 2.60 \\
\hline Posterior Cingulate L & $31,23,118$ & 0 & 0.00 & 4 & 2.74 & 0 & 0 & 0 & 0 \\
\hline Posterior Cingulate R & $31,23,118$ & 40 & 3.01 & 23 & 2.91 & 0 & 0 & 5 & 2.63 \\
\hline Middle Frontal L & $6,8,9,10$ & 259 & 2.88 & 696 & 3.29 & 0 & 0 & 469 & 3.36 \\
\hline Middle Frontal R & $6,8,9,10$ & 211 & 3.04 & 361 & 3.31 & 363 & 3.09 & 398 & 3.32 \\
\hline Superior Frontal L & $6,8,9,10$ & 132 & 2.97 & 413 & 3.20 & 0 & 0 & 196 & 3.12 \\
\hline Superior Frontal R & $6,8,9,10$ & 257 & 2.91 & 356 & 3.05 & 158 & 2.99 & 233 & 3.24 \\
\hline Superior Occipital L & $18,19,7,31$ & 187 & 2.98 & 130 & 3.04 & 0 & 0 & 144 & 3.07 \\
\hline Superior Occipital R & $18,19,7,31$ & 53 & 2.81 & 39 & 2.91 & 0 & 0 & 0 & 0 \\
\hline Superior Parietal L & 7,19 & 167 & 2.91 & 207 & 2.90 & 0 & 0 & 162 & 3.35 \\
\hline Superior Parietal R & 7,19 & 115 & 2.95 & 17 & 2.77 & 0 & 0 & 191 & 3.12 \\
\hline Postcentral gyrus L & $1,2,3,40,43$ & 201 & 2.90 & 235 & 2.86 & 0 & 0 & 373 & 3.23 \\
\hline Postcentral gyrus R & $1,2,3,40,43$ & 121 & 2.93 & 134 & 3.11 & 0 & 0 & 164 & 3.19 \\
\hline Precentral gyrus L & $3,4,6$ & 15 & 2.54 & 154 & 2.99 & 0 & 0 & 370 & 3.38 \\
\hline Precentral gyrus R & $3,4,6$ & 61 & 3.05 & 264 & 3.08 & 11 & 2.61 & 250 & 3.46 \\
\hline Precuneus L & $31,7,23,29$ & 57 & 2.79 & 59 & 2.96 & 0 & 0 & 8 & 2.46 \\
\hline Precuneus R & $31,7,23,29$ & 141 & 2.85 & 6 & 2.35 & 0 & 0 & 62 & 2.81 \\
\hline SMA L & 6 & 18 & 2.81 & 150 & 3.30 & 0 & 0 & 57 & 2.88 \\
\hline SMAR & 6 & 1 & 2.33 & 58 & 3.25 & 0 & 0 & 97 & 2.84 \\
\hline
\end{tabular}

L, left; $R$, right; SMA, supplementary motor area; PG, precentral gyrus. 


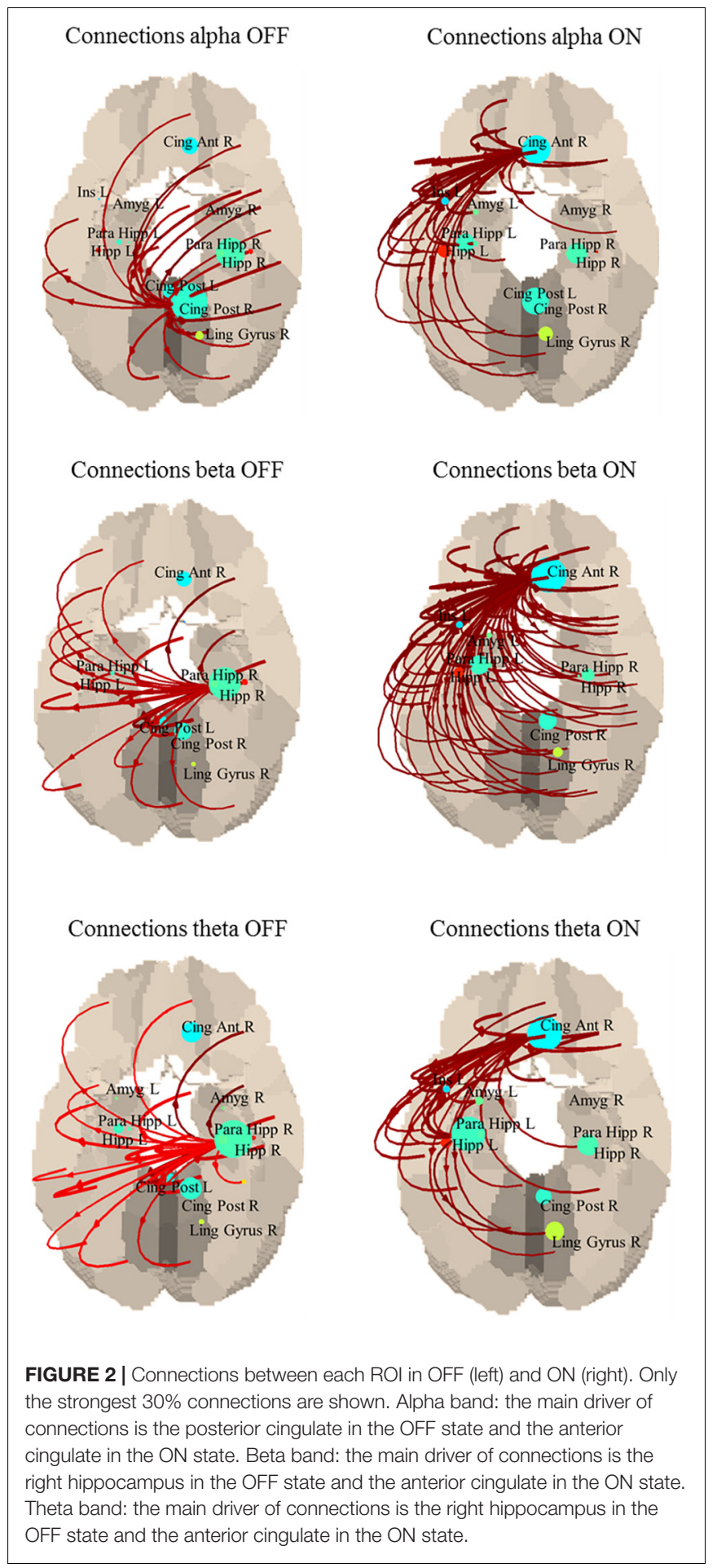

the frontal eye field, the junction of precentral and superior frontal sulci, and middle and superior frontal gyri (Damoiseaux et al., 2006). In our study, structures of attentive and executive systems showed increased functional connectivity with cortical motor areas after L-dopa administration. This may suggest that dopaminergic medication could be of help to PD patients as for their executive dysfunction, by improving the cognitive, attentional and executive steps, essential for movements preparation. When activations and connectivity were investigated during the performance of a motor-attentional task (Rowe et al., 2002), an attentional modulation of connectivity was observed only in the healthy subjects, but not in PD patients. In particular, the attention to action led to further activations of prefrontal, parietal, and para-cingulate cortices and SMA. The engagement of the attentional control network was also investigated in PD and a lower activation of frontal and parietal hubs of the dorsal attention network was described (Shine et al., 2014). Reduced functional connectivity has been reported within both the executive-attention and visual networks in PD patients with freezing compared to those without (Tessitore et al., 2012a), suggesting that freezing may be related to a dysfunction of the visuo-spatial network. As none of our early-stage PD patients presented freezing, the freezing related network may be impaired before clinical manifestation.

Partial directed coherence analysis of EEG data acquired during the fMRI scans revealed no statistically significant motor network changes when comparing ON vs. OFF. Nevertheless, the results suggest an interesting tendency toward a decreased coherence after the intake of L-dopa within the motor system and between the motor system and the rest of the brain. However, the effect of L-dopa on cortico-cortical coherence is still debated and opposing results can be found in the literature. Previous resting state scalp EEG studies (Silberstein et al., 2005; Hirschmann et al., 2013) have shown that STN-cortical and cortico-cortical coherence correlate with the severity of parkinsonism and that this coupling was modulated by L-dopa therapy and STN stimulation. When MEG and subthalamic local field potential recordings were used to investigate connectivity in PD patients (Litvak et al., 2011), two major spatio-temporal patterns of coupling between cortex and STN were observed, in alpha and beta bands. In the alpha band, coherence between STN and bilateral temporoparietal cortex and brainstem has been described, suggesting a possible attentional role; in the beta band the pattern involved the STN and the ipsilateral anterior parietal and frontal cortices, suggesting an involvement in the executive functions. In this study, dopaminergic medication increased beta coherence between STN and prefrontal cortex. However, later studies did not confirm this effect of pharmacological intervention on cortical coherence. For example, the evaluation of the direct transfer function between STN and cortex at rest and during movement (Lalo et al., 2008), with and without pharmacological dopaminergic input, showed that the beta band coherence did not change after dopaminergic therapy. MEG coherence among SMA and other motor areas has also been investigated during resting and during isometric muscular contraction (Pollok et al., 2013), before and after L-dopa intake. Interestingly, an increased SMA-M1 coherence in OFF during isometric contraction was found, and it was remedied by L-dopa. Nevertheless, coherence strength for the resting state did not differ after L-dopa intake, suggesting that SMA-M1 coherence might be more related to movement execution than to the rest condition. Besides, 
a high-density EEG study investigated the effect of acute L-dopa administration on movement-related cortical oscillations, showing that L-dopa reduce the exaggerated movementrelated beta-band desynchronization in the SMA that was observed in PD patients compared to healthy subjects (Chung et al., 2018) and that functional brain activity in the basal ganglia pathways relate to the response of beta-band cortical activity to levodopa.

When whole brain outflow was considered, the regions that showed a stronger tendency to change their connectivity are the posterior and anterior cingulate, and the right hippocampus, mainly ascribable to the DMN. This is quite interesting as changes in the connectivity with the DMN were also observed in our fMRI results, and we discussed above the crucial role of this network and its connections with the sensorimotor areas in PD patients. Moreover, there seems to be a shift of the involvement in connections toward the anterior subpart of DMN after the intake of L-dopa. Structural and functional alterations of cingulate cortex has been previously shown in PD patients. For example, increased functional connectivity and higher eigenvector centrality (a network measure that allow to identify prominent nodes in the whole brain network) in the posterior cingulate gyrus and lower centrality for the anterior cingulate gyrus (de Schipper et al., 2018), white matter microstructural alterations in the cingulate bundle near the orbital and anterior cingulate gyrus (Albrecht et al., 2019), or a loss of integrity in cingulate structural covariance network, for both anterior and posterior cingulate cortices (de Schipper et al., 2017). It would be suggestive to hypothesize that what we see is a relative normalization of the connectivity pattern, speculating about different roles of anterior and posterior cingulate cortices, but it might be a rash speculation, as a group of healthy controls is not available and there are no similar findings in literature to compare with.

The absence of clear PDC findings in the present study may be related to methodological limitations. Simultaneous recordings to MRI induces artifacts in the EEG, reducing the quality of the trace. The gradient artifact is the most important one, with an amplitude of about 50 times the background EEG. The most widely used method to remove it consists of estimating the artifact and subtracting it from each frame (Bénar et al., 2003). In our case, the frequency removed by the gradient artifact correction was within the beta band. Furthermore, when removing the cardioballistic artifact by ICA, there is also a risk of removing components of signal which are actually coming from the brain. A precise description of the influence of MRI gradients and ECG artifacts on multivariate measures performed on EEG signals, such as PDC, is still lacking. Further exploration of these effects by using various acquisition protocols and scanning equipment are necessary to properly evaluate the method's sensitivity to these artifacts.

The main limit of this exploratory study is the small number of recruited patients. This poses substantial limitation to the generalizability of the results, indeed the present study should be considered a pilot investigation, that might show the potentialities of EEG and fMRI combination in PD patients, but without any presumptions of fully reliable and solid conclusions. Further studies in larger samples are needed to confirm and expand the present results. However, the sample is very homogeneous as all $\mathrm{PD}$ patients are in an early stage of the disease course with comparable motor impairment. Despite the small cohort, both fMRI and EEG findings are directionally similar, showing increased connectivity after L-dopa intake in PD patients, particularly for motor areas and their connections with dorsal attention and DMN areas. Taking into consideration the novelty of this approach in PD patients, this pilot study showed the potentialities of this methodology to better understand the mechanisms underlying electrical and hemodynamic functional connectivity changes in these patients.

\section{DATA AVAILABILITY}

The datasets generated for this study are available on request to the corresponding author.

\section{ETHICS STATEMENT}

This study was carried out in accordance with the Declaration of Helsinki. The protocol was approved by the Local Public Health Service (AUSL) of Bologna Ethics Committee and all participants provided written informed consent.

\section{AUTHOR CONTRIBUTIONS}

FP, ClT, GR, PC, GC-B, CaT, RL, and PT contributed to the conception of the study. SE, FP, ClT, GR, LG, LF, FB, CB, DM, and CaT contributed to the acquisitions. SE, FP, ClT, LF, AC, and LT contributed to the data analysis. SE, FP, ClT, GR, LF, AC, PC, GC, CaT, RL, and PT contributed to the results interpretation. SE, $\mathrm{FP}$, and ClT contributed to manuscript preparation. All authors contributed to the manuscript revision and approved the final version of the manuscript.

\section{FUNDING}

CSI “Central Nervous System Imaging," Project co-funded by the ENIAC Joint Undertaking under the SUB-PROGRAMME SP1 - Nanoelectronics for Health and Wellness ENIAC JU Grant Agreement No. 120209.

\section{SUPPLEMENTARY MATERIAL}

The Supplementary Material for this article can be found online at: https://www.frontiersin.org/articles/10.3389/fnins.2019. 00611/full\#supplementary-material 


\section{REFERENCES}

Akram, H., Wu, C., Hyam, J., Foltynie, T., Limousin, P., De Vita, E., et al. (2017). 1-Dopa responsiveness is associated with distinctive connectivity patterns in advanced Parkinson's disease. Mov. Disord. 32, 874-883. doi: 10.1002/mds. 27017

Albrecht, F., Ballarini, T., Neumann, J., and Schroeter, M. L. (2019). FDG-PET hypometabolism is more sensitive than MRI atrophy in Parkinson's disease: a whole-brain multimodal imaging meta-analysis. Neuroimage Clin. 21:101594. doi: 10.1016/j.nicl.2018.11.004

Allen, P. J., Josephs, O., and Turner, R. (2000). A method for removing imaging artifact from continuous EEG recorded during functional MRI. NeuroImage 12, 230-239. doi: 10.1006/nimg.2000.0599

Astolfi, L., Cincotti, F., Mattia, D., De Vico Fallani, F., Tocci, A., Colosimo, A., et al. (2008). Tracking the time-varying cortical connectivity patterns by adaptive multivariate estimators. IEEE Trans. Biomed. Eng. 55, 902-913. doi: 10.1109/ TBME.2007.905419

Bajaj, S., Krismer, F., Palma, J. A., Wenning, G. K., Kaufmann, H., Poewe, W., et al. (2017). Diffusion-weighted MRI distinguishes Parkinson disease from the parkinsonian variant of multiple system atrophy: a systematic review and meta-analysis. PLoS One 12:e0189897. doi: 10.1371/journal.pone.0189897

Beall, E. B., and Lowe, M. J. (2007). Isolating physiologic noise sources with independently determined spatial measures. NeuroImage 37, 1286-1300. doi: 10.1016/j.neuroimage.2007.07.004

Bénar, C., Aghakhani, Y., Wang, Y., Izenberg, A., Al-Asmi, A., Dubeau, F., et al. (2003). Quality of EEG in simultaneous EEG-fMRI for epilepsy. Clin. Neurophysiol. 114, 569-580. doi: 10.1016/S1388-2457(02)00383-8

Birot, G., Spinelli, L., Vulliémoz, S., Mégevand, P., Brunet, D., Seeck, M., et al. (2014). Head model and electrical source imaging: a study of 38 epileptic patients. Neuroimage Clin. 5, 77-83. doi: 10.1016/j.nicl.2014.06.005

Boecker, R., Holz, N. E., Buchmann, A. F., Blomeyer, D., Plichta, M. M., Wolf, I., et al. (2014). Impact of early life adversity on reward processing in young adults: EEG-fMRI results from a prospective study over 25 years. PLoS One 9:e104185. doi: 10.1371/journal.pone.0104185

Brown, P. (2003). Oscillatory nature of human basal ganglia activity: relationship to the pathophysiology of Parkinson's disease. Mov. Disord. 18, 357-363. doi: $10.1002 / \mathrm{mds} .10358$

Brown, P., and Marsden, C. D. (1998). What do the basal ganglia do? Lancet 351, 1801-1804. doi: 10.1016/S0140-6736(97)11225-9

Brown, P., Oliviero, A., Mazzone, P., Insola, A., Tonali, P., and Di Lazarro, V. (2001). Dopamine dependency of oscillations between subthalamic nucleus and pallidum in Parkinson's disease. J. Neurosci. 21, 1033-1038. doi: 10.1523/ JNEUROSCI.21-03-01033.2001

Brueggen, K., Fiala, C., Berger, C., Ochmann, S., Babiloni, C., and Teipel, S. J. (2017). Early changes in alpha band power and DMN BOLD activity in alzheimer's disease: a simultaneous resting state EEG-fMRI study. Front. Aging Neurosci. 6:319. doi: 10.3389/fnagi.2017.00319

Buhmann, C., Glauche, V., Sturenburg, H. J., Oechsner, M., Weiller, C., and Buchel, C. (2003). Pharmacologically modulated fMRI-cortical responiveness to levodopa in drug-naive hemiparkinsonian patients. Brain 126, 451-461. doi: 10.1093/brain/awg033

Chung, J. W., Burciu, R. G., Ofori, E., Coombes, S. A., Christou, E. A., Okun, M. S., et al. (2018). Beta-band oscillations in the supplementary motor cortex are modulated by levodopa and associated with functional activity in the basal ganglia. Neuroimage Clin. 18, 559-571. doi: 10.1016/j.nicl.2018.05.021

Coito, A., Genetti, M., Pittau, F., Iannotti, G. R., Thomschewski, A., Höller, Y., et al. (2016). Altered directed functional connectivity in temporal lobe epilepsy in the absence of interictal spikes: a high density EEG study. Epilepsia 57, 402-411. doi: $10.1111 /$ epi.13308

Coito, A., Plomp, G., Genetti, M., Abela, E., Wiest, R., Seeck, M., et al. (2015). Dynamic directed interictal connectivity in left and right temporal lobe epilepsy. Epilepsia 56, 207-217. doi: 10.1111/epi.12904

Damoiseaux, J. S., Rombouts, S. A., Barkhof, F., Scheltens, P., Stam, C. J., Smith, S. M., et al. (2006). Consistent resting-state networks across healthy subjects. PNAS 103, 13848-13853. doi: 10.1073/pnas.0601417103

de Schipper, L. J., Hafkemeijer, A., van der Grond, J., Marinus, J., Henselmans, J. M. L., and van Hilten, J. J. (2018). Altered whole-brain and network-based functional connectivity in parkinson's disease. Front. Neurol. 9:419. doi: 10. 3389/fneur.2018.00419

de Schipper, L. J., van der Grond, J., Marinus, J., Henselmans, J. M. L., and van Hilten, J. J. (2017). Loss of integrity and atrophy in cingulate structural covariance networks in Parkinson's disease. Neuroimage Clin. 15, 587-593. doi: 10.1016/j.nicl.2017.05.012

Drissi, N. M., Szakács, A., Witt, S. T., Wretman, A., Ulander, M., Ståhlbrandt, H., et al. (2016). Altered brain microstate dynamics in adolescents with narcolepsy. Front. Hum. Neurosci. 3:369. doi: 10.3389/fnhum.2016.00369

Esposito, F., Tessitore, A., Giordano, A., De Micco, R., Paccone, A., Conforti, R., et al. (2013). Rhythm-specific modulation of the sensorimotor network in drug-naive patients with Parkinson's disease by levodopa. Brain 136, 710-725. doi: 10.1093/brain/awt007

Gelb, D. J., Oliver, E., and Gilman, S. (1999). Diagnostic criteria for Parkinson disease. Arch Neurol. 56, 33-39.

Glover, G. H., Li, T. Q., and Ress, D. (2000). Image-based method for retrospective correction of physiological motion effects in fMRI: RETROICOR. Magn. Reson. Med. 44, 162-167. doi: 10.1002/1522-2594(200007)44:1<162::aid-mrm23>3.3. co;2-5

Grave de Peralta Menendez, R., Murray, M. M., Michel, C. M., Martuzzi, R., and Gonzalez Andino, S. L. (2004). Electrical neuroimaging based on biophysical constraints. Neuroimage 21, 527-539. doi: 10.1016/j.neuroimage.2003.09.051

Haslinger, B., Erhard, P., Kampfe, N., Boecker, H., Rummeny, E., Schwaiger, M., et al. (2001). Event-related functional magnetic resonance imaging in Parkinson's disease before and after levodopa. Brain 124, 558-570. doi: 10.1093/ brain/124.3.558

Heim, B., Krismer, F., De Marzi, R., and Seppi, K. (2017). Magnetic resonance imaging for the diagnosis of Parkinson's disease. J. Neural Transm. 124, 915964. doi: 10.1007/s00702-017-1717-8

Hirschmann, J., Özkurt, T. E., Butz, M., Homburger, M., Elben, S., Hartmann, C. J., et al. (2011). Distinct oscillatory STN-cortical loops revealed by simultaneous MEG and local field potential recordings in patients with Parkinson's disease. NeuroImage 55, 1159-1168. doi: 10.1016/j.neuroimage.2010.11.063

Hirschmann, J., Özkurt, T. E., Butz, M., Homburger, M., Elben, S., Hartmann, C. J., et al. (2013). Differential modulation of STN-cortical and cortico-muscular coherence by movement and levodopa in Parkinson's disease. NeuroImage 68, 203-213. doi: 10.1016/j.neuroimage.2012.11.036

Hoehn, M. M., and Yahr, M. D. (1967). Parkinsonism: onset, progression and mortality. Neurology 17, 427-442.

Huster, R. J., Debener, S., Eichele, T., and Herrmann, C. S. (2012). Methods for simultaneous EEG-fMRI: an introductory review. J. Neurosci. 32, 6053-6060. doi: 10.1523/JNEUROSCI.0447-12.2012

Jankovic, J. (2008). Parkinson's disease: clinical features and diagnosis. J. Neurol. Neurosurg. Psychiatry 4, 368-376. doi: 10.1136/jnnp.2007.131045

Jenkinson, M., Beckmann, C. F., Behrens, T. E., Woolrich, M. W., and Smith, S. M. (2012). FSL. NeuroImage 62, 782-790. doi: 10.1016/j.neuroimage.2011.09.015

Krajcovicova, L., Mikl, M., Marecek, R., and Rektorova, I. (2012). The default mode network integrity in patients with Parkinson's disease is levodopa equivalent dose-dependent. J. Neural Transm. 119, 443-454. doi: 10.1007/s00702-0110723-5

Kwak, Y., Peltier, S. J., Bohnen, N. I., Müller, M. L., Dayalu, P., and Seidler, R. D. (2012). L-DOPA changes spontaneous low-frequency BOLD signal oscillations in Parkinson's disease: a resting state fMRI study. Front. Syst. Neurosci. 6:52. doi: $10.3389 /$ fnsys.2012.00052

Lalo, E., Thobois, S., Sharott, A., Polo, G., Mertens, P., Pogosyan, A., et al. (2008). Patterns of bidirectional communication between cortex and basal ganglia during movement in patients with Parkinson disease. J. Neurosci. 28, 3008-3016. doi: 10.1523/JNEUROSCI.5295-07.2008

Lei, X., Wang, Y., Yuan, H., and Mantini, D. (2014). Neuronal oscillations and functional interactions between resting state networks. Hum. Brain Mapp. 35, 3517-3528. doi: 10.1002/hbm.22418

Levy, R., Ashby, P., Hutchison, W. D., Lang, A. E., Lozano, A. M., and Dostrovsky, J. O. (2002a). Dependence of subthalamic nucleus oscillations on movement and dopamine in Parkinson's disease. Brain 125, 1196-1209. doi: 10.1093/brain/ awf128

Levy, R., Hutchison, W. D., Lozano, A. M., and Dostrovsky, J. O. (2002b). Synchronized neuronal discharge in the basal ganglia of parkinsonian patients 
is limited to oscillatory activity. J. Neurosci. 22, 2855-2861. doi: 10.1523/ JNEUROSCI.22-07-02855.2002

Litvak, V., Jha, A., Eusebio, A., Oostenveld, R., Foltynie, T., Limousin, P., et al. (2011). Resting oscillatory corticosubthalamic connectivity in patients with Parkinson's disease. Brain 134, 359-374. doi: 10.1093/brain/awq332

Marsden, C. D., and Obeso, J. A. (1994). The functions of the basal ganglia and the paradox of stereotaxic surgery in Parkinson's disease. Brain 117, 877-897. doi: 10.1093/brain/117.4.877

McPherson, A. (1970). Convulsive seizures and electroencephalogram changes in three patients during levodopa therapy. Neurology 20, 41-45. doi: 10.1212/ WNL.20.12_Part_2.41

Morisi, R., Manners, D. N., Gnecco, G., Lanconelli, N., Testa, C., Evangelisti, S., et al. (2018). Multi-class parkinsonian disorders classification with quantitative MR markers and graph-based features using support vector machines. Parkinson. Relat. Disord. 47, 64-70. doi: 10.1016/j.parkreldis.2017.11.343

Neufeld, M. Y., Inzelberg, R., and Korczyn, A. D. (1988). EEG in demented and non-demented parkinsonian patients. Acta Neurol Scand. 78, 1-5. doi: 10.1111/ j.1600-0404.1988.tb03609.x

Obeso, J. A., Rodriguez, M. C., and DeLong, M. R. (1997). Basal ganglia pathophysiology. a critical review. Adv. Neurol. 74, 3-18.

Ohara, S., Mima, T., Baba, K., Ikeda, A., Kunieda, T., Matsumoto, R., et al. (2001). Increased synchronization of cortical oscillatory activities between human supplementary motor and primary sensorimotor areas during voluntary movements. J. Neurosci. 21, 9377-9393. doi: 10.1523/JNEUROSCI.21-2309377.2001

Plomp, G., Quairiaux, C., Michel, C. M., and Astolfi, L. (2014). The physiological plausibility of time-varying Granger-causal modeling: normalization and weighting by spectral power. Neuroimage 97, 206-216. doi: 10.1016/j. neuroimage.2014.04.016

Pollok, B., Kamp, D., Butz, M., Wojtecki, L., Timmermann, L., Südmeyer, M., et al. (2013). Increased SMA-M1 coherence in Parkinson's disease - Pathophysiology or compensation? Exp. Neurol. 247, 178-181. doi: 10.1016/j.expneurol.2013. 04.013

Priori, A., Foffani, G., Pesenti, A., Bianchi, A., Chiesa, V., Baselli, G., et al. (2002). Movement-related modulation of neural activity in human basal ganglia and its L-DOPA dependency: recordings from deep brain stimulation electrodes in patients with Parkinson's disease. Neurol Sci. 23(Suppl. 2), S101-S102. doi: $10.1007 /$ s100720200089

Rizzo, G., Tonon, C., and Lodi, R. (2012). Looking into the brain: How can conventional, morphometric and functional MRI help in diagnosing and understanding PD? Basal Ganglia 2, 175-182. doi: 10.1016/j.baga.2012.06.001

Rosenkranz, K., and Lemieux, L. (2010). Present and future of simultaneous EEG-fMRI. MAGMA 23, 309-316. doi: 10.1007/s10334-009-0196-9

Rowe, J., Stephan, K. E., Friston, K., Frackowiak, R., Lees, A., and Passingham, R. (2002). Attention to action in Parkinson's disease. Impaired effective connectivity among frontal cortical regions. Brain 125, 276-289. doi: 10.1093/ brain/awf036

Schneider, S., Wagels, L., Haeussinger, F. B., Fallgatter, A. J., Ehlis, A. C., and Rapp, A. M. (2015). Haemodynamic and electrophysiological markers of pragmatic language comprehension in schizophrenia. World J. Biol. Psychiatry 16, 398410. doi: 10.3109/15622975.2015.1019359

Serrien, D. J., and Brown, P. (2003). The integration of cortical and behavioural dynamics during initial learning of a motor task. Eur. J. Neurosci. 17, 10981104. doi: 10.1046/j.1460-9568.2003.02534.x

Sharman, M., Valabregue, R., Perlbarg, V., Marrakchi-Kacem, L., Vidailhet, M., Benali, H., et al. (2013). Parkinson's disease patients show reduced corticalsubcortical sensorimotor connectivity. Mov. Disord. 28, 447-454. doi: 10.1002/ mds. 25255

Shine, J. M., Halliday, G. M., Gilat, M., Matar, E., Bolitho, S. J., Carlos, M., et al. (2014). The role of dysfunctional attentional control networks in visual misperceptions in parkinson's disease. Hum. Brain Mapp. 35, 2206-2219. doi: 10.1002/hbm.22321

Silberstein, P., Oliviero, A., Di Lazzaro, V., Insola, A., Mazzone, P., and Brown, P. (2005). Oscillatory pallidal local field potential activity inversely correlates with limb dyskinesias in Parkinson's disease. Exp. Neurol. 194, 523-529. doi: 10.1016/j.expneurol.2005.03.014

Syed Nasser, N., Ibrahim, B., Sharifat, H., Abdul Rashid, A., and Suppiah, S. (2019). Incremental benefits of EEG informed fMRI in the study of disorders related to meso-corticolimbic dopamine pathway dysfunction: a systematic review of recent literature. J. Clin. Neurosci. doi: 10.1016/j.jocn.2019.03.054 [Epub ahead of print].

Tagliazucchi, E., and Laufs, H. (2014). Decoding wakefulness levels from typical fMRI resting-state data reveals reliable drifts between wakefulness and sleep. Neuron 82, 695-708. doi: 10.1016/j.neuron.2014.03.020

Tessitore, A., Amboni, M., Esposito, F., Russo, A., Picillo, M., Marcuccio, L., et al. (2012a). Resting state brain connectivity in patients with Parkinson's disease and freezing of gait. Parkinson. Relat. Disord. 18, 781-787. doi: 10.1016/j. parkreldis.2012.03.018

Tessitore, A., Esposito, F., Vitale, C., Santangelo, G., Amboni, M., Russo, A., et al. (2012b). Default-mode network connectivity in cognitively unimpaired patients with Parkinson disease. Neurology 79, 2226-2232. doi: 10.1212/WNL. 0b013e31827689d6

Van Eimeren, T., Monchi, O., Ballanger, B., and Strafella, A. P. (2009). Dysfunction of the default mode network in parkinson disease. Arch. Neurol. 66, 877-883. doi: 10.1001/archneurol.2009.97

Van Mierlo, P., Carrette, E., Hallez, H., Vonck, K., Van Roost, D., Boon, P., et al. (2011). Accurate epileptogenic focus localization through time-variant functional connectivity analysis of intracranial electroencephalographic signals. Neuroimage 56, 1122-1133. doi: 10.1016/j.neuroimage.2011.02.009

Vitali, P., Di Perri, C., Vaudano, A. E., Meletti, S., and Villani, F. (2015). Integration of multimodal neuroimaging methods: a rationale for clinical applications of simultaneous EEG-fMRI. Funct. Neurol. 30, 9-20.

Williams, D., Tijssen, M., Van Bruggen, G., Bosch, A., Insola, A., Di Lazzaro, V., et al. (2002). Dopamine-dependent changes in the functional connectivity between basal ganglia and cerebral cortex in humans. Brain 125, 1558-1569. doi: 10.1093/brain/awf156

Wu, T., and Hallett, M. (2005). A functional MRI study of automatic movements in patients with Parkinson's disease. Brain 128, 2250-2259. doi: 10.1093/brain/ awh569

Wu, T., Long, X., Wang, L., Hallett, M., Zang, Y., Li, K., et al. (2011). Functional connectivity of cortical motor areas in the resting state in parkinson's disease. Hum. Brain Mapp. 32, 1443-1457. doi: 10.1002/hbm.21118

Wu, T., Wang, J., Wang, C., Hallett, M., Zang, Y., Wu, X., et al. (2012). Basal ganglia circuits changes in Parkinson's disease patients. Neurosci. Lett. 524:55. doi: 10.1016/j.neulet.2012.07.012

Wu, T., Wang, L., Chena, Y., Zhaob, C., Li, K., and Chana, P. (2009). Changes of functional connectivity of the motor network in the resting state in Parkinson's disease. NeuroImage 460, 6-10. doi: 10.1016/j.neulet.2009.05.046

Yeager, C. L., Alberts, W., and Delattre, L. D. (1966). Effect of stereotaxic surgery upon electroencephalographic status of parkinsonian patients. Neurology 16, 904-910.

Zanigni, S., Evangelisti, S., Testa, C., Manners, D. N., Calandra-Buonaura, G., Guarino, M., et al. (2017). White matter and cortical changes in atypical parkinsonisms: a multimodal quantitative MR study. Parkinson. Relat. Disord. 39, 44-51. doi: 10.1016/j.parkreldis.2017.03.001

Conflict of Interest Statement: The authors declare that the research was conducted in the absence of any commercial or financial relationships that could be construed as a potential conflict of interest.

Copyright (C) 2019 Evangelisti, Pittau, Testa, Rizzo, Gramegna, Ferri, Coito, Cortelli, Calandra-Buonaura, Bisquoli, Bianchini, Manners, Talozzi, Tonon, Lodi and Tinuper. This is an open-access article distributed under the terms of the Creative Commons Attribution License (CC BY). The use, distribution or reproduction in other forums is permitted, provided the original author(s) and the copyright owner(s) are credited and that the original publication in this journal is cited, in accordance with accepted academic practice. No use, distribution or reproduction is permitted which does not comply with these terms. 
OPEN ACCESS

Edited by:

Roberto Esposito,

A.O. Ospedali Riuniti Marche Nord,

Italy

Reviewed by:

Abraham Z. Snyder,

Washington University in St. Louis,

United States

Darya Chyzhyk,

Inria Saclay - île-de-France, France

*Correspondence:

Massimo Filippi

filippi.massimo@hsr.it

Specialty section: This article was submitted to Brain Imaging Methods, a section of the journal Frontiers in Neuroscience

Received: 05 April 2019 Accepted: 07 June 2019

Published: 20 June 2019

Citation:

Filippi M, Spinelli EG, Cividini C and Agosta F (2019) Resting State

Dynamic Functional Connectivity in Neurodegenerative Conditions: A Review of Magnetic Resonance Imaging Findings.

Front. Neurosci. 13:657. doi: 10.3389/fnins.2019.00657

\section{Resting State Dynamic Functional Connectivity in Neurodegenerative Conditions: A Review of Magnetic Resonance Imaging Findings}

\author{
Massimo Filippi1,2,3*, Edoardo G. Spinelli ${ }^{1}$, Camilla Cividini and Federica Agosta ${ }^{1,3}$ \\ ${ }^{1}$ Neuroimaging Research Unit, Institute of Experimental Neurology, Division of Neuroscience, IRCCS San Raffaele Scientific \\ Institute, Milan, Italy, ${ }^{2}$ Neurology Unit, IRCCS San Raffaele Scientific Institute, Milan, Italy, ${ }^{3}$ Vita-Salute San Raffaele \\ University, Milan, Italy
}

In the last few decades, brain functional connectivity $(\mathrm{FC})$ has been extensively assessed using resting-state functional magnetic resonance imaging (RS-fMRI), which is able to identify temporally correlated brain regions known as RS functional networks. Fundamental insights into the pathophysiology of several neurodegenerative conditions have been provided by studies in this field. However, most of these studies are based on the assumption of temporal stationarity of RS functional networks, despite recent evidence suggests that the spatial patterns of RS networks may change periodically over the time of an $\mathrm{fMRI}$ scan acquisition. For this reason, dynamic functional connectivity (dFC) analysis has been recently implemented and proposed in order to consider the temporal fluctuations of FC. These approaches hold promise to provide fundamental information for the identification of pathophysiological and diagnostic markers in the vast field of neurodegenerative diseases. This review summarizes the main currently available approaches for dFC analysis and reports their recent applications for the assessment of the most common neurodegenerative conditions, including Alzheimer's disease, Parkinson's disease, dementia with Lewy bodies, and frontotemporal dementia. Critical state-of-the-art findings, limitations, and future perspectives regarding the analysis of $\mathrm{dFC}$ in these diseases are provided from both a clinical and a technical point of view.

\footnotetext{
Keywords: dynamic functional connectivity, fMRI, neurodegeneration, dementia, Alzheimer's disease, Parkinson's disease, Lewy bodies, frontotemporal dementia
}

\section{INTRODUCTION: FROM STATIC TO DYNAMIC FUNCTIONAL CONNECTIVITY}

Neurodegenerative diseases are characterized by a progressive loss of neurons associated with deposition of aberrant proteins, leading to alterations of the structural and functional properties of the brain (Kovacs, 2017). In the last decades, the non-invasive restingstate (RS) functional magnetic resonance imaging (RS-fMRI) technique has been widely applied in these clinical populations, to investigate more in depth the spatial topology and strength of interactions between brain networks (Smitha et al., 2017; Hohenfeld et al., 2018). 
Functional magnetic resonance imaging uses the bloodoxygenation-level dependent (BOLD) signal, which is sensitive to spontaneous neural activity. In particular, low-frequency oscillations $(<0.1 \mathrm{~Hz})$ of the BOLD signal are analyzed to obtain functional information of brain networks. Functional connectivity (FC) quantifies the temporal correlation of functional activation in different brain regions and can be expressed in terms of pairwise Pearson's correlation coefficients, covariance, or mutual information between time series, revealing specific networks (Smitha et al., 2017). FC has been recognized as an important biomarker for better understanding the pathophysiological mechanisms of numerous neurodegenerative diseases, including Alzheimer's disease (AD) (Filippi et al., 2017), Parkinson's disease (PD) (Baggio et al., 2015; Filippi et al., 2019), and frontotemporal dementia (FTD) (Filippi et al., 2017).

So far, the implicit hypothesis on which FC analysis has been based is the assumption of temporal stationarity of the functional interaction between connections. Considering the dynamic nature of brain activity, a novel approach is provided by dynamic functional connectivity $(\mathrm{dFC})$, which considers the temporal fluctuations of functional connections in faster timescales (Hutchison et al., 2013). Unlike conventional static FC, which is obtained from the correlation within an entire time series, dFC refers to the brain activity within sub-portions of time series (Menon and Krishnamurthy, 2019). Major efforts have been made to identify and analyze time-varying, but recurring, FC sub-patterns of coupling among brain regions, constituting the brain "chronnectome" (Calhoun et al., 2014).

The aim of this review is to describe the contribution of $\mathrm{dFC}$ studies in RS conditions for a better understanding of neurodegenerative diseases. We are going to focus on the most common approaches to analyze $\mathrm{dFC}$ and review recent findings in this field concerning $\mathrm{AD}, \mathrm{PD}$ and other parkinsonisms, and FTD. We conclude this work summarizing caveats, limitations and future perspectives regarding $\mathrm{dFC}$ analysis.

\section{METHODOLOGICAL OVERVIEW}

Several computational strategies have been implemented to characterize temporal and spatial variations of BOLD signal (Wee et al., 2016; Jie et al., 2018; Liu et al., 2018). The most common approach is provided by the sliding-window technique (Chen et al., 2016, 2017; de Vos et al., 2018; Diez-Cirarda et al., 2018), characterized by the selection of a time window - shorter than the whole-scan time - whose data points are used to calculate FC metrics. The window is shifted in time by a fixed number of data points, referred as step, which defines the overlap between two successive windows. The step duration ranges from one single data point to the length of the window (i.e., non-overlapping windows) (Jie et al., 2018; Park et al., 2018).

In combination with the sliding-window approach, several studies have applied clustering methods to identify reproducible, transient patterns and to evaluate the commonly used graph metrics, the dwell time, defined as the number of consecutive windows in a specific state, and the number of transitions between states (Allen et al., 2014).
Since a subject could be in more than one state at a given point, the concept of "meta-states" and meta-state measures has been introduced to intuitively characterize the dynamic fluidity in FC (Miller et al., 2016; Premi et al., 2019). Meta-state measures include the number of occupied meta-states, number of switches between meta-states, greatest distance between two meta-states and overall distance (Premi et al., 2019). Furthermore, the sliding-window approach can be integrated with the application of independent component analysis (ICA) to identify spatial maps in the windowed BOLD signal and assess variability or graph theoretical metrics (Jones et al., 2012; de Vos et al., 2018; Premi et al., 2019). The sliding-window approach can also be used jointly with classification algorithms to exploit the information resulting from patterns of dFC (Chen et al., 2016; Guo et al., 2017; Figure 1).

Alternative approaches to evaluate $\mathrm{dFC}$ are represented by time-frequency analysis, dynamic connectivity regression (DCR) and dynamic connectivity detection (DCD), which are datadriven techniques for detecting FC change points within RS FMRI time series, and derive dynamic information from such points (Xu and Lindquist, 2015).

\section{ALZHEIMER'S DISEASE}

Alzheimer's disease is the most common neurodegenerative cause of dementia (Alzheimer's, 2016) and has been extensively studied by means of advanced MRI techniques. The inclusion of RS fMRI into imaging protocols in AD has been particularly advantageous, as the difficulty to obtain subjects' cooperation could influence task-related fMRI results. Conspicuous evidence has shown decreased FC of the default mode network (DMN) across the $\mathrm{AD}$ continuum, including patients with full-blown $\mathrm{AD}$ dementia and amnestic mild cognitive impairment (MCI) (Greicius et al., 2004; Bai et al., 2008; Agosta et al., 2012; Koch et al., 2012). Decreased connectivity within the DMN - consisting of the posterior cingulate, inferior parietal, inferolateral temporal, anterior cingulate, prefrontal, and hippocampal regions - is often accompanied by increased connectivity in the attentional frontoparietal and salience networks, likely mirroring compensatory mechanisms (Agosta et al., 2012; Badhwar et al., 2017). Disconnection between posterior (i.e., posterior cingulate and parietal regions) and anterior DMN nodes (i.e., anterior cingulate and prefrontal regions) was found to cause relative decreased connectivity within the posterior DMN and increased connectivity within the anterior DMN (Jones et al., 2011). Functional rearrangements have demonstrated clinical usefulness for predicting conversion to AD in MCI patients (Bai et al., 2011; Petrella et al., 2011; Li et al., 2016).

Given the high consistency of these findings across static FC studies, AD represents a good candidate to apply dFC approaches to the field of neurodegenerative disorders, since capturing the evolving architecture of brain networks over short periods of time might provide further pathophysiological insights into these conditions, and eventually better diagnostic or prognostic indicators. The first study investigating $\mathrm{dFC}$ of $\mathrm{AD}$ patients examined changes over time of a modularity 


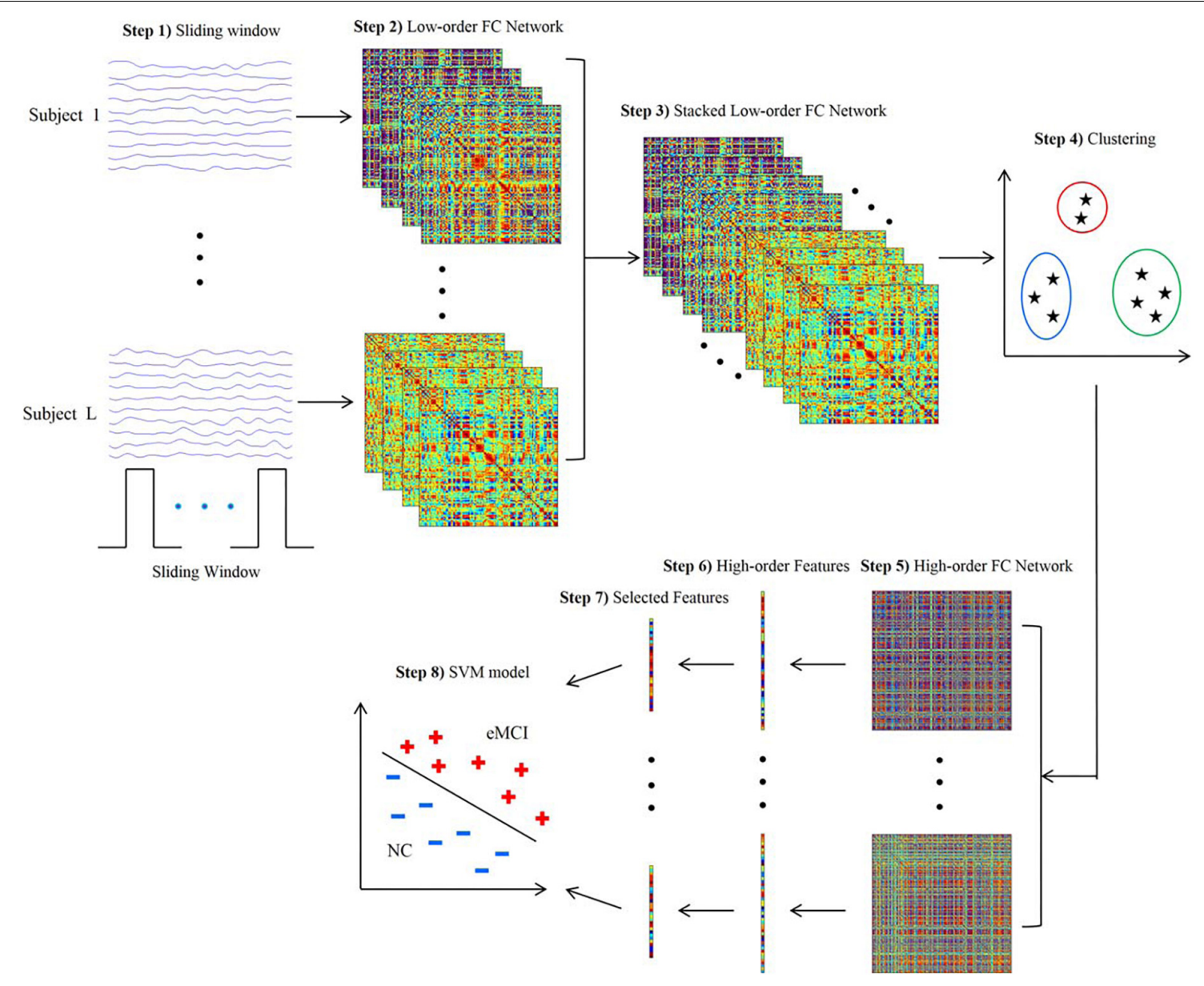

FIGURE 1 | Framework for construction of high-order functional connectivity (FC) network. (1) Partition of the RS-fMRI time series into multiple overlapping segments of subseries applying sliding-window technique; (2) collection of low-order FC matrices, one for each subseries; (3) stack of all matrices of all subjects together to obtain correlation time series for each element; (4) application of the clustering algorithm to group all the correlation time series; (5) construction of high-order FC network, considering the mean correlation time series for each cluster as vertex and the pairwise Pearson's correlation coefficient between each pair of vertices as weight; (6) calculation of local clustering coefficients; (7) selection of a discriminative feature subset from the local clustering coefficients; (8) implementation of support vector machine (SVM) model for classification. RS-fMRI, resting-state functional magnetic resonance imaging; FC, functional connectivity (reproduced with permission from Chen et al., 2016).

metric using a sliding-window analysis (Jones et al., 2012). The non-stationary nature of the brain modular organization was demonstrated and related with significant variations of the dwell time within different sub-network configurations of the DMN in subjects with $\mathrm{AD}$ dementia compared with healthy controls; specifically, $\mathrm{AD}$ patients spent less time in brain functional states with strong posterior DMN region contribution and more time in states with greater anterior DMN region contribution (Jones et al., 2012). A subsequent study investigated the evolution of $\mathrm{dFC}$ disruptions across the $\mathrm{AD}$ spectrum, showing alterations in patients with dementia compared to MCI and subjective cognitive decline (SCD) in terms of local dFC within the temporal, frontal-superior and default-mode networks; decreased global metastability between functional states was also found, supporting the hypothesis that oscillatory patterns are progressively altered over the $\mathrm{AD}$ continuum, eventually leading to a shrinkage of the "dynamic repertoire" (i.e., a smaller set of functional configurations) in the brain of AD patients (CordovaPalomera et al., 2017). Consistently, another study showed a progressive loss of whole-brain metastability according to the severity of cognitive impairment along the $\mathrm{AD}$ continuum, reaching statistical significance only in patients with dementia, when compared with healthy controls (Demirtas et al., 2017).

Researchers have also aimed to identify dFC alterations that may represent candidate non-invasive diagnostic biomarkers in the early stages of AD. A sparse temporal network-based framework has been tested for the classification of patients with early MCI by means of support vector machine (SVM) algorithms, yielding an accuracy of approximately $80 \%$ in the discrimination from healthy controls, compared with accuracies ranging 62-72\% using FC static approaches (Wee et al., 2016). Another recent study aimed to integrate both temporal and spatial properties of dFC networks for the classification of early and late MCI patients (Jie et al., 2018). Accuracies of 


\section{A Group-specific cluster centroids State I}

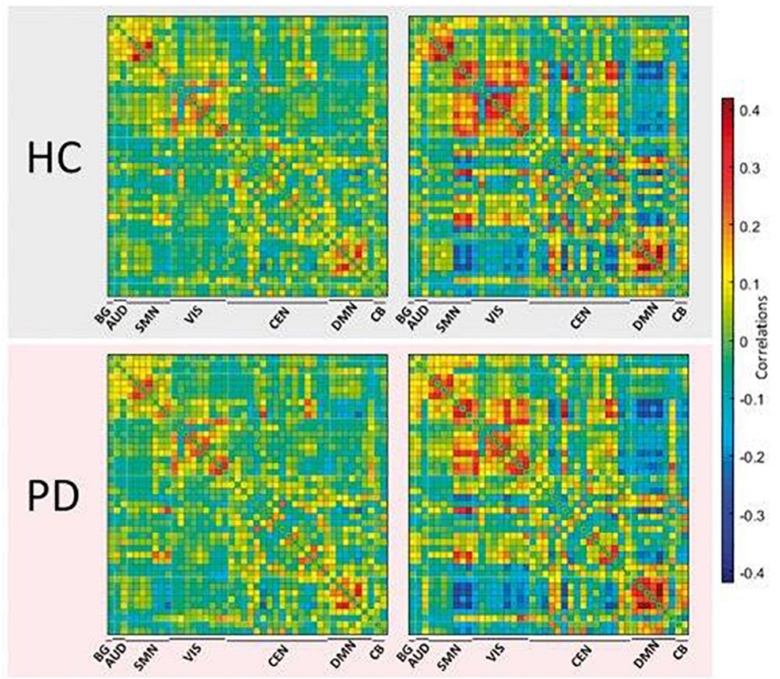

B Functional connectivity

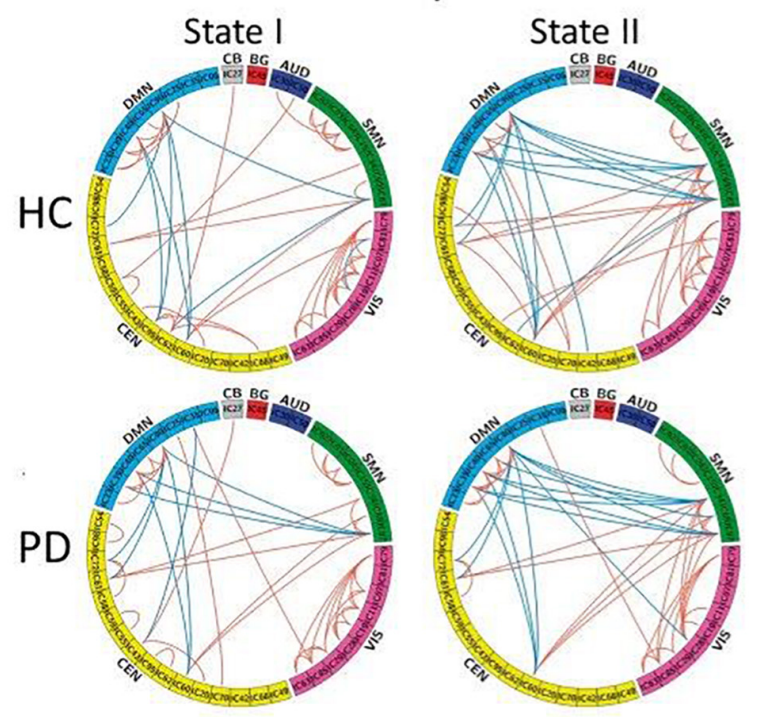

FIGURE 2 | Functional connectivity state results. (A) Group-specific cluster centroid for each state, averaged across subject-specific median cluster centroids of each group [percentage of total occurrences for stage I and II: $83.4 \%$ and $16.6 \%$ in the healthy controls (HC) and $70.8 \%$ and $29.2 \%$ in the Parkinson's disease (PD) group, respectively]. (B) Functional connectivity in each state is shown for healthy controls and Parkinson's disease groups, representing the $5 \%$ of the functional connectivity network with the strongest connections. BG, basal ganglia; AUD, auditory; SMN, sensorimotor; VIS, visual; CEN, cognitive executive; CB, cerebellar network (reproduced with permission from Kim et al., 2017).

approximately $78 \%$ were obtained when SVM algorithms were trained based on the dFC patterns of components of the DMN and temporal and cerebellar regions (Jie et al., 2018). An attempt to combine multiple dFC parameters for an automated classification of early MCI patients was recently proposed by applying a tensor model of spatio-temporal BOLD signal to each voxel of the white matter (WM), to be integrated with the information provided by the FC of grey matter (GM) regions (Chen et al., 2017). Such combined GM and WM approach yielded an accuracy of almost $79 \%$ in discriminating early MCI patients from healthy subjects, compared with $74 \%$ provided by GM dFC measures alone (Chen et al., 2017). Another promising approach is the clustering of standard-correlation time series for all pairs of brain regions (i.e., the classic "low-order" FC networks) into a smaller set of "high-order" dFC networks according to their intrinsic common patterns. The combination of high-order dFC with the conventional low-order analysis allowed SVM-based discrimination of early MCI patients from healthy subjects with an accuracy of $88 \%$, outperforming other previous approaches (Chen et al., 2016; Figure 1).

\section{PARKINSON'S DISEASE}

Parkinson's disease (PD) is the second most common neurodegenerative disorder and is characterized by dopamine depletion in the nigro-striatal system leading to progressive functional impairment (Poewe et al., 2017). Widespread functional rearrangements related to the development of motor and non-motor symptoms occur over the clinical progression in PD patients (Filippi et al., 2019). Several RS fMRI studies have identified alterations of the cerebello-thalamo-cortical circuit as a key hallmark of PD (Helmich et al., 2010; Hacker et al., 2012; Agosta et al., 2014; Akram et al., 2017), with reduced activation of the posterior putamen correlating with motor impairment as the most consistent finding (Herz et al., 2014). Furthermore, disrupted FC in the DMN, fronto-parietal, salience and associative visual networks has been linked to the development of cognitive deficits in PD (Amboni et al., 2015; Baggio et al., 2015; Putcha et al., 2015; Zhan et al., 2018). Particularly, normal decoupling between the DMN and fronto-parietal networks was reduced in PD patients with MCI (PD-MCI) (Baggio et al., 2015; Putcha et al., 2015), and FC alterations within the DMN were able to predict subsequent cognitive decline in cognitively unimpaired PD patients (van Eimeren et al., 2009; Tessitore et al., 2012; Putcha et al., 2015).

The first study assessing the dynamic functional properties of RS networks in PD patients identified two main FC configurations: a more frequent and strongly segregated state (defined as "State I") and a less frequent, more integrated "State II" (Kim et al., 2017). Compared with healthy subjects, PD patients showed a significant decrease of dwell time in State I, with a proportional increase of dwell time in State II that was correlated with the severity of motor symptoms, indicating that the loss of functional segregation between brain networks might represent a key element in PD pathogenesis (Kim et al., 2017; Figure 2). To eliminate the possible influence of long-term dopaminergic therapy, dFC alterations were also 
assessed in early stage, drug-naïve PD patients, who showed decreased switching rate between dynamic states correlating with disease severity, supporting the view that a limited dynamic range of whole-brain FC might represent an early PD marker (Cordes et al., 2018; Zhuang et al., 2018). Another recent study focused on the spatial configuration of dFC alterations within two homogeneous subunits of the putamen of PD subjects, demonstrating a degradation of subregional specificity between the anterior and posterior putaminal subunits that was related with disease severity (Liu et al., 2018).

Along with motor impairment, $\mathrm{dFC}$ has also provided significant information regarding the underpinnings of cognitive symptoms in PD. Decreased dwell time in a more segregated state and increased state transitions have been demonstrated in PD-MCI patients compared with healthy controls, configuring a pattern that PD patients with normal cognition lacked (DiezCirarda et al., 2018). Increased dFC within the dorsal-attention network was found to predict attention performance in $\mathrm{PD}$ patients (Madhyastha et al., 2015), whereas a positive correlation between $\mathrm{dFC}$ of the DMN and performance on a visuospatial memory task has also been recently reported (Engels et al., 2018).

\section{OTHER NEURODEGENERATIVE CONDITIONS}

To date, most dFC studies assessing patients with neurodegenerative conditions have focused on the two most common diseases, i.e., $\mathrm{AD}$ and $\mathrm{PD}$. Considering also the novelty of these approaches, evidence regarding other pathological entities is currently scarce, but in rapid development.

Dementia with Lewy bodies (DLB) is among the most common causes of dementia after $\mathrm{AD}$, and is characterized by cognitive fluctuations, parkinsonism, and visual hallucinations (McKeith et al., 2017). Classic RS-fMRI static studies have shown FC reductions in widespread brain networks in DLB subjects, with desynchronization of cortical and subcortical areas within the attention-executive networks correlating with cognitive fluctuations (Lowther et al., 2014; Peraza et al., 2014). Considering the transient nature of some of the main features of DLB (i.e., cognitive fluctuations and hallucinations), dFC studies are expected to provide fundamental insights into the pathophysiology of this disease. Indeed, $\mathrm{dFC}$ has demonstrated significant differences in DLB patients compared with healthy subjects in visual (i.e., the occipito-parieto-frontal and medial occipital networks) and attentional networks (i.e., the right fronto-parietal control network), which also showed decreased mutual dependency, suggesting that temporal disconnection between these networks might be relevant for DLB pathogenesis (Sourty et al., 2016).

Frontotemporal dementia is another frequent neurodegenerative condition encompassing a wide range of clinical presentations, including behavioral, executive, language and motor deficits (Olney et al., 2017). To our knowledge, no study has assessed dFC alterations in patients with FTD. However, a recent work focused on presymptomatic carriers of FTD-causing mutations (Premi et al., 2019), being FTD an inherited autosomal disorder in $30-40 \%$ of cases (Rohrer and Warren, 2011). Mutation carriers showed lower number of meta-states, decreased switching rate between meta-states and shorter meta-state total distance compared with healthy controls, demonstrating that a reduced dynamic fluidity and restricted dynamic range in brain functional "chronnectome" is an early event in the development of FTD (Premi et al., 2019). The assessment of such alterations in the phase which is closest to clinical conversion might be a promising research field for the development of biomarkers to be used in clinical trials of FTD.

\section{CAVEATS, LIMITATIONS AND FUTURE DIRECTIONS}

Based on the evidence here reviewed, dFC studies have shed new light on the pathophysiological alterations underlying the most common neurodegenerative diseases. However, important concerns remain regarding the possible influence of vigilance fluctuations during the fMRI scan. Although patients are routinely instructed to stay awake for the whole scan duration, sleep disturbances are frequent clinical features of dementias and parkinsonian syndromes (Malhotra, 2018), and fluctuating alertness will affect FC (Tagliazucchi et al., 2012; Haimovici et al., 2017). This important issue would be overcome by simultaneous EEG-fMRI acquisition (Tagliazucchi and Laufs, 2014; Allen et al., 2018), although this approach is technically challenging and has not been explored in neurodegenerative conditions yet.

Some technical caveats also need to be considered. The slidingwindow technique has been repeatedly applied because of its analytical simplicity and easy implementation: in most studies, the number of BOLD signal subseries $\mathrm{K}$ is decided based on the window length $\mathrm{W}$, the number of temporal image volumes $\mathrm{N}$ and the step, according to the formulation $\mathrm{K}=[(\mathrm{N}-\mathrm{W}) / \mathrm{s}]$ +1 (Chen et al., 2016; Wee et al., 2016; Guo et al., 2017). However, the window length, as well as the step parameter, are matter of debate: choosing a short window could increase the risk of misleading spurious fluctuations, while choosing a long window could fail to identify state transitions (Preti et al., 2017). A trade-off must be reached: at present, different studies suggested a window length of 30-60 $\mathrm{s}$ as sufficient for detecting dFC changes (Jones et al., 2012; Diez-Cirarda et al., 2018; Premi et al., 2019), even though some studies opted for longer windows (Chen et al., 2016; Wee et al., 2016). An alternative way to find the optimum window length is represented by the timefrequency analysis (Cordes et al., 2018). The step parameter is also chosen arbitrarily, commonly ranging one to two volumes for overlapping windows (Quevenco et al., 2017; Liu et al., 2018), although a few studies adopted non-overlapping windows (Jie et al., 2018; Park et al., 2018). Another crucial point is the choice of window shape: rectangular, modulated rectangular or tapered windows are the most used (Diez-Cirarda et al., 2018; Premi et al., 2019).

Beyond these methodological aspects, there are still some unsolved questions regarding dFC. Neurobiological underpinnings and mechanisms of dynamic states have to be 
clarified (Smith et al., 2011), as the possibility that reported changes may be driven by signal noise or sampling variability needs to be considered (Laumann et al., 2017). Indeed, the fluctuations in the sliding-window correlation time series can be associated to $\mathrm{dFC}$ or simply generated by random noise, so more complex statistical models are required to deal with this issue (Hindriks et al., 2016). The artifact problem that applies to conventional resting-state fMRI is also a crucial aspect for dFC analysis (Nalci et al., 2019), as the BOLD signal is sensitive to non-stationary physiological processes, such as head motion (Power et al., 2014) and blood partial pressure of carbon dioxide (pCO2) due to respiration (Power et al., 2018). Moreover, important factors to take into account for a correct interpretation of $\mathrm{dFC}$ results are the selection of the a priori atlas or ICA algorithm used to obtain regions of interest and the assessment of specific FC metrics. Finally, the reliability and reproducibility of dFC patterns are still a challenge, although some efforts have been made on solving this issue (Abrol et al., 2017).

\section{REFERENCES}

Abrol, A., Damaraju, E., Miller, R. L., Stephen, J. M., Claus, E. D., Mayer, A. R., et al. (2017). Replicability of time-varying connectivity patterns in large resting state fMRI samples. Neuroimage 163, 160-176. doi: 10.1016/j.neuroimage.2017. 09.020

Agosta, F., Caso, F., Stankovic, I., Inuggi, A., Petrovic, I., Svetel, M., et al. (2014). Cortico-striatal-thalamic network functional connectivity in hemiparkinsonism. Neurobiol. Aging 35, 2592-2602. doi: 10.1016/j. neurobiolaging.2014.05.032

Agosta, F., Pievani, M., Geroldi, C., Copetti, M., Frisoni, G. B., and Filippi, M. (2012). Resting state fMRI in Alzheimer's disease: beyond the default mode network. Neurobiol. Aging 33, 1564-1578. doi: 10.1016/j.neurobiolaging.2011. 06.007

Akram, H., Wu, C., Hyam, J., Foltynie, T., Limousin, P., De Vita, E., et al. (2017). 1-Dopa responsiveness is associated with distinctive connectivity patterns in advanced Parkinson's disease. Mov. Disord. 32, 874-883. doi: 10.1002/mds. 27017

Allen, E. A., Damaraju, E., Eichele, T., Wu, L., and Calhoun, V. D. (2018). EEG signatures of dynamic functional network connectivity states. Brain Topogr. 31, 101-116. doi: 10.1007/s10548-017-0546-542

Allen, E. A., Damaraju, E., Plis, S. M., Erhardt, E. B., Eichele, T., and Calhoun, V. D. (2014). Tracking whole-brain connectivity dynamics in the resting state. Cereb. Cortex 24, 663-676. doi: 10.1093/cercor/bhs352

Alzheimer's, A. (2016). 2016 Alzheimer's disease facts and figures. Alzheimers Dement 12, 459-509. doi: 10.1016/j.jalz.2016.03.001

Amboni, M., Tessitore, A., Esposito, F., Santangelo, G., Picillo, M., Vitale, C., et al. (2015). Resting-state functional connectivity associated with mild cognitive impairment in Parkinson's disease. J. Neurol. 262, 425-434. doi: 10.1007/ s00415-014-7591-7595

Badhwar, A., Tam, A., Dansereau, C., Orban, P., Hoffstaedter, F., and Bellec, P. (2017). Resting-state network dysfunction in Alzheimer's disease: a systematic review and meta-analysis. Alzheimers Dement 8, 73-85. doi: 10.1016/j.dadm. 2017.03.007

Baggio, H. C., Segura, B., Sala-Llonch, R., Marti, M. J., Valldeoriola, F., Compta, Y., et al. (2015). Cognitive impairment and resting-state network connectivity in Parkinson's disease. Hum. Brain Mapp. 36, 199-212. doi: 10.1002/hbm.22622

Bai, F., Liao, W., Watson, D. R., Shi, Y., Wang, Y., Yue, C., et al. (2011). Abnormal whole-brain functional connection in amnestic mild cognitive impairment patients. Behav. Brain Res. 216, 666-672. doi: 10.1016/j.bbr.2010.09.010

Bai, F., Zhang, Z., Yu, H., Shi, Y., Yuan, Y., Zhu, W., et al. (2008). Default-mode network activity distinguishes amnestic type mild cognitive impairment from

\section{CONCLUSION}

Assessing $\mathrm{dFC}$ is a promising way to better understand neurodegenerative processes and investigate novel disease diagnostic and prognostic biomarkers. However, future developments are needed to rule out the influence of vigilance fluctuations, overcome the limitations of the sliding-window approach - possibly using other methods as time-frequency analysis -, identify the most informative dFC metrics, and minimize artifacts by means of adequate preprocessing, so as to be more confident in the description and interpretation of these findings.

\section{AUTHOR CONTRIBUTIONS}

MF contributed to the study concept and acted as study supervisor. All authors contributed to writing, reading, and approving the final version of the manuscript.

healthy aging: a combined structural and resting-state functional MRI study. Neurosci. Lett. 438, 111-115. doi: 10.1016/j.neulet.2008.04.021

Calhoun, V. D., Miller, R., Pearlson, G., and Adali, T. (2014). The chronnectome: time-varying connectivity networks as the next frontier in fMRI data discovery. Neuron 84, 262-274. doi: 10.1016/j.neuron.2014.10.015

Chen, X., Zhang, H., Gao, Y., Wee, C. Y., Li, G., Shen, D., et al. (2016). High-order resting-state functional connectivity network for MCI classification. Hum. Brain Mapp. 37, 3282-3296. doi: 10.1002/hbm.23240

Chen, X., Zhang, H., Zhang, L., Shen, C., Lee, S. W., and Shen, D. (2017). Extraction of dynamic functional connectivity from brain grey matter and white matter for MCI classification. Hum. Brain Mapp. 38, 5019-5034. doi: 10.1002/hbm.23711

Cordes, D., Zhuang, X., Kaleem, M., Sreenivasan, K., Yang, Z., Mishra, V., et al. (2018). Advances in functional magnetic resonance imaging data analysis methods using empirical mode decomposition to investigate temporal changes in early Parkinson's disease. Alzheimers Dement 4, 372-386. doi: 10.1016/j.trci. 2018.04.009

Cordova-Palomera, A., Kaufmann, T., Persson, K., Alnaes, D., Doan, N. T., Moberget, T., et al. (2017). Disrupted global metastability and static and dynamic brain connectivity across individuals in the Alzheimer's disease continuum. Sci. Rep. 7:40268. doi: 10.1038/srep40268

de Vos, F., Koini, M., Schouten, T. M., Seiler, S., van der Grond, J., Lechner, A., et al. (2018). A comprehensive analysis of resting state fMRI measures to classify individual patients with Alzheimer's disease. Neuroimage 167, 62-72. doi: 10.1016/j.neuroimage.2017.11.025

Demirtas, M., Falcon, C., Tucholka, A., Gispert, J. D., Molinuevo, J. L., and Deco, G. (2017). A whole-brain computational modeling approach to explain the alterations in resting-state functional connectivity during progression of Alzheimer's disease. Neuroimage Clin. 16, 343-354. doi: 10.1016/j.nicl.2017. 08.006

Diez-Cirarda, M., Strafella, A. P., Kim, J., Pena, J., Ojeda, N., Cabrera-Zubizarreta, A., et al. (2018). Dynamic functional connectivity in Parkinson's disease patients with mild cognitive impairment and normal cognition. Neuroimage Clin. 17, 847-855. doi: 10.1016/j.nicl.2017.12.013

Engels, G., Vlaar, A., McCoy, B., Scherder, E., and Douw, L. (2018). Dynamic functional connectivity and symptoms of Parkinson's disease: a resting-state fMRI study. Front. Aging Neurosci. 10:388. doi: 10.3389/fnagi.2018.00388

Filippi, M., Basaia, S., Canu, E., Imperiale, F., Meani, A., Caso, F., et al. (2017). Brain network connectivity differs in early-onset neurodegenerative dementia. Neurology 89, 1764-1772. doi: 10.1212/WNL.0000000000004577

Filippi, M., Sarasso, E., and Agosta, F. (2019). Resting-state functional MRI in Parkinsonian syndromes. Mov. Disord. Clin. Pract. 6, 104-117. doi: 10.1002/ mdc3.12730 
Greicius, M. D., Srivastava, G., Reiss, A. L., and Menon, V. (2004). Defaultmode network activity distinguishes Alzheimer's disease from healthy aging: evidence from functional MRI. Proc. Natl. Acad Sci. U.S.A. 101, 4637-4642. doi: 10.1073/pnas.0308627101

Guo, H., Liu, L., Chen, J., Xu, Y., and Jie, X. (2017). Alzheimer classification using a minimum spanning tree of high-order functional network on fMRI dataset. Front. Neurosci. 11:639. doi: 10.3389/fnins.2017.00639

Hacker, C. D., Perlmutter, J. S., Criswell, S. R., Ances, B. M., and Snyder, A. Z. (2012). Resting state functional connectivity of the striatum in Parkinson's disease. Brain 135(Pt 12), 3699-3711. doi: 10.1093/brain/ aws 281

Haimovici, A., Tagliazucchi, E., Balenzuela, P., and Laufs, H. (2017). On wakefulness fluctuations as a source of BOLD functional connectivity dynamics. Sci. Rep. 7:5908. doi: 10.1038/s41598-017-06389-6384

Helmich, R. C., Derikx, L. C., Bakker, M., Scheeringa, R., Bloem, B. R., and Toni, I. (2010). Spatial remapping of cortico-striatal connectivity in Parkinson's disease. Cereb. Cortex 20, 1175-1186. doi: 10.1093/cercor/ bhp 178

Herz, D. M., Eickhoff, S. B., Lokkegaard, A., and Siebner, H. R. (2014). Functional neuroimaging of motor control in Parkinson's disease: a meta-analysis. Hum. Brain Mapp. 35, 3227-3237. doi: 10.1002/hbm.22397

Hindriks, R., Adhikari, M. H., Murayama, Y., Ganzetti, M., Mantini, D., Logothetis, N. K., et al. (2016). Can sliding-window correlations reveal dynamic functional connectivity in resting-state fMRI? Neuroimage 127, 242-256. doi: 10.1016/j. neuroimage.2015.11.055

Hohenfeld, C., Werner, C. J., and Reetz, K. (2018). Resting-state connectivity in neurodegenerative disorders: is there potential for an imaging biomarker? Neuroimage Clin. 18, 849-870. doi: 10.1016/j.nicl.2018.03.013

Hutchison, R. M., Womelsdorf, T., Allen, E. A., Bandettini, P. A., Calhoun, V. D., Corbetta, M., et al. (2013). Dynamic functional connectivity: promise, issues, and interpretations. Neuroimage 80, 360-378. doi: 10.1016/j.neuroimage.2013. 05.079

Jie, B., Liu, M., and Shen, D. (2018). Integration of temporal and spatial properties of dynamic connectivity networks for automatic diagnosis of brain disease. Med. Image Anal. 47, 81-94. doi: 10.1016/j.media.2018. 03.013

Jones, D. T., Machulda, M. M., Vemuri, P., McDade, E. M., Zeng, G., Senjem, M. L., et al. (2011). Age-related changes in the default mode network are more advanced in Alzheimer disease. Neurology 77, 1524-1531. doi: 10.1212/WNL. 0b013e318233b33d

Jones, D. T., Vemuri, P., Murphy, M. C., Gunter, J. L., Senjem, M. L., Machulda, M. M., et al. (2012). Non-stationarity in the "resting brain's" modular architecture. PLoS One 7:e39731. doi: 10.1371/journal.pone.003 9731

Kim, J., Criaud, M., Cho, S. S., Diez-Cirarda, M., Mihaescu, A., Coakeley, S., et al. (2017). Abnormal intrinsic brain functional network dynamics in Parkinson's disease. Brain 140, 2955-2967. doi: 10.1093/brain/awx233

Koch, W., Teipel, S., Mueller, S., Benninghoff, J., Wagner, M., Bokde, A. L., et al. (2012). Diagnostic power of default mode network resting state fMRI in the detection of Alzheimer's disease. Neurobiol. Aging 33, 466-478. doi: 10.1016/j. neurobiolaging.2010.04.013

Kovacs, G. G. (2017). Concepts and classification of neurodegenerative diseases. Handb. Clin. Neurol. 145, 301-307. doi: 10.1016/B978-0-12-802395-2. 00021-23

Laumann, T. O., Snyder, A. Z., Mitra, A., Gordon, E. M., Gratton, C., Adeyemo, B., et al. (2017). On the stability of BOLD fMRI correlations. Cereb. Cortex 27, 4719-4732. doi: 10.1093/cercor/bhw265

Li, Y., Wang, X., Li, Y., Sun, Y., Sheng, C., Li, H., et al. (2016). Abnormal restingstate functional connectivity strength in mild cognitive impairment and its conversion to Alzheimer's Disease. Neural. Plast. 2016:4680972. doi: 10.1155/ 2016/4680972

Liu, A., Lin, S. J., Mi, T., Chen, X., Chan, P., Wang, Z. J., et al. (2018). Decreased subregional specificity of the putamen in Parkinson's disease revealed by dynamic connectivity-derived parcellation. Neuroimage Clin. 20, 1163-1175. doi: $10.1016 /$ j.nicl.2018.10.022

Lowther, E. R., O’Brien, J. T., Firbank, M. J., and Blamire, A. M. (2014). Lewy body compared with Alzheimer dementia is associated with decreased functional connectivity in resting state networks. Psychiatry Res. 223, 192-201. doi: 10. 1016/j.pscychresns.2014.06.004

Madhyastha, T. M., Askren, M. K., Boord, P., and Grabowski, T. J. (2015). Dynamic connectivity at rest predicts attention task performance. Brain Connect. 5, 45-59. doi: 10.1089/brain.2014.0248

Malhotra, R. K. (2018). Neurodegenerative disorders and sleep. Sleep Med. Clin. 13, 63-70. doi: 10.1016/j.jsmc.2017.09.006

McKeith, I. G., Boeve, B. F., Dickson, D. W., Halliday, G., Taylor, J. P., Weintraub, D., et al. (2017). Diagnosis and management of dementia with Lewy bodies: fourth consensus report of the DLB consortium. Neurology 89, 88-100. doi: 10.1212/WNL.0000000000004058

Menon, S. S., and Krishnamurthy, K. (2019). A comparison of static and dynamic functional connectivities for identifying subjects and biological sex using intrinsic individual brain connectivity. Sci. Rep. 9:5729. doi: 10.1038/s41598019-42090-42094

Miller, R. L., Yaesoubi, M., Turner, J. A., Mathalon, D., Preda, A., Pearlson, G., et al. (2016). Higher dimensional meta-state analysis reveals reduced resting fMRI connectivity dynamism in schizophrenia patients. PLoS One 11:e0149849. doi: 10.1371/journal.pone.0149849

Nalci, A., Rao, B. D., and Liu, T. T. (2019). Nuisance effects and the limitations of nuisance regression in dynamic functional connectivity fMRI. Neuroimage 184, 1005-1031. doi: 10.1016/j.neuroimage.2018.09.024

Olney, N. T., Spina, S., and Miller, B. L. (2017). Frontotemporal dementia. Neurol. Clin. 35, 339-374. doi: 10.1016/j.ncl.2017.01.008

Park, H. J., Friston, K. J., Pae, C., Park, B., and Razi, A. (2018). Dynamic effective connectivity in resting state fMRI. Neuroimage 180(Pt B), 594-608. doi: 10. 1016/j.neuroimage.2017.11.033

Peraza, L. R., Kaiser, M., Firbank, M., Graziadio, S., Bonanni, L., Onofrj, M., et al. (2014). fMRI resting state networks and their association with cognitive fluctuations in dementia with Lewy bodies. Neuroimage Clin. 4, 558-565. doi: 10.1016/j.nicl.2014.03.013

Petrella, J. R., Sheldon, F. C., Prince, S. E., Calhoun, V. D., and Doraiswamy, P. M. (2011). Default mode network connectivity in stable vs progressive mild cognitive impairment. Neurology 76, 511-517. doi: 10.1212/WNL. 0b013e31820af94e

Poewe, W., Seppi, K., Tanner, C. M., Halliday, G. M., Brundin, P., Volkmann, J., et al. (2017). Parkinson disease. Nat. Rev. Dis. Primers 3:17013. doi: 10.1038/ nrdp. 2017.13

Power, J. D., Mitra, A., Laumann, T. O., Snyder, A. Z., Schlaggar, B. L., and Petersen, S. E. (2014). Methods to detect, characterize, and remove motion artifact in resting state fMRI. Neuroimage 84, 320-341. doi: 10.1016/j.neuroimage.2013. 08.048

Power, J. D., Plitt, M., Gotts, S. J., Kundu, P., Voon, V., Bandettini, P. A., et al. (2018). Ridding fMRI data of motion-related influences: removal of signals with distinct spatial and physical bases in multiecho data. Proc. Natl. Acad Sci. U.S.A. 115, E2105-E2114. doi: 10.1073/pnas.1720985115

Premi, E., Calhoun, V. D., Diano, M., Gazzina, S., Cosseddu, M., Alberici, A., et al. (2019). The inner fluctuations of the brain in presymptomatic Frontotemporal Dementia: the chronnectome fingerprint. Neuroimage 189, 645-654. doi: 10.1016/j.neuroimage.2019.01.080

Preti, M. G., Bolton, T. A., and Van De Ville, D. (2017). The dynamic functional connectome: state-of-the-art and perspectives. Neuroimage 160, 41-54. doi: 10.1016/j.neuroimage.2016.12.061

Putcha, D., Ross, R. S., Cronin-Golomb, A., Janes, A. C., and Stern, C. E. (2015). Altered intrinsic functional coupling between core neurocognitive networks in Parkinson's disease. Neuroimage Clin. 7, 449-455. doi: 10.1016/j.nicl.2015. 01.012

Quevenco, F. C., Preti, M. G., van Bergen, J. M., Hua, J., Wyss, M., Li, X., et al. (2017). Memory performance-related dynamic brain connectivity indicates pathological burden and genetic risk for Alzheimer's disease. Alzheimers Res. Ther. 9:24. doi: 10.1186/s13195-017-0249-247

Rohrer, J. D., and Warren, J. D. (2011). Phenotypic signatures of genetic frontotemporal dementia. Curr. Opin. Neurol. 24, 542-549. doi: 10.1097/WCO. 0b013e32834cd442

Smith, S. M., Miller, K. L., Salimi-Khorshidi, G., Webster, M., Beckmann, C. F., Nichols, T. E., et al. (2011). Network modelling methods for FMRI. Neuroimage 54, 875-891. doi: 10.1016/j.neuroimage.2010.08.063 
Smitha, K. A., Akhil Raja, K., Arun, K. M., Rajesh, P. G., Thomas, B., Kapilamoorthy, T. R., et al. (2017). Resting state fMRI: a review on methods in resting state connectivity analysis and resting state networks. Neuroradiol. J. 30, 305-317. doi: 10.1177/1971400917697342

Sourty, M., Thoraval, L., Roquet, D., Armspach, J. P., Foucher, J., and Blanc, F. (2016). Identifying dynamic functional connectivity changes in dementia with lewy bodies based on product hidden markov models. Front. Comput. Neurosci. 10:60. doi: 10.3389/fncom.2016.00060

Tagliazucchi, E., and Laufs, H. (2014). Decoding wakefulness levels from typical fMRI resting-state data reveals reliable drifts between wakefulness and sleep. Neuron 82, 695-708. doi: 10.1016/j.neuron.2014.03.020

Tagliazucchi, E., von Wegner, F., Morzelewski, A., Brodbeck, V., and Laufs, H. (2012). Dynamic BOLD functional connectivity in humans and its electrophysiological correlates. Front. Hum. Neurosci. 6:339. doi: 10.3389/ fnhum.2012.00339

Tessitore, A., Esposito, F., Vitale, C., Santangelo, G., Amboni, M., Russo, A., et al. (2012). Default-mode network connectivity in cognitively unimpaired patients with Parkinson disease. Neurology 79, 2226-2232. doi: 10.1212/WNL. 0b013e31827689d6

van Eimeren, T., Monchi, O., Ballanger, B., and Strafella, A. P. (2009). Dysfunction of the default mode network in Parkinson disease: a functional magnetic resonance imaging study. Arch. Neurol. 66, 877-883. doi: 10.1001/archneurol. 2009.97

Wee, C. Y., Yang, S., Yap, P. T., Shen, D., and Alzheimer's Disease Neuroimaging Initiative (2016). Sparse temporally dynamic resting-state functional connectivity networks for early MCI identification. Brain Imaging Behav. 10, 342-356. doi: 10.1007/s11682-015-9408-9402

Xu, Y., and Lindquist, M. A. (2015). Dynamic connectivity detection: an algorithm for determining functional connectivity change points in fMRI data. Front. Neurosci. 9:285. doi: 10.3389/fnins.2015.00285
Zhan, Z. W., Lin, L. Z., Yu, E. H., Xin, J. W., Lin, L., Lin, H. L., et al. (2018). Abnormal resting-state functional connectivity in posterior cingulate cortex of Parkinson's disease with mild cognitive impairment and dementia. CNS Neurosci. Ther. 24, 897-905. doi: 10.1111/cns.12838

Zhuang, X., Walsh, R. R., Sreenivasan, K., Yang, Z., Mishra, V., and Cordes, D. (2018). Incorporating spatial constraint in co-activation pattern analysis to explore the dynamics of resting-state networks: an application to Parkinson's disease. Neuroimage 172, 64-84. doi: 10.1016/j.neuroimage.2018.01.019

Conflict of Interest Statement: MF is Editor-in-Chief of the Journal of Neurology; has received compensation for consulting services and/or speaking activities from Biogen Idec, Merck-Serono, Novartis, Teva Pharmaceutical Industries; and has received research support from Biogen Idec, Merck-Serono, Novartis, Teva Pharmaceutical Industries, Roche, Italian Ministry of Health, Fondazione Italiana Sclerosi Multipla, and ARiSLA (Fondazione Italiana di Ricerca per la SLA). FA is Section Editor of NeuroImage: Clinical; has received speaker honoraria from Biogen Idec and Novartis; and receives or has received research supports from the Italian Ministry of Health, AriSLA (Fondazione Italiana di Ricerca per la SLA), and the European Research Council.

The remaining authors declare that the research was conducted in the absence of any commercial or financial relationships that could be construed as a potential conflict of interest.

Copyright () 2019 Filippi, Spinelli, Cividini and Agosta. This is an open-access article distributed under the terms of the Creative Commons Attribution License (CC BY). The use, distribution or reproduction in other forums is permitted, provided the original author(s) and the copyright owner(s) are credited and that the original publication in this journal is cited, in accordance with accepted academic practice. No use, distribution or reproduction is permitted which does not comply with these terms. 


\section{OPEN ACCESS}

Edited by:

Nicoletta Cera,

University of Porto, Portugal

Reviewed by:

Christopher C. Abbott,

University of New Mexico Health

Science Center, United States

Zhi-De Deng,

National Institute of Mental Health

(NIMH), United States

${ }^{*}$ Correspondence:

Yong Xu

xuyongsmu@vip.163.com

tThese authors have contributed equally to this work

Specialty section:

This article was submitted to Applied Neuroimaging,

a section of the journal

Frontiers in Neurology

Received: 15 March 2019

Accepted: 13 June 2019

Published: 27 June 2019

Citation:

Li Q, Liu S, Guo M, Yang C-X and Xu Y (2019) The Principles of Electroconvulsive Therapy Based on

Correlations of Schizophrenia and Epilepsy: A View From Brain Networks. Front. Neurol. 10:688 doi: 10.3389/fneur.2019.00688

\section{The Principles of Electroconvulsive Therapy Based on Correlations of Schizophrenia and Epilepsy: A View From Brain Networks}

\author{
Qi $\mathrm{Li}^{1 \dagger}$, Sha Liu ${ }^{1 \dagger}$, Meng Guo ${ }^{1}$, Cheng-Xiang Yang ${ }^{1}$ and Yong $\mathrm{Xu}{ }^{1,2,3,4 *}$ \\ ${ }^{1}$ Department of Psychiatry, First Hospital/First Clinical Medical College of Shanxi Medical University, Taiyuan, China, ${ }^{2}$ MDT \\ Center for Cognitive Impairment and Sleep Disorders, First Hospital of Shanxi Medical University, Taiyuan, China, ${ }^{3}$ National \\ Key Disciplines, Key Laboratory for Cellular Physiology of Ministry of Education, Department of Neurobiology, Shanxi Medical \\ University, Taiyuan, China, ${ }^{4}$ Department of Humanities and Social Science, Shanxi Medical University, Taiyuan, China
}

Electroconvulsive therapy (ECT) was established based on Meduna's hypothesis that there is an antagonism between schizophrenia and epilepsy, and that the induction of a seizure could alleviate the symptoms of schizophrenia. However, subsequent investigations of the mechanisms of ECT have largely ignored this originally established relationship between these two disorders. With the development of functional magnetic resonance imaging (fMRI), brain-network studies have demonstrated that schizophrenia and epilepsy share common dysfunctions in the default-mode network (DMN), saliency network (SN), dorsal-attention network (DAN), and central-executive network (CEN). Additionally, fMRI-defined brain networks have also been shown to be useful in the evaluation of the treatment efficacy of ECT. Here, we compared the ECT-induced changes in the pathological conditions between schizophrenia and epilepsy in order to offer further insight as to whether the mechanisms of ECT are truly based on antagonistic and/or affinitive relationships between these two disorders.

Keywords: electroconvulsive therapy (ECT), schizophrenia, temporal lobe epilepsy (TLE), brain networks, graph theory

\section{INTRODUCTION}

Electroconvulsive therapy (ECT) is one of the oldest therapeutic modalities in psychiatric clinical practice. The therapeutic effects of ECT putatively depend on the ECT-induced manifestation of seizure-like states, which was first proposed by the Hungarian neuropsychiatrist Ladislas Meduna in 1934 (1). This hypothesis arose from conspicuously opposite pathological results in epileptic vs. schizophrenic patients; Meduna observed an excess of glial cells in the brain tissue of epileptic patients (2), while his colleague found a reduction of glial cells in the brain tissue of schizophrenic patients (3). This findings convinced Meduna that there was an antagonism between schizophrenia and epilepsy, and gave birth to the idea that induction of seizure might help to alleviate the symptoms of schizophrenia. Subsequently, Meduna conducted the first human experiment by using camphor intramuscular injections (4). Finally, camphor was replaced by electricity to achieve more stable therapeutic effects by Ugo Cerletti and Lucio Bini, from which ECT was born (5). 
Although Meduna' hypothesis has since been refuted by the finding that different glial subtypes have different pathological features and do not exhibit a homogeneous opposing relationship between epilepsy and schizophrenia $(6,7)$, the relationship between these two disorders is still an interesting topic and has been debated for many years $(8,9)$. Schizophrenia is a serious psychiatric disorder that is always characterized by positive symptoms (hallucinations and delusions), negative symptoms (emotional disorders and impaired motivations), and cognitive impairment (10). Temporal lobe epilepsy (TLE) is the most widely studied and specific subtype of epilepsy $(11,12)$. Since these two neuropsychiatric disorders always shared some common symptoms, such as psychosis (13), emotion recognition disorders (14), and cognitive impairment (15), TLE showed a tight relation with schizophrenia in clinical diagnosis. The primary point of controversy is whether these two disorders have biological antagonism or affinity with one another. Some recent neuropathological studies still supported the antagonistic hypothesis for decreased astrocyte numbers in schizophrenia $(16,17)$ and increased astrocyte numbers and size in epilepsy (7). However, increasing sources of data also indicate that these two disorders may share some similarity. One study reported that there were some overlapping etiological factors between epilepsy and schizophrenia based on a population-based family study (18). Another study reported that schizophrenia and epilepsy share common features at the genetic level (19). As such, the relationship between epilepsy and schizophrenia is still an open question and requires further exploration for its elucidation.

Originating from the putative relationship between epilepsy and schizophrenia, ECT is now widely used for depression, acute manic episodes, catatonia, and treatment-resistant schizophrenia (20). Over the past 80 years, numerous psychological, psychoanalytical, and biological theories have been built to posit the potential therapeutic mechanisms of ECT (5). The present putative mechanisms of ECT have been primarily focused on structural, functional, and compositional changes of the brain after ECT treatment (21). Among these phenomena, the roles of cerebral blood flow (22), the blood-brain barrier (23), neurotransmitters (24), and the immune system (25) during ECT have been investigated. However, the potential mechanisms of ECT based on the correlation of epilepsy and schizophrenia remain largely unknown.

With the development of structural magnetic resonance imaging (sMRI) and functional MRI (fMRI), both structural and functional neuroimaging studies have provided more information to help better understand the relationship between schizophrenia and epilepsy $(11,26)$. Based on neuroimaging data, brain networks have been defined as correlational networks between several related brain regions in resting or task conditions. Disrupted brain networks-including the defaultmode network (DMN), dorsal-attention network (DAN), centralexecutive network (CEN), and saliency network (SN)-have been detected in both schizophrenic $(27,28)$ and epileptic patients (29-31). These shared brain networks may help to better understand the relationship between schizophrenia and epilepsy. These disease-disrupted brain networks can also be used as valuable biomarkers for assaying the therapeutic effects of ECT (32-34), which have shown apparent changes after ECT treatment at both structural and functional levels. Thus, comparison of the ECT-induced changes in the pathological conditions of schizophrenia and epilepsy may offer further insight as to whether the mechanisms of ECT are truly based on antagonistic and/or affinitive relationships between these two disorders.

This review will focus on brain-network changes in schizophrenia and epilepsy to discuss the affinity and/or antagonism between these two disorders. Our view was synthesized based on brain networks including the DMN, DAN, $\mathrm{CEN}$, and $\mathrm{SN}$, and a more large scale assessment of networks from graph theory. Additionally, changes in brain networks after ECT treatment will be compared under these two pathological conditions to help better understand the principles of ECT. All of the cited articles included in this review were written and published in English and were published before January 2019. The search engine, PubMed, was used with MESH terms.

\section{DEFAULT-MODE NETWORK}

Initial fMRI studies mainly focused on task-induced increases in regional brain activities during goal-directed behaviors. Subsequently, it was found that there are also consistent and task-independent decreases in regional brain activities, which is a phenomenon that has been defined as the baseline or default mode of brain function (35). Since then, the default mode of brain function has generated far more interest, discussion, and controversy, and it has called more attention to the importance of intrinsic brain activities (36). Combined with blood-oxygen-level-dependent (BOLD) signals and diffusiontensor imaging (DTI) data, these intrinsic brain activities have been shown to depend on networks across several brain regions, which collectively has been termed the DMN and consists of the following: the posterior cingulate cortex (PCC); adjacent precuneus (PCUN); medial prefrontal cortex (mPFC); mesial and inferior temporal lobes (mTL/iTL); and the inferior parietal lobe (iPL) (37) (Figure 1A). Functionally, these intrinsic brain activities of the DMN are activated at rest, become deactivated at the initiation of a task, and play an important role in cognitive functions and emotional processing (38). In the $\mathrm{N}$ back working-memory task, when cognition load increased, the functional connectivity within the DMN concomitantly decreased (39). Additionally, during long-term stabilization of memory, the intra-network synchrony of the DMN was positively corelated to individual performance (40). In emotional processing, less decreased DMN activity was highly related to poor emotionality (41). These physiological signatures of the DMN have attracted more and more attention in terms of their promising clinical applications in assessing and treating neuropsychiatric disorders.

Brain network studies have demonstrated increasing evidence of correlations between the DMN and some symptoms of schizophrenia (42). Auditory verbal hallucinations represent the most common positive symptom of schizophrenia and induce alterations of functional connectivity within the 
A

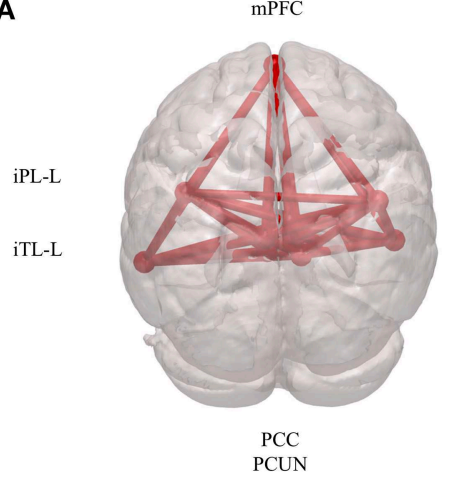

C

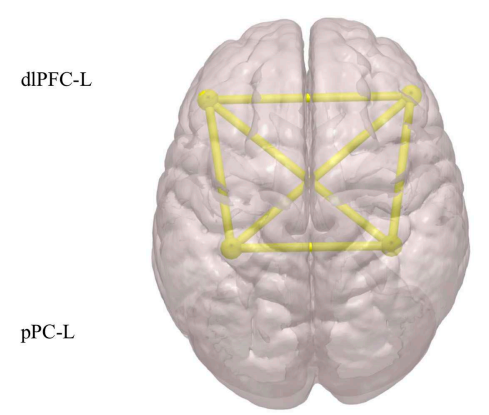

B

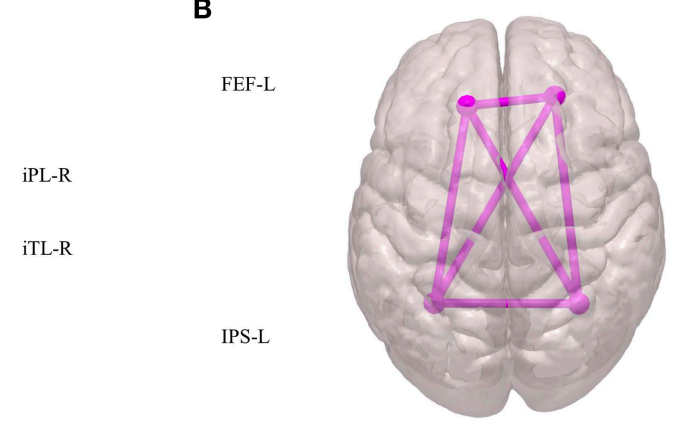

FEF-R

IPS-R

D

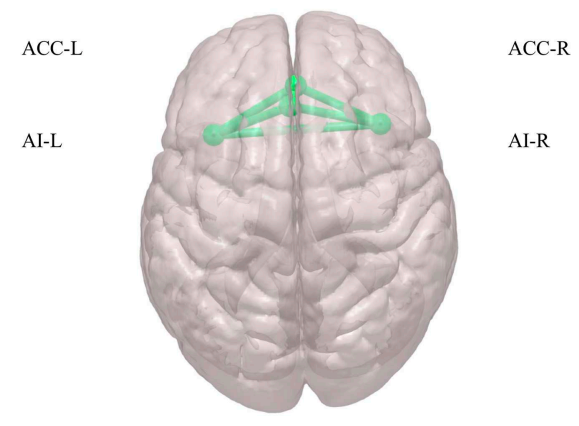

FIGURE 1 | Components of DMN, DAN, CEN, and SN. (A) Spatial components of DMN. (B) Spatial components of DAN. (C) Spatial components of CEN. (D) Spatial components of SN. DMN, default mode network; DAN, dorsal attention network; CEN, central executive network; SN, salience network; mPFC, medial prefrontal cortex; iPL, inferior parietal lobe; iTL, inferior temporal lobes; PCC, posterior cingulate cortex; PCUN, adjacent precuneus; FEF, frontal eye fields; IPS, intraparietal sulcus; dIPFC, dorsal lateral prefrontal cortex; pPC, posterior parietal cortex; ACC, anterior cingulate cortex; Al, anterior insula.

DMN (43). Additionally, patients with delusions also exhibit reduced regional deactivation of the DMN (44). Besides typical symptoms, positive symptoms scored by Positive and Negative Syndrome Scale (PANSS) have also been shown to be positively correlated with increased deactivation of brain regions, including the medial frontal, temporal, and cingulate gyri (45). For negative symptoms, emotional disorders of patients were found to be positively related to the magnitude of deactivation of rAC and mPFC regions (46). Additionally, negative symptoms scored by SANS have been shown to have a linear correlation to the functional connectivity within the DMN (47). These results indicate that changes of deactivation and functional connectivity within the DMN may represent a valuable means for assessing positive and negative schizophrenic symptoms; however, further evidence is required before this potential utility is sufficiently verified. Since the DMN is an important brain network that participates in cognitive processes, the relationship between the DMN and cognitive impairment of schizophrenia has also been widely studied. In a working memory task, the mPFC showed less deactivation (48) and greater activation (49). When the task load changed, DMN functions were over-recruited during a lowtask load, and hyper-deactivated during a high-task load (50). Other brain regions, such as the left-superior temporal gyrus, have also been shown to be positively correlated to cognitive impairment (51). In addition to BOLD-based functional studies, DTI anatomical data have also revealed that altered frontal structural connectivity is corelated to cognitive ability as well as schizophrenic symptoms (52).

Studies of the DMN in TLE have indicated altered taskrelated deactivations compared with those of healthy controls (53) and reduced functional connectivity (54). However, it may be difficult to confirm the affinity or antagonistic relationship of psychiatric manifestations between schizophrenia and TLE based on the present DMN results. More well-designed investigations are needed in terms of elucidating the short-term and long-term effects of seizure on the DMN, and for the comparison of the symptoms of these two disorders. Similar to schizophrenia, the role of the DMN in TLE-induced cognitive impairment has also been comprehensively studied. In the N-back working-memory task, the ACC showed greater deactivation in TLE patients (55). Additionally, there was decreased functional connectivity between the mPFC and mTL/iTL (54), as well as the mPFC and hippocampus (56). Interestingly, comparison of the cognitiverelated DMN changes between TLE and schizophrenia suggests an affinity between these two disorders. This relationship may be due to similar pathological changes in terms of the hippocampus exhibiting overlapping pathological features between the two disorders (57). 
Clinical practices have demonstrated that ECT can effectively ameliorate the positive symptoms of schizophrenia (58) and significantly reduce PANSS scores (59). Additionally, a brain network study revealed that increased $\mathrm{mTL}$ connectivity and PCC volume were accompanied by clinical improvement (33). These results may suggest that the therapeutic effects of ECT are achieved by an opposite regulation of the DMN in schizophrenia. However, this hypothesis still requires additional evidence from large-sample investigations. Additionally, whether this opposing change of the DMN can be detected in TLE patients requires further investigation. By exploring seizure-induced changes in TLE patients, we may better elucidate the relationship between schizophrenia and TLE and any therapeutic outcomes of ECT. Although ECT is effective, the side effects of ECT still harbor great concerns (60). Also, although similar cognitive impairments between schizophrenia and TLE have been documented, further studies are required to determine whether the side effects of ECT are related to seizure-induced hippocampal dysfunction.

\section{DORSAL ATTENTION NETWORK}

The DAN has been defined as a network that is comprised of the intraparietal sulcus (IPS) and the frontal eye fields (FEF) (61) (Figure 1B). Both IPS and FEF within the DAN play an important role in the maintenance of spatial attention, saccade planning, and visual working memory (62). The DAN is also activated during feature-based attention and provides a spatial coding in multiple reference frames (63). Furthermore, there is an interaction between the DAN and the DMN to carry out brain functions (64).

The DAN has been found to be altered in schizophrenic patients while carrying out several tasks that are mainly related to cognitive impairment. In visual attention and motor learning tasks, patients had reduced activation in the dorsal neocortical visual attention network (65). In a visual oddball task, connectivity between the right IPS (intraparietal sulcus) and right anterior insula (AI, a component of the ventral network) was significantly decreased in schizophrenic patients (66). In an Nback task, patients with schizophrenia had decreased inhibitory self-connections within the DAN regions, particularly in the left FEF and the left SPL (67). The interacting changes between the DAN and other networks have also been found. In schizophrenics during a working memory task, the DMN connectivity with the DAN was decreased $(68,69)$. In contrast to the default mode, patients demonstrated less connectivity in the executive control and dorsal attention networks (70).

Patients with TLE presented decreased functional connectivity in almost all of the regions within the $\operatorname{DAN}(31,71)$. For example, the FC values of the bilateral frontal eye field (FEF) and left intraparietal sulcus (IPS) were decreased (72). Thus, the DAN can also be regarded as a biomarker to explain the common cognition pathological mechanisms between schizophrenia and TLE, for which neuroimaging studies have revealed similar connectivity changes. ECT has also been shown to influence the DAN in depressed patients (73). However, the influence of the DAN in schizophrenia requires further investigation.

\section{CENTRAL-EXECUTIVE NETWORKS}

The CEN is a brain network that links the dorsal lateral prefrontal cortex (dlPFC) and posterior parietal cortex (pPC) (74) (Figure 1C). The CEN is frequently activated during typical fMRI executive tasks and its activity is often contrary to that of DMN activity (75). Brain imaging studies have shown that intelligence differences are positively correlated to functional interactions within the CEN, in both children and adults (76). Additionally, another important role of the CEN is to inhibit the DMN functions under certain conditions (77). This coordination between the CEN and the DMN is important in many neuropsychiatric disorders.

From recent literature, most results have reported that the CEN participates in a triple network including the DMN, the $\mathrm{CEN}$, and the salience network (SN) rather than serving an isolated role in schizophrenia (78). Thus, we will be discuss this topic after introducing the SN.

\section{SALIENCE NETWORK}

The $\mathrm{SN}$ is defined as a brain network comprised of the anterior insula (AI) and anterior cingulate cortex (ACC) (79) (Figure 1D). Physically, there is a strong functional connectivity within the SN, which is important for sensory perception and the coordination of behavioral responses $(80,81)$. Additionally, during many forms of emotional processing, the brain regions within the SN exhibit increased activity (82). The SN can also interact with the DMN and CEN to form a triple network, which participates in many mental process and disorders (83-85).

Schizophrenic patients show consistent abnormalities of insular signatures in both structural and functional neuroimaging studies, which indicates that the $\mathrm{SN}$ is involved in pathological processes (86). The $\mathrm{SN}$ has a direct correlation to schizophrenia symptoms, in which both auditory verbal hallucinations and delusions were detected to induce aberrant $\mathrm{SN}$ functional connectivity $(87,88)$. However, the strengths of these functional connectivities are not always homogeneous, because some studies have found a reduction in such connectivities (86), whereas other studies have reported a mixed pattern of increased and decreased connections (89). The interaction of the SN and DMN showed delayed communication that was directly correlated to positive and negative symptoms of schizophrenia (90). Additionally, a disrupted SN-CEN circuit also accounted for these schizophrenic symptoms (91). The triple network formed by SN-CEN-DMN nodes has shown dysregulated connections in schizophrenia (92) and mainly contributes to positive symptoms (93).

In TLE patients, one study reported that there was decreased connectivity to insula and ACC, suggesting a reduced SN (31). However, this result still needs to be further confirmed by more neuroimaging studies. When compared to schizophrenia, TLE showed a similar changes of the SN activity. However, 
it is difficult to confirm this hypothesis since there is still not enough neuroimaging evidence to properly assess this phenomenon. After ECT treatment, enhanced inter-network connectivity between the SN and the DMN has been found (73). However, whether this enhanced connectivity would be found in TLE patients requires further investigation.

\section{THE EMERGING ROLE OF GRAPH THEORY}

An increasing number of studies has supported that schizophrenia and TLE are disorders involving abnormal brain networks rather than several abnormal discrete brain regions $(29,70)$. It may be difficult to reach a unifying result by analysis of regional activation/deactivation or connectivity abnormalities. Therefore, graph theory, which is a mathematical framework that allows for the quantitative modeling and analysis of networks, has been applied with increasing success to neuroimaging data (94). In graph theory, the brain can be represented as a graph, and the set of nodes may be composed of brain regions or voxels (on a macroscopic level) or individual neurons (on a cellular level). Thus, edges will represent the connections between these brain regions/voxels or individual neurons depending on the conditions (95). Then, this information can be encoded in a mathematical data structure called a connectivity matrix. Based on the connectivity matrix, graph theory provides a more large-scale assessment of the human brain and can provide more integrative information of various diseases (96).

The results of graph theoretical analysis on schizophrenia are still inconsistent, but there has been some convergence around the concept of topological randomization $(97,98)$. Previous studies have shown that the functional brain networks of schizophrenia are relatively shifted toward the randomness of small-world topology (99). Another study also confirmed that schizophrenics demonstrate significant randomization of global network metrics (100). Additionally, the parameters of the global-network topology of schizophrenia have been found to be decreased in both functional and anatomical networks $(96,101)$.

On the contrary, graph theoretical analyses of networks have provided evidence generally suggesting a shift toward a more regularized topology in TLE patients (102). Within the range of small-world topologies, a more regularized network topology is present in TLE patients (103). The increased path length and clustering in TLE patients supports this more regularized arrangement (104). Thus, interestingly, topological characteristics have revealed an antagonistic feature between schizophrenia and epilepsy, although these studies of schizophrenia and epilepsy were independently conducted.

\section{REFERENCES}

1. Fink M. Meduna and the origins of convulsive therapy. Am J Psychiatry. (1984) 141:1034-41. doi: 10.1176/ajp.141.9.1034

2. Meduna Lv. Beiträge zur Histopathologie der Mikroglia. Arch Psychiatr Nervenkrankheiten. (1928) 82, 123-193. doi: 10.1007/bf01828859
However, there are limited reports regarding the effects of ECT on brain-network dynamics in schizophrenia. Whether ECT treatment reverses randomized brain networks to a more regularized pattern will require further investigations. As for the side effects of ECT, graph analysis of TLE patients showed that individuals with poor seizure control experienced more severe memory impairment (105). These results may indicate that a well-organized ECT practice may reduce side effects, but further neuroimaging evidence is still needed to validate or refute this hypothesis.

\section{CONCLUSIONS}

Functional brain networks, such as the DMN, the SN and the DAN, have provided a new perspective to understand the relationship between schizophrenia and epilepsy. At this level, these two diseases show similar connectivity changes and suggest that they have more of an affinity-type relationship due to their similar pathological features. At larger scale, graph theoretical analysis has indicated an antagonistic relationship between these two diseases, although more evidence is needed to determine the validity of these findings. In addition, ECT treatment has been shown to modify the dynamics of these brain networks. If future studies verify that ECT treatment can reverse randomized brain networks in schizophrenia to more regularized patterns, the original premise for the creation of ECT may be further corroborated and better understood.

\section{AUTHOR CONTRIBUTIONS}

YX designed and supervised the study. QL and SL drafted the manuscript. MG and C-XY collected some literature.

\section{FUNDING}

This work was supported by the National Key Research and Development Program of China (2016YFC1307004), the National Natural Science Foundation of China (81571319 and 81701326), the Multidisciplinary Team for Cognitive Impairment of Shanxi Science and Technology Innovation Training Team (201705D131027), the Support Program of the Youth Sanjin Scholars, and the 136 Medical Rejuvenation Project of Shanxi Province.

\section{ACKNOWLEDGMENTS}

We thank LetPub (www.letpub.com) for its linguistic assistance during the preparation of this manuscript. Psychiatr. (1931) 134:163-267. doi: 10.1007/bf02896997

4. Gazdag G, Bitter I, Ungvari GS, Baran B, Fink M. Laszlo Meduna's pilot studies with camphor inductions of seizures: the first 11 patients. J ECT. (2009) 25:3-11. doi: 10.1097/YCT.0b013e31819359fc 
5. Gazdag G, Ungvari GS. Electroconvulsive therapy: 80 years old and still going strong. World J Psychiatry. (2019) 9:1-6. doi: 10.5498/wjp.v9.i1.1

6. Bernstein HG, Steiner J, Guest PC, Dobrowolny H, Bogerts B. Glial cells as key players in schizophrenia pathology: recent insights and concepts of therapy. Schizophr Res. (2015) 161:4-18. doi: 10.1016/j.schres.2014. 03.035

7. Farrell JS, Wolff MD, Teskey GC. Neurodegeneration and pathology in epilepsy: clinical and basic perspectives. Adv Neurobiol. (2017) 15:317-34. doi: 10.1007/978-3-319-57193-5_12

8. Reynolds EH. Epilepsy and schizophrenia: relationship and biochemistry. Lancet. (1968) 1:398-401.

9. Cascella NG, Schretlen DJ, Sawa A. Schizophrenia and epilepsy: is there a shared susceptibility? Neurosci Res. (2009) 63:227-35. doi: 10.1016/j.neures.2009.01.002

10. Owen MJ, Sawa A, Mortensen PB. Schizophrenia. Lancet. (2016) 388:86-97. doi: 10.1016/s0140-6736(15)01121-6

11. Butler T, Weisholtz D, Isenberg N, Harding E, Epstein J, Stern E, et al. Neuroimaging of frontal-limbic dysfunction in schizophrenia and epilepsyrelated psychosis: toward a convergent neurobiology. Epilepsy Behav. (2012) 23:113-22. doi: 10.1016/j.yebeh.2011.11.004

12. Kandratavicius L, Hallak JE, Leite JP. What are the similarities and differences between schizophrenia and schizophrenia-like psychosis of epilepsy? A neuropathological approach to the understanding of schizophrenia spectrum and epilepsy. Epilepsy Behav. (2014) 38:143-7. doi: 10.1016/j.yebeh.2014.01.005

13. Hilger E, Zimprich F, Pataraia E, Aull-Watschinger S, Jung R, Baumgartner C, et al. Psychoses in epilepsy: a comparison of postictal and interictal psychoses. Epilepsy Behav. (2016) 60:58-62. doi: 10.1016/j.yebeh.2016.04.005

14. Monti G, Meletti S. Emotion recognition in temporal lobe epilepsy: a systematic review. Neurosci Biobehav Rev. (2015) 55:280-93. doi: 10.1016/j.neubiorev.2015.05.009

15. Allone C, Lo Buono V, Corallo F, Pisani LR, Pollicino P, Bramanti P, et al. Neuroimaging and cognitive functions in temporal lobe epilepsy: a review of the literature. J Neurol Sci. (2017) 381:7-15. doi: 10.1016/j.jns.2017.08.007

16. Steffek AE, McCullumsmith RE, Haroutunian V, Meador-Woodruff JH. Cortical expression of glial fibrillary acidic protein and glutamine synthetase is decreased in schizophrenia. Schizophr Res. (2008) 103:71-82. doi: 10.1016/j.schres.2008.04.032

17. Williams MR, Hampton T, Pearce RK, Hirsch SR, Ansorge O, Thom M, et al. Astrocyte decrease in the subgenual cingulate and callosal genu in schizophrenia. Eur Arch Psychiatry Clin Neurosci. (2013) 263:41-52. doi: 10.1007/s00406-012-0328-5

18. Clarke MC, Tanskanen A, Huttunen MO, Clancy M, Cotter DR, Cannon M. Evidence for shared susceptibility to epilepsy and psychosis: a population-based family study. Biol Psychiatry. (2012) 71:836-9. doi: 10.1016/j.biopsych.2012.01.011

19. Vonberg FW, Bigdeli TB. Genetic correlation between schizophrenia and epilepsy. JAMA Neurol. (2016) 73:125-6. doi: 10.1001/jamaneurol.2015.3480

20. Silvia HC, Mezzasalma MAU, Nardi AE. Pivotal role of tissue plasminogen activator in the mechanism of action of electroconvulsive therapy. J Psychopharmacol. (2014) 28:99-105. doi: 10.1177/02698811135 07639

21. Singh A, Kar SK. How electroconvulsive therapy works?: understanding the neurobiological mechanisms. Clin Psychopharmacol Neurosci. (2017) 15:210-21. doi: 10.9758/cpn.2017.15.3.210

22. Takano H, Motohashi N, Uema T, Ogawa K, Ohnishi T, Nishikawa M, et al. Changes in regional cerebral blood flow during acute electroconvulsive therapy in patients with depression: positron emission tomographic study. Br J Psychiatry. (2007) 190:63-8. doi: 10.1192/bjp.bp.106.023036

23. Andrade C, Bolwig TG. Electroconvulsive therapy, hypertensive surge, blood-brain barrier breach, and amnesia: exploring the evidence for a connection. J ECT. (2014) 30:160-4. doi: 10.1097/yct.0000000000 000133

24. Baldinger P, Lotan A, Frey R, Kasper S, Lerer B, Lanzenberger R. Neurotransmitters and electroconvulsive therapy. J ECT. (2014) 30:116-21. doi: $10.1097 /$ yct.0000000000000138

25. Yrondi A, Sporer M, Peran P, Schmitt L, Arbus C, Sauvaget A. Electroconvulsive therapy, depression, the immune system and inflammation: a systematic review. Brain Stimul. (2018) 11:29-51. doi: 10.1016/j.brs.2017.10.013

26. Marsh L, Sullivan EV, Morrell M, Lim KO, Pfefferbaum A. Structural brain abnormalities in patients with schizophrenia, epilepsy, and epilepsy with chronic interictal psychosis. Psychiatry Res. (2001) 108:1-15. doi: 10.1016/s0925-4927(01)00115-9

27. Lee WH, Doucet GE, Leibu E, Frangou S. Resting-state network connectivity and metastability predict clinical symptoms in schizophrenia. Schizophr Res. (2018) 201:208-16. doi: 10.1016/j.schres.2018.04.029

28. Lefort-Besnard J, Bassett DS, Smallwood J, Margulies DS, Derntl B, Gruber $\mathrm{O}$, et al. Different shades of default mode disturbance in schizophrenia: subnodal covariance estimation in structure and function. Hum Brain Mapp. (2018) 39:644-61. doi: 10.1002/hbm.23870

29. Cataldi M, Avoli M, de Villers-Sidani E. Resting state networks in temporal lobe epilepsy. Epilepsia. (2013) 54:2048-59. doi: 10.1111/epi.12400

30. Wei H, An J, Shen H, Zeng LL, Qiu S, Hu D. Altered effective connectivity among core neurocognitive networks in idiopathic generalized epilepsy: an fMRI evidence. Front Hum Neurosci. (2016) 10:447. doi: 10.3389/fnhum.2016.00447

31. Burianova H, Faizo NL, Gray M, Hocking J, Galloway G, Reutens D. Altered functional connectivity in mesial temporal lobe epilepsy. Epilepsy Res. (2017) 137:45-52. doi: 10.1016/j.eplepsyres.2017.09.001

32. Mulders PC, van Eijndhoven PF, Pluijmen J, Schene AH, Tendolkar I, Beckmann CF. Default mode network coherence in treatment-resistant major depressive disorder during electroconvulsive therapy. J Affect Disord. (2016) 205:130-7. doi: 10.1016/j.jad.2016.06.059

33. Wolf RC, Nolte HM, Hirjak D, Hofer S, Seidl U, Depping MS, et al. Structural network changes in patients with major depression and schizophrenia treated with electroconvulsive therapy. Eur Neuropsychopharmacol. (2016) 26:1465-74. doi: 10.1016/j.euroneuro.2016.06.008

34. Chen GD, Ji F, Li GY, Lyu BX, Hu W, Zhuo CJ. Antidepressant effects of electroconvulsive therapy unrelated to the brain's functional network connectivity alterations at an individual level. Chin Med J. (2017) 130:414-9. doi: 10.4103/0366-6999.199845

35. Raichle ME, MacLeod AM, Snyder AZ, Powers WJ, Gusnard DA, Shulman GL. A default mode of brain function. Proc Natl Acad Sci USA. (2001) 98:676-82. doi: 10.1073/pnas.98.2.676

36. Raichle ME, Snyder AZ. A default mode of brain function: a brief history of an evolving idea. Neuroimage. (2007) 37:1083-90; discussion 1097-89. doi: 10.1016/j.neuroimage.2007.02.041

37. Greicius MD, Supekar K, Menon V, Dougherty RF. Resting-state functional connectivity reflects structural connectivity in the default mode network. Cereb Cortex. (2009) 19:72-8. doi: 10.1093/cercor/bhn059

38. Mohan A, Roberto AJ, Mohan A, Lorenzo A, Jones K, Carney MJ, et al. The significance of the default mode network (DMN) in neurological and neuropsychiatric disorders: a review. Yale J Biol Med. (2016) 89:49-57.

39. Liang X, Zou Q, He Y, Yang Y. Topologically reorganized connectivity architecture of default-mode, executive-control, and salience networks across working memory task loads. Cereb Cortex. (2016) 26:1501-11. doi: 10.1093/cercor/bhu316

40. Sneve MH, Grydeland H, Amlien IK, Langnes E, Walhovd KB, Fjell AM. Decoupling of large-scale brain networks supports the consolidation of durable episodic memories. Neuroimage. (2017) 153:336-45. doi: 10.1016/j.neuroimage.2016.05.048

41. Ho TC, Connolly CG, Henje Blom E, LeWinn KZ, Strigo IA, Paulus MP, et al. Emotion-dependent functional connectivity of the default mode network in adolescent depression. Biol Psychiatry. (2015) 78:635-46. doi: 10.1016/j.biopsych.2014.09.002

42. Hu ML, Zong XF, Mann JJ, Zheng JJ, Liao YH, Li ZC, et al. A review of the functional and anatomical default mode network in schizophrenia. Neurosci Bull. (2017) 33:73-84. doi: 10.1007/s12264-016-0090-1

43. Alonso-Solis A, Vives-Gilabert Y, Grasa E, Portella MJ, Rabella M, Sauras RB, et al. Resting-state functional connectivity alterations in the default network of schizophrenia patients with persistent auditory verbal hallucinations. Schizophr Res. (2015) 161:261-8. doi: 10.1016/j.schres.2014.10.047

44. Lavigne KM, Menon M, Woodward TS. Functional brain networks underlying evidence integration and delusions in schizophrenia. Schizophr Bull. (2019). doi: 10.1093/schbul/sbz032. [Epub ahead of print]. 
45. Garrity AG, Pearlson GD, McKiernan K, Lloyd D, Kiehl KA, Calhoun VD. Aberrant "default mode" functional connectivity in schizophrenia. Am J Psychiatry. (2007) 164:450-7. doi: 10.1176/ajp.2007.164.3.450

46. Harrison BJ, Yucel M, Pujol J, Pantelis C. Task-induced deactivation of midline cortical regions in schizophrenia assessed with fMRI. Schizophr Res. (2007) 91:82-6. doi: 10.1016/j.schres.2006.12.027

47. Bluhm RL, Miller J, Lanius RA, Osuch EA, Boksman K, Neufeld RW, et al. Spontaneous low-frequency fluctuations in the BOLD signal in schizophrenic patients: anomalies in the default network. Schizophr Bull. (2007) 33:1004-12. doi: 10.1093/schbul/sbm052

48. Pomarol-Clotet E, Salvador R, Sarro S, Gomar J, Vila F, Martinez A, et al. Failure to deactivate in the prefrontal cortex in schizophrenia: dysfunction of the default mode network? Psychol Med. (2008) 38:1185-93. doi: $10.1017 / \mathrm{s} 0033291708003565$

49. Whitfield-Gabrieli S, Thermenos HW, Milanovic S, Tsuang MT, Faraone SV, McCarley RW, et al. Hyperactivity and hyperconnectivity of the default network in schizophrenia and in first-degree relatives of persons with schizophrenia. Proc Natl Acad Sci USA. (2009) 106:1279-84. doi: 10.1073/pnas.0809141106

50. Hahn B, Harvey AN, Gold JM, Ross TJ, Stein EA. Load-dependent hyperdeactivation of the default mode network in people with schizophrenia. Schizophr Res. (2017) 185:190-6. doi: 10.1016/j.schres.2017.01.001

51. Wang S, Zhan Y, Zhang Y, Lyu L, Lyu H, Wang G, et al. Abnormal longand short-range functional connectivity in adolescent-onset schizophrenia patients: a resting-state fMRI study. Prog Neuropsychopharmacol Biol Psychiatry. (2018) 81:445-51. doi: 10.1016/j.pnpbp.2017.08.012

52. Camchong J, MacDonald AW III, Bell C, Mueller BA, Lim KO. Altered functional and anatomical connectivity in schizophrenia. Schizophr Bull. (2011) 37:640-50. doi: 10.1093/schbul/sbp131

53. Stretton J, Winston G, Sidhu M, Centeno M, Vollmar C, Bonelli $\mathrm{S}$, et al. Neural correlates of working memory in Temporal Lobe Epilepsy-an fMRI study. Neuroimage. (2012) 60:1696-703. doi: 10.1016/j.neuroimage.2012.01.126

54. Zhang Z, Lu G, Zhong Y, Tan Q, Liao W, Wang Z, et al. Altered spontaneous neuronal activity of the default-mode network in mesial temporal lobe epilepsy. Brain Res. (2010) 1323:152-60. doi: 10.1016/j.brainres.2010. 01.042

55. Stretton J, Pope RA, Winston GP, Sidhu MK, Symms M, Duncan JS, et al. Temporal lobe epilepsy and affective disorders: the role of the subgenual anterior cingulate cortex. J Neurol Neurosurg Psychiatry. (2015) 86:144-51. doi: 10.1136/jnnp-2013-306966

56. Pittau F, Grova C, Moeller F, Dubeau F, Gotman J. Patterns of altered functional connectivity in mesial temporal lobe epilepsy. Epilepsia. (2012) 53:1013-23. doi: 10.1111/j.1528-1167.2012.03464.x

57. Nakahara S, Adachi $M$, Ito $H$, Matsumoto $M$, Tajinda $K$, van Erp TGM. Hippocampal pathophysiology: commonality shared by temporal lobe epilepsy and psychiatric disorders. Neurosci J. (2018) 2018:4852359. doi: 10.1155/2018/4852359

58. Wang J, Tang Y, Curtin A, Xia M, Tang X, Zhao Y, et al. ECT-induced brain plasticity correlates with positive symptom improvement in schizophrenia by voxel-based morphometry analysis of grey matter. Brain Stimul. (2019) 12:319-28. doi: 10.1016/j.brs.2018.11.006

59. Kim HS, Kim SH, Lee NY, Youn $\mathrm{T}$, Lee JH, Chung $\mathrm{S}$, et al. Effectiveness of electroconvulsive therapy augmentation on clozapineresistant schizophrenia. Psychiatry Investig. (2017) 14:58-62. doi: 10.4306/pi.2017.14.1.58

60. Sanghani SN, Petrides G, Kellner CH. Electroconvulsive therapy (ECT) in schizophrenia: a review of recent literature. Curr Opin Psychiatry. (2018) 31:213-22. doi: 10.1097/yco.0000000000000418

61. Vossel S, Geng JJ, Fink GR. Dorsal and ventral attention systems: distinct neural circuits but collaborative roles. Neuroscientist. (2014) 20:150-9. doi: $10.1177 / 1073858413494269$

62. Jerde TA, Merriam EP, Riggall AC, Hedges JH, Curtis CE. Prioritized maps of space in human frontoparietal cortex. J Neurosci. (2012) 32:17382-90. doi: 10.1523/jneurosci.3810-12.2012

63. Ptak R. The frontoparietal attention network of the human brain: action, saliency, and a priority map of the environment. Neuroscientist. (2012) 18:502-15. doi: 10.1177/1073858411409051
64. Dixon ML, Andrews-Hanna JR, Spreng RN, Irving ZC, Mills C, Girn M, et al. Interactions between the default network and dorsal attention network vary across default subsystems, time, and cognitive states. Neuroimage. (2017) 147:632-49. doi: 10.1016/j.neuroimage.2016.12.073

65. Keedy SK, Reilly JL, Bishop JR, Weiden PJ, Sweeney JA. Impact of antipsychotic treatment on attention and motor learning systems in first-episode schizophrenia. Schizophr Bull. (2015) 41:355-65. doi: 10.1093/schbul/sbu071

66. Jimenez AM, Lee J, Green MF, Wynn JK. Functional connectivity when detecting rare visual targets in schizophrenia. Psychiatry Res Neuroimaging. (2017) 261:35-43. doi: 10.1016/j.pscychresns.2017.01.007

67. Zhou Y, Zeidman P, Wu S, Razi A, Chen C, Yang L, et al. Altered intrinsic and extrinsic connectivity in schizophrenia. Neuroimage Clin. (2018) 17:704-16. doi: 10.1016/j.nicl.2017.12.006

68. Woodward TS, Leong K, Sanford N, Tipper CM, Lavigne KM. Altered balance of functional brain networks in Schizophrenia. Psychiatry Res Neuroimaging. (2016) 248:94-104. doi: 10.1016/j.pscychresns.2016. 01.003

69. Godwin D, Ji A, Kandala S, Mamah D. Functional connectivity of cognitive brain networks in schizophrenia during a working memory task. Front Psychiatry. (2017) 8:294. doi: 10.3389/fpsyt.2017.00294

70. Woodward ND, Rogers B, Heckers S. Functional resting-state networks are differentially affected in schizophrenia. Schizophr Res. (2011) 130:86-93. doi: 10.1016/j.schres.2011.03.010

71. Zhang Z, Lu G, Zhong Y, Tan Q, Yang Z, Liao W, et al. Impaired attention network in temporal lobe epilepsy: a resting FMRI study. Neurosci Lett. (2009) 458:97-101. doi: 10.1016/j.neulet.2009.04.040

72. Jiang LW, Qian RB, Fu XM, Zhang D, Peng N, Niu CS, et al. Altered attention networks and DMN in refractory epilepsy: a resting-state functional and causal connectivity study. Epilepsy Behav. (2018) 88:81-6. doi: 10.1016/j.yebeh.2018.06.045

73. Wang J, Wei Q, Wang L, Zhang H, Bai T, Cheng L, et al. Functional reorganization of intra- and internetwork connectivity in major depressive disorder after electroconvulsive therapy. Hum Brain Mapp. (2018) 39:140311. doi: $10.1002 / \mathrm{hbm} .23928$

74. Seeley WW, Menon V, Schatzberg AF, Keller J, Glover GH, Kenna $\mathrm{H}$, et al. Dissociable intrinsic connectivity networks for salience processing and executive control. J Neurosci. (2007) 27:2349-56. doi: 10.1523/jneurosci.5587-06.2007

75. Sherman LE, Rudie JD, Pfeifer JH, Masten CL, McNealy K, Dapretto M. Development of the default mode and central executive networks across early adolescence: a longitudinal study. Dev Cogn Neurosci. (2014) 10:148-59. doi: $10.1016 /$ j.dcn.2014.08.002

76. Langeslag SJ, Schmidt M, Ghassabian A, Jaddoe VW, Hofman A, van der Lugt A, et al. Functional connectivity between parietal and frontal brain regions and intelligence in young children: the Generation R study. Hum Brain Mapp. (2013) 34:3299-307. doi: 10.1002/hbm.22143

77. Chen AC, Oathes DJ, Chang C, Bradley T, Zhou ZW, Williams LM, et al. Causal interactions between fronto-parietal central executive and defaultmode networks in humans. Proc Natl Acad Sci USA. (2013) 110:19944-9. doi: 10.1073/pnas.1311772110

78. Kang Y, Huang $\mathrm{K}$, Cai S, Wang $\mathrm{H}$, Liu J, Wang $\mathrm{Y}$, et al. Association between function and structure of the triple network and catechol-O-methyltransferase val(158)met polymorphism in the first episode schizophrenia. Neurosci Lett. (2018) 687:65-70. doi: 10.1016/j.neulet.2018.09.033

79. Palaniyappan L, White TP, Liddle PF. The concept of salience network dysfunction in schizophrenia: from neuroimaging observations to therapeutic opportunities. Curr Top Med Chem. (2012) 12:2324-38. doi: $10.2174 / 156802612805289881$

80. Lamichhane B, Dhamala M. The salience network and its functional architecture in a perceptual decision: an effective connectivity study. Brain Connect. (2015) 5:362-70. doi: 10.1089/brain.2014.0282

81. Lamichhane B, Adhikari BM, Dhamala M. Salience network activity in perceptual decisions. Brain Connect. (2016) 6:558-71. doi: 10.1089/brain.2015.0392

82. Barrett LF, Satpute AB. Large-scale brain networks in affective and social neuroscience: towards an integrative functional architecture of the 
brain. Curr Opin Neurobiol. (2013) 23:361-72. doi: 10.1016/j.conb.2012. 12.012

83. Chand GB, Dhamala M. Interactions among the brain defaultmode, salience, and central-executive networks during perceptual decision-making of moving dots. Brain Connect. (2016) 6:249-54. doi: 10.1089/brain.2015.0379

84. Fan J, Zhong M, Gan J, Liu W, Niu C, Liao H, et al. Altered connectivity within and between the default mode, central executive, and salience networks in obsessive-compulsive disorder. J Affect Disord. (2017) 223:10614. doi: 10.1016/j.jad.2017.07.041

85. Liu Y, Li L, Li B, Feng N, Li L, Zhang X, et al. Decreased triple network connectivity in patients with recent onset post-traumatic stress disorder after a single prolonged trauma exposure. Sci Rep. (2017) 7:12625. doi: 10.1038/s41598-017-12964-6

86. Palaniyappan L, Liddle PF. Does the salience network play a cardinal role in psychosis? An emerging hypothesis of insular dysfunction. J Psychiatry Neurosci. (2012) 37:17-27. doi: 10.1503/jpn. 100176

87. Orliac F, Naveau M, Joliot M, Delcroix N, Razafimandimby A, Brazo P, et al. Links among resting-state default-mode network, salience network, and symptomatology in schizophrenia. Schizophr Res. (2013) 148:74-80. doi: 10.1016/j.schres.2013.05.007

88. Mallikarjun PK, Lalousis PA, Dunne TF, Heinze K, Reniers RL, Broome MR, et al. Aberrant salience network functional connectivity in auditory verbal hallucinations: a first episode psychosis sample. Transl Psychiatry. (2018) 8:69. doi: 10.1038/s41398-018-0118-6

89. White TP, Gilleen J, Shergill SS. Dysregulated but not decreased salience network activity in schizophrenia. Front Hum Neurosci. (2013) 7:65. doi: 10.3389/fnhum.2013.00065

90. Hare SM, Ford JM, Mathalon DH, Damaraju E, Bustillo J, Belger A, et al. Salience-default mode functional network connectivity linked to positive and negative symptoms of schizophrenia. Schizophr Bull. (2018) 45:892-901. doi: $10.1093 /$ schbul/sby112

91. Chen Q, Chen X, He X, Wang L, Wang K, Qiu B. Aberrant structural and functional connectivity in the salience network and central executive network circuit in schizophrenia. Neurosci Lett. (2016) 627:178-84. doi: 10.1016/j.neulet.2016.05.035

92. Jiang Y, Duan M, Chen X, Chang X, He H, Li Y, et al. Common and distinct dysfunctional patterns contribute to triple network model in schizophrenia and depression: a preliminary study. Prog Neuropsychopharmacol Biol Psychiatry. (2017) 79(Pt B):302-10. doi: 10.1016/j.pnpbp.2017. 07.007

93. Supekar K, Cai W, Krishnadas R, Palaniyappan L, Menon V. Dysregulated brain dynamics in a triple-network saliency model of schizophrenia and its relation to psychosis. Biol Psychiatry. (2019) 85:60-9. doi: 10.1016/j.biopsych.2018.07.020

94. Bullmore E, Sporns O. Complex brain networks: graph theoretical analysis of structural and functional systems. Nat Rev Neurosci. (2009) 10:186-98. doi: $10.1038 / \mathrm{nrn} 2575$
95. Chiang S, Haneef Z. Graph theory findings in the pathophysiology of temporal lobe epilepsy. Clin Neurophysiol. (2014) 125:1295-305. doi: 10.1016/j.clinph.2014.04.004

96. Nelson BG, Bassett DS, Camchong J, Bullmore ET, Lim KO. Comparison of large-scale human brain functional and anatomical networks in schizophrenia. Neuroimage Clin. (2017) 15:439-48. doi: 10.1016/j.nicl.2017.05.007

97. Stam CJ, Reijneveld JC. Graph theoretical analysis of complex networks in the brain. Nonlinear Biomed Phys. (2007) 1:3. doi: 10.1186/1753-4631-1-3

98. Rubinov M, Knock SA, Stam CJ, Micheloyannis S, Harris AW, Williams LM, et al. Small-world properties of nonlinear brain activity in schizophrenia. Hum Brain Mapp. (2009) 30:403-16. doi: 10.1002/hbm.20517

99. Liu Y, Liang M, Zhou Y, He Y, Hao Y, Song M, et al. Disrupted small-world networks in schizophrenia. Brain. (2008) 131(Pt 4):945-61. doi: 10.1093/brain/awn018

100. Lo CY, Su TW, Huang CC, Hung CC, Chen WL, Lan TH, et al. Randomization and resilience of brain functional networks as systemslevel endophenotypes of schizophrenia. Proc Natl Acad Sci USA. (2015) 112:9123-8. doi: 10.1073/pnas.1502052112

101. Erdeniz B, Serin E, Ibadi Y, Tas C. Decreased functional connectivity in schizophrenia: the relationship between social functioning, social cognition and graph theoretical network measures. Psychiatry Res Neuroimaging. (2017) 270:22-31. doi: 10.1016/j.pscychresns.2017.09.011

102. Bernhardt BC, Chen Z, He Y, Evans AC, Bernasconi N. Graph-theoretical analysis reveals disrupted small-world organization of cortical thickness correlation networks in temporal lobe epilepsy. Cereb Cortex. (2011) 21:2147-57. doi: 10.1093/cercor/bhq291

103. Bernhardt BC, Bonilha L, Gross DW. Network analysis for a network disorder: the emerging role of graph theory in the study of epilepsy. Epilepsy Behav. (2015) 50:162-70. doi: 10.1016/j.yebeh.2015.06.005

104. Bernhardt BC, Bernasconi N, Hong SJ, Dery S, Bernasconi A. Subregional mesiotemporal network topology is altered in temporal lobe epilepsy. Cereb Cortex. (2016) 26:3237-48. doi: 10.1093/cercor/bhv166

105. Park CH, Choi YS, Jung AR, Chung HK, Kim HJ, Yoo JH, et al. Seizure control and memory impairment are related to disrupted brain functional integration in temporal lobe epilepsy. J Neuropsychiatry Clin Neurosci. (2017) 29:343-50. doi: 10.1176/appi.neuropsych.16100216

Conflict of Interest Statement: The authors declare that the research was conducted in the absence of any commercial or financial relationships that could be construed as a potential conflict of interest.

Copyright (c) $2019 \mathrm{Li}$, Liu, Guo, Yang and Xu. This is an open-access article distributed under the terms of the Creative Commons Attribution License (CC BY). The use, distribution or reproduction in other forums is permitted, provided the original author(s) and the copyright owner(s) are credited and that the original publication in this journal is cited, in accordance with accepted academic practice. No use, distribution or reproduction is permitted which does not comply with these terms. 


\section{Eigenvector Centrality Dynamics From Resting-State fMRI: Gender and Age Differences in Healthy Subjects}

\section{OPEN ACCESS}

Edited by:

Roberto Esposito, Azienda Ospedaliera Ospedali Riuniti

Marche Nord, Italy

Reviewed by:

William Hedley Thompson, Karolinska Institute (KI), Sweden

Xiang $L i$,

Massachusetts General Hospital, Harvard Medical School,

United States

*Correspondence: Alle Meije Wink a.wink@amsterdamumc.n

Specialty section: This article was submitted to Brain Imaging Methods, a section of the journal Frontiers in Neuroscience

Received: 25 March 2019 Accepted: 06 June 2019

Published: 27 June 2019

Citation:

Wink AM (2019) Eigenvector Centrality Dynamics From Resting-State fMRl: Gender and Age Differences in Healthy Subjects. Front. Neurosci. 13:648. doi: 10.3389/fnins.2019.00648

\author{
Alle Meije Wink* \\ Radiology and Nuclear Medicine, Amsterdam University Medical Center, Amsterdam, Netherlands
}

With the increasing use of functional brain network properties as markers of brain disorders, efficient visualization and evaluation methods have become essential. Eigenvector centrality mapping (ECM) of functional MRI (fMRI) data enables the representation of per-node graph theoretical measures as brain maps. This paper studies the use of centrality dynamics for measuring group differences in imaging studies. Imaging data were used from a publicly available imaging study, which included resting $\mathrm{fMRI}$ data. After warping the images to a standard space and masking cortical regions, ECM were computed in a sliding window. The dual regression method was used to identify dynamic centrality differences inside well-known resting-state networks between gender and age groups. Gender-related differences were found in the medial and lateral visual, motor, default mode, and executive control RSN, where male subjects had more consistent centrality variations within the network. Age-related differences between the youngest and oldest subjects, based on a median split, were found in the medial visual, executive control and left frontoparietal networks, where younger subjects had more consistent centrality variations within the network. Our findings show that centrality dynamics can be used to identify between-group functional brain network centrality differences, and that age and gender distributions studies need to be taken into account in functional imaging studies.

\footnotetext{
Keywords: graph theory - graph algorithms, trees, functional MRI (fMRI) methods, imaging studies, age-related, gender-related
}

\section{INTRODUCTION}

Functional brain network properties are increasingly studied as markers of neurological and psychiatric disorders. With the availability of fast computer hardware and increased memory, analyses have been focusing on the dynamics of networks. We describe an efficient implementation and application of dynamic centrality and its differences between males and females.

Resting functional magnetic resonance imaging (RfMRI) acquires MR images of the brain that are sensitive to blood oxygenation, which serves as a proxy for local brain activity. In the absence of a task, a number of recurring patterns in RfMRI data (Smith et al., 2009) are referred to as 
resting-state networks (RSNs) computed from pairwise similarity matrices between voxel time series (Damoiseaux et al., 2006). In the case of these non-evoked, non-causal, undirected similarities, the term 'functional connectivity' is used, and (Pearson) correlation is a common connectivity measure.

Visualization and interpretation of complete network connections matrices is increasingly problematic with increasing network size. Voxelwise whole-brain network analyses therefore focus on properties of network nodes. The concept of centrality of a node in a network represents the proportion of the network traffic involving that node. It can be expressed in a number of ways, for example:

- the number of connections of a node (degree centrality)

- the number of times a node appears in the shortest path between two other nodes (betweenness centrality).

For eigenvector centrality EC, this measure is the coefficient in the dominant eigenvector (the one with the largest eigenvalue) of the connections matrix. It can be computed iteratively as the weighted sum of centralities of a node's neighbors. Voxelwise EC computation, or eigenvector centrality mapping (ECM) (Lohmann et al., 2010), has shown to provide a measure for functional brain network analysis that is robust with respect to physiological and technical confounding factors and sensitive to changes correlated with pathology.

Eigenvector centrality mapping has been used to identify functional brain network changes in patients, e.g., with Alzheimer's disease (AD) (Binnewijzend et al., 2014), multiple sclerosis (Eijlers et al., 2017), and healthy subjects at risk for AD (Wink et al., 2018).

The observation that functional brain properties vary over time led to the emergence of dynamic functional connectivity (Tagliazucchi et al., 2016; Preti et al., 2017). Unlike dynamic causal modeling experiments, where a change in cognitive tasks leads to a different connection pattern, dynamic functional connectivity itself is also non-evoked and non-causal. Functional similarities such as the correlation between time series, are measured inside a sampling interval, and network dynamics are often measured by shifting a fixed-size time window across the scan duration. The result is a time series of network measures which can be further analyzed.

This makes dynamic functional connectivity as a method bit of a double-edged sword: properties of temporal connectivity can be studied in greater detail, but the method produces an enormous amount of intermediate results, requiring an extra step in analysis, visualization, and interpretation.

We propose dynamic eigenvector centrality mapping (dECM), which produces a time series of centrality maps, each of which is an image of the same size as the original volumes. This allows easy and intuitive visualization of each window position for which centrality is computed. This technique has been used before as an intermediate result (Preti and Ville, 2017), which showed that dominant EC patterns over time are similar to RSN. This paper uses those previous findings to detect group-based differences in EC variability.

We use dual regression, an analysis used for functional connectivity studies (Nickerson et al., 2017), to demonstrate how differences between males and females and age groups can be found in a public example data set.

\section{MATERIALS AND METHODS}

\section{Data From OpenNeuro}

Imaging data was downloaded from the OpenNeuro project ${ }^{1}$. The "Washington 120" data set includes structural T1-weighted MR images and resting fMRI time series of 120 healthy subjects (59 male), recruited from Washington University. Subjects were right-handed native English speakers, reported no neurological or psychiatric disease, were not on medication and had previously given written informed consent (Power et al., 2013). Mean age was 24.7 years at scanning (median: 24.5) and standard deviation 2.4 years (interquartile range: 3.1 ). Ages were not significantly different between males and females (Kruskal-Wallis $p=0.5$ ).

Imaging was done on a Siemens MAGNETOM Trio 3T scanner (Erlangen, Germany) with a Siemens 12 channel Head Matrix Coil. The T1-weighted scans used the MP-RAGE sequence with $\mathrm{TE}=3.06 \mathrm{~ms}$, TR-partition $=2.4 \mathrm{~s}, \mathrm{TI}=1000 \mathrm{~ms}$, flip angle $=8^{\circ}$ and 127 slices with $1 \mathrm{~mm} \times 1 \mathrm{~mm} \times 1 \mathrm{~mm}$ voxels. Resting fMRI (RfMRI) was acquired using an EPI sequence with $\mathrm{TE}=27 \mathrm{~ms}, \mathrm{TR}=2.5 \mathrm{~s}$, flip angle $=90^{\circ}$ and 32 contiguous interleaved $4 \mathrm{~mm}$ axial slices with in-plane resolution $=4 \mathrm{~mm} \times 4 \mathrm{~mm}$. Subjects kept their eyes open during scanning, fixating on a cross displayed on the screen. The duration of scan varied from 130 volumes $\sim 5 \mathrm{~min}$, to 720 volumes $\sim 30 \mathrm{~min}$.

The OpenNeuro website provides the preprocessed data, using $\mathrm{SPM}^{2}$ version 8 (Ashburner and Friston, 2005), in standard space. The original scans had been corrected for slice timing to align the start times of all slices per volume, spatially realigned to correct head motion, and their intensities normalized to a mean of 1000. Images were then resampled to MNI-space on an isotropic $3 \mathrm{~mm}$ grid (Power et al., 2014). The preprocessing using mriqc (Esteban et al., 2017) reported five subjects with a mean frame displacement FD (Power et al., 2012) of $0.2 \mathrm{~mm}$. We excluded these five subjects from our analysis. Remaining motion-related effects were removed from the standard-space time series by computing the effects of motion using single-subject GLMs with the motion parameters as covariates, and subtracting these from the data (Soares et al., 2016). The resulting data set were 115 subjects ( 57 male) with median age 24.5 years ( 57 below) whose time series had the linear effects of rigid-body motion parameters for removed for each volume.

The T1-weigted images available from the OpenNeuro website were accompanied by segmentations into tissue types and parameters for mapping those to the MNI space in SPM. We

\footnotetext{
${ }^{1}$ https://openneuro.org

${ }^{2}$ https://www.fil.ion.ucl.ac.uk/spm
} 
applied these parameters to the anatomical images to bring them in the same space as the RfMRI data.

The gray matter masks were thresholded at $20 \%$ gray matter density and binarized before being mapped into MNI space. The MNI-space masks were then thresholded again at 0.2 and binarized, to create one gray matter mask for the group. A mask of each of the functional images was created by taking the time series' minimum at each voxel, and thresholding the map of temporal minima at the $20^{\text {th }}$ percentile, yielding a binary mask. The group mean over all subjects' binary masks was thresholded at $75 \%$ yielding one binary mask for the group. Every RfMRI data set was masked by the intersection of the group gray matter and functional masks and an additional mask to exclude the cerebellum. Using the same mask for every subject ensured that sizes between the RfMRI networks did not vary, which would have been a source of variance (Van Wijk et al., 2010).

\section{Eigenvector Centrality Mapping}

Most connectivity analyses for RfMRI are based on the matrix $\boldsymbol{R}$ of pairwise correlations between voxels or brain regions. In the voxelwise case, the size of this matrix poses two problems: (i) the matrix is too big for the working memory of standard computers and (ii) the total connectome, i.e., the set of connections describing a condition or an experiment, is to big to visualize or interpret. A common solution is to spatially downsample the data or to group the voxels into subnetworks or parcellations. Another approach is to look at node properties instead of connections, with the benefit of keeping the full imaging resolution. The centrality property signifies the prominence of a node in terms of the participation in the connections of the network.

Centrality can be expressed using a growing number of measures. The simplest is degree: the number of connections of a node to others. One of the more intuitive measures of a node's centrality is betweenness: the proportion of all the shortest paths between pairs of nodes in the network on which it lies (Fletcher and Wennekers, 2016).

A node's eigenvector centrality (EC) is defined as the sum of its neighbors' centralities (Lohmann et al., 2010) and can be efficiently computed from the connections matrix $\boldsymbol{R}$ using power iteration if this matrix is semi-definite and positive (or irreducible and non-negative). Power iteration starts with an estimate vector $v_{0}$ and computes

$$
v_{i+1}=\mathbf{R}^{*} v_{i}
$$

followed by $L_{2}$ normalization, for subsequent steps, until a convergence criterion is met. A computationally efficient definition of voxelwise ECM uses $\boldsymbol{R}+1$ as the connections matrix to ensure positivity and the fact that for intensity-normalized scaled fMRI data $\boldsymbol{Y}_{[N \times T]}$ of $N$ voxels and $T$ time points (Equation 1) can be re-written as

$$
\nu_{i+1}=\mathbf{Y}_{[N \times T]} *\left(\mathbf{Y}_{[T \times N]}^{\mathbf{T}} * \nu_{i[N \times 1]}\right)
$$

so that it needs to store at most NT values during computation instead of $N^{2}$ (Wink et al., 2012). The ECM technique has since been used in a number of settings to differentiate between experimental conditions and patient groups (Lohmann et al., 2010; Wink et al., 2012, 2018; Binnewijzend et al., 2014; Schoonheim et al., 2014; Duinkerken et al., 2017; Eijlers et al., 2017).

Correlations of signals measured during a time interval are static measures, yet we know that functional connections in the brain are dynamic in that their strength varies in time (Tagliazucchi et al., 2012; Zalesky and Breakspear, 2015; Breakspear, 2017). To capture the temporal evolution of centrality, we computed voxelwise EC in a sliding window, resulting in a time series of 3-dimensional EC maps. We used the fastECM matlab toolbox ${ }^{3}$ for ECM computations, which enables the computation of centrality time series as follows: given the number $T$ of volumes in the input time series and a requested number $M$ of centrality maps, it moves a window of $T-M+1$ volumes over all $M$ different positions and returns the time series of centrality maps. For the data used in this paper, with input time series of different lengths, this meant that the size of the window for EC computation varied between subjects.

We used $M=100$, resulting in a time series of 100 ECMs per subject (see Figure 1), containing each voxel's centrality given the starting time of the interval in which it was computed. Maps were multiplied to have intensities around 4500.

\section{Statistics of EC Variability Over Time}

Each map of the EC time series shows the spatial pattern of the voxelwise temporal correlations inside its time window, and if well-defined RSNs such as the default mode network (DMN) and visual networks (Damoiseaux et al., 2006) vary over time, these variations should be visible in the time-varying EC maps. One way to determine this temporal variability is using dual regressions, DR (Nickerson et al., 2017). This method first regresses each fMRI data set onto a set of spatial patterns, e.g., ones that represent $\mathrm{RSN}$, resulting in a representative time series per pattern, per subject. After that, the fMRI data are regressed onto these representative time series, yielding a voxelwise measure of temporal correspondence with each pattern, for each subject. Both steps are generalized linear models: for data $\boldsymbol{Y}_{[N \times T]}$ and a set $\boldsymbol{C}_{[N \times M]}$ of $M$ spatial patterns, the first step solves

$$
\mathbf{Y}_{[N \times T]}=\mathbf{C}_{[N \times M]} * \mathbf{S}_{[M \times T]}+\varepsilon_{[N \times T]}
$$

for the pattern-specific time series $S[\mathrm{M} \times \mathrm{T}]$, and the next step solves

$$
\mathbf{Y}_{[N \times T]}=\mathbf{D}_{[N \times M]} * \mathbf{S}_{[M \times T]}+\varepsilon_{[N \times T]}
$$

for the voxelwise correspondence $D_{[N \times M]}$ to $S_{[M \times T]}$, and can be computed with a least squares method. The dual regression method, first introduced in 2009 (Beckmann, 2009) has been used to identify between-group RSNs differences (Smith et al., 2014; Nickerson et al., 2017). We computed DR using 10 spatial components linked to RSN by performing

\footnotetext{
${ }^{3}$ https://github.com/amwink/bias/tree/master/matlab/fastECM
} 


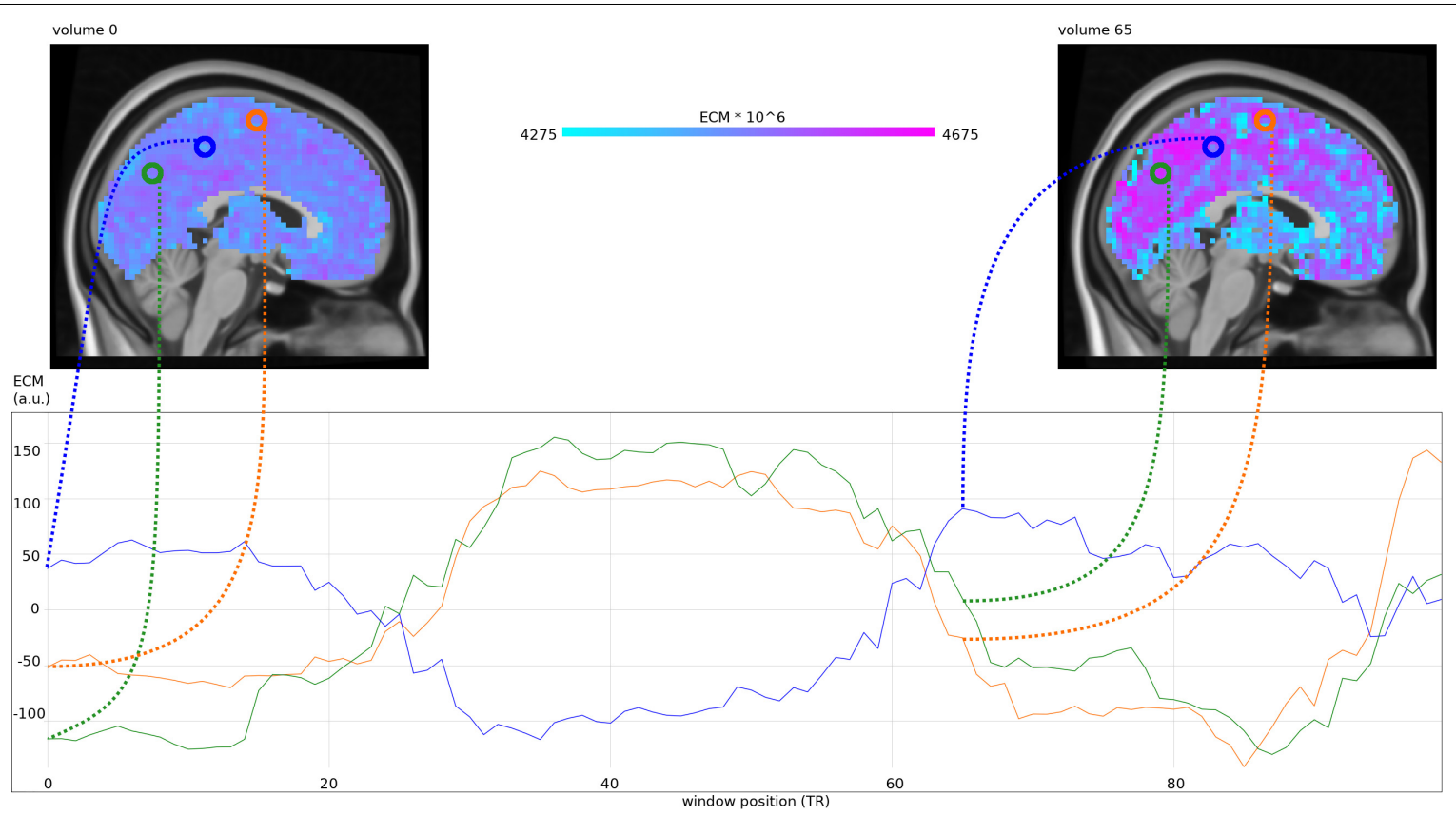

FIGURE 1 | (Top) Eigenvector centrality maps (ECM) computed in a sliding window at 100 different positions, at window position 0*TR (a) and 65*TR (b). (Bottom) Mean-centered centrality timecourses for voxels at position $(3,-67,35)$ in $\mathrm{MNI}$ space (green), position $(3,-37,50)$ in $\mathrm{MNI}$ space (blue) and position $(3,-7,65)$ in $\mathrm{MNI}$ space (orange).

independent component analysis (ICA) on the results of 7,342 fMRI analyses (Smith et al., 2009), resulting in 10 regression maps per subject between-group differences in temporal EC variations between female and male participants, and young vs. old participants, respectively, were assessed for each RSN mask except the 'cerebellar' RSN with permutation statistics, using 10000 permutations and family-wise error (FWE) control with threshold-free cluster enhancement (TFCE) and a significance threshold of $p<0.05$. FWE was done by re-computing the statistics (Equation) after group/age permutation, respectively and storing the maximum statistic found (Nichols and Holmes, 2002). Observed statistics were compared with the histogram of these maximum statistics, thus achieving location independence, and therefore, FWE control. TFCE (Smith and Nichols, 2009) increases the statistic values of each peak that is part of a spatial cluster by integrating the cluster's spatial extent at different levels below the peak, providing a balance between correction for multiple voxelwise tests and relaxing the correction for voxels that are part of a cluster (and therefore not independent). Effects of gender and age were investigated in the between-group design using a contrast between male and female participants and younger and older participants, respectively, the latter being defined by a median split. A covariate for scan length (and thus, window length) was also included in the model. The dual regression script was slightly modified to only test for EC variations with each RSN pattern inside the mask (of Z-scores for that pattern above Section "Statistics of EC Variability Over Time"), as used previously (Binnewijzend et al., 2012). Analysis scripts for this paper are available at https://github.com/amwink/openNeuro.

\section{RESULTS}

\section{Participant Sub-groups}

The median(mean) age of the female and male participants, respectively, were $24.4(24.5)$ and $24.9(24.5)$, and their standard deviations (interquartile ranges) were 1.9(3.1) and 2.6(3.2), respectively. The median split of the subjects into younger and older resulted in a 'younger' and 'older' group with mean (median) age of 22.8(23.2) and 26.5(26.3), respectively, and standard deviations (interquartile ranges) of 1.1(1.3) and $1.5(2.1)$, respectively. The 'younger' and 'older' group both consisted of 28 males and 29 females. The distributions of ages did not differ significantly for males and females in the young subjects (Kruskal-Wallis $p=0.39$; it did so in the old subjects (Kruskal-Wallis $p=0.04$ ) where the males were older than the females. Across the whole group there was no effect (Kruskal-Wallis $p=0.32$ ).

\section{Eigenvector Centrality Mapping}

Time averages of each subject's voxelwise EC time series were combined for four subgroups: young-old $\times$ male-female. The averages across these groups are shown in Figure 2 The overall features of the maps are very similar in the groups and to individual EC maps per volume (see Figure 1), indicating robust features and temporal stability.

\section{Statistics of EC Variability Over Time}

The dual regression analysis and non-parametric testing showed effects for a number of resting network patterns. Many of these 


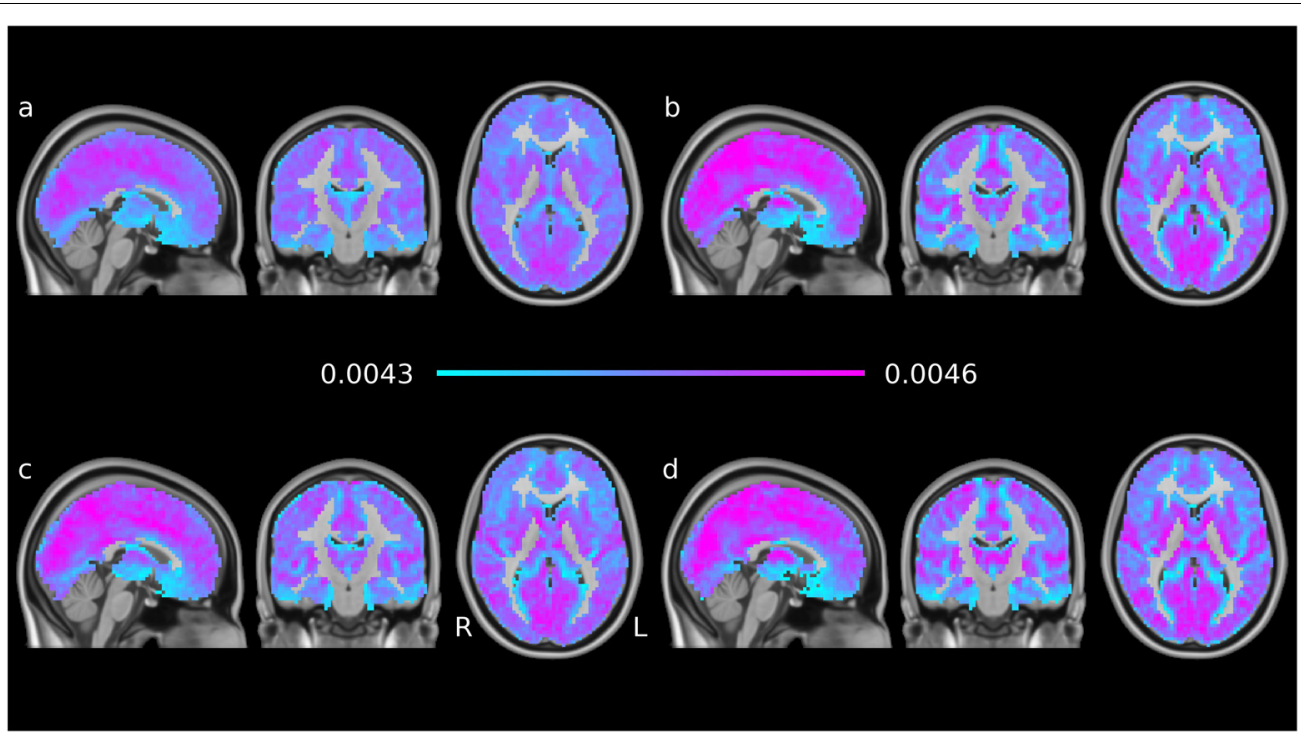

FIGURE 2 | EC time series means, averaged per subgroup: (a) younger males, (b) younger females, (c) older males, (d) older females. The groups are generally similar; the female participants show more contrast than the males participants and the older groups show higher peaks than the younger groups of the same gender.

were limited to single voxels; we report only the clusters of more than 10 voxels. These were found in the medial and lateral visual networks (see Figures $\mathbf{3 a}, \mathbf{b}$ ), the default mode network (Figure 3c), the sensorimotor network (Figure 3d), the auditory network (Figure $\mathbf{3 e}$ ), and the left fronto-parietal network (Figure 3f). The EC variations in the other networks (occipital visual, lateral visual, cerebellum, executive control and right fronto-parietal) did not differ between male and female subjects or between the two age groups.

In the medial visual network (Figure 3a), EC covariations with the mean time course were lower in females than in males (blue voxels) in the precuneus. In a partly overlapping region they were also lower in the older group than the younger (green voxels). This region was concentrated in the cuneus and precuneus, touching visual cortices V1 (Brodmann area 18) and, to a lesser extent, V2 (Brodmann area 17).

Two small clusters in the lateral visual network (Figure $3 \mathbf{b}$ ) showed lower correlations in the female group compared to male (blue voxels). These voxels were in the right medial temporal gyrus and bilateral inferior temporal gyrus.

In the default mode network, DMN (Figure 3c), two voxel locations showed lower covariatons in EC with its mean time course in the female group compared to the male (blue voxels). These voxels were contained in the parietal lobe: precuneus and posterior cingulate cortex.

EC covariations with the sensorimotor network (Figure 3d) were lower in females than in males (blue voxels) in a small region in the primary motor cortex (Brodmann area 4) and premotor cortex (Brodmann area 6) and primary somatosenory cortex (BA 2).

Smaller clusters were also found in the executive control network (Figure 3e), with lower covariations in females than males in the right medial frontal gyrus (blue voxels) and lower covariations in the older group than the younger in the left and right middle and superior frontal gyrus (green voxels).

In the left frontoparietal network (Figure 3f), EC covariations with the mean time course were lower in the older group than the younger in the left lateral occippital cortex (green voxels). No corresponding clusters were found in the right frontoparietal network.

Plots of the group mean network time courses found during dual regression were made by averaging the result of the spatial regressions (yielding a time course per network mask) of subjects by group, and then plotting the winsorized mean time course for each network (with a trim of 0.1 ), and the standard deviation divided by 5 as a ribbon to show the relative variability inside the group for each network (see Figure 4).

Although no obvious difference is visible between males and females, the older groups on the right show less EC variability than the younger groups on the left. There is a partial ordering in the networks in average centrality that is consistent across groups: the DMN has the highest mean centrality in all four groups, followed by the medial and lateral visual networks. The right frontoparietal and occipital visual networks are generally next, with the sensorimotor network being in different positions around them. The auditory and executive control networks are below those. Interestingly, the left frontoparietal network, consistently consistently has the lowest mean EC in all groups, showing a large difference with its right counterpart.

\section{DISCUSSION}

The method of dynamic voxelwise brain network centrality computed from fMRI combines the advantage of ECM of being able to investigate functional brain network properties on the 


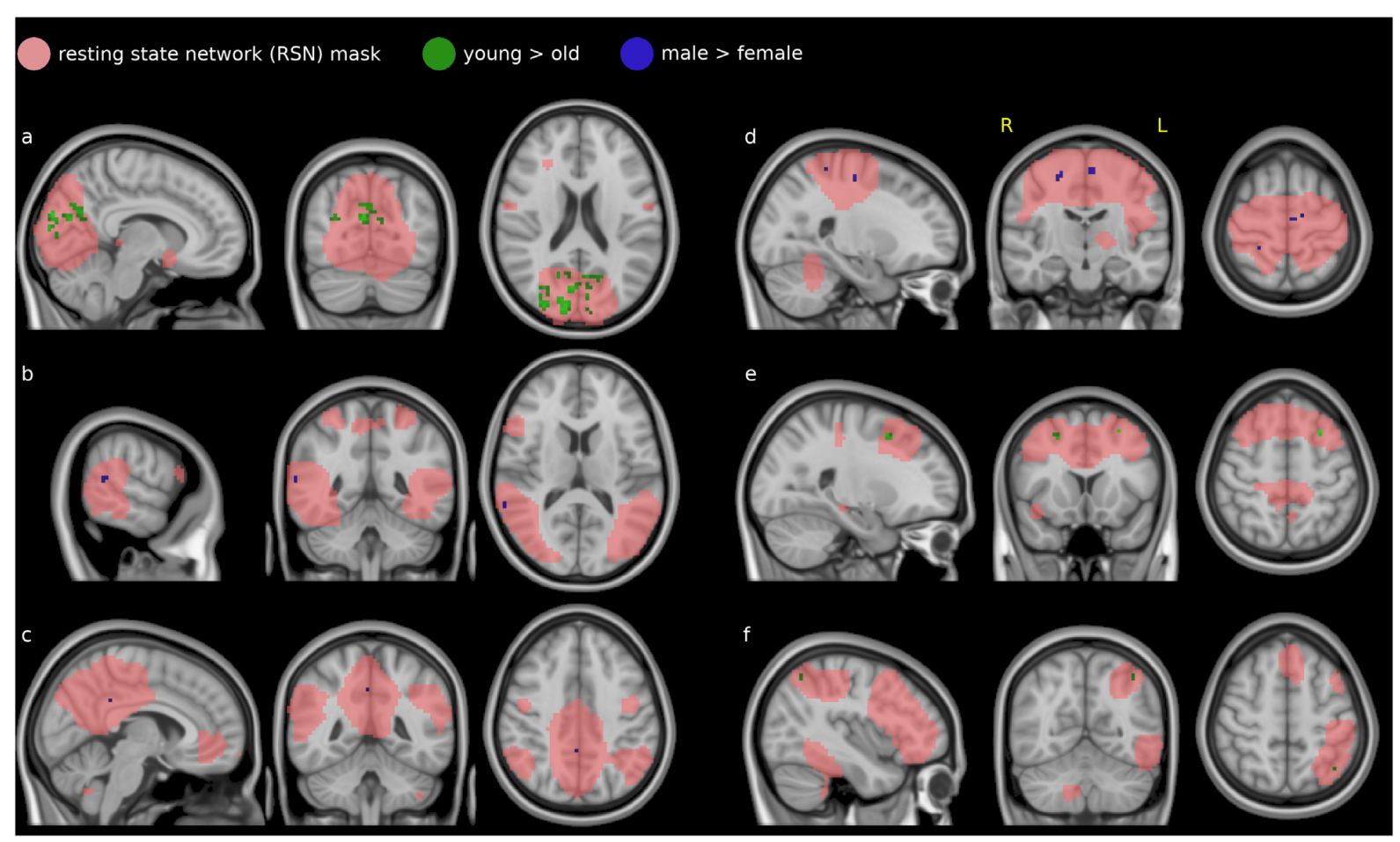

FIGURE 3 | Difference in covariability of EC with established resting-state networks (RSN, salmon) between males and females (blue) and younger and older subjects (green): the medial visual network (a), the lateral visual network (b) the default mode network (c), the sensorimotor network (d), the executive control network (e), and the left fronto-parietal network (f).

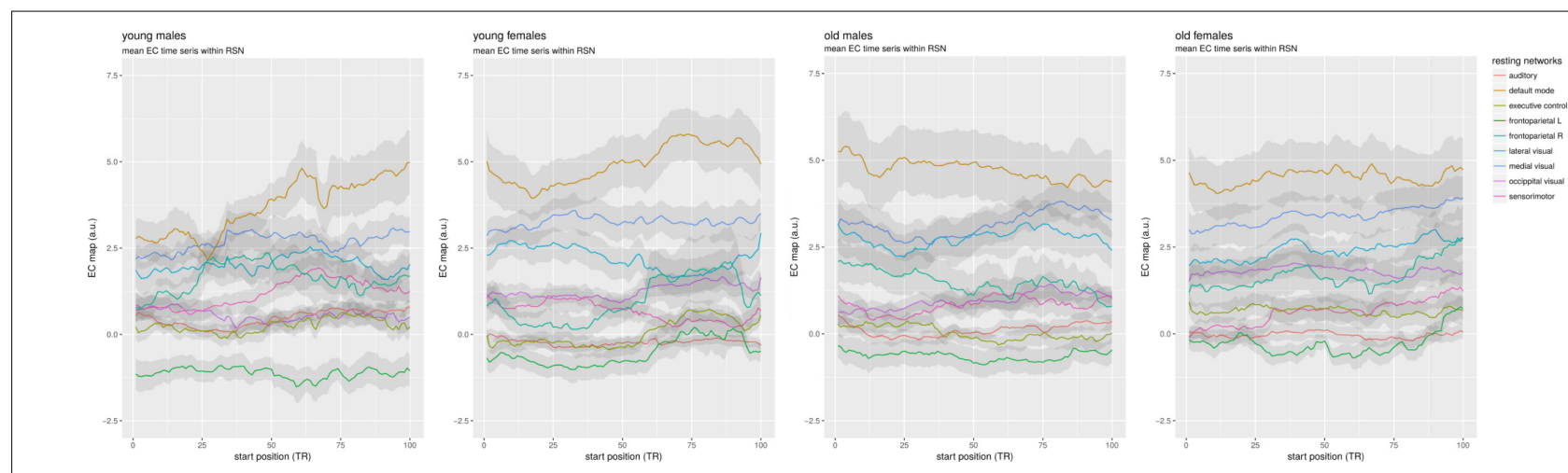

FIGURE 4 | Mean time courses per resting network. Average time series were made from the spatial regression maps minus the cerebellum, which were then averaged per group using a winsorized mean.

voxel level so that the results can be presented together with other brain image statistic maps, and the sensitivity to time-dependent changes of sliding-window correlation-based analyses of fMRI experimental data.

Comparisons of single-window ECM (Figure 1) with the mean ECM taken over all windows (Figure 2), and their comparison with single 'static' ECM from previous studies (Binnewijzend et al., 2014; Duinkerken et al., 2017; Eijlers et al., 2017) show that the pattern of centralities is relatively stable across windows; changes over time are mainly fluctuations around the pattern represented by the mean ECM. This is also in line with the dominant patterns found previously in EC time series (Preti and Ville, 2017).

In line with earlier findings in resting fMRI data network analysis (Achard et al., 2006; Kiviniemi et al., 2011), we found slowly varying variability in our centrality maps. Other methods for studying functional brain network dynamics (Fraiman et al., 2009; Allan et al., 2015; Thompson et al., 2018) find different spatiotemporal dynamics. As our method uses a sliding window, slow temporal variability is expected (Hindriks et al., 2016).

Differences in resting fMRI connectivity between males and females have been described before (Gong et al., 2011) but 
gender differences in the stability/rigidity of functional brain networks have had less attention. Earlier studies have found lower connectivity with the primary visual and auditory cortex in female participants (Filippi et al., 2013; Smith et al., 2014), indicating that their strength is lower than in men; but this does not mean that centrality in these networks is lower. Synaptic density in the visual cortex has been reported to be higher in male subjects than female (Alonso-Nanclares et al., 2008), although it is not immediately clear that this would lead to stronger covariations within the visual cortex.

Not much is known about resting between-gender functional connectivity differences in the motor network. Ritchie et al. (2018) found stronger functional connectivity in males than in females between the sensorimotor network and visual and prefrontal areas. This could indicate an increased centrality, which could in turn lead to increased temporal correspondence in centrality variations within the motor network. Another study found higher fractional amplitudes of low-frequency fluctuations (fALFF) in the sensorimotor cortex in males than females (Biswal et al., 2010). These slower oscillations may cause a more stable temporal behavior in signal, connectivity, and centrality.

The effect of age on resting-state connectivity has been studied extensively, although this has concentrated more on older subjects. The regions in the first network that shows differences between the age groups in temporal EC variability, medial visual (Figure 3a), have been previously reported as having decreased centrality in patients with Alzheimer's disease (AD) compared with controls (Binnewijzend et al., 2014) and in subjects with elevated risk factors for $\mathrm{AD}$, including age (Wink et al., 2018). If synchronization in these regions with the rest of the visual network deteriorates, this may cause a lower covariability with the centrality of the rest of the network as well. The fact that an age effect can already be measured in the young adults included here, confirms previous findings of the age-sensitivity of eigenvector centrality in the visual cortex.

Decreased connectivity with age has been measured in the DMN (Vidal-Piñeiro et al., 2014; Sala-Llonch et al., 2015). Geerligs et al. (2015) report age-related changes in the same networks found in this study, where segregation of networks is higher in younger participants: the networks can more clearly be separated. The DMN and the frontoparietal network showed a reduction in local efficiency, which me be related to our intra-network reductions in connectivity and centrality with age. A difference was also found in visual and motor networks between the 'participation index' between younger and older subjects, that is: the proportion of signal that is directed toward other RSN. This was higher in older subjects. Our findings may relate to this, in that better concordance with external networks may lead to weaker intra-network connectivities and centralities.

Older adults have also been shown to use the fronto-parietal network more 'efficiently' in cognitive tasks to reduce the influence of external distractions, and this network showed lower connectivity at rest (Campbell et al., 2012, 2013). There is also evidence that connectivity between the DMN and frontoparietal networks is an indication of cognitive flexibility (Douw et al., 2016), suggesting mental agility but also distractability, which would decrease with age. However, this combination of terminology is speculative at the moment.

Mean network-specific centralities as measured with the sliding-window approach (Figure 4) show smooth and slow dynamics, indicating that they are relatively stable on the time scale of an fMRI experiment, in agreement with earlier findings on time-varying centrality (Liao et al., 2015), indicating that functional networks are associated with structural connections. This is also reflected by the fact that relative mean network centralities are stable within and between age and gender groups, with the DMN having the highest average centralities in all cases.

The differences in dynamic eigenvector centralities between gender and age groups enjoin graph theoretic analyses of fMRI data studies to model or correct for these factors.

\section{Limitations}

One of the aims of this paper was inviting others to reproduce these analyses. For this reason, we used a publicly available data set with fully pre-processed images to minimize differences in outcomes to pre-processing. A disadvantage of this strategy is the reduced level of control over these previous steps (such as the correction of motion effects between realignment and standard space mapping).

Not all subjects were scanned for the same amount of time, which yields variable underlying signal to noise ratios for the volumes of different ECM time series. At the moment it is not known how this influences the ECM time series dynamics; this is the subject of ongoing work.

The connections matrix used for ECM is the correlations matrix, increased with 1 to guarantee non-negativity (Wink et al., 2012). This equation, which negatively correlates with the squared L2-norm of signals with a Gaussian distribution, cannot be as trivially used for more advanced measures of connections, such as partial correlation (Zhen et al., 2007).

The statistical tests between groups are corrected for multiple voxel comparisons, but not for multiple contrasts (resting networks). If this correction is applied $(p=0.05 / 10)$ then none of the RSNs show significant differences in dynamics.

\section{CONCLUSION}

We have presented dynamic ECM, provide an implementation in the fastECM package and demonstrate its application in a publicly available dataset, demonstrating changes in EC variability inside resting networks between gender and age groups.

\section{DATA AVAILABILITY}

The datasets generated for this study are available on request to the corresponding author.

\section{AUTHOR CONTRIBUTIONS}

AW performed the analyses and wrote the manuscript. 


\section{FUNDING}

AW received funding from EuroPOND (Horizon 2020 Grant No. 666992), EP-AD (IMI Grant No. 115736), and AMYPAD (IMI Grant No. 115962).

\section{REFERENCES}

Achard, S., Salvador, R., Whitcher, B., Suckling, J., and Bullmore, E. (2006). A resilient and low-frequency and small-world human brain functional network with highly connected association cortical hubs. J. Neurosci. 26, 63-72. doi: 10.1523/JNEUROSCI.3874-05.2006

Allan, T. W., Francis, S. T., Caballero-Gaudes, C., Morris, P. G., Liddle, E. B., Liddle, P. F., et al. (2015). Functional connectivity in MRI is driven by spontaneous BOLD events. PLoS One 10:e0124577. doi: 10.1371/journal.pone. 0124577

Alonso-Nanclares, L., Gonzalez-Soriano, J., Rodriguez, J. R., and DeFelipe, J. (2008). Gender differences in human cortical synaptic density. PNAS 105, 14615-14619. doi: 10.1073/pnas.0803652105

Ashburner, J., and Friston, K. J. (2005). Unified segmentation. Neuroimage 26, 839-851. doi: 10.1016/j.neuroimage.2005.02.018

Beckmann, C. (2009). Group comparison of resting-state FMRI data using multisubject ICA and dual regression. NeuroImage 47, S148.

Binnewijzend, M. A. A., Adriaanse, S. M., Van der Flier, W. M., Teunissen, C. E., de Munck, J. C., Stam, C. J., et al. (2014). Brain network alterations in Alzheimer's disease measured by Eigenvector centrality in fMRI are related to cognition and CSF biomarkers. Hum. Brain Mapp. 35, 2383-2393. doi: 10.1002/hbm.22335

Binnewijzend, M. A. A., Schoonheim, M. M., Sanz-Arigita, E., Wink, A. M., van der Flier, W. M., Tolboom, N., et al. (2012). Resting-state fMRI changes in Alzheimer's disease and mild cognitive impairment. Neurobiol. Aging 33, 2018-2028. doi: 10.1016/j.neurobiolaging.2011.07.003

Biswal, B. B., Mennes, M., Zuo, X.-N., Gohel, S., Kelly, C., Smith, S. M., et al. (2010). Toward discovery science of human brain function. PNAS 107, 4734-4739. doi: 10.1073/pnas.0911855107

Breakspear, M. (2017). Dynamic models of large-scale brain activity. Nat. Neurosci. 20, 340-352. doi: 10.1038/nn.4497

Campbell, K., Grigg, O., Saverino, C., Churchill, N., and Grady, C. (2013). Age differences in the intrinsic functional connectivity of default network subsystems. Front. Aging Neurosci. 5:73. doi: 10.3389/fnagi.2013.00073

Campbell, K. L., Grady, C. L., Ng, C., and Hasher, L. (2012). Age differences in the frontoparietal cognitive control network: implications for distractibility. Neuropsychologia 50, 2212-2223. doi: 10.1016/j.neuropsychologia.2012.05.025

Damoiseaux, J. S., Rombouts, S. A. R., Barkhof, F., Scheltens, P., Stam, C. J., Smith, S. M., et al. (2006). Consistent resting-state networks across healthy subjects. Proc. Natl. Acad. Sci. U.S.A. 103, 13848-13853. doi: 10.1073/pnas.0601417103

Douw, L., Wakeman, D. G., Tanaka, N., Liu, H., and Stufflebeam, S. M. (2016). State-dependent variability of dynamic functional connectivity between frontoparietal and default networks relates to cognitive flexibility. Neuroscience 339, 12-21. doi: 10.1016/j.neuroscience.2016.09.034

Duinkerken, E., van Schoonheim, M. M., IJzerman, R. G., Moll, A. C., LandeiraFernandez, J., Klein, M., et al. (2017). Altered eigenvector centrality is related to local resting-state network functional connectivity in patients with longstanding type 1 diabetes mellitus. Hum. Brain Mapp. 38, 3623-3636. doi: $10.1002 / \mathrm{hbm} .23617$

Eijlers, A. J. C., Meijer, K. A., Wassenaar, T. M., Steenwijk, M. D., Uitdehaag, B. M. J., Barkhof, F., et al. (2017). Increased default-mode network centrality in cognitively impaired multiple sclerosis patients. Neurology 88, 952-960. doi: 10.1212/WNL.0000000000003689

Esteban, O., Birman, D., Schaer, M., Koyejo, O. O., Poldrack, R. A., and Gorgolewski, K. J. (2017). MRIQC: advancing the automatic prediction of image quality in MRI from unseen sites. PLoS One 12:e0184661. doi: 10.1371/ journal.pone.0184661

Filippi, M., Valsasina, P., Misci, P., Falini, A., Comi, G., and Rocca, M. A. (2013). The organization of intrinsic brain activity differs between genders: a restingstate fMRI study in a large cohort of young healthy subjects. Hum. Brain Map. 34, 1330-1343. doi: 10.1002/hbm.21514

\section{ACKNOWLEDGMENTS}

The author thanks to OpenNeuro and Jonathan Power, Steve Petersen and Brad Schlaggar from Washington University for distributing the data used in this study.

Fletcher, J. M., and Wennekers, T. (2016). From structure to activity: using centrality measures to predict neuronal activity. Int. J. Neur. Syst. 28:1750013. doi: 10.1142/S0129065717500137

Fraiman, D., Balenzuela, P., Foss, J., and Chialvo, D. R. (2009). Ising-like dynamics in large-scale functional brain networks. Phys. Rev. E Stat. Nonlin. Soft Matter Phys. 79(6 Pt 1):061922.

Geerligs, L., Renken, R. J., Saliasi, E., Maurits, N. M., and Lorist, M. M. (2015). A brain-wide study of age-related changes in functional connectivity. Cereb. Cortex 25, 1987-1999. doi: 10.1093/cercor/bhu012

Gong, G., He, Y., and Evans, A. C. (2011). Brain connectivity: gender makes a difference. Neuroscientist 17, 575-591. doi: 10.1177/1073858410386492

Hindriks, R., Adhikari, M. H., Murayama, Y., Ganzetti, M., Mantini, D., Logothetis, N. K., et al. (2016). Can sliding-window correlations reveal dynamic functional connectivity in resting-state fMRI? NeuroImage 127, 242-256. doi: 10.1016/j. neuroimage.2015.11.055

Kiviniemi, V., Vire, T., Remes, J., Elseoud, A. A., Starck, T., Tervonen, O., et al. (2011). A sliding time-window ICA reveals spatial variability of the default mode network in time. Brain Connect. 1, 339-347. doi: 10.1089/brain.2011. 0036

Liao, X., Yuan, L., Zhao, T., Dai, Z., Shu, N., Xia, M., et al. (2015). Spontaneous functional network dynamics and associated structural substrates in the human brain. Front. Hum. Neurosci 9:478. doi: 10.3389/fnhum.2015.00478

Lohmann, G., Margulies, D. S., Horstmann, A., Pleger, B., Lepsien, J., Goldhahn, D., et al. (2010). Eigenvector centrality mapping for analyzing connectivity patterns in FMRI data of the human brain. PLoS One 5:e10232. doi: 10.1371/journal. pone.0010232

Nichols, T. E., and Holmes, A. P. (2002). Nonparametric permutation tests for functional neuroimaging: a primer with examples. Hum. Brain Mapp. 15, 1-25. doi: $10.1002 / \mathrm{hbm} .1058$

Nickerson, L. D., Smith, S. M., Öngür, D., and Beckmann, C. F. (2017). Using dual regression to investigate network shape and amplitude in functional connectivity analyses. Front Neurosci 11:115. doi: 10.3389/fnins.2017.00115

Power, J. D., Barnes, K. A., Snyder, A. Z., Schlaggar, B. L., and Petersen, S. E. (2012). Spurious but systematic correlations in functional connectivity MRI networks arise from subject motion. Neuroimage 59, 2142-2154. doi: 10.1016/j. neuroimage.2011.10.018

Power, J. D., Mitra, A., Laumann, T. O., Snyder, A. Z., Schlaggar, B. L., and Petersen, S. E. (2014). Methods to detect, characterize, and remove motion artifact in resting state fMRI. NeuroImage 84, 320-341. doi: 10.1016/j.neuroimage.2013. 08.048

Power, J. D., Schlaggar, B. L., Lessov-Schlaggar, C. N., and Petersen, S. E. (2013). Evidence for hubs in human functional brain networks. Neuron 79, 798-813. doi: 10.1016/j.neuron.2013.07.035

Preti, M. G., Bolton, T. A., and Van De Ville, D. (2017). The dynamic functional connectome: state-of-the-art and perspectives. NeuroImage 160, 41-54. doi: 10.1016/j.neuroimage.2016.12.061

Preti, M. G., and Ville, D. V. D. (2017). Dynamics of functional connectivity at high spatial resolution reveal long-range interactions and fine-scale organization. Sci. Rep. 7:12773. doi: 10.1038/s41598-017-12993-12991

Ritchie, S. J., Cox, S. R., Shen, X., Lombardo, M. V., Reus, L. M., Alloza, C., et al. (2018). Sex differences in the adult human brain: evidence from $5216 \mathrm{UK}$ biobank participants. Cereb. Cortex 28, 2959-2975. doi: 10.1093/cercor/bhy109

Sala-Llonch, R., Bartrés-Faz, D., and Junqué, C. (2015). Reorganization of brain networks in aging: a review of functional connectivity studies. Front. Psychol 6:663. doi: 10.3389/fpsyg.2015.00663

Schoonheim, M. M., Geurts, J. J. G., Wiebenga, O. T., De Munck, J. C. Polman, C. H., Stam, C. J., et al. (2014). Changes in functional network centrality underlie cognitive dysfunction and physical disability in multiple sclerosis. Mult. Scler. J. 20, 1058-1065. doi: 10.1177/135245851351 6892 
Smith, D. V., Utevsky, A. V., Bland, A. R., Clement, N., Clithero, J. A., Harsch, A. E. W., et al. (2014). Characterizing individual differences in functional connectivity using dual-regression and seed-based approaches. Neuroimage 95, 1-12. doi: 10.1016/j.neuroimage.2014.03.042

Smith, S. M., Fox, P. T., Miller, K. L., Glahn, D. C., Fox, P. M., Mackay, C. E., et al. (2009). Correspondence of the brain's functional architecture during activation and rest. PNAS 106, 13040-13045. doi: 10.1073/pnas.0905267106

Smith, S. M., and Nichols, T. E. (2009). Threshold-free cluster enhancement: addressing problems of smoothing, threshold dependence and localisation in cluster inference. NeuroImage 44, 83-98. doi: 10.1016/j.neuroimage.2008.03.061

Soares, J. M., Magalhães, R., Moreira, P. S., Sousa, A., Ganz, E., Sampaio, A., et al. (2016). A hitchhiker's guide to functional magnetic resonance imaging. Front. Neurosci. 10:515. doi: 10.3389/fnins.2016.00515

Tagliazucchi, E., Balenzuela, P., Fraiman, D., and Chialvo, D. R. (2012). Criticality in large-scale brain fMRI dynamics unveiled by a novel point process analysis. Front. Physiol 3:15. doi: 10.3389/fphys.2012.00015

Tagliazucchi, E., Siniatchkin, M., Laufs, H., and Chialvo, D. R. (2016). The voxelwise functional connectome can be efficiently derived from co-activations in a sparse spatio-temporal point-process. Front. Neurosci 10:381. doi: 10.3389/ fnins.2016.00381

Thompson, W. H., Richter, C. G., Plavén-Sigray, P., and Fransson, P. (2018). Simulations to benchmark time-varying connectivity methods for fMRI. PLoS Comput. Biol. 14:e1006196. doi: 10.1371/journal.pcbi.1006196

Van Wijk, B. C. M., Stam, C. J., and Daffertshofer, A. (2010). Comparing brain networks of different size and connectivity density using graph theory. PLoS One 5:e13701. doi: 10.1371/journal.pone.0013701

Vidal-Piñeiro, D., Valls-Pedret, C., Fernández-Cabello, S., Arenaza-Urquijo, E. M., Sala-Llonch, R., Solana, E., et al. (2014). Decreased default mode network connectivity correlates with age-associated structural and cognitive changes. Front. Aging Neurosci 6:256. doi: 10.3389/fnagi.2014.00256

Wink, A. M., de Munck, J. C., van der Werf, Y. D., van den Heuvel, O. A., and Barkhof, F. (2012). Fast eigenvector centrality mapping of voxel-wise connectivity in functional magnetic resonance imaging: implementation, validation, and interpretation. Brain Connect. 2, 265-274. doi: 10.1089/brain. 2012.0087

Wink, A. M., Tijms, B. M., Kate, M., ten Raspor, E., Munck, J. C., de Altena, E., et al. (2018). Functional brain network centrality is related to APOE genotype in cognitively normal elderly. Brain Behav. 8:e01080. doi: 10.1002/brb3.1080

Zalesky, A., and Breakspear, M. (2015). Towards a statistical test for functional connectivity dynamics. NeuroImage 114, 466-470. doi: 10.1016/j.neuroimage. 2015.03.047

Zhen, Z., Tian, J., Qin, W., and Zhang, H. (2007). "Partial correlation mapping of brain functional connectivity with resting state fMRI," in Proceedings of the SPIE 6511, Medical Imaging 2007: Physiology, Function, and Structure from Medical Images, eds A. Manduca and X. P. Hu (San Diego, CA: SPIE), 651112 .

Conflict of Interest Statement: The author declares that the research was conducted in the absence of any commercial or financial relationships that could be construed as a potential conflict of interest.

Copyright (c) 2019 Wink. This is an open-access article distributed under the terms of the Creative Commons Attribution License (CC BY). The use, distribution or reproduction in other forums is permitted, provided the original author(s) and the copyright owner(s) are credited and that the original publication in this journal is cited, in accordance with accepted academic practice. No use, distribution or reproduction is permitted which does not comply with these terms. 


\section{OPEN ACCESS}

Edited by:

Roberto Esposito,

A.O. Ospedali Riuniti Marche

Nord, Italy

Reviewed by:

Hui Shen,

National University of Defense Technology, China

Vesa J. Kiviniemi,

University of Oulu, Finland

${ }^{*}$ Correspondence:

Maria A. Rocca

rocca.mara@hsr.it

Specialty section: This article was submitted to Brain Imaging Methods, a section of the journal Frontiers in Neuroscience

Received: 25 February 2019 Accepted: 29 May 2019

Published: 10 July 2019

Citation:

Valsasina P, Hidalgo de la Cruz M

Filippi M and Rocca MA (2019)

Characterizing Rapid Fluctuations of Resting State Functional Connectivity in Demyelinating, Neurodegenerative,

and Psychiatric Conditions: From

Static to Time-Varying Analysis.

Front. Neurosci. 13:618

doi: 10.3389/fnins.2019.00618

\section{Characterizing Rapid Fluctuations of Resting State Functional Connectivity in Demyelinating, Neurodegenerative, and Psychiatric Conditions: From Static to Time-Varying Analysis}

\author{
Paola Valsasina ${ }^{1}$, Milagros Hidalgo de la Cruz ${ }^{1,2}$, Massimo Filippi ${ }^{1,2,3}$ and \\ Maria A. Rocca ${ }^{1,3 *}$
}

${ }^{1}$ Neuroimaging Research Unit, Institute of Experimental Neurology, Division of Neuroscience, IRCCS San Raffaele Scientific Institute, Milan, Italy, ${ }^{2}$ Vita-Salute San Raffaele University, Milan, Italy, ${ }^{3}$ Neurology Unit, IRCCS San Raffaele Scientific Institute, Milan, Italy

Functional magnetic resonance imaging (fMRI) at resting state (RS) has been widely used to characterize the main brain networks. Functional connectivity (FC) has been mostly assessed assuming that FC is static across the whole fMRI examination. However, FC is highly variable at a very fast time-scale, as demonstrated by neurophysiological techniques. Time-varying functional connectivity (TVC) is a novel approach that allows capturing reoccurring patterns of interaction among functional brain networks. Aim of this review is to provide a description of the methods currently used to assess TVC on RS FMRI data, and to summarize the main results of studies applying TVC in healthy controls and patients with multiple sclerosis (MS). An overview of the main results obtained in neurodegenerative and psychiatric conditions is also provided. The most popular TVC approach is based on the so-called "sliding windows," in which the RS $\mathrm{fMRl}$ acquisition is divided in small temporal segments (windows). A window of fixed length is shifted over RS fMRI time courses, and data within each window are used to calculate FC and its variability over time. Sliding windows can be combined with clustering techniques to identify recurring FC states or used to assess global TVC properties of large-scale functional networks or specific brain regions. TVC studies have used heterogeneous methodologies so far. Despite this, similar results have been obtained across investigations. In healthy subjects, the default-mode network (DMN) exhibited the highest degree of connectivity dynamism. In MS patients, abnormal global TVC properties and TVC strengths were found mainly in sensorimotor, DMN and salience networks, and were associated with more severe structural MRI damage and with more severe physical and cognitive disability. Conversely, abnormal TVC measures of the temporal network were correlated with better cognitive performances and less severe fatigue. In patients with neurodegenerative and psychiatric conditions, TVC abnormalities of the DMN, attention and executive networks were associated to more 
severe clinical manifestations. TVC helps to provide novel insights into fundamental properties of functional networks, and improves the understanding of brain reorganization mechanisms. Future technical advances might help to clarify TVC association with disease prognosis and response to treatment.

Keywords: multiple sclerosis, neurodegenerative conditions, time-varying, functional connectivity, resting state, fMRI

\section{INTRODUCTION}

The human brain at resting state (RS) exhibits highly structured spontaneous fluctuations in functional magnetic resonance imaging (fMRI) data, which reflect the underlying network architecture (Biswal et al., 2010). RS functional connectivity (FC) captures the temporal associations between such fluctuations, and has been successfully used to characterize the main networks of the brain and map abnormalities of functional network architecture occurring in different neurological conditions. In healthy controls, RS FC strength was found to be associated to age, with RS fluctuations being strongest in adulthood and lowest in children and elderly (Mak et al., 2017). A dependency of connectivity from sex (Biswal et al., 2010; Mak et al., 2017), as well as from cognitive, emotional, and behavioral variables was also detected (Kelly et al., 2012).

Multiple sclerosis (MS) is an inflammatory and neurodegenerative disease of the central nervous system leading to a progressive increase over time of clinical disability and cognitive impairment (Filippi et al., 2017, 2018). Reorganization of brain functional networks in MS has been shown from the first RS fMRI studies (Lowe et al., 2002, 2008; Rocca et al., 2010; Roosendaal et al., 2010), which is thought to limit the clinical consequences of widespread tissue damage (Filippi et al., 2013a; Sbardella et al., 2015). Cortical reorganization has been demonstrated to be variable across the different stages of the disease, and a progressive exhaustion or inefficiency of the adaptive properties of the cerebral cortex is likely to be among the factors responsible for the worsening of clinical disability (Rocca et al., 2010, 2018; Roosendaal et al., 2010; Loitfelder et al., 2011). In neurodegenerative conditions, RS FC studies showed a progressive and gradual spreading of connectivity changes from a target brain network, reflecting specific behavioral and cognitive dysfunctions (Zhou et al., 2017). In psychiatric diseases, disruption of fronto-parietal network connectivity seems to be the common fingerprint across distinct forms of pathology (Baker et al., 2019).

However, current understanding of the role of functional abnormalities in neurological and psychiatric disorders is still incomplete, mostly due to inconsistencies in the findings from several studies. Specifically, in MS some investigations found trends toward lower RS FC vs. healthy controls in the defaultmode (Rocca et al., 2010, 2012, 2018; Bonavita et al., 2011), sensorimotor (Rocca et al., 2018) and subcortical (Liu et al., 2011; Rocca et al., 2018) networks, while in other studies the opposite trends were observed (Roosendaal et al., 2010; Tona et al., 2014; Schoonheim et al., 2015). Similarly, even if RS FC abnormalities were principally located in the core regions hit by pathology, a certain variability of brain areas involved by RS FC changes was detected in neurodegenerative and psychiatric conditions (Busatto, 2013; Weiner et al., 2017).

The wide spectrum of clinical characteristics of MS patients has been considered as one of the main causes for the discrepancies described in RS fMRI literature (Filippi et al., 2013a; Sbardella et al., 2015). However, technical factors might also bias connectivity estimation, including scanner-related signal instabilities, an inappropriate control of confounding covariates, and the application of analysis methods based on inaccurate assumptions.

For instance, one of the main assumptions of classical RS FC assessment methods is that connectivity is static across the entire fMRI examination, e.g., it can be assessed by calculating the mean correlation between whole-length RS fMRI time series (Biswal et al., 2010). However, as widely evident by neurophysiological techniques, brain FC is highly variable at a very fast time-scale. The functioning human brain during any state of wakefulness repeatedly changes between different combinations of cognitive, sensorimotor, attentional, emotional, auditory, and visual-related tasks. Notably, the majority of brain regions experience continuous functional changes even during sleep (Tagliazucchi and van Someren, 2017). Thus, studying time-varying RS FC patterns is likely to shed light not only on physiological processes occurring in healthy subjects, but also to understand clinical manifestations of different neurological and psychiatric conditions. In fact, clinical symptoms associated to these diseases are likely to depend not only from damage to specific brain regions, but also from delayed (or abnormal) communication between brain areas. The study of the temporal reconfigurations of FC occurring within RS fMRI sessions has been defined as time-varying functional connectivity (TVC) (Hutchison et al., 2013; Calhoun et al., 2014; Preti et al., 2017).

The main goal of this review is to summarize the main results obtained using TVC in healthy and diseased populations. A particular focus is given to studies of patients with MS; however, the main findings of investigations performed in neurodegenerative and psychiatric conditions are also reported. The review is structured as follows: in section Methods Used to Assess Time-Varying Functional Connectivity, we present the main approaches developed to investigate TVC using fMRI data, with a main emphasis on the methods applied to study MS patients. Then, we summarize the results obtained applying these methods in healthy controls (section Application of TVC to Healthy Subjects) and in patients with MS (section Application of TVC Techniques to MS). An overview of the results derived from other neurological and psychiatric conditions is also given (section Application 
of Time-Varying FC Techniques to Psychiatric and Other Neurological Diseases). In the final part (section Current Limitations and Future Directions), current TVC methodological limitations are discussed and possible future developments are presented.

\section{METHODS USED TO ASSESS TIME-VARYING FUNCTIONAL CONNECTIVITY}

Several analysis strategies have been applied so far to quantify temporal variations of blood oxygenation level dependent (BOLD) signal fluctuations (Hutchison et al., 2013; Preti et al., 2017). Some strategies aim at capturing variations in interregional associations between pairs of brain areas (Sakoglu et al., 2010; Allen et al., 2014), while others try to detect changing patterns of temporal synchrony at a multivariate level, e.g., considering all brain regions at once (Tagliazucchi et al., 2012; Liu and Duyn, 2013). One of the most popular methods for TVC analysis, which is based on the use of the so-called "sliding windows" (Sakoglu et al., 2010; Allen et al., 2014), belongs to the first category, since it relies on the calculation of a series of pairwise correlation coefficients over small shifting segments of fMRI time series.

Despite the great variability of available pipelines, TVC analysis usually requires the performance of the following steps: (1) selection of a set of regions of interest (ROIs) in the brain; (2) assessment of time-varying correlations among the selected ROIs; and (3) extraction of features quantifying connectivity changes over time, as described in details in the next paragraphs.

\section{Selection of Regions of Interest for TVC Analysis}

It is important to properly identify the ROIs (which may be areas of the brain, or even entire functional networks) that will be included in TVC analysis. Several factors can influence the choice of ROIs: spatial resolution, the use of a priori hypotheses or datadriven strategies, and the rationale of the experiment, which may focus on selected functional circuits or on the whole brain.

The large majority of studies assessing TVC in MS patients mainly relied on the use of a priori atlases, such as the Automatic Anatomical Labeling (AAL) (Tzourio-Mazoyer et al., 2002) or the Desikan (Desikan et al., 2006) cortical atlas (Leonardi et al., 2013; Lin et al., 2018; van Geest et al., 2018a,b). Some studies built ad-hoc ROIs centered in critical nodes of large-scale brain networks (Bosma et al., 2018). However, a widely used approach in previous literature consists in a ROI data-driven selection through independent component analysis (ICA; Rocca et al., 2010, 2019; Sakoglu et al., 2010; Filippi et al., 2013b; Allen et al., 2014; Damaraju et al., 2014; Yang et al., 2014; Zalesky et al., 2014; Bisecco et al., 2018; Castellazzi et al., 2018; d'Ambrosio et al., 2019) (Figure 1). The broad application of ICA in previous TVC studies can be explained by the flexibility of this approach, which allows to extract ROIs at different spatial resolution according to ICA dimensionality, to perfectly fit the data (avoiding non-linear registrations with a priori atlases, which may be challenging in diseased populations) and to reduce the impact of physiological and motion-related noise.

Since ROIs identified by "static" a priori atlases may not reflect significant connectivity variations occurring within brain regions at short time scales (Ryyppo et al., 2018), recent studies have suggested that incorporating information of time-varying connectivity between neighboring voxels to parcellate the brain may improve accuracy of TVC analyses (Preti and Van De Ville, 2017; Ryyppo et al., 2018).

\section{Assessment of Time-Varying Correlations Among Brain Regions Sliding Window Analysis}

The most popular strategy used to examine time-varying correlations between RS fMRI time series relies on the use of sliding windows (Sakoglu et al., 2010; Allen et al., 2014). In this approach, a time window of fixed length is selected, and correlations between pairs of fMRI time series are calculated using data within that window. Then, the window is shifted in time by a certain number of time points, and correlations are re-assessed on the new data. This procedure results in a series of pair-wise correlation matrices that describe the time-resolved behavior of connectivity over the entire duration of the fMRI experiment (Allen et al., 2014; Figure 1).

The choice of an appropriate length for sliding windows is crucial: too short time segments may introduce spurious fluctuations associated with intrinsic fMRI signal instability, while with increased window size TVC estimation may become too similar to the classic static FC (Leonardi and Van De Ville, 2015; Preti et al., 2017). Different validation analyses recommended to set window length around 30-60 s (or the equivalent time expressed as repetition times, TRs), demonstrating consistent reproducibility of the obtained results (Allen et al., 2014; Damaraju et al., 2014; Rashid et al., 2014, 2016; Zalesky et al., 2014; Leonardi and Van De Ville, 2015; Qin et al., 2015; Zalesky and Breakspear, 2015; Choe et al., 2017; Zhang C. et al., 2018).

Once sliding windows correlation matrices have been produced, different strategies can be applied to extract features describing connectivity reorganization through time inside the data (Leonardi et al., 2013; Allen et al., 2014; Miller et al., 2016), as described in details in section Extraction of Features Quantifying Time-Varying Connectivity.

\section{Beyond Sliding-Window Analysis}

A variety of approaches alternative to sliding windows have been developed to quantify TVC in fMRI data (Preti et al., 2017). For instance, time-frequency decomposition has been used to represent correlations between two fMRI time series in the joint time and frequency domain (Chang and Glover, 2010; Yaesoubi et al., 2015a; Figure 1). Point-process analysis allowed to detect recurring patterns of co-activation between brain regions from a small fraction of the total scans of a RS fMRI experiment (Tagliazucchi et al., 2012; Liu and Duyn, 2013). Phase coherence connectivity has been proposed to calculate RS FC at each recorded fMRI time point (Deco and Kringelbach, 2016).

In MS studies, two alternative methods to sliding windows have been applied. One study (Bosma et al., 2018) used dynamic 
Selection of regions of interest

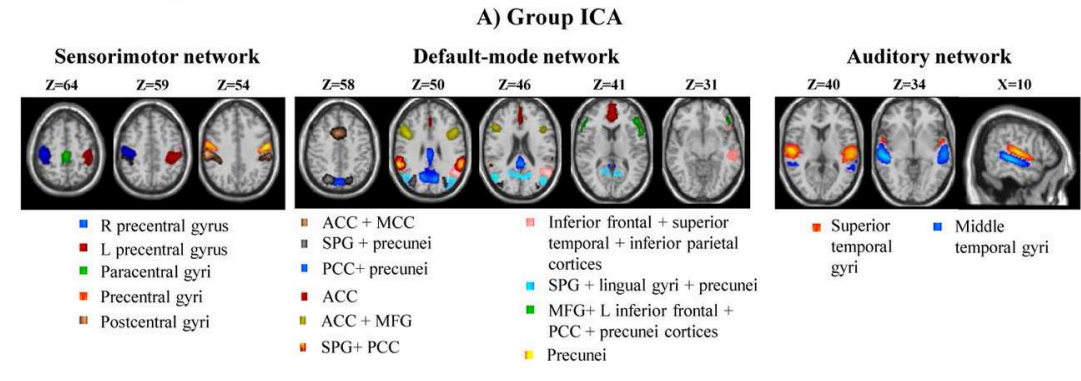

B) A priori atlases

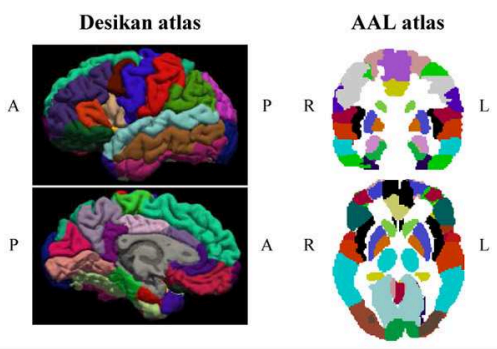

Assessment of time-varying correlations

C) Sliding windows analysis

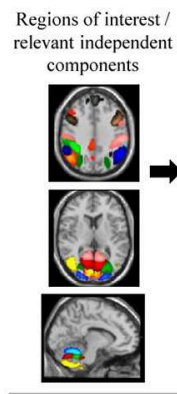

D) Time-frequency analysis

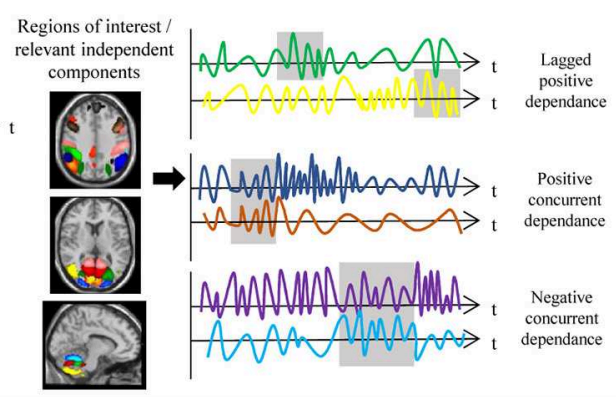

E) Point process analysis

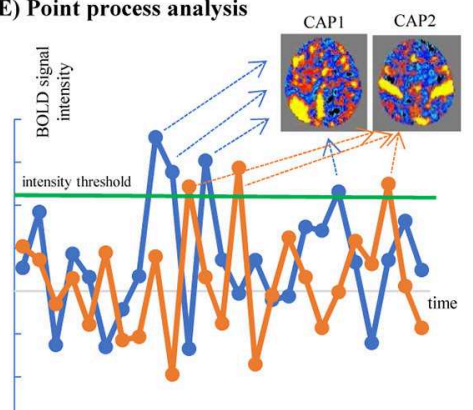

Extraction of features quantifying connectivity dynamics

F) Graph theoretical analysis

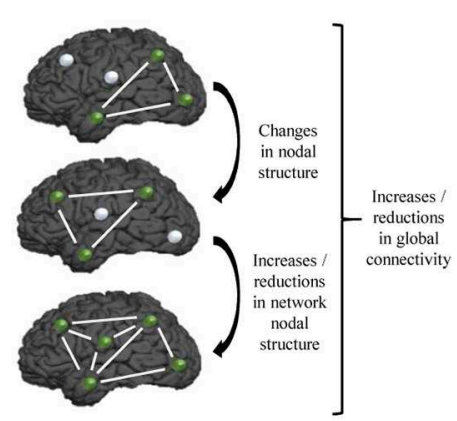

G) k-means clustering analysis

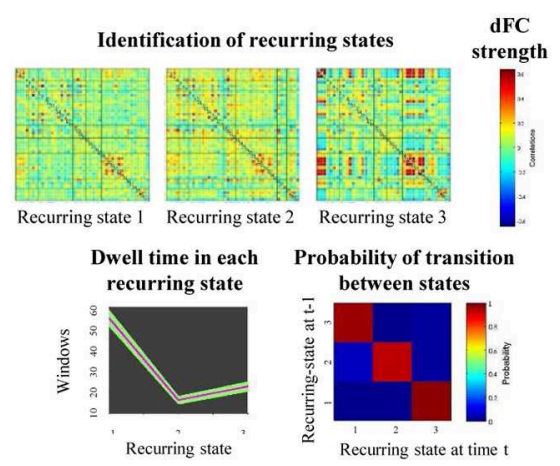

H) Fuzzy meta-states analysis

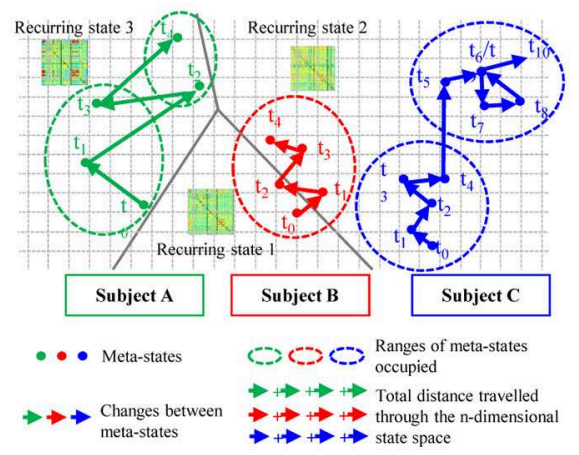

FIGURE 1 | Schematic representation of the post-processing steps used in the assessment of time-varying functional connectivity (TVC). Top row: selection of regions of interest for TVC analysis, which can be done using data-driven approaches (e.g., independent component analysis, A) or using a priori atlases (B). Middle row: assessment of time-varying correlations between fMRI time series. The most popular approach consists in using a sliding-window analysis (C); alternative approaches, such as time-frequency analysis (D) or point-process analysis (E) have been also proposed. Bottom row: extraction of features quantifying connectivity changes over time, which can be done using several techniques, such as graph theory (F), k-means clustering to estimate recurring TVC states (G), or fuzzy meta-state analysis (H). ICA, independent component analysis; AAL, automatic anatomical labeling; ACC, anterior cingulate cortex; CAP, co-activation pattern; MCC, middle cingulate cortex; PCC, posterior cingulate cortex; SPG, superior parietal gyrus; MFG, middle frontal gyrus; R, right; L, left.

conditional correlations (DCC) to quantify TVC. DCC were originally proposed to study fluctuations of financial time series (Engle, 2002) and subsequently adapted to neuroimaging data to quantify time-varying variances and correlations between multivariate RS fMRI time series (Lindquist et al., 2014). DCC overcome some limitations intrinsic to sliding-window techniques, since they do not depend from any arbitrary window length and do not give the same weight to all time points within the window, ignoring older observations. Moreover, DCC are not easily confused by changes of correlation occurring in fMRI time series merely due to random noise (Lindquist et al., 2014).
Another study (Zhou et al., 2016) quantified connectivity reorganization over time using brain entropy (BEN). Entropy is a statistical and physical index that measures irregularity of a time-varying system (Sandler, 2006). In RS fMRI data, voxel-wise assessments of entropy were performed by calculating sample entropy, defined as the negative logarithm of the probability that if two time series of length $m$ have a correlation $<r$, then two time series of length $m+1$ also have a correlation $<r$. A higher entropy indicates increased randomness of a system, meaning that the time-varying system activity is less predictable and less organized (Wang et al., 2014). 


\section{Extraction of Features Quantifying Time-Varying Connectivity}

Sliding-window (or alternative) techniques produce a large amount of correlation data, calculated on several time segments. Some features have then to be extracted from this big data mass, to summarize to what extent functional relationships reorganize through time. The simplest summary TVC statistic is standard deviation (or variance) of sliding-window correlation time series (Sakoglu et al., 2010; Choe et al., 2017) or of DCC time series (Lindquist et al., 2014; Bosma et al., 2018). The mean TVC (Huang et al., 2019) or the sum of absolute differences in pairwise connectivity between consecutive windows have also been used as a summary TVC measure (van Geest et al., 2018a,b). Another interesting metric assessing temporal variability of BOLD fluctuations is the so-called amplitude of low-frequency fluctuations functional connectivity (ALFF-FC) (Shen et al., 2016), which sums up the spectral content of low-frequency RS fluctuations through consecutive sliding windows.

Flexibility metrics quantifying time-varying global and regional network properties were also calculated using a graph theory framework (Lin et al., 2018), as described in section Graph Theoretical Analysis. More complex strategies rely on the identification of connectivity patterns that reoccur over time during the course of the experiment. Reoccurring RS FC patterns, often called "states," can be determined using clustering techniques (Allen et al., 2014), principal component analysis (Leonardi et al., 2013) or tensor decomposition (Mokhtari et al., 2018a), as detailed in section Definition of Reoccurring Connectivity States. Finally, approaches overcoming a rigid data decomposition into "fixed" connectivity states have been recently proposed (Miller et al., 2016), as described in detail in section Fuzzy Meta-State Analysis.

\section{Graph Theoretical Analysis}

Graph theory analysis can be applied to series of matrices derived from sliding-window analysis (Figure 1). Besides the classical network metrics (Rubinov and Sporns, 2010), which can be quantified as a function of time (Fukushima and Sporns, 2018), more specific metrics can be used to assess time-varying network structure. For instance, network power measures the summed values of TVC pairs in all windows, and density estimates how dense, on average, connections are over time. Specific timeresolved network features include network variation, which describes how different are connectivity values between two adjacent windows, flexibility of homologous, non-homologous and intra-hemispheric connections, which quantify connectivity differences between two consecutive windows for the specified type of connections (Lin et al., 2018), or the Fiedler value, which summarizes how well-connected a network is (Cai J. et al., 2018). Recently, novel approaches have been proposed to improve modeling of brain network TVC using graph theory (Khambhati et al., 2018). Such modeling strategies aim to assess time-varying patterns of connectivity (e.g., dynamic community detection or non-negative matrix factorization), time-varying patterns of activity, or a combination of both. A detailed review of these methods is reported in Khambhati et al. (2018).

\section{Definition of Reoccurring Connectivity States}

One of the most diffuse approaches used to identify reoccurring FC states from sliding-window matrices is based on hardclustering algorithms (Preti et al., 2017), such as the kmeans algorithm (Allen et al., 2014). In this approach, data are partitioned into different connectivity states by maximizing a cluster validity index, which describes the betweencluster/within-cluster distance ratio. In this way, identified recurring connectivity states have a minimal degree of overlap (Allen et al., 2014). The amount of time spent in each recurring state (dwell time) and the number of transitions between states can be calculated and compared between groups (Figure 1). Between-group comparisons can be also performed on pair-wise TVC strengths within each detected state (Allen et al., 2014).

Other ways to identify FC states from sliding-window data rely on principal component analysis (PCA) (Leonardi et al., 2013) or tensor decomposition (Mokhtari et al., 2018a). PCA is able to decompose sliding-window matrices into patterns of correlated connectivities (called "eigenconnectivities") between brain regions. Each eigenconnectivity pattern is characterized by a "contribution" (which can be thought as the equivalent of dwell times for k-means clustering analysis), which varies over time across subjects. Between-group comparisons of such contributions may allow to characterize TVC abnormalities in patients' populations (Leonardi et al., 2013). Similarly, tensor decomposition (Mokhtari et al., 2018a) is able to decompose sliding-window connectivity matrices in a set of components, each with an associated weight, which explain the majority of data content.

\section{Fuzzy Meta-State Analysis}

In hard-clustering analysis, windowed correlation matrices are forced to fit into determined TVC recurring states. However, the existence of just one state at each time point may be a too rigid assumption. A more flexible approach is to consider the possibility that multiple states might be represented to varying degrees at the same time point. The contribution of each state for a specific time is characterized by a vector that is called a "meta-state" (Miller et al., 2016). Four different measures of neural reorganization over time can be associated to such meta-states and can be calculated for each study subject: (1) the total number of distinct meta-states that a subject assumes during the experiment; (2) the number of changes between distinct meta-states; (3) the range of meta-states occupied in the $\mathrm{n}$-dimensional meta-state space during the entire RS fMRI experiment; and (4) the total distance traveled in the n-dimensional state space (Figure 1).

\section{APPLICATION OF TVC TO HEALTHY SUBJECTS}

\section{Main TVC Findings in Healthy Subjects}

The results of the main studies assessing TVC in healthy controls are summarized in Table $\mathbf{1 .}$

In healthy subjects, it was always possible to identify a certain number of recurring connectivity configurations (from 3 to 12, depending on the method applied and on RS fMRI sequence 
TABLE 1 | Summary of studies assessing time-varying resting state functional connectivity in healthy subjects and simulated data.

\begin{tabular}{|c|c|c|c|c|}
\hline Study & $\begin{array}{l}\text { RS fMRI acquisition } \\
\text { parameters } \Omega\end{array}$ & TVC analysis approach $^{\wedge}$ & Study subjects ${ }^{\theta}$ & Main findings \\
\hline Allen et al. (2014) & $\begin{array}{l}\text { Siemens Trio } 3 \mathrm{~T} \\
152 \text { volumes } \\
\mathrm{TR}=2 \mathrm{~s}\end{array}$ & $\begin{array}{l}\text { 1. Group ICA decomposition in } 50 \\
\text { relevant independent } \\
\text { components of interest, } \\
\text { classified into } 7 \text { different } \\
\text { functional networks } \\
\text { 2. Sliding-window analysis, window } \\
\text { length }=22 \text { TRs ( } 44 \mathrm{~s}) \text {, steps = } \\
1 \text { TR ( } 2 \text { s). } \\
\text { 3. k-means clustering ( } 7 \\
\text { recurring states) }\end{array}$ & $\begin{array}{l}405 \text { healthy adults } \\
200 \text { females }(49.4 \%) \\
\text { mean age }=21.0 \text { years } \\
\text { age range }=12-35 \text { years }\end{array}$ & $\begin{array}{l}\text { - Identification of recurring TVC } \\
\text { states that partially diverge from } \\
\text { static connectivity patterns } \\
\text { - Regions belonging to the DMN } \\
\text { have highly variable connectivity } \\
\text { over time, while regions of the } \\
\text { sensory and motor networks } \\
\text { exhibit more stable } \\
\text { connectivity configurations }\end{array}$ \\
\hline Allen et al. (2017) & $\begin{array}{l}\text { Siemens Sonata } 1.5 \mathrm{~T} \\
\text { Eyes open/Eyes closed } \\
255 \text { volumes } \\
\text { TR }=2 \mathrm{~s}\end{array}$ & $\begin{array}{l}\text { 1. Group ICA decomposition in } 43 \\
\text { relevant independent } \\
\text { components of interest, } \\
\text { classified into seven different } \\
\text { functional networks } \\
\text { 2. Sliding-window analysis, window } \\
\text { length }=30 \text { TRs ( } 60 \mathrm{~s} \text { ), steps = } \\
1 \text { TR ( } 2 \mathrm{~s} \text { ) } \\
\text { 3. k-means clustering ( } 5 \text { recurring } \\
\text { states) } \\
\text { 4. Correlations with EEG data }\end{array}$ & $\begin{array}{l}23 \text { healthy adults } \\
7 \text { females }(30.4 \%) \\
\text { mean age }=29 \text { years } \\
\mathrm{SD}=8.8 \text { years }\end{array}$ & $\begin{array}{l}\text { - States were replicable with those } \\
\text { of Allen et al. (2014) } \\
\text { - TVC states correspond to } \\
\text { neurophysiological mental states } \\
\text { detected with EEG } \\
\text { - Eyes open/eyes closed conditions } \\
\text { show some common and some } \\
\text { different connectivity patterns } \\
\text { Connectivity between the } \\
\text { thalamus and the cortex changes } \\
\text { from positive to negative in eyes } \\
\text { closed vs. open condition }\end{array}$ \\
\hline Cabral et al. (2017) & $\begin{array}{l}\text { Siemens Avanto } 1.5 \mathrm{~T} \\
180 \text { volumes } \\
\mathrm{TR}=2 \mathrm{~s}\end{array}$ & $\begin{array}{l}\text { 1. Segmentation in } 90 \text { cortical brain } \\
\text { regions of the } A A L \text { atlas } \\
\text { 2. Phase-coherence connectivity at } \\
\text { each time point } \\
\text { 3. Leading eigenvectors and } \\
\text { subsequent k-means clustering } \\
\text { (five recurring states) }\end{array}$ & $\begin{array}{l}55 \text { healthy adults with good } \\
\text { cognitive performance } \\
31 \text { females }(44.6 \%) \\
\text { mean age }=64 \text { years } \\
\text { SD }=9 \text { years } \\
43 \text { healthy adults with poor } \\
\text { cognitive performance } \\
29 \text { females ( } 66 \%) \\
\text { mean age }=66 \\
\text { SD }=8 \text { years }\end{array}$ & $\begin{array}{l}\text { - More frequent switches in subjects } \\
\text { with poor cognitive vs. good } \\
\text { cognitive performances } \\
\text { - The lower occurrence of a state } \\
\text { of global, positive coherence is } \\
\text { associated with worse } \\
\text { cognitive performances }\end{array}$ \\
\hline Cai B. et al. (2018) & $\begin{array}{l}\text { Siemens Trio 3T } \\
126 \text { volumes } \\
\mathrm{TR}=3 \mathrm{~s}\end{array}$ & $\begin{array}{l}\text { 1. Segmentation in } 264 \text { regions of } \\
\text { the Power atlas (Power et al., } \\
\text { 2011), grouped into } 10 \\
\text { functional networks } \\
\text { 2. Sliding-window analysis, window } \\
\text { length = } 50 \text { TRs ( } 150 \mathrm{~s} \text { ), steps = } \\
1 \mathrm{TR} \text { ( } 3 \mathrm{~s} \text { ) and dynamic sparse } \\
\text { connectivity models } \\
\text { 3. k-means clustering analysis (4 } \\
\text { recurring states) }\end{array}$ & $\begin{array}{l}\text { Philadelphia } \\
\text { neurodevelopmental } \\
\text { cohort database } \\
240 \text { young adults } \\
146 \text { females }(60.8 \%) \\
\text { mean age }=18.99 \text { years } \\
\text { SD }=1.12 \text { years } \\
232 \text { children } \\
123 \text { females }(53 \%) \\
\text { mean age }=10.67 \text { years } \\
\text { SD }=1.09 \text { years }\end{array}$ & $\begin{array}{l}\text { - Compared with young adults, } \\
\text { children had increased } \\
\text { connectivity between the DMN } \\
\text { and other subnetworks } \\
\text { - Children had reduced connectivity } \\
\text { among sensorimotor, executive } \\
\text { control and auditory networks vs. } \\
\text { young adults } \\
\text { - Young adults spent more time in } \\
\text { the most connected state }\end{array}$ \\
\hline Chang and Glover (2010) & $\begin{array}{l}\text { GE Signa HDx or Signa } 750 \text { 3T } \\
360 \text { volumes } \\
\text { TR }=2 \mathrm{~s}\end{array}$ & $\begin{array}{l}\text { 1. ROls in crucial nodes of the } \\
\text { DMN and of the "task-positive" } \\
\text { (executive control) network } \\
\text { 2. Time-frequency decomposition } \\
\text { using Wavelet transform } \\
\text { coherence; } \\
\text { sliding-window analysis }\end{array}$ & $\begin{array}{l}12 \text { healthy adults } \\
6 \text { females }(50 \%) \\
\text { mean age }=27.7 \text { years } \\
\text { SD }=12.4 \text { years }\end{array}$ & $\begin{array}{l}\text { - Coherence and phase between } \\
\text { the PCC and nodes of the } \\
\text { executive control network } \\
\text { significantly vary in time and } \\
\text { frequency } \\
\text { - High variability over time was } \\
\text { observed between the PCC and } \\
\text { brain areas involved in higher-level } \\
\text { cognitive functions }\end{array}$ \\
\hline Chen T. et al. (2016) & $\begin{array}{l}\text { Siemens Skyra 3T } \\
\text { Eyes open } \\
1,200 \text { volumes } \\
\text { TR }=0.72 \mathrm{~s} \\
\text { Test-retest data }\end{array}$ & $\begin{array}{l}\text { 1. Segmentation in } 264 \text { regions of } \\
\text { the Power atlas (Power et al., } \\
\text { 2011) } \\
\text { 2. Sliding-window analysis, window } \\
\text { length }=55 \text { TRs }(40 \mathrm{~s}) \text {, steps = } \\
1 \mathrm{TR}(0.72 \mathrm{~s}) \\
\text { 3. Graph theoretical analysis }\end{array}$ & $\begin{array}{l}\text { Human Connectome } \\
\text { Project dataset } \\
77 \text { healthy adults } \\
50 \text { females }(64.1 \%) \\
\text { age range }=22-35 \text { years }\end{array}$ & $\begin{array}{l}\text { - The salience network showed } \\
\text { highly flexible connectivity } \\
\text { with fronto-parietal, cingulate- } \\
\text { opercular, and attention networks } \\
\text { - The salience network maintained } \\
\text { a consistently high level of } \\
\text { network centrality over time }\end{array}$ \\
\hline
\end{tabular}


TABLE 1 | Continued

\begin{tabular}{|c|c|c|c|c|}
\hline Study & $\begin{array}{l}\text { RS fMRI acquisition } \\
\text { parameters }{ }^{\Omega}\end{array}$ & TVC analysis approach ${ }^{\wedge}$ & Study subjects ${ }^{\theta}$ & Main findings \\
\hline Choe et al. (2017) & $\begin{array}{l}\text { Multi-Modal MRI } \\
\text { Reproducibility Resource } \\
\text { (Kirby) data set } \\
\text { Philips Achieva 3T } \\
210 \text { volumes } \\
\text { TR }=2 \mathrm{~s} \\
\text { Test-retest data } \\
\text { Human Connectome Project } \\
\text { S500 Data dataset } \\
\text { Siemens Skyra 3T } \\
\text { 1,200 volumes } \\
\text { TR = 0.72 s } \\
\text { Test-retest data }\end{array}$ & $\begin{array}{l}\text { Kirby dataset: } \\
\text { 1. Group ICA decomposition in } 39 \\
\text { relevant components of interest, } \\
\text { classified into } 7 \text { functional } \\
\text { networks } \\
\text { 2. Sliding-window analysis, window } \\
\text { length = } 30 \text { TRs ( } 60 \text { s) } \\
\text { Human Connectome Project } \\
\text { S500 Data dataset: } \\
\text { 1. Group ICA decomposition in } 50 \\
\text { relevant components of interest } \\
\text { 2. Sliding-window analysis, window } \\
\text { lengths = 15, 30, } 60, \text { and } 120 \\
\text { TRs ( } 11,22,43 \text {, and } 86 \text { s) } \\
\text { 3. TVC mean and variance, } \\
\text { k-means clustering (three } \\
\text { recurring states) and dynamic } \\
\text { conditional } \\
\text { correlation approaches }\end{array}$ & $\begin{array}{l}\text { Kirby dataset } \\
20 \text { healthy adults Human } \\
\text { Connectome Project } \mathbf{5 0 0} \\
\text { Data dataset } \\
523 \text { healthy adults }\end{array}$ & $\begin{array}{l}\text { - TVC can be reliably estimated in } \\
\text { test-retest data } \\
\text { - The dynamic conditional } \\
\text { correlation method seems to be } \\
\text { more reliable than } \\
\text { sliding-window analysis }\end{array}$ \\
\hline Lim et al. (2018) & $\begin{array}{l}\text { Siemens Prisma 3T } \\
\text { Eyes open } \\
250 \text { volumes } \\
\text { TR }=2 \text { s }\end{array}$ & $\begin{array}{l}\text { 1. Segmentation of } 114 \text { regions of } \\
\text { the Yeo atlas (Yeo et al., 2011), } \\
\text { classified into } 17 \text { functional } \\
\text { networks } \\
\text { 2. Sliding-window analysis, window } \\
\text { length }=7 \text { TRs ( } 14 \mathrm{~s}) \text {, steps }=1 \\
\text { TR ( } 2 \mathrm{~s} \text { ) } \\
\text { 3. k-means clustering (3-7 } \\
\text { recurring states) }\end{array}$ & $\begin{array}{l}21 \text { healthy adults with } \\
\text { high-trait mindfulness } \\
13 \text { females }(61.9 \%) \\
\text { mean age }=23.7 \text { years } \\
\mathrm{SD}=3.4 \text { years } \\
18 \text { healthy adults with } \\
\text { low-trait mindfulness } \\
13 \text { females }(72.2 \%) \\
\text { mean age }=21.9 \text { years } \\
\mathrm{SD}=2.3 \text { years }\end{array}$ & $\begin{array}{l}\text { - High trait mindfulness subjects } \\
\text { spent significantly more time in a } \\
\text { high within-network connectivity } \\
\text { state, characterized by greater } \\
\text { anti-correlations between task- } \\
\text { positive networks and the } \\
\text { DMN } \\
\text { - Transitions between brain states } \\
\text { was more frequent in high vs. low } \\
\text { trait mindfulness subjects }\end{array}$ \\
\hline Lindquist et al. (2014) & $\begin{array}{l}\text { Philips Achieva } 3 T \\
210 \text { volumes } \\
\text { TR }=2 \text { s } \\
\text { Test-retest data }\end{array}$ & $\begin{array}{l}\text { 1. Segmentation of six spherical } \\
\text { ROls (radius }=3 \mathrm{~mm} \text { ) containing } \\
\text { regions of the DMN } \\
\text { 2. Point-process analysis } \\
\text { 3. Estimation of variance of } \\
\text { dynamic connectivity } \\
\text { correlations, compared with } \\
\text { traditional } \\
\text { sliding-window analysis }\end{array}$ & $\begin{array}{l}\text { Multimodal MRI } \\
\text { Reproducibility Resource } \\
\text { (Kirby21) dataset } \\
21 \text { healthy adults } \\
10 \text { females }(47.6 \%) \\
\text { mean age }=31.76 \text { years } \\
\text { SD }=9.47 \text { years }\end{array}$ & $\begin{array}{l}\text { - Dynamic conditional correlations } \\
\text { are able to quantify dynamics of } \\
\text { RS fMRI data } \\
\text { - Dynamic conditional correlations } \\
\text { have a similar performance as } \\
\text { sliding-window analysis in } \\
\text { quantifying TVC between } \\
\text { brain regions }\end{array}$ \\
\hline Liu and Duyn (2013) & $\begin{array}{l}\text { Multicenter } 3 T \text { scanners } \\
\text { Volumes varying from } 119 \text { to } 195 \\
\text { TR varying from } 2.3 \text { to } 3 \mathrm{~s}\end{array}$ & $\begin{array}{l}\text { 1. Segmentation of two spherical } \\
\text { ROls (radius }=6 \mathrm{~mm} \text { ) containing } \\
\text { the PCC and left intraparietal } \\
\text { sulcus } \\
\text { 2. Point-process analysis } \\
\text { 3. k-means clustering of } \\
\text { coactivation patterns (eight } \\
\text { coactivation patterns for the } \\
\text { PCC and } 12 \text { for the left } \\
\text { intraparietal sulcus) }\end{array}$ & $\begin{array}{l}1000 \text { Functional } \\
\text { Connectomes Project (FCP) } \\
247 \text { healthy adults } \\
151 \text { females }(61.1 \%) \\
\text { mean age }=22.72 \text { years } \\
S D=4.61 \text { years } \\
\text { age range }=18-44 \text { years }\end{array}$ & $\begin{array}{l}\text { - Point-process analysis was able } \\
\text { to extract correlational patterns in } \\
\text { RS fMRI data from relatively brief } \\
\text { periods of co-activation (or co- } \\
\text { deactivation) of brain regions } \\
\text { - Co-activation patterns resembled } \\
\text { classical networks derived from } \\
\text { static RS FC analysis, while more } \\
\text { fine-grained co-activation } \\
\text { patterns were detected }\end{array}$ \\
\hline Marusak et al. (2017) & $\begin{array}{l}\text { GE Signa 3T } \\
\text { Siemens Verio 3T } \\
\text { both scanners: } \\
180 \text { volumes } \\
\text { TR }=2 \mathrm{~s}\end{array}$ & $\begin{array}{l}\text { 1. Group ICA decomposition in } 25 \\
\text { relevant independent } \\
\text { components of interest, } \\
\text { classified into } 3 \text { functional } \\
\text { networks } \\
\text { 2. Sliding-window analysis, window } \\
\text { length = } 22 \text { TRs ( } 44 \mathrm{~s} \text { ), steps = } \\
\text { 1 TR ( } 2 \mathrm{~s} \text { ) } \\
\text { 3. k-means clustering (six recurring } \\
\text { states) } \\
\text { 4. Correlation with age and } \\
\text { internal thoughts }\end{array}$ & $\begin{array}{l}\text { Stanford University dataset } \\
73 \text { normally } \\
\text { developing children } \\
34 \text { females }(46.57 \%) \\
\text { mean age }=12.47 \\
\text { SD }=1.88 \text { years Wayne State } \\
\text { University dataset } \\
73 \text { normally } \\
\text { developing children } \\
49 \text { females ( } 67.12 \%) \\
\text { mean age }=12.09 \text { years } \\
\text { SD }=2.54 \text { years }\end{array}$ & $\begin{array}{l}\text { - The occurrence and amount of } \\
\text { time spent in specific TVC states } \\
\text { are related to the content of self- } \\
\text { generated thought during the scan } \\
\text { - Temporal variability of TVC among } \\
\text { cognitive networks increases with } \\
\text { age } \\
\text { - Regions showing the highest TVC } \\
\text { include multi-modal areas } \\
\text { associated with high-order } \\
\text { cognitive functions, such as the } \\
\text { precuneus and inferior } \\
\text { parietal lobe }\end{array}$ \\
\hline
\end{tabular}


TABLE 1 | Continued

\begin{tabular}{|c|c|c|c|c|}
\hline Study & $\begin{array}{l}\text { RS fMRI acquisition } \\
\text { parameters } \Omega\end{array}$ & TVC analysis approach ${ }^{\wedge}$ & Study subjects ${ }^{\theta}$ & Main findings \\
\hline Marusak et al. (2018) & $\begin{array}{l}\text { Siemens Verio } 3 \mathrm{~T} \\
390 \text { volumes } \mathrm{TR}=1.5 \mathrm{~s}\end{array}$ & $\begin{array}{l}\text { 1. Group ICA decomposition in four } \\
\text { relevant independent } \\
\text { components of interest } \\
\text { 2. Sliding-window analysis, window } \\
\text { length = } 30 \text { TRs ( } 45 \mathrm{~s} \text { ), steps = } \\
1 \text { TR ( } 1.5 \mathrm{~s} \text { ) } \\
\text { 3. k-means clustering (5 recurring } \\
\text { states) } \\
\text { 4. Correlations with } \\
\text { mindfulness scores }\end{array}$ & $\begin{array}{l}42 \text { children } \\
23 \text { females }(54.8 \%) \\
\text { mean age }=10.3 \text { years } \\
S D=2.9 \text { years } \\
\text { age range }=6-17 \text { years }\end{array}$ & $\begin{array}{l}\text { - High-mindfulness children had a } \\
\text { greater number of transitions } \\
\text { between states than } \\
\text { low-mindfulness children and } \\
\text { showed a state-specific reduction } \\
\text { in connectivity between } \\
\text { salience/emotion and central } \\
\text { executive networks }\end{array}$ \\
\hline Nini et al. (2017) & $\begin{array}{l}\text { Siemens Trio } 3 \mathrm{~T} \\
225 \text { volumes } \\
\mathrm{TR}=2.48 \mathrm{~s}\end{array}$ & $\begin{array}{l}\text { 1. Segmentation in } 90 \text { regions of } \\
\text { the } A A L \text { atlas } \\
\text { 2. Sliding-window analysis, window } \\
\text { length }=25 \mathrm{~s} \text {, steps }=0.6 \mathrm{~s} \\
\text { 3. Graph theory analysis: flexibility } \\
\text { and variance }\end{array}$ & $\begin{array}{l}\mathbf{1 , 0 0 0} \text { Functional } \\
\text { Connectomes Project } \\
148 \text { healthy young adults } \\
74 \text { females }(50 \%) \\
\text { age range }=18-26 \text { years }\end{array}$ & $\begin{array}{l}\text { - Flexibility of amygdala, } \\
\text { hippocampus, fusiform gyrus, } \\
\text { and temporal gyrus was higher in } \\
\text { males than in females } \\
\text { - Flexibility of middle cingulate } \\
\text { cortex, thalamus, precuneus, and } \\
\text { temporo-occipital regions was } \\
\text { higher in females than in males }\end{array}$ \\
\hline Shi et al. (2018) & $\begin{array}{l}\text { Siemens Trio } 3 \mathrm{~T} \\
232 \text { volumes } \\
\mathrm{TR}=2 \mathrm{~s}\end{array}$ & $\begin{array}{l}\text { 1. Group ICA decomposition in } 5 \\
\text { relevant independent } \\
\text { components of interest } \\
\text { 2. Sliding-window analysis, window } \\
\text { length }=30 \text { TRs ( } 60 \mathrm{~s} \text { ), steps = } \\
1 \text { TR ( } 2 \mathrm{~s} \text { ) } \\
\text { 3. k-means clustering (four } \\
\text { recurring states) and fuzzy-meta } \\
\text { states analyses }\end{array}$ & $\begin{array}{l}\text { Southwest University } \\
\text { Longitudinal Imaging } \\
\text { Multimodal dataset } \\
331 \text { healthy young adults } \\
247 \text { females }(74.6 \%) \\
\text { mean age }=20.20 \text { years } \\
\text { SD }=1.34 \text { years } \\
212 \text { healthy young adults } \\
115 \text { females }(54.2 \%) \text { mean age } \\
=22.36 \text { years } \\
\text { SD }=1.49 \text { years }\end{array}$ & $\begin{array}{l}\text { - Subjects having a high score in } \\
\text { subjective well being spent less } \\
\text { time in a state characterized by } \\
\text { low cross-network connectivity } \\
\text { and strong within-network } \\
\text { connectivity } \\
\text { - The total number of transitions } \\
\text { across states was correlated with } \\
\text { a higher subjective } \\
\text { well-being score }\end{array}$ \\
\hline Smith et al. (2018) & $\begin{array}{l}\text { Siemens Skyra 3T scanner } \\
\text { Eyes open } \\
1,200 \text { volumes } \\
\text { TR }=0.72 \mathrm{~s} \\
\text { Test-retest data }\end{array}$ & $\begin{array}{l}\text { 1. Segmentation of } 90 \text { regions from } \\
\text { Shirer et al. (Shirer et al., 2012) } \\
\text { 2. Point-process analysis } \\
\text { 3. k-means clustering of } \\
\text { coactivation patterns (four } \\
\text { recurring states) }\end{array}$ & $\begin{array}{l}\text { Human Connectome } \\
\text { Project S500 Data dataset } \\
100 \text { healthy adults } \\
54 \text { females }(54 \%)\end{array}$ & $\begin{array}{l}\text { - Brain state- properties were } \\
\text { reliable across days } \\
\text { - Summary metrics of brain } \\
\text { connectivity dynamics had an } \\
\text { adequate test-retest reliability }\end{array}$ \\
\hline Tagliazucchi et al. (2013) & $\begin{array}{l}\text { Siemens Trio } 3 \mathrm{~T} \\
1,505 \text { volumes } \\
\mathrm{TR}=2.08 \mathrm{~s}\end{array}$ & $\begin{array}{l}\text { 1. Group ICA decomposition in six } \\
\text { relevant independent } \\
\text { components of interest } \\
\text { 2. Detrended fluctuation analysis } \\
\text { 3. Hurst exponent (measuring } \\
\text { long-range } \\
\text { temporal dependence) }\end{array}$ & 39 healthy adults & $\begin{array}{l}\text { - Temporal memory of RS fMRI } \\
\text { time series decreases from } \\
\text { wakefulness to deep non-rapid } \\
\text { eye movement sleep } \\
\text { - Long-range temporal } \\
\text { dependence decreases especially } \\
\text { in regions of the DMN and } \\
\text { attention network }\end{array}$ \\
\hline Vidaurre et al. (2018) & $\begin{array}{l}\text { Human Connectome Project } \\
\text { dataset } \\
\text { Siemens Skyra 3T } \\
\text { Eyes open } \\
1,200 \text { volumes } \\
\text { TR }=0.72 \mathrm{~s} \\
\text { UK Biobank dataset } \\
\text { Siemens Skyra 3T } \\
\text { Eyes open } \\
490 \text { volumes } \\
\text { TR }=0.735 \mathrm{~s}\end{array}$ & $\begin{array}{l}\text { 1. Group ICA decomposition in } 50 \\
\text { relevant independent } \\
\text { components of interest from the } \\
\text { HCP dataset, in } 55 \text { relevant } \\
\text { independent components of } \\
\text { interest from the UK Biobank } \\
\text { dataset } \\
\text { 2. Hidden Markov model } \\
\text { 3. Stochastic inference (12 } \\
\text { recurring states) }\end{array}$ & $\begin{array}{l}\text { Human Connectome } \\
\text { Project dataset } \\
820 \text { healthy adults } \\
453 \text { females }(55.2 \%) \\
\text { age range }=22-35 \text { years } \\
\text { UK Biobank dataset } \\
5847 \text { healthy adults } \\
\text { age range }=40-69 \text { years }\end{array}$ & $\begin{array}{l}\text { - Hidden Markov models allow to } \\
\text { model resting (or task-related) } \\
\text { brain activity as a time-varying } \\
\text { sequence of distinct brain } \\
\text { networks, also when analyzing } \\
\text { very large amounts of data }\end{array}$ \\
\hline Yaesoubi et al. (2015a) & $\begin{array}{l}\text { Data from Allen et al., } 2014 \\
\text { Siemens Trio 3T } \\
152 \text { volumes } \\
\text { TR }=2 \mathrm{~s}\end{array}$ & $\begin{array}{l}\text { 1. Group ICA decomposition in } 50 \\
\text { relevant independent } \\
\text { components of interest } \\
\text { Time-frequency decomposition } \\
\text { 2. k-means clustering (five } \\
\text { recurring states) }\end{array}$ & $\begin{array}{l}\text { Data from Allen et al. ( } 2014) \\
405 \text { healthy adults } \\
\text { 3. } 200 \text { females }(49.4 \%) \\
\text { 4. mean age }=21.0 \text { years } \\
\text { 5. age range }=12-35 \text { years }\end{array}$ & $\begin{array}{l}\text { - A new time-frequency } \\
\text { decomposition approach, based } \\
\text { on wavelet transform coherence, } \\
\text { detected time-frequency } \\
\text { connectivity variations in RS fMRI } \\
\text { data }\end{array}$ \\
\hline
\end{tabular}


TABLE 1 | Continued

\begin{tabular}{|c|c|c|c|c|}
\hline Study & $\begin{array}{l}\text { RS fMRI acquisition } \\
\text { parameters }{ }^{\Omega}\end{array}$ & TVC analysis approach ${ }^{\wedge}$ & Study subjects ${ }^{\theta}$ & Main findings \\
\hline
\end{tabular}

Yaesoubi et al. (2015b)

Data from Allen et al. (2014) Siemens Trio 3T

152 volumes

$\mathrm{TR}=2 \mathrm{~s}$

Yaesoubi et al. (2017b)

Siemens Trio 3T

152 volumes

$\mathrm{TR}=2 \mathrm{~s}$
Data from Allen et al. (2014)

1. Group decomposition in 50 relevant components of interest classified into seven different functional networks

2. Sliding-window analysis, window length $=32$ TRs $(44 \mathrm{~s})$, steps $=$ 1 TR (2s)

3. Clustering of sliding-window matrices using temporal ICA to find maximally mutually temporally independent connectivity patterns (five recurring states)

4. Sex differences

1. Group ICA decomposition in 50 relevant independent components of interest

2. Time-frequency decomposition

3. k-means clustering of z-scored time-frequency decompositions to find recurring frequency modes (four recurring modes)

Yang et al. (2014)

Zalesky et al. (2014)

Siemens Skyra 3T 1,200 volumes $\mathrm{TR}=0.72 \mathrm{~s}$

Zhang C. et al. (2018)
Siemens Trio 3T

884 volumes

$\mathrm{TR}=0.645 \mathrm{~s}$

Test-retest data length $=60 \mathrm{~s}$, steps $=1 \mathrm{TR}$
1. Four spherical ROIs (radius $=$ $3 \mathrm{~mm}$ ) in crucial nodes of the posteromedial cortex; segmentation of 156 regions from Craddock et al. (2012)

2. Sliding-window analysis, window length $=69$ TRs $(44 \mathrm{~s})$, steps = 3 TRs (2s)

3. Hierarchical clustering (five recurring states)

1. Segmentation in different numbers of ROls (from 90 to 4,000) (Zalesky et al., 2010)

2. Sliding-window analysis, window (0.72s)

3. Non-stationarity of RS fMRI fluctuations measured using an ad hoc test statistic

1. Segmentation in 116 regions of the AAL atlas and 160 regions of the Dosenbach atlas (Dosenbach et al., 2010) Sliding-window analysis, window age range $=22-37$ years length $=$ from 20 TRs to 200 TRs

2. Standard deviation from the mean and excursion from the median. Amplitude of low-frequency fluctuations across sliding windows

22 healthy adults

4. 6 females (27.3\%)

5. mean age $=33.5$ years

6. $S D=12.5$ years

7. age range $=19-60$ years Q2 Data dataset

10 healthy adults 6 females (60\%) age range $=22-35$ years 820 healthy adults 454 females (55.4\%)
Data from Allen et al. (2014)

405 healthy adults 200 females (49.4\%) mean age $=21.0$ years

age range $=12-35$ years

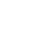

- Recurring connectivity patterns in time-frequency domain revealed significant between-group differences based on sex

- A method alternative to k-means clustering is proposed, based on temporal ICA. This method allowed to detect temporally independent connectivity states

- Frequency of occupancy of such states was not different between genders

Data from Allen et al. (2014) 405 healthy adults 200 females (49.4\%) mean age $=21.0$ years age range $=12-35$ years
- Time-frequency decomposition allowed to capture frequency variations in individual network time courses

- Frequency modes represent "periodic" activities consisting of instantaneous activations and deactivations

- Each subregion of the posteromedial cortex was associated with five recurring connectivity states Each subregion possessed a unique preferred state and distinct transition patterns

Human connectome project - A consistent set of functional connections had pronounced fluctuations over time

- The most dynamic connections were inter-modular and involved hubs of the DMN and fronto-parietal network

Human connectome project - TVC was reliable, especially when S900 Data dataset windows size was between 30 and 50 TRs, but less reliable than static FC

- The highest reliability for static and dynamic FC analysis was found for intra-network connections in the fronto-parietal, DMN, sensorimotor, and occipital networks

\footnotetext{
${ }^{\Omega}$ All RS scans were acquired in the eyes-closed condition, except where indicated.

^TVC analysis approach summarizes: (1) ROIs used; (2) assessment of time-varying correlations between brain regions; (3) features extracted for assessing TVC.

${ }^{\theta}$ For each study group of healthy subjects, sex is represented as number of females (\%), mean age and standard deviation (SD).

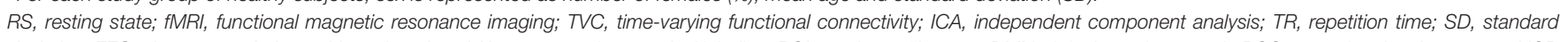

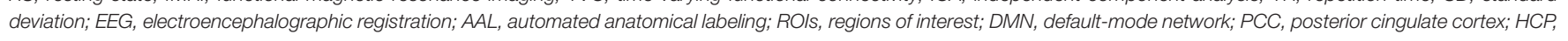
Human connectome project; UK, United Kingdom; FC, functional connectivity.
} 
settings). The DMN was one of the functional networks showing the highest degree of connectivity change over time, both when analyzing within-DMN TVC (Chang and Glover, 2010; Liu and Duyn, 2013; Lindquist et al., 2014; Zalesky et al., 2014) and when considering connections between the DMN and other crucial cognitive networks (Chang and Glover, 2010; Liu and Duyn, 2013; Allen et al., 2014; Marusak et al., 2017; Vidaurre et al., 2018). High dynamism was also observed in multimodal brain regions, involved in high-order emotional and cognitive processing (Yang et al., 2014; Zalesky et al., 2014; Chen S. et al., 2016; Marusak et al., 2017; Vidaurre et al., 2018). Such quick temporal reconfigurations may be required to facilitate transient psychological states between different brain functions (starting, maintenance or conclusion of the different attentional, cognitive, and executive tasks). Conversely, networks involved in sensory and motor processing showed more "static" connectivity profiles (Allen et al., 2014; Zalesky et al., 2014).

TVC was also useful to characterize age- and sex-related features. For instance, it was shown that children have higher TVC between the DMN and other subnetworks than young adults, but that young adults have stronger TVC than children among sensorimotor, executive control, and auditory networks (Cai B. et al., 2018). Moreover, variability of TVC among cognitive networks increased with age (Marusak et al., 2017). Overall, these results suggest that maturation is associated with a higher flexibility of functional connections. More discrepancies were found when analyzing sex-related characteristics of connectivity dynamics (Yaesoubi et al., 2015a,b; Nini et al., 2017). While some studies found no differences in TVC configurations between males and females (Yaesoubi et al., 2015b), other studies found that connectivity configurations were different between genders (Yaesoubi et al., 2015a,b; Nini et al., 2017): males showed a higher connectivity flexibility than females in the amygdala, hippocampus, fusiform, and temporal gyrus, whereas the opposite trend was found in the middle cingulate cortex, thalamus, precuneus, and some temporaloccipital regions (Nini et al., 2017).

TVC constitutes a complex and novel methodology. Studies from healthy controls also served to test how reliable and reproducible TVC results were across scanning sessions. This was the goal of some recent investigations (Choe et al., 2017; Smith et al., 2018; Zhang C. et al., 2018), which found that TVC metrics were reliable across days (Smith et al., 2018) and had an overall good reproducibility (Choe et al., 2017; Smith et al., 2018; Zhang C. et al., 2018), even if lower than that of the corresponding static FC metrics (Zhang C. et al., 2018). The highest reliability was found for intra-network connections in the DMN, fronto-parietal, sensorimotor, and occipital networks (Zhang C. et al., 2018).

To better investigate the intrinsic nature of TVC states and their electrophysiological correlates, simultaneously acquired electroencephalography (EEG) and RS fMRI data were analyzed and concurrent temporal variations were assessed (Allen et al., 2017). Results indicated that connectivity states detected by TVC analysis correspond to neuro-electric brain activity with distinct spectral signatures. Moreover, eyes open/eyes closed conditions show some common and some different connectivity patterns, with a greater integration within sensory systems, as well as reduced modularity and increased global efficiency, in the eyes-closed compared to the eyes-open condition (Allen et al., 2017). These results integrate and complete previous EGG/RS fMRI studies, which showed a variable TVC configuration between wakefulness and different stages of sleep (Tagliazucchi et al., 2013), with temporal memory and long-range temporal dependencies decreasing from wakefulness to deep non-rapid eye movement sleep.

\section{Correlations Between TVC and Behavioral/Neuropsychological Performances in Healthy Subjects}

To date, correlations between TVC measures and cognitive performances in healthy controls have been evaluated by one study (Cabral et al., 2017), which found that worse cognitive performance in healthy elderly was associated with a lower permanence in a TVC state characterized by strong, positive connectivity. These results suggest that a more static pattern of TVC may characterize poor vs. good performers.

Another study (Shi et al., 2018) analyzed the correlation between TVC and scores obtained at questionnaires of subjective well-being, and found that subjects with higher well-being scores spent less time in low cross-network and strong within-network connectivity states. The total number of transitions between states was also higher in subjects with high well-being scores, suggesting a more efficient transfer of information between networks in this group (Shi et al., 2018). Finally, two studies assessing the relationship between TVC and mindfulness in healthy adults (Lim et al., 2018) and children (Marusak et al., 2018) had similar conclusions, showing that high-mindfulness subjects spent more time in highly-connected states and switched more frequently between states than low-mindfulness subjects, suggesting a more efficient and flexible connectivity in the first group.

\section{APPLICATION OF TVC TECHNIQUES TO MS}

\section{Main TVC Findings in MS Patients}

The main studies assessing TVC abnormalities in MS patients are summarized in Table 2. As it is evident from this table, TVC methodologies applied in MS investigations were quite heterogeneous. Despite this, results of different studies share some common points.

First of all, networks showing the greatest amount of TVC abnormalities in MS patients in comparison to healthy subjects were the DMN, salience, executive and sensorimotor networks (Leonardi et al., 2013; Zhou et al., 2016; Bosma et al., 2018; Lin et al., 2018; d'Ambrosio et al., 2019; Rocca et al., 2019).

The regional pattern of TVC abnormalities was quite complex, and regions involved by TVC changes were variable across studies, probably depending from the used TVC approach and patients' clinical characteristics. The analysis of eigenconnectivity patterns helped to identify the presence of stronger TVC in parietal regions and weaker TVC in frontal/subcortical regions 
TABLE 2 | Summary of studies assessing time-varying resting state functional connectivity modifications in multiple sclerosis (MS).

\begin{tabular}{|c|c|c|c|c|}
\hline Study & $\begin{array}{l}\text { RS fMRI acquisition } \\
\text { parameters } \Omega\end{array}$ & TVC analysis approach ${ }^{\wedge}$ & Study subjects $^{\theta}$ & Main findings \\
\hline Bosma et al. (2018) & $\begin{array}{l}\text { GE } 3 T \\
277 \text { volumes } \\
\mathrm{TR}=2 \mathrm{~s}\end{array}$ & $\begin{array}{l}\text { 1. Segmentation of } 5 \text { cortical } \\
\text { regions belonging to the DMN, } \\
\text { salience network, ascending and } \\
\text { descending nociceptive network } \\
\text { (according with Hemington et al., } \\
\text { 2016) and the primary sensory } \\
\text { area (Harvard Oxford Cortical } \\
\text { Structural Atlas, Desikan et al., } \\
\text { 2006) } \\
\text { 2. Dynamic conditional correlations } \\
\text { 3. Standard deviation of dynamic } \\
\text { conditional correlation and of RS } \\
\text { fMRI time series }\end{array}$ & $\begin{array}{l}31 \text { MS patients ( } 25 \\
\text { relapsing-remitting MS, } 4 \\
\text { secondary progressive MS, } \\
3 \text { unknown) } \\
20 \text { females }(64.5 \%) \\
\text { mean age }=39 \text { years } \\
\mathrm{SD}=10 \text { years } \\
31 \text { healthy controls } \\
20 \text { females }(64.5 \%) \\
\text { mean age }=38 \text { years } \\
\mathrm{SD}=11 \text { years }\end{array}$ & $\begin{array}{l}\text { - Greater TVC between the salience } \\
\text { and ascending nociceptive } \\
\text { network in MS patients vs. healthy } \\
\text { controls } \\
\text { - Greater variability of RS FC in MS } \\
\text { patients vs. healthy controls } \\
\text { - Patients with neuropathic pain } \\
\text { had abnormal cross-network } \\
\text { connectivity between the salience } \\
\text { and DMN }\end{array}$ \\
\hline d'Ambrosio et al. (2019) & $\begin{array}{l}\text { Multicenter setting: seven } \\
\text { centers } \\
\text { 3T } \\
200 \text { volumes } \\
\text { TR }=3 \mathrm{~s}\end{array}$ & $\begin{array}{l}\text { 1. Group ICA decomposition in } 43 \\
\text { relevant independent } \\
\text { components of interest, } \\
\text { classified into seven different } \\
\text { functional networks } \\
\text { 2. Sliding-window analysis, window } \\
\text { length = } 22 \text { TRs ( } 66 \mathrm{~s}) \text {, step }=1 \\
\text { TR ( } 3 \mathrm{~s} \text { ) } \\
\text { 3. k-means clustering analysis } \\
\text { (three recurring states); fuzzy } \\
\text { meta-state analysis } \\
\text { 4. Correlations with clinical } \\
\text { variables, cognitive } \\
\text { performance, T2 lesion volume, } \\
\text { and brain volume }\end{array}$ & $\begin{array}{l}\text { MAGNIMS Cognition study } \\
62 \text { relapsing-remitting MS } \\
\text { patients ( } 23 \text { with cognitive } \\
\text { impairment, } 39 \text { without } \\
\text { cognitive impairment) } \\
40 \text { females ( } 64.5 \%) \\
\text { mean age }=39.5 \text { years } \\
\text { SD }=8.5 \text { years } \\
65 \text { healthy controls } \\
38 \text { females ( } 58 \%) \\
\text { mean age }=35.8 \text { years } \\
\text { SD }=9.4 \text { years }\end{array}$ & $\begin{array}{l}\text { - MS patients, compared to healthy } \\
\text { controls, showed: (i) reduced } \\
\text { TVC between subcortical and } \\
\text { visual/cognitive networks, as well } \\
\text { as between visual and cognitive } \\
\text { networks; and (ii) increased } \\
\text { TVC between subcortical and } \\
\text { sensorimotor networks } \\
\text { - Compared to cognitively } \\
\text { preserved, cognitively impaired } \\
\text { MS patients showed reduced } \\
\text { TVC between subcortical and } \\
\text { DMN, lower dwell time in a } \\
\text { state characterized by high intra- } \\
\text { and inter-network connectivity, } \\
\text { and lower global connectivity } \\
\text { variations over time } \\
\text { - In patients with cognitive } \\
\text { impairment, reduced global } \\
\text { dynamism correlated with } \\
\text { brain atrophy }\end{array}$ \\
\hline Huang et al. (2019) & $\begin{array}{l}\text { Siemens Trio } 3 \mathrm{~T} \\
240 \text { volumes } \\
\mathrm{TR}=2 \mathrm{~s}\end{array}$ & $\begin{array}{l}\text { 1. Segmentation of six regions of } \\
\text { interest belonging to the } \\
\text { attention network } \\
\text { 2. Sliding-window analysis, window } \\
\text { length }=40 \text { TRs }(80 \mathrm{~s}) \text {, and } 20 \\
\text { TRs }(40 \mathrm{~s}) \text {, steps }=1 \mathrm{TR}(2 \mathrm{~s}) \\
\text { 3. Estimation of the temporal } \\
\text { correlation coefficient between } \\
\text { truncated time courses }\end{array}$ & $\begin{array}{l}22 \text { relapsing-remitting } \\
\text { MS patients } \\
15 \text { females }(68.2 \%) \\
\text { mean age }=39.1 \text { years } \\
\text { age range }=20-58 \text { years } \\
22 \text { healthy controls } \\
15 \text { females }(68.2 \%) \\
\text { mean age }=39.6 \text { years } \\
\text { age range }=26-56 \text { years }\end{array}$ & $\begin{array}{l}\text { - Compared to controls, decreased } \\
\text { TVC within the dorsal and ventral } \\
\text { attention networks, as well as } \\
\text { increased TVC between the dorsal } \\
\text { and ventral attention networks } \\
\text { was detected } \\
\text { - Decreased TVC within parietal } \\
\text { and between fronto-temporal } \\
\text { regions was correlated with a } \\
\text { higher white matter lesion load }\end{array}$ \\
\hline Leonardi et al. (2013) & $\begin{array}{l}\text { Siemens Trio 3T } \\
450 \text { volumes } \\
\mathrm{TR}=1.1 \mathrm{~s}\end{array}$ & $\begin{array}{l}\text { 1. Segmentation of } 88 \text { brain } \\
\text { regions from the AAL atlas } \\
\text { (Tzourio-Mazoyer et al., 2002) } \\
\text { 2. Sliding-window analysis, window } \\
\text { length }=30 \text { TRs ( } 33 \mathrm{~s}), 40 \text { TRs } \\
\text { (44 s), } 60 \text { TRs }(66 \mathrm{~s} \text { ) and } 120 \mathrm{TRs} \\
\text { (132 s), steps }=2 \text { TRs }(2.2 \mathrm{~s}) \\
\text { 3. Principal component analysis: } \\
10 \text { eigenconnectivity } \\
\text { patterns (states) }\end{array}$ & $\begin{array}{l}22 \text { relapsing-remitting } \\
\text { MS patients } \\
14 \text { females }(63.6 \%) \\
\text { mean age }=36.8 \text { years } \\
S D=8 \text { years } \\
14 \text { healthy controls } \\
9 \text { females }(64.2 \%) \\
\text { mean age }=38.4 \text { years } \\
S D=6 \text { years }\end{array}$ & $\begin{array}{l}\text { - A novel data-driven approach, } \\
\text { based on principal component } \\
\text { analysis, was able to detect } \\
\text { large-scale recurring connectivity } \\
\text { patterns with similar dynamics } \\
\text { - Compared to controls, MS } \\
\text { patients showed more frequently } \\
\text { strong connections in parietal } \\
\text { regions (PCC, superior parietal } \\
\text { and angular gyrus) and more } \\
\text { frequently weak connections in } \\
\text { prefrontal regions and in } \\
\text { the amygdala }\end{array}$ \\
\hline Lin et al. (2018) & $\begin{array}{l}\text { Philips Achieva } 3 \mathrm{~T} \\
240 \text { volumes } \\
\mathrm{TR}=2 \mathrm{~s}\end{array}$ & $\begin{array}{l}\text { 1. Segmentation of } 18 \text { cortical } \\
\text { regions from the Freesurfer } \\
\text { Desikan atlas (Desikan et al., } \\
2006 \text { ) } \\
\text { 2. Sliding-window analysis, window } \\
\text { length }=20 \text { TRs ( } 40 \mathrm{~s} \text { ), steps = } \\
1 \mathrm{TR}(2 \mathrm{~s})\end{array}$ & $\begin{array}{l}37 \text { relapsing-remitting } \\
\text { MS patients } \\
28 \text { females }(75.7 \%) \\
\text { mean age }=42.57 \text { years } \\
\text { SD }=11.4 \text { years }\end{array}$ & $\begin{array}{l}\text { - Lower network variations } \\
\text { and higher flexibility of inter- } \\
\text { hemispheric connections in MS } \\
\text { patients compared with controls } \\
\text { - Better executive functions on } \\
\text { cognitive testing were associated } \\
\text { to higher connectivity dynamics }\end{array}$ \\
\hline
\end{tabular}


TABLE 2 | Continued

\begin{tabular}{|c|c|c|c|c|}
\hline Study & $\begin{array}{l}\text { RS fMRI acquisition } \\
\text { parameters }^{\Omega}\end{array}$ & TVC analysis approach $^{\wedge}$ & Study subjects ${ }^{\theta}$ & Main findings \\
\hline & & $\begin{array}{l}\text { 3. Graph theory: network } \\
\text { variations, flexibility of } \\
\text { inter-hemispheric, } \\
\text { cross-hemispheric, and } \\
\text { intra-hemispheric connections }\end{array}$ & $\begin{array}{l}18 \text { relapsing-remitting } \mathrm{MS} \\
\text { patients (matched with healthy } \\
\text { controls) } 15 \text { females } \\
(83.3 \%) \text { mean age }=32 \text { years } \\
\mathrm{SD}=4.9 \text { years } \\
15 \text { healthy controls } \\
8 \text { females }(53.3 \%) \\
\text { mean age }=28.93 \text { years } \\
\mathrm{SD}=5 \text { years }\end{array}$ & \\
\hline Rocca et al. (2019) & $\begin{array}{l}\text { Philips Achieva } 1.5 \mathrm{~T} \\
200 \text { volumes } \\
\text { TR }=3 \mathrm{~s}\end{array}$ & $\begin{array}{l}\text { 1. Group ICA decomposition in } 43 \\
\text { relevant independent } \\
\text { components of interest, } \\
\text { classified into seven different } \\
\text { functional networks } \\
\text { 2. Sliding-window analysis, window } \\
\text { length = } 22 \text { TRs ( } 66 \mathrm{~s} \text { ), step =1 } \\
\text { TR ( } 3 \mathrm{~s} \text { ) } \\
\text { 3. k-means clustering analysis (two } \\
\text { recurring states) } \\
\text { 4. Fuzzy meta-state analysis } \\
\text { 5. Correlations with clinical } \\
\text { variables, cognitive } \\
\text { performance, T2 lesion volume, } \\
\text { and brain volume }\end{array}$ & $\begin{array}{l}50 \text { patients with CIS } \\
\text { suggestive of } \mathrm{MS} \\
30 \text { females }(60 \%) \\
\text { mean age }=30.5 \text { years } \\
\mathrm{SD}=7.7 \text { years } \\
13 \text { healthy controls } \\
9 \text { females }(69.2 \%) \\
\text { mean age }=33.1 \text { years } \\
\mathrm{SD}=7.8 \text { years }\end{array}$ & $\begin{array}{l}\text { - At baseline, compared to healthy } \\
\text { controls, CIS patients showed } \\
\text { TVC abnormalities between } \\
\text { sensorimotor and DMN with the } \\
\text { remaining networks } \\
\text { - According to type of onset, } \\
\text { selective baseline RS FC decrease } \\
\text { was detected in functional } \\
\text { networks more affected by the } \\
\text { clinical attack } \\
\text { - At follow-up, increased } \\
\text { connectivity strength and global } \\
\text { connectivity dynamism was } \\
\text { observed in patients vs. healthy } \\
\text { controls - In CIS patients, higher TVC at } \\
\text { year } 2 \text { correlated with lower white } \\
\text { matter lesion volume changes } \\
\text { at follow-up }\end{array}$ \\
\hline van Geest et al. (2018a) & $\begin{array}{l}\text { GE Signa HDxt } 3 T \\
\text { RS fMRI: } \\
202 \text { volumes } \\
\text { TR }=2.2 \mathrm{~s} \\
\text { Task-related (SDMT): } \\
460 \text { volumes } \\
\text { TR }=2 \mathrm{~s}\end{array}$ & $\begin{array}{l}\text { 1. Segmentation of } 224 \text { regions } \\
\text { from the Brainnettome atlas (Fan } \\
\text { et al., 2016), Yeo atlas (Yeo } \\
\text { et al., 2011) and from FSL FIRST } \\
\text { segmentation } \\
\text { 2. RS fMRI: sliding-window } \\
\text { analysis, window length = } 27 \\
\text { TRs (59.4 s), steps }=5 \text { TRs } \\
\text { (11s) Task-related fMRI: } \\
\text { sliding-window analysis, window } \\
\text { length = } 30 \text { TRs ( } 60 \text { s), steps = } \\
5 \text { TRs (10 s) } \\
\text { 3. Sum of the absolute differences } \\
\text { in RS and task-related FC } \\
\text { between consecutive windows }\end{array}$ & $\begin{array}{l}29 \text { MS patients } \\
18 \text { females }(62 \%) \\
\text { mean age }=41.25 \text { years } \\
S D=9.34 \text { years } \\
18 \text { healthy controls } \\
11 \text { females }(61.1 \%) \\
\text { mean age }=40.68 \text { years } \\
S D=13.29 \text { years }\end{array}$ & $\begin{array}{l}\text { - TVC in the DMN increased during } \\
\text { the task vs. rest in both controls } \\
\text { and MS patients } \\
\text { - A higher increase of TVC in the } \\
\text { DMN during the task vs. rest was } \\
\text { associated with better information } \\
\text { processing speed in MS patients }\end{array}$ \\
\hline van Geest et al. (2018b) & $\begin{array}{l}\text { Siemens Sonata } 1.5 \mathrm{~T} \\
\text { RS fMRI: } \\
200 \text { volumes } \\
\text { TR }=2.85 \mathrm{~s} \\
\text { Task-related fMRI (episodic } \\
\text { memory): } \\
208 \text { volumes } \\
\text { TR }=2.22 \mathrm{~s}\end{array}$ & $\begin{array}{l}\text { 1. Segmentation of } 92 \text { brain } \\
\text { regions from the AAL atlas } \\
\text { (Tzourio-Mazoyer et al., 2002) } \\
\text { 2. Task-related fMRI: } \\
\text { sliding-window analysis, window } \\
\text { length }=27 \text { volumes }(59.9 \mathrm{~s}) \text {, } \\
\text { steps }=5 \text { TRs ( } 11.1 \mathrm{~s} \text { ) } \\
\text { 3. Sum of the absolute differences } \\
\text { in FC between } \\
\text { consecutive windows }\end{array}$ & $\begin{array}{l}38 \text { MS patients } \\
26 \text { females }(68.4 \%) \\
\text { mean age }=47.2 \text { years } \\
\text { SD }=8 \text { years } \\
29 \text { healthy controls } \\
18 \text { females }(62.1 \%) \\
\text { mean age }=43.9 \text { years } \\
S D=8.4 \text { years }\end{array}$ & $\begin{array}{l}\text { - TVC of the left and right } \\
\text { hippocampus, as well as TVC } \\
\text { of the entire brain, did not differ } \\
\text { between healthy controls and MS } \\
\text { patients } \\
\text { - Lower hippocampal TVC was } \\
\text { associated with better verbal } \\
\text { learning and memory, and with } \\
\text { better visuospatial learning and } \\
\text { memory performances }\end{array}$ \\
\hline Zhou et al. (2016) & $\begin{array}{l}\text { Siemens Trio } 3 \mathrm{~T} \\
240 \text { volumes } \\
\mathrm{TR}=2 \mathrm{~s}\end{array}$ & $\begin{array}{l}\text { 1. Voxel-wise analysis (no ROI } \\
\text { selection necessary) } \\
\text { 2. Calculation of brain entropy and } \\
\text { amplitude of low frequency } \\
\text { fluctuations } \\
\text { 3. Voxel-wise comparison of brain } \\
\text { entropy and amplitude of low } \\
\text { frequency fluctuations }\end{array}$ & $\begin{array}{l}34 \text { relapsing-remitting } \\
\text { MS patients } \\
21 \text { females }(61.8 \%) \\
\text { mean age }=42.1 \text { years } \\
\text { age range }=20-58 \text { years } \\
34 \text { healthy controls } \\
21 \text { females }(61.8 \%) \\
\text { mean age }=41.8 \text { years } \\
\text { age range }=21-58 \text { years }\end{array}$ & $\begin{array}{l}\text { - Brain entropy was increased } \\
\text { in MS patients compared to } \\
\text { controls, especially in regions } \\
\text { related to motor, executive, } \\
\text { spatial coordination and memory } \\
\text { functions } \\
\text { - More severe brain entropy was } \\
\text { correlated with a higher } \\
\text { clinical disability }\end{array}$ \\
\hline
\end{tabular}

${ }^{2}$ All RS scans were acquired in the eyes-closed condition.

^TVC analysis approach summarizes: (1) ROls used; (2) assessment of time-varying correlations between brain regions; (3) features extracted for assessing TVC.

${ }^{\theta}$ For each study group of healthy subjects, sex is represented as number of females (\%), mean age and standard deviation (SD).

$R S$, resting state; $\mathrm{FMRI}$, functional magnetic resonance imaging; TVC, time-varying functional connectivity; TR, repetition time; DMN, default-mode network; MS, multiple sclerosis; SD, standard deviation; AAL, automated anatomical labeling; PCC, posterior cingulate cortex; CIS, clinically isolated syndrome. 
in relapsing-remitting MS patients with mild to moderate disability compared to healthy controls (Leonardi et al., 2013). These patients also showed more frequently strong connections in temporal and parietal (angular gyrus) regions as well as weaker connections in motor and amygdalar regions vs. control subjects (Leonardi et al., 2013). Another study assessing TVC abnormalities in relapsing-remitting MS patients with mild disability found an increased BEN (corresponding to an increased connectivity disorganization) of regions involved in motor, executive and spatial coordination, as well as reduced BEN in memory brain areas (including temporal and hippocampal cortices) and relay areas as the cerebellum or the brainstem compared to healthy subjects (Zhou et al., 2016).

A recent study using DCC to quantify TVC (Bosma et al., 2018) confirmed the results obtained by Leonardi et al. (2013), and found an increased BOLD signal variability in posterior regions of the DMN in MS patients vs. controls. The same study also found an increased TVC between the salience network and the ascending nociceptive pathway. Conversely, divergent results were obtained by Lin et al. (2018), who showed an overall reduction of network variation in MS patients compared to healthy controls, suggesting a globally more "static" FC configuration, but at the same time found an increased flexibility of interhemispheric connections, which was interpreted as a compensatory mechanism for the decreased global connectivity. A complex pattern of increased and decreased TVC was also shown by d'Ambrosio et al. (2019), who found, in a multicenter study, a selective TVC increase between subcortical and visual/cognitive networks, and a TVC decrease between subcortical and sensorimotor networks in relapsing-remitting MS patients compared to healthy controls (d'Ambrosio et al., 2019).

Specific investigations of crucial systems involved in cognitive functions were performed by Van Geest et al., who studied TVC of the hippocampal network (Lin et al., 2018; van Geest et al., 2018a,b) and of the DMN (Lin et al., 2018; van Geest et al., 2018a,b) and by Huang et al., who investigated the attention network (Huang et al., 2019). Overall, hippocampal and DMN TVC were not different between MS patients and control subjects; however, including TVC measures in multivariate statistical models contributed to explain the performance of MS patients at visuospatial memory (Lin et al., 2018; van Geest et al., 2018a,b) and information processing speed (Lin et al., 2018; van Geest et al., 2018a,b) tasks. Huang et al. detected a complex pattern of TVC abnormalities, which was characterized by a TVC decrease within the dorsal and ventral attention networks, as well as TVC increase between the same networks (Huang et al., 2019).

Changes in TVC at the earliest stages of the disease have been rarely assessed, but interesting results have been observed. Patients with clinically isolated syndrome (CIS) suggestive of MS exhibited, early after the first demyelinating attack, reduced TVC in the functional networks more affected by the clinical onset, compared to healthy controls (Rocca et al., 2019). These patients also showed, in the first 2 years after the clinical event, a progressive increase over time of TVC strength, mainly between the DMN and sensorimotor/visual/cognitive networks, combined with a progressive increase over time of global fuzzy meta-state dynamism (Rocca et al., 2019).

Overall, these results suggest that, at the beginning of the disease, TVC dysfunctions have a specific correspondence with clinical symptoms. Then, a progressive increase of TVC oscillations occurs, probably trying to compensate disease-related damage. This initial phase seems to be followed by a loss of coordination and flexibility among brain regions in MS patients (Leonardi et al., 2013; Zhou et al., 2016; Lin et al., 2018; d'Ambrosio et al., 2019), which may be compensated by local increased fluctuations between specific areas (Lin et al., 2018; van Geest et al., 2018a,b).

Recent studies tried to investigate TVC changes in MS populations affected by specific clinical manifestations. In details, cognitive impairment in patients with MS was associated to reduced TVC between subcortical and DMN areas, as well as to reduced global dynamism, compared to cognitively preserved patients (d'Ambrosio et al., 2019). Patients with MS suffering from neuropathic pain expressed selectively reduced TVC strength in the salience-descending nociceptive circuit (Bosma et al., 2018), whereas in patients without such neuropathic pain, TVC strength was increased in the same network (Bosma et al., 2018).

The large majority of the above-mentioned studies assessed TVC changes in relapsing-remitting MS patients, while detailed investigations of TVC abnormalities occurring in progressive MS phenotypes or over the course of the disease, are still missing.

\section{Correlations Between TVC and Clinical, Neuropsychological, and Structural MRI Variables in MS}

Different correlation analyses have been performed in MS patients, in order to understand the possible association between TVC abnormalities and motor and cognitive performances, as well as with specific clinical symptoms such as fatigue.

A higher expanded disability status scale (EDSS) score, reflecting more severe clinical disability, was found to be correlated with increased BEN in the bilateral supplementary motor area and in the right precentral operculum (Zhou et al., 2016), as well as with a more rigid (less fluid) global connectivity in MS patients (Lin et al., 2018). Conversely, other studies failed to show significant associations between TVC abnormalities and disability, probably because of the relatively low sample size and/or a narrow EDSS range (Leonardi et al., 2013).

Several correlations have been detected between TVC abnormalities and MS patients' cognitive performances. In particular, better scores in tests involving executive control functions and processing speed ability were correlated with a higher global network dynamism (Lin et al., 2018). Similar findings were shown by van Geest et al. (2018a), who found that a higher dynamism in the DMN during an information processing speed task vs. a resting state condition was associated with better information processing speed performances. These results are in agreement with the reduced network dynamics observed in cognitively impaired vs. preserved MS patients (d'Ambrosio 
et al., 2019). Conversely, a lower hippocampal TVC contributed to explain, at least partially, better verbal learning, visuospatial learning, and memory performances (van Geest et al., 2018b).

Lower fatigue was associated with reduced TVC in the parahippocampal gyrus, right posterior cerebellum, and brainstem (Zhou et al., 2016). Pain interference has been associated with increased TVC in the posterior cingulate cortex, an associative region involved in the salience and nociceptive networks and DMN (Bosma et al., 2018).

A few studies investigated the relationship between TVC and white matter lesions or MS-related structural damage. Decreased TVC between parietal and fronto-temporal regions of the attention network was associated with an higher lesion load in relapsing-remitting MS patients (Huang et al., 2019). A significant association has been demonstrated between reduced global dynamism in cognitively impaired MS patients and brain atrophy (d'Ambrosio et al., 2019), as well as between increased TVC and diffuse microstructural damage in relapsing-remitting MS patients, quantified as a higher mean diffusivity on diffusion-tensor MRI (Zhou et al., 2016). At the earliest stages of MS, a progressive increase of TVC over 2 years of follow-up was associated with a lower white matter lesion volume change over the same period of time (Rocca et al., 2019).

In summary, in MS patients, abnormal global TVC properties of the sensorimotor, DMN and salience networks were associated with more severe tissue damage at structural MRI, more severe clinical disability, worse cognitive performance and pain interference, evidencing a maladaptive neuronal response to direct disease-related damage. Conversely, abnormal TVC properties of the temporal network and relay areas as the cerebellum and brainstem were correlated with better cognitive performances and less severe fatigue, suggesting a compensatory role of TVC changes.

\section{APPLICATION OF TIME-VARYING FC TECHNIQUES TO PSYCHIATRIC AND OTHER NEUROLOGICAL DISEASES}

\section{Main Findings in Psychiatric and Other Neurological Diseases}

The main studies discussed in this section are summarized in Table 3.

Several studies tried to characterize TVC abnormalities present in different psychiatric and neurological diseases, sometimes looking for an early diagnostic biomarker (Du et al., 2018; Mennigen et al., 2018). Modification of TVC strength, dwell time or number of transitions between states varied according to the disease status in patients affected by bipolar disorder (Rashid et al., 2014, 2016), schizophrenia (Yu et al., 2015; Cetin et al., 2016; Rashid et al., 2016; Gazula et al., 2018; Yue et al., 2018; Zhang W. et al., 2018), depression (Liao et al., 2018; Qiu et al., 2018; Zhi et al., 2018), autism (He et al., 2018; Rashid et al., 2018a), stroke (Chen et al., 2018), mild traumatic brain injury (Vergara et al., 2018), epilepsy (Ridley et al., 2017; Klugah-Brown et al., 2018), Alzheimer's disease (Quevenco et al., 2017; Jie et al., 2018), and Parkinson's disease (Engels et al., 2018).

In psychiatric diseases, widespread TVC abnormalities have been found. Patients with bipolar disorder and major depression expressed TVC abnormalities mainly in executive (Rashid et al., 2014; Du et al., 2017), amygdala/salience (Qiu et al., 2018; Zhi et al., 2018), and salience/executive regions (Mokhtari et al., 2018a,b). Schizophrenia patients showed a complex pattern of decreased and increased TVC mainly in the DMN (Sakoglu et al., 2010; Abrol et al., 2017), and in frontal, parietal, auditory (Damaraju et al., 2014; Rashid et al., 2014; Du et al., 2017, 2018; Sun et al., 2018), visual (Fu et al., 2018; Rashid et al., 2018b; Sun et al., 2018), and thalamic areas (Damaraju et al., 2014; Rashid et al., 2014, 2018b; Du et al., 2018). Schizophrenia patients spent less time and made fewer transitions between states characterized by weak correlations between the thalami and sense-related brain regions (Damaraju et al., 2014). They also showed more lagged correlations between the DMN and sensory networks (Yaesoubi et al., 2017a) and a higher occupancy rate of globally disconnected states (Yu et al., 2015; Cetin et al., 2016; Rashid et al., 2016; Gazula et al., 2018; Yue et al., 2018; Zhang W. et al., 2018). In children with autism spectrum disorders, TVC was mainly decreased in DMN and insular areas (Falahpour et al., 2016; Guo et al., 2018; He et al., 2018; Rashid et al., 2018a).

In neurological disorders, TVC abnormalities have been mainly observed in areas directly affected by the disease. For example, subcortical stroke and mild traumatic brain injury patients showed TVC abnormalities in sensorimotor networks (Chen et al., 2018; Vergara et al., 2018). Patients with myoclonic/frontal lobe epilepsy showed reduced TVC mainly in frontal and parietal brain regions, whereas patients with temporal lobe epilepsy experienced TVC decrease mainly in temporal regions (Ridley et al., 2017; Klugah-Brown et al., 2018; Wang et al., 2018). Generalized epilepsy was related to TVC strength changes mainly in the DMN and cognitive networks (Liu et al., 2017; Li et al., 2018). Patients suffering from Alzheimer's disease had reduced regional (nodal) TVC (Alderson et al., 2018) and alterations in inter-network TVC of the anterior and posterior regions of the DMN (Jones et al., 2012; Quevenco et al., 2017), the frontal cortex and temporal areas (Jie et al., 2018). Patients with Parkinson's disease showed TVC changes mainly in sensorimotor, executive, cognitive (Liu et al., 2018), visual, and DMN areas (Diez-Cirarda et al., 2018), combined with reduced global and nodal TVC (Cai J. et al., 2018; Diez-Cirarda et al., 2018).

\section{Correlations Between TVC and Clinical, Neuropsychological, and Structural MRI Variables in Psychiatric and Other Neurological Diseases}

In schizophrenia patients, reduced global time-resolved graph metrics have been related to structural disease-related damage (Yu et al., 2015), while abnormalities in TVC of auditory brain regions have been correlated with the presence of auditory hallucinations (Sun et al., 2018). Hallucinations were also 
TABLE 3 | Summary of studies assessing time-varying resting state functional connectivity modifications in different psychiatric and neurological pathologies (excluding multiple sclerosis).

\begin{tabular}{|c|c|c|c|c|}
\hline Study & $\begin{array}{l}\text { RS fMRI acquisition } \\
\text { parameters }^{\Omega}\end{array}$ & TVC analysis approach ${ }^{\wedge}$ & Study subjects ${ }^{\theta}$ & Main findings \\
\hline Abrol et al. (2017) & $\begin{array}{l}\text { Six sites: Siemens Tim Trio 3T } \\
\text { One site: GE Discovery MR750 } \\
\text { 3T } \\
162 \text { volumes } \\
\text { TR }=2 \mathrm{~s}\end{array}$ & $\begin{array}{l}\text { 1. Group ICA decomposition in } 47 \\
\text { relevant independent } \\
\text { components of interest, } \\
\text { classified into } 7 \text { functional } \\
\text { networks } \\
\text { 2. Sliding-window analysis, window } \\
\text { length = } 22 \text { TRs ( } 44 \mathrm{~s} \text { ), steps = } \\
1 \text { TR ( } 2 \mathrm{~s} \text { ) } \\
\text { 4. Clustering (five recurring states) } \\
\text { performed using temporal ICA } \\
\text { 3. Correlations with gray } \\
\text { matter volumes }\end{array}$ & $\begin{array}{l}\text { FBIRN Data Repository } 151 \\
\text { schizophrenia patients } \\
37 \text { females }(24.5 \%) \\
\text { mean age }=37.8 \text { years } \\
163 \text { healthy subjects } \\
46 \text { females }(28.2 \%) \\
\text { mean age }=36.9 \text { years }\end{array}$ & $\begin{array}{l}\text { - Compared to healthy subjects, } \\
\text { patients with schizophrenia } \\
\text { exhibited higher TVC strength } \\
\text { between: i) sensorimotor, } \\
\text { precuneus and parietal areas; } \\
\text { and ii) frontal, temporal and insular } \\
\text { cortices } \\
\text { - In patients, TVC abnormalities } \\
\text { correlated with lower gray } \\
\text { matter volumes }\end{array}$ \\
\hline Alderson et al. (2018) & $\begin{array}{l}\text { Philips Intera MR } 3 \mathrm{~T} \\
140 \text { volumes } \\
\mathrm{TR}=3 \mathrm{~s}\end{array}$ & $\begin{array}{l}\text { 1. Group ICA decomposition in } \\
\text { nine relevant independent } \\
\text { components of interest, } \\
\text { subsequently segmented in } 148 \\
\text { cortical regions from the } \\
\text { Destrieux atlas (Destrieux et al., } \\
\text { 2010) } \\
\text { 2. Time-frequency analysis } \\
\text { 3. Graph theory: synchrony, global } \\
\text { metastability, eigenvector } \\
\text { centrality, clustering coefficient, } \\
\text { local efficiency, and participation } \\
\text { coefficient } \\
\text { 4. Correlation with } \\
\text { structural abnormalities }\end{array}$ & $\begin{array}{l}\text { ADNI database } 34 \text { patients } \\
\text { with Alzheimer's disease } \\
18 \text { females }(52.9 \%) \\
\text { mean age }=73.79 \text { years } \\
\text { SD }=6.14 \text { years } \\
33 \text { patients with mild } \\
\text { cognitive impairment } \\
13 \text { females (39.4\%) } \\
\text { mean age }=73.61 \text { years } \\
\text { SD }=5.6 \text { years } \\
36 \text { healthy controls } \\
19 \text { females (52.8\%) } \\
\text { mean age }=74.46 \text { years } \\
\text { SD }=5.51 \text { years }\end{array}$ & $\begin{array}{l}\text { - In Alzheimer's disease patients, } \\
\text { reduced synchrony was observed } \\
\text { between right fronto-parietal } \\
\text { regions, sensorimotor regions } \\
\text { and DMN, together with overall } \\
\text { reduced metastability } \\
\text { - In patients, increased eigenvector } \\
\text { centrality, clustering coefficient, } \\
\text { local efficiency, and participation } \\
\text { coefficient correlated with more } \\
\text { severe structural damage }\end{array}$ \\
\hline Cai J. et al. (2018) & $\begin{array}{l}\text { Siemens Trio 3T } \\
\text { Unspecified volumes } \\
\text { TR }=2 \mathrm{~s}\end{array}$ & $\begin{array}{l}\text { 1. Segmentation of } 76 \text { brain } \\
\text { regions from the Desikan atlas } \\
\text { (Desikan et al., 2006) } \\
\text { 2. Sliding-window analysis, window } \\
\text { length = } 30 \text { TRs ( } 60 \text { s), steps = } \\
2 \text { TRs (4 s) } \\
\text { 3. Graph theoretical analysis: } \\
\text { global efficiency, clustering } \\
\text { efficiency, modularity, } \\
\text { assortativity, Fiedler value }\end{array}$ & $\begin{array}{l}69 \text { Parkinson's } \\
\text { disease patients } \\
30 \text { females }(43.5 \%) \\
\text { mean age }=60 \text { years } \\
\text { SD }=9.8 \text { years } \\
29 \text { healthy controls } \\
13 \text { females }(43.5 \%) \\
\text { mean age }=58.3 \text { years } \\
\text { SD }=97.5 \text { years }\end{array}$ & $\begin{array}{l}\text { - Compared to healthy subjects, } \\
\text { patients with Parkinson's } \\
\text { disease showed lower network } \\
\text { connections (Fiedler value), } \\
\text { modularity and global efficiency } \\
\text { - Lower network connections in } \\
\text { patients with Parkinson's disease } \\
\text { correlated with disease severity }\end{array}$ \\
\hline Cetin et al. (2016) & $\begin{array}{l}\text { Siemens Trio } 3 \mathrm{~T} \\
149 \text { volumes } \\
\mathrm{TR}=2 \mathrm{~s}\end{array}$ & $\begin{array}{l}\text { 1. Group ICA decomposition in } 39 \\
\text { relevant independent } \\
\text { components of interest } \\
\text { 2. Sliding-window analysis, window } \\
\text { length }=31 \text { TRs }(62 \mathrm{~s}) \text {, steps = } \\
1 \text { TRs }(2 \mathrm{~s}) \\
\text { k-means clustering (five } \\
\text { recurring states) } \\
\text { 3. Correlations with } \\
\text { magnetoencephalography data } \\
\text { and classification performance } \\
\text { compared to static FC } \\
\text { and magnetoencephalography }\end{array}$ & $\begin{array}{l}47 \text { schizophrenia patients } \\
13 \text { females }(27.7 \%) \\
\text { mean age }=35.18 \text { years } \\
\text { SD }=11.83 \text { years } \\
45 \text { healthy controls } \\
7 \text { females }(15.6 \%) \\
\text { mean age }=37.28 \text { years } \\
\text { SD }=13.86 \text { years }\end{array}$ & $\begin{array}{l}\text { - Classification between } \\
\text { schizophrenia patients and } \\
\text { healthy controls improved with } \\
\text { TVC (accuracy = 82.79\%) } \\
\text { compared to static FC metrics } \\
\text { (accuracy = 70.33\%) } \\
\text { - Classification performance did } \\
\text { not improve when using a } \\
\text { combination of TVC and } \\
\text { magnetoencephalography } \\
\text { metrics (accuracy = 85.35\%), } \\
\text { compared to the combination of } \\
\text { static FC and } \\
\text { magnetoencephalography } \\
\text { metrics (accuracy = 87.91\%) }\end{array}$ \\
\hline Chen et al. (2018) & $\begin{array}{l}\text { Philips Achieva } 3 \mathrm{~T} \\
170 \text { volumes } \\
\mathrm{TR}=2 \mathrm{~s}\end{array}$ & $\begin{array}{l}\text { 1. Segmentation of left and right } \\
\text { primary motor area, premotor } \\
\text { cortex and supplementary motor } \\
\text { area (spherical ROls, radius = } \\
5 \mathrm{~mm} \text { ) } \\
\text { Sliding-window analysis, window } \\
\text { length = 32TRs ( } 64 \mathrm{~s} \text { ), steps = } 1 \\
\text { TR ( } 2 \mathrm{~s} \text { ) } \\
\text { 2. Standard deviation of TVC } \\
\text { across windows }\end{array}$ & $\begin{array}{l}70 \text { stroke patients } \\
45 \text { right-sided lesions } \\
23 \text { females }(32.9 \%) \\
\text { mean age }=58.44 \text { years } \\
\mathrm{SD}=11.43 \text { years } \\
25 \text { left-sided lesions } \\
8 \text { females }(11.4 \%) \\
\text { mean age }=59.88 \text { years } \\
\mathrm{SD}=12.96 \text { years } \\
55 \text { healthy controls }\end{array}$ & $\begin{array}{l}\text { - Compared to healthy controls, } \\
\text { stroke patients showed TVC } \\
\text { reductions between sensorimotor } \\
\text { and visual-related cortices and } \\
\text { between the sensorimotor and } \\
\text { the limbic system } \\
\text { - In stroke patients with right-sided } \\
\text { lesions, reduced TVC between } \\
\text { the right primary motor area and } \\
\text { the left precentral gyrus correlated } \\
\text { with more severe disability }\end{array}$ \\
\hline
\end{tabular}


TABLE 3 | Continued

\begin{tabular}{|c|c|c|c|c|}
\hline Study & $\begin{array}{l}\text { RS fMRI acquisition } \\
\text { parameters }^{\Omega}\end{array}$ & TVC analysis approach^^ & Study subjects ${ }^{\theta}$ & Main findings \\
\hline & & & $\begin{array}{l}26 \text { females }(37.1 \%) \\
\text { mean age }=56.73 \text { years } \\
S D=10.21 \text { years }\end{array}$ & \\
\hline Damaraju et al. (2014) & $\begin{array}{l}6 \text { sites: Siemens Tim Trio 3T } \\
1 \text { site: GE Discovery MR750 3T } \\
162 \text { volumes } \\
\text { TR }=2 \mathrm{~s}\end{array}$ & $\begin{array}{l}\text { 1. Group ICA decomposition in } 50 \\
\text { relevant independent } \\
\text { components of interest, } \\
\text { classified into } 7 \text { different } \\
\text { functional networks } \\
\text { 2. Sliding-window analysis, window } \\
\text { length = } 22 \text { TRs ( } 44 \mathrm{~s}), \text { steps = } \\
1 \text { TR ( } 2 \mathrm{~s} \text { ) } \\
\text { 3. k-means clustering (five } \\
\text { recurring states) }\end{array}$ & $\begin{array}{l}151 \text { schizophrenia patients } \\
37 \text { females }(24.5 \%) \\
\text { mean age }=37.8 \text { years } \\
163 \text { healthy subjects } \\
46 \text { females }(28.2 \%) \\
\text { mean age }=36.9 \text { years }\end{array}$ & $\begin{array}{l}\text { - Compared to healthy controls, } \\
\text { schizophrenia patients showed: (i) } \\
\text { higher dwell time in states } \\
\text { characterized by overall low inter- } \\
\text { and intra-network TVC strength; } \\
\text { (ii) lower dwell time in states } \\
\text { characterized by high correlations } \\
\text { between visual, motor and } \\
\text { auditory networks; and (iii) } \\
\text { increased TVC between thalami } \\
\text { and sensory networks }\end{array}$ \\
\hline Diez-Cirarda et al. (2018) & $\begin{array}{l}\text { Philips Achieva TX } 3 \mathrm{~T} \\
214 \text { volumes } \\
\mathrm{TR}=2.1 \mathrm{~s}\end{array}$ & $\begin{array}{l}\text { 1. Group ICA decomposition in } 29 \\
\text { relevant independent } \\
\text { components of interest, } \\
\text { classified into seven functional } \\
\text { networks } \\
\text { 2. Sliding-window analysis, window } \\
\text { length = } 22 \text { TRs ( } 44.2 \mathrm{~s} \text { ), steps } \\
=1 \text { TR ( } 2.1 \mathrm{~s} \text { ) } \\
\text { 3. k-means clustering (two } \\
\text { recurring states). } \\
\text { Graph theory: global efficiency, } \\
\text { local efficiency, clustering } \\
\text { coefficient, } \\
\text { betweenness centrality }\end{array}$ & $\begin{array}{l}37 \text { patients with } \\
\text { Parkinson's disease } \\
12 \text { with normal cognition } \\
6 \text { females }(50 \%) \\
\text { mean age }=65.17 \text { years } \\
\text { SD }=8.31 \text { years } \\
23 \text { with mild cognitive } \\
\text { impairment } \\
10 \text { females ( } 44 \% \text { ) } \\
\text { mean age }=69.17 \text { years } \\
\text { SD }=4.48 \text { years } \\
26 \text { healthy controls } \\
8 \text { females ( } 31 \% \text { ) } \\
\text { mean age }=68.31 \text { years } \\
\text { SD }=7.52 \text { years }\end{array}$ & $\begin{array}{l}\text { - Compared to healthy controls, } \\
\text { Parkinson's disease patients } \\
\text { with mild cognitive impairment } \\
\text { showed lower dwell time in a } \\
\text { state characterized by overall } \\
\text { low strength of inter- and intra- } \\
\text { network connections, as well } \\
\text { as higher number of transitions } \\
\text { between states } \\
\text { - Parkinson's disease patients with } \\
\text { cognitive impairment also } \\
\text { showed: (i) reduced clustering } \\
\text { coefficient in the right precentral } \\
\text { gyrus vs. healthy controls; and (ii) } \\
\text { reduced betweenness centrality } \\
\text { of the left paracentral gyrus vs. } \\
\text { patients without } \\
\text { cognitive impairment }\end{array}$ \\
\hline Du et al. (2017) & $\begin{array}{l}3 \text { sites: Siemens Trio Tim 3T } \\
2 \text { sites: GE Signa HDx 3T } \\
1 \text { site: Siemens Allegra 3T } \\
1 \text { site: Philips 3T } \\
\text { 100-210 volumes } \\
\text { TR ranging from } 1.5 \text { to } 3 \mathrm{~s}\end{array}$ & $\begin{array}{l}\text { 1. Segmentation of } 116 \text { brain } \\
\text { regions from the AAL atlas } \\
\text { (Tzourio-Mazoyer et al., 2002) } \\
\text { 2. Sliding-window analysis, window } \\
\text { length = } 20 \text { TRs (ranging from } \\
30 \text { to } 60 \text { s) } \\
\text { 3. GIG-ICA clustering (five } \\
\text { recurring-states) } \\
\text { 4. Correlations with } \\
\text { cognitive scores }\end{array}$ & $\begin{array}{l}\text { Bipolar and schizophrenia } \\
\text { network on } \\
\text { intermediate phenotypes } \\
113 \text { schizophrenia patients } \\
57 \text { females }(50 \%) \\
\text { mean age }=35.57 \text { years } \\
S D=12.29 \text { years } \\
132 \text { schizoaffective } \\
\text { disorder patients } \\
75 \text { females ( } 57 \%) \\
\text { mean age }=36.23 \text { years } \\
\text { SD }=12.23 \text { years } \\
140 \text { bipolar disorder with } \\
\text { psychosis patients } \\
87 \text { females ( } 62 \%) \\
\text { mean age }=36 \text { years } \\
\text { SD }=12.57 \text { years } \\
238 \text { healthy controls } \\
138 \text { females (58\%) } \\
\text { mean age = } 38.15 \text { years } \\
\text { SD = } 12.55 \text { years }\end{array}$ & $\begin{array}{l}\text { - Compared to healthy controls (and } \\
\text { bipolar patients), schizophrenia } \\
\text { and schizoaffective disorder } \\
\text { patients showed increased TVC } \\
\text { between frontal with angular and } \\
\text { postcentral areas, and reduced } \\
\text { TVC between temporal and frontal } \\
\text { areas } \\
\text { - Compared with all remaining } \\
\text { study groups, schizophrenia } \\
\text { patients also showed reduced } \\
\text { TVC between the cerebellum and } \\
\text { subcortical and frontal areas } \\
\text { - Reduced TVC between cerebellar } \\
\text { and frontal areas correlated with } \\
\text { higher symptom severity scores }\end{array}$ \\
\hline Du et al. (2018) & $\begin{array}{l}\text { Siemens Tim Trio 3T } \\
180 \text { volumes } \\
\text { TR }=2 \mathrm{~s}\end{array}$ & $\begin{array}{l}\text { 1. Segmentation of } 116 \text { brain } \\
\text { regions from the AAL atlas } \\
\text { (Tzourio-Mazoyer et al., 2002) } \\
\text { 2. Sliding-window analysis, window } \\
\text { length }=20 \text { TRs ( } 40 \mathrm{~s} \text { ), steps = } \\
1 \text { TR }(2 \mathrm{~s} \text { ) } \\
\text { 3. GIG-ICA clustering } \\
\text { (five recurring-states) }\end{array}$ & $\begin{array}{l}58 \text { schizophrenia patients } \\
20 \text { females }(35 \%) \\
\text { mean age }=21.8 \text { years } \\
S D=3.8 \text { years } \\
53 \text { adults at high risk of } \\
\text { developing schizophrenia } \\
21 \text { females }(38 \%) \\
\text { mean age }=20.4 \text { years }\end{array}$ & $\begin{array}{l}\text { - Compared to healthy controls, } \\
\text { schizophrenia patients and adults } \\
\text { with high risk of developing } \\
\text { schizophrenia showed TVC } \\
\text { alterations between motor, } \\
\text { temporal, cerebellar, frontal and } \\
\text { thalamic areas } \\
\text { - Schizophrenia patients, } \\
\text { compared }\end{array}$ \\
\hline
\end{tabular}


TABLE 3 | Continued

\begin{tabular}{|c|c|c|c|c|}
\hline Study & $\begin{array}{l}\text { RS fMRI acquisition } \\
\text { parameters } \Omega\end{array}$ & TVC analysis approach $^{\wedge}$ & Study subjects ${ }^{\theta}$ & Main findings \\
\hline & & & $\begin{array}{l}\mathrm{SD}=4.5 \text { years } \\
70 \text { healthy controls } \\
29 \text { females }(41 \%) \\
\text { mean age }=21.9 \text { years } \\
\mathrm{SD}=5.6 \text { years }\end{array}$ & $\begin{array}{l}\text { to adults with high risk of } \\
\text { developing schizophrenia, also } \\
\text { showed increased TVC between } \\
\text { the cerebellum, temporal cortex, } \\
\text { frontal gyri and thalami } \\
\text { - Increased TVC between temporal } \\
\text { and cerebellar areas correlated } \\
\text { with higher symptom } \\
\text { severity scores }\end{array}$ \\
\hline Engels et al. (2018) & $\begin{array}{l}\text { GE Signa HDxT 3T } \\
202 \text { volumes } \\
\text { TR }=2.15 \mathrm{~s}\end{array}$ & $\begin{array}{l}\text { 1. Segmentation of } 264 \text { brain } \\
\text { regions from the Power atlas } \\
\text { (Power et al., } 2011) \\
\text { 2. Sliding-window analysis, window } \\
\text { length }=28 \text { TRs }(60.2 \mathrm{~s}) \text {, steps } \\
=5 \text { TRs }(10.75 \mathrm{~s}) \\
\text { 3. Standard deviation of TVC } \\
\text { across windows }\end{array}$ & $\begin{array}{l}24 \text { Parkinson's } \\
\text { disease patients } \\
7 \text { females }(29.2 \%) \\
\text { mean age }=63.42 \text { years } \\
\mathrm{SD}=7.93 \text { years } \\
27 \text { healthy controls } \\
11 \text { females }(40.1 \%) \\
\text { mean age }=59.37 \text { years } \\
\mathrm{SD}=8.54 \text { years }\end{array}$ & $\begin{array}{l}\text { - Compared with patients without } \\
\text { cognitive impairment, Parkinson's } \\
\text { disease patients with mild } \\
\text { cognitive impairment showed } \\
\text { higher TVC between the DMN } \\
\text { and the rest of the brain } \\
\text { - In patients, no correlation was } \\
\text { found between TVC abnormalities } \\
\text { and motor severity }\end{array}$ \\
\hline Falahpour et al. (2016) & $\begin{array}{l}17 \text { sites } \\
T R=2 s\end{array}$ & $\begin{array}{l}\text { 1. Manual segmentation of } 10 \\
\text { spherical ROls (radius }=6 \text { and } \\
10 \mathrm{~mm} \text { ) } \\
\text { 2. Sliding-window analysis, window } \\
\text { length }=15 \mathrm{TRs}(30 \mathrm{~s}) \text {, steps }= \\
4 \text { TRs }(8 \mathrm{~s}) \\
\text { 3. Standard deviation of TVC } \\
\text { across windows }\end{array}$ & $\begin{array}{l}\text { Autism Brain Imaging Data } \\
\text { Exchange (ABIDE) } \\
76 \text { autism spectrum disorders } \\
9 \text { females }(11.8 \%) \\
\text { mean age }=16.1 \text { years } \\
\mathrm{SD}=4.9 \text { years } \\
\text { range }=7-29.9 \text { years } \\
76 \text { typically development } \\
\text { young adults } \\
12 \text { females }(15.8 \%) \text { mean age } \\
=15.8 \text { years } \\
\mathrm{SD}=4.5 \text { years } \\
\text { range }=8-29.9 \text { years }\end{array}$ & $\begin{array}{l}\text { - No between-group differences } \\
\text { were observed in TVC }\end{array}$ \\
\hline Fu et al. (2018) & $\begin{array}{l}6 \text { sites: Siemens Tim Trio 3T } \\
1 \text { site: GE Discovery MR750 3T } \\
162 \text { volumes } \\
\text { TR }=2 \mathrm{~s}\end{array}$ & $\begin{array}{l}\text { 1. Group ICA decomposition in } 48 \\
\text { relevant independent } \\
\text { components of interest, } \\
\text { classified into seven functional } \\
\text { networks } \\
\text { 2. Sliding-window analysis, window } \\
\text { length = } 20 \text { TRs ( } 40 \text { s), steps = } \\
1 \text { TR ( } 2 \text { s) } \\
\text { 3. k-means clustering of dynamic } \\
\text { amplitude of low-frequency } \\
\text { fluctuations (six recurring states) }\end{array}$ & $\begin{array}{l}\text { FBIRN Data Repository } \\
151 \text { schizophrenia patients } \\
37 \text { females }(24.5 \%) \\
\text { mean age }=37.8 \text { years } \\
\text { SD }=11.4 \text { years } 163 \\
\text { healthy controls } \\
46 \text { females }(28.2 \%) \\
\text { mean age }=36.9 \text { years } \\
\text { SD }=11 \text { years }\end{array}$ & $\begin{array}{l}\text { - Compared to healthy controls, } \\
\text { schizophrenia patients showed } \\
\text { increased dynamic amplitude } \\
\text { of low-frequency fluctuations in } \\
\text { states characterized by strong } \\
\text { TVC between the thalami and } \\
\text { sensory regions } \\
\text { - Patients also showed reduced } \\
\text { dynamic amplitude of } \\
\text { low-frequency fluctuations in } \\
\text { states characterized by weak } \\
\text { TVC between the thalami and } \\
\text { sensory regions }\end{array}$ \\
\hline Guo et al. (2018) & 14 sites & $\begin{array}{l}\text { 1. Manual segmentation of three } \\
\text { spherical ROls (radius }=6 \mathrm{~mm} \text { ) } \\
\text { 2. Flexible least squares to } \\
\text { construct a TVC map at each } \\
\text { timepoint } \\
\text { 3. k-means clustering (five } \\
\text { recurring states) } \\
\text { Correlations with clinical scores }\end{array}$ & $\begin{array}{l}\text { Autism Brain Imaging Data } \\
\text { Exchange (ABIDE) } \\
209 \text { autism spectrum } \\
\text { disorder adolescents } \\
0 \text { females }(0 \%) \\
\text { mean age }=16.5 \text { years } \\
\mathrm{SD}=6.2 \text { years } \\
298 \text { typical } \\
\text { development adolescents } \\
0 \text { females }(0 \%) \\
\text { mean age }=16.8 \text { years } \\
\mathrm{SD}=6.2 \text { years }\end{array}$ & $\begin{array}{l}\text { - Compared to typically developing } \\
\text { adolescents, autism spectrum } \\
\text { disorder adolescents showed } \\
\text { reduced TVC among the right } \\
\text { anterior insula, ventromedial } \\
\text { prefrontal cortex and the posterior } \\
\text { central cortex } \\
\text { - Reduced TVC between the right } \\
\text { anterior insula and the } \\
\text { ventromedial prefrontal cortex } \\
\text { correlated with higher } \\
\text { symptom severity }\end{array}$ \\
\hline He et al. (2018) & $\begin{array}{l}\text { Philips Achieva 3T } \\
\mathrm{TR}=2 \mathrm{~s}\end{array}$ & $\begin{array}{l}\text { 1. Group ICA decomposition of the } \\
\text { DMN, used to select the PCC for } \\
\text { subsequent analyses } \\
\text { 2. Sliding-window analysis, window } \\
\text { length }=50 \text { TRs (100 s), steps = } \\
2 \text { TRs (4 s) }\end{array}$ & $\begin{array}{l}\text { Autism Brain Imaging Data } \\
\text { Exchange (ABIDE) } \\
38 \text { autism spectrum disorders } \\
0 \text { females }(0 \%) \\
\text { age range }=3-7 \text { years } \\
41 \text { typical } \\
\text { development children }\end{array}$ & $\begin{array}{l}\text { - Compared to typically developing } \\
\text { children, Autism spectrum } \\
\text { disorders children showed } \\
\text { differences in TVC variance } \\
\text { between the PCC and: (1) the } \\
\text { whole brain; (2) the right } \\
\text { precentral gyrus; and (3) visual }\end{array}$ \\
\hline
\end{tabular}


TABLE 3 | Continued

\begin{tabular}{|c|c|c|c|c|}
\hline Study & $\begin{array}{l}\text { RS fMRI acquisition } \\
\text { parameters }^{\Omega}\end{array}$ & TVC analysis approach ${ }^{\wedge}$ & Study subjects ${ }^{\theta}$ & Main findings \\
\hline & & $\begin{array}{l}\text { 3. Calculation of TVC map between } \\
\text { the PCC and the rest of the brain } \\
\text { in each window; calculation of } \\
\text { variance of FC across windows } \\
\text { k-means clustering analysis (six } \\
\text { recurring states) } \\
\text { Correlation with social } \\
\text { behavior scales }\end{array}$ & $\begin{array}{l}0 \text { females }(0 \%) \\
\text { age range }=3-6 \text { years }\end{array}$ & $\begin{array}{l}\text { areas } \\
\text { - In autism spectrum disorder } \\
\text { children, lower TVC variance } \\
\text { between the PCC and the right } \\
\text { precentral gyrus negatively } \\
\text { correlated with social motivation }\end{array}$ \\
\hline Jie et al. (2018) & $\begin{array}{l}\text { Philips } 3 T \text { scanners } \\
140 \text { volumes } \\
\text { TR ranging from } 2.2 \text { to } 3.1 \mathrm{~s}\end{array}$ & $\begin{array}{l}\text { 1. Segmentation of } 116 \text { brain } \\
\text { regions from the AAL atlas } \\
\text { (Tzourio-Mazoyer et al., 2002) } \\
\text { 2. Sliding-window analysis, } \\
\text { non-overlapping windows, } \\
\text { window length ranging from } 30 \\
\text { to } 60 \mathrm{~s} \\
\text { 3. Metrics of temporal and spatial } \\
\text { variability of TVC across } \\
\text { windows } \\
\text { 4. Six machine-learning } \\
\text { classification algorithms }\end{array}$ & $\begin{array}{l}\text { ADNI database } \\
43 \text { patients with mild cognitive } \\
\text { impairment with late onset } \\
17 \text { females }(39.5 \%) \\
\text { mean age }=72.1 \text { years } \\
\text { SD }=8.2 \text { years } \\
56 \text { patients with mild cognitive } \\
\text { impairment with early onset } \\
35 \text { females }(62.5 \%) \\
\text { mean age }=71.1 \text { years } \\
\text { SD }=6.8 \text { years } \\
50 \text { healthy controls } \\
29 \text { females ( } 58 \%) \\
\text { mean age }=75 \text { years } \\
\mathrm{SD}=6.9 \text { years }\end{array}$ & $\begin{array}{l}\text { - Patients with early mild cognitive } \\
\text { impairment, compared to healthy } \\
\text { controls, showed increased TVC } \\
\text { variability } \\
\text { - TVC abnormalities helped to } \\
\text { identify patients with early-onset } \\
\text { mild cognitive impairment from } \\
\text { patients with late-onset mild } \\
\text { cognitive impairment and healthy } \\
\text { controls (accuracy }=74.7 \text { and } \\
\text { 73.6\%, respectively) }\end{array}$ \\
\hline Jones et al. (2012) & $\begin{array}{l}\text { GE Signa HDx } 3 T \\
100 \text { volumes } \\
\text { TR }=3 \mathrm{~s}\end{array}$ & $\begin{array}{l}\text { 1. Group ICA decomposition in } 54 \\
\text { relevant independent } \\
\text { components of interest, used to } \\
\text { develop } 68 \text { cubical ROls (edge } \\
=10 \mathrm{~mm} \text { ) } \\
\text { 2. Sliding-window analysis, window } \\
\text { length }=9 \text { TRs ( } 27 \mathrm{~s} \text { ) and } 11 \\
\text { TRs ( } 33 \mathrm{~s} \text { ) } \\
\text { 3. Graph theory: variability of } \\
\text { modularity across windows }\end{array}$ & $\begin{array}{l}28 \text { patients with } \\
\text { Alzheimer's disease } \\
\text { Unspecified sex and age } \\
892 \text { healthy controls } \\
438 \text { females }(49 \%) \\
\text { median years }=79 \text { years } \\
\text { range }=75-83 \text { years }\end{array}$ & $\begin{array}{l}\text { - Patients with Alzheimer's disease } \\
\text { showed lower dwell time in brain } \\
\text { states with strong contributions of } \\
\text { the posterior areas of the DMN, } \\
\text { and higher dwell time in states } \\
\text { with strong contributions of the } \\
\text { anterior areas of the DMN }\end{array}$ \\
\hline $\begin{array}{l}\text { Klugah-Brown et al. } \\
\text { (2018) }\end{array}$ & $\begin{array}{l}\text { GE Discovery MR750 3T } \\
250 \text { volumes } \\
\text { TR }=2 \mathrm{~s}\end{array}$ & $\begin{array}{l}\text { 1. Group ICA decomposition in } 50 \\
\text { relevant independent } \\
\text { components of interest, } \\
\text { classified into seven functional } \\
\text { networks } \\
\text { 2. Sliding-window analysis, window } \\
\text { length = } 22 \text { TRs ( } 44 \mathrm{~s} \text { ), steps = } \\
1 \mathrm{TR} \text { ( } 2 \mathrm{~s} \text { ) } \\
\text { 3. k-means clustering (four } \\
\text { recurring states) } \\
\text { Individual reconstruction of TVC } \\
\text { states using dual regression }\end{array}$ & $\begin{array}{l}19 \text { frontal lobe } \\
\text { epilepsy patients } \\
9 \text { females }(47.4 \%) \\
\text { median age }=24.2 \text { years } \\
\text { range }=13-51 \text { years } \\
18 \text { healthy controls } \\
5 \text { females }(27.8 \%) \\
\text { median age }=23.9 \text { years } \\
\text { range }=11-41 \text { years }\end{array}$ & $\begin{array}{l}\text { - Compared to healthy subjects, } \\
\text { epilepsy patients showed } \\
\text { reduced TVC between the } \\
\text { fronto-parietal network and } \\
\text { cerebellar/subcortical networks } \\
\text { - They also spent less time in } \\
\text { the most fundamental connectivity } \\
\text { state } \\
\text { - A lower dwell time in this state } \\
\text { correlated with age of } \\
\text { seizure onset }\end{array}$ \\
\hline Li et al. (2018) & $\begin{array}{l}\text { GE Discovery } 7503 T \\
240 \text { volumes } \\
\text { TR }=2 \mathrm{~s}\end{array}$ & $\begin{array}{l}\text { 1. Segmentation of cortical brain } \\
\text { regions from the AAL atlas } \\
\text { (Tzourio-Mazoyer et al., 2002) } \\
\text { 2. Sliding-window analysis, window } \\
\text { length }=50 \text { TRs ( } 100 \mathrm{~s} \text { ), steps = } \\
10 \text { TRs ( } 20 \mathrm{~s} \text { ) } \\
\text { 3. Standard deviation of TVC } \\
\text { density (proportional to the } \\
\text { number of functional } \\
\text { connections) across windows }\end{array}$ & $\begin{array}{l}43 \text { children with benign } \\
\text { epilepsy } \\
\text { (centrotemporal spikes) } \\
19 \text { females }(44.2 \%) \\
\text { mean age }=9.61 \text { years } \\
\text { SD }=2.04 \text { years } \\
28 \text { typically developing children } \\
13 \text { females ( } 46.4 \%) \\
\text { mean age }=10 \text { years } \\
\text { SD }=2.31 \text { years }\end{array}$ & $\begin{array}{l}\text { - Compared to typically developing } \\
\text { children, epilepsy children showed } \\
\text { decreased TVC variability in the } \\
\text { orbital inferior frontal gyrus and } \\
\text { increased TVC variability in the } \\
\text { precuneus } \\
\text { - Patients with interictal epileptiform } \\
\text { discharges, compared to patients } \\
\text { without interictal epileptiform } \\
\text { discharges, showed higher TVC } \\
\text { variability in the supramarginal } \\
\text { gyrus } \\
\text { - Excessive TVC variability of the } \\
\text { precuneus correlated with a } \\
\text { younger onset age of seizure }\end{array}$ \\
\hline Liao et al. (2018) & $\begin{array}{l}\text { Unspecified GE 3T } \\
240 \text { volumes } \\
\text { TR }=2 \mathrm{~s}\end{array}$ & $\begin{array}{l}\text { 1. Segmentation of } 200 \text { brain } \\
\text { regions using the Craddock atlas } \\
\text { (Craddock et al., 2012) }\end{array}$ & $\begin{array}{l}48 \text { major depressive disorder } \\
37 \text { females }(77.1 \%) \\
\text { mean age }=34.8 \text { years }\end{array}$ & $\begin{array}{l}\text { - Increased network strength and } \\
\text { efficiency in patients with suicide } \\
\text { ideation compared to healthy }\end{array}$ \\
\hline
\end{tabular}


TABLE 3 | Continued

\begin{tabular}{|c|c|c|c|}
\hline Study & $\begin{array}{l}\text { RS fMRI acquisition } \\
\text { parameters }^{\Omega}\end{array}$ & TVC analysis approach^^ & Study subjects ${ }^{\theta}$ \\
\hline & & $\begin{array}{l}\text { 2. Sliding-window analysis, window } \\
\text { length = 50TRs (100 s), steps = } \\
\text { 5TRs (10 s) } \\
\text { 3. Graph theory analyses: network } \\
\text { strength, network efficiency, } \\
\text { nodal efficiency, small worldness } \\
\text { Variance of area-under-the-curve } \\
\text { of graph metrics } \\
\text { 4. Correlation with suicide } \\
\text { ideation scores }\end{array}$ & $\begin{array}{l}\mathrm{SD}=10.3 \text { years } \\
30 \text { healthy controls } \\
18 \text { females }(60 \%) \\
\text { mean age }=35.7 \text { years } \\
\mathrm{SD}=10.2 \text { years }\end{array}$ \\
\hline Liu et al. (2017) & $\begin{array}{l}\text { Siemens Trio } 3 T \\
250 \text { volumes } \\
\text { TR }=2 \mathrm{~s}\end{array}$ & $\begin{array}{l}\text { 1. Group ICA decomposition in } 21 \\
\text { relevant independent } \\
\text { components of interest } \\
\text { 2. Sliding-window analysis, window } \\
\text { length }=55 \text { TRs (110 s), steps = } \\
1 \text { TR }(2 \mathrm{~s}) \\
\text { 3. k-means clustering (six recurring } \\
\text { states) } \\
\text { 4. Correlations with } \\
\text { disease duration }\end{array}$ & $\begin{array}{l}43 \text { patients with idiopathic } \\
\text { generalized epilepsy } \\
15 \text { females }(34.8 \%) \\
\text { mean age }=23.12 \text { years } \\
\text { SD }=4.8 \text { years } \\
48 \text { healthy controls } \\
19 \text { females }(39.5 \%) \\
\text { mean age }=23.02 \text { years } \\
\text { SD }=1.49 \text { years }\end{array}$ \\
\hline
\end{tabular}

$\begin{array}{ll}\text { Liu et al. (2018) } & \text { Siemens Trio 3T } \\ & \text { Unspecified volumes } \\ & \text { TR }=2 \mathrm{~s}\end{array}$
Siemens Trio 3T

170 volumes

$\mathrm{TR}=2 \mathrm{~s}$
1. Segmentation of bilateral $\quad 30$ patients with putamen and 56 brain regions Parkinson's disease from the Desikan atlas (Desikan 11 females (36.7\%) et al., 2006)

2. Sliding-window analysis, window $\mathrm{SD}=9.9$ years length $=30$ TRs $(60 \mathrm{~s})$, steps $=28$ healthy controls 2 TRs (4s)

3. Standard deviation of TVC strength

4. Correlations with clinical scores

1. Group ICA decomposition in 4753 patients with clinical relevant independent components of interest, classified into eight functional networks high-risk for psychosis 21 females (39.6\%) mean age $=20.4$ years $\mathrm{SD}=4.5$ years

2. Sliding-window analysis, window 58 schizophrenia patients length $=22$ TRs (44 s), steps $=20$ females $(34.5 \%)$ $1 \mathrm{TR}(2 \mathrm{~s})$

3. k-means clustering (five recurring states). Fuzzy meta-state analysis

1. Segmentation of three amygdalar subregions in each hemisphere, following the JuBrain Cytoarchitectonic Atlas (Zilles and Amunts, 2010) mean age $=21.8$ years $\mathrm{SD}=3.8$ years 70 healthy controls 41 females (58.6\%) mean age $=21.9$ years $\mathrm{SD}=5.6$ years 30 patients with major depression disorder 20 females (66.7\%) mean age $=36.1$ years $\mathrm{SD}=12.3$ years

2. Sliding-window analysis, window range $=18-60$ years length $=100$ TRs $(200 \mathrm{~s})$, step $=62$ healthy controls $1 \mathrm{TR}(2 \mathrm{~s}) \quad 33$ females $(53.2 \%)$
Main findings

subjects and major depressed patients without suicide ideation

- Patients without suicide ideation showed TVC alterations within the left middle/inferior frontal gyrus, right superior parietal gyrus, right postcentral gyrus and right fusiform gyrus

- TVC network strength distinguished patients with and without suicide ideation from healthy subjects

- Patients with idiopathic generalized epilepsy showed reduced dwell time in a state characterized by strong correlations between visual and remaining sense-related networks, as well as increased dwell time in a state characterized by strong correlations between cognitive and sense-related networks

- In patients with idiopathic generalized epilepsy, reduced dwell time in the first above-mentioned state was correlated with a higher seizure frequency

- Compared to healthy controls, Parkinson's disease patients showed reduced TVC between the posterior subunit in the left putamen with the left superior frontal gyrus, right putamen and the right precentral gyrus, as well as between the right posterior putamen and bilateral pallidum nuclei

- TVC abnormalities correlated with more severe disability

- Compared to healthy subjects, schizophrenia patients showed significantly lower global meta-state dynamism

- Compared to healthy controls, patients with high-risk for psychosis showed significantly lower meta-state dynamism

\footnotetext{
- Compared to healthy controls, patients with major depression disorder exhibited decreased positive TVC correlations between the amygdala and left centromedial and superficial subregions, primarily in the brainstem, decreased positive fronto-thalamic TVC, and
} 
TABLE 3 | Continued

\begin{tabular}{|c|c|c|c|}
\hline Study & $\begin{array}{l}\text { RS fMRI acquisition } \\
\text { parameters } \Omega\end{array}$ & TVC analysis approach^^ & Study subjects ${ }^{\theta}$ \\
\hline & & $\begin{array}{l}\text { 3. Voxel-wise maps of variance of } \\
\text { amygdalar TVC across windows }\end{array}$ & $\begin{array}{l}\text { mean age }=35.1 \text { years } \\
\text { - } S D=15.9 \text { years } \\
\text { - range }=16-81 \text { years }\end{array}$ \\
\hline
\end{tabular}

Quevenco et al. (2017) Philips Achieva 7T 200 volumes

$\mathrm{TR}=2 \mathrm{~s}$

Rashid et al. (2014)

Siemens Allegra 3T

Eyes open

202 volumes

$\mathrm{TR}=1.5 \mathrm{~s}$
1. Segmentation of 90 brain cortical regions from the AAL atlas (Tzourio-Mazoyer et al., 2002)

2. Sliding-window analysis, window length $=30$ TRs $(60 \mathrm{~s})$, steps $=$ $1 \mathrm{TR}$ (2s)

3. Principal components analysis: eigen-connectivity patterns (states)

1. Group ICA decomposition in 49 relevant independent components of interest, classified into 7 functional networks

2. Sliding-window analysis, window length $=22$ TRs (33 s), steps = $1 \mathrm{TR}(1.5 \mathrm{~s})$

3. k-means clustering (five recurring states)
37 healthy controls divided according to presence/absence of memory decline

13 females (35.1\%)

mean age $=73$ years $\mathrm{SD}=6.6$ years
60 schizophrenia patients 13 females (21.7\%) mean age $=35.85$ years $\mathrm{SD}=12.01$ years 38 bipolar disorder patients 20 females (52.6\%) mean age $=38.96$ years $\mathrm{SD}=10.9$ years 61 healthy controls 28 females (45.9\%) mean age $=35.4$ years $\mathrm{SD}=11.57$ years
Rashid et al. (2016)

Siemens Allegra 3T

Eyes open

202 volumes

$\mathrm{TR}=1.5 \mathrm{~s}$

\begin{abstract}
Rashid et al. (2018a) GE Discovery 3T
160 volumes

$\mathrm{TR}=2 \mathrm{~s}$
\end{abstract}

1. Group ICA decomposition in 4960 schizophrenia patients relevant independent components of interest, classified into seven functional networks

2. Sliding-window analysis, window length $=22$ TRs (33s), steps $=$ $1 \mathrm{TR}(1.5 \mathrm{~s})$

3. k-means clustering (five recurring-states)

4. Machine learning classification of mean age $=35.4$ years the study subgroups

1. Group ICA decomposition in 38 relevant independent components of interest

2. Sliding-window analysis, window length $=22$ TRs (44 s), steps $=$ 1 TR (2s)

3. k-means clustering (four recurring states)
13 females $(21.7 \%)$ mean age $=35.85$ years $\mathrm{SD}=12.01$ years 38 bipolar disorder patients

20 females $(52.6 \%)$ mean age $=38.96$ years $\mathrm{SD}=10.9$ years 61 healthy controls 28 females (45.9\%)

\section{$\mathrm{SD}=11.57$ years}

774 children 22 children diagnosed with autism spectrum disorders 15 children with autistic traits age range $=4.89-8.90$ years 774 typical development children
Main findings

decreased negative TVC of the left centromedial subregion with the right superior frontal gyrus

- In patients, mean positive TVC strength between the left centromedial region and brainstem was positively correlated with the age of onset of major depression disorder

- Subjects with memory decline showed reduced TVC between anterior and posterior brain areas

- Increased global connectivity, reduced TVC between anterior and posterior brain areas, increased TVC between interhemispheric fronto-temporal areas and reduced TVC between parietal and temporal areas correlated with memory decline and apoprotein E- $\varepsilon 4$ carrier status

- Compared to controls, schizophrenia patients showed increased TVC between: (i) temporal regions; (ii) frontal regions; (iii) subcortical regions; iv) temporal and parietal regions, and reduced TVC between: (i) frontal and parietal regions and (ii) frontal and occipital areas

- Compared to bipolar patients, schizophrenia patients showed increased TVC between: (i) frontal and parietal areas; (ii) sensorimotor areas; (iii) sensorimotor and parietal areas

- Compared to healthy controls, bipolar disorder patients showed increased TVC between temporal and parietal areas, as well as reduced TVC within parietal regions

- TVC improved classification between patients with schizophrenia, patients with bipolar disorder and healthy controls: TVC overall classification accuracy (84.28\%) was significantly higher than overall classification accuracy of static FC metrics (59.12\%)

\footnotetext{
- In typically developing children, TVC globally increased with age in fronto-temporal, fronto-parietal and temporo-parietal networks

- Compared to typically developing children, autism spectrum disorder children showed: (i) increased TVC between the right insula and left
} 
TABLE 3 | Continued

\begin{tabular}{|c|c|c|c|c|}
\hline Study & $\begin{array}{l}\text { RS fMRI acquisition } \\
\text { parameters }^{\Omega}\end{array}$ & TVC analysis approach $^{\wedge}$ & Study subjects ${ }^{\theta}$ & Main findings \\
\hline & & $\begin{array}{l}\text { 4. Sex and age association } \\
\text { with recurring-states }\end{array}$ & & $\begin{array}{l}\text { superior frontal gyrus, right } \\
\text { supramarginal gyrus and left } \\
\text { precuneus; and (ii) reduced TVC } \\
\text { between the right insula and the } \\
\text { right supramarginal gyrus, the left } \\
\text { supplementary motor area and } \\
\text { right supramarginal gyrus } \\
\text { - Autism spectrum disorder } \\
\text { patients with high level of autistic } \\
\text { traits showed longer dwell times } \\
\text { in a globally disconnected state }\end{array}$ \\
\hline Rashid et al. (2018b) & $\begin{array}{l}\text { Siemens Trio 3T } \\
\text { GE Discovery MR750 3T } \\
162 \text { volumes } \\
\text { TR }=2 \mathrm{~s}\end{array}$ & $\begin{array}{l}\text { 1. Group ICA decomposition in } 7 \\
\text { relevant independent } \\
\text { components of interest } \\
\text { 2. Sliding-window analysis, window } \\
\text { length }=22 \text { TRs ( } 44 \mathrm{~s}) \text {, steps = } \\
1 \mathrm{TR}(2 \mathrm{~s}) \\
\text { 3. k-means clustering (five } \\
\text { recurring states) } \\
\text { Correlation with peak weights of } \\
\text { single nucleotide polymorphism } \\
\text { mostly located in chromosome } 6\end{array}$ & $\begin{array}{l}\text { FBIRN Data Repository } \\
61 \text { schizophrenia patients } \\
9 \text { females }(14.8 \%) \\
\text { mean age }=38.4 \text { years } \\
87 \text { healthy controls } \\
26 \text { females }(29.9 \%) \\
\text { mean age }=36.8 \text { years }\end{array}$ & $\begin{array}{l}\text { - Schizophrenia patients showed } \\
\text { a lower occupancy rate of a } \\
\text { state characterized by high TVC } \\
\text { in temporal, parietal, limbic and } \\
\text { occipital regions (state 1), as well } \\
\text { as a higher occupancy rate of a } \\
\text { state characterized by increased } \\
\text { fronto-limbic and intra-occipital } \\
\text { TVC (state 5) vs. healthy subjects } \\
\text { - Schizophrenia patients with } \\
\text { increased gene polymorphism } \\
\text { had stronger disrupted TVC in } \\
\text { states } 1 \text { and } 5\end{array}$ \\
\hline Ridley et al. (2017) & $\begin{array}{l}\text { Siemens Avanto } 1.5 \mathrm{~T} \\
200 \text { volumes } \\
\mathrm{TR}=3 \mathrm{~s}\end{array}$ & $\begin{array}{l}\text { 1. Segmentation of spherical ROls } \\
\text { (radius }=5 \mathrm{~mm} \text { ), defined by their } \\
\text { contact to implanted electrodes } \\
\text { 2. Sliding-window analysis, window } \\
\text { length }=30 \text { TRs ( } 90 \mathrm{~s} \text { ), steps = } \\
\text { 0.66 TR ( } 2 \mathrm{~s} \text { ) } \\
\text { 3. Correlation with EEG data }\end{array}$ & $\begin{array}{l}9 \text { patients with } \\
\text { drug-resistant epilepsy } \\
3 \text { females }(33.3 \%) \\
\text { mean age }=30.4 \text { years } \\
\mathrm{SD}=4.5 \text { years } \\
\text { range }=24-38 \text { years } \\
\text { No control group }\end{array}$ & $\begin{array}{l}\text { - In cortices not involved by } \\
\text { epilepsy, TVC was correlated with } \\
\text { EEG registration of all frequency } \\
\text { bands } \\
\text { - In epileptic cortices, TVC } \\
\text { correlated with EEG in alpha band }\end{array}$ \\
\hline Sakoglu et al. (2010) & $\begin{array}{l}\text { Siemens Allegra } 3 \mathrm{~T} \\
\text { Active } \mathrm{fMRl} \text { (auditory oddball } \\
\text { task): } \\
\text { Two consecutive runs } \\
249 \text { volumes } \\
\mathrm{TR}=1.5 \mathrm{~s}\end{array}$ & $\begin{array}{l}\text { 1. Group ICA decomposition in } 10 \\
\text { relevant independent } \\
\text { components of interest } \\
\text { 2. Sliding-window analysis, window } \\
\text { length }=64 \text { TRs ( } 96 \mathrm{~s} \text { ), steps = } \\
2 \text { TRs ( } 3 \mathrm{~s}) \\
\text { 3. Time-frequency analysis } \\
\text { 4. Standard deviation of TVC } \\
\text { across windows, between-group } \\
\text { comparison of TVC in } \\
\text { each window }\end{array}$ & $\begin{array}{l}28 \text { schizophrenia patients } \\
5 \text { females }(17.9 \%) \\
\text { mean age }=36.4 \text { years } \\
\text { SD }=12.43 \text { years } \\
28 \text { healthy controls } \\
9 \text { females }(32.1 \%) \\
\text { mean age }=28.8 \text { years } \\
\text { SD }=10.7 \text { years }\end{array}$ & $\begin{array}{l}\text { - Compared to controls, } \\
\text { schizophrenia patients exhibited } \\
\text { reduced TVC task-modulation } \\
\text { between the medial temporal } \\
\text { network and the right lateral } \\
\text { fronto-parietal/frontal networks. } \\
\text { They also showed increased TVC } \\
\text { task-modulation between the } \\
\text { motor and frontal networks, and } \\
\text { between the posterior DMN and } \\
\text { orbitofrontal/parietal networks }\end{array}$ \\
\hline Sun et al. (2018) & $\begin{array}{l}\text { Laboratory dataset } \\
\text { Philips Achieva 3T } \\
\text { Eyes open } \\
240 \text { volumes } \\
\text { TR }=2 \mathrm{~s} \\
\text { COBRE dataset } \\
\text { Siemens Trio 3T } \\
150 \text { volumes } \\
\text { TR }=2 \mathrm{~s}\end{array}$ & $\begin{array}{l}\text { 1. Segmentation of } 90 \text { brain } \\
\text { regions from the AAL atlas } \\
\text { (Tzourio-Mazoyer et al., 2002) } \\
\text { 2. Sliding-window analysis, window } \\
\text { length }=50 \text { TRs (100 s), steps = } \\
\text { 3 TRs (6s) } \\
\text { 3. Graph theory analysis: temporal } \\
\text { global/local efficiency, richness, } \\
\text { sparsity range of } \\
\text { temporal networks }\end{array}$ & $\begin{array}{l}\text { Laboratory dataset } \\
18 \text { schizophrenia patients } \\
8 \text { females }(44.4 \%) \\
\text { mean age }=38.8 \text { years } \\
\text { SD }=9.9 \text { years } \\
\text { range }=24-56 \text { years } \\
19 \text { healthy controls } \\
9 \text { females ( } 47.4 \% \text { ) } \\
\text { mean age }=37.7 \text { years } \\
\text { SD }=9.0 \text { years } \\
\text { range }=28-59 \text { years } \\
\text { COBRE dataset } \\
53 \text { schizophrenia patients } \\
12 \text { females ( } 22.6 \% \text { ) } \\
\text { mean age }=38.3 \text { years } \\
\text { SD }=13.9 \text { years } \\
\text { range }=18-65 \text { years } \\
57 \text { healthy controls } \\
20 \text { females ( } 35.1 \% \text { ) } \\
\text { mean age = } 35.4 \text { years } \\
\text { SD = } 11.9 \text { years } \\
\text { range }=18-62 \text { years }\end{array}$ & $\begin{array}{l}\text { - Compared to healthy controls, } \\
\text { schizophrenia patients showed } \\
\text { higher temporal regional efficiency } \\
\text { with left frontal, right medial } \\
\text { parietal and bilateral subcortical } \\
\text { areas } \\
\text { - Abnormalities of temporal } \\
\text { network efficiency correlated with } \\
\text { a higher presence of } \\
\text { schizophrenia positive and } \\
\text { negative symptoms }\end{array}$ \\
\hline
\end{tabular}


TABLE 3 | Continued

\begin{tabular}{|c|c|c|c|c|}
\hline Study & $\begin{array}{l}\text { RS fMRI acquisition } \\
\text { parameters }^{\Omega}\end{array}$ & TVC analysis approach^${ }^{\wedge}$ & Study subjects ${ }^{\theta}$ & Main findings \\
\hline Vergara et al. (2018) & $\begin{array}{l}\text { Siemens Trio } 3 \mathrm{~T} \\
145 \text { volumes } \\
\mathrm{TR}=2 \mathrm{~s}\end{array}$ & $\begin{array}{l}\text { 1. Group ICA decomposition in } 48 \\
\text { relevant independent } \\
\text { components of interest, } \\
\text { classified in nine functional } \\
\text { networks } \\
\text { 2. Sliding-window analysis, window } \\
\text { length }=15 \text { TRs ( } 30 \mathrm{~s} \text { ) } \\
\text { 3. k-means clustering (four } \\
\text { recurring states) } \\
\text { 4. Machine learning for } \\
\text { group classification }\end{array}$ & $\begin{array}{l}48 \text { patients with mild traumatic } \\
\text { brain injury } \\
25 \text { females }(52.1 \%) \\
\text { mean age }=27.79 \text { years } \\
\mathrm{SD}=9.18 \text { years } \\
48 \text { healthy controls } \\
25 \text { females }(52.1 \%) \\
\text { mean age }=27.40 \text { years } \\
\mathrm{SD}=8.96 \text { years }\end{array}$ & $\begin{array}{l}\text { - Compared to healthy controls, } \\
\text { mild traumatic brain injury patients } \\
\text { showed stronger TVC between } \\
\text { the cerebellum and sensorimotor } \\
\text { areas, as well as a trend toward } \\
\text { increased connectivity between } \\
\text { the cerebellum and almost all } \\
\text { cortical areas } \\
\text { - Results were similar to those } \\
\text { obtained with the study of static } \\
\text { FC (Vergara et al., 2017) }\end{array}$ \\
\hline Wang et al. (2018) & $\begin{array}{l}\text { Siemens TIM Trio 3T } \\
300 \text { volumes } \\
\text { TR }=2 \mathrm{~s}\end{array}$ & $\begin{array}{l}\text { 1. Voxel-by-voxel calculation of } \\
\text { connection strength index (CSI) } \\
\text { and connection count index } \\
\text { (CCl) within a whole gray matter } \\
\text { from the MNI template (Evans } \\
\text { et al., 1992) } \\
\text { 2. Sliding-window analysis, window } \\
\text { length = } 60 \text { TRs (120 s), steps = } \\
\text { 1 TR ( } 2 \text { s) } \\
\text { 3. Mean of CSI and CCl } \\
\text { across windows }\end{array}$ & $\begin{array}{l}18 \text { patients with juvenile } \\
\text { myoclonic epilepsy } \\
15 \text { females }(83.3 \%) \\
\text { mean age }=30.11 \text { years } \\
\text { SD }=7.73 \text { years } \\
\text { range }=20-48 \text { years } \\
25 \text { young adults } \\
10 \text { females ( } 40 \% \text { ) } \\
\text { mean age }=33.2 \text { years } \\
\text { SD }=13.5 \text { years }\end{array}$ & $\begin{array}{l}\text { - Patients with juvenile myoclonic } \\
\text { epilepsy showed increased TVC } \\
\text { in the left dorsolateral prefrontal } \\
\text { cortex, dorsal striatum, precentral } \\
\text { and middle temporal gyri }\end{array}$ \\
\hline Yaesoubi et al. (2017a) & $\begin{array}{l}\text { Siemens Tim Trio 3T } \\
\text { GE Discovery MR750 3T } \\
162 \text { volumes } \\
\text { TR }=2 \mathrm{~s}\end{array}$ & $\begin{array}{l}\text { 1. Group ICA decomposition in } 50 \\
\text { relevant independent } \\
\text { components of interest, using } \\
\text { data from a subgroup of } 120 \\
\text { healthy subjects } \\
\text { 2. Time-frequency analysis } \\
\text { 3. k-means clustering (five } \\
\text { recurring states) }\end{array}$ & $\begin{array}{l}\text { FBIRN Data Repository } \\
163 \text { healthy subjects } \\
46 \text { females }(28.2 \%) \\
\text { mean age }=36.9 \text { years } \\
151 \text { schizophrenia patients } \\
37 \text { females }(24.5 \%) \\
\text { mean age }=37.8 \text { years }\end{array}$ & $\begin{array}{l}\text { - Using temporal and frequency } \\
\text { information, it was possible to } \\
\text { estimate TVC states present } \\
\text { both in healthy controls } \\
\text { and schizophrenia patients } \\
\text { (characterized by very high or very } \\
\text { low frequency profiles), and states } \\
\text { present just in one group } \\
\text { - Compared to controls, } \\
\text { schizophrenia patients showed } \\
\text { more connectivity patterns } \\
\text { characterized by anti-correlations } \\
\text { between the sensorimotor and } \\
\text { visual/auditory/subcortical } \\
\text { networks, as well as more lagged } \\
\text { correlation between the DMN and } \\
\text { sensory networks }\end{array}$ \\
\hline Yu et al. (2015) & $\begin{array}{l}\text { Siemens Trio 3T } \\
\text { Eyes open } \\
150 \text { volumes } \\
\text { TR }=2 \mathrm{~s}\end{array}$ & $\begin{array}{l}\text { 1. Group ICA decomposition in } 48 \\
\text { relevant independent } \\
\text { components of interest, } \\
\text { classified into six functional } \\
\text { networks } \\
\text { 2. Sliding-window analysis, window } \\
\text { length = } 20 \text { TRs ( } 40 \text { s), step =1 } \\
\text { TR ( } 2 \text { s) } \\
\text { 3. Graph theory: connectivity } \\
\text { strength, clustering coefficient, } \\
\text { global efficiency; variance of } \\
\text { graph metrics over time. } \\
\text { 4. Assessment of reoccurring } \\
\text { connectivity states based on } \\
\text { graph metrics (four } \\
\text { recurring states) }\end{array}$ & $\begin{array}{l}82 \text { schizophrenia patients } \\
17 \text { females }(20.7 \%) \\
\text { mean age }=38 \text { years } \\
S D=14 \text { years } \\
82 \text { healthy controls } \\
19 \text { females }(23.2 \%) \\
\text { mean age }=37.7 \text { years } \\
S D=10.8 \text { years }\end{array}$ & $\begin{array}{l}\text { - Compared to controls, } \\
\text { schizophrenia patients showed } \\
\text { lower connectivity strength, } \\
\text { clustering coefficient and global } \\
\text { efficiency, as well as higher } \\
\text { occupancy rate of a state } \\
\text { characterized by disconnection } \\
\text { between the sensorimotor, the } \\
\text { cognitive control, and the DMN }\end{array}$ \\
\hline Yue et al. (2018) & $\begin{array}{l}\text { Siemens Trio } 3 \mathrm{~T} \\
240 \text { volumes } \\
\mathrm{TR}=2 \mathrm{~s}\end{array}$ & $\begin{array}{l}\text { 1. Segmentation of bilateral } \\
\text { amygdalae, using stereotaxic } \\
\text { and probabilistic maps of } \\
\text { cytoarchitectonic boundaries }\end{array}$ & $\begin{array}{l}33 \text { schizophrenia patients } \\
22 \text { females }(66.7 \%) \\
\text { mean age }=30.6 \text { years } \\
\mathrm{SD}=8.13 \text { years } \\
34 \text { healthy controls }\end{array}$ & $\begin{array}{l}\text { - Compared to controls, } \\
\text { schizophrenia patients showed } \\
\text { increased TVC between the left } \\
\text { amygdala and orbitofrontal } \\
\text { regions }\end{array}$ \\
\hline
\end{tabular}


TABLE 3 | Continued

\begin{tabular}{|c|c|c|c|c|}
\hline Study & $\begin{array}{l}\text { RS fMRI acquisition } \\
\text { parameters } \Omega\end{array}$ & TVC analysis approach ${ }^{\wedge}$ & Study subjects ${ }^{\theta}$ & Main findings \\
\hline & & $\begin{array}{l}\text { length }=18 \text { TRs (36 s) } \\
\text { 3. Standard deviation of voxel-wise } \\
\text { amygdalar TVC across windows }\end{array}$ & $\begin{array}{l}20 \text { females }(58.8 \%) \\
\text { mean age }=28.12 \text { years } \\
S D=6.5 \text { years }\end{array}$ & $\begin{array}{l}\text { - In schizophrenia patients, } \\
\text { variability of TVC correlated with } \\
\text { worse information processing and } \\
\text { attention performance, as well as } \\
\text { with more severe disease severity }\end{array}$ \\
\hline Zhang W. et al. (2018) & $\begin{array}{l}\text { Siemens Trio } 3 \mathrm{~T} \\
1,000 \text { volumes } \\
\mathrm{TR}=0.427 \mathrm{~s}\end{array}$ & $\begin{array}{l}\text { 1. Segmentation of Brodmann } \\
\text { areas 44, } 45 \text { (frontal), 22, } 40 \\
\text { (auditory) (Zilles and Amunts, } \\
\text { 2010) } \\
\text { 2. Sliding-window analysis, window } \\
\text { length } 100 \text { TRs ( } 42.7 \mathrm{~s} \text { ), steps = } \\
2 \text { TRs ( } 0.85 \mathrm{~s} \text { ) } \\
\text { 3. k-means clustering (5 recurring } \\
\text { states) } \\
\text { 4. Variance of TVC strength } \\
\text { between ROls across windows. } \\
\text { 5. Correlation with clinical scales }\end{array}$ & $\begin{array}{l}35 \text { schizophrenia patients } \\
14 \text { females }(40 \%) \\
\text { mean age }=32.61 \text { years } \\
S D=11.58 \text { years } \\
22 \text { healthy controls } \\
13 \text { females }(60 \%) \\
\text { mean age }=34.91 \text { years } \\
S D=13.34 \text { years }\end{array}$ & $\begin{array}{l}\text { - Schizophrenia patients with } \\
\text { auditory hallucinations showed } \\
\text { decreased TVC between the left } \\
\text { frontal speech and left temporal } \\
\text { auditory areas vs. healthy controls }\end{array}$ \\
\hline Zhi et al. (2018) & $\begin{array}{l}\text { Multicenter setting } \\
\text { Philips Achieva 3T } \\
\text { Siemens Verio 3T } \\
\text { Siemens Prisma 3T } \\
240 \text { volumes } \\
\text { TR }=2 \mathrm{~s}\end{array}$ & $\begin{array}{l}\text { 1. Group ICA decomposition in } 49 \\
\text { relevant independent } \\
\text { components of interest, } \\
\text { classified into eight functional } \\
\text { networks } \\
\text { 2. Sliding-window analysis, window } \\
\text { length = } 22 \text { TRs ( } 44 \mathrm{~s} \text { ), steps = } \\
1 \text { TR ( } 2 \text { s) } \\
\text { 3. k-means clustering (five } \\
\text { recurring states) } \\
\text { 4. Graph theory: global and node } \\
\text { properties in each connectivity } \\
\text { state } \\
\text { 5. Correlations with depression } \\
\text { severity and cognitive score }\end{array}$ & $\begin{array}{l}182 \text { major depressive } \\
\text { disorder patients } \\
119 \text { females }(65.4 \%) \\
\text { mean age }=32.0 \text { years } \\
\text { SD }=10.3 \text { years } \\
218 \text { healthy controls } \\
142 \text { females }(65.2 \%) \\
\text { mean age }=29.5 \text { years } \\
S D=8.3 \text { years }\end{array}$ & $\begin{array}{l}\text { - Compared to controls, major } \\
\text { depressive disorder patients } \\
\text { showed: (i) higher TVC strength } \\
\text { between the superior frontal and } \\
\text { middle frontal gyrus; (ii) decreased } \\
\text { TVC between the lingual gyrus } \\
\text { and middle occipital gyrus; and } \\
\text { (iii) decreased TVC between the } \\
\text { superior parietal lobe and middle } \\
\text { frontal gyrus } \\
\text { - Correlation between TVC } \\
\text { abnormalities and: (i) more severe } \\
\text { depressive symptoms, impaired } \\
\text { attention and worse executive } \\
\text { functions; (ii) lower attention; and } \\
\text { (iii) worse performances at } \\
\text { working memory and } \\
\text { executive functions }\end{array}$ \\
\hline
\end{tabular}

$\Omega_{A}$ All RS scans were acquired in the eyes-closed condition, except where indicated.

^TVC analysis approach summarizes: (1) ROls used; (2) assessment of time-varying correlations between brain regions; (3) features extracted for assessing TVC.

${ }^{\theta}$ For each study group of healthy subjects, sex is represented as number of females (\%), mean age and standard deviation (SD).

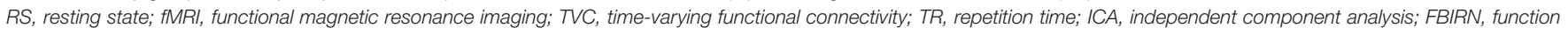

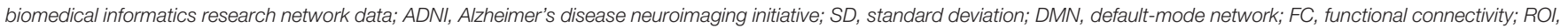

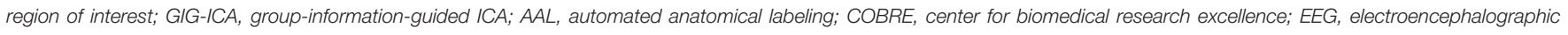
registration; PCC, posterior cingulate cortex; CSI, connection strength index; CCI, connection count index; MNI, Montreal Neurological Institute.

correlated with a more rigid, reduced global dynamism (Miller et al., 2016; Mennigen et al., 2018). Autistic behavior and diagnosis were associated with longer dwell times in a globally disconnected state (Rashid et al., 2018a).

In patients with temporal lobe epilepsy, recurring states characterized by high inter-network TVC expressed reduced dwell time and correlated with an early seizure onset (KlugahBrown et al., 2018). Interestingly, reduced TVC in the ictal irritative zone was associated to an intracranial EEG connectivity increase in the same epileptic region in alpha, beta and gamma bands (Ridley et al., 2017). In patients with Alzheimer's disease, TVC abnormalities between the anterior and the posterior DMN areas correlated with poorer episodic memory performance (Quevenco et al., 2017), while reductions in global TVC were associated with microstructural tissue damage (Alderson et al., 2018). In patients with Parkinson's disease, TVC abnormalities in the DMN have been associated with memory performance
(Engels et al., 2018), while TVC alterations in the putamen were associated with clinical disability (Liu et al., 2018).

At this moment, TVC approaches are applicable only at a group level. However, some preliminary investigations have successfully used TVC abnormalities to classify schizophrenia patients from bipolar patients and/or healthy controls (Cetin et al., 2016; Rashid et al., 2016), suggesting a future application of TVC at an individual level.

\section{CURRENT LIMITATIONS AND FUTURE DIRECTIONS}

The field of TVC is relatively new: all main technical developments have been achieved in the last 9 years. Nonetheless, in such short period of time TVC has provided greater 
insights into fundamental properties of functional networks, and has improved knowledge of the pathophysiological brain reorganization occurring in MS and other neurological and psychiatric diseases.

However, TVC methodology presents some inherent limitations that are likely to be overcome in the next future. Further investigations are also needed to better understand the physiological meaning of TVC fluctuations and their electrophysiological correlates.

\section{How Reliably Can Time-Varying Fluctuations Be Detected From fMRI Data?}

One of the main pitfalls of current TVC analysis approaches consists in the fact that the mere presence of signal fluctuations in an fMRI time series is often taken as an evidence of TVC (Hindriks et al., 2016). This might be not necessarily true: FC values fluctuating over time might be observed just because of noise, or statistical uncertainty. Several measures have been employed to test the effective presence of FC variability in fMRI time series, including variance (Sakoglu et al., 2010), standard deviation (Chang and Glover, 2010), kurtosis (Laumann et al., 2017), or more complex, non-linear measures (Zalesky et al., 2014). Usually, these metrics are compared between real fMRI data and simulated data, constructed ad-hoc to have a static FC. If the test is significant, the null hypothesis of stationarity can be rejected, and TVC can be considered to be effectively present in the data.

Results of studies assessing evidence of TVC in RS fMRI data were quite disappointing, showing that the power of TVC detection in typical 10-min RS fMRI acquisitions was relatively low (Leonardi and Van De Ville, 2015; Hindriks et al., 2016; Zhang C. et al., 2018). Solutions to improve the likelihood of detecting TVC might be the choice of appropriate lengths for sliding windows (Leonardi and Van De Ville, 2015) or the concatenation of more RS fMRI sessions (Hindriks et al., 2016). On the other hand, it is possible that measures used to test the hypothesis of dynamism so far might be not fully appropriate (Miller et al., 2018). Indeed, novel wavelet-based metrics (Miller et al., 2018) seem to be more sensitive to capture non-stationarities present in real RS fMRI data.

\section{Improving Temporal Resolution of fMRI Acquisitions}

Results of TVC also depend upon the temporal resolution used to acquire fMRI data. TVC studies usually investigate modifications in RS FC occurring within seconds, by using fMRI volumes acquired with TRs ranging from $1 \mathrm{~s}$ to $3 \mathrm{~s}$ (Chang and Glover, 2010; Allen et al., 2017; Cabral et al., 2017; Nini et al., 2017; Yaesoubi et al., 2017b; Marusak et al., 2018). Investigations performed on RS fMRI data acquired with a higher sampling rate, e.g., thanks to the use of simultaneous multislice imaging techniques, may be more powerful in detecting changing connectivity reconfigurations over time (Choe et al., 2017). Also, the use of ultra-fast fMRI acquisition techniques, such as inverse imaging (Lin et al., 2006), generalized inverse imaging (Boyacioglu and Barth, 2013), or multi-slab echovolumar imaging (Posse et al., 2013), might constitute an important improvement for TVC. Ultra-fast fMRI allows to acquire a single functional volume covering the whole brain in $<300 \mathrm{~ms}$, resembling the results of magnetoencephalography studies (Asslander et al., 2013). Therefore, fMRI scans acquired with ultra-fast techniques do not include physiological aliasing and allow the detection of more accurate BOLD signal responses to neural activity. Seminal studies already showed that ultra-fast fMRI significantly enhanced the sensitivity of mapping RS FC dynamics (Posse et al., 2013).

\section{Improving TVC Pre- and Post-processing}

Regardless of the analysis method, the signal-to-noise ratio of the BOLD signal in RS fMRI is low, especially in small temporal segments (Handwerker et al., 2012). Non-neural processes contaminating RS fMRI time series can affect TVC estimates (Hutchison et al., 2013; Murphy et al., 2013; Preti and Van De Ville, 2017). These confounds often include the effects of motion, cardiac and respiratory activity, and fluctuations in arterial $\mathrm{CO}_{2}$ concentration (Hutchison et al., 2013; Murphy et al., 2013; Nikolaou et al., 2016; Glomb et al., 2018). Global signal regression (GSR) may be useful to better denoise RS fMRI time series (Murphy and Fox, 2017); however, it was shown to slightly reduce reliability of the estimated TVC connectivity states (Smith et al., 2018). Moreover, the impact of GSR was spatially heterogeneous across brain regions and was dependent from the amount of global signal magnitude across windows (Xu et al., 2018). As such, caution is suggested in applying GSR to slidingwindow correlation analyses, and a control of subjects' mental fluctuations during RS fMRI scanning is recommended (Xu et al., 2018). By applying accurate pre-processing steps on the fMRI data, the rate of artifacts present in the TVC analyses will be minimized (Murphy et al., 2013), thus increasing the quality of the observed findings.

Improvements can still be done not only to pre-processing of RS fMRI time series, but also to TVC post-processing, e.g., by implementing new, accurate methods to estimate changing connectivity over time. Recent papers proposed new approaches to analyse TVC, which aim at capturing change points of connectivity in functional correlation matrices (Cribben et al., 2012; Jeong et al., 2016; Kundu et al., 2018). Other studies introduced tensor-based multilayer community detection algorithms, which are able to describe how organization of functional networks evolves over time (Al-Sharoa et al., 2019). All these methods might be useful to complement TVC information obtained by using more standard, state-of-art methods, such as sliding-window analysis. Finally, improvements can still be done in statistical thresholding strategies. TVC assessment relies on the use of a massive amount of pairwise correlations, stored in series of connectivity matrices, and the best way to perform a proper adjustment for multiple comparisons is still an open issue. Traditional methods of correction for multiple comparisons (Friston et al., 1994) may be too conservative and may suppress all significant results; therefore, different approaches of adjustment for multiple comparisons might be more suitable. For instance, network-based statistic (NBS, 
Zalesky et al., 2010) was proposed as an alternative method of multiple comparison correction in studies using graph theory, which suffers of similar drawbacks as TVC. NBS has been rarely applied in TVC studies (Diez-Cirarda et al., 2018), probably because the process to construct "components" is not straightforward when connectivity matrices change over time. Future studies investigating new strategies of adjustment for multiple comparisons may propose new solutions for this issue.

\section{Functional Interpretation of TVC Findings}

Although some studies have tried to provide a functional interpretation of TVC output, several questions remain to be answered by future work. Preliminary data found some degree of correspondence between EEG rhythms and TVC frequency content (Allen et al., 2017) and hypothesized that some of the TVC states observed in healthy subjects, especially at the end of RS fMRI sessions, might be related to drowsiness or light sleep (Allen et al., 2014, 2017). A preliminary study assessing contemporary TVC and EEG registrations confirmed the presence of connectivity changes over different phases of sleep, with long-range temporal dependencies becoming weaker during deep sleep (Tagliazucchi et al., 2013). Still, it is not clear why larger TVC oscillations have been registered in functional networks at the beginning of RS fMRI sessions (Allen et al., 2014, 2017). Theories hypothesizing the brain functional "anticipation" (e.g., brain predisposition to switch quickly between different psychological states; Zalesky et al., 2014) might partially explain why more "specialized" functional networks (sensorimotor, auditory, visual) express more constant TVC behavior, while more complex, multi-modal networks express more dynamism. On the other hand, constant TVC oscillations in sensorimotor, auditory and visual networks might only reflect their lower activity during RS (Syed et al., 2017).

From this perspective, additional multi-modal studies integrating information from imaging and electrophysiological modalities are necessary for a better comprehension of the neural origin, mechanisms and function of temporal FC variations, as well as of the physiological meaning of TVC states.

\section{CONCLUSIONS}

The analysis of time-varying FC has contributed to provide significant information on intrinsic brain functional

\section{REFERENCES}

Abrol, A., Rashid, B., Rachakonda, S., Damaraju, E., and Calhoun, V. D. (2017). Schizophrenia shows disrupted links between brain volume and dynamic functional connectivity. Front. Neurosci. 11:624. doi: 10.3389/fnins.2017. 00624

Alderson, T. H., Bokde, A. L. W., Kelso, J. A. S., Maguire, L., and Coyle, D. (2018). Metastable neural dynamics in Alzheimer's disease are disrupted by lesions to the structural connectome. Neuroimage 183, 438-455. doi: 10.1016/j.neuroimage.2018. 08.033

Allen, E. A., Damaraju, E., Eichele, T., Wu, L., and Calhoun, V. D. (2017). EEG signatures of dynamic functional network connectivity states. Brain Topogr. 31, 101-116. doi: 10.1007/s10548-017-0546-2 organization, both in healthy and diseased conditions, which complements data produced by static FC approaches. TVC seems to be an intrinsic property of the brain with a neural origin, although some open questions still remain about the correct interpretation of TVC output.

In MS patients, TVC helped to better understand the pathophysiological functional reorganization occurring in the brain, with a peculiar involvement of the DMN, salience, sensorimotor, and fronto-temporal networks. TVC abnormalities were partially correlated with more severe tissue damage and more severe clinical disability, while more extensive correlations were found with abnormal cognitive performances. In patients with neurodegenerative and psychiatric conditions, TVC abnormalities of the DMN, attention and executive networks were also associated to stronger clinical manifestations. Overall, these results suggest a maladaptive neuronal response to disease-related damage.

There are still several unmet needs in neurological and psychiatric conditions that TVC analysis may help to address. First, TVC may be useful to identify multi-modal regions, crucial for functional network plasticity, which may constitute possible targets for motor and cognitive neurorehabilitation protocols, as well as for symptomatic or disease-modifying treatments. Second, trajectories of TVC changes over time during the disease course need to be better defined, both in MS and in psychiatric/other neurodegenerative disorders. This may be the topic of future longitudinal studies, or of cross-sectional studies enrolling patients at different disease phases. Finally, it is still unclear whether TVC abnormalities may have a prognostic value on future disease course. The collection of clinical data at medium- or longterm follow-up may allow to define whether some TVC abnormalities are associated with a more favorable/worse disease prognosis.

\section{AUTHOR CONTRIBUTIONS}

MH wrote the first draft of the manuscript. PV, MF, and MR drafted/revised all sections of the manuscript. MR contributed to the study concept and acted as study supervisor. All authors contributed intellectually to manuscript revision, read, and approved the submitted version.

Allen, E. A., Damaraju, E., Plis, S. M., Erhardt, E. B., Eichele, T., and Calhoun, V. D. (2014). Tracking whole-brain connectivity dynamics in the resting state. Cereb. Cortex 24, 663-676. doi: 10.1093/cercor/bhs352

Al-Sharoa, E., Al-Khassaweneh, M., and Aviyente, S. (2019). Tensor based temporal and multi-layer community detection for studying brain dynamics during resting state fMRI. IEEE Trans. Biomed. Eng. 66, 659-709. doi: 10.1109/TBME.2018.2854676

Asslander, J., Zahneisen, B., Hugger, T., Reisert, M., Lee, H. L., LeVan, P., et al. (2013). Single shot whole brain imaging using spherical stack of spirals trajectories. Neuroimage 73, 59-70. doi: 10.1016/j.neuroimage.2013.01.065

Baker, J. T., Dillon, D. G., Patrick, L. M., Roffman, J. L., Brady, R. O. Jr., Pizzagalli, D. A., et al. (2019). Functional connectomics of affective and psychotic pathology. Proc. Natl. Acad. Sci. U.S.A. 116, 9050-9059. doi: $10.1073 /$ pnas. 1820780116 
Bisecco, A., Nardo, F. D., Docimo, R., Caiazzo, G., d’Ambrosio, A., Bonavita, S., et al. (2018). Fatigue in multiple sclerosis: the contribution of restingstate functional connectivity reorganization. Mult. Scler. 24, 1696-1705. doi: $10.1177 / 1352458517730932$

Biswal, B. B., Mennes, M., Zuo, X. N., Gohel, S., Kelly, C., Smith, S. M., et al. (2010). Toward discovery science of human brain function. Proc. Natl. Acad. Sci. U.S.A. 107, 4734-4739. doi: 10.1073/pnas.0911855107

Bonavita, S., Gallo, A., Sacco, R., Corte, M. D., Bisecco, A., Docimo, R., et al. (2011). Distributed changes in default-mode resting-state connectivity in multiple sclerosis. Mult. Scler. 17, 411-422. doi: 10.1177/13524585103 94609

Bosma, R. L., Kim, J. A., Cheng, J. C., Rogachov, A., Hemington, K. S., Osborne, N. R., et al. (2018). Dynamic pain connectome functional connectivity and oscillations reflect multiple sclerosis pain. Pain 159, 2267-2276. doi: $10.1097 /$ j.pain.0000000000001332

Boyacioglu, R., and Barth, M. (2013). Generalized INverse imaging (GIN): ultrafast fMRI with physiological noise correction. Magn. Reson. Med. 70, 962-971. doi: $10.1002 / \mathrm{mrm} .24528$

Busatto, G. F. (2013). Structural and functional neuroimaging studies in major depressive disorder with psychotic features: a critical review. Schizophr. Bull. 39, 776-786. doi: 10.1093/schbul/sbt054

Cabral, J., Vidaurre, D., Marques, P., Magalhaes, R., Silva Moreira, P., Miguel Soares, J., et al. (2017). Cognitive performance in healthy older adults relates to spontaneous switching between states of functional connectivity during rest. Sci. Rep. 7:5135. doi: 10.1038/s41598-01705425-7

Cai, B., Zille, P., Stephen, J. M., Wilson, T. W., Calhoun, V. D., and Wang, Y. P. (2018). Estimation of dynamic sparse connectivity patterns from resting state fMRI. IEEE Trans. Med. Imaging 37, 1224-1234. doi: 10.1109/TMI.2017.2786553

Cai, J., Liu, A., Mi, T., Garg, S., Trappe, W., McKeown, M. J., et al. (2018). Dynamic graph theoretical analysis of functional connectivity in parkinson's disease: the importance of fiedler value. IEEE J. Biomed. Health Inform. doi: 10.1109/JBHI.2018.2875456. [Epub ahead of print]

Calhoun, V. D., Miller, R., Pearlson, G., and Adali, T. (2014). The chronnectome: time-varying connectivity networks as the next frontier in fMRI data discovery. Neuron 84, 262-274. doi: 10.1016/j.neuron.2014.10.015

Castellazzi, G., Debernard, L., Melzer, T. R., Dalrymple-Alford, J. C., D’Angelo, E., Miller, D. H., et al. (2018). Functional connectivity alterations reveal complex mechanisms based on clinical and radiological status in mild relapsing remitting multiple sclerosis. Front. Neurol. 9:690. doi: 10.3389/fneur.2018.00690

Cetin, M. S., Houck, J. M., Rashid, B., Agacoglu, O., Stephen, J. M., Sui, J., et al. (2016). Multimodal classification of schizophrenia patients with MEG and fMRI data using static and dynamic connectivity measures. Front. Neurosci. 10:466. doi: 10.3389/fnins.2016.00466

Chang, C., and Glover, G. H. (2010). Time-frequency dynamics of restingstate brain connectivity measured with fMRI. Neuroimage 50, 81-98. doi: 10.1016/j.neuroimage.2009.12.011

Chen, J., Sun, D., Shi, Y., Jin, W., Wang, Y., Xi, Q., et al. (2018). Alterations of static functional connectivity and dynamic functional connectivity in motor execution regions after stroke. Neurosci. Lett. 686, 112-121. doi: 10.1016/j.neulet.2018.09.008

Chen, S., Langley, J., Chen, X., and Hu, X. (2016). Spatiotemporal modeling of brain dynamics using resting-state functional magnetic resonance imaging with gaussian hidden markov model. Brain Connect. 6, 326-334. doi: 10.1089/brain.2015.0398

Chen, T., Cai, W., Ryali, S., Supekar, K., and Menon, V. (2016). Distinct global brain dynamics and spatiotemporal organization of the salience network. PLoS Biol. 14:e1002469. doi: 10.1371/journal.pbio.1002469

Choe, A. S., Nebel, M. B., Barber, A. D., Cohen, J. R., Xu, Y., Pekar, J. J., et al. (2017). Comparing test-retest reliability of dynamic functional connectivity methods. Neuroimage 158, 155-175. doi: 10.1016/j.neuroimage.2017. 07.005

Craddock, R. C., James, G. A., Holtzheimer, P. E. 3rd, Hu, X. P., and Mayberg, H. S. (2012). A whole brain fMRI atlas generated via spatially constrained spectral clustering. Hum. Brain Mapp. 33, 1914-1928. doi: 10.1002/hbm.2 1333
Cribben, I., Haraldsdottir, R., Atlas, L. Y., Wager, T. D., and Lindquist, M. A. (2012). Dynamic connectivity regression: determining staterelated changes in brain connectivity. Neuroimage 61, 907-920. doi: 10.1016/j.neuroimage.2012.03.070

Damaraju, E., Allen, E. A., Belger, A., Ford, J. M., McEwen, S., Mathalon, D. H., et al. (2014). Dynamic functional connectivity analysis reveals transient states of dysconnectivity in schizophrenia. Neuroimage Clin. 5, 298-308. doi: 10.1016/j.nicl.2014.07.003

d'Ambrosio, A., Valsasina, P., Gallo, A., De Stefano, N., Pareto, D., Barkhof, F., et al. (2019). Reduced dynamics of functional connectivity and cognitive impairment in multiple sclerosis. Mult. Scler. doi: 10.1177/1352458519837707. [Epub ahead of print].

Deco, G., and Kringelbach, M. L. (2016). Metastability and coherence: extending the communication through coherence hypothesis using a whole-brain computational perspective. Trends Neurosci. 39, 125-135. doi: 10.1016/j.tins.2016.01.001

Desikan, R. S., Segonne, F., Fischl, B., Quinn, B. T., Dickerson, B. C., Blacker, D., et al. (2006). An automated labeling system for subdividing the human cerebral cortex on MRI scans into gyral based regions of interest. Neuroimage 31, 968-980. doi: 10.1016/j.neuroimage.2006.01.021

Destrieux, C., Fischl, B., Dale, A., and Halgren, E. (2010). Automatic parcellation of human cortical gyri and sulci using standard anatomical nomenclature. Neuroimage 53, 1-15. doi: 10.1016/j.neuroimage.2010.06.010

Diez-Cirarda, M., Strafella, A. P., Kim, J., Pena, J., Ojeda, N., Cabrera-Zubizarreta, A., et al. (2018). Dynamic functional connectivity in Parkinson's disease patients with mild cognitive impairment and normal cognition. Neuroimage Clin. 17, 847-855. doi: 10.1016/j.nicl.2017.12.013

Dosenbach, N. U., Nardos, B., Cohen, A. L., Fair, D. A., Power, J. D., Church, J. A., et al. (2010). Prediction of individual brain maturity using fMRI. Science 329, 1358-1361. doi: 10.1126/science.1194144

Du, Y., Fryer, S. L., Fu, Z., Lin, D., Sui, J., Chen, J., et al. (2018). Dynamic functional connectivity impairments in early schizophrenia and clinical high-risk for psychosis. Neuroimage 180(Pt B), 632-645. doi: 10.1016/j.neuroimage.2017.10.022

Du, Y., Pearlson, G. D., Lin, D., Sui, J., Chen, J., Salman, M., et al. (2017). Identifying dynamic functional connectivity biomarkers using GIG-ICA: application to schizophrenia, schizoaffective disorder, and psychotic bipolar disorder. Hum. Brain Mapp. 38, 2683-2708. doi: 10.1002/hbm.23553

Engels, G., Vlaar, A., McCoy, B., Scherder, E., and Douw, L. (2018). Dynamic functional connectivity and symptoms of parkinson's disease: a resting-state fMRI study. Front. Aging Neurosci. 10:388. doi: 10.3389/fnagi.2018.00388

Engle, R. (2002). Dynamic conditional correlation: a simple class of multivariate generalized autoregressive conditional heteroskedasticity models. J. Bus. Econ. Stat. 20, 339-350. doi: 10.1198/073500102288618487

Evans, A. C., Collins, D. L., and Milner, B. (1992). An MRI-based stereotactic atlas from 250 young normal subjects. Soc. Neurosci. 18:408.

Falahpour, M., Thompson, W. K., Abbott, A. E., Jahedi, A., Mulvey, M. E., Datko, M., et al. (2016). Underconnected, but not broken? Dynamic functional connectivity MRI shows underconnectivity in autism is linked to increased intra-individual variability across time. Brain Connect. 6, 403-414. doi: 10.1089/brain.2015.0389

Fan, L., Chu, C., Li, H., Chen, L., Xie, S., Zhang, Y., et al. (2016). The human brainnetome atlas: a new brain atlas based on connectional architecture. Cereb. Cortex 26, 3508-3526. doi: 10.1093/cercor/bhw157

Filippi, M., Agosta, F., Spinelli, E. G., and Rocca, M. A. (2013a). Imaging resting state brain function in multiple sclerosis. J. Neurol. 260, 1709-1713. doi: 10.1007/s00415-012-6695-z

Filippi, M., Bar-Or, A., Piehl, F., Preziosa, P., Solari, A., Vukusic, S., et al. (2018). Multiple sclerosis. Nat. Rev. Dis. Primers 4:43. doi: 10.1038/s41572-018-0050-3

Filippi, M., Preziosa, P., and Rocca, M. A. (2017). Brain mapping in multiple sclerosis: Lessons learned about the human brain. Neuroimage 190, 32-45. doi: 10.1016/j.neuroimage.2017. 09.021

Filippi, M., Valsasina, P., Misci, P., Falini, A., Comi, G., and Rocca, M. A. (2013b). The organization of intrinsic brain activity differs between genders: a resting-state fMRI study in a large cohort of young healthy subjects. Hum. Brain Mapp. 34, 1330-1343. doi: 10.1002/hbm. 21514 
Friston, K. J., Worsley, K. J., Frackowiak, R. S., Mazziotta, J. C., and Evans, A. C. (1994). Assessing the significance of focal activations using their spatial extent. Hum. Brain Mapp. 1, 210-220. doi: 10.1002/hbm.460010306

Fu, Z., Tu, Y., Di, X., Du, Y., Pearlson, G. D., Turner, J. A., et al. (2018). Characterizing dynamic amplitude of low-frequency fluctuation and its relationship with dynamic functional connectivity: an application to schizophrenia. Neuroimage 180(Pt B), 619-631. doi: 10.1016/j.neuroimage.2017.09.035

Fukushima, M., and Sporns, O. (2018). Comparison of fluctuations in global network topology of modeled and empirical brain functional connectivity. PLoS Comput. Biol. 14:e1006497. doi: 10.1371/journal.pcbi.1006497

Gazula, H., Baker, B. T., Damaraju, E., Plis, S. M., Panta, S. R., Silva, R. F., et al. (2018). Decentralized analysis of brain imaging data: voxel-based morphometry and dynamic functional network connectivity. Front. Neuroinform. 12:55. doi: 10.3389/fninf.2018.00055

Glomb, K., Ponce-Alvarez, A., Gilson, M., Ritter, P., and Deco, G. (2018). Stereotypical modulations in dynamic functional connectivity explained by changes in BOLD variance. Neuroimage 171, 40-54. doi: 10.1016/j.neuroimage.2017.12.074

Guo, X., Duan, X., Suckling, J., Chen, H., Liao, W., Cui, Q., et al. (2018). Partially impaired functional connectivity states between right anterior insula and default mode network in autism spectrum disorder. Hum. Brain Mapp. 40, 1264-1275. doi: 10.1002/hbm.24447

Handwerker, D. A., Roopchansingh, V., Gonzalez-Castillo, J., and Bandettini, P. A. (2012). Periodic changes in fMRI connectivity. Neuroimage 63, 1712-1719. doi: 10.1016/j.neuroimage.2012.06.078

He, C., Chen, Y., Jian, T., Chen, H., Guo, X., Wang, J., et al. (2018). Dynamic functional connectivity analysis reveals decreased variability of the defaultmode network in developing autistic brain. Autism Res. 11, 1479-1493. doi: 10.1002/aur.2020

Hemington, K. S., Wu, Q., Kucyi, A., Inman, R. D., and Davis, K. D. (2016). Abnormal cross-network functional connectivity in chronic pain and its association with clinical symptoms. Brain Struct. Funct. 221, 4203-4219. doi: 10.1007/s00429-015-1161-1

Hindriks, R., Adhikari, M. H., Murayama, Y., Ganzetti, M., Mantini, D., Logothetis, N. K., et al. (2016). Can sliding-window correlations reveal dynamic functional connectivity in resting-state fMRI? Neuroimage 127, 242-256. doi: 10.1016/j.neuroimage.2015.11.055

Huang, M., Zhou, F., Wu, L., Wang, B., Guo, L., Zhao, Y., et al. (2019). White matter lesion loads associated with dynamic functional connectivity within attention network in patients with relapsing-remitting multiple sclerosis. J. Clin. Neurosci. 65, 59-65. doi: 10.1016/j.jocn.2019.03.034

Hutchison, R. M., Womelsdorf, T., Allen, E. A., Bandettini, P. A., Calhoun, V. D., Corbetta, M., et al. (2013). Dynamic functional connectivity: promise, issues, and interpretations. Neuroimage 80, 360-378. doi: 10.1016/j.neuroimage.2013.05.079

Jeong, S. O., Pae, C., and Park, H. J. (2016). Connectivity-based change point detection for large-size functional networks. Neuroimage 143, 353-363. doi: 10.1016/j.neuroimage.2016.09.019

Jie, B., Liu, M., and Shen, D. (2018). Integration of temporal and spatial properties of dynamic connectivity networks for automatic diagnosis of brain disease. Med. Image Anal. 47, 81-94. doi: 10.1016/j.media.2018.03.013

Jones, D. T., Vemuri, P., Murphy, M. C., Gunter, J. L., Senjem, M. L., Machulda, M. M., et al. (2012). Non-stationarity in the "resting brain's" modular architecture. PLoS ONE 7:e39731. doi: 10.1371/journal.pone.0039731

Kelly, C., Biswal, B. B., Craddock, R. C., Castellanos, F. X., and Milham, M. P. (2012). Characterizing variation in the functional connectome: promise and pitfalls. Trends Cogn. Sci. 16, 181-188. doi: 10.1016/j.tics.2012. 02.001

Khambhati, A. N., Sizemore, A. E., Betzel, R. F., and Bassett, D. S. (2018). Modeling and interpreting mesoscale network dynamics. Neuroimage 180(Pt B), 337-349. doi: 10.1016/j.neuroimage.2017.06.029

Klugah-Brown, B., Luo, C., He, H., Jiang, S., Armah, G. K., Wu, Y., et al. (2018). Altered dynamic functional network connectivity in frontal lobe epilepsy. Brain Topogr. 32, 394-404. doi: 10.1007/s10548-018-0678-z

Kundu, S., Ming, J., Pierce, J., McDowell, J., and Guo, Y. (2018). Estimating dynamic brain functional networks using multi-subject fMRI data. Neuroimage 183, 635-649. doi: 10.1016/j.neuroimage.2018.07.045
Laumann, T. O., Snyder, A. Z., Mitra, A., Gordon, E. M., Gratton, C., Adeyemo, B., et al. (2017). On the stability of BOLD fMRI correlations. Cereb. Cortex 27, 4719-4732. doi: 10.1093/cercor/bhw265

Leonardi, N., Richiardi, J., Gschwind, M., Simioni, S., Annoni, J. M., Schluep, M., et al. (2013). Principal components of functional connectivity: a new approach to study dynamic brain connectivity during rest. Neuroimage 83, 937-950. doi: 10.1016/j.neuroimage.2013.07.019

Leonardi, N., and Van De Ville, D. (2015). On spurious and real fluctuations of dynamic functional connectivity during rest. Neuroimage 104, 430-436. doi: 10.1016/j.neuroimage.2014.09.007

Li, R., Wang, L., Chen, H., Guo, X., Liao, W., Tang, Y. L., et al. (2018). Abnormal dynamics of functional connectivity density in children with benign epilepsy with centrotemporal spikes. Brain Imaging Behav. doi: 10.1007/s11682-018-9914-0. [Epub ahead of print].

Liao, W., Li, J., Duan, X., Cui, Q., Chen, H., and Chen, H. (2018). Static and dynamic connectomics differentiate between depressed patients with and without suicidal ideation. Hum. Brain Mapp. 39, 4105-4118. doi: 10.1002/hbm.24235

Lim, J., Teng, J., Patanaik, A., Tandi, J., and Massar, S. A. A. (2018). Dynamic functional connectivity markers of objective trait mindfulness. Neuroimage 176, 193-202. doi: 10.1016/j.neuroimage.2018.04.056

Lin, F. H., Wald, L. L., Ahlfors, S. P., Hamalainen, M. S., Kwong, K. K., and Belliveau, J. W. (2006). Dynamic magnetic resonance inverse imaging of human brain function. Magn. Reson. Med. 56, 787-802. doi: 10.1002/mrm.20997

Lin, S. J., Vavasour, I., Kosaka, B., Li, D. K. B., Traboulsee, A., MacKay, A., et al. (2018). Education, and the balance between dynamic and stationary functional connectivity jointly support executive functions in relapsing-remitting multiple sclerosis. Hum. Brain Map. 39, 5039-5049. doi: 10.1002/hbm.24343

Lindquist, M. A., Xu, Y., Nebel, M. B., and Caffo, B. S. (2014). Evaluating dynamic bivariate correlations in resting-state fMRI: a comparison study and a new approach. Neuroimage 101, 531-546. doi: 10.1016/j.neuroimage.2014.06.052

Liu, A., Lin, S. J., Mi, T., Chen, X., Chan, P., Wang, Z. J., et al. (2018). Decreased subregional specificity of the putamen in Parkinson's Disease revealed by dynamic connectivity-derived parcellation. Neuroimage Clin. 20, 1163-1175. doi: 10.1016/j.nicl.2018.10.022

Liu, F., Wang, Y., Li, M., Wang, W., Li, R., Zhang, Z., et al. (2017). Dynamic functional network connectivity in idiopathic generalized epilepsy with generalized tonic-clonic seizure. Hum. Brain Mapp. 38, 957-973. doi: $10.1002 / \mathrm{hbm} .23430$

Liu, X., and Duyn, J. H. (2013). Time-varying functional network information extracted from brief instances of spontaneous brain activity. Proc. Natl. Acad. Sci. U.S.A. 110, 4392-4397. doi: 10.1073/pnas.1216856110

Liu, Y., Liang, P., Duan, Y., Jia, X., Yu, C., Zhang, M., et al. (2011). Brain plasticity in relapsing-remitting multiple sclerosis: evidence from resting-state fMRI. $J$. Neurol. Sci. 304, 127-131. doi: 10.1016/j.jns.2011.01.023

Loitfelder, M., Fazekas, F., Petrovic, K., Fuchs, S., Ropele, S., WallnerBlazek, M., et al. (2011). Reorganization in cognitive networks with progression of multiple sclerosis: insights from fMRI. Neurology 76, 526-533. doi: 10.1212/WNL.0b013e31820b75cf

Lowe, M. J., Beall, E. B., Sakaie, K. E., Koenig, K. A., Stone, L., Marrie, R. A., et al. (2008). Resting state sensorimotor functional connectivity in multiple sclerosis inversely correlates with transcallosal motor pathway transverse diffusivity. Hum. Brain Mapp. 29, 818-827. doi: 10.1002/hbm.20576

Lowe, M. J., Phillips, M. D., Lurito, J. T., Mattson, D., Dzemidzic, M., and Mathews, V. P. (2002). Multiple sclerosis: low-frequency temporal blood oxygen leveldependent fluctuations indicate reduced functional connectivity initial results. Radiology 224, 184-192. doi: 10.1148/radiol.2241011005

Mak, L. E., Minuzzi, L., MacQueen, G., Hall, G., Kennedy, S. H., and Milev, R. (2017). The default mode network in healthy individuals: a systematic review and meta-analysis. Brain Connect. 7, 25-33. doi: 10.1089/brain.2016.0438

Marusak, H. A., Calhoun, V. D., Brown, S., Crespo, L. M., Sala-Hamrick, K., Gotlib, I. H., et al. (2017). Dynamic functional connectivity of neurocognitive networks in children. Hum. Brain Mapp. 38, 97-108. doi: 10.1002/hbm. 23346

Marusak, H. A., Elrahal, F., Peters, C. A., Kundu, P., Lombardo, M. V., Calhoun, V. D., et al. (2018). Mindfulness and dynamic functional neural connectivity in children and adolescents. Behav. Brain Res. 336, 211-218. doi: 10.1016/j.bbr.2017.09.010 
Mennigen, E., Miller, R. L., Rashid, B., Fryer, S. L., Loewy, R. L., Stuart, B. K., et al. (2018). Reduced higher-dimensional resting state fMRI dynamism in clinical high-risk individuals for schizophrenia identified by meta-state analysis. Schizophr. Res. 201, 217-223. doi: 10.1016/j.schres.2018.06.007

Miller, R. L., Abrol, A., Adali, T., Levin-Schwarz, Y., and Calhoun, V. D. (2018). Resting-state fMRI dynamics and null models: perspectives, sampling variability, and simulations. Front. Neurosci. 12:551. doi: 10.3389/fnins.2018.00551

Miller, R. L., Yaesoubi, M., Turner, J. A., Mathalon, D., Preda, A., Pearlson, G., et al. (2016). Higher Dimensional meta-state analysis reveals reduced resting fMRI connectivity dynamism in schizophrenia patients. PLoS ONE 11:e0149849. doi: 10.1371/journal.pone.0149849

Mokhtari, F., Laurienti, P. J., Rejeski, W. J., and Ballard, G. (2018a). Dynamic fMRI connectivity tensor decomposition: a new approach to analyze and interpret dynamic brain connectivity. Brain Connect. 9, 95-112. doi: 10.1089/brain.2018.0605

Mokhtari, F., Rejeski, W. J., Zhu, Y., Wu, G., Simpson, S. L., Burdette, J. H., et al. (2018b). Dynamic fMRI networks predict success in a behavioral weight loss program among older adults. Neuroimage 173, 421-433. doi: 10.1016/j.neuroimage.2018.02.025

Murphy, K., Birn, R. M., and Bandettini, P. A. (2013). Restingstate fMRI confounds and cleanup. Neuroimage 80, 349-359. doi: 10.1016/j.neuroimage.2013.04.001

Murphy, K., and Fox, M. D. (2017). Towards a consensus regarding global signal regression for resting state functional connectivity MRI. Neuroimage 154, 169-173. doi: 10.1016/j.neuroimage.2016.11.052

Nikolaou, F., Orphanidou, C., Papakyriakou, P., Murphy, K., Wise, R. G., and Mitsis, G. D. (2016). Spontaneous physiological variability modulates dynamic functional connectivity in resting-state functional magnetic resonance imaging. Philos. Trans. Ser. A Math. Phys. Eng. Sci. 374:20150183. doi: $10.1098 /$ rsta.2015.0183

Nini, M., Hongna, Z., Zhiying, L., Li, Y., and Xia, W. (2017). "Gender differences in dynamic functional connectivity based on resting-state fMRI," in Conference proceedings: Annual International Conference of the IEEE Engineering in Medicine and Biology Society (Stougthon, WI: The Printing House, Inc.) 2017, 2940-2943.

Posse, S., Ackley, E., Mutihac, R., Zhang, T., Hummatov, R., Akhtari, M., et al. (2013). High-speed real-time resting-state FMRI using multi-slab echovolumar imaging. Front. Hum. Neurosci. 7:479. doi: 10.3389/fnhum.2013.00479

Power, J. D., Cohen, A. L., Nelson, S. M., Wig, G. S., Barnes, K. A., Church, J. A., et al. (2011). Functional network organization of the human brain. Neuron 72, 665-678. doi: 10.1016/j.neuron.2011.09.006

Preti, M. G., Bolton, T. A., and Van De Ville, D. (2017). The dynamic functional connectome: State-of-the-art and perspectives. Neuroimage 160, 41-54. doi: 10.1016/j.neuroimage.2016.12.061

Preti, M. G., and Van De Ville, D. (2017). Dynamics of functional connectivity at high spatial resolution reveal long-range interactions and fine-scale organization. Sci. Rep. 7:12773. doi: 10.1038/s41598-017-12993-1

Qin, J., Chen, S. G., Hu, D., Zeng, L. L., Fan, Y. M., Chen, X. P., et al. (2015). Predicting individual brain maturity using dynamic functional connectivity. Front. Hum. Neurosci. 9:418. doi: 10.3389/fnhum.2015.00418

Qiu, L., Xia, M., Cheng, B., Yuan, L., Kuang, W., Bi, F., et al. (2018). Abnormal dynamic functional connectivity of amygdalar subregions in untreated patients with first-episode major depressive disorder. J. Psychiatry Neurosci. 43, 262-272. doi: 10.1503/jpn.170112

Quevenco, F. C., Preti, M. G., van Bergen, J. M., Hua, J., Wyss, M., Li, X., et al. (2017). Memory performance-related dynamic brain connectivity indicates pathological burden and genetic risk for Alzheimer's disease. Alzheimers Res. Ther. 9:24. doi: 10.1186/s13195-017-0249-7

Rashid, B., Arbabshirani, M. R., Damaraju, E., Cetin, M. S., Miller, R., Pearlson, G. D., et al. (2016). Classification of schizophrenia and bipolar patients using static and dynamic resting-state fMRI brain connectivity. Neuroimage 134, 645-657. doi: 10.1016/j.neuroimage.2016.04.051

Rashid, B., Blanken, L. M. E., Muetzel, R. L., Miller, R., Damaraju, E., Arbabshirani, M. R., et al. (2018a). Connectivity dynamics in typical development and its relationship to autistic traits and autism spectrum disorder. Hum. Brain Mapp. 39, 3127-3142. doi: 10.1002/hbm.24064
Rashid, B., Chen, J., Rashid, I., Damaraju, E., Liu, J., Miller, R., et al. (2018b). A framework for linking resting-state chronnectome/genome features in schizophrenia: a pilot study. Neuroimage 184, 843-854. doi: 10.1016/j.neuroimage.2018.10.004

Rashid, B., Damaraju, E., Pearlson, G. D., and Calhoun, V. D. (2014). Dynamic connectivity states estimated from resting fMRI Identify differences among Schizophrenia, bipolar disorder, and healthy control subjects. Front. Hum. Neurosci. 8:897. doi: 10.3389/fnhum.2014.00897

Ridley, B., Wirsich, J., Bettus, G., Rodionov, R., Murta, T., Chaudhary, U., et al. (2017). Simultaneous intracranial EEG-fMRI shows inter-modality correlation in time-resolved connectivity within normal areas but not within epileptic regions. Brain Topogr. 30, 639-655. doi: 10.1007/s10548-017-0551-5

Rocca, M., Hidalgo de la Cruz, M., Valsasina, P., Mesaros, S., Martinovic, V., Ivanovic, J., et al. (2019). Two-year dynamic functional network connectivity in clinically isolated syndrome. Mult. Scler. doi: 10.1177/1352458519837704. [Epub ahead of print].

Rocca, M. A., Valsasina, P., Absinta, M., Riccitelli, G., Rodegher, M. E., Misci, P., et al. (2010). Default-mode network dysfunction and cognitive impairment in progressive MS. Neurology 74, 1252-1259. doi: 10.1212/WNL.0b013e3181d9ed91

Rocca, M. A., Valsasina, P., Leavitt, V. M., Rodegher, M., Radaelli, M., Riccitelli, G. C., et al. (2018). Functional network connectivity abnormalities in multiple sclerosis: correlations with disability and cognitive impairment. Mult. Scler. 24, 459-471. doi: 10.1177/1352458517699875

Rocca, M. A., Valsasina, P., Martinelli, V., Misci, P., Falini, A., Comi, G., et al. (2012). Large-scale neuronal network dysfunction in relapsing-remitting multiple sclerosis. Neurology 79, 1449-1457. doi: 10.1212/WNL.0b013e31826d5f10

Roosendaal, S. D., Schoonheim, M. M., Hulst, H. E., Sanz-Arigita, E. J., Smith, S M., Geurts, J. J., et al. (2010). Resting state networks change in clinically isolated syndrome. Brain 133(Pt 6), 1612-1621. doi: 10.1093/brain/awq058

Rubinov, M., and Sporns, O. (2010). Complex network measures of brain connectivity: uses and interpretations. Neuroimage 52, 1059-1069. doi: 10.1016/j.neuroimage.2009.10.003

Ryyppo, E., Glerean, E., Brattico, E., Saramaki, J., and Korhonen, O. (2018). Regions of Interest as nodes of dynamic functional brain networks. Netw. Neurosci. 2, 513-535. doi: 10.1162/netn_a_00047

Sakoglu, U., Pearlson, G. D., Kiehl, K. A., Wang, Y. M., Michael, A. M., and Calhoun, V. D. (2010). A method for evaluating dynamic functional network connectivity and task-modulation: application to schizophrenia. Magma 23, 351-366. doi: 10.1007/s10334-010-0197-8

Sandler, S. I. (2006). Chemical, Biochemical and Engineering Thermodynamics, 4th Edn. Hoboken, NJ: John Wiley \& Sons, Inc.

Sbardella, E., Petsas, N., Tona, F., and Pantano, P. (2015). Resting-State fMRI in MS: general concepts and brief overview of its application. Biomed Res. Int. 2015:212693. doi: 10.1155/2015/212693

Schoonheim, M. M., Hulst, H. E., Brandt, R. B., Strik, M., Wink, A. M., Uitdehaag, B. M., et al. (2015). Thalamus structure and function determine severity of cognitive impairment in multiple sclerosis. Neurology 84, 776-783. doi: 10.1212/WNL.0000000000001285

Shen, H., Li, Z., Qin, J., Liu, Q., Wang, L., Zeng, L. L., et al. (2016). Changes in functional connectivity dynamics associated with vigilance network in taxi drivers. Neuroimage 124( $\mathrm{Pt}$ A), 367-378. doi: 10.1016/j.neuroimage.2015.09.010

Shi, L., Sun, J., Wu, X., Wei, D., Chen, Q., Yang, W., et al. (2018). Brain networks of happiness: dynamic functional connectivity among the default, cognitive and salience networks relates to subjective well-being. Soc. Cogn. Affect. Neurosci. 13, 851-862. doi: 10.1093/scan/nsy059

Shirer, W. R., Ryali, S., Rykhlevskaia, E., Menon, V., and Greicius, M. D. (2012). Decoding subject-driven cognitive states with whole-brain connectivity patterns. Cereb. Cortex 22, 158-165. doi: 10.1093/cercor/bhr099

Smith, D. M., Zhao, Y., Keilholz, S. D., and Schumacher, E. H. (2018). Investigating the intersession reliability of dynamic brain-state properties. Brain Connect. 8, 255-267. doi: 10.1089/brain.2017.0571

Sun, Y., Collinson, S. L., Suckling, J., and Sim, K. (2018). Dynamic reorganization of functional connectivity reveals abnormal temporal efficiency in schizophrenia. Schizophr. Bull. 45, 659-669. doi: 10.1093/schbul/sby077 
Syed, M. F., Lindquist, M. A., Pillai, J. J., Agarwal, S., Gujar, S. K., Choe, A. S., et al. (2017). Dynamic functional connectivity states between the dorsal and ventral sensorimotor networks revealed by dynamic conditional correlation analysis of resting-state functional magnetic resonance imaging. Brain Connect. 7, 635-642. doi: 10.1089/brain.2017.0533

Tagliazucchi, E., Balenzuela, P., Fraiman, D., and Chialvo, D. R. (2012). Criticality in large-scale brain FMRI dynamics unveiled by a novel point process analysis. Front. Physiol. 3:15. doi: 10.3389/fphys.2012.00015

Tagliazucchi, E., and van Someren, E. J. W. (2017). The large-scale functional connectivity correlates of consciousness and arousal during the healthy and pathological human sleep cycle. Neuroimage 160, 55-72. doi: 10.1016/j.neuroimage.2017.06.026

Tagliazucchi, E., von Wegner, F., Morzelewski, A., Brodbeck, V., Jahnke, K., and Laufs, H. (2013). Breakdown of long-range temporal dependence in default mode and attention networks during deep sleep. Proc. Natl. Acad. Sci. U.S.A. 110, 15419-15424. doi: 10.1073/pnas.1312848110

Tona, F., Petsas, N., Sbardella, E., Prosperini, L., Carmellini, M., Pozzilli, C., et al. (2014). Multiple sclerosis: altered thalamic resting-state functional connectivity and its effect on cognitive function. Radiology 271, 814-821. doi: 10.1148/radiol.14131688

Tzourio-Mazoyer, N., Landeau, B., Papathanassiou, D., Crivello, F., Etard, O., Delcroix, N., et al. (2002). Automated anatomical labeling of activations in SPM using a macroscopic anatomical parcellation of the MNI MRI single-subject brain. Neuroimage 15, 273-289. doi: 10.1006/nimg.2001.0978

van Geest, Q., Douw, L., van 't Klooster, S., Leurs, C. E., Genova, H. M., Wylie, G. R., et al. (2018a). Information processing speed in multiple sclerosis: Relevance of default mode network dynamics. Neuroimage Clin. 19, 507-515. doi: 10.1016/j.nicl.2018.05.015

van Geest, Q., Hulst, H. E., Meijer, K. A., Hoyng, L., Geurts, J. J. G., and Douw, L. (2018b). The importance of hippocampal dynamic connectivity in explaining memory function in multiple sclerosis. Brain Behav. 8:e00954. doi: 10.1002/brb3.954

Vergara, V. M., Mayer, A. R., Damaraju, E., Kiehl, K. A., and Calhoun, V. (2017). Detection of mild traumatic brain injury by machine learning classification using resting state functional network connectivity and fractional anisotropy. J. Neurotrauma 34, 1045-1053. doi: 10.1089/neu.2016.4526

Vergara, V. M., Mayer, A. R., Kiehl, K. A., and Calhoun, V. D. (2018). Dynamic functional network connectivity discriminates mild traumatic brain injury through machine learning. Neuroimage Clin. 19, 30-37. doi: 10.1016/j.nicl.2018.03.017

Vidaurre, D., Abeysuriya, R., Becker, R., Quinn, A. J., Alfaro-Almagro, F., Smith, S. M., et al. (2018). Discovering dynamic brain networks from big data in rest and task. Neuroimage 180(Pt B), 646-656. doi: 10.1016/j.neuroimage.2017.06.077

Wang, Y., Berglund, I. S., Uppman, M., and Li, T. Q. (2018). Juvenile myoclonic epilepsy has hyper dynamic functional connectivity in the dorsolateral frontal cortex. Neuroimage Clin. 21:101604. doi: 10.1016/j.nicl.2018.11.014

Wang, Z., Li, Y., Childress, A. R., and Detre, J. A. (2014). Brain entropy mapping using fMRI. PLoS ONE 9:e89948. doi: 10.1371/journal.pone.0089948

Weiner, M. W., Veitch, D. P., Aisen, P. S., Beckett, L. A., Cairns, N. J., Green, R. C., et al. (2017). Recent publications from the Alzheimer's disease neuroimaging initiative: reviewing progress toward improved $\mathrm{AD}$ clinical trials. Alzheimers Dement. 13, e1-e85. doi: 10.1016/j.jalz.2016.11.007

Xu, H., Su, J., Qin, J., Li, M., Zeng, L. L., Hu, D., et al. (2018). Impact of global signal regression on characterizing dynamic functional connectivity and brain states. Neuroimage 173, 127-145. doi: 10.1016/j.neuroimage.2018.02.036

Yaesoubi, M., Allen, E. A., Miller, R. L., and Calhoun, V. D. (2015a). Dynamic coherence analysis of resting fMRI data to jointly capture state-based phase, frequency, and time-domain information. Neuroimage 120, 133-142. doi: 10.1016/j.neuroimage.2015.07.002

Yaesoubi, M., Miller, R. L., Bustillo, J., Lim, K. O., Vaidya, J., and Calhoun, V. D. (2017a). A joint time-frequency analysis of resting-state functional connectivity reveals novel patterns of connectivity shared between or unique to schizophrenia patients and healthy controls. Neuroimage Clin. 15, 761-768. doi: 10.1016/j.nicl.2017.06.023

Yaesoubi, M., Miller, R. L., and Calhoun, V. D. (2015b). Mutually temporally independent connectivity patterns: a new framework to study the dynamics of brain connectivity at rest with application to explain group difference based on gender. Neuroimage 107, 85-94. doi: 10.1016/j.neuroimage.2014.11.054

Yaesoubi, M., Miller, R. L., and Calhoun, V. D. (2017b). Time-varying spectral power of resting-state fMRI networks reveal cross-frequency dependence in dynamic connectivity. PLoS ONE 12:e0171647. doi: 10.1371/journal.pone.0171647

Yang, Z., Craddock, R. C., Margulies, D. S., Yan, C. G., and Milham, M. P. (2014). Common intrinsic connectivity states among posteromedial cortex subdivisions: Insights from analysis of temporal dynamics. Neuroimage 93( $\mathrm{Pt}$ 1), 124-137. doi: 10.1016/j.neuroimage.2014.02.014

Yeo, B. T., Krienen, F. M., Sepulcre, J., Sabuncu, M. R., Lashkari, D., Hollinshead, M., et al. (2011). The organization of the human cerebral cortex estimated by intrinsic functional connectivity. J. Neurophysiol. 106, 1125-1165. doi: 10.1152/jn.00338.2011

Yu, Q., Erhardt, E. B., Sui, J., Du, Y., He, H., Hjelm, D., et al. (2015). Assessing dynamic brain graphs of time-varying connectivity in fMRI data: application to healthy controls and patients with schizophrenia. Neuroimage 107, 345-355. doi: 10.1016/j.neuroimage.2014.12.020

Yue, J. L., Li, P., Shi, L., Lin, X., Sun, H. Q., and Lu, L. (2018). Enhanced temporal variability of amygdala-frontal functional connectivity in patients with schizophrenia. Neuroimage Clin. 18, 527-532. doi: 10.1016/j.nicl.2018.02.025

Zalesky, A., and Breakspear, M. (2015). Towards a statistical test for functional connectivity dynamics. Neuroimage 114, 466-470. doi: 10.1016/j.neuroimage.2015.03.047

Zalesky, A., Fornito, A., and Bullmore, E. T. (2010). Network-based statistic: identifying differences in brain networks. Neuroimage 53, 1197-1207. doi: 10.1016/j.neuroimage.2010.06.041

Zalesky, A., Fornito, A., Cocchi, L., Gollo, L. L., and Breakspear, M. (2014). Time-resolved resting-state brain networks. Proc. Natl. Acad. Sci. U.S.A. 111, 10341-10346. doi: 10.1073/pnas.1400181111

Zhang, C., Baum, S. A., Adduru, V. R., Biswal, B. B., and Michael, A. M. (2018). Test-retest reliability of dynamic functional connectivity in resting state fMRI. Neuroimage 183, 907-918. doi: 10.1016/j.neuroimage.2018.08.021

Zhang, W., Li, S., Wang, X., Gong, Y., Yao, L., Xiao, Y., et al. (2018). Abnormal dynamic functional connectivity between speech and auditory areas in schizophrenia patients with auditory hallucinations. Neuroimage Clin. 19 , 918-924. doi: 10.1016/j.nicl.2018.06.018

Zhi, D., Calhoun, V. D., Lv, L., Ma, X., Ke, Q., Fu, Z., et al. (2018) Aberrant dynamic functional network connectivity and graph properties in major depressive disorder. Front. Psychiatry 9:339. doi: 10.3389/fpsyt.2018. 00339

Zhou, F., Zhuang, Y., Gong, H., Zhan, J., Grossman, M., and Wang, Z. (2016). Resting state brain entropy alterations in relapsing remitting multiple sclerosis. PLoS ONE 11:e0146080. doi: 10.1371/journal.pone.01 46080

Zhou, J., Liu, S., Ng, K. K., and Wang, J. (2017). Applications of resting-state functional connectivity to neurodegenerative disease. Neuroimaging Clin. N. Am. 27, 663-683. doi: 10.1016/j.nic.2017. 06.007

Zilles, K., and Amunts, K. (2010). Centenary of Brodmann's map-conception and fate. Nat. Rev. Neurosci. 11, 139-145. doi: 10.1038/nrn2776

Conflict of Interest Statement: PV received speakers' honoraria from ExceMed. MF is Editor-in-Chief of the Journal of Neurology; received compensation for consulting services and/or speaking activities from Biogen Idec, Merck-Serono, Novartis, Teva Pharmaceutical Industries; and receives research support from Biogen Idec, Merck-Serono, Novartis, Teva Pharmaceutical Industries, Roche, Italian Ministry of Health, Fondazione Italiana Sclerosi Multipla, and ARiSLA (Fondazione Italiana di Ricerca per la SLA). MR received speakers honoraria from Biogen Idec, Novartis, Genzyme, Sanofi-Aventis, Teva, Merck Serono, and Roche and receives research support from the Italian Ministry of Health and Fondazione Italiana Sclerosi Multipla.

The remaining author declares that the research was conducted in the absence of any commercial or financial relationships that could be construed as a potential conflict of interest.

Copyright (c) 2019 Valsasina, Hidalgo de la Cruz, Filippi and Rocca. This is an open-access article distributed under the terms of the Creative Commons Attribution License (CC BY). The use, distribution or reproduction in other forums is permitted, provided the original author(s) and the copyright owner(s) are credited and that the original publication in this journal is cited, in accordance with accepted academic practice. No use, distribution or reproduction is permitted which does not comply with these terms. 


\section{OPEN ACCESS}

Edited by:

Nicoletta Cera

University of Porto, Portugal

Reviewed by:

Anne Lise Pitel,

University of Caen Normandy, France Maria Mathew D'Souza,

Institute of Nuclear Medicine \& Allied

Sciences (DRDO), India

*Correspondence:

Tadashi Watabe

watabe@tracer.med.osaka-u.ac.jp

${ }^{{ } \text {Present address: }}$ Jun Hatazawa

Joint Research Division for the Quantum Cancer Therapy, Research Center for Nuclear Physics, Osaka University, Osaka, Japan

Specialty section:

This article was submitted to Brain Imaging Methods, a section of the journal Frontiers in Neuroscience

Received: 22 February 2019 Accepted: 10 July 2019 Published: 26 July 2019

Citation:

Watabe T and Hatazawa J (2019) Evaluation of Functional Connectivity in the Brain Using Positron Emission

Tomography: A Mini-Review. Front. Neurosci. 13:775. doi: 10.3389/fnins.2019.00775

\section{Evaluation of Functional Connectivity in the Brain Using Positron Emission Tomography: A Mini-Review}

\author{
Tadashi Watabe ${ }^{1,2 *}$ and Jun Hatazawa ${ }^{1,2+}$ \\ ${ }^{1}$ Department of Nuclear Medicine and Tracer Kinetics, Graduate School of Medicine, Osaka University, Osaka, Japan, \\ ${ }^{2}$ Institute for Radiation Sciences, Osaka University, Osaka, Japan
}

Resting-state networks (RSNs) exhibit spontaneous functional connectivity in the resting state. Previous studies have evaluated RSNs mainly based on spontaneous fluctuations in blood oxygenation level-dependent (BOLD) signals during functional magnetic resonance imaging (fMRI). However, separation between regional increases in cerebral blood flow (CBF) and oxygen consumption is theoretically difficult using BOLDfMRI. Such separation can be achieved using quantitative ${ }^{15} \mathrm{O}$-gas and water positron emission tomography (PET). In addition, ${ }^{18} \mathrm{~F}$-FDG PET can be used to investigate functional connectivity based on changes in glucose metabolism, which reflects local brain activity. Previous studies have highlighted the feasibility and clinical usefulness of ${ }^{18}$ F-FDG-PET for the analysis of RSNs, and recent studies have utilized simultaneous PET/fMRI for such analyses. While PET provides seed information regarding the focus of the abnormalities (e.g., hypometabolism and reduced target binding), $\mathrm{fMRI}$ is used for the analysis of functional connectivity. Thus, as PET and fMRI provide different types of information, integrating these modalities may aid in elucidating the pathological mechanisms underlying certain diseases, and in characterizing individual patients.

Keywords: PET, functional connectivity, default mode network, blood flow, metabolism

\section{INTRODUCTION}

Resting-state networks (RSNs) exhibit spontaneous functional connectivity in the resting state. Among these is the default mode network (DMN), in which the posterior cingulate cortex (PCC) plays a central role, connecting with the medial-prefrontal, hippocampal, and lateral temporal areas (Raichle et al., 2001; Buckner et al., 2008; Raichle, 2015). While the DMN is active during the resting state, it becomes deactivated during task states (McCormick and Telzer, 2018). Previous studies have demonstrated that the DMN is related to cognitive functions including memory, thinking, and the integration of social information. Furthermore, DMN abnormalities have been observed in patients with autism, schizophrenia, and Alzheimer's disease (AD) (Buckner et al., 2008).

The default mode network was first discovered by Raichle et al. using positron emission tomography (PET) (2001). The authors focused on the regional oxygen extraction fraction (OEF), and which refers to the ratio of the cerebral metabolic rate of oxygen $\left(\mathrm{CMRO}_{2}\right)$ to cerebral blood flow $(\mathrm{CBF})$. Increases in $\mathrm{CBF}$ during task-related brain activation occur in conjunction with smaller changes in $\mathrm{CMRO}_{2}$, resulting in a relative decrease in the OEF. In contrast, the OEF increases during the deactivated state. Raichle et al. (2001) reported that the OEF increases in the visual cortex of healthy adults during the resting state (i.e., awake with eyes closed). Eye closure usually 
induces deactivation in the visual cortex and corresponding increases in OEF. However, many brain regions exhibit decreases in OEF when compared to the mean hemispheric value, suggesting that some areas are activated even in the baseline or "default" state of the brain.

Since the discovery of the DMN, several studies have used functional magnetic resonance imaging (fMRI) to analyze functional connectivity based on spontaneous fluctuations in blood oxygenation-level dependent (BOLD) signals (Greicius et al., 2003). When an area of the brain is activated, the BOLD signal of the corresponding region increases, reflecting a larger increase in regional CBF than in regional $\mathrm{CMRO}_{2}$ (Raichle et al., 2001). Therefore, functional connectivity among brain regions can be estimated by evaluating the correlations among the timecourses of changes in BOLD signals. While the methodologies for detecting decreased OEF on PET and increased BOLD signaling on fMRI are similar, PET is associated with superior quantitative accuracy for the evaluation of $\mathrm{CBF}$ and $\mathrm{CMRO}_{2}$, which are determined separately in the calculation of OEF.

In the present study, we summarized recent advances in the evaluation of functional connectivity using PET. Since the methodological aspects of connectivity analysis have been summarized by Aiello et al. (2016), Yakushev et al. (2017), and Verger and Guedj (2018), we mainly focused on how PET data beyond fluorodeoxyglucose (FDG) can be utilized for functional connectivity analyses. Using the term "functional connectivity PET," we searched PubMed for articles published between February 2016 and February 2019. Similarly, we searched PubMed using the term "functional connectivity FDG PET" for articles published between February 2014 and February 2019. We included clinical studies involving patients or healthy volunteers in which PET was used for connectivity analyses. Preliminary studies and those with incomplete methodological information were excluded from our analysis.

\section{EVALUATION OF FUNCTIONAL CORRELATIONS USING ${ }^{15} \mathrm{O}-\mathrm{GAS}$ PET}

Regional $\mathrm{CBF}$ and $\mathrm{CMRO}_{2}$ can be quantitatively evaluated via ${ }^{15} \mathrm{O}$-labeled gas and water PET (Hatazawa et al., 1996; Watabe et al., 2014). Our group recently reported that functional correlations in the DMN can be estimated using quantitative ${ }^{15}$ O-PET (Aoe et al., 2018). Our findings indicated that evaluation based on changes in CBF revealed a larger number of brain networks than evaluation based on changes in $\mathrm{CMRO}_{2}$, suggesting that the contribution of blood flow to functional correlations in the DMN is greater than that of oxygen consumption (Figure 1). In this method, two brain regions are considered functionally correlated when their quantitative $\mathrm{CBF}$ or $\mathrm{CMRO}_{2}$ values exhibit a significant positive intersubject correlation. Similar methods were also adopted in another previous study (Di et al., 2012).

Unlike BOLD-fMRI, ${ }^{15} \mathrm{O}$-gas and water PET is advantageous in that $\mathrm{CBF}$, and $\mathrm{CMRO}_{2}$ can be evaluated separately. Local brain activation first increases regional $\mathrm{CMRO}_{2}$, reflecting increases in energy metabolism, following which CBF increases (Figure 2).
However, one disadvantage of PET is that it is difficult to clarify temporal changes due to the long scan duration of each acquisition and the time required for radioactive decay when compared with fMRI, which exhibits a temporal resolution on the order of seconds. Therefore, researchers should remain aware that the theoretical bases of determining functional correlations via PET and determining functional connectivity via BOLDfMRI are different.

\section{EVALUATION OF FUNCTIONAL CONNECTIVITY VIA ${ }^{18}$ F-FDG PET}

Glucose is the fundamental metabolic substrate in the brain. Given that glucose metabolism reflects local brain activity, this measure can be used to analyze functional connectivity. Passow et al. (2015) reported a close association between local metabolic activity and functional connectivity by comparing fluctuations in FDG-uptake and BOLD signals, suggesting that ${ }^{18}$ F-FDGPET is feasible and reliable for functional connectivity analyses in the brain. In their study, FDG-PET images were acquired in 12 frames ( 5 min each) following intravenous injection of FDG, and fluctuations among these frames were evaluated based on global normalization. Wehrl et al. (2013) also utilized temporal information from dynamic PET, while additional studies have highlighted the utility of static PET data for the evaluation of metabolic functional connectivity. In a graph theory-based network analysis, Vanicek et al. (2016) evaluated preoperative FDG-PET data for patients with right-sided temporal lobe epilepsy (TLE) (RTLE; $n=30$ ), left-sided TLE (LTLE; $n=32$ ), and healthy controls $(n=31)$. FDG-PET has also been used in clinical practice to detect seizure lateralization in patients with TLE. Indeed, patients with RTLE exhibit higher lobar connectivity weights than those with LTLE for connections involving the temporal and parietal lobes of the contralateral hemisphere. Although the mechanisms underlying such differences remain to be fully elucidated, the authors suspected that compensatory mechanisms were more prominent in patients with RTLE than in those with LTLE.

Pagani et al. (2017) performed independent-component analysis of ${ }^{18} \mathrm{~F}$-FDG PET data, revealing that patients with $\mathrm{AD}$ exhibit gradual disruptions in functional brain connectivity during the progression of cognitive decline. Chen et al. (2018) investigated alterations in whole-brain intrinsic functional connectivity in patients with dementia with Lewy Bodies (DLB). In their study, the DLB group exhibited increases in the strength of some connections when compared to the healthy control group. These findings suggest that alterations in functional connectivity can be evaluated with a certain level of sensitivity in patients with dementia using ${ }^{18}$ F-FDG PET.

Another study highlighted the usefulness of functional connectivity analysis for predicting responses to vagus nerve stimulation (VNS) in patients with epileptic seizures ( $\mathrm{Yu}$ et al., 2018). Yu et al. (2018) performed preoperative FDGPET in pediatric patients with refractory epilepsy undergoing VNS and analyzed metabolic connectivity via an independent component analysis. The authors reported significant differences 

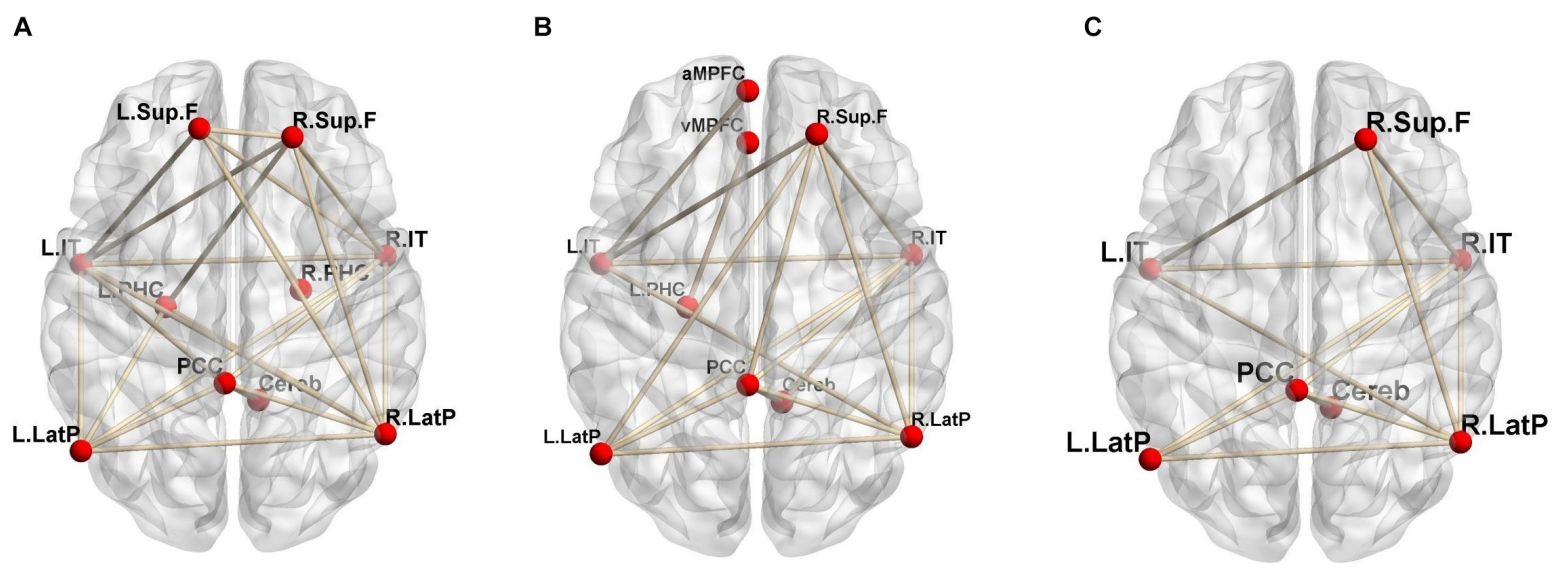

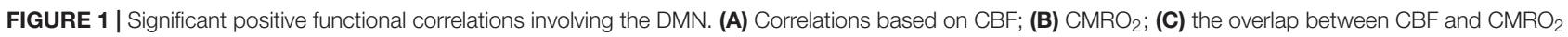
(reprint from Aoe et al., 2018; permission obtained from the Annals of Nuclear Medicine in accordance with their open access policy).

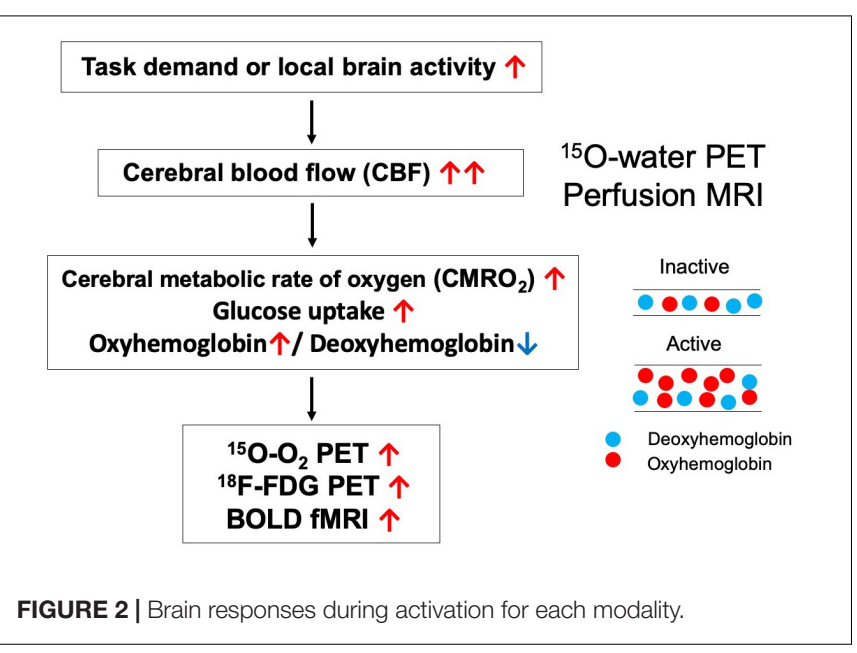

in metabolic connectivity between the VNS-effective and VNSineffective groups. Relative changes in glucose metabolism were strongly connected among areas of the brainstem, cingulate gyrus, cerebellum, bilateral insula, and putamen in the VNSeffective group. Thus, these studies demonstrate that ${ }^{18}$ F-FDGPET analyses of functional connectivity are clinically useful for identifying potential responders to therapy.

In, Villien et al., 2014 reported a new method for overcoming the temporal limitations of ${ }^{18} \mathrm{~F}-\mathrm{FDG}-\mathrm{PET}$, observing that temporal changes in glucose metabolism can be evaluated during continuous injection of FDG. Since FDG is metabolically trapped in neurons or glial cells in the brain after being metabolized into glucose-6-phosphate by hexokinase, the upslope of the time-activity curves reflects real-time glucose metabolism. This technique can used to detect focal brain regions exhibiting task- or stimulus-related activation, similar to BOLD-fMRI (Jamadar et al., 2019). Although further technical adjustments are required to improve the analysis of functional connectivity during continuous-infusion PET, this technique is promising for overcoming the temporal limitations of PET.

\section{INTEGRATED DETECTION VIA PET AND MRI}

Recent studies have utilized simultaneous or combined PET/MRI for functional connectivity analysis, allowing for perfect coregistration in terms of spatial, and temporal matching (Table 1). Using an independent component analysis, Savio et al. (2017) compared the detection of RSNs between resting-state fMRI and ${ }^{18}$ F-FDG PET using simultaneous PET/MR in healthy volunteers (2017). Similar networks were detected using the two modalities, suggesting that coupling between glucose metabolism and blood oxygenation responses is preserved in healthy individuals. Marchitelli et al. (2018) reported that bioenergetic coupling between glucose utilization and neurovascular transmission decreases with age in healthy adults, and that more marked decreases are observed in patients with amnestic mild cognitive impairment (MCI) and mild-to-moderate AD (2018). In their study, ${ }^{18}$ F-FDG-PET was used to detect regions exhibiting hypometabolism, rather than for connectivity analysis. Withinsubject analysis, in which correlations between glucose utilization (PET) and connectivity (fMRI) were evaluated for each participant, revealed that patients with MCI/AD exhibited significant decreases in such correlations. For between-subjects comparisons of PET/fMRI data, in which each imaging modality and metric (volumes) were concatenated across all participants, overlap between the two modalities varied depending on fMRI connectivity parameters.

\section{UTILITY OF PET AS A SEED FOR fMRI ANALYSIS}

Previous studies have compared the correlations between PET and fMRI data using simultaneous PET/MRI. Wehrl et al. (2014) proposed the concept of cometomics, which combines connectivity data and metabolic information. When comparing patients with healthy controls, regions exhibiting 
TABLE 1 | Main findings of functional connectivity analyses using PET and fMRI.

\begin{tabular}{|c|c|c|}
\hline Authors (year) & Participants & Method of analysis and main findings \\
\hline Savio et al., 2017 & $\begin{array}{l}\text { A total of } 22 \text { healthy, right-handed participants } \\
\text { (15 men, } 7 \text { women; mean age: } \\
54.5 \pm 10.0 \text { years) }\end{array}$ & $\begin{array}{l}\text { Acquisition: Simultaneous PET/MRI with static }{ }^{18} \text { F-FDG-PET. Analysis: ICA ( } 20 \\
\text { components). Results: Similar RSNs were detected via fMRI and }{ }^{18} \text { F-FDG PET when } \\
\text { resting-state } \mathrm{AMRI} \text { and }{ }^{18} \text { F-FDG-PET data were acquired simultaneously. }\end{array}$ \\
\hline Marchitelli et al., 2018 & $\begin{array}{l}\text { A total of } 23 \text { patients with } \mathrm{aMCl} / \mathrm{AD} \text { and } 23 \\
\text { healthy older adults }\end{array}$ & $\begin{array}{l}\text { Acquisition: Simultaneous PET/MRI with static }{ }^{18} \text { F-FDG-PET. Analysis: Uptake on } \\
{ }^{18} \text { F-FDG-PET and resting-state fMRI metrics were compared between patients with } \\
\text { aMCI/AD and controls and expressed as } Z \text { scores. Results: For within-subject } \\
\text { PET/fMRI comparisons, correlations were high overall in healthy controls but } 17 \% \text { lower } \\
\text { in patients with aMCI/AD (significant at } p<0.05 \text { ). For between-subject comparisons, } \\
\text { FDG/gICA-DR exhibited the greatest overlap around the posterior DMN nodes. }\end{array}$ \\
\hline Yokoi et al., 2018 & $\begin{array}{l}\text { Amyloid-positive patients with early } \operatorname{AD}(n=23) \\
\text { and amyloid-negative control participants } \\
(n=24)\end{array}$ & $\begin{array}{l}\text { Acquisition: Separate PET ( }{ }^{18} \mathrm{~F}-\mathrm{THK} 5351 \text {, static) and MRI (3T). Analysis: Seed-based } \\
\text { connectivity analysis was performed by generating seed ROls based on regions } \\
\text { exhibiting the most significant differences in }{ }^{18} \mathrm{~F}-\mathrm{THK} 5351 \text { retention between patients } \\
\text { with AD and controls. Results: Patients with AD exhibited significant decreases in } \\
\text { connectivity between the PCC and widespread brain regions. }\end{array}$ \\
\hline Kim et al., 2019 & $\begin{array}{l}\text { A total of } 16 \text { patients with MDD without } \\
\text { comorbidities and } 15 \text { healthy controls }\end{array}$ & $\begin{array}{l}\text { Acquisition: Separate PET }\left[\left({ }^{11} \mathrm{C}\right) \mathrm{ABP} 688 \text {, dynamic] and MRI }(3 \mathrm{~T}) \text {. Analysis: The BP } \mathrm{ND}\right. \\
\text { of }\left[{ }^{11} \mathrm{C}\right] \mathrm{ABP} 688 \text { was quantified using the SRTM for mGluR5 availability. Seed-based } \\
\text { functional connectivity analysis was performed using resting-state fMRI data with } \\
\text { regions derived from quantitative }\left[{ }^{11} \mathrm{C}\right] \mathrm{ABP} 688 \mathrm{PET} \text { as seeds. Results: Patterns of } \\
\text { correlation between }\left[{ }^{11} \mathrm{C}\right] \mathrm{ABP} 688 \mathrm{BP} \\
\text { involving the superior prefrontal cortex were opposite in the depression and control } \\
\text { groups. }\end{array}$ \\
\hline Hamilton et al., 2018 & A total of 16 patients with MDD and 14 controls & $\begin{array}{l}\text { Acquisition: Simultaneous } \mathrm{fMRI} \text { and }{ }^{11} \mathrm{C} \text {-raclopride } \mathrm{PET} \text { targeting the dopamine } \mathrm{D}_{2} \\
\text { receptor. Analysis: } \mathrm{BP}_{\mathrm{ND}} \text { was estimated via the MRTM, using the cerebellum as the } \\
\text { reference tissue. Functional connectivity analysis was performed using striatal regions } \\
\text { exhibiting significant between-group } \mathrm{BP}_{\mathrm{ND}} \text { differences as seeds. Results: Increased } \\
\mathrm{BP}_{\mathrm{ND}} \text { and decreased connectivity were observed in the striatum. The BP } \mathrm{ND}_{\mathrm{N}} \text { was } \\
\text { increased in both the left ventral striatum and right dorsal striatum in patients with MDD. } \\
\text { Connectivity between these regions and cortical targets was also decreased in the } \\
\text { MDD group. }\end{array}$ \\
\hline
\end{tabular}

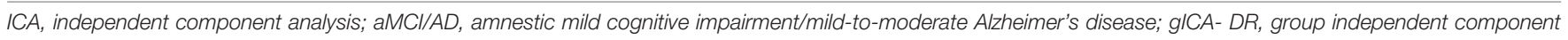

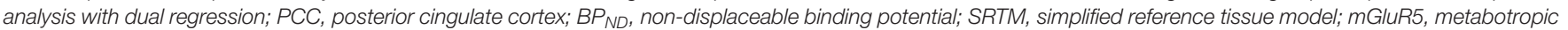
glutamate receptor-5; MRTM, multi-linear reference tissue model; MDD, major depressive disorder.

hypometabolism or reduced target binding on PET can be identified via statistical parametric mapping or region-of-interest (ROI)-based analyses. Resting-state BOLD-fMRI data can be used to derive RSN maps or related parameters during functional connectivity analyses. When combining these methods, regions exhibiting abnormalities on PET images are used as seed regions for the connectivity analysis.

Yokoi et al. (2018) performed resting-state fMRI and ${ }^{18} \mathrm{~F}$ THK5351 PET in amyloid-positive patients with early AD $(n=23)$ and healthy, amyloid-negative controls $(n=24)$. To perform seed-based connectivity analysis, they generated several seed ROIs based on significant differences in ${ }^{18} \mathrm{~F}$-THK5351 retention between the $\mathrm{AD}$ and control groups. Histological evaluations at autopsy indicated that ${ }^{18} \mathrm{~F}$-THK5351 retention corresponded to tau deposition, monoamine oxidase-B (MAOB) levels, and astrogliosis in the brains of patients with AD. Furthermore, seed-based connectivity analysis revealed significant decreases in connectivity between the posterior cingulate cortex (PCC) and widespread brain regions in patients with $\mathrm{AD}$.

Kim et al. (2019) performed seed-based functional connectivity analysis using resting-state fMRI data with seed regions derived from quantitative $\left[{ }^{11} \mathrm{C}\right] \mathrm{ABP} 688$ PET analysis in patients with major depression. To determine metabotropic glutamate receptor-5 (mGluR5) availability, the binding potential $\left(\mathrm{BP}_{\mathrm{ND}}\right)$ of $\left[{ }^{11} \mathrm{C}\right] \mathrm{ABP} 688$ was quantified using the simplified reference tissue model. ROI-based analysis revealed that the $\left[{ }^{11} \mathrm{C}\right] \mathrm{ABP} 688 \mathrm{BP}_{\mathrm{ND}}$ in the prefrontal cortex was significantly lower in patients than in controls. $\mathrm{BP}_{\mathrm{ND}}$ seed-based functional connectivity analysis revealed significantly reduced connectivity between the inferior parietal cortex and fusiform gyrus/inferior occipital cortex in patients, relative to that observed in controls. Patterns of correlation between $\left[{ }^{11} \mathrm{C}\right] \mathrm{ABP} 688 \mathrm{BP}_{\mathrm{ND}}$ and the strength of functional connectivity involving the superior prefrontal cortex seed were opposite in the depression and control groups. Thus, using PET to determine mGluR5 availability revealed related alterations in functional connectivity in the patient group. In addition, Hamilton et al. (2018) conducted whole-brain functional connectivity analysis via $\mathrm{fMRI}$ using striatal regions exhibiting significant $\mathrm{BP}_{\mathrm{ND}}$ differences on ${ }^{11} \mathrm{C}$-raclopride $\mathrm{PET}$ as seeds. They examined dopamine $\mathrm{D}_{2}$ receptors using ${ }^{11} \mathrm{C}$-raclopride $\mathrm{PET}$ in patients with major depressive disorder $(n=16)$ and controls $(n=14)$. Increased $\mathrm{BP}_{\mathrm{ND}}$ in both the left ventral striatum and right dorsal striatum and decreased functional connectivity between the affected striatal areas and their respective cortical targets were observed in patients with major depressive disorder.

Although previous reports on functional connectivity determined using simultaneous PET/MR are still limited (Table 1), a combination of connectivity data and 
metabolic/receptor information will be the major approach in future research.

\section{CONCLUSION}

Functional correlations between blood flow and oxygen consumption can be separately estimated using quantitative ${ }^{15} \mathrm{O}$ gas and water PET. Glucose metabolism during ${ }^{18}$ F-FDG PET can also be used for functional connectivity analysis. Moreover, PET can provide information regarding the focus of abnormalities,

\section{REFERENCES}

Aiello, M., Cavaliere, C., and Salvatore, M. (2016). Hybrid PET/MR imaging and brain connectivity. Front. Neurosci. 10:64. doi: 10.3389/fnins.2016.00064

Aoe, J., Watabe, T., Shimosegawa, E., Kato, H., Kanai, Y., Naka, S., et al. (2018). Evaluation of the default-mode network by quantitative (15)O-PET: comparative study between cerebral blood flow and oxygen consumption. Ann. Nucl. Med. 32, 485-491. doi: 10.1007/s12149-018-1272-x

Buckner, R. L., Andrews-Hanna, J. R., and Schacter, D. L. (2008). The brain's default network: anatomy, function, and relevance to disease. Ann. N. Y. Acad. Sci. 1124, 1-38. doi: 10.1196/annals.1440.011

Chen, D., Lu, J., Zhou, H., Jiang, J., Wu, P., Guo, Q., et al. (2018). Glucose metabolic brain network differences between chinese patients with lewy body dementia and healthy control. Behav. Neurol. 2018:8420658. doi: 10.1155/2018/8420658

Di, X., Biswal, B. B., and Alzheimer's Disease Neuroimaging Initiative. (2012). Metabolic brain covariant networks as revealed by FDG-PET with reference to resting-state fMRI networks. Brain Connect. 2, 275-283. doi: 10.1089/brain. 2012.0086

Greicius, M. D., Krasnow, B., Reiss, A. L., and Menon, V. (2003). Functional connectivity in the resting brain: a network analysis of the default mode hypothesis. Proc. Natl. Acad. Sci. U.S.A. 100, 253-258. doi: 10.1073/pnas. 0135058100

Hamilton, J. P., Sacchet, M. D., Hjornevik, T., Chin, F. T., Shen, B., Kampe, R., et al. (2018). Striatal dopamine deficits predict reductions in striatal functional connectivity in major depression: a concurrent (11)C-raclopride positron emission tomography and functional magnetic resonance imaging investigation. Transl. Psychiatry 8:264. doi: 10.1038/s41398-018-0316-2

Hatazawa, J., Sasajima, T., Shimosegawa, E., Fujita, H., Okudera, T., Kanno, I., et al. (1996). Regional cerebral blood flow response in gray matter heterotopia during finger tapping: an activation study with positron emission tomography. AJNR Am. J. Neuroradiol. 17, 479-482.

Jamadar, S. D., Ward, P. G., Li, S., Sforazzini, F., Baran, J., Chen, Z., et al. (2019). Simultaneous task-based BOLD-fMRI and [18-F] FDG functional PET for measurement of neuronal metabolism in the human visual cortex. Neuroimage 189, 258-266. doi: 10.1016/j.neuroimage.2019.01.003

Kim, J. H., Joo, Y. H., Son, Y. D., Kim, J. H., Kim, Y. K., Kim, H. K., et al. (2019). In vivo metabotropic glutamate receptor 5 availability-associated functional connectivity alterations in drug-naive young adults with major depression. Eur. Neuropsychopharmacol. 29, 278-290. doi: 10.1016/j.euroneuro.2018.12.001

Marchitelli, R., Aiello, M., Cachia, A., Quarantelli, M., Cavaliere, C., Postiglione, A., et al. (2018). Simultaneous resting-state FDG-PET/fMRI in alzheimer disease: relationship between glucose metabolism and intrinsic activity. Neuroimage 176, 246-258. doi: 10.1016/j.neuroimage.2018.04.048

McCormick, E. M., and Telzer, E. H. (2018). Contributions of default mode network stability and deactivation to adolescent task engagement. Sci. Rep. 8:18049. doi: 10.1038/s41598-018-36269-4

Pagani, M., Giuliani, A., Oberg, J., De Carli, F., Morbelli, S., Girtler, N., et al. (2017). Progressive disintegration of brain networking from normal aging to alzheimer disease: analysis of independent components of (18)F-FDG PET data. J. Nucl. Med. 58, 1132-1139. doi: 10.2967/jnumed.116.184309

Passow, S., Specht, K., Adamsen, T. C., Biermann, M., Brekke, N., Craven, A. R., et al. (2015). Default-mode network functional connectivity is closely related to metabolic activity. Hum. Brain Mapp. 36, 2027-2038. doi: 10.1002/hbm.22753 which can then be used for functional connectivity analyses via fMRI. Comparisons between PET and fMRI data may thus allow for improved understanding of the pathophysiology of several brain disorders. Such comparisons may also aid in characterizing individual patients with these disorders.

\section{AUTHOR CONTRIBUTIONS}

Both authors wrote and approved the final version of the manuscript.

Raichle, M. E. (2015). The brain's default mode network. Annu. Rev. Neurosci. 38, 433-447. doi: 10.1146/annurev-neuro-071013-014030

Raichle, M. E., MacLeod, A. M., Snyder, A. Z., Powers, W. J., Gusnard, D. A., and Shulman, G. L. (2001). A default mode of brain function. Proc. Natl. Acad. Sci. U.S.A. 98, 676-682. doi: 10.1073/pnas.98.2.676

Savio, A., Funger, S., Tahmasian, M., Rachakonda, S., Manoliu, A., Sorg, C., et al. (2017). Resting-state networks as simultaneously measured with functional MRI and PET. J. Nucl. Med. 58, 1314-1317. doi: 10.2967/jnumed.116.185835

Vanicek, T., Hahn, A., Traub-Weidinger, T., Hilger, E., Spies, M., Wadsak, W., et al. (2016). Insights into intrinsic brain networks based on graph theory and PET in right- compared to left-sided temporal lobe epilepsy. Sci. Rep. 6:28513. doi: $10.1038 /$ srep 28513

Verger, A., and Guedj, E. (2018). The renaissance of functional (18)F-FDG PET brain activation imaging. Eur. J. Nucl. Med. Mol. Imaging. 45, 2338-2341. doi: 10.1007/s00259-018-4165-2

Villien, M., Wey, H. Y., Mandeville, J. B., Catana, C., Polimeni, J. R., Sander, C. Y., et al. (2014). Dynamic functional imaging of brain glucose utilization using fPET-FDG. Neuroimage 100, 192-199. doi: 10.1016/j.neuroimage.2014.06.025

Watabe, T., Shimosegawa, E., Kato, H., Isohashi, K., Ishibashi, M., Tatsumi, M., et al. (2014). Paradoxical reduction of cerebral blood flow after acetazolamide loading: a hemodynamic and metabolic study with (15)O PET. Neurosci. Bull. 30, 845-856. doi: 10.1007/s12264-013-1459-z

Wehrl, H. F., Hossain, M., Lankes, K., Liu, C. C., Bezrukov, I., Martirosian, P., et al. (2013). Simultaneous PET-MRI reveals brain function in activated and resting state on metabolic, hemodynamic and multiple temporal scales. Nat. Med. 19, 1184-1189. doi: 10.1038/nm.3290

Wehrl, H. F., Wiehr, S., Divine, M. R., Gatidis, S., Gullberg, G. T., Maier, F. C., et al. (2014). Preclinical and translational PET/MR imaging. J. Nucl. Med. 55(Suppl. 2), 11S-18S. doi: 10.2967/jnumed.113.129221

Yakushev, I., Drzezga, A., and Habeck, C. (2017). Metabolic connectivity: methods and applications. Curr. Opin. Neurol. 30, 677-685. doi: 10.1097/WCO. 0000000000000494

Yokoi, T., Watanabe, H., Yamaguchi, H., Bagarinao, E., Masuda, M., Imai, K., et al. (2018). Involvement of the precuneus/posterior cingulate cortex is significant for the development of alzheimer's disease: a PET (THK5351, PiB) and resting fMRI study. Front. Aging Neurosci. 10:304. doi: 10.3389/fnagi.2018. 00304

Yu, R., Park, H. J., Cho, H., Ko, A., Pae, C., Oh, M. K., et al. (2018). Interregional metabolic connectivity of 2-deoxy-2[(18) F]fluoro-D-glucose positron emission tomography in vagus nerve stimulation for pediatric patients with epilepsy: a retrospective cross-sectional study. Epilepsia 59, 2249-2259. doi: 10.1111/epi. 14590

Conflict of Interest Statement: The authors declare that the research was conducted in the absence of any commercial or financial relationships that could be construed as a potential conflict of interest.

Copyright (c) 2019 Watabe and Hatazawa. This is an open-access article distributed under the terms of the Creative Commons Attribution License (CC BY). The use, distribution or reproduction in other forums is permitted, provided the original author(s) and the copyright owner(s) are credited and that the original publication in this journal is cited, in accordance with accepted academic practice. No use, distribution or reproduction is permitted which does not comply with these terms. 


\section{How Sensitive Are Conventional} MEG Functional Connectivity Metrics With Sliding Windows to Detect Genuine Fluctuations in Dynamic Functional Connectivity?

\author{
Lucrezia Liuzzi', Andrew J. Quinn'2, George C. O'Neill', Mark W. Woolrich', \\ Matthew J. Brookes ${ }^{1}$, Arjan Hillebrand ${ }^{4}$ and Prejaas Tewarie ${ }^{1,4 *}$
}

${ }^{1}$ Sir Peter Mansfield Imaging Centre, School of Physics and Astronomy, University of Nottingham, Nottingham, United Kingdom, ${ }^{2}$ Oxford Centre for Human Brain Activity, University of Oxford, Warneford Hospital, Oxford, United Kingdom, ${ }^{3}$ Oxford Centre for Functional MRI of the Brain, University of Oxford, John Radcliffe Hospital, Oxford, United Kingdom, ${ }^{4}$ Department of Clinical Neurophysiology and MEG Center, Amsterdam Neuroscience, Amsterdam UMC, Vrije Universiteit Amsterdam, Amsterdam, Netherlands

Despite advances in the field of dynamic connectivity, fixed sliding window approaches for the detection of fluctuations in functional connectivity are still widely used. The use of conventional connectivity metrics in conjunction with a fixed sliding window comes with the arbitrariness of the chosen window lengths. In this paper we use multivariate autoregressive and neural mass models with a priori defined ground truths to systematically analyze the sensitivity of conventional metrics in combination with different window lengths to detect genuine fluctuations in connectivity for various underlying state durations. Metrics of interest are the coherence, imaginary coherence, phase lag index, phase locking value and the amplitude envelope correlation. We performed analysis for two nodes and at the network level. We demonstrate that these metrics show indeed higher variability for genuine temporal fluctuations in connectivity compared to a static connectivity state superimposed by noise. Overall, the error of the connectivity estimates themselves decreases for longer state durations (order of seconds), while correlations of the connectivity fluctuations with the ground truth was higher for longer state durations. In general, metrics, in combination with a sliding window, perform poorly for very short state durations. Increasing the SNR of the system only leads to a moderate improvement. In addition, at the network level, only longer window widths were sufficient to detect plausible resting state networks that matched the underlying ground truth, especially for the phase locking value, amplitude envelope correlation and coherence. The length of these longer window widths did not necessarily correspond to the underlying state durations. For short window widths resting state network connectivity patterns could not be retrieved. We conclude that fixed sliding window approaches for connectivity can detect modulations of connectivity, but mostly if the underlying dynamics operate on moderate to slow timescales. In practice, this can be a drawback, as state durations can vary significantly in empirical data.

Keywords: dynamic functional connectivity, magnetoencephalography, multivariate autoregressive models, sliding window, neural mass model 


\section{INTRODUCTION}

Large-scale functional interactions in the brain are assumed to be mirrored by statistical dependencies between time evolving activity of neuronal populations, which can be quantified by magnetoencephalography (MEG), electroencephalography (EEG) and functional (f)MRI. The spatiotemporal patterns of these functional interactions (functional connectivity) are still commonly treated in a "static connectivity" sense, i.e., functional connectivity estimates are obtained by collapsing connectivity over windows. There is ample evidence that neuronal interactions appear in spatial clusters or subnetworks that form and dissolute over time (Breakspear et al., 2004; O’Neill et al., 2015, 2017a; de Pasquale et al., 2016). By collapsing connectivity over time windows, we potentially miss crucial information about the temporal evolution of these clusters. Furthermore, recent electrophysiological studies have demonstrated that taking into account the temporal domain of connectivity may give insight into abnormal brain function in neurological diseases (Stam et al., 2005; Carbo et al., 2017; Kim et al., 2017; Watanabe and Rees, 2017). Therefore, there is a need to include the dynamics of functional interactions into our analysis. In addition, given the superior temporal resolution of MEG and EEG to fMRI, these modalities could offer a means to analyze the dynamics of functional interactions in more detail.

A conventional approach to tackle dynamic functional connectivity is to use fixed sliding window approaches, i.e., a window of fixed length is moved in time, using an overlapping or non-overlapping approach, and "static" connectivity is estimated within every fixed window (O’Neill et al., 2017b). This approach comes with the arbitrariness of the window length. Recent approaches have demonstrated that dynamic connectivity can be well-described at a variety of time-scales, therefore a fixed window length may not be appropriate to capture the underlying fluctuations in connectivity (Baker et al., 2014; Vidaurre et al., 2016, 2018; Brookes et al., 2018; Tewarie et al., 2018; Tronarp et al., 2018). In other words, there may be a mismatch between the temporal scale of the underlying fluctuations and the predefined fixed window length, potentially leading to erroneous estimates of connectivity. Nevertheless, studies have used the sliding window approach during cognitive tasks and also in neurological disease (O’Neill et al., 2017b). Of special interest in these studies was the variability of connectivity, which was shown to relate better to outcome measures, such as cognitive decline after neurosurgical intervention (Carbo et al., 2017), than static connectivity information. However, it is known from the fMRI literature that variability of connectivity does not necessarily imply that the underlying system is non-stationary or dynamic (Hindriks et al., 2016; Laumann et al., 2016). Furthermore, most of the popular functional connectivity metrics have mainly been systematically evaluated in the static connectivity sense (David et al., 2004; Wang et al., 2014; Colclough et al., 2016; Dimitriadis et al., 2018). Therefore, there is a need to understand the strengths and limitations of commonly used metrics of functional connectivity when used with a fixed sliding window approach.

In the current work, we systematically analyze the sensitivity and specificity of the fixed sliding window approach in conjunction with commonly used connectivity metrics to detect genuine fluctuations in connectivity. We opted to include connectivity metrics based on the two important intrinsic modes of connectivity, i.e., phase- and amplitude based methods (Siegel et al., 2012). In order to perform this analysis, we require a system with a known ground truth regarding the strength and duration of the time varying connections. Given the lack of this information in empirical MEG data, we employ two models: a parameterized neural mass model (NMM) (Jansen and Rit, 1995) and a parameterized multivariate autoregressive model (MAR) (Neumaier and Schneider, 2001) that both provide a priori defined ground truths. Simulations are performed for a two node system and a large scale network. The former allows us to test the performance of the metrics without any external nuisance factors, while the latter allows us to test the performance of the metrics in a more realistic scenario. We first test in a two node system the null hypothesis that the observed variability of connectivity estimates can merely be understood in terms of an underlying static system superimposed by noise. Secondly, we analyze the correlation between, and error in, the connectivity estimates compared to the underlying ground truth for different state durations and window lengths. In other words, are the different metrics sensitive to the same or different time-scales of dynamic connectivity? Are results from connectivity metrics only valid within a limited temporal range? We then test the ability of metrics to capture the underlying fluctuations in states for different signal-to-noise ratios (SNR), since shorter window lengths also come with the limitation of lower SNR. We finally evaluate in large scale network simulations if connectivity metrics can retrieve switching between a priori defined resting state networks over time and whether the spatial connectivity patterns can be retrieved using non-negative tensor factorization. This is performed in a system without and with linear mixing (in order to model signal leakage).

\section{MATERIALS AND METHODS}

\section{Multivariate Autoregressive Model}

The multivariate autoregressive (MAR) model describes data observations of a system at time $t\left(X_{\mathrm{t}}\right)$ as a linear mapping $(A)$ of $p$ past observations

$$
X_{t}=\sum_{\tau=1}^{p} A_{\tau} X_{t-\tau}+e,
$$

where $e$ is additive Gaussian noise, and $p=2$. This autoregressive model is an infinite impulse response or all-pole linear filter whose frequency content is determined by the roots of the polynomial $A$. These roots can be estimated by an eigendecomposition of the companion form of the parameter matrix (Neumaier and Schneider, 2001). Each mode of the decomposition is defined by a resonant frequency described by the eigenvalue and a projection into the channels in $X$ described by the eigenvectors. Each state in the simulations is defined by a 2-node MAR model with a single resonance defined by its eigenparameters with a frequency, magnitude and weight in each node. 
The weight into node 1 is held fixed whilst the weight in node 2 varies between 0 and 0.9 to create a phase locked coupling of different strengths. These eigen-parameters are then transformed back into the temporal parameter matrix $A$. New realizations of the system can then be generated by filtering white noise with the pre-defined $A$ matrix. Each parameter matrix A produces time-series with a fixed phase lag between nodes 1 and 2 at a given coupling strength. Note that this will inherently favor phase based metrics over amplitude metrics in our simulations. Three parameter settings were created, corresponding to three states with low, medium and high coupling strength. Duration of the states, i.e., duration of ground truth connectivity was determined by a Gamma distribution. Figure 1 shows an example of the output of the MAR model. We generated $300 \mathrm{~s}$ of data for each simulation and iteration and all data were band pass filtered into the beta band $(15-25 \mathrm{~Hz})$.

\section{Parameterized Neural Mass Model}

Since simulations are characterized by model specific features of the data that will inherently influence connectivity estimates, we opted to also use a different model to generate data to assess generalizability of results across models for the two node system. At the same time, this gave us the opportunity to analyze a large scale network with conduction delays. We employed the wellknown Jansen and Rit NMM (Jansen and Rit, 1995). Every unit in the NMM consisted of an excitatory population, an inhibitory population, and a pyramidal population whose activity mimicked the MEG/EEG signal. Simulations were fed with a Gaussian white-noise process and equations were solved using a stochastic Heun's integration scheme with a time step of $1 \times 10^{-4}$ (Tewarie et al., 2019b). Parameters were the same as used in Grimbert and Faugeras (2006). We generated data for the working point of the model well beyond a Hopf bifurcation (i.e., in the limit cycle regime), with the rationale that no unpredictable switching could occur between the limit cycle regime and the linear regime due to noise. Hence, in this way a controllable system for the analysis of dynamic functional connectivity was achieved. We first connected two nodes using a structural coupling parameter $k$. For the two node system, coupling $k$ was parameterized as a time-series, instead of a constant as is typically done. Duration of the states for $k$, and hence the duration of ground truth connectivity was again determined by a Gamma distribution. The parameterization of $k$ was realized in the same way as described above for the MAR model (with fast, medium, slow and mixed states). We generated $300 \mathrm{~s}$ of data for each simulation and iteration and all data were band pass filtered into the alpha band $(8-13 \mathrm{~Hz})$. In the second part of the simulations, we simulated activity for $N=78$ nodes, corresponding to the cortical regions in the automated anatomical atlas (AAL) (Tzourio-Mazoyer et al., 2002). This number of nodes also roughly matches the potential number of independent sources for the relatively low spatial dimensionality of MEG/EEG data (Farahibozorg et al., 2018). To boost neurobiological realism, we included distance dependent conduction delays in our simulations (Cabral et al., 2014; Tewarie et al., 2019b). To this end, the Euclidean distances between centroids of the parcels in the AAL atlas was used and divided by conduction velocity $(v=10 \mathrm{~m} / \mathrm{s})$. For the large scale network simulations, we kept $k$ constant throughout the simulations, but fed the simulations with a structural connectivity tensor (instead of a structural connectivity matrix). This structural connectivity tensor had dimensions $N \times N \times T$, where refers $T$ to the duration of the simulation. At every time point the structural connectivity tensor was characterized by a resting state subnetwork connectivity state. The resting state networks of interest were the default mode network (DMN), sensorimotor network (SMN), the frontoparietal networks (FPN), and the visual network. The duration of the states was again determined by a Gamma distribution (with fast, medium, slow and mixed states). For every time point only one resting state network was active (see Figure 2), with the other nodes being active, but not connected. The resting state networks were obtained from independent component analysis of fMRI data (at the voxel level) projected onto the AAL atlas in standard MNI space [data obtained from Tewarie et al. (2016)].

\section{MEG Connectivity Metrics}

We employ methods that capture information from two modes of connectivity, i.e., amplitude and phase based metrics. We apply connectivity metrics to beta band (MAR) and alpha band (NMM) filtered timecourses for both amplitude and phase based metrics (i.e., amplitude envelope correlation, phase locking value, phase lag index, coherence and imaginary coherence). The implementation of all the metrics is exactly the same as in Liuzzi et al. (2017).

1. Amplitude envelope correlation (AEC) (Brookes et al., 2011; Hipp et al., 2012): The amplitude envelopes are extracted using the Hilbert transform of band pass filtered data and the Pearson correlation is computed between the amplitude envelopes of two pair of regions.

2. Coherence (COH) and Imaginary Coherence (iCO) (Nolte et al., 2004): Coherency is computed on band pass filtered timecourses by evaluating

$$
C(f)=\frac{S_{x y}(f)}{\sqrt{S_{x x}(f) S_{y y}(f)}},
$$

where $S_{\mathrm{xy}}$ denotes the cross spectral density for two timecourses when $x \neq y$ or the auto spectral densities when $x=y$. The coherence was computed from the absolute value of coherency and by averaging over the frequency band of interest. Imaginary Coherence can also be extracted from Eq. 2 by simply calculating the imaginary part of coherency $C(f)$ thus removing zero lag phase relationships.

3. Phase Locking Value (PLV) (Lachaux et al., 1999) characterizes a stable phase relationship between two timecourses within a predefined window. The instantaneous phases are derived using the Hilbert transform and the difference between the instantaneous phases $i$ and $j$ at time $t$ denoted as $\Delta \varphi_{i j}(t)$. Phase locking is subsequently defined as

$$
P L V=\left|\left\langle e^{i \Delta \varphi_{i j}(t)}\right\rangle\right|
$$



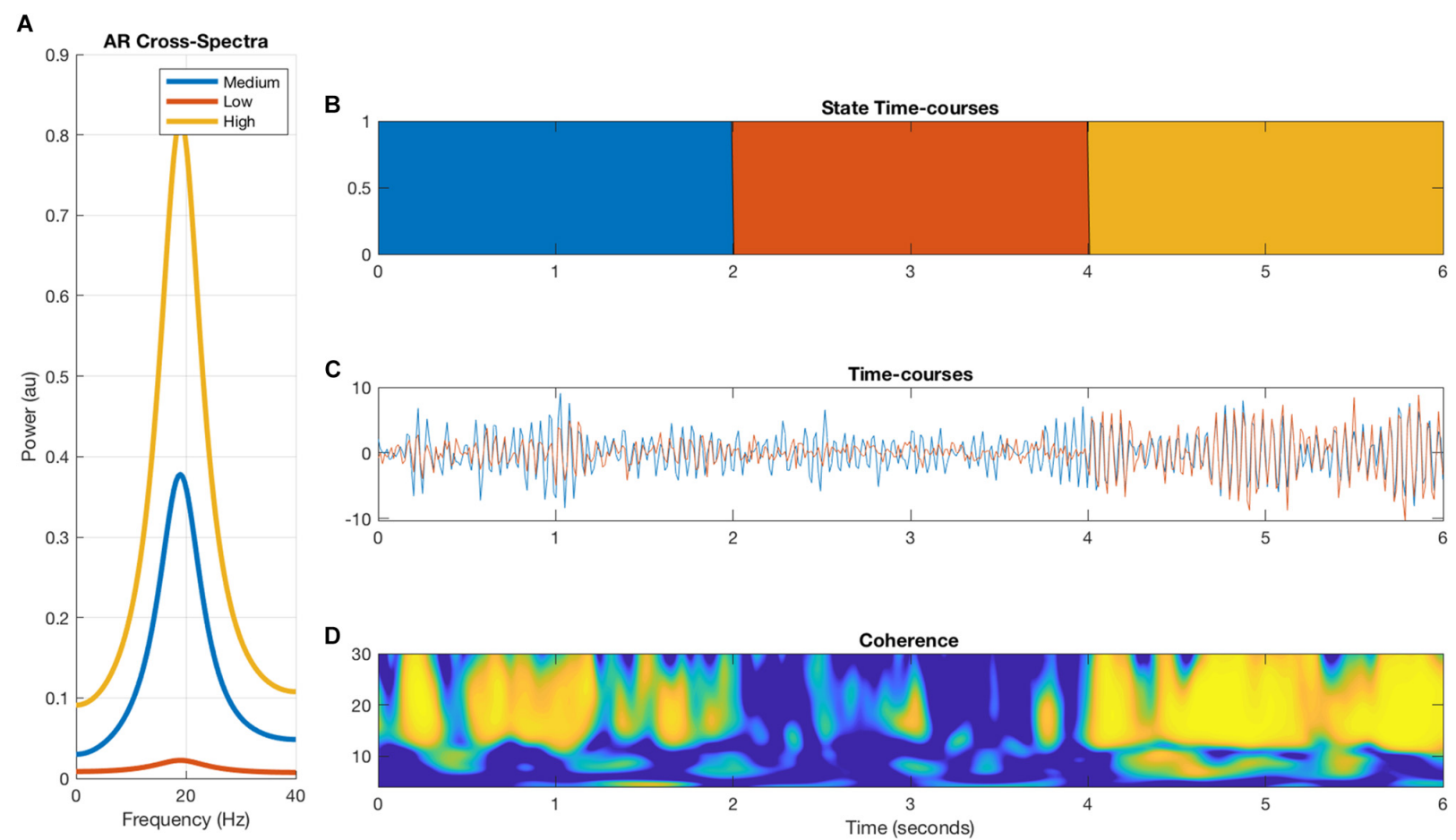

E
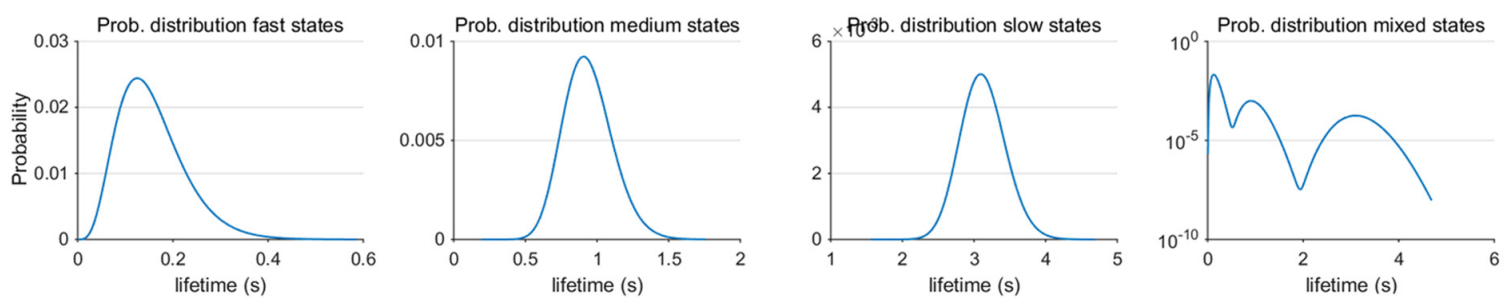

FIGURE 1 | Overview of the multivariate autoregressive model. The cross-spectra (A), state timecourses (B), the two nodal timecourses (C), the coherence between the two nodes (D) are illustrated. Panel (E) shows the ground-truth probability distributions of the state lifetimes for fast, medium, slow and mixed states, which are input to the model (note that the $y$-axis of the mixed states is in logarithmic scale).

4. Phase Lag index (PLI) (Stam et al., 2007): The PLI uses similar phase information as the PLV but discards zero-lag phase differences. Unlike the PLV, it merely quantifies the asymmetry of the phase difference distribution

$$
P L I=\left|\left\langle\operatorname{sign}\left(\mathfrak{s}\left[e^{i \Delta \varphi_{i j}(t)}\right]\right)\right\rangle\right|
$$

\section{Extracting Time Varying Networks Using Non-negative Tensor Factorization}

We extracted time evolving subnetworks using non-negative tensor factorization. This method can be considered as a higher order principal component analysis and decomposes a third order tensor into a set of basis vectors (Bro, 1997; Gauvin et al., 2014)

$$
T=\sum_{l=1}^{L} a_{l} \times b_{l} \times c_{l}
$$

Here $\times$ corresponds to the outer product, and $a_{l}, b_{l}$ and $c_{l}$ correspond to basis vectors of component $l$ and have dimensions $N$ (network size) and $t$ (number of time points). In other words, the outer product of $a_{l}$ and $b_{l}$ reflects the connectivity patterns characterized by the time-series $c_{l}$. The factorization of tensor $T$ is found by solving the optimization problem $\min _{\mathrm{A}, \mathrm{B}, \mathrm{C}}\left\|\mathrm{T}-\mathrm{T}_{\mathrm{A}, \mathrm{B}, \mathrm{C}}^{\prime}\right\|$, with the constraints of orthogonality of the first two basis vectors $\left(a_{k}, b_{k}\right)$, and non-negativity of the last vectors $c_{k}$ (where $T_{A, B, C}^{\prime}$ is the approximation of $T_{A, B, C}$ ). The $\mathrm{N}$-way toolbox (version 1.8) in Matlab was used for this analysis (Andersson and Bro, 2000). We set $L=4$, given the four resting state networks that were used in the simulations.

\section{Analysis Steps}

In order to analyze the sensitivity of our connectivity metrics to genuine fluctuations in connectivity, we follow a stepby-step approach. Analysis is divided into two parts: (1) simulations for a two node system and (2) simulations for a large scale network. 

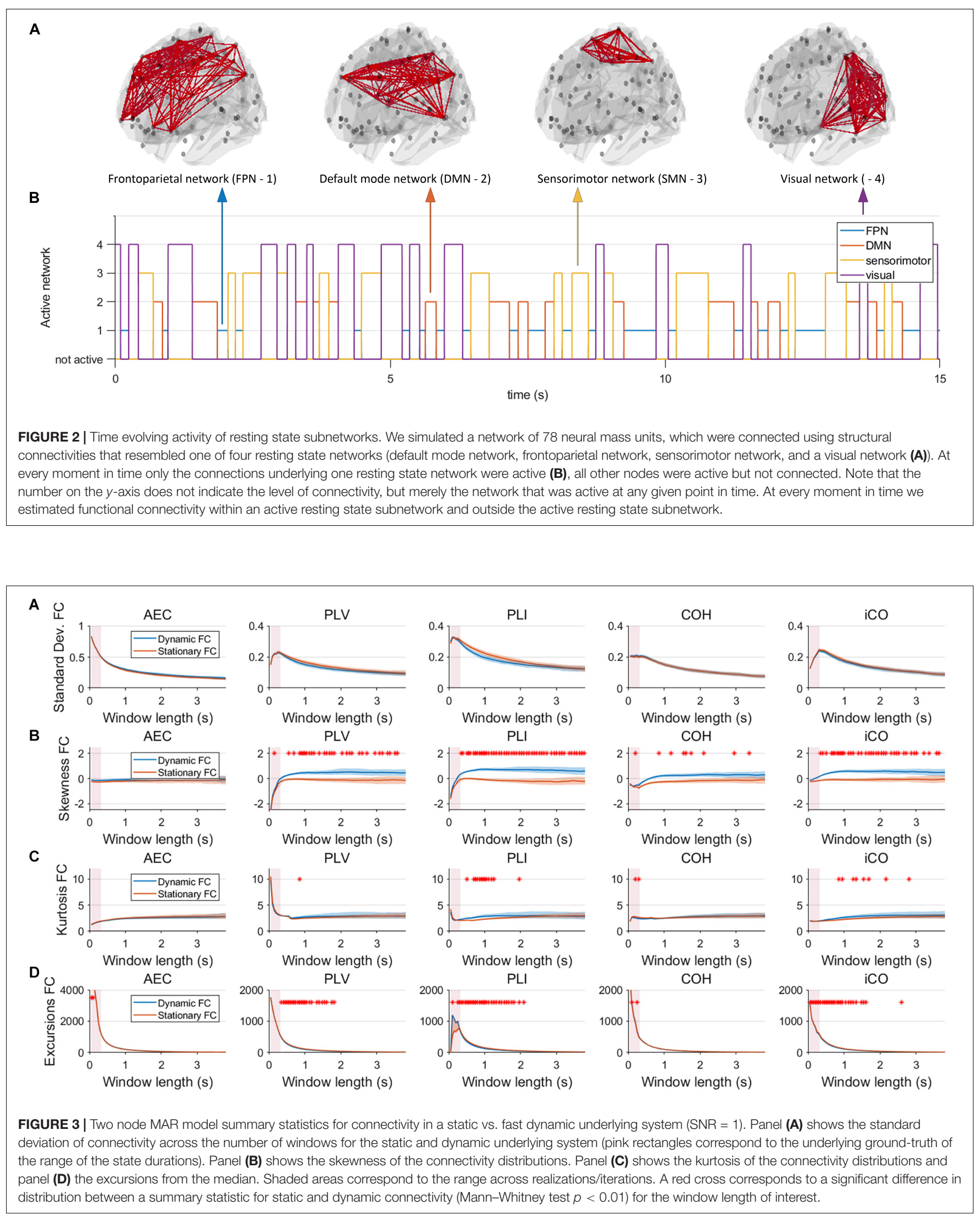


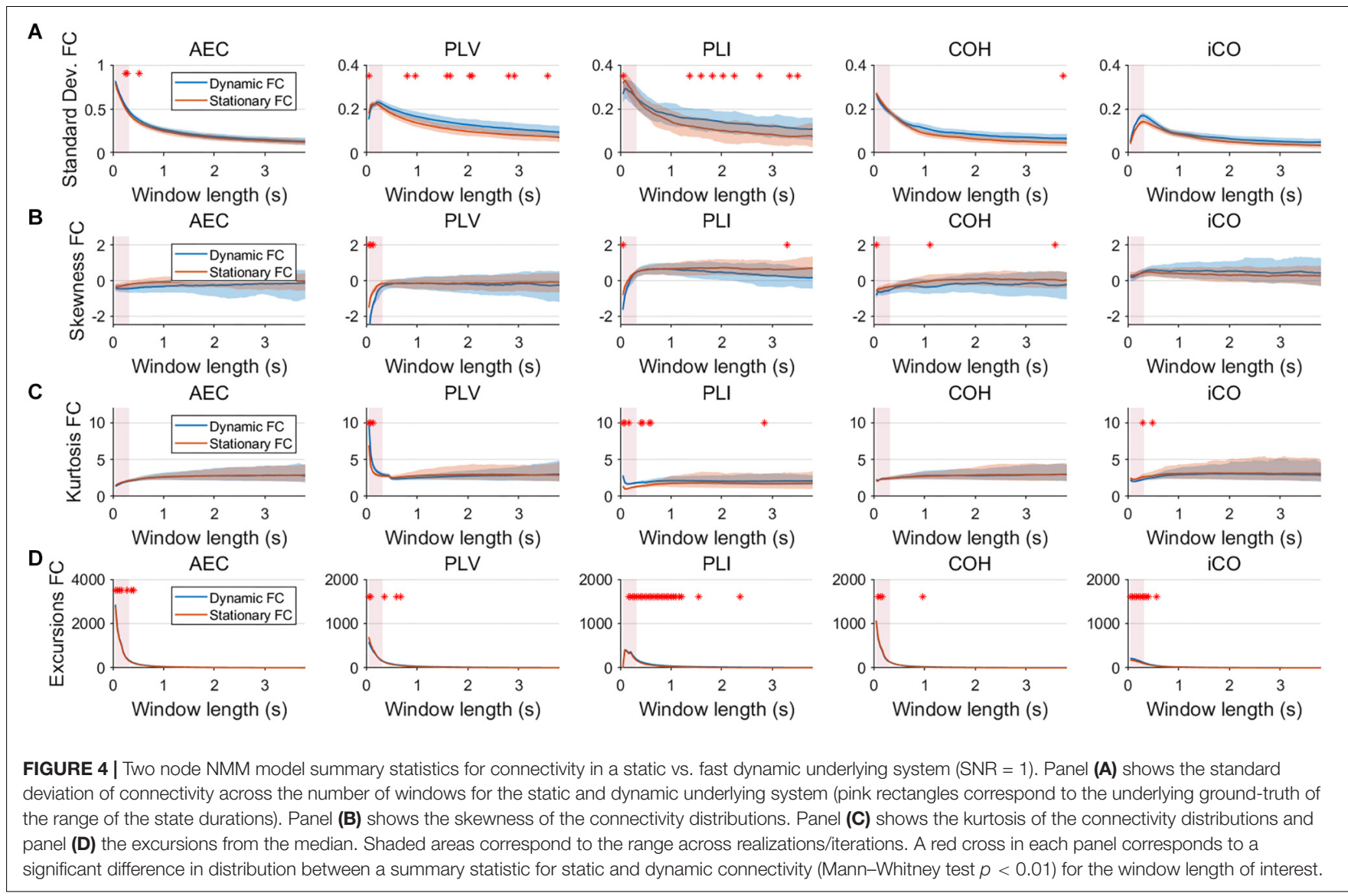

\section{Two Node System Analysis}

Dynamic vs. static connectivity

We first simulated two conditions: (1) two timecourses with an underlying static connectivity between them, superimposed by noise, and (2) two timecourses with underlying fluctuations in connectivity for three different mean state durations $(125 \mathrm{~ms}$, $1 \mathrm{~s}, 3 \mathrm{~s}$, mixed state durations based on the latter three mean state durations). These different state lifetimes and mean state durations were obtained by tuning the scale and shape parameters for a Gamma distribution. Then, for the different metrics we computed the connectivity using a sliding window approach with windows $50 \%$ overlapping in time, resulting in a distribution of connectivity values for the entire simulation. We calculated four summary statistics from the distribution: (1) standard deviation, (2) skewness, (3) kurtosis, (4) excursions from the median. The latter three statistics were selected as resulting connectivity distributions, especially for the "dynamic connectivity case" can be non-Gaussian. The metric "excursions from the median" has extensively been described in Zalesky et al. (2014) and Hindriks et al. (2016), and captures the length and height of all excursions from the median. The rationale is that, the longer and larger the excursions from the median, the greater the evidence for nonstationarity of connectivity. We a priori expected all four metrics to be larger in the dynamic connectivity case than in the static connectivity case. A range of window lengths were chosen for this analysis. For every window we ran twenty iterations. This was done for both the static connectivity case as well as for the dynamic connectivity case. The null hypothesis (i.e., observed variability of connectivity estimates can be fully accounted for by an underlying static system superimposed by noise) can be rejected if the summary statistic in the dynamic system exceeds the summary statistic of the static system. For every window length we computed the Mann-Whitney test to reject the null hypotheses.

\section{Detecting genuine fluctuations in connectivity}

We evaluated for the different metrics the mean error of the connectivity estimate. The error was defined as the mean absolute difference between underlying ground truth and connectivity estimates. This was again done for different mean state durations and for a range of window lengths. Similarly, we computed the Pearson correlation coefficient between the underlying ground truth and the connectivity fluctuations obtained with the different metrics. For both outcome measures, prior to calculation, we interpolated the connectivity estimates using a cubic spline interpolation. Interpolation was necessary since there was a dimension mismatch between the underlying ground truth connectivity timecourses and the connectivity estimates obtained with the five metrics [i.e., the latter were based on one estimate for every window, whereas in that window the underlying connectivity was fully sampled as the data timecourse (i.e., the ground truth)]. We used non-parametric Friedman 

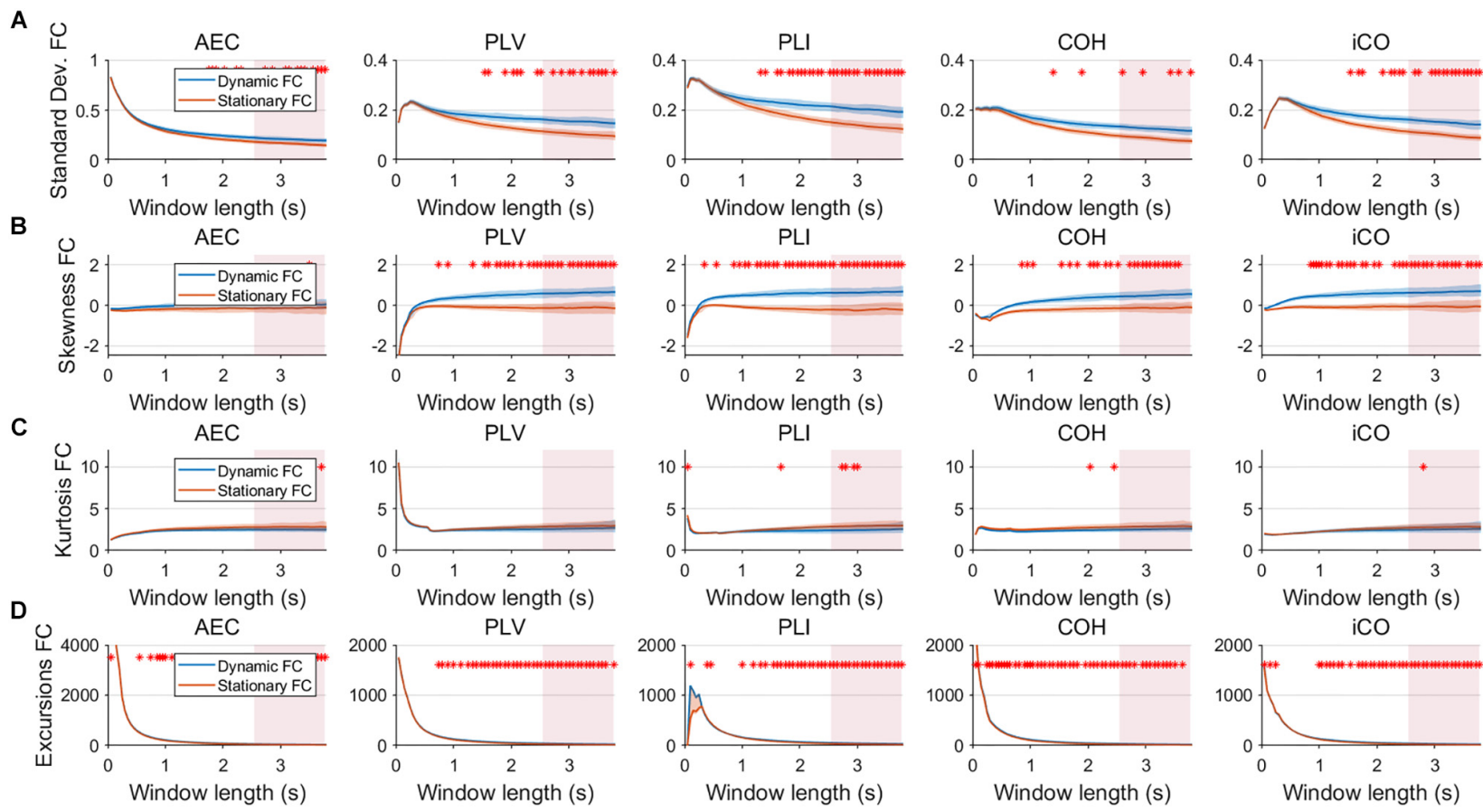

PLV

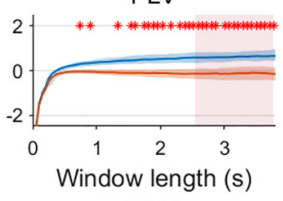

PLI

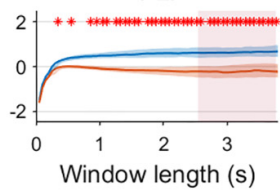

$\mathrm{COH}$
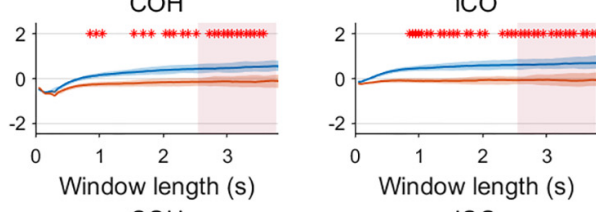

$\mathrm{COH}$

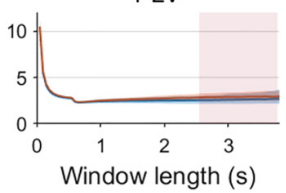

PLI

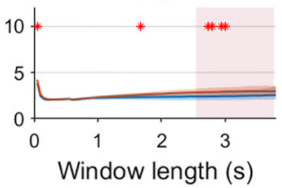

PLI
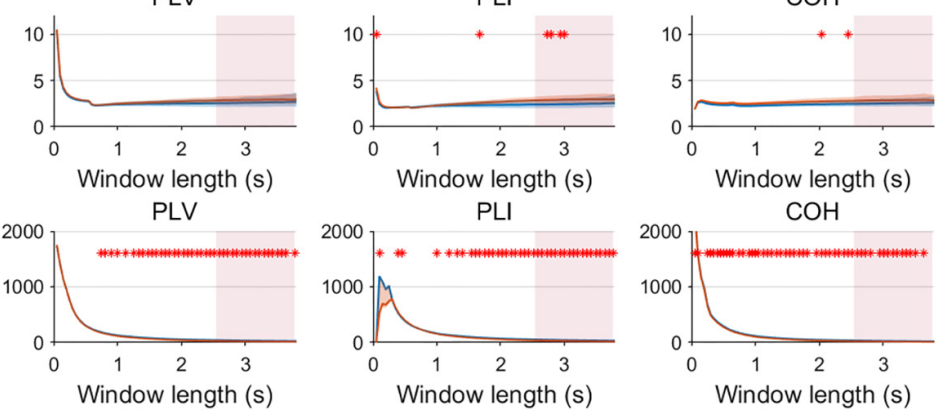

$\mathrm{iCO}$

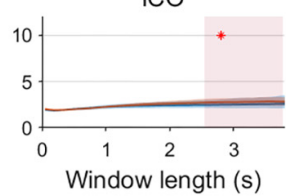

$\mathrm{COH}$
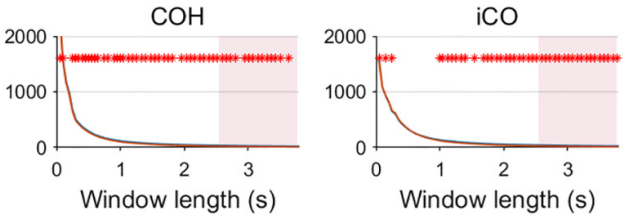

FIGURE 5 | Two node MAR model summary statistics in connectivity in a static vs. slow dynamic underlying system (SNR = 1). Panel (A) shows the standard deviation of connectivity across the number of windows for the static and dynamic underlying system (pink rectangles correspond to the underlying ground-truth of the range of the state durations). Panel (B) shows the skewness of the connectivity distributions. Panel (C) shows the kurtosis of the connectivity distributions and panel (D) the excursions from the median. Shaded areas correspond to the range across realizations/iterations. A red cross in each panel corresponds to a significant difference in distribution between a summary statistic for static and dynamic connectivity (Mann-Whitney test $p<0.01$ ) for the window length of interest.

tests to test the effect of window length on the correlations and mean error (test for repeated measures). This was done in order to analyze whether a change in the distributions of the correlations or mean absolute error was significantly different for subsequent window lengths.

\section{The effect of SNR}

We evaluated the effect of different SNR for different mean state durations on the ability of connectivity metrics to detect the underlying ground-truth connectivity. Again the correlation between the estimates and the underlying ground truths was evaluated.

\section{Network Analysis}

Detecting temporal fluctuations of resting state subnetworks

We evaluated whether the connectivity metrics are sensitive to detect switching of resting state subnetworks over time. Similar as for the two node system analysis, we temporally interpolated the estimated functional connectivity data since there was a dimension mismatch with the dimension of the structural connectivity tensor (time interval of a window is reduced to a single estimate for functional connectivity). Again note that there was only one resting state network active at every time point (DMN, SMN, FPN or visual), and the duration of the states (fast, medium, slow, mixed) determined the switching between four different resting state subnetworks. For every time point $t$ in the simulation, we tested whether the strength of within resting state network connectivity exceeded the magnitude of connectivity outside the resting state network at every point in time. This was tested for the a priori defined connections belonging to a resting state subnetwork.

\section{Detecting temporal fluctuations of resting state subnetworks with linear mixing}

The former analysis was repeated in a more realistic scenario. Source localization for empirical MEG/EEG data usually involves signal leakage, which manifests itself as a zero-time lag linear summation of underlying signals. Here, instead of a forward projection in our simulations from the network source nodes to sensors and an inverse projection to the sources (Chella et al., 2019), we opted to implement linear mixing to model such signal leakage (Lobier et al., 2014). Linear mixing was induced by adding for a time-series of a node $i$ the weighted activity of all other nodes using a linear combination, $\tilde{x}_{i}=x_{i}+\sum_{j, j \neq i}^{N} \frac{1}{\mathrm{~d}_{\mathrm{ij}}} \mathrm{x}_{\mathrm{j}}$, where the weights were chosen as the inverse Euclidean distance between two nodes $i$ and $j$. Lastly, prior to the calculation of the connectivity-metrics that are sensitive to signal leakage (coherence, amplitude envelope correlation and phase locking value), we used a symmetric multivariate leakage correction method to reduce the effects of signal leakage (Colclough et al., 2015). For the windows and metrics that did show significant differences in connectivity for the a priori defined connections within subnetworks and outside subnetworks, we also tested 


\section{A}

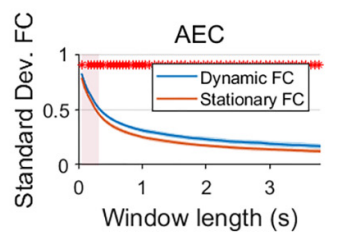

B

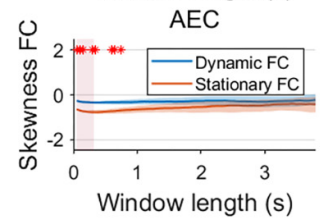

C

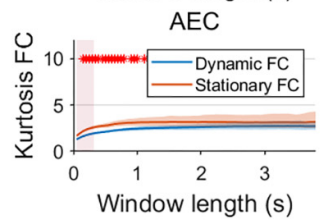

D

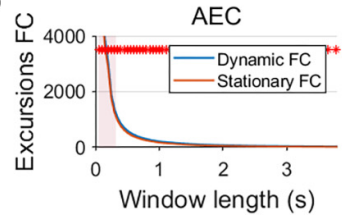

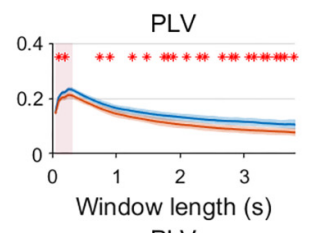

PLV

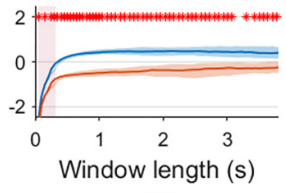

PLV

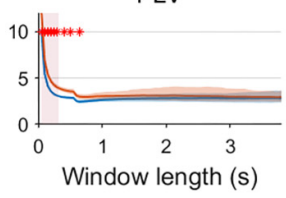

PLV

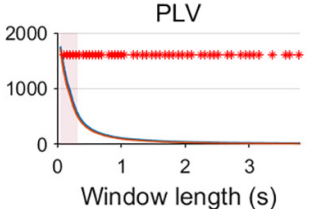

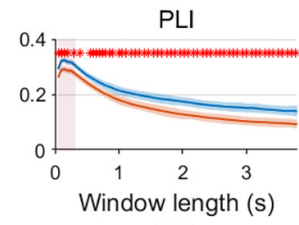

PLI

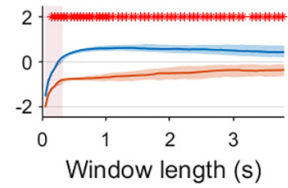

$\mathrm{PLI}$

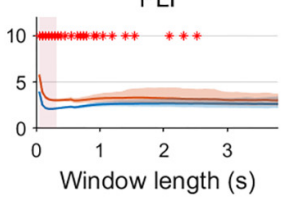

PLI

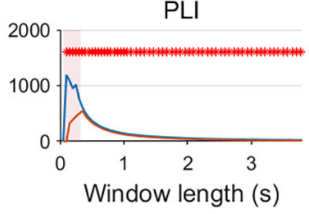

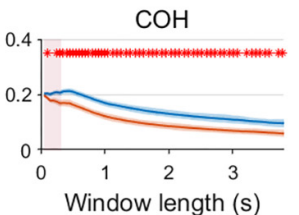

$\mathrm{COH}$

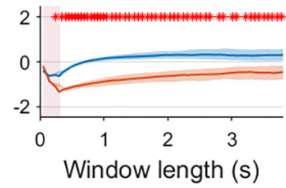

$\mathrm{COH}$

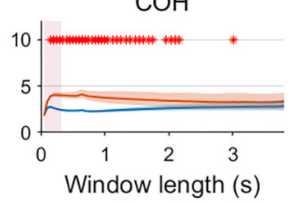

$\mathrm{COH}$

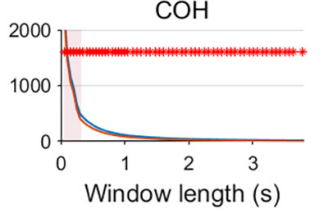

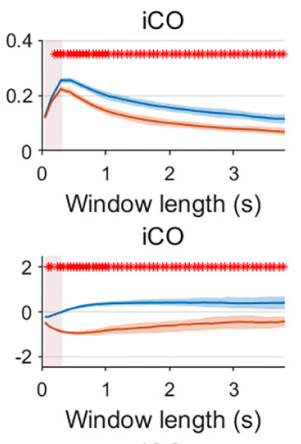

iCO

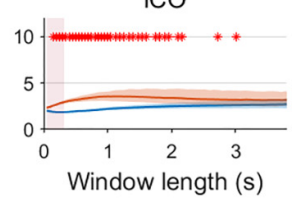

iCO

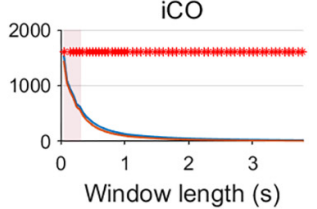

FIGURE 6 | Two node MAR model summary statistics for connectivity in a static vs. fast dynamic underlying system (SNR = 3). Panel (A) shows the standard deviation of connectivity across the number of windows for the static and dynamic underlying system (pink rectangles correspond to the underlying ground-truth of the range of the state durations). Panel (B) shows the skewness of the connectivity distributions. Panel (C) shows the kurtosis of the connectivity distributions and panel (D) the excursions from the median. Shaded areas correspond to the range across realizations/iterations. A red cross in each panel corresponds to a significant difference in distribution between a summary statistic for static and dynamic connectivity (Mann-Whitney test $p<0.01$ ) for the window length of interest.

whether time varying subnetworks could be retrieved from the data itself using non-negative tensor factorization.

\section{RESULTS}

\section{Two Node System: Estimates of Static vs. Dynamic Connectivity}

Fluctuations for dynamic connectivity in our simulations were characterized by fast, medium, slow and mixed states. Results are shown for the MAR and NMM models and for an SNR of 1 (Figures 3-5) and for an SNR of 3 (Figure 6) for the MAR model for the fast states. Figure $\mathbf{3 A}$ shows the variability (standard deviation) of connectivity estimates for the fast dynamic and the static system as a function of window length. We a priori expected higher variability for the fast dynamic case, since in its underlying timecourse of connectivity there was a frequent switch between three coupling values (low, medium, high connectivity), whereas for the static case there was constant coupling during the whole simulation. For all metrics, we could see that for an SNR of 1 connectivity estimates from a genuine dynamic connectivity timecourse showed the same variability as connectivity estimates based on the static connectivity timecourse (Mann-Whitney test for all windows $p>0.01$ ). For both the static and dynamic case, variability generally decreased as a function of the window length. A difference in skewness was observed between static connectivity (Figure 3B) and dynamic connectivity with higher skewness for dynamic connectivity for all measures but the AEC, but usually in window lengths that did not match the underlying state durations. Note that the positive skewness for the dynamic case for most metrics indicates that the connectivity distribution was non-Gaussian. Kurtosis and excursions from the median were significantly larger for the dynamic connectivity than for the static connectivity (Figures 3C,D), although for both, mostly not for the window widths that matched the underlying state durations. Although note that for the excursions from the median this was also significant for the correct window lengths. Simulations based on the NMM for an SNR of 1 and fast state durations showed similar results for most metrics and summary statistics (Figure 4). Again, excursions from the median was significantly higher for dynamic connectivity, especially for the window widths that matched the underlying state durations. Similar curves were also obtained for medium (Supplementary Figure S1), mixed (Supplementary Figure S2) and slow states (Figure 5) for the MAR model and NMM model (Supplementary Figures S3-S5), with the difference that especially for slow states there was a clear divergence in variability, skewness, kurtosis and excursions from the median between static and dynamic connectivity (Figure 5) for several window lengths, including the longer window lengths that matched the underlying state durations. Note that curves for fast states, medium and slow states have very similar shapes for both MAR and NMM, indicating that the form of these curves are largely determined by window length rather than state durations. 


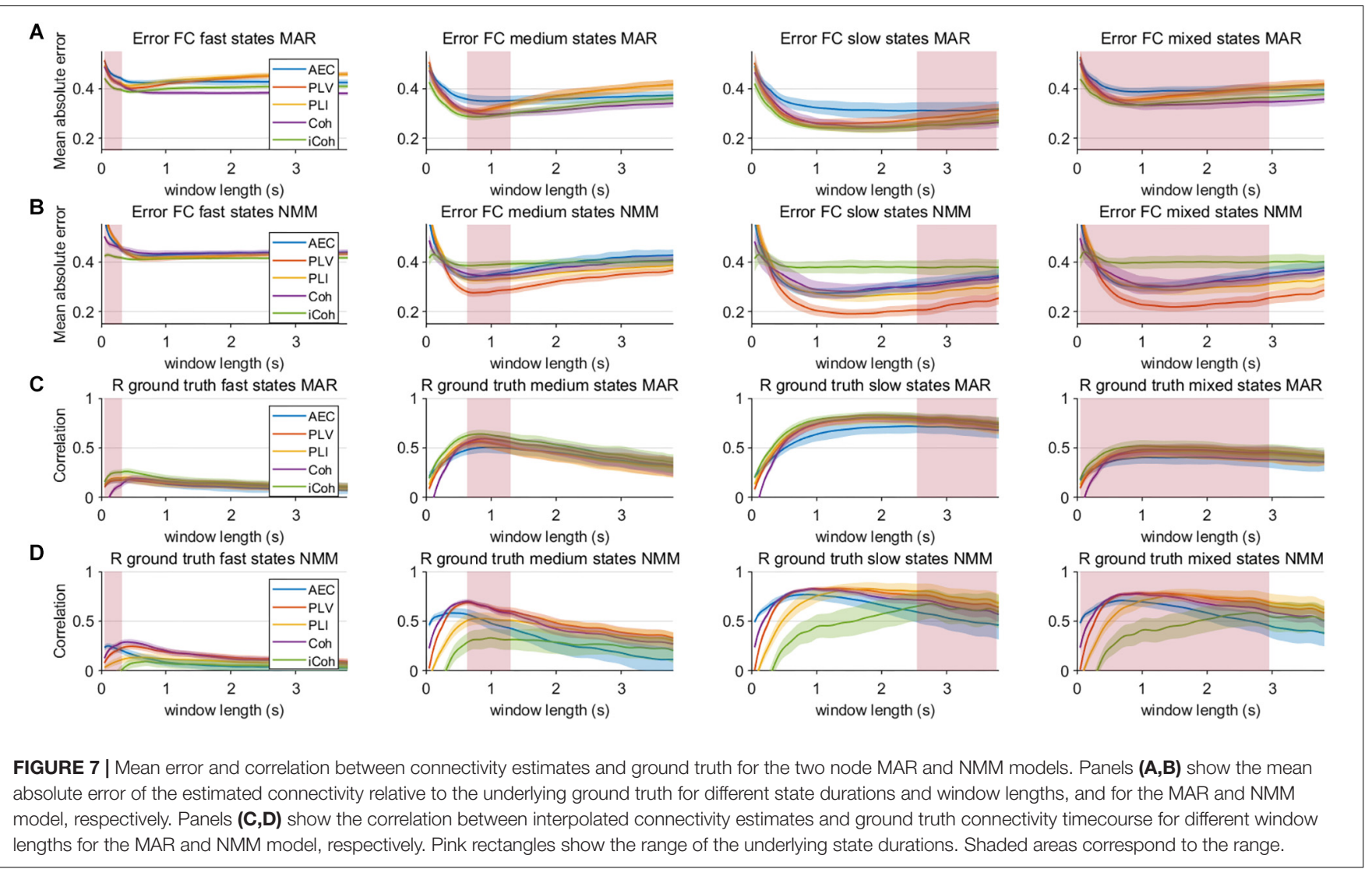

TABLE 1 | Non-parametric Friedman statistics for all correlation and mean absolute error for all metrics.

\begin{tabular}{|c|c|c|c|c|c|c|c|c|c|c|}
\hline & \multicolumn{2}{|c|}{ AEC } & \multicolumn{2}{|c|}{ PLV } & \multicolumn{2}{|c|}{ PLI } & \multicolumn{2}{|c|}{$\mathrm{COH}$} & \multicolumn{2}{|c|}{ ico } \\
\hline & $\chi^{2}(79)$ & $p$ & $\chi^{2}(79)$ & $p$ & $\chi^{2}(79)$ & $p$ & $\chi^{2}(79)$ & $p$ & $\chi^{2}(79)$ & $p$ \\
\hline Fast-states R & 213 & $<0.001$ & 560 & $<0.001$ & 523 & $<0.001$ & 234 & $<0.001$ & 429 & $<0.001$ \\
\hline Fast-states Error & 441 & $<0.001$ & 383 & $<0.001$ & 413 & $<0.001$ & 453 & $<0.001$ & 510 & $<0.001$ \\
\hline Medium-states R & 327 & $<0.001$ & 576 & $<0.001$ & 575 & $<0.001$ & 504 & $<0.001$ & 574 & $<0.001$ \\
\hline Slow-states R & 299 & $<0.001$ & 444 & $<0.001$ & 471 & $<0.001$ & 417 & $<0.001$ & 462 & $<0.001$ \\
\hline Slow-states Error & 451 & $<0.001$ & 510 & $<0.001$ & 489 & $<0.001$ & 515 & $<0.001$ & 514 & $<0.001$ \\
\hline Mixed-states R & 223 & $<0.001$ & 525 & $<0.001$ & 523 & $<0.001$ & 367 & $<0.001$ & 505 & $<0.001$ \\
\hline Mixed-states Error & 261 & $<0.001$ & 323 & $<0.001$ & 313 & $<0.001$ & 369 & $<0.001$ & 373 & $<0.001$ \\
\hline \multicolumn{11}{|l|}{ NMM model } \\
\hline Fast-states R & 472 & $<0.001$ & 595 & $<0.001$ & 278 & $<0.001$ & 644 & $<0.001$ & 259 & $<0.001$ \\
\hline Slow-states R & 536 & $<0.001$ & 657 & $<0.001$ & 541 & $<0.001$ & 694 & $<0.001$ & 514 & $<0.001$ \\
\hline Slow-states Error & 518 & $<0.001$ & 559 & $<0.001$ & 445 & $<0.001$ & 427 & $<0.001$ & 175 & $<0.001$ \\
\hline Mixed-states R & 572 & $<0.001$ & 652 & $<0.001$ & 506 & $<0.001$ & 691 & $<0.001$ & 483 & $<0.001$ \\
\hline Mixed-states Error & 543 & $<0.001$ & 531 & $<0.001$ & 422 & $<0.001$ & 412 & $<0.001$ & 174 & $<0.001$ \\
\hline
\end{tabular}

Significance corresponds to a significant change in modulation of the error or correlation with ground truth (R) for different window lengths. 
Having said this, increasing the SNR resulted in a better disentanglement of dynamic vs. static connectivity with our conventional metrics. Figure $\mathbf{6}$ shows the results for fast states for an SNR of 3. In contrast to results for an SNR of 1 (Figure 3 ), the range of the standard deviation for static and dynamic connectivity start to diverge for most metrics for even short window lengths (Figure 6A). A clear divergence between static and dynamic connectivity was also observed for skewness (Figure 6B), as well as for kurtosis (Figure 6C) and excursions from the median. Results for medium, slow and mixed states for an SNR of 3 showed similar disentanglement of dynamic vs. static connectivity (see Supplementary Figures S6-S8).

\section{Two Node System: Identifying Genuine Fluctuations in Dynamic Connectivity}

Results in the previous section show that especially for sufficient long window lengths, metrics could identify higher and genuine variability for the dynamic underlying system. However, this only indicates that metrics have some sensitivity to pick up differences between coupling and uncoupling, it does not indicate whether the correct duration of the state lifetimes could be captured. In order to address this, we computed the mean error and the correlation coefficient between ground truth connectivity timecourse and (interpolated) estimated connectivity timecourse. Figures 7A,B displays the mean error for the connectivity estimates for different metrics and state durations and for the different models (pink rectangle shows the range of true underlying state durations). This shows that the error increases for shorter state durations and for shorter window lengths for both the MAR and NMM simulations. For medium and slow state durations there seems to occur a plateau or minimum for the window lengths that roughly match the duration of the states. For fast states, there is no clear difference between the metrics in the window lengths that matches the underlying ground truth connectivity modulations. However, for slow states the error of the AEC is larger than for the other metrics for the MAR model, while for the NMM model the error of iCO is larger than for other metrics. There was a significant effect of window length for all metrics and state durations on the mean absolute error (see Table 1). Similar results can be observed for the correlation with the ground truth, i.e., the shorter the state durations, the lower the correlation of the connectivity estimates with the ground truth (Figures 7C,D). For short state durations, there is a poor correlation with the ground truth for all the metrics and for both NMM and MAR simulations. Again, there are maxima at, or around, (e.g., for short state durations) the window lengths that match the underlying state durations. There is no clear difference in performance for the different metrics in the MAR simulations, apart from the observation that the AEC underperforms for longer state durations. Whereas for the NMM simulations the iCO seem to perform worse for longer and mixed state durations. Again, there was a significant effect of window length for all metrics and state durations on the correlation with the ground truth (see Table 1).

\section{Two Node System: Sensitivity in Detecting Dynamic Connectivity for Different SNRs}

An important hurdle in the estimation of dynamic connectivity is the limited SNR of the data due to the inclusion only a relatively small number of samples. We therefore calculated the correlation of the connectivity estimates with the ground truth for various SNRs, and for both models (Figure 8). Results are only shown for the window length that matches the underlying state duration, i.e., the most optimal condition. For correlation with the ground truth the most important observation is that increases in SNR only lead to moderate increase of the correlation. Note that for all metrics, state durations and models, an increase in SNR eventually leads to a plateau where further increases in SNR hardly affects the correlation with the ground truth. The imaginary coherence seem to outperform the other metrics for fast state durations for the MAR model, which is the opposite for the NMM model. For the MAR model, for lower values of SNR the AEC seems to perform worse for slow states compared to the other metrics.

\section{Network Analysis: Detecting Temporal Fluctuations of Resting State Networks}

We extended our two node analysis to a large scale network analysis. Figure 9 shows the results for neural mass simulations with an SNR $=3$ and without linear mixing. For every mean resting state subnetwork duration of activity (fast - A, medium B, slow - C, mixed states - D), we show functional connectivity within the active resting state subnetworks and functional connectivity outside of the resting state subnetworks. For the AEC, PLV and $\mathrm{COH}$, we can observe that the estimate of connectivity within the resting state networks is higher than outside of these networks, especially for longer window lengths. PLI and iCO fail to show significant higher connectivity within resting state networks compared to outside network connectivity. Similarly as for the two node system, the curve of connectivity versus window length strongly depends on the selected window length rather than on the underlying duration of activity of the resting state subnetworks. Note that mostly there was a mismatch between the window length that showed significant differences and the underlying state durations, e.g., PLV and $\mathrm{COH}$ showed higher connectivity for resting state subnetworks compared to connectivity outside the subnetworks for longer window lengths than the underlying state durations (see Figure 9A). However, for AEC and PLV this also happens for longer window lengths that match the underlying dynamics of the slow states.

\section{Network Analysis: Detecting Temporal Fluctuations of Resting State Networks With Linear Mixing}

We repeated the same analysis as in the previous section for a connected network of neural mass with linear mixing for different resting state network durations (fast - A, medium - B, slow C, mixed states $-\mathrm{D}$ ). Results for an $\mathrm{SNR}=3$ did not show any significant difference between connectivity within resting 


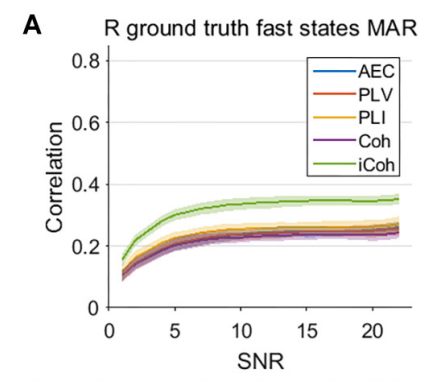

B $\quad \mathrm{R}$ ground truth fast states NMM

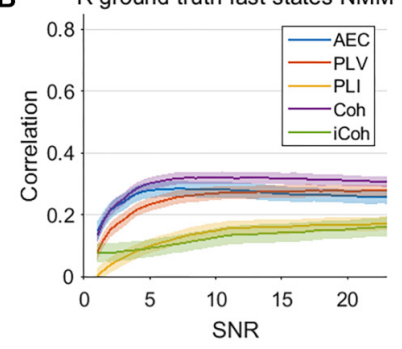

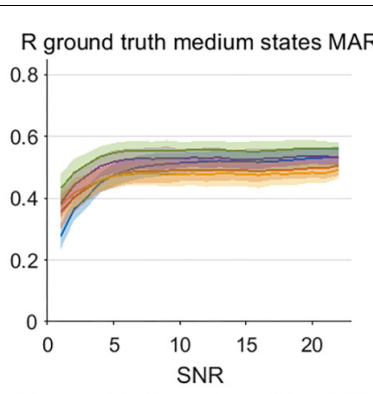

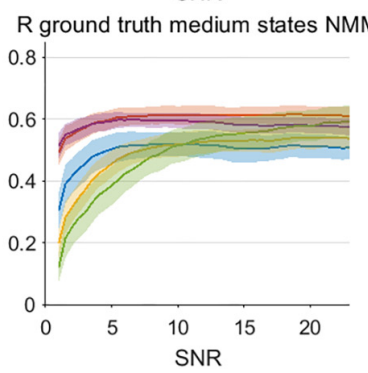

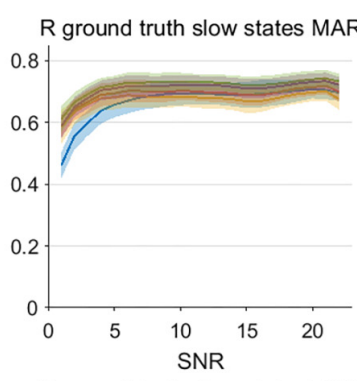

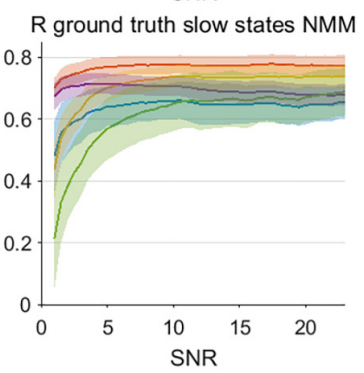

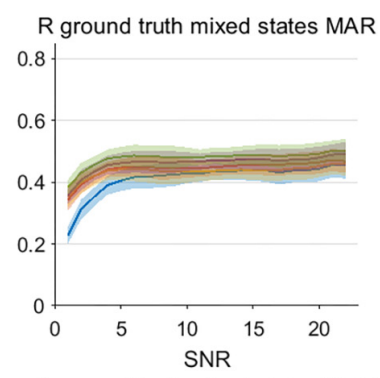

$\mathrm{R}$ ground truth mixed states NMM

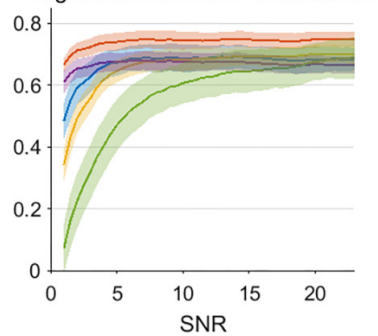

FIGURE 8 | Correlation between connectivity estimates and ground truth for different levels of SNR for the two node MAR and NMM models. Panels (A,B) show the correlation between interpolated connectivity estimates and ground truth connectivity timecourse for different levels of SNR for the MAR and NMM model, respectively. Shaded areas correspond to the range of correlation values.

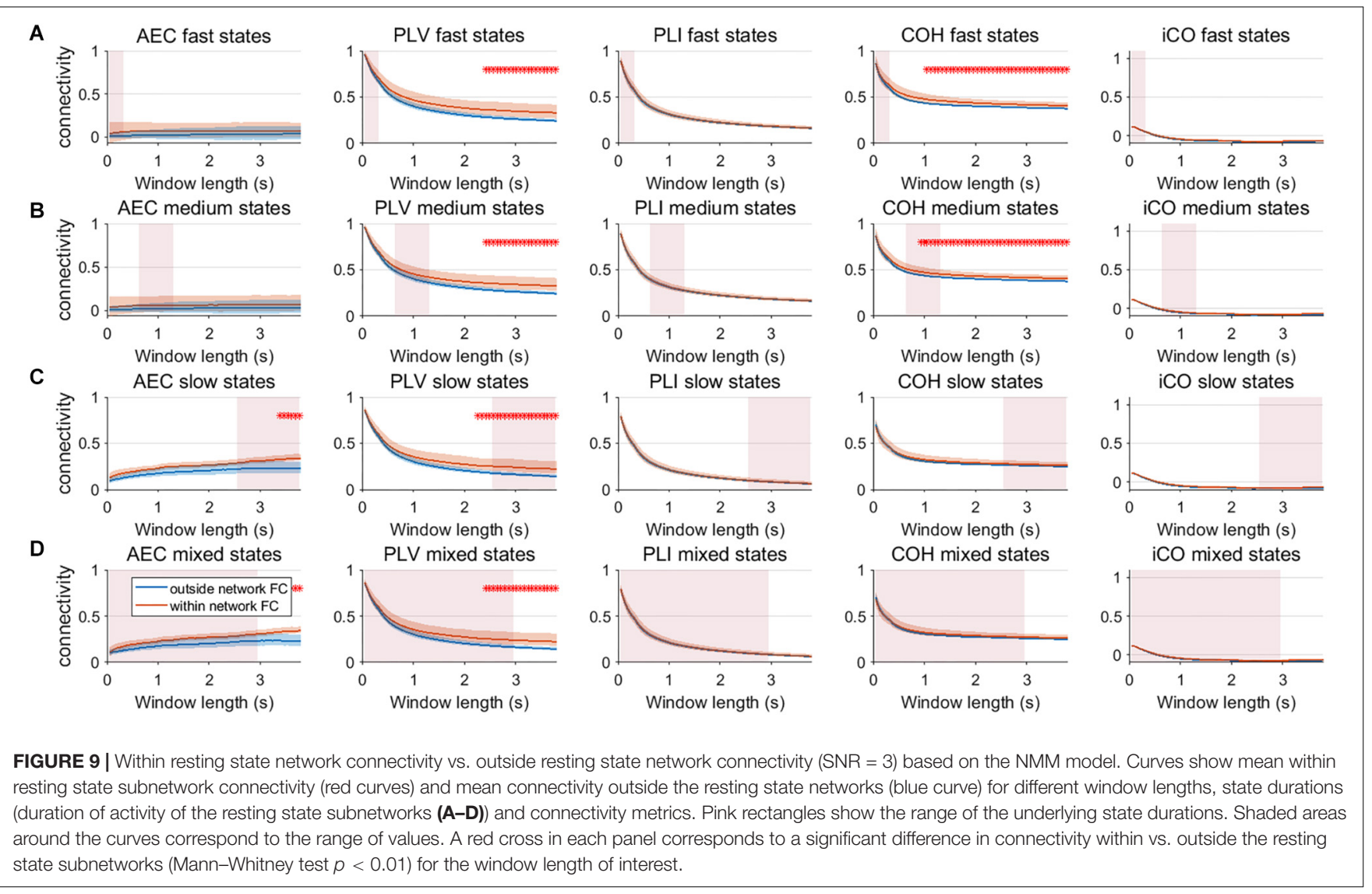

state networks and connectivity outside resting state networks (Supplementary Figure S9). Figure 10 shows the result for $\mathrm{SNR}=5$. Again, the curve for connectivity versus window is for all metrics very similar for different state durations, i.e., again the window length has a larger effect on estimations of connectivity than the underlying state durations. Within resting 
A

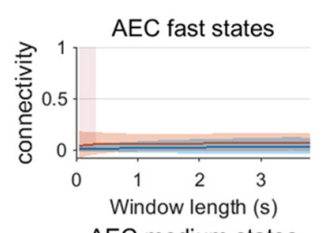

B

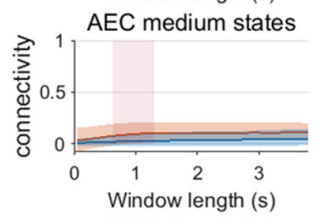

C

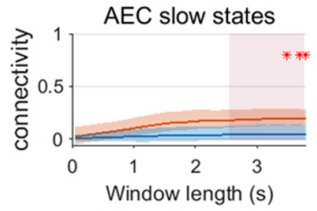

D

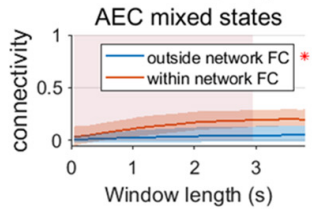

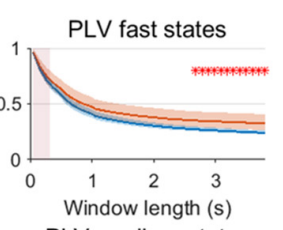

PLV medium states

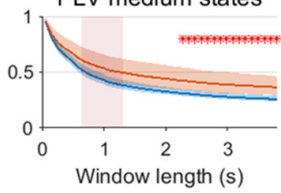

PLV slow states
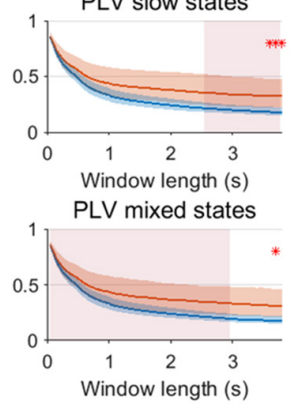

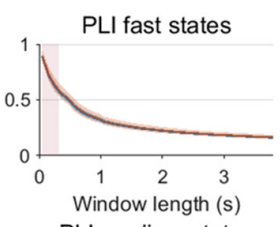

PLI medium states

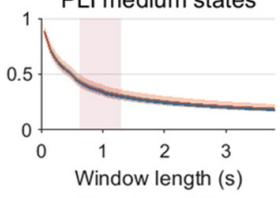

PLI slow states

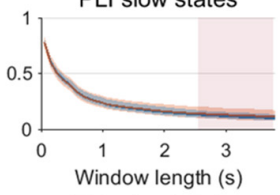

PLI mixed states

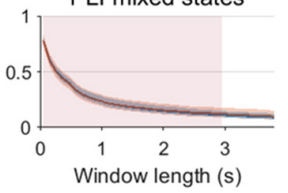

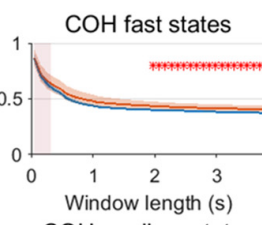
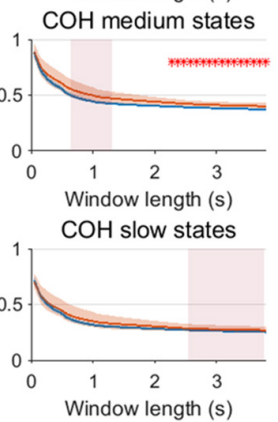

$\mathrm{COH}$ mixed states

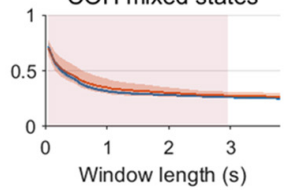

iCO fast states
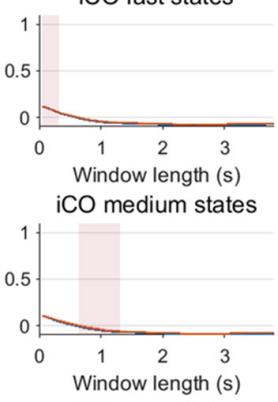

iCO slow states

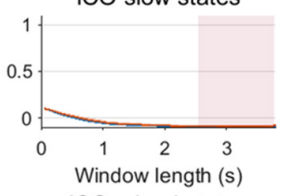

iCO mixed states

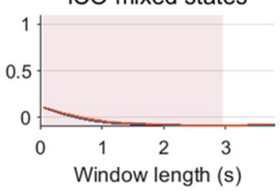

FIGURE 10 | Within resting state network connectivity vs. outside resting state network connectivity $(\mathrm{SNR}=5)$ based on the NMM model with linear mixing and symmetric leakage correction. Curves show mean within resting state subnetwork connectivity (red curves) and mean connectivity outside the resting state networks (blue curve) for different window lengths, state durations (duration of activity of the resting state subnetworks (A-D)) and connectivity metrics. Pink rectangles show the range of the underlying state durations. Shaded areas around the curves correspond to the range of values. A red cross in each panel corresponds to a significant difference in connectivity within vs. outside the resting state subnetworks (Mann-Whitney test $p<0.01$ ) for the window length of interest.

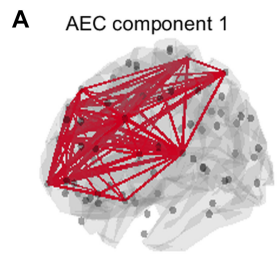

B

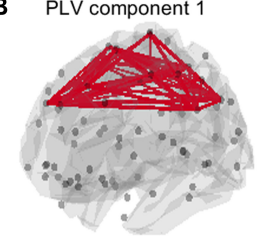

C

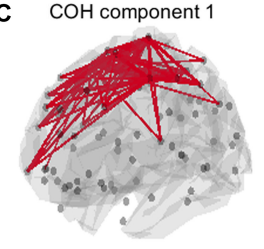

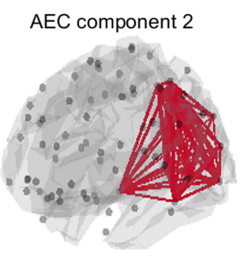

PLV component 2

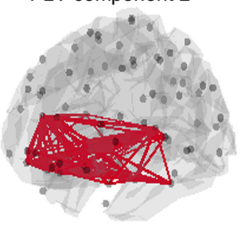

COH component 2

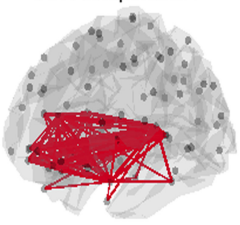

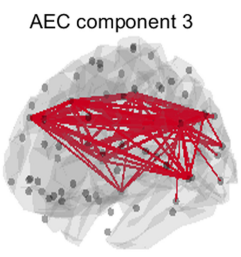

PLV component 3

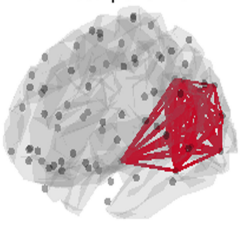

$\mathrm{COH}$ component 3

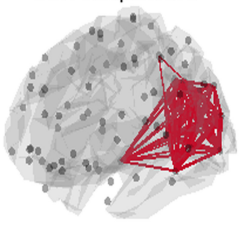

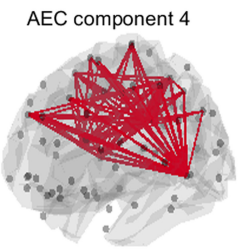

PLV component 4

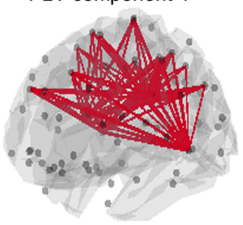

$\mathrm{COH}$ component 4

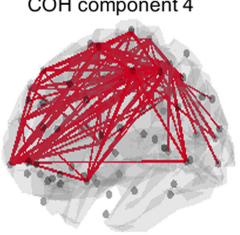

FIGURE 11 | Estimated spatial patterns of time varying networks using non-negative tensor factorization. Results are shown for an SNR $=5$ in a NMM model with linear mixing and symmetric leakage correction and for a window length of $4 \mathrm{~s}$ with slow underlying state durations. The upper row (A) shows estimated networks for the AEC (amplitude envelope correlation). The second row (B) shows estimated networks for the PLV (phase locking value), while the third row (C) shows estimated networks for the $\mathrm{COH}$ (Coherence). The upper 3\% of the connections within each component is illustrated. Note that for all metrics some of the a priori defined networks could be retrieved. A priori defined networks were the default mode network (DMN), the sensorimotor network (SMN), the frontoparietal networks (FPN) and the visual network (see Figure 2). 
state connectivity is again higher for the AEC, PLV and $\mathrm{COH}$, especially for longer window lengths. The window length for which there is significant difference between within resting state connectivity and connectivity outside the networks is on average longer than without linear mixing and symmetric leakage.

For the AEC, PLV and $\mathrm{COH}$, we tested for window lengths of $4 \mathrm{~s}$ whether we could retrieve the spatial patterns of the a-prior defined resting state networks. i.e., the DMN, the SMN, the FPN and the visual network (Figure 2). Figure 11 shows the spatial patterns of the estimated time varying networks for the slow states. Components estimated from AEC resembled three a priori defined networks, while components extracted from the PLV resembled two apparent resting state networks, and for $\mathrm{COH}$ three clearly recognizable networks could be obtained. For example, for the AEC, nonnegative tensor factorization retrieved a FPN with predominantly connections on the right, the visual network, default mode network, but no clear sensorimotor network. For the PLV we could observe a sensorimotor network and visual network, and a network reminiscent of the default mode network. Lastly, for the $\mathrm{COH}$ we could observe a clear visual network and two networks that had some spatial characteristics of the sensorimotor and FPN. To emphasize our previous analysis (shown in Figure 10), we could not identify clearcut networks for medium states and window lengths of $1 \mathrm{~s}$ for all the metrics, though the visual network was an exception (see Supplementary Figure S10). As was also hinted in Figure 10, for medium states, extraction of resting state networks improved for using longer windows (see Supplementary Figure S11), though networks were not as clearly recognizable as for slow states.

\section{DISCUSSION}

Despite advances in the field of dynamic connectivity, fixed sliding window approaches in conjunction with conventional metrics are still widely used to identify dynamic connectivity. Given the lack of ground truth in empirical MEG data, we used simulations based on parameterized MAR and NMM models informed by predefined timecourses of connectivity. We performed a step by step analysis in a two node system and in a large scale network. Two node analysis revealed that SNR should be sufficiently large in order to distinguish static connectivity from dynamic connectivity. Especially excursions from the median was the most sensitive measure to distinguish dynamic connectivity from static connectivity. All connectivity metrics, performed well to detect fluctuations in slow dynamic states and to some extent medium dynamic states. However, the identification of fast underlying ground truth states (mean state duration $125 \mathrm{~ms}$ ) was poor for all metrics. An increase in SNR resulted in only a moderate increase in identification of the ground truth connectivity timecourses. Network analysis indeed underscored that resting state networks could only be retrieved for sufficient levels of SNR and long state durations.

Variability has often been used as the outcome measure to quantify the dynamics of connectivity. In the current work variability was quantified by the standard deviation of connectivity across estimates from all sliding windows (with a constant width). Here, we show that variability unequal to zero in itself does not necessarily imply evidence for a dynamic underlying system (Figure 3). For both static and dynamic connectivity there was high variability for short window lengths, indicating that high variability can merely be an artifact of the window length. Also note that high variability for short states (Figure 3A) could co-occur with poor identification of the underlying connectivity timecourse (Figures 7C,D). Especially, for low SNR (SNR < 3), for fast and medium states variability could hardly distinguish genuine dynamic connectivity from static connectivity. Another hurdle with the use of the standard deviation of connectivity is that the connectivity distributions are mostly non-Gaussian, as evidenced by non-zero skewness. The skewness of the distribution was a more sensitive statistic to detect differences in static vs. dynamic connectivity, especially for the phase based metrics, though the window lengths for which this occurred did not necessarily match the underlying state durations. The kurtosis was found to be less useful in disentangling dynamic from static connectivity, while excursions from the median was sensitive for detecting genuine fluctuations in connectivity, especially for the window widths that match the underlying state duration. However, note that for both skewness and excursion, their magnitude is highly influenced by the width of the window.

Unlike in empirical data, we could test the performance of the metrics with an underlying ground truth. Results were fairly similar for the MAR and NMM simulations. All conventional metrics performed poorly when the ground truth contained fast states, as their correlation with the underlying ground truth timecourse was low. In other words, metrics are unable to quantify very brief states in a fixed sliding window approach. This therefore limits the use of fixed sliding window approaches for the detection of fast states. Our results showed that sliding window approaches are safe to use if the underlying states are at least of medium duration. Even an increase in SNR could not significantly improve the performance of the metrics for the fast states, implying that the low performance is not only related to SNR but also to the properties of the metrics. Recent studies have shown that in empirical resting state data fluctuations in amplitude and phase coupling can be well-described in a range from a few hundred milliseconds to seconds (Baker et al., 2014; Tewarie et al., 2018; Vidaurre et al., 2018). We mimicked this by the mixed state condition, which actually also show very moderate correlation for different metrics with the underlying ground truth, indicating that there is high uncertainty in detection of the underlying connectivity. Finally, the correlation with the ground truth was usually maximal for window lengths that more or less matched the state durations (except for longer state durations). A mismatch between window length and underlying state duration also led to a drop in correlation with the ground truth. This emphasizes the problem of an arbitrary window length for the estimation of dynamic connectivity with unknown, and most likely varying, state durations.

Another important observation is that one would beforehand expect that phase-based metrics would perform better for 
faster states, whereas the amplitude envelope correlation would perform better for slower states, since the amplitude envelopes modulates on slower timescales than the phases. This was especially obvious for the network simulations, where PLV and coherence were sensitive in detecting medium state durations, while AEC was only able to detect network connectivity for slow and mixed state durations. However, at the same time, this notion requires caution since performance of metrics seem to depend on the type of simulation (MAR vs. NMM). For example, in the MAR model, there is explicit parameterization of phase locking, which would favor estimates of phase locking over amplitude-amplitude coupling. Potentially, differences in MAR and NMM results could also be explained by differences in the intrinsic frequencies of the oscillations, i.e., beta band oscillations in MAR vs. alpha band oscillations in NMM. This may also explain the observed difference in performance of imaginary coherence for NMM vs. MAR simulations. Also note, that for most simulations, performance of metrics nearly converge for sufficient SNR or longer window lengths (see Figures 7, 8).

Our two node analysis was extended to a more realistic large scale network scenario. For simulations without linear mixing and leakage correction, metrics that were inherently sensitive for leakage, AEC, PLV and coherence were sensitive in detecting genuine fluctuations in connectivity. Coherence was even sensitive to detect within resting state network connectivity for medium state durations for windows that matched these state durations. However, this was also the case for longer window lengths that did not match the underlying state duration. Thus this indicates that significant higher connectivity, for a resting state network for a specific window length, does not necessarily imply that the underlying duration of temporal varying connectivity has the same temporal scale as the window width. Especially slow states were detectable by the AEC, PLV and $\mathrm{COH}$ for longer window widths. For the scenario with linear mixing and symmetric leakage correction, results showed that to extract meaningful resting state networks, we usually required longer windows, especially for the AEC. This finding is in line with empirical work, where meaningful time varying networks for AEC could be extracted during a working memory task for longer window widths (O’Neill et al., 2017a).

Some methodological issues warrant further discussion. A limitation of the current work is that our simulations are not necessarily a direct representation of electrophysiological data. However, a methodological issue with empirical data is its lack of ground truth. Connectivity analysis with empirical data would require the use of surrogate data (Dimitriadis et al., 2018). Previous work has illustrated that the choice and selection of surrogate methods is not trivial (O'Neill et al., 2017b) and conclusions regarding non-stationarity of connectivity based on surrogate data can be highly biased by the selection of the method itself. Extensive analysis of various surrogate methods in the context of dynamic connectivity is beyond the scope of this paper, but should surely be explored in future work (Dimitriadis et al., 2018). Another limitation is that other metrics of functional connectivity such as mutual information (Palus,
1997) or measures that characterize generalized synchronization, such the synchronization likelihood (Stam and Van Dijk, 2002), as well as metrics that estimate directed connectivity (Nolte et al., 2010; Lobier et al., 2014) were not included in our analysis. Here, we restricted our analysis to frequently used (and computationally inexpensive) phase- and amplitudebased functional connectivity metrics. In addition, ideally the effects of co-registration, lead field inaccuracies, and effects of inverse operator would also be simulated (Hincapié et al., 2017; Chella et al., 2019). However, these errors lead to reduced amplitude of the reconstructed signal. As a surrogate, and in order to reduce the number of simulations, these effects can be observed by examining the effects of SNR on our results. Lastly, network simulations were only performed for the NMM, since to boost neurobiological realism, we could implement distance dependent conduction delays. Implementing these distance dependent delays is not trivial in a linear MAR model, and therefore the MAR model was not used for this purpose.

In conclusion, we have demonstrated the strengths and limitations of metrics based on the two intrinsic modes of coupling (amplitude and phase) with regards to the detection of genuine fluctuations in functional connectivity. Fixed sliding window approaches have difficulty in detecting brief states, even when using short window lengths. Increasing SNR does not mitigate this sufficiently, especially for large scale networks. We therefore recommend the use of longer window lengths (at least 3-4 s) to estimate fluctuations in functional connectivity of resting state networks. Our simulations showed that PLV, AEC and coherence outperform imaginary coherence and PLI, which might advocate for the use of the former three metrics for the estimation of dynamic functional connectivity. Furthermore, an often used metric to quantify dynamic FC, variability, also comes with difficulties: high variability could co-occur with low correlation with the ground truth and can be an artifact of the used window length. Given the non-Gaussianity of most connectivity distributions, skewness may be a more appropriate metric to quantify genuine fluctuations in connectivity and in addition, excursions from the median could also be used in this context. However, again the magnitude of these metrics can be merely an artifact of the selected window length, and these metrics provide only meaningful information if they are tested against a null-hypothesis of static connectivity. Caution is therefore warranted when using these outcome measures in empirical data.

\section{DATA AVAILABILITY}

All datasets generated for this study are included in the manuscript and/or the Supplementary Files.

\section{AUTHOR CONTRIBUTIONS}

LL, AQ, MW, MB, and GO designed the study. AQ and LL wrote the code. LL, PT, and AQ analyzed the data. LL, AQ, GO, 
MW, AH, and PT wrote and edited the manuscript. All authors contributed to interpretation of results.

\section{FUNDING}

This work was funded by a Medical Research Council (MRC) New Investigator Research Grant (MR/M006301/1) and an associated studentship (to LL) funded by the University of

\section{REFERENCES}

Andersson, C. A., and Bro, R. (2000). The N-way toolbox for MATLAB. Chemom. Intell. Lab. Syst. 52, 1-4. doi: 10.1016/j.aca.2016.01.020

Baker, A. P., Brookes, M. J., Rezek, I. A., Smith, S. M., Behrens, T., Smith, P. J. P., et al. (2014). Fast transient networks in spontaneous human brain activity. Elife 3:e01867. doi: 10.7554/eLife.01867

Breakspear, M., Williams, L. M., and Stam, C. J. (2004). A novel method for the topographic analysis of neural activity reveals formation and dissolution of 'dynamic cell assemblies.'. J. Comput. Neurosci. 16, 49-68. doi: 10.1023/b: jcns.0000004841.66897.7d

Bro, R. (1997). PARAFAC. tutorial and applications. Chemom. Intell. Lab. Syst. 38, 149-171. doi: 10.1016/s0169-7439(97)00032-4

Brookes, M. J., Groom, M. J., Liuzzi, L., Hill, R. M., Smith, H. J. F., Briley, P. M., et al. (2018). Altered temporal stability in dynamic neural networks underlies connectivity changes in neurodevelopment. Neuroimage 174, 563-575. doi: 10.1016/j.neuroimage.2018.03.008

Brookes, M. J., Hale, J. R., Zumer, J. M., Stevenson, C. M., Francis, S. T., Barnes, G. R., et al. (2011). Measuring functional connectivity using MEG: methodology and comparison with fcMRI. Neuroimage 56, 1082-1104. doi: 10.1016/j.neuroimage.2011.02.054

Cabral, J., Luckhoo, H., Woolrich, M., Joensson, M., Mohseni, H., Baker, A., et al. (2014). Exploring mechanisms of spontaneous functional connectivity in MEG: how delayed network interactions lead to structured amplitude envelopes of band-pass filtered oscillations. Neuroimage 90, 423-435. doi: 10.1016/j. neuroimage.2013.11.047

Carbo, E. W. S., Hillebrand, A., Van Dellen, E., Tewarie, P., de Witt Hamer, P. C., Baayen, J. C., et al. (2017). Dynamic hub load predicts cognitive decline after resective neurosurgery. Sci. Rep. 7:42117. doi: 10.1038/srep42117

Chella, F., Marzetti, L., Stenroos, M., Parkkonen, L., Ilmoniemi, R. J., Romani, G. L., et al. (2019). The impact of improved MEG-MRI co-registration on MEG connectivity analysis. Neuroimage 197, 354-367. doi: 10.1016/j.neuroimage. 2019.04.061

Colclough, G. L., Brookes, M. J., Smith, S. M., and Woolrich, M. W. (2015). A symmetric multivariate leakage correction for MEG connectomes. Neuroimage 117, 439-448. doi: 10.1016/j.neuroimage.2015.03.071

Colclough, G. L., Woolrich, M. W., Tewarie, P. K., Brookes, M. J., Quinn, A. J., and Smith, S. M. (2016). How reliable are MEG resting-state connectivity metrics? Neuroimage 138, 284-293. doi: 10.1016/j.neuroimage.2016.05.070

David, O., Cosmelli, D., and Friston, K. J. (2004). Evaluation of different measures of functional connectivity using a neural mass model. Neuroimage 21, 659-673. doi: 10.1016/j.neuroimage.2003.10.006

de Pasquale, F., Della Penna, S., Sporns, O., Romani, G. L., and Corbetta, M. (2016). A dynamic core network and global efficiency in the resting human brain. Cereb. Cortex 26, 4015-4033. doi: 10.1093/cercor/bhv185

Dimitriadis, S. I., Routley, B., Linden, D. E., and Singh, K. D. (2018). Reliability of static and dynamic network metrics in the resting-state: a MEG-beamformed connectivity analysis. Front. Neurosci. 12:506. doi: 10.3389/fnins.2018.00506

Farahibozorg, S.-R., Henson, R. N., and Hauk, O. (2018). Adaptive cortical parcellations for source reconstructed EEG/MEG connectomes. Neuroimage 169, 23-45. doi: 10.1016/j.neuroimage.2017.09.009

Gauvin, L., Panisson, A., and Cattuto, C. (2014). Detecting the community structure and activity patterns of temporal networks: a non-negative tensor factorization approach. PLoS One 9:e86028. doi: 10.1371/journal.pone.008 6028
Nottingham. We also acknowledge Medical Research Council Partnership Grant (MR/K005464/1).

\section{SUPPLEMENTARY MATERIAL}

The Supplementary Material for this article can be found online at: https://www.frontiersin.org/articles/10.3389/fnins. 2019.00797/full\#supplementary-material

Grimbert, F., and Faugeras, O. (2006). Bifurcation analysis of Jansen's neural mass model. Neural Comput. 18, 3052-3068. doi: 10.1162/neco.2006.18.12.3052

Hincapié, A.-S., Kujala, J., Mattout, J., Pascarella, A., Daligault, S., Delpuech, C., et al. (2017). The impact of MEG source reconstruction method on sourcespace connectivity estimation: a comparison between minimum-norm solution and beamforming. Neuroimage 156, 29-42. doi: 10.1016/j.neuroimage.2017. 04.038

Hindriks, R., Adhikari, M. H., Murayama, Y., Ganzetti, M., Mantini, D., Logothetis, N. K., et al. (2016). Can sliding-window correlations reveal dynamic functional connectivity in resting-state fMRI? Neuroimage 127, 242-256. doi: 10.1016/j. neuroimage.2015.11.055

Hipp, J. F., Hawellek, D. J., Corbetta, M., Siegel, M., and Engel, A. K. (2012). Large-scale cortical correlation structure of spontaneous oscillatory activity. Nat. Neurosci. 15, 884-890. doi: 10.1038/nn.3101

Jansen, B. H., and Rit, V. G. (1995). Electroencephalogram and visual evoked potential generation in a mathematical model of coupled cortical columns. Biol. Cybern. 73, 357-366. doi: 10.1007/s004220050191

Kim, J., Criaud, M., Cho, S. S., Díez-Cirarda, M., Mihaescu, A., Coakeley, S., et al. (2017). Abnormal intrinsic brain functional network dynamics in Parkinson's disease. Brain 140, 2955-2967. doi: 10.1093/brain/awx233

Lachaux, J.-P., Rodriguez, E., Martinerie, J., and Varela, F. J. (1999). Measuring phase synchrony in brain signals. Hum. Brain Mapp. 8, 194-208. doi: 10.1002/ (sici)1097-0193(1999)8:4<194::aid-hbm4>3.0.co;2-c

Laumann, T. O., Snyder, A. Z., Mitra, A., Gordon, E. M., Gratton, C., Adeyemo, B., et al. (2016). On the stability of BOLD fMRI correlations. Cereb. cortex 27, 4719-4732. doi: 10.1093/cercor/bhw265

Liuzzi, L., Gascoyne, L. E., Tewarie, P. K., Barratt, E. L., Boto, E., and Brookes, M. J. (2017). Optimising experimental design for MEG resting state functional connectivity measurement. Neuroimage 155, 565-576. doi: 10.1016/ j.neuroimage.2016.11.064

Lobier, M., Siebenhühner, F., Palva, S., and Palva, J. M. (2014). Phase transfer entropy: a novel phase-based measure for directed connectivity in networks coupled by oscillatory interactions. Neuroimage 85, 853-872. doi: 10.1016/j. neuroimage.2013.08.056

Neumaier, A., and Schneider, T. (2001). Estimation of parameters and eigenmodes of multivariate autoregressive models. ACM Trans. Math. Softw. 27, 27-57. doi: $10.1145 / 382043.382304$

Nolte, G., Bai, O., Wheaton, L., Mari, Z., Vorbach, S., and Hallett, M. (2004). Identifying true brain interaction from EEG data using the imaginary part of coherency. Clin. Neurophysiol. 115, 2292-2307. doi: 10.1016/j.clinph.2004.04. 029

Nolte, G., Ziehe, A., Krämer, N., Popescu, F., and Müller, K.-R. (2010). Comparison of granger causality and phase slope index. JMLR Workshop Con. Proc. 6, 267-276.

O’Neill, G. C., Bauer, M., Woolrich, M. W., Morris, P. G., Barnes, G. R., and Brookes, M. J. (2015). Dynamic recruitment of resting state sub-networks. Neuroimage 115, 85-95. doi: 10.1016/j.neuroimage.2015.04.030

O’Neill, G. C., Tewarie, P. K., Colclough, G. L., Gascoyne, L. E., Hunt, B. A. E., Morris, P. G., et al. (2017a). Measurement of dynamic task related functional networks using MEG. Neuroimage 146, 667-678. doi: 10.1016/j.neuroimage. 2016.08.061

O’Neill, G. C., Tewarie, P., Vidaurre, D., Liuzzi, L., Woolrich, M. W., and Brookes, M. J. (2017b). Dynamics of large-scale electrophysiological networks: a technical review. Neuroimage 180(Pt B), 559-576. doi: 10.1016/j.neuroimage. 2017.10.003 
Palus, M. (1997). Detecting phase synchronization in noisy systems. Phys. Lett. A 235, 341-351. doi: 10.1016/s0375-9601(97)00635-x

Siegel, M., Donner, T. H., and Engel, A. K. (2012). Spectral fingerprints of largescale neuronal interactions. Nat. Rev. Neurosci. 13, 121-134. doi: 10.1038/ nrn3137

Stam, C. J., Montez, T., Jones, B. F., Rombouts, S., Van Der Made, Y., Pijnenburg, Y. A. L., et al. (2005). Disturbed fluctuations of resting state EEG synchronization in Alzheimer's disease. Clin. Neurophysiol. 116, 708-715. doi: 10.1016/j.clinph.2004.09.022

Stam, C. J., Nolte, G., and Daffertshofer, A. (2007). Phase lag index: assessment of functional connectivity from multi channel EEG and MEG with diminished bias from common sources. Hum. Brain Mapp. 28, 1178-1193. doi: 10.1002/ hbm.20346

Stam, C. J., and Van Dijk, B. W. (2002). Synchronization likelihood: an unbiased measure of generalized synchronization in multivariate data sets. Phys. D Nonlinear Phenom. 163, 236-251. doi: 10.1016/s0167-2789(01)00386-4

Tewarie, P., Abeysuriya, R., Byrne, Á, O’Neill, G. C., Sotiropoulos, S. N., Brookes, M. J., et al. (2019a). ). How do spatially distinct frequency specific MEG networks emerge from one underlying structural connectome? The role of the structural eigenmodes. Neuroimage 186, 211-220. doi: 10.1016/j.neuroimage. 2018.10.079

Tewarie, P., Liuzzi, L., O’Neill, G. C., Quinn, A. J., Griffa, A., Woolrich, M. W., et al. (2019b). Tracking dynamic brain networks using high temporal resolution MEG measures of functional connectivity. Neuroimage. 200, 38-50. doi: 10 . 1016/j.neuroimage.2019.06.006

Tewarie, P., Bright, M. G., Hillebrand, A., Robson, S. E., Gascoyne, L. E., Morris, P. G., et al. (2016). Predicting haemodynamic networks using electrophysiology: the role of non-linear and cross-frequency interactions. Neuroimage 130, $273-$ 292. doi: 10.1016/j.neuroimage.2016.01.053

Tewarie, P., Hunt, B. A. E., O’Neill, G. C., Byrne, Á, Aquino, K. M., Bauer, M., et al. (2018). Relationships between neuronal oscillatory amplitude and dynamic functional connectivity. Cereb. Cortex 29, 2668-2681. doi: 10.1093/ cercor/bhy136

Tronarp, F., Subramaniyam, N. P., Särkkä, S., and Parkkonen, L. (2018). “Tracking of dynamic functional connectivity from MEG data with Kalman filtering," in
2018 40th Annual International Conference of the IEEE Engineering in Medicine and Biology Society (EMBC), (Piscataway, NJ: IEEE), 1003-1006.

Tzourio-Mazoyer, N., Landeau, B., Papathanassiou, D., Crivello, F., Etard, O., Delcroix, N., et al. (2002). Automated anatomical labeling of activations in SPM using a macroscopic anatomical parcellation of the MNI MRI single-subject brain. Neuroimage 15, 273-289. doi: 10.1006/nimg.2001. 0978

Vidaurre, D., Hunt, L. T., Quinn, A. J., Hunt, B. A. E., Brookes, M. J., Nobre, A. C., et al. (2018). Spontaneous cortical activity transiently organises into frequency specific phase-coupling networks. Nat. Commun. 9:2987. doi: 10.1038/s41467018-05316-z

Vidaurre, D., Quinn, A. J., Baker, A. P., Dupret, D., Tejero-Cantero, A., and Woolrich, M. W. (2016). Spectrally resolved fast transient brain states in electrophysiological data. Neuroimage 126, 81-95. doi: 10.1016/j.neuroimage. 2015.11.047

Wang, H. E., Bénar, C. G., Quilichini, P. P., Friston, K. J., Jirsa, V. K., and Bernard, C. (2014). A systematic framework for functional connectivity measures. Front. Neurosci. 8:405. doi: 10.3389/fnins.2014.00405

Watanabe, T., and Rees, G. (2017). Brain network dynamics in high-functioning individuals with autism. Nat. Commun. 8:16048. doi: 10.1038/ncomms16048

Zalesky, A., Fornito, A., Cocchi, L., Gollo, L. L., and Breakspear, M. (2014). Time-resolved resting-state brain networks. Proc. Natl. Acad. Sci. U.S.A. 111, 10341-10346. doi: 10.1073/pnas.1400181111

Conflict of Interest Statement: The authors declare that the research was conducted in the absence of any commercial or financial relationships that could be construed as a potential conflict of interest.

Copyright (C) 2019 Liuzzi, Quinn, O’Neill, Woolrich, Brookes, Hillebrand and Tewarie. This is an open-access article distributed under the terms of the Creative Commons Attribution License (CC BY). The use, distribution or reproduction in other forums is permitted, provided the original author(s) and the copyright owner(s) are credited and that the original publication in this journal is cited, in accordance with accepted academic practice. No use, distribution or reproduction is permitted which does not comply with these terms. 


\section{OPEN ACCESS}

Edited by:

Roberto Esposito

A.O. Ospedali Riuniti Marche

Nord, Italy

Reviewed by:

Olga V. Martynova,

Institute of Higher Nervous Activity and Neurophysiology (RAS), Russia

Jarod L. Roland,

Washington University in St. Louis,

United States

*Correspondence:

Alena Damborská

adambor@med.muni.cz

Specialty section:

This article was submitted to

Neuroimaging and Stimulation,

a section of the journal

Frontiers in Psychiatry

Received: 23 March 2019

Accepted: 15 July 2019

Published: 09 August 2019

Citation:

Damborská A, Tomescu Ml,

Honzírková E, Barteček $R$,

Hořínková J, Fedorová S, Ondruš Š and Michel CM (2019)

EEG Resting-State Large-Scale Brain

Network Dynamics Are Related to

Depressive Symptoms.

Front. Psychiatry 10:548.

doi: 10.3389/fpsyt.2019.00548

\section{EEG Resting-State Large-Scale Brain Network Dynamics Are Related to Depressive Symptoms}

\author{
Alena Damborská 1,2*, Miralena I. Tomescu ${ }^{1}$, Eliška Honzirková2, Richard Barteček², \\ Jana Hořínková ${ }^{2}$, Sylvie Fedorová ${ }^{2}$, Šimon Ondruš ${ }^{2}$ and Christoph M. Michel ${ }^{1,3}$ \\ ${ }^{1}$ Department of Basic Neurosciences, Campus Biotech, University of Geneva, Geneva, Switzerland, ${ }^{2}$ Department of \\ Psychiatry, Faculty of Medicine, Masaryk University and University Hospital Brno, Brno, Czechia, ${ }^{3}$ Lemanic Biomedical \\ Imaging Centre (CIBM), Geneva, Switzerland
}

Background: The few previous studies on resting-state electroencephalography (EEG) microstates in depressive patients suggest altered temporal characteristics of microstates compared to those of healthy subjects. We tested whether resting-state microstate temporal characteristics could capture large-scale brain network dynamic activity relevant to depressive symptomatology.

Methods: To evaluate a possible relationship between the resting-state large-scale brain network dynamics and depressive symptoms, we performed EEG microstate analysis in 19 patients with moderate to severe depression in bipolar affective disorder, depressive episode, and recurrent depressive disorder and in 19 healthy controls.

Results: Microstate analysis revealed six classes of microstates (A-F) in global clustering across all subjects. There were no between-group differences in the temporal characteristics of microstates. In the patient group, higher depressive symptomatology on the Montgomery-Åsberg Depression Rating Scale correlated with higher occurrence of microstate A (Spearman's rank correlation, $r=0.70, p<0.01$ ).

Conclusion: Our results suggest that the observed interindividual differences in restingstate EEG microstate parameters could reflect altered large-scale brain network dynamics relevant to depressive symptomatology during depressive episodes. Replication in larger cohort is needed to assess the utility of the microstate analysis approach in an objective depression assessment at the individual level.

Keywords: EEG microstates, large-scale brain networks, resting state, dynamic brain activity, major depressive disorder, bipolar disorder

\section{INTRODUCTION}

Major depressive disorder (MDD) and bipolar disorder are among the most serious psychiatric disorders with high prevalence and illness-related disability (1-3). Despite growing evidence for the spectrum concept of mood disorders (4), and even with the advanced neuroimaging methods developed in recent years, the underlying pathophysiological mechanisms of depression remain poorly understood. Evidence across resting-state functional magnetic resonance (fMRI) studies consistently points to an impairment of large-scale resting-state brain networks in MDD rather than a disruption of discrete brain regions (5-8). Consistent with the neurobiological model of depression (9), 
numerous resting-state fMRI studies show decreased frontal cortex function and increased limbic system function in patients with MDD (10). Functional abnormalities in large-scale brain networks include hypoconnectivity within the frontoparietal network (7) and the reward circuitry, centered around the ventral striatum (11). Reduced functional connectivity in first-episode drug-naïve patients with MDD was also recently reported between the frontoparietal and cingulo-opercular networks (12). Moreover, hyperconnectivity of the default mode network (13) and amygdala hyperconnectivity with the affective salience network $(14,15)$ were shown to be characteristic features of depression.

In general, large-scale networks dynamically re-organize themselves on sub-second temporal scales to enable efficient functioning $(16,17)$. Fast temporal dynamics of large-scale neural networks, not accessible with the low temporal resolution of the fMRI technique, can be investigated by analyzing the temporal characteristics of "EEG microstates" $(18,19)$. Scalp EEG measures the electric potential generated by the neuronal activity in the brain with a temporal resolution in the millisecond range. A sufficient number of electrodes distributed over the scalp, i.e., high density-EEG (HD-EEG), allows for the reconstruction of a scalp potential map representing the global brain activity (20). Any change in the map topography reflects a change in the distribution and/or orientation of the active sources in the brain (21). Already in 1987 (22), Lehmann et al. observed that in spontaneous resting-state EEG, the topography of the scalp potential map remains stable for a short period of time and then rapidly switches to a new topography in which it remains stable again. Ignoring map polarity, the duration of these stable topographies is around 80-120 ms. Lehmann called these short periods of stability EEG microstates and attributed them to periods of synchronized activity within large-scale brain networks. For a recent review, see Ref. (19). Assessment of the temporal characteristics of these microstates provides information about the dynamics of large-scale brain networks, because this technique simultaneously considers signals recorded from all areas of the cortex. Since the temporal variation in resting-state brain network dynamics may be a significant biomarker of illness and therapeutic outcome (23-25), microstate analysis is a highly suitable tool for this purpose.

Numerous studies demonstrated changes in EEG microstates in patients with neuropsychiatric disorders such as schizophrenia, dementia, panic disorder, multiple sclerosis, and others [for reviews see Refs. $(19,26)]$. Despite the potential of microstate analysis for detecting global brain dynamic impairment, microstates were not investigated in depressive patients, except for three studies that provided inconsistent results. Using adaptive segmentation of resting state EEG in depressive patients, two early studies showed abnormal microstate topographies and reduced overall average microstate duration (27) but unchanged numbers of different microstates per second (28). In a more recent study using a topographical atomize-agglomerate hierarchical clustering algorithm, abnormally increased overall microstate duration and decreased overall microstate occurrence per second were reported in treatment-resistant depression (29).
A better understanding of disruption and changes in brain network dynamics in depression is critical for developing novel and targeted treatments, e.g., deep brain stimulation in treatmentresistant depression (30). Furthermore, microstate features reflecting the disruption of brain network dynamics might be later tested as candidate biomarkers of depressive disorder and predictors of treatment response. Thus, the main goal of our study was to explore how resting-state microstate dynamics are affected in depressive patients as compared to healthy individuals. We hypothesized that patients with depression will show different microstate dynamics than healthy controls in terms of the temporal characteristics of EEG microstates such as duration, coverage, and occurrence. We also hypothesized that microstate dynamics will be related to the overall clinical severity of depression.

\section{MATERIALS AND METHODS}

\section{Subjects}

Data was collected from 19 depressive patients (age in years: mean = 53.0, standard deviation $=9.8 ; 6$ females ) and 19 healthy control $(\mathrm{HC})$ subjects (age in years: mean $=51.4$, standard deviation $=9.1$; 6 females). Education was classified into three levels: $1=$ no high school, 2 = high school, 3 = university studies in the depressed $($ mean $=1.9$, standard deviation $=0.9)$ and $\mathrm{HC}($ mean $=2.2$, standard deviation $=0.7$ ) groups. There were no differences in gender, and an independent sample $t$-test also showed no significant differences in age [ $t$-value $(\mathrm{df} 36)=0.45, p>0.05]$ or education [ $t$-value $(\mathrm{df} 36)=-1.5, p>0.05]$ between the two groups. The patients were recruited at the Department of Psychiatry, Faculty of Medicine, Masaryk University and University Hospital Brno. The diagnostic process had two steps and was determined based on the clinical evaluation by two board-certified psychiatrists. First, the diagnosis was made according to the criteria for research of the International Classification of Disorders (ICD-10). Second, the diagnosis was confirmed by the Mini International Neuropsychiatric interview (M.I.N.I.) according to the Diagnostic and Statistical Manual of Mental Disorders, Fifth Edition (DSM-V). All patients were examined in the shortest time period after the admission and before the stabilisation of treatment, typically during their first week of hospitalization. All patients met the criteria for at least a moderate degree of depression within the following affective disorders: bipolar affective disorder (F31), depressive episode (F32), and recurrent depressive disorder (F33). Exclusion criteria for patients were any psychiatric or neurological comorbidity, IQ $<70$, organic disorder with influence on the brain function, alcohol dependence, or other substance dependence. All patients were in the on-medication state with marked interindividual variability in specific medicaments received. The patient characteristics are summarized in Table 1. Control subjects were recruited by general practitioners from their database of clients. Control subjects underwent the M.I.N.I. by board-certified psychiatrists to ensure that they had no previous or current psychiatric disorder according to the DSM-V criteria. The scores on the Montgomery-Åsberg Depression Rating Scale (MADRS), a specific questionnaire validated for patients with mood disorders 
TABLE 1 | Patient characteristics.

\begin{tabular}{|c|c|c|c|c|c|c|c|c|}
\hline Patient & $\begin{array}{c}\text { ICD-10 } \\
\text { diagnose }\end{array}$ & $\begin{array}{l}\text { Number of } \\
\text { episodes }\end{array}$ & $\begin{array}{c}\text { Illness } \\
\text { duration } \\
\text { (years) }\end{array}$ & $\begin{array}{l}\text { MADRS } \\
\text { score }\end{array}$ & CGI score & BZD & AD/AP/MS & $\begin{array}{c}\text { Medication } \\
\text { scale AD/AP/ } \\
\text { MS }\end{array}$ \\
\hline 1 & F31.4 & 3 & 2 & 27 & 4 & 2 & $\mathrm{AD}, \mathrm{AP}, \mathrm{MS}$ & 3 \\
\hline 2 & F32.2 & 1 & 0.5 & 24 & 5 & 0 & $A D$ & 2 \\
\hline 3 & F32.1 & 1 & 1 & 15 & 4 & 2 & $A D$ & 2 \\
\hline 4 & F31.5 & 5 & 20 & 39 & 6 & 0 & $\mathrm{AP}$ & 2 \\
\hline 5 & F33.1 & 3 & 7 & 18 & 4 & 0 & $A D$ & 1 \\
\hline 6 & F33.1 & 2 & 8 & 9 & 3 & 1.33 & $A D$ & 1 \\
\hline 7 & F32.1 & 1 & 1 & 24 & 4 & 1.33 & $A D, A P$ & 3 \\
\hline 8 & F31.4 & 4 & 27 & 29 & 5 & 2 & $\mathrm{AP}$ & 2 \\
\hline 9 & F33.3 & 2 & 5 & 36 & 6 & 1 & $A D, A P$ & 4 \\
\hline 10 & F33.1 & 3 & 19 & 21 & 4 & 1 & $A D$ & 1 \\
\hline 11 & F33.3 & 2 & 2 & 38 & 5 & 6 & $A D, A P$ & 4 \\
\hline 12 & F33.2 & 2 & 1 & 39 & 5 & 3 & $A D, A P$ & 4 \\
\hline 13 & F32.3 & 1 & 0.08 & 21 & 5 & 2 & $A D, A P$ & 4 \\
\hline 14 & F33.2 & 5 & 21 & 32 & 5 & 0 & $A D, A P$ & 3 \\
\hline 15 & F33.3 & 2 & 2 & 38 & 6 & 3 & $A D, A P$ & 4 \\
\hline 16 & F32.3 & 1 & 0.08 & 37 & 6 & 2 & $A D, A P$ & 4 \\
\hline 17 & F33.1 & 3 & 4 & 18 & 4 & 0 & $A D, A P$ & 4 \\
\hline 18 & F31.3 & 2 & 16 & 28 & 4 & 0 & AP, MS & 4 \\
\hline 19 & F31.3 & 11 & 24 & 23 & 4 & 1 & AP, MS & 4 \\
\hline
\end{tabular}

F31.3, Bipolar affective disorder, current episode mild or moderate depression; F31.4, Bipolar affective disorder, current episode severe depression without psychotic symptoms; F31.5, Bipolar affective disorder, current episode severe depression with psychotic symptoms; F32.1, Moderate depressive episode; F32.2, Severe depressive episode without psychotic symptoms; F32.3, Severe depressive episode with psychotic symptoms; F33.1, Recurrent depressive disorder, current episode moderate; F33.2, Recurrent depressive disorder, current episode severe without psychotic symptoms; F33.3, Recurrent depressive disorder, current episode severe with psychotic symptoms; BZD, benzodiazepine equivalent dose (31); AD, antidepressants (mirtazapine, citalopram, venlafaxine, vortioxetine, sertraline); AP, antipsychotics (risperidone, olanzapine, quetiapine, amisulpride, aripiprazole); MS, mood stabilizers (valproate, lamotrigine); medication scale AD/AP/MS: 1, one medication in sub-therapeutic doses; 2 , one medication in therapeutic doses; 3, combination of medications with one in therapeutic doses; 4, combination of medications with more than one in therapeutic doses; MADRS (Montgomery-Åsberg Depression Rating Scale): score is between 0 and 60, the higher the score the higher the depressive symptom severity; CGI (Clinical Global Impression) scale: healthy (1) - most extremely ill (7). Four patients were undergoing the first (patient 3) and second (patients 4 and 9) week of electroconvulsive therapy and the first week of repetitive transcranial magnetic stimulation (patient 5). No clinical effect of these neurostimulation treatments was apparent.

(32), and Clinical Global Impression (CGI) (33), a general test validated for mental disorders, were used to evaluate the severity of depressive symptoms in patients. The status of depression was further described with lifetime count of depressive episodes and illness duration in years. Medication in $24 \mathrm{~h}$ preceding the EEG examination was also recorded (see Table 1). This study was carried out in accordance with the recommendations of Ethics Committee of University Hospital Brno with written informed consent from all subjects. All subjects gave written informed consent in accordance with the Declaration of Helsinki. The protocol was approved by the Ethics Committee of University Hospital Brno, Czech Republic.

\section{EEG Recording and Pre-processing}

Subjects were sitting in a comfortable upright position in an electrically shielded room with dimmed light. They were instructed to stay as calm as possible to keep their eyes closed and to relax for $15 \mathrm{~min}$. They were asked to stay awake. All participants were monitored by the cameras, and in the event of signs of nodding off or EEG signs of drowsiness detected by online visual inspection, the recording was stopped. The EEG was recorded with a high density 128-channel system (EGI System 400; Electrical Geodesic Inc., OR, USA), a sampling rate of $1 \mathrm{kHz}$, and $\mathrm{Cz}$ as acquisition reference.

Five minutes of the EEG data were selected based on visual assessment of the artifacts. The EEG was band-pass filtered between 1 and $40 \mathrm{~Hz}$. Subsequently, in order to remove ballistocardiogram and oculo-motor artifacts, infomax-based Independent Component Analysis (34) was applied to all but one or two channels rejected due to abundant artifacts. Only components related to ballistocardiogram, saccadic eye movements, and eye blinking were removed based on the waveform, topography, and time course of the component. The cleaned EEG recording was down-sampled to $125 \mathrm{~Hz}$, and the previously identified noisy channels were interpolated using a three-dimensional spherical spline (35) and re-referenced to the average reference. For subsequent analyses, the EEG data was reduced to 110 channels to remove muscular artifacts originating in the neck and face. All the preprocessing steps were done using the freely available Cartool Software 3.70, programmed by Denis Brunet Cartool (https://sites.google.com/site/cartoolcommunity/ home) and MATLAB.

\section{Microstate Analysis}

The microstate analysis (see Figure 1) followed the standard procedure using $k$-means clustering method to estimate the optimal set of topographies explaining the EEG signal (36-38). The polarity of the maps was ignored in this clustering procedure. To determine the optimal number of clusters, we applied a metacriterion that is a combination of seven independent optimization criteria [for details see Ref. (39)]. In order to improve the signalto-noise ratio, only the data at the time points of the local 
A

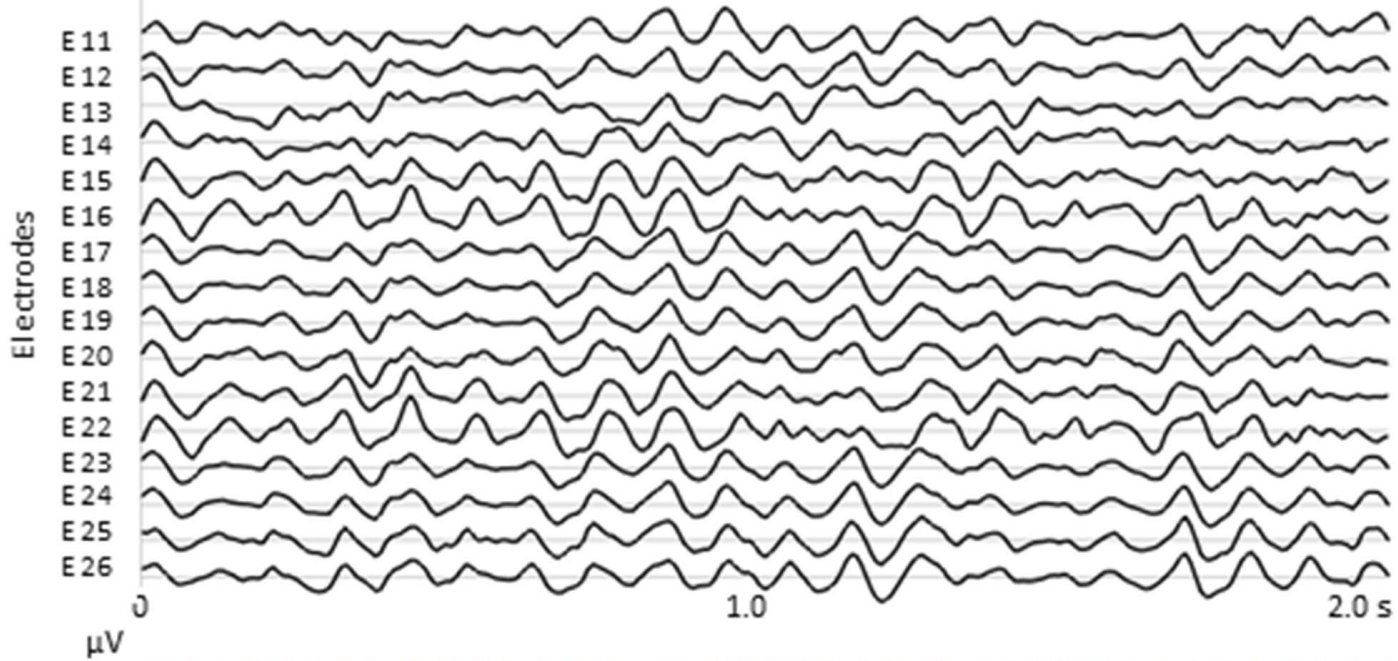

B : ${ }_{0}^{10} \mathrm{~m}$ 0

C

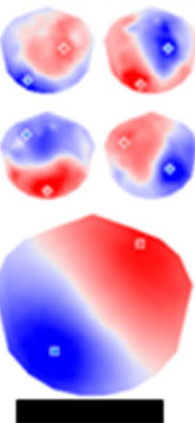

A

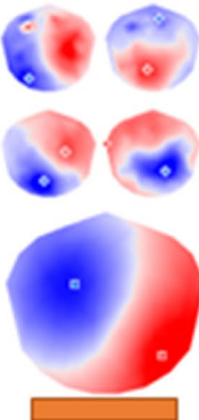

B

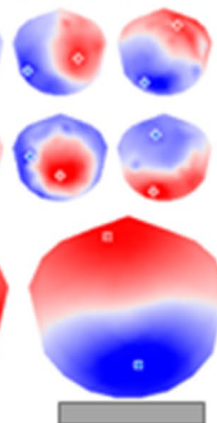

C
1,0

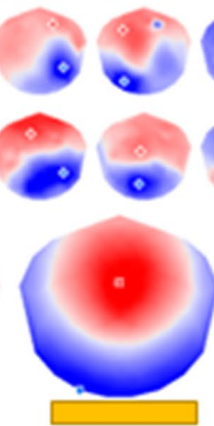

D

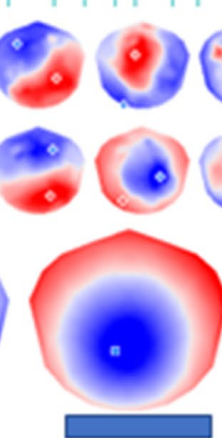

$\mathrm{E}$
$2.0 \mathrm{~s}$

30
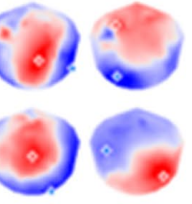
$-30$

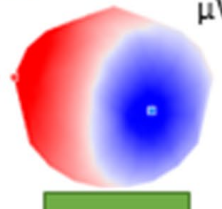

$\mathrm{F}$

$\mathbf{E}$

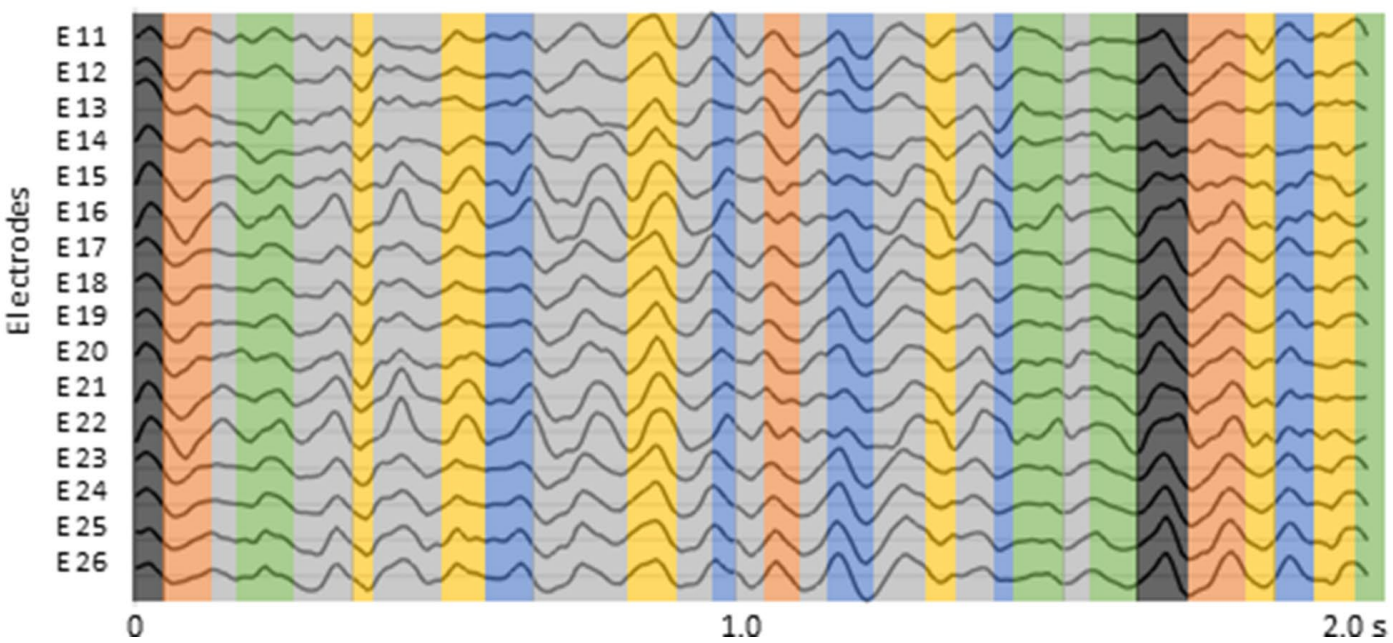

FIGURE 1 | Microstate analysis: (A) resting-state EEG from subsample of 16 out of 110 electrodes; (B) global field power (GFP) curve with the GFP peaks (vertical lines) in the same EEG period as shown in (A); (C) potential maps at successive GFP peaks, indicated in (B), from the first $1 \mathrm{~s}$ period of the recording; (D) set of six cluster maps best explaining the data as revealed by K-means clustering of the maps at the GFP peaks; (E) the original EEG recording shown in (A) with superimposed color-coded microstate segments. Note that each time point of the EEG recording was labelled with the cluster map, shown in (D), with which the instant map correlated best. The duration of segments, occurrence, and coverage for all microstates were computed on thus labeled EEG recording. 
maximum of the global field power (GFP) were clustered (38, 40-42). The GFP is a scalar measure of the strength of the scalp potential field and is calculated as the standard deviation of all electrodes at a given time point $(36,37,43)$. The cluster analysis was first computed at the individual level and then at global level across all participants (patients and controls).

Spatial correlation was calculated between every map identified at the global level and the individual subject's topographical map in every instant of the pre-processed EEG recording. Each continuous time point of the subject's EEG (not only the GFP peaks) was then assigned to the microstate class of the highest correlation, again ignoring polarity $(19,36,39,44)$. Temporal smoothing parameters [window half size $=3$, strength (Besag Factor $)=10$ ] ensured that the noise during low GFP did not artificially interrupt the temporal segments of stable topography $(36,38)$. For each subject, three temporal parameters were then calculated for each of the previously identified microstates: i) occurrence, ii) coverage, and iii) duration. Occurrence indicates how many times a microstate class recurs in $1 \mathrm{~s}$. The coverage represents the summed amount of time spent in a given microstate class. The duration in milliseconds for a given microstate class indicates the amount of time that a given microstate class is continuously present. In order to assess the extent to which the representative microstate topographies explain the original EEG data, the global explained variance (GEV) was calculated as the sum of the explained variances of each microstate weighted by the GFP. Microstate analysis was performed using the freely available Cartool Software 3.70, programmed by Denis Brunet Cartool (https://sites.google.com/site/cartoolcommunity/home).

\section{Statistical Analysis}

To investigate group differences, independent $t$-tests were used for temporal parameters of each microstate. Comparisons were corrected using the false discovery rate (FDR) method (45). In order to evaluate the possible relation of microstate dynamics to severity of depression, we computed Spearman's rank correlation coefficients of all microstate parameters with the MADRS and CGI scores and number of episodes. In order to evaluate possible influence of medication on microstate dynamics, we calculated Spearman's rank correlation coefficients between all microstate parameters and medication that patients received during $24 \mathrm{~h}$ preceding the EEG measurement. Intake of antidepressants, antipsychotics, and mood stabilizers was indicated as a single ordinal variable taking into account the number of medicaments and their dosages. Intake of benzodiazepines was expressed with the benzodiazepine equivalent dose (33). A significance level of $a<0.01$ was used for all correlations. Statistical evaluation of the results was performed by the routines included in the program package Statistica'13 (1984-2018, TIBCO, Software Inc, Version 13.4.0.14).

\section{RESULTS}

The meta-criterion used to determine the most dominant topographies revealed six microstates explaining $82.6 \%$ of the global variance. Four topographies resembled those previously reported in the literature as A, B, C, and D maps $(19,29,40,41)$ and two topographies resembled the recently identified (46) resting-state microstate maps. We labeled these maps as A-D, in accordance with previous literature, and as $\mathrm{E}$ and $\mathrm{F}$ (Figure 2).

The groups did not differ in any temporal parameter in any microstate. The depressive group was indistinguishable from the control group (all absolute $t$-values $<2.5$ ). The FDR-corrected $p$-values (six comparisons for the six microstate classes) were not significant between the patients and controls for any microstate in the duration $(\mathrm{A}: p=0.39 ; \mathrm{B}: p=0.39 ; \mathrm{C}: p=0.30 ; \mathrm{D}: p=0.39$; $\mathrm{E}: p=0.77$; F: $p=0.68$ ), occurrence (A: $p=0.13$; B: $p=0.92$; C: $p=0.92 ; \mathrm{D}: p=0.92 ; \mathrm{E}: p=0.13 ; \mathrm{F}: p=0.29)$, or coverage $(\mathrm{A}: p=$ $0.44 ; \mathrm{B}: p=0.75$; $: p=0.44 ; \mathrm{D}: p=0.75 ; \mathrm{E}: p=0.16$; F $: p=0.44)$.

The results of Spearman's rank correlation revealed a positive association of the depression severity with the presence of microstate A but not with the presence of other microstates. The occurrence of microstate A significantly correlated with the MADRS scores $(r=0.70, p<0.01$; Figure 3), but not with the CGI score $(r=0.40)$, illness duration $(r=0.06)$, or the number of episodes $(r=0.08)$. There were no significant associations between the depression severity and the duration or coverage of microstate A (all absolute $r$-values < 0.55).

The results of Spearman's rank correlation revealed a significant positive association between the medication status and the presence of microstate $\mathrm{E}$ but not with the presence of other microstates. The occurrence of microstate E significantly correlated with the intake of antidepressants, antipsychotics, and mood stabilizers $(r=0.65, p<0.01$; Figure 4$)$, but not with the intake of benzodiazepines $(r=0.20)$. There were no significant associations between the medication status and the duration or coverage of microstate $\mathrm{E}$ (all absolute $r$-values $<0.45$ ).

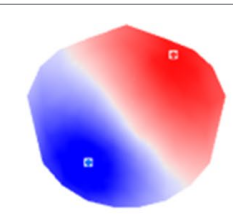

A

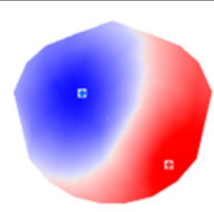

B

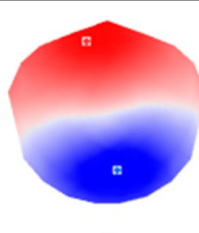

C

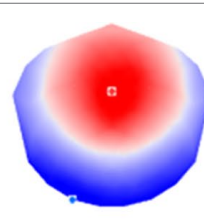

D

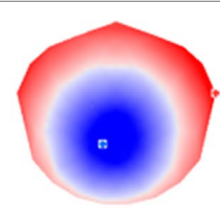

$\mathrm{E}$

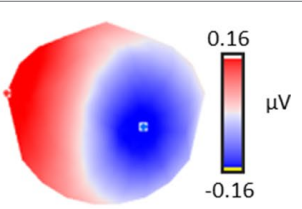

$\mathrm{F}$

FIGURE 2 | The six microstate topographies identified in the global clustering across all subjects 


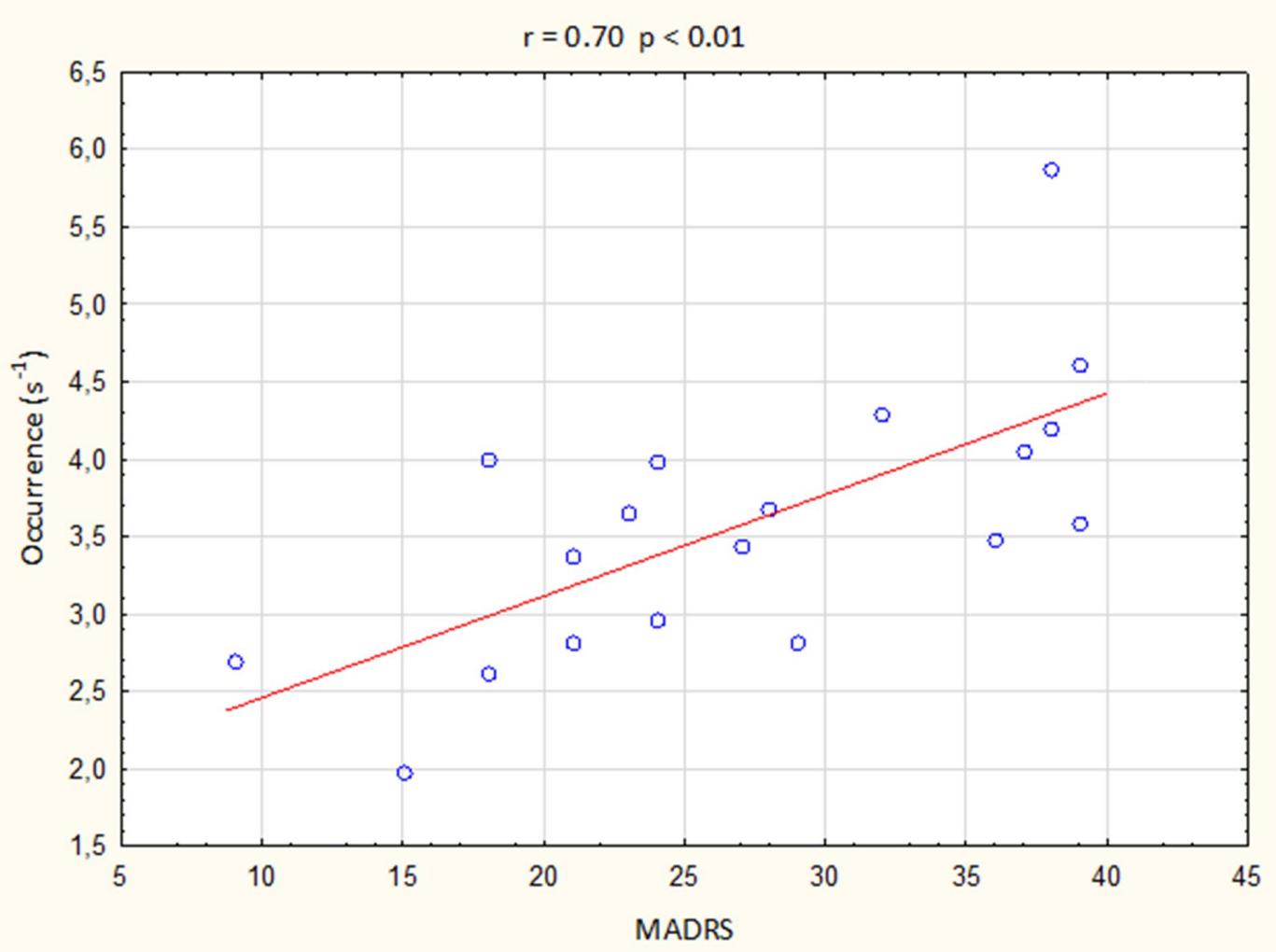

FIGURE 3 | Correlation between the occurrence of microstate A and Montgomery-Åsberg Depression Rating Scale (MADRS) score.

$$
r=0.65 p<0.01
$$

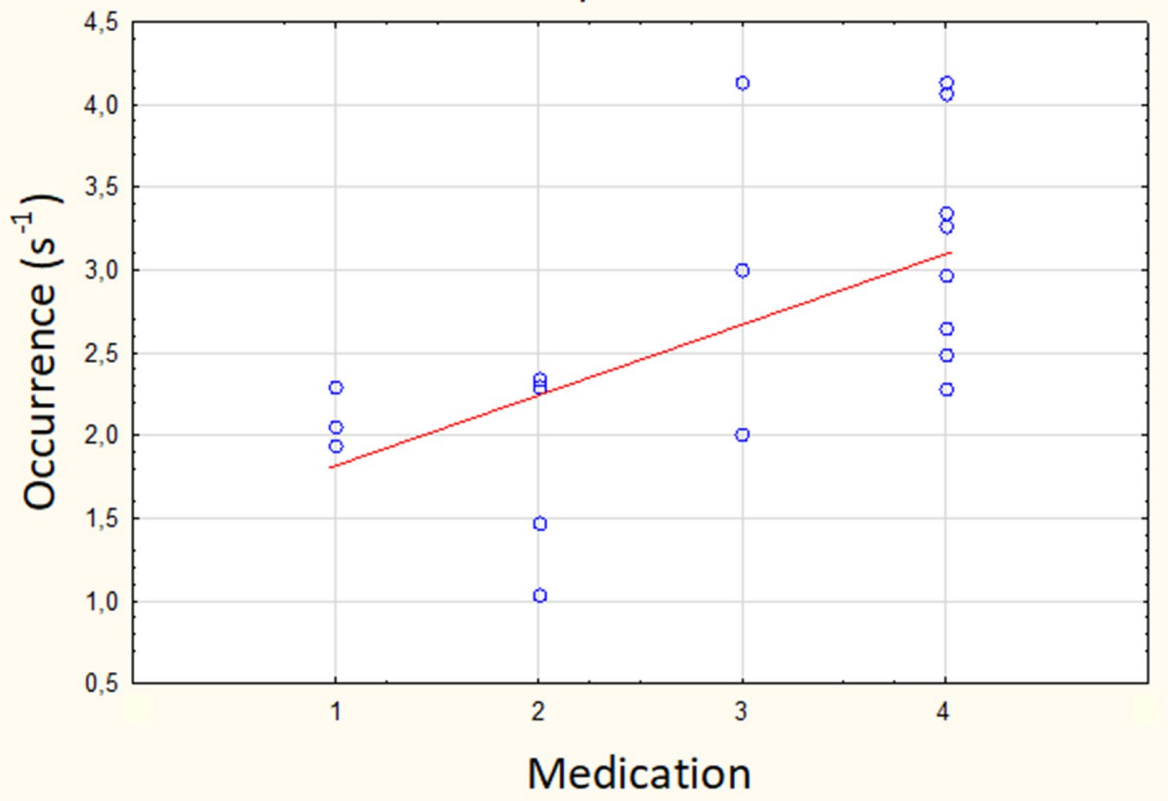

FIGURE 4 | Correlation between the occurrence of microstate $E$ and the intake of antidepressants, antipsychotics, and mood stabilizers. Medication scale: 1 , one medication in sub-therapeutic doses; 2 , one medication in therapeutic doses; 3 , combination of medications with one in therapeutic doses; 4 , combination of medications with more than one in therapeutic doses. 


\section{DISCUSSION}

In this report, the dynamics of resting-state large-scale brain network activity are depicted in the form of functional EEG brain microstates. We demonstrated that microstate temporal dynamics are sensitive to interindividual differences in depressive symptom severity in patients with moderate to severe depression. Particularly, we showed that severity of depressive symptoms correlated with higher occurrence of the microstate A. This finding suggests that microstate analysis-based neural markers might represent a largely untapped resource for understanding the neurobiology of depression. Since the between-group differences were absent in EEG dynamic, it is, however, unknown, if higher occurrence of microstate A is a complex expression of depressive symptomatology or if it reflects a latent risk factor. The here demonstrated symptomrelated interindividual differences in microstate dynamics need further research to test its utility in an objective depression assessment. The present study is the first in a planned longitudinal study series with depressive patients recruited at the University Hospital Brno that will help further investigate the microstate parameters as possible predictors of treatment response to both medication and neurostimulation methods including the electroconvulsive therapy.

Only three studies examined microstate duration and/or occurrence in depressive patients. The earliest study showed lower duration in the depressive group than in controls (27). In a subsequent study, between-group differences in occurrence were found neither in young nor aged depressive patients as compared to healthy controls (28). Contrary to these early findings, longer duration and lower occurrence of microstates in treatment-resistant depressed patients as compared to healthy subjects were demonstrated recently (29). The authors suggested that the increased duration and decreased occurrence in microstates could reflect modulation of global brain dynamics with neurotropic medications previously taken by patients resistant to antidepressant treatment. In the current study, we found an increased microstate A occurrence with depression only as an effect related to the symptom severity and not as a between-group difference. This finding is, despite different analytical approaches used in the studies, consistent with the previously reported lowering of microstate occurrence following magnetic seizure therapy and electroconvulsive therapy that in fact might represent a normalization of occurrence with successful treatment (29).

Different methodological approaches might have, however, led to discrepant findings in terms of duration of microstates among the current and the three previous studies. The methodological differences include different frequency bands examined (28), different clustering algorithms applied (27-29), different numbers of maps used for backfitting to the EEG (29), and analyzing all data points (e.g., in current study) or only those with the local maxima of the global field power [e.g., in Ref. (29)].

Discrepant findings may also reflect the pathophysiologic heterogeneity of depression. Similarly to the current sample, the experimental group in the study by Strik et al. (27) included depressive patients who met the criteria for unipolar or bipolar mood disorders or for dysthymia. The other two studies both focused on unipolar depression $(28,29)$, the more recent one was even restricted only to the treatment-resistant form of depression (29). With respect to the symptom variations in patients meeting criteria for depression, currently based solely on the clinical interviews and diagnostic questionnaires, such heterogeneity in findings could be expected.

In the current study, the topography of microstate $\mathrm{A}$ strongly resembled the topography of one of the four canonical microstates, i.e., microstate A, earlier described in the literature $(19,26)$. Using resting-state fMRI, this microstate was previously linked to the auditory brain network (41), involving bilateral superior and middle temporal gyri, regions associated with phonological processing (47). In addition to this indirect identification of involved brain structures, the sources generating microstate scalp topographies were directly estimated $(39,46)$. The left temporal lobe and left insula were identified as the major generators of microstate A (47). Additionally, left-lateralized activity in the medial prefrontal cortex and the occipital gyri was most recently reported to underlie this microstate (39).

Evidence from the meta-analysis of functional neuroimaging studies suggests resting-state functional alterations in firstepisode drug-naïv MDD patients in the fronto-limbic system, including the dorsolateral prefrontal cortex and putamen, and in the default mode network, namely, the precuneus and superior and middle temporal gyri (48). Altered activity in the superior temporal gyrus in patients with MDD was reported repeatedly in fMRI studies (49-52) and was also suggested to be responsible for the abnormal processing of negative mood and cognition in first-episode, drug-naive patients with MDD (48). Our findings of positive associations of depressive symptoms with the occurrence of microstate A that is related to temporal lobe activity are thus in line with these studies.

It has been shown that benzodiazepines and antipsychotics may modulate microstate dynamics (53). Accordingly, we observed the effect of medication on the presence of microstate E. The topography of this microstate strongly resembled one of the newly reported microstates, the generators of which were identified in the dorsal anterior cingulate cortex, superior and middle frontal gyri, and insula (46). The cingulo-opercular network (CON), comprising regions in the thalamus as well as frontal operculum/anterior insula and anterior cingulate cortex, is considered to have a central role in sustaining alertness (54) or in general for maintaining perceptual readiness (55). An important role in the pathophysiological mechanisms of depression was suggested for the CON, whose disrupted functional connectivity was observed in first-episode drug-naïve patients with MDD (12). Since the medication status in our study was only roughly defined, it is rather questionable whether the observed correlation between the medication scoring and the occurrence of microstate E could be related to the pharmacological effect on the activity of structures constituting the $\mathrm{CON}$.

In the current study, we decided to use the resting-state condition rather than employing any cognitive task. Depression affects not only emotional and cognitive mental operations but also motivational processes. Therefore, the task performance 
differences between patients and healthy controls may relate to different levels of motivation rather than information processing per se. Using a resting-state condition makes it possible to avoid some task-related confounds and makes the application of noninvasive neuroimaging techniques a powerful tool for measuring baseline brain activity (56). Moreover, if the research outputs such as those presented here lead to developing a new diagnostic tool for depressive disorder, such a tool, based on evaluating the restingstate scalp EEG, will be easy to use and require only minimal cooperation from the patients.

It is important to note that our data may have limitations. First, our sample included mixed diagnoses, with both bipolar and unipolar disorders. The observed relationship between the microstate A occurrence and depressive symptomatology should therefore be considered as a state rather than as a trait marker of depression. Second, the low sample size and great variability in medication made it impossible to examine any potential influence of medication on the microstate parameters by comparing patients receiving a specific drug with those not receiving it. To summarize the various medications, an ordinal variable was used that is only a rough measurement of medication usage. Therefore, the observed relationship between the microstate E occurrence and medications should be viewed with caution.

\section{CONCLUSIONS}

The study presented here provides insights into global brain dynamics of the resting-state in depressive patients. The identified depressive symptom-related changes in resting-state large-scale brain dynamics suggest the utility of the microstate analysis approach in an objective depression assessment. On the other side, using this analysis at the individual level could prove challenging. To test the observed microstate changes as possible biomarkers of illness and/or treatment response at individual level is the next step for future research in depressive patients.

\section{DATA AVAILABILITY}

The raw data supporting the conclusions of this manuscript will be made available by the authors, without undue reservation, to any qualified researcher.

\section{REFERENCES}

1. Andrade L, Caraveo-Anduaga JJ, Berglund P, Bijl RV, De Graaf R, Vollebergh W, et al. The epidemiology of major depressive episodes: results from the International Consortium of Psychiatric Epidemiology (ICPE) Surveys. Int J Methods Psychiatr Res (2003) 12:3-21. doi: 10.1002/mpr.138

2. Eaton WW, Alexandre P, Bienvenu OJ, Clarke D, Martins SS, Nestadt G, et al. The burden of mental disorders. Public Mental Health, Oxford University Press, Oxford, UK (2012). doi: 10.1093/acprof:oso/9780195390445.003.0001

3. Cloutier M, Greene M, Guerin A, Touya M, Wu E. The economic burden of bipolar I disorder in the United States in 2015. J Affective Disord (2018) 226:45-51. doi: 10.1016/j.jad.2017.09.011

4. Angst J, Merikangas KR, Cui L, Van Meter A, Ajdacic-Gross V, Rössler W. Bipolar spectrum in major depressive disorders. Eur Arch Psychiatry Clin Neurosci (2018) 268:741-48. doi: 10.1007/s00406-018-0927-x

\section{ETHICS STATEMENT}

This study was carried out in accordance with the recommendations of Ethics Committee of University Hospital Brno with written informed consent from all subjects. All subjects gave written informed consent in accordance with the Declaration of Helsinki. The protocol was approved by the Ethics Committee of University Hospital in Brno, Czech Republic.

\section{AUTHOR CONTRIBUTIONS}

$\mathrm{AD}$ designed the study, performed the analysis, and wrote the initial draft. MT served as consultant for the data analysis. RB and $\mathrm{JH}$ were responsible for patient recruitment and clinical assessment. EH collected the HD-EEG data. SF and ŠO were involved in the clinical assessment. CM served as an advisor and was responsible for the overall oversight of the study. All authors revised the manuscript.

\section{FUNDING}

This project received funding from the European Union Horizon 2020 research and innovation program under the Marie Skłodowska-Curie grant agreement No. 739939. The study was also supported by Ministry of Health, Czech Republic conceptual development of research organization (University Hospital Brno - FNBr, 65269705). These funding sources had no role in the design, collection, analysis, or interpretation of the study. CM was supported by the Swiss National Science Foundation (grant No. 320030_184677), by the National Centre of Competence in Research (NCCR) "SYNAPSY-The Synaptic Basis of Mental Diseases" (NCCR Synapsy Grant \# "51NF40 185897), and by the Swiss National Science Foundation (Sinergia project CRSII5_170873).

\section{ACKNOWLEDGMENTS}

The authors wish to thank Anne Meredith Johnson for providing language help.

5. Gong Q, He Y. Depression, neuroimaging and connectomics: a selective overview. Biol Psychiatry (2015) 77:223-35. doi: 10.1016/j.biopsych.2014. 08.009

6. Iwabuchi SJ, Krishnadas R, Li C, Auer DP, Radua J, Palaniyappan L. Localized connectivity in depression: a meta-analysis of resting state functional imaging studies. Neurosci Biobehav Rev (2015) 51:77-86. doi: 10.1016/j. neubiorev.2015.01.006

7. Kaiser RH, Andrews-Hanna JR, Wager TD, Pizzagalli DA. Large-scale network dysfunction in major depressive disorder: a meta-analysis of resting-state functional connectivity. JAMA Psychiatry (2015) 72:603-11. doi: 10.1001/jamapsychiatry.2015.0071

8. Peng D, Liddle EB, Iwabuchi SJ, Zhang C, Wu Z, Liu J, et al. Dissociated largescale functional connectivity networks of the precuneus in medication-naïve first-episode depression. Psychiatry Res Neuroimaging (2015) 232:250-56. doi: 10.1016/j.pscychresns.2015.03.003 
9. Mayberg HS. Limbic-cortical dysregulation: a proposed model of depression. J Neuropsychiatry Clin Neurosci (1997) 9:471-81. doi: 10.1176/jnp.9.3.471

10. Fischer AS, Keller CJ, Etkin A. The clinical applicability of functional connectivity in depression: pathways toward more targeted intervention. Biol Psychiatry Cogn Neurosci Neuroimaging (2016) 1:262-70. doi: 10.1016/j. bpsc.2016.02.004

11. Satterthwaite TD, Kable JW, Vandekar L, Katchmar N, Bassett DS, Baldassano CF, et al. Common and dissociable dysfunction of the reward system in bipolar and unipolar depression. Neuropsychopharmacol (2015) 40:2258-68. doi: 10.1038/npp.2015.75

12. Wu X, Lin P, Yang J, Song H, Yang R, Yang J. Dysfunction of the cinguloopercular network in first-episode medication-naive patients with major depressive disorder. J Affective Disord (2016) 200:275-83. doi: 10.1016/j. jad.2016.04.046

13. Greicius MD, Flores BH, Menon V, Glover GH, Solvason HB, Kenna H, et al. Resting-state functional connectivity in major depression: abnormally increased contributions from subgenual cingulate cortex and thalamus. Biol Psychiatry (2007) 62:429-37. doi: 10.1016/j.biopsych.2006.09.020

14. Price JL, Drevets WC. Neurocircuitry of mood disorders. Neuropsychopharmacol (2010) 35:192-16. doi: 10.1038/npp.2009.104

15. Hamilton JP, Chen MC, Gotlib IH. Neural systems approaches to understanding major depressive disorder: an intrinsic functional organization perspective. Neurobiol Dis (2013) 52:4-11. doi: 10.1016/j.nbd.2012.01.015

16. de Pasquale. F, Corbetta M, Betti. V, Della Penna S. Cortical cores in network dynamics. Neuroimage (2018) 180:370-82. doi: 10.1016/j.neuroimage. 2017.09.063

17. Bressler SL, Menon V. Large-scale brain networks in cognition: emerging methods and principles. Trends Cogn Sci (2010) 14(6):277-90. doi: 10.1016/j. tics.2010.04.004

18. Van De Ville D, Britz J, Michel CM. EEG microstate sequences in healthy humans at rest reveal scale-free dynamics. Proc Natl Acad Sci (2010) 107:18179-84. doi: 10.1073/pnas.1007841107

19. Michel CM, Koenig T. EEG microstates as a tool for studying the temporal dynamics of whole-brain neuronal networks: a review. Neuroimage (2018) 180:577-93. doi: 10.1016/j.neuroimage.2017.11.062

20. Michel CM, Murray MM. Towards the utilization of EEG as a brain imaging tool. Neuroimage (2012) 61:371-85. doi: 10.1016/j.neuroimage.2011.12.039

21. Lehmann D. Principles of spatial analysis. In: Gevins AS, Remont A, editors. Methods of analysis of brain electrical and magnetic signals. Elsevier (1987). p. 309-54.

22. Lehmann D, Ozaki H, Pal I. EEG alpha map series: brain micro-states by space-oriented adaptive segmentation. Electroencephalogr Clin Neurophysiol (1987) 67:271-88. doi: 10.1016/0013-4694(87)90025-3

23. Hutchison RM, Womelsdorf T, Allen EA, Bandettini PA, Calhoun VD, Corbetta $\mathrm{M}$, et al. Dynamic functional connectivity: promise, issues, and interpretations. NeuroImage (2013) 80:360-78. doi: 10.1016/j.neuroimage. 2013.05.079

24. Chang C, Glover GH. Time-frequency dynamics of resting-state brain connectivity measured with fMRI. NeuroImage (2010) 50:81-98. doi: 10.1016/j.neuroimage.2009.12.011

25. Honey CJ, Kötter R, Breakspear M, Sporns O. Network structure of cerebral cortex shapes functional connectivity on multiple time scales. Proc Natl Acad Sci (2007) 104:10240-45. doi: 10.1073/pnas.0701519104

26. Khanna A, Pascual-Leone A, Michel CM, Farzan F. Microstates in restingstate EEG: current status and future directions. Neurosci Biobehav Rev (2015) 49:105-13. doi: 10.1016/j.neubiorev.2014.12.010

27. Strik WK, Dierks T, Becker T, Lehmann D. Larger topographical variance and decreased duration of brain electric microstates in depression. J Neural Transm (1995) 99:213-22. doi: 10.1007/BF01271480

28. Ihl R, Brinkmeyer J. Differential diagnosis of aging, dementia of the Alzheimer type and depression with EEG-segmentation. Dement Geriatr Cogn Disord (1999) 10:64-9. doi: 10.1159/000017103

29. Atluri S, Wong W, Moreno S, Blumberger DM, Daskalakis ZJ, Farzan F. Selective modulation of brain network dynamics by seizure therapy in treatment-resistant depression. NeuroImage Clin (2018) 20:1176-90. doi: 10.1016/j.nicl.2018.10.015

30. Drobisz D, Damborská. A. Deep brain stimulation targets for treating depression. Behav Brain Res (2019) 359:266-73. doi: 10.1016/j.bbr.2018.11.004
31. Bazire S. Benzodiazepine equivalent doses. In: Psychotropic Drug Directory. Cheltenham, UK: Lloyd-Reinhold Communications (2014).

32. Williams JBW, Kobak KA. Development and reliability of a structured interview guide for the Montgomery Asberg Depression Rating Scale (SIGMA). Br J Psychiatry (2008) 192:52-8. doi: 10.1192/bjp.bp.106.032532

33. Guy W ed. ECDEU Assessment Manual for Psychopharmacology. Rockville, MD: US Department of Health, Education, and Welfare Public Health Service Alcohol, Drug Abuse, and Mental Health Administration (1976).

34. Jung T, Makeig S, Westerfield M, Townsend J, Courchesne E.c., Sejnowski TJ. Removal of eye activity artifacts from visual event-related potentials in normal and clinical subjects. Clin Neurophysiol (2000) 111:1745-58. doi: 10.1016/S1388-2457(00)00386-2

35. Perrin F, Pernier J, Bertrand O, Echallier JF. Spherical splines for scalp potential and current density mapping. Electroencephalogr Clin Neurophysiol (1989) 72:184-87. doi: 10.1016/0013-4694(89)90180-6

36. Brunet D, Murray MM, Michel CM. Spatiotemporal analysis of multichannel EEG: CARTOOL. Comput Intell Neurosci (2011) 813870:1-15. doi: 10.1155/ 2011/813870

37. Murray MM, Brunet D, Michel CM. Topographic ERP analyses: a stepby-step tutorial review. Brain Topogr (2008) 20:249-64. doi: 10.1007/ s10548-008-0054-5

38. Pascual-Marqui RD, Michel CM, Lehmann D. Segmentation of brain electrical activity into microstates; model estimation and validation. IEEE Trans Biomed Eng (1995) 42:658-65. doi: 10.1109/10.391164

39. Bréchet L, Brunet D, Birot G, Gruetter R, Michel CM, Jorge J. Capturing the spatiotemporal dynamics of self-generated, task-initiated thoughts with EEG and fMRI. Neuroimage (2019) 194:82-92. doi: 10.1016/j.neuroimage.2019. 03.029

40. Koenig T, Prichep L, Lehmann D, Sosa PV, Braeker E, Kleinlogel H, et al. Millisecond by millisecond, year by year: normative EEG microstates and developmental stages. Neuroimage (2002) 16:41-8. doi: 10.1006/nimg. 2002.1070

41. Britz J, Van De Ville D, Michel CM. BOLD correlates of EEG topography reveal rapid resting-state network dynamics. NeuroImage (2010) 52:1162-70. doi: 10.1016/j.neuroimage.2010.02.052

42. Tomescu MI, Rihs TA, Becker R, Britz J, Custo A, Grouiller F, et al. Deviant dynamics of EEG resting state pattern in 22q11.2 deletion syndrome adolescents: a vulnerability marker of schizophrenia? Schizophr Res (2014) 157:175-81. doi: 10.1016/j.schres.2014.05.036

43. Michel CM, Brandeis D, Skrandies W, Pascual R, Strik WK, Dierks T. Global field power: a 'time-honoured' index for EEG/EP map analysis. Int $J$ Psychophysiol (1993) 15:1-2. doi: 10.1016/0167-8760(93)90088-7

44. Santarnecchi E, Khanna AR, Musaeus CS, Benwell CSY, Davila P, Farzan F, et al. EEG microstate correlates of fluid intelligence and response to cognitive training. Brain Topogr (2017) 30:502-20. doi: 10.1007/s10548-017-0565-Z

45. Benjamini Y. Discovering the false discovery rate. J R Stat Soc Ser B Stat Methodol (2010) 72:405-16. doi: 10.1111/j.1467-9868.2010.00746.x

46. Custo A, Van De Ville D, Wells WM, Tomescu MI, Brunet D, Michel CM. Electroencephalographic resting-state networks: source localization of microstates. Brain Connect (2017) 7:671-82. doi: 10.1089/brain.2016.0476

47. Buchsbaum BR, Hickok G, Humphries C. Role of left posterior superior temporal gyrus in phonological processing for speech perception and production. Cogn Sci (2001) 25:663-78. doi: 10.1207/s15516709cog2505_2

48. Zhong X, Pu W, Yao S. Functional alterations of fronto-limbic circuit and default mode network systems in first-episode, drug-naïve patients with major depressive disorder: a meta-analysis of resting-state fMRI data. J Affective Disord (2016) 206:280-86. doi: 10.1016/j.jad.2016.09.005

49. Ke Z, Qing G, Zhiliang L, Fei X, Xiao S, Huafu C, et al. Abnormal functional connectivity density in first-episode, drug-naive adult patients with major depressive disorder. J Affect Disord (2016) 194:153-8. doi: 10.1016/j.jad. 2015.12.081

50. Liu Z, Xu C, Xu Y, Wang Y, Zhao B, Lv Y, et al. Decreased regional homogeneity in insula and cerebellum: a resting-state fMRI study in patients with major depression and subjects at high risk for major depression. Psychiatry Res (2010) 182:211-15. doi: 10.1016/j.pscychresns.2010.03.004

51. Shen T, Qiu M, Li C, Zhang J, Wu Z, Wang B, et al. Altered spontaneous neural activity in first-episode, unmedicated patients with major depressive disorder. Neuroreport (2014) 25:1302-07. doi: 10.1097/WNR.0000000000000263 
52. Tadayonnejad R, Yang S, Kumar A, Ajilore O. Clinical, cognitive, and functional connectivity correlations of resting-state intrinsic brain activity alterations in unmedicated depression. J Affective Disord (2015) 172:241-50. doi: 10.1016/j.jad.2014.10.017

53. Kinoshita T, Strik WK, Michel CM, Yagyu T, Saito M, Lehmann D. Microstate segmentation of spontaneous multichannel EEG map series under diazepam and sulpiride. Pharmacopsychiatry (1995) 28:51-5. doi: 10.1055/s-2007-979588

54. Coste CP, Kleinschmidt A. Cingulo-opercular network activity maintains alertness. Neuroimage (2016) 128:264-72. doi: 10.1016/j.neuroimage.2016. 01.026

55. Sadaghiani S, D'Esposito M. Functional characterization of the cinguloopercular network in the maintenance of tonic alertness. Cereb Cortex (2015) 25:2763-73. doi: 10.1093/cercor/bhu072
56. Gusnard DA, Raichle ME. Searching for a baseline: Functional imaging and the resting human brain. Nat Rev Neurosci (2001) 2:685-694.

Conflict of Interest Statement: The authors declare that the research was conducted in the absence of any commercial or financial relationships that could be construed as a potential conflict of interest.

Copyright (c) 2019 Damborská, Tomescu, Honzírková, Barteček, Hořínková, Fedorová, Ondruš and Michel. This is an open-access article distributed under the terms of the Creative Commons Attribution License (CC BY). The use, distribution or reproduction in other forums is permitted, provided the original author(s) and the copyright owner(s) are credited and that the original publication in this journal is cited, in accordance with accepted academic practice. No use, distribution or reproduction is permitted which does not comply with these terms. 


\title{
Lower Functional Connectivity in Vestibular-Limbic Networks in Individuals With Subclinical Agoraphobia
}

\begin{abstract}
Iole Indovina ${ }^{1,2 * t}$, Allegra Conti ${ }^{2 \dagger}$, Francesco Lacquaniti ${ }^{2,3}$, Jeffrey P. Staab ${ }^{4}$, Luca Passamonti ${ }^{5,6 *}$ and Nicola Toschi ${ }^{7,8}$

${ }^{1}$ Department of Medicine and Surgery, Saint Camillus International University of Health and Medical Sciences, Rome, Italy, ${ }^{2}$ Laboratory of Neuromotor Physiology, IRCCS Santa Lucia Foundation, Rome, Italy, ${ }^{3}$ Department of Systems Medicine and Centre of Space BioMedicine, University of Rome Tor Vergata, Rome, Italy, ${ }^{4}$ Departments of Psychiatry and Psychology and Otorhinolaryngology-Head and Neck Surgery, Mayo Clinic, Rochester, MN, United States, ${ }^{5}$ Department of Clinical Neurosciences, University of Cambridge, Cambridge, United Kingdom, ${ }^{6}$ Institute of Bioimaging and Molecular Physiology, National Research Council, Milan, Italy, ${ }^{7}$ Department of Biomedicine and Prevention, University of Rome Tor Vergata, Rome, Italy, ${ }^{8}$ Department of Radiology, Athinoula A. Martinos Center for Biomedical Imaging, Boston, MA, United States
\end{abstract}

OPEN ACCESS

Edited by:

Roberto Esposito,

A.O. Ospedali Riuniti Marche

Nord, Italy

Reviewed by:

Liang Zhan

University of Pittsburgh, United States

Hui Shen,

National University of Defense Technology, China

*Correspondence:

lole Indovina

iole.indovina@unicamillus.it Luca Passamonti

Ip337@medschl.cam.ac.uk

†These authors have contributed equally to this work

Specialty section:

This article was submitted to Applied Neuroimaging, a section of the journal Frontiers in Neurology

Received: 30 April 2019

Accepted: 26 July 2019

Published: 13 August 2019

Citation:

Indovina I, Conti A, Lacquaniti F Staab JP, Passamonti L and Toschi N (2019) Lower Functional Connectivity in Vestibular-Limbic Networks in Individuals With Subclinical Agoraphobia. Front. Neurol. 10:874. doi: 10.3389/fneur.2019.00874
Background: Agoraphobia was described in 1871 as a condition of fear-related alterations in spatial orientation and locomotor control triggered by places or situations that might cause a patient to panic and feel trapped. In contemporary nosology, however, this original concept of agoraphobia was split into two diagnostic entities, i.e., the modern anxiety disorder of agoraphobia, consisting solely of phobic/avoidant symptoms in public spaces, and the recently defined vestibular disorder of persistent postural perceptual dizziness (PPPD), characterized by dizziness, and unsteadiness exacerbated by visual motion stimuli. Previous neuroimaging studies found altered brain activity and connectivity in visual-vestibular networks of patients with PPPD vs. healthy controls. Neuroticism and introversion, which pre-dispose to both agoraphobia and PPPD, influenced brain responses to vestibular and visual motion stimuli in patients with PPPD. Similar neuroimaging studies have not been undertaken in patients with agoraphobia in its current definition. Given their shared history and pre-disposing factors, we sought to test the hypotheses that individuals with agoraphobic symptoms have alterations in visual-vestibular networks similar to those of patients with PPPD, and that these alterations are influenced by neuroticism and introversion.

Methods: Drawing from the Human Connectome Project $(\mathrm{HCP})$ database, we matched 52 participants with sub-clinical agoraphobia and 52 control subjects without agoraphobic symptoms on 19 demographic and psychological/psychiatric variables. We then employed a graph-theoretical framework to compare resting-state functional magnetic resonance images between groups and evaluated the interactive effects of neuroticism and introversion on the brain signatures of agoraphobia.

Results: Individuals with subclinical agoraphobia had lower global clustering, efficiency and transitivity relative to controls. They also had lower connectivity metrics in two brain networks, one positioned to process incoming visual space-motion information, assess threat, and initiate/inhibit behavioral responses (visuospatial-emotional network) and one positioned to control and monitor locomotion (vestibular-navigational network). 
Introversion interacted with agoraphobic symptoms to lower the connectivity of the visuospatial-emotional network. This contrasted with previous findings describing neuroticism-associated higher connectivity in a narrower visual-spatial-frontal network in patients with PPPD.

Conclusion: Functional connectivity was lower in two brain networks in subclinical agoraphobia as compared to healthy controls. These networks integrate visual vestibular and emotional response to guide movement in space.

Keywords: agoraphobia, persistent postural perceptual dizziness, vestibular network, functional connectivity, resting state fMRI, graphs theory

\section{INTRODUCTION}

Agoraphobia (fear of the marketplace), was first described by the German neuroscientist C.F. Westphal in 1871 as a syndrome of altered spatial perception, cognitive distortions about safe locomotion, and fear-driven limitations of mobility (1). Westphal called this condition agoraphobia because he observed it in the busy marketplaces of town squares in nineteenth century European villages. Critically, he considered anxiety, altered spatial perception, and restricted mobility to be "part of one process." In the modern era, agoraphobia is conceptualized as an anxiety disorder defined solely by fear and avoidance of public places outside of home (2), and the space and motion symptoms that concerned Westphal are no longer part of the disorder. For nearly a century, such symptoms were relegated to the vague notion of psychogenic dizziness, until conditions such as supermarket syndrome (3) and space phobia (4) appeared in the medical literature in the 1970's. These were followed by the syndromes of phobic postural vertigo (5), space-motion phobia (6), and chronic subjective dizziness (7) in which vestibular symptoms and difficulties with exposure to space and motion stimuli were core elements, either co-existing with or predisposed and precipitated by anxiety. All of these syndromes provided the background for the recently described vestibular disorder called persistent postural-perceptual dizziness (PPPD), which is defined solely by vestibular symptoms and sensitivity to visual space-motion stimuli (8). Thus, contemporary diagnostic nomenclature (9) separated Westphal's agoraphobia into two conditions, one with anxiety-phobic symptoms and one with vestibular-motion symptoms. This distinction is clinically useful, as agoraphobia and PPPD can be seen quite independently of one another in medical settings, but it should not be interpreted as a formal severing of potential etio-pathogenetic mechanisms. Indeed, Westphal and his contemporaries engaged in lively debates about the relative contributions of visual, vestibular, and psychological processes to the original concept of agoraphobia (10).

Several lines of evidence suggest a shared pre-disposition and partially overlapping clinical manifestations of agoraphobia and PPPD. For example, the personality traits of neuroticism and introversion may be associated with both conditions. A study of twin siblings found independent genetic contributions of neuroticism and introversion to agoraphobia (11). Studies of chronic subjective dizziness found that neurotic and introverted traits were significantly more common in patients with this precursor of PPPD than in patients with other chronic vestibular disorders who had similar levels of dizziness and anxiety or normative samples $(12,13)$. Studies of PPPD itself also identified levels of neuroticism that were higher than normal (14), and structural vestibular disorders may trigger both PPPD and agoraphobia $(8,15)$. Investigations of patients with panic disorder and agoraphobia found increased rates of vestibular symptoms, sensitivity to visual motion stimuli, alterations in postural control, and subtle abnormalities in vestibular laboratory tests compared to normal controls (1621). Conversely, research in patients with the precursors of PPPD found that $60 \%$ had anxiety disorders, and in particular panic disorder and agoraphobia (22). Treatment with the selective serotonin reuptake inhibitor (SSRI) paroxetine was shown to normalize changes in postural control in patients with panic disorder (23). Indeed, SSRIs are the mainstay of pharmacological treatment of PPPD, even in patients without psychiatric comorbidity (24).

Given their shared history and the overlapping clinical features, it is possible for agoraphobia and PPPD to share underlying brain mechanisms. Neuroanatomical studies identified extensive connections between vestibular and anxiety systems, extending from the brainstem to the cortex (25), and functional magnetic resonance imaging studies in normal humans revealed significant effects of vestibular stimulation on activity and connectivity in both vestibular and anxiety regions, modulated by neuroticism and introversion $(26,27)$. Space and motion information in the brain is processed in a widely distributed network, and there is no unimodal primary sensory cortex for vestibular inputs as there are for other sensory modalities. Instead, visual space-motion data are processed by a "multimodal vestibular network" of brain areas that contain neurons that receive combinations of vestibular, visual, and somatosensory stimuli. These multimodal neurons have been found in several regions centered around the parietal opercula, posterior insula, and adjacent posterior perisylvian regions of the parietal and temporal cortex, which constitute the central connectivity nodes of the multimodal vestibular cortex $(28,29)$ and contribute to perception of gravity (30-32). This network extends to the medial superior temporal area (MST) and posterior inferior temporal gyrus, ventral intraparietal area, 
superior parietal lobe, somato-motor cortex, hippocampal formation, anterior insula, inferior frontal gyrus, and cingulate cortex (28-32). Some of these multimodal areas (e.g., anterior insula and hippocampus) are critically involved in emotional processing, which may underlie clinical observations of close associations between anxiety and vestibular disorders, including agoraphobia and PPPD (10, 25, 33-37).

The structure and function of brain regions that comprise the multimodal visuo-vestibular network have been studied in patients with PPPD and its precursors using various neuroimaging methods (38-44). In studies that controlled for group-averaged anxiety-related variables but not for their variances, patients with PPPD compared to healthy controls had lower activity, functional connectivity, and cortical folding within the parietal opercula (OP1-4), as well as in a wider network of visuo-vestibular regions including the posterior insula, posterior superior temporal sulcus, superior parietal cortex and motor vestibular regions (38-40). In contrast, patients with PPPD compared to healthy controls had higher frontooccipital connectivity linked to state and trait anxiety $(41,45)$. Furthermore, patients with phobic postural vertigo (essentially PPPD plus phobic/avoidance symptoms related to visual spacemotion stimuli) had increased connectivity between motor cortex (Broadman area 4-BA4) and orbitofrontal, fronto-polar, and anterior cingulate cortices compared to healthy controls (44).

The brain mechanisms presumed to underlie agoraphobic anxiety have been described extensively $(46,47)$. In recent neuroimaging studies, patients with panic disorder and agoraphobia showed greater activation than normal controls in the insular cortices bilaterally as well as in the left inferior frontal gyrus, dorsomedial pre-frontal cortex, caudate and hippocampus in response to exposure to symptom-specific pictures compared to healthy controls (48). Activations in the striatum and insula may be stronger in anticipation than actual viewing of agoraphobia-specific stimuli (49). In a nonclinical sample, subclinical agoraphobic symptoms correlated positively with alterations in cortical volumes of the right lingual gyrus, left superior, middle, and inferior temporal gyri, and bilateral calcarine sulci. Exploratory analyses extended those findings to the left pre-central and post-central gyri, the right orbitofrontal cortex, insula, and posterior cingulate gyrus, and bilateral precunei (50). A comparison of these results to those from patients with PPPD suggests potential similarities and differences in task-driven activation (e.g., visual cortical areas-positively correlated with symptom severity in both PPPD and agoraphobia; insula-decreased in PPPD, increased in agoraphobia). However, it is not known if connectivity differs between these disorders. Furthermore, most agoraphobia studies were not controlled for the potential confounds of neuroticism and introversion, which pre-dispose to both conditions $(11-14,45)$.

In this study we aimed to explore the functional brain connectivity signatures of sub-clinical agoraphobia through a graph-theoretical framework applied to task-free functional MRI in a group of healthy participants selected from the database of the Human Connectome Project (HCP) (51). The extensive information available on subjects in the HCP allowed us to select subjects who reported agoraphobic symptoms and carefully match them to a comparison group with no agoraphobic symptoms on demographics, handedness, and 16 other variables that could interact with agoraphobia such as levels of panic, anxiety, and depressive symptoms, perceived stress, personality traits, negative affect, and self-efficacy. We compared the two groups that we selected from the HCP to test the hypothesis that people with agoraphobic symptoms show lower connectivity in areas of the multimodal vestibular network previously identified in patients with PPPD. In addition, we aimed to assess whether anxiety-related personality traits interact with agoraphobic symptoms to further decrease connectivity.

\section{MATERIALS AND METHODS}

\section{Participants}

We used rsfMRI data from the $\$ 1200$ HCP data release, which comprises MRI data and psychological assessments from 1,003 healthy volunteers (http://www.humanconnectome.org/ documentation/S1200/). Due to the dominance of the right hemisphere representation of the vestibular function in righthanded individuals (and of the left hemisphere in left-handers) (52) we selected only right-handed individuals, i.e., individuals with handedness score higher than 50 (-100/100 range) (53), to avoid confounds due to different lateralization of functions. Among right-handers, 52 individuals reported agoraphobia symptoms. Experience of at least one episode of agoraphobia, panic or major depression and the number of lifetime depressive symptoms were evaluated by the HCP consortium through the Semi-Structured Assessment for the Genetics of Alcoholism (SSAGA) (54) in agreement with DSM-5 Criteria (2). Anger, aggression, hostility and fear affect were assessed via the NIH Toolbox Fear-Affect Survey, comprising items from the PROMIS Anxiety Item Bank (55). Fear-somatic arousal and sadness were assessed through the Mood and Anxiety Symptom Questionnaire $(56,57)$ and PROMIS Depression Item Bank $(58)$, respectively. Five Factor Model personality traits were assessed via the NEO five-factor inventory (NEO-FFI) (59). Perceived stress and selfefficacy were scored on the Perceived Stress Scale (60) and on the General Self-Efficacy Scale (http://userpage.fu-berlin.de/ health/ selfscal.htm).

\section{Matching Procedure}

We matched agoraphobic subjects to 52 healthy right-handed individuals by age, gender, handedness, psychological variables (anger-affect, anger-hostility, anger-aggressivity, fear-affect, fearsomatic arousal, sadness, perceived stress, self-efficacy), presence of psychiatric disorders (panic disorder, one major depressive episode over lifetime, total number of depressive symptoms over lifetime), and personality scores (neuroticism, extraversion, openness, conscientiousness, agreeableness). The matching procedure was as follows: (i) repeated random sampling (with replacement) of 52 non-agoraphobic subjects from the whole ( $n=1,003$ HCP database; (ii) for each sample, group-wise comparison (against the agoraphobia group) of mean values (Mann-Whitney $U$-Test for continuous variables and ChiSquared test for dichotomous variables) and variances (Brown Forsythe test) for all matching variables, and (iii) acceptance of the first "matched" sample of 52 healthy subjects when 


\section{A}

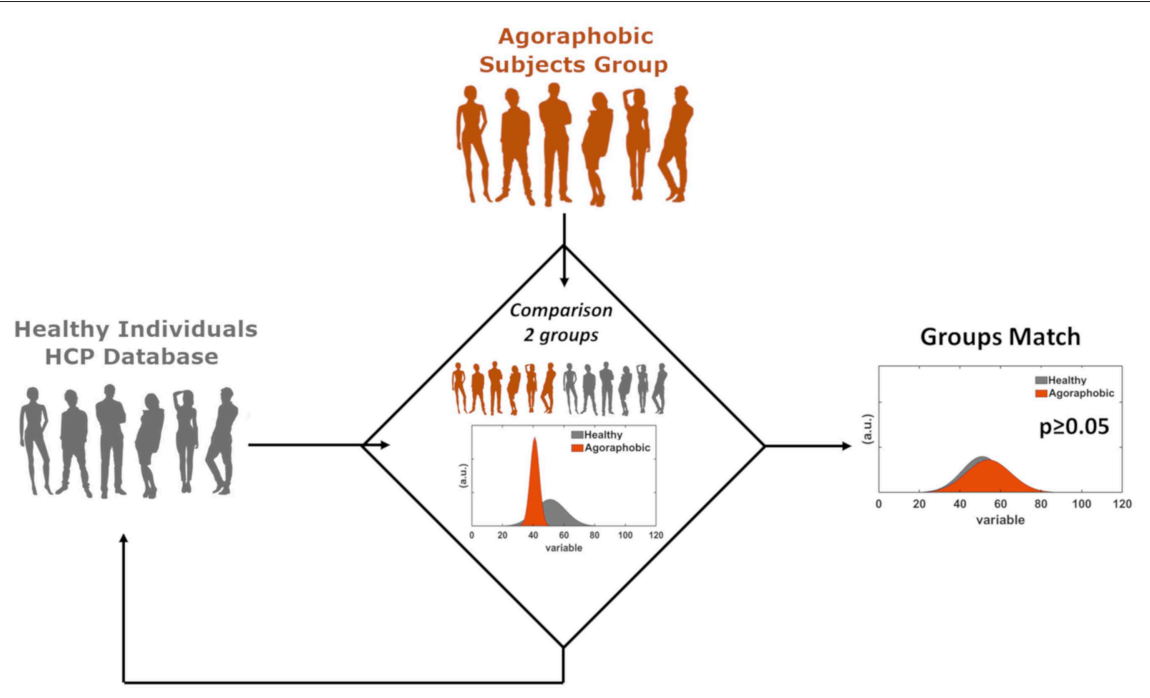

B
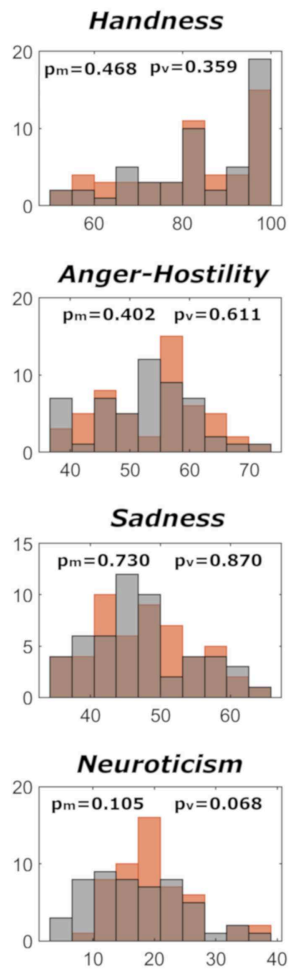

Panic Disorder

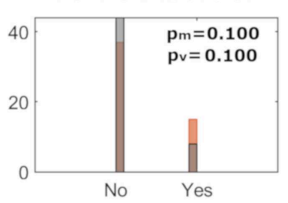

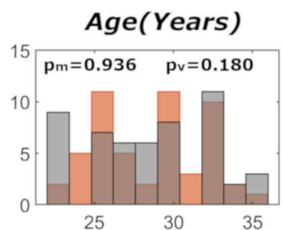
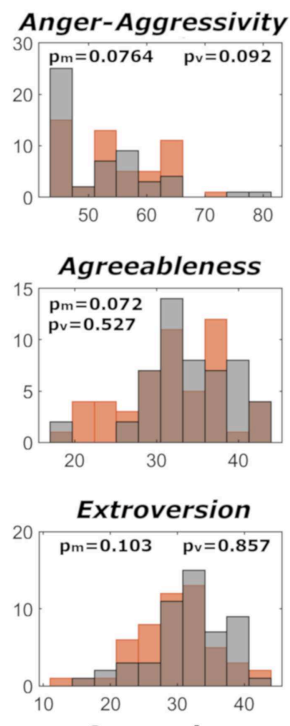

One major
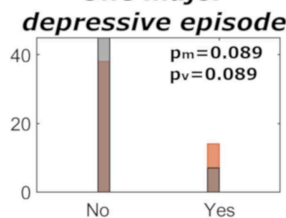
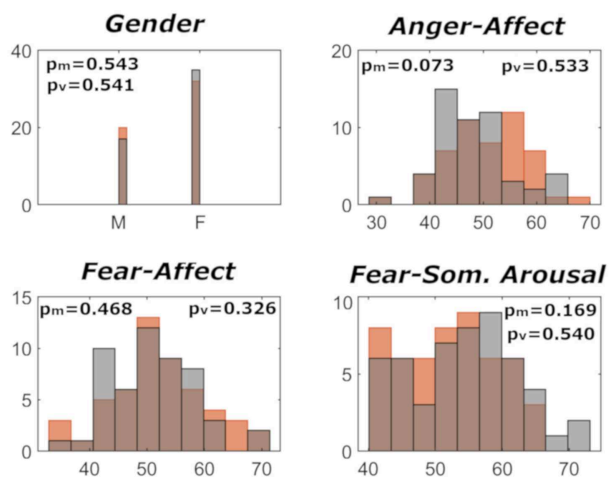

Fear-Som. Arousal
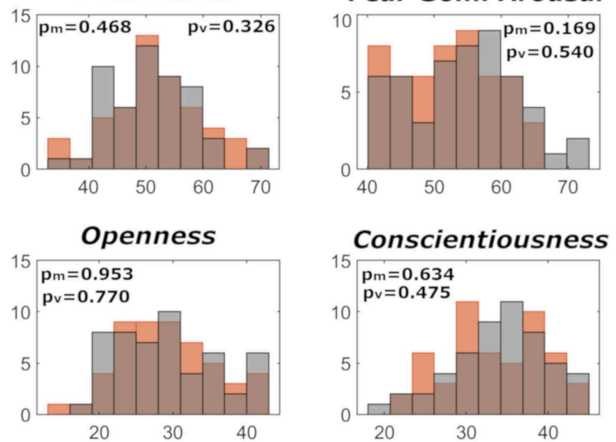

Conscientiousness
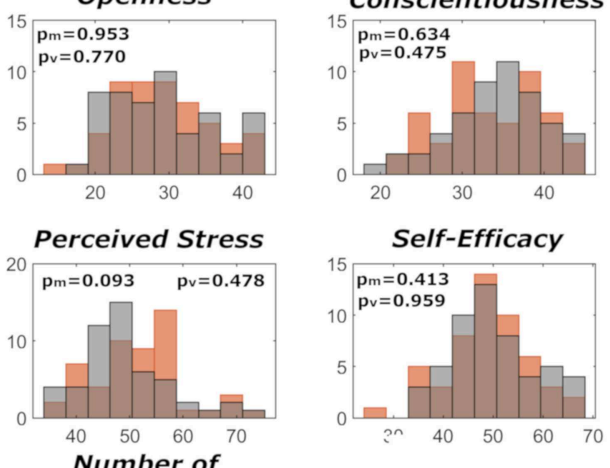

depressive symptoms
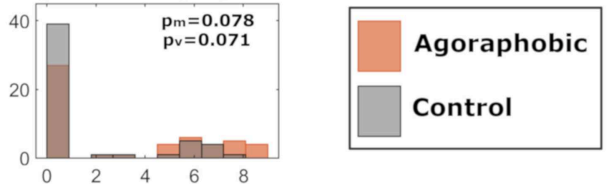

FIGURE 1 | (A) Matching of agoraphic and healthy control groups was performed through repeated random sampling (with replacement) of 52 non-agoraphobic subjects and subsequent statistical testing on matching variables. (B) Distributions of the demographic, psychological, psychiatric, and personality variables for the two matched groups. Variables relative to agoraphobic and healthy subjects are shown in orange and in gray, respectively. $p_{\mathrm{m}}$ and $p_{\mathrm{v}}$ refer to median and variance comparison, respectively. 
all resulting $p$-values (19 comparisons for both means and variances) were $p \geq 0.05$ (Figure 1).

\section{Magnetic Resonance Imaging (MRI) Scanning and Definition of Nodes}

Within HCP scanning procedures, $\mathrm{fMRI}$ data were acquired on a Siemens Skyra 3T in four runs of $\sim 15 \mathrm{~min}$ each, through a Gradient-echo EPI sequence (1,200 volume per run, $\mathrm{T}_{\mathrm{R}}=$ $720 \mathrm{~ms} ; \mathrm{T}_{\mathrm{E}}=33.1 \mathrm{~ms}, \mathrm{FA}=52 \mathrm{deg}, \mathrm{FOV}=208 \times 180 \mathrm{~mm}(\mathrm{RO}$ $\times \mathrm{PE})$, resolution $(\mathrm{x}, \mathrm{y}, \mathrm{z})=2.0 \times 2.0 \times 2.0 \mathrm{~mm}^{3}$, Multiband factor $=8$, bandwidth $=2290 \mathrm{~Hz} / \mathrm{Px}$ ).

Each 15-min run of each subject's rfMRI data was pre-processed according to Smith et al. (61); it was minimallypre-processed (62), and had artifacts removed using ICA + FIX $(63,64)$. ICA + FIX is a data-driven algorithm, which is based on an automated classifier specifically and manually trained to discern, amongst independent component analysis (ICA) results, diverse sources of noise (acquisition, movement, physiological artifacts). The version trained for HPC data has been seen to guarantee an accuracy (as well as sensitivity and specificity) of around 99\% $(61,63,64)$. Inter-subject registration of cerebral cortex was carried out using areal feature-based alignment and the Multimodal Surface Matching algorithm ("MSMAll") $(65,66)$. For feeding into group-PCA, each dataset was then temporally demeaned and had variance normalization applied according to Beckmann and Smith (67), after which group-PCA output was generated by MIGP (MELODIC's Incremental Group-PCA) from 1,003 subjects (68). More details can be found at https://www.humanconnectome.org/study/hcp-young-adult/ article/release-s1200-extensively-processed-rfmri-data. While each resulting spatial component map (node map) is obtained through regression and therefore defined over the whole brain, there is a steep roll-off in areas which are mostly related to other components. In order to highlight the brain areas which are dominant/unique in each component, for visualization and description purposes in this paper we thresholded each map at the 98th percentile. For analysis, we employed the individual adjacency matrices computed by the HCP consortium based on group-PCA at dimensionality 100 . These which were based on partial correlation coefficients between node timeseries ("netmats2"). The graph representation of each adjacency matrix therefore comprised 100 nodes. Each node can be considered a resting state network in itself [see e.g., (69) for an example at dimensionality 15].

\section{Graph Theoretical Metrics}

After matching, in all adjacency matrices negative correlations were set to zero. For each subject matrix, we calculated nodewise, local graph metrics quantifying the centrality of a node within a network (local strength and betweenness centrality), its ability to transmit information at local level (local efficiency), its integration or segregation properties (clustering coefficient), and its overall influence in a network (Eigenvector centrality) (70). Similarly, for each subject we calculated three overall graph metrics: strength, efficiency and transitivity. These are called global graph metrics and provide information about the general topological properties of each subject's connectivity matrix. The first two (strength and efficiency) are calculated by averaging over all nodes, while transitivity is defined for the whole graph. The clustering coefficient of a node estimates how much a single node tends to aggregate, through edges (i.e., connections), with its nearest neighbors. Transitivity is a variant of the clustering coefficient in which a networkwide normalization strategy mitigates the influence of outliers (e.g., of nodes with very low number of connections) on the clustering coefficient. Local efficiency is a similar measure, which estimates, in each location within the graph, how well a node's neighbors can exchange information when the node itself is removed (i.e., it can also be thought of as a measure of resilience). All graph-theoretical measures were computed via the Brain Connectivity Toolbox (70) (https://sites.google. $\mathrm{com} / \mathrm{site} / \mathrm{bctnet} /$ ). In addition, given that no consensus exist on thresholding strategies before computing graph-theoretical computations, we repeated the main analyses after thresholding all adjacency matrices at 50\% density.

\section{Statistical Analysis}

All metrics were compared between groups (agoraphobia vs. healthy controls) through the non-parametric Mann-Whitney $U$ test. Results were corrected for multiple comparisons across the number of nodes (100) using an FDR approach at $p<$ 0.05 . Whenever a significant group effect was found $(p<$ 0.05 , FDR-corrected), we separately tested the interaction of agoraphobia and anxiety-related personality traits (neuroticism and introversion) using a general linear model which included group, trait and group*trait interaction. Additionally, whenever a significant group effect was found in local graph metrics in a certain node ( $p<0.05$, corrected), we separately ranked the adjacency matrix elements for the agoraphobic and control group in order to identify, for each group, nodes with greatest overall connectivity to the rest of the brain.

\section{RESULTS}

Globally, we found lower clustering coefficient, efficiency and transitivity in the agoraphobic group compared to the matched control group ( $p=0.01,0.01$, and 0.02 , respectively, MannWhitney $U$ test) (Figure 2). These results were confirmed when using matrices thresholded at $50 \%$ density. In this case, the $p$-values resulting from the Mann-Whitney $U$ tests are $p=0.014 ; 0.018 ; 0.010$ for the comparison between global clustering coefficient, efficiency and transitivity of the two groups, respectively. We found two separate networks (ICA components) with significant lower local clustering coefficient and efficiency in subjects with agoraphobic symptoms vs. matched controls. These extended across multiple cortical regions.

The first of these composite networks spanned visual, vestibular, motor, navigation and emotion processing areas (component 23, depicted in red in Figure 3A). The clustering coefficient $\left(p_{\text {corr }}=0.0002\right)$ and efficiency $\left(p_{\text {corr }}=0.0003\right)$ of this network were both lower in the agoraphobic than control groups (Figures 3B,C). This component contained a small area at the interface between V1, V2, and MST and extended anteriorly into the ventral visual stream in the fundus of the superior temporal 

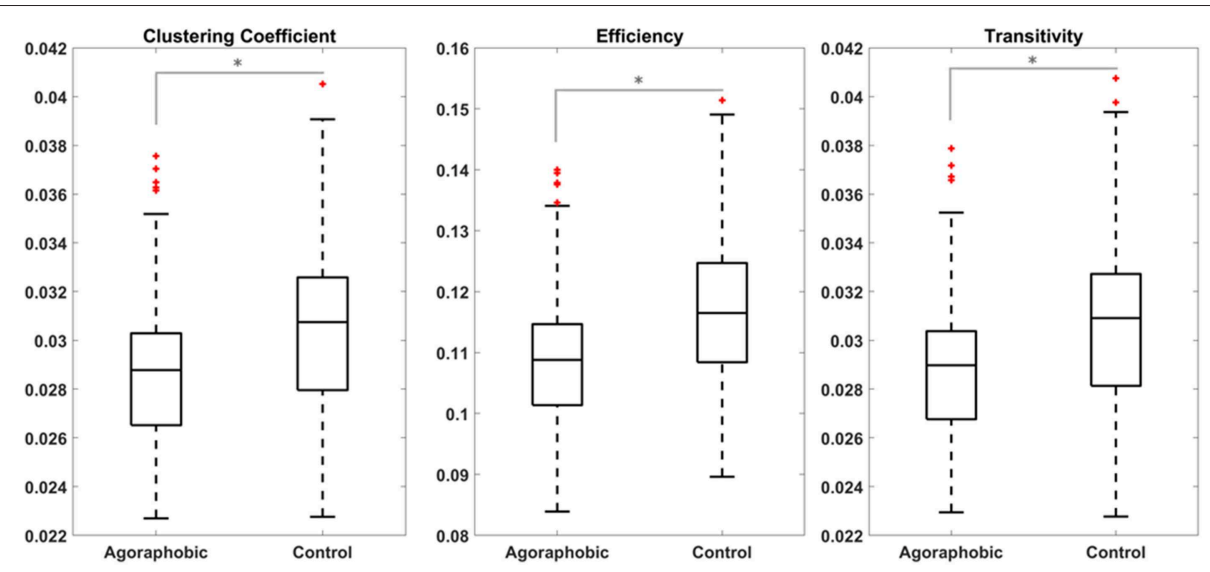

FIGURE 2 | Global clustering coefficient, efficiency, and transitivity were lower in patients with subclinical agoraphobic compared to controls. Figures show the median value (line), quartiles (boxes) and extremes (whiskers) of the three metrics for the agoraphobic and control groups. Comparison between the two subjects groups were performed through non-parametric Mann-Whitney $U$ tests.

sulcus (FST), basal parietal regions (area parietalis basalis in limine temporali PHT, PH) and posterior area temporalis proper (TE1p, TE2p) [Von Economo nomenclature (71)]. Medially, it encompassed the parieto-occipital sulcus POS1 in the precuneus on the left. Additional parietal regions included the posterior and superior angular gyrus (PGp andPGs) in the caudal inferiorparietal lobe (cIPL); the supramarginal gyrus (PF), $\mathrm{PF}$ opercularis (PFop), and tenuicorticalis (PFt) in the rostral IPL and lateral, medial and ventral intra-parietal area (LIP,MIP, and VIP) in the superior parietal lobe (SPL); and area 3a. The posterior insula was included on the right (PoI1-2). This composite network extended into the rostral and ventral parts of area BA $6(6 \mathrm{r}$ and $6 \mathrm{v})$ in the pre-motor cortex and into the frontal operculum (Fop2) and inferior frontal sulcus (IFS) in the prefrontal cortex. Subcortical structures, specifically the amygdala, head of the caudate nucleus, putamen, and anterior thalamus also were part of the network. We termed this component the "visuospatial-emotional network" because it encompassed brain regions that integrate incoming multi-modality space and motion information (V1/V2, ventral visual stream, cIPL, and posterior insula) $(72,73)$, areas that process emotions (amygdala and associated subcortical nuclei), and regions that initiate/inhibit movements (pre-motor and pre-frontal cortices).

The second composite network encompassed the left posterior insula, retroinsula, and granular insula, and bilateral parietal operculum (OP1,2,3,4), primary somatosensory and motor cortex (BA 1, 2, 3b, 4), pre-motor cortex (6d), medial SPL, cingulate cortex (5m, 5L, 24), and rostral hippocampus (component 41, depicted in green in Figure 3A). In this network, only the clustering coefficient $\left(p_{\text {corr }}=0.0009\right)$ was lower in subjects with agoraphobic symptoms compared to controls (Figure 3B). We named this component the "vestibularnavigational network" because it linked the principal vestibular cortical regions and the hippocampus to somatosensory and motor cortices. In summary the visuospatial-emotional and vestibular-navigational networks both extended across parietal, insular and pre-frontal cortices of the brain.
These networks are mostly connected to three common components (in yellow in Figure 4) plus additional components that spread again over the parietal, insular and pre-frontal cortices (Figure 4).

These results were confirmed when using matrices thresholded at 50\% density. In this case, individuals with subclinical agoraphobia were found to have lower clustering coefficient $\left(p_{\text {corr }}=0.023\right)$ and local efficiency $\left(p_{\text {corr }}=0.023\right)$ in visuospatial-emotional network (node number 23) as compared to controls. In addition, in subclinical agoraphobia the local clustering coefficient was lower (as compared to controls, $p_{\text {corr }}=$ 0.026 ) also in the vestibular-navigational network (node 41).

We did not find a main effect of introversion (all $p$ 's > 0.6 ) or neuroticism (all $p$ 's $>0.1$ ) on graph theoretical-metrics. In the visuospatial-emotional network, however, introversion interacted significantly with the main effect of agoraphobia to reduce the clustering coefficient and efficiency of subjects with agoraphobic symptoms compared to controls $(F=7.9, p=0.006$ and $F=7.0, p=0.009$, respectively) (Figure 5). Thus, individuals with agoraphobic symptoms and introverted personality traits had the lowest connectivity metrics. Introversion did not interact significantly with agoraphobia in the vestibular-navigational network. Neuroticism had no effect on either network.

These results were confirmed when using matrices thresholded at 50\% density. Also in this case, in the visuospatialemotional network both local efficiency $(p=0.012)$ and clustering coefficient $(p=0.009)$ were significantly affected by the interaction of agoraphobic group and introversion.

\section{DISCUSSION}

In this study, we were able to utilize the detailed information of the HCP database to identify a group of 52 subjects with sub-clinical agoraphobic symptoms and match them closely to a control group of 52 subjects across a large number of potential confounds. Critically, matching was done not only for mean values of potentially confounding variables, but also for their 


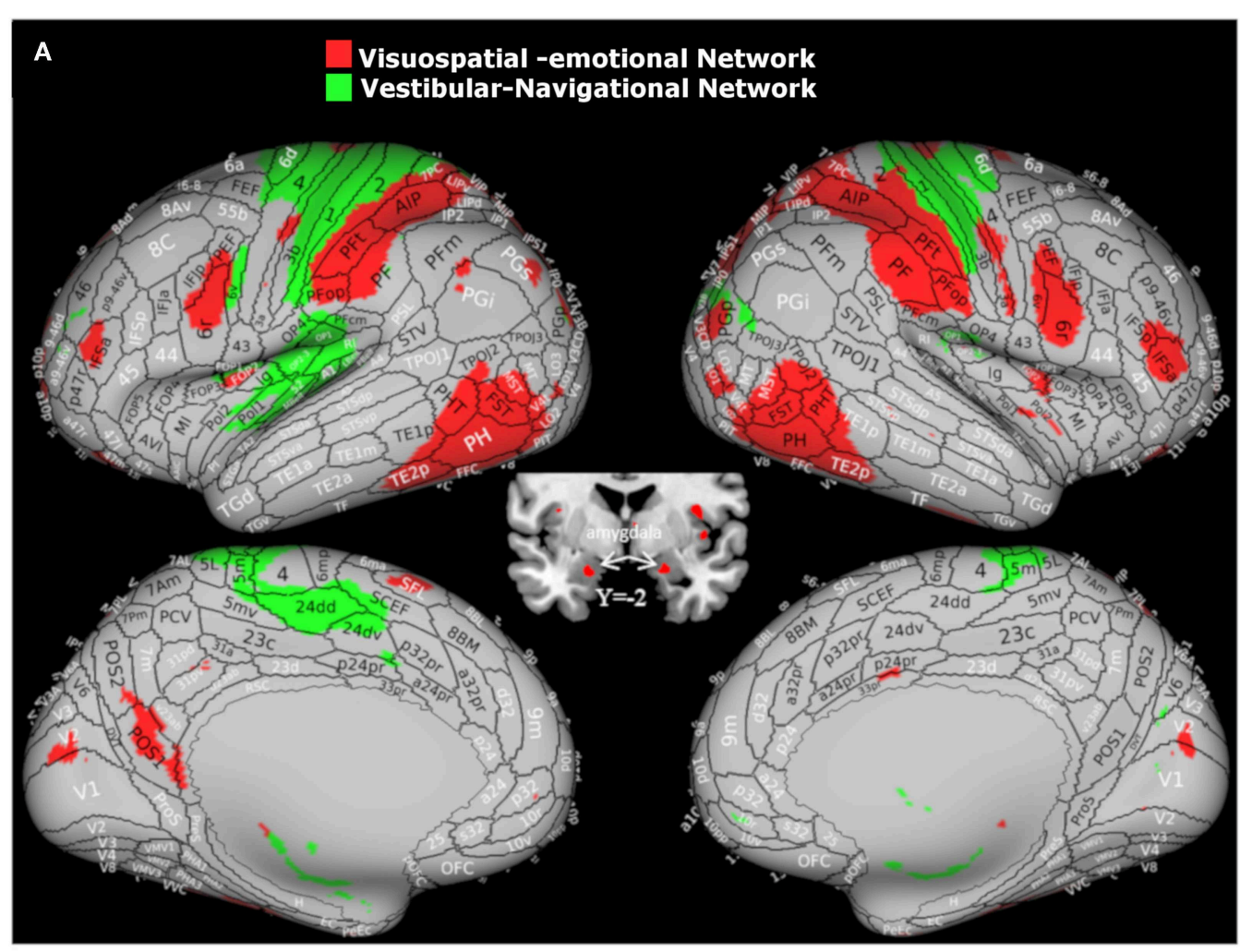

B

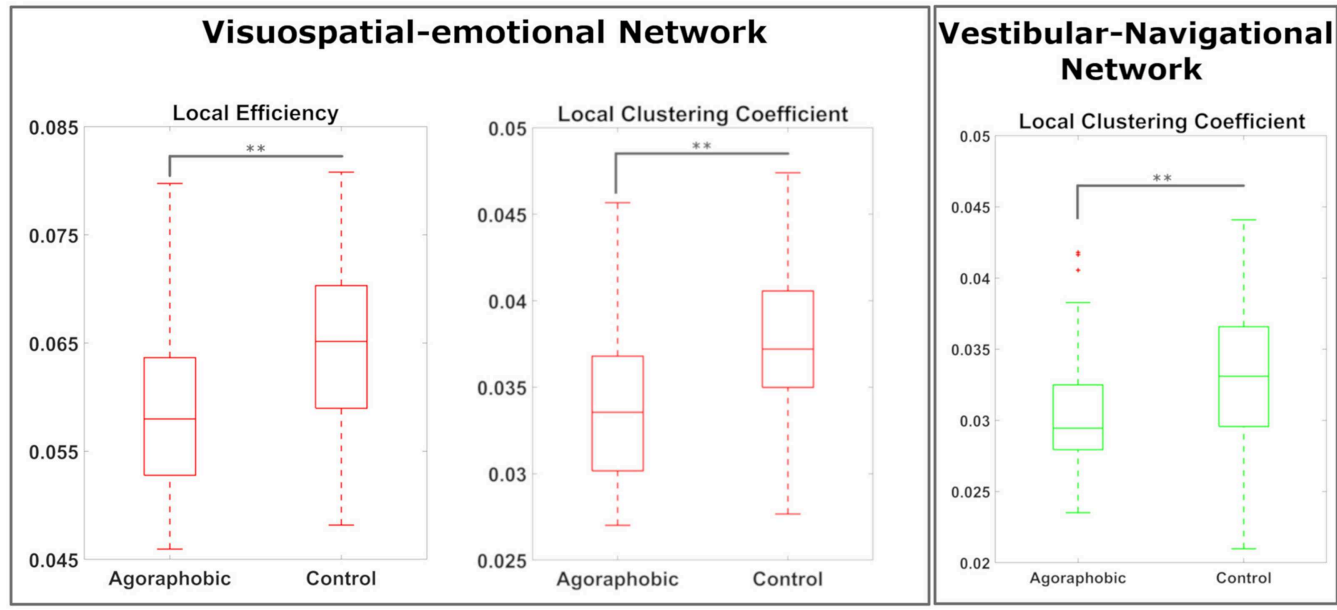

C
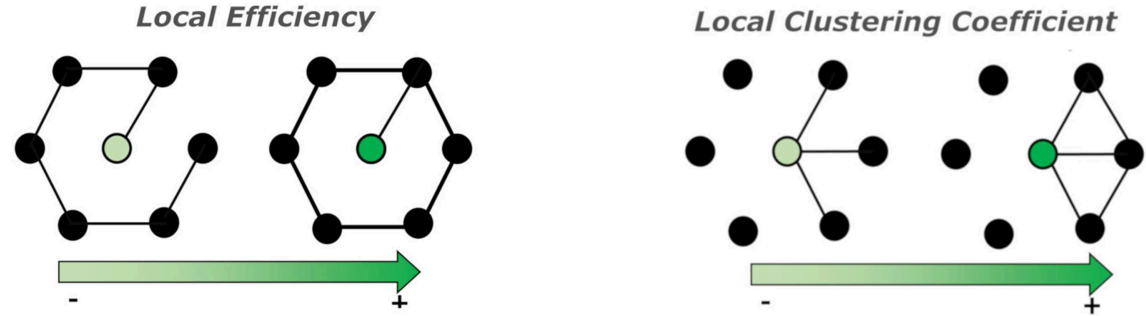

FIGURE 3 | (A) The visuospatial-emotional network [node 23, shown in red on the inflated brain (66)] and vestibular-navigational network (node 41, in green) showed reduced clustering coefficients in patients with subclinical agoraphobic symptoms compared to the control group. The visuospatial-emotional network also had a 
FIGURE 3 | lower efficiency subjects with agoraphobic symptoms. ICAs include values higher than the 98'th percentile. (B) The median value (line), quartiles (boxes), and extremes (whiskers) of the local metrics for both nodes are shown. The comparisons between the two subject groups were performed using non-parametric Mann-Whitney $U$ tests. P-values were corrected for the number of nodes (100) using FDR ( $p$-value t* $_{<}$0.01). (C) Examples of networks in which local efficiency and clustering coefficient vary as a function of connections.
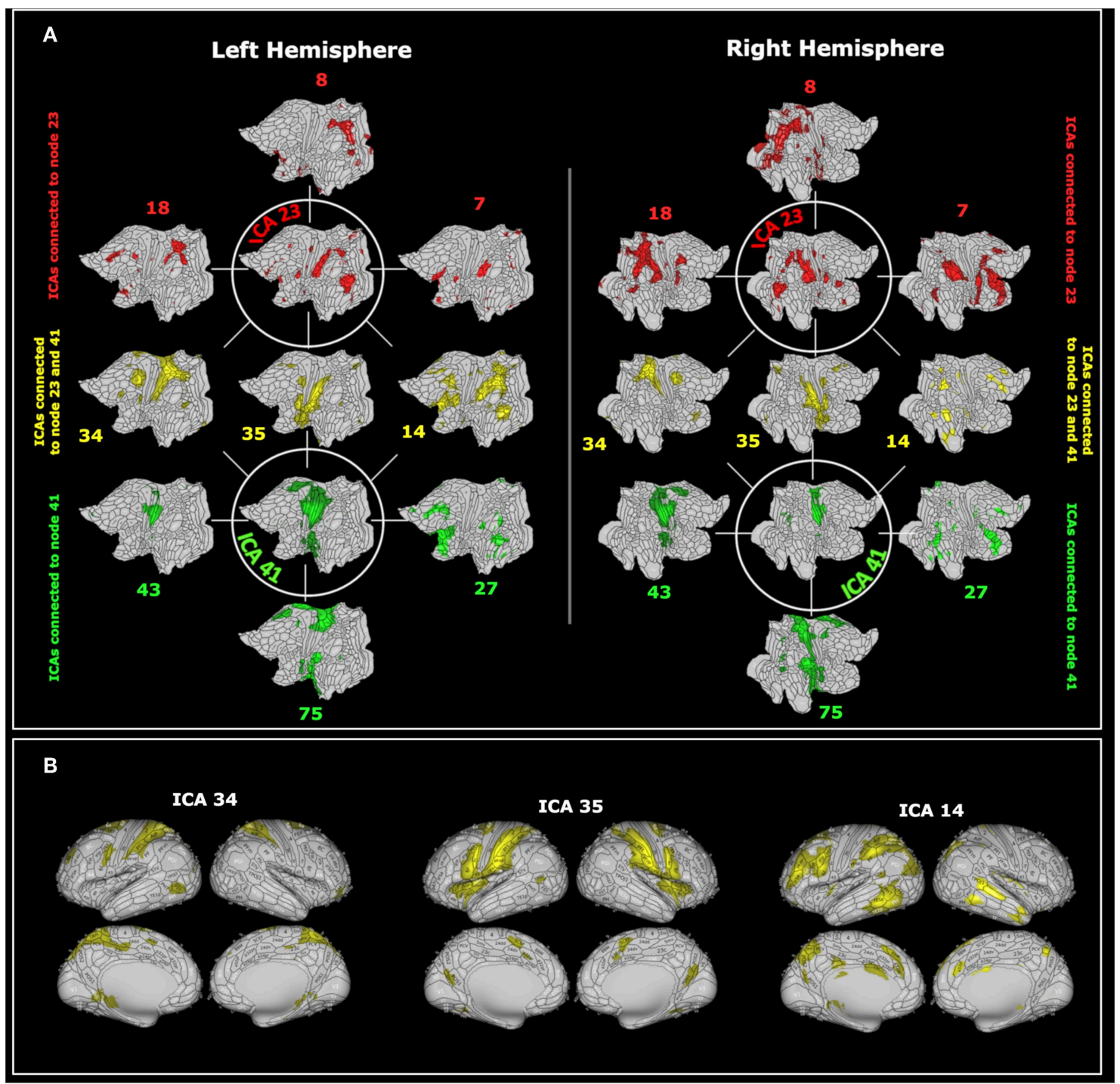

FIGURE 4 | (A) The visuospatial-emotional network centered about ICA 23 (in green) and the vestibular-navigational network centered about ICA 41 (in red) are superimposed on flat brain (66). The visuospatial-emotional network was strongly connected with regions 18, 8, 7, whereas the vestibular-navigational network was connected with regions 43, 75, 27. The two networks overlapped at nodes 34, 35, and 14 (in yellow). (B) The shared nodes are depicted again superimposed on the inflated brain (66). For visualization purposes we only reported the 6 nodes most closely connected to ICA 23 or 41 . ICAs include values higher than the 98 'th percentile.

variances. These strict criteria enabled us to study the neural correlates of agoraphobic symptoms far more independently of confounding influences than previous studies of agoraphobia and PPPD. In the best controlled investigations of PPPD published to date, for example, patient and control groups were matched for mean scores of measures of state anxiety and depression and five personality factors $(39,40,45)$. We also were able to exploit the image quality of the HCP database to extract a high granularity yet robust decomposition of brain nodes and networks through group-ICA (dimensionality 100).

First, we found that agoraphobic symptoms were related to lower global efficiency, clustering coefficient and transitivity, reflecting overall lower integrative functioning across the entire brain. Second, through analyses of local, component-wise effects, 


\section{Visuospatial-Emotional Network}
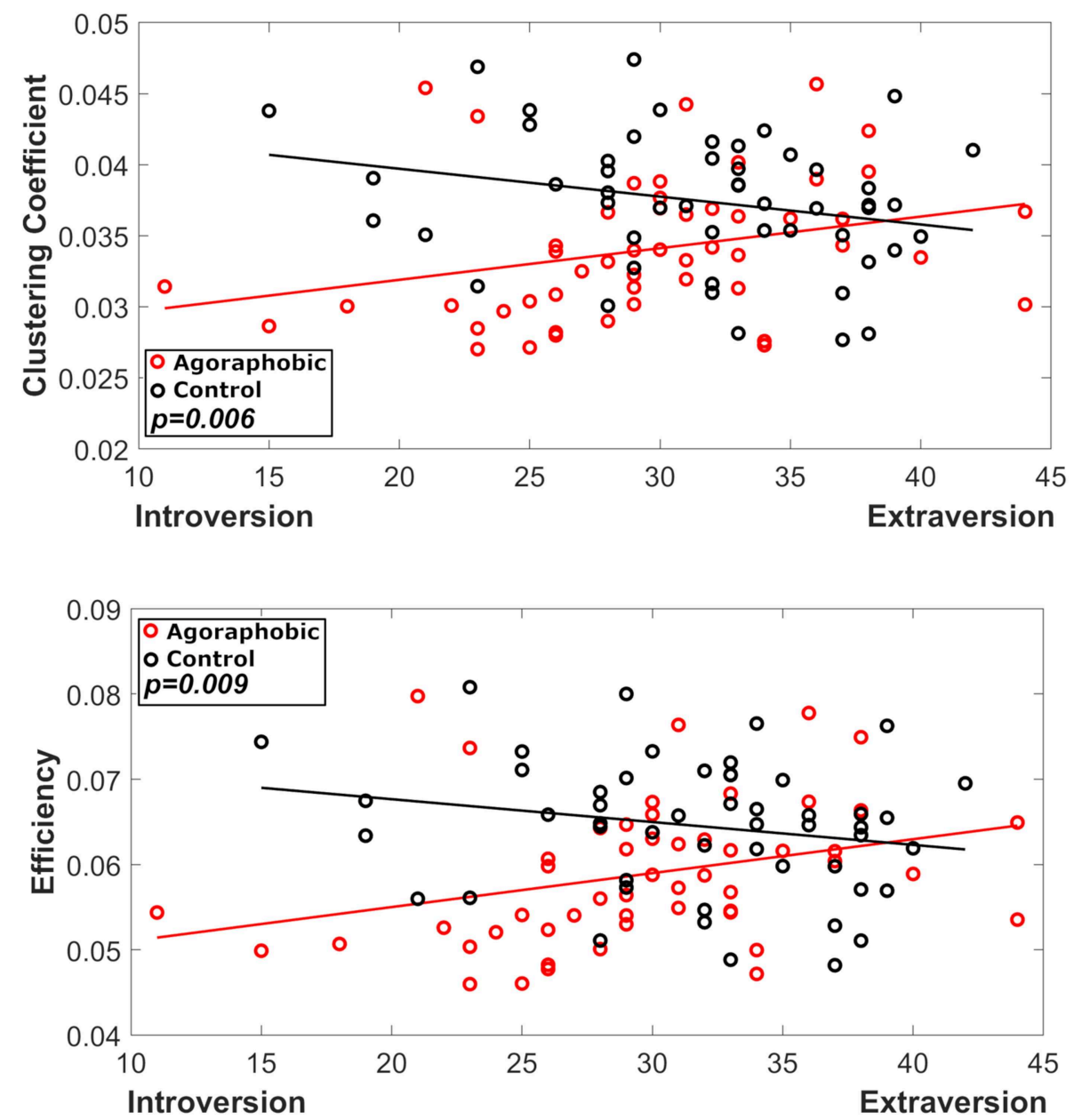

FIGURE 5 | Results of the interaction analysis of agoraphobic group and introversion, where red and black symbols refer to the agoraphobic and control groups, respectively. In node 23 both local efficiency $(p=0.009)$ and clustering coefficient $(p=0.006)$ were significantly affected by the interaction of agoraphobic group and introversion.

we identified two networks which, when comparing patients with agoraphobic symptoms to healthy controls, scored lower in at least one connectivity measure. The visuospatial-emotional network included portions of the primary visual cortex, ventral visual stream, multiple regions of the parietal lobe, and parts of the pre-motor and pre-frontal cortices as well as the anterior thalamus, basal ganglia, and amygdala. This network would be well-positioned to process incoming visual stimuli (occipital lobe and visual stream), link them to vestibular and somatosensory inputs (parietal association areas and posterior insula), and use this information to plan and initiate or inhibit locomotor commands (pre-motor and pre-frontal regions) in response to desires and threats in the environment (amygdala and associated subcortical structures). However, the lower level of efficiency and clustering of this network suggests that patients with subclinical agoraphobic symptoms may not incorporate all of this information into high level management of their behaviors, but perhaps react more instinctively to agoraphobic stimuli. The fact that introversion further reduced efficiency and clustering of this network raises the possibility that individuals with this personality trait are even more strongly driven by innately determined reactions to agoraphobic stimuli.

The vestibular-navigational network encompassed core regions of the multimodal vestibular cortex in the parietal operculum bilaterally plus areas of the primary somatosensory cortex, motor cortex, pre-motor cortex, cingulate and hippocampus. This suggests a pre-dominant role on the output side of locomotor control. The vestibular-navigational network is well-positioned to control (motor and pre-motor cortices) and monitor (somatosensory and vestibular cortices) movements in space (hippocampus) and compare intended to actual outcomes (cingulate cortex). The lower level of clustering 
of this network corroborates the fact that agoraphobic stimuli are salient for networks that are dedicated to control of movement in space.

Spatial and navigation systems are characterized by a high degree of redundancy. Detection of movement of self is supported by the multimodal nature of the vestibular cortex in which vestibular signals, somatosensory inputs, and optic flow all provide information about self-motion (74). Detection of movement in the environment is much more dependent on visual information (hearing plays a lesser role in humans), but vestibular and somatosensory inputs are necessary to stabilize the eyes on targets of interest. Navigation is supported by neurons that are sensitive to the direction and speed of self-motion. These are redundantly located not only in the hippocampal formation and entorhinal cortex, but also across the parietal cortex and subcortical structures (73). Thus, reductions in integrative functioning of the visuospatial-emotional and vestibular-navigational networks in individuals with self-reported agoraphobic symptoms does not preclude adequate processing of visual space-motion data or acceptable control of movement but indicates that these networks may weigh data that they process differently than normal. One example is visual dependency, i.e., the tendency to rely more strongly on visual than vestibular or somatosensory information for spatial orientation. This over-reliance on visual inputs has been reported in patients with agoraphobia, perhaps because vision can detect threats at a distance whereas vestibular and somatosensory systems require contact with the body. Regardless, over-weighting of visual information may cause spatial disorientation in environments with complex patterns or multiple moving objects (75).

The visuospatial-emotional and vestibular-navigational networks identified in patients with sub-clinical agoraphobia have comparable, though not identical, counterparts in patients with PPPD. Studies using sound-evoked vestibular stimuli found reduced activity and connectivity in a network encompassing the parietal opercula, posterior insula, posterior superior temporal sulcus, superior parietal cortex and motor vestibular regions in patients with chronic subjective dizziness, a PPPD precursor, compared to healthy controls (38). This is roughly analogous to the vestibular-navigational network identified in this study of patients with agoraphobic symptoms. In addition to the overlapping brain structures that comprised these networks, neither was influenced by neuroticism or introversion. Investigations employing visual motion stimuli in patients with PPPD identified a link between visual (V3 and middle occipital gyrus) and frontal regions (inferior frontal gyrus/anterior insula) (40), but this was more limited than the visuospatial-emotional network found in patients with agoraphobic symptoms in that it did not extend into the parietal lobe, posterior insula or pre-motor areas. Furthermore, neuroticism was associated with increased connectivity in the visual-frontal network of patients with PPPD (45) whereas introversion interacted with agoraphobic symptoms to decrease connectivity in the visuospatial-emotional network of patients with subclinical agoraphobia. Interestingly, neuroimaging findings in a study of patients with phobic postural vertigo (i.e., individuals with PPPD plus substantial phobic/avoidant symptoms) were centered about vestibular cortical regions of the parietal operculum and insula, but also extended into the motor cortex, orbitofrontal, and anterior cingulate cortices (44). Those results were not adjusted for state anxiety though half of the patients were too anxious to tolerate the confined spaces of the MRI scanner, suggesting that the associated phobic avoidant symptoms rather than core elements of PPPD were responsible for the broad changes in brain structure and function.

Taken together with previous neuroimaging work on PPPD and its precursors (38-45), the results of this investigation provide information about the architecture of brain networks subserving spatial orientation, control of locomotion, and threat assessment as they relate to PPPD and agoraphobic symptoms. They indicate that Westphal's observation that one process linked alterations in sense of space, control of movement in motionrich environments, and instinctive fear reactions (1) was not an error but may involve two linked networks in the brain. At the same time, they also suggest that Westphal's agoraphobia had two components that reflect the contemporary separation of agoraphobia and PPPD into separate clinical entities. The first component involves the detection and processing of afferent visual space-motion information and any threats contained therein, plus initiation or inhibition of behavioral responses. The brain network underlying these functions is less wellconnected in patients with agoraphobic symptoms than in normal individuals, an effect that is amplified by the severity of introversion. In contrast, an overlapping, but narrower network of visual-frontal regions shows greater connectivity in patients with PPPD compared to normal controls in proportion to the severity of neuroticism (45). With regard to this component, patients with phobic postural vertigo (i.e., PPPD plus phobic/avoidance symptoms) seem to possess alterations in brain functioning that are closer to agoraphobia than PPPD alone (44). The second component involves the motor control and monitoring of locomotion. The brain network underlying these functions is similar in patients with subclinical agoraphobia and PPPD. It is less well-connected in both disorders, but not affected by anxiety-related personality traits in either one.

\section{LIMITATIONS OF THE STUDY}

The investigation of a cohort of individuals with subclinical agoraphobic symptoms may be considered a strength and a weakness of study design. On the positive side, we were able to study the neural correlates of visual space-motion processing in our subjects without the potential confounds of serious panic/phobic symptoms. On the negative side, our findings are limited to sub-threshold symptoms and may not apply to patients with fully developed diagnoses of agoraphobia. This limitation is mitigated by the observation of other investigators that agoraphobic symptoms lie on a continuum from normal to pathological levels (50); that is, patients with the clinical diagnosis of agoraphobia have quantitatively, not qualitatively, different symptoms than individuals with subclinical presentations. Thus, 
we likely investigated one end of a continuous spectrum, not a distinct entity. In the HCP database, agoraphobia was evaluated through the SSAGA interview; however, we did not have access to the complete results of the interview, which examines autonomic and vestibular symptoms associated with avoidance in detail. Thus, our findings may be limited by the extent of symptoms that were available to us to characterize our agoraphobic group.

\section{DATA AVAILABILITY}

Publicly available datasets were analyzed in this study. This data can be found here: http://www.humanconnectome.org/.

\section{ETHICS STATEMENT}

Data were provided by the Human Connectome Project, WUMinn Consortium (Principal Investigators: David Van Essen and Kamil Ugurbil; 1U54MH091657).

\section{AUTHOR CONTRIBUTIONS}

II and NT managed the overall project (conceptualization and methodology). AC performed graph metric calculations. AC and

\section{REFERENCES}

1. Westphal C. Die Agoraphobie, eine neuropathische Erscheinung. Zeitschrift für Psychiatr. (1871) 3:138-61. doi: 10.1007/BF02156040

2. American Psychiatric Association. Diagnostic and Statistical Manual of Mental Disorders. 5. Washington, DC: American Psychiatric Association (2013). doi: 10.1176/appi.books.9780890425596

3. McCabe BF. Diseases of the end organ and vestibular nerve. In: Naunton RF, editor. The Vestibular System. New York, NY: Academic Press (1975). p. 299-302. doi: 10.1016/B978-0-12-514950-1.50027-4

4. Marks IM, Bebbington P. Space phobia: syndrome or agoraphobic variant? $\mathrm{Br}$ Med J. (1976) 2:345-7. doi: 10.1136/bmj.2.6031.345

5. Brandt T, Dieterich M. Phobischer Attacken Schwankschwindel, ein neues Syndrom? Munch Med Wschr. (1986) 28:247-50.

6. Jacob RG, Lilienfeld SO, Furman JMR, Durrant JD, Turner SM. Panic disorder with vestibular dysfunc- tion: Further clinical observation and description of space and motion phobic stimuli. J Anxiety Disorders. (1989) 3:11730. doi: 10.1016/0887-6185(89)90006-6

7. Staab JP, Ruckenstein MJ. Expanding the differen- tial diagnosis of dizziness. Arch Otolaryngol Head Neck Surg. (2007) 133:1706. doi: 10.1001/archotol.133.2.170

8. Staab JP, Eckhardt-Henn A, Horii A, Jacob R, Strupp M, Brandt T, et al. Diagnostic criteria for persistent postural-perceptual dizziness (PPPD): Consensus document of the committee for the Classification of Vestibular Disorders of the Bárány Society. J Vestib Res Equilib Orientat. (2017) 27:191208. doi: 10.3233/VES-170622

9. World Health Organization. International Classification of Diseases. $11^{\text {th }}$ Edition for Mortality and Morbidity Statistics. Available online at: https://icd. who.int/browse11/l-m/en (accessed July 31, 2019).

10. Balaban CD, Jacob RG. Background and history of the interface between anxiety and vertigo. J Anxiety Disorders. (2001) 15:27-51. doi: 10.1016/S0887-6185(00)00041-4

11. Bienvenu OJ, Hettema JM, Neale MC, Prescott CA, Kendler KS. Low extraversion and high neuroticism as indices of genetic and environmental risk for social phobia, agoraphobia, and animal phobia. Am J Psychiatry. (2007) 164:1714-21. doi: 10.1176/appi.ajp.2007.06101667
NT performed statistical analysis. II, AC, and NT prepared the original draft, critically reviewed the manuscript, and approved the final manuscript as submitted. JS, FL, and LP provided methodological perspective on the study, critically reviewed, and edited the initial manuscript and approved the final manuscript as submitted. All authors contributed to manuscript revision and editing.

\section{FUNDING}

Data were provided by the Human Connectome Project, WU-Minn Consortium (Principal Investigators: David Van Essen and Kamil Ugurbil; 1U54MH091657) funded by the $16 \mathrm{NIH}$ Institutes and Centers that support the $\mathrm{NIH}$ Blueprint for Neuroscience Research; and by the McDonnell Center for Systems Neuroscience at Washington University. Research supported by the Italian Ministry of Health (PE-2013-02355372 and IRCCS Fondazione Santa Lucia Ricerca corrente) and by the U.S. Department of Defense Congressionally Directed Medical Research Program W81XWH1810760 PT170028. LP was funded by the Medical Research Council (MRC) (MR/P01271X/1) at the University of Cambridge.

12. Staab JP, Rohe DE, Eggers SD, Shepard NT. Anxious, introverted personality traits in patients with chronic subjective dizziness. J Psychosom Res. (2014) 76:80-3. doi: 10.1016/j.jpsychores.2013.11.008

13. Chiarella G, Petrolo C, Riccelli R, Giofrè L, Olivadese G, Gioacchini FM, et al. Chronic subjective dizziness: analysis of underlying personality factors. $J$ Vestib Res. (2016) 26:403-8. doi: 10.3233/VES-160590

14. Yan Z, Cui L, Yu T, Liang H, Wang Y, Chen CC. Analysis of the characteristics of persistent postural- perceptual dizziness: a clinical-based study in China. Int J Audiol. (2016) 56:1-5. doi: 10.1080/14992027.2016.1211763

15. Staab JP, Ruckenstein MJ. Which comes first? Psy- chogenic dizziness versus otogenic anxiety. Laryngoscope. (2003) 113:17148. doi: 10.1097/00005537-200310000-00010

16. Yardley L, Britton J, Lear S, Bird J, Luxon LM. Relationship between balance system function and agoraphobic avoidance. Behav Res Ther. (1995) 33:4359. doi: 10.1016/0005-7967(94)00060-W

17. Jacob RG, Furman JM, Durrant JD, Turner SM. Panic, agoraphobia, and vestibular dysfunction. Am J Psychiatry. (1996) 153:50312. doi: 10.1176/ajp.153.4.503

18. Perna G, Dario A, Caldirola D, Stefania B, Cesarani A, Bellodi L. Panic disorder: the role of the balance system. J Psychiatr Res. (2001) 35:27986. doi: 10.1016/S0022-3956(01)00031-0

19. Jacob RG, Redfern MS, Furman JM. Space and motion discomfort and abnormal balance control in patients with anxiety disorders. J Neurol Neurosurg Psychiatry. (2009) 80:74-8. doi: 10.1136/jnnp.2007.136432

20. Jacob RG, Furman JM, Durrant JD, Turner SM. Surface dependence: a balance control strategy in panic disorder with agoraphobia. Psychosom Med. (1997) 59:323-30. doi: 10.1097/00006842-199705000-00016

21. Hoffman DL, O'Leary DP, Munjack DJ. Autorotation test abnormalities of the horizontal and vertical vestibulo-ocular reflexes in panic disorder. Otolaryngol Head Neck Surg. (1994) 110:259-69. doi: 10.1016/S0194-5998(94)70768-5

22. Staab JP. Chronic subjective dizziness, Continuum: Life- long Learning in Neurology (Minneap Minn). Neuro Otol. (2012) 18:1118-41. doi: 10.1212/01.CON.0000421622.56525.58

23. Perna G, Alpini D, Caldirola D, Raponi G, Cesarani A, Bellodi L. Serotonergic modulation of the balance system in panic disorder: an open study. Depress Anxiety. (2003) 17:101-6. doi: 10.1002/da.10092 
24. Popkirov S, Staab JP, Stone J. Persistent postural-perceptual dizziness (PPPD): a common, characteristic and treatable cause of chronic dizziness. Pract Neurol. (2018) 18:5-13. doi: 10.1136/practneurol-2017-001809

25. Staab JP, Balaban CD, Furman JM. Threat assessment and locomotion: clinical applications of an integrated model of anxiety and postural control. Semin Neurol. (2013) 33:297-306. doi: 10.1055/s-0033-1356462

26. Indovina I, Riccelli R, Staab JP, Lacquaniti F, Passamonti L. Personality traits modulate subcortical and cortical vestibular and anxiety responses to sound-evoked otolithic receptor stimulation. J Psychosom Res. (2014) 77:391400. doi: 10.1016/j.jpsychores.2014.09.005

27. Riccelli R, Indovina I, Staab JP, Nigro S, Augimeri A, Lacquaniti F, et al. Neuroticism modulates brain visuo-vestibular and anxiety systems during a virtual rollercoaster task. Hum Brain Mapp. (2016) 38:71526. doi: $10.1002 / \mathrm{hbm} .23411$

28. Lopez C, Blanke O, Mast FW. The human vestibular cortex revealed by coordinate-based activation likelihood estimation meta-analysis. Neuroscience. (2012) 212:159-79. doi: 10.1016/j.neuroscience.2012.03.028

29. zu Eulenburg P, Caspers S, Roski C, Eickhoff SB. Meta-analytical definition and functional connectivity of the human vestibular cortex. NeuroImage. (2012) 60:162-9. doi: 10.1016/j.neuroimage.2011.12.032

30. Indovina I, Maffei V, Pauwels K, Macaluso E, Orban GA, Lacquaniti F. Simulated self-motion in a visual gravity field: Sensitivity to vertical and horizontal heading in the human brain. Neuroimage. (2013) 71:11424. doi: 10.1016/j.neuroimage.2013.01.005

31. Lacquaniti F, Bosco G, Gravano S, Indovina I, La Scaleia B, Maffei V, et al. Multisensory integration and internal models for sensing gravity effects in primates. Biomed Res Int. (2014) 2014:615854. doi: 10.1155/2014/615854

32. Maffei V, Indovina I, Macaluso E, Ivanenko YP, A Orban G, Lacquaniti F. Visual gravity cues in the interpretation of biological movements: neural correlates in humans. Neuroimage. (2015) 104:221-30. doi: 10.1016/j.neuroimage.2014.10.006

33. Balaban $\mathrm{CD}$. Projections from the parabrachial nucleus to the vestibular nuclei: potential substrates for autonomic and limbic influences on vestibular responses. Brain Res. (2004) 996:126-37. doi: 10.1016/j.brainres.2003.10.026

34. Balaban CD. Neural substrates linking balance control and anxiety. Physiol Behav. (2002) 77:469-75. doi: 10.1016/S0031-9384(02)00935-6

35. Balaban CD, Thayer JF. Neurological bases for balance-anxiety links. J Anxiety Disord. (2001) 15:53-79. doi: 10.1016/S0887-6185(00)00042-6

36. Craig ADB. How do you feel-now? The anterior insula and human awareness. Nat Rev Neurosci. (2009) 10:59-70. doi: 10.1038/nrn2555

37. Indovina I, Robbins TW, Núñez-Elizalde AO, Dunn BD, Bishop SJ. Fearconditioning mechanisms associated with trait vulnerability to anxiety in humans. Neuron. (2011) 69:563-71. doi: 10.1016/j.neuron.2010.12.034

38. Indovina I, Riccelli R, Chiarella G, Petrolo C, Augimeri A, Giofrè L, et al. Role of the Insula and vestibular system in patients with chronic subjective dizziness: an fMRI Study Using Sound-Evoked Vestibular Stimulation. Front Behav Neurosci. (2015) 9:334. doi: 10.3389/fnbeh.2015.00334

39. Nigro S, Indovina I, Riccelli R, Chiarella G, Petrolo C, Lacquaniti F, et al. Reduced cortical folding in multi-modal vestibular regions in persistent postural perceptual dizziness. Brain Imaging Behav. (2018) 13:798809. doi: 10.1007/s11682-018-9900-6

40. Riccelli R, Passamonti L, Toschi N, Nigro S, Chiarella G, Petrolo C, et al. Indovina Altered I insular and occipital responses to simulated vertical selfmotion in patients with persistent postural-perceptual dizziness. Front Neurol. (2017) 8:529. doi: 10.3389/fneur.2017.00529

41. Lee J-O, Lee E-S, Kim J-S, Lee Y-B, Jeong Y, Choi BS, et al. Altered brain function in persistent postural perceptual dizziness: A study on resting state functional connectivity. Hum Brain Mapp. (2018) 39:334053. doi: $10.1002 / \mathrm{hbm} .24080$

42. Na S, Im JJ, Jeong H, Lee E-S, Lee T-K, Chung Y-A, et al. Cerebral perfusion abnormalities in patients with persistent postural-perceptual dizziness (PPPD): a SPECT study. J Neural Transm Vienna Austria. (2019) 126:123-9. doi: 10.1007/s00702-018-1948-3

43. Wurthmann S, Naegel S, Schulte Steinberg B, Theysohn N, Diener H-C, Kleinschnitz C, et al. Cerebral gray matter changes in persistent postural perceptual dizziness. J Psychosom Res. (2017) 103:95-101. doi: 10.1016/j.jpsychores.2017.10.007
44. Popp P, Zu Eulenburg P, Stephan T, Bögle R, Habs M, Henningsen P, et al. Cortical alterations in phobic postural vertigo - a multimodal imaging approach. Ann Clin Transl Neurol. (2018) 5:717-29. doi: 10.1002/acn3.570

45. Passamonti L, Riccelli R, Lacquaniti F, Staab JP, Indovina I. Brain responses to virtual reality visual motion stimulation are affected by neurotic personality traits in patients with persistent postural-perceptual dizziness. J Vestib Res. Equilib Orientat. (2019) 28:369-78. doi: 10.3233/VES-190653

46. Damsa C, Kosel M, Moussally J. Current status of brain imaging in anxiety disorders. Curr Opin Psychiatry. (2009) 22:96-110. doi: 10.1097/YCO.0b013e328319bd10

47. Ferrari MCF, Busatto GF, McGuire PK, Crippa JAS. Structural magnetic resonance imaging in anxiety disorders: an update of research findings. Rev Bras Psiquiatr São Paulo Braz. (2008) 30:251-64. doi: 10.1590/S1516-44462008000300013

48. Engel KR, Obst K, Bandelow B, Dechent P, Gruber O, Zerr I, et al. Functional MRI activation in response to panic-specific, non-panic aversive, and neutral pictures in patients with panic disorder and healthy controls. Eur Arch Psychiatry Clin Neurosci. (2016) 266:557-66. doi: 10.1007/s00406-015-0653-6

49. Wittmann A, Schlagenhauf F, Guhn A, Lueken U, Gaehlsdorf C, Stoy $\mathrm{M}$, et al. Anticipating agoraphobic situations: the neural correlates of panic disorder with agoraphobia. Psychol Med. (2014) 44:238596. doi: 10.1017/S0033291713003085

50. Besteher B, Squarcina L, Spalthoff R, Bellani M, Gaser C, Nenadić I, et al. Subclinical agoraphobia symptoms and regional brain volumes in non-clinical subjects: between compensation and resilience? Front Psychiatry. (2018) 9: 541. doi: 10.3389/fpsyt.2018.00541

51. Van Essen DC, Smith SM, Barch DM, Behrens TEJ, Yacoub E, Ugurbil K, et al. The WU-Minn Human Connectome Project: an overview. Neuroimage. (2013) 80:62-79. doi: 10.1016/j.neuroimage.2013.05.041

52. Dieterich M, Bense S, Lutz S, Drzezga A, Stephan T, Bartenstein P, et al. Dominance for vestibular cortical function in the non-dominant hemisphere. Cereb Cortex N Y N. (2003) 13:994-1007. doi: 10.1093/cercor/13.9.994

53. Oldfield RC. The assessment and analysis of handedness: the Edinburgh inventory. Neuropsychologia. (1971) 9:97113. doi: 10.1016/0028-3932(71)90067-4

54. Bucholz KK, Cadoret R, Cloninger CR, Dinwiddie SH, Hesselbrock VM, Nurnberger JI, et al. A new, semi-structured psychiatric interview for use in genetic linkage studies: a report on the reliability of the SSAGA. J Stud Alcohol. (1994) 55:149-58. doi: 10.15288/jsa.1994.55.149

55. Schalet BD, Pilkonis PA, Yu L, Dodds N, Johnston KL, Yount $\mathrm{S}$, et al. Clinical validity of PROMIS Depression, Anxiety, and Anger across diverse clinical samples. J Clin Epidemiol. (2016) 73:119-27. doi: 10.1016/j.jclinepi.2015.08.036

56. Clark LA, Watson D. Tripartite model of anxiety and depression: psychometric evidence and taxonomic implications. J Abnorm Psychol. (1991) 100:316-36. doi: 10.1037//0021-843X.100.3.316

57. Lee S-A, Kim K-H, Cho S-M. Validation of the mood and anxiety symptom questionnaire in Korean adolescents. Psychiatry Invest. (2015) 12:21826. doi: 10.4306/pi.2015.12.2.218

58. Pilkonis PA, Yu L, Dodds NE, Johnston KL, Maihoefer CC, Lawrence SM. Validation of the depression item bank from the Patient-Reported Outcomes Measurement Information System (PROMIS) in a three-month observational study. J Psychiatr Res. (2014) 56:112-19. doi: 10.1016/j.jpsychires.2014.05.010

59. Costa PT Jr., McCrae RR. Stability and change in personality assessment: the revised NEO Personality Inventory in the year 2000. J Pers Assess. (1997) 68:86-94. doi: 10.1207/s15327752jpa6801_7

60. Cohen S, Kamarck T, Mermelstein R. A global measure of perceived stress. $J$ Health Soc Behav. (1983) 24:385-96. doi: 10.2307/2136404

61. Smith SM, Beckmann CF, Andersson J, Auerbach EJ, Bijsterbosch J, Douaud G, et al. Resting-state fMRI in the Human Connectome Project. Neuroimage. (2013) 80:144-68. doi: 10.1016/j.neuroimage.2013.05.039

62. Glasser MF, Sotiropoulos SN, Wilson JA, Coalson TS, Fischl B, Andersson JL, et al. The minimal preprocessing pipelines for the Human Connectome Project. Neuroimage. (2013) 80:10524. doi: 10.1016/j.neuroimage.2013.04.127

63. Salimi-Khorshidi G, Douaud G, Beckmann CF, Glasser MF, Griffanti L, Smith SM. Automatic denoising of functional MRI data: combining independent 
component analysis and hierarchical fusion of classifiers. Neuroimage. (2014) 90:449-68. doi: 10.1016/j.neuroimage.2013.11.046

64. Griffanti L, Salimi-Khorshidi G, Beckmann CF, Auerbach EJ, Douaud G, Sexton CE, et al. ICA-based artefact removal and accelerated fMRI acquisition for improved resting state network imaging. Neuroimage. (2014) 95:23247. doi: 10.1016/j.neuroimage.2014.03.034

65. Robinson EC, Jbabdi S, Glasser MF, Andersson J, Burgess GC, Harms MP, et al. MSM: a new flexible framework for Multimodal Surface Matching. Neuroimage. (2014) 100:414-26. doi: 10.1016/j.neuroimage.2014.05.069

66. Glasser MF, Coalson TS, Robinson EC, Hacker CD, Harwell J, Yacoub E, et al. A multi-modal parcellation of human cerebral cortex. Nature. (2016) 536:171-8. doi: 10.1038/nature18933

67. Beckmann CF, Smith SM. Probabilistic independent component analysis for functional magnetic resonance imaging. IEEE Trans Med Imaging. (2004) 23:137-52. doi: 10.1109/TMI.2003.822821

68. Smith SM, Hyvärinen A, Varoquaux G, Miller KL, Beckmann CF. Group-PCA for very large fMRI datasets. Neuroimage. (2014) 101:73849. doi: 10.1016/j.neuroimage.2014.07.051

69. Toschi N, Duggento A, Passamonti L. Functional connectivity in amygdalarsensory/(pre)motor networks at rest: new evidence from the Human Connectome Project. Eur J Neurosci. (2017) 45:1224-9. doi: 10.1111/ejn.13544

70. Rubinov M, Sporns O. Complex network measures of brain connectivity: uses and interpretations. Neuroimage. (2010) 52:1059-69. doi: 10.1016/j.neuroimage.2009.10.003

71. von Economo C, Koskinas GN. Chapter 2: Atlas of cytoarchitectonics of the adult human cerebral cortex. In: Geyer S, Turner R, editors. Microstructural
Parcellation of the Human Cerebral Cortex. Basel: Karger (2008). p. 33-53.

72. Kravitz DJ, Saleem KS, Baker CI, Mishkin M. A new neural framework for visuospatial processing. Nat Rev Neurosci. (2011) 12:217-30. doi: $10.1038 / \mathrm{nrn} 3008$

73. Indovina I, Maffei V, Mazzarella E, Sulpizio V, Galati G, Lacquaniti F. Path integration in 3D from visual motion cues: a human fMRI study. Neuroimage. (2016) 142:512-21. doi: 10.1016/j.neuroimage.2016.07.008

74. Britten KH. Mechanisms of self-motion perception. Annu Rev Neurosci. (2008) 31:389-410. doi: 10.1146/annurev.neuro.29.051605. 112953

75. Redfern MS, Furman JM, Jacob RG. Visually induced postural sway in anxiety disorders. J Anxiety Disord. (2007) 21:70416. doi: 10.1016/j.janxdis.2006.09.002

Conflict of Interest Statement: The authors declare that the research was conducted in the absence of any commercial or financial relationships that could be construed as a potential conflict of interest.

Copyright () 2019 Indovina, Conti, Lacquaniti, Staab, Passamonti and Toschi. This is an open-access article distributed under the terms of the Creative Commons Attribution License (CC BY). The use, distribution or reproduction in other forums is permitted, provided the original author(s) and the copyright owner(s) are credited and that the original publication in this journal is cited, in accordance with accepted academic practice. No use, distribution or reproduction is permitted which does not comply with these terms. 


\section{OPEN ACCESS}

Edited by:

Roberto Esposito,

A.O. Ospedali Riuniti Marche Nord,

Reviewed by:

Rongfeng Qi,

Nanjing University, China

Dahua Yu,

Inner Mongolia University of Science and Technology, China

*Correspondence:

Wenbin Guo

guowenbin76@csu.edu.cn

Specialty section:

This article was submitted to Neuroimaging and Stimulation,

a section of the journal

Frontiers in Psychiatry

Received: 15 May 2019

Accepted: 25 July 2019

Published: 16 August 2019

Citation:

Ou Y, SU Q, Liu F, Ding Y, Chen J,

Zhang Z, Zhao J and Guo W (2019) Increased Nucleus Accumbens Connectivity in Resting-State Patients With Drug-Naive, First-Episode

Somatization Disorder.

Front. Psychiatry 10:585.

doi: 10.3389/fpsyt.2019.00585

\section{Increased Nucleus Accumbens Connectivity in Resting-State Patients With Drug-Naive, First- Episode Somatization Disorder}

\author{
Yangpan Ou ${ }^{1,2}$, Qinji Su ${ }^{3}$, Feng Liu', Yudan Ding ${ }^{1,2}$, Jindong Chen ${ }^{1,2}$, Zhikun Zhang ${ }^{3}$, \\ Jingping Zhao ${ }^{1,2}$ and Wenbin Guo ${ }^{1,2 *}$ \\ ${ }^{1}$ Department of Psychiatry, The Second Xiangya Hospital of Central South University, Changsha, China, ${ }^{2}$ National Clinical \\ Research Center on Mental Disorders, Changsha, China, ${ }^{3}$ Mental Health Center, The First Affiliated Hospital of Guangxi \\ Medical University, Nanning, China, ${ }^{4}$ Department of Radiology, Tianjin Medical University General Hospital, Tianjin, China
}

The nucleus accumbens (NAc) plays an important role in the reward circuit, and abnormal regional activities of the reward circuit have been reported in various psychiatric disorders including somatization disorder (SD). However, few researches are designed to analyze the NAc connectivity in SD. This study was designed to explore the NAc connectivity in first-episode, drug-naive patients with SD using the bilateral NAc as seeds. Twentyfive first-episode, drug-naive patients with SD and 28 healthy controls were recruited. Functional connectivity (FC) was designed to analyze the images. LIBSVM (a library for support vector machines) was used to identify whether abnormal FC could be utilized to discriminate the patients from the controls. The patients showed significantly increased FC between the left NAc and the right gyrus rectus and left medial prefrontal cortex/anterior cingulate cortex (MPFC/ACC), and between the right NAc and the left gyrus rectus and left MPFC/ACC compared with the controls. The patients could be separated from the controls through increased FC between the left NAc and the right gyrus rectus with a sensitivity of $88.00 \%$ and a specificity of $82.14 \%$. The findings reveal that patients with SD have increased NAc connectivity with the frontal regions of the reward circuit. Increased left NAc-right gyrus rectus connectivity can be used as a potential marker to discriminate patients with SD from healthy controls. The study thus highlights the importance of the reward circuit in the neuropathology of SD.

Keywords: somatization disorder, functional connectivity, reward circuit, functional magnetic resonance imaging, support vector machine

\section{INTRODUCTION}

Somatization disorder (SD) is a psychiatric disorder characterized by multiple, recurrent, and clinically significant complaints of somatic symptoms. Patients with SD usually undergo numerous medical examinations without an accurate diagnosis. Consequently, their medical cost increases dramatically (1).

In recent years, neuroimaging techniques provide us with new ways to analyze changes of brain function and structure in psychiatric patients (2). Anatomical alterations and connectivities have been revealed in patients with SD using structural imaging techniques. For example, patients with 
SD showed decreased fractional anisotropy in the right cingulum and right inferior fronto-occipital fasciculus (3). Atmaca et al. found that patients with SD had significantly small amygdala relative to controls (4). By contrast, increased bilateral caudate nuclei volumes have been detected in patients with SD compared with controls (5).

Previously, abnormal brain regional activities have been found in SD using functional neuroimaging methods. For example, patients with SD showed increased coherence-based regional homogeneity (Cohe-ReHo) in the left medial prefrontal cortex/ anterior cingulate cortex (MPFC/ACC) (6), and increased regional activity in the bilateral MPFC has been detected in patients with SD (7). Patients with SD also showed abnormal functional connectivity (FC) between the cingulate-insular network and sensorimotor network (SMN)/anterior default-mode network (DMN), between the posterior DMN and SMN, and between the anterior DMN and posterior DMN/SMN compared with healthy controls (8). Increased FC strength in the right inferior temporal gyrus (ITG) has been found in patients with SD (9). Moreover, patients with SD exhibited increased cerebellar-DMN connectivity, which was correlated to the somatization severity and personality (10). However, little attention has been focused on the dysconnectivity of the reward circuit in SD.

The reward circuit is a group of neural structures related to associative learning, incentive salience, and positive emotions (11). The mesolimbic reward circuit comprises the NAc, ventral tegmental area (VTA), prefrontal cortex (PFC), and hippocampus $(12,13)$. Located in the ventral striatum, the NAc is an important brain reward region that integrates different inhibitory and excitatory inputs to salience signal of rewarding stimuli (14). In a previous study, patients with SD presented hypoperfusion in the frontal and prefrontal areas using the single-photon emission computed tomography (SPECT) scan (15). Moreover, Hakala et al. revealed regional cerebral hypometabolism in the caudate nuclei, right precentral gyrus, and left putamen in patients with SD (16). These findings suggest that reward circuit is involved in the pathophysiology of SD.

SVM (support vector machine) is a supervised learning model with correlated learning algorithms that analyzes data used for regression and classification analysis (17). Given a pieces of training examples, an SVM training algorithm creates a model that deals new examples to one sort or the other, making it a nonprobabilistic binary linear classifier. SVM structures a hyperplane or set of hyperplanes in a high- or infinite-dimensional space, which can be applied for regression, classification, or other roles like outlier detection. In particular, SVM utilizes a training dataset to get differences between the patients and the controls, and a testing dataset is used to assess classification performance on uncharted data. The classifier algorithm is applied with a leave-pair-out cross-validation (LPO-CV) method to acquire the highest specificity and sensitivity (18). SVM has been widely performed in medical disease. For example, SVM was applied to identify patients with coronary heart disease (CHD) from nonCHD individuals (19). Wang et al. revealed that SVM model could diagnose lymph node metastasis better than preoperative short axis size of largest lymph node on computed tomography (20). In our previous study, SVM analysis could be used to discriminate patients with SD from healthy controls with proper sensitivity and specificity (6). In this study, SVM was used to examine whether abnormal NAc connectivity could be applied to distinguish the patients from the controls.

So far, few studies have analyzed abnormal FC of the reward circuit in SD using the seed-based FC method, which is conducted by calculating the correlations between the preselected brain regions (seeds) and the rest brain regions. This method has been used in subjects with high social anhedonia, and the cortico-striatal abnormalities in the reward-related symptomatology have been revealed (21). In this study, we employed bilateral NAc (from the Harvard Oxford Atlases) as seeds. Then, the seed-based FC method was used to identify abnormal connectivity between the seeds and other regions of brain. Based on abovementioned findings, we hypothesized that increased NAc connectivity would be detected in SD, particularly within the reward circuit, which could be used to discriminate the patients from the controls. We also expected there were some correlations between abnormal FCs and clinical variables in the patients.

\section{MATERIALS AND METHODS}

\section{Participants}

Twenty-five right-handed patients with first-episode and drug-naive SD were recruited from the First Affiliated Hospital of Guangxi Medical University. Twenty-eight healthy controls were recruited from the community. The controls were screened by using the Structured Clinical Interview of the Diagnostic and Statistical Manual of Mental Disorders-IV (SCID), non-patient edition (22), and no neuropsychiatric disorders in their first-degree relatives. Patients with SD should meet the criteria of the SCID, patient edition (22). Somatic symptoms of patients with SD should originate from several specific origins (i.e., at least four pain symptoms, two gastro-intestinal symptoms, one sexual symptom, and one pseudo-neurological symptom), and the symptoms were in the absence of a medical explanation, factitious disorder, or malingering (23). Participants were excluded according to the following criteria: other psychiatric disorders (e.g., bipolar disorders, schizophrenia, or personality disorders), severe medical diseases, substance abuse disorders, mental retardation, and any limits for MRI.

The Hamilton Anxiety Scale (HAMA) (24), Hamilton Depression Scale (HAMD, 17 items) (25), and somatization subscale of Symptom Checklist-90 (SCL-90) (26) were used to assess the symptomatic severity of anxiety, depression, and somatization. Eysenck Personality Questionnaire (EPQ) (27) was used to evaluate personality dimensions. Wisconsin Card Sorting Test (WCST) (28) and digit symbol coding of Wechsler Adult Intelligence Scale (WAIS) were applied to identify cognitive functions.

After given detailed knowledge of the contents, all the participants signed a written informed consent. The local ethics committee of the First Affiliated Hospital of Guangxi Medical University approved this study. 


\section{MRI Acquisition}

Functional MRI scans were obtained with a Siemens 3T scanner. During the procedures, the participants were asked to remain motionless and awake with their eye closed. Soft earplugs and foam pads were used to reduce scanner noise and head motion. Resting-state functional scans were obtained with a gradientecho echo-planar imaging sequence using the following parameters: repetition time/echo time $=2,000 / 30 \mathrm{~ms}, 30$ slices, $64 \times 64$ matrix, $90^{\circ}$ flip angle, $24-\mathrm{cm}$ FOV, 4-mm slice thickness, 0.4-mm gap, and 250 volumes (500 s).

\section{Data Preprocessing}

We preprocessed the imaging data with Data Processing \& Analysis for (resting-state) Brain Imaging (29) in MATLAB. Slice timing and head movement were first corrected, and no participant had more than $2 \mathrm{~mm}$ of maximal displacement in any direction of $\mathrm{x}, \mathrm{y}$, and $\mathrm{z}$ and more than $2^{\circ}$ in any angular dimension. After that, the images were normalized in the standard Montreal Neurological Institute (MNI) EPI space and resampled with $3 \times 3 \times 3-\mathrm{mm}^{3}$ resolution. The obtained images were then smoothed with a 4 -mm full width at half-maximum Gaussian kernel, bandpass filtered $(0.01-0.08 \mathrm{~Hz})$, and linearly detrended. In addition, framewise displacement (FD) was computed as described in a previous study (30). The mean FD is a covariate of no interest to handle the residual effects caused by head motion. We removed time points with $\mathrm{FD}>0.2 \mathrm{~mm}$ to control aggressive head motion. We did not regress out the global signal since it was suggested to be saved in processing the FC data (31).

\section{FC Processing}

Bilateral NAc from the Harvard Oxford Atlases were selected as seeds for the whole-brain FC processing with the software REST (32). For each participant, seed-based FC was computed as Pearson correlation coefficients between the seeds and other voxels of the whole brain. The correlation coefficients were then z-transformed for standard purpose, and seed-based FC maps were generated.

\section{Statistical Analysis}

Two-sample $t$ tests were performed to compare the distribution of age, years of education, and clinical scales between patients with SD and healthy controls. A chi-square test was used to judge sex distributions.

Group differences were compared using voxel-wise two sample t-tests. Age and the mean FD values were used as covariates to minimize the potential effects of these variables. The significance level was set at $p<0.05$ for multiple comparisons corrected by Gaussian random field (GRF) theory (voxel significance: $p<0.001$, cluster significance: $p<0.05)$.

LIBSVM (33) was performed to examine whether abnormal FC between bilateral NAc and other brain regions could distinguish patients with SD from healthy controls.

To explore the correlations between abnormal FC values and clinical variables, voxel-based correlations were conducted. The correlation results were Bonferroni corrected at $p<0.05$.

\section{RESULTS}

\section{Characteristics of the Participants}

General information of the participants is shown in Table 1, and no difference was observed regarding age, sex ratio, education level, EPQ extraversion/lie scores, digit symbol coding of WAIS, and WCST between the two groups. The scores of HAMA, HAMD, EPQ psychoticism/neuroticism, and somatization subscale of SCL-90 of the patients were higher than those of the controls (Table 1).

\section{Group Differences in Seed-Based FC Analyses}

The patients showed significantly increased FC between the left NAc and the right gyrus rectus $(t=4.2239, p<0.001)$ and left MPFC/ACC $(t=3.9208, p<0.001)$, and between the right NAc and the left gyrus rectus $(t=5.7374, p<0.001)$ and left MPFC/ ACC $(t=4.3168, p<0.001)$ compared with the controls (Figure 1 and Table 2).

\section{Correlations Between Abnormal FC and Clinical or Personality or Cognitive Variables in the Patients}

No correlations were detected between increased FC between the left NAc and the right gyrus rectus and left MPFC/ACC, and between the right NAc and the left gyrus rectus and left MPFC/ ACC and clinical or personality or cognitive variables (WCST and digit symbol coding of WAIS) in the patients.

TABLE 1 | Characteristics of participants.

\begin{tabular}{lccc}
\hline Variables & $\begin{array}{c}\text { Patients } \\
(\mathbf{n}=\mathbf{2 5})\end{array}$ & $\begin{array}{c}\text { Controls } \\
\mathbf{( n = 2 8 )}\end{array}$ & $\boldsymbol{p}$ value \\
\hline Age (years) & $41.00 \pm 10.76$ & $38.71 \pm 9.59$ & $0.42^{\mathrm{b}}$ \\
Sex (male/female) & $4 / 21$ & $6 / 22$ & $0.73^{\mathrm{a}}$ \\
Years of education (years) & $7.72 \pm 4.39$ & $7.82 \pm 2.59$ & $0.92^{\mathrm{b}}$ \\
FD (mm) & $0.08 \pm 0.03$ & $0.10 \pm 0.05$ & $0.02^{\mathrm{b}}$ \\
Illness duration (months) & $59.12 \pm 62.22$ & & \\
Somatization subscale of & $28.48 \pm 10.37$ & $14.32 \pm 3.44$ & $<0.001^{\mathrm{b}}$ \\
SCL-90 & & & \\
HAMD & $18.84 \pm 7.31$ & $2.60 \pm 1.83$ & $<0.001^{\mathrm{b}}$ \\
HAMA & $22.96 \pm 10.95$ & $0.53 \pm 0.99$ & $<0.001^{\mathrm{b}}$ \\
Digit symbol coding of WAIS & $8.28 \pm 2.87$ & $9.64 \pm 2.15$ & $0.06^{\mathrm{b}}$ \\
EPQ & & & \\
Extraversion & $46.84 \pm 11.02$ & $49.75 \pm 9.65$ & $0.31^{\mathrm{b}}$ \\
Psychoticism & $50.52 \pm 9.01$ & $45.00 \pm 8.54$ & $0.03^{\mathrm{b}}$ \\
Neuroticism & $57.36 \pm 9.18$ & $46.78 \pm 10.24$ & $<0.001^{\mathrm{b}}$ \\
Lie & $49.44 \pm 12.31$ & $47.96 \pm 11.01$ & $0.65^{\mathrm{b}}$ \\
WCST & & & \\
Number of categories achieved & $3.52 \pm 1.76$ & $3.89 \pm 1.66$ & $0.43^{\mathrm{b}}$ \\
Number of errors & $22.84 \pm 9.12$ & $24.71 \pm 8.91$ & $0.45^{\mathrm{b}}$ \\
Number of perseverative errors & $20.04 \pm 9.48$ & $22.82 \pm 8.72$ & $0.27^{\mathrm{b}}$ \\
\hline
\end{tabular}

aThe $p$ value for sex distribution was obtained by a chi-square test.

${ }^{b}$ The $p$ values were obtained by two samples t-tests.

FD, Framewise displacement; HAMD, Hamilton depression scale; HAMA, Hamilton

Anxiety Scale; SCL-90, Symptom Checklist-90; EPQ, Eysenck Personality

Questionnaire; WAIS, Wechsler Adult Intelligence Scale; WCST, Wisconsin Card

Sorting Test. 


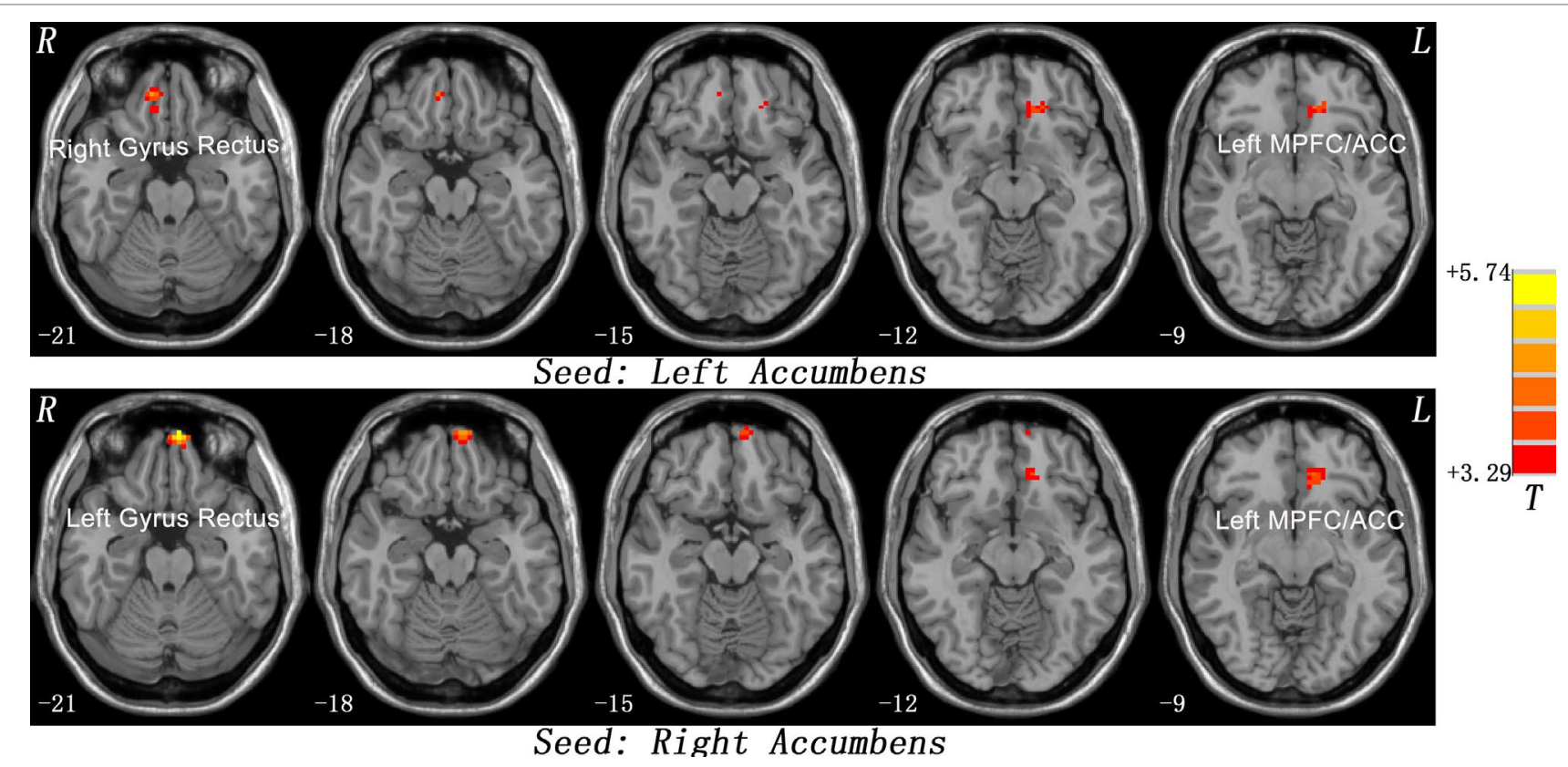

FIGURE 1 | Statistical maps showing seed-based functional connectivity differences between subject groups. The patients showed significantly increased FC between the left NAc and the right gyrus rectus and left MPFC/ACC, and between the right NAc and the left gyrus rectus and left MPFC/ACC compared with the controls. Red denotes high FC values in the patients, and the color bar indicates the T values from two-sample t-tests. FC, functional connectivity; NAc, nucleus accumbens; MPFC/ACC, medial prefrontal cortex/anterior cingulate cortex.

TABLE 2 | Regions with increased functional connectivity with the accumbens in patients.

\begin{tabular}{lccccc}
\hline Cluster location & \multicolumn{3}{c}{ Peak (MNI) } & $\begin{array}{c}\text { Number } \\
\text { of voxels }\end{array}$ & T value \\
\cline { 2 - 4 } & $\mathbf{x}$ & $\mathbf{y}$ & $\mathbf{z}$ & & \\
\hline Seed: Left Accumbens & & & & & \\
Right Gyrus Rectus & 12 & 45 & -24 & 38 & 4.2239 \\
Left MPFC/ACC & -12 & 36 & -9 & 25 & 3.9208 \\
Seed: Right & & & & & \\
Accumbens & -6 & 63 & -21 & 38 & 5.7374 \\
Left Gyrus Rectus & -12 & 36 & -9 & 39 & 4.3168 \\
Left MPFC/ACC & & & & & \\
\hline
\end{tabular}

MNI, Montreal Neurological Institute; MPFC, medial prefrontal cortex; ACC, anterior cingulate cortex.

\section{LIBSVM Analysis}

As shown in Figure 2, the FC values between the left NAc and the right gyrus rectus could correctly classify 22 of 25 patients and 23 of the 28 controls, resulting in an optimal sensitivity of $88.00 \%$ and an optimal specificity of $82.14 \%$ (Figure 2 ).

\section{DISCUSSION}

In this study, we used bilateral NAc as seeds to analyze the seed-based FC in first-episode and drug-naive SD. The primary finding is that patients showed significantly increased FC values between the left NAc and the right gyrus rectus and left MPFC/ ACC, and between the right NAc and the left gyrus rectus and left $\mathrm{MPFC} / \mathrm{ACC}$ compared with the controls. Increased connectivity between the left NAc and the right gyrus rectus can be used as a potential marker to discriminate patients with SD from healthy controls with optimal sensitivity and specificity. There are no correlations between abnormal FC values and clinical variables in the patients.

Increased NAc connectivity with other brains have been found in this study. The NAc receives heterogeneous gamma-aminobutyric acid (GABAergic) and dopaminergic projections from the VTA $(34,35)$ as well as glutamatergic afferents from the PFC (36), hippocampus $(37,38)$, thalamus (39), and amygdala (40). The NAc is a complex, integral hub in the reward circuit (41). For example, patients with SD commonly have pain symptoms, and the NAc plays an important role in reward-aversion processing during pain perception (42). Baliki et al. found that the NAc showed abnormal activities when patients were in the presence of chronic pain, and the NAc activity could anticipate analgesic potential on chronic pain (43).

The MPFC/ACC plays an important role in the reward circuit, which generates emotional and cognitive information (44), and abnormal activity within the MPFC areas may be related to augment pain perception in patients with SD (45). Furthermore, a study showed that negative emotional stimuli could activate the MPFC/ACC, which revealed that the MPFC/ACC might be involved in appraisal and expression of negative emotion (46).

The gyrus rectus, also named straight gyrus, is located at the medial most margin of the inferior surface of frontal lobe and is continuous with the superior frontal gyrus on the medial surface. Up to now, the function of the gyrus rectus 


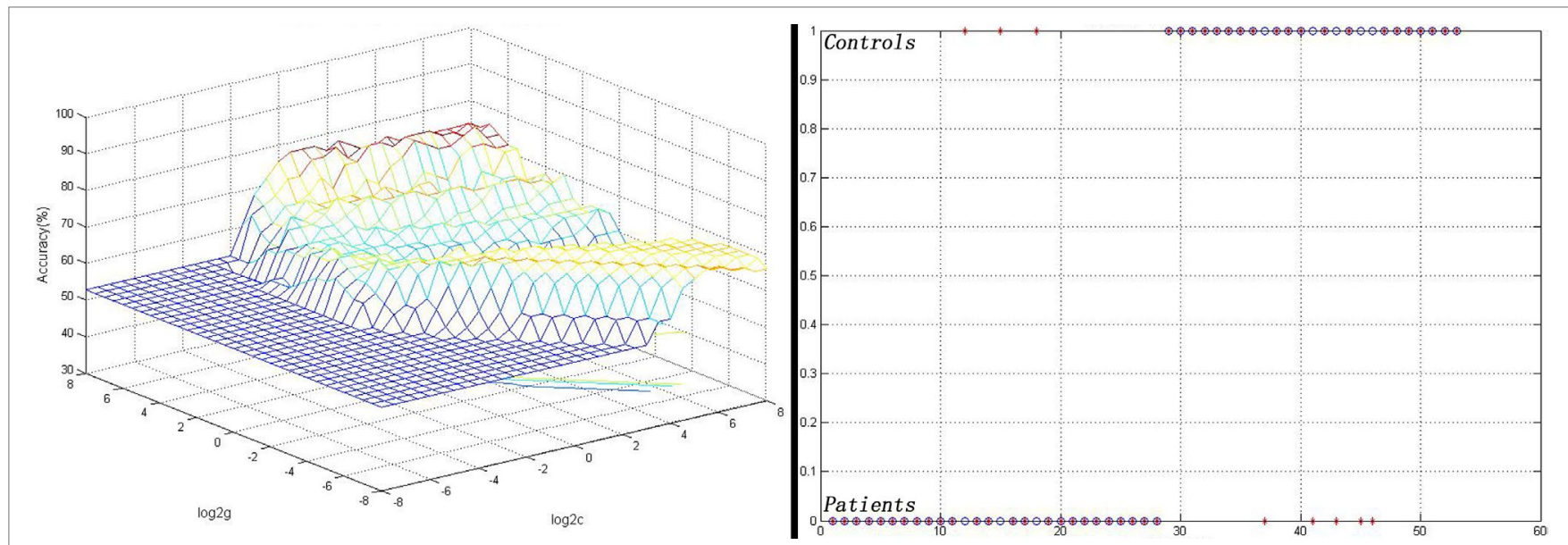

FIGURE 2 | Visualization of the SVM results for identifying patients from controls using the FC values between the left NAc and the right gyrus rectus. Left: 3D view of the classified accuracy with the best parameters; right: classified map of the FC values between the left NAc and the right gyrus rectus. SVM, Support vector machine; FC, functional connectivity; NAc, nucleus accumbens.

is unclear. However, a research suggested that patients with obsessive-compulsive disorder have decreased prefrontal hemodynamic response (47). In our study, patients with SD showed significantly increased FC values between the left NAc and the right gyrus rectus and left $\mathrm{MPFC} / \mathrm{ACC}$, and between the right NAc and the left gyrus rectus and left MPFC/ACC compared with the controls.

Increased FC is usually considered as compensatory reallocation or dedifferentiation to functional deficits in the brain regions $(48,49)$. Patients with SD may have deficits in emotional processing, and the MPFC/ACC is related to the negative emotion (46). Du et al. found that the stimulated dorsolateral PFC-NAc FC can predict the anti-depressant and anti-anxiety effects of repeated transcranial magnetic stimulation (rTMS) (50). Furthermore, deep brain stimulation (DBS) targeting the NAc and rTMS about the left dorsolateral PFC also exhibited antidepressant and antianxiety effects (51-53). Therefore, increased NAc connectivity in the present study may be a compensatory effort to functional deficits in these regions.

In a previous study, a significantly positive correlation has been found between increased activity in the bilateral superior MPFC and the somatization subscale scores of SCL90 in patients with SD (7). We hypothesized that correlations would be detected between increased NAc connectivity and clinical parameters. Therefore, no correlation in the present study is somewhat surprised. There are several possibilities account for this issue. First, sample size of this research may be small to establish a correlation. Second, increased NAc connectivity may be an internal alteration for patients with SD independent of symptomatic severity. Third, the clinical parameters are concentrated, such as the scores of the digit symbol coding of WAIS of the patients with SD are centered at 8.28 points.

SVM analysis suggests that the increased FC values between the left NAc and the right gyrus rectus could be used to discriminate patients with SD from healthy controls with a sensitivity of $88.00 \%$ and a specificity of $82.14 \%$. A highly credible research is characterized by specificity and sensitivity above $70 \%$ in the medical domain (54). Interpretation of the high discriminative power result must think about the multivariate nature of the SVM method. SVM, a multivariate method, has been additionally based on inter-regional correlations, while standard quality univariate techniques regard each voxel as a spatially independent unit (55). Therefore, increased FC values may be used as a potential marker to discriminate patients with SD from healthy controls.

Our study has several limitations. First, this research is a cross-sectional one, and it is unclear how the NAc connectivity will alter after treatment. A longitudinal study is needed to clarify this issue. Second, some studies showed that abnormal FC was correlated to anhedonia (56). However, psychological tests about anhedonia were not assessed in this study. The relationship between abnormal FC and anhedonia remains unknown. Third, the sample size in our study is relatively small, which may minimize the translational value of our findings. Fourth, the HAMA scores and HAMD scores were significantly different between the SD group and HC group. Therefore, there is a possibility that the present findings may be affected by the HAMA scores and HAMD scores. To clarify this issue, we reanalyzed the data with age, mean FD values, HAMA scores, and HAMD scores as covariates and obtained similar results as previously reported. Therefore, the present findings seemed impossible to be affected by HAMA scores and HAMD scores. Finally, the confounding effects of scans, such as respiratory and cardiac rhythm, could not be completely eliminated.

Despite the limitations, the current research first examines the NAc connectivity in resting-state patients with first-episode, drug-naive SD. The findings reveal that patients with SD have increased NAc connectivity with some regions of the reward circuit. Increased NAc connectivity can be used as a potential 
marker to discriminate patients with SD from healthy controls. This study thus highlights the importance of the reward circuit in the neuropathology of SD.

\section{DATA AVAILABILITY}

All datasets generated for this study are included in the manuscript and/or the Supplementary files.

\section{AUTHOR CONTRIBUTIONS}

WG and JZ designed the study. WG, FL, QS, and ZZ collected the original imaging data. WG, FL, YD, and JC managed and analyzed the imaging data. YO wrote the first draft of the manuscript. All the authors contributed to and approved the final manuscript.

\section{REFERENCES}

1. Krishnan V, Sood M, Chadda RK. Caregiver burden and disability in somatization disorder. J Psychosom Res (2013) 75(4):376-80. doi: 10.1016/j. jpsychores.2013.08.020

2. Browning M, Fletcher P, Sharpe M. Can neuroimaging help us to understand and classify somatoform disorders? A systematic and critical review. Psychosom Med (2011) 73(2):173-84. doi: 10.1097/PSY.0b013e31820824f6

3. Zhang J, Jiang M, Yao D, Dai Y, Long L, Yu M, et al. Alterations in white matter integrity in first-episode, treatment-naive patients with somatization disorder. Neurosci Lett (2015) 599:102-8. doi: 10.1016/j.neulet.2015.05.037

4. Atmaca M, Sirlier B, Yildirim H, Kayali A. Hippocampus and amygdalar volumes in patients with somatization disorder. Prog Neuropsychopharmacol Biol Psychiatry (2011) 35(7):1699-703. doi: 10.1016/j.pnpbp.2011.05.016

5. Hakala M, Karlsson H, Kurki T, Aalto S, Koponen S, Vahlberg T, et al. Volumes of the caudate nuclei in women with somatization disorder and healthy women. Psychiatry Res (2004) 131(1):71-8. doi: 10.1016/j.pscychresns.2004.03.001

6. Ou Y, Liu F, Chen J, Pan P, Wu R, Su Q, et al. Increased coherence-based regional homogeneity in resting-state patients with first-episode, drug-naive somatization disorder. J Affect Disord (2018) 235:150-4. doi: 10.1016/j. jad.2018.04.036

7. Su Q, Yao D, Jiang M, Liu F, Jiang J, Xu C, et al. Dissociation of regional activity in default mode network in medication-naive, first-episode somatization disorder. PLoS One (2014) 9(7):e99273. doi: 10.1371/journal. pone.0099273

8. Otti A, Guendel H, Henningsen P, Zimmer C, Wohlschlaeger AM, NollHussong M. Functional network connectivity of pain-related resting state networks in somatoform pain disorder: an exploratory fMRI study. J Psychiatry Neurosci (2013) 38(1):57-65. doi: 10.1503/jpn.110187

9. Su Q, Yao D, Jiang M, Liu F, Jiang J, Xu C, et al. Increased functional connectivity strength of right inferior temporal gyrus in first-episode, drug-naive somatization disorder. Aust N Z J Psychiatry (2015) 49(1):7481. doi: $10.1177 / 0004867414553949$

10. Wang H, Guo W, Liu F, Chen J, Wu R, Zhang Z, et al. Clinical significance of increased cerebellar default-mode network connectivity in resting-state patients with drug-naive somatization disorder. Medicine (Baltimore) (2016) 95(28):e4043. doi: 10.1097/MD.0000000000004043

11. Schultz W. Neuronal reward and decision signals: from theories to data. Physiol Rev (2015) 95(3):853-951. doi: 10.1152/physrev.00023.2014

12. Russo SJ, Nestler EJ. The brain reward circuitry in mood disorders. Nat Rev Neurosci (2013) 14(9):609-25. doi: 10.1038/nrn3381

13. Sesack SR and Grace AA. Cortico-Basal ganglia reward network: microcircuitry. Neuropsychopharmacology (2010) 35(1):27-47. doi: 10.1038/npp.2009.93

\section{FUNDING}

This study was supported by grants from the National Key R\&D Program of China (Grant Nos. 2016YFC1307100 and 2016YFC1306900) and the National Natural Science Foundation of China (Grant Nos. 81571310, 817714477, and 81630033).

\section{ACKNOWLEDGMENTS}

The authors thank all individuals who served as the research participants.

\section{SUPPLEMENTARY MATERIAL}

The Supplementary Material for this article can be found online at: https://www.frontiersin.org/articles/10.3389/fpsyt.2019.00585/ full\#supplementary-material

14. Smith KS, Berridge KC and Aldridge JW. Disentangling pleasure from incentive salience and learning signals in brain reward circuitry. Proc Natl Acad Sci U S A (2011) 108(27):E255-64. doi: 10.1073/pnas.1101920108

15. Garcia-Campayo J, Sanz-Carrillo C, Baringo T, Ceballos C. SPECT scan in somatization disorder patients: an exploratory study of eleven cases. Aust $N$ Z J Psychiatry (2001) 35(3):359-63. doi: 10.1046/j.1440-1614.2001.00909.x

16. Hakala M, Karlsson H, Ruotsalainen U, Koponen S, Bergman J, Stenman H, et al. Severe somatization in women is associated with altered cerebral glucose metabolism. Psychol Med (2002) 32(8):1379-85. doi: 10.1017/ S0033291702006578

17. Cortes CV, Vladimir N. Support-vector networks. Mach Learn (1995) 20(3):273-97. doi: 10.1007/BF00994018

18. Wang S, Zhan Y, Zhang Y, Lyu L, Lyu H, Wang G, et al. Abnormal longand short-range functional connectivity in adolescent-onset schizophrenia patients: aresting-state fMRI study. Prog Neuropsychopharmacol Biol Psychiatry (2018) 81:445-51. doi: 10.1016/j.pnpbp.2017.08.012

19. Zhu Y, Wu J, Fang Y. Study on application of SVM in prediction of coronary heart disease. Sheng Wu Yi Xue Gong Cheng Xue Za Zhi (2013) 30(6):1180-5.

20. Wang ZL, Zhou ZG, Chen Y, Li XT, Sun YS. Support Vector machines model of computed tomography for assessing lymph node metastasis in esophageal cancer with neoadjuvant chemotherapy. J Comput Assist Tomogr (2017) 41(3):455-60. doi: 10.1097/RCT.0000000000000555

21. Wang Y, Liu WH, Li Z, Wei XH, Jiang XQ, Geng FL, et al. Altered corticostriatal functional connectivity in individuals with high social anhedonia. Psychol Med (2016) 46(1):125-35. doi: 10.1017/S0033291715001592

22. First MB, Spitzer RL, Gibbon M, Williams JBW. Structured clinical interview for dsm-iv axis $i$ disorders, clinician version (SCID-CV). New York: Biometric Research Department (1997) p. 132-2.

23. Claassen-van Dessel N, van der Wouden JC, Dekker J, van der Horst HE. Clinical value of DSM IV and DSM 5 criteria for diagnosing the most prevalent somatoform disorders in patients with medically unexplained physical symptoms (MUPS). J Psychosom Res (2016) 82:4-10. doi: 10.1016/j. jpsychores.2016.01.004

24. Hamilton M. The assessment of anxiety states by rating. Br J Med Psychol (1959) 32(1):50-5. doi: 10.1111/j.2044-8341.1959.tb00467.x

25. Hamilton M. A rating scale for depression. J Neurol Neurosurg Psychiatry (1960) 23:56-62. doi: 10.1136/jnnp.23.1.56

26. Derogatis LR, Rickels K, Rock AF. The SCL-90 and the MMPI: a step in the validation of a new self-report scale. Br J Psychiatry (1976) 128:280-9. doi: 10.1192/bjp.128.3.280

27. Eysenck SB, Eysenck HJ. The questionnaire measurement of psychoticism. Psychol Med (1972) 2(1):50-5. doi: 10.1017/S0033291700045608 
28. Greve KW, Stickle TR, Love JM, Bianchini KJ, Stanford MS. Latent structure of the Wisconsin Card Sorting Test: a confirmatory factor analytic study. Arch Clin Neuropsychol (2005) 20(3):355-64. doi: 10.1016/j.acn.2004.09.004

29. Yan CG, Wang XD, Zuo XN, Zang YF. et al. DPABI: data processing and analysis for (resting-state) Brain Imaging. Neuroinformatics (2016) 14(3):339-51. doi: 10.1007/s12021-016-9299-4

30. Power JD, Barnes KA, Snyder AZ, Schlaggar BL, Petersen SE. Spurious but systematic correlations in functional connectivity MRI networks arise from subject motion. Neuroimage (2012) 59(3):2142-54. doi: 10.1016/j. neuroimage.2011.10.018

31. Hahamy A, Calhoun V, Pearlson G, Harel M, Stern N, Attar F, et al. Save the global: global signal connectivity as a tool for studying clinical populations with functional magnetic resonance imaging. Brain Connect (2014) 4(6):395-403. doi: 10.1089/brain.2014.0244

32. Song XW, Dong ZY, Long XY, Li SF, Zuo XN, Zhu CZ, et al. REST: a toolkit for resting-state functional magnetic resonance imaging data processing. PLoS One (2011) 6(9):e25031. doi: 10.1371/journal.pone.0025031

33. Chang CC, Hsu CW, Lin CJ. The analysis of decomposition methods for support vector machines. IEEE Trans Neural Netw (2000) 11(4):1003-8. doi: $10.1109 / 72.857780$

34. Brown MT, Tan KR, O'Connor EC, Nikonenko I, Muller D, Luscher C. Ventral tegmental area GABA projections pause accumbal cholinergic interneurons to enhance associative learning. Nature (2012) 492(7429):4526. doi: $10.1038 /$ nature 11657

35. Tritsch NX, Ding JB, Sabatini BL. Dopaminergic neurons inhibit striatal output through non-canonical release of GABA. Nature (2012) 490(7419):262-6. doi: 10.1038/nature11466

36. Montaron MF, Deniau JM, Menetrey A, Glowinski J, Thierry AM. Prefrontal cortex inputs of the nucleus accumbens-nigro-thalamic circuit. Neuroscience (1996) 71(2):371-82. doi: 10.1016/0306-4522(95)00455-6

37. Bagot RC, Parise EM, Pena CJ, Zhang HX, Maze I, Chaudhury D, et al. Ventral hippocampal afferents to the nucleus accumbens regulate susceptibility to depression. Nat Commun (2015) 6:7062. doi: 10.1038/ncomms8626

38. Britt JP, Benaliouad F, McDevitt RA, Stuber GD, Wise RA, Bonci A. Synaptic and behavioral profile of multiple glutamatergic inputs to the nucleus accumbens. Neuron (2012) 76(4):790-803. doi: 10.1016/j. neuron.2012.09.040

39. Christoffel DJ, Golden SA, Walsh JJ, Guise KG, Heshmati M, Friedman $\mathrm{AK}$, et al. Excitatory transmission at thalamo-striatal synapses mediates susceptibility to social stress. Nat Neurosci (2015) 18(7):962-4. doi: 10.1038/ nn.4034

40. Stuber GD, Sparta DR, Stamatakis AM, van Leeuwen WA, Hardjoprajitno JE, Cho S, et al. Excitatory transmission from the amygdala to nucleus accumbens facilitates reward seeking. Nature (2011) 475(7356):377-80. doi: 10.1038 /nature 10194

41. Heshmati M, Russo SJ. Anhedonia and the brain reward circuitry in depression. Curr Behav Neurosci Rep (2015) 2(3):146-53. doi: 10.1007/s40473-015-0044-3

42. Borsook D, Upadhyay J, Chudler EH, Becerra L. A key role of the basal ganglia in pain and analgesia-insights gained through human functional imaging. Mol Pain (2010) 6:27. doi: 10.1186/1744-8069-6-27

43. Baliki MN, Geha PY, Fields HL, Apkarian AV. Predicting value of pain and analgesia: nucleus accumbens response to noxious stimuli changes in the presence of chronic pain. Neuron (2010) 66(1):149-60. doi: 10.1016/j. neuron.2010.03.002

44. Mayberg HS, Liotti M, Brannan SK, McGinnis S, Mahurin RK, Jerabek PA, et al. Reciprocal limbic-cortical function and negative mood: converging
PET findings in depression and normal sadness. Am J Psychiatry (1999) 156(5):675-82. doi: 10.1176/ajp.156.5.675

45. Gracely RH, Geisser ME, Giesecke T, Grant MA, Petzke F, Williams DA, et al. Pain catastrophizing and neural responses to pain among persons with fibromyalgia. Brain (2004) 127(Pt 4):835-43. doi: 10.1093/brain/awh098

46. Etkin A, Egner T, Kalisch R. Emotional processing in anterior cingulate and medial prefrontal cortex. Trends Cogn Sci (2011) 15(2):85-93. doi: 10.1016/j. tics.2010.11.004

47. Okada K, Ota T, Iida J, Kishimoto N, Kishimoto T. Lower prefrontal activity in adults with obsessive-compulsive disorder as measured by near-infrared spectroscopy. Prog Neuropsychopharmacol Biol Psychiatry (2013) 43:7-13. doi: 10.1016/j.pnpbp.2012.11.013

48. Cabeza R, Anderson ND, Locantore JK, McIntosh AR. Aging gracefully: compensatory brain activity in high-performing older adults. Neuroimage (2002) 17(3):1394-402. doi: 10.1006/nimg.2002.1280

49. Guo W, Liu F, Liu J, Yu L, Zhang Z, Zhang J, et al. Is there a cerebellar compensatory effort in first-episode, treatment-naive major depressive disorder at rest? Prog Neuropsychopharmacol Biol Psychiatry (2013) 46:13-8. doi: 10.1016/j.pnpbp.2013.06.009

50. Du L, Liu H, Du W, Chao F, Zhang L, Wang K, et al. Stimulated left DLPFCnucleus accumbens functional connectivity predicts the anti-depression and anti-anxiety effects of rTMS for depression. Transl Psychiatry (2018) 7(11):3. doi: 10.1038/s41398-017-0005-6

51. Schlaepfer TE, Bewernick BH, Kayser S, Hurlemann R, Coenen VA. Deep brain stimulation of the human reward system for major depressionrationale, outcomes and outlook. Neuropsychopharmacology (2014) 39(6):1303-14. doi: 10.1038/npp.2014.28

52. Schlaepfer TE, Cohen MX, Frick C, Kosel M, Brodesser D, Axmacher N, et al. Deep brain stimulation to reward circuitry alleviates anhedonia in refractory major depression. Neuropsychopharmacology (2008) 33(2):36877. doi: 10.1038/sj.npp.1301408

53. Diefenbach GJ, Bragdon L, Goethe JW. Treating anxious depression using repetitive transcranial magnetic stimulation. J Affect Disord (2013) 151(1):365-8. doi: 10.1016/j.jad.2013.05.094

54. Gong Q, Li L, Tognin S, Wu Q, Pettersson-Yeo W, Lui S, et al. Using structural neuroanatomy to identify trauma survivors with and without post-traumatic stress disorder at the individual level. Psychol Med (2014) 44(1):195-203. doi: $10.1017 /$ S0033291713000561

55. Gong Q, Wu Q, Scarpazza C, Lui S, Jia Z, Marquand A, et al. Prognostic prediction of therapeutic response in depression using high-field MR imaging. Neuroimage (2011) 55(4):1497-503. doi: 10.1016/j.neuroimage.2010.11.079

56. Sharma A, Wolf DH, Ciric R, Kable JW, Moore TM, Vandekar SN, et al. Common dimensional reward deficits across mood and psychotic disorders: a connectome-wide association study. Am J Psychiatry (2017) 174(7):657-66. doi: 10.1176/appi.ajp.2016.16070774

Conflict of Interest Statement: The authors declare that the research was conducted in the absence of any commercial or financial relationships that could be construed as a potential conflict of interest.

Copyright (c) 2019 Ou, Su, Liu, Ding, Chen, Zhang, Zhao and Guo. This is an openaccess article distributed under the terms of the Creative Commons Attribution License (CC BY). The use, distribution or reproduction in other forums is permitted, provided the original author(s) and the copyright owner(s) are credited and that the original publication in this journal is cited, in accordance with accepted academic practice. No use, distribution or reproduction is permitted which does not comply with these terms. 


\section{Sex Moderates Amyloid and Apolipoprotein $\varepsilon 4$ Effects on Default Mode Network Connectivity at Rest}

\section{OPEN ACCESS}

Edited by:

Nicoletta Cera

University of Porto, Portugal

Reviewed by:

Gabriel Gonzalez-Escamilla,

Johannes Gutenberg University

Mainz, Germany

Nicola Filippini,

University of Oxford, United Kingdom

*Correspondence:

Jessica Z. K. Caldwell

caldwej5@ccf.org

${ }^{\dagger}$ Data used in preparation of this article were obtained from the Alzheimer's Disease Neuroimaging Initiative (ADNI) database

(adni.loni.usc.edu). As such, the investigators within the ADNI contributed to the design and

implementation of $A D N I$ and/or provided data but did not participate in analysis or writing of this report. A complete listing of $A D N I$ investigators can be found at: http://adni.Ioni.usc.

edu/wp-content/uploads/ how_to_apply/

ADNI_Acknowledgement_List.pdf

Specialty section

This article was submitted to Applied Neuroimaging,

a section of the journal

Frontiers in Neurology

Received: 07 May 2019 Accepted: 02 August 2019

Published: 20 August 2019

Citation:

Caldwell JZK, Zhuang X, Leavitt MJ, Banks SJ, Cummings $J$ and Cordes D (2019) Sex Moderates Amyloid and Apolipoprotein 44 Effects on Default Mode Network Connectivity at Rest.

Front. Neurol. 10:900.

doi: 10.3389/fneur.2019.00900

\author{
Jessica Z. K. Caldwell ${ }^{1 *}$, Xiaowei Zhuang ${ }^{1}$, MacKenzie J. Leavitt ${ }^{1}$, Sarah J. Banks ${ }^{2}$, \\ Jeffrey Cummings ${ }^{1,3}$ and Dietmar Cordes ${ }^{1}$, for the Alzheimer's Disease \\ Neuroimaging Initiative ${ }^{\dagger}$ \\ ${ }^{1}$ Cleveland Clinic Lou Ruvo Center for Brain Health, Las Vegas, NV, United States, ${ }^{2}$ Department of Neurosciences, \\ University of California, San Diego, San Diego, CA, United States, ${ }^{3}$ UNLV School of Allied Health Sciences, \\ Las Vegas, NV, United States
}

Women are more likely to have Alzheimer's disease (AD) and decline more rapidly once diagnosed despite greater verbal memory early in the disease compared to men-an advantage that has been termed "memory reserve." Resting state functional MRI (fMRI) investigations demonstrate interactions between sex and AD risk factors in default mode network (DMN) connectivity, a network of brain regions showing progressive dysfunction in AD. Separate work suggests connectivity of left prefrontal cortex (PFC) may correlate with more general cognitive reserve in healthy aging. It is unknown whether left prefrontal functional connectivity with anterior and posterior default mode network (aDMN, pDMN) might differ by sex in AD. This study employed group independent component analysis (ICA) to analyze resting state $\mathrm{AMRI}$ data from 158 participants from the Alzheimer's Disease Neuroimaging Initiative (ADNI) with baseline diagnoses of normal cognition or early mild cognitive impairment (eMCl). pDMN and aDMN were defined on a subject-specific basis; prefrontal areas were selected from the Brodmann atlas (BA 6, 44, 8, and 9). Moderation regression analyses examined whether sex and amyloid PET positivity $(\mathrm{A}+/-)$ moderated effects of apolipoprotein $\varepsilon 4$ (APOE $\varepsilon 4)$ on connectivity between left PFC, aDMN, and pDMN; and between aDMN and pDMN. Significant analyses were followed up with partial correlations assessing relationship of connectivity to verbal memory on the Rey Auditory Verbal Learning Test (RAVLT), and with preliminary analyses within $\mathrm{NC}$ and $\mathrm{eMCl}$ groups separately. Results showed no sex moderation of effects of $\mathrm{A}+$ and $A P O E \varepsilon 4$ on left prefrontal/DMN connectivity in the full sample. However, sex significantly moderated impact of $A+$ and $A P O E \varepsilon 4$ on connectivity between aDMN and pDMN ( $p<0.01)$. Women with an APOE allele $(\varepsilon 4+)$ and $A+$ showed greater aDMN/pDMN connectivity than their $\varepsilon 4$ - counterparts. No significant results were observed in men. Subgroup analyses suggested the aDMN/pDMN finding was true for those with $\mathrm{NC}$, not eMCl. Partial correlations controlling for age and education showed increased aDMN/pDMN connectivity related to better verbal learning in women $(p<0.01)$ and not men $(p=0.18)$. In women at risk for AD or in early symptomatic stages who also have evidence of amyloid burden, stronger aDMN/pDMN connectivity may support verbal learning.

Keywords: aging, degenerative disease, functional MRI, memory, positron emission tomography (PET) 


\section{INTRODUCTION}

Alzheimer's disease (AD) is a progressive neurodegenerative disease that causes characteristic memory decline. Current estimates are that 5.7 million people in the US have $\mathrm{AD}$, and $\sim 2 / 3$ of those with the disease are women (1). These statistics have led to a resurgence of research in sex-based differences in $\mathrm{AD}$ (2). Much of this work has delineated ways in which women show vulnerabilities and more precipitous decline due to $\operatorname{AD}(3,4)$. However, a number of investigations have shown that women have some early resilience to AD-related changes, and specifically that their verbal memory remains intact despite positive amyloid positron emission tomography (PET) scan evidence of brain amyloidosis $(5,6)$, mild to moderate changes in hippocampal volumes (7), and reduced brain fluorodeoxyglucose (FDG) PET activity (8). This initial maintenance of memory despite pathological burden has been called memory reserve, and has been thought to reflect women's lifetime history of stronger verbal memory when compared to men (9).

Moving beyond sex-based memory reserve, a separate body of literature has delineated ways in which general cognitive reserve-often indexed by higher education levels or intelligence, and presumed to involve cognition broadly - can delay onset of AD symptoms (10). Similar to the sex-focused literature showing that women ultimately decline faster, literature on cognitive reserve in $\mathrm{AD}$ has shown that some individuals have a more rapid decline after an extended period of reserve-related stability (11).

The underlying neural correlates of the established concept of cognitive reserve and the newer proposal of women's memory reserve remain incompletely understood. With specific respect to women's memory reserve, work from our group suggests that compared to men, women at risk for $\mathrm{AD}$ may have measureable differences in volume of the hippocampus (12), a region known to be critical for both memory and $\mathrm{AD}$ (13). However, the hippocampus is only one potential contributor. It is possible that women's early memory resilience relates more to structural integrity of cortical networks important for memory storage and retrieval or to differences in neural function rather than structure.

In line with this, research in aging and mild cognitive impairment (MCI) suggests that a potential neural correlate of general cognitive reserve is functional resting state connectivity of the left prefrontal cortex-both globally $(14,15)$ and specifically with the brain's default mode network (DMN) (16). The DMN is a network of brain regions shown to be more active when people are not engaged in a particular cognitive task, i.e., at "rest" $(17,18)$. Brain regions connected by the DMN are among the first to be implicated in $\mathrm{AD}$, and spread of $\mathrm{AD}$ pathology follows the $\operatorname{DMN}(19,20)$. Longitudinal changes in $\mathrm{DMN}$ function related to $\mathrm{AD}$ have been delineated, such that individuals at risk or in very early stages of $\mathrm{AD}$ show dysfunction in posterior aspects of the DMN (pDMN) (21-25), and as AD progresses, the pattern shifts to predominant dysfunction of the anterior DMN (aDMN). This pattern has been hypothesized to reflect progressive network overload and failure (26). Interactions of sex and $\mathrm{AD}$ risk factors (i.e., apolipoprotein $\varepsilon 4$ status; $A P O E$ $\varepsilon 4)$ have also been observed in the DMN, with healthy older women with an $\varepsilon 4$ allele showing decreased posterior DMN connectivity compared to non-carriers (27).
The left prefrontal cortex (PFC) is a large region comprised of subregions with distinct functionality and connectivity. Generally, verbal functions including verbal memory lateralize to the brain's left hemisphere in most individuals, highlighting the potential importance of this hemisphere when considering women's verbal memory reserve (28). Of particular interest to the general cognitive reserve literature are left lateral PFC (i.e., Brodmann area 6 and 44) $(15,16)$, and left dorsolateral prefrontal cortex (dlPFC; including BA 8 and 9). BA 6 and 44 have been shown in resting state functional connectivity studies to have anticorrelation-or negative correlation-with the DMN, with maintenance of anticorrelation marking cognitive reserve in amnestic MCI (15). The dlPFC has been implicated as a site of cognitive reserve-based structural integrity (29) and compensatory activation, such that maintained functionality on FDG PET relates to maintained cognition (30). Of note, many other brain regions have been suggested to play a role in general cognitive reserve, including right prefrontal cortex $(31,32)$ and inferior and middle temporal cortex (33).

No studies to date have examined whether resting state functional connectivity of left prefrontal cortex with aDMN and pDMN might serve to support women's memory reserve in AD. The present analysis sought to follow the demonstrated importance of looking at sex in $\mathrm{AD}$ by evaluating sex differences in the functional connectivity between left prefrontal cortex and $\mathrm{aDMN}$ and $\mathrm{pDMN}$, and between aDMN and $\mathrm{pDMN}$, in individuals with normal cognition (NC) and early mild cognitive impairment (eMCI), who were participants in a large, national study of AD (the Alzheimer's Disease Neuroimaging Initiative or $\mathrm{ADNI}$ ) and either had or did not have known $\mathrm{AD}$ risk factors (i.e., $A P O E \varepsilon 4$ allele) and pathological burden (i.e., positive amyloid PET). Due to reduced power, a secondary and preliminary analysis examined connectivity in the diagnostic subgroups separately, as to date findings of women's memory reserve have been at the NC stage. We specifically hypothesized that sex would moderate the effects of the presence of an APOE $\varepsilon 4$ allele and positive amyloid PET on functional connectivity within the DMN and between left prefrontal cortex and the DMN, and that this altered connectivity would relate to women's stronger preserved memory abilities.

\section{MATERIALS AND METHODS}

\section{Participants}

Data were included from 158 participants from the ADNI (http://adni-info.org) who had available resting state functional magnetic resonance imaging (fMRI) data and were diagnosed at baseline as having normal cognition [including those with subjective memory complaints (SMC)] or eMCI. As described on the ADNI website, ADNI defined Normal cognition as no SMC, Mini Mental State Exam [MMSE; (34)] of 24-30, Clinical Dementia Rating (CDR) (35), and memory box $=0$, educationadjusted raw scores on the Wechsler Memory Scale Logical Memory II test (Raw $\geq 9$ for $>16$ years education; $\geq 5$ for $8-15$ years education; $\geq 3$ for $0-7$ years education), and no significant impairment in cognitive functions or activities of daily living. Criteria for SMC were the same as for normal cognition, but with the addition of self-reported memory problems on the Cognitive 
Change Index ( $>16)$. In the present analysis, individuals with SMC were combined with NC. Early MCI was defined by ADNI as SMC, MMSE of 24-30, CDR of 0.5, CDR memory box of $\geq 0.5$, education-adjusted raw scores on the Wechsler Memory Scale Logical Memory II test (Raw $=9-11$ for $>16$ years education; 59 for 8-15 years education; 3-6 for 0-7 years education), and not meeting criteria for dementia (36). Individuals with two copies of the apolipoprotein $\varepsilon 4$ allele ( $A P O E \varepsilon 4 / 4)$ were excluded. Analyses were conducted in the full sample, and repeated excluding individuals with eMCI.

The study was approved by each participating ADNI site's local Institutional Review Boards, as documented on the ADNI website. All participants gave written, informed consent.

\section{Verbal Memory Assessment}

Verbal learning and memory were assessed using the Rey Auditory Verbal Learning Test (RAVLT) (37). Specifically, we analyzed total learning and delayed free recall scores.

\section{Resting State fMRI Processing}

For each subject, the first available resting-state fMRI scan was used for the analysis.

The first five time frames (15s) were removed to allow the MR signal to achieve T1 equilibrium. Remaining time frames were slice-timing corrected, realigned to the mean echo-planar image using SPM12 (http://www.fil.ion.ucl.ac.uk/spm/), co-registered to the subject T1 space, normalized to the standard MNI-152 $2 \mathrm{~mm}$-template using Advanced Normalization Tools software
(http://stnava.github.io/ANTs/) and then spatially smoothed using a 3D Gaussian filter with full-width-half-maximum (FWHM) equals $8 \mathrm{~mm}$. The T1 image for each subject was segmented into gray matter, white matter and cerebrospinal fluid (CSF) to generate subject specific white matter and CSF masks. These masks were further normalized to the standard MNI-152 $2 \mathrm{~mm}$ space. Signals from subject white matter and CSF (average time series within white matter and CSF masks), as well as six head motion parameters were regressed out from each dataset. All voxel time courses were further band pass filtered $(0.008 \mathrm{~Hz}$ $<\mathrm{f}<0.1 \mathrm{~Hz}$ ) and variance normalized.

The root-mean-square (RMS) motion was computed for each subject (38). Specifically, rotational displacements were converted to translational displacements by projection to a surface of a $50 \mathrm{~mm}$ radius sphere and RMS head motion was then computed from both the original translational displacements and the converted rotational displacements. All subjects had less than a voxel-size RMS motion $(0.30 \pm 0.23 \mathrm{~mm})$.

Resting-state networks were obtained through a spatial group independent component analysis (ICA). Principle component analysis (PCA) was first carried out for data reduction and the first 100 PCA components were retained for each subject. Data from all subjects were concatenated in time and input to the group ICA program. The ICA was carried out with in-house MATLAB scripts using the fast-ICA algorithm (39), repeated 20 times to output stable components; 30 stable ICA components were finally obtained. Out of 30 ICA components, posterior and anterior DMN were manually identified and
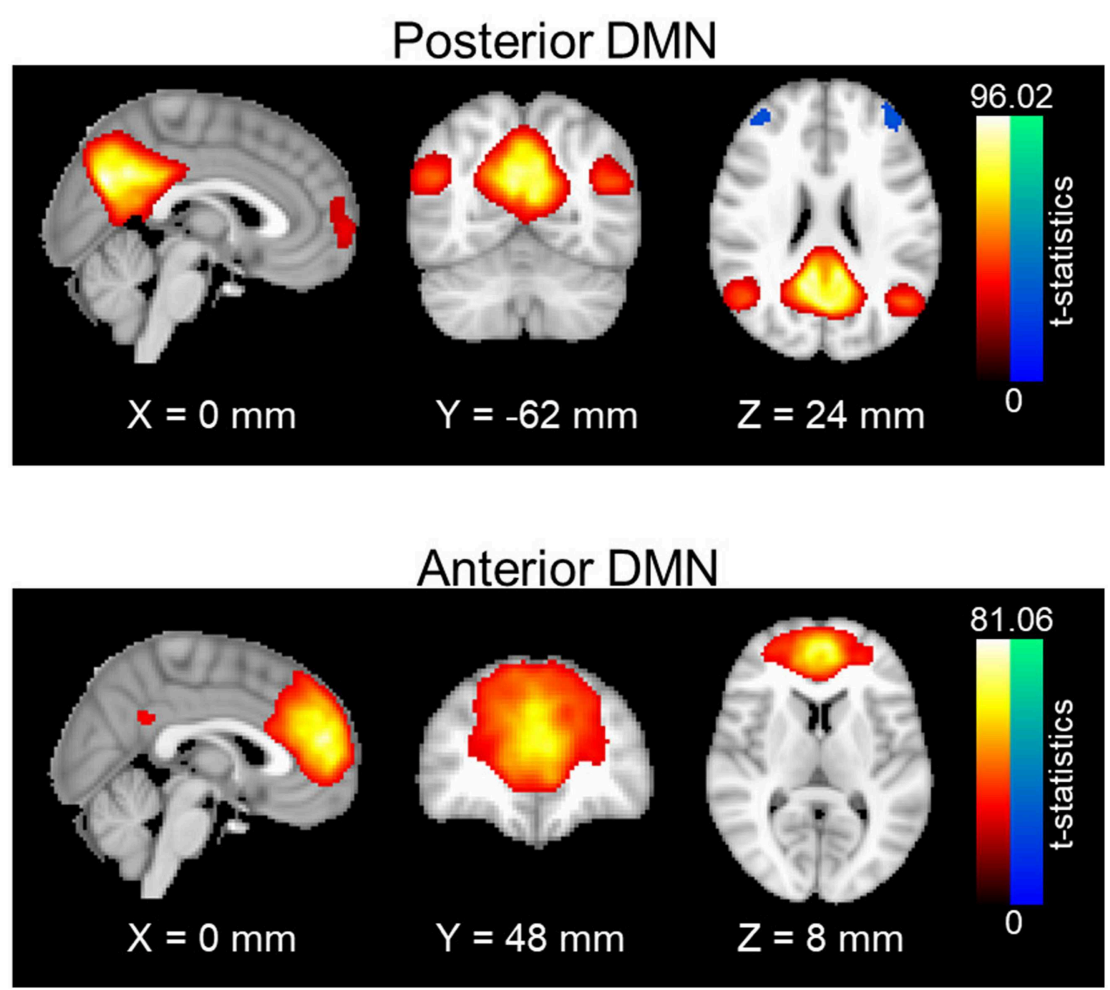

FIGURE 1 | Spatial maps of anterior and posterior DMN. One-sample t-statistics have been computed across the single-subject spatial maps and thresholded at $t=2$. DMN, Default mode network; std, standard deviation. 
selected (see Figure 1). Posterior DMN mainly includes posterior cingulate cortex, precuneus, bilateral angular gyrus, and bilateral middle temporal gyrus, whereas anterior DMN majorly consists of superior frontal gyrus, superior medial gyrus and anterior cingulate cortex Subject-specific spatial maps and time-courses for these two networks were reconstructed using GIG-ICA (40). Prefrontal areas were selected from the Brodmann atlas, as left Brodmann Areas (BA) 6, 44, 8, and 9. For each subject, average time courses were computed for each BA area. Functional connectivity between each prefrontal area and the DMN pair were accessed using the Pearson's correlation value between the average time courses in each BA area and the subject-specific network time series. Fisher z-transformation was then applied to the Pearson's correlation values so that computed $z$-values are normally distributed.

\section{Florbetapir PET Image Processing}

Amyloid status $(\mathrm{A}+/-)$ was determined from the PET study most proximal to participant fMRI scan date. The summarized standardized uptake value ratio (SUVR) normalized to the cerebellum were obtained from the ADNI database and the amyloid status $(\mathrm{A}+/-)$ was further determined using a 1.11 cut-off.

\section{APOE Genotype}

APOE genotype was downloaded from the ADNI website. After exclusion of individuals with two copies of the $\varepsilon 4$ allele, a binary variable was created, coding individuals as either $\varepsilon 4+$ or $\varepsilon 4-$.

\section{Statistical Methods}

Analyses were performed using Statistical Package for the Social Sciences (SPSS) and the Process Macro $(41,42)$.

$T$-tests or Mann-Whitney $U$-tests (the latter where group sizes differed) were used to explore group differences in demographic variables.

Moderation regressions examined whether sex and amyloid PET positivity $(\mathrm{A}+/-)$ moderated effects of a single copy of the $A P O E \& 4$ allele on connectivity between left prefrontal cortex regions of interest (i.e., BA 6, 44, 8, and 9) and the posterior and anterior default mode network (pDMN; aDMN), as well as between aDMN and pDMN. For all analyses, connectivity was assessed as using the Fisher $\mathrm{z}$ transformed correlation values, $A P O E \& 4$ carrier status was treated as an independent variable, $\mathrm{A}+/-$ as a moderator, and sex as a secondary moderator. Age at screening visit and education were included as covariates.

Significant moderation regression analyses were followed up with correlation analyses assessing the relationship of connectivity values with performance on the RAVLT immediate and delayed recall, partialling out effects of age and education.

Significant moderation regression analyses were also followed up with preliminary analyses in the NC and eMCI groups separately. All regressions and correlations were repeated; data are considered preliminary due to reduced power.

Significant models were corrected for multiple comparisons within each model using a Bonferroni correction based on the number of comparisons of interest in the model for the hypothesized three-way interaction. We are only interested in the following four comparisons: (1) A+ vs. A- APOE $\varepsilon 4$ carrying women; (2) A+ vs. A- APOE $\varepsilon 4$ carrying men; (3) $\mathrm{A}+/ A P O E$ $\varepsilon 4$ carrying women vs. $\mathrm{A}+/ A P O E \varepsilon 4$ carrying men; and (4) $\mathrm{A}-/ A P O E \varepsilon 4$ carrying women vs. A-/APOE $\varepsilon 4$ carrying men. Therefore, the corrected $p$-value threshold is $p=0.05 / 4$.

\section{RESULTS}

\section{Demographics and Descriptives}

Demographics and sex differences in demographic factors and memory for the full sample are summarized in Table 1. Demographics are additionally broken down by diagnosis in Tables S1, S2, and diagnosis and amyloid status in Table S3. Descriptive statistics for functional correlations (Pearson's $r$ values) between left prefrontal and DMN and between aDMN and pDMN are summarized in Table S4, broken down by sex, diagnosis, amyloid status, and APOE $\varepsilon 4$ status.

\section{Sex Does Not Moderate Left Prefrontal/DMN Connectivity Across Diagnoses}

Moderation regression models for the aDMN showed only one significant result, for the overall model predicting aDMN connectivity with BA44 ( $p=0.05)$; however, within this model, there were no significant main or interaction effects. Models predicting aDMN connectivity with other prefrontal regions were not significant (BA6: $p=0.44$; BA8: $p=0.23$; BA9: $p=$ 0.19; see Table 2).

For the pDMN, the overall model predicting BA9/pDMN connectivity was significant $(p=0.003)$, but the 3 -way sex by $\mathrm{A}+/$ - by APOE $\varepsilon 4$ interaction was not $(p=0.09)$. Main effects of age $(p=0.002)$, sex $(p=0.03)$, and education $(p=0.04)$ were noted, with women showing greater connectivity and older and more educated individuals showing less connectivity. Models

TABLE 1 | Participant demographics and group differences by sex (NC + MCl).

\begin{tabular}{lccc}
\hline & Male & Female & $\begin{array}{c}\text { Group } \\
\text { difference } \\
\text { (p-value }\end{array}$ \\
& & & from T-test) \\
\hline Number of subjects & 79 & 79 & 0.427 \\
Amyloid status & 33 positive & 38 positive & 0.734 \\
APOE4 & 24 & 26 & 0.039 \\
Age & $76.54 \pm 6.57$ & $74.15 \pm 7.76$ & 1.000 \\
& Range: $65.6-$ & Range: $56.4-$ & 0.031 \\
Handedness (right/left) & 95.4 & 95.8 & 0.180 \\
Years of education & $72 / 7$ & $72 / 7$ & 0.180 \\
RMS motion (mm) & $0.31 \pm 0.24$ & $0.27 \pm 0.22$ & 0.001 \\
RMS motion (mm) & $0.31 \pm 0.24$ & $0.27 \pm 0.22$ & \\
RAVLT immediate & $38.59 \pm$ & $44.65 \pm$ & 11.24 \\
RAVLT delay & 11.09 & $6.75 \pm 4.51$ & 0.041 \\
\hline
\end{tabular}

APOE4, Apolipoprotein $\varepsilon 4$ allele; RAVLT, Rey Auditory Verbal Learning Test; RMS, Root Mean Squared.

p-values reflect significance of Mann-Whitney tests. 
TABLE 2 | Summary of regression analyses for the connectivity between left prefrontal regions and anterior default mode network.

\begin{tabular}{|c|c|c|c|c|}
\hline Variable & $B$ & $p$ & \multicolumn{2}{|c|}{ 95\% Confidence interva } \\
\hline \multicolumn{5}{|c|}{ CONNECTIVITY BETWEEN LEFT BA6 AND ANTERIOR DMN } \\
\hline Overall model & & 0.44 & & \\
\hline APOE4 & -0.093 & 0.75 & -0.681 & 0.495 \\
\hline Amyloid & -0.030 & 0.87 & -0.396 & 0.337 \\
\hline Sex & -0.091 & 0.15 & -0.216 & 0.033 \\
\hline APOE4*amyloid & -0.008 & 0.98 & -0.728 & 0.710 \\
\hline APOE4*sex & 0.036 & 0.84 & -0.306 & 0.379 \\
\hline Amyloid*sex & 0.047 & 0.68 & -0.180 & 0.273 \\
\hline APOE4*amyloid*sex & -0.019 & 0.93 & -0.447 & 0.408 \\
\hline Age & 0.005 & 0.10 & -0.0009 & 0.011 \\
\hline Education & -0.001 & 0.90 & -0.019 & 0.016 \\
\hline \multicolumn{5}{|c|}{ CONNECTIVITY BETWEEN LEFT BA44 AND ANTERIOR DMN } \\
\hline Overall model & & 0.05 & & \\
\hline APOE4 & -0.378 & 0.20 & -0.964 & 0.208 \\
\hline Amyloid & -0.265 & 0.15 & -0.630 & 0.010 \\
\hline Sex & -0.100 & 0.11 & -0.224 & 0.024 \\
\hline APOE4*amyloid & 0.260 & 0.47 & -0.456 & 0.976 \\
\hline APOE4*sex & 0.156 & 0.37 & -0.185 & 0.497 \\
\hline Amyloid*sex & 0.178 & 0.12 & -0.047 & 0.404 \\
\hline APOE4*amyloid*sex & -0.163 & 0.45 & -0.589 & 0.263 \\
\hline Age & 0.005 & 0.09 & -0.0009 & 0.011 \\
\hline Education & 0.007 & 0.44 & -0.010 & 0.024 \\
\hline \multicolumn{5}{|c|}{ CONNECTIVITY BETWEEN LEFT BA8 AND ANTERIOR DMN } \\
\hline Overall model & & 0.23 & & \\
\hline APOE4 & 0.169 & 0.50 & -0.330 & 0.669 \\
\hline Amyloid & 0.252 & 0.11 & -0.059 & 0.563 \\
\hline Sex & -0.038 & 0.48 & -0.144 & 0.067 \\
\hline APOE4*amyloid & -0.507 & 0.10 & -1.118 & 0.104 \\
\hline APOE4*sex & -0.126 & 0.39 & -0.417 & 0.165 \\
\hline Amyloid*sex & -0.160 & 0.10 & -0.352 & 0.032 \\
\hline APOE4*amyloid ${ }^{\star}$ sex & 0.287 & 0.12 & -0.076 & 0.650 \\
\hline Age & 0.000 & 0.99 & -0.005 & 0.005 \\
\hline Education & -0.003 & 0.69 & -0.018 & 0.012 \\
\hline \multicolumn{5}{|c|}{ CONNECTIVITY BETWEEN LEFT BA9 AND ANTERIOR DMN } \\
\hline Overall model & & 0.19 & & \\
\hline APOE4 & 0.055 & 0.83 & -0.450 & 0.559 \\
\hline Amyloid & 0.351 & 0.03 & 0.037 & 0.666 \\
\hline Sex & -0.018 & 0.74 & -0.125 & 0.089 \\
\hline APOE4*amyloid & -0.572 & 0.07 & -1.188 & 0.045 \\
\hline APOE4*sex & -0.081 & 0.59 & -0.374 & 0.213 \\
\hline Amyloidsex & -0.224 & 0.02 & -0.418 & -0.030 \\
\hline APOE4ªmyloidsex & 0.411 & 0.03 & 0.045 & 0.777 \\
\hline Age & -0.002 & 0.52 & -0.007 & 0.004 \\
\hline Education & -0.001 & 0.89 & -0.016 & 0.014 \\
\hline
\end{tabular}

${ }^{*}$ Connectivity in Fisher's z. APOE4, Apolipoprotein $\varepsilon 4$ allele; BA, Brodmann area; DMN, Default Mode Network.

predicting connectivity of other prefrontal regions to $\mathrm{pDMN}$ were not significant (BA6: $p=0.93$; BA44: $p=0.76$; BA8: $p=$ 0.21; Table 3).
TABLE 3 | Summary of regression analyses for connectivity between left prefrontal regions and the posterior default mode network.

\begin{tabular}{lcccc}
\hline Variable & $\boldsymbol{B}$ & $\boldsymbol{p}$ & \multicolumn{2}{c}{$95 \%$ Confidence interval } \\
\hline CONNECTIVITY BETWEEN LEFT BA6 AND POSTERIOR DMN \\
Overall model & \multicolumn{3}{c}{0.93} \\
APOE4 & -0.232 & 0.36 & -0.726 & 0.263 \\
Amyloid & 0.030 & 0.16 & -0.279 & 0.338 \\
Sex & 0.007 & 0.90 & -0.098 & 0.111 \\
APOE4 ${ }^{*}$ amyloid & 0.254 & 0.41 & -0.350 & 0.858 \\
APOE4 ${ }^{*}$ sex & 0.115 & 0.43 & -0.173 & 0.403 \\
Amyloid ${ }^{*}$ sex & 0.022 & 0.82 & -0.168 & 0.212 \\
APOE4 ${ }^{*}$ amyloid ${ }^{*}$ sex & -0.153 & 0.40 & -0.513 & 0.206 \\
Age & -0.001 & 0.59 & -0.007 & 0.004 \\
Education & 0.003 & 0.67 & -0.012 & 0.018
\end{tabular}

\section{CONNECTIVITY BETWEEN LEFT BA44 AND POSTERIOR DMN}

\begin{tabular}{|c|c|c|c|c|}
\hline Overall model & & 0.76 & & \\
\hline APOE4 & -0.343 & 0.15 & -0.805 & 0.120 \\
\hline Amyloid & -0.050 & 0.73 & -0.338 & 0.239 \\
\hline Sex & 0.035 & 0.48 & -0.063 & 0.133 \\
\hline APOE4*amyloid & 0.464 & 0.11 & -0.102 & 1.029 \\
\hline APOE4*sex & 0.167 & 0.22 & -0.102 & 0.436 \\
\hline Amyloid*sex & 0.025 & 0.78 & -0.153 & 0.203 \\
\hline APOE4*amyloid*sex & -0.265 & 0.12 & -0.602 & 0.071 \\
\hline Age & -0.0007 & 0.78 & -0.005 & 0.004 \\
\hline Education & 0.006 & 0.39 & -0.008 & 0.020 \\
\hline
\end{tabular}

\section{CONNECTIVITY BETWEEN LEFT BA8 AND POSTERIOR DMN}

$\begin{array}{lcccc}\text { Overall model } & & 0.20 & & \\ \text { APOE4 } & 0.117 & 0.71 & -0.499 & 0.734 \\ \text { Amyloid } & 0.181 & 0.35 & -0.203 & 0.565 \\ \text { Sex } & 0.082 & 0.22 & -0.048 & 0.212 \\ \text { APOE4*amyloid } & -0.197 & 0.61 & -0.951 & 0.557 \\ \text { APOE4*sex }^{*} & -0.077 & 0.67 & -0.435 & 0.282 \\ \text { Amyloid*sex } & -0.169 & 0.16 & -0.406 & 0.069 \\ \text { APOE4*amyloid }{ }^{*} \text { sex } & 0.168 & 0.46 & -0.280 & 0.616 \\ \text { Age } & -0.008 & 0.02 & -0.014 & -0.001 \\ \text { Education } & -0.015 & 0.12 & -0.033 & 0.004\end{array}$

\section{CONNECTIVITY BETWEEN LEFT BA9 AND POSTERIOR DMN}

Overall model

$\begin{array}{lllll}\text { APOE4 } & 0.338 & 0.30 & -0.304 & 0.979\end{array}$

$\begin{array}{lllll}\text { Amyloid } & 0.252 & 0.21 & -0.148 & 0.652\end{array}$

$\begin{array}{lllll}\text { Sex } & 0.154 & 0.03 & 0.019 & 0.290\end{array}$

$\begin{array}{lllll}\text { APOE4}^{*} \text { amyloid } & -0.467 & 0.24 & -1.251 & 0.317\end{array}$

$\begin{array}{lllll}\text { APOE4*sex } & -0.230 & 0.23 & -0.603 & 0.143\end{array}$

$\begin{array}{lllll}\text { Amyloid*sex }^{*} & -0.229 & 0.07 & -0.475 & 0.018\end{array}$

$\begin{array}{lllll}\text { APOE4*amyloid }^{*} \text { sex } & 0.405 & 0.09 & -0.061 & 0.872\end{array}$

$\begin{array}{lllll}\text { Age } & -0.011 & 0.002 & -0.018 & -0.004\end{array}$

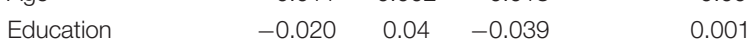

${ }^{*}$ Connectivity in Fisher's z. APOE4, Apolipoprotein $\varepsilon 4$ allele; BA, Brodmann area; DMN, Default Mode Network.

Secondary analyses within NC showed the model for BA9/pDMN was significant $(p=0.008)$ and the uncorrected three-way sex by $\mathrm{A}+/-$ by $A P O E \varepsilon 4$ interaction was also significant $(p=0.03)$. The interaction was not significant after multiple comparison correction; however, parsing the interaction suggested an $A P O E \varepsilon 4$ by $\mathrm{A}+/$ - effect on connectivity only in women $(p=0.005)$ and not men $(p=0.56)$, with lowest connectivity in $\mathrm{A}-/ \varepsilon 4+$ women. 
TABLE 4 | Summary of regression analyses for connectivity between anterior and posterior default mode network.

\begin{tabular}{lllll}
\hline Variable & B & $\boldsymbol{p}$ & \multicolumn{2}{c}{$95 \%$ Confidence interval } \\
\hline CONNECTIVITY BETWEEN ANTERIOR AND POSTERIOR DMN \\
Overall model \\
APOE4 & \multicolumn{3}{c}{0.0003} \\
Amyloid & 0.652 & 0.03 & 0.047 & 1.256 \\
Sex & 0.373 & 0.05 & -0.004 & 0.750 \\
APOE4*amyloid & -0.851 & 0.02 & -1.591 & -0.112 \\
APOE4*sex & -0.385 & 0.03 & -0.737 & -0.033 \\
Amyloid*sex & -0.305 & 0.01 & -0.538 & -0.072 \\
APOE4*amyloid ${ }^{*}$ sex & 0.569 & 0.01 & 0.129 & 1.008 \\
Age & -0.012 & 0.0002 & -0.019 & -0.006 \\
Education & -0.018 & 0.06 & -0.036 & 0.0004 \\
\hline
\end{tabular}

${ }^{\star}$ Connectivity in Fisher's Z.

The secondary analysis within eMCI individuals showed the model was not significant for BA9/pDMN $(p=0.15)$.

\section{Sex Moderates aDMN/pDMN Connectivity Across Diagnoses}

Moderation regression results showed a significant overall model predicting connectivity from aDMN to $\mathrm{pDMN}(p=0.0003)$. This model also evidenced a significant uncorrected 3-way interaction effect of sex, $\mathrm{A}+/-$, and $A P O E \varepsilon 4$ status on connectivity $(p=0.01$; significant after correction for multiple comparisons correction). Specifically, there was an $A P O E \varepsilon 4$ by $\mathrm{A}+/$ - interaction effect on aDMN/pDMN connectivity only in women $(p=0.04$; not significant after multiple comparison correction) and not men $(p=0.11)$. Parsing this interaction showed a trend toward $A P O E \& 4$ effect on aDMN/pDMN connectivity in women who were $\mathrm{A}+(p=0.07)$, and not in those who were $\mathrm{A}-(p$ $=0.26)$. Specifically, $A+, \varepsilon 4+$ women showed more positive $\mathrm{aDMN} / \mathrm{pDMN}$ connectivity. For men, the trend-level result suggested the opposite pattern (i.e., $\mathrm{A}-[p=0.07]$ rather than $\mathrm{A}+[p=0.93]$ men showing a relationship of $A P O E \varepsilon 4$ to greater $\mathrm{aDMN} / \mathrm{pDMN}$ connectivity). This model also showed a main effect of age $(p<0.001)$, with older individuals showing less connectivity (Table 4; Figure 2).

Secondary analyses showed that for NC, aDMN/pDMN connectivity findings were similar to those for the full sample, though did not pass multiple comparison correction (overall model $p=0.0004$; 3-way interaction $p=0.014$; interaction of $A P O E \& 4$ by $\mathrm{A}+/-$ significant in women $[p=0.02]$ and not men $[p=0.20])$. The model for MCI was not significant $(p=0.09)$.

\section{aDMN/pDMN Connectivity Relates to Verbal Memory}

Partial correlations controlling for age and education showed that increased $\mathrm{aDMN} / \mathrm{pDMN}$ connectivity related to better verbal learning in women $(p=0.006$; simple Pearson correlation values: $r=0.39, p<0.001)$ and not men $(p=0.18$; simple Pearson correlation values: $r=0.14, p=0.24)$. Increased aDMN $/ \mathrm{pDMN}$

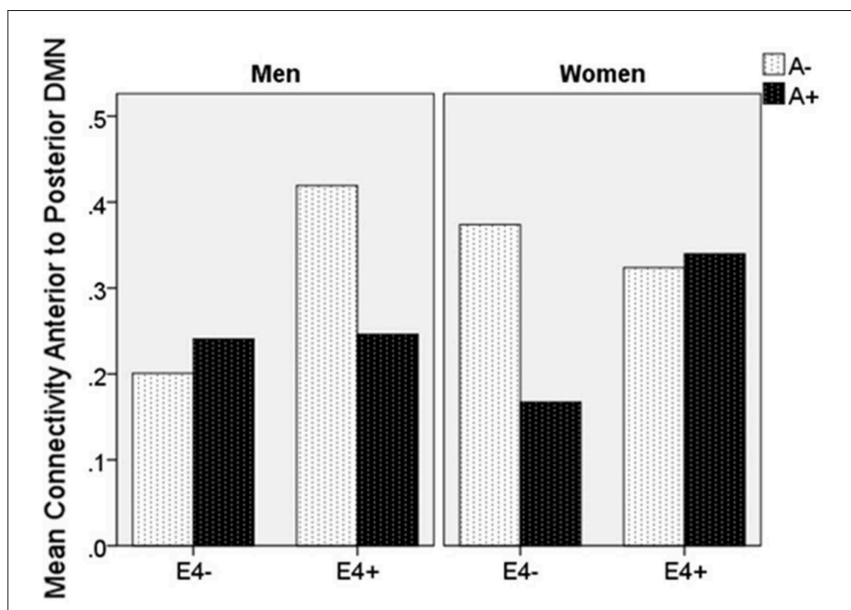

FIGURE 2 | Mean resting state functional connectivity by sex, APOE $\varepsilon 4$ carrier status, and amyloid status. APOE $\varepsilon 4$ : Apolipoprotein 4. $\varepsilon 4(+/-)$ indicates presence or absence of gene. $A(+/-)$ indicates presence or absence of positive amyloid PET scan. Units are Pearson's r-values.

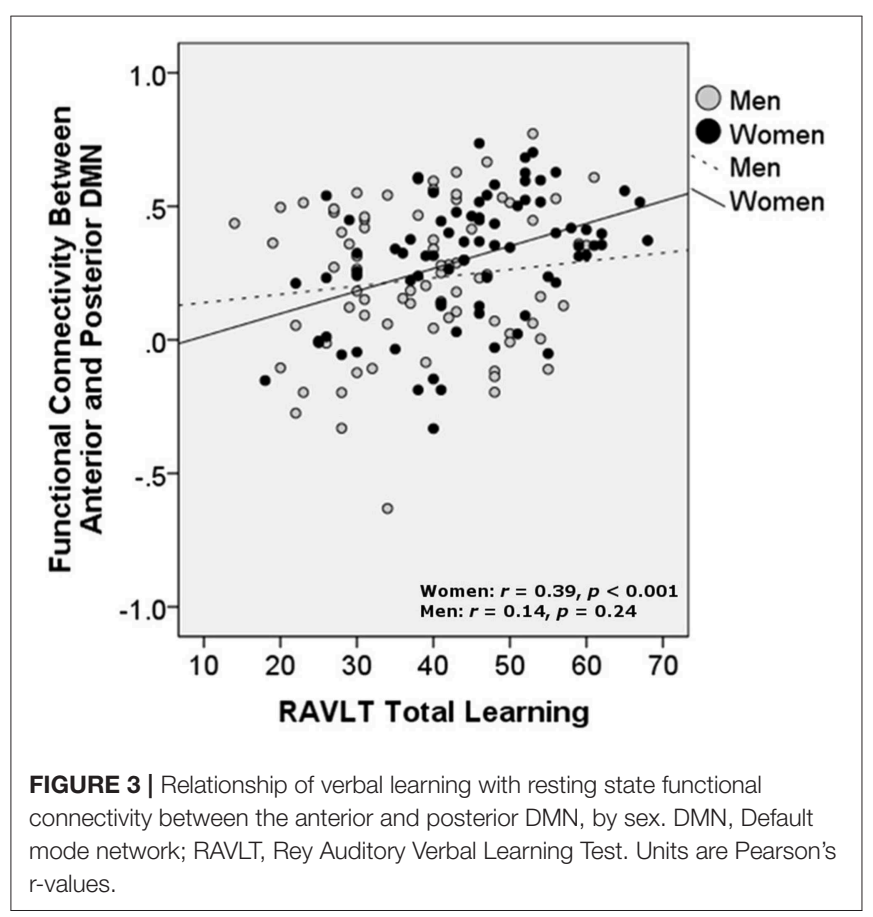

connectivity did not significantly relate to delayed recall after partialling out age and education (women: $p=0.13$; men: $p=$ 0.43 ; Figure 3 ). aDMN/pDMN connectivity was not correlated with education alone $(p=0.53)$.

\section{DISCUSSION}

The current investigation showed that sex did not moderate the impact of $A P O E \& 4$ genotype and amyloid status on left prefrontal/DMN connectivity across NC and MCI cohorts. However, sex did moderate the impact of $A P O E \varepsilon 4$ genotype and amyloid positivity on functional connectivity between anterior 
and posterior $\mathrm{DMN}$. In particular, aDMN/pDMN connectivity was greater in the presence of an APOE $\varepsilon 4$ genotype, but only in women with a positive amyloid scan. In other words, connectivity was highest in those with most $\mathrm{AD}$ risk and burden. Interestingly, for women only, greater aDMN/pDMN connectivity was correlated with better verbal learning. Men showed trend-level results suggesting that $A P O E \& 4$ genotype was associated with greater connectivity only for individuals who were amyloid negative-the opposite pattern to women.

The primary hypotheses of this investigation were not supported, suggesting that women's verbal memory reserve does not relate to left prefrontal functional connectivity with the DMN, which may underlie more general cognitive reserve. In our sample, left prefrontal and DMN connectivity were also not correlated with education, a typical marker of general cognitive reserve, and this may explain our overall negative finding. Lack of findings may also relate to sample-size or methodological choices (i.e., excluding right prefrontal and bilateral temporal cortex; defining DMN on a subject-specific basis but left PFC based on the Brodmann atlas). Another factor to consider is that work has shown that beyond cognitive reserve and underlying neural reserve, complex genetic interactions may have a role in resilience to $\mathrm{AD}$. Assessing the wide array of genetic contributors, some of which are known to differ by sex (43-45), was beyond the scope of this analysis, but may be key in understanding the neural basis of women's memory reserve in AD. Despite these caveats, secondary analyses suggested that BA9/pDMN connectivity may be moderated by sex in individuals with normal cognition. Given reduced power in our secondary analyses, this finding warrants follow-up in larger samples.

Our finding that sex moderated the impact of $\mathrm{A}+$ and $A P O E \varepsilon 4$ status on connectivity between anterior and posterior $\mathrm{DMN}$, and that greater aDMN/pDMN connectivity related to better verbal learning in women overall, will also require followup. This pattern could suggest reserve in verbal learning or compensation in women, and this could be further evaluated using longitudinal approaches. In the context of prior work showing an $A P O E \& 4$ by sex interaction in the $\mathrm{pDMN}$ resulting in less global pDMN connectivity in $\varepsilon 4$ positive healthy women (27), the present results could suggest that specific aspects of pDMN connectivity are maintained or increased in women at highest risk, while global pDMN connectivity declines. Our findings for $\mathrm{aDMN} / \mathrm{pDMN}$ connectivity appear to be driven by the NC group, which could be consistent with early resilience to overall DMN dysfunction over time. At the same time, tighter aDMN/pDMN coupling in the most at risk individuals is unlikely to be advantageous in the long term [i.e., (26)] and may play a role in women's steeper decline after the MCI stage. Interestingly, the pattern in $\varepsilon 4+/ A+$ women was opposite of the pattern in men and also opposite of the age effect, which predicted less connectivity. Given the age of our sample, our age findings are consistent with recent studies in healthy aging (46), but complex interaction of age and other risk factors may warrant additional assessment. Of note, the lack of interaction in men appears to be due to amyloid positivity having an effect on connectivity that does not differ by $\varepsilon 4$ status. In contrast, a main effect of $\varepsilon 4$ allele presence on greater connectivity in men is suggested. While this could not be directly assessed in the current model, it deserves attention in future investigations.

Limitations of the present study include the high education level of the ADNI sample and relatively small sample size for examination of complex interactive effects. Future efforts should aim to replicate these findings in larger and longitudinal samples, and to carefully consider effects of diagnosis. It will also be key to examine functional connectivity at rest of other regions suggested to be important for cognitive reserve, such as the right prefrontal cortex and left inferior temporal cortex. In addition, the present analysis included a small number of individuals with SMC but no cognitive impairment in the normal control group. As SMC have been associated with cognitive decline, this analytical choice may have impacted our findings, and it will be important to further assess the SMC group independently in the future. Moreover, given the relatively large number of variables of interest in the present analysis, diagnostic group was not treated formally as a measure of interest in the primary analysis. This is a limitation that warrants further assessment. Beyond resting-state investigations, it may also be informative to examine sex-based reserve correlates in task-based fMRI, focusing on memory.

Overall, the strengths of the present investigation lie in its novel integration of the otherwise separate research literatures on general cognitive reserve and sex-based memory reserve as assessed by functional resting state connectivity. We use a relatively large and well-characterized sample to show that women's memory reserve may be supported in part by complex interplay between networks typically most active at rest and prefrontal brain regions. These findings lend support for the continued importance of considering sex as a factor of importance in $\mathrm{AD}$ neural correlates. Better understanding of these correlates may in turn be helpful in delineating targets for treatment.

\section{DATA AVAILABILITY}

The datasets analyzed for this study can be found at the Alzheimer's Disease Neuroimaging Initiative website (ADNI; http://adni-info.org).

\section{ETHICS STATEMENT}

This study was carried out in accordance with the recommendations of ADNI local site Institutional Review Boards, with written informed consent from all subjects. All subjects gave written informed consent in accordance with the Declaration of Helsinki. The study was approved by each participating ADNI site's local Institutional Review Boards.

\section{AUTHOR CONTRIBUTIONS}

JCa designed the study, interpreted data, and prepared the manuscript. XZ analyzed and interpreted data. ML acquired data and prepared the manuscript. JCu and SB provided critical revision of the manuscript for intellectual content. DC provided 
critical input to study design and data interpretation, and also provided critical revision of the manuscript for intellectual content. All authors read and approved the final manuscript.

\section{FUNDING}

Research reported in this publication was supported by an Institutional Development Award (IDeA) from the National Institute of General Medical Sciences of the National Institutes of Health under grant number 5P20GM109025. In addition, research reported in this publication was supported in part by a grant from A Woman's Nation/Maria Shriver to JCa and SB. The Alzheimer's Disease Neuroimaging Initiative (ADNI) (National Institutes of Health Grant U01 AG024904) and DOD ADNI (Department of Defense award number W81XWH12-2-0012). ADNI was funded by the National Institute on Aging, the National Institute of Biomedical Imaging and Bioengineering, and through generous contributions from the following: AbbVie, Alzheimer's Association; Alzheimer's Drug Discovery Foundation; Araclon Biotech; BioClinica, Inc.; Biogen; Bristol-Myers Squibb Company; CereSpir, Inc.; Eisai Inc.; Elan Pharmaceuticals, Inc.; Eli Lilly and Company; EuroImmun; F. Hoffmann-La Roche Ltd. and its affiliated company Genentech, Inc.; Fujirebio; GE Healthcare; IXICO Ltd.; Janssen Alzheimer Immunotherapy Research \& Development, LLC.; Johnson

\section{REFERENCES}

1. Herbert LE, Weuve J, Scherr PA, Evans DA. Alzheimer disease in the United States (2010-2050) estimated using the 2010 Census. Neurology. (2013) 80:1778-83. doi: 10.1212/WNL.0b013e31828726f5

2. Nebel RA, Aggarwal NT, Barnes LL, Gallagher A, Goldstein JM, Kantraci $\mathrm{K}$, et al. Understanding the impact of sex and gender in Alzheimer's disease: a call to action. Alzheimers Dement. (2018) 14:1171-83. doi: 10.1016/j.jalz.2018.04.008

3. Buckley RF, Mormino EC, Amariglio RE, Properzi MJ, Rabin JS, Lim YY, et al. Sex, amyloid, and $A P O E \& 4$ and risk of cognitive decline in preclinical Alzheimer's disease: findings from three well-characterized cohorts. Alzheimers Dement. (2018) 14:1193-203. doi: 10.1016/j.jalz.2018. 04.010

4. Koran MEI, Wagener M, Hohman TJ. Sex differences in the association between AD biomarkers and cognitive decline. Brain Imaging Behav. (2017) 11:205-13. doi: 10.1007/s11682-016-9523-8

5. Sundermann EE, Biegon A, Rubin LH, Lipton RB, Landau S, Maki PM. Does the female advantage in verbal memory contribute to underestimating Alzheimer's disease pathology in women versus men? J Alzheimer's Dis. (2017) 56:947-57. doi: 10.3233/JAD-160716

6. Caldwell JZK, Berg JL, Cummings JL, Banks SJ. Sex moderates theimpact of diagnosis and amyloid PET positivity on hippocampal subfield volume. J Alzheimer's Dis. (2018) 64:79-89. doi: 10.3233/JAD180028

7. Sundermann EE, Biegon A, Rubin LH, Lipton RB, Mowrey W, Landau $S$, et al. Better verbal memory in women than men in MCI despite similar levels of hippocampal atrophy. Neurology. (2016) 86:1368-76. doi: 10.1212/WNL.0000000000002570

8. Sundermann EE, Maki PM, Rubin LH, Lipton RB, Landau S, Biegon A. Female advantage in verbal memory Evidence of sex-specific cognitive reserve. Neurology. (2016) 87:1916-24. doi: 10.1212/WNL.0000000000003288

9. McCarrey AC, An Y, Kitner-Triolo MH, Ferrucci L, Resnick SM. Sex differences in cognitive trajectories in clinicallynormal older adults. Psychol Aging. (2016) 31:166-75. doi: 10.1037/pag0000070
\& Johnson Pharmaceutical Research \& Development LLC.; Lumosity; Lundbeck; Merck \& Co., Inc.; Meso Scale Diagnostics, LLC.; NeuroRx Research; Neurotrack Technologies; Novartis Pharmaceuticals Corporation; Pfizer Inc.; Piramal Imaging; Servier; Takeda Pharmaceutical Company; and Transition Therapeutics. The Canadian Institutes of Health Research is providing funds to support ADNI clinical sites in Canada. Private sector contributions are facilitated by the Foundation for the National Institutes of Health (www.fnih.org). The grantee organization is the Northern California Institute for Research and Education, and the study is coordinated by the Alzheimer's Disease Cooperative Study at the University of California, San Diego. ADNI data are disseminated by the Laboratory for Neuro Imaging at the University of Southern California.

\section{ACKNOWLEDGMENTS}

We thank the ADNI for their contribution to the field and for specific use of these data.

\section{SUPPLEMENTARY MATERIAL}

The Supplementary Material for this article can be found online at: https://www.frontiersin.org/articles/10.3389/fneur. 2019.00900/full\#supplementary-material
10. Stern Y. Cognitive reserve in ageing and Alzheimer's disease. Lancet Neurol. (2012) 11:1006-2. doi: 10.1016/S1474-4422(12)70191-6

11. Mungas D, Gavett B, Fletcher E, Farias ST, DeCarli C, Reed B. Education amplifies brain atrophy effect on cognitive decline: implications for cognitive reserve. Neurobiol Aging. (2018) 68:142-50. doi: 10.1016/j.neurobiolaging.2018.04.002

12. Caldwell JZK, Berg J-L, Cummings JL, Banks SJ. Moderating effects of sex on the impact of diagnosis and amyloid positivity on verbal memory and hippocampal volume. Alzheimers Res Ther. (2017) 9:72. doi: 10.1186/s13195-017-0300-8

13. Braak H, Braak E. Neuropathological staging of Alzheimer-related changes. Acta Neuropathol. (1991) 82:239-59. doi: 10.1007/BF00308809

14. Franzmeier N, Caballero MAA, Taylor ANW, Simon-Vermot L, Buerger $\mathrm{K}$, Ertl-Wagner B, et al. Resting-state global functional connectivity as a biomarker of cognitive reserve in mild cognitive impairment. Brain Imaging Behav. (2017) 11:368-82. doi: 10.1007/s11682-016-9599-1

15. Franzmeier N, Buerger K, Teipel S, Stern Y, Dichgans $M$, Ewers $M$. Cognitive reserve moderates the association between functional network anti-correlations and memory in MCI. Neurobiol Aging. (2017) 50:152-62. doi: 10.1016/j.neurobiolaging.2016.11.013

16. Franzmeier N, Gottler J, Grimmer T, Drzezga A, Araque-Caballero MA, Simon-Vermot L, et al. Resting-state connectivity of the left frontal cortex to the default mode and dorsal attention network supports reserve in mild cognitive impairment. Front Aging Neurosci. (2017) 9:264. doi: 10.3389/fnagi.2017.00264

17. Raichle ME, MacLeod AM, Snyder AZ, Powers WJ, Gusnard DA, Shulman GL. A default mode of brain function. Proc Natl Acad Sci USA. (2001) 98:676-82. doi: 10.1073/pnas.98.2.676

18. Buckner RL, Andrews-Hanna JR, Schacter DL. The brain's default network: anatomy, function, and relevance to disease. Ann NY Acad Sci. (2008) 1124:138. doi: 10.1196/annals.1440.011

19. Greicius MD, Srivastava G, Reiss AL, Menon V. Default-mode network activity distinguishes Alzheimer's disease from healthy aging: evidence from functional MRI. Proc Natl Acad Sci USA. (2004) 101:4637-2. doi: $10.1073 /$ pnas.0308627101 
20. Sorg C, Riedl V, Muhlau M, Calhoun VD, Eichele T, Laer L, et al. Selective changes of resting state networks in individuals at risk for Alzheimer's disease. Proc Natl Acad Sci USA. (2007) 104:18760-5. doi: 10.1073/pnas.0708803104

21. Damoiseaux JS, Prater KE, Miller BL, Greicius MD. Functional connectivity tracks clinical deterioration in Alzheimer's disease. Neurobiol Aging. (2012) 33:e19-30. doi: 10.1016/j.neurobiolaging.2011.06.024

22. Wang $Z$, Liang $P$, Jia $X$, Jin $G$, Song $H$, Han $Y$, et al. The baseline and longitudinal changes of PCC connectivity in mild cognitive impairment: a combined structure and resting-state fMRI study. PLoS ONE. (2012) 7:e36838. doi: 10.1371/journal.pone.0036838

23. Hedden T, Van Dijk KR, Becker JA, Mehta A, Sperling RA, Johnson $\mathrm{KA}$, et al. Disruption of functional connectivity in clinically normal older adults harboring amyloid burden. J Neurosci. (2009) 29:12686-94. doi: 10.1523/JNEUROSCI.3189-09.2009

24. Sheline YI, Raichle ME, Snyder AZ, Morris JC, Head D, Wang S, et al. Amyloid plaques disrupt resting state default mode network connectivity in cognitively normal elderly. Biol Psychiatry. (2010) 67:584-7. doi: 10.1016/j.biopsych.2009.08.024

25. Sheline YI, Morris JC, Snyder AZ, Price JL, Yan Z, D'Angelo G, et al. $A P O E \& 4$ allele disrupts resting state fMRI connectivity in the absence of amyloid plaques or decreased CSF Aß42. J Neurosci. (2010) 30:17035-40. doi: 10.1523/JNEUROSCI.3987-10.2010

26. Jones DT, Knopman DS, Gunter JL, Graff-Radford J, Vemuri P, Boeve BF, et al. Cascading network failure across the Alzheimer's disease spectrum. Brain. (2016) 139:547-62. doi: 10.1093/brain/awv338

27. Damoiseaux JS, Seeley WW, Zhou J, Shirer WR, Coppola G, Karydas A, et al. Gender modulates the $A P O E \& 4$ effect in healthy older adults: convergent evidence from functional brain connectivity and spinal fluid tau levels. $J$ Neurosci. (2012) 32:8254-62. doi: 10.1523/JNEUROSCI.0305-12.2012

28. Smith EE, Jonides J, Koeppe RA. Dissociating verbal and spatial working memory using PET. Cereb Cortex. (1996) 6:11-20. doi: 10.1093/cercor/6.1.11

29. van Veluw SJ, Sawyer EK, Clover L, Cousijn H, De Jager C, Esiri MM, et al. Prefrontal cortex cytoarchitechture in normal aging and Alzheimer's disease: a relationship with IQ. Brain Struct Funct. (2012) 217:797-808. doi: 10.1007/s00429-012-0381-x

30. Morbelli S, Perneczky R, Drzezga A, Frisoni GB, Caroli A, van Berckel $\mathrm{BN}$, et al. Metabolic networks underlying cognitive reserve in prodromal Alzheimer disease: a European Alzheimer disease consortium project. J Nucl Med. (2013) 54:894-902. doi: 10.2967/jnumed.112.113928

31. Brosnan MB, Demaria G, Petersen A, Dockree PM, Robertson IH, Wiegand I. Plasticity of the right-lateralized cognitive reserve network in ageing. Cereb Cortex. (2018) 28:1749-59. doi: 10.1093/cercor/bhx085

32. Robertson IH. Right hemisphere role in cognitive reserve. Neurobiol Aging. (2014) 35:1375-85. doi: 10.1016/j.neurobiolaging.2013.11.028

33. Bauckneht M, Chincarini A, Piva R, Arnaldi D, Girtler N, Massa $\mathrm{F}$, et al. Metabolic correlates of reserve and resilience in MCI due to Alzheimer's Disease (AD). Alzheimers Res Ther. (2018) 10:35. doi: 10.1186/s13195-018-0366-y

34. Folstein MF, Folstein SE, McHugh PR. "Mini-mental state." A practical method for grading the cognitive state of patients for the clinician. J Psychiatr Res. (1975) 12:189-98.

35. Morris JC. Clinical dementia rating: current version and scoring rules. Neurology. (1993) 43:2412-4. doi: 10.1212/WNL.43.11.2412-a
36. Petersen RC, Aisen PS, Beckett LA, Donohue MC, Gamst AC, Harvey DJ, et al. Alzheimer's Disease Neuroimaging Initiative (ADNI): clinical characterization. Neurology. (2010) 74:201-9. doi: 10.1212/WNL.0b013e3181cb3e25

37. Rey A. L'examen Clinique En Psychologie. Paris: Presses Universitaires de France (1964).

38. Power JD, Mitra A, Laumann TO, Snyder AZ, Schlaggar BL, Petersen SE, et al Methods to detect, characterize, and remove motion artifact in resting state fMRI. Neuroimage. (2014) 84:320-41. doi: 10.1016/j.neuroimage.2013.08.048

39. Hyvärinen A, Karhunen J, Oja E. Independent Component Analysis. John Wiley \& Sons, Inc. (2002).

40. Du Y, Fan Y. Group information guided ICA for fMRI data analysis. Neuroimage. (2013) 69:157-97. doi: 10.1016/j.neuroimage.2012.11.008

41. Hayes AF. Introduction to Mediation, Moderation, and Conditional Process Analysis: A Regression Based-Approach. New York, NY: Guillford Press (2013).

42. IBM Corp Released. IBM SPSS Statistics for Windows, Version 23.0. Armonk, NY: IBM Corp (2015).

43. Hohman TJ, McLaren DG, Mormino EC, Gifford KA, Libon DJ, Jefferson AL. Asymptomatic Alzheimer disease: defining resilience. Neurology. (2016) 87:2443-50. doi: 10.1212/WNL.0000000000003397

44. Hohman TJ, Dumitrescu L, Cox NJ, Jefferson AL. Genetic resilience to amyloid related cognitive decline. Brain Imaging Behav. (2017) 11:401-9. doi: 10.1007/s11682-016-9615-5

45. Deming Y, Dumitrescu L, Barnes LL, Thambisetty M, Kunkle B, Gifford KA, et al. Sex-specific genetic predictors of Alzheimer's disease biomarkers. Acta Neuropathol. (2018) 136:857-72. doi: 10.1007/s00401-0181881-4

46. Staffaroni AM, Brown JA, Casaletto KB, Elahi FM, Deng J, Neuhaus J, et al. The longitudinal trajectory of default mode network connectivity in healthy older adults varies as a function of age and is associated with changes in episodic memory and processing speed. J Neurosci. (2018) 38:2809-17. doi: 10.1523/JNEUROSCI.3067-17.2018

Conflict of Interest Statement: JCu has provided consultation to Acadia, Accera, Actinogen, Alkahest, Allergan, Alzheon, Avanir, Axsome, BiOasis Technologies, Biogen, Diadem, EIP Pharma, Eisai, Genentech, Green Valley, Grifols, Hisun, Idorsia, Kyowa Kirin, Lilly, Lundbeck, Merck, Otsuka, Proclara, QR, Resverlogix, Roche, Samus, Samumed, Sunovion, Suven, Takeda, Teva, Toyama, and United Neuroscience pharmaceutical and assessment companies. JCu acknowledges funding from the National Institute of General Medical Sciences (Grant: P20GM109025) and support from Keep Memory Alive.

The remaining authors declare that the research was conducted in the absence of any commercial or financial relationships that could be construed as a potential conflict of interest.

Copyright (c) 2019 Caldwell, Zhuang, Leavitt, Banks, Cummings and Cordes. This is an open-access article distributed under the terms of the Creative Commons Attribution License (CC BY). The use, distribution or reproduction in other forums is permitted, provided the original author(s) and the copyright owner(s) are credited and that the original publication in this journal is cited, in accordance with accepted academic practice. No use, distribution or reproduction is permitted which does not comply with these terms. 


\section{Decreased Cross-Domain Mutual Information in Schizophrenia From Dynamic Connectivity States}

\section{OPEN ACCESS}

Edited by:

Filippo Cieri,

Cleveland Clinic Lou Ruvo Center for Brain Health, United States

Reviewed by:

Zhikun Zhang,

Guangxi Medical University, China

Raymond Salvador,

FIDMAG Hermanas Hospitalarias

Research Foundation, Spain

*Correspondence:

Mustafa S. Salman

msalman@gsu.org;

esalman@gatech.edu

Specialty section

This article was submitted to

Brain Imaging Methods,

a section of the journal

Frontiers in Neuroscience

Received: 01 March 2019

Accepted: 02 August 2019

Published: 22 August 2019

Citation:

Salman MS, Vergara VM,

Damaraju E and Calhoun VD (2019) Decreased Cross-Domain Mutual Information in Schizophrenia From

Dynamic Connectivity States.

Front. Neurosci. 13:873.

doi: 10.3389/fnins.2019.00873

\begin{abstract}
Mustafa S. Salman ${ }^{1,2 *}$, Victor M. Vergara², Eswar Damaraju² and Vince D. Calhoun ${ }^{1,2}$
${ }^{1}$ School of Electrical and Computer Engineering, Georgia Institute of Technology, Atlanta, GA, United States, ${ }^{2}$ Tri-Institutional Center for Translational Research in Neuroimaging and Data Science (TReNDS), Georgia State University, Georgia Institute of Technology and Emory University, Atlanta, GA, United States
\end{abstract}

The study of dynamic functional network connectivity ( $\mathrm{dFNC}$ ) has been important to understand the healthy and diseased brain. Recent developments model groups of functionally related brain structures (defined as functional domains) as entities that can send and receive information. A domain analysis starts by detecting a finite set of connectivity patterns known as domain states within each functional domain. Dynamic functional domain connectivity (DFDC) is a novel information theoretic framework for studying the temporal sequence of the domain states and the amount of information shared among domains. In this setting, the information flow among functional domains can be compared to the flow of bits among entities in a digital network. Schizophrenia is a chronic psychiatric disorder which is associated with how the brain processes information. Here, we employed the DFDC framework to analyze a dataset containing resting-state fMRI scans from 163 healthy controls (HCs) and 151 schizophrenia patients (SZs). As in other information theory methods, this study measured domain state probabilities, entropy within each DFDC and the cross-domain mutual information (CDMI) between pairs of DFDC. Results indicate that SZs show significantly higher (transformed) entropy than HCs in subcortical (SC)-SC; default mode network (DMN)visual (VIS) and frontoparietal (FRN)-VIS DFDCs. SZs also show lower (transformed) CDMI between SC-VIS vs. SC-sensorimotor (SM), attention (ATTN)-VIS vs. ATTN-SM and ATTN-SM vs. ATTN-ATTN DFDC pairs after correcting for multiple comparisons. These results imply that different DFDC pairs function in a more independent manner in SZs compared to HCs. Our findings present evidence of higher uncertainty and randomness in SZ brain function.

\section{Keywords: fMRI, functional network connectivity, functional domain, ICA, schizophrenia, information theory}

Abbreviations: ATTN, attention; BOLD, blood oxygenation level dependent; CDMI, cross-domain mutual information; DFDC, dynamic functional domain connectivity; dFNC, dynamic functional network connectivity; DMN, default mode network; EPI, echo planar imaging; FBIRN, functional biomedical informatics research network; FDR, false discovery rate; fMRI, functional magnetic resonance imaging; FNC, functional network connectivity; FRN, frontoparietal; FWHM, full width at half maximum; GIFT, group ICA of fMRI; HCs, healthy controls; ICA, independent component analysis; ICs, independent components; MI, mutual information; MNI, montreal neurological institute; PCA, principal component analysis; PCs, principal components; RMS, root mean square; RSN, resting-state networks; SC, subcortical; SFNR, signal-tofluctuation noise ratio; SM, sensorimotor; STR, spatio-temporal regression; SZs, schizophrenia patients; TCs, time courses; VIS, visual. 


\section{INTRODUCTION}

Schizophrenia is a chronic psychiatric disorder whose mechanism is not well understood yet. Its pathological and genetic background is complex and has not provided a clear understanding of the cause of the disorder. As a result, interest is growing in the functional neuroimaging of schizophrenia to provide additional clues about how this disorder impacts brain function (Kahn et al., 2015). The symptoms of schizophrenia can be broadly divided into positive, negative and cognitive categories. For instance, positive symptoms, characterized by abnormal salience processing and hallucinations, have shown links to abnormal functional activation in midbrain, speech and auditory cortices in SZs (Murray et al., 2008; Sommer et al., 2008). Negative symptoms related to reward processing and social cognition have shown association with reduced activation in amygdala in SZs (Aleman and Kahn, 2005; Juckel et al., 2006). Broad impairment of cognitive function, such as working and episodic memory, manifest as abnormal activation in dorsolateral prefrontal cortex in SZs (Brunet-Gouet and Decety, 2006; FusarPoli et al., 2013). Recent results suggest that these regional alterations are best understood as abnormalities in functional connectivity. For example, dorsolateral prefrontal cortex connectivity is altered in SZs and individuals at risk (Wolf et al., 2009; Esslinger et al., 2013). Methods from network topology has shown that small-world properties may be altered in SZs (Bassett et al., 2008; van den Heuvel et al., 2013). Schizophrenia is therefore associated with subtle changes in neural cell population and cell to cell communication and the study of brain functional connectivity is critical in understanding its causes.

Functional magnetic resonance imaging is a non-invasive imaging technique which can be used to study normal brain function in healthy individuals and disrupted brain function in patients with brain disorders. It captures signals from brain regions using a BOLD contrast in response to tasks or at rest. Resting-state fMRI reveals that even in the absence of a task, anatomically separate brain regions show large-scale neuronal activity which are functionally synchronized to one another (Biswal et al., 1995; Greicius et al., 2003). FNC refers to the temporal coherence of low-frequency BOLD activity between separate networks of neurons. The temporal coherence also shows variation with time thus characterizing a dFNC signal (Allen et al., 2014). Two of the most widely used methods for the analysis of FNC are seed-based approaches (Biswal et al., 1995) and data-driven approaches. Among the data driven approaches, ICA is in widespread use (Calhoun et al., 2009). The spatial ICA approach expresses the temporal fMRI data as a linear combination of spatially (statistically) independent sources known as functional networks (hereafter referred to as simply "networks") (Erhardt et al., 2011a). Unlike a seedbased approach, ICA requires no prior information about the pattern of the networks or their TCs. In this work, we perform ICA analysis of multi-subject fMRI data using the group ICA approach (Calhoun et al., 2001; Calhoun and Adali, 2012). This approach allows for estimation of the spatial networks common across subjects, and the associated TCs. These TCs can then be used to study dynamic brain function in different populations.
A growing number of studies of dynamic brain function using data-driven analysis of fMRI have shown links to the genetic risks, brain biology and clinical state of schizophrenia. Common approaches for studying dFNC of the human brain identifies replicable patterns of correlation among brain regions, known as brain states (Allen et al., 2014; Barttfeld et al., 2015; Leonardi and Van De Ville, 2015; Abrol et al., 2016; Chen et al., 2017). It has been found that SZs tend to linger in a state of weak connectivity at rest (Damaraju et al., 2014; Du et al., 2016; Lottman et al., 2017; Rabany et al., 2018; Sanfratello et al., 2019). Similar findings have been reported in patients with bipolar disorder and mild cognitive impairment (Rashid et al., 2016; Zhi et al., 2018). SZs also demonstrate reduced connectivity dynamism in the higher dimensional meta-state space compared to the HCs (Miller et al., 2016b). Another prior study indicated that the combined auditory-visual-sensorimotor network in HCs show increased sensitivity to connectivity in the other functional domains (groups of functionally related brain networks) in terms of measures derived by taking the transition probabilities of the inter-domain and intra-domain correlation patterns into account (Miller et al., 2016a). Group ICA methods have also identified potential biomarkers for schizophrenia, bipolar disorder and schizoaffective disorder (Du et al., 2015) and links with reduced brain volume and dFNC in schizophrenia (Abrol et al., 2017). These studies have demonstrated widespread disruption of interaction within the brain in schizophrenia and other mental disorders by employing whole-brain dFNC analysis.

Recently, Vergara et al. proposed an information theoretic framework to study communication between brain functional domains (Vergara and Calhoun, 2017; Vergara et al., 2017). Functional domains are groups of anatomically and functionally coherent networks. This approach allows for investigation of the flow of information between the functional domains by assigning binary digit values, or bits, to DFDC properties of the brain and estimating the quantity of bits required for this communication in terms of entropy and MI. The human brain is a large and complicated system and information theory can be a very useful statistical tool for studying such a system. Information theory was first described in Shannon's seminal paper "A Mathematical Theory of Communication" (Shannon, 1948) and since then has been applied to diverse fields in science because of its significance and flexibility. The word "information" may convey different meaning in different context. In information theory, a random variable $X$ provides (mutual) information about another random variable $Y$ when the knowledge about $X$ reduces the average uncertainty about $Y$ (Cover and Thomas, 2006). MI is non-negative and symmetric, i.e., $X$ says as much about $Y$ as says about $X$. The average uncertainty in a random variable is known as entropy. These basic concepts of information theory may enable us to assign relative measures to how information is stored and transferred within the human brain. Indeed, much of the application of information theory in neuroscience is on neural information flow, which concerns the transmission and constraints of information flowing through the nervous system (Borst and Theunissen, 1999; Dimitrov et al., 2011). However, apart from the study of neural 
information flow, there is little application of information theory in the study of healthy and diseased brain function in the existing literature.

In this work, we use the information theoretic framework of DFDC to examine group differences between HCs and SZs. We first use the spatial group ICA technique to estimate spatially ICs and associated TCs from the fMRI scans of $163 \mathrm{HCs}$ and 151 SZs. Then we use the TCs to estimate the whole-brain $\mathrm{dFNC}$, from which we extract the DFDC states. We study various information theoretic properties of these time-varying DFDC states, such as state probabilities, entropy and CDMI. Finally, we conduct some statistical analysis on these properties to find links to schizophrenia.

\section{MATERIALS AND METHODS}

\section{Data}

The data used in this study were collected under the FBIRN phase-III study at the following sites across the United States: University of California, Irvine (UCI), University of California, Los Angeles (UCLA); The University of New Mexico (NM), The University of Iowa (IA), University of Minnesota (MN), Duke University/University of North Carolina, University of California, San Diego (UCSD; healthy subjects only), and the University of California San Francisco (UCSF). Informed consent was obtained from the participants according to the guidelines set by the Internal Review Boards at each site. Resting-state fMRI data were originally collected from 186 HCs and 176 SZs. The SZ subjects were diagnosed using Structured Clinical Interview for DSM-IV-TR Axis I Disorders (First et al., 2002). The SZ subjects were excluded if they had a history of major medical illness and tardive dyskinesia or significant extrapyramidal symptoms or significant changes in psychotropic medications in the previous 2 months before the scan. Any healthy subject who had a first degree relative with a psychotic illness diagnosis or history of major neurological or psychiatric medical illness was also excluded. Subjects were also excluded if they did not have any of the following: sufficient eyesight to see visual displays, normal hearing levels, fluency in English and ability to perform the study tasks, IQ greater than 75 or if they had previous head injury or prolonged unconsciousness, substance or alcohol dependence, migraine treatments or MRI contradictions. Prior to participating in scanning procedures, all subjects received extensive diagnostic evaluations by experienced raters. All patients received the following symptom ratings: Scales for the Assessment of Positive (SAPS) and Negative Symptoms (SANS) and a modified Positive and Negative Symptom Scale (PANSS). The SZs were age and gender-matched. Imaging data for six of the seven sites were collected on a 3T Siemens Tim Trio System and on a 3T General Electric Discovery MR750 scanner at one site. 162 volumes of EPI, BOLD, fMRI data were collected from each participant using $3 \mathrm{~T}$ scanners with the following imaging parameters: $\mathrm{FOV}=220 \mathrm{~mm} \times 220 \mathrm{~mm}(64 \times 64$ matrix $), \mathrm{TR}=2 \mathrm{~s}, \mathrm{TE}=30 \mathrm{~ms}$, $\mathrm{FA}=770,32$ sequential ascending axial slices with thickness of 4 and $1 \mathrm{~mm}$ skip.

\section{Medication Information}

Anti-psychotic data was available for 129 patients in the FBIRN phase-III dataset. We used their respective chlorpromazine (CPZ) dosage equivalents for the patients with available doselevel medication data, as specified by Andreasen et al. (2010).

\section{Preprocessing}

We preprocessed the fMRI data using the SPM (Friston, 2007) and AFNI (Cox, 1996) toolboxes and scripts written in the MATLAB $^{\mathrm{TM}}$ software. At first, we measured the SFNR (Friedman et al., 2006) and maximum RMS translation using the INRIAlign toolbox in SPM (Freire et al., 2002) for all subjects. We excluded subjects with SFNR $<150$ and RMS translation $>4 \mathrm{~mm}$, which left us with 314 subjects for analysis, including $163 \mathrm{HCs}$ (mean age 36.9, 46 women) and $151 \mathrm{SZs}$ (mean age 37.8, 37 women). Next, we performed slice-timing correction using the middle slice as reference, followed by despiking using AFNI's 3dDespike algorithm to reduce the effect of outliers, normalization to the standard MNI space, resampling to $3 \mathrm{~mm} \times 3 \mathrm{~mm} \times 3 \mathrm{~mm}$ voxels, and smoothing to $6 \mathrm{~mm}$ FWHM kernel. Finally, we performed variance normalization on each voxel TC. More details about the data acquisition, preprocessing and quality control can be found in prior work (Damaraju et al., 2014; Keator et al., 2016).

\section{Group ICA}

We performed group-level spatial ICA (Calhoun et al., 2001; Calhoun and Adali, 2012) to decompose the temporally concatenated fMRI data of all subjects into 100 spatially independent group-level components. This was achieved in two steps. At first, we performed a subject-level PCA with the number of PCs set at 120, followed by a group-level PCA on the reduced and concatenated data with the number of PCs set at 100 . We chose a high number of PCs as it has been shown to stabilize the subsequent back-reconstruction process and produce refined ICs corresponding to known anatomical and functional segmentation (Allen et al., 2011; Erhardt et al., 2011b). Next, we performed ICA on the PCA-reduced data using the Infomax algorithm (Bell and Sejnowski, 1995) to estimate the group-level ICs. We repeated ICA 20 times and selected the most representative solution to ensure the stability of the IC estimation (Ma et al., 2011). We identified 100 most reliable components as the final group-level ICs.

After discarding the artifact-related ICs, we characterized 47 of the group-level ICs as RSN. An IC was identified as an RSN if its peak activation cluster was in the gray matter, there was minimal overlap with known vascular, susceptibility, ventricular and edge regions, and the mean power spectra of the IC showed higher low frequency spectral power (Allen et al., 2011). We grouped these networks into SC, auditory (AUD), SM, VIS, ATTN, frontal (FRN), DMN, and cerebellar (CB) functional domains based on their anatomical and presumed functional properties (Allen et al., 2014). Next, we estimated the subject-specific networks and their associated TCs based on the group-level ICs using the STR approach (Beckmann et al., 2009; Erhardt et al., 2011b). Finally, we post-processed the subject-level TCs of these networks by 
removing linear, quadratic and cubic trends, regressing out the head motion parameters and despiking using AFNI's 3dDespike algorithm (Cox, 1996). Correlation among brain networks are known to be driven primarily by low frequency fluctuations in the BOLD fMRI data (Cordes et al., 2001). Therefore, we filtered the TCs using a 5th order Butterworth filter with a passband frequency of $0.01-0.15 \mathrm{~Hz}$.

\section{dFNC Estimation}

We proceeded to analyze dFNC for each subject using a sliding window method (Sakoğlu et al., 2010; Allen et al., 2014; Damaraju et al., 2014). We used a sliding window with size of 22 TR (44 s) and convolved it with a Gaussian of $\sigma=3 \mathrm{TR}$ to obtain tapering along the edges. We used the sliding window in steps of 1 TR to estimate the dFNC among the TCs from a regularized inverse covariance matrix using a graphical LASSO framework (Friedman et al., 2008; Varoquaux et al., 2010). We transformed the dFNC values for each subject using the Fisher$\mathrm{Z}$ transformation and residualized them with respect to the gender, age and site variables. The dFNC analysis was performed using the temporal dFNC toolbox included in the GIFT software (Calhoun, 2004). Prior work can be consulted to obtain more details on the group ICA analysis of the fMRI data and the AFNC estimation steps (Damaraju et al., 2014).

\section{Dynamic Functional Domain Connectivity}

Figure 1 shows a flowchart of the analysis undertaken to investigate schizophrenia using the information theoretic framework. At first, for each window, we used every connectivity between the networks belonging to each pair of domains $Z[t]$ and $Y[t]$ to determine the temporal DFDC matrix $\mathrm{D}_{(Z, Y)}[t]$ (Vergara et al., 2017). Note that the FNC between two TCs is a scalar quantity, but the DFDC between two functional domains is not scalar unless both contain one network each. Furthermore, the FNC of a network with itself is 1 (one). In contrast, it is possible to have a multi-dimensional DFDC for a functional domain with itself if it contains more than two networks. In the two-network case, the DFDC is a scalar quantity. In our analysis, there were two such functional domains. The next step in our framework is to run a clustering algorithm on the DFDC. We chose not to apply clustering on the two DFDC with scalar quantities and excluded them from further analysis. We applied $K$-means clustering to the DFDC $\mathrm{D}_{(Z, Y)}[t]$ across all windows and all subjects with 10 replicates. We set the number of clusters at $K=3$ using the elbow criterion on the cluster validity index (Allen et al., 2014). Thus we identified the cluster centroids $C_{(Z, Y)}^{k}=\left[D_{(Z, Y)}^{1}, D_{(Z, Y)}^{2}, D_{(Z, Y)}^{3}\right]$ and a membership function $m_{(Z, Y)}[t] \in C_{(Z, Y)}^{k}$ corresponding to each windowed DFDC. The DFDC analysis was performed using custom scripts written in MATLAB ${ }^{\text {TM }}$.

Each cluster represents a dynamic state in DFDC. This method is analogous to the whole-brain dynamic state identification method (Allen et al., 2014), but estimates the dynamic states at the functional domain level. Therefore, as shown in Figure 1, DFDC is essentially a sub-matrix of the whole-brain dFNC matrix. The membership function expresses the closest cluster centroid based on an arbitrary distance measure. Here, we used correlation as the distance metric as it is a normalized metric showing more sensitivity to the DFDC patterns, although other possible choices such as $L_{1}, L_{2}$ norms will likely be highly similar (Damaraju et al., 2014).

\section{DFDC State Probabilities and Entropy}

Next, we conceptualized the data within an information theoretic framework. In this framework, each of the DFDC states in the set $C^{k}(Z, Y)$ is an element from an alphabet. The corresponding membership function $m_{(z, y)}[t]$ defines the frequency of occurrence of each dynamic state $D^{i(z, y)}$ (alphabet element). Thus, we can estimate the probability, $p^{i(z, y)}$, of a given state $D^{i(z, y)}$ from the membership function (also known as the occupancy rate). The DFDC entropy is then computed as:

$$
H_{(Z, Y)}=-\sum_{i} p_{(Z, Y)}^{i} \log _{2}\left(p_{(Z, Y)}^{i}\right)
$$

Entropy provides a measure of uncertainty. Higher entropy indicates that all possible states of a DFDC are nearly equally likely to be observed. Conversely, zero entropy implies maximum predictability of an outcome state.

\section{dFNC Entropy}

Just as for the DFDC states, we can also capture uncertainly at the whole-brain level by measuring entropy of the dFNC states. It is computed as:

$$
H_{(X)}=-\sum_{i} p_{(X)}^{i} \log _{2}\left(p_{(X)}^{i}\right)
$$

Here, $p^{i}(x)$ is the probability of a given dFNC state for a subject, where the states are estimated by running $K$-means clustering on the dFNC matrices across all subjects with $K=3$. This is useful for interpreting the findings based on DFDC and dFNC.

\section{Cross-Domain Mutual Information}

The other information theoretic measure we were interested in is the CDMI. CDMI is defined as:

$$
I_{(A B, Z Y)}=\sum p_{(A B, Z Y)}^{(i, j)} \log _{2} \frac{p_{(A B, Z Y)}^{(i, j)}}{p_{(A, B)}^{i} p_{(Z, Y)}^{j}}
$$

where $A$ and $B$ are two other different domains than $Z$ and $Y$. $p_{(A B, Z Y)}^{(i, j)}$ indicates the joint probability based on the temporal cooccurrence of the membership states $m_{(Z, Y)}[t], m_{(A, B)}[t]$ and is given by:

$$
\begin{gathered}
p_{(A B, Z Y)}^{i}=p\left(m_{(Z, Y)}=D_{(Z, Y)}^{i}, m_{(A, B)}=D_{(A, B)}^{i}\right) \\
D_{(Z, Y)}^{i} \in C_{(Z, Y)}^{\left(k_{1}\right)}, D_{(A, B)}^{i} \in C_{(A, B)}^{\left(k_{1}\right)}
\end{gathered}
$$

It can be shown from Eq. 2 that $I_{(A B, Z Y)}=H_{(Z, Y)}-H_{(Z, Y \mid A, B)}$, where $H_{(Z, Y \mid A, B)}$ is the conditional entropy of the DFDC $D_{(Z, Y)}$ given $D_{(A, B)}$. MI serves as a measure of dependence (or lack thereof) between two random variables. MI is symmetric and 


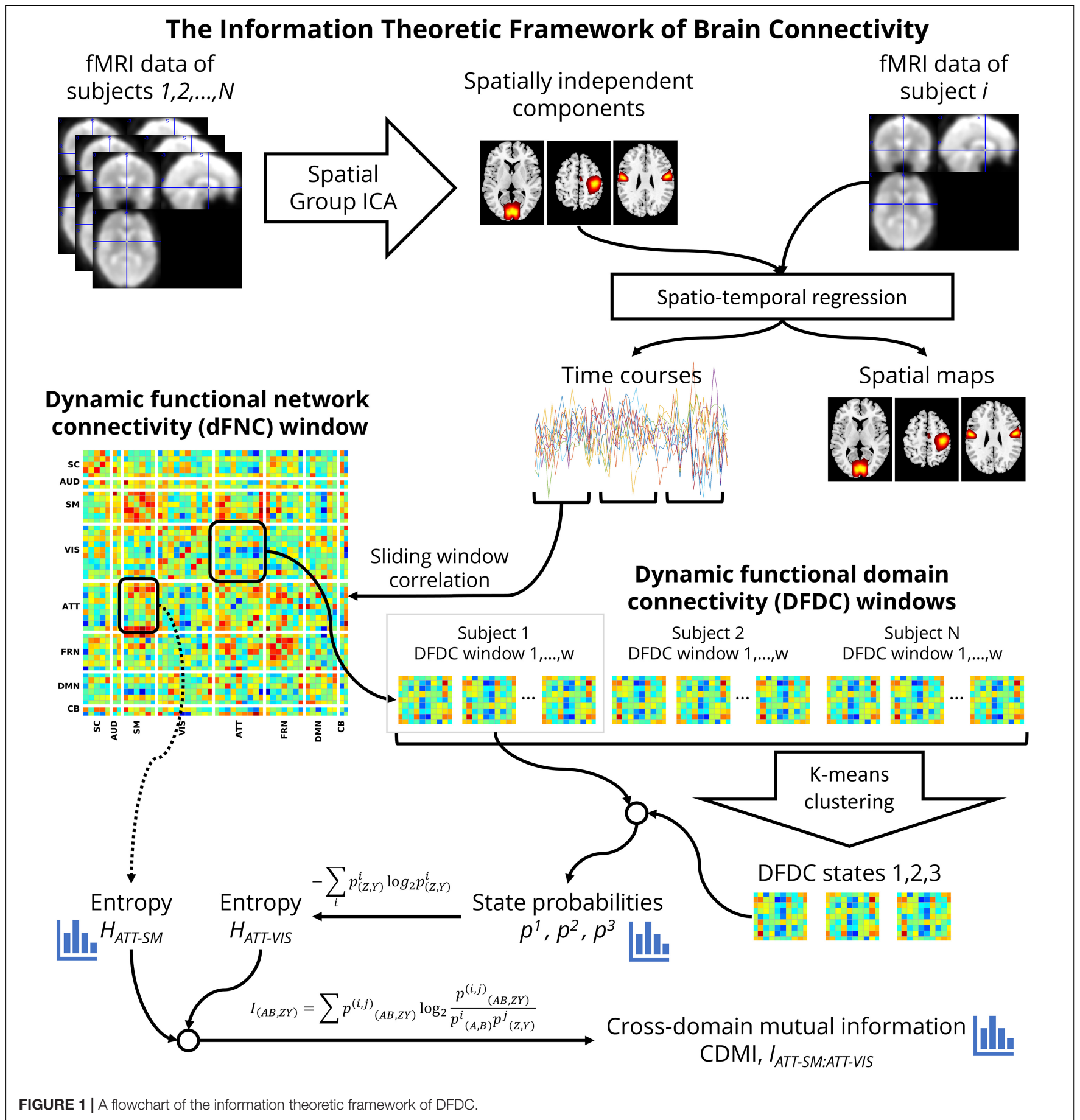

non-negative, and zero if and only if the two random variables are statistically independent. Note that if $Z=A$ and $Y=B$, then $I_{(A B, Z Y)}$ simply indicates the entropy of $D_{(Z, Y)}$, or $H_{(Z, Y)}$.

\section{Statistical Analysis}

We performed statistical analysis on various information theoretic measures, i.e., state probabilities, entropy and CDMI, with the intent of testing for group differences. We observed that the DFDC state probabilities had positively skewed distribution (see Supplementary Figures 2, 4, 6). In case of DFDC entropy, the distributions were negatively skewed (see Supplementary Figure 8). Note that the number of maximum possible dynamic states for each DFDC was set at 3, which translates to a maximum achievable entropy value of 1.585 bits/sample in the event of an equal occupancy rate $(33.33 \%)$ for each state. We transformed the DFDC state probabilities and entropy data across all subjects in the following manner to obtain Gaussian distributions. At first the data was scaled between $[-(1-K),(1-K)]$. Here, $K$ is a small 
number, introduced so that we could subsequently apply the Fisher-Z transformation on the scaled data without encountering discontinuity. Next, we used a one-sample Kolmogorov-Smirnov test to confirm that the transformed quantities came from a standard normal distribution and hence satisfied the assumptions of relevant statistical analyses. We fit a linear model separately to each transformed DFDC state probability and entropy with the subject diagnosis (HC-SZ) as the independent categorical variable and examined the group difference. We also looked at group difference in the whole-brain dFNC entropy using a similar test.

We observed that most of the CDMI were very low, indicating high statistical independence between the corresponding DFDC pairs. We used the following bootstrapping technique to select the significantly high CDMIs for further investigation (Vergara et al., 2017). We first computed the state probabilities and joint probabilities across all data (subject $\times$ window) from the clustering results on each DFDC. We used these values to computer one CDMI for each DFDC pair. Note that the CDMI of a DFDC with itself was ignored, as it is merely the entropy for that DFDC. We bootstrapped these CDMI values using $10^{9}$ iterations. The significant CDMI threshold was chosen at 5\% level. The DFDC pairs with CDMI values higher than this threshold were selected for further analysis. The CDMIs also had right skewed distribution and were transformed similar to the state probabilities and entropy, before being fit to a linear model to examine group difference.

We also assessed the relationship of the SZ's DFDC entropy and the significant CDMIs with both the patient PANSS scores and $\mathrm{CPZ}$ dosage equivalents. We correlated the $\mathrm{CPZ}$ dosage equivalents (in log-scale) with each DFDC entropy and significant CDMI. The CPZ dosage equivalents were logtransformed because their original distribution had very low "peakedness" or kurtosis. We also correlated the patient PANSS scores (positive, negative and general separately) with each DFDC entropy and the significant CDMI.

\section{RESULTS}

We set out to examine group differences between HCs and SZs using the information theoretic framework of brain connectivity. The first step in this process was to estimate the brain networks using group ICA. Figure 2 shows the 47 networks grouped into eight functional domains, i.e., SC (5 networks), AUD (2), SM (6), VIS (10), ATTN (9), FRN (7), DMN (6), and CB (2) domains. Out of these, AUD and $\mathrm{CB}$, having two ICs each, were excluded from further analysis. More details such as the anatomical labels and peak activation coordinate of these networks can be found in prior work (Damaraju et al., 2014) and in the Supplementary Table 1.

\section{Group Difference in DFDC State Probabilities}

We determined the DFDC states via $K$-means clustering and examined the group differences in the subject state probabilities. The six domains under experiment generated $\left(\begin{array}{l}6 \\ 2\end{array}\right)=21$ domain pairs. Supplementary Figure 1 shows the three cluster centroids or states for each of the 21 DFDC. Figure 3A shows the mean probabilities for the three states across all subjects. The DFDC are sorted from top to bottom in the order of increasing entropy, and the mean probabilities in each row are sorted from left to right in decreasing order. We observed that the state probabilities had a right-skewed distribution (see Supplementary Figures 2, 4, 6) and transformed them to standard normal distributions (see Supplementary Figures 3, 5, 7). Figure 3B shows the result of linear regression on the transformed state probabilities with diagnosis (HC-SZ) as the independent categorical variable. The color intensity indicates $\operatorname{sign}(\beta) \times|\log 10(p)|$ where $\beta$ is the coefficient of regression and $p$ is the $p$-value corresponding to the t-statistic of the coefficient. Results indicate that SZs have significantly higher (transformed) state 1 probabilities in the SM-DMN DFDC and (transformed) state 2 probabilities in VISDMN DFDC at $p<0.05$ level (uncorrected).

\section{Group Difference in DFDC and dFNC Entropy}

Next, we computed the DFDC entropies from the state probabilities using Eq. 1 and examined the group differences in the entropy of the 21 DFDCs. We observed that the entropy had left-skewed distribution (see Supplementary Figure 8) and transformed them to standard normal distributions (see Supplementary Figure 9). Figures 4A,B show the mean entropy across all HCs and SZs for different DFDC. Figure 4C shows the result of linear regression on the transformed entropy with diagnosis (HC-SZ) as the independent categorical variable. The color intensity indicates $\operatorname{sign}(\beta) \times|\log 10(p)|$ where $\beta$ is the coefficient of regression and $p$ is the $p$-value corresponding to the $t$-statistic of the coefficient. Results indicate that SZs have significantly higher entropy than HCs in the SC-SC, FRNVIS and DMN-VIS DFDC at $p<0.05$ level (uncorrected). Figure 4D shows the entropy for HCs and SZs computed from the whole-brain dFNC. We observed that the dFNC entropy is also significantly higher in SZs $(p=0.0025)$, commensurate with the majority of the DFDC entropy.

\section{Group Difference in Cross-Domain Mutual Information}

The last information theoretic measure we examined was the CDMI between each pair of DFDC. We used the state probabilities in Eq. 3 to determine the CDMI between two DFDC and examined the group differences. Pairwise CDMI between 21 DFDC under experiment resulted in $\left(\begin{array}{c}21 \\ 2\end{array}\right)=210$ CDMI values. Figure 5 show the mean CDMI across HCs and SZs. We observed that when measured across all subjects, the CDMI values were below or equal to 0.2871 (see Supplementary Figure 10). Using bootstrapping, we chose the minimum significant CDMI threshold of 0.099612 at $5 \%$ level and performed statistical analysis on the 10 DFDC pairs whose CDMI were higher than this threshold. More details about these 10 CDMI are provided in Supplementary Table 2. We observed that the CDMI showed a right-skewed distribution (see Supplementary Figure 11) and hence transformed them to standard normal distributions (see Supplementary Figure 12). Figure 6 shows a chord diagram of 

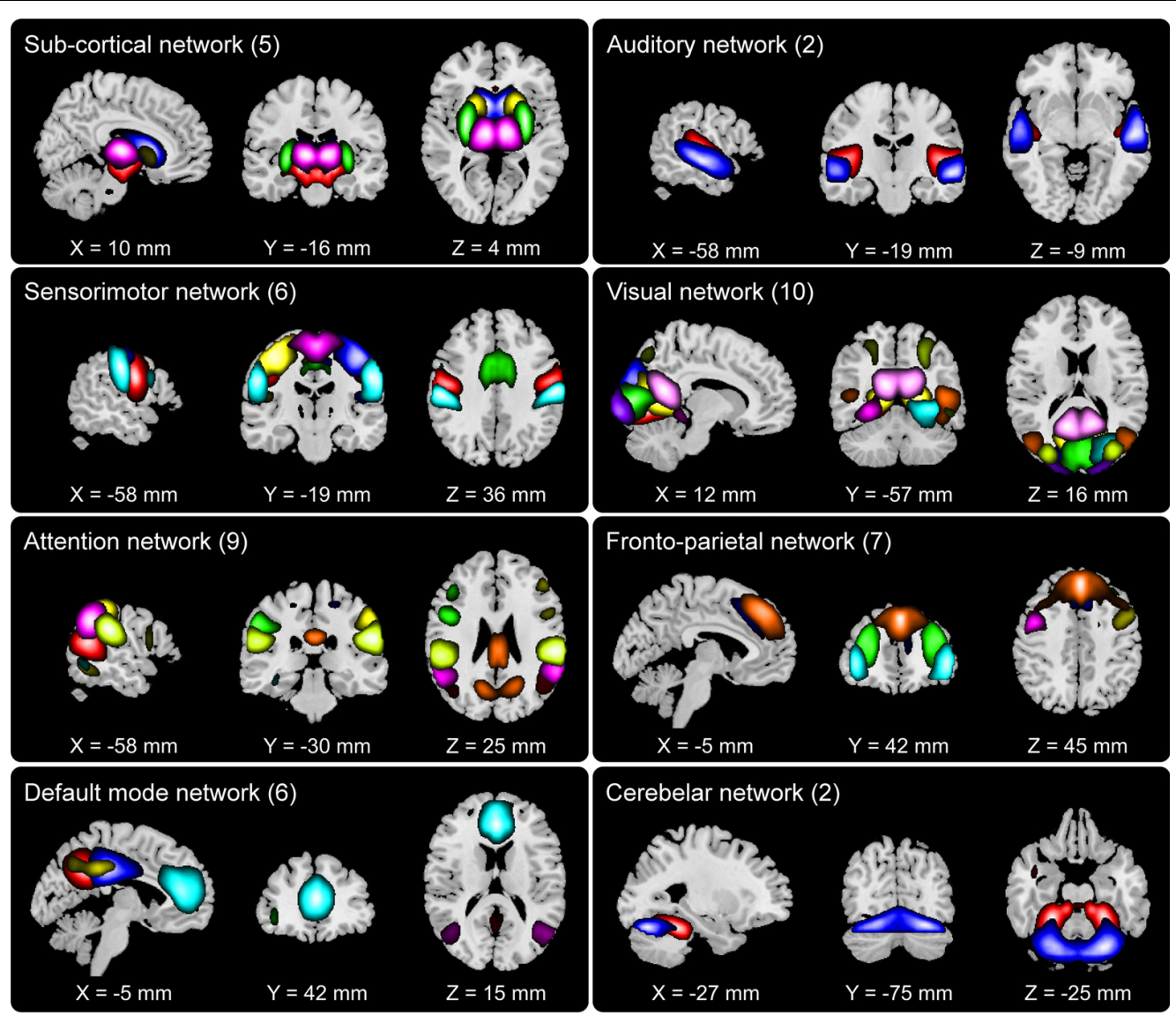

FIGURE 2 | Composite view of the 47 spatially independent group-level functional networks categorized into eight functional domains: subcortical (SC, 5 networks), auditory (AUD, 2), sensorimotor (SM, 6), visual (VIS, 10), attention (ATTN, 9), frontoparietal (FRN, 7), default mode (DMN, 6) and cerebellar (CB, 2). Intensity of the color represents z-scores. Functional network labels and peak activation coordinates can be found in prior work (Damaraju et al., 2014).

the results of linear regression on the transformed CDMI values with subject diagnosis (HC-SZ) as the independent categorical variable. The color intensity of the connecting links between a pair of DFDC indicates sign $(\beta)$ and the width of the links indicates $\operatorname{sign}(\beta) \times|\log 10(p)|$ where $\beta$ is the coefficient of regression and $p$ is the $p$-value corresponding to the $t$-statistic of the coefficient. The $p$-values were controlled for FDR when conducting multiple comparisons. Results indicate that in 7 out of 10 DFDC pairs under consideration, SZs have lower CDMI than HCs. Three of those are statistically significant at $p<0.05$ level after FDR correction, which are SC-SM vs. SC-VIS, SM-ATTN vs. VIS-ATTN and SM-ATTN vs. ATTN-ATTN DFDC pairs. This enables us to draw the conclusion that SZs demonstrate significantly lower CDMI, and higher independence, than HCs between these DFDC states.

\section{Correlation With Medication and Symptoms}

We found that the CPZ dosage equivalent of the patients was not significantly correlated with any of the DFDC entropy or the significant CDMIs of the patients. The linear correlation coefficient between the two and the FDR-corrected $p$-values for testing the hypothesis of no correlation are presented in Supplementary Figures 14, 15. We found that the PANSS positive scores of the patients were significantly correlated with the VIS-FRN vs. VIS-DMN CDMI $(R=0.27, p=0.0084$, FDR corrected). The linear correlation coefficients and FDR corrected $p$-values are added in Supplementary Figures 16, 17. We did not find any significant correlation between the PANSS positive scores and any DFDC entropy, or PANSS general and negative scores of the patients and any of the DFDC entropy or the significant CDMIs after FDR correction for multiple comparisons.

\section{DISCUSSION}

We set out to utilize the information theoretic framework of DFDC (Vergara et al., 2017) to examine how the information processing between the brain functional domains are impacted in schizophrenia. We specifically looked at the transformed entropy in the subject-wise time-varying DFDC patterns, and the CDMI between each pair of those DFDC patterns. We found that SZs show significantly higher entropy than HCs within multiple DFDC. It suggests that there is higher uncertainty or randomness 

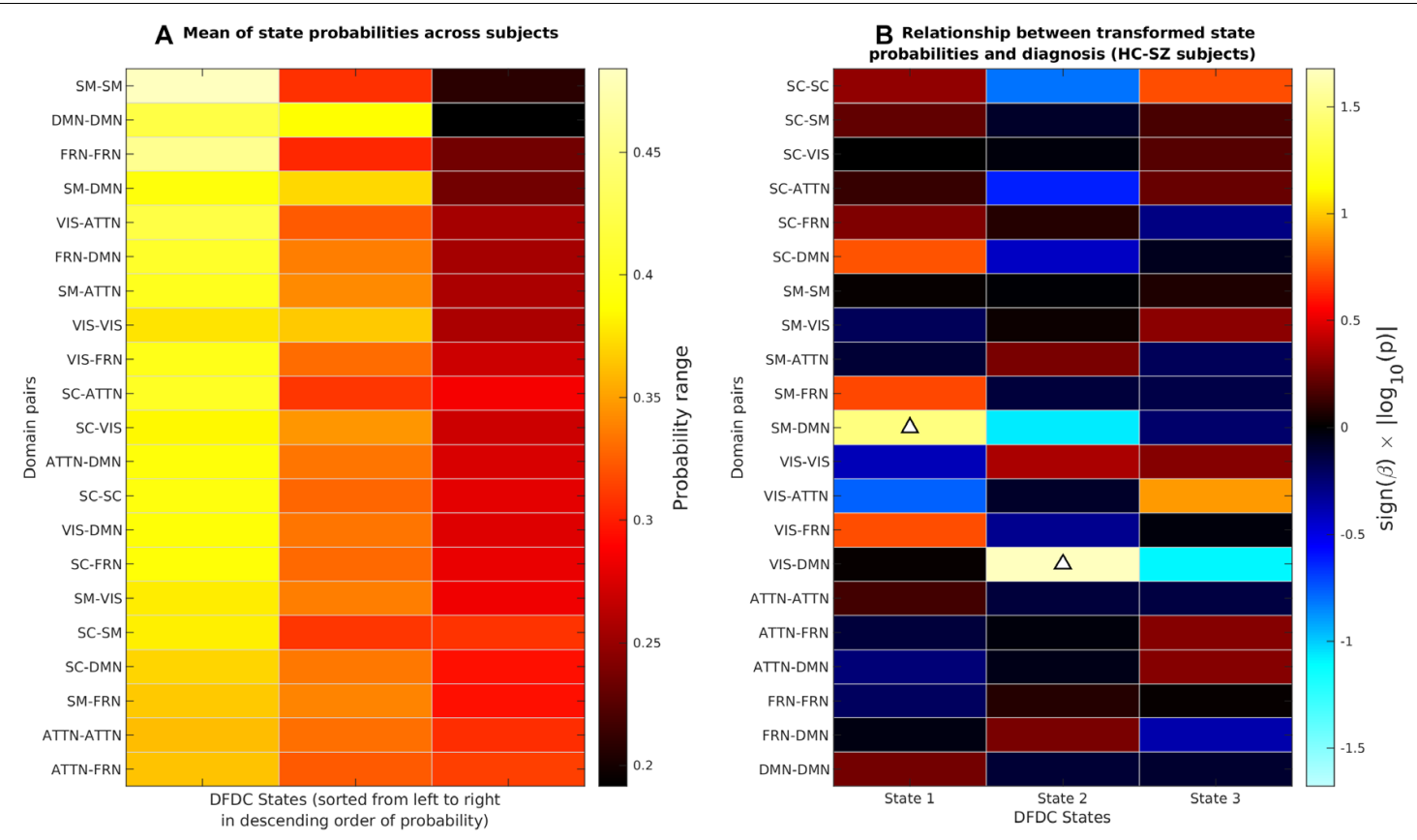

FIGURE 3 | (A) The DFDC state probabilities averaged across all subjects for each of the $K=3$ states and DFDC domain pairs obtained using $K$-means clustering. The domain pairs are sorted from top to bottom in the order of increasing entropy. (B) Results obtained by regressing the diagnosis labels (HC-SZ) of the subjects on each of the DFDC state probabilities. The color intensity indicates sign $(\beta) \times|\log 10(p)|$ where $\beta$ is the coefficient of regression and $p$ is the $p$-value corresponding to the $t$-statistic of the coefficient. Upward arrows indicate that in two cases SZ have significantly higher state probabilities than HCs ( $p<0.05$, uncorrected).

of the DFDC patterns in the diseased brain. Furthermore, SZs demonstrate significantly lower CDMI than HCs between several DFDC pairs. It presents a compelling evidence of higher statistical independence between the activity of different DFDC in the diseased brain.

There are numerous studies hypothesizing aberrant brain function in schizophrenia (Aleman and Kahn, 2005; BrunetGouet and Decety, 2006; Juckel et al., 2006; Bassett et al., 2008; Murray et al., 2008; Sommer et al., 2008; Wolf et al., 2009; Esslinger et al., 2013; Fusar-Poli et al., 2013; van den Heuvel et al., 2013; Kahn et al., 2015). Our work is one of the first to explore information sharing at the functional domain level in schizophrenia, and our findings corroborate the notion of the aberrant nature of functional connectivity. We also examined entropy in whole-brain $\mathrm{dFNC}$ which has not been reported in the literature. We found that SZs have significantly higher entropy than HCs at both whole-brain and functional domain level.

Evidence of weaker CDMI in SZs implies that connectivity among different pairs of functional domains, or DFDC, have less influence on each other's domain activation patterns in SZs. Note that we selected a significant CDMI threshold via bootstrapping and ran linear regression on the selected CDMI, because the CDMI for most of the DFDC pairs are very low (indicating higher independence). It can be argued that the results passed the test for multiple comparisons only because of the lower number of comparisons. Hence, we also examined the group difference in every CDMI and corrected for multiple comparisons, instead of selecting a few based on a bootstrapped threshold. We still found that SZs have significantly lower
CDMI than HCs in the SM-ATTN vs. ATTN-ATTN CDMI, while showing a general trend toward lower CDMI in SZs compared to HCs (Supplementary Figure 13). Miller et al. (2016a) has previously explored information processing among functional domains in schizophrenia and found that auditoryvisual-sensorimotor network in the healthy population showed increased sensitivity to connectivity in the other functional domains. The authors derived two novel metrics (distributional dissimilarity and specificity) based on the transition probabilities of the inter-domain and intra-domain correlation patterns which can capture information flow among the domains. Their major finding - that these metrics are reduced in SZs - is in accordance with our findings. However, we have come to this conclusion by employing information theoretic concepts which are well-defined and broadly used in other fields. Moreover, previous authors used metrics based on transition probability of the connectivity states, whereas we have employed the entropy of the whole sequence of the connectivity states.

We ran some additional tests to investigate the site effect on CDMI and entropy. Specifically, we extended our statistical models to include both diagnosis (2 levels) and site (7 levels) factors, then performed an $\mathrm{N}$-way analysis of covariance using Matlab "anovan" function, followed by a multiple comparison of the stats using the "multcompare" function. We have added the results in Supplementary Figure 18 which shows the 2 cases where we found significant differences between the marginal CDMI means with the site variable considered. In our original manuscript, we identified both these CDMIs to be significantly different between $\mathrm{HC}$ and SZ with the site effect not considered. 


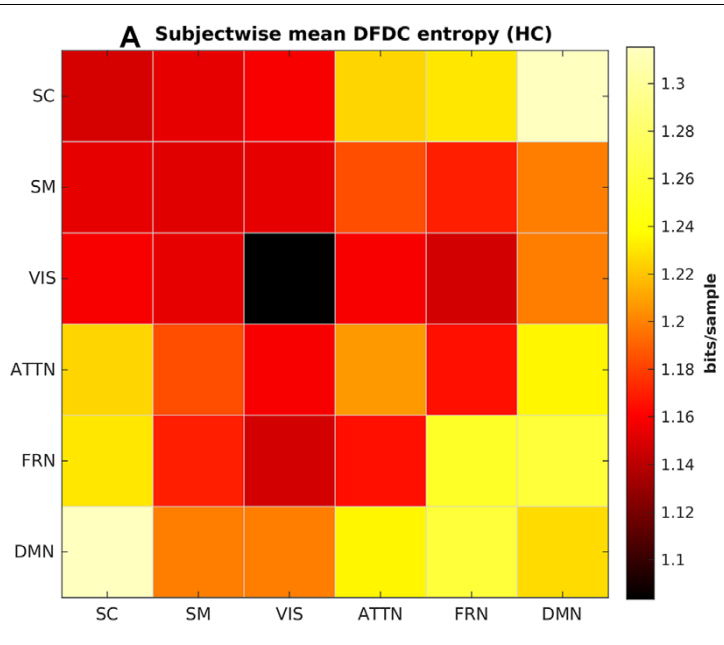

C Relationship between transformed

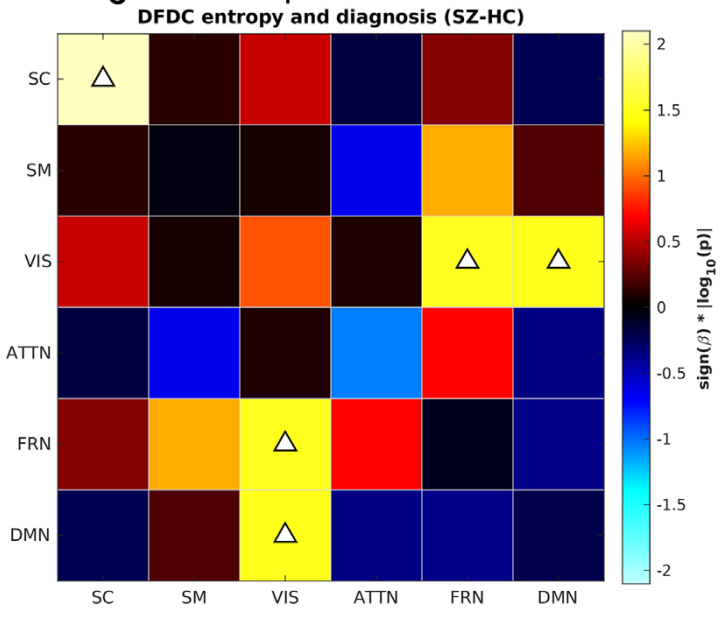

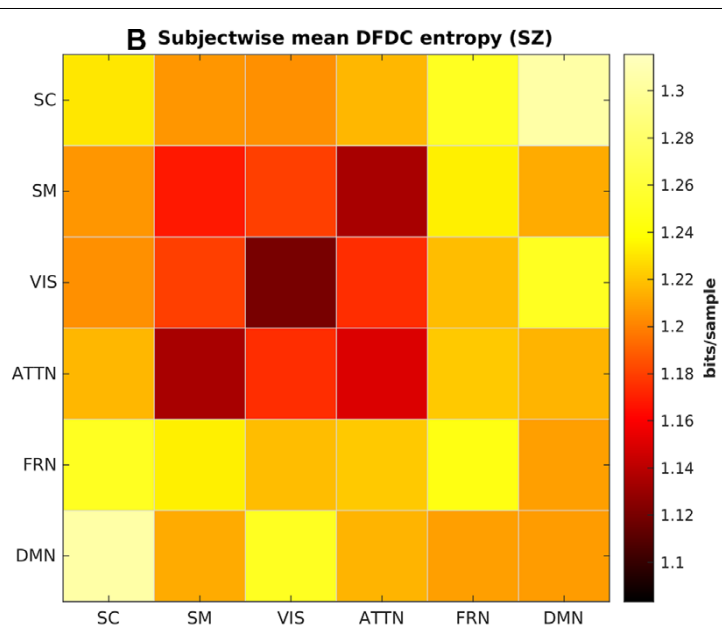

D Group difference in whole-brain dFNC entropy

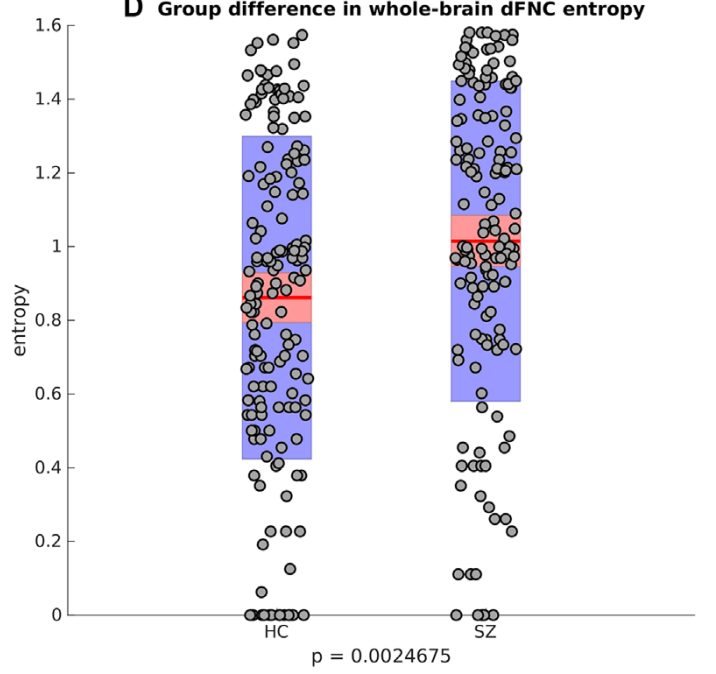

FIGURE 4 | (A) Subject-wise mean entropy for HCs, (B) subject-wise mean entropy for SZs, (C) group difference results from regressing diagnosis on subject-wise entropy. The color intensity indicates sign $(\beta) \times|\log 10(p)|$ where $\beta$ is the coefficient of regression and $p$ is the $p$-value corresponding to the $t$-statistic of the coefficient. Upward arrows on the positive quantities indicate SZs have significantly higher entropy than HCs ( $p<0.05$, uncorrected). (D) Boxplots showing whole-brain dFNC entropy in HC vs. SZ. SZs have significantly higher dFNC entropy than HCs.
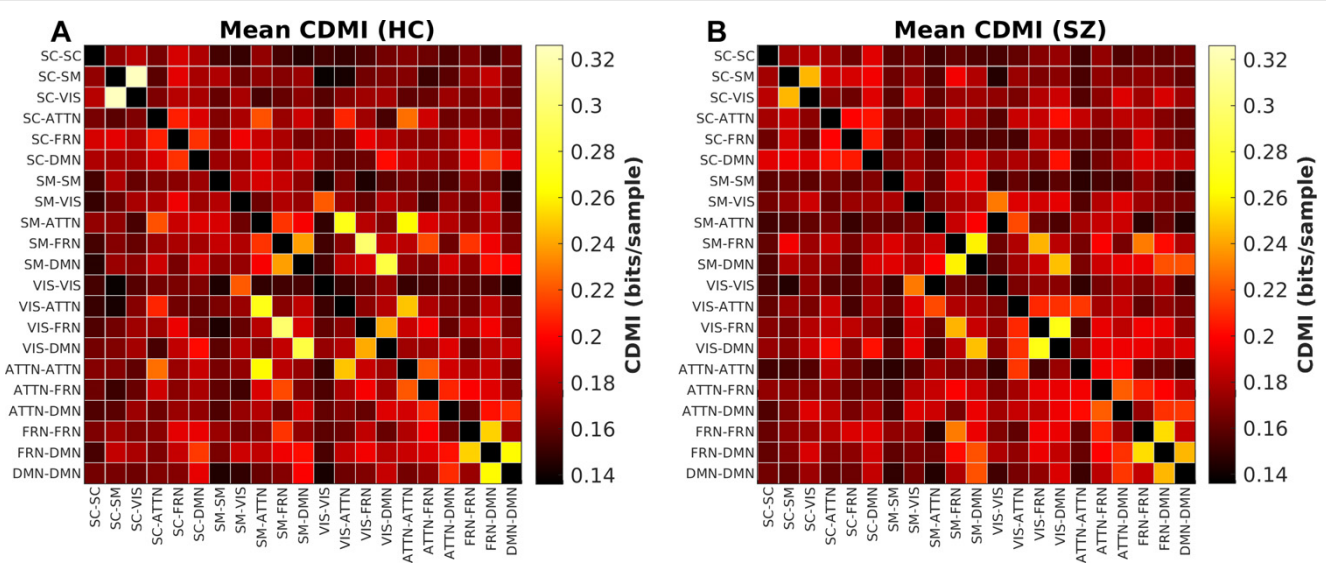

FIGURE 5 | (A) Mean CDMl between different DFDC for HCs, (B) for SZs. 


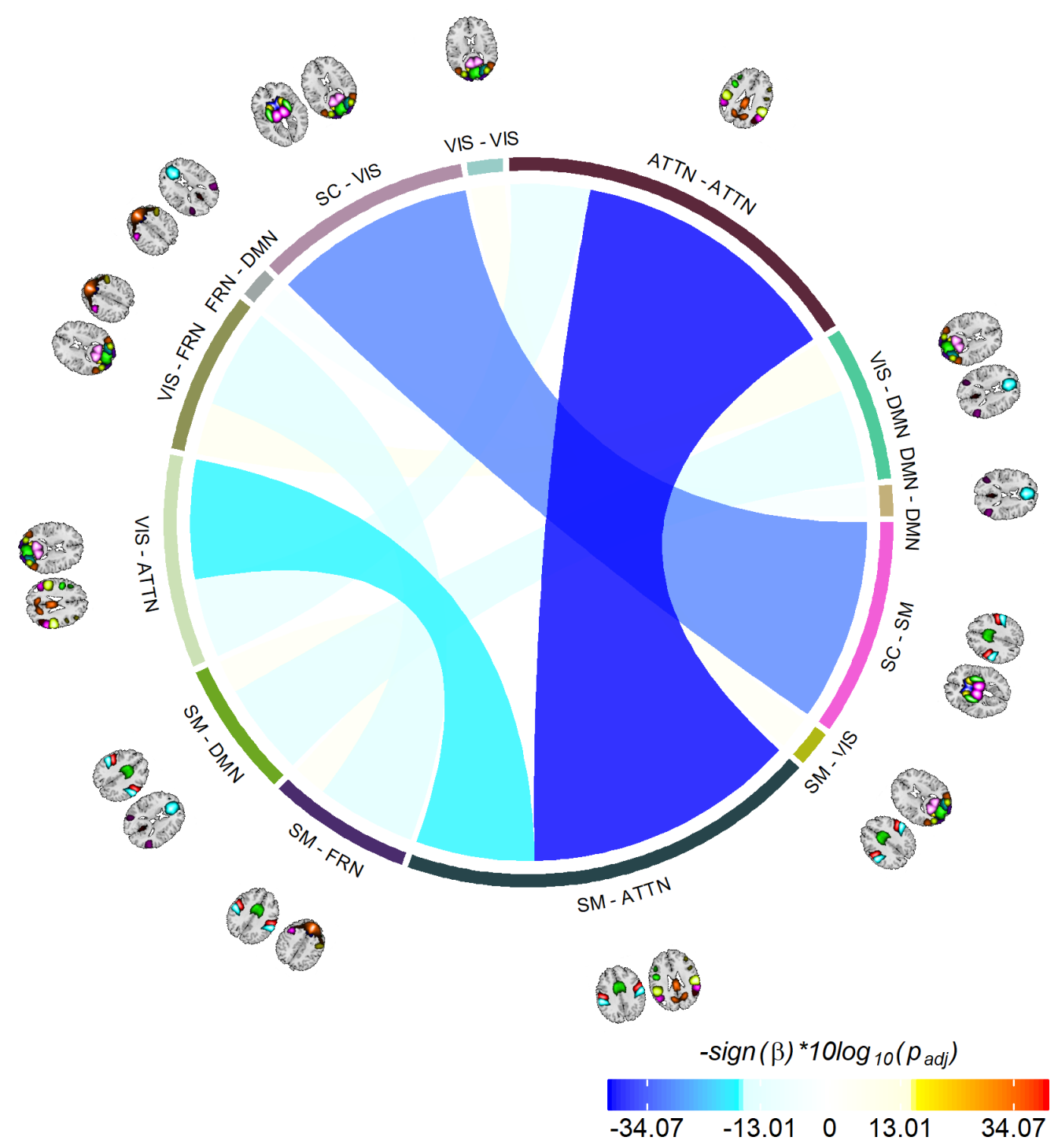

FIGURE 6 | Significant group differences between HCs and SZs. Showing 10 CDMI between 13 different pairs of DFDC which had significantly high CDMI value (determined via bootstrapping). The color of the links indicates sign $(\beta)$ and the width indicates sign ( $\beta$ ) $\times|\log 10(p)|$, where $\beta$ is the regression slope and $p$ is the $p$-value corresponding to the diagnosis variable corrected using FDR correction for multiple comparisons. Negative $\beta$ values indicate decreased CDMI in SZs, with the three darkest/widest links showing the pairs of DFDC which show significant group difference in CDMl at $p<0.05$ level.

From similar analysis on entropy, site difference was observed in the SC-VIS entropy. Given these results, we do not see significant influence of site on the results in the current setting.

It bears discussion what our findings imply for SZ and brain disorders. From previous literature we know of hyper connectivity abnormalities in the SC region (Damaraju et al., 2014; Vergara et al., 2019) and thus it is not a surprise that we see entropy effects in the SC domain. Our results indicate that SC hyper connectivity in SZ translates into higher uncertainty (higher entropy). Given that the SC domain encompass thalamus and putamen, this could point to an increase of information relay in the cortico-thalamic loops. A possible explanation is that the cortical regions in charge of sensory processing are sending an extra amount of signaling to the cortico-thalamic loops in SZ. This might overwork the information relay functions of the striatal and thalamic regions which responds to this demand by enhancing functioning and increasing the amount of information (larger entropy) processed. This overworking of the brain seems to be determined between the SC and VIS regions and two domains (FRN and DMN). Similar impaired VIS-SC-FRN processing has been previously reported in SZ (Sehatpour et al., 2010; John et al., 2018). In our study, we also discover impaired VIS-DMN processing. The patient PANSS positive score shows significant correlation with the VIS-FRN vs. VIS-DMN CDMI. The literature supports this evidence of link between positive symptoms such as visual hallucination and abnormal DMN 
activity in SZ (Jardri et al., 2013) as well as Parkinson's disease (Yao et al., 2014; Shine et al., 2015). It is possible that overworking the SC domain enhances important information among VIS, DMN and FRN domains, but reduces information sharing among other domains in order to compensate the workload. Example of this reduction was observed in the SM-SC vs. SC-VIS CDMI which might have diminished workload to be able to dedicate more resources for other areas more active during resting state. By leveraging information theoretic principles, our work provides some novel insights about the uncertainty of the dFNC and DFDC states in SZ.

Next, we discuss some of the weaknesses and limitations in this study. Firstly, we have imposed the number of clusters, $K=3$ when clustering the DFDC patterns using the $K$-means algorithm. The number $K$ was chosen by observing the elbow criterion. We also experimented by varying $K$ between $\{3,4,5\}$, as well as by including the AUD and CB functional domains in the analysis. The results agree for various settings, i.e., SZs tend to have higher entropy and lower CDMI than HCs. For different settings we found different sets of DFDC to be significant, but some of the DFDCs, such as SC-SC show higher entropy in SZs, and SM-ATTN vs. ATTN-ATTN CDMI tends to be lower in SZs irrespective of different settings. Secondly, the assignment of different networks to the functional domains tends to be subjective and consensus-based among researchers depending on the anatomy and prevalent knowledge about the function of such networks. An important future direction will be to motivate optimal choice of the number of clusters, clustering method, and a replicable method of assignment of functional domains in the information theoretic framework and beyond. Finally, we observed that the effect sizes of the entropy/CDMI group differences are not very large (see Supplementary Figures 9, 12). The effect sizes range between 0.09 and 0.30 for the DFDCs showing significant group differences in entropy, and the linear regression results do not pass correction for multiple comparisons. However, for the CDMI group comparisons, the effect sizes are higher $(0.29-0.46)$ and the linear regression results indeed pass FDR correction for multiple comparisons to corroborate our conclusions.

Since its introduction 70 years ago, information theory has been adopted and appropriated in many different scientific domains, including neuroscience where the study of neural information flow sees major application of the information theoretic principles. In recent years the interest in dynamic nature of the human brain and its implication in brain disorders has peaked. Brain disorders such as schizophrenia as well as aging and dementia are strongly indicative of the deterioration of storage and transmission mechanism of information in the human brain. In this work, we have generated novel insights about the uncertainty of the dFNC and DFDC states in schizophrenia by leveraging information theoretic principles. Further work in this area may help us decipher what language distant brain regions use to "talk" to each other, as well as how this interaction is impaired in brain diseases (Vergara and Calhoun, 2017). The study of the complex yet orderly, dynamic function of the healthy human brain and the lack thereof in mental illness can hopefully benefit from the application of the simple, yet elegant information theoretic framework such as one introduced in our work.

\section{DATA AVAILABILITY}

The datasets for this manuscript are not publicly available. We plan to make all of the connectivity matrices, results, and code available to the public. While we would also like to make the raw data available, it is not our study and unfortunately there are still IRB restrictions which prevent such sharing for now. Requests to access the datasets should be directed to https://www.nitrc.org/ projects/fbirn.

\section{ETHICS STATEMENT}

The data used in this study were collected under the Functional Biomedical Informatics Research Network (FBIRN) phase-III study at the following sites: University of California, Irvine (UCI), University of California, Los Angeles (UCLA), The University of New Mexico (NM), The University of Iowa (IA), University of Minnesota (MN), Duke University/University of North Carolina, University of California, San Diego (UCSD; healthy subjects only), and the University of California, San Francisco (UCSF). Informed consent was obtained from the participants according to the guidelines set by the Internal Review Boards at each site.

\section{AUTHOR CONTRIBUTIONS}

MS, VV, and VC designed the project. MS wrote the manuscript. ED performed the preprocessing, group ICA, and $\mathrm{dFNC}$ analyses. MS, VV, and VC performed the DFDC and statistical analyses, interpreted the results, and edited the manuscript.

\section{FUNDING}

This work was supported by the National Institutes of Health grants (R01EB020407, P20GM103472, and P30GM122734) and the National Science Foundation grant 1539067 (to VC).

\section{ACKNOWLEDGMENTS}

We would like to thank Zening Fu, Ph.D. for help with the preparation of figures.

\section{SUPPLEMENTARY MATERIAL}

The Supplementary Material for this article can be found online at: https://www.frontiersin.org/articles/10.3389/fnins. 2019.00873/full\#supplementary-material 


\section{REFERENCES}

Abrol, A., Chaze, C., Damaraju, E., and Calhoun, V. D. (2016). "The chronnectome: evaluating replicability of dynamic connectivity patterns in 7500 resting fmri datasets," in Proceedings of the 2016 38th Annual International Conference of the IEEE Engineering in Medicine and Biology Society $(E M B C)$, Orlando, FL.

Abrol, A., Rashid, B., Rachakonda, S., Damaraju, E., and Calhoun, V. D. (2017). Schizophrenia shows disrupted links between brain volume and dynamic functional connectivity. Front. Neurosci. 11:624. doi: 10.3389/fnins.2017.00624

Aleman, A., and Kahn, R. S. (2005). Strange feelings: do amygdala abnormalities dysregulate the emotional brain in schizophrenia? Prog. Neurobiol. 77, 283-298. doi: 10.1016/j.pneurobio.2005.11.005

Allen, E. A., Damaraju, E., Plis, S. M., Erhardt, E. B., Eichele, T., and Calhoun, V. D. (2014). Tracking whole-brain connectivity dynamics in the resting state. Cereb. Cortex 24, 663-676. doi: 10.1093/cercor/bhs352

Allen, E. A., Erhardt, E. B., Damaraju, E., Gruner, W., Segall, J. M., Silva, R. F., et al. (2011). A baseline for the multivariate comparison of resting-state networks. Front. Syst. Neurosci. 5:2. doi: 10.3389/fnsys.2011.00002

Andreasen, N. C., Pressler, M., Nopoulos, P., Miller, D., and Ho, B.-C. (2010). Antipsychotic dose equivalents and dose-years: a standardized method for comparing exposure to different drugs. Biol. Psychiatry 67, 255-262. doi: 10. 1016/j.biopsych.2009.08.040

Barttfeld, P., Uhrig, L., Sitt, J. D., Sigman, M., Jarraya, B., and Dehaene, S. (2015). Signature of consciousness in the dynamics of resting-state brain activity. PNAS 112, 887-892. doi: 10.1073/pnas.1418031112

Bassett, D. S., Bullmore, E., Verchinski, B. A., Mattay, V. S., Weinberger, D. R., and Meyer-Lindenberg, A. (2008). Hierarchical organization of human cortical networks in health and schizophrenia. J. Neurosci. 28, 9239-9248. doi: 10.1523/ JNEUROSCI.1929-08.2008

Beckmann, C. F., Mackay, C. E., Filippini, N., and Smith, S. M. (2009). Group comparison of resting-state FMRI data using multi-subject ICA and dual regression. NeuroImage 47:S148.

Bell, A. J., and Sejnowski, T. J. (1995). An information-maximization approach to blind separation and blind deconvolution. Neural Comput. 7, 1129-1159. doi: 10.1162/neco.1995.7.6.1129

Biswal, B., Zerrin Yetkin, F., Haughton, V. M., and Hyde, J. S. (1995). Functional connectivity in the motor cortex of resting human brain using echo-planar mri. Magn. Reson. Med. 34, 537-541. doi: 10.1002/mrm.1910340409

Borst, A., and Theunissen, F. E. (1999). Information theory and neural coding. Nat. Neurosci. 2, 947-957. doi: 10.1038/14731

Brunet-Gouet, E., and Decety, J. (2006). Social brain dysfunctions in schizophrenia: a review of neuroimaging studies. Psychiatry Res. 148, 75-92. doi: 10.1016/j. pscychresns.2006.05.001

Calhoun, V. D. (2004). GIFT Software. Available at: http://mialab.mrn.org/ software/gift/index.html (accessed September 13, 2017).

Calhoun, V. D., and Adali, T. (2012). Multisubject independent component analysis of fmri: a decade of intrinsic networks, default mode, and neurodiagnostic discovery. IEEE Rev. Biomed. Eng. 5, 60-73. doi: 10.1109/ RBME.2012.2211076

Calhoun, V. D., Adali, T., Pearlson, G. D., and Pekar, J. J. (2001). A method for making group inferences from functional MRI data using independent component analysis. Hum. Brain Mapp. 14, 140-151. doi: 10.1002/hbm. 1048

Calhoun, V. D., Liu, J., and Adal, T. (2009). A review of group ICA for fMRI data and ICA for joint inference of imaging, genetic, and ERP data. NeuroImage 45, S163-S172. doi: 10.1016/j.neuroimage.2008.10.057

Chen, X., Zhang, H., Zhang, L., Shen, C., Lee, S.-W., and Shen, D. (2017). Extraction of dynamic functional connectivity from brain grey matter and white matter for MCI classification: dynamic connectivity for MCI classification. Hum. Brain Mapp. 38, 5019-5034. doi: 10.1002/hbm.23711

Cordes, D., Haughton, V. M., Arfanakis, K., Carew, J. D., Turski, P. A., Moritz, C. H., et al. (2001). Frequencies contributing to functional connectivity in the cerebral cortex in "resting-state" data. AJNR Am. J. Neuroradiol. 22, 1326-1333.

Cover, T. M., and Thomas, J. A. (2006). Elements of Information Theory, 2nd Edn. Hoboken, NJ: Wiley-Interscience.

Cox, R. W. (1996). AFNI: software for analysis and visualization of functional magnetic resonance neuroimages. Comput. Biomed. Res. 29, 162-173. doi: 10.1006/cbmr.1996.0014
Damaraju, E., Allen, E. A., Belger, A., Ford, J. M., McEwen, S., Mathalon, D. H., et al. (2014). Dynamic functional connectivity analysis reveals transient states of dysconnectivity in schizophrenia. NeuroImage 5, 298-308. doi: 10.1016/j.nicl. 2014.07.003

Dimitrov, A. G., Lazar, A. A., and Victor, J. D. (2011). Information theory in neuroscience. J. Comput. Neurosci. 30, 1-5.

Du, Y., Pearlson, G. D., Liu, J., Sui, J., Yu, Q., He, H., et al. (2015). A group ICA based framework for evaluating resting fMRI markers when disease categories are unclear: application to schizophrenia, bipolar, and schizoaffective disorders. NeuroImage 122, 272-280. doi: 10.1016/j.neuroimage.2015.07.054

Du, Y., Pearlson, G. D., Yu, Q., He, H., Lin, D., Sui, J., et al. (2016). Interaction among subsystems within default mode network diminished in schizophrenia patients: a dynamic connectivity approach. Schizophrenia Res. 170, 55-65. doi: 10.1016/j.schres.2015.11.021

Erhardt, E. B., Allen, E. A., Damaraju, E., and Calhoun, V. D. (2011a). On network derivation, classification, and visualization: a response to habeck and moeller. Brain Connect. 1, 105-110. doi: 10.1089/brain.2011.0022

Erhardt, E. B., Rachakonda, S., Bedrick, E. J., Allen, E. A., Adali, T., and Calhoun, V. D. (2011b). Comparison of multi-subject ICA methods for analysis of fMRI data. Hum. Brain Mapp. 32, 2075-2095. doi: 10.1002/hbm. 21170

Esslinger, C., Braun, U., Schirmbeck, F., Santos, A., Meyer-Lindenberg, A., Zink, M., et al. (2013). Activation of midbrain and ventral striatal regions implicates salience processing during a modified beads task. PLoS One 8:e58536. doi: 10.1371/journal.pone.0058536

First, M., Spitzer, R., Gibbon, M., and Williams, J. (2002). Structured Clinical Interview for DSM-IV-TR Axis I Disorders, Research Version, Non-patient Edition. (SCID-I/NP). New York, NY: NewYork State Psychiatric Institute.

Freire, L., Roche, A., and Mangin, J. F. (2002). What is the best similarity measure for motion correction in fMRI time series? IEEE Trans. Med. Imag. 21, 470-484. doi: 10.1109/TMI.2002.1009383

Friedman, J., Hastie, T., and Tibshirani, R. (2008). Sparse inverse covariance estimation with the graphical lasso. Biostatistics 9, 432-441. doi: 10.1093/ biostatistics/kxm045

Friedman, L., Glover, G. H., and Fbirn Consortium (2006). Reducing interscanner variability of activation in a multicenter fMRI study: controlling for signalto-fluctuation-noise-ratio (SFNR) differences. NeuroImage 33, 471-481. doi: 10.1016/j.neuroimage.2006.07.012

Friston, K. J. (2007). Statistical Parametric Mapping: the Analysis of Funtional Brain Images, 1st Edn. Amsterdam: Elsevier/Academic Press.

Fusar-Poli, P., Borgwardt, S., Bechdolf, A., Addington, J., Riecher-Rössler, A., Schultze-Lutter, F., et al. (2013). The psychosis high-risk state: a comprehensive state-of-the-art review. JAMA Psychiatry 70, 107-120. doi: 10. 1001/jamapsychiatry.2013.269

Greicius, M. D., Krasnow, B., Reiss, A. L., and Menon, V. (2003). Functional connectivity in the resting brain: a network analysis of the default mode hypothesis. Proc. Natl. Acad. Sci. U.S.A. 100, 253-258. doi: 10.1073/pnas. 0135058100

Jardri, R., Thomas, P., Delmaire, C., Delion, P., and Pins, D. (2013). The neurodynamic organization of modality-dependent hallucinations. Cereb. Cortex 23, 1108-1117. doi: 10.1093/cercor/bhs082

John, Y. J., Zikopoulos, B., Bullock, D., and Barbas, H. (2018). Visual attention deficits in schizophrenia can arise from inhibitory dysfunction in thalamus or cortex. Comput. Psychiatry 2, 223-257. doi: 10.1162/y_a_00023

Juckel, G., Schlagenhauf, F., Koslowski, M., Wüstenberg, T., Villringer, A., Knutson, B., et al. (2006). Dysfunction of ventral striatal reward prediction in schizophrenia. Neuroimage 29, 409-416. doi: 10.1016/j.neuroimage.2005. 07.051

Kahn, R. S., Sommer, I. E., Murray, R. M., Meyer-Lindenberg, A., Weinberger, D. R., Cannon, T. D., et al. (2015). Schizophrenia. Nat. Rev. Dis. Primers 1:15067. doi: 10.1038/nrdp.2015.67

Keator, D. B., van Erp, T. G. M., Turner, J. A., Glover, G. H., Mueller, B. A., Liu, T. T., et al. (2016). The function biomedical informatics research network data repository. Neuroimage 124, 1074-1079. doi: 10.1016/j.neuroimage.2015. 09.003

Leonardi, N., and Van De Ville, D. (2015). On spurious and real fluctuations of dynamic functional connectivity during rest. NeuroImage 104, 430-436. doi: 10.1016/j.neuroimage.2014.09.007 
Lottman, K. K. M., Kraguljac, N. V. M., White, D. M. M., Morgan, C. J. P., Calhoun, V. D. P., Butt, A. M., et al. (2017). Risperidone effects on brain dynamic connectivity- a prospective resting state fMRI study in schizophrenia. Front. Psychiatry 8:14. doi: 10.3389/fpsyt.2017.00014

Ma, S., Correa, N. M., Li, X.-L., Eichele, T., Calhoun, V. D., and Adalı, T. (2011). Automatic identification of functional clusters in fMRI data using spatial information. IEEE Trans. Biomed. Eng. 58, 3406-3417. doi: 10.1109/TBME. 2011.2167149

Miller, R. L., Vergara, V. M., Keator, D. B., and Calhoun, V. D. (2016a). A method for intertemporal functional-domain connectivity analysis: application to schizophrenia reveals distorted directional information flow. IEEE Trans. Biomed. Eng. 63, 2525-2539. doi: 10.1109/TBME.2016.2600637

Miller, R. L., Yaesoubi, M., Turner, J. A., Mathalon, D., Preda, A., Pearlson, G., et al. (2016b). Higher dimensional meta-state analysis reveals reduced resting fmri connectivity dynamism in schizophrenia patients. PLoS One 11:e0149849. doi: 10.1371/journal.pone.0149849

Murray, G., Corlett, P., Clark, L., Pessiglione, M., Blackwell, A., Honey, G., et al. (2008). Substantia nigra/ventral tegmental reward prediction error disruption in psychosis. Mol. Psychiatry 13, 239-276. doi: 10.1038/sj.mp.4002058

Rabany, L., Brocke, S., Calhoun, V. D., Pittman, B., Corbera, S., Wexler, B. E., et al. (2018). S224. dynamic functional network connectivity in schizophrenia and autism spectrum disorder. Biol. Psychiatry 83:S435. doi: 10.1016/j.biopsych. 2018.02.1116

Rashid, B., Arbabshirani, M. R., Damaraju, E., Cetin, M. S., Miller, R., Pearlson, G. D., et al. (2016). Classification of schizophrenia and bipolar patients using static and dynamic resting-state fMRI brain connectivity. NeuroImage 134, 645-657. doi: 10.1016/j.neuroimage.2016.04.051

Sakoğlu, Ü, Pearlson, G. D., Kiehl, K. A., Wang, Y. M., Michael, A. M., and Calhoun, V. D. (2010). A method for evaluating dynamic functional network connectivity and task-modulation: application to schizophrenia. Magn. Reson. Mater. Phys. Biol. Med. 23, 351-366. doi: 10.1007/s10334-010-0197-8

Sanfratello, L., Houck, J., and Calhoun, V. D. (2019). Dynamic functional network connectivity in schizophrenia with meg and fmri, do different time scales tell a different story? Brain Connect. 9, 251-262. doi: 10.1089/brain.2018. 0608

Sehatpour, P., Dias, E. C., Butler, P. D., Revheim, N., Guilfoyle, D. N., Foxe, J. J., et al. (2010). Impaired visual object processing across an occipital-frontalhippocampal brain network in schizophrenia: an integrated neuroimaging study. Arch. Gen. Psychiatry 67:772. doi: 10.1001/archgenpsychiatry.2010.85

Shannon, C. E. (1948). A mathematical theory of communication. Bell. Syst. Tech. J. 27, 379-423. doi: 10.1002/j.1538-7305.1948.tb01338.x

Shine, J. M., Muller, A. J., O’Callaghan, C., Hornberger, M., Halliday, G. M., and Lewis, S. J. (2015). Abnormal connectivity between the default mode and the visual system underlies the manifestation of visual hallucinations in Parkinson's disease: a task-based fMRI study. NPJ Parkinson's Dis. 1:15003. doi: 10.1038/ npjparkd.2015.3
Sommer, I. E. C., Diederen, K. M. J., Blom, J.-D., Willems, A., Kushan, L., Slotema, K., et al. (2008). Auditory verbal hallucinations predominantly activate the right inferior frontal area. Brain 131, 3169-3177. doi: 10.1093/brain/awn251

van den Heuvel, M. P., Sporns, O., Collin, G., Scheewe, T., Mandl, R. C. W., Cahn, W., et al. (2013). Abnormal rich club organization and functional brain dynamics in schizophrenia. JAMA Psychiatry 70, 783-792. doi: 10.1001/ jamapsychiatry.2013.1328

Varoquaux, G., Gramfort, A., Poline, J.-B., and Thirion, B. (2010). Brain Covariance Selection: Better Individual Functional Connectivity Models Using Population Prior. Available at: http://papers.nips.cc/paper/4080-braincovariance-selection-better-individual-functional-connectivity-modelsusing-population-prior (accessed February 19, 2016).

Vergara, V. M., and Calhoun, V. D. (2017). "Brain language: uncovering functional connectivity codes," in Proceedings Of the 2017 51st Asilomar Conference On Signals, Systems, and Computers, (Piscataway, NJ).

Vergara, V. M., Damaraju, E., Turner, J. A., Pearlson, G., Belger, A., Mathalon, D. H., et al. (2019). Altered domain functional network connectivity strength and randomness in schizophrenia. Front. Psychiatry 10:499. doi: 10.3389/fpsyt. 2019.00499

Vergara, V. M., Miller, R. L., and Calhoun, V. D. (2017). An information theory framework for dynamic functional domain connectivity. J. Neurosci. Methods 284, 103-111. doi: 10.1016/j.jneumeth.2017.04.009

Wolf, R. C., Vasic, N., Sambataro, F., Höse, A., Frasch, K., Schmid, M., et al. (2009). Temporally anticorrelated brain networks during working memory performance reveal aberrant prefrontal and hippocampal connectivity in patients with schizophrenia. Prog. Neuropsychopharmacol. Biol. Psychiatry 33, 1464-1473. doi: 10.1016/j.pnpbp.2009.07.032

Yao, N., Chang, R. S.-K., Cheung, C., Pang, S., Lau, K. K., Suckling, J., et al. (2014). The default mode network is disrupted in parkinson's disease with visual hallucinations. Hum. Brain Mapp. 35, 5658-5666. doi: 10.1002/hbm. 22577

Zhi, D., Calhoun, V. D., Lv, L., Ma, X., Ke, Q., Fu, Z., et al. (2018). Aberrant dynamic functional network connectivity and graph properties in major depressive disorder. Front. Psychiatry 9:339. doi: 10.3389/fpsyt.2018. 00339

Conflict of Interest Statement: The authors declare that the research was conducted in the absence of any commercial or financial relationships that could be construed as a potential conflict of interest.

Copyright (c) 2019 Salman, Vergara, Damaraju and Calhoun. This is an open-access article distributed under the terms of the Creative Commons Attribution License (CC BY). The use, distribution or reproduction in other forums is permitted, provided the original author(s) and the copyright owner(s) are credited and that the original publication in this journal is cited, in accordance with accepted academic practice. No use, distribution or reproduction is permitted which does not comply with these terms. 


\section{OPEN ACCESS}

Edited by:

Alessandra Griffa,

VU University Amsterdam,

Netherlands

Reviewed by:

Lorenzo Fabrizi,

University College London,

United Kingdom

Chiara Nosarti,

King's College London,

United Kingdom

Ruth Eckstein Grunau,

University of British Columbia,

Canada

Lynne Joanne Williams, BC Children's Hospital MRI Research Facility, Canada

*Correspondence:

Domenico Tortora domenicotortora@gaslini.org Andrea Rossi

andrearossi@gaslini.org

Specialty section

This article was submitted to

Brain Imaging Methods,

a section of the journal

Frontiers in Neuroscience

Received: 14 April 2019

Accepted: 12 August 2019

Published: 23 August 2019

Citation:

Tortora D, Severino M, Di Biase C,

Malova M, Parodi A, Minghetti D,

Traggiai $C$, Uccella $S$, Boeri L, Morana G, Rossi A and Ramenghi LA (2019) Early Pain Exposure Influences

Functional Brain Connectivity in Very

Preterm Neonates.

Front. Neurosci. 13:899 doi: 10.3389/fnins.2019.00899

\section{Early Pain Exposure Influences Functional Brain Connectivity in Very Preterm Neonates}

\author{
Domenico Tortora ${ }^{1 *}$, Mariasavina Severino', Carlo Di Biase², Maryia Malova², \\ Alessandro Parodi ${ }^{2}$, Diego Minghetti ${ }^{2}$, Cristina Traggiai ${ }^{2}$, Sara Uccella ${ }^{3}$, Luca Boeri ${ }^{3}$, \\ Giovanni Morana ${ }^{1}$, Andrea Rossi ${ }^{1 *}$ and Luca Antonio Ramenghi \\ ${ }^{1}$ Neuroradiology Unit, IRCCS Istituto Giannina Gaslini, Genoa, Italy, ${ }^{2}$ Neonatal Intensive Care Unit, IRCCS Istituto Giannina \\ Gaslini, Genoa, Italy, ${ }^{3}$ Child Neuropsychiatry Unit, IRCCS Istituto Giannina Gaslini, Genoa, Italy
}

Background: Early exposure to nociceptive events may cause brain structural alterations in preterm neonates, with long-lasting consequences on neurodevelopmental outcome. Little is known on the extent to which early pain may affect brain connectivity. We aim to evaluate brain functional connectivity changes in preterm neonate that underwent multiple invasive procedures during the postnatal period, and to correlate them with the neurodevelopmental outcome at 24 months.

Methods: In this prospective case-control study, we collected information about exposure to painful events during the early postnatal period and resting-state BOLD$\mathrm{fMRI}$ data at term equivalent age from two groups of preterm neonate: 33 subjected to painful procedures during the neonatal intensive care (mean gestational age $27.9 \pm 1.8$ weeks) and 13 who did not require invasive procedures (average gestational age $31.2 \pm 2.1$ weeks). A data-driven principal-component-based multivariate pattern analysis (MVPA) was used to investigate the effect of early pain exposure on brain functional connectivity, and the relationship between connectivity changes and neurodevelopmental outcome at 24 months, assessed with Griffiths, Developmental Scale-Revised: 0-2.

Results: Early pain was associated with decreased functional connectivity between thalami and bilateral somatosensory cortex, and between the right insular cortex and ipsilateral amygdala and hippocampal regions, with a more evident effect in preterm neonate undergoing more invasive procedures. Functional connectivity of the right thalamocortical pathway was related to neuromotor outcome at 24 months $(P=0.003)$.

Conclusion: Early exposure to pain is associated with abnormal functional connectivity of developing networks involved in the modulation of noxious stimuli in preterm neonate, contributing to the neurodevelopmental consequence of preterm birth.

Keywords: preterm neonates, fMRI, pain, functional connectivity, neonatal neuroimaging, nociceptive modulations, brain connectivity, resting state 


\section{INTRODUCTION}

The survival rates for preterm infants have improved considerably in recent decades due to the advances in perinatal and neonatal care (Lea et al., 2017). On the other hand, preterm neonates necessitate invasive treatments as part of their essential intensive care and can be subjected to multiple painful procedures requiring skin breaks (i.e., heel lances, intravenous or central line insertion, intramuscular injection, and surgical interventions) or intubations that may have a relevant influence on normal brain maturation and developmental neuroplasticity especially at low gestational ages (Carbajal et al., 2008). Indeed, in the early phases of development, the brain is extremely adaptable to novel sensory information inducing experiencedependent plasticity mechanisms (i.e., adaptive neuroplasticity) and vulnerable to several different insults leading to reactive post-injury neuroplasticity (Ismail et al., 2017).

In this regards, it has been recently demonstrated that exposure to nociceptive stimuli at earlier gestational ages may have several effects on the developing brain, with more severe alterations of multiple cerebral structures in extremely preterm neonates exposed to multiple painful procedures ( $\leq 28$ weeks of gestational age) (Brummelte et al., 2015; Duerden et al., 2018). In particular, pain in preterm neonates may induce somatosensory changes (Grunau et al., 1994; Goffaux et al., 2008; Walker et al., 2009a), with longlasting consequences upon subcortical gray and white-matter development subserving different somatosensory, cognitive, and motor processes (Brummelte et al., 2012; Ranger et al., 2013).

In preterm neonates, altered brain structure contributes to poorer cognitive, and motor outcomes during the first years of life (Grunau et al., 2009; Brummelte et al., 2012; Vinall et al., 2014; Duerden et al., 2018).

Despite the increasing evidence that repeated painful experiences during the first days of life in the neonatal intensive care environment may contribute to structural alterations of the preterm neonatal brain, little research has focused on the relationship between painful stressors and functional activity of the preterm brain at term corrected age (Smith et al., 2011). Recent studies demonstrated weaker functional connectivity in preterm neonates evaluated at term corrected age, with more severe alterations of network complexity in neonates with white matter injuries (Ball et al., 2016; Scheinost et al., 2016; Duerden et al., 2019). Nevertheless, little is known on the extent to which painful stressors may affect brain connectivity in these early phases, with potential consequences on future brain development, and plasticity.

In this study, we hypothesized that early exposure of preterm infants to painful stimuli in the neonatal intensive care setting could influence the functional connectivity of brain regions involved into pain processing. Accordingly, we aimed to explore differences in functional brain connectivity among preterm neonates that underwent multiple invasive procedures during the perinatal period and a control group of gestationalage and sex-matched neonates who experienced less than 5 procedures and no intubation nor surgical interventions. Additionally, we aimed to assess if abnormalities of brain connectivity are related to the invasiveness of treatments, correlating brain connectivity features with the number, and type of painful events. Finally, we aimed to explore the association between functional connectivity alterations at termcorrected age in preterm neonates both exposed and not exposed to painful stimuli and their neurodevelopmental outcome at 24 months.

\section{MATERIALS AND METHODS}

The local ethics committee (Comitato Etico Regione Liguria, Genoa, Italy) approved this study and parents provided written informed consent in accordance with the Declaration of Helsinki.

\section{Participants}

We retrospectively identified all 112 preterm neonates with birth weight $<1500 \mathrm{~g}$ who underwent brain MRI at term equivalent age within our institutional screening program for identification of prematurity-related lesions between July 2016 and January 2017. Clinical data were systematically collected from the neonates' charts by a neonatologist. As a measure of pain, each individual invasive procedure involving skin breaks was considered, including heel lances, intubations, intravenous or central line insertion, intramuscular injection, and surgical interventions. Other clinical variables included gestational age at birth, postmenstrual age at MRI scan, gender, APGAR scores, morphine dose, midazolam dose, dexamethasone dose, and days of mechanical ventilation.

Neonates were first divided into two groups: (i) those who were subjected to invasive procedures during the neonatal period (IP-group) and (ii) those who were not subjected to invasive procedures (control group). Subsequently, we elaborated a neonatal intensive care invasiveness (NICI) score based on the type and number of invasive procedures: 0 , no painful events during the early post-natal period or $<5$ skin breaks (i.e., heel lances, central line insertion, and intramuscular injection); I, $>5$ skin breaks or neonatal endotracheal intubation; II, $>5$ skin breaks and neonatal endotracheal intubation; III, surgical interventions (i.e., patent ductus arteriosus ligation and surgical necrotizing enterocolitis). Each neonate was assigned to one of these categories by the neonatologist (AP) who assisted all the infants during the postnatal period.

Exclusion criteria were: (i) presence of significant cerebral injuries (congenital malformations/syndromes, antenatal infections, ischemic lesions, hemorrhagic infarctions, or intraventricular-germinal matrix hemorrhage), (ii) absence of resting state fMRI in the study protocol, and (iii) poor quality of MR images due to motion artifacts.

\section{Neurodevelopmental Outcome}

The neurodevelopmental assessment was performed at 24 months of age between July 2018 and January 2019, using the Griffiths Mental Development Scales-Revised: Birth to 2 years (Griffiths et al., 2007). Developmental quotient, locomotor, personal-social, hearing-speech, eye-hand coordination, and performance outcomes subscales were assessed by 2 
testers (LB and SU), unaware of brain imaging findings (Griffiths et al., 2007).

\section{Brain MRI}

MR imaging was performed on a 3T Philips MR scanner (Ingenia 3T; Philips Healthcare, Best, Netherlands) using a 32channel parallel imaging head coil. Anatomical MR imaging was acquired with a high-resolution 3D T1-weighted image sequence $\left(1 \mathrm{~mm}^{3} \times 1 \mathrm{~mm}^{3} \times 1 \mathrm{~mm}^{3}\right.$ voxel size). For each subject we performed a resting state scan consisting of 250 volumes (5 min and $45 \mathrm{~s}$ ), using an echo-planar imaging with $\mathrm{TR} / \mathrm{TE}=2500 / 30 \mathrm{~ms}$, flip angle $=90^{\circ}, 53$ slices, $96 \times 96$ matrix size, $\mathrm{FOV}=288 \mathrm{~mm} \times 288 \mathrm{~mm}$, voxel size $=3 \mathrm{~mm}$ isotropic, and an interleaved mode of slice acquisition. Volumetric T1weighted sequence, axial and coronal T2-weighted sequences and susceptibility-weighted imaging (SWI) scans were also evaluated for brain lesions.

All patients were fed before MRI examination in order to achieve spontaneous sleep and were spontaneously breathing during examination. Hearing protection was used in all patients. Heart rate and oxygen saturation were non-invasively monitored by pulse-oximetry during examination.

\section{Structural Analysis}

Brain segmentation was performed on 3D T1-weighted sequences calculating gray matter, white matter, and cerebrospinal fluid maps. Non-brain tissue components were removed by using Skull stripping toolbox of BrainSuite version $15 c^{1}$. Extracted images were then segmented into tissue classes using unified segmentation as implemented in the "Segment" option of SPM12 (Wellcome Department, University College London, United Kingdom). For guiding segmentation, we used tissue probability maps from preterm neonates scanned at term age (Kuklisova-Murgasova et al., 2011). A neuroradiologist with 16 years of experience (GM) validated brain segmentation results by qualitative assessment.

\section{Resting State Analysis}

Prior to functional data analysis, every BOLD sequence was preprocessed using SPM12 software (Welcome Trust Center of Neuroimaging, University College London, United Kingdom). The first four scans were not considered for the analysis. Image preprocessing included slice-time corrections, image realignment to median image of each sequence, co-registration of functional images and structural 3D T1-weighted images, and normalization of structural and functional scans to a neonatal template (Shi et al., 2011). Functional volumes were spatially smoothed using an $8 \mathrm{~mm}$ FWHM Gaussian kernel to address the anatomical variability that characterizes the neonatal brain. Subject level connectivity analysis was performed using the Conn toolbox v18b (Whitfield-Gabrieli and Nieto-Castanon, 2012) on Matlab. Functional volumes were band pass filtered at default values of $0.008-0.09 \mathrm{~Hz}$ simultaneously with nuisance regression. Subject specific nuisance regressors included 6 movement regressors and their time derivatives, and 5 regressors pertaining to

${ }^{1}$ http://brainsuite.org white matter and CSF signals sources, respectively, using a principal component (PCA) based noise correction approach (Behzadi et al., 2007).

Differences in functional connectivity among the two groups of preterm neonates were investigated using a datadriven principal component based multivariate pattern analysis $(\text { MVPA })^{2}$. The MVPA provides a regionally unbiased mapping of brain areas that show abnormal whole brain connectivity patterns according to the exposure to early procedural pain events. In particular, we tested for clusters of different brain connectivity that differed between neonates with exposure to early procedural pain and controls, as represented by PCA component volumes. For the analysis we considered 8 PCA components $(C=8)$ and we maintained the four principal components that explained most of the variance of the connectivity matrix (Thompson et al., 2016). Subsequently, we performed a whole-brain post hoc seed correlation analysis using spherical seeds placed at the peak voxels of the major clusters obtained in the MVPA, in order to investigate differences in connectivity patterns of these regions against all other voxels in the brain. This latter post hoc analysis aims to characterize the observed patterns of connectivity between the found MVPA seeds and the rest of the brain, testing which areas of the brain changes its connectivity in relation to exposure to procedural pain. Additionally, a seedto-voxel analysis, considering the same seeds placed at the peak voxels of the major clusters obtained in the MVPA, was also used to explore the relationship between the brain connectivity of regions influenced by early exposure to pain stimuli and the NICI score. Fisher-transformed correlation coefficient values were considered as the metric of functional connectivity. All group analyses were controlled for mean frame wise displacement, gestational age at birth, gender and postmenstrual age at MRI. Results were thresholded at a voxel-wise $p<0.001$ (FWEcorrected) two-sided level and cluster-level $p<0.05$ (FWEcorrected) level.

\section{Brain Connectivity and Neurodevelopment}

We evaluated the relationship between the brain connectivity of regions influenced by early pain exposure and the neurodevelopmental outcome at 24 months of corrected age, extracting connectivity values from the suprathreshold clusters obtained at the previous post hoc seed-to-voxel analysis. An exploratory analysis was conducted to test the relationship between these extracted functional connectivity values and outcome scores using a Spearman Rho correlation test. Subsequently, the effects of functional connectivity (independent variable) on the neurodevelopmental outcome scores at 24 months corrected-age (dependent variables) were examined using a general linear regression model. Variables known to influence brain development and clinical outcome were entered as covariates, including gestational age at birth and MRI (weeks), gender, total morphine dose, and days of intubation. Preliminary checks were conducted to ensure that there was no violation of the assumptions of normality, linearity,

${ }^{2}$ http://www.nitrc.org/projects/conn 


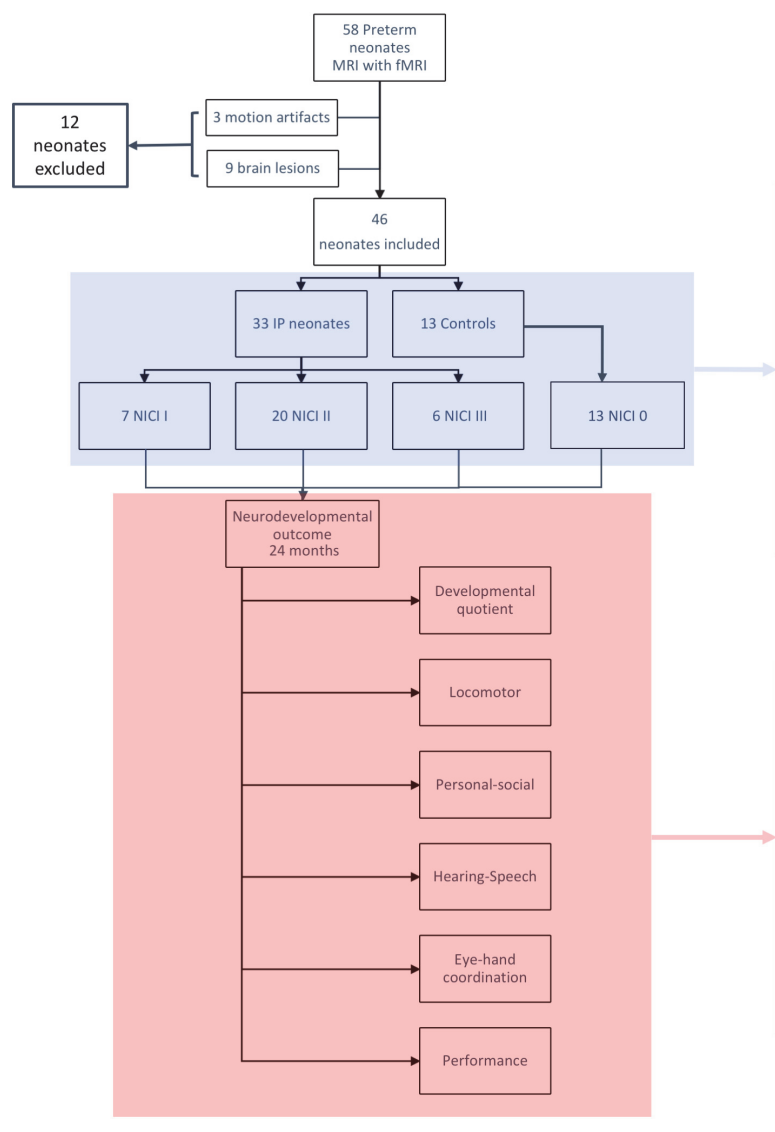

\section{fMRI ANALYSIS}

1) Data-driven principal component based multivariate pattern analysis (MVPA) to identify brain regions with different pattern of brain connectivity between IP neonates and controls

2) Post-hoc seed-to-voxel analysis to investigate differences between IP neonates and controls in functional connectivity of regions of the major clusters obtained in the MVPA (seeds) against all other voxels in the brain

3) Post-hoc seed-to-voxel analysis to investigate the relationship between the $\mathrm{NICl}$ score and functional connectivity of regions of the major clusters obtained in the MVPA (seeds) against all other voxels in the brain

4) Spearman Rho Correlation analysis to test the relationship between 24 months Griffith's subscales outcome score and connectivity values obtained from the post-hoc seed-to-voxel analysis comparing functional connectivity between IP neonates and controls

5) General linear regression model to explore the effects of functional connectivity values obtained from the post-hoc seed-to-voxel analysis comparing functional connectivity between IP neonates and controls (independent variable) on the 24 months Griffith's subscales outcome score (dependent variables)

FIGURE 1 | Flow-chart summarizing the fMRI analysis. Control group, neonates not requiring invasive procedures; IP group, neonates requiring invasive procedures; $\mathrm{NICl}$ score, neonatal intensive care invasiveness score; 0 category, no painful events during the early post-natal period or $<5$ skin breaks (i.e., heel lances, central line insertion, and intramuscular injection); I category, $>5$ skin breaks or neonatal endotracheal intubation; // category, $>5$ skin breaks and neonatal endotracheal intubation; III category, surgical interventions (i.e., patent ductus arteriosus ligation and surgical necrotizing enterocolitis).

homogeneity of variances, homogeneity of regression slopes, and reliable measurement of the covariate (Figure 1).

Statistical analyses were performed using SPSS Statistics software, v21 (IBM, Armonk, NY, United States).

\section{RESULTS}

\section{Clinical Characteristics}

Brain MRI studies of 112 consecutive preterm neonates were retrospectively evaluated. The BOLD fMRI sequence was performed in 58 neonates. The MRI studies of 12/58 patients (20.7\%) were excluded: brain lesions were present in 9 cases (8 GMH-IVH, 2 arteriovenous malformations, and 2 brain malformations), and fMRI sequences were affected by motion artifacts in the remaining 3 neonates. Thus, the MRI studies of $46 / 58(79.3 \%)$ preterm neonates were included in the study. Thirty-three $(71.7 \%, 33 / 46)$ neonates were subjected to painful procedures during the neonatal intensive care (IPgroup): $7 / 33$ (21\%) were assigned to the NICI I category, 20/33 $(60 \%)$ to the NICI II category, and 6/33 (19\%) to the NICI III category. Conversely, 13 preterm neonates $(28.3 \%, 13 / 46)$ required less than 5 procedures during the perinatal period (control group) (Table 1).

\section{Neurodevelopmental Outcome}

Table 2 summarize results of neurodevelopmental outcome evaluation at 24 months. An abnormal neurodevelopmental outcome occurred more frequently in neonates who required significant invasive procedures in the neonatal period compared to the control group ( $P=0.001)$. In particular, $20 / 46(43.4 \%)$ ex-preterm children (all belonging to the IP group) obtained neurodevelopmental scores lower than 85 in at least one of the 6 developmental domains of the Griffiths scale: 3/7 $(42.9 \%)$ of the NICI I category neonates, $12 / 20(60 \%)$ of the NICI II category neonates, and 5/6 (83.3\%) of the NICI III category neonates. Conversely, the performance of the remaining 26/46 (56.6\%) children, including 13 IP neonates and all control children, was higher than 85 in all domains (Supplementary Figure S1).

\section{Functional Brain Connectivity}

The multivariate pattern analysis analysis showed group differences with regard to whole brain functional connectivity 
TABLE 1 | Demographic and clinical characteristics of neonates divided according to the NICl score categories.

\begin{tabular}{|c|c|c|c|c|c|}
\hline \multirow[b]{2}{*}{ NICl score } & \multirow{2}{*}{$\begin{array}{c}\text { Control-group } \\
0\end{array}$} & \multicolumn{3}{|c|}{ IP-group } & \multirow[t]{2}{*}{$P$} \\
\hline & & I & II & III & \\
\hline$N^{\circ}$ neonates & 13 & 7 & 20 & 6 & \\
\hline $\begin{array}{l}\text { Gestational age (week) Median } \\
\text { (range) }\end{array}$ & $31.2(27.1-32.8)$ & $28.2(27.3-29.1)$ & $27.1(26.3-30.2)$ & $27.9(25.3-31.7)$ & 0.085 \\
\hline $\begin{array}{l}\text { Postmenstrual age at MRI } \\
\text { (week) Median (range) }\end{array}$ & $40.1(39.1-41.2)$ & $40.1(39.3-40.3)$ & $40.8(38.9-41.3)$ & $40.3(39.4-41.9)$ & 0.832 \\
\hline Birth weight (gr) Median (range) & 1189.25 (863.81-1514.21) & 1090.88 (844.32-1427.32) & $1060.18(713.41-1523.78)$ & $1190.21(756.54-1497.59)$ & 0.743 \\
\hline Gender $N^{\circ}$ neonates (\%) & & & & & 0.833 \\
\hline Male & $5(38 \%)$ & $4(57 \%)$ & $8(40 \%)$ & $3(50 \%)$ & \\
\hline Female & $8(62 \%)$ & $3(43 \%)$ & $12(60 \%)$ & $3(50 \%)$ & \\
\hline APGAR 1' Median (range) & $8.81(5-10)$ & $6.97(5-9)$ & $6.43(5-8)$ & $6.58(5-7)$ & 0.011 \\
\hline APGAR 5' Median (range) & $9.15(7-10)$ & $8.91(7-10)$ & $8.21(6-9)$ & $7.22(6-8)$ & 0.003 \\
\hline $\begin{array}{l}\text { Days of mechanical ventilation } \\
\text { Median (range) }\end{array}$ & $0(0)$ & $2(0-4)$ & $6(2-15)$ & $9(3-21)$ & $<0.001$ \\
\hline $\begin{array}{l}\text { Morphine dose* Median } \\
\text { (range); } N^{\circ} \text { neonates }\end{array}$ & $0(0) ; 0$ & $0.15(0.13-0.19) ; 4$ & $1.8(0.51-3.9) ; 16$ & $2.93(0.6-10.5) ; 6$ & $<0.001$ \\
\hline $\begin{array}{l}\text { Midazolam dose* Median } \\
\text { (range); } N^{\circ} \text { neonates }\end{array}$ & $0(0) ; 0$ & $1.2(1-3.5) ; 4$ & $2.3(1.5-6.7) ; 7$ & $6.5(3.9-11.7) ; 5$ & $<0.001$ \\
\hline $\begin{array}{l}\text { Dexamethasone* dose Median } \\
\text { (range); } N^{\circ} \text { neonates }\end{array}$ & $0(0) ; 0$ & $0.94(0.8-1.2) ; 3$ & $0.88(0.7-1.2) ; 7$ & $1.2(0.75-2.1) ; 4$ & $<0.001$ \\
\hline $\begin{array}{l}\text { Total skin breaks Median } \\
\text { (range) }\end{array}$ & $1(1-3)$ & $9(5-12)$ & $13(7-19)$ & $28(9-41)$ & $<0.001$ \\
\hline $\begin{array}{l}\text { Endotracheal intubation } \\
N^{\circ} \text { neonates (\%) }\end{array}$ & $0(0 \%)$ & $0(0 \%)$ & $20(100 \%)$ & $6(100 \%)$ & $<0.001$ \\
\hline Surgery $N^{\circ}$ neonates (\%) & $0(0 \%)$ & $0(0 \%)$ & $0(0 \%)$ & $6(100 \%)$ & $<0.001$ \\
\hline
\end{tabular}

TABLE 2 | Clinical outcome at 24 months and NICl score categories.

\begin{tabular}{|c|c|c|c|c|c|c|c|}
\hline \multicolumn{2}{|c|}{$\mathrm{NICl}$ score } & \multicolumn{6}{|c|}{ Griffith's developmental scale scores (mean \pm std.dev) } \\
\hline & & $\begin{array}{l}\text { Developmental } \\
\text { quotient }\end{array}$ & Locomotor & Personal-social & Hearing-speech & Eye-hand coordination & Performance \\
\hline Control group & 0 category $N=13$ & $101.3 \pm 3.1$ & $101.5 \pm 3.2$ & $98.9 \pm 4.2$ & $101.9 \pm 3.6$ & $101.9 \pm 3.7$ & $99.9 \pm 3.1$ \\
\hline \multirow[t]{3}{*}{ IP group } & I category $N=7$ & $98.4 \pm 10.1$ & $99.1 \pm 5.7$ & $90.1 \pm 9.2$ & $94.2 \pm 9.6$ & $91.3 \pm 8.4$ & $94.7 \pm 7.2$ \\
\hline & II category $N=20$ & $90.2 \pm 10.9$ & $93.1 \pm 11.2$ & $87.6 \pm 9.3$ & $94.1 \pm 7.4$ & $90.1 \pm 9.2$ & $92.5 \pm 8.5$ \\
\hline & III category $N=6$ & $87.8 \pm 8.3^{*}$ & $84.5 \pm 5.3^{*}$ & $83.5 \pm 8.3^{*}$ & $86.8 \pm 4.6^{*}$ & $86.7 \pm 4.1^{*}$ & $87.8 \pm 3.1^{*}$ \\
\hline
\end{tabular}

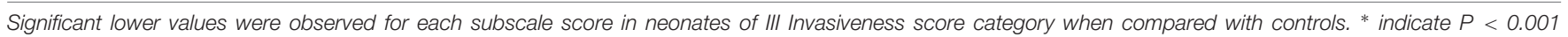

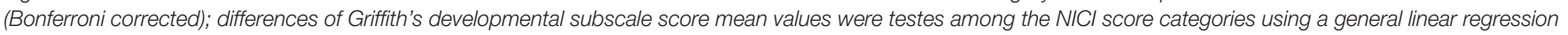

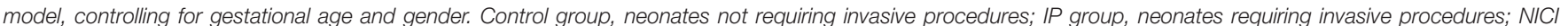

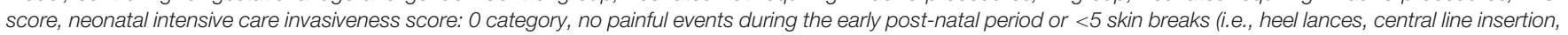

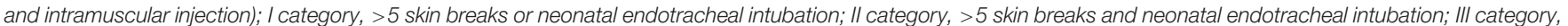
surgical interventions (i.e., patent ductus arteriosus ligation and surgical necrotizing enterocolitis).

patterns in three regions: the right insular cortex (rIC) and right and left thalami (Figure 2). In the post hoc seed correlation analyses, performed using seed regions based on the MVPA results, we found decreased connectivity in the IP group compared to control neonates between both thalami and bilateral somatosensory regions (pre-central and postcentral gyri) (Figure 3), while we found stronger connectivity between right thalamus and the right superior temporal gyrus (Figure 4).
The post hoc seed-to-voxel analysis performed from the seed region of right insular cortex revealed a weaker connectivity with the right amygdala and the right hippocampal and parahippocampal regions (Figure 5 and Table 3).

The correlation analysis between the NICI score and the functional connectivity revealed a significant negative correlation between the NICI score and the connectivity strength in the right thalamus and ipsilateral somatosensory cortex, indicating weaker functional connectivity in thalamocortical circuits in neonates 


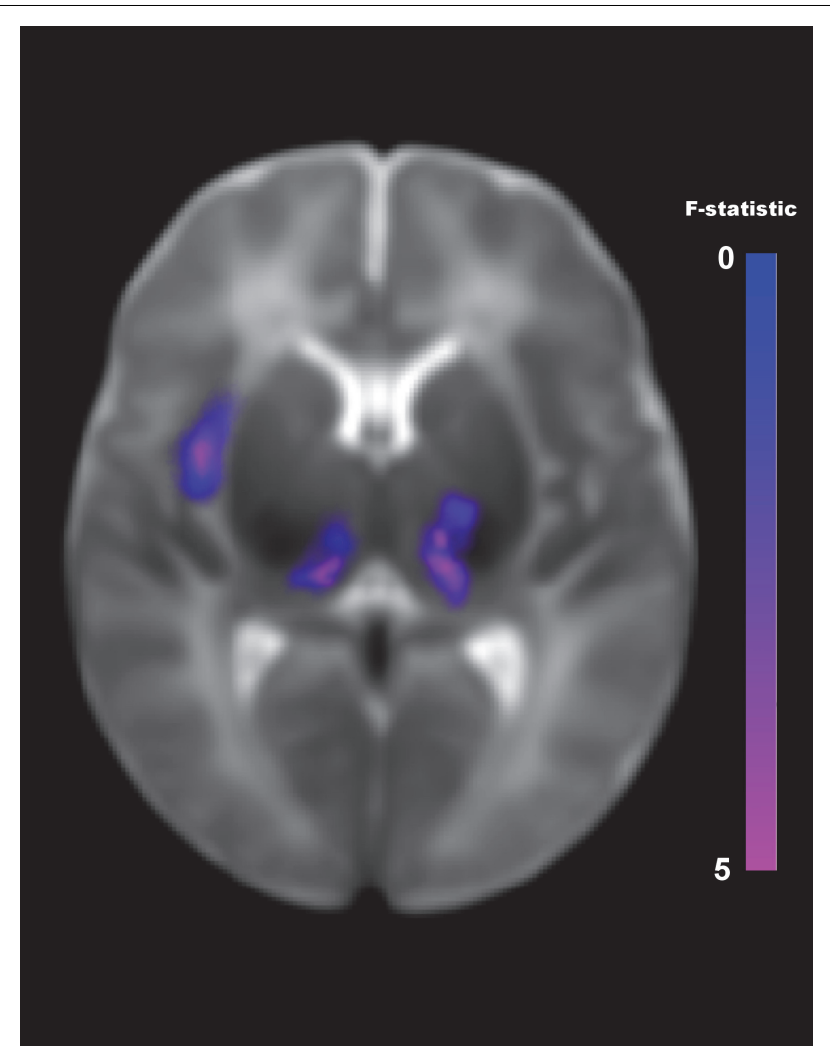

FIGURE 2 | Differences in brain connectivity profile indexed at a voxel level using multivariate pattern analysis (MVPA). MVPA results are overlaid on a representative neonatal axial T2-weighted image. Blue-violet clusters indicates brain regions (thalami and right insular cortex) that show different pattern of functional connectivity between IP neonates and controls. Colorbar indicates F-statistic of between group differences with regard to the spatial maps of the four principal components. Three clusters were identified: in both thalami and in the right insula. The F-maps are threshold voxelwise at a $p<0.001$ FWE-corrected and at $p<0.05$ FWE-corrected at cluster-level.

who experienced a greater number of invasive procedures $(r=-0.651 ; P=0.0003)$.

\section{Functional Brain Connectivity and Neurodevelopmental Outcome}

The exploratory correlation analysis revealed a significant positive correlation between the locomotor subscale scores and the functional connectivity between the right thalamus and ipsilateral somatosensory cortices $(r=0.642 ; P<0.001)$. We also observed a positive correlation between the locomotor score and the functional connectivity between the right insula and the ipsilateral amygdala and hippocampal/parahippocampal areas $(r=0.510 ; P<0.001)$ (Figure 6). No significant correlations were observed between the functional connectivity of these regions and other neurodevelopmental subscale scores, nor between the functional connectivity of the left thalamus and all neurodevelopmental scores.

The general linear regression analyses exploring the effects of functional connectivity of the right thalamus and right insula at

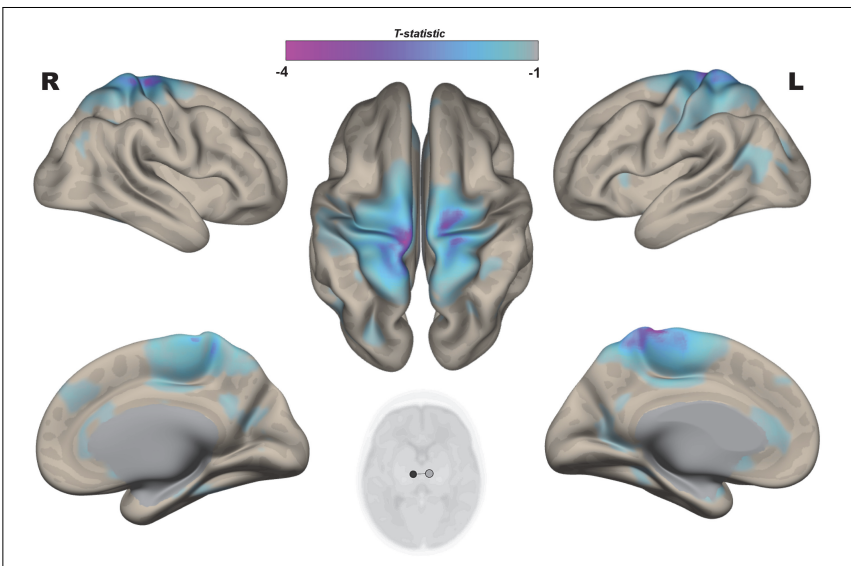

FIGURE 3 | Post hoc Seed-to-Voxel analysis using seed regions at level of thalami at the peak coordinates from MVPA. This analysis identified decreased functional connectivity between thalami and somatosensory areas (blue-violet clusters) in the IP neonates when compared with controls. Colorbar indicates T-statistic of between groups differences (IP neonates < controls), the T-map is thresholded at voxelwise $p<0.001$ FWE-corrected, and $p<0.05$ FWE-corrected clusters level and at voxel level.

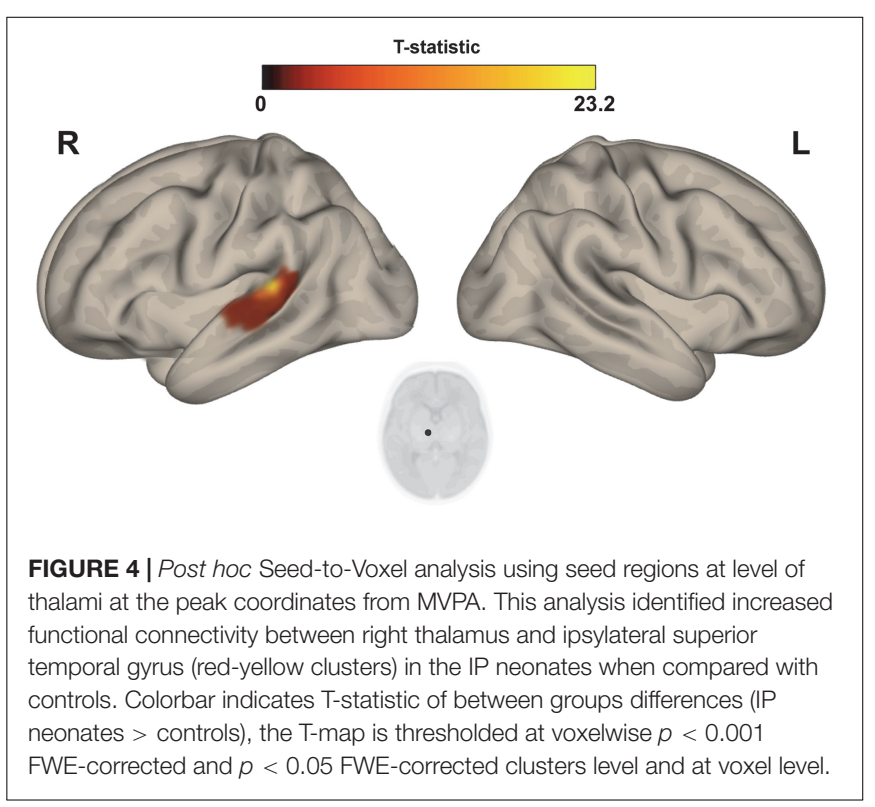

term equivalent age on the locomotor outcome at 24 months of corrected age yielded the following results: the locomotor score was predicted by the functional connectivity of right thalamus and perirolandic cortex $(B=23.506 ; P=0.003)$, while the functional connectivity of the right insula was not a significant predictor of the locomotor outcome $(ß=15.785 ; P=0.085)$.

\section{DISCUSSION}

This fMRI study unravels altered brain functional connectivity at term equivalent age in preterm neonates who experienced significant painful events during neonatal intensive care, and demonstrates a relationship of these fMRI abnormalities with the 


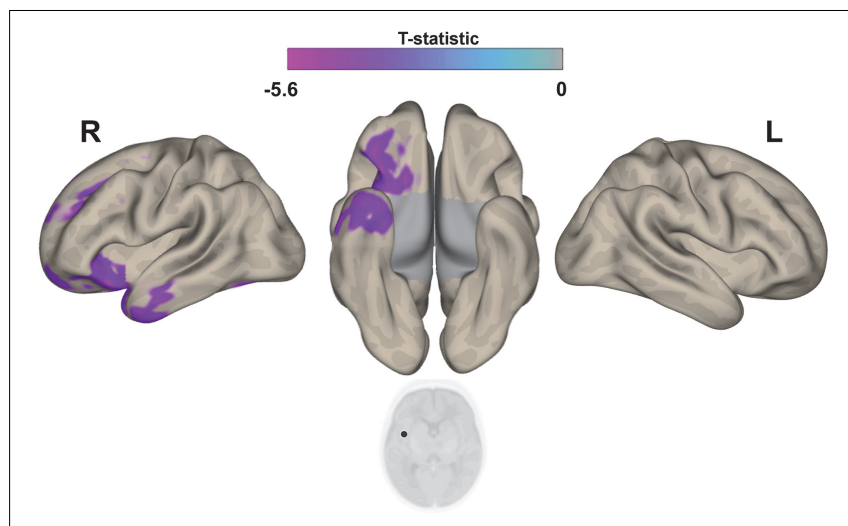

FIGURE 5 | Post hoc Seed-to-Voxel analysis using seed regions at level of right insular cortex at the peak coordinates from MVPA. This analysis identified decreased functional connectivity between right insular cortex and ipsilateral amygdala/hyppocampus (blue-violet clusters) in the IP neonates when compared with controls. Colorbar indicates T-statistic of between groups differences (IP neonates < controls), the T-map is thresholded at voxelwise $p<0.001$ FWE-corrected and $p<0.05$ FWE-corrected clusters level and at voxel level.

motor outcome at 24 months of corrected age. In particular, in these neonates we demonstrated weaker functional connectivity in the thalamocortical pathways and between the right insula and limbic system. Of note, the thalamic nuclei, thalamocortical pathways and limbic structures play a crucial role in encoding noxious stimuli (Caulo et al., 2019). More in detail, the thalamus receives nociceptive signals via two major ascending pathways: the spinothalamic and spino-reticulo-thalamic tracts. The spinothalamic tract conveys information about noxious stimuli from the dorsal horn and spinal trigeminal nucleus to both the lateral and medial thalamus. In contrast, the spino-reticulo-thalamic tract is thought to carry nociceptive information specifically to the medial thalamus via an additional synaptic relay within the medullary reticular formation of the brainstem (Gauriau and Bernard, 2002). These thalamic regions innervate specific sets of cortical target areas, including the somatosensory and limbic cortices (Groh et al., 2018), with different functions of thalamocortical pathways in the regulation of both sensory-discriminative and emotional aspects of pain stimuli. The transmission of pain impulses to cerebral cortex and subcortical gray matters represents an important requirement for the descending modulation of the spinal response to painful events (Groh et al., 2018). Of note, this nociceptive circuitry is functionally immature in neonates, especially during the early post-natal period (Fitzgerald, 2005).

Due to the immaturity of the descending modulation of nociceptive activity (Hatfield, 2014), preterm neonates may demonstrate central sensitization to repeated painful stimuli, which may in turn alter the brain microstructure and production of stress hormones, with poorer cognitive, motor, and behavioral neurodevelopment (Fitzgerald et al., 1989; Walker et al., 2009b).

Interestingly, previous studies examining the effects of early exposition to painful events on brain development in preterm neonates showed that neonatal pain was associated with decreased brain volume particularly impacting frontal and parietal lobes at term equivalent age (Smith et al., 2011; Brummelte et al., 2012). More recently, Duerden et al. demonstrated that also subcortical structures may be vulnerable to procedural pain corroborating the original finding of impaired white matter and subcortical gray matter with early pain exposure of premature babies. In particular, they found that preterm neonates requiring invasive procedures during the early postnatal period showed decreased thalamic NAA/Cho and microstructural alterations in thalamocortical pathways. Remarkably, these structural and metabolic thalamic abnormalities correlated with poorer cognitive and motor outcomes (Duerden et al., 2018).

Our findings confirm the association between early invasive procedures and altered development of the thalamus and thalamocortical pathways in preterm neonates. Indeed, we speculate that weaker functional connectivity observed in IP neonates may derive from the impaired development of thalami and thalamocortical connections during the early postnatal period of very premature babies. Of note, during brain development, maturation and topographic organization of afferent thalamocortical projections occur through an activitydependent manner (Shatz, 1990; Goodman and Shatz, 1993), with axons from the thalamus contending for representation in the cortex in response to both external stimuli and endogenous NMDA-dependent mechanisms (Crair and Malenka, 1995). Abnormal amplified external stimuli related to pain may disrupt the activity of this pathway during early brain development, and may determine abnormal distribution of thalamocortical projections and atypical somatosensory cortex development.

Recently, it has been demonstrated that early procedural pain induces a greater release of glutamate, since NMDA receptors involved in the transmission of pain signals are more active during early life (Vinall and Grunau, 2014). Excessive release of glutamate may thus determine further trigger to both oxidative stress and inflammatory reactions in the premature neonatal brain (Panfoli et al., 2018), that may arrest the development of subplate neurons and preoligodendrocytes, which are particularly vulnerable to reactive oxygen, nitrogen species, and cytokines secreted by microglia (Back et al., 1998; Haynes et al., 2003; Slater et al., 2012; Panfoli et al., 2018; Tortora et al., 2018a,b). Taken together, these data suggest that repeated exposure to neonatal procedural pain is associated with alterations of both neuronal structure and function with potential relevant consequences onto neurodevelopmental outcome (Boardman et al., 2010; Chau et al., 2013; Duerden et al., 2018).

Another important result of our study was the weaker functional connectivity between the insular cortex and the limbic structures (i.e., amygdala and hyppocampus) in IP neonates. Interestingly, the limbic system and hypothalamus, together with several other cortical and subcortical brain regions, are fundamental in creating a descending feedback loop that modulates spinal dorsal horn activity in response to external stimuli (Beggs, 2015). In particular, the segregation of afferent inputs to the spinal cord from the peripheral central nervous system allows to distinguish sensory information as tactile, thermal, or nociceptive. This process occurs postnatally and 
TABLE 3 | Group differences in functional connectivity.

\begin{tabular}{|c|c|c|c|c|}
\hline Contrast & Seed (center of sphere) & Target (peak coordinates) & Cluster size (\# voxels) & Cluster p-FWE \\
\hline \multirow[t]{5}{*}{ Controls > IP } & Left thalamus $(-7,-15,2)$ & Left Somatosensory $(-4,-27,34)$ & 1873 & $<0.000001$ \\
\hline & & Right Somatosensory $(6,-25,42)$ & 973 & 0.00006 \\
\hline & Right thalamus $(8,-18,5)$ & Right Somatosensory $(5,-26,37)$ & 2373 & $<0.000001$ \\
\hline & & Left Somatosensory $(-11,-26,42)$ & 665 & 0.00008 \\
\hline & Right insula $(26,-2,4)$ & Right amygdala/hippocampus $(14,-10,15)$ & 871 & 0.00007 \\
\hline $\mathrm{IP}>$ Controls & Right thalamus $(8,-18,5)$ & Right superior temporal gyrus $(40,-19,3)$ & 672 & 0.00027 \\
\hline
\end{tabular}

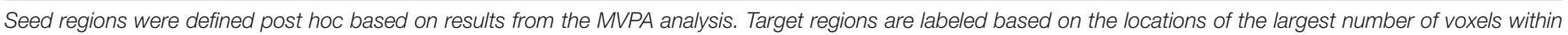
significant cluster. All results are significant on a corrected cluster level ( $p<0.0005$ FDR). Controls, non-invasive procedure group; IP, invasive procedure group.

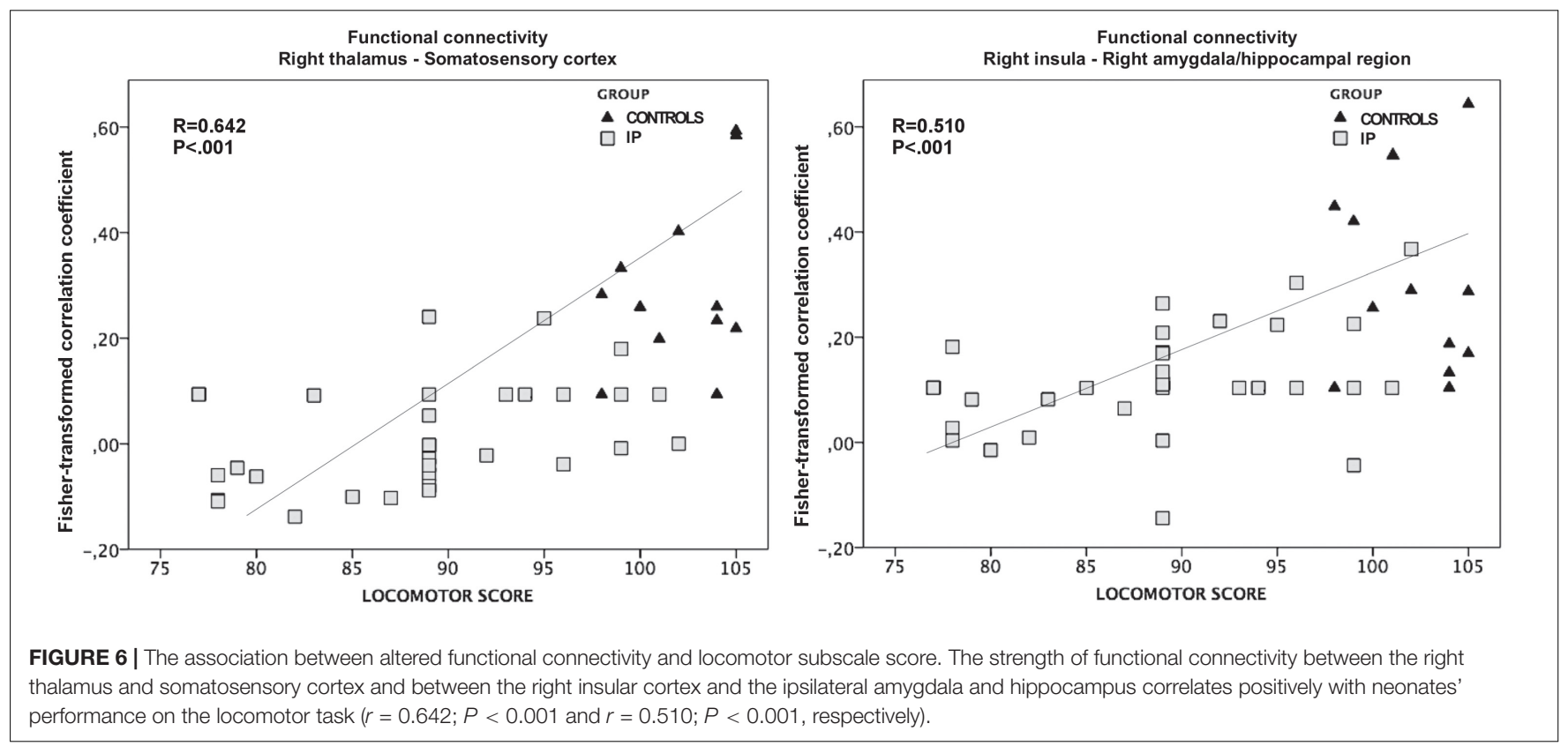

requires several modulation processes at different levels of the central nervous system. The dorsal horn of the spinal cord is the first point of modulation of sensory information from the periphery, acting as a site of complex integration of sensorial information. In addition to this local circuitry, brain modulatory networks including the limbic system and hypothalamus regulate the spinal activity through both facilitatory and inhibitory inputs (Beggs, 2015). In early postnatal life, these descending pathways are almost exclusively facilitatory and targeted to low-threshold (tactile) input, promoting an ongoing activitydependent maturation of dorsal horn nociceptive circuitry. On the other hand, the descending inhibitory control, which is pivotal for modulating pain perception being selectively targeted to high-threshold (painful) stimuli, develops later (Koch and Fitzgerald, 2014). Here, we postulate that the lower functional connectivity observed between the insular cortex and limbic system in IP neonates may reflect abnormal maturation of these cortical networks due to the exposure to repeated painful stimuli during the early postnatal period, particularly in the premature brains.

Finally, fMRI analysis demonstrated a relationship between connectivity alterations of the thalamus and insular cortex and specific neurodevelopmental impairments at 24 months of age. In particular, weaker intrinsic functional connectivity of the thalamocortical pathways, and the insular cortex-limbic connections was associated with poorer locomotor outcome. This finding is corroborated by prior reports demonstrating that preterm neonates exposed to early postnatal painful events showed macro- and microstructural alterations in thalamocortical projections and other cortical regions (i.e., frontal and parietal cortex) that were associated with adverse motor and cognitive neurodevelopmental outcome in the first years of life, involving also the mechanisms of anxiety and emotion regulation (Duerden et al., 2015; Tortora et al., 2015; Navarra et al., 2016; Malavolti et al., 2017; Filippa et al., 2019). Moreover, Duerden et al. (2018) demonstrated that early pain exposure can influence thalamocortical pathways maturation, especially in extremely preterm born neonates, and that the thalamic volume growth during the first weeks of life correlated with cognitive and motor scores at 36 months of age. Intriguingly, we found that functional connectivity of thalamocortical pathways remains the single factor able to predict the locomotor outcome at 24 months of age after controlling for the confounding effects of the clinical factors, thus supporting 
the important role of thalami in the development of motor functions in preterm neonates (Lao et al., 2016). Further studies on cognitive functions and emotional processing of preterm neonates studied at school age are necessary to elucidate whether these functional connectivity changes may impact also on academic or social/behavioral performances.

\section{LIMITATIONS}

Limitations of this study include the relatively small sample size, albeit justified by the small incidence of normal MRI examination in very early preterm neonates. Moreover the short neurodevelopmental follow-up performed at 24 months of corrected age, the lack of information about the socioeconomic status and educational level of parents, and the use of a limited numbers of clinical confounders in the fMRI analysis could represent a source of bias. In addition, the difficulty of distinguishing between direct effects of exposure to painful stimuli and the confounding clinical and demographic variables may play a role. In this study, we considered the confounding effects of gestational age, gender, age at MRI scan and the length of stay in the neonatal intensive care unit. Moreover, we included in the analysis several clinical factors, such as total morphine and days of intubation, which may have influenced functional brain development, the degree of neuroinflammation, and clinical outcome. Finally, we selected only patients with normal brain MRI to avoid the confounding effect of brain lesions on functional connectivity.

\section{CONCLUSION}

In summary, our findings suggest that early exposure to pain is associated with abnormal functional connectivity of developing networks involved in the modulation of noxious stimuli in preterm neonates, contributing to the neurodevelopmental consequence of preterm birth. Future follow-up studies are needed to determine how the consequences of early painful events may affect the late developmental outcome within groups

\section{REFERENCES}

Back, S. A., Gan, X., Li, Y., Rosenberg, P. A., and Volpe, J. J. (1998). Maturationdependent vulnerability of oligodendrocytes to oxidative stress-induced death caused by glutathione depletion. J. Neurosci. 18, 6241-6253. doi: 10.1523/ jneurosci.18-16-06241.1998

Ball, G., Aljabar, P., Arichi, T., Tusor, N., Cox, D., Merchant, N., et al. (2016). Machine-learning to characterise neonatal functional connectivity in the preterm brain. Neuroimage 124, 267-275. doi: 10.1016/j.neuroimage.2015. 08.055

Beggs, S. (2015). Long-term consequences of neonatal injury. Can. J. Psychiatry 60, 176-180. doi: 10.1177/070674371506000404

Behzadi, Y., Restom, K., Liau, J., and Liu, T. T. (2007). A component based noise correction method (CompCor) for BOLD asnd perfusion based fMRI. Neuroimage 37, 90-101. doi: 10.1016/j.neuroimage.2007.04.042

Boardman, J. P., Craven, C., Valappil, S., Counsell, S. J., Dyet, L. E., Rueckert, D., et al. (2010). A common neonatal image phenotype predicts adverse of babies of the same gestational age and how it can be mitigated by appropriate pharmacological analgesia or by other positive systematic sensorial stimulation known to reduce nociceptive reaction of preterm neonates (Ramenghi et al., 1999).

\section{DATA AVAILABILITY}

The raw data supporting the conclusions of this manuscript will be made available by the authors, without undue reservation, to any qualified researcher.

\section{ETHICS STATEMENT}

The local ethics committee (Comitato Etico Regione Liguria, Genoa, Italy) approved this study and parents provided written informed consent in accordance with the Declaration of Helsinki.

\section{AUTHOR CONTRIBUTIONS}

DT analyzed and interpreted the data, and drafted the manuscript. MS, MM, CD, AP, DM, CT, SU, LB, and GM collected the data and revised the manuscript. AR and LR designed the study, interpreted the data, and revised the manuscript. All authors discussed the results and commented on the manuscript.

\section{FUNDING}

This work was supported by funds from the Current Research for the 2018-2020 triennium - Ministry of Health - Italy and Eu-Brain Non-profit Association, Genoa, Italy.

\section{SUPPLEMENTARY MATERIAL}

The Supplementary Material for this article can be found online at: https://www.frontiersin.org/articles/10.3389/fnins. 2019.00899/full\#supplementary-material

neurodevelopmental outcome in children born preterm. Neuroimage 52, 409414. doi: 10.1016/j.neuroimage.2010.04.261

Brummelte, S., Chau, C. M. Y., Cepeda, I. L., Degenhardt, A., Weinberg, J., Synnes, A. R., et al. (2015). Cortisol levels in former preterm children at school age are predicted by neonatal procedural pain-related stress. Psychoneuroendocrinology 51, 151-163. doi: 10.1016/j.psyneuen.2014. 09.018

Brummelte, S., Grunau, R. E., Chau, V., Poskitt, K. J., Brant, R., Vinall, J., et al. (2012). Procedural pain and brain development in premature newborns. Ann. Neurol. 71, 385-396. doi: 10.1002/ana.22267

Carbajal, R., Nguyen-Bourgain, C., and Armengaud, J.-B. (2008). How can we improve pain relief in neonates? Expert Rev. Neurother. 8, 1617-1620. doi: 10.1586/14737175.8.11.1617

Caulo, M., Maruotti, V., and Ferretti, A. (2019). "Brain Imaging of Pain," in Pain Imaging. Cham: Springer International Publishing, 7-22.

Chau, V., Synnes, A., Grunau, R. E., Poskitt, K. J., Brant, R., and Miller, S. P. (2013). Abnormal brain maturation in preterm neonates associated with 
adverse developmental outcomes. Neurology 81, 2082-2089. doi: 10.1212/01. wnl.0000437298.43688.b9

Crair, M. C., and Malenka, R. C. (1995). A critical period for long-term potentiation at thalamocortical synapses. Nature 375, 325-328. doi: 10.1038/375 $325 \mathrm{a} 0$

Duerden, E. G., Foong, J., Chau, V., Branson, H., Poskitt, K. J., Grunau, R. E., et al. (2015). Tract-based spatial statistics in preterm-born neonates predicts cognitive and motor outcomes at 18 months. Am. J. Neuroradiol. 36, 1565-1571. doi: 10.3174/ajnr.A4312

Duerden, E. G., Grunau, R. E., Guo, T., Foong, J., Pearson, A., Au-Young, S., et al. (2018). Early procedural pain is associated with regionally-specific alterations in thalamic development in preterm neonates. J. Neurosci. 38, 878-886. doi: 10.1523/JNEUROSCI.0867-17.2017

Duerden, E. G., Halani, S., Ng, K., Guo, T., Foong, J., Glass, T. J. A., et al. (2019). White matter injury predicts disrupted functional connectivity and microstructure in very preterm born neonates. Neuro Image. Clin. 21:101596. doi: 10.1016/j.nicl.2018.11.006

Filippa, M., Poisbeau, P., Mairesse, J., Monaci, M. G., Baud, O., Hüppi, P., et al. (2019). Pain, parental involvement, and oxytocin in the neonatal intensive care unit. Front. Psychol. 10:715. doi: 10.3389/fpsyg.2019.00715

Fitzgerald, M. (2005). The development of nociceptive circuits. Nat. Rev. Neurosci. 6, 507-520. doi: 10.1038/nrn1701

Fitzgerald, M., Millard, C., and McIntosh, N. (1989). Cutaneous hypersensitivity following peripheral tissue damage in newborn infants and its reversal with topical anaesthesia. Pain 39, 31-36. doi: 10.1016/0304-3959(89)90 $172-3$

Gauriau, C., and Bernard, J.-F. (2002). Pain pathways and parabrachial circuits in the rat. Exp. Physiol. 87, 251-258. doi: 10.1113/eph87 02357

Goffaux, P., Lafrenaye, S., Morin, M., Patural, H., Demers, G., and Marchand, S. (2008). Preterm births: can neonatal pain alter the development of endogenous gating systems? Eur. J. Pain 12, 945-951. doi: 10.1016/j.ejpain.2008.01.003

Goodman, C. S., and Shatz, C. J. (1993). Developmental mechanisms that generate precise patterns of neuronal connectivity. Cell 72(Suppl.)), 77-98. doi: 10.1016/ s0092-8674(05)80030-3

Griffiths, R., Battaglia, F., Savoini, M., and Huntley, M. (2007). GMDSR: Griffiths Mental Development Scales, Revised: O-2 Anni: Manuale. Available at: https://scholar.google.com/scholar_lookup?title=GMDSRGriffithsmentaldevelopmentscales-revised0--2Anni\&author=R.Griffiths\& author=M.Huntley\&publication_year=2007 (accessed February 23, 2019).

Groh, A., Krieger, P., Mease, R. A., and Henderson, L. (2018). Acute and chronic pain processing in the thalamocortical system of humans and animal models. Neuroscience 387, 58-71. doi: 10.1016/j.neuroscience.2017. 09.042

Grunau, R. E., Whitfield, M. F., Petrie-Thomas, J., Synnes, A. R., Cepeda, I. L., Keidar, A., et al. (2009). Neonatal pain, parenting stress and interaction, in relation to cognitive and motor development at 8 and 18 months in preterm infants. Pain 143, 138-146. doi: 10.1016/j.pain.2009.02.014

Grunau, R. V., Whitfield, M. F., Petrie, J. H., and Fryer, E. L. (1994). Early pain experience, child and family factors, as precursors of somatization: a prospective study of extremely premature and fullterm children. Pain 56, 353-359. doi: 10.1016/0304-3959(94)90174-0

Hatfield, L. A. (2014). Neonatal pain: what's age got to do with it? Surg. Neurol. Int. 5, S479-S489. doi: 10.4103/2152-7806.144630

Haynes, R. L., Folkerth, R. D., Keefe, R. J., Sung, I., Swzeda, L. I., Rosenberg, P. A., et al. (2003). Nitrosative and oxidative injury to premyelinating oligodendrocytes in periventricular leukomalacia. J. Neuropathol. Exp. Neurol. 62, 441-450. doi: 10.1093/jnen/62.5.441

Ismail, F. Y., Fatemi, A., and Johnston, M. V. (2017). Cerebral plasticity: windows of opportunity in the developing brain. Eur. J. Paediatr. Neurol. 21, 23-48. doi: 10.1016/j.ejpn.2016.07.007

Koch, S. C., and Fitzgerald, M. (2014). The selectivity of rostroventral medulla descending control of spinal sensory inputs shifts postnatally from A fibre to C fibre evoked activity. J. Physiol. 592, 1535-1544. doi: 10.1113/jphysiol.2013. 267518

Kuklisova-Murgasova, M., Aljabar, P., Srinivasan, L., Counsell, S. J., Doria, V., Serag, A., et al. (2011). A dynamic 4D probabilistic atlas of the developing brain. Neuroimage 54, 2750-2763. doi: 10.1016/j.neuroimage.2010.10.019
Lao, Y., Wang, Y., Shi, J., Ceschin, R., Nelson, M. D., Panigrahy, A., et al. (2016). Thalamic alterations in preterm neonates and their relation to ventral striatum disturbances revealed by a combined shape and pose analysis. Brain Struct. Funct. 221, 487-506. doi: 10.1007/s00429-014-0921-7

Lea, C. L., Smith-Collins, A., and Luyt, K. (2017). Protecting the premature brain: current evidence-based strategies for minimising perinatal brain injury in preterm infants. Arch. Dis. Child. Fetal Neonatal Ed. 102, F176-F182. doi: 10.1136/archdischild-2016-311949

Malavolti, A. M., Chau, V., Brown-Lum, M., Poskitt, K. J., Brant, R., Synnes, A., et al. (2017). Association between corpus callosum development on magnetic resonance imaging and diffusion tensor imaging, and neurodevelopmental outcome in neonates born very preterm. Dev. Med. Child Neurol. 59, 433-440. doi: $10.1111 / \mathrm{dmcn} .13364$

Navarra, R., Sestieri, C., Conte, E., Salomone, R., Mattei, P. A., Romani, G. L., et al. (2016). Perinatal MRI diffusivity is related to early assessment of motor performance in preterm neonates. Neuroradiol. J. 29, 137-145. doi: 10.1177/ 1971400915628019

Panfoli, I., Candiano, G., Malova, M., De Angelis, L., Cardiello, V., Buonocore, G., et al. (2018). Oxidative stress as a primary risk factor for brain damage in preterm newborns. Front. Pediatr. 6:369. doi: 10.3389/fped.2018.00369

Ramenghi, L. A., Evans, D. J., and Levene, M. I. (1999). Quot;Sucrose analgesia;: absorptive mechanism or taste perception? Arch. Dis. Child. Fetal Neonatal Ed. 80, F146-F147.

Ranger, M., Chau, C. M. Y., Garg, A., Woodward, T. S., Beg, M. F., Bjornson, B., et al. (2013). Neonatal pain-related stress predicts cortical thickness at age 7 years in children born very preterm. PLoS One 8:e76702. doi: 10.1371/journal. pone. 0076702

Scheinost, D., Kwon, S. H., Shen, X., Lacadie, C., Schneider, K. C., Dai, F., et al. (2016). Preterm birth alters neonatal, functional rich club organization. Brain Struct. Funct. 221, 3211-3222. doi: 10.1007/s00429-0151096-6

Shatz, C. J. (1990). Impulse activity and the patterning of connections during CNS development. Neuron 5, 745-756. doi: 10.1016/0896-6273(90)90333-b

Shi, F., Yap, P.-T., Wu, G., Jia, H., Gilmore, J. H., Lin, W., et al. (2011). Infant brain atlases from neonates to 1- and 2-year-olds. PLoS One 6:e18746. doi: 10.1371/journal.pone.0018746

Slater, L., Asmerom, Y., Boskovic, D. S., Bahjri, K., Plank, M. S., Angeles, K. R., et al. (2012). Procedural pain and oxidative stress in premature neonates. J. Pain 13, 590-597. doi: 10.1016/j.jpain.2012.03.010

Smith, G. C., Gutovich, J., Smyser, C., Pineda, R., Newnham, C., Tjoeng, T. H., et al. (2011). Neonatal intensive care unit stress is associated with brain development in preterm infants. Ann. Neurol. 70, 541-549. doi: 10.1002/ana. 22545

Thompson, W. H., Thelin, E. P., Lilja, A., Bellander, B.-M., and Fransson, P. (2016). Functional resting-state fMRI connectivity correlates with serum levels of the S100B protein in the acute phase of traumatic brain injury. Neuro Image. Clin. 12, 1004-1012. doi: 10.1016/j.nicl.2016. 05.005

Tortora, D., Martinetti, C., Severino, M., Uccella, S., Malova, M., Parodi, A., et al. (2018a). The effects of mild germinal matrix-intraventricular haemorrhage on the developmental white matter microstructure of preterm neonates: a DTI study. Eur. Radiol. 28, 1157-1166. doi: 10.1007/s00330-0175060-0

Tortora, D., Severino, M., Sedlacik, J., Toselli, B., Malova, M., Parodi, A., et al. (2018b). Quantitative susceptibility map analysis in preterm neonates with germinal matrix-intraventricular hemorrhage. J. Magn. Reson. Imaging 48, 1199-1207. doi: 10.1002/jmri.26163

Tortora, D., Panara, V., Mattei, P. A., Tartaro, A., Salomone, R., Domizio, S., et al. (2015). Comparing 3T T1-weighted sequences in identifying hyperintense punctate lesions in preterm neonates. Am. J. Neuroradiol. 36, 581-586. doi: 10.3174/ajnr.A4144

Vinall, J., and Grunau, R. E. (2014). Impact of repeated procedural pain-related stress in infants born very preterm. Pediatr. Res. 75, 584-587. doi: 10.1038/pr. 2014.16

Vinall, J., Miller, S. P., Bjornson, B. H., Fitzpatrick, K. P. V., Poskitt, K. J., Brant, R., et al. (2014). invasive procedures in preterm children: brain and cognitive development at school age. Pediatrics 133, 412-421. doi: 10.1542/peds.20131863 
Walker, S. M., Franck, L. S., Fitzgerald, M., Myles, J., Stocks, J., and Marlow, N. (2009a). Long-term impact of neonatal intensive care and surgery on somatosensory perception in children born extremely preterm. Pain 141, 79-87. doi: $10.1016 /$ j.pain.2008.10.012

Walker, S. M., Tochiki, K. K., and Fitzgerald, M. (2009b). Hindpaw incision in early life increases the hyperalgesic response to repeat surgical injury: critical period and dependence on initial afferent activity. Pain 147, 99-106. doi: 10.1016/j. pain.2009.08.017

Whitfield-Gabrieli, S., and Nieto-Castanon, A. (2012). Conn: a functional connectivity toolbox for correlated and anticorrelated brain networks. Brain Connect. 2, 125-141. doi: 10.1089/brain.2012.0073
Conflict of Interest Statement: The authors declare that the research was conducted in the absence of any commercial or financial relationships that could be construed as a potential conflict of interest.

Copyright (c) 2019 Tortora, Severino, Di Biase, Malova, Parodi, Minghetti, Traggiai, Uccella, Boeri, Morana, Rossi and Ramenghi. This is an open-access article distributed under the terms of the Creative Commons Attribution License (CC BY). The use, distribution or reproduction in other forums is permitted, provided the original author(s) and the copyright owner(s) are credited and that the original publication in this journal is cited, in accordance with accepted academic practice. No use, distribution or reproduction is permitted which does not comply with these terms. 
OPEN ACCESS

Edited by:

Filippo Cieri,

Cleveland Clinic, United States

Reviewed by:

Arun Bokde,

Trinity College Dublin, Ireland

Jianhui Zhong,

University of Rochester, United States

${ }^{*}$ Correspondence:

Abbas Babajani-Feremi ababajan@uthsc.edu

${ }^{\dagger}$ Data used in preparation of this article were obtained from the

Alzheimer's Disease Neuroimaging Initiative (ADNI) database

(adni.loni.usc.edu). As such, the

investigators within the ADNI

contributed to the design and

implementation of ADNI and/or provided data but did not participate in analysis or writing of this report. A complete listing of $A D N I$ investigators can be found at: http://adni./oni.usc. edu/wp-content/uploads/ how_to_apply/

ADNI_Acknowledgment_List.pdf

Specialty section:

This article was submitted to Applied Neuroimaging,

a section of the journal

Frontiers in Neurology

Received: 04 April 2019 Accepted: 05 August 2019

Published: 30 August 2019

Citation:

Hojjati SH, Ebrahimzadeh A and

Babajani-Feremi A (2019) Identification of the Early Stage of Alzheimer's Disease Using Structural

MRI and Resting-State fMRI.

Front. Neurol. 10:904

doi: 10.3389/fneur.2019.00904

\section{Identification of the Early Stage of Alzheimer's Disease Using Structural MRI and Resting-State fMRI}

\author{
Seyed Hani Hojjati ${ }^{1,2,3}$, Ata Ebrahimzadeh ${ }^{2}$ and Abbas Babajani-Feremi ${ }^{1,3,4 *}$ \\ for the Alzheimer's Disease Neuroimaging Initiative ${ }^{\dagger}$
}

\begin{abstract}
${ }^{1}$ Department of Pediatrics, University of Tennessee Health Science Center, Memphis, TN, United States, ${ }^{2}$ Department of Electrical Engineering, Babol University of Technology, Babol, Iran, ${ }^{3}$ Neuroscience Institute and Children's Foundation Research Institute, Le Bonheur Children's Hospital, Memphis, TN, United States, ${ }^{4}$ Department of Anatomy and Neurobiology, University of Tennessee Health Science Center, Memphis, TN, United States
\end{abstract}

Accurate prediction of the early stage of Alzheimer's disease (AD) is important but very challenging. The goal of this study was to utilize predictors for diagnosis conversion to AD based on integrating resting-state functional MRI (rs-fMRI) connectivity analysis and structural MRI (sMRI). We included 177 subjects in this study and aimed at identifying patients with mild cognitive impairment $(\mathrm{MCl})$ who progress to $A D, M C l$ converter ( $\mathrm{MCl}-\mathrm{C})$, patients with $\mathrm{MCl}$ who do not progress to $\mathrm{AD}, \mathrm{MCl}$ non-converter (MCl-NC), patients with $A D$, and healthy controls $(\mathrm{HC})$. The graph theory was used to characterize different aspects of the rs-fMRI brain network by calculating measures of integration and segregation. The cortical and subcortical measurements, e.g., cortical thickness, were extracted from sMRI data. The rs-fMRI graph measures were combined with the sMRI measures to construct input features of a support vector machine (SVM) and classify different groups of subjects. Two feature selection algorithms [i.e., the discriminant correlation analysis (DCA) and sequential feature collection (SFC)] were used for feature reduction and selecting a subset of optimal features. Maximum accuracy of 67 and $56 \%$ for three-group ("AD, MCl-C, and $\mathrm{MCl}-\mathrm{NC}$ " or "MCl-C, MCl-NC, and $\mathrm{HC}$ ") and four-group ("AD, MCl-C, MCl-NC, and HC") classification, respectively, were obtained with the SFC feature selection algorithm. We also identified hub nodes in the rs-fMRI brain network which were associated with the early stage of $A D$. Our results demonstrated the potential of the proposed method based on integration of the functional and structural MRI for identification of the early stage of $A D$.

Keywords: Alzheimer's disease (AD), mild cognitive impairment (MCI), resting-state fMRI, graph theory, machine learning, hub nodes

\section{INTRODUCTION}

Alzheimer's disease $(\mathrm{AD})$ is a neurodegenerative disorder, known as a disconnection syndrome that disturbs communication between different brain regions (1). It implies that the brain network is changed during the transition from healthy condition to mild cognitive impairment (MCI) and AD. Since intervention prior to the occurrence of overt and irreversible neuronal loss is critical for the maintenance of normal brain function, prediction of $\mathrm{MCI}$, and conversion to $\mathrm{AD}$ in cognitively normal older adults is a priority for $\mathrm{AD}$ research. 
Approximately 15\% of adults older than 65 years old suffer from $\mathrm{MCI}$ and from these more than half progress to $\mathrm{AD}$ within 5 years (2). Prediction of early stage of $\mathrm{AD}$ is important and several studies have focused on investigating this prediction. Structural magnetic resonance imaging (sMRI) can be utilized to reliably characterize brain volumes, areas, cortical thickness, and curvature (3), and has been widely used to investigate alteration of these brain measures in transition from normal aging to AD. Patients with AD have diminished memory and executive function. Patients with early stage $\mathrm{AD}$ can have MCI, and although impaired, may perform similarly to normal older adults on easier memory tasks. Prognostic predictions of subjects from prodromal stages such as MCI is an area of great clinical interest $(4,5)$. However, prediction of symptomatic progression remains a relatively unexplored task. Memory impairment and dementia are common in the elderly population. Prognostic forecasting of symptom severity is complicated not only by the heterogeneity in demographics and clinical presentation, but also highly variable and non-linear symptom patterns exhibited in those suffering from $\mathrm{MCI}(6,7)$. In a recent study, Eskildsen et al. used patterns of cortical thickness and identified cortical regions potentially discriminative for separating MCI patients into converters (MCI$\mathrm{C}$ ), who received a diagnosis of $\mathrm{AD}$ dementia within 2 years, and non-converters (MCI-NC), who remained stable for 3 years (8). They reported promising results for the prediction of patients with prodromal $\mathrm{AD}$ progressing to probable $\mathrm{AD}$. Eskildsen et al. considered "time to conversion" and separated patients in different groups based on this time, and reported $<80 \%$ accuracy for predicting conversion to AD. Beheshti et al. utilized a voxel based morphometric technique to extract the global and local gray matter volumes, and then used these volumes to classify AD and healthy controls (HC) (9).

Several studies have investigated AD induced alterations of the brain network using the resting-state functional MRI (rsfMRI) (10-16). rs-fMRI has been shown to be a powerful tool for identifying the pathophysiology of functional connectivity not only in patients with $\mathrm{AD}$ but also in patients with other neurological or neuropsychiatric conditions (17). Accumulating evidence suggests that intrinsic connectivity at rest provides the communication channels of task information (18). rs-fMRI networks have been shown to be highly sensitive to AD (19). We and other investigators have reported the ability of rs-fMRI in identification of patients with MCI and AD $(13-15,20)$. We demonstrated potential of rs-fMRI in prediction of the early stage of AD (10). Grieder et al. suggested that cognitive decrease symptoms in $\mathrm{AD}$ is directly related to reduction of complexity in the brain network (21). It was reported that rs-fMRI functional connectivity can show AD-related cognitive impairment in an aging population with health, MCI, and AD (16).

Neuroimaging modalities, such as positron emission tomography (PET), diffusion-tensor imaging (DTI), rs-fMRI, and sMRI, have been found informative in providing biomarkers of conversion from MCI to AD (22-27). While most of previous studies considered a single modality approach for diagnosis of $\mathrm{AD}$ (28), it is expected that a multi-modal approach can improve accuracy of prediction of conversion to AD compared to a single-modality approach (29). Tong et al. utilized features extracted from sMRI, PET, cerebrospinal fluid (CSF) biomarkers, and categorical genetic information, and classify $\mathrm{HC}, \mathrm{MCI}$ and AD with an accuracy of 60.2\% (29). Peng et al. developed a kernel-learning-based method to combine sMRI, PET, and genetic information for $\mathrm{AD}$ and $\mathrm{MCI}$ diagnosis, and reported classification accuracies of $96.1 \%, 80.3 \%$, and $76.9 \%$ for $\mathrm{AD}$ vs. HC, MCI vs. HC, and AD vs. MCI, respectively (30). Ahmed et al. combined DTI with sMRI to improve accuracy of AD, MCI and $\mathrm{HC}$ classification, and obtained an accuracy between $76 \%$ (AD vs. $\mathrm{MCI}$ ) to $90 \%$ (AD vs. $\mathrm{HC}$ ) for two-group classification. Dyrba et al. applied a multimodal approach based on sMRI, DTI, and rs-fMRI and classify $\mathrm{AD}$ from $\mathrm{HC}$ with an area under curve (AUC) of the receiver operating characteristic of $82 \%$ (31).

The brain topology analysis based on the graph theory provides powerful tools to study structural and functional characteristics of the brain network. Graph theory is a mathematical tool that is capable of concisely quantifying the properties of complex systems and modeling interrelationships between the brain regions. Since a large number of local and global graph measures, i.e., features, can be extracted from the brain networks, reducing dimension of features is an essential process for identifying optimal subset of features. In this study, we employed two feature selection algorithms in a machine learning approach to identify discriminative features for classifying $\mathrm{AD}, \mathrm{MCI}$, and normal aging. We developed an automatic classification algorithm that combined information from sMRI with rs-fMRI graph measures to classify four groups of subjects (AD, MCI-C, MCI-NC, and $\mathrm{HC}$ ). We used baseline rs-fMRI and sMRI data for MCI-C and MCI-NC patients. The MCI-NC patients did not convert to $\mathrm{AD}$ in 36 months after the baseline rs-fMRI, although MCI-C patients converted to AD from 6 to 36 months after the baseline rs-fMRI. We did not use the "time to conversion" of MCI-C patients in our algorithm to test performance of the proposed method in a challenging condition where this information is unknown in real clinical application. Our aim was to develop a method with an ability to distinguish potential "MCI-decliners" from those who remain stable. To our knowledge, this is the first study that investigated integration of rs-fMRI and sMRI for four-group classification (i.e., AD, MCI-C, MCI-NC and HC).

\section{MATERIALS AND METHODS Overall Procedure}

The overall procedure of our proposed method is shown in Figure 1. Structural MRI (T1-weighted images) and rs-fMRI data of 177 subjects (34 AD, 25 MCI-C, 69 MCI-NC, and 49 $\mathrm{HC})$ were used in this study. After preprocessing of rs-fMRI data, we used the Dosenbach atlas (32) to parcellate the brain into 160 region of interests (ROIs), and the adjacency matrix was calculated using the Pearson correlation between the time series of each pair of brain regions. We calculated 10 local and 13 global graph measures based on the adjacency matrix in each patient. FreeSurfer was utilized for preprocessing, cortical reconstruction, and volumetric segmentation of sMRI images. Volumes of the subcortical structures in addition to the surface area, curvature, thickness, and volume of 148 cortical areas, based 


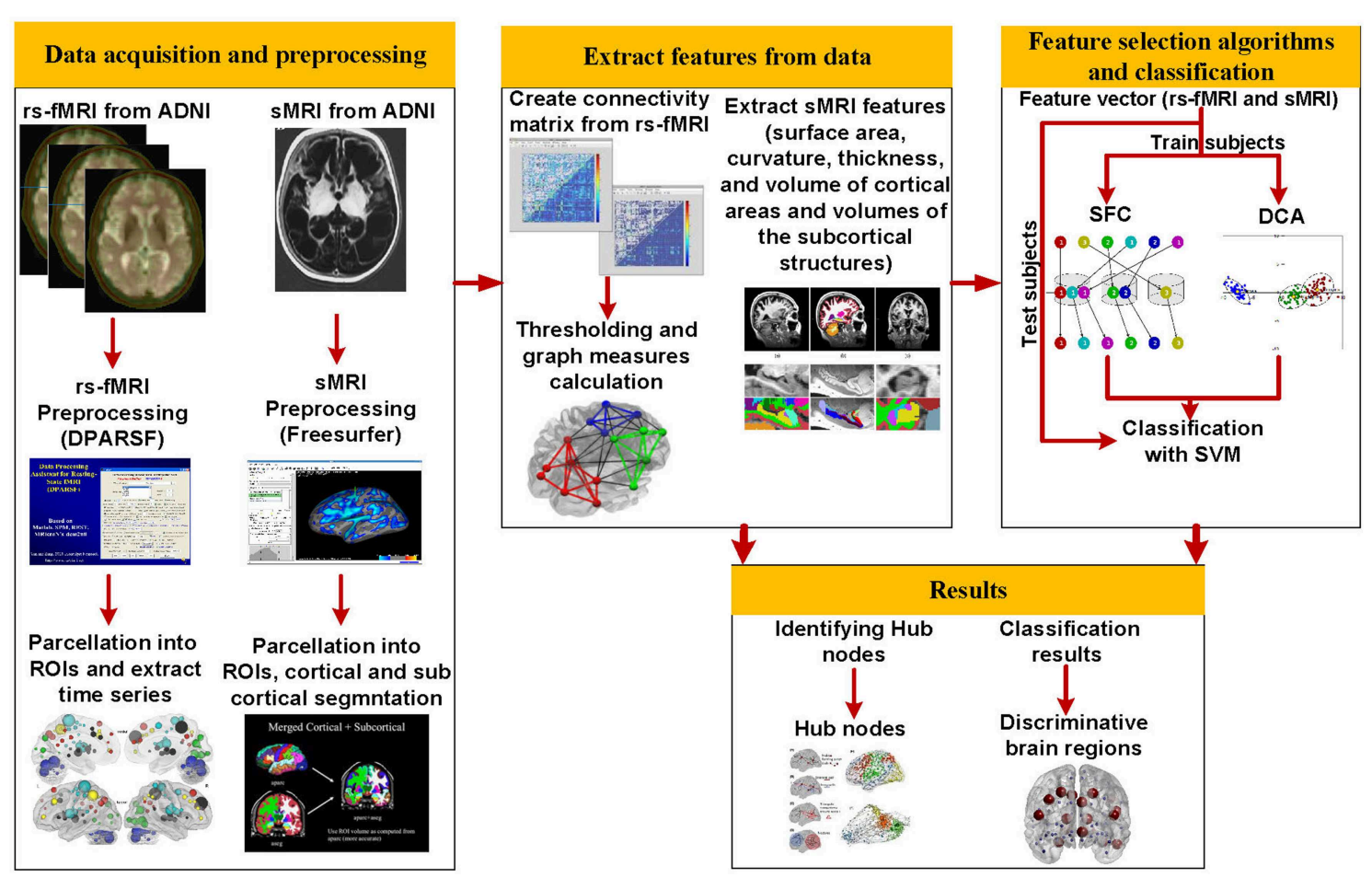

FIGURE 1 | The overall procedures of this study.

on the Destrieux atlas (33), were calculated and used as sMRI features in our algorithm. The rs-fMRI local and global graph measures were combined with sMRI measures to generate a feature vector in each patient. Two feature selection algorithms were applied to find an optimal subset of features for support vector machine (SVM) (10). We trained, cross-validated, and tested SVM to classify AD, MCI-C, MCI-NC, and HC using the selected rs-fMRI and sMRI features. We performed a second analysis on rs-fMRI data to identify hub nodes of the brain network and identify alteration of hubs in transient from healthy aging to $\mathrm{AD}$.

\section{Subjects}

We included 34 patients with $\mathrm{AD}$ (average age 72.5 years, 18 female), 25 patients with MCI-C (average age 73 years, 11 female), 69 patients with MCI-NC (average age 72.9 years, 37 female), and $49 \mathrm{HC}$ (average age 74.4 years, 28 female) from Alzheimer's Disease Neuroimaging Initiative (ADNI) database in this study. Subjects of this study were selected based on availability of both rs-fMRI and sMRI datasets in ADNI. We used the diagnosis variables to select the converted and nonconverted subjects. In the current study, we tried to include all subjects, listed in ADNI database and had a complete set of sMRI and rs-fMRI data. The MCI-C patients were converted to $\mathrm{AD}$ between 6 and 36 months. The MCI-NC patients did not convert to $\mathrm{AD}$ after 36 months of follow-up. The patients with AD had a Mini-Mental State Examination (MMSE) score of 2026, a Clinical Dementia Rating (CDR) of 0.5 or 1.0 , and met the
National Institute of Neurological and Communicative Disorders and Stroke and the $\mathrm{AD}$ and Related Disorders Association (NINCDS/ADRDA) criteria for probable AD. The patients with MCI had MMSE scores between 24 and 30, a memory complaint, objective memory loss measured by education adjusted scores on Wechsler Memory Scale Logical Memory II, a CDR of 0.5, absence of significant levels of impairment in other cognitive domains, essentially preserved activities of daily living, and an absence of dementia. The normal subjects were non-depressed, non-MCI, and non-demented, and had a MMSE score of 24-30 and a CDR close to zero. Demographic information of subjects is summarized in Table 1. Subjects for this study were selected based on availability of both rs-fMRI and sMRI datasets.

\section{Data Acquisition and Preprocessing}

The functional and structural MRI images were collected according to the ADNI acquisition protocol (34) ${ }^{1}$. A total of 140 functional volumes (TR/TE $3000 / 30 \mathrm{~ms}$, flip angle $=80^{\circ}$, $3.313 \mathrm{~mm}$ slice thickness, 48 slices) were obtained. Standard preprocessing routines were applied on rs-fMRI dataset using Data Processing Assistant for Resting State fMRI (DPARSF)

\footnotetext{
${ }^{1}$ Data used in the preparation of this article were obtained from the Alzheimer's Disease Neuroimaging Initiative (ADNI) database (adni.loni.usc.edu) that was launched in 2003 and led by Principal Investigator Michael W. Weiner, MD. The primary goal of ADNI has been to test whether serial MRI, positron emission tomography (PET), other biological markers, and clinical and neuropsychological assessment can be combined to measure the progression of mild cognitive MCI and early AD. For up-to-date information, see www.adni-info.org.
} 
TABLE 1 | Demographic and clinical information.

\begin{tabular}{|c|c|c|c|c|c|}
\hline & $\mathrm{HC}$ & MCI-NC & $\mathrm{MCl}-\mathrm{C}$ & $A D$ & $P$-value \\
\hline Number & 49 & 69 & 25 & 34 & \\
\hline Male/Female & $21 / 28$ & $32 / 37$ & $14 / 11$ & $16 / 18$ & $0.76^{\mathrm{a}}$ \\
\hline MMSE score & $29.35 \pm 1.63$ & $27.57 \pm 2.21$ & $26.64 \pm 1.85$ & $21.24 \pm 3.37$ & $0.0003^{b}$ \\
\hline CDR score & $0.035 \pm 0.21$ & $0.5 \pm 0.0$ & $0.5 \pm 0.0$ & $0.92 \pm 0.31$ & $0.0001^{b}$ \\
\hline
\end{tabular}

MMSE, mini-mental state examination; CDR, clinical dementia rating; ${ }^{a}$ Fisher extract test; ${ }^{b}$ ANOVA test.

package (35) and SPM12 toolbox (http://www.fil.ion.ucl.ac.uk/ spm). Slice-timing correction to the last slice was performed. The fMRI time-series realigned using a six-parameter rigid-body spatial transformation to compensate for head movement effects. Then all images were normalized into the Montreal Neurological Institute (MNI) space, resampled to $3-\mathrm{mm}$ isotropic voxels, detrended, smoothed using a Gaussian filter with FWHM = $4 \mathrm{~mm}$, and band-pass filtered $(0.01-0.08 \mathrm{~Hz})$. To reduce the effect of the physiological artifacts, the whole-brain signal was removed by a multiple linear regression analysis. In addition to the global mean signal, six head motion parameters, the cerebrospinal fluid (CSF), and the white matter signals were removed as nuisance covariates to reduce the effects of motion and non-neuronal BOLD fluctuations (36).

T1-weighted MRI of all subjects were processed using FreeSurfer (version 4.5.0). Cortical reconstruction and volumetric segmentation were performed using the following procedures: removal of non-brain tissue using a hybrid watershed/surface deformation procedure (37); automated Talairach transformation; segmentation of the subcortical white matter and deep gray matter volumetric structures (including hippocampus, amygdala, caudate, putamen, and ventricles) (37-39); intensity normalization; tessellation of gray matter and white matter boundary; automated topology correction (40); and deformation following intensity gradients to optimally place the gray/white and gray/cerebrospinal fluid borders at the location where the greatest shift in intensity defines the transition to the other tissue class $(41,42)$. After completing the cortical models, registration to a spherical atlas was performed (43), followed by cortical parcellation based on Destrieux atlas (33), and subcortical segmentation.

\section{Extracting Features From rs-fMRI and sMRI Data}

An adjacency matrix was calculated using the Pearson's correlation between the time series of the fMRI signals of all pairs of 160 ROIs of Dosenbach atlas. We used a similar method detailed in our previous studies (12-15) and converted the weighted adjacency matrices to binary ones by applying an optimal threshold (44). By maximizing the global cost efficiency (GCE) of the brain network (44), we identified an optimal threshold for each adjacency matrix. To have the same number of connections after thresholding of the adjacency matrices of all subjects, we used an average optimal threshold across subjects at $19.2 \%$, and then computed the graph measures. Then 10 local and 13 global graph measures were calculated based on rs-fMRI adjacency matrix. The local graph measures were betweenness centrality, clustering coefficient, characteristic path, community structure Newman, community structure Louvain, eccentricity, eigenvector centrality, rich club coefficient, sub graph centrality, and participation coefficient (45). The global graph measures were assortativity, clustering coefficient, characteristic path, community structure Newman output, community structure Louvain output, cost efficiency (two measures), density, efficiency, graph radius, graph diameter, transitivity, and small-worldness (45).

The surface area, average cortical curvature, average and standard deviation of thickness, and volume of gray matter of 148 cortical areas (according to Destrieux atlas) were considered as sMRI features in our algorithm. We also considered 34 features corresponding to volumes of subcortical structures in our algorithm. To make measurements comparable between subjects, an anatomical normalization was performed. For each subject, all volume quantifications were divided by the corresponding estimated intracranial volume (eTIV) and area quantifications were divided by the total area of the same hemisphere. Neither cortical thickness nor curvature needed to be anatomically normalized. After the feature extracting step, the features were normalized in each subject individually.

\section{Feature Selection}

Solving pattern recognition or classification problems with data of high dimensionality is a challenging issue, particularly in neuroimaging applications with limited samples, and large number of features. The learning models tend to overfit and become less generalizable if input features are redundant or irrelevant to classification. Feature selection is usually performed to identify relevant features, reduce dimensionality of the trained model, and improve generalization of the model (12). An efficient feature selection algorithm is the essential part of a machine learning approach in case of high dimensional features. We utilized two feature selection algorithms in this study: discriminant correlation analysis (DCA) and sequential feature collection (SFC). We have shown efficiency of the SFC feature selection algorithm in identifying the early stage of $\mathrm{AD}(10,11)$. Efficiency and reliability of the DCA feature selection algorithm have also been demonstrated in several previous studies $(46,47)$.

DCA has been used in pattern recognition applications for fusing the features extracted from multiple modalities or combining different feature vectors extracted from a single 
modality (48). DCA has a low computational load and can be employed in real-time applications. DAC is a variant of principal component analysis (PCA). PCA is the most prominent tool for reducing size of a high-dimensional feature vector, especially in unsupervised learning. DCA was developed for supervised learning environment, as a supervised PCA, to maximize the discriminant capability of classification (49). The DCA transforms features space into signal and noise subspaces. The signal subspace of DCA is associated with classification effectiveness and the noise subspace is not related to the discriminant power of the classification. For a feature vector in DCA, a within-class and a between-class scatter matrices are constructed to represent the noise and signal subspaces, respectively. Then transformed features to signal space are calculated by maximizing a signal-to-noise ratio based on the within-class and between-class scatter matrices (49). The DCA can also perform an effective feature fusion by maximizing the pairwise correlations across the two feature sets and, at the same time, eliminating the between-class correlations and restricting the correlations to be within the classes.

We developed the SFC algorithm to find an optimal subset of features (with a small number of features) (10). The SFC algorithm sorts all features using the multivariate minimal redundancy maximal relevance (MRMR) feature selection algorithm. The MRMR feature selection algorithm selects features that have maximal statistical dependency based on mutual information by considering relevant and redundant features simultaneously (50). The MRMR is defined as:

$$
M R M R=M A X_{s}\left\{\frac{1}{|S|} \sum_{f_{i} \in S} I\left(f_{i} ; c\right)-\frac{1}{|S|^{2}} \sum_{f_{i}, f_{j} \in S} I\left(f_{i} ; f_{j}\right)\right\}
$$

where the relevance of a feature set $S$ for class $C$ is defined by the average value of mutual information $I(.$, .) between the individual feature $f_{i}$ and the class $C$, and the redundancy of all features in the feature set $S$ is the average value of mutual information between the features $f_{i}$ and $f_{j}$. The SFC algorithm is described in detail previously (10). Briefly, features were first sorted based on their MRMR scores. The first and the last features in the sorted feature vector had maximum and minimum discrimination ability, respectively, in classification. Then a combination of filter and wrapper feature selection algorithms were used to find optimal subset of features with best classification accuracy.

\section{Classification}

To evaluate performance of the prosed method for classification of four groups (AD, MCI-C, MCI-NC, and HC), we used the $k$-fold cross-validation (KCV) which is one of the most widely used resampling techniques (51), and its estimates for the cross-validation errors nearly agree with the true errors (52). In addition, we evaluated performance of our classification algorithm using independent and non-training test samples. To this end, we used a 5 -fold approach and assigned $80 \%$ of subjects in each of four groups to train/cross-validation set $(n=141 ; 27$ $\mathrm{AD}, 20 \mathrm{MCI}-\mathrm{C}, 55 \mathrm{MCI}-\mathrm{NC}$, and $39 \mathrm{HC}$ ) and $20 \%$ of subjects to independent and non-training test set $(n=36$; $7 \mathrm{AD}, 5 \mathrm{MCI}-$ C, 14 MCI-NC, and $10 \mathrm{HC}$ ). We then used another 5-fold for cross-validation and further divided the train/cross-validation set to $80 \%$ for training $(n=113 ; 22 \mathrm{AD}, 16 \mathrm{MCI}-\mathrm{C}, 44 \mathrm{MCI}-\mathrm{NC}$, and $31 \mathrm{HC}$ ) and $20 \%$ for cross-validation ( $n=26$; 5 AD, 4 MCI-C, $11 \mathrm{MCI}-\mathrm{NC}$, and $8 \mathrm{HC}$ ). We used SFC or DCA algorithms for feature selection based on a combined sMRI and rs-fMRI features of $n=141$ subjects in train/cross-validation set. An unequal sample size may cause bias in results of a classifier. We prevented this possible bias by using equal number of training and test samples in four groups based on a similar approach described in details in our previous study (11). Since MCI-C group has minimum number of subjects, we randomly selected a subset of subjects in HC, MCI-NC, and AD groups equal to the number of MCI-C subjects. This random selection was repeated 1,000 times and average performance of the classifier across this repetition was calculated.

The selected features were used to train and cross-validate an SVM to classify four groups of subjects (AD, MCI-C, MCI-NC, and HC) in the train/cross-validation set. We used SVM for classification in this study. The SVM classifier was implemented in MATLAB using LIBSVM toolbox (53). After training and cross-validating the SVM, the accuracy, sensitivity, specificity, positive predictively, and the area under curve (ROC) of the receiver operating characteristic of the trained SVM were calculated for subjects in the test sets.

\section{Hub Node Identification}

We calculated betweenness centrality and eigenvector centrality graph measures of the rs-fMRI brain network to identify hub regions. It is noteworthy that the number of hub nodes can be highly influenced by several factors, including type(s) of the centrality measure, and the value of threshold applied on the adjacency matrix. We selected the betweenness centrality and eigenvector centrality graph measures because these measures were more frequently selected by our SFC algorithm compared to other centrality measures. In addition, these measures conceptually aligned with the integrative role ascribed to hubs, as they reflect the diversity of a region's cross-network connections. Brain regions (i.e., nodes) with betweenness centrality or eigenvector centrality larger than mean plus two standard deviation across all nodes were identified as hub nodes. Since the values of graph measures depend on the level of threshold applied on the adjacency matrix, we calculated the centrality measures by using the threshold in a range from 0.1 to 0.3 with a step of 0.01 ( 21 thresholds). For each value of the threshold, the betweenness centrality and eigenvector centrality of nodes were calculated and then hub nodes were identified. Next, a percentage for identification of a node as a hub node in different threshold values was calculated. Finally, we reported hub nodes that were identified in more than $85 \%$ of thresholds.

\section{RESULTS}

\section{Three- and Four-Group Classification}

We performed three- and four-group classification using the SFC and DCA feature selection algorithms. Our results revealed that SFC outperforms DCA for feature selection in three- and four-group classification with an extra accuracy $>7 \%$ (Table 2). 
TABLE 2 | Accuracy of three- and four-group classification using the SFC and DCA feature selection algorithms.

\begin{tabular}{lll}
\hline Three-group classification & SFC & $67.6 \%$ \\
(AD, MCl-C and MCl-NC) & DCA & $57.6 \%$ \\
Three-group classification & SFC & $66.0 \%$ \\
(HC, MCl-C and MCl-NC) & DCA & $58.2 \%$ \\
Four-group classification & SFC & $56.1 \%$ \\
(AD, HC, MCl-C and MCl-NC) & DCA & $48.8 \%$
\end{tabular}

DCA, discriminant correlation analysis; SFC, sequential feature collection.

The accuracies of SVM with SFC feature selection algorithm for three-group classification ("AD, MCI-C, MCI-NC" or "MCI$\mathrm{C}, \mathrm{MCI}-\mathrm{NC}, \mathrm{HC}$ ”) and four-group classification (“AD, MCI-C, MCI-NC, HC”) were $\sim 66$ and 56\%, respectively (accuracy by chance is 33 and $25 \%$, respectively). The sensitivity, specificity, positive predictive value (PPV) and AUC of SFC algorithm are listed in Table 3, and the confusion matrix is shown in Figure 2. Our algorithm is very specific (>96\%) but not sensitive (24\%) in identifying MCI-C patients. In fact, the majority of missclassified MCI-C patients (48\%) were identified as MCI-NC, which indicates similarity of the brain network and structural abnormalities of MCI-C patients with that of MCI-NC patients. Our proposed method has a good sensitivity (62\%) and specificity (72\%) for identifying MCI-NC patients. The majority of missclassified MCI-NC patients (21\%) were identified as HC, which points to a mild abnormalities of the brain of MCI-NC patients compared to that of normal aging subjects.

\section{Important Features for Four-Group Classification}

Top features that were selected by the SFC feature selection algorithm in at least $80 \%$ of training folds were listed in Table 4 that consisted of only rs-fMRI graph measures. We, however, found that eight sMRI features, including thickness of five cortical areas, were selected by the SFC algorithm in at least $60 \%$ of training folds. The top six features listed in Table 4 correspond to the rs-fMRI graph measures and represent modularity of the brain network in six brain regions (Figure 3). We used analysis of variance (ANOVA) and found a significant between-group difference $(P<0.01)$ in three features, i.e., community structure Louvain in medial cerebellum and superior frontal cortex and community structure Newman in post occipital (Table 4). Values of these three features in $\mathrm{AD} v s$. MCI-C and $\mathrm{HC}$ vs. MC-NC are plotted in Figure 4. Results of this figure show that values of these features are clustered in $\mathrm{AD}$ and $\mathrm{HC}$ but are scattered in MCI-C and MCI-NC, indicating a similar modularity of the brain network across subjects in $\mathrm{AD}$ or $\mathrm{HC}$ group but a diverse modularity in patients with MCI corresponding to different rates of dementia in these patients.

\section{Hub Analysis}

Hub nodes of the rs-fMRI brain network in four groups of subjects (AD, MCI-C, MCI-NC, and HC) are listed in Table 5. We identified 11 nodes as the hub nodes based on two centrality measures, betweenness centrality, and eigenvector centrality, in
TABLE 3 | Sensitivity, specificity, positive predictive value (PPV), AUC, and accuracy of three- and four-group classification based on the SFC feature selection algorithm.

\begin{tabular}{|c|c|c|c|c|}
\hline & & $\begin{array}{l}\text { Three group } \\
\text { classification } \\
\text { (AD, MCl-C, } \\
\text { MCl-NC) }\end{array}$ & $\begin{array}{l}\text { Three group } \\
\text { classification } \\
\text { (MCl-C, } \\
\mathrm{MCl}-\mathrm{NC}, \\
\mathrm{HC} \text { ) }\end{array}$ & $\begin{array}{c}\text { Four group } \\
\text { classification } \\
\text { (AD, MCl-C, } \\
\mathrm{MCl}-\mathrm{NC}, \\
\mathrm{HC})\end{array}$ \\
\hline \multirow[t]{4}{*}{ Sensitivity (\%) } & $A D$ & 52.3 & - & 46.1 \\
\hline & $\mathrm{MCl}-\mathrm{C}$ & 36.0 & 44.0 & 24.0 \\
\hline & $\mathrm{MCl}-\mathrm{NC}$ & 89.6 & 71.7 & 61.8 \\
\hline & $\mathrm{HC}$ & - & 69.5 & 75.5 \\
\hline \multirow[t]{4}{*}{ Specificity (\%) } & $A D$ & 91.1 & - & 85.0 \\
\hline & $\mathrm{MCl}-\mathrm{C}$ & 97.7 & 90.8 & 96.1 \\
\hline & $\mathrm{MCl}-\mathrm{NC}$ & 47.5 & 74.7 & 72.0 \\
\hline & $\mathrm{HC}$ & - & 72.6 & 66.3 \\
\hline \multirow[t]{4}{*}{ PPV (\%) } & $A D$ & 77.3 & - & 49.7 \\
\hline & $\mathrm{MCl}-\mathrm{C}$ & 85 & 52.8 & 76.6 \\
\hline & $\mathrm{MCl}-\mathrm{NC}$ & 67.3 & 75.6 & 65.5 \\
\hline & $\mathrm{HC}$ & - & 63.5 & 53.5 \\
\hline \multirow[t]{4}{*}{ AUC } & $A D$ & 0.72 & - & 0.65 \\
\hline & $\mathrm{MCl}-\mathrm{C}$ & 0.67 & 0.68 & 0.60 \\
\hline & $\mathrm{MCl}-\mathrm{NC}$ & 0.69 & 0.74 & 0.66 \\
\hline & $\mathrm{HC}$ & - & 0.72 & 0.70 \\
\hline \multirow[t]{4}{*}{ Accuracy (\%) } & $A D$ & 53 & - & 47 \\
\hline & $\mathrm{MCl}-\mathrm{C}$ & 36 & 44 & 24 \\
\hline & $\mathrm{MCl}-\mathrm{NC}$ & 89 & 72 & 62 \\
\hline & $\mathrm{HC}$ & - & 69 & 75 \\
\hline \multirow{2}{*}{$\begin{array}{l}\text { Number of } \\
\text { selected } \\
\text { features }\end{array}$} & rs-fMRI & 12 & 25 & 25 \\
\hline & sMRI & 8 & 5 & 7 \\
\hline
\end{tabular}

The average number of selected rs-fMRI and sMRI features (across 5-folds of training data) by the SFC algorithm are listed in two bottom rows.

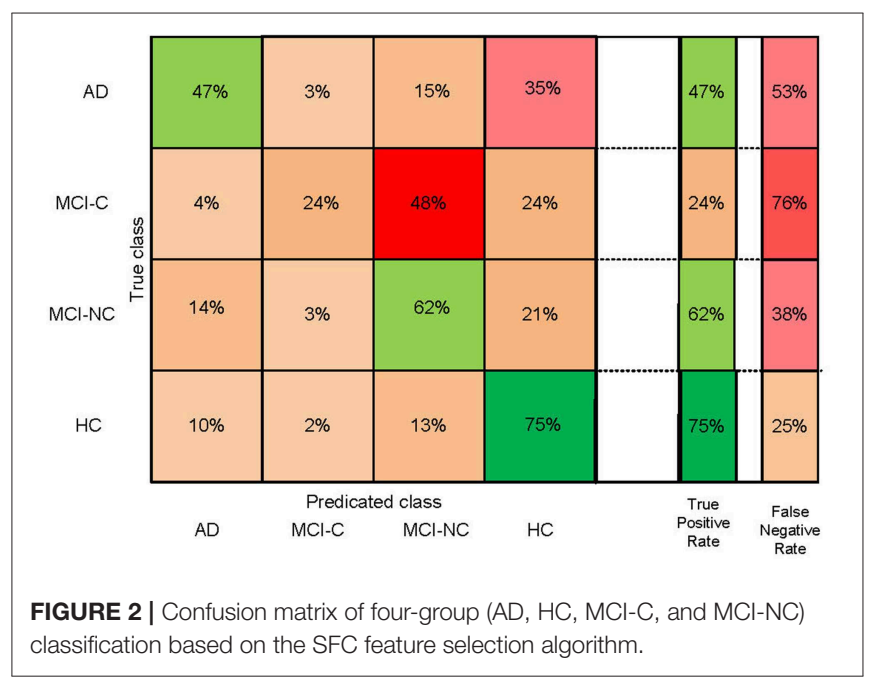

AD (6 hubs), MCI-C (5 hubs), MCI-NC (2 hubs), and HC (2 hubs). Patients with AD and MCI-C had a common hub in the basal ganglia, and this region was not a hub in MCI-NC 
and HC. MCI patients (but not AD and HC) had a hub in the parietal cortex. Insular cortex was a common hub in AD, MCI$\mathrm{C}$, and HC. On the other hand, there were group-specific hubs in each group (e.g., anterior cingulate cortex in $\mathrm{AD}$, occipital cortex in MCI-C, precentral gyrus in MCI-NC, and posterior cingulate in $\mathrm{HC}$ ) which were not identified in other groups as a hub node. It is interesting to mention that 4 out of 6 features with the most discriminant information in four-group classification (Table 4) were associated with four hub regions (i.e., insular cortex, occipital cortex, cerebellum, and precentral gyrus) listed in Table 5.

\section{DISCUSSION}

We proposed a machine learning algorithm to classify patients in the early stage of $\mathrm{AD}$ (MCI-C and MCI-NC), patients with AD, and normal aging subjects $(\mathrm{HC})$ by integrating rs-fMRI and sMRI data. This study provided three main results: (1) we examined

TABLE 4 | Top six features selected by the SFC algorithm for the four-group (AD, $\mathrm{HC}, \mathrm{MCl}-\mathrm{C}$, and $\mathrm{MCl}-\mathrm{NC}$ ) classification.

\begin{tabular}{llc}
\hline rs-fMRI graph measure & Brain area & $\boldsymbol{P}$-value \\
\hline CSL modularity & Median cerebellum & $8.7 \times 10^{-5}$ \\
CSN modularity & Post occipital & $5 \times 10^{-4}$ \\
CSL modularity & Superior frontal cortex & $8.9 \times 10^{-3}$ \\
CSN modularity & Occipital & $1.1 \times 10^{-2}$ \\
CSL modularity & Middle insula & $1.4 \times 10^{-2}$ \\
CSN modularity & Precentral gyrus & $2 \times 10^{-1}$ \\
\hline
\end{tabular}

$P$-values were calculated using the analysis of variance (ANOVA) to find a significant difference among four groups of subjects.

CSL, Community structure Louvain; CSN, Community structure Newman. the capability of integrating rs-fMRI and sMRI in a bi-modal approach to identify conversion from MCI to AD by evaluating performances of three- and four-group classifications (AD, MCI$\mathrm{C}, \mathrm{MCI}-\mathrm{NC}$, and $\mathrm{HC}$ ); (2) we evaluated performances of SFC and DCA feature selection algorithms in identification of optimal features from a large number of rs-fMRI and sMRI features. Our results revealed that the SFC algorithm outperformed the DCA feature selection algorithm by providing an extra accuracy of $>7 \%$ in four-group classification; and (3) we identified hub nodes of the rs-fMRI brain network in AD, MCI-C, MCI-NC, and HC, and found different hubs in patients within the early stage of AD.

Prediction of the early stage of $\mathrm{AD}$ using rs-fMRI and sMRI data based on a four-group classification (AD, MCI-C, MCI-NC, and HC) remains a relatively unexplored task. We performed three- and four-group classifications by integrating rs-fMRI and sMRI features, and observed that combining structural and functional MRI features improves performance of classification. We demonstrated in our previous study that a bimodal (sMRI and rs-fMRI) approach outperformed a unimodal (sMRI or rs-fMRI) approach for a two-group classification (MCI-C and MCI-NC) with an increased accuracy up to $17 \%$ (11). Therefore, we decided to use a bi-modal approach (sMRI and rs-fMRI) for three- and four-groups classification in the current study. Schouten et al. utilized functional and structural MRI and classified $16 \mathrm{AD}$ patients from 22 healthy controls, found that combining features of two modalities improves performance of classification, and achieved an accuracy of $89.5 \%$ in two-group (AD vs. HC) calcification (54). In another study, Canu et al. combined features extracted from structural MRI (cortical thickness in 68 cortical regions) and diffusion tractography (white matter microstructure) to classify 62 early onset $\mathrm{AD}$ and 27 behavioral variant of frontotemporal dementia patients, and reported $82 \%$ classification accuracy by integrating features of two modalities (55). Suk et al. integrated sMRI and
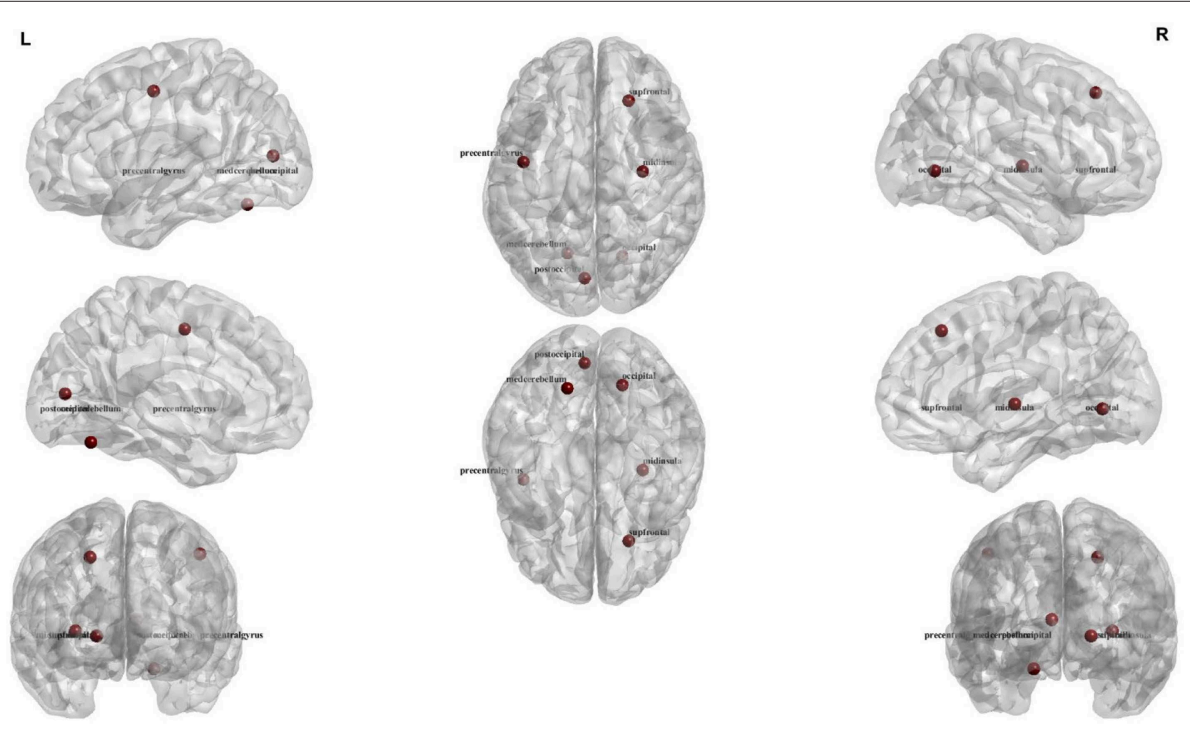

FIGURE 3 | |llustration of the six regions corresponding to top six rs-fMRI features for four-group (AD, HC, MCl-C, and MCl-NC) classification. 

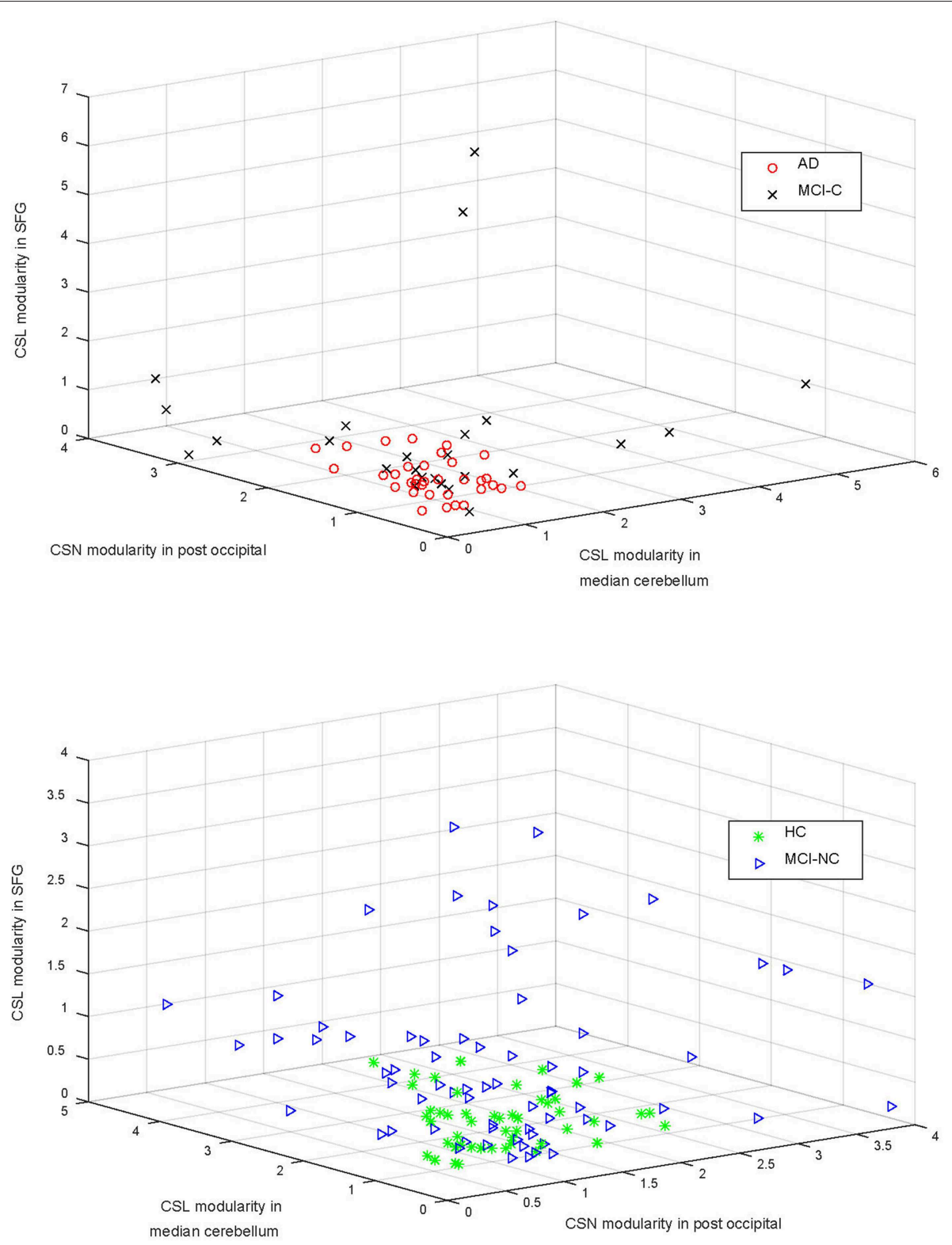

FIGURE 4 | The community structure Louvain (CSL) modularity and community structure Newman (CSN) modularity in three areas (i.e., superior frontal gyrus - SFG, median cerebellum, and post occipital cortex) are compared in the top and bottom panels for AD vs. MCl-C and HC vs. MCI-NC, respectively. Modularity of the brain network in these regions were significantly different in four groups $(P<0.01$; Table 4).

positron emission tomography (PET) features in a deep learning algorithm, and reported $98.8,90.7,83.7$, and $83.3 \%$ accuracies in binary (two-group) classification $\mathrm{AD} / \mathrm{HC}, \mathrm{MCI} / \mathrm{HC}, \mathrm{AD} / \mathrm{MCI}$, and MCI-C/MCI-NC, respectively (56). It is noteworthy that Suk et al. did not test performance of their proposed algorithm in three- or four-group classification, as we did. To our knowledge, this is the first study which integrated rs-fMRI with sMRI data in a four group (AD, MCI-C, MCI-NC, and HC) classification approach. For the binary classification MCI-C vs. MCI-NC, Zhang et al. (57) achieved 73.9\% accuracy by utilizing a multi-modal neuroimaging approach using FDG-PET, sMRI, and cerebrospinal fluid (CSF) data. Beheshti et al. used sMRI features in a discriminative feature ranking method to find the most discriminative feature set, and reported 75\% accuracy in MCI-C vs. MCI-NC binary calcification (9). In another study, Young et al. provided AD prediction model by adding the apolipoprotein E (ApoE) genotype to FDG-PET, sMRI, and CSF data, and reported $74 \%$ accuracy in classification of MCI-C vs. MCI-NC (58). It is noteworthy that previous studies have shown that MCI is a heterogeneous condition where MCI-NC subjects appear more healthy and MCI-C subjects appear more in AD condition (59).

Figure 2 shows that our algorithm was more accurate in identification of HC compared to other groups of subjects, which is expected due to the similarity of the brain structure and network of patients with MCI and AD compared to that of $\mathrm{HC}$. 
TABLE 5 | The rs-fMRI hub nodes in four groups of subjects (AD, HC, MCl-C, and $\mathrm{MCl}-\mathrm{NC})$.

\begin{tabular}{lcccc}
\hline Hub nodes & AD & MCI-C & MCI-NC & HC \\
\hline Basal ganglia & $X$ & $X$ & - & - \\
Temporal & $X$ & - & - & - \\
Anterior cingulate cortex (ACC) & $X$ & - & - & - \\
Medial frontal cortex (mFC) & $X$ & - & - & - \\
Thalamus & $X$ & - & - & - \\
Parietal & - & $X$ & $X$ & - \\
Insula & $X$ & $X$ & - & X \\
Cerebellum & - & $X$ & - & - \\
Occipital & - & $X$ & - & - \\
Precentral gyrus & - & - & $X$ & X \\
Posterior cingulate & - & - & - & \\
\hline
\end{tabular}

The listed areas are based on the Dosenbach atlas.

The best and worst accuracies of our algorithm were $75 \%$ for $\mathrm{HC}$ and 24\% for MCI-C groups, respectively (Figure 2). Results in Table 3 show that our algorithm was specific (>90\%) but not sensitive $(<44 \%)$ in identifying MCI-C group compared to other groups, in both three- and four-group classification. The majority of miss-classified MCI-C patients were identified as MCI-NC patients (Figure 2), indicating similarity of the rs-fMRI and sMRI features of patients in the former group with that of the latter group. On the other hand, our algorithm had a good sensitivity and specificity in identifying MCI-NC patients.

Identifying MCI-C patients is a difficult task since we used the baseline rs-fMRI and sMRI data in these patients and they converted to AD 6 to 36 months after that. In addition to using the baseline data in these patients, they had a heterogeneity in their conversion time to AD from 6 to 36 months. MCI$\mathrm{C}$ patients who converted to $\mathrm{AD}$ in a longer time (e.g., at 36 months) after baseline may have a similar brain network and structure at baseline compared to MCI-NC patients, who did not convert to $\mathrm{AD}$. On the other hand, brain network and structure of the MCI-C patients who converted to AD in a shorter time (e.g., at 6 months) may be similar to that of the $\mathrm{AD}$ patients. Furthermore, MCI-C patients were the only unstable group of patients who had a change of status from MCI to AD during 36 months follow-up. In fact, subjects in HC, MCI-NC, and $\mathrm{AD}$ groups were stable and did not convert to another group during at least 36 months. Moreover, we observed instability in the MCI-C patients that some of them had conversion to $\mathrm{AD}$ and then revision to MCI during 36 months follow-up. It is noteworthy that we excluded MCI-C patients with multiple conversion and revision in our analysis. Therefore, it is expected that a classifier has a lower performance in identifying MCI$\mathrm{C}$ compared to other groups. In line with this expectation and as shown in Figure 2 and Table 3, our proposed algorithm provided a superior performance in classifying HC, MCI-NC, and $\mathrm{AD}$ compared to MCI-C. It is noteworthy that we evaluated our algorithm in identification of the early stage of $\mathrm{AD}$ based on three- and four-group classification (AD, MCI-C, MCI-NC, and $\mathrm{HC}$ ), while most previous studied investigated a two-group classification (e.g., $\mathrm{AD}$ vs. $\mathrm{HC}$ and $\mathrm{AD}$ vs. $\mathrm{MCI})(5,60,61)$.
Top six features and brain regions that were selected by SFC in four-group classification are listed in Table 4 and Figure 3. These features were all related to rs-fMRI graph measures and represent modularity of the brain network in six cortical regions (median cerebellum, post occipital, superior frontal gyrus, occipital, middle insula, and precentral gyrus). The modularity was calculated based on the community structure Louvain or community structure Newman graph measures. A modular network has an arrangement of nodes in large modules such that maximum possible number of edges lies within groups and minimum possible number of edges lies between groups (62). Modularity of the brain network has been showed to be informative in the early stages of the neurodegenerative disease (63). A recent study demonstrated the ability of modularity of the brain network in discriminating $\mathrm{AD}$ and $\mathrm{HC}$ (63). Results of ANOVA in Table 4 show that the modularity in three brain regions, i.e., median cerebellum, post occipital and superior frontal gyrus, were significantly different in four groups of subjects $(P<0.01)$. In agreement with our results, previous studies reported association of posterior occipital, superior frontal cortex, occipital, and middle insula with AD (64-66). Another observation from Table 4 is that there was no sMRI feature among the top six features which may indicate that rs-fMRI is more informative than sMRI in identification of the early stage of $\mathrm{AD}$. This observation was in agreement with previous studies reported that biomarkers based on the functional brain network may outperform biomarkers based on the structural measures in predicting the early stage of $\operatorname{AD}(11,67)$.

In Figure 4, we compared four groups based on modularity of the brain network in three regions (i.e., median cerebellum, post occipital, and superior frontal gyrus) which were significantly different in four groups $(P<0.01)$. Figure 4 shows that modularity in $\mathrm{AD}$ or $\mathrm{HC}$ was clustered across subjects but the modularity in MCI-C or MCI-NC was scattered across subjects. This scatter of modularity in patients with MCI may be related to the inhomogeneity in MCI group, as a transitional state between normal aging and $\mathrm{AD}$, in that characteristic of their brain may vary between two extremes from $\mathrm{HC}$ to $\mathrm{AD}$.

Results of our hub analyses in Table $\mathbf{5}$ are generally consistent with previous findings in terms of localization, and provide additional support for the underlying topological organization of the early stage of AD. Interestingly, some hub areas in Table 5 (i.e., insular cortex, occipital, cerebellum, and precentral gyrus) were also identified by the SFC algorithm as important areas in classification of four groups. We found that the insular cortex was a common hub node in $\mathrm{AD}, \mathrm{MCI}-\mathrm{C}$, and $\mathrm{HC}$, and our finding is in agreement with previous studies showing importance of this area in $\operatorname{AD}(68,69)$. Our finding that Basal ganglia was a common hub in AD and MCI-C is in agreement with a recent study showing pivotal role of this area in patients with earlyonset $\mathrm{AD}$ (70). We found the parietal cortex as a common hub in MCI-C and MCI-NC patients. In agreement with our finding, association of the parietal cortex with $\mathrm{AD}$ has been reported in previous studies $(71,72)$. Our results revealed that $A D$ and MCI-C subjects had larger number of hub nodes compared to MCI-NC and HC subjects which may relate to the abnormalities 
in largescale network connectivity in $\mathrm{AD}$ and MCI-C brain regions (73).

\section{DATA AVAILABILITY}

Publicly available datasets were analyzed in this study. This data can be found here: http://adni.loni.usc.edu/.

\section{ETHICS STATEMENT}

Data used in this paper were obtained from the Alzheimer's Disease Neuroimaging Initiative (ADNI) database (http://ADNI. loni.usc.edu). Data collection and sharing for this project was funded by the Alzheimer's Disease Neuroimaging Initiative (ADNI) (National Institutes of Health Grant U01 AG024904).

\section{AUTHOR CONTRIBUTIONS}

SH: preparing required data from ADNI, data analysis, statistical analysis, and drafting and revision of the manuscript. AE: interpreting results and revision of the manuscript. AB-F: study design and conceptualization, data analysis and interpretation of the results, drafting, and revision of the manuscript.

\section{FUNDING}

This study was funded by Le Bonheur Children's Hospital, the Children's Foundation Research Institute, and the Le Bonheur

\section{REFERENCES}

1. Serrano-Pozo A, FrAosch MP, Masliah E, Hyman BT. Neuropathological alterations in Alzheimer disease. Cold Spring Harb Perspect Med. (2011) 1:a006189. doi: 10.1101/cshperspect.a006189

2. Farlow M. Treatment of mild cognitive impairment (MCI). Curr Alzheimer Res. (2009) 6:362-7. doi: 10.2174/1567205097889 29282

3. He Y, Chen Z, Evans A. Structural insights into aberrant topological patterns of large-scale cortical networks in Alzheimer's disease. J Neurosci. (2008) 28:4756-66. doi: 10.1523/JNEUROSCI.0141-08.2008

4. Huang L, Jin Y, Gao Y, Thung KH, Shen D. Longitudinal clinical score prediction in Alzheimer's disease with soft-split sparse regression based random forest. Neurobiol Aging. (2016) 46:180-91. doi: 10.1016/j.neurobiolaging.2016.07.005

5. Moradi E, Pepe A, Gaser C, Huttunen H, Tohka JI. Alzheimer's disease neuroimaging, machine learning framework for early MRI-based Alzheimer's conversion prediction in MCI subjects. Neuroimage. (2015) 104:398-412. doi: 10.1016/j.neuroimage.2014.10.002

6. Jack CR Jr, Vemuri P, Wiste HJ, Weigand SD, Lesnick TG, Lowe V, et al. Shapes of the trajectories of 5 major biomarkers of Alzheimer disease. Arch Neurol. (2012) 69:856-67. doi: 10.1001/archneurol.2011.3405

7. Sabuncu MR, Desikan RS, Sepulcre J, Yeo BT, Liu H, Schmansky NJ, et al. The dynamics of cortical and hippocampal atrophy in Alzheimer disease. Arch Neurol. (2011) 68:1040-8. doi: 10.1001/archneurol.2011.167

8. Eskildsen SF, Coupé P, García-Lorenzo D, Fonov V, Pruessner JC, Collins DL, et al. Prediction of Alzheimer's disease in subjects with mild cognitive impairment from the ADNI cohort using patterns of cortical thinning, Neuroimage. (2013) 65:511-21. doi: 10.1016/j.neuroimage.2012.09.058

9. Beheshti I, Demirel H, Matsuda H, Alzheimer's Disease Neuroimaging Initiative. Classification of Alzheimer's disease and prediction of mild cognitive impairment-to-Alzheimer's conversion from structural magnetic
Associate Board, Memphis, TN. Data collection and sharing for this project was funded by the Alzheimer's Disease Neuroimaging Initiative (ADNI) (National Institutes of Health Grant U01 AG024904). ADNI was funded by the National Institute on Aging, the National Institute of Biomedical Imaging and Bioengineering, and through generous contributions from the following: AbbVie, Alzheimer's Association; Alzheimer's Drug Discovery Foundation; Araclon Biotech; BioClinica, Inc.; Biogen; Bristol-Myers Squibb Company; CereSpir, Inc.; Eisai Inc.; Elan Pharmaceuticals, Inc.; Eli Lilly and Company; EuroImmun; F. Hoffmann-La Roche Ltd and its affiliated company Genentech, Inc.; Fujirebio; GE Healthcare; IXICO Ltd.; Janssen Alzheimer Immunotherapy Research \& Development, LLC.; Johnson \& Johnson Pharmaceutical Research \& Development LLC.; Lumosity; Lundbeck; Merck \& Co., Inc.; Meso Scale Diagnostics, LLC.; NeuroRx Research; Neurotrack Technologies; Novartis Pharmaceuticals Corporation; Pfizer Inc.; Piramal Imaging; Servier; Takeda Pharmaceutical Company; and Transition Therapeutics. The Canadian Institutes of Health Research is providing funds to support ADNI clinical sites in Canada. Private sector contributions are facilitated by the Foundation for the National Institutes of Health (www.fnih.org). The grantee organization is the Northern California Institute for Research and Education, and the study is coordinated by the Alzheimer's Disease Cooperative Study at the University of California, San Diego. ADNI data are disseminated by the Laboratory for Neuro Imaging at the University of Southern California. resource imaging using feature ranking and a genetic algorithm. Comput Biol Med. (2017) 83:109-19. doi: 10.1016/j.compbiomed.2017.02.011

10. Hojjati SH, Ebrahimzadeh A, Khazaee A, Babajani-Feremi AI. Alzheimer's disease neuroimaging, predicting conversion from $\mathrm{MCI}$ to $\mathrm{AD}$ using restingstate fMRI, graph theoretical approach and SVM. J Neurosci Methods. (2017) 282:69-80. doi: 10.1016/j.jneumeth.2017.03.006

11. Hojjati SH, Ebrahimzadeh A, Khazaee A, Babajani-Feremi AI. Alzheimer's disease neuroimaging, predicting conversion from $\mathrm{MCI}$ to $\mathrm{AD}$ by integrating rs-fMRI and structural MRI. Comput Biol Med. (2018) 102:30-9. doi: 10.1016/j.compbiomed.2018.09.004

12. Khazaee A, Ebrahimzadeh A, Babajani-Feremi A. Identifying patients with Alzheimer's disease using resting-state fMRI and graph theory. Clin Neurophysiol. (2015) 126:2132-41. doi: 10.1016/j.clinph.2015.02.060

13. Khazaee A, Ebrahimzadeh A, Babajani-Feremi A. Application of pattern recognition and graph theoretical approaches to analysis of brain network in Alzheimer's disease. J Med Imag Health Informat. (2015) 5:1145-155. doi: 10.1166/jmihi.2015.1513

14. Khazaee A, Ebrahimzadeh A, Babajani-Feremi A. Application of advanced machine learning methods on resting-state fMRI network for identification of mild cognitive impairment and Alzheimer's disease. Brain Imaging Behav. (2016) 10:799-817. doi: 10.1007/s11682-015-9448-7

15. Khazaee A, Ebrahimzadeh A, Babajani-Feremi AI. Alzheimer's disease neuroimaging, classification of patients with MCI and AD from healthy controls using directed graph measures of resting-state fMRI. Behav Brain Res. (2017) 322:339-50. doi: 10.1016/j.bbr.2016.06.043

16. Lin Q, Rosenberg MD, Yoo K, Hsu TW, O'Connell TP, Chun MM. Resting-state functional connectivity predicts cognitive impairment related to Alzheimer's disease. Front Aging Neurosci. (2018) 10:94. doi: 10.3389/fnagi.2018.00094

17. Greicius M. Resting-state functional connectivity in neuropsychiatric disorders. Curr Opin Neurol. (2008) 21:424-30. doi: 10.1097/WCO.0b013e328306f2c5 
18. Ito T, Kulkarni KR, Schultz DH, Mill RD, Chen RH, Solomyak LI, et al. Cognitive task information is transferred between brain regions via resting-state network topology. Nature Commun. (2017) 8:1027. doi: 10.1038/s41467-017-01000-w

19. Sheline YI, Morris JC, Snyder AZ, Price JL, Yan Z, D’Angelo G, et al. APOE4 allele disrupts resting state fMRI connectivity in the absence of amyloid plaques or decreased CSF A 342 . J Neurosci. (2010) 30:17035-40. doi: 10.1523/JNEUROSCI.3987-10.2010

20. Stam CJ, Jones BF, Nolte G, Breakspear M, Scheltens P. Small-world networks and functional connectivity in Alzheimer's disease. Cereb Cortex. (2007) 17:92-9. doi: 10.1093/cercor/bhj127

21. Grieder M, Wang DJJ, Dierks T, Wahlund LO, Jann K. Default mode network complexity and cognitive decline in mild Alzheimer's disease. Front Neurosci. (2018) 12:770. doi: 10.3389/fnins.2018.00770

22. Binnewijzend MA, Schoonheim MM, Sanz-Arigita E, Wink AM, van der Flier WM, Tolboom N, et al. Resting-state fMRI changes in Alzheimer's disease and mild cognitive impairment. Neurobiol Aging. (2012) 33:2018-28. doi: 10.1016/j.neurobiolaging.2011.07.003

23. Bosch B, Arenaza-Urquijo EM, Rami L, Sala-Llonch R, Junqué C, SoléPadullés C, et al. Multiple DTI index analysis in normal aging, amnestic MCI and AD. Relationship with neuropsychological performance. Neurobiol Aging. (2012) 33:61-74. doi: 10.1016/j.neurobiolaging.2010.02.004

24. Lindemer ER, Salat DH, Smith EE, Nguyen K, Fischl B, Greve DN, et al. White matter signal abnormality quality differentiates mild cognitive impairment that converts to Alzheimer's disease from nonconverters. Neurobiol Aging. (2015) 36:2447-57. doi: 10.1016/j.neurobiolaging.2015.05.011

25. Okello A, Koivunen J, Edison P, Archer H, Turkheimer FK. Någren $\mathrm{K}$, et al. Conversion of amyloid positive and negative MCI to AD over 3 years: an 11C-PIB PET study. Neurology. (2009) 73:754-60. doi: 10.1212/WNL.0b013e3181b23564

26. Pagani M, Giuliani A, Oberg J, Chincarini A, Morbelli S, Brugnolo A, et al. Predicting the transition from normal aging to Alzheimer's disease: a statistical mechanistic evaluation of FDG-PET data. Neuroimage. (2016) 141:282-90. doi: 10.1016/j.neuroimage.2016.07.043

27. Risacher SL, Saykin AJ, Wes JD, Shen L, Firpi HA, McDonald BC. Baseline MRI predictors of conversion from MCI to probable $\mathrm{AD}$ in the ADNI cohort. Curr Alzheimer Res. (2009) 6:347-61. doi: 10.2174/156720509788 929273

28. Mateos-Perez JM, Dadar M, Lacalle-Aurioles M, Iturria-Medina Y, Zeighami Y, Evans AC. Structural neuroimaging as clinical predictor: a review of machine learning applications. Neuroimage Clin. (2018) 20:506-22. doi: 10.1016/j.nicl.2018.08.019

29. Tong T, Gray K, Gao QQ, Chen L, Rueckert D, Initia AD N. Multi-modal classification of Alzheimer's disease using nonlinear graph fusion. Pattern Recogn. (2017) 63:171-81. doi: 10.1016/j.patcog.2016.10.009

30. Peng JL, Zhu XF, Wang Y, An L, Shen DG. Structured sparsity regularized multiple kernel learning for Alzheimer's disease diagnosis. Pattern Recogn. (2019) 88:370-82. doi: 10.1016/j.patcog.2018.11.027

31. Dyrba M, Grothe M, Kirste T, Teipel SJ. Multimodal analysis of functional and structural disconnection in Alzheimer's disease using multiple kernel SVM. Hum Brain Mapp. (2015) 36:2118-31. doi: 10.1002/hbm.22759

32. Dosenbach NU F, Nardos B, Cohen AL, Fair DA, Power JD, Church JA, et al. Prediction of individual brain maturity using Fmri. Science. (2010) 329:1358-61. doi: 10.1126/science.1194144

33. Fischl B, van der Kouwe A, Destrieux C, Halgren E, Segonne F, Salat DH, et al. Automatically parcellating the human cerebral cortex. Cerebral Cortex. (2004) 14:11-22. doi: 10.1093/cercor/bhg087

34. Jack CR Jr, Bernstein MA, Fox NC, Thompson P, Alexander G, Harvey $\mathrm{D}$, et al. The Alzheimer's Disease Neuroimaging Initiative (ADNI): MRI methods. J Magn Reson Imaging. (2008) 27:685-91. doi: 10.1002/jmri. 21049

35. Chao-Gan Y, Yu-Feng Z. DPARSF: a MATLAB toolbox for pipeline data analysis of resting-state fMRI. Front Syst Neurosci. (2010) 4:13. doi: 10.3389/fnsys.2010.00013

36. Greicius MD, Krasnow B, Reiss AL, Menon V. Functional connectivity in the resting brain: a network analysis of the default mode hypothesis. Proc Natl Acad Sci USA. (2003) 100:253-8. doi: 10.1073/pnas.01350 58100
37. Segonne F, Dale AM, Busa E, Glessner M, Salat D, Hahn HK, et al. A hybrid approach to the skull stripping problem in MRI. Neuroimage. (2004) 22:1060-75. doi: 10.1016/j.neuroimage.2004.03.032

38. Fischl B, Salat DH, Busa E, Albert M, Dieterich M, Haselgrove $\mathrm{C}$, et al. Whole brain segmentation: automated labeling of neuroanatomical structures in the human brain. Neuron. (2002) 33:341-55. doi: 10.1016/S0896-6273(02)00569-X

39. Fischl B, Salat DH, van der Kouwe AJ, Makris N, Segonne F, Quinn BT, et al. Sequence-independent segmentation of magnetic resonance images. Neuroimage. (2004) 23(Suppl. 1):S69-84. doi: 10.1016/j.neuroimage.2004.07.016

40. Fischl B, Liu A, Dale AM. Automated manifold surgery: constructing geometrically accurate and topologically correct models of the human cerebral cortex. IEEE Trans Med Imaging. (2001) 20:70-80. doi: 10.1109/42.906426

41. Fischl B, Dale AM. Measuring the thickness of the human cerebral cortex from magnetic resonance images. Proc Natl Acad Sci USA. (2000) 97:11050-5. doi: 10.1073/pnas.200033797

42. Dale AM, Fischl B, Sereno MI. Cortical surface-based analysis. Segmentation I, surface reconstruction. Neuroimage. (1999) 9:179-94. doi: 10.1006/nimg.1998.0395

43. Fischl B, Sereno MI, Dale AM. Cortical surface-based analysis. II: inflation, flattening, a surface-based coordinate system. Neuroimage. (1999) 9:195-207. doi: 10.1006/nimg.1998.0396

44. Bassett DS, Bullmore ET, Meyer-Lindenberg A, Apud JA, Weinberger DR, Coppola R. Cognitive fitness of cost-efficient brain functional networks. Proc Natl Acad Sci USA. (2009) 106:11747-52. doi: 10.1073/pnas.0903641106

45. Rubinov M, Sporns O. Complex network measures of brain connectivity: uses and interpretations. Neuroimage. (2010) 52:1059-69. doi: 10.1016/j.neuroimage.2009.10.003

46. Soleymani S, Dabouei A, Kazemi H, Dawson J, Nasrabadi NM. Multi-level feature abstraction from convolutional neural networks for multimodal biometric identification. In: 2018 24th International Conference on Pattern Recognition (ICPR). (Beijing) (2018), 3469-76. doi: 10.1109/ICPR.2018.8545061

47. Boyaci E, Sert M. Video classification based on ConvNet collaboration and feature selection. In: 2017 25th Signal Processing and Communications Applications Conference (SIU). (Antalya) (2017), 1-4. doi: 10.1109/SIU.2017.7960515

48. Haghighat M, Abdel-Mottaleb M, Alhalabi W. Discriminant correlation analysis: real-time feature level fusion for multimodal biometric recognition. IEEE Trans Informat Forens Secur. (2016) 11:1984-96. doi: 10.1109/TIFS.2016.2569061

49. Kung SY, Chanyaswad T, Chang JM, Wu PY. Collaborative PCA/DCA learning methods for compressive privacy. In: Acm Transactions on Embedded Computing Systems (New York, NY: ACM) (2017). doi: 10.1145/2996460

50. Yang J, Zhu Z, He S, Ji Z. Minimal-redundancy-maximal-relevance feature selection using different relevance measures for omics data classification. In: Computational Intelligence in Bioinformatics and Computational Biology (CIBCB) (Singapore: IEEE) (2013). p. 246-51. doi: 10.1109/CIBCB.2013.6595417

51. Anguita D, Ghio A, Ridella S, Sterpi D. K-fold cross validation for error rate estimate in support vector machines. in: Proceedings of The 2009 International Conference on Data Mining, DMIN (Las Vegas, NV) (2009). p. 291-97.

52. Braga-Neto UM, Dougherty ER. Is cross-validation valid for smallsample microarray classification? Bioinformatics. (2004) 20:374-80. doi: 10.1093/bioinformatics/btg419

53. Chang CC, Lin CJ. LIBSVM: a library for support vector machines. ACM Trans Intell Syst Technol. (2011) 2:27. doi: 10.1145/1961189.1961199

54. Schouten TM, Koini M, de Vos F, Seiler S, van der Grond J, Lechner A, et al. Combining anatomical, diffusion, resting state functional magnetic resonance imaging for individual classification of mild and moderate Alzheimer's disease. NeuroImage Clin. (2016) 11:46-51. doi: 10.1016/j.nicl.2016.01.002

55. Canu E, Agosta F, Mandic-Stojmenovic G, Stojkovic T, Stefanova E, Inuggi A, et al. Multiparametric MRI to distinguish early onset alzheimer's disease and behavioural variant of frontotemporal dementia. Neuroimage Clin. (2017) 15:428-38. doi: 10.1016/j.nicl.2017.05.018

56. Suk HI, Lee SW, Shen D, The Alzheimer's Disease Neuroimaging Initiative. Latent feature representation with stacked auto-encoder 
for AD/MCI diagnosis. Brain Struct Funct. (2015) 220:841-59. doi: 10.1007/s00429-013-0687-3

57. Zhang D, Shen DI. Alzheimer's Disease Neuroimaging. Multi-modal multi-task learning for joint prediction of multiple regression and classification variables in Alzheimer's disease. Neuroimage. (2012) 59:895-907. doi: 10.1016/j.neuroimage.2011.09.069

58. Young J, Modat M, Cardoso MJ, Mendelson A, Cash D, Ourselin S, et al. Accurate multimodal probabilistic prediction of conversion to Alzheimer's disease in patients with mild cognitive impairment, Neuroimage Clin. (2013) 2:735-45. doi: 10.1016/j.nicl.2013.05.004

59. Cheng B, Liu M, Zhang D, Munsell BC, Shen D. Domain transfer learning for MCI conversion prediction. IEEE Trans Biomed Eng. (2015) 62:1805-17. doi: 10.1109/TBME.2015.2404809

60. Misra C, Fan Y, Davatzikos C. Baseline and longitudinal patterns of brain atrophy in MCI patients, their use in prediction of short-term conversion to AD: results from ADNI. Neuroimage. (2009) 44:1415-22. doi: 10.1016/j.neuroimage.2008.10.031

61. Wolz R, Julkunen V, Koikkalainen J, Niskanen E, Zhang DP, Rueckert $\mathrm{D}$, et al. Multi-method analysis of MRI images in early diagnostics of Alzheimer's disease. PLoS ONE. (2011) 6:e25446. doi: 10.1371/journal.pone.0 025446

62. Rubinov $M$, Sporns $\mathrm{O}$. Weight-conserving characterization of complex functional brain networks. Neuroimage. (2011) 56:2068-79. doi: 10.1016/j.neuroimage.2011.03.069

63. Jalili M. Graph theoretical analysis of Alzheimer's disease: discrimination of AD patients from healthy subjects. Informat Sci. (2017) 384:145-56. doi: 10.1016/j.ins.2016.08.047

64. Arnold SE, Hyman BT, Flory J, Damasio AR, Van Hoesen GW. The topographical and neuroanatomical distribution of neurofibrillary tangles and neuritic plaques in the cerebral cortex of patients with Alzheimer's disease. Cerebral Cortex. (1991) 1:103-16. doi: 10.1093/cercor/1.1.103

65. Bai F, Xie C, Yuan Y, Shi Y, Zhang Z. Promoter haplotypes of interleukin-10 gene linked to cortex plasticity in subjects with risk of Alzheimer's disease. Neuroimage Clin. (2018) 17:587-95. doi: 10.1016/j.nicl.2017.11.019

66. Chen B, Zhong X, Mai N, Peng Q, Wu Z, Ouyang C, et al. Cognitive impairment and structural abnormalities in late life depression with olfactory identification impairment: an Alzheimer's disease-like pattern. Int J Neuropsychopharmacol. (2018) 21:640-48. doi: 10.1093/ijnp/pyy016
67. Coleman P, Federoff H, Kurlan R. A focus on the synapse for neuroprotection in Alzheimer disease and other dementias. Neurology. (2004) 63:1155-162. doi: 10.1212/01.WNL.0000140626.48118.0A

68. Davatzikos C, Bhatt P, Shaw LM, Batmanghelich KN, Trojanowski JQ. Prediction of MCI to AD conversion, via MRI, CSF biomarkers, pattern classification. Neurobiol Aging. (2011) 32:2322.e19-2322.e27. doi: 10.1016/j.neurobiolaging.2010.05.023

69. Bechdolf A, Wood SJ, Nelson B, Velakoulis D, Yucel M, Takahashi T, et al. Amygdala and insula volumes prior to illness onset in bipolar disorder: a magnetic resonance imaging study. Psychiatry Res. (2012) 201:34-9. doi: 10.1016/j.pscychresns.2011.06.010

70. McGough EL, Kelly VE, Weaver KE, Logsdon RG, McCurry SM, Pike $\mathrm{KC}$, et al. Limbic and basal ganglia neuroanatomical correlates of gait and executive function: older adults with mild cognitive impairment and intact cognition. Am J Phys Med Rehabil. (2018) 97:229-35. doi: 10.1097/PHM.0000000000000881

71. Abu-Akel A, Shamay-Tsoory S. Neuroanatomical and neurochemical bases of theory of mind. Neuropsychologia. (2011) 49:2971-84. doi: 10.1016/j.neuropsychologia.2011.07.012

72. Li R, Wu X, Fleisher AS, Reiman EM, Chen K, Yao L. Attention-related networks in Alzheimer's disease: a resting functional MRI study. Hum Brain Mapp. (2012) 33:1076-88. doi: 10.1002/hbm.21269

73. Tijms BM, Wink AM, de Haan WW, van der Flier M, Stam CJ, Scheltens $\mathrm{P}$, et al. Alzheimer's disease: connecting findings from graph theoretical studies of brain networks. Neurobiol Aging. (2013) 34:2023-36. doi: 10.1016/j.neurobiolaging.2013.02.020

Conflict of Interest Statement: The authors declare that the research was conducted in the absence of any commercial or financial relationships that could be construed as a potential conflict of interest.

Copyright (๑ 2019 Hojjati, Ebrahimzadeh and Babajani-Feremi. This is an openaccess article distributed under the terms of the Creative Commons Attribution License (CC BY). The use, distribution or reproduction in other forums is permitted, provided the original author(s) and the copyright owner(s) are credited and that the original publication in this journal is cited, in accordance with accepted academic practice. No use, distribution or reproduction is permitted which does not comply with these terms. 


\section{OPEN ACCESS}

Edited by:

Nicoletta Cera

University of Porto, Portugal

Reviewed by:

Simon Maier,

University of Freiburg, Germany

Yanbin Jia,

The First Affiliated Hospital of Jinan

University, China

Roberta Rossi,

IRCCS Istituto Centro San Giovanni di

Dio Fatebenefratelli, Italy

*Correspondence:

Changlian Tan

tanchanglian@csu.edu.cn;

tanchanglianxy@aliyun.com

Jinyao Yi

jinyaoyi@csu.edu.cn

${ }^{\dagger}$ These authors have contributed equally to this work as co-first authors

Specialty section:

This article was submitted to

Brain Imaging Methods,

a section of the journal

Frontiers in Neuroscience

Received: 09 January 2019

Accepted: 29 August 2019

Published: 13 September 2019

Citation:

Lei X, Zhong M, Zhang B, Yang $H$, Peng W, Liu Q, Zhang Y, Yao S, Tan C

and Yi J (2019) Structural

and Functional Connectivity of the Anterior Cingulate Cortex in Patients With Borderline Personality Disorder.

Front. Neurosci. 13:971.

doi: 10.3389/fnins.2019.00971

\section{Structural and Functional Connectivity of the Anterior Cingulate Cortex in Patients With Borderline Personality Disorder}

\author{
Xiaoxia Lei'1t, Mingtian Zhong ${ }^{2 \dagger}$, Bowen Zhang ${ }^{2}$, Huihui Yang ${ }^{1}$, Wanrong Peng ${ }^{1}$, \\ Qian Liu ${ }^{2}$, Yu Zhang ${ }^{2}$, Shuqiao Yao ${ }^{1,3}$, Changlian Tan ${ }^{4 *}$ and Jinyao Yi1,3*
}

${ }^{1}$ Medical Psychological Center, The Second Xiangya Hospital, Central South University, Changsha, China, ${ }^{2}$ Center for Studies of Psychological Application, School of Psychology, South China Normal University, Guangzhou, China, ${ }^{3}$ Medical Psychological Institute, Central South University, Changsha, China, ${ }^{4}$ Department of Radiology, The Second Xiangya Hospital, Central South University, Changsha, China

Background: Emerging evidences supported the hypothesis that emotional dysregulation results from aberrant connectivity within the fronto-limbic neural networks in patients with borderline personality disorder (BPD). Considering its important role in emotional regulation, the anterior cingulate cortex (ACC) has not yet been fully explored in BPD patients. Therefore, using the seed-based resting state functional connectivity (rsFC) and probabilistic fiber tracking, we aimed to explore the alterations of functional and structural connectivity (SC) of the ACC in patients with BPD.

Methods: A cohort of 50 unmedicated, young BPD patients and 54 sex-, age-, and education-matched healthy controls (HCs) completed psychological tests and underwent rs-fMRI and diffuse tensor imaging (DTI) scanning. Rs-FC analysis and probabilistic fiber tracking were used to plot SC and FC of the ACC.

Results: With the left ACC selected as a seed, BPD patients exhibited increased rsFC and abnormal SC with the right middle frontal gyrus (MFG), and decreased rSFC with the left middle temporal gyrus (MTG), compared with HCs. Additionally, negative cognitive emotion regulation and depressive symptoms both correlated negatively with the rsFC of the left ACC in BPD patients.

Conclusion: Abnormal SC and FC of the ACC underlie the deficient emotional regulation circuitry in BPD patients. Such alterations may be important biomarkers of BPD and thus could point to potential BPD treatment targets.

\footnotetext{
Keywords: borderline personality disorder, emotion, anterior cingulate cortex, resting state functional connectivity, probabilistic fiber tracking
}

Abbreviations: ACC, anterior cingulate cortex; BPD, borderline personality disorder; CC, corpus collosum; DTI, diffusion tensor imaging; FA, fractional anisotropy; FC, functional connectivity; HCs, healthy controls; MD, mean diffusivity; MFG, middle frontal gyrus; MTG, middle temporal gyrus; PFC, prefrontal cortex; rs-fMRI, resting state functional magnetic resonance imaging; SC, structural connectivity; WM, white matter. 


\section{INTRODUCTION}

Borderline personality disorder is a common psychiatric disorder, characterized by a pervasive pattern of emotional lability, impulsivity, interpersonal difficulties, identity disturbances, and disturbed cognition (Leichsenring et al., 2011). Emotional dysregulation, which is considered a core pathological feature of BPD (Gratz and Roemer, 2004; Dsm-5, 2013), has been attributed to weakened inhibitory effects of the prefrontal cortex (PFC) on hyperactive limbic brain regions in response to emotional stimuli (Krause-Utz et al., 2014b; Schulze et al., 2016).

The PFC involvement in emotion regulation has been supported by neuroimaging studies in which control of emotionrelated behavior was related to the activation of several frontal regions, including the ACC, dorsolateral PFC, and ventral medial PFC (Koenigsberg et al., 2009; Krause-Utz et al., 2012; Kamphausen et al., 2013; Ruocco et al., 2013). For example, activation of the ACC and other prefrontal structures (e.g., dorsolateral PFC and medial or orbital PFC) has been found to be consistently decreased during emotional processing in $\mathrm{BPD}$ patients compared with HCs (Silbersweig et al., 2007; Kraus et al., 2010; Smoski et al., 2011). Importantly, decreased PFC activation was reported to be more pronounced in response to negative emotional stimuli than to neutral stimuli (Schulze et al., 2016).

Apart from aberrant functional alterations of the ACC and other frontal structures, there also have been reported structural and biochemical aberration in these frontal lobes in BPD studies. Decreased gray matter volume (Hazlett et al., 2005; Minzenberg et al., 2008; Schulze et al., 2016) and thickness (Rüsch et al., 2007) in ACC, and reduced gray matter volumes in the orbitofrontal cortex and dorsolateral PFC (Chanen et al., 2008; Soloff et al., 2012), have been reported in patients with BPD, compared with HCs. In addition, BPD patients have increased glutamate and $\mathrm{N}$-acetylaspartate concentrations in the left ACC (Hoerst et al., 2010; Rüsch et al., 2010a) and decreased $\mathrm{N}$-acetylaspartate concentrations in the dorsolateral PFC (Tebartz van Elst et al., 2001).

Previous neuroimaging studies on BPD suggested that abnormal functional activity in frontal regions might be interrelated rather than independent (New et al., 2007; Niedtfeld et al., 2012; Kamphausen et al., 2013; Krause-Utz et al., 2014a). The frontal lobes and the ACC in particular played important roles in emotional cognitive control, and their deactivation was related strongly to negative emotional processing in BPD patients (Bush et al., 2000). Anatomically, the ACC has rich structural and functional connections with other regions in the frontal lobes. For example, the subgenual cingulate gyrus (part of the ventral medial cingulate gyrus) receives afferent projections from the orbital cortex and has a close relationship with part of the orbitalfrontal cortex (Rüsch et al., 2010b). These connections help to integrate sensory information, coordinate autonomic nervous responses, and regulate emotion and behavior (Kringelbach, 2005). However, few studies have investigated alterations in SC or FC of emotion-related brain regions in BPD, especially the ACC. In one study investigating interhemispheric SC in BPD with a novel fiber-tracking approach, impaired connectivity between the left and right ACCs was observed (Rüsch et al., 2010b). Further investigations focusing on the ACC and other prefrontal areas involved in emotion regulation should be performed to confirm whether there is impaired interhemispheric connectivity in these patients (Rüsch et al., 2007, 2010b).

In this study, we used a multimodal approach combining rsFC and probabilistic fiber tracking with the bilateral ACC as seeds, to explore SC and FC of the ACC with other frontal regions, and how they relate to BPD-relevant psychological variables, namely negative affect intensity, negative cognitive emotion regulation strategies, depression, and anxiety. To exclude the influence of age, drug use, and comorbidity, we used a young BPD group with no antipsychotic drug use and no comorbidities. The severity of depression and anxiety symptoms was controlled as covariates to limit the influence of possible confounding effects.

\section{MATERIALS AND METHODS}

\section{Subjects}

Patients diagnosed with BPD were recruited from an outpatient clinic affiliated with the Second Xiangya Hospital of Central South University in Changsha, Hunan province, China. HCs were recruited by advertisements from the surrounding communities. BPD diagnostic assessments were performed by two wellqualified psychiatrists using the structured clinical interview for axis II disorders (SCID II, First and Gibbon, 2004) of the Diagnostic and Statistical Manual of Mental Disorders, Fourth Edition (DSM-IV). Each patient received the Structured Clinical Interview for DSM-IV Axis I disorders (SCID I, First and Gibbon, 2004) to exclude past or current Axis I diagnoses (e.g., major depressive disorder, bipolar disorder, schizophrenia, delusional disorder, and schizo-affective disorder). The exclusion criteria for HCs were past or current history of any DSM-IV axis I or axis II disorder, a history of epilepsy or serious trauma, current medical problems, and family history of psychiatric disorders among first-degree relatives.

The study cohort included 50 patients with BPD and 54 age-, sex-, and education-matched HCs. All participants completed the rs-fMRI and T1 scanning; and of the 104 participants, 46 BPD patients and 44 HCs were scanned with DTI sequences. This study was approved by the ethics committee of the Central South University, and each participant signed an informed consent form at enrollment.

\section{Scales}

Prior to neuroimaging, all participants underwent several psychometric assessments, including the Personality Diagnostic Questionnaire (PDQ-4+), Short Affect Intensity Scale (SAIS), Cognitive Emotion Regulation Questionnaire (CERQ), Center for Epidemiologic Studies Depression Scale (CES-D), and State-Trait Anxiety Inventory (STAI). The PDQ-4+, a selfrated personality disorder questionnaire, includes 12 subscales corresponding to 12 kinds of personality disorders. The borderline sub-scale contains nine items adopting 0-1 scoring method (i.e., Yes or No), and the total score ranges from 0 to 9 , which $\geq 5$ indicates a screened positive BPD (Hyler et al., 1992). The SAIS was used to assess affect intensity, which includes 
three dimensions (positive intensity, negative affectivity, and serenity; Geuens and De Pelsmacker, 2002; Zhong et al., 2010). Only the negative affectivity score (range, 1-6) assessing negative emotional reactions (e.g., nervousness, worry, sadness, and fear) was used in the current study. The CERQ is used to measure cognitive emotion regulation strategies with positive and negative subscales (Zhu et al., 2008). In the current study, only the negative subscale was used to assess subjects' negative emotion regulation strategies (e.g., self-blame, rumination, catastrophization, and blaming others). The STAI and the CES-D were used to assess anxiety and depression levels, respectively (Shek, 1993; Xiao et al., 2016).

\section{MRI Parameters}

Magnetic resonance imaging data were collected on a 3.0-T Philips Ingenia, in which foam pads were used to position and immobilize each subject's head within the coil. Highresolution anatomical T1-weighted scans were obtained with the following parameters: repetition time $(\mathrm{TR})=7.44 \mathrm{~ms}$; echo time $(\mathrm{TE})=3.46 \mathrm{~ms}$; flip angle $=8^{\circ}$; sagittal slices $=301$; slice thickness $=1.2 \mathrm{~mm}$; slice spacing $=0.6 \mathrm{~mm}$; matrix size $=240 \times 240$; volume $=1$; and voxel size $=1 \times 1 \times 1 \mathrm{~mm}^{3}$.

Resting state functional magnetic resonance imaging images were obtained with following parameters: TR $=2000 \mathrm{~ms}$; $\mathrm{TE}=30 \mathrm{~ms}$; flip angle $=90^{\circ}$; slices $=36$; slice thickness $=4 \mathrm{~mm}$; slice spacing $=4 \mathrm{~mm}$; matrix size $=128 \times 128$; volumes $=200$; volume interval $=2 \mathrm{~s}$; and voxel size $=2 \times 2 \times 2 \mathrm{~mm}^{3}$. While being scanned, all participants were asked to relax, keep their eyes closed, and stay awake.

Diffuse tensor imaging images were acquired with the following parameters: $\mathrm{TR}=6176 \mathrm{~ms}$; $\mathrm{TE}=79 \mathrm{~ms}$; flip angle $=90^{\circ}$; slices $=60$; slice thickness $=2.5 \mathrm{~mm}$; slice spacing $=2.5 \mathrm{~mm}$; and matrix size $=144 \times 144$, voxel size $=2 \times 2 \times 2 \mathrm{~mm}^{3}$. Diffusionsensitizing gradient encoding was applied in 32 directions with a diffusion-weighted factor of $b=700 \mathrm{~s} / \mathrm{mm}^{2}$ and two b0 $(b=0)$ images. Images were acquired parallel to the anterior and posterior commissure.

\section{Imaging Data Processing Rs-fMRI Processing}

Resting state functional magnetic resonance imaging data were analyzed in DPARSF (Data Processing Assistant for rsfMRI software ${ }^{1}$; Yan and Zang, 2010), based on Statistical Parametric Mapping $12\left(\mathrm{SPM}_{12}{ }^{2}\right.$ ) in MATLAB (Release 2017, The MathWorks, Inc., Natick, MA, United States). Data processing included the following steps: convert DICOM images to NIfTI files; remove the first 10 time points to minimize the influence of instability in the initial signals; slice timing with the 18th slice as the reference; spatial realignment for head motion correction (subjects with head motion $>2.0 \mathrm{~mm}$ or $>2.0^{\circ}$ were excluded); register each subject's fMRI images to their segmented high-resolution T1-weighted anatomical images; regress nuisance variables, including white matter (WM) and cerebral spinal fluid signals; normalize fMRI images to

${ }^{1}$ http://rfmri.org/DPARSF

${ }^{2}$ http://www.fil.ion.ucl.ac.uk/spm/ standard Montreal Neurological Institute (MNI) templates with a resolution of $3 \times 3 \times 3 \mathrm{~mm}^{3}$; smooth with a 4 - $\mathrm{mm}$ fullwidth-half-maximum Gaussian kernel; linear detrend to discard physiological noise and drift from scanner instabilities and head motion; band-pass filtering $(0.01-0.08 \mathrm{~Hz})$; and calculate rsFC with the bilateral ACC selected as seeds, which were made by the Anatomical Automatic Labeling 90 regions (AAL90) templates to quantify the relationship between the seeds and other brain regions.

\section{DTI Processing}

Diffuse tensor imaging data were processed on the FMRIB's Diffusion Toolbox-FDT v2.0 toolbox from FSL-FMRIB Software Library, FMRIB, Oxford, United Kingdom ${ }^{3}$ (Jenkinson et al., 2012). DTI data processing included the following steps: correct for eddy current distortion and head motion; fit a diffusion tensor model using the DTI fit in the FMRIB Diffusion Toolbox generating individual FA and MD; calculate withinvoxel probability density functions of the principal diffusion direction using FSL's BEDPOSTX tool using Markov Chain Monte Carlo sampling, which also accounts for the possibility of crossing fibers within a voxel (Woolrich et al., 2009); conduct probabilistic fiber tracking with PROBTRACKX implemented in FSL (Behrens et al., 2007), which repeatedly samples the distribution at each voxel to produce "streamlines" that connect voxels between selected seed regions (5000 streamline samples, $0.5 \mathrm{~mm}$ step length, curvature threshold $=0.2$ ).

\section{Probabilistic Fiber Tracking Analysis}

Probabilistic fiber tracking analysis was based on the rsFC results and mainly investigated SC between which had abnormal functional connectivities in rs-fMRI analysis, i.e., the left ACC was selected as the seed, and the right MFG and left MTG as the targets. All selections were made by the AAL90 templates in FSL. A single image (fdt_paths) per participant was generated to visualize WM tracts connecting seed and target regions after data processing. The total number of seed-to-target WM tracts (i.e., waytotal) was also generated to calculate the connective probability or tract strength, which defined as the number of tracts from the seed, divided by the number of tracts that reach the target (van den Bos et al., 2014; Yuan et al., 2018).

After probabilistic tracking, generated fdt_paths images were further thresholded based on the individual maximum connectivity value within a tract. The maximum connectivity value was obtained with fslstats and voxels, which had values of $>5 \%$ of the maximum connectivity value were kept in the analysis (Bennett et al., 2011; Hirsiger et al., 2016; Yuan et al., 2017). A common tract was created from tracts existed in $\geq 75 \%$ of the participants. Then, we extracted individual FA and MD of the WM tracts by de-projecting the common tracts to individual diffusion images (Ethofer et al., 2012; Gschwind et al., 2012).

\section{Statistical Analysis}

Statistical analyses were conducted in SPSS 20.0 (SPSS Inc., Chicago, IL, United States) and the SPM12 toolbox of MATLAB.

${ }^{3}$ https://fsl.fmrib.ox.ac.uk/fsl/fslwiki/FSL 
To compare group differences in demographic and psychological variables, Chi-squared tests and independent-sample $t$-tests were conducted. To investigate the significantly altered rsFC, two sample $t$-tests were performed in SPM12 with depression and anxiety scores as covariates. All resulting maps were familywise error (FWE) corrected for the whole brain. To explore potential group differences in SC (i.e., probability) and integrity (i.e., FA and MD), analysis of covariance (ANCOVA) was performed with age, depression, and anxiety levels controlled. Pearson's correlations were conducted to investigate potential associations between emotional characteristics (i.e., psychometric scores including SAIS_N, CERQ_N, CES-D, TAI, and SAI) and neuroimaging indices (i.e., rsFC values, tract probability, mean $\mathrm{FA}$, and $\mathrm{MD}$ ) in the BPD group. A threshold of $P<0.05$ was considered significant.

\section{RESULTS}

\section{Demographic and Psychological Characteristics}

Age, sex, and education level did not differ significantly between the BPD and HC groups. The BPD group had significantly higher SAIS negative affectivity, CERQ negative subscale, CES-D, and STAI scores than the HC group (Table 1).

\section{RsFC}

Borderline personality disorder patients exhibited significantly increased rsFC of the ACC with the right MFG and decreased rsFC with the left MTG compared with HCs. In addition, the BPD group had lower connectivities between both ACC sides and the corpus collosum (CC) than HCs (Table 2 and Figure 1).

\section{SC}

Compared with HCs, the BPD group had significantly decreased FA values in left ACC-to-right MFG tract (Table 3 and

TABLE 1 | Inter-group comparisons of demographic and psychological characteristics.

\begin{tabular}{|c|c|c|c|c|}
\hline Characteristic & $\begin{array}{l}\text { BPD group } \\
(N=50)\end{array}$ & $\begin{array}{l}\text { HC group } \\
(N=54)\end{array}$ & $t / \chi^{2}$ & $P$ \\
\hline Age & $25.33 \pm 2.93$ & $24.83 \pm 1.37$ & 1.11 & 0.27 \\
\hline Sex ratio, male/female & $25 / 25$ & $21 / 33$ & 1.3 & 0.25 \\
\hline Education (y) & $15.82 \pm 1.05$ & $16.21 \pm 1.28$ & -1.68 & 0.09 \\
\hline PDQ-4+_borderline & $3.89 \pm 1.98$ & $0.76 \pm 0.97$ & 9.42 & $<0.01$ \\
\hline SAIS_N & $4.29 \pm 0.82$ & $3.49 \pm 0.65$ & 5.53 & $<0.01$ \\
\hline CERQ_N & $40.70 \pm 5.66$ & $34.78 \pm 5.04$ & 8.07 & $<0.01$ \\
\hline CES-D & $37.32 \pm 10.20$ & $28.70 \pm 5.67$ & 4.42 & $<0.01$ \\
\hline SAI & $37.72 \pm 10.09$ & $29.36 \pm 6.07$ & 4.35 & $<0.01$ \\
\hline TAl & $43.57 \pm 8.71$ & $32.78 \pm 5.22$ & 6.21 & $<0.01$ \\
\hline
\end{tabular}

PDQ-4+, Personality Diagnostic Questionnaire; SAIS_N, negative affectivity score of Short Affect Intensity Scale; CERQ_N, negative subscale of Cognitive Emotion Regulation Questionnaire; CES-D, Center for Epidemiologic Studies Depression Scale; SAI, State Anxiety Inventory; TAl, Trait Anxiety Inventory. ${ }^{a}$ Chi-square test; all other t-tests.
TABLE 2 | Significant differences in FC between BPD patients and HCs.

\begin{tabular}{|c|c|c|c|c|c|}
\hline \multirow[t]{2}{*}{ Cluster } & \multirow[t]{2}{*}{$P_{-\mathrm{FWE}-\mathrm{corr}}$} & \multirow[t]{2}{*}{$\begin{array}{l}\text { Brain region/ } \\
\text { fasciculus }\end{array}$} & \multirow[t]{2}{*}{ Voxels } & \multirow[t]{2}{*}{$t$} & \multirow{2}{*}{$\begin{array}{c}\text { MNI } \\
\text { coordinates } \\
(X, Y, Z)\end{array}$} \\
\hline & & & & & \\
\hline \multicolumn{6}{|c|}{ ACC_L as a seed } \\
\hline $\mathrm{FC} \uparrow$ Cluster1 & 0.041 & Frontal_Mid_R & 42 & 4.85 & $27,36,24$ \\
\hline $\mathrm{FC} \downarrow$ Cluster2 & 0.037 & Temporal_Mid_L & 43 & -5.41 & $-45,-45,0$ \\
\hline FC $\downarrow$ Cluster3 & 0.026 & Corpus callosum & 47 & -4.85 & $6,3,24$ \\
\hline \multicolumn{6}{|c|}{ ACC_R as a seed } \\
\hline FC $\downarrow$ Cluster1 & 0.013 & Corpus callosum & 56 & -5.13 & $6,3,24$ \\
\hline
\end{tabular}

Figure 2), findings suggestive of impaired fiber integrity. The tract probability of left ACC-to-right MFG and left ACC-to-left MTG tract did not differ between the two groups.

\section{Associations Between Imaging Parameters and Psychological Variables in BPD Patients}

Pearson correlation analyses showed that CERQ-negative subscale scores correlated negatively with rsFC between the left ACC and CC $(r=-0.59, P=0.032)$, and CES-D scores correlated negatively with rsFC between the left ACC and right MFG $(r=-0.37, P=0.037)$ after multiple comparisons with Bonferroni correction. There were no other significant correlations between psychological variables (negative affective SAIS or STAI) and imaging parameters (rsFC values, tract probability, mean FA, or MD).

\section{DISCUSSION}

In the current study, combining rsFC and probabilistic fiber tracking with the bilateral ACC as seeds, we explored the SC and FC of the ACC and examined correlations between abnormal neuroimaging indices and psychological variables in BPD patients. We found that patients with BPD exhibited abnormal SC and FC in the emotion-regulation-related ACC$\mathrm{CC}$-frontal neural circuit. These findings suggest that abnormal SC and FC in frontal-limbic structures of the brain could mediate emotional dysregulation in patients with BPD.

\section{SC and FC Between the Left ACC and Right MFG}

The ACC and PFC are key brain regions in emotional regulation and impulsiveness (Davidson and Irwin, 1999). Previous ontask fMRI studies utilizing emotion regulation strategies (e.g., restraint and reappraisal) have suggested that the ACC and other frontal regions (i.e., orbital frontal cortex, dorsolateral PFC, dorsomedial PFC, and ventrolateral PFC) are involved in emotional regulation processing (Beauregard et al., 2001; Lévesque et al., 2003; Ochsner et al., 2004; Phan et al., 2005; 


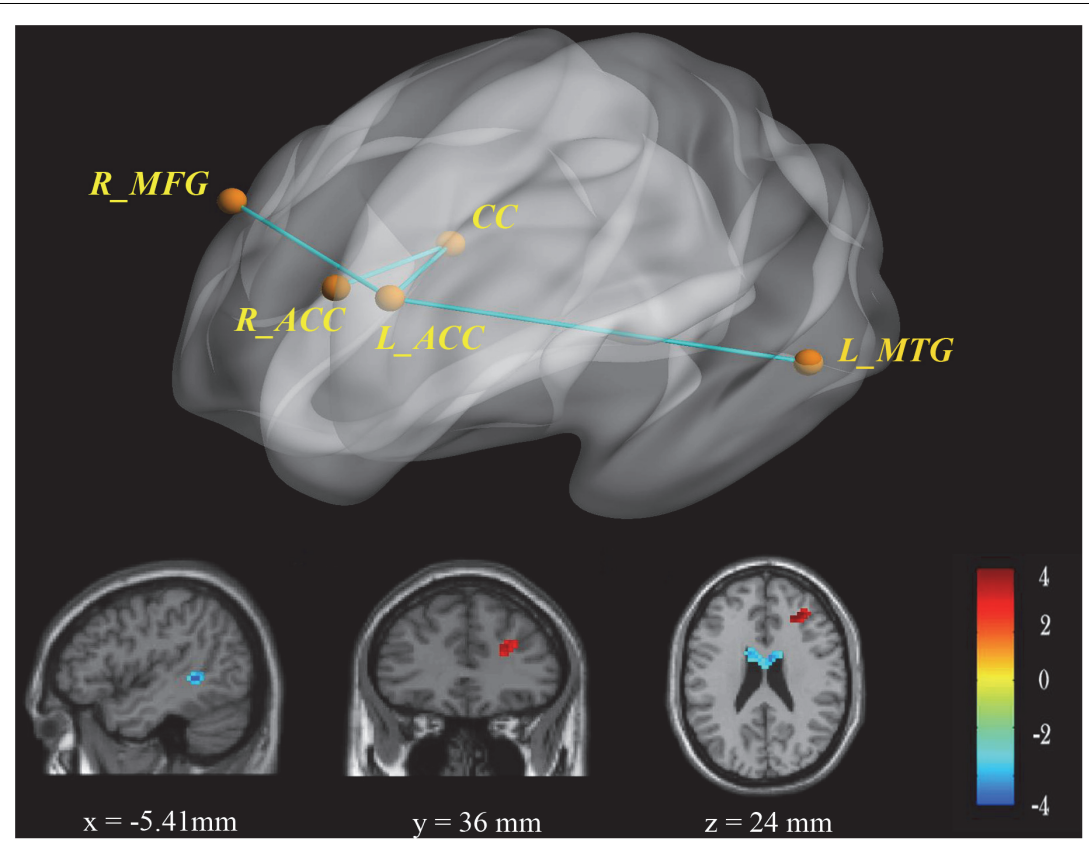

FIGURE 1 | RsFC of the bilateral ACC as seeds. There was increased (red) rsFC between the left ACC and right MFG, as well as decreased (blue) rsFC between the left ACC and left MTG in the BPD group compared with the HC group. FWE-corrected $p<0.05$ significance criterion. Color bar indicates the T-score.

Urry et al., 2006). More specifically, the MFG, a component of the dorsolateral PFC, is particularly important in mood regulation and suppression of unwanted memories (Kluetsch et al., 2012; Krause-Utz et al., 2014a). In the current study, we found increased left ACC-to-right MFG FC in patients with BPD, consistent with previous findings (Krause-Utz et al., 2012). Based on rsFC data, we plotted the left ACC-to-right MFG fiber bundle in BPD patients using probabilistic fiber tracking and found that left ACC efferents passed through the CC and projected to the contralateral MFG, consistent with normal anatomy (Rüsch et al., 2010b). Importantly, we found that WM integrity from the left ACC to the right MFG was impaired with decreased FA in the BPD group compared with the HC group. The presently observed altered rsFC and impaired WM fasciculus integrity between the ACC and MFG suggest that emotional regulation circuitry may be deficient in BPD patients.

\section{FC Between the Left ACC and Left MTG}

Alterations in the brain default model network (DMN), including the medial PFC, ACC, cuneus/precuneus, MTG, thalamus, and insular cortex, which are located mainly along the midline of the brain, were found in a prior rsfMRI study in BPD patients (Wolf et al., 2011). The ACC and MTG are important for numerous important DMN functions, including: separating internal cognition from external stimulus processing; autobiographical memory processing; and monitoring of cognitive, emotional, and somatosensory states (Buckner et al., 2008; Spreng et al., 2009; Napadow et al., 2010). In a previous rs-fMRI study, we found decreased connectivityamplitude coupling in the left MTG, suggesting that the left MTG may be a functionally impaired hub in BPD (Lei et al., 2018).
The MTG also has decreased rs activation (Wingenfeld et al., 2009) and increased activity during emotional processing in BPD patients (Guitart-Masip et al., 2009). Together with previous studies (Wolf et al., 2011; Lei et al., 2017), our study found decreased rsFC between the left ACC and the left MTG in BPD patients, which suggested disturbed DMN and emotional processing in patients with BPD.

\section{SC and FC Between the ACC and CC}

The CC mediates communication between the hemispheres (Luders et al., 2010). The WM integrity of the CC genu and body has previously been shown to be impaired in BPD patients (Salvador et al., 2016). Moreover, the CC isthmus was found to

TABLE 3 | Comparison of probabilistic fiber tracking between BPD patients and $\mathrm{HCs}$.

\begin{tabular}{llcccc}
\hline Seed to target & $\begin{array}{l}\text { Fiber } \\
\text { metric }\end{array}$ & $\begin{array}{c}\text { BPD } \\
\text { group } \\
(\mathbf{N}=\mathbf{4 6})\end{array}$ & $\begin{array}{c}\text { HC group } \\
(\mathbf{N}=\mathbf{4 4})\end{array}$ & $\boldsymbol{F}$ & $\boldsymbol{P}$ \\
\hline ACC_Lto & FA & $0.35 \pm 0.10$ & $0.37 \pm 0.02$ & -7.85 & $<0.01$ \\
Frontal_mid_R & MD $\left(\times 10^{-3}\right)$ & $0.89 \pm 0.03$ & $0.91 \pm 0.05$ & -3.04 & 0.08 \\
& Probability & $0.11 \pm 0.16$ & $0.09 \pm 0.11$ & 0.23 & 0.6 \\
& $\left(\times 10^{-1}\right)$ & & & & \\
ACC_Lto & FA & $0.31 \pm 0.01$ & $0.32 \pm 0.01$ & 0.11 & 0.74 \\
Temporal_mid_L & MD $\left(\times 10^{-3}\right)$ & $0.81 \pm 0.02$ & $0.81 \pm 0.02$ & -2.63 & 0.11 \\
& Probability & $0.02 \pm 0.42$ & $0.02 \pm 0.06$ & -1.52 & 0.22 \\
& $\left(\times 10^{-2}\right)$ & & & & \\
\hline
\end{tabular}

$B P D$, borderline personality disorder; HC, healthy controls; $A C C \_L$, left anterior cingulate cortex; Frontal_Mid_R, right frontal middle gyrus; Temporal_Mid_L, left temporal middle gyrus; FA, fractional anisotropy; $M D$, mean diffusivity. 


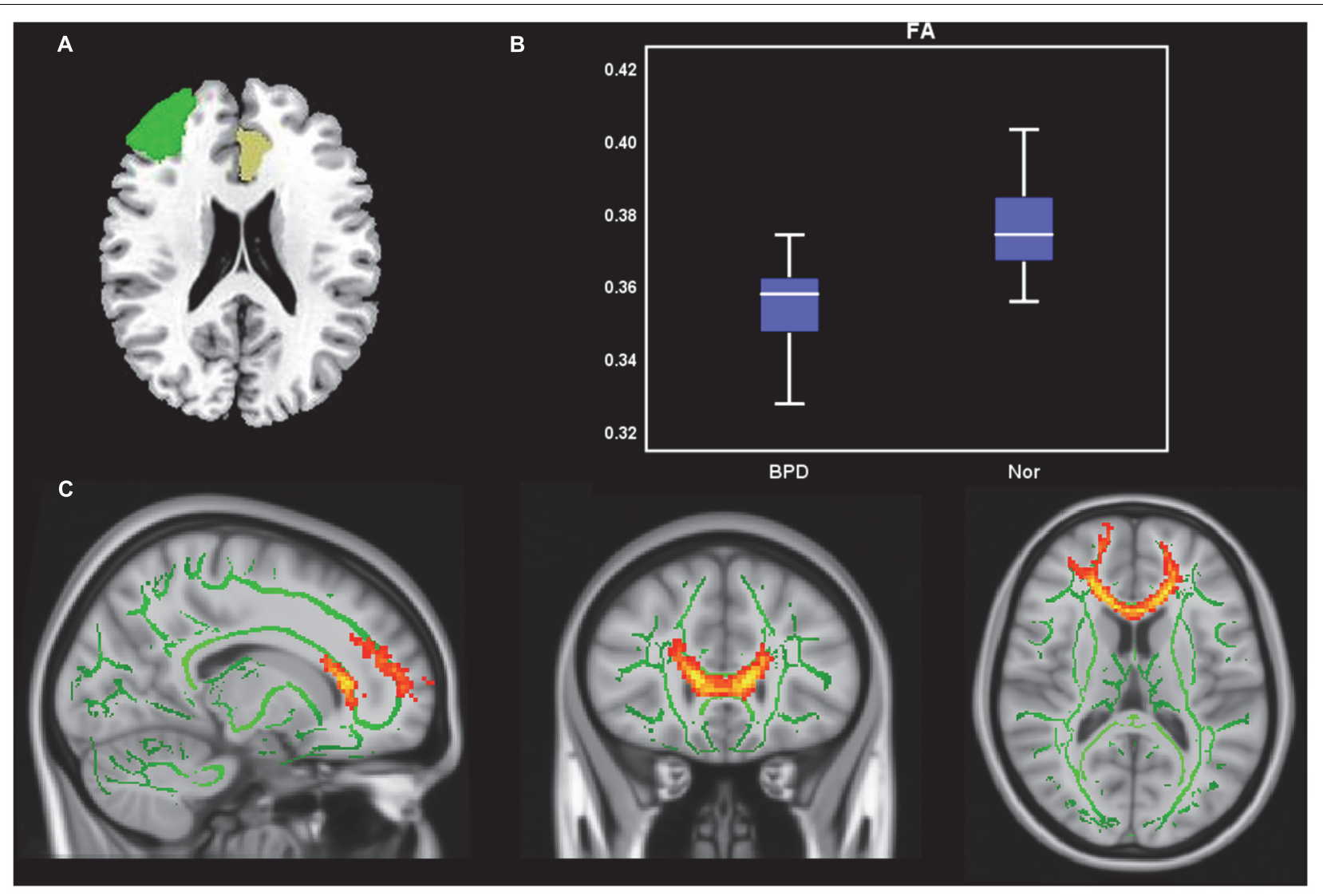

FIGURE 2 | Probabilistic fiber tracking from the left ACC to the right MFG. (A) The seed (left ACC, yellow) and target (right MFG, green) were based on the AAL90 template and back-projected to the individual's native space in sagittal, coronal, and axial planes. (B) Group comparisons of FA and MD values for fiber bundles passing though the left ACC to the right MFG $(p<0.05)$. $Y$-axis presents the mean of FA and MD values. The error bars present the standard error of mean. (C) Fiber distribution: fibers starting from the left ACC passed through the CC and the right ACC and then entered the right MFG.

be thinner in BPD patients than in HCs, suggesting that interhemispheric SC may be affected in BPD (Rüsch et al., 2007). The present finding of decreased rsFC between the bilateral ACC and the CC is consistent with the possibility that SC between the cerebral hemispheres may be abnormal in BPD patients. Because the ACC is involved in regulating emotion and behavior (Minzenberg et al., 2007; Silbersweig et al., 2007; Koenigsberg et al., 2009; Niedtfeld et al., 2012; Perez et al., 2016; Schulze et al., 2016), decreased interhemispheric connectivity between the left and right ACCs may be a pathological correlate of BPD.

\section{Psychometric Correlates of ACC Abnormalities in BPD}

Patients with BPD have been shown consistently to be hypersensitive to negative emotional stimuli, with emotional dysregulation and negative regulatory strategies that are often accompanied by anxiety and depression (Yen et al., 2002; Niedtfeld et al., 2010; Schulze et al., 2016; Yang et al., 2018). The present findings of significantly higher SAISnegative affectivity, CERQ-negative subscale, CES-D, and STAI scores in BPD patients than HCs are consistent with heightened negative intensity, depression, and anxiety levels as well as reliance on negative emotion cognition regulation strategies in BPD.

A frontal-limbic system suppression model in which there is reduced PFC control of hyperactive limbic areas has emerged as a favored hypothesis of the neurological basis of emotional dysregulation in BPD (Agrawal et al., 2004). More specifically, amygdalar hyperactivity with hypoactivity in the ACC and PFC (medial, orbital, and dorsolateral) is typical during negative emotional processes in BPD (Silbersweig et al., 2007; Perez et al., 2016; Baczkowski et al., 2017). In this study, cognitive emotion regulation scores correlated negatively with left ACC$\mathrm{CC}$ rsFC, while depressive scores correlated negatively with left ACC-right MFG rsFC. These results provide further support for the view that emotional regulation circuitry may be impaired in BPD patients.

\section{Limitations}

Although we combined rsFC and SC analyses to investigate links between function and structure in BPD, which had not been thoroughly assessed before, this study had some limitations. Firstly, FC was assessed in a rs rather than in an on-task state. We are planning future studies that will focus on FC 
during the performance of emotion-related tasks. Secondly, because this study focused primarily on the emotion-related ACC and associated frontal regions, connectivities among other emotion-related limbic structures (e.g., amygdala) remain to be investigated further to provide a more comprehensive understanding of brain connectivity in BPD.

\section{CONCLUSION}

In this study, using rs-fMRI and probabilistic fiber tracking, we combined SC and FC data to investigate BPD alterations in ACC-based circuitry. With the left ACC selected as a seed, BPD patients exhibited increased rsFC and abnormal SC with the right MFG, and decreased rsFC with the left MTG, compared with HCs. Further, rsFC of the left ACC correlated negatively with negative cognitive emotion regulation and depressive symptoms in BPD patients. Given that the ACC plays an important role in emotional cognitive control, abnormal connectivity of the ACC supports the possibility that BPD may be characterized by deficient emotional regulation circuitry. Collectively, our data suggest that connectivity of these brain regions may be important

\section{REFERENCES}

Agrawal, H. R., Gunderson, J., Holmes, B. M., and Lyons-Ruth, K. (2004). Attachment studies with borderline patients: a review. Harv. Rev. Psychiatry 12, 94-104. doi: 10.1080/10673220490447218

Baczkowski, B. M., van Zutphen, L., Siep, N., Jacob, G. A., Domes, G., Maier, S., et al. (2017). Deficient amygdala-prefrontal intrinsic connectivity after effortful emotion regulation in borderline personality disorder. Eur. Arch. Psychiatry Clin. Neurosci. 267, 551-565. doi: 10.1007/s00406-016-0760-z

Beauregard, M., Lévesque, J., and Bourgouin, P. (2001). Neural correlates of conscious self-regulation of emotion. J. Neurosci. 21, 1-6.

Behrens, T. E. J., Berg, H. J., Jbabdi, S., Rushworth, M. F. S., and Woolrich, M. W. (2007). Probabilistic diffusion tractography with multiple fibre orientations: what can we gain? Neuroimage 34, 144-155. doi: 10.1016/j.neuroimage.2006. 09.018

Bennett, I. J., Madden, D. J., Vaidya, C. J., Howard, J. H., and Howard, D. V. (2011). White matter integrity correlates of implicit sequence learning in healthy aging. Neurobiol. Aging 32, 2317.e1-2317.e12. doi: 10.1016/j.neurobiolaging.2010. 03.017

Buckner, R. L., Andrews-Hanna, J. R., and Schacter, D. L. (2008). The brain's default network: anatomy, function, and relevance to disease. Ann. N. Y. Acad. Sci. 1124, 1-38. doi: 10.1196/annals.1440.011

Bush, G., Luu, P., and Posner, M. I. (2000). Bush_2000_conitive and emotional influence in anterior cingulate cortex. Trends Cogn. Sci. 4, 215-222. doi: 10. 1016/s1364-6613(00)01483-2

Chanen, A. M., Velakoulis, D., Carison, K., Gaunson, K., Wood, S. J., Yuen, H. P., et al. (2008). Orbitofrontal, amygdala and hippocampal volumes in teenagers with first-presentation borderline personality disorder. Psychiatry Res. - Neuroimaging 163, 116-125. doi: 10.1016/j.pscychresns.2007.08.007

Davidson, R. J., and Irwin, W. (1999). The functional neuroanatomy of emotion and affective style. Trends Cogn. Sci. 3, 11-21. doi: 10.1016/S1364-6613(98) $01265-1260$

Dsm-5 (2013). Diagnostic and Statistical Manual of Mental Disorders (DSM-5), 5th Edn. Washington, DC: American Psychiatric Association.

Ethofer, T., Bretscher, J., Gschwind, M., Kreifelts, B., Wildgruber, D., and Vuilleumier, P. (2012). Emotional voice areas: anatomic location, functional properties, and structural connections revealed by combined fMRI/DTI. Cereb. Cortex 22, 191-200. doi: 10.1093/cercor/bhr113 imaging biomarkers in BPD populations, which could have clinical implications for treatment of the disorder.

\section{ETHICS STATEMENT}

This study was approved by the ethics committee of the Central South University, and each participant signed an informed consent form at enrollment.

\section{AUTHOR CONTRIBUTIONS}

$\mathrm{XL}$ analyzed the data and wrote the manuscript. BZ, HY, WP, QL, and YZ collected the data. MZ, CT, and JY gave the idea and revised the manuscript. SY also helped the manuscript in revision.

\section{FUNDING}

This study was supported by grant from the National Natural Science Foundation of China (Grant No. 81370034).

First, M. B., and Gibbon, M. (2004). "The structured clinical interview for DSMIV axis i disorders (SCID-I) and the structured clinical interview for DSMIV axis II disorders (SCID-II),"in Comprehensive Handbook Of Psychological Assessment Personality Assessment, M. J. Hilsenroth, D. L. Segal, (Hoboken, NJ: John Wiley \& Sons Inc), 134-143.

Geuens, M., and De Pelsmacker, P. (2002). Developing a short affect intensity scale. Psychol. Rep. 91, 657-670. doi: 10.2466/pr0.2002.91.2.657

Gratz, K. L., and Roemer, L. (2004). Multidimensional assessment of emotion regulation and dysregulation: development, factor structure, and initial validation of the difficulties in emotion regulation scale. J. Psychopathol. Behav. Assess. 26, 41-54. doi: 10.1023/B:JOBA.0000007455.08539.94

Gschwind, M., Pourtois, G., Schwartz, S., Van De Ville, D., and Vuilleumier, P. (2012). White-matter connectivity between face-responsive regions in the human brain. Cereb. Cortex 22, 1564-1576. doi: 10.1093/cercor/bhr226

Guitart-Masip, M., Pascual, J. C., Carmona, S., Hoekzema, E., Bergé, D., Pérez, V., et al. (2009). Neural correlates of impaired emotional discrimination in borderline personality disorder: an fMRI study. Prog. Neuro Psychopharmacol. Biol. Psychiatry 33, 1537-1545. doi: 10.1016/j.pnpbp.2009.08.022

Hazlett, E. A., New, A. S., Newmark, R., Haznedar, M. M., Lo, J. N., Speiser, L. J., et al. (2005). Reduced anterior and posterior cingulate gray matter in borderline personality disorder. Biol. Psychiatry 58, 614-623. doi: 10.1016/j.biopsych.2005. 04.029

Hirsiger, S., Koppelmans, V., Mérillat, S., Liem, F., Erdeniz, B., Seidler, R. D., et al. (2016). Structural and functional connectivity in healthy aging: associations for cognition and motor behavior. Hum. Brain Mapp. 37, 855-867. doi: 10.1002/ hbm. 23067

Hoerst, M., Weber-Fahr, W., Tunc-Skarka, N., Ruf, M., Bohus, M., Schmahl, C., et al. (2010). Correlation of glutamate levels in the anterior cingulate cortex with self-reported impulsivity in patients with borderline personality disorder and healthy controls. Arch. Gen. Psychiatry 67, 946-954. doi: 10.1001/ archgenpsychiatry.2010.93

Hyler, S. E., Skodol, A. E., Oldham, J. M., Kellman, H. D., and Doidge, N. (1992). Validity of the personality diagnostic questionnaire-revised: a replication in an outpatient sample. Compr. Psychiatry 33, 73-77. doi: 10.1016/0010-440X(92) 90001-90007

Jenkinson, M., Beckmann, C. F., Behrens, T. E. J., Woolrich, M. W., and Smith, S. M. (2012). FSL. Neuroimage 62, 782-790. doi: 10.1016/j.neuroimage.2011. 09.015 
Kamphausen, S., Schröder, P., Maier, S., Bader, K., Feige, B., Kaller, C. P., et al. (2013). Medial prefrontal dysfunction and prolonged amygdala response during instructed fear processing in borderline personality disorder. World J. Biol. Psychiatry 14, 307-318. doi: 10.3109/15622975.2012. 665174

Kluetsch, R. C., Schmahl, C., Niedtfeld, I., Densmore, M., Calhoun, V. D., Daniels, J., et al. (2012). Alterations in default mode network connectivity during pain processing in borderline personality disorder. Arch. Gen. Psychiatry 69, 993-1002. doi: 10.1001/archgenpsychiatry.2012.476

Koenigsberg, H. W., Siever, L. J., Lee, H., Pizzarello, S., New, A. S., Goodman, M., et al. (2009). Neural correlates of emotion processing in borderline personality disorder. Psychiatry Res. Neuroimaging 172, 192-199. doi: 10.1016/ j.pscychresns.2008.07.010

Kraus, A., Valerius, G., Seifritz, E., Ruf, M., Bremner, J. D., Bohus, M., et al. (2010). Script-driven imagery of self-injurious behavior in patients with borderline personality disorder: a pilot FMRI study. Acta Psychiatr. Scand. 121, 41-51. doi: 10.1111/j.1600-0447.2009.01417.x

Krause-Utz, A., Oei, N. Y. L., Niedtfeld, I., Bohus, M., Spinhoven, P., Schmahl, C., et al. (2012). Influence of emotional distraction on working memory performance in borderline personality disorder. Psychol. Med. 42, 2181-2192. doi: 10.1017/S0033291712000153

Krause-Utz, A., Veer, I. M., Rombouts, S. A. R. B., Bohus, M., Schmahl, C., and Elzinga, B. M. (2014a). Amygdala and anterior cingulate resting-state functional connectivity in borderline personality disorder patients with a history of interpersonal trauma. Psychol. Med. 44, 2889-2901. doi: 10.1017/ S0033291714000324

Krause-Utz, A., Winter, D., Niedtfeld, I., and Schmahl, C. (2014b). The latest neuroimaging findings in borderline personality disorder topical collection on personality disorders. Curr. Psychiatry Rep. 16:348. doi: 10.1007/s11920-0140438-z

Kringelbach, M. L. (2005). The human orbitofrontal cortex: linking reward to hedonic experience. Nat. Rev. Neurosci. 6, 691-702. doi: 10.1038/nrn1747

Lei, X., Liao, Y., Zhong, M., Peng, W., Liu, Q., Yao, S., et al. (2018). Functional connectivity density, local brain spontaneous activity, and their coupling strengths in patients with borderline personality disorder. Front. Psychiatry 9:342. doi: 10.3389/fpsyt.2018.00342

Lei, X., Zhong, M., Liu, Y., Jin, X., Zhou, Q., Xi, C., et al. (2017). A restingstate fMRI study in borderline personality disorder combining amplitude of low frequency fluctuation, regional homogeneity and seed based functional connectivity. J. Affect. Disord. 218, 299-305. doi: 10.1016/j.jad.2017.04.067

Leichsenring, F., Leibing, E., Kruse, J., New, A. S., and Leweke, F. (2011). Borderline personality disorder. Lancet 377, 74-84. doi: 10.1016/S0140-6736(10)6142261425

Lévesque, J., Eugène, F., Joanette, Y., Paquette, V., Mensour, B., Beaudoin, G., et al. (2003). Neural circuitry underlying voluntary suppression of sadness. Biol. Psychiatry 53, 502-510. doi: 10.1016/S0006-3223(02)01817-1816

Luders, E., Thompson, P. M., and Toga, A. W. (2010). The development of the corpus callosum in the healthy human brain. J. Neurosci. 30, 10985-10990. doi: 10.1523/JNEUROSCI.5122-09.2010

Minzenberg, M. J., Fan, J., New, A. S., Tang, C. Y., and Siever, L. J. (2007). Frontolimbic dysfunction in response to facial emotion in borderline personality disorder: an event-related fMRI study. Psychiatry Res. Neuroimaging 155, 231243. doi: 10.1016/j.pscychresns.2007.03.006

Minzenberg, M. J., Fan, J., New, A. S., Tang, C. Y., and Siever, L. J. (2008). Frontolimbic structural changes in borderline personality disorder. J. Psychiatr. Res. 42, 727-733. doi: 10.1016/j.jpsychires.2007.07.015

Napadow, V., LaCount, L., Park, K., As-Sanie, S., Clauw, D. J., and Harris, R. E. (2010). Intrinsic brain connectivity in fibromyalgia is associated with chronic pain intensity. Arthritis Rheum. 62, 2545-2555. doi: 10.1002/art. 27497

New, A. S., Hazlett, E. A., Buchsbaum, M. S., Goodman, M., Mitelman, S. A., Newmark, R., et al. (2007). Amygdala-prefrontal disconnection in borderline personality disorder. Neuropsychopharmacology 32, 1629-1640. doi: 10.1038/sj. npp. 1301283

Niedtfeld, I., Kirsch, P., Schulze, L., Herpertz, S. C., Bohus, M., and Schmahl, C. (2012). Functional connectivity of pain-mediated affect regulation in borderline personality disorder. PLoS One 7:e33293. doi: 10.1371/journal.pone.003 3293
Niedtfeld, I., Schulze, L., Kirsch, P., Herpertz, S. C., Bohus, M., and Schmahl, C. (2010). Affect regulation and pain in borderline personality disorder: a possible link to the understanding of self-injury. Biol. Psychiatry 68, 383-391. doi: 10.1016/j.biopsych.2010.04.015

Ochsner, K. N., Ray, R. D., Cooper, J. C., Robertson, E. R., Chopra, S., Gabrieli, J. D. E., et al. (2004). For better or for worse: neural systems supporting the cognitive down- and up-regulation of negative emotion. Neuroimage 23, 483-499. doi: 10.1016/j.neuroimage.2004.06.030

Perez, D. L., Vago, D. R., Pan, H., Root, J., Tuescher, O., Fuchs, B. H., et al. (2016). Frontolimbic neural circuit changes in emotional processing and inhibitory control associated with clinical improvement following transference-focused psychotherapy in borderline personality disorder. Psychiatry Clin. Neurosci. 70, 51-61. doi: 10.1111/pcn.12357

Phan, K. L., Fitzgerald, D. A., Nathan, P. J., Moore, G. J., Uhde, T. W., and Tancer, M. E. (2005). Neural substrates for voluntary suppression of negative affect: a functional magnetic resonance imaging study. Biol. Psychiatry 57, 210-219. doi: 10.1016/j.biopsych.2004.10.030

Ruocco, A. C., Amirthavasagam, S., Choi-Kain, L. W., and McMain, S. F. (2013). Neural correlates of negative emotionality in borderline personality disorder: an activation-likelihood-estimation meta-analysis. Biol. Psychiatry 73, 153-160. doi: 10.1016/j.biopsych.2012.07.014

Rüsch, N., Boeker, M., Büchert, M., Glauche, V., Bohrmann, C., Ebert, D., et al. (2010a). Neurochemical alterations in women with borderline personality disorder and comorbid attention-deficit hyperactivity disorder. World J. Biol. Psychiatry 11, 372-381. doi: 10.3109/15622970801958331

Rüsch, N., Bracht, T., Kreher, B. W., Schnell, S., Glauche, V., Il'yasov, K. A., et al. (2010b). Reduced interhemispheric structural connectivity between anterior cingulate cortices in borderline personality disorder. Psychiatry Res. Neuroimaging 181, 151-154. doi: 10.1016/j.pscychresns.2009.08.004

Rüsch, N., Luders, E., Lieb, K., Zahn, R., Ebert, D., Thompson, P. M., et al. (2007). Corpus callosum abnormalities in women with borderline personality disorder and comorbid attention-deficit hyperactivity disorder. J. Psychiatry Neurosci. 32, 417-422.

Salvador, R., Vega, D., Pascual, J. C., Marco, J., Canales-Rodríguez, E. J., Aguilar, S., et al. (2016). Converging medial frontal resting state and diffusion-based abnormalities in borderline personality disorder. Biol. Psychiatry 79, 107-116. doi: 10.1016/j.biopsych.2014.08.026

Schulze, L., Schmahl, C., and Niedtfeld, I. (2016). Neural correlates of disturbed emotion processing in borderline personality disorder: a multimodal metaanalysis. Biol. Psychiatry 79, 97-106. doi: 10.1016/j.biopsych.2015.03.027

Shek, D. T. L. (1993). The chinese version of the state-trait anxiety inventory: its relationship to different measures of psychological well-being. J. Clin. Psychol. 49, 349-358. doi: 10.1002/1097-4679(199305)49:3<349::aid-jclp2270490308> 3.0.co; $2-\mathrm{j}$

Silbersweig, D., Clarkin, J. F., Goldstein, M., Kernberg, O. F., Tuescher, O., Levy, K. N., et al. (2007). Failure of frontolimbic inhibitory function in the context of negative emotion in borderline personality disorder. Am. J. Psychiatry 164, 1832-1841. doi: 10.1176/appi.ajp.2007.06010126

Smoski, M. J., Salsman, N., Wang, L., Smith, V., Lynch, T. R., Dager, S. R., et al. (2011). Functional imaging of emotion reactivity in opiate-dependent borderline personality disorder. Personal. Disord. Theory, Res. Treat. 2, 230241. doi: 10.1037/a0022228

Soloff, P. H., Pruitt, P., Sharma, M., Radwan, J., White, R., and Diwadkar, V. A. (2012). Structural brain abnormalities and suicidal behavior in borderline personality disorder. J. Psychiatr. Res. 46, 516-525. doi: 10.1016/j.jpsychires. 2012.01.003

Spreng, R. N., Mar, R. A., and Kim, A. S. N. (2009). The common neural basis of autobiographical memory, prospection, navigation, theory of mind, and the default mode: a quantitative meta-analysis. J. Cogn. Neurosci. 21, 489-510. doi: 10.1162/jocn.2008.21029

Tebartz van Elst, L., Thiel, T., Hesslinger, B., Lieb, K., Bohus, M., Hennig, J., et al. (2001). Subtle prefrontal neuropathology in a pilot magnetic resonance spectroscopy study in patients with borderline personality disorder. J. Neuropsychiatry Clin. Neurosci. 13, 511-514. doi: 10.1176/appi.neuropsych. 13.4.511

Urry, H. L., Van Reekum, C. M., Johnstone, T., Kalin, N. H., Thurow, M. E., Schaefer, H. S., et al. (2006). Amygdala and ventromedial prefrontal cortex are inversely coupled during regulation of negative affect and predict the diurnal 
pattern of cortisol secretion among older adults. J. Neurosci. 26, 4415-4425. doi: 10.1523/JNEUROSCI.3215-05.2006

van den Bos, W., Rodriguez, C. A., Schweitzer, J. B., and McClure, S. M. (2014). Connectivity strength of dissociable striatal tracts predict individual differences in temporal discounting. J. Neurosci. 34, 10298-10310. doi: 10.1523/ JNEUROSCI.4105-13.2014

Wingenfeld, K., Rullkoetter, N., Mensebach, C., Beblo, T., Mertens, M., Kreisel, S., et al. (2009). Neural correlates of the individual emotional stroop in borderline personality disorder. Psychoneuroendocrinology 34, 571-586. doi: 10.1016/j. psyneuen.2008.10.024

Wolf, R. C., Sambataro, F., Vasic, N., Schmid, M., Thomann, P. A., Bienentreu, S. D., et al. (2011). Aberrant connectivity of resting-state networks in borderline personality disorder. J. Psychiatry Neurosci. 36, 402-411. doi: 10.1503/jpn. 100150

Woolrich, M. W., Jbabdi, S., Patenaude, B., Chappell, M., Makni, S., Behrens, T., et al. (2009). Bayesian analysis of neuroimaging data in FSL. Neuroimage 45, S173-S186. doi: 10.1016/j.neuroimage.2008.10.055

Xiao, J., Qiu, Y., He, Y., Cui, L., Auerbach, R. P., McWhinnie, C. M., et al. (2016). "Weakest link" as a cognitive vulnerability within the hopelessness theory of depression in chinese university students. Stress Heal. 32, 20-27. doi: 10.1002/ smi.2571

Yan, C., and Zang, Y. (2010). DPARSF: a MATLAB toolbox for "pipeline" data analysis of resting-state fMRI. Front. Syst. Neurosci. 4:13. doi: 10.3389/fnsys. 2010.00013

Yang, H., Lei, X., Zhong, M., Zhou, Q., Ling, Y., Jungkunz, M., et al. (2018). Psychometric properties of the Chinese version of the brief borderline symptom list in undergraduate students and clinical patients. Front. Psychol. 9:605. doi: 10.3389/fpsyg.2018.00605
Yen, S., Shea, M., and Battle, C. (2002). Traumatic exposure and posttraumatic stress disorder in borderline, schizotypal, avoidant, and obsessive-compulsive. J. Nerv Ment Dis. 190, 510-518. doi: 10.1097/01.NMD.0000026620.66764.78

Yuan, K., Yu, D., Bi, Y., Wang, R., Li, M., Zhang, Y., et al. (2017). The left dorsolateral prefrontal cortex and caudate pathway: new evidence for cueinduced craving of smokers. Hum. Brain Mapp. 38, 4644-4656. doi: 10.1002/ hbm. 23690

Yuan, K., Zhao, M., Yu, D., Manza, P., Volkow, N. D., Wang, G. J., et al. (2018). Striato-cortical tracts predict 12-h abstinence-induced lapse in smokers. Neuropsychopharmacology 43, 2452-2458. doi: 10.1038/s41386-018-0182-x

Zhong, M., Yi, J., Ling, Y., Zhu, X., and Yao, S. (2010). The reliability and validity of the short affect intensity scale-chinese version. J. Chin. Clin. Psychol. 18, 556-558.

Zhu, X., Auerbach, R. P., Yao, S., Abela, J. J. R. Z., Xiao, J., and Tong, X. (2008). Psychometric properties of the cognitive emotion regulation questionnaire: chinese version. Cogn. Emot. 22, 288-307. doi: 10.1080/02699930701369035

Conflict of Interest Statement: The authors declare that the research was conducted in the absence of any commercial or financial relationships that could be construed as a potential conflict of interest.

Copyright (c) 2019 Lei, Zhong, Zhang, Yang, Peng, Liu, Zhang, Yao, Tan and Yi. This is an open-access article distributed under the terms of the Creative Commons Attribution License (CC BY). The use, distribution or reproduction in other forums is permitted, provided the original author(s) and the copyright owner(s) are credited and that the original publication in this journal is cited, in accordance with accepted academic practice. No use, distribution or reproduction is permitted which does not comply with these terms. 
OPEN ACCESS

Edited by:

Roberto Esposito,

A.O. Ospedali Riuniti Marche Nord,

Italy

Reviewed by:

Katharine Dunlop,

University of Toronto, Canada

Lubin Wang,

Beijing Institute of Basic Medical

Sciences, China

*Correspondence:

Yingying Tang

yytang0522@gmail.com

Liu Liu

shadow_elve@sina.cn

Jijun Wang

jijunwang27@163.com

Specialty section:

This article was submitted to

Brain Imaging Methods,

a section of the journal

Frontiers in Neuroscience

Received: 25 February 2019

Accepted: 21 August 2019

Published: 13 September 2019

Citation:

Tang Y, Jiao $X$, Wang J, Zhu T, Zhou J, Qian Z, Zhang T, Cui H, Li H,

Tang $X, X u L$, Zhang L, Wei $Y$,

Sheng J, Liu L and Wang J (2019)

Dynamic Functional Connectivity

Within the Fronto-Limbic Network Induced by Intermittent Theta-Burst

Stimulation: A Pilot Study.

Front. Neurosci. 13:944.

doi: 10.3389/fnins.2019.00944
Dynamic Functional Connectivity Within the Fronto-Limbic Network Induced by Intermittent Theta-Burst Stimulation: A Pilot Study

Yingying Tang ${ }^{1,2 *}$, Xiong Jiao ${ }^{1,3}$, Junjie Wang ${ }^{1,4}$, Tianyuan Zhu' ${ }^{1}$, Jie Zhou ${ }^{1,3}$, Zhenying Qian', Tianhong Zhang ${ }^{1}$, Huiru Cui', Hui Li', Xiaochen Tang ${ }^{1}$, Lihua Xu', Ling Zhang ${ }^{4}$, Yanyan Wei ${ }^{1}$, Jianhua Sheng ${ }^{1}$, Liu Liu${ }^{5 *}$ and Jijun Wang ${ }^{1,6,7 *}$

${ }^{1}$ Shanghai Key Laboratory of Psychotic Disorders, Shanghai Mental Health Center, Shanghai Jiao Tong University School of Medicine, Shanghai, China, ${ }^{2}$ Key Laboratory of Embedded System and Service Computing, Ministry of Education, Tongji University, Shanghai, China, ${ }^{3}$ School of Biomedical Engineering, Shanghai Jiao Tong University, Shanghai, China, ${ }^{4}$ Institute of Mental Health, Suzhou Guangji Hospital, The Affiliated Guangji Hospital of Soochow University, Suzhou, China, ${ }^{5}$ Department of Nuclear Medicine, Shanghai Chest Hospital, Shanghai Jiao Tong University, Shanghai, China, ${ }^{6}$ CAS Center for Excellence in Brain Science and Intelligence Technology (CEBSIT), Chinese Academy of Sciences, Shanghai, China,

7 Institute of Psychology and Behavioral Science, Shanghai Jiao Tong University, Shanghai, China

Purpose: The utility of transcranial magnetic stimulation (TMS) has been growing rapidly in both neurocognitive studies and clinical applications in decades. However, it remains unclear how the responses of the stimulated site and the site-related functional network to the external TMS manipulation dynamically change over time.

Methods: A multi-session combining TMS-fMRI experiment was conducted to explore the spatiotemporal effects of TMS within the fronto-limbic network. Ten healthy volunteers were modulated by intermittent theta-burst stimulation (iTBS) at a precise site within the left dorsolateral prefrontal cortex (DLPFC, MNI coordinate [-44 36 20]), navigated by individual structural MRI image. Three-session resting-state $\mathrm{AMRI}$ images were acquired before iTBS (TP1), immediately after iTBS (TP2), and 15 min after iTBS (TP3) for each participant. Seventy-four regions of interests (ROIs) within the frontolimbic network were chosen including the bilateral superior frontal gyrus (SFG), middle frontal gyrus (MidFG), inferior frontal gyrus (IFG), orbital gyrus (OrG), cingulate gyrus (CG), and subcortical nuclei (hippocampus and amygdala). Regional fractional amplitude of low-frequency fluctuation (fALFF) and ROI-to-ROI functional connectivity (FC) were compared among TP1, TP2, and TP3.

Results: The immediate iTBS effect was observed at the stimulated site. FC between the left dorsolateral SFG and left dorsal IFG and between the left rostral IFG and right MidFG increased at TP2 as compared to at TP1 (all FDR- $p<0.05$ ), while FC within the left OrG decreased. The relatively long-term iTBS effect transmitted with decreased FC between the left IFG and right amygdala, increased FC between the left MidFG and left OrG, and decreased FC between bilateral IFG and OrG at TP3 than at TP1 (all FDR$p<0.05)$. Meanwhile, mean fALFF values over the left SFG, MidFG, ventral CG, and 
IFG were significantly increased at TP3 as compared to those at TP2 (all $p<0.05$ with Bonferroni correction).

Conclusion: By combining TMS and $\mathrm{fMRI}$, it becomes possible to track the spatiotemporal dynamics of TMS after-effects within the fronto-limbic network. Our findings suggested that the iTBS effect dynamically changed over time from the local neural activation at the stimulated site to its connected remote regions within the fronto-limbic network.

Keywords: transcranial magnetic stimulation, intermittent theta-burst stimulation, after-effects, functional connectivity, the fronto-limbic network

\section{INTRODUCTION}

Transcranial magnetic stimulation (TMS) provides a noninvasive way to explore the brain function in both basic neuroscience and clinical applications (Lefaucheur et al., 2014). TMS pulses induce current within the cortex underneath the site of stimulation, leading to local neural activation (Allen et al., 2007) as well as consequent alterations within a distributed network (Chen et al., 2013). After one-session TMS modulation, i.e., intermittent theta burst stimulation (iTBS), the after-effects can be beyond the duration of stimulation and may last about 30 min (Huang et al., 2005). Therefore, the TMS after-effects should be temporally and spatially dynamic over the local region and within the whole-brain network (Ruff et al., 2009; Hawco et al., 2017, 2018). However, the pattern of tempo-spatial dynamics induced by TMS manipulation remains unclear.

There is few evidence of the duration of TMS after-effects over the primary motor cortex in humans. Huang et al. examined the after-effects induced by different TMS paradigms (iTBS, continuous TBS, intermediate TBS, and $15 \mathrm{~Hz}$ TMS) using motor evoked potential (MEP) amplitude (Huang et al., 2005). The enhanced MEP amplitudes last about $20 \mathrm{~min}$ after 600-pulse iTBS, but 60 min after 600-pulse cTBS (Huang et al., 2005). Peinemann et al. (2004) found one-session $5 \mathrm{~Hz}$ repetitive TMS with 1,800 pulses rather than 150 pulses induced stable MEP facilitatory for at least $30 \mathrm{~min}$. To further clarify the TMS aftereffects beyond the motor cortex, other neuroimaging techniques are required to measure the TMS induced neural activity.

Combining EEG with TMS is one way to characterize the temporal neural processing following TMS manipulation in non-motor regions (Chung et al., 2015). A review by Chung et al. proposed the TMS-evoked potential (TEP) as a sensitive measure of cortical excitation and inhibition (Chung et al., 2015). Indicators such as $\mathrm{N} 100$ and long-interval cortical inhibition (LICI) developed from TEP over the dorsolateral prefrontal cortex (DLPFC) were proven to be helpful for reflecting the integrity of the frontal cortex and had potentials for predicting the outcome of treatment in depression (Sun et al., 2016). When the time varied after a TMS pulse, distinctive TEP components occurred with dynamic topographic representation (Chung et al., 2015). However, it is lack of multi-session off-line EEG-TMS to monitor the affect-effects along with time following TMS. On the other hand, although topographic distribution for different TEP components suggested that neural activations not only at the site of stimulation but also at the distal sites could be influenced by TMS, more direct evidence of the precise spatial dynamics induced by TMS is further needed.

Some additional attempts have been made by combining resting-state fMRI (rsfMRI) with TMS to obtain a better spatial resolution. Wang et al. (2014) aimed to enhanced the hippocampus-related associative memory that was achieved by delivering multi-session $20 \mathrm{~Hz}$ rTMS over the lateral parietal cortex with strong functional connectivity (FC) with hippocampus. Chen et al. directly examined the dynamic interaction within three large-scale neural networks, i.e., the default mode network (DMN), the salience network (SN), and the central executive network (ECN) (Chen et al., 2013). TMS targets in different network nodes had causal influence to both within-network and between-network connectivity (Chen et al., 2013). Hawco et al. suggested spread TMS-induced cortical changes that were related to the FC between the stimulated site and SN (Hawco et al., 2018). Moreover, combining TMS and fMRI has attracted increasing attention in optimizing the rTMS treatments (Fox et al., 2012a,b, 2014), with the most mature example of major depressive disorder (MDD). As the left DLPFC was one of the most popular targets for rTMS treatment in MDD, individuals' intrinsic FC between this site and the subgenual anterior cingulate cortex (sgACC) predicted the efficacy of their rTMS treatments (Weigand et al., 2018). Therefore, it is worthy of uncovering the spatial propagation of TMS after-effects within the cortico-subcortical networks to improve the therapeutic outcome.

In the present study, we performed single-session rsfMRI acquisition before iTBS and two-session rsfMRI acquisitions after iTBS to characterize the immediate and long-term after-effects within and beyond the site of stimulation in healthy volunteers. Precise neuroimaging-guided iTBS was delivered over the left DLPFC (the most popular TMS target) using an MRI-compatible TMS coil within the MRI scanner, which made it possible to monitor the immediate iTBS effect. Considering the limitation of a small sample size, we restricted our analysis within the fronto-limbic network to reflect the spatial propagation following iTBS. Firstly, the fronto-limbic network is well known, consisting of a dorsal pathway with DLPFC and anterior cingulate cortex (ACC) for emotional regulation and executive control, as well as a ventral pathway with orbital frontal cortex, hippocampus, and amygdala for reward processing, both playing an important role in the etiology of MDD (Mayberg, 2003). Secondly, previous 
studies on TMS over the left DLPFC suggested a prominent effect within the fronto-limbic network, especially the FC between the left DLPFC and ACC, in both healthy controls and patients with depression (Fox et al., 2012a; Tik et al., 2017). We hypothesized that (1) the immediate iTBS effect may be stronger around the site of stimulation than in remote sites, and (2) the long-term iTBS effect may spread beyond the site of stimulation and be stronger in the other node(s) within the fronto-limbic network.

\section{MATERIALS AND METHODS}

\section{Participants}

The experimental protocol was approved by the Ethics Committee at Shanghai Mental Health Center. Written informed consent was obtained from each participant. Ten healthy volunteers [ 4 females and 6 males; age (mean \pm SD): $25.5 \pm 2.8$ years old, education (mean $\pm \mathrm{SD}$ ): $17.0 \pm 0.8$ years] were recruited from the community by online advertisement. All participants were screened by a psychiatrist. Exclusion criteria include a personal or family history of mental illness, a history of any substance or alcohol abuse, severe physical disease, loss of consciousness, any foreign metallic objects in their head or stimulator in their body, or any other contraindication for MRI examination or TMS intervention. All participants received one-session TMS modulation and completed MRI image acquisitions (Figure 1).

\section{TMS Procedure}

Transcranial magnetic stimulation stimuli were delivered using MagPro X100 magnetic stimulator (Medtronic Co., Denmark). Individual resting motor threshold (MT) was examined with a figure-eight coil (MC-B70) outside the MRI scanner. MEP was measured from the left abductor pillicis brevis (APB) muscle with surface electrodes and then amplified and quantified using Keypoint (Medtronic Co., Denmark). The "hotspot" for each participant was determined over the primary motor area where the largest MEP was evoked (Tang et al., 2018). In 5 out of 10 trials, the lowest intensity that succeeded in evoking peak-to-peak MEP exceeding $50 \mu \mathrm{V}$ was defined as individual MT.

One-session iTBS was applied in the MRI scanner using an MRI-compatible coil (MRi-B91). The iTBS applied three $50-\mathrm{Hz}$ pulses every $200 \mathrm{~ms}$ as one burst and delivered for $2 \mathrm{~s}$ with 10-s intervals (Huang et al., 2005). In total, there were 600 pulses for each session. The intensity of iTBS was set as $80 \%$ of individual resting MT (Grossheinrich et al., 2009; Rossi et al., 2009; Lefaucheur et al., 2014). The target was precisely localized over individual left DLPFC determined by the MNI coordinate $(x=-44, y=36, z=20)$ using LOCALITE TMS Navigator (LOCALITE GmbH, Schloss Birlinghoven, Germany) (Lerman et al., 2014). The localization of left DLPFC was at the border of BA 9 and 46 defined by Rajkowska and Goldman-Rakic (1995). The TMS coil was fixed inside the MRI head coil.

\section{MRI Data Acquisition}

All MRI images were obtained in Shanghai Mental Health Center on a Siemens 3T Verio MRI system (MR B17, Siemens AG, Erlangen, Germany) with an open 1-channel head coil. Each subject completed two-step MRI scans depicted in Figure 1: (1) the first MRI scan only included structural T1-weighted images for the localization of individual precise TMS target (the left DLPFC); 2) after the fixation of TMS coil, the second MRI scan included structural T1-weighted images, the first resting-state fMRI images before iTBS modulation, magnetic resonance spectroscopy (MRS), the second resting-state fMRI images immediately after iTBS modulation, MRS and the third resting-state fMRI images $15 \mathrm{~min}$ after iTBS modulation. MRS data analysis was not included in the current study.

T1-weighted images were acquired using a magnetizationprepared rapid acquisition gradient-echo (MPRAGE) sequence with repetition time $(\mathrm{TR})=2530 \mathrm{~ms}$, echo time $(\mathrm{TE})=3.65 \mathrm{~ms}$, field of view $(\mathrm{FOV})=256 \mathrm{~mm}$, matrix $=256 \times 256$, slice thickness $=1 \mathrm{~mm}, 224$ coronal slices, flip angle $=7^{\circ}$, and generalized autocalibrating partial parallel acquisition (GRAPPA) with acceleration factor 2 .

\begin{tabular}{c|c|c|}
\cline { 2 - 2 } Step 1: Localization & $\begin{array}{c}\text { sMRI } \\
\text { of precise TMS target } \\
\text { (T1 weighted } \\
\text { images) }\end{array}$ & $\begin{array}{c}\text { Neuroimaging- } \\
\text { guided precise } \\
\text { TMS target }\end{array}$ \\
\hline
\end{tabular}

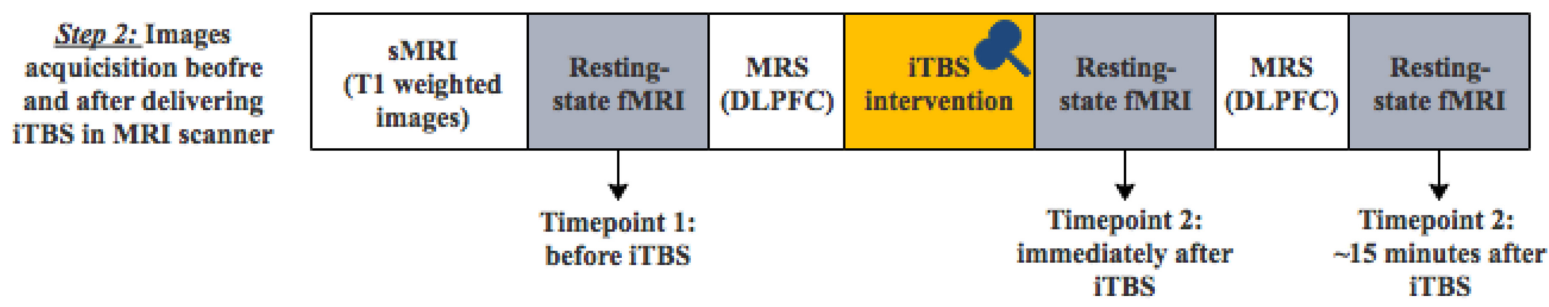

FIGURE 1 | The diagram of the two-step experimental procedure (sMRI, structural MRI; TMS, transcranial magnetic stimulation; MRS, magnetic resonance spectroscopy). 
Resting-stage fMRI images were acquired using an echo planar scanning sequence with $\mathrm{TR}=2,000 \mathrm{~ms}, \mathrm{TE}=30 \mathrm{~ms}$, $\mathrm{FOV}=220 \mathrm{~mm}$, matrix $=64 \times 64$, slice thickness $=4 \mathrm{~mm}, 30$ axial slices with a between-slice gap of $1 \mathrm{~mm}$, flip angle $=90^{\circ}$, and total data volume $=180$. Subjects were asked to close their eyes, relax, and think of nothing during the rsfMRI acquisitions.

\section{Resting-State fMRI Data Processing}

Resting-state fMRI data were preprocessed including slice timing correction, realignment, normalization, and smoothing (8-mm FWHM Gaussian filter) using CONN v.18.b ${ }^{1}$ (WhitfieldGabrieli and Nieto-Castanon, 2012). All subjects' functional data were co-registered to their structural data with a linear transformation and then normalized to MNI space with a nonlinear transformation. The Artifact Detection Tools (ART) were embedded in CONN to identify outlier images if the head motion in $x, y$, or $z$ direction was over $1 \mathrm{~mm}$ or the global mean intensity in the image was over three standard deviations from the mean image intensity for all images. Individual T1weighted images were segmented into gray matter, white matter, and CSF and generated three masks (Whitfield-Gabrieli et al., 2016). Linear regression was applied to remove the confounding effects including (1) BOLD signals from white matter and CSF, which were used for aCompCor; (2) head motion confound defined by six rigid-body motion parameters and six firstorder temporal derivatives; (3) ART-based scrubbing parameters containing invalid scans. Then a band-pass filter $(0.01-0.08 \mathrm{~Hz})$ and linear detrending were applied to the resulting residual BOLD time series.

Firstly, ROI-to-ROI FC analysis was performed at individual level using Conn. Seventy-four frontal, limbic, and subcortical ROIs were selected from the Human Brainnetome Atlas (Fan et al., 2016), including 14 ROIs in superior frontal gyrus (SFG), 14 ROIs in middle frontal gyrus (MidFG), 12 ROIs in inferior frontal gyrus (IFG), 12 ROIs in orbital gyrus (OrG), 14 ROIs in limbic lobe/cingulate gyrus (CG), and 8 subcortical nuclei (4 in amygdala and 4 in hippocampus) with detailed information in Supplementary Material.

Secondly, amplitude of low-frequency fluctuation (ALFF) and fractional ALFF (fALFF) were calculated within each voxel using Conn (Zang et al., 2007; Zou et al., 2008). The fALFF at each voxel was the relative amplitude of BOLD signal fluctuation in the frequency band of $0.01-0.08 \mathrm{~Hz}$ compared to the entire frequency band before filtering (Whitfield-Gabrieli and Nieto-Castanon, 2012). Further, mean fALFF values were calculated within each of the above 74 ROIs.

\section{Statistical Analysis}

There were three conditions for each participant including before the iTBS (TP1), immediately after the iTBS (TP2), and $15 \mathrm{~min}$ after the iTBS (TP3). For ROI-to-ROI FC values, the immediate iTBS effect was examined by comparing FC values at TP2 with those at TP1, while the long-term iTBS effect was examined by comparing FC values at TP3 with those at TP1. Two-sided pairedsample $t$ tests were performed for both the ROI-to-ROI FC. The

\footnotetext{
${ }^{1}$ https://www.nitrc.org/projects/conn
}

statistical significance was set at a false discover rate-corrected $p$ $($ FDR- $p)<0.05$.

Repeated-measure analyses of variance (ANOVAs) were performed for mean regional fALFF values with two withingroup factors of time (TP1, TP2, and TP3) and region (74 ROIs). Simple-effect tests were further performed to examine regional fALFF differences across TP1, TP2, and TP3. Multiple comparisons were controlled using Bonferroni correction.

\section{RESULTS}

\section{The iTBS Effect on ROI-to-ROI FC}

The iTBS effect was dynamic along with the time and spread within the fronto-limbic network as shown in Figure 2 and Table 1.

The immediate iTBS effect was observed within the left frontal areas and between the bilateral frontal areas. FC between the left dorsolateral SFG (A6dl_l) and left dorsal IFG (A44d, $0.110 \pm 0.055$, FDR- $p=0.005)$ and between the left rostral IFG (A45r_l) and right MidFG (A10l_r, $0.353 \pm 0.032$, FDR$p=0.032)$ increased at TP2 as compared to those at TP1 $(-0.038 \pm 0.064$ and $0.170 \pm 0.043)$. FC between the left OrG areas (A13_1 and A12_471_l, $-0.047 \pm 0.059$, FDR- $p=0.018)$ decreased at TP2 as compared to that at TP1 (0.178 \pm 0.043$)$. The increased FC between the left dorsal SFG and left dorsal IFG and decreased FC between the left OrG areas were presented in each of the 10 participants, suggesting a high consistency at the individual level (Figure 2C). However, the above iTBS effect was attenuated not significant around $15 \mathrm{~min}$ after the iTBS modulation.

The long-term iTBS effect on the FC was more widespread within the bilateral frontal areas and between left IFG and amygdala as shown in Figure 2B. FC between the left caudal IFG (A45c_l) and right medial amygdala (mAmyg_r) significantly decreased around $15 \mathrm{~min}$ after the iTBS $(-0.215 \pm 0.057$, FDR$p=0.015)$ as compared to that before the iTBS $(0.035 \pm 0.063)$, but not significantly changed immediately after the iTBS (FDR$p>0.05)$. FC between the left caudal IFG (A45c_l) and left medial amygdala (mAmyg_l) had a tendency to decrease in a similar way (FDR- $p=0.094)$. FC between the left MidFG (A46_l) and left OrG increased at TP3 (A12_47o_l: $0.152 \pm 0.041$, FDR- $p=0.042$; A12_471_l: $0.190 \pm 0.063$, FDR- $p=0.045)$ as compared to that at TP1 (A12_47o_l: $-0.048 \pm 0.048$; A12_471_l: $0.037 \pm 0.053$ ), whereas FC between the left caudal IFG (A45c_l) and right medial OrG (A14m_r, TP1: $-0.059 \pm 0.048$; TP3: $-0.271 \pm 0.037$; FDR- $p=0.015$ ) and between the right opercular IFG (A44op_r) and left medial OrG (A11m_l, TP1: $-0.127 \pm 0.055$; TP3: $-0.382 \pm 0.059$; FDR- $p=0.045)$ decreased. Additionally, FC between the right OrG (A12_47o_r) and bilateral cingulate gyrus also had a tendency to be significantly changed around $15 \mathrm{~min}$ after iTBS (left CG: A23d_l, FDR- $p=0.057$; right CG: A23d_r, FDR- $p=0.098)$.

\section{The iTBS Effect on Regional fALFF}

There were significant main effects of both time $[F(2,18)=3.881$, $p=0.047]$ and region $[F(73,657)=19.353, p<0.001]$ on regional 


\section{A Immediate iTBS effect}
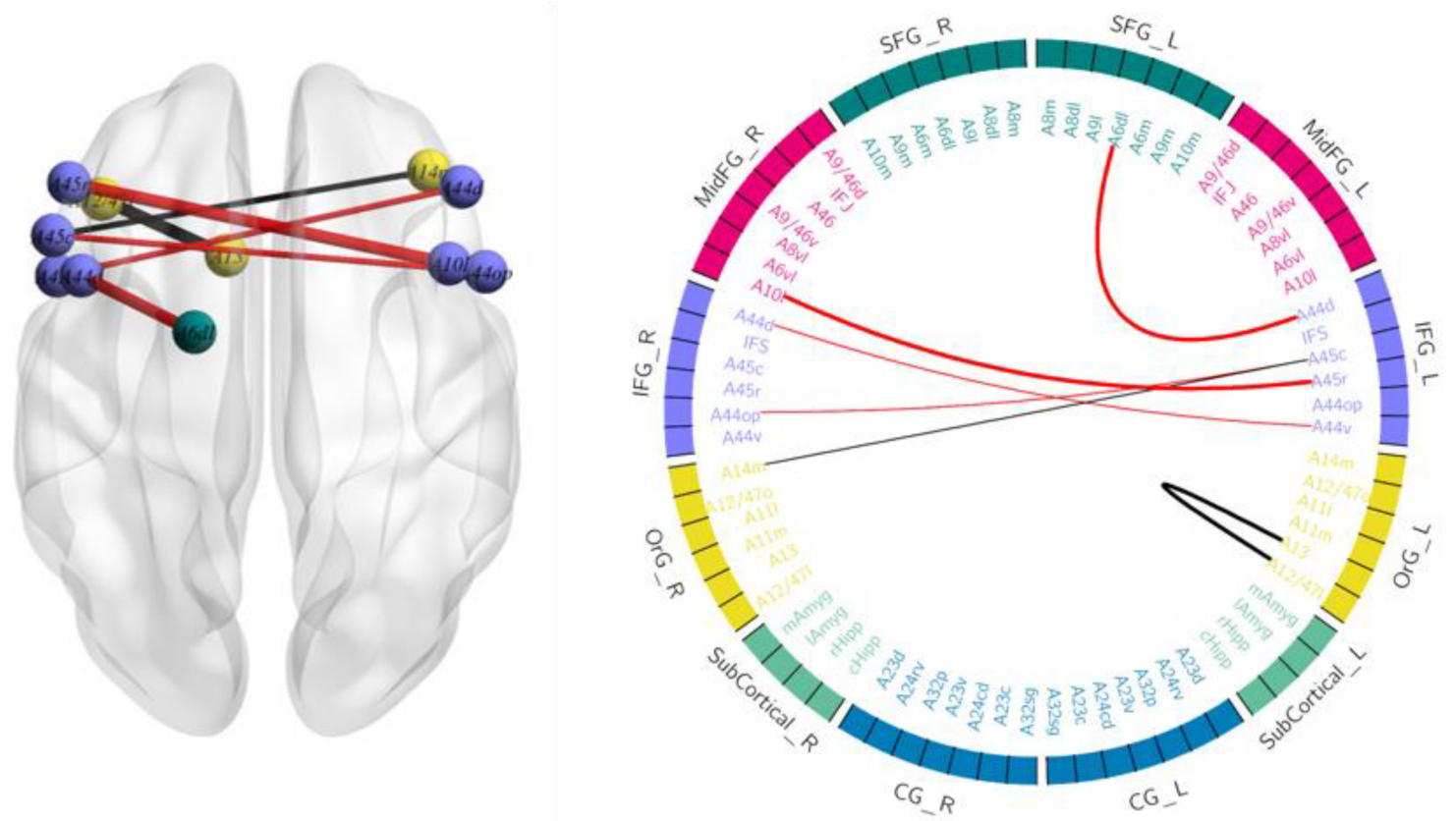

\section{B Long-term iTBS effect}
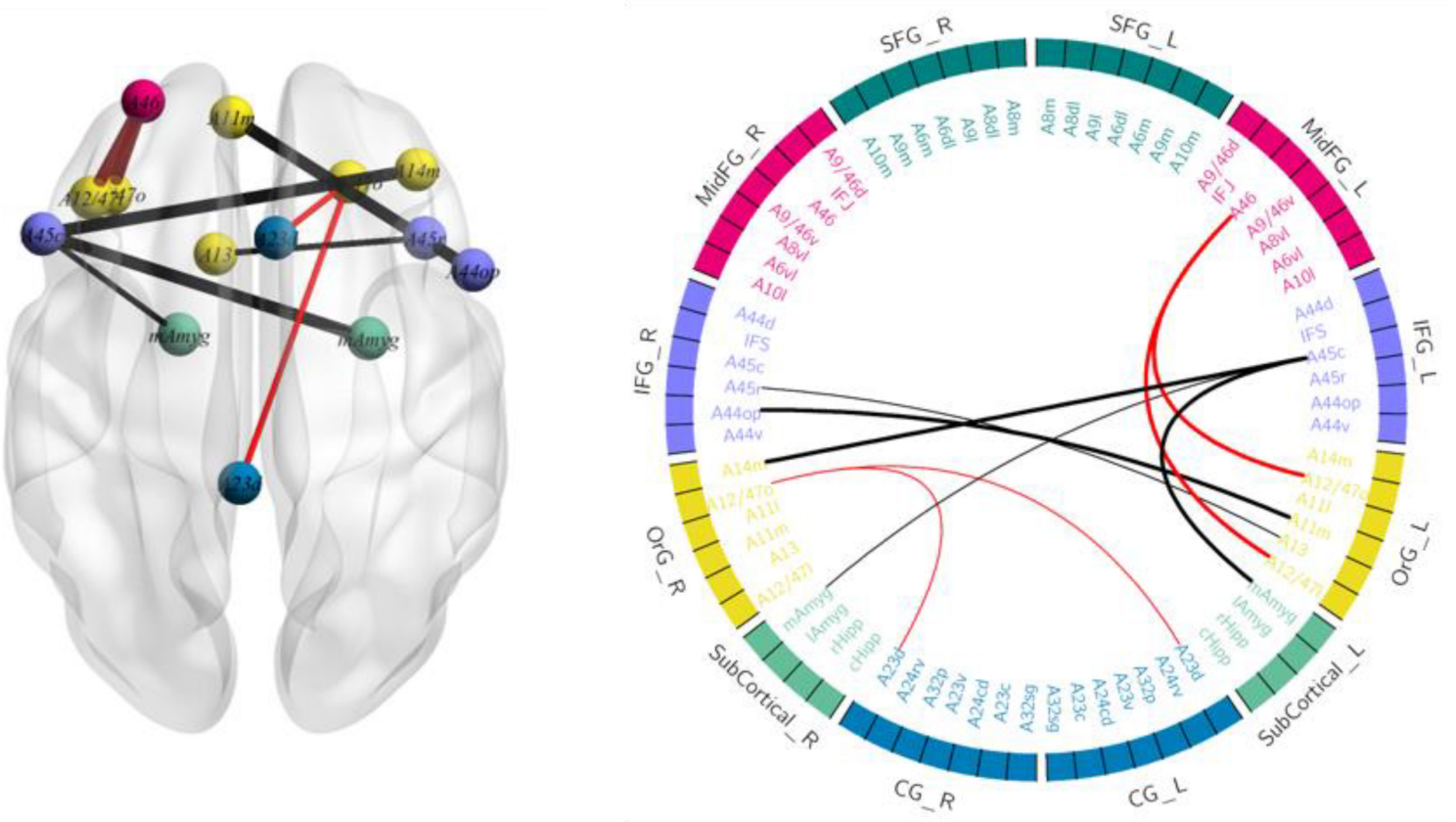

FIGURE 2 | The immediate and long-term iTBS after-effects on the ROI-to-ROI functional connectivity (FC). (A) FC within the left frontal regions and between the bilateral areas changed at timepoint 2 as compared to that at timepoint 1. (B) FC within the bilateral frontal areas and between the left IFG and amygdala changed at timepoint 3 as compared to that at timepoint 1. FC increases are in red and FC decreases are in black (thick line: $p<0.05$; thin line: $p<0.1$ ).

fALFF. As shown in Figure 3, simple effect tests further suggested that regional fALFF values over the left medial SFG (A9m_l, $p=0.010)$, left dorsal MidFG (A9_46d_l, $p=0.049)$, left ventral cingulate gyrus (A23v_l, $p=0.011$ ), and left opercular IFG
(A44op_l, $p=0.004$ ) increased at TP3 as compared to those at TP2 (all $p$ values $<0.05$ adjusted using Bonferroni correction). There were no significant differences of mean fALFF values between TP1 and TP 2 or between TP1 and TP3. 
TABLE 1 | The ROI-to-ROI functional connectivity significantly changed after the intermittent theta-burst stimulation (iTBS) immediately and 15 min later.

\begin{tabular}{|c|c|c|c|}
\hline $\begin{array}{l}\text { ROI-to-ROI functional } \\
\text { connectivity }\end{array}$ & Statistic & $\begin{array}{l}\text { Uncorrected } \\
p \text { value }\end{array}$ & $\begin{array}{c}\text { FDR- } \\
\text { corrected } \\
p \text { value }\end{array}$ \\
\hline \multicolumn{4}{|l|}{ Immediate iTBS effect (TP2 vs. TP1): } \\
\hline $\begin{array}{l}\text { Left dorsolateral SFG-left dorsal } \\
\text { IFG }\end{array}$ & $F(1,9)=48.92$ & 0.0001 & 0.0046 \\
\hline Left OrG-left lateral OrG & $F(1,9)=33.97$ & 0.0003 & 0.0183 \\
\hline $\begin{array}{l}\text { Left rostral IFG-right lateral } \\
\text { MidFG }\end{array}$ & $F(1,9)=28.98$ & 0.0004 & 0.0323 \\
\hline Left ventral IFG-right dorsal IFG & $F(1,9)=24.90$ & 0.0007 & 0.0547 \\
\hline Left caudal IFG-right opercular IFG & $F(1,9)=19.89$ & 0.0016 & 0.0576 \\
\hline Left caudal IFG-right medial OrG & $F(1,9)=20.82$ & 0.0014 & 0.0993 \\
\hline \multicolumn{4}{|l|}{ Long-term iTBS effect (TP3 vs. TP1): } \\
\hline $\begin{array}{l}\text { Left caudal IFG-right medial } \\
\text { amygdala }\end{array}$ & $F(1,9)=29.45$ & 0.0004 & 0.0153 \\
\hline Left caudal IFG-right medial OrG & $F(1,9)=31.58$ & 0.0003 & 0.0153 \\
\hline $\begin{array}{l}\text { Left medial OrG-right } \\
\text { opercular IFG }\end{array}$ & $F(1,9)=26.44$ & 0.0006 & 0.0445 \\
\hline Left MidFG-left lateral OrG & $F(1,9)=26.37$ & 0.0006 & 0.0449 \\
\hline Left MidFG-left orbital OrG & $F(1,9)=26.98$ & 0.0006 & 0.0415 \\
\hline Right orbital OrG-left dorsal CG & $F(1,9)=24.58$ & 0.0008 & 0.0571 \\
\hline Left caudal IFG-left medial amygdala & $F(1,9)=14.87$ & 0.0039 & 0.0941 \\
\hline Left OrG-right rostral IFG & $F(1,9)=20.92$ & 0.0013 & 0.0977 \\
\hline Right orbital OrG-right CG & $F(1,9)=16.78$ & 0.0027 & 0.0982 \\
\hline
\end{tabular}

CG, cingulate gyrus; FDR, false discovery rate; FG, inferior frontal gyrus; iTBS, intermittent theta-burst stimulation; MidFG, middle frontal gyrus; OrG, orbital gyrus; $R O I$, region of interest; SFG, superior frontal gyrus; TP, timepoint. Significant FC changes with FDR-p $<0.05$ are in bold.

\section{DISCUSSION}

By combining TMS with multi-session resting-state fMRI, the present study examined the dynamic changes within the frontolimbic network induced by precise iTBS modulation. As we hypothesized, iTBS after-effects were observed not only around the stimulated region but also at remote regions interconnected with the target. Immediately after iTBS, FC changed significantly between the left frontal areas and between bilateral frontal areas. About 15 min later, the alteration of FC transmitted and was more widespread between bilateral frontal areas and between left IFG and amygdala. Further, fALFF measurements at two different time windows after iTBS showed increases at the left SFG, MidFG, CG, and IFG. More importantly, these spreading patterns after precise neuroimaging-guided iTBS manipulation had a high consistency at the individual level. Our findings suggested TMS-fMRI as an advanced technique to determine the dynamic patterns of TMS-induced effects within the brain network.

We delivered TMS pulses precisely at the dorsolateral prefrontal node (MNI coordinate, $x=-44, y=36, z=20$ ) in the left MidFG (A9_46v_l), which had structural connections with the right frontal regions and subcortical regions and FC with the fronto-parietal network according to the Human Brainnetome Atlas (Yeo et al., 2011; Fan et al., 2016). Immediately after singlesession excitatory iTBS, increased FC between the left dorsal SFG and IFG and between left IFG and right MidFG may suggest an enhancement in the dorsal pathway, whereas decreased FC between the left OrG areas suggested an attenuation in the ventral pathway (Mayberg, 2003; Ruff et al., 2009; Eldaief et al., 2011). Consistent with previous findings, Hawco et al. found the prominent TMS-induced FC changes between the DLPFC target and salience network (SN) (Hawco et al., 2018). Chen et al. found excitatory single pulse over the anterior media frontal cortex increased within-SN FC (Chen et al., 2013). A previous TMSPET study also showed significant dopaminergic changes in the medial orbitofrontal cortex and ACC after $10 \mathrm{~Hz}$ TMS over the left DLPFC in healthy volunteers (Cho and Strafella, 2009). An iTBS effect could spread out immediately from the target to the left SFG, IFG, and OrG.

The iTBS after-effect continued its propagation from the left frontal regions to bilateral amygdala and to right frontal regions along with time. These remote iTBS effects could be based on anatomical connectivity by white matter tracts, such as the uncinate fasciculus connecting the frontal region and the limbic system as well as the genu of corpus callosum connecting bilateral IFG (Kier et al., 2004; Fan et al., 2016). About 15 min later, decreased FC between the left IFG and bilateral amygdala suggested a subsequent change in the ventral pathway, which is critical for the generation and regulation of negative emotion (Hiser and Koenigs, 2018). More supports of remote effects induced by TMS were provided by the changed functional covariation between different regions. Wang et al. enhanced the cortico-hippocampal FC by multi-session excitatory TMS over the lateral parietal cortex (Wang et al., 2014). Other concurrent TMS-fMRI studies also found TMS-induced changes of remote FC, such as that between the frontal cortex/parietal and visual occipital regions (Ruff et al., 2006; Sack et al., 2007). Since the iTBS effect became more prominent within the left IFG later, we inferred that neural activation at the left IFG may be indirect as a consequence of limbic activation.

Regional fALFF values increased at the left SFG, MidFG, IFG, and ventral CG later after iTBS, which further supported the continuous excitatory effect at $15 \mathrm{~min}$ after iTBS. Few other studies examined the regional activity after TMS or iTBS modulation. Only Chen et al. reported decreased low-frequency signal amplitude within the DMN network after inhibitory rTMS (Chen et al., 2013). The finding of increased fALFF value in the left MidFG, SFG, and IFG was consistent with the more widespread FC connectivity induced by long-term iTBS effect. Additionally, the regional fALFF increased in the left ventral CG, and FC between the right OrG and bilateral CG also had a tendency to be significantly changed around $15 \mathrm{~min}$ after iTBS. Cingulate cortex was a hot hub for the connectivity within the DLPFC and limbic network (Tik et al., 2017). Fox et al. suggested that FC between the DLPFC and subgenual ACC could be a valuable predictor for the TMS effects in depression (Fox et al., 2012a). Tik et al. found only the connectivity to the ACC increased at $15 \mathrm{~min}$ after $10-\mathrm{Hz}$ TMS modulation over the left DLPFC, and this effect disappeared at $30 \mathrm{~min}$ after TMS (Tik et al., 2017).

As shown in Figure 3, FC and fALFF changes from TP1 to TP2 varied among subjects, but the changes from TP2 to 


\section{A iTBS effect on functional connectivity}
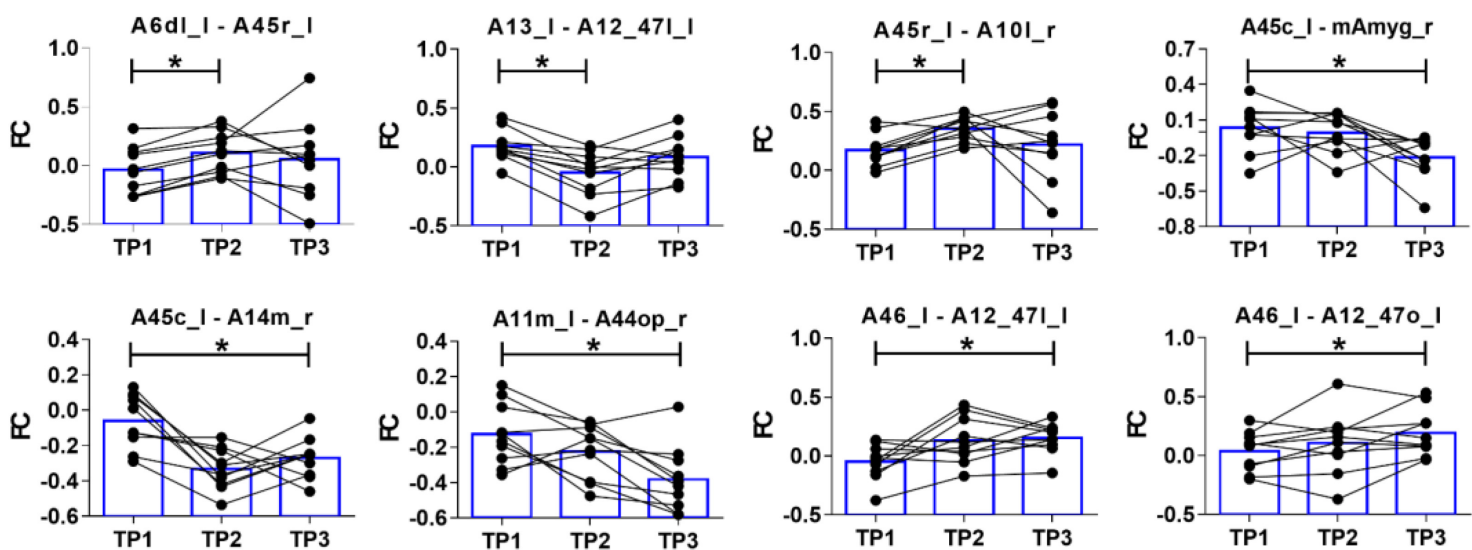

B iTBS effect on regional fALFF
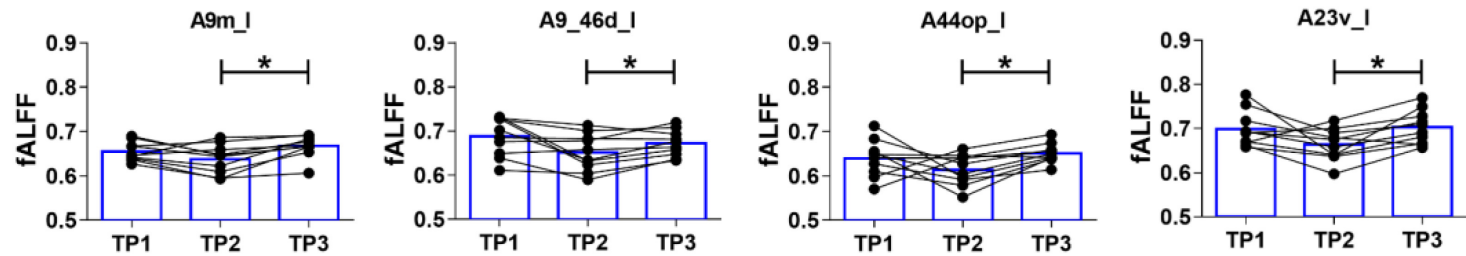

FIGURE 3 | The iTBS-induced changes of functional connectivity and fractional amplitude of low-frequency fluctuation (fALFF) at timepoint 1 (TP1), timepoint 2 (TP2), and timepoint 3 (TP3). (A) Immediate iTBS effect (TP1 vs. TP2) was observed within the left frontal areas and between the bilateral frontal areas, while long-term iTBS effects (TP1 vs. TP3) was more widespread within the bilateral frontal areas and between the left caudal IFG (A45c_I) and right medial amygdala (mAmyg_r). (B) Regional fALFF changed from TP2 to TP3 at the left medial SFG (A9m_l), left dorsal MidFG (A9_46d_I), left opercular IFG (A44op_l), and left ventral cingulate gyrus (A23v_l).

TP3 had a high inter-subject consistency. One critical factor contributing to the heterogeneity of TMS after-effects could be the different location of individual TMS targets (Fox et al., 2012a; Lefaucheur et al., 2014; Hawco et al., 2018). The use of individual T1-image-guided localization for the TMS target in the present study could reduce the heterogeneity of the iTBS effect. The other factor may be the different latency of individual TMS-induced response. A higher consistency for FC and fALFF changes among subjects at TP3 suggested a convergence of the remote iTBS effect within the fronto-limbic network after a longer time. However, more evidence is required in the future to prove our intention.

There were several limitations in the present study. Firstly, only 10 healthy volunteers were recruited for the experiments. The small sample size made it a bit difficult to obtain strong statistical significance. Secondly, there was no control or sham condition. As an exploratory study, only iTBS over the left DLPFC was used. The observed iTBS-induced dynamic patterns needed to be interpreted in caution. Thirdly, we narrowed our observation in the fronto-limbic network to tract the iTBS effects. There could be other regions or networks also influenced by iTBS that were omitted here. Further, more works with experimental designs including control/sham TMS condition, different intensities, and stimulated sites are needed to predict the iTBS-induced effects within the large-scale brain networks.
In conclusion, to the best of our knowledge, there were a few studies characterizing the temporal and spatial dynamics induced by single-session iTBS manipulation. The FC and fALFF measurements consistently demonstrated a significant immediate iTBS effect around the stimulated DLPFC site, while a long-term iTBS effect was prominent between the left IFG and amygdala as well as between bilateral frontal regions. Thus, our findings suggested that the combination of TMS-fMRI should be an advanced technique to clarify the TMS-induced effect, as well as to optimize the clinical application for treatments.

\section{DATA AVAILABILITY}

The datasets generated for this study are available on request to the corresponding author.

\section{ETHICS STATEMENT}

The experimental protocol was approved by the Ethics Committee at Shanghai Mental Health Center. Written informed consent was obtained from each participant. 


\section{AUTHOR CONTRIBUTIONS}

JiW and YT conceptualized and designed the study. TZha, HC, HL, LX, and YW recruited the participants and completed the screening assessments. YT, JuW, TZhu, and ZQ performed the TMS manipulation. YT, XJ, XT, JS, and JiW analyzed the data and performed the statistical analysis. YT, JS, and JiW wrote the first draft of the manuscript. All authors revised the manuscript and approved the final manuscript.

\section{FUNDING}

This work was supported by the Ministry of Science and Technology of China, the National Key R\&D Program of China (2016YFC1306803), the National Natural Science Foundation of China (81871050, 81671332, and 81602415), the Shanghai Science and Technology Committee (17411953100 and 18411952400), the Shanghai Key Basic Research Project (16JC1420201), the Natural Science Foundation of Shanghai (16ZR1446600 and 18ZR1432600), the Shanghai Jiao Tong

\section{REFERENCES}

Allen, E. A., Pasley, B. N., Duong, T., and Freeman, R. D. (2007). Transcranial magnetic stimulation elicits coupled neural and hemodynamic consequences. Science 317, 1918-1921. doi: 10.1126/science.1146426

Chen, A. C., Oathes, D. J., Chang, C., Bradley, T., Zhou, Z. W., Williams, L. M., et al. (2013). Causal interactions between fronto-parietal central executive and default-mode networks in humans. Proc. Natl. Acad. Sci. U.S.A. 110, 1994419949. doi: 10.1073/pnas.1311772110

Cho, S. S., and Strafella, A. P. (2009). rTMS of the left dorsolateral prefrontal cortex modulates dopamine release in the ipsilateral anterior cingulate cortex and orbitofrontal cortex. PLoS One 4:e6725. doi: 10.1371/journal.pone.0006725

Chung, S. W., Rogasch, N. C., Hoy, K. E., and Fitzgerald, P. B. (2015). Measuring brain stimulation induced changes in cortical properties using TMS-EEG. Brain Stimul. 8, 1010-1020. doi: 10.1016/j.brs.2015.07.029

Eldaief, M. C., Halko, M. A., Buckner, R. L., and Pascual-Leone, A. (2011). Transcranial magnetic stimulation modulates the brain's intrinsic activity in a frequency-dependent manner. Proc. Natl. Acad. Sci. U.S.A. 108, 21229-21234. doi: 10.1073/pnas.1113103109

Fan, L., Li, H., Zhuo, J., Zhang, Y., Wang, J., Chen, L., et al. (2016). The human brainnetome atlas: a new brain atlas based on connectional architecture. Cereb. Cortex 26, 3508-3526. doi: 10.1093/cercor/bhw157

Fox, M. D., Buckner, R. L., Liu, H., Chakravarty, M. M., Lozano, A. M., and PascualLeone, A. (2014). Resting-state networks link invasive and noninvasive brain stimulation across diverse psychiatric and neurological diseases. Proc. Natl. Acad. Sci. U.S.A. 111, E4367-E4375. doi: 10.1073/pnas.1405003111

Fox, M. D., Buckner, R. L., White, M. P., Greicius, M. D., and Pascual-Leone, A. (2012a). Efficacy of transcranial magnetic stimulation targets for depression is related to intrinsic functional connectivity with the subgenual cingulate. Biol. Psychiatry 72, 595-603. doi: 10.1016/j.biopsych.2012.04.028

Fox, M. D., Halko, M. A., Eldaief, M. C., and Pascual-Leone, A. (2012b). Measuring and manipulating brain connectivity with resting state functional connectivity magnetic resonance imaging (fcMRI) and transcranial magnetic stimulation (TMS). Neuroimage 62, 2232-2243. doi: 10.1016/j.neuroimage.2012.03.035

Grossheinrich, N., Rau, A., Pogarell, O., Hennig-Fast, K., Reinl, M., Karch, S., et al. (2009). Theta burst stimulation of the prefrontal cortex: safety and impact on cognition, mood, and resting electroencephalogram. Biol. Psychiatry 65, 778-784. doi: 10.1016/j.biopsych.2008.10.029

Hawco, C., Armony, J. L., Daskalakis, Z. J., Berlim, M. T., Chakravarty, M. M., Pike, G. B., et al. (2017). Differing time of onset of concurrent TMS-fMRI during
University (YG2016MS36), the Clinical Research Center at Shanghai Mental Health Center (CRC2018ZD01), the Shanghai Municipal Commission of Health and Family Planning (201640050), and the Fund of Humanities and Social Sciences of Shanghai Jiao Tong University (16JXRZ11). YT was funded by a Municipal Human Resources Development Program for Outstanding Young Talents in Medical and Health Sciences in Shanghai (2017YQ069). JuW was funded by the Suzhou Municipal Science and Technology Bureau, China (sys2018097).

\section{ACKNOWLEDGMENTS}

We thank all the healthy volunteers for their participation.

\section{SUPPLEMENTARY MATERIAL}

The Supplementary Material for this article can be found online at: https://www.frontiersin.org/articles/10.3389/fnins. 2019.00944/full\#supplementary-material

associative memory encoding: a measure of dynamic connectivity. Front. Hum. Neurosci. 11:404. doi: 10.3389/fnhum.2017.00404

Hawco, C., Voineskos, A. N., Steeves, J. K. E., Dickie, E. W., Viviano, J. D., Downar, J., et al. (2018). Spread of activity following TMS is related to intrinsic resting connectivity to the salience network: a concurrent TMS-fMRI study. Cortex 108, 160-172. doi: 10.1016/j.cortex.2018.07.010

Hiser, J., and Koenigs, M. (2018). The multifaceted role of the ventromedial prefrontal cortex in emotion, decision making, social cognition, and psychopathology. Biol. Psychiatry 83, 638-647. doi: 10.1016/j.biopsych.2017. 10.030

Huang, Y. Z., Edwards, M. J., Rounis, E., Bhatia, K. P., and Rothwell, J. C. (2005). Theta burst stimulation of the human motor cortex. Neuron 45, 201-206. doi: 10.1016/j.neuron.2004.12.033

Kier, E. L., Staib, L. H., Davis, L. M., and Bronen, R. A. (2004). MR imaging of the temporal stem: anatomic dissection tractography of the uncinate fasciculus, inferior occipitofrontal fasciculus, and Meyer's loop of the optic radiation. AJNR Am. J. Neuroradiol. 25, 677-691.

Lefaucheur, J. P., Andre-Obadia, N., Antal, A., Ayache, S. S., Baeken, C., Benninger, D. H., et al. (2014). Evidence-based guidelines on the therapeutic use of repetitive transcranial magnetic stimulation (rTMS). Clin. Neurophysiol. 125, 2150-2206. doi: 10.1016/j.clinph.2014.05.021

Lerman, C., Gu, H., Loughead, J., Ruparel, K., Yang, Y., and Stein, E. A. (2014). Large-scale brain network coupling predicts acute nicotine abstinence effects on craving and cognitive function. JAMA Psychiatry 71, 523-530. doi: 10.1001/ jamapsychiatry.2013.4091

Mayberg, H. S. (2003). Modulating dysfunctional limbic-cortical circuits in depression: towards development of brain-based algorithms for diagnosis and optimised treatment. Br. Med. Bull. 65, 193-207. doi: 10.1093/bmb/65.1.193

Peinemann, A., Reimer, B., Loer, C., Quartarone, A., Munchau, A., Conrad, B., et al. (2004). Long-lasting increase in corticospinal excitability after 1800 pulses of subthreshold $5 \mathrm{~Hz}$ repetitive TMS to the primary motor cortex. Clin. Neurophysiol. 115, 1519-1526. doi: 10.1016/j.clinph.2004.02.005

Rajkowska, G., and Goldman-Rakic, P. S. (1995). Cytoarchitectonic definition of prefrontal areas in the normal human cortex: II. variability in locations of areas 9 and 46 and relationship to the talairach coordinate system. Cereb. Cortex 5, 323-337. doi: 10.1093/cercor/5.4.323

Rossi, S., Hallett, M., Rossini, P. M., and Pascual-Leone, A. (2009). Safety, ethical considerations, and application guidelines for the use of transcranial magnetic stimulation in clinical practice and research. Clin. Neurophysiol. 120, 20082039. doi: 10.1016/j.clinph.2009.08.016 
Ruff, C. C., Blankenburg, F., Bjoertomt, O., Bestmann, S., Freeman, E., Haynes, J. D., et al. (2006). Concurrent TMS-fMRI and psychophysics reveal frontal influences on human retinotopic visual cortex. Curr. Biol. 16, 1479-1488. doi: 10.1016/j.cub.2006.06.057

Ruff, C. C., Driver, J., and Bestmann, S. (2009). Combining TMS and fMRI: From 'virtual lesions' to functional-network accounts of cognition. Cortex 45, 1043-1049. doi: 10.1016/j.cortex.2008.10.012

Sack, A. T., Kohler, A., Bestmann, S., Linden, D. E., Dechent, P., Goebel, R., et al. (2007). Imaging the brain activity changes underlying impaired visuospatial judgments: simultaneous FMRI, TMS, and behavioral studies. Cereb. Cortex 17, 2841-2852. doi: 10.1093/cercor/bhm013

Sun, Y., Farzan, F., Mulsant, B. H., Rajji, T. K., Fitzgerald, P. B., Barr, M. S., et al. (2016). Indicators for remission of suicidal ideation following magnetic seizure therapy in patients with treatment-resistant depression. JAMA Psychiatry 73, 337-345. doi: 10.1001/jamapsychiatry.2015.3097

Tang, Y., Ying, C., Wang, J., Jiao, X., Qian, Z., Zhang, T., et al. (2018). Precise theta burst transcranial magnetic stimulation selectively reduced duration-related mismatch negativity. Biol. Psychol. 137, 125-132. doi: 10.1016/j.biopsycho.2018. 08.001

Tik, M., Hoffmann, A., Sladky, R., Tomova, L., Hummer, A., Navarro de Lara, L., et al. (2017). Towards understanding rTMS mechanism of action: stimulation of the DLPFC causes network-specific increase in functional connectivity. Neuroimage 162, 289-296. doi: 10.1016/j.neuroimage.2017.09.022

Wang, J. X., Rogers, L. M., Gross, E. Z., Ryals, A. J., Dokucu, M. E., Brandstatt, K. L., et al. (2014). Targeted enhancement of cortical-hippocampal brain networks and associative memory. Science 345, 1054-1057. doi: 10.1126/science. 1252900

Weigand, A., Horn, A., Caballero, R., Cooke, D., Stern, A. P., Taylor, S. F., et al. (2018). Prospective validation that subgenual connectivity predicts antidepressant efficacy of transcranial magnetic stimulation sites. Biol. Psychiatry 84, 28-37. doi: 10.1016/j.biopsych.2017.10.028
Whitfield-Gabrieli, S., Ghosh, S. S., Nieto-Castanon, A., Saygin, Z., Doehrmann, O., Chai, X. J., et al. (2016). Brain connectomics predict response to treatment in social anxiety disorder. Mol. Psychiatry 21, 680-685. doi: 10.1038/mp. 2015.109

Whitfield-Gabrieli, S., and Nieto-Castanon, A. (2012). Conn: a functional connectivity toolbox for correlated and anticorrelated brain networks. Brain Connect. 2, 125-141. doi: 10.1089/brain.2012.0073

Yeo, B. T., Krienen, F. M., Sepulcre, J., Sabuncu, M. R., Lashkari, D., Hollinshead, M., et al. (2011). The organization of the human cerebral cortex estimated by intrinsic functional connectivity. J. Neurophysiol. 106, 1125-1165. doi: 10.1152/ jn.00338.2011

Zang, Y. F., He, Y., Zhu, C. Z., Cao, Q. J., Sui, M. Q., Liang, M., et al. (2007). Altered baseline brain activity in children with ADHD revealed by resting-state functional MRI. Brain Dev. 29, 83-91. doi: 10.1016/j.braindev.2006.07.002

Zou, Q. H., Zhu, C. Z., Yang, Y., Zuo, X. N., Long, X. Y., Cao, Q. J., et al. (2008). An improved approach to detection of amplitude of low-frequency fluctuation (ALFF) for resting-state fMRI: fractional ALFF. J. Neurosci. Methods 172, 137-141. doi: 10.1016/j.jneumeth.2008.04.012

Conflict of Interest Statement: The authors declare that the research was conducted in the absence of any commercial or financial relationships that could be construed as a potential conflict of interest.

Copyright (c) 2019 Tang, Jiao, Wang, Zhu, Zhou, Qian, Zhang, Cui, Li, Tang, $X u$, Zhang, Wei, Sheng, Liu and Wang. This is an open-access article distributed under the terms of the Creative Commons Attribution License (CC BY). The use, distribution or reproduction in other forums is permitted, provided the original author(s) and the copyright owner(s) are credited and that the original publication in this journal is cited, in accordance with accepted academic practice. No use, distribution or reproduction is permitted which does not comply with these terms. 


\section{OPEN ACCESS}

Edited by:

Dong Song,

University of Southern California,

United States

Reviewed by:

Zhikun Zhang,

Guangxi Medical University, China

Chu-Chung Huang,

National Yang-Ming University, Taiwan

Michal Mikl,

Masaryk University, Czechia

*Correspondence:

Armando Tartaro

a.tartaro@radiol.unich.it

${ }^{\dagger}$ These authors have contributed

equally to this work

Specialty section:

This article was submitted to

Brain Imaging Methods,

a section of the journal

Frontiers in Neuroscience

Received: 30 March 2019

Accepted: 30 July 2019

Published: 13 September 2019

Citation:

Cera N, Esposito R, Cieri F and

Tartaro A (2019) Altered Cingulate

Cortex Functional Connectivity

in Normal Aging and Mild Cognitive

Impairment. Front. Neurosci. 13:857.

doi: 10.3389/fnins.2019.00857

\section{Altered Cingulate Cortex Functional Connectivity in Normal Aging and Mild Cognitive Impairment}

\author{
Nicoletta Cera ${ }^{1 \dagger}$, Roberto Esposito ${ }^{2 t}$, Filippo Cierit and Armando Tartaro ${ }^{4 *}$ \\ ${ }^{1}$ Faculty of Psychology and Educational Science, University of Porto, Porto, Portugal, ${ }^{2}$ Radiology Unit, Azienda Ospedaliera \\ Ospedali Riuniti Marche Nord, Pesaro, Italy, ${ }^{3}$ Department of Neurology, Cleveland Clinic Lou Ruvo Center for Brain Health, \\ Las Vegas, NV, United States, ${ }^{4}$ Department of Neuroscience, Imaging and Clinical Sciences, Institute of Advanced \\ Biomedical Technologies, D'Annunzio University of Chieti-Pescara, Chieti, Italy
}

Purpose: Resting-state functional Magnetic Resonance Imaging studies revealed that the brain is organized into specialized networks constituted by regions that show a coherent fluctuation of spontaneous activity. Among these networks, the cingulate cortex appears to play a crucial role, particularly in the default mode network, the dorsal attention network and the salience network. In the present study, we mapped the functional connectivity (FC) pattern of different regions of the cingulate cortex: the anterior cingulate cortex, midcingulate cortex and posterior cingulate cortex/retro splenial cortex, which have been in turn divided into a total of 9 subregions. We compared FC patterns of the cingulate subregions in a sample of mild cognitive impairment patients and healthy elderly subjects.

Methods: We enrolled 19 healthy elders (age range: 61-72 y.o.) and 16 Mild cognitive impairment patients (age range 64-87 y.o.). All participants had comparable levels of education (8-10 years) and were neurologically examined to exclude visual and motor impairments, major medical conditions, psychiatric or neurological disorders and consumption of psychotropic drugs. The diagnosis of mild cognitive impairment was performed according to Petersen criteria. Subjects were evaluated with MiniMental State Examination, Frontal Assessment Battery, and prose memory (Babcock story) tests. In addition, with functional Magnetic Resonance Imaging, we investigated resting-state network activities.

Results: Healthy elderly, compared to mild cognitive impairment, showed significant increased level of FC for the ventral part of the anterior cingulate cortex in correspondence to the bilateral caudate and ventromedial prefrontal cortex. Moreover, for the midcingulate cortex the healthy elderly group showed increased levels of FC in the somatomotor region, prefrontal cortex, and superior parietal lobule. Meanwhile, the mild cognitive impairment group showed an increased level of FC for the superior frontal gyrus, frontal eye field and orbitofrontal cortex compared to the healthy elderly group. 
Conclusion: Our findings indicate that cognitive decline observed in mild cognitive impairment patients damages the global FC of the cingulate cortex, supporting the idea that abnormalities in resting-state activities of the cingulate cortex could be a useful additional tool in order to better understand the brain mechanisms of $\mathrm{MCl}$.

Keywords: resting-state FC-fMRI, cingulate cortex, aging, MCI, functional connectivity, fMRI

\section{INTRODUCTION}

In the last decade, a growing interest has been shown in age related abnormal changes in brain structures and functions underlying Mild cognitive impairment (MCI) and Alzheimer Disease (AD). During normal and pathological aging several cognitive, emotional and motor changes occur (Dubois and Albert, 2004; Lyketsos et al., 2011; Minhas et al., 2018). These brain age-related changes have a significant impact on individual quality of life and affect several domains (Petersen, 2004; Petersen and Negash, 2008; Metzler-Baddeley et al., 2012; Esposito et al., 2013; Cieri et al., 2017). Although the neural mechanisms underlying age-related dementias have not been well understood, several cognitive and emotional deficits can accelerate the risk of conversion to dementia. Previous studies have revealed that many brain regions are involved in emotional and cognitive age-related changes (Rombouts et al., 2005; Cieri et al., 2017).

Functional neuroimaging, in particular functional MRI (fMRI), has been extensively used to investigate the changes in brain function due to aging (Ferreira and Busatto, 2013; Gröschel et al., 2013; Brier et al., 2014) and functional changes possibly related to pre-symptomatic clinical stages of dementia, such as MCI (Fleisher et al., 2009; Greene and Killiany, 2010; Esposito et al., 2013). The functional correlation among several brain regions of the resting-state fMRI (rsfMRI) signal acquired during the resting state is considered a reliable tool to study and understand the age-related changes in several domains of cognitive functions and emotional processing. More specifically, rsfMRI studies revealed a brain organization of specialized networks constituted by regions that show a coherent fluctuation of spontaneous activity (Deco et al., 2011; Hansen et al., 2015). Indeed, several resting-state networks (RSNs) have been identified, such as the Default Mode Network (DMN), Salience Network (SN); Frontoparietal Network (FPN), primary Sensory Motor Network (SMN), Exastrastriate Visual System (EVS), and Dorsal Attention Network (DAN; Van den Heuvel et al., 2009; Van den Heuvel and Hulshoff Pol, 2010). Among these, DMN has been considered the most studied network and includes the following main nodes: the medial frontal gyrus (MedFG), posterior cingulate cortex (PCC), bilateral angular gyrus (AG) and the hippocampus (Hp; Mevel et al., 2013; Passow et al., 2015). This network is also known as the task-negative network because its regions are typically deactivated during execution of attention demanding tasks (Passow et al., 2015).

DAN, conversely to DMN - is called a task-positive network, being active during cognitive tasks which demand attention and mental control, and its function is considered fundamental during the neuroaging process. It consists of the following regions: the inferior parietal sulcus (IPS), frontal eye field (FEF), anterior cingulate cortex (ACC) and bilateral middle temporal gyrus (MidTempG; Corbetta and Shulman, 2002; Fox et al., 2005). Another RSN that received attention for its implications in the emotional processing is the $\mathrm{SN}$, composed of the bilateral anterior insular cortices and dorsal cingulate cortex (DCC). During aging and in the elderly neurodegenerative conditions as $\mathrm{MCI}$, these RSNs showed an altered pattern of functional connectivity (FC).

Among these networks, the cingulate cortex seems to play a key role in many cognitive, motor and emotional functions. In humans and other primates, the cingulate cortex is a thick belt of cortex encircling the corpus callosum, considered one of the most prominent features on the mesial surface of the brain (Shackman et al., 2011). fMRI studies showed the importance of this structure's involvement among others functions with the insular cortex, the secondary somatosensory cortex, the nuclei in the tegmentum and the hypothalamus in the regulation of attentional focus by integrating external and internal stimuli, and in the expression of emotional states, thus modulating a motivational state that obtains homeostasis (Damasio et al., 2000).

Mega et al. (1996), in their proposed divisions of the limbic system, described on one hand the paleocortex part, including the amygdala, the orbitofrontal cortex, the temporal polar and anterior insula, and on the other hand the archicortical portion, including the hippocampus and ACC. The first component is responsible for the implicit integration of affects, drives and object associations; the second deals with explicit sensory processing, encoding and attentional control (Mayberg, 1994). Although divided into two sub-divisions, the paleocortex and archicortical cortex remain integrated during learning, forming a fundamentally integrated system in which the cingulate cortex seems to play a key role in emotional and affective processes, cognitive control, cognition, executing goal-directed behavior and motivation, having an important effect on social behavior (Behrens et al., 2009), psychopathology (Shin and Liberzon, 2010) and neurological disorders (Vogt, 2009).

This structure considered as a whole or considered in its subdivision into subregions seems to be involved and impaired in its functions in the pathological aging processes, playing a key role in many cognitive, motor and emotional functions, often involved in MCI. Its subregional specialization is probably related to its anatomical differentiation into cytoarchitectonically distinct subregions. According to this view, Vogt (2005) was able to isolate four principal cingulate subregions, showing an unvarying cytoarchitectonic structure and a specific pattern of connectivity, involved in different functions. The first region is the ACC, which plays an important role in affective evaluation (Allman et al., 2001), conflict monitoring, detection (Botvinick et al., 2004), response selection (Awh and Gehring, 1999) and 
attentional control (Posner, 1994). The second subregion is the midcingulate cortex (MCC), involved in the attention monitoring for action (Pardo et al., 1991), response selection (Corbetta et al., 1991), anticipation and working memory. The PCC appears to be more involved in the mechanisms of visuospatial orientation and body-navigation (Vogt, 2005) and in self-reflection and autobiographical memories (Spreng et al., 2009). The last subregion, the retrosplenial cingulate cortex (RSCC), plays a role in memory and visuospatial functions (Burgess et al., 2001; Keene and Bucci, 2008; Vann et al., 2009). This segregationist model attributes a single cognitive or emotional function to each single subregion.

Beckmann et al. (2009) proposed a division of the cingulate cortex into more than four regions: in a diffusion tractography study he isolated nine subregions and found that the pattern of structural connectivity, for more than one cingulate region, was overlapping.

Yu et al. (2011) tested the four-region model of the cingulate cortex, decomposing the FC pattern in seven different seeds regions, confirming the reliability of the four-region model and showing specific correlations between the several seed regions defined in the cingulate cortex and the principal RSNs.

Specifically, the ACC showed positive correlations with the DMN and anti-correlations with the visual network. The MCC was correlated with the cognitive and sensorimotor networks and anti-correlated with the visual, affective and DMN.

In a recent meta-analytic study, Torta et al. (2013) found a tripartite subdivision of the cingulate cortex during restingstate, whereas a different subdivision was observed during tasks. This tripartite subdivision involved the anterior, middle and posterior regions. This result is important for the FC during rest, and it is in line with studies on the alteration observed in the principal resting-state networks in patients affected by $\mathrm{MCI}$ and other neurodegenerative disorders. In fact, rs-networks, such as DMN, FPN and DAN, involve different subregions of the cingulate cortex: for instance, anterior and posterior regions of the cingulate cortex are key nodes of DMN, whereas FPN involves the midcingulate cortex (Vincent et al., 2008; Gilmore et al., 2015). Furthermore, according to Torta et al. (2013), the cingulate cortex modifies its connectivity from a resting-state to an active or "working state." The FC pattern of the cingulate cortex appears more defined than observed during a task. Given the hypotheses from Torta and colleagues about the tripartite rs-dependent parcellation of the cingulate cortex into anterior, middle and posterior portions, we have decided to apply the same tripartition. Subsequently, according to the methods described in Yu et al. (2011), we have selected a total of 9 ROIs to study their FC pattern (and alterations) in a group of MCI patients and a group of healthy elderly. Given the subregional differentiation of the cingulate cortex and its role in several RSNs, such as DMN, DAN and SN, that suffer from aging-related cognitive and emotional changes in healthy subjects and MCI patients, we decided to compare the FC patterns of the cingulate subregions in a sample of MCI and healthy elderly subjects.

We observed specific alterations in the FC pattern in subregions of the anterior, posterior and middle cingulate. These results are globally in line with the hypothesis from
Torta et al. (2013), even though we did not test our patients with cognitive or emotional tasks.

\section{MATERIALS AND METHODS}

\section{Participants}

We conducted all procedures following Helsinki Declaration principles, and the study was approved by the Institutional and Ethics Committee of the University "G. d'Annunzio" of Chieti-Pescara. Nineteen healthy elders (age range: 61-72 y.o.; mean age: 65.2) and 16 MCI patients (age range 64-87 y.o.; mean age: 73.6$)$ were included. All participants had comparable levels of education (8-10 years) and were neurologically examined to exclude visual and motor impairments, major medical conditions, psychiatric or neurological disorders and consumption of psychotropic drugs.

\section{Neuropsychological Assessment}

MCI status was assigned according to the Petersen criteria (Petersen and Negash, 2008). Cognitive status of elders and MCI patients was evaluated using the following neuropsychological tests: Mini-Mental State Examination (MMSE) to evaluate the global cognitive status (26-30: normal; 25-23: MCI; <22: cognitive impairment); prose memory test (Babcock story) to evaluate prose memory (immediate recall cut-off: 5.33; delayed recall cut-off: 5.07) and the Frontal Assessment Battery (FAB; Appollonio et al., 2005) to screen for global executive functions (14-18: normal; <13: cognitive impairment). The sample was composed of 4 a-MCI single domains, 6 a-MCI multi domains and 6 non-amnestic MCI domains.

\section{Resting-State fMRI Acquisition}

Functional and structural fMRI imaging was performed by means of a Philips Achieva 3 T Scanner (Philips Medical Systems, Best, Netherlands) using a whole-body radiofrequency coil for signal excitation and an eight-channel head coil for signal reception. During the fMRI/MRI scans, all participants were instructed to relax while fixating a white dot in the center of a gray-background screen, projected via an LCD projector and viewed via a mirror placed above the subject's head. To minimize involuntary and voluntary head movement, a foam pad was employed. Restingstate fMRI data were collected in four runs lasting 4 min each. The small pauses between runs were used to check that patients and subjects did not fall asleep. Blood Oxygen Level Dependent data were acquired by means of $\mathrm{T} 2 *$-weighted echo planar imaging (EPI) sequences with the following parameters: TE $35 \mathrm{~ms}$, matrix size $64 \times 64$, FOV $256 \mathrm{~mm}$, in-plane voxel size $4 \times 4 \mathrm{~mm}$, Sensitivity Encoding (SENSE) factor 1.8 anterior-posterior, flip angle $75^{\circ}$, slice thickness $4 \mathrm{~mm}$ and no gap. Functional volumes consisted of 30 trans-axial slices, acquired with a volume TR of $1700 \mathrm{~ms}$ (145 volumes per run). At the end of the session, a highresolution structural volume was acquired via a $3 \mathrm{D}$ fast field echo T1-weighted sequence (sagittal, matrix $256 \times 256$, FOV $256 \mathrm{~mm}$, slice thickness $1 \mathrm{~mm}$, no gap, in-plane voxel size $1 \mathrm{~mm} \times 1 \mathrm{~mm}$, flip angle $12^{\circ}$, TR $9.7 \mathrm{~ms}$ and TE $4 \mathrm{~ms}$ ). 


\section{Data Analysis: Socio-Demographic, Neuropsychological Scores, fMRI}

For the fMRI/MRI data set, the following procedure was applied. The first four volumes of each functional run were discarded to allow T1 equilibration of the MR signal. MRI and fMRI data were analyzed by means of Brain Voyager QX 2.4 software (Brain Innovation, Maastricht, Netherlands). Preprocessing of functional data was performed by sequentially applying slice scan time correction, three-dimensional motion correction, and removal of linear trends from voxel time series. Motion correction was performed using a rigid body registration of EPI images to a reference image represented by the fifth volume of the first run. The root mean square (RMS) of the six realignment parameters (three translations and three rotations) was considered, in order to inspect for runs affected by excessive movement. One run for one, one run for two elders and one run for one MCI patient exceeded a root mean square (RMS) movement of $1.5 \mathrm{~mm}$ and were discarded from further analysis. Preprocessed functional volumes were then co-registered with the corresponding structural dataset. Both structural and functional volumes were then transformed into the Talairach space using a piecewise affine and continuous transformation. Functional volumes were re-sampled at a voxel size of $3 \mathrm{~mm} \times 3 \mathrm{~mm} \times 3 \mathrm{~mm}$. Spatial smoothing with a Gaussian kernel of $6.0 \mathrm{~mm}$ full-width half-maximum was applied to functional images corresponding to two voxels in the resampled data to account for intersubjective variability while maintaining a relatively high spatial resolution. Finally, the four runs of each subject were concatenated, resulting in voxel time courses with 564 time points (423 for the few subjects that contributed with three runs). The realignment to a common reference image and the scaling to a common mean performed in the previous preprocessing steps prevented discontinuities in the concatenated timeseries.

\section{Definition of the Seed-ROIs}

The cingulate cortex has been divided into 9 regions, according to the methods described in Yu et al. (2011). Since several authors did not refer to inter-hemisphere differences, we have decided to select bilateral seed regions of interest (seed-ROIs) according to the cytoarchitectonic structural findings (Vogt and Vogt, 2003; Vogt et al., 2006). Nine bilateral regions of interest (ROIs) were determined using Talairach coordinates (Table 1 and Figure 1). Each ROI was created by means of TalCoord2VOI plug-in with a radius of $5 \mathrm{~mm}$. Each ROI has been drawn in order to include the cytoarchitectonic part of the cortex and avoid the overlapping between two different ones. In particular, the seedROIs have been selected in order to include the anterior, middle and posterior portions of the cingulate cortex. ROI1, 2 and 3 represent the anterior and posterior portions of ACC. ROI1 is situated in correspondence with the middle BA 24 of the ACC, whereas ROI2 is positioned in correspondence with the ventral BA24 of the ACC. BA 32, the most anterior part of the ACC, is represented by the ROI3. ROI4, 5 and 6 have been selected to include the MCC. Specifically, ROI4 represents the ventral part of BA 24 in correspondence of the MCC. ROI5 includes the dorsal part of BA 24 in the MCC and the posterior part of BA 24 is represented by ROI6. ROI7, 8 and 9 include the posterior portion of the cingulate. The ventral area of PCC (BA 23) is represented by ROI7, whereas ROI8 includes the RSCC (BA 29). Finally, the dorsal PCC, also known as BA 31, is included in ROI9.

\section{Statistical Analysis \\ Analysis of Neuropsychological Scores and Sociodemographic Data}

To assess between-group differences of sociodemographic data and neuropsychological scores, a series of two-tailed $t$ tests, corrected for multiple comparisons, has been applied. The Healthy Elderly group was significant younger than the MCI group $(t=-5.11, p<0.001)$, but the two groups showed the same levels of education $(t=0.70, p=0.48)$.

The Levene test showed non-homogeneous variances for immediate recall and delayed recall $(p<0.01$ and $p<0.005$ respectively). Thus, a non-parametric Mann-Whitney test has been applied to assess significant differences for immediate recall $(U=32, p<0.05)$ and delayed recall $(U=51, p<0.05)$. The $t$-test applied for MMSE and FAB showed significant between-group differences $(t=9.53, p<0.001$ and $t=6.94$, $p<0.001$ respectively).

Finally, a one-way MANCOVA was performed to assess the effect of age on the testing results, showing no significant effect for the age as covariate $[\lambda=0.94 ; F(4,21)=0,29 ; P=0,88]$. To assess between-group differences for the dichotomous variable of sex, a series of $\chi^{2}$ tests with Yates correction were performed. No significant differences were observed between Healthy Elderly and $\operatorname{MCI}\left(\chi^{2}=0.6 ; p<0.81\right.$ Yates corrected $)$.

\section{fMRI Data Analysis}

Whole brain seed-based connectivity maps, related to the cingulate cortex ROIs, were created for all subjects. Then, correlations were calculated between ROI time-courses (i.e., the time-course in each of the ROI) and all the time-courses of the brain voxels. BOLD time-courses were extracted from each ROI by obtaining an average value for each voxel of the ROI modeled for each single subject. To reveal FC patterns that were consistent for the two groups (Healthy Elderly and MCI) in relation to each of the nine cingulate subregions, we proceeded in the following way: after applying Fisher's r-to-z transformation to each correlation map, random-effect analysis was independently performed for both study groups. FC maps were computed according to the guidelines from Margulies et al. (2007). Nuisance covariates were included in the analyses to reduce effects of physiological processes such as fluctuations related to cardiac and respiratory cycles, or to motion. To this aim, we included eight additional covariates that modeled nuisance signals sampled from white matter (WM) and cerebrospinal fluid (CSF), as well as from six motion parameters (3 rotations and 3 translations as saved by the $3 \mathrm{D}$ motion correction). We derived WM/CSF nuisance signals averaging voxel time courses from each subject's whole brain WM/CSF masks. These masks were generated by the segmentation process of each subject's brain by means of brain voyager QX. After a z-normalization applied to the all seedbased predictors, the analyses, repeated with each subdivision of 
TABLE 1 | Regions of Interest in the Cingulate cortex according the Talairach coordinates and number of voxels for MCl and Healthy Elderly.

\begin{tabular}{lcccc}
\hline & \multicolumn{3}{c}{ Cingulate cortex - seed regions } & \\
\hline Seed regions & Mean $\boldsymbol{x}$ & Mean $\boldsymbol{y}$ & Mean $\boldsymbol{z}$ & Number of voxels \\
\hline BA 24 cV & 0 & 20 & 24 & 257 \\
BA24a & 0 & 27 & 20 & 257 \\
BA 32 & 0 & 31 & 30 & 257 \\
BA a24b & 0 & 17 & 40 & 257 \\
BA p24a & 0 & -7 & 34 & 257 \\
BA p24b' & 0 & -13 & 29 & 257 \\
BA 23 & 0 & -26 & 23 & 257 \\
BA 29 & 0 & -46 & 33 & 257 \\
BA 31 & 0 & -34 & 257 \\
\hline
\end{tabular}

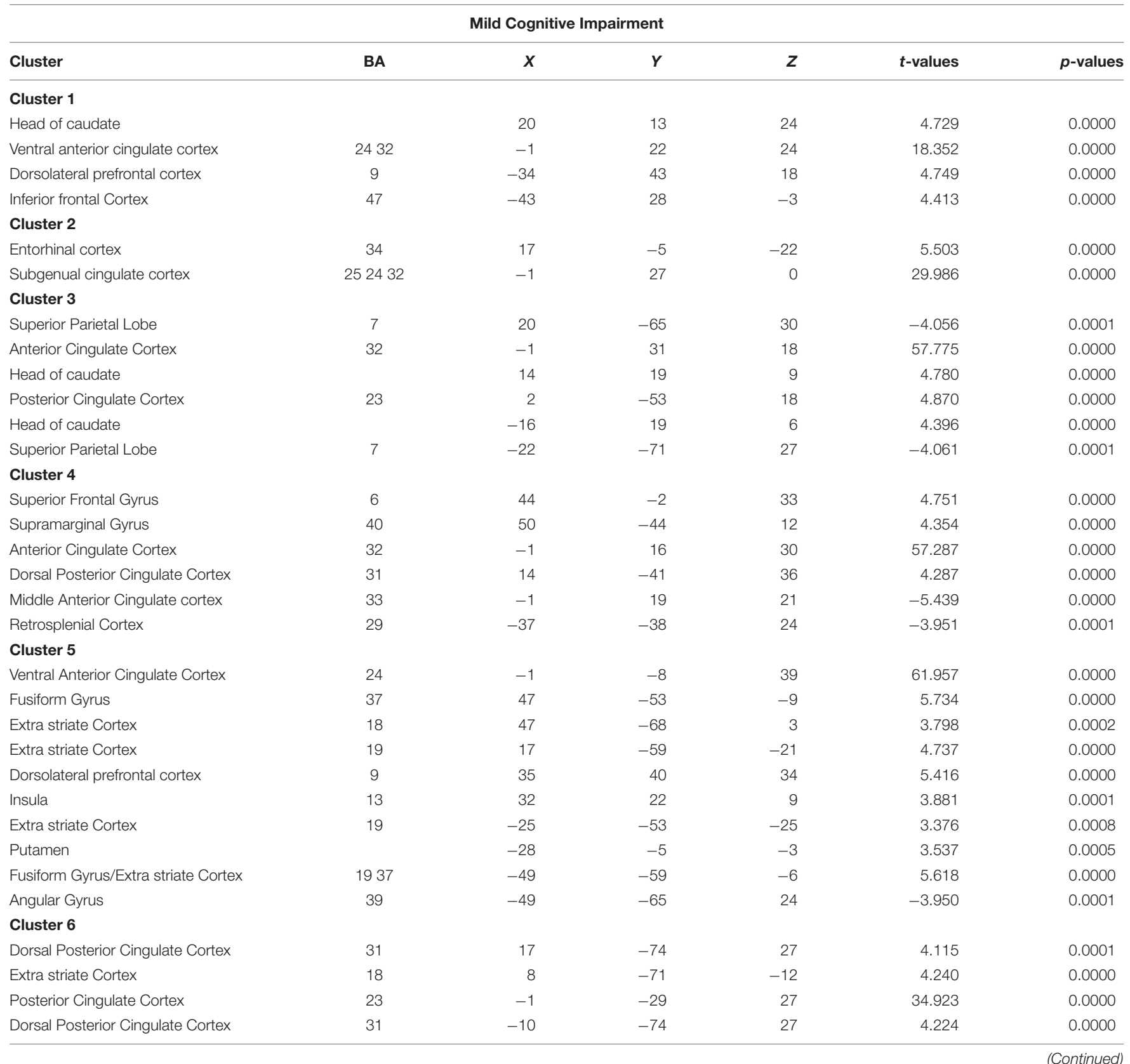


TABLE 1 | Continued

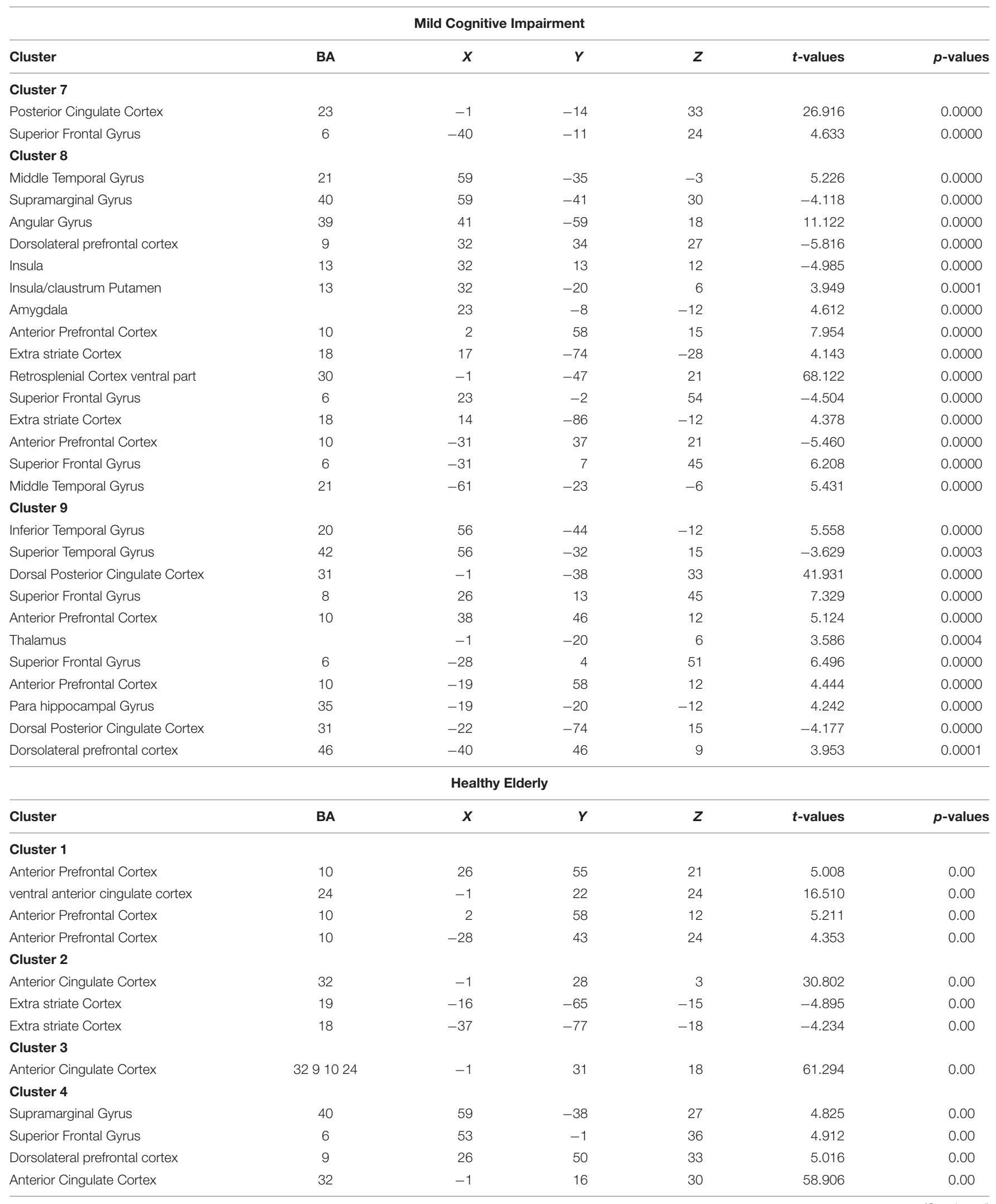


TABLE 1 | Continued

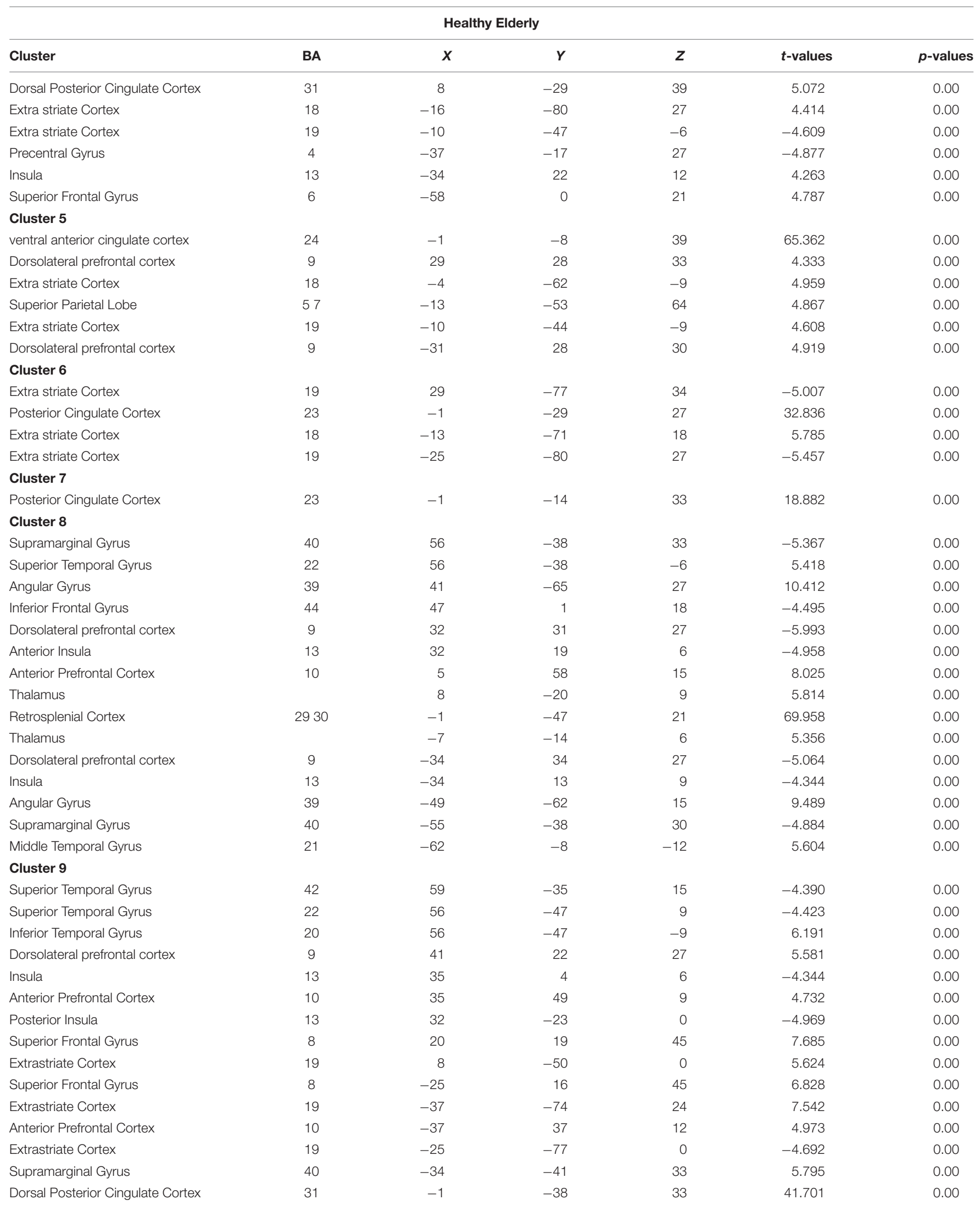




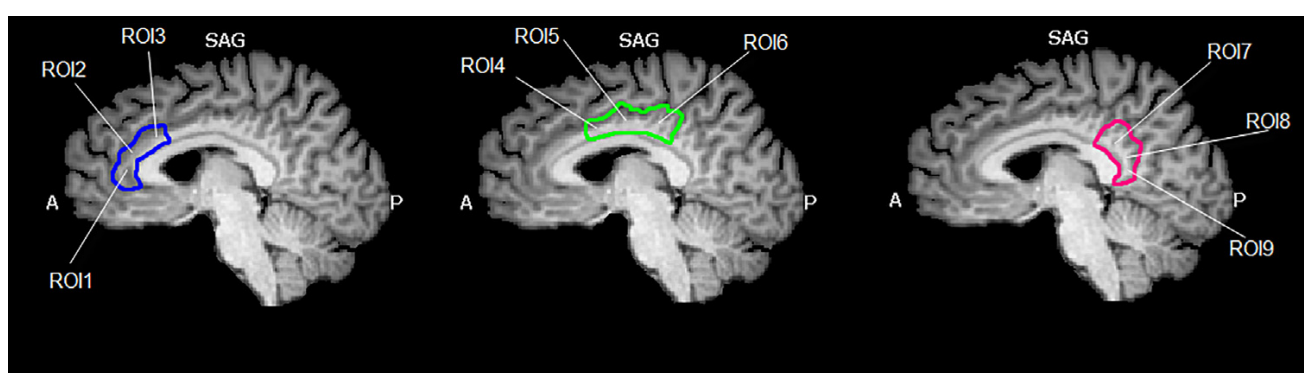

FIGURE 1 | Region of interest (ROIs) used. Image shows the ROls used for the FC analysis and their spatial location in the cingulate cortex. The cingulate cortex has been divided into three functional and anatomical parts. The blue part comprises the anterior cingulate cortex, the green part represents the set of ROls included in the midcingulate cortex and the red one represents the posterior cingulate cortex. Images have been drawn on a Talairach atlas and in radiological convention.

the cingulate cortex, were inserted in a regression model. For each seed ROI, a subject and group FC map were computed on a voxel-wise basis. For each subject, the General Linear Model (GLM) for multiple regression analysis produced 9 ROI-based t-maps and a global map based on the whole cingulate cortex. To assess between-group differences, an RFX-GLM was carried out and a mixed-design voxel-wise ANOVA, with 2 between-group levels for each seed-ROI, was performed. Statistical significance has been assessed by setting a threshold that was corrected by the False Discovery Rate (FDR) with $q<0.05$ and $p<0.05$. To avoid circularity effects, statistical analyses were performed following guidelines form Kriegeskorte et al. (2009). Circularity in the statistical, and specifically in the fMRI data analysis, is considered a logical fallacy occurring when the same data are used two or more times in the same analysis. This effect affects the results creating false-positive and violates the assumption of statistical independence. For this reason, we opted for anatomical selection of the ROIs and between-group comparison. However, given the significant between-group difference in the age and to study the contribution of the neuropsychological test scores to FC patterns, we performed a series of MANCOVAs on the BOLD signal extracted from the clusters resulting from the betweengroup comparison.

\section{RESULTS}

\section{Healthy Elderly Group FC of the Cingulate Cortex}

The FC maps for each seed-ROI of the cingulate cortex in the healthy elderly group are shown in the Figure $\mathbf{2}$ and detailed described in Table 1. ROI1, middle BA 24 of the ACC: the FC pattern included the involvement of the bilateral anterior dorsal portion of the ACC, in particular BA 32 and the left dorsolateral prefrontal cortex (dlPFC) and the inferior frontal gyrus (IFG). Moreover, this region established a FC with the head of the caudate. ROI2, ventral BA24 of the ACC: the ventral part of BA 24 in the ACC established a FC pattern with the entorhinal cortex, in which a peak has been observed at the ventral part of BA 32 and subgenual anterior cingulate cortex (sgACC). ROI3, the most anterior part of the ACC (BA 32): BA32 showed negative FC with bilateral superior parietal lobules (SPL), whereas positive FC has been observed with the anterior part of the ACC and posterior part of the cingulate cortex, and subcortically with the head of caudate. ROI4, the ventral part of the MCC (BA 24): The pattern observed involves principally the right supramarginal gyrus and the other subregions of the cingulate cortex, like the left RSCC, MCC and ACC and the right dorsal PCC. ROI5, dorsal part of the MCC (BA 24): the dorsal regions of the MCC showed positive FC with the bilateral extrastriate cortices and the bilateral fusiform gyrus, right insula, right dlPFC and left putamen. Negative FC was observed with the left AG. ROI6, posterior part of the MCC (BA24): Established FC with extra striate cortex and posterior and dorsal PCC. ROI7, ventral area of PCC (BA 23): The ventral part of BA 23 established a FC with the left superior frontal gyrus (SFG). ROI8, retrosplenial cortex (BA 29): The RSCC showed positive FC in the right hemisphere with the MidTempG, the angular gyrus, the amygdala, the anterior prefrontal cortex, the SFG and the extra striate cortex. The same hemisphere showed negative FC with the supramarginal gyrus, the dlPFC and the insula. In the left hemisphere BA 29 showed a consistent positive FC pattern with the SFG, the MidTempG and the ventral portion of the retrosplenial cortex. Negative FC has been observed with the anterior prefrontal cortex and the SFG. ROI9- PCC: the PCC in the healthy elderly group showed positive $\mathrm{FC}$ in the right hemisphere with the inferior temporal gyrus, the superior frontal gyrus and the anterior prefrontal cortex. In the left hemisphere the same positive pattern has been observed with the dorsal PCC, the thalamus and the SFG. The only negative correlation has been found in correspondence with the right superior temporal gyrus.

\section{Cingulate Cortex FC in the $\mathrm{MCl}$ Group}

The FC maps for each seed-ROI of the Cingulate Cortex in the MCI group are shown in Figure 2 and described in Table 1. ROI1, middle BA 24 of the ACC: The FC pattern observed in the MCI group showed the involvement of the right head of the caudate, and in the left hemisphere the ventral ACC, the dlPFC and the inferior frontal cortex. ROI2, ventral BA 24 of the ACC: The FC pattern was observed in correspondence with the right entorhinal cortex and the left sgACC. ROI3, the most anterior part of the ACC (BA 32): A positive FC pattern was found in the bilateral head of the caudate and PCC; negative FC was observed in 
correspondence with the bilateral SPL. ROI4, the ventral part of the MCC (BA 24): A positive FC pattern was observed in the right hemisphere in the SFG, the supramarginal gyrus and the dorsal PCC. In the left hemisphere, a positive FC pattern was observed in the MCC and retrosplenial cortex. ROI5, dorsal part of the MCC (BA 24): The FC pattern observed for the right hemisphere involved the ventral ACC, the fusiform gyrus, the extra striate cortex, the dlPFC and the insula. A positive FC pattern was also found in the left putamen, left fusiform gyrus. Meanwhile, we found negative FC in correspondence with the left angular gyrus. ROI6, posterior part of the MCC (BA24): We observed a positive FC with the bilateral dorsal PCC and the extra striate cortex in the right hemisphere. ROI7, ventral area of posterior cingulate cortex (BA 23): A positive FC was observed with the PCC and the left SFG. ROI8, retrosplenial cortex (BA 29): A positive FC pattern was observed in the right hemisphere with the MidTempG, the $\mathrm{AG}$, the right insula/claustrum putamen, the amygdala, anterior PFC, extra striate cortex and ventral portion of the retrosplenial cortex. In the same hemisphere, a negative pattern has been found with supramarginal and superior prefrontal cortex and anterior insula. ROI9, PCC: A positive FC pattern was observed between the PCC and the right inferior temporal gyrus, dlPFC, anterior prefrontal cortex, SFG and extrastriate cortex. Conversely, negative correlations were observed with the right superior temporal gyrus, IFG, and anterior and posterior insula. In the left hemisphere, a positive significant FC pattern was found with the superior temporal gyrus, extrastriate cortex, anterior prefrontal cortex, suparmarginal gyrus and dorsal PCC, whereas a negative pattern was observed with the extrastriate cortex (Table 1 and Figure 2).

\section{Between-Group Results}

Among the FC patterns, we found only three between-group differences. Given the exploratory purpose of the present study, all the differences have been calculated on a whole brain manner, avoiding putting the focus only on specific regions involved in a given pathway. After baseline analysis, we then performed the between-group analysis for each seed ROI map, setting the significant $p$ level at $p<0.05$ FDR corrected. The comparison between healthy elderly compared with the MCI group showed a significant increased level of FC for the ROI2 in the elderly healthy group in correspondence with the bilateral caudate and left central ventromedial prefrontal cortex (vmPFC), identified with BA 10-32. Meanwhile, for ROI5 the healthy group showed increased levels of FC in correspondence with the motor cortex, S2, bilateral and right SPL. The comparison MCI with healthy elders highlighted the increased level of FC in the MCI group for ROI9 in correspondence with the middle frontal cortex, ACC and precentral sulcus (Table 2 and Figure 3 ). The MANCOVA was preformed to assess the effect of age on the clusters from the FC pattern of ROI2, ROI5 and ROI9 but showed no significant results $(\lambda=0.55, p=0.11, \lambda=0.49$, $p=0.07 ; \lambda=0.86, p=0.75)$. No significant correlations have been observed between BOLD signal extracted for the resulting clusters of the above-mentioned ROIs and neuropsychological test scores.

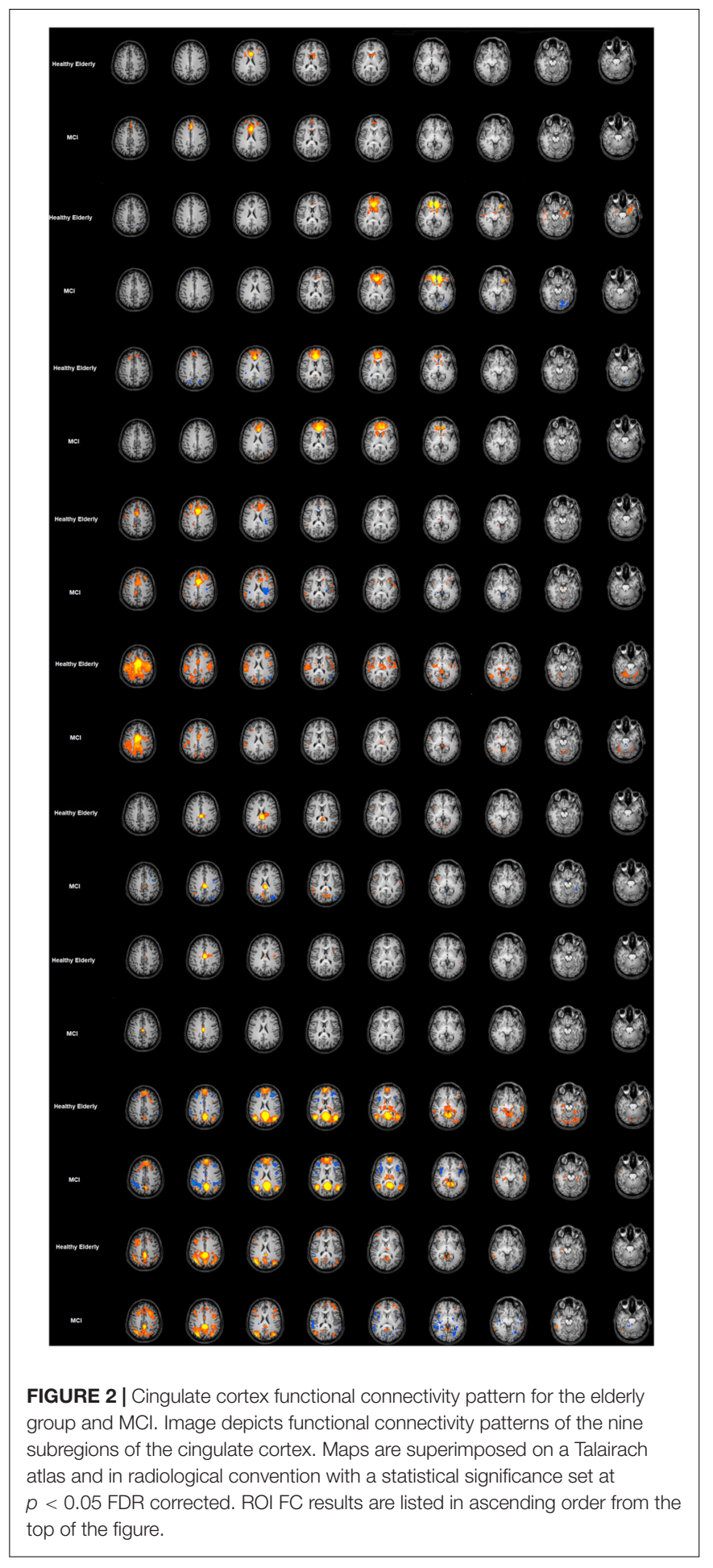

\section{DISCUSSION}

The cingulate cortex has historically been conceived of as a multifaceted brain structure in both cytoarchitectural and functional framework. Its high inner variability should be reflected in the processes underlying the aging and related clinical condition. 
TABLE 2 | Between group comparison ( $\mathrm{MCl}$ > Elderly) for the three seed ROls.

\begin{tabular}{|c|c|c|c|c|c|c|c|c|}
\hline & BA & Hemisphere & Peak X & Peak Y & Peak Z & Number of voxels & $t$ & $p$ \\
\hline \multicolumn{9}{|l|}{ ROI2: Clusters } \\
\hline Occipital Lobe & $18 / 19$ & $\mathrm{R}$ & 23 & -80 & 37 & 509 & -5.35 & 0.00 \\
\hline Caudate Nucleus & & $\mathrm{R}$ & 11 & 7 & 9 & 426 & -5.07 & 0.00 \\
\hline Caudate Nucleus & & $L$ & -10 & 16 & 12 & 1358 & -5.36 & 0.00 \\
\hline Lateral occipital cortex & $18 / 19$ & $\mathrm{~L}$ & -28 & -86 & 15 & 674 & -7.25 & 0.00 \\
\hline $\mathrm{IFG}$ & 10 & $\mathrm{~L}$ & -34 & 22 & 24 & 1262 & -4.80 & 0.00 \\
\hline Rolandic Operculum & 22 & $\mathrm{~L}$ & -46 & -8 & 6 & 375 & -5.07 & 0.00 \\
\hline Ventral IFG & 47 & $\mathrm{~L}$ & -40 & 28 & -3 & 314 & -6.91 & 0.00 \\
\hline ITG & 21 & $\mathrm{~L}$ & -46 & -53 & -9 & 549 & -5.56 & 0.00 \\
\hline STG & 41 & $\mathrm{~L}$ & -55 & -17 & 12 & 1569 & -5.50 & 0.00 \\
\hline Lateral occipital cortex & 1819 & $\mathrm{~L}$ & -55 & -59 & 0 & 1428 & -6.14 & 0.00 \\
\hline Ventromedial prefrontal cortex & 1032 & $L$ & -17 & 38 & 6 & 624 & -4.34 & 0.00 \\
\hline \multicolumn{9}{|l|}{ ROI5: Clusters } \\
\hline IPL & 41 & $\mathrm{R}$ & 38 & -35 & 9 & 397 & -6.22 & 0.00 \\
\hline anterior Intraparietal Sulcus & 6 & $\mathrm{R}$ & 17 & -17 & 45 & 2204 & -6.66 & 0.00 \\
\hline Thalamus & & $\mathrm{R}$ & 20 & -14 & 0 & 459 & -6.70 & 0.00 \\
\hline Sensory-motor cortex & 34 & $\mathrm{R}$ & 2 & -35 & 60 & 3689 & -6.92 & 0.00 \\
\hline Retrosplenial cortex/Lingual Gyrus & 30 & $\mathrm{R}$ & 5 & -65 & 9 & 462 & -5.01 & 0.00 \\
\hline Occipital cortex & 1819 & $L$ & -16 & -71 & -6 & 2588 & -7.89 & 0.00 \\
\hline Precuneus & 3029 & $\mathrm{~L}$ & -16 & -62 & 6 & 639 & -5.37 & 0.00 \\
\hline Caudal cingulate & 326 & $L$ & -7 & 7 & 39 & 316 & -5.45 & 0.00 \\
\hline Middle Frontal Gyrus & 6 & $\mathrm{~L}$ & -13 & -17 & 63 & 633 & -5.67 & 0.00 \\
\hline Medial Frontal Gyrus & 6 & $L$ & -13 & 1 & 63 & 330 & -5.26 & 0.00 \\
\hline Anterior Insula/ Putamen & 13 & $L$ & -25 & 22 & 6 & 487 & -4.78 & 0.00 \\
\hline \multicolumn{9}{|l|}{ ROI9: Clusters } \\
\hline Superior Temporal Gyrus & 22 & $\mathrm{R}$ & 53 & -50 & 9 & 337 & -6.32 & 0.00 \\
\hline Lateral Thalamus & & $\mathrm{R}$ & 32 & -23 & -3 & 684 & -6.80 & 0.00 \\
\hline Posterior Cingulate & 31 & $\mathrm{R}$ & 5 & -32 & 36 & 586 & -5.55 & 0.00 \\
\hline Superior Frontal Gyrus & 8 & $L$ & -25 & 19 & 39 & 677 & 6.11 & 0.00 \\
\hline Orbitofrontral/ventrolateral prefrontal cortex & 11 & $\mathrm{~L}$ & -40 & 34 & -6 & 430 & 6.24 & 0.00 \\
\hline Frontal eye fields & 6 & $\mathrm{~L}$ & -37 & -5 & 36 & 488 & 7.32 & 0.00 \\
\hline Inferior Middle Temporal Sulcus & 37 & $\mathrm{~L}$ & -49 & -62 & 3 & 314 & -6.85 & 0.00 \\
\hline
\end{tabular}

BA, Broadmann Areas; IPL, Inferior parietal lobe; IFG, Inferior Frontal Gyrus; ITG, Inferior Temporal Gyrus; STG, Superior Temporal Gyrus.

In healthy young subjects it is possible to distinguish several regions based on the classification performed by Broadman in the early 1900. This classification has been used and adapted to the subsequent fMRI findings. One of the most popular classifications of the cingulate cortex, in terms of functions and anatomy, is the four-regions model theorized and studied by Vogt (2005).

We analyzed the resting-state FC patterns of the human cingulate cortex in healthy elderly and MCI individuals, for its anatomically and functionally predefined subregions (Vogt, 2005). Using a seed-based FC approach, we found that each cingulate subregion establishes connections with specific brain regions susceptible to be altered by the mechanisms underlying pathological aging decline.

Vogt's (2005) four-region model was proposed based on integrated neurobiological assessments, and it represents a highly detailed neurobiological and anatomical model of this brain structure. The borders of the regions were defined according anatomical markers. Each region has similar cytoarchitectural structure and a common circuitry and functions. This model allows the investigation of the cingulate cortex's functions in healthy brain, several neuropsychiatric diseases and neurodegenerative processes.

Both the ventral part of the ACC and the vmPFC are anterior parts of the DMN, and functional brain imaging studies have often shown increased activity in prefrontal brain regions in older adults. This has been proposed to reflect a compensatory shift to greater reliance on PFC, helping to maintain cognitive function (Morcom and Henson, 2018). In fact, one specific approach, the posterior-to-anterior shift in aging (PASA) theory, states that during cognitively demanding tasks the recruitment of anterior regions such as PFC contributes to maintenance of cognitive performance when posterior cortical function is impaired (Davis et al., 2008; Grady, 2012). Our results show that during resting-state the MCI group have increased FC in regions more dorsal-posterior - such as the precentral sulcus and middle frontal cortex - compared to the healthy elderly group, in which we observed a significant increased level of FC 


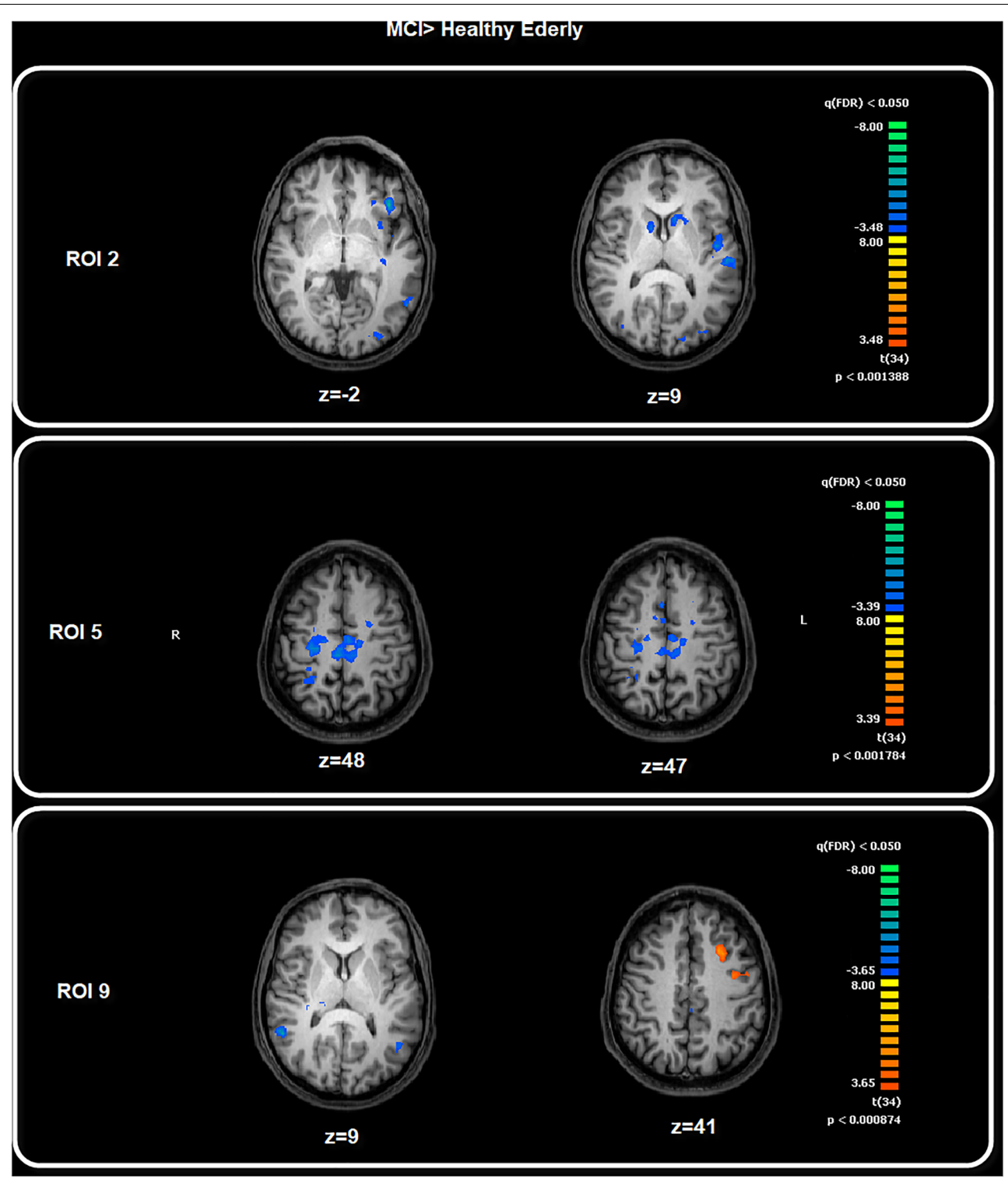

FIGURE 3 | Between-group comparison: $\mathrm{MCl}>$ Healthy Elderly. Image depicts the significant between-group differences for the FC pattern. Maps are superimposed on a Talairach atlas and in radiological convention with a statistical significance set at $p<0.05$ FDR corrected.

for the ventral part of ACC (ROI2) with the bilateral caudate, the vmPFC and also in the dorsal part of the MCC (ROI5), the somatomotor regions and the SPL, showing a greater ability to connect regions that are more anterior-posterior. The increased FC in regions more dorsal-posterior could be interpreted as a compensation mechanism to counteract decay affecting posterior regions primarily affected by impairment in neurodegenerative processes such as MCI and AD.

Morcom and Henson (2018) claim alternatively that activity may become less specific as people age. The authors pointed out that nevertheless that the increased activity in PFC in older adults carried less information about memory outcomes than activity in visual regions, underlying that the optimal function depends on successful brain maintenance rather than compensation.
The vmPFC is an important region connected to the ventral tegmental area (VTA), amygdala, temporal lobe, olfactory system and the dorsomedial thalamus. It sends signals to many different brain regions, including the temporal lobe, amygdala, lateral hypothalamus, hippocampal formation and cingulate cortex (Decety and Michalska, 2010). This fundamental network of connections affords to the vmPFC the ability to receive and monitor large amounts of sensory data and to affect and influence a plethora of other brain regions, particularly the amygdala. We can speculate that this increased FC with this area can support the maintenance of a better cognitive state in the healthy elderly.

In our case, FC increased in the healthy elderly group in the caudate nucleus as well as part of striatum, which coordinates multiple aspects of cognition, including motor and 
action planning, decision-making, motivation, reinforcement and reward perception (Ferré et al., 2010; Taylor et al., 2013; Yager et al., 2015). Striatal outputs from both the dorsal and ventral components are primarily composed of medium spiny neurons (MSNs), a type of projection neuron, which have two primary phenotypes: "indirect" MSNs that express D2-type receptors and "direct" MSNs that express D1-type receptors (Ferré et al., 2010; Yager et al., 2015). The increased FC in this area could be interpreted as good brain maintenance and an implicit monitor capable of screening the healthy elderly group regarding regions important in many aspects of cognition, motor, action planning and motivation. If damaged in their connections and in their networks, compromises typical of MCI can occur.

The caudate nucleus contributes to behavior through the excitation of correct action schemas and selection of appropriate sub-goals based on an evaluation of action-outcomes; both processes are fundamental to successful goal-directed action (Grahn et al., 2009) and our result of increased FC of ventral ACC with the caudate could be interpreted as an adaptive and successful behavior of healthy elderly in evaluation and selection tasks, an important feature for healthy cognition.

We found that cognitive decline observed in the MCI affected the global FC of the cingulate cortex and noteworthily the dorsal posterior MCC, showing a significant difference in the FC pattern in comparison to the healthy elders. The MCC can be sub-divided into two regions, which are, according to Vogt (2016), anterior and posterior. The MCI showed decreased FC in correspondence with the motor and premotor regions: the pattern of the seed ROI we defined as the "dorsal posterior MCC" was in correspondence with these bilateral motor and premotor regions. Connection with the parietal cortex plays a crucial role in modulating MCC during multisensory action monitoring, and the posterior MCC plays a primary role in the spatial reorientation of body and motor reaction to sensory and noxious stimuli (Morrison et al., 2007). In particular, the MCC processes statistical information that tracks the extent to which the outcomes of decisions meet goals when interacting with others and processes information about rewards that guide decision-making (Apps et al., 2013). This statistical processing of information seems to link this region to the "reduction of complexity" (Friston, 2010), following the approach in which the brain-mind system is perpetually committed in active inference by integrating external and internal stimuli, analyzing data from the sensorium and from external reality, trying to keep down the entropy levels and minimizing the possibility of surprise, thus modulating a motivational state that obtains homeostasis (Damasio et al., 2000). In our study, the MCC established FC connections with different portions of the parietal cortex, which as we noticed plays a crucial role in modulating the MCC during multisensory action monitoring. Recent findings suggest the existence of a frontoparietal control system consisting of flexible hubs that regulate distributed systems (e.g., visual, limbic, motor) according to current task goals (Cole et al., 2014; Cieri et al., 2017). The decreased FC in correspondence with these regions from the MCI group could represent one of the causes at the base of their cognitive impairment, being impaired in their statistical processing of information.
As proposed by Apps et al. (2013), the MCC plays an important role in processing information about the rewards others will receive and the decisions that lead to others' rewarding outcomes.

During aging, the pattern of movements can be altered by several acquired peripheral difficulties that could affect the organization of the somatomotor brain regions. This is more evident in the MCI group. The motor cortex has been found to be involved in the programming of movements (Rizzolatti et al., 1996) and in recognition of others' actions (Gallese et al., 2004; Fadiga et al., 2005; Goldman and Sripada, 2005; Keysers and Gazzola, 2009; Keysers et al., 2010). Perhaps in the MCI, these mechanisms implemented in the motor cortex and related to the activity of MCC should be affected by the alterations concurrent in the MCI patients. MCI subjects showed impairment in the recognition of emotional expression, particularly happy, sad and fearful facial expressions (Weiss et al., 2008). Interestingly, Weiss et al. (2008) observed the worst performance in the MCI multiple domain and not in the group of single-domain MCI patients. The functional circuit between the MCC and motor regions could play a role in the interface between the motor and emotion systems.

Recognition of an emotional expression could be affected by impairment of the memory retrieval processes, which in turn affects the planning of emotion-related body/face action/reaction. Our hypothesis is coherent with the transient impairment of motor cortex excitability, caused by transcranial magnetic stimulation (TMS), during emotion processing (Hajcak et al., 2007). Moreover, previous findings reported an indirect effect of unpleasant pictures on motor cortex excitability (Oliveri et al., 2003), which pointed out a facilitatory effect of the action on the emotions. It is plausible that the MCC and its subdivision could play a role in emotion recognition, working memory and emotion expression by means of their connections with the motor and premotor cortex, again facilitating the system in statistical information processing.

Our study highlights a new role of the cingulate cortex and in particular the MCC in the processes related to normal and pathological aging. To disentangle the role played by these subregions in mechanisms like motivation, action planning and emotions, future tasks related to fMRI and TMS studies would be helpful, possibly through a larger and more homogenous sample.

The RSCC is considered one of the key regions involved in a series of cognitive functions, such as memory and visuospatial functions (Vann et al., 2009). We observed a specific increased FC connection between retrosplenial and FEF cortices in elders.

The RSCC has been classically found to be involved during spatial navigation (Maguire, 2001) and episodic memory tasks. The FEF (Vernet et al., 2014) is responsible for saccadic movements and awareness. The increased FC between the two regions may reflect the effort to store new spatial information about the environment relevant for the spatial information recall (Hort et al., 2007; Rankin et al., 2007); moreover, it plays an important role during integration and updating of different spatial strategies. Among the functions in which it is involved, the RSCC has a key role in autobiographical memory and emotions. A recent FC study (Cheng et al., 2018) found an increased connectivity between posterior cingulate regions and 
lateral orbitofrontal cortex in depressed patients. It is possible that this posterior cingulate - orbitofrontal system is related to the sad memories, given that the orbitofrontal regions are involved in the non-reward system.

\section{LIMITS OF THE STUDY}

Our work has some limitations that must be acknowledged. The first and most important concerns the relatively small sample size. Furthermore, another important limitation includes the heterogeneity of the MCI sample, a limitation linked to our small sample size. Increasing the sample size, homogeneity of the MCI group and improving neuropsychological evaluation in future studies would allow researchers to better address the relationship between variation in FC and cognitive performance, enhancing the significance of the present results. Moreover, cross-sectional studies could be useful to disentangle the role played by several functional brain circuits relevant in the MCI pre-AD stage, and future studies such as task-related fMRI and TMS studies would be needed to enlighten the role played by resting-state subregions in mechanisms like motivation, action planning and emotions in MCI patients.

\section{CONCLUSION}

Our findings support the idea that abnormalities in restingstate activities of specific brain regions, such as the cingulate cortex, could be a useful additional tool in order to better understand the brain mechanisms of the MCI. Alterations of functional brain activity has been detected in several studies in cognitively impaired individuals. Although the modification of FC still remains unclear, it could be seen as a cerebral plastic reorganization to maintain cognitive functions (Wen et al., 2013) or instead as a neural excitotoxicity representing impending neuronal failure. The posterior-to-anterior shift in aging (PASA) theory describes the recruitment of anterior regions such as the PFC as being able to help the maintenance of cognitive performance during a cognitive task, when posterior cortical function is impaired (Davis et al., 2008; Grady, 2012). In our resting-state study the results show that the MCI group had increased FC in regions more dorsal-posterior such as the precentral sulcus and middle frontal cortex compared to the healthy elderly group in which we observed a significant increased level of FC for the ventral part of the ACC (ROI2) with the bilateral caudate, the vmPFC and also in

\section{REFERENCES}

Allman, J. M., Hakeem, A., Erwin, J. M., Nimchinsky, E., and Hof, P. (2001). bThe anterior cingulate cortex. The evolution of an interface between emotion and cognition. Ann. N.Y.Acad. Sci. 935, 107-117. doi: 10.1111/j.1749-6632.2001. tb03476.x

Appollonio, I., Leone, M., Isella, V., Piamarta, F., Consoli, T., Villa, M. L., et al. (2005). The Frontal Assessment Battery (FAB): normative values in an Italian population sample. Neurol. Sci. 26, 108-116. doi: 10.1007/s10072-0050443-4 the dorsal part of the MCC (ROI5), the somatomotor regions, and the SPL, showing a greater ability to connect regions more anterior-posterior. The increased FC in regions more dorsalposterior could be interpreted as a compensation mechanism to counteract decay affecting posterior regions primarily affected by impairment in neurodegenerative processes such as MCI and Alzheimer's. Otherwise it could implicate an altered FC mechanism, not necessarily of a compensatory nature. We also found decreased FC in correspondence with the motor and premotor regions, the "dorsal posterior MCC." Connection with the parietal cortex plays a crucial role in modulating MCC during multisensory action monitoring. As we noted, the MCC could be linked to the statistical processing of information toward a "reduction of complexity" (Friston, 2010) through the integration of external and internal stimuli. This decreased FC in the MCI subjects could be linked to their impairment in the information processing and their consequent reduced cognitive ability. Longitudinal neuroimaging studies, homogeneity in the MCI sample, improvement of sample size and improving neuropsychological evaluation in future studies would allow researchers to better address the relationship between variation in FC and cognitive performance, enhancing the significance of the present results.

\section{DATA AVAILABILITY}

The datasets for this manuscript are not publicly available. Data used in the manuscript are covered by privacy because they include sensitive patient information. Requests to access the datasets should be directed to Armando Tartaro (a.tartaro@radiol.unich.it).

\section{ETHICS STATEMENT}

All participants gave written informed consent, and this study was approved by our local ethics committee.

\section{AUTHOR CONTRIBUTIONS}

NC, RE, FC, and AT conceived and designed the study. RE and $\mathrm{FC}$ acquired and collected the clinical data. NC analyzed the data. NC, RE, and FC wrote the manuscript. AT revised the manuscript.

Apps, M. A., Lockwood, P. L., and Balsters, J. H. (2013). The role of the midcingulate cortex in monitoring others' decisions. Front. Neurosci. 7:251. doi: 10.3389/fnins.2013.00251

Awh, E., and Gehring, W. J. (1999). The anterior cingulate cortex lends a hand in response selection. Nat. Neurosci. 2, 853-854. doi: 10.1038/ 13145

Beckmann, M., Johansen-Berg, H., and Rushworth, M. F. (2009). Connectivitybased parcellation of human cingulate cortex and its relation to functional specialization. J. Neurosci. 29, 1175-1190. doi: 10.1523/JNEUROSCI.3328-08. 2009 
Behrens, T. E. J., Hunt, L. T., and Rushworth, M. F. S. (2009). The computation of social behavior. Science 324, 1160-1164. doi: 10.1126/science.1169694

Botvinick, M. M., Cohen, J. D., and Carter, C. S. (2004). Conflict monitoring and anterior cingulate cortex: an update. Trends Cogn. Sci. 8, 539-546. doi: 10.1016/j.tics.2004.10.003

Brier, M. R., Thomas, J. B., Fagan, A. M., Hassenstab, J., Holtzman, D. M., Benzinger, T. L., et al. (2014). Functional connectivity and graph theory in preclinical Alzheimer's disease. Neurobiol. Aging 35, 757-768. doi: 10.1016/j. neurobiolaging.2013.10.081

Burgess, N., Maguire, E. A., Spiers, H. J., and O'Keefe, J. (2001). A temporoparietal and prefrontal network for retrieving the spatial context of lifelike events. Neuroimage. 14, 439-453. doi: 10.1006/nimg.2001.0806

Cheng, W., Rolls, E. T., Qiu, J., Xie, X., Wei, D., Huang, C. C., et al. (2018). Increased functional connectivity of the posterior cingulate cortex with the lateral orbitofrontal cortex in depression. Transl Psychiatry. 8:90. doi: 10.1038/ s41398-018-0139-1

Cieri, F., Esposito, R., Cera, N., Pieramico, V., Tartaro, A., and Di Giannantonio, M. (2017). Late-life depression: modifications of brain resting state activity. J. Geriatr. Psychiatry Neurol. 30, 140-150. doi: 10.1177/08919887177 00509

Cole, M. C., Bassett, D. S., Power, J. D., Braver, T. S., and Petersen, S. E. (2014). Intrinsic and task-evoked network architectures of the human brain. Neuron 83, 238-251. doi: 10.1016/j.neuron.2014.05.014

Corbetta, M., Miezin, F. M., Dobmeyer, S., Shulman, G. L., and Petersen, S. E. (1991). Selective and divided attention during visual discriminations of shape, color, and speed: functional anatomy by positron emission tomography. J. Neurosci. 11, 2383-2402. doi: 10.1523/jneurosci.11-08-02383.1991

Corbetta, M., and Shulman, G. L. (2002). Control of goal-directed and stimulusdriven attention in the brain. Nat. Rev. Neurosci. 3, 201-215. doi: 10.1038/ nrn755

Damasio, A. R., Grabowski, T. J., Bechara, A., Damasio, H., Ponto, L. L. B., Parvizi, J. et al. (2000). Subcortical and cortical brain activity during the feeling of self-generated emotions. Nat. Neurosci. 3, 1049-1056.

Davis, S. W., Dennis, N. A., Daselaar, S. M., Fleck, M. S., and Cabeza, R. (2008). Que PASA? The posterior-anterior shift in aging. Cereb. Cortex 8, 1201-1209. doi: $10.1093 /$ cercor/bhm155

Decety, J., and Michalska, K. J. (2010). Neurodevelopmental changes in the circuits underlying empathy and sympathy from childhood to adulthood. Dev. Sci. 13, 886-899. doi: $10.1111 / j .1467-7687.2009 .00940 . x$

Deco, G., Jirsa, V. K., and McIntosh, A. R. (2011). Emerging concepts for the dynamical organization of resting-state activity in the brain. Nat. Rev. Neurosci. 12, 43-56. doi: 10.1038/nrn2961

Dubois, B., and Albert, M. L. (2004). Amnestic MCI or prodromal Alzheimer's disease? Lancet Neurol. 3, 246-248. doi: 10.1016/s1474-4422(04)00710-0

Esposito, R., Mosca, A., Pieramico, V., Cieri, F., Cera, N., and Sensi, S. L. (2013). Characterization of resting state activity in MCI individuals. PeerJ 1:e135. doi: 10.7717/peerj.135

Fadiga, L., Craighero, L., and Olivier, E. (2005). Human motor cortex excitability during the perception of others' action. Curr. Opin. Neurobiol. 15, 213-218. doi: 10.1016/j.conb.2005.03.013

Ferré, S., Lluís, C., Justinova, Z., Quiroz, C., Orru, M., Navarro, G., et al. (2010). Adenosine-cannabinoid receptor interactions. Implications for striatal function. Br. J. Pharmacol. 160, 443-453. doi: 10.1111/j.1476-5381.2010. 00723.x

Ferreira, L. K., and Busatto, G. F. (2013). Resting-state functional connectivity in normal brain aging. Neurosci. Biobehav. Rev. 37, 384-400. doi: 10.1016/j. neubiorev.2013.01.017

Fleisher, A. S., Sherzai, A., Taylor, C., Langbaum, J. B., Chen, K., and Buxton, R. B. (2009). Resting-state BOLD networks versus task-associated functional MRI for distinguishing Alzheimer's disease risk groups. Neuroimage 47, 1678-1690. doi: 10.1016/j.neuroimage.2009.06.021

Fox, M. D., Snyder, A. Z., Vincent, J. L., Corbetta, M., Van Essen, D. C., and Raichle, M. E. (2005). The human brain is intrinsically organized into dynamic, anticorrelated functionalnetworks. Proc. Natl. Acad. Sci. U.S.A. 102, 9673-9678. doi: $10.1073 /$ pnas. 0504136102

Friston, K. (2010). The free-energy principle: a unified brain theory? Nat. Rev. Neurosci. 11, 127-138. doi: 10.1038/nrn2787
Gallese, V., Keysers, C., and Rizzolatti, G. (2004). A unifying view of the basis of social cognition. Trends Cogn. Sci. 8, 396-403. doi: 10.1016/j.tics.2004.07.002

Gilmore, A. W., Nelson, S. M., and McDermott, K. B. (2015). A parietal memory network revealed by multiple MRI methods. Trends Cogn. Sci. 19, 534-543. doi: 10.1016/j.tics.2015.07.004

Goldman, A. I., and Sripada, C. S. (2005). Simulationist models of face-based emotion recognition. Cognition 94, 193-213. doi: 10.1016/j.cognition.2004. 01.005

Grady, C. (2012). The cognitive neuroscience of ageing. Nat. Rev. Neurosci. 13, 491-505. doi: 10.1038/nrn3256

Grahn, J. A., Parkinson, J. A., and Owen, A. M. (2009). The role of the basal ganglia in learning and memory: neuropsychological studies. Behav. Brain Res. 199, 53-60. doi: 10.1016/j.bbr.2008.11.020

Greene, S. J., and Killiany, R. J. (2010). Alzheimer's Disease Neuroimaging Initiative.subregions of the inferior parietal lobule are affected in the progression to Alzheimer's disease. Neurobiol. Aging. 31, 1304-1311. doi: 10. 1016/j.neurobiolaging.2010.04.026

Gröschel, S., Sohns, J. M., Schmidt-Samoa, C., Baudewig, J., Becker, L., Dechent, P., et al. (2013). Effects of age on negative BOLD signal changes in the primary somatosensory cortex. Neuroimage. 71, 10-18. doi: 10.1016/j.neuroimage.2012. 12.039

Hajcak, G., Molnar, C., George, M. S., Bolger, K., Koola, J., and Nahas, Z. (2007). Emotion facilitates action: a transcranial magnetic stimulation study of motor cortex excitability during picture viewing. Psychophysiology 44, 91-97. doi: 10.1111/j.1469-8986.2006.00487.x

Hansen, E. C., Battaglia, D., Spiegler, A., Deco, G., and Jirsa, V. K. (2015). Functional connectivity dynamics: modeling the switching behavior of the resting state. Neuroimage 105, 525-535. doi: 10.1016/j.neuroimage.2014.11.001

Hort, J., Laczó, J., Vyhnálek, M., Bojar, M., Bureš, J., and Vlček, K. (2007). Spatial navigation deficit in amnestic mild cognitive impairment. Proc. Natl. Acad. Sci. U.S.A. 104, 4042-4047. doi: 10.1073/pnas.0611314104

Keene, C. S., and Bucci, D. J. (2008). Involvement of the retrosplenial cortex in processing multiple conditioned stimuli. Behav. Neurosci. 122, 651-658. doi: 10.1037/0735-7044.122.3.651

Keysers, C., and Gazzola, V. (2009). Expanding the mirror: vicarious activity for actions, emotions, and sensations. Curr. Opin. Neurobiol. 19, 666-671. doi: 10.1016/j.conb.2009.10.006

Keysers, C., Kaas, J. H., and Gazzola, V. (2010). Somatosensation in social perception. Nat. Rev. Neurosci. 11, 417-428. doi: 10.1038/nrn2833

Kriegeskorte, N., Simmons, W. K., Bellgowan, P. S., and Baker, C. I. (2009). Circular analysis in systems neuroscience: the dangers of double dipping. Nat. Neurosci. 12, 535-540. doi: 10.1038/nn.2303

Lyketsos, C. G., Carrillo, M. C., Ryan, J. M., Khachaturian, A. S., Trzepacz, P., Amatniek, J., et al. (2011). Neuropsychiatric symptoms in Alzheimer's disease. Alzheimers Dement. 7, 532-539. doi: 10.1016/j.jalz.2011.05.2410

Maguire, E. A. (2001). The retrosplenial contribution to human navigation: a review of lesion and neuroimaging findings. Scand. J. Psychol. 42, 225-238. doi: 10.1111/1467-9450.00233

Margulies, D. S., Kelly, A. M., Uddin, L. Q., Biswal, B. B., Castellanos, F. X., and Milham, M. P. (2007). Mapping the functional connectivity of anterior cingulate cortex. Neuroimage 37, 579-588. doi: 10.1016/j.neuroimage.2007.05.019

Mayberg, H. S. (1994). Frontal lobe dysfunction in secondary depression. J. Neuropsychiatry Clin. Neurosci. 6, 428-442. doi: 10.1176/jnp.6.4.428

Mega, M. S., Cummings, J. L., Fiorello, T., and Gornbein, J. (1996). The spectrum of behavioral changes in Alzheimer's disease. Neurology 46, 130-135. doi: 10. 1212/WNL.46.1.130

Metzler-Baddeley, C., Jones, D. K., Steventon, J., Westacott, L., Aggleton, J. P., and O'Sullivan, M. J. (2012). Cingulum microstructure predicts cognitive control in older age and mild cognitive impairment. J. Neurosci. 32, 17612-17619. doi: 10.1523/JNEUROSCI.3299-12.2012

Mevel, K., Landeau, B., Fouquet, M., La Joie, R., Villain, N., Mézenge, F., et al. (2013). Age effect on the default mode network, inner thoughts, and cognitive abilities. Neurobiol Aging. 34, 1292-1301. doi: 10.1016/j.neurobiolaging.2012. 08.018

Minhas, S., Khanum, A., Riaz, F., Khan, S. A., and Alvi, A. (2018). Predicting progression from mild cognitive impairment to Alzheimer's disease using autoregressive modelling of longitudinal and multimodal biomarkers. IEEE 
J. Biomed Health Inform. 22, 818-825. doi: 10.1109/JBHI.2017.2703918 doi: 10.1109/jbhi.2017.2703918

Morcom, A. M., and Henson, R. N. A. (2018). Increased Prefrontal Activity with Aging Reflects Nonspecific Neural Responses Rather than Compensation. J. Neurosci. 38, 7303-7313. doi: 10.1523/JNEUROSCI.1701-17.2018

Morrison, I., Peelen, M. V., and Downing, P. E. (2007). The sight of others' pain modulates motor processing in human cingulate cortex. Cereb. Cortex 17, 2214-2222. doi: 10.1093/cercor/bhl129

Oliveri, M., Babiloni, C., Filippi, M. M., Caltagirone, C., Babiloni, F., Cicinelli, P., et al. (2003). Influence of the supplementary motor area on primary motor cortex excitability during movements triggered by neutral or emotionally unpleasant visual cues. Exp. Brain Res. 149, 214-221. doi: 10.1007/s00221-0021346-8

Pardo, J. V., Fox, P. T., and Raichle, M. E. (1991). Localization of a human system for sustained attention by positron emission tomography. Nature 349, 61-64. doi: 10.1038/349061a0

Passow, S., Specht, K., Adamsen, T. C., Biermann, M., Brekke, N., Craven, A. R., et al. (2015). Default-mode network functional connectivity is closely related to metabolic activity. Hum. Brain Mapp. 36, 2027-2038. doi: 10.1002/hbm.22753

Petersen, R. C. (2004). Mild cognitive impairment as a diagnostic entity. J. Int. Med. 256, 183-194. doi: 10.1111/j.1365-2796.2004.01388.x

Petersen, R. C., and Negash, S. (2008). Mild cognitive impairment: an overview. CNS Spectr 13, 45-53. doi: 10.1017/s1092852900016151

Posner, M. I. (1994). Attention: the mechanisms of consciousness. Proc. Natl. Acad. Sci. U.S.A. 91, 7398-7403.

Rankin, K. P., Mucke, L., Miller, B. L., and Gorno-Tempini, M. L. (2007). Spatial cognition and the human navigation network in AD and MCI. Neurology 69, 986-997. doi: 10.1212/01.wnl.0000271376.19515.c6

Rizzolatti, G., Fadiga, L., Gallese, V., and Fogassi, L. (1996). Premotor cortex and the recognition of motor actions. Brain Res. Cogn. Brain Res. 3, 131-141. doi: 10.1016/0926-6410(95)00038-0

Rombouts, S. A., Barkhof, F., Goekoop, R., Stam, C. J., and Scheltens, P. (2005). Altered resting state networks in mild cognitive impairment and mild Alzheimer's disease: an fMRI study. Hum. Brain Mapp. 26, 231-239. doi: $10.1002 / \mathrm{hbm} .20160$

Shackman, A. J., Salomons, T. V., Slagter, H. A., Fox, A. S., Winter, J. J., and Davidson, R. J. (2011). The integration of negative affect, pain and cognitive control in the cingulate cortex. Nat. Rev. Neurosci. 2011 12, 154-167. doi: $10.1038 /$ nrn2994

Shin, L. M., and Liberzon, I. (2010). The neurocircuitry of fear, stress, and anxiety disorders. Neuropsychopharmacology 35, 169-191. doi: 10.1038/npp.2009.83

Spreng, R. N., Mar, R. A., and Kim, A. S. (2009). The common neural basis of autobiographical memory, prospection, navigation, theory of mind, and the default mode: a quantitative meta-analysis. J. Cogn. Neurosci. 21, 489-510. doi: 10.1162 /jocn.2008.21029

Taylor, S. B., Lewis, C. R., and Olive, M. F. (2013). The neurocircuitry of illicit psychostimulant addiction: acute and chronic effects in humans. Subst Abuse Rehabil 4, 29-43. doi: 10.2147/SAR.S39684

Torta, D. M., Costa, T., Duca, S., Fox, P. T., and Cauda, F. (2013). Parcellation of the cingulate cortex at rest and during tasks: a meta-analytic clustering and experimental study. Front. Hum. Neurosci. 7:275. doi: 10.3389/fnhum.2013. 00275

Van den Heuvel, M. P., and Hulshoff Pol, H. E. (2010). Exploring the brain network: a review on resting-state fMRI functional connectivity. Eur. Neuropsychopharmacol. 20, 519-534. doi: 10.1016/j.euroneuro.2010.03.008
Van den Heuvel, M. P., Mandl, R. C., Kahn, R. S., and Hulshoff Pol, H. E. (2009). Functionally linked resting-state networks reflect the underlying structural connectivity architecture of the human brain. Hum. Brain Mapp. 30, 3127-3141. doi: $10.1002 / \mathrm{hbm} .20737$

Vann, S. D., Aggleton, J. P., and Maguire, E. A. (2009). What does the retrosplenial cortex do? Nat. Rev. Neurosci. 10, 792-802. doi: 10.1038/nrn2733

Vernet, M., Quentin, R., Chanes, L., Mitsumasu, A., and Valero-Cabré, A. (2014). Frontal eye field, where art thou? Anatomy, function, and non-invasive manipulation of frontal regions involved in eye movements and associated cognitive operations. Front. Integr. Neurosci. 8:66. doi: 10.3389/fnint.2014. 00066

Vincent, J. L., Kahn, I., Snyder, A. Z., Raichle, M. E., and Buckner, R. L. (2008). Evidence for a frontoparietal control system revealed by intrinsic functional connectivity. J. Neurophysiol. 100, 3328-3342. doi: 10.1152/jn.90355. 2008

Vogt, B. A. (2005). Pain and emotion interactions in subregions of the cingulate gyrus. Nat. Rev. Neurosci. 6, 533-544. doi: 10.1038/nrn1704

Vogt, B. A. (2009). Cingulate Neurobiology and Disease. Oxford: Oxford University Press.

Vogt, B. A. (2016). Midcingulate cortex: structure, connections, homologies, functions and diseases. J. Chem. Neuroanat. 74, 28-46. doi: 10.1016/j.jchemneu. 2016.01.010

Vogt, B. A., and Vogt, L. (2003). Cytology of human dorsal midcingulate and supplementary motor cortices. J. Chem. Neuroanat. 26, 301-309. doi: 10.1016/ j.jchemneu.2003.09.004

Vogt, B. A., Vogt, L., and Laureys, S. (2006). Cytology and functionally correlated circuits of human posterior cingulate areas. Neuroimage 29, 452-466. doi: 10.1016/j.neuroimage.2005.07.048

Weiss, E. M., Kohler, C. G., Vonbank, J., Stadelmann, E., Kemmler, G., Hinterhuber, H., et al. (2008). Impairment in emotion recognition abilities in patients with mild cognitive impairment, early and moderate Alzheimer disease compared with healthy comparison subjects. Am. J. Geriatr. Psychiatry 16, 974-980. doi: 10.1097/JGP.0b013e318186bd53

Wen, X., Liu, Y., Yao, L., and Ding, M. (2013). Top-down regulation of default mode activity in spatial visual attention. J. Neurosci. 33, 6444-6453. doi: 10. 1523/JNEUROSCI.4939-12.2013

Yager, L. M., Garcia, A. F., Wunsch, A. M., and Ferguson, S. M. (2015). The ins and outs of the striatum: role in drug addiction. Neuroscience 2015, 529-541. doi: 10.1016/j.neuroscience. 06.033

Yu, C., Zhou, Y., Liu, Y., Jiang, T., Dong, H., Zhang, Y., et al. (2011). Functional segregation of the human cingulate cortex is confirmed by functional connectivity based neuroanatomical parcellation. Neuroimage 54, 2571-2581. doi: 10.1016/j.neuroimage.2010.11.018

Conflict of Interest Statement: The authors declare that the research was conducted in the absence of any commercial or financial relationships that could be construed as a potential conflict of interest.

Copyright $\odot 2019$ Cera, Esposito, Cieri and Tartaro. This is an open-access article distributed under the terms of the Creative Commons Attribution License (CC BY). The use, distribution or reproduction in other forums is permitted, provided the original author(s) and the copyright owner(s) are credited and that the original publication in this journal is cited, in accordance with accepted academic practice. No use, distribution or reproduction is permitted which does not comply with these terms. 


\section{OPEN ACCESS}

Edited by:

Roberto Esposito,

A.O. Ospedali Riuniti Marche

Nord, Italy

Reviewed by:

Adam Bauer,

Washington University in St. Louis,

United States

Martin Havlicek,

Maastricht University, Netherlands

*Correspondence:

D. Blair Jovellar

blairjovellar@alumni.ubc.ca

Doris J. Doudet

ddoudet@mail.ubc.ca

Specialty section:

This article was submitted to Brain Imaging Methods,

a section of the journal

Frontiers in Neuroscience

Received: 08 February 2019

Accepted: 30 August 2019

Published: 18 September 2019

Citation:

Jovellar DB and Doudet DJ (2019) fMRI in Non-human Primate: A Review

on Factors That Can Affect

Interpretation and Dynamic Causal

Modeling Application.

Front. Neurosci. 13:973.

doi: 10.3389/fnins.2019.00973

\section{fMRI in Non-human Primate: A Review on Factors That Can Affect Interpretation and Dynamic Causal Modeling Application}

\section{Blair Jovellar ${ }^{1,2 *}$ and Doris J. Doudet ${ }^{1 *}$}

${ }^{1}$ Division of Neurology, Department of Medicine, University of British Columbia, Vancouver, BC, Canada, ${ }^{2}$ Center of Neurology, Hertie Institute for Clinical Brain Research, University Hospital, Tuebingen, Germany

Dynamic causal modeling (DCM) - a framework for inferring hidden neuronal states from brain activity measurements (e. g., fMRI) and their context-dependent modulation-was developed for human neuroimaging, and has not been optimized for non-human primate (NHP) studies, which are usually done under anesthesia. Animal neuroimaging studies offer the potential to improve effective connectivity modeling using DCM through combining functional imaging with invasive procedures such as in vivo optogenetic or electrical stimulation. Employing a Bayesian approach, model parameters are estimated based on prior knowledge of conditions that might be related to neural and BOLD dynamics (e.g., requires empirical knowledge about the range of plausible parameter values). As such, we address the following questions in this review: What factors need to be considered when applying DCM to NHP data? What differences in functional networks, cerebrovascular architecture and physiology exist between human and NHPs that are relevant for DCM application? How do anesthetics affect vascular physiology, BOLD contrast, and neural dynamics - particularly, effective communication within, and between networks? Considering the factors that are relevant for DCM application to NHP neuroimaging, we propose a strategy for modeling effective connectivity under anesthesia using an integrated physiologic-stochastic DCM (IPS-DCM).

Keywords: non-human primate, fMRI, effective connectivity, dynamic causal modeling, image analysis, DCM, anesthesia, BOLD

\section{INTRODUCTION}

Neuroimaging analyses in humans and non-human primates (NHP) have become increasingly sophisticated. One such innovative image analysis technique is dynamic causal modeling (DCM) which has been applied to human fMRI data (Friston et al., 2003; Rowe et al., 2010; Boly et al., 2012; Havlicek et al., 2017; Park et al., 2018; Tak et al., 2018). DCM is a method for inferring hidden neuronal states from brain activity measurements (e.g., fMRI) and their context-dependent modulation (Stephan et al., 2010). Using a Bayesian framework, DCM generates a predicted time serious using a set of differential equations to model neural dynamics. Then, one estimates model parameter by optimally fitting the predicted time series with the observed data. Using DCM, one can test mechanistic hypotheses about how the observed data was generated. 
Utilizing a Bayesian approach, model parameters are estimated based on prior knowledge of conditions that might be related to neural and BOLD dynamics (e.g., requires empirical knowledge about the range of plausible parameter values). As such, we discuss: (1) what factors need to be considered when applying DCM to NHP (keeping in mind that it was designed for human fMRI data); and (2) considering said factors, what strategies can one implement when modeling effective connectivity to fMRI data recorded under anesthesia. The latter is an important consideration in NHP fMRI, since most imaging experiments are done under anesthesia and anesthetics have been demonstrated to impart changes in BOLD and neural dynamics, particularly the inhibitory drive (Martin et al., 2006; Masamoto et al., 2007; Moran et al., 2011; Aksenov et al., 2015; Paasonen et al., 2018).

Herein we discuss the following: (1) DCM overview and motivation for NHP data application; (2) comparison of cerebrovascular architecture and functional networks between humans and NHPs; (3) effects of anesthetics on vascular physiology, BOLD contrast, and neural dynamics-focusing on effective communication within and between networks. Lastly, we propose a strategy for modeling effective connectivity under anesthesia using an integrated physiologic-stochastic DCM (IPSDCM). Taking all these together, we can avoid pitfalls in DCM application in NHP data and facilitate more accurate interpretation of the observed neural dynamics as measured through BOLD fMRI.

\section{DYNAMIC CAUSAL MODELING (DCM) IN NON-HUMAN PRIMATES \\ DCM Overview}

This section discusses an overview of DCM, including its assumptions, and the motivations for NHP data application. DCM, developed by Friston et al. (2003), is a method that allows estimation of network dynamics and how the dynamic neuronal states give rise to the measured data (Heinzle and Stephan, 2018), as well as how functional coupling is affected by experimental factors. When applied to fMRI data, the BOLD signal is considered a measurable (observed) variable (y) of the underlying neural activity $(\mathrm{z})$ that is not directly measured with fMRI; hence, neural activity is considered a "hidden state variable" (Kahan and Foltynie, 2013). As a measure of effective connectivity (EC), DCM quantifies the directed (causal) influence between regions (Friston, 2009) and considers the rate of change of neural activity over time $(\mathrm{z})$ in response to incoming signals-afferents from other brain regions, experimental manipulation, or both. This is done by creating generative models which are plausible models of how the observed BOLD signal may be generated by those influences.

In DCM, one needs to specify which regions to include in the overall model. As such, DCM follows an analysis that can address which regions in the brain an experimental manipulation induces changes in BOLD response such as a general linear model (GLM) (Stephan et al., 2004). In GLM, time series from each voxel are fitted with an experimental design matrix wherein each condition is specified and the relationship between neural and BOLD response is modeled via a hemodynamic response function (Pernet, 2014). When there are no contrast differences between conditions, there is no motivation to do DCM. Once the relevant regions are identified with GLM, the time series from each region of interest is extracted. After which, one specifies the model architecture-the location of intrinsic connections, driving, and modulatory inputs. Then, one proceeds to estimate the parameters of the generative model.

DCM can offer a more accurate modeling of network dynamics from fMRI data as it considers both the distributed neuronal interactions, and the transformation of the neuronal dynamics to the measured (BOLD) signal (Havlicek et al., 2015). This is accomplished through three fundamental components of the DCM generative model: (1) neuronal (connectivity) model, (2) hemodynamic "Balloon" model, and (3) BOLD signal change equation (Stephan et al., 2007; Havlicek et al., 2015; Friston et al., 2017). A discussion on strategies for modifying the generative model for NHP fMRI under, particularly under anesthesia, will be discussed in a later section.

Each region or node is represented by (differential) neural state equations that provide an abstraction of the summed activity of a neuronal population. The neural state equation is comprised of three parameters that embody: (1) intrinsic connectivity among regions in the absence of input (A matrix); (2) direct influence of extrinsic input on neuronal activity ( $\mathrm{C}$ matrix); and (3) context-dependent change in connectivity induced by the input (B matrix). Neural dynamics in DCM have been characterized by single-state, two-state, and adaptive twostate equations. The single-state neural equation (Friston et al., 2003) of the classical DCM models synaptic activity of a single excitatory neuronal population in a cortical region-with the rationale that most cortico-cortical connections are excitatory. Two-state (Marreiros et al., 2008) and adaptive two-state (Havlicek et al., 2015) neural equations model both excitatory (glutamatergic) and inhibitory (GABAergic) connections within each region, which makes it a more physiological representation of neural population dynamics. In contrast to the two-state model, the adaptive two-state model includes parameters which allow for adaptation and refractory effects of the neuronal response (Havlicek et al., 2015). These are described in detail in Friston et al. (2003), Marreiros et al. (2008), Havlicek et al. (2015).

The neural model is combined with the hemodynamic model (Friston et al., 2003)—which incorporates the dynamics of neurovascular coupling and the Balloon-Windkessel model (Buxton et al., 1998; Mandeville et al., 1999). This biophysical forward model provides region-specific estimates of the translation of neuronal activity into a predicted BOLD response-as such, hemodynamic states are a function of the neuronal state/s of each region (Friston et al., 2003). The hemodynamic model is comprised of four differential equations that characterize how for each region pre-synaptic activity drives hemodynamic responses-which are mediated by astrocytic $\mathrm{Ca}^{2+}$ signaling whose endfeet release vasodilatory metabolites (MacVicar and Newman, 2015). This vasodilatory signal is subject to auto-regulatory feedback (Attwell and Iadecola, 2002; Friston et al., 2003) and blood flow changes proportionately 
to the vasodilatory signal which leads to an increase in blood volume with concomitant decrease in deoxyhemoglobin (Stephan et al., 2004).

The output signal is defined by the BOLD signal change equation-which links blood volume and deoxyhemoglobin content to the BOLD signal change (Stephan et al., 2007). The resulting $B O L D$ signal depends on the depends on the relative contributions of intra- and extravascular signals (Buxton et al., 2004; Friston et al., 2017) and follows the flow dynamics with a delay of $\sim 1$ s (Stephan et al., 2004). The BOLD signal change equation completes the DCM generative model.

In standard DCM, the parameters of the full forward model (combined neural and hemodynamic models) is estimated from the measured BOLD data using is estimated iteratively from the measured BOLD data using Variational Bayesian (VB) algorithm (Friston et al., 2007) to produce probabilistic estimates of the expected value of each parameter given the data. Details of the parameter estimation process are beyond the scope of this review and the reader is referred to Friston et al. (2002). The objective of the estimation process is to generate a predicted signal that closely matches the observed BOLD data (Kahan and Foltynie, 2013). From the DCM parameter estimates, one can then quantify and make inferences about connection strength and direction between regions based on prior assumptions about connectivity architecture.

Finally, Bayesian model selection (BMS) is done to compare a series of models that represent different prior hypotheses of connectivity architecture to examine which of the competing models most likely generated the observed data. This is done by comparing the evidence for each model. Model evidence-the normalization constant for the product of the likelihood of the data and prior probability of the parametersis approximated using Laplace approximation in standard DCM (Stephan et al., 2007). This yields the log-evidence for each model, characterized as the conditional probability (posterior probability) of competing models given the observed data (Kahan and Foltynie, 2013). The optimal model is one that represents the best compromise between model fit (accuracy) and complexity (characterized by the number of free parameters in the model) (Stephan et al., 2007) —it is the best fitting, yet, most parsimonious model. As such, this model is more generalizable.

\section{DCM Assumptions}

There are three main assumptions in DCM: (1) deterministic assumption on the inputs that enter the system; (2) Gaussian assumption on the posterior density; and (3) assumption of equal detection of BOLD signal changes throughout the brain. Firstly, in classical DCM, all processes in the system are considered deterministic such that it is presumed that neural dynamics in a region is entirely due to incoming afferents from other regions and/ or experimental inputs. This assumption is not always tenable in NHP fMRI studies which are mostly done under anesthesia-this restricts task-based imaging to simpler visual/ auditory experiments and many imaging experiments are task-free. However, extensions of DCM in humans have allowed its application in resting state studies: (1) using classical DCM, one can stimulate nodes with fluctuations of specific frequencies
(Di and Biswal, 2014); (2) modeling random fluctuations in neural dynamics explicitly using stochastic DCM (Li et al., 2011; Daunizeau et al., 2012); or (3) estimating the spectral density of neuronal fluctuations such that effective connectivity of hidden neuronal states is a function of observed functional connectivity from hemodynamic responses (Friston et al., 2014; Park et al., 2018). Secondly, parameter estimates of the generative model are assumed to be Gaussian-i.e., that the values are normally distributed. This may be a concern when using twostate DCM which use exponentiated scale parameters that introduce positivity constraints as these values likely do not have a normal distribution (Hillebrandt et al., 2014). Lastly, DCM rests on the assumption that BOLD signal detection is equally sensitive across brain regions. This was validated by Friston et al. (2003) by simulating region-specific dropout wherein they found that DCM does not accommodate substantial signal dropout (e.g., 50\%). In the absence of profound dropout, DCM is robust to regional variations in sensitivity to BOLD signal changes.

\section{Why Apply DCM to NHP FMRI?}

The motivations for applying DCM to NHP data are founded on the prospective to make DCM models more accurate with animal experiments that are not yet feasible or are considered unethical in humans, as well as on being a more physiologicallyinformed characterization of network dynamics vs. previous effective connectivity models.

Animal imaging data and experiments possess the potential to make DCM models more accurate. Invasive procedures such as in vivo optogenetic and electrical stimulation can be combined with functional imaging to examine how disruptions at the microscale can affect whole brain network dynamics. Additionally, one can also investigate the temporal evolution of the effects of drugs (e.g., administered intravenously or through gas inhalation). Moreover, advances in high-field fMRI at the sub-millimeter scale, allows imaging and modeling at laminar resolutions (Heinzle et al., 2016; Friston et al., 2017).

In order to accurately disentangle neuronal- and hemodynamics, experimental manipulation can provide necessary constraints to estimate hemodynamic parameters, which may explain discrepancies in response shape between neuronal and BOLD response. This can be addressed by using multi-modal recordings (e.g., simultaneous measurements of $\mathrm{CBF}, \mathrm{CBV}$, and BOLD), which is often more feasible in animal studies, including NHP. For instance, Havlicek et al. (2017) showed that combined analysis of BOLD and $\mathrm{CBF}$ data yields more robust effective connectivity estimates.

Furthermore, DCM was originally developed specifically for fMRI data (Friston et al., 2003) which gives it an edge over other models such as Granger causality and structural equation modeling (SEM) that were initially applied in the fields of economics, psychology, and genetics (Wright, 1920; Granger, 1969). Compared with previous EC measures, DCM allows a more physiologically-informed characterization of network dynamics as it incorporates a hemodynamic model that has previously been experimentally validated (Buxton et al., 1998; Friston et al., 2003). On the other hand, the 
disadvantages of Granger causality in fMRI application is plentiful. Using four different algorithms, Smith et al. (2011) demonstrated that Granger causality exhibits poor $(<20 \%)$ sensitivity in connection link detection, false positive identification and directionality estimation (Smith et al., 2011). Moreover, Witt and Meyerand (2009) found that Granger causality has poor sensitivity and specificity (close to chance levels) when modeling data including intrinsic variance from trimmed time series. While DCM is computationally costly, its ability to model non-linear and dynamic neuronal interactions (Bielczyk et al., 2019), as well as both unidirectional and bidirectional connections (Vaudano et al., 2013; Buijink et al., 2015) give a more accurate picture of underlying neuronal activity. While originally designed for task fMRI, recent developments such as stochastic or spectral DCM allow modeling of resting state fMRI (Li et al., 2011; Daunizeau et al., 2012; Friston et al., 2014; Park et al., 2018). Classical DCM (Friston et al., 2003) pose restrictions on network size as increasing the number of nodes considerably increases computational time (Bielczyk et al., 2019). However, ensuing extensions allow exploratory studies involving larger networks such as spectral DCM (Friston, 2011) for resting-state fMRI and DCM with sparsity constraints for task fMRI (Frässle et al., 2018). The many advantages of DCM over previous effective connectivity measures and the ability to combine invasive procedures in neuroimaging make DCM application to NHP data an exciting endeavor and may offer the possibility to improve the accuracy of causal models. A summary of the advantages and disadvantages of the commonly used effective connectivity models (structural equation modeling, Granger causality, transfer entropy, and dynamic causal modeling) is outlined in Table 1.

\section{CONSIDERATIONS SPECIFIC TO DCM APPLICATION IN NHP}

\section{Functional Networks and Cerebrovascular Architecture: Human vs. NHP Cerebrovascular Architecture and Physiology}

The cerebral vasculature of NHP is largely similar to humans in terms of the architecture of superficial pial vessels and intracortical vessels (Duvernoy et al., 1981; Weber et al., 2008). Similarly found in humans (Duvernoy et al., 1981), large vessels are found on the surface of the macaque brain (Weber et al., 2008). The density of superficial vessels have been found to vary across cortical regions - the occipital lobe surface is highly vascularized compared to the less dense vascularization at the top of the hemispheres near the interhemispheric fissure (Scharrer, 1960; Duvernoy et al., 1981). In terms of vessel diameter, pial veins generally have a larger diameter than arteries - central veins have an average diameter of $280-380 \mu \mathrm{m}$ and peripheral veins average $130 \mu \mathrm{m}$, while central arteries have a diameter of $260-280 \mu \mathrm{m}$ and peripheral arteries average $150-180 \mu \mathrm{m}$ (Duvernoy et al., 1981; Guibert et al., 2010). As for intracortical vessels, the laminar distribution of vessels in NHP are similar to that found in humans. Studying the primate visual cortex, Bell and Ball (1985) found a high density of cortical vessels in layer IVC that ends at the boundary between primary and secondary visual cortices-akin to the vascular distribution in humans.

The fluctuations in deoxyhemoglobin concentration detected by the BOLD contrast depends on the combined changes in cerebral blood volume (CBV), cerebral blood flow (CBF), and cerebral metabolic rate of oxygen (CMRO2) (Buxton et al., 2014). In general, the values for each hemodynamic component is comparable between humans and NHP. Van Aken et al. (1986) found $\mathrm{CBF}$ at $48 \pm 4 \mathrm{~mL} / 100 \mathrm{~g} / \mathrm{min}$ in baboons. In humans, Olsen et al. (1994) demonstrated $51 \mathrm{~mL} / 100 \mathrm{~g} / \mathrm{min}$, while Ito et al. (2004) found $69.8 \pm 15.4 \mathrm{~mL} / 100 \mathrm{~mL} / \mathrm{min} \mathrm{CBF}$ values. CBV ranged from 3.5 to $4.7 \mathrm{ml} / 100 \mathrm{~g}$ of brain tissue in the macaque (Phelps et al., 1973; Grubb et al., 1974; Eichling et al., 1975) while $\mathrm{CBV}=3.8 \pm 0.7 \mathrm{ml} / 100 \mathrm{ml}^{-1}$ (Ito et al., 2004). As for CMRO2, Van Aken et al. (1986) obtained $3.64 \pm 1$ $(\mathrm{ml} / 100 \mathrm{~g} / \mathrm{min})$ in baboons and Olsen et al. (1994) found 3.5 $(\mathrm{ml} / 100 \mathrm{~g} / \mathrm{min})$ in humans. The values for $\mathrm{CBF}$ and $\mathrm{CMRO} 2$ are modulated by anesthetics and this will be discussed in an ensuing section.

\section{Functional Differences}

Resting-state fMRI studies comparing human and NHP connectivity have revealed three main differences. First, there are differences in specific connectivity patterns that may potentially indicate cognitive specializations in humans. For example, comparing the organization of the dorsal frontal cortex between humans and macaques (Sallet et al., 2013), found a high degree of similarity in functional coupling patterns between the medial frontal cortex and other regions (i.e., frontal pole, medial prefrontal, and dorsal frontal convexity) in both. However, certain regions in the dorsolateral prefrontal cortex (areas 9/46) were coupled with the superior and medial parietal cortex in humans but not in macaques. Further, Mars et al. (2011) demonstrated resting-state functional connections between anterior prefrontal cortex and central inferior parietal lobule (IPL) in humans which are not found in macaques. Second, there may be species-specific differences in cortical hub distribution. Upon mapping putative hubs in humans, chimpanzees, and macaques, Li L. et al. (2013) demonstrated the ventrolateral prefrontal, medial parietal and retrosplenial cortices are hubs across three species. In contrast, medial prefrontal, inferior parietal, and V1 cortices were hubs in macaques and chimpanzees and not in humans. Additionally, superior parietal and medial premotor cortices were hubs in humans and not in the NHPs. Third, though it has been demonstrated that there are 11 functionally correspondent networks in both humans and macaques, three networks were found in humans that are missing in the latter (Mantini et al., 2013). While sensory-motor, attention, language, and default mode networks are evolutionarily conserved, two lateralized fronto-parietal networks are unique to humans (Van Essen and Dierker, 2007). These have been implicated in general intelligence (Duncan et al., 2000), abstract reasoning (Dehaene et al., 2003), and tool 
TABLE 1 | Comparison of commonly used effective connectivity models.

\begin{tabular}{|c|c|c|}
\hline & Pros & Cons \\
\hline $\begin{array}{l}\text { Structural equation } \\
\text { modeling }\end{array}$ & $\begin{array}{l}\text { * Can detect excitatory and inhibitory connections and connection } \\
\text { strength (Bielczyk et al., 2019) } \\
\text { * Sensitivity to small changes in path weight values due to large } \\
\text { dynamic range } \\
\text { (Witt and Meyerand, 2009) }\end{array}$ & $\begin{array}{l}\text { * Difficulty in estimating reciprocal and cyclic connections (physiologically, } \\
\text { reciprocal connections are ubiquitous in the brain) (Friston, 2011) } \\
\text { * May not be as suitable to event-related design due to the assumption that } \\
\text { random fluctuations change very slowly in relation to neuronal dynamics } \\
\text { such that neuronal dynamics has already reached steady-state at the time } \\
\text { of recording (Friston, 2011) } \\
\text { * May be inappropriate in the context of disease or pharmacologic } \\
\text { experiments that can affect hemodynamic response function } \\
\text { (Rowe et al., 2010) }\end{array}$ \\
\hline Granger causality & $\begin{array}{l}\text { * Can detect excitatory and inhibitory connections and connection } \\
\text { strength (Bielczyk et al., 2019) } \\
\text { * Yields bidirectional connections (Bielczyk et al., 2019) } \\
\text { * Results can be mapped onto the brain similar to fMRI (Goebel } \\
\text { et al., 2003; Roebroeck et al., 2005; Witt and Meyerand, 2009) }\end{array}$ & $\begin{array}{l}\text { * Poor sensitivity and specificty (close to chance levels) when modeling data } \\
\text { including intrinsic variance from trimmed time series (Witt and Meyerand, } \\
\text { 2009) } \\
\text { * Assumption of signal stationarity (Seth et al., 2015) } \\
\text { * Restriction on network size-the number of nodes divided by the number of } \\
\text { shifts can never exceed the number of time points (Bielczyk et al., 2019) } \\
\text { * Markovian assumption that random terms in the vector autoregression } \\
\text { model are serially independent may not hold when the terms become } \\
\text { temporally correlated upon converting from continuous to discrete time } \\
\text { formulations (Friston, 2011) } \\
\text { * The spatial distribution of GC has been associated with the Circle of Willis } \\
\text { and identifies major arteries and veins as causal hubs (Webb et al., 2013) } \\
\text { * Assumption of uniform hemodynamic response function across regions } \\
\text { may elicit spurious causal relationship when one region has faster } \\
\text { hemodynamic activity-the temporal precedence of the peak in one region } \\
\text { may be mistaken for Granger causing the other (Bielczyk et al., 2019) } \\
\text { * fMRI temporal resolution may be too slow for accurate depiction of neural } \\
\text { dynamics using Granger causality (Witt and Meyerand, 2009) } \\
\text { * Poor (<20\%) sensitivity in connection link detection, false positive } \\
\text { identification and directionality estimation (Smith et al., 2011) }\end{array}$ \\
\hline Transfer entropy & $\begin{array}{l}\text { * Can detect excitatory and inhibitory connections and connection } \\
\text { strength (Bielczyk et al., 2019) } \\
\text { * Captures linear and non-linear interactions between nodes } \\
\text { (Bielczyk et al., 2019) } \\
\text { * Computationally cost-efficient (Vicente et al., 2011) }\end{array}$ & $\begin{array}{l}\text { * Restriction on network size-the number of nodes divided by the number of } \\
\text { shifts can never exceed the number of time points (Bielczyk et al., 2019) } \\
\text { * Imposes a time-lag in the inference procedure with similar disadvantages } \\
\text { as Granger Causality in fMRI application (Schreiber, 2000) }\end{array}$ \\
\hline $\begin{array}{l}\text { Dynamic causal } \\
\text { modeling }\end{array}$ & $\begin{array}{l}\text { * Developed specifically for fMRI (Friston et al., 2003) data and } \\
\text { incorporates a biologically-informed model of BOLD dynamics } \\
\text { (Buxton et al., 1998), unlike other models Granger causality and } \\
\text { SEM were originally applied in the fields of economics, } \\
\text { psychology, and genetics (Wright, 1920; Granger, 1969) } \\
\text { * Can detect excitatory and inhibitory connections and connection } \\
\text { strength (Bielczyk et al., 2019) } \\
\text { * Can model both unidirectional and bidirectional connections } \\
\text { Naudano et al., 2013; Buijink et al., 2015) } \\
\text { * Models nonlinear and dynamic neuronal interactions (Bielczyk } \\
\text { et al., 2019) } \\
\text { * Classical DCM is suitable for event-related designs (Rowe et al., } \\
\text { 2010) } \\
\text { * Stochastic or spectral DCM is suitable for resting state studies } \\
\text { (Li et al., 2011; Daunizeau et al., 2012; Friston et al., 2014; Park } \\
\text { et al., 2018) } \\
\text { * For exploratory studies involving larger networks, spectral DCM } \\
\text { (Friston, 2011) can be applied for resting-state fMRI while DCM } \\
\text { with sparsity constraints can be applied for task fMRI (Frässle } \\
\text { et al., 2018) }\end{array}$ & $\begin{array}{l}\text { * Computationally-expensive (Bielczyk et al., 2019) } \\
\text { * Restriction on network size (using classical DCM)-increasing the number } \\
\text { of nodes considerably increases computational time (Bielczyk et al., 2019) } \\
\text { * Depends on prior assumptions on connectivity architecture } \\
\text { (Friston et al., 2003) } \\
\text { * Assumes all models are equally likely (even implausible models) (Lohmann } \\
\text { et al., 2012); hence, substantial knowledge is needed to define all } \\
\text { plausible causal connections between nodes }\end{array}$ \\
\hline
\end{tabular}

use, particularly retrieving and planning transitive actions for subsequent hand motor behavior (Frey, 2008). A third human-specific network includes the anterior insula and dorsal anterior cingulate cortex-both putatively involved in empathy (Singer and Lamm, 2009).

\section{The Anesthetized Brain} Anesthetic Effect on Vascular Physiology and BOLD Contrast

Anesthetics have been found to impart changes in cerebrovascular function and the BOLD signal. As these are 
incorporated in the DCM generative model, it is important to examine how these are affected in the anesthetized brain, particularly since most NHP neuroimaging is done under anesthesia.

There are conflicting results in the regional distribution of anesthetic-induced changes in vascular physiology. Li C.-X. et al. (2013) examined the dose-dependent effect of isoflurane on regional $\mathrm{CBF}$ of cortical and subcortical structures in macaques. They found that high isoflurane concentrations (i.e., 1.5\%) resulted in global $\mathrm{CBF}$ increase which was most evident in subcortical structures-specifically in the thalamus and cerebellum in macaques. Interestingly, under the $0.75-1.5 \%$ isoflurane maintenance doses, there were no observable CBF changes in cortical regions (i.e., anterior cingulated cortex, motor cortex, medial prefrontal cortex) and the caudate. These indicate that while CBF auto-regulation is intact in cortical regions and the caudate under isoflurane maintenance dose, it is impaired in the thalamus and cerebellum, and suggest that subcortical structures contribute the most to the increase in global CBF. On the other hand, Långsjö et al. (2005) demonstrated that ketamine increased whole brain CBF in humans-with the highest increase in the anterior cingulate. They found that $\mathrm{CMRO} 2$ increased only in the frontal cortex while glucose metabolism increased only the in the thalamus. Långsjö et al. (2005) assert that this indicates that majority of the increases in CBF most likely do not indicate neuronal activation. Another study by Van Aken et al. (1986) showed that the impact of isoflurane on CBF was biphasiclow levels $(0.5 \pm 0.35$ vol\%) resulted in vasoconstriction and decreased CBF while higher concentrations $(0.95 \pm 0.7$ vol\% and $1.4 \pm 1$ vol\%) caused vasodilation and increased $C B F$ to baseline levels (no anesthesia). While they found that the effect of isoflurane on $\mathrm{CBF}$ was biphasic, CMRO2 continually decreased in a dose-dependent manner. The disparity in the findings of these studies may be attributed to methodological differences in anesthetic (isoflurane vs. ketamine), species (human vs. primate), and imaging modality (arterial spin labeling MRI vs. PET). Nonetheless, the results of these studies indicate anestheticrelated disruption of CBF-metabolism coupling characterized by more widespread $\mathrm{CBF}$ increases with minor changes in CMRO2 and glucose metabolism, with dose-related variabilities in response.

As for the impact of anesthetics on BOLD contrast, anesthetics have been demonstrated to cause changes in the spectral components and decrease the activated area and magnitude of the signal, as well as change the hemodynamic response temporal structure (Martin et al., 2006; Aksenov et al., 2015; Paasonen et al., 2018). Paasonen et al. (2018) found that awake mice exhibit higher spectral BOLD power at a wide frequency range. In contrast, they found that all six anesthetic conditions studied strongly suppressed power and BOLD fluctuations occurred at narrower frequency ranges, which potentially reflect more homogeneous activity. This was a predictable outcome as by definition, anesthetics exert an inhibitory effect on brain function. Aksenov et al. (2015) suggested that enhanced GABA-A receptor inhibition and diminished afferent input reduces the spread of stimulus-related activity, which results in more focal activation and decreased BOLD response area.
Further, they surmised that decreased BOLD response magnitude reflects reduced thalamic input and intra-cortical processing associated with decreased neuronal excitation. A decrease in hemodynamic response magnitude under anesthesia has also been demonstrated in previous studies employing optical imaging spectroscopy (OIS) (Berwick et al., 2002). Aside from decreased duration of the BOLD signal reported by Aksenov et al. (2015), Martin et al. (2006) found that anesthesia increased hemodynamic response latency ( $\sim 2 \mathrm{~s}$ awake vs. $4 \mathrm{~s}$ anesthetized).

\section{Anesthetic Impact on Effective Communication Within and Between Networks}

It is now widely acknowledged that anesthetics modulate communication within and between networks. Anestheticinduced perturbations in effective connectivity have been observed across multiple neuroimaging techniques, species (humans and rodent models), and anesthetics. In particular, effective connectivity changes have been demonstrated in thalamo-cortical, cortico-thalamic, and cortico-cortical (both association and sensory cortices) connections. Under propofol, ketamine, or isoflurane, selective disruption of frontal to parietal feedback has been widely replicated in human subjects and rats (Imas et al., 2005; Lee et al., 2009; Boly et al., 2012) particularly gamma frequencies $(50 \mathrm{~Hz})$ (Imas et al., 2005). In addition, Gómez et al. (2013) found that propofol decreased feedback from middle frontal gyrus to superior temporal gyrus. As for sensory-motor regions, multiple studies found impaired effective connectivity under anesthesia. White and Alkire (2003) demonstrated impaired effective drive from the supplementary motor area (SMA) to the primary motor (M1) cortex in humans, while Kang et al. (2016) showed abolished causal flow of $7-12 \mathrm{~Hz}$ activity from primary sensory (S1) to M1 and ventrobasal thalamic nucleus in mice. Decreased feedforward and feedback connections were found within auditory cortical regions (Heschl's gyrus and superior temporal gyrus) in humans under propofol (Gómez et al., 2013). Pertaining to anestheticrelated changes in thalamocortical effective connectivity, the results are more heterogeneous. Causal flow from the thalamus to the anterior cingulate and posterior parietal cortices was intact under propofol (Boly et al., 2012). On the other hand, effective connectivity from the thalamus to the SMA was impaired under halothane or isoflurane (White and Alkire, 2003). However, effective thalamic drive to M1 has shown variable resultsremaining unchanged under ketamine in mice (Kang et al., 2016) compared to the awake state, while decreasing under halothane or isoflurane in humans (White and Alkire, 2003). This discrepancy may indicate species-specific differences in thalamicmotor connectivity in response to anesthetics, differences in regional distribution of different anesthetics (ketamine vs. halothane or isoflurane), or both. Altogether, these findings indicate that anesthetics induce: (1) disruption of higher-order information processing; and (2) reduced capacity for sensorymotor integration.

\section{Relevance to DCM in NHP}

For DCM to generate reliable neuronal signal estimates, having an accurate model of the hemodynamic response is crucial; thus, 
it is important to assess whether differences in cerebrovascular architecture and physiology in NHP-factors that contribute to the BOLD contrast-may affect priors on the biophysical parameters of the model.

The cerebrovascular architecture and laminar distribution of blood vessels in NHPs are similar to that of humans. The fMRI BOLD signal indicates changes in deoxyhemoglobin and the signal is sensitive to fluctuations in venous blood volumes. The cerebral vasculature of NHP is largely similar to humans in terms of the architecture of superficial pial vessels and intracortical vessels (Duvernoy et al., 1981; Weber et al., 2008; Adams et al., 2015). By extension, the BOLD signal-to-noise ratio in superficial to deep brain areas is expected to have similar distribution in both.

More importantly, the BOLD signal is a function of CBV, CBF, and $\mathrm{CMRO} 2$ - which reflects fluctuations in deoxyhemoglobin content (Buxton et al., 2014), and DCM includes priors on these hemodynamic parameters. Since the CBV, CBF, and CMRO2 in NHP are comparable to human values (Phelps et al., 1973; Grubb et al., 1974; Eichling et al., 1975; Van Aken et al., 1986; Olsen et al., 1994), it is tempting to presume that one can proceed with applying DCM in NHP without adjustments on the biophysical parameters if one solely considers cerebrovascular architecture and physiology.

Notably, anesthetics such as isoflurane and ketamine have been found to increase blood flow (Van Aken and van Hemelrijck, 1991; Långsjö et al., 2005; Slupe and Kirsch, 2018), which is relevant in primate neuroimaging as it is usually done under anesthesia. The decision on how to proceed with DCM in the anesthetized brain depends on the research question. Upon comparing eight different hemodynamic models by modifying the coefficients of the BOLD signal change equation, Stephan et al. (2007) assert that variations in the hemodynamic model are relatively inconsequential when making inferences on underlying neuronal dynamics and their causal influences. This is due to the relative independence of the parameters of the neural state equation-characterizing intrinsic connectivity and their context-dependent modulation (A and B matrices)-from the amplitude of the hemodynamic response and the direct influence of extrinsic inputs on neuronal activity (matrix C). On the other hand, if the research question focuses on regional variations in hemodynamic parameters (e.g., in healthy vs. clinical populations), then preliminary tests need to be performed to determine the effect of variations on the priors of the biophysical model-related to anesthetic influence. For instance, if the experimenter can identify the baseline $\mathrm{CBF}$ and $\mathrm{CBV}$ using multi-modal recordings, the mean transit time (i.e., baseline $\mathrm{CBV} /$ baseline $\mathrm{CBF}$ ) - which scales the $\mathrm{CBV}$ and deoxyhemoglobin changes in the hemodynamic modelcould be directly calculated. Other strategies for modifying the hemodynamic model are discussed in the last paragraph of the next section on IPS-DCM parameters.

Moreover, caution is necessary when interpreting results if the model includes regions or networks in which effective connectivity may be modulated by anesthetics. Regions most affected by anesthetics are: (1) fronto-parietal (Ku et al., 2011; Boly et al., 2012; Kim et al., 2017); (2) sensory-motor (i.e., S1 to
M1, SMA to M1, auditory cortical regions) (White and Alkire, 2003; Gómez et al., 2013); and (3) thalamocortical networks specifically involving somatosensory and motor function (White and Alkire, 2003; Kang et al., 2016). Since effective drive of these networks are reduced or abolished by anesthetics, it may result in underestimation of the effect of interventions (e.g., medications, brain stimulation) on connectivity within and between affected networks. It may also result in the overestimation of the modulatory effects of different interventions if aimed at reducing hyperconnectivity.

Additionally, anesthetics can modulate neural dynamics by altering inhibitory drive, neural refractory period, and cortical adaptation (Masamoto et al., 2007; Moran et al., 2011). These artifacts can be addressed by using an adaptive two-state neural connectivity model (Havlicek et al., 2015). This and other strategies for applying DCM in NHP under anesthesia are discussed in the succeeding section.

\section{INTEGRATED PHYSIOLOGIC-STOCHASTIC DCM: MODELING EFFECTIVE CONNECTIVITY UNDER ANESTHESIA}

\section{IPS-DCM Parameters}

An important factor to consider upon implementing DCM in the anesthetized NHP is the type of DCM to use. Anesthetics can decrease BOLD signal-to-noise ratio and artificially lower spontaneous fluctuations and activity correlations (Hutchison et al., 2014). In addition to dose-dependent linear decrease in glutamatergic excitatory postsynaptic potentials (EPSPs) together with non-linear increase (saturating) in GABAergic inhibitory postsynaptic potentials (IPSPs) (increasing local inhibitory drive) (Moran et al., 2011), anesthetics modulate the neural refractory period and cortical adaptation with varying degrees depending on anesthetic type and dose (Masamoto et al., 2007). To overcome these limitations, we propose the combined use of two DCM extensions: stochastic DCM (Li et al., 2011) and physiologically-informed DCM (P-DCM) which has an adaptive two-state neural connectivity equation (Havlicek et al., 2015).

Stochastic DCM models fluctuations in hidden states (e.g., neuronal or hemodynamic states) that are due to endogenous (autonomous) dynamics, not exogenous experimental inputs (Li et al., 2011). By accounting for noise in the model using stochastic DCM, the risk of under-estimating effective connectivity parameters can be reduced (Gómez et al., 2013). Additionally, stochastic DCM is more useful when there are non-linear interactions among hidden states, such as the nonlinear increase in GABAergic inhibitory drive (Moran et al., 2011; Daunizeau et al., 2012). Finally, Li et al. (2011) demonstrated that stochastic DCM using the generalized filtering (GF) inversion method showed higher sensitivity in detecting group differences compared to the variational Bayesian (VB) algorithm (Friston et al., 2007) of standard deterministic DCM.

Two-state (Marreiros et al., 2008) and adaptive two-state (Havlicek et al., 2015) neural equations model both excitatory (glutamatergic) and inhibitory (GABAergic) connections within each region, which makes it a more physiological representation 
of neural population dynamics. In contrast to the two-state model, the adaptive two-state model includes parameters which allow for adaptation and refractory effects of the neuronal response (Havlicek et al., 2015). This extension is relevant since anesthetics induce changes to the neural refractory period and cortical adaptation (Ogawa et al., 1992; Masamoto et al., 2007). Furthermore, explicitly modeling both excitatory and inhibitory signals may help capture the potentiation of GABAergic inhibition under anesthetics (Moran et al., 2011). This may be elucidated in the dynamics of the inhibitory subpopulation or the interaction between excitatory and inhibitory subpopulations (Gómez et al., 2013).

The adaptive two-state DCM is the neuronal connectivity component of P-DCM developed by Havlicek et al. (2015). The parameters that incorporate adaptation and refractory effects to neuronal response are: (1) the inhibitory gain factor $\lambda$, which modulates the amplitude and temporal smoothness of the inhibitory activity in relation to the excitatory drive, and (2) the inhibitory-excitatory connection $\mu$ which reflects the temporary imbalance in temporal smoothness between excitatory and inhibitory activity that can result in neuronal adaptation.

The second modification in P-DCM involves the hemodynamic model. The changes are 2-fold: (1) modeling feedforward neurovascular coupling (vs. feedback NVC in classical DCM); and (2) incorporating a viscoelastic effect in the Balloon model. Experiments by Lindauer et al. (2010) and Powers et al. (1996) show that manipulating oxygen and glucose levels in the blood do not regulate blood flow as per negative feedback hypotheses (Attwell et al., 2010; Havlicek et al., 2015). Additionally, hypercapnia experiments demonstrated that higher baseline CBF has minimal impact on the absolute stimulus-induced $\mathrm{CBF}$ change (while relative $\mathrm{CBF}$ became smaller) (Li et al., 2000; Brown et al., 2003; Zappe et al., 2008). This latter point is pertinent to DCM application in NHP since isoflurane-the most commonly used anesthetic in animal experiments-is a potent vasodilator and causes higher baseline $\mathrm{CBF}$ that may be uncoupled from cerebral energy metabolism (Van Aken and van Hemelrijck, 1991). Thus, feedforward neurovascular coupling is more relevant in NHP image analysis, particularly under anesthesia. On the other hand, the viscoelastic component in the hemodynamic model was added to account for transient responses (i.e., BOLD post-stimulus undershoot and overshoot) outside of the steady-state relationship between $\mathrm{CBF}$ and $\mathrm{CBV}$, described by the power law where $\alpha=0.38$ (Grubb et al., 1974). The duration of the transient adjustment period is regulated by viscoelastic time constant $\tau$-which allows for variations in outflow curve during balloon inflation and deflation, corresponding to BOLD response overshoot and undershoot (Buxton et al., 1998, 2004). The BOLD transients are then presumed to reflect both neuronal post-stimulus deactivation and vascular uncoupling related to slow recovery of venous CBV (Havlicek et al., 2015).

Lastly, the BOLD signal change equation was modified to accommodate different magnetic field strengths. k1, k2, and k3 are parameters that reflect baseline physiological properties of brain tissue and acquisition parameters and have been adjusted to depend on different magnetic field strengths. Moreover, they also suggest revised values for $\varepsilon$ (ratio of intra-and extravascular signal) and r0 (regression slope of changes in intra-vascular signal relaxation rate with changes in oxygen saturation) depending on acquisition sequence (gradient echo vs. spin echo) (Uludag et al., 2009; Havlicek et al., 2015).

As stated above, we propose to combine P-DCM with stochastic DCM. Since stochastic DCM uses the same biophysical forward model as classical DCM, the changes in biophysical parameters in P-DCM can be integrated in the stochastic extension (Havlicek et al., 2015). Thus, in this application of DCM on NHP fMRI, we suggest estimating the full forward model using the biophysical parameters of P-DCM applying the generalized filtering (GF) inversion method of stochastic DCM (Li et al., 2011). A schematic of IPS-DCM is illustrated in Figure 1. A salient consideration here is that stochastic DCM tends to emphasize neuronal dynamics over hemodynamics (Reviewer 2 comment). Nonetheless, as aforementioned, stochastic DCM is a more accurate model when there are non-linear interactions among hidden states, such as the non-linear increase in GABAergic inhibitory drive (Moran et al., 2011; Daunizeau et al., 2012) which is relevant when imaging under anesthesia. For NHP neuroimaging under anesthesia, this is useful for task-based imaging with simple visual/ auditory experiments, and even more so for resting state studies. Therefore, in order to more accurately estimate both neuronal and hemodynamic changes, future extensions of PDCM (which is so far formulated and applied as a deterministic model) to fully stochastic or partially stochastic (stochastic neuronal model and deterministic hemodynamic model) may be relevant.

With this integrated physiologic-stochastic DCM, one can examine anesthetic impact on effective connectivity in NHP. This can be done by first building generative models of fMRI done pre and post anesthesia. Then, Bayesian model comparison allows one to examine which model best explains the changes in effective connectivity between awake and anesthetized states.

An important consideration here is that anesthetics such as isoflurane and ketamine increase baseline CBF due to increased vasodilation (Van Aken and van Hemelrijck, 1991; Långsjö et al., 2005; Slupe and Kirsch, 2018). It is also a common observation that increased baseline $\mathrm{CBF}$ results in smaller relative $\mathrm{CBF}$ change (Li et al., 2000; Cohen et al., 2002; Zappe et al., 2008). While current DCMs model relative CBF changes (Friston et al., 2003; Havlicek et al., 2015), the baseline CBF is not directly expressed. Thus, in order to account for the effect that anesthetics have on relative change in $\mathrm{CBF}$, the equation modeling $\mathrm{CBF}$ change in DCM models could be further modified to reflect also baseline CBF values. It would be then also beneficial to consider multi-modal data that measure both CBF and BOLD signals (Havlicek et al., 2017). Even if CBF is not measured directly, it is helpful to have baseline $\mathrm{CBF}$ as a parameter. Additionally, as mentioned above, anesthesia can the increase latency of $\mathrm{CBF}$ response (also later reflected in BOLD response), e.g., from $\sim 2$ to $\sim 4 \mathrm{~s}$ (Martin et al., 2006). Latency of CBF response can be controlled in DCM models. For example, Havlicek et al. (2017) accounted for differences between latency of positive and negative $\mathrm{CBF}$ responses measured in anesthesized NHP 


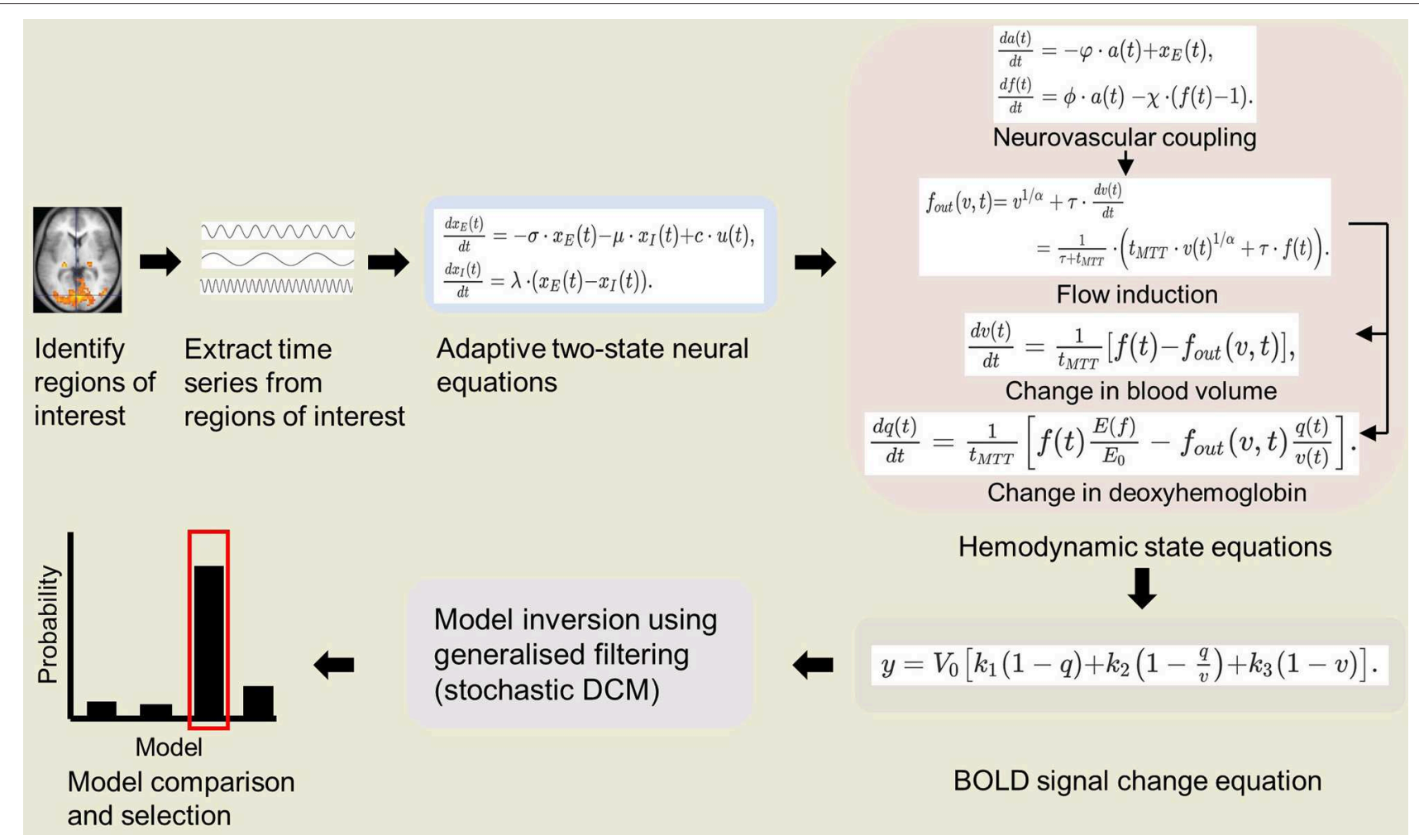

FIGURE 1 | Schematic of integrated physiologic-stochastic DCM (IPS-DCM). After identifying and extracting the time series from regions of interest, model parameters are then estimated using P-DCM equations (Havlicek et al., 2015). P-DCM incorporates: (1) an adaptive two-state neuronal model that allows adaptation and refractory effects to neuronal response; (2) a hemodynamic model that implements feedforward neurovascular coupling and a viscoelastic effect on the Balloon model; (3) a BOLD signal change equation that accounts for magnetic field differences. The model inversion is done using generalized filtering (stochastic DCM) (Li et al., 2011). Lastly, one proceeds to model comparison and selection of the winning model.

by optimizing parameter $\chi$ in the feedforward neurovascular coupling of the P-DCM, while other parameters $(\phi, \varphi)$ could remain fixed. These considerations could permit more physiologically accurate evaluation of the effect of pre- and postanesthesia on effective connectivity, as modeled using IPS-DCM.

\section{Comparison With Other DCM Applications Under Anesthesia}

DCM has been applied in two other studies examining connectivity changes under propofol-induced loss of consciousness in fronto-parietal (Boly et al., 2012) and auditory (Gómez et al., 2013) networks. Boly et al. (2012) applied DCM for steady-state responses (SSR) and used neural mass models for each region of the fronto-parietal network (plus thalamic source)-three subpopulations in cortical regions (one excitatory and two inhibitor) and two for the thalamic source (excitatory relay cells and inhibitory reticular cells). On the other hand, Gómez et al. (2013) utilized combined stochastic (Daunizeau et al., 2009) and two-state (Marreiros et al., 2008) DCM to model one frontal and two temporal (auditory) cortical regions. In this section, we discuss the main caveats of both studies and how IPS-DCM may address said limitations.

The main weakness of both studies is the steady-state assumption of both DCMs which may not hold under anesthesia.
Boly et al. (2012) found in initial increase in beta and gamma rhythms while delta to alpha frequencies continually increased as loss of consciousness emerged and acknowledge that this may reflect neuronal up and down states. Gómez et al. (2013) asserts that they maintained steady-state by keeping the anesthetic dose fixed after Ramsay evaluation during fMRI recordings. However, Yeom et al. (2017) demonstrated that even when patient-controlled anesthetic levels (propofol and midazolam) were unchanged once consciousness was lost, over time, there was gradually increasing power in frequencies $<15 \mathrm{~Hz}$ together with decreasing power at $>15 \mathrm{~Hz}$. Increased delta and alpha power were most evident in frontal and parieto-occipital regions. Thus, the steady-state assumption of both DCMs may not hold in both studies with anesthetics.

The combined use of the adaptive two-state DCM by Havlicek et al. (2015) and stochastic DCM by Li et al. (2011) offers a number of advantages over the previous two DCM applications. Li et al. (2011) demonstrated that the generalized filtering inversion method in this DCM extension provides better effective connectivity estimates and higher sensitivity to detecting group differences than the expectation maximization (EM) or dynamic expectation maximization (DEM) of classical and variational Bayes stochastic DCM (i.e., it detected two additional connections exhibiting group differences). 
As for the neuronal model, Havlicek et al. (2015) performed simulations comparing standard two-state DCM and P-DCM (adaptive two-state) and showed time courses of responses to either $1 \mathrm{~s}$ or $30 \mathrm{~s}$ stimulation in one region, as well as connectivity dynamics of a three-region network in response to a $30 \mathrm{~s}$ stimulation. They demonstrated that with standard two-state DCM, varying the neuronal post-stimulus deactivation does not translate to the BOLD undershoot, while P-DCM accurately models post-stimulus neuronal deactivation, both in response to $1 \mathrm{~s}$ or $30 \mathrm{~s}$ stimulation. This ability also holds even when CBV and $\mathrm{CBF}$ are uncoupled-the BOLD post-stimulus undershoot is stronger than the $\mathrm{CBF}$ response which resembles experimental results (Chen and Pike, 2009).

Pertaining to the connectivity dynamics of the simulated neuronal network, standard two-state DCM was unable to capture decreased neuronal activity below baseline-which suggests poor signal variance when both activations and deactivations are in the measured BOLD data. Meanwhile, PDCM effectively expresses transients in neuronal and BOLD responses during faster and slower neuronal dynamics. This holds in both positive and negative responses (activation and deactivation).

\section{CONCLUSION}

In summary, the most important factors to consider when applying DCM in NHP under anesthesia are cerebrovascular physiology as well as anesthetic-induced changes in neural and BOLD response dynamics. Moreover, caution is necessary when interpreting results if the model includes regions or networks in which effective connectivity may be modulated by anesthetics such as: (1) fronto-parietal; (2) sensory-motor (i.e., S1-M1, SMA-M1, auditory cortical regions); and (3) thalamocortical networks specifically involving somatosensory and motor function.

The integrated P-DCM (Havlicek et al., 2015) and stochastic DCM (Li et al., 2011) (IPS-DCM) aims to address the modulatory effects of anesthetics on neural activity and the BOLD response

\section{REFERENCES}

Adams, D. L., Piserchia, V., Economides, J. R., and Horton, J. C. (2015). Vascular supply of the cerebral cortex is specialized for cell layers but not columns. Cereb. Cortex 25, 3673-3681. doi: 10.1093/cercor/bhu221

Aksenov, D. P., Li, L., Miller, M. J., Iordanescu, G., and Wyrwicz, A. M. (2015). Effects of anesthesia on BOLD signal and neuronal activity in the somatosensory cortex. J. Cereb. Blood Flow Metab. 35, 1819-1826. doi: $10.1038 /$ jcbfm. 2015.130

Attwell, D., Buchan, A. M., Charpak, S., Lauritzen, M., MacVicar, B., and Newman, E. (2010). Glial and neuronal control of brain blood flow. Nature 468, 232-243. doi: $10.1038 /$ nature 09613

Attwell, D., and Iadecola, C. (2002). The neural basis of functional brain imaging signals. Trends Neurosci. 25, 621-625. doi: 10.1016/S0166-2236(02) 02264-6

Bell, M. A., and Ball, M. J. (1985). Laminar variation in the microvascular architecture of normal human visual cortex (area 17). Brain Res. 335, 139-143.

Bernal-Casas, D., Balaguer-Ballester, E., Gerchen, M. F., Iglesias, S., Walter, H., Heinz, A., et al. (2013). Multi-site reproducibility such as changes in inhibitory drive, neural refractory period, and cortical adaptation (Masamoto et al., 2007; Moran et al., 2011). This is accomplished through: (1) an adaptive two-state neuronal model that incorporates adaptation and refractory effects to neuronal response; (2) a hemodynamic model that incorporates feedforward neurovascular coupling and a viscoelastic effect on the Balloon model; (3) a BOLD signal change equation that accounts for magnetic field differences; and (4) stochastic (generalized filtering) model inversion that addresses non-linear interactions among hidden states, such as the non-linear increase in GABAergic inhibitory drive (Moran et al., 2011; Daunizeau et al., 2012) under anesthesia.

This paper presents the motivations for applying DCM to NHP fMRI and potential strategies for addressing anesthetic effects on neuronal activity and BOLD response, which is pertinent in primate neuroimaging under anesthesia. Clearly, a number of challenges remain. For example, the validity of this physiologic-stochastic DCM integration needs to be established. Additionally, its applicability to resting state data is also an interesting consideration. Stochastic DCM has been validated in resting-state fMRI (Razi et al., 2015); however, P-DCM has only been applied to task-based recordings (Havlicek et al., 2017). Future investigations are needed to examine the face and construct validity of IPS-DCM, as well as its applicability to resting-state data.

\section{AUTHOR CONTRIBUTIONS}

DJ and DD conceived the review focus, reviewed the literature, finalized the manuscript, and approved the final version of the manuscript. DJ summarized the literature review and wrote the first draft.

\section{ACKNOWLEDGMENTS}

We are grateful to Bernard Ng, Ph.D. and Rafael von Känel, Ph.D. for the stimulating discussions and the reviewers for their detailed comments. of prefrontal-hippocampal connectivity estimates by stochastic DCM. NeuroImage 82, 555-563. doi: 10.1016/j.neuroimage.2013. 05.120

Berwick, J., Martin, C., Martindale, J., Jones, M., Johnston, D., Zheng, Y., et al. (2002). Hemodynamic response in the unanesthetized rat: intrinsic optical imaging and spectroscopy of the barrel cortex. J. Cereb. Blood Flow Metab. 22, 670-679. doi: 10.1097/00004647-200206000-00005

Bielczyk, N. Z., Uithol, S., van Mourik, T., Anderson, P., Glennon, J. C., and Buitelaar, J. K. (2019). Disentangling causal webs in the brain using functional magnetic resonance imaging: A review of current approaches. Netw. Neurosci. 3, 237-273. doi: 10.1162/netn_a_00062

Boly, M., Moran, R., Murphy, M., Boveroux, P., Bruno, M. A., Noirhomme, Q., et al. (2012). Connectivity changes underlying spectral EEG changes during propofol-induced loss of consciousness. J. Neurosci. 32, 7082-7090. doi: 10.1523/JNEUROSCI.3769-1 1.2012

Brown, G. G., Eyler Zorrilla, L. T., Georgy, B., Kindermann, S. S., Wong, E. C., and Buxton, R. B. (2003). BOLD and perfusion response to finger-thumb apposition after acetazolamide administration: differential relationship to global perfusion. 
J. Cereb. Blood Flow Metab. 23, 829-837. doi: 10.1097/01.WCB.0000071887.63 724.B2

Buijink, A. W. G., van der Stouwe, A. M. M., Broersma, M., Sharifi, S., Groot, P. F. C., Speelman, J. D., et al. (2015). Motor network disruption in essential tremor: a functional and effective connectivity study. Brain 138, 2934-2947. doi: 10.1093/brain/awv225

Buxton, R. B., Griffeth, V. E., Simon, A. B., Moradi, F., and Shmuel, A. (2014). Variability of the coupling of blood flow and oxygen metabolism responses in the brain: a problem for interpreting BOLD studies but potentially a new window on the underlying neural activity. Front. Neurosci. 8:139. doi: 10.3389/fnins.2014.00139

Buxton, R. B., Uludag K., Dubowitz, D. J., and Liu, T. T. (2004). Modeling the hemodynamic response to brain activation. Neuroimage 23 (Suppl. 1), S220-S233. doi: 10.1016/j.neuroimage.2004.07.013

Buxton, R. B., Wong, E. C., and Frank, L. R. (1998). Dynamics of blood flow and oxygenation changes during brain activation: the balloon model. Magn. Reson. Med. 39, 855-864. doi: 10.1002/mrm.1910390602

Chen, J. J., and Pike, G. B. (2009). Origins of the BOLD post-stimulus undershoot. Neuroimage. 46, 559-568. doi: 10.1016/j.neuroimage.2009.03.015

Cohen, E. R., Ugurbil, K., and Kim, S.-G. (2002). Effect of basal conditions on the magnitude and dynamics of the blood oxygenation leveldependent fMRI response. J. Cerebr. Blood Flow Metabol. 22, 1042-1053. doi: 10.1097/00004647-200209000-00002

Daunizeau, J., Friston, K., and Kiebel, S. (2009). Variational Bayesian identification and prediction of stochastic nonlinear dynamic causal models. Phys. D 238, 2089-2118. doi: 10.1016/j.physd.2009.08.002

Daunizeau, J., Stephan, K. E., and Friston, K. J. (2012). Stochastic dynamic causal modelling of fMRI data: should we care about neural noise? Neuroimage 62, 464-481. doi: 10.1016/j.neuroimage.2012.04.061

Dehaene, S., Piazza, M., Pinel, P., and Cohen, L. (2003). Three parietal circuits for number processing. Cogn. Neuropsychol. 20, 487-506. doi: 10.1080/02643290244000239

Di, X., and Biswal, B. B. (2014). Identifying the default mode network structure using dynamic causal modeling on resting-state functional magnetic resonance imaging. Neuroimage 86:53e59. doi: 10.1016/j.neuroimage.2013.07.071

Duncan, J., Seitz, R. J., Kolodny, J., Bor, D., Herzog, H., Ahmed, A., et al. (2000). A neural basis for general intelligence. Science 289, 457-460. doi: 10.1126/science.289.5478.457

Duvernoy, H. M., Delon, S., and Vannson, J. L. (1981). Cortical blood vessels of the human brain. Brain Res. Bull. 7, 519-579. doi: 10.1016/0361-9230(81)90007-1

Eichling, J. O., Raichle, M. E., Grubb, R. L Jr., Larson, K. B., and Ter-Pogossian, M. M. (1975). In vivo determination of cerebral blood volume with radioactive oxygen-15 in the monkey. Circ. Res. 37, 707-714. doi: 10.1161/01.RES.37.6.707

Frässle, S., Lomakina, E. I., Kasper, L., Manjaly, Z. M., Leffe, A., Pruessmann, K. P., et al. (2018). A generative model of whole-brain effective connectivity. NeuroImage 179, 505-529. doi: 10.1016/j.neuroimage.2018.05.058

Frey, S. H. (2008). Tool use, communicative gesture, and cerebral asymmetries in the modern human brain. Philos. Trans. R. Soc. Lond. B. Biol. Sci. 363, 1951-1957. doi: 10.1098/rstb.2008.0008

Friston, K. (2009). Causal modelling and brain connectivity in functional magnetic resonance imaging. PLoS Biol. 7:e1000033. doi: 10.1371/journal.pbio.1000033

Friston, K., Mattout, J., Trujillo-Barreto, N., Ashburner, J., and Penny, W. (2007). Variational free energy and the Laplace approximation. Neuroimage 34, 220-234. doi: 10.1016/j.neuroimage.2006.08.035

Friston, K. J. (2011). Functional and effective connectivity: a review. Brain Connect. 1, 13-36. doi: 10.1089/brain.2011.0008

Friston, K. J., Glaser, D. E., Henson, R. N., Kiebel, S., Phillips, C., and Ashburner, J. (2002). Classical and Bayesian inference in neuroimaging: applications. Neuroimage 16, 484-512. doi: 10.1006/nimg.2002.1091

Friston, K. J., Harrison, L., and Penny, W. (2003). Dynamic causal modelling. Neuroimage. 19, 1273-1302. doi: 10.1016/S1053-8119(03)00202-7

Friston, K. J., Kahan, J., Biswal, B., and Razi, A. (2014). A DCM for resting state fMRI. Neuroimage 94, 396-407. doi: 10.1016/j.neuroimage.2013.12.009

Friston, K. J., Preller, K. H., Mathys, C., Cagnan, H., Heinzle, J., Razi, A., et al. (2017). Dymamic causal modelling revisited. Neuroimage. 199, 730-744. doi: 10.1016/j.neuroimage.2017.02.045

Goebel, R., Roebroeck, A., Kim, D. S., and Formisano, E. (2003). Investigating directed cortical interactions in time-resolved fMRI data using vector autoregressive modeling and Granger causality mapping. Magn. Reson. Imaging 21, 1251-1261. doi: 10.1016/j.mri.2003.08.026

Gómez, F., Phillips, C., Soddu, A., Boly, M., Boveroux, P., Vanhaudenhuyse, A., et al. (2013). Changes in effective connectivity by propofol sedation. PLOS ONE 8:e71370. doi: 10.1371/journal.pone.0071370

Granger, C. W. J. (1969). Investigating causal relations by econometric models and cross-spectral methods. Econometrica 37, 424-438. doi: 10.2307/1912791

Grubb, R. L Jr., Raichle, M. E., Eichling, J. O., and Ter-Pogossian, M. M. (1974). The effects of changes in $\mathrm{PaCO}_{2}$ on cerebral blood volume, blood flow, and vascular mean transit time. Stroke 5, 630-639. doi: 10.1161/01.STR.5.5.630

Guibert, R., Fonta, C., and Plouraboué, F. (2010). Cerebral blood flow modeling in primate cortex. J. Cereb. Blood Flow Metab. 30, 1860-1873. doi: $10.1038 /$ jcbfm.2010.105

Havlicek, M., Ivanov, D., Roebroeck, A., and Uludag, K. (2017). Determining excitatory and inhibitory neuronal activity from multimodal fMRI data using a generative hemodynamic model. Front. Neurosci. 11:616. doi: $10.3389 /$ fnins.2017.00616

Havlicek, M., Roebroeck, A., Friston, K., Gardumi, A., Ivanov, D., and Uludag, K. (2015). Physiologically informed dynamic causal modeling of fMRI data. Neuroimage 122, 355-372. doi: 10.1016/j.neuroimage.2015.07.078

Heinzle, J., Koopmans, P. J., den Ouden, H. E., Raman, S., and Stephan, K. E. (2016). A hemodynamic model for layered BOLD signals. Neuroimage 125, 556-570. doi: 10.1016/j.neuroimage.2015.10.025

Heinzle, J., and Stephan, K. (2018). "Chapter 5: Dynamic causal modeling and its application to psychiatric disorders," in Computational Psychiatry, eds A. Anticevic and J. D. Murray (Cambirdge, MA: Academic Press), 117-144.

Hillebrandt, H., Friston, K. J., and Blakemore, S. J. (2014). Effective connectivity during animacy perception-dynamic causal modelling of Human Connectome Project data. Sci. Rep. 4:6240. doi: 10.1038/srep06240

Hutchison, R. M., Hutchison, M., Manning, K. Y., Menon, R. S., and Everling, S. (2014). Isoflurane induces dose-dependent alterations in the cortical connectivity profiles and dynamic properties of the brain's functional architecture. Hum. Brain Mapp. 35, 5754-5775. doi: 10.1002/hbm.22583

Imas, O. A., Ropella, K. M., Ward, B. D., Wood, J. D., and Hudetz, A. G. (2005). Volatile anesthetics disrupt frontal-posterior recurrent information transfer at gamma frequencies in rat. Neurosci. Lett. 387, 145-150. doi: 10.1016/j.neulet.2005.06.018

Ito, H., Kanno, I., Kato, C., Sasaki, T., Ishii, K., Ouchi, Y. et al. (2004). Database of normal human cerebral blood flow, cerebral blood volume, cerebral oxygen extraction fraction and cerebral metabolic rate of oxygen measured by positron emission tomography with 15O-labelled carbon dioxide or water, carbon monoxide and oxygen: a multicentre study in Japan. Eur. J. Nucl. Med. Mol. Imaging 31, 635-643. doi: 10.1007/s00259-003-1430-8

Kahan, J., and Foltynie, T. (2013). Understanding DCM: ten simple rules for the clinician. Neuroimage 83, 542-549. doi: 10.1016/j.neuroimage.2013.07.008

Kang, J. H., Choi, J. H., Hwang, E., and Kim, S. P. (2016). Changes in effective connectivity of sensorimotor rhythms in thalamocortical circuits during the induction and recovery of anesthesia in mice. J. Neurol. Sci. 369, 165-175. doi: 10.1016/j.jns.2016.08.031

Kim, P. J., Kim, H. G., Noh, G. J., Koo, Y. S., and Shin, T. J. (2017). Disruption of frontal-parietal connectivity during conscious sedation by propofol administration. Neuroreport 28, 896-902. doi: 10.1097/WNR.0000000000000853

Ku, S. W., Lee, U., Noh, G. J., Jun, I. G., and Mashour, G. A. (2011). Preferential inhibition of frontal-to-parietal feedback connectivity is a neurophysiologic correlate of general anesthesia in surgical patients. PLOS ONE 6:e25155. doi: 10.1371/journal.pone.0025155

Långsjö, J. W., Maksimow, A., Salmi, E., Kaisti, K., Aalto, S., Oikonen, V., et al. (2005). S-ketamine anesthesia increases cerebral blood flow in excess of the metabolic needs in humans. Anesthesiology 103, 258-268. doi: 10.1097/00000542-200508000-00008

Lee, U., Kim, S., Noh, G. J., Choi, B. M., Hwang, E., and Mashour, G. A. (2009). The directionality and functional organization of frontoparietal connectivity during consciousness and anesthesia in humans. Conscious. Cogn. 18, 1069-1078. doi: 10.1016/j.concog.2009.04.004

Li, B., Daunizeau, J., Stephan, K. E., Penny, W., Hu, D., and Friston, K. (2011). Generalised filtering and stochastic DCM for fMRI. Neuroimage 58, 442-457. doi: 10.1016/j.neuroimage.2011.01.085 
Li, C.-X., Patel, S., Auerbach, E. J., and Zhang, X. (2013). Dose-dependent effect of isoflurane on regional cerebral blood flow in anesthetized macaque monkeys. Neurosci. Lett. 541, 58-62. doi: 10.1016/j.neulet.2013.02.007

Li, L., Hu, X., Preuss, T. M., Glasser, M. F., Damen, F. W., Qiu, Y., et al. (2013). Mapping putative hubs in human, chimpanzee and rhesus macaque connectomes via diffusion tractography. Neuroimage 80, 462-474. doi: 10.1016/j.neuroimage.2013.04.024

Li, T.-Q., Kastrup, A., Moseley, M. E., and Glover, G. H. (2000). Changes in baseline cerebral blood flow in humans do not influence regional cerebral blood flow response to photic stimulation. J. Magn. Reson. Imaging 12, 757-762. doi: 10.1002/1522-2586(200011)12:53.0.CO;2-4

Lindauer, U., Leithner, C., Kaasch, H., Rohrer, B., Foddis, M., Füchtemeier, M., et al. (2010). Neurovascular coupling in rat brain operates independent of hemoglobin deoxygenation. J. Cereb Blood Flow Metab. 30, 757-768. doi: $10.1038 /$ jcbfm. 2009.259

Lohmann, G., Erfurth, K., Muller, K., and Turner, R. (2012). Critical comments on dynamic causal modelling. Neuroimage 59, 2322-2329. doi: 10.1016/j.neuroimage.2011.09.025

MacVicar, B. A., and Newman, E. A. (2015). Astrocyte regulation of blood flow in the brain. Cold Spring Harb Perspect. Biol. 7:a020388. doi: 10.1101/cshperspect.a020388

Mandeville, J. B., Marota, J. J., Ayata, C., Zararchuk, G., Moskowitz, M. A., Rosen, B., et al. (1999). Evidence of a cerebrovascular postarteriole Windkessel with delayed compliance. J. Cereb. Blood Flow Metab. 19, 679-689. doi: 10.1097/00004647-199906000-00012

Mantini, D., Corbetta, M., Romani, G. L., Orban, G. A., and Vanduffel, W. (2013). Evolutionarily novel functional networks in the human brain? J. Neurosci. 33, 3259-3275. doi: 10.1523/JNEUROSCI.4392-12.2013

Marreiros, A. C., Kiebel, S. J., and Friston, K. J. (2008). Dynamic causal modelling for fMRI: a two-state model. Neuroimage 39, 269-278. doi: 10.1016/j.neuroimage.2007.08.019

Mars, R. B., Jbabdi, S., Sallet, J., O’Reilly, J. X., Croxson, P. L., Olivier, E., et al. (2011). Diffusion-weighted imaging tractography-based parcellation of the human parietal cortex and comparison with human and macaque resting-state functional connectivity. J. Neurosci. 31, 4087-4100. doi: 10.1523/JNEUROSCI.5102-10.2011

Martin, C., Martindale, J., Berwick, J., and Mayhew, J. (2006). Investigating neuralhemodynamic coupling and the hemodynamic response function in the awake rat. Neuroimage 32, 33-48. doi: 10.1016/j.neuroimage.2006.02.021

Masamoto, K., Kim, T., Fukuda, M., Wang, P., and Kim, S. G. (2007). Relationship between neural, vascular, and BOLD signals in isoflurane-anesthetized rat somatosensory cortex. Cereb. Cortex 17, 942-950. doi: 10.1093/cercor/bhl005

Moran, R. J., Jung, F., Kumagai, T., Endepols, H., Graf, R., Dolan, R. J., et al. (2011). Dynamic causal models and physiological inference: a validation study using isoflurane anaesthesia in rodents. PLOS ONE 6:e22790. doi: 10.1371/journal.pone.0022790

Ogawa, S., Tank, D. W., Menon, R., Ellermann, J. M., Kim, S. G., Merkle, H., et al. (1992). Intrinsic signal changes accompanying sensory stimulation: functional brain mapping with magnetic resonance imaging. Proc. Natl. Acad. Sci. U.S.A. 89:5951-5955. doi: 10.1073/pnas.89.13.5951

Olsen, K. S., Henriksen, L., Owen-Falkenberg, A., Dige-Petersen, H., Rosenorn, J., and Chraemmer-Jorgensen, B. (1994). Effect of 1 or 2 MAC isoflurane with or without ketanserin on cerebral blood flow autoregulation in man. Br. J. Anaesth. 72, 66-71. doi: 10.1093/bja/72.1.66

Paasonen, J., Stenroos, P., Salo, R. A., Kiviniemi, V., and Gröhn, O. (2018). Functional connectivity under six anesthesia protocols and the awake condition in rat brain. Neuroimage 172, 9-20. doi: 10.1016/j.neuroimage.2018. 01.014

Park, H. J., Friston, K. J., Pae, C., Park, B., and Razi, A. (2018). Dynamic effective connectivity in resting state fMRI. Neuroimage $180(\mathrm{Pt} \mathrm{B}), 594-608$. doi: 10.1016/j.neuroimage.2017.11.033

Pernet, C. R. (2014). Misconceptions in the use of the General Linear Model applied to functional MRI: a tutorial for junior neuro-imagers. Front. Neurosci. 8:1. doi: 10.3389/fnins.2014.00001

Phelps, M. E., Grubb, R. L Jr., and Ter-Pogossian, M. M. (1973). In vivo regional cerebral blood volume by x-ray fluorescence: validation of method. J. Appl. Physiol. 35, 741-747. doi: 10.1152/jappl.1973.35.5.741
Powers, W. J., Hirsch, I. B., and Cryer, P. E. (1996). Effect of stepped hypoglycemia on regional cerebral blood flow response to physiological brain activation. Am. J. Physiol. 270, H554-H559. doi: 10.1152/ajpheart.1996.270.2.H554

Razi, A., Kahan, J., Rees, G., and Friston, K. J. (2015). Construct validation of a DCM for resting state fMRI. Neuroimage 106, 1-14. doi: 10.1016/j.neuroimage.2014.11.027

Roebroeck, A., Formisano, E., and Goebel, R. (2005). Mapping directed influence over the brain using Granger causality and fMRI. Neuroimage 25, 230-242. doi: 10.1016/j.neuroimage.2004.11.017

Rowe, J. B., Hughes, L. E., Barker, R. A., and Owen, A. M. (2010). Dynamic causal modelling of effective connectivity from fMRI: are results reproducible and sensitive to Parkinson's disease and its treatment? Neuroimage 52, 1015-1026. doi: 10.1016/j.neuroimage.2009. 12.080

Sallet, J., Mars, R. B., Noonan, M. P., Neubert, F.-X., Jbabdi, S., O’Reilly, J. X., et al. (2013). The organization of dorsal frontal cortex in humans and macaques. J. Neurosci. 33, 12255-12274. doi: 10.1523/JNEUROSCI.5108-12.2013

Scharrer, E. (1960). Brain Function and the Evolution of Cerebral Vascularization. James Arthur Lecture on the Evolution of the Human Brain. New York: The American Museum of Natural History, f-32.

Schreiber, T. (2000). Measuring information transfer. Phys. Rev. Lett. 85, 461-464. doi: 10.1103/PhysRevLett.85.461

Schuyler, B., Ollinger, J. M., Oakes, T. R., Johnstone, T., and Davidson, R. J. (2010). Dynamic causal modeling applied to fMRI data shows high reliability. Neuroimage 49, 603-611. doi: 10.1016/j.neuroimage.2009.07.015

Seth, A. K., Barrett, A. B., and Barnett, L. (2015). Granger causality analysis in neuroscience and neuroimaging. J. Neurosci. (2015) 35, 3293-3297. doi: 10.1523/JNEUROSCI.4399-14.2015

Singer, T., and Lamm, C. (2009). The social neuroscience of empathy. Ann. N. Y. Acad. Sci. 1156, 81-96. doi: 10.1111/j.1749-6632.2009.04418.x

Slupe, A. M., and Kirsch, J. R. (2018). Effects of anesthesia on cerebral blood flow, metabolism, and neuroprotection. J. Cereb. Blood Flow Metabol. 38, 2192-2208. doi: 10.1177/0271678X18789273

Smith, S., Miller, K., Salimi-Khorshidi, G., Webster, M., Beckmann, C., Nichols, T., and Woolrich, M. (2011). Network modelling methods for fMRI. NeuroImage 54, 875-891. doi: 10.1016/j.neuroimage.2010.08.063

Stephan, K. E., Harrison, L. M., Penny, W. D., and Friston, K. J. (2004). Biophysical models of fMRI responses. Curr. Opin. Neurobiol. 14, 629-635. doi: 10.1016/j.conb.2004.08.006

Stephan, K. E., Penny, W. D., Moran, R. J., den Ouden, H. E., Daunizeau, J., and Friston, K. J. (2010). Ten simple rules for dynamic causal modeling. Neuroimage 49, 3099-3109. doi: 10.1016/j.neuroimage.2009.11.015

Stephan, K. E., Weiskopf, N., Drysdale, P. M., Robinson, P. A., and Friston, K. J. (2007). Comparing hemodynamic models with DCM. Neuroimage 38, 387-401. doi: 10.1016/j.neuroimage.2007.07.040

Tak, S., Noh, J., Cheong, C., Zeidman, P., Razi, A., Penny, W. D., et al. (2018). A validation of dynamic causal modelling for 7T fMRI. J. Neurosci. Methods 305, 36-45. doi: 10.1016/j.jneumeth.2018.05.002

Uludag, K., Müller-Bierl, B., and Ugurbil, K. (2009). An integrative model for neuronal activity-induced signal changes for gradient and spin echo functional imaging. Neuroimage 48, 150-165. doi: 10.1016/j.neuroimage.2009.05.051

Van Aken, H., Fitch, W., Graham, D. I., Brussel, T., and Themann, H. (1986). Cardiovascular and cerebrovascular effects of isofluraneinduced hypotension in the baboon. Anesth. Analg. 65, 565-574. doi: 10.1213/00000539-198606000-00003

Van Aken, H., and van Hemelrijck, J. (1991). Influence of anesthesia on cerebral blood flow and cerebral metabolism: an overview. Agressologie 32, 303-306.

Van Essen, D. C., and Dierker, D. L. (2007). Surface-based and probabilistic atlases of primate cerebral cortex. Neuron 56, 209-225. doi: 10.1016/j.neuron.2007.10.015

Vaudano, A. E., Avanzini, P., Tassi, L., Ruggieri, A., Cantalupo, G., Benuzzi, F., and ..., Meletti S (2013). Causality within the epileptic network: an EEG-fMRI study validated by intracranial EEG. Front. Neurol. 14:185. doi: 10.3389/fneur.2013.00185

Vicente, R., Wibral, M., Lindner, M., and Pipa, G. (2011). Transfer entropy-a model-free measure of effective connectivity for the neurosciences. J. Comput. Neurosci. 30, 45-67. doi: 10.1007/s10827-010-0262-3 
Webb, J. T., Ferguson, M. A., Nielsen, J. A., and Anderson, J. S. (2013). BOLD Granger causality reflects vascular anatomy. PLOS ONE 8:e84279. doi: 10.1371/journal.pone.0084279

Weber, B., Keller, A. L., Reichold, J., and Logothetis, N. K. (2008). The microvascular system of the striate and extrastriate visual cortex of the macaque. Cereb. Cortex. 18:2318-2330. doi: 10.1093/cercor/bhm259

White, N. S., and Alkire, M. T. (2003). Impaired thalamocortical connectivity in humans during general-anesthetic-induced unconsciousness. Neuroimage 19(2 Pt 1), 402-411. doi: 10.1016/S1053-8119(03)00103-4

Witt, S. T., and Meyerand, M. E. (2009). The effects of computational method, data modeling, and TR on effective connectivity results. Brain Imaging Behav. 3, 220-231. doi: 10.1007/s11682-009-9064-5

Wright, S. (1920). The relative importance of heredity and environment in determining the piebald pattern of guinea-pigs. Proc. Natl. Acad. Sci. U.S.A. 6, 320-332. doi: 10.1073/pnas.6.6.320

Yeom, S. K., Won, D. O., Chi, S. I., Seo, K. S., Kim, H. J., Müller, K. R., et al. (2017). Spatio-temporal dynamics of multimodal EEG-fNIRS signals in the loss and recovery of consciousness under sedation using midazolam and propofol. PLoS ONE 12:e0187743. doi: 10.1371/journal.pone.0187743

Zappe, A. C., Uludag, K., Oeltermann, A., Ugurbil, K., and Logothetis, N. K. (2008) The influence of moderate hypercapnia on neural activity in the anesthetized non-human primate. Cereb. Cortex 18, 2666-2673. doi: 10.1093/cercor/bhn023

Conflict of Interest Statement: The authors declare that the research was conducted in the absence of any commercial or financial relationships that could be construed as a potential conflict of interest.

Copyright $\odot 2019$ Jovellar and Doudet. This is an open-access article distributed under the terms of the Creative Commons Attribution License (CC BY). The use, distribution or reproduction in other forums is permitted, provided the original author(s) and the copyright owner(s) are credited and that the original publication in this journal is cited, in accordance with accepted academic practice. No use, distribution or reproduction is permitted which does not comply with these terms. 


\section{OPEN ACCESS}

Edited by:

Roberto Esposito,

A.O. Ospedali Riuniti Marche Nord,

Italy

Reviewed by: Heinz Boeker,

University of Zurich, Switzerland Karl Friston,

University College London, United Kingdom

${ }^{*}$ Correspondence: Georg Northoff georg.northoff@theroyal.ca

Specialty section:

This article was submitted to

Cognitive Neuroscience,

a section of the journal

Frontiers in Human Neuroscience

Received: 03 July 2019

Accepted: 29 August 2019 Published: 19 September 2019

Citation: Northoff G (2019) Lessons From Astronomy and Biology for the Mind-Copernican Revolution in Neuroscience.

Front. Hum. Neurosci. 13:319. doi: 10.3389/fnhum.2019.00319

\section{Lessons From Astronomy and Biology for the Mind-Copernican Revolution in Neuroscience}

\author{
Georg Northoff * \\ Cellular and Molecular Medicine Faculty of Medicine, University of Ottawa Institute of Mental Health Research, Ottawa, ON, \\ Canada
}

Neuroscience made major progress in unravelling the neural basis of mental features like self, consciousness, affect, etc. However, we nevertheless lack what recently has been described as "missing ingredient" or "common currency" in the relationship between neuronal and mental activity. Rather than putting forward yet another theory of the neural basis of mental features, I here suggest a change in our methodological strategy how to approach the brain, that is, our view or vantage point of the brain. Learning from astronomy (Copernicus) and biology (Darwin), I suggest that we may want to change our currently pre-Copernican vantage point from within brain to a post-Copernican vantage point from beyond brain. Such post-Copernican vantage point from beyond brain allows us taking into view that what happens beyond the brain itself, e.g., the world, and how that shapes the brain and its neural activity, e.g., world-brain relation. We then lend empirical support to the world-brain relation by converging it with Karl Friston's free energy principle that, as we see it, provides a neuro-ecological and therefore post-Copernican view of the brain. That, in turn, allows us taking into view that mental features are shaped by both world and brain and are therefore truly neuroecological rather than merely neuronal. This raises the question for the link, e.g., the "missing ingredient" or "common currency" of world brain relation and mental features. Recent empirical evidence suggests that temporo-spatial dynamics may provide such link as it characterizes both the world-brain relation's free energy and mental features, e.g., their spatiotemporality as described in philosophy. Taken together, I here advocate a change in our methodological strategy on how to approach the brain, that is, a shift from a pre-Copernican vantage point from within brain to a post-Copernican vantage point from beyond brain. The latter allows us taking into view that what happens beyond the brain in the world and how that shapes the brain in such a way that it can yield mental features. This amounts to nothing less than a Copernican turn or revolution in neuroscience akin to the ones in astronomy (Copernicus) and biology (Darwin).

Keywords: physics, biology, neuroscience, common currency, copernican turn 


\section{INTRODUCTION}

\section{Mental Features-How Can We Reconcile World and Brain?}

The mind and its various mental features present us with a puzzle. On the one hand, mental features like self, consciousness, and affect (and others) can be characterized by an experience or perception of specific events or objects and even the own self as part of the wider world beyond ourselves, e.g., body and brain. Taken in such sense, mental features exhibit a strong ecological component. On the other hand, recent research in neuroscience clearly demonstrates a neural basis of the various mental features in the brain. One would consequently assume that mental features are neuronal rather than ecological.

How can we reconcile both mental, e.g., ecological and neuronal views of mental features in our empirical research in neuroscience (while refraining from any ontological metaphysical assumptions; see below)? One way to do so is to reduce mental features to the neuronal mechanisms of the brain. This is reflected in various excellent neuroscientific theories of mental features like consciousness and self. Among others, these include the Integrated Information Theory (IIT; Tononi et al., 2016), the Global Neuronal Workspace Theory (GNWT; Dehaene et al., 2014, 2017), and the Temporo-spatial Theory of consciousness (TTC; Northoff, 2013, 2014a,b, 2016a,b,c,d, 2018; Northoff and Huang, 2017).

The same also holds for other mental features like self where neuronal accounts of cognitive (Churchland, 2002), dynamic pattern (Gallagher, 2005), affective (Panksepp, 1998a,b), attentional (Sui and Humphreys, 2015), embodied (Gallagher, 2005; Thompson, 2007; Hu et al., 2016), and temporo-spatial (Northoff, 2016a,b,c,d, 2017) theories of self have been suggested. Finally, affect has also been the focus where, rather than reducing it, neuronal and mental features are conceived as two sides of one and the same activity (which, metaphysically, presupposes dual-aspect monism) - this has recently been suggested by Mark Solms who conceives affect as most basic and primary manifestation of consciousness and mental features (Solms, 2017, 2018, 2019; see also Damasio, 2018).

Yet another methodological strategy on how to reconcile ecological and neuronal views of mental features is to change our approach to the brain. Specifically, one may want to take into view that what happens beyond the brain itself, e.g., in world and body, and how that shapes the brain itself in such way that it can yield mental features. Such neuro-ecological (rather than purely neuronal) view of the brain, in turn, may allow us to account for both ecological and neuronal aspects of mental features. Even more important, we can then take into view that what has been recently described as "missing ingredient" (Lamme, 2018) or "common currency" (Northoff, 2019) of neuronal and mental features. The main goal in the present article consists in sketching such alternative methodological strategy in our approach to the brain and how it yields mental features.

\section{Main and Specific Aims-Copernican Revolution in Neuroscience}

My main aim is to demonstrate that neuroscience can learn from both astronomy and biology in their Copernican turns. Copernicus changed our view of earth (see below) which allowed him to take into view a novel and different relation of universe and earth. Analogously so in the case of Darwin. He changed our view of human species which enabled him to take into view our relation to evolution (see below for details on both Copernicus and Darwin). I now suggest the same kind of Copernican turn or revolution with regard to the brain. We may want to change our currently pre-Copernican view of the brain and replace it by a post-Copernican view. That, as I suggest, will enable us to take a novel post-Copernican view how the brain is related to the world, e.g., world-brain relation (see below for details; Northoff, 2016a,b,c,d, 2018). Importantly, this, in turn, makes possible to reconcile ecological and neuronal view of mental features as truly neuro-ecological rather than as merely neuronal.

My suggestion amounts to nothing less than the claim for a Copernican revolution in neuroscience (analogous to the ones in astronomy and biology). Note that I conceive such Copernican revolution in merely empirical terms of neuroscience. Hence, I only focus on the methodological strategy, e.g., our view or vantage point (see below) with regard to the brain in purely empirical terms, that is, how we can approach and understand the brain's neuronal features as we observe and investigate them in neuroscience. In contrast, I refrain from more philosophical claims of an epistemological (as, for instance, Kant suggests; Kant, 1781/1998), metaphysical, or ontological (Whitehead, 1929/1978; Sherburne, 1983; Northoff, 2016a,b,c,d). Copernican revolution (see also Northoff, 2018, for discussing the Copernican revolution in more detail in the context of the mind-body problem or world-brain problem).

Refraining from such wider senses of the Copernican revolution beyond the merely empirical territory of neuroscience entails that I here do not address any kind of philosophical problems like the "explanatory gap" (Levine, 1983), "hard problem" (Chalmers, 1996), or mind-body problem (see also Northoff, 2018 for the dissolution of the mind-body problem and its replacement by the worldbrain problem). Accordingly, my understanding of the Copernican revolution in this article is purely empirical and limited to neuroscience (rather than psychology as Sigmund Freud has also been attributed a Copernican revolution with respect to the relation of consciousness and unconsciousness; see Weinert, 2013) which, as I see it, is akin to the revolutions in both astronomy (Copernicus) and biology (Darwin).

The first specific aim consists in briefly describing the basic features of the Copernican revolutions in both astronomy (Copernicus) and biology (Darwin). That will provide the basis for the second specific aim, that is, the comparison of pre- and post-Copernican views of the brain. The third specific aim is to sketch a post-Copernican view of the brain by suggesting a 
neuro-ecological view of the brain in terms of Karl Friston's free energy principle (Friston and Stephan, 2007; Friston, 2010; Bruineberg et al., 2018a,b). That sets the basis for the fourth specific aim that consists in outlining (albeit tentatively) a post-Copernican view of mental features as neuro-ecological and temporo-spatial.

\section{PART I: PRE- VS. POST-COPERNICAN VANTAGE POINTS IN ASTRONOMY AND BIOLOGY}

\section{Vantage Point-Egocentric vs. Allocentric}

What is a vantage point? I here consider the concept of vantage point in its original definition as a "position or stand point from which something is viewed or considered" (Oxford Dictionary). Taken in this sense, the concept of vantage point comes close to those of point of view or viewpoint. The chosen vantage point may provide a specific view or viewpoint that includes a wide range of phenomena while excluding others.

Let us take the example of viewing a city. One walks around within a city. That allow us to see the details of, for instance, the mosaic on the door of the big gothic cathedral. In contrast, we remain unable to take into view the cathedral as such and how it is integrated and thus fits into its respective context, i.e., the city as whole. Specifically, the relationship between city as whole and the cathedral as part of it remains opaque to us. The cathedral looks very special to us in such viewpoint since it is not related at all to the rest of the city and its spatiotemporal coordinates-our view thus highlights the specialness of the cathedral and its dichotomy with the city. Such vantage point from within city takes the city itself including the cathedral as center thus entailing what can be described as "vantage point from within city (and cathedral)." Such viewpoint corresponds well to the egocentric "vantage points from within earth and humans" in astronomy and biology prior to Copernicus and Darwin (see below).

That changes, once one moves to the mountain nearby from which one can view the city as a whole including the big cathedral. Now, we can take into view how the big cathedral is part of the overall spatiotemporal coordinates of the city that is, how well it integrates and aligns to its respective context and the city as a whole. The spatiotemporal scope and range of our view is thus extended beyond the cathedral-this is possible by taking a what we describe as "vantage point from beyond city (and cathedral)." As we will see below, that corresponds well to the allocentric vantage points Copernicus and Darwin introduced, the "vantage points from beyond earth and humans."

\section{Pre- vs. Post-Copernican Vantage Points in Astronomy-Earth and Universe}

Before Copernicus, the universe did include earth and other planets. However, the earth was not just another planet besides the others within the universe as a whole. Instead, earth was conceived special when compared to the other planets and the rest of the universe. Specifically, the earth was supposed to hold together the universe when being attributed the role of the center around which all other planets move in the periphery.

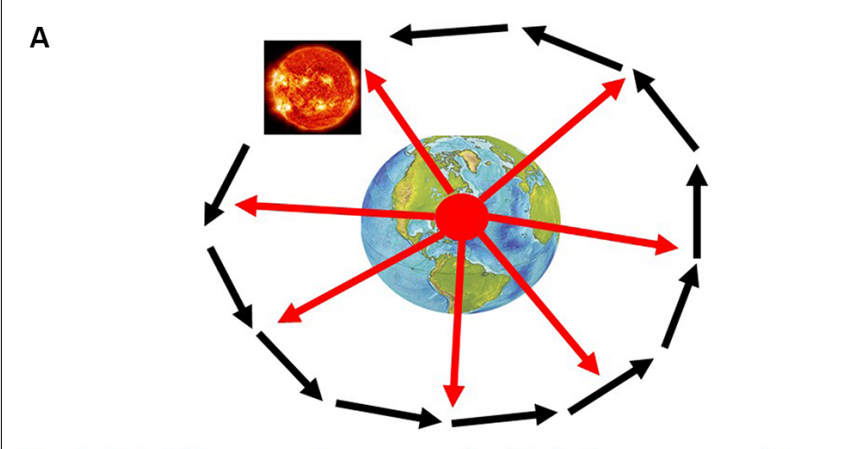

Earth/World as centre around which the sun revolves

B

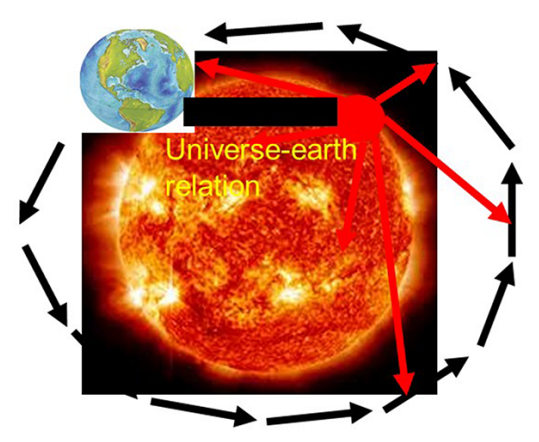

FIGURE 1 | (A) Pre-Copernican-Geo-centric view with Vantage point from within earth. (B) Post-Copernican-Helio-centric view with Vantage point from beyond earth.

There was thus a clear center-periphery dichotomy between earth and non-earth-the earth was deemed special entailing a geocentric and consequently egocentric view of the universe, a "vantage point from within earth" (see also Northoff, 2018; see Figure 1A).

The specialness of earth was further supported by its non-moving character. Being conceived as center that holds the whole universe together, the earth itself was not supposed to change as any such change would lead to the breakdown of the universe. The earth was thus conceived in an a-temporal way-the earth was assumed to be eternally present without any change in space and time. Together, the earth was characterized by specialness, e.g., its role as center, and its dichotomy to the universe, e.g., a-spatiotemporal vs. spatiotemporal.

The view of the universe changed with Copernicus which was empirically confirmed by his successors Kepler, Bruno, Gallilei, and Newton (Weinert, 2013; Northoff, 2018). Copernicus introduced a different view of earth within the universe. $\mathrm{He}$ attributed the observed movements to earth itself. Rather than sun and all other planets revolving around earth as center of the universe, he suggested the opposite namely that the earth revolves around the sun as center of universe. This led to the replacement of the geocentric and egocentric view of the universe by a heliocentric and allocentric view of the relationship of earth and universe.

In the case of a heliocentric framework, the earth loses its specialness in terms of its position, its role, and its temporal 
features. The earth is no longer the center of the universe around which all other planets revolve. Instead, the earth is replaced by the sun-the special position of earth is thus lost. Moreover, it is no longer the earth that holds together the universe but the sun-the central role of earth for the universe is also lost. Finally, the a-spatiotemporal nature of earth as not being subject to change is replaced by attributing movements to earth as it revolves around the sun-the earth is thus characterized by the same spatiotemporal features as the rest of the universe albeit in different degrees, e.g., in a smaller scale. Together, the specialness and dichotomy of earth in the pre-Copernican view are replaced by non-specialness of earth and intrinsic relationship of earth and universe.

How was it possible for Copernicus to take into view the non-specialness of earth and its intrinsic relationship with the universe? He abandoned the traditional vantage point from within earth and replaced it by one that allowed him to take into view that what happens beyond the earth itself within the universe and how that shapes the earth itself, e.g., its movements within the universe. Like the tourist walking to the nearby hill to view city and cathedral, Copernicus shifted the vantage point from within earth to what I describe as "vantage point from beyond earth" (see Figure 1B).

\section{Pre- vs. Post-Copernican Vantage Points in Biology-Human and Non-human Species}

How about biology? Darwin is often credited with bringing about a Copernican turn which lead to a scientific (and metaphysical) revolution in biology (Ruse, 2009; Weinert, 2013). Before Darwin, humans were considered special when compared to non-human species. Humans were regarded the center of the world with capacities vastly superior to the ones of non-human species. This led to the assumption of a special role of humans as only they were attributed soul and mind which enabled them to be in special contact with God as creator of the world. Since God does not change and is therefore a-spatiotemporal, humans and, more specifically, their soul or mind must also be a-spatiotemporal (as otherwise they could not be in contact with God) this entailed dichotomy of mind and world.

Both specialness of humans and their dichotomy to the rest of the world can be taken into view only when presupposing a "vantage point from within humans." That all changed with Darwin though. He presented empirical evidence that humans are part of the same evolution as non-human species. The specialness of humans was thus lost and replaced by their non-specialness.

Even more important, Darwin showed that both humans and non-humans are subject to the same principles in the world, e.g., natural selection, throughout space and time in their evolution. This allowed Darwin to take into view the intrinsic relationship of humans and non-humans including their commonly shared spatiotemporal features. Accordingly, Darwin thus able to take into view that happens beyond human species in the world including non-human species and how that shapes and relates to the humans themselves.

Look beyond humans themselves which presupposes a "vantage point from beyond human."

\section{PART II: PRE- VS. POST-COPERNICAN VANTAGE POINT IN NEUROSCIENCE}

\section{Vantage Point From Within Mind-From Philosophy to Psychology and Cognitive Science}

The famous 17 th century philosopher Descartes is considered the main source of the dualism of mind and body. The body as part of the wider world can be observed in time and space, that is, at discrete points in time and space, and operates in a purely mechanical way like a machine. That, in contrast, is not the case in consciousness and other mental features like self. The mental features are neither spatiotemporal, e.g., they a-spatiotemporal as traditionally conceived in philosophy. Nor are mental features mechanical like body and world. We, therefore, cannot attribute mental features to the body as part of the world but to the mind.

Together, the mind is special as it alone can mediate mental features like consciousness, affect/emotional feelings, and self-this reflects the specialness of mind. At the same time, the mind can be characterized by its dichotomy to body/world with seemingly no relationship between both. For that reason, Descartes conceived mind and body/world as separate existences and realities - this led him to the famous mind-body dualism which, at the same time, implies dualism of mind and world (McDowell, 1994; Northoff, 2018).

How is it possible to conceive the possibility of mind-body dualism including specialness of mind and its dichotomy to body and world? This presupposes a vantage point that takes the mind itself as its center or "primary location" in the same way pre-Copernican astronomists and biologists took earth and humans as their viewpoints. The assumption of the specialness of mind and its dichotomy to body/world thus presupposes a "vantage point from within mind" (Northoff, 2018). Taking the mind as reference for mental features, such vantage point from within mind leads to a mento-centric and ego-centric view of mental features as being special and dichotomous to body and world (see Figure 2A).

One may now want to argue that such mind-body dualism is just a merely philosophical problem. Current research in psychology and cognitive science goes beyond that by showing how the mind operates, displays different functions like consciousness, cognition, self, and affect/emotion, and uses certain computational principles and mechanisms. That does not change the basic methodological presupposition though, that is, the egocentric vantage point from within mind. True psychology and cognitive science shift from the metaphysical domain of philosophy to the empirical domain. That by itself does not change the methodological strategy how to approach the mind though.

Even an empirical approach to the mind can still take the mind itself as center and conceive the latter as special. That is, for instance, the case when one attributes specific psychological processes or computational mechanisms to mental features which stand in dichotomous relation to those of non-mental features in body and world. The mento- and thus egocentric character of the methodological approach to the mind is thus 


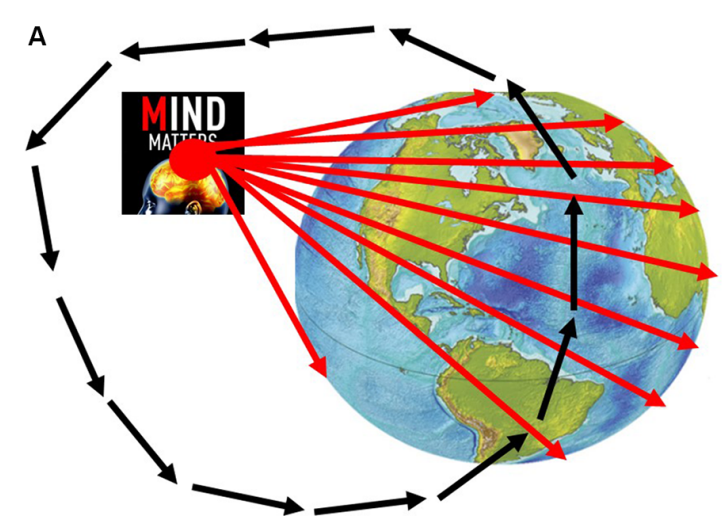

C

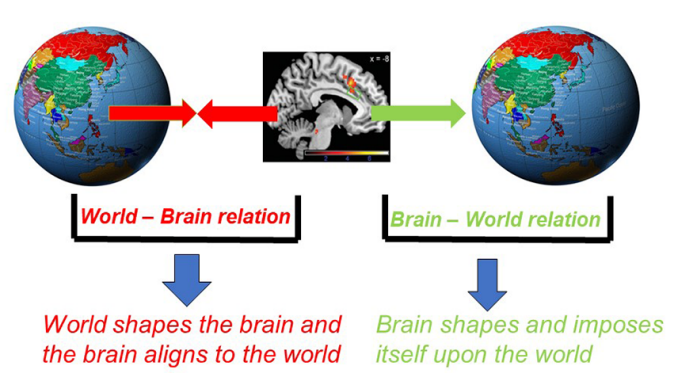

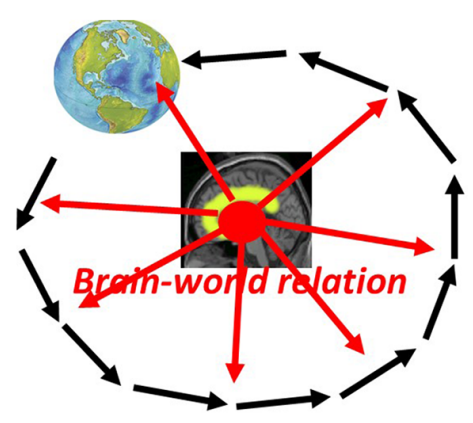

D

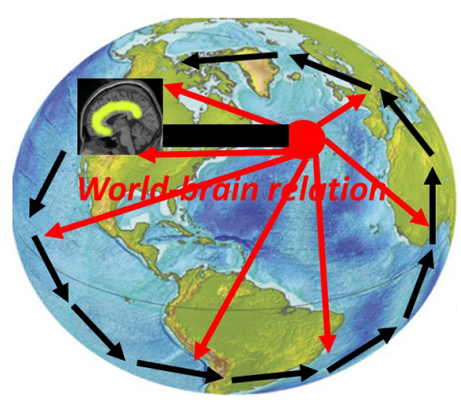

FIGURE 2 | (A) Vantage point from within mind: mento-centric and pre-Copernican stance. (B) Vantage point from with brain: neuro-centric and ego-centric view with brain as center of the world. (C) World-brain relation vs. brain-world relation. (D) Vantage point from beyond brain: allo-centric view of the brain-post-Copernican.

more or less preserved in psychology and cognitive science-they thus presuppose a vantage point from within the mind.

\section{Vantage Point From Within Brain-Neuroscience}

One may now be tempted to say that we know better these days. The assumption of mind has been disputed in both philosophy and even more so in neuroscience in our time. There is no mind anymore, mental features like consciousness and self are based on the brain and are thus physical or better neuronal rather than primarily mental, e.g., non-physical. Mind-body dualism is thus replaced by monism with either materialism/physicalism, panpsychism (Tononi and Koch, 2015), or dual-aspect monism (Solms, 2017, 2018, 2019).

The recognition of the neural basis of mental features has led to a search for their neuronal mechanisms. In the debate about, for instance, consciousness, this has led to the search for the neural correlates of consciousness (the NCC; Chalmers, 2001; Crick and Koch, 2003; Koch, 2004; Aru et al., 2012; de Graaf et al., 2012; Northoff, 2013, 2014a,b; Solms, 2018, 2019). The NCC has been defined as the minimum neuronal mechanisms jointly sufficient for any one specific conscious percept (Crick and Koch, 1990; Koch, 2004). Recent progress in consciousness research further introduces two refined interpretations of the NCC as: (1) the content-specific NCC, which determines a particular phenomenal distinction with an experience; and (2) the full NCC, which supports conscious experiences in their entirety, irrespective of the contents (Koch et al., 2016).

The NCC assumes special neuronal features within the brain itself. These special yet not fully clear neuronal features (see below) are supposed to underlie consciousness; this distinguishes them from other neuronal features that only mediate unconsciousness. The NCC thus signify a special neuronal role for consciousness and entail a neuronal dichotomy of consciousness vs. unconsciousness. Specialness and dichotomy are thus now "located" within the brain itself, that is, in terms of two sets of neuronal features and mechanisms.

Without going into detail, these specific neuronal mechanism include, to name just a few of the various suggested ones, information integration (Tononi et al., 2016), recurrent processing (Lamme, 2018), access to global workspace (Dehaene et al., 2017), embodiment (Tallon-Baudry et al., 2018), higherorder cognition (Lau and Rosenthal, 2011), predictive error minimization (Hohwy, 2013), temporo-spatial dynamics (Northoff and Huang, 2017; Northoff, 2019), or subcortical mechanisms and affect/emotion as emphasized by Panksepp (1998a,b), Damasio (2018) and Solms (2018, 2019).

How can we take into view the specialness of the neuronal mechanisms of mental features and their dichotomy to those 
of non-mental features? That is possible only by taking a view from within the brain itself and, more specifically, from within the neuronal mechanisms supposedly underlying mental features. One thus presupposes a "vantage point from within brain" in neuroscience. The mento-centric view of psychology is thus replaced by a neuro-centric view of mental features in neuroscience. However, despite the difference between mento- and neuro-centric views of mental features, both psychology/cognitive science and neuroscience still presuppose a rather egocentric vantage point, e.g., that is, from within either mind or brain which entails what, further down, I describe as brain-world relation. This marks both approaches to mental features as pre-Copernican (see Figure 2B).

Some approaches may want to argue that they are not neurocentric as they, instead of the brain, presuppose information (Tononi et al., 2016), cognition (Lau and Rosenthal, 2011), or the body (Noe, 2004; Thompson, 2007; Blanke et al., 2015; Tallon-Baudry et al., 2018) as primary basis of mental features like consciousness. True indeed, these approaches are no longer neuro-centric. That does not relieve them of their ego-centric character though. The ego-centric approach is now transferred from the brain to body, cognition, or information-they are thus body-centric, information-centric, and cognition-centric and therefore be characterized by "vantage points from within information, cognition or body." As in the neuro-centric approaches, both specialness of mental features and their dichotomy to non-mental features are still preserved in these approaches (which shall not be elaborated in detail here) this marks them as pre-Copernican.

\section{Vantage Point From Beyond Brain I-Brain-World Relation vs. World-Brain Relation}

One may now want to raise the question for a post-Copernican approach to the brain, a vantage point from beyond brain, and how that will look like. Like earth and human species, the brain and mental features would then no longer be conceived as special nor as dichotomous to the world. I here briefly want to formulate the criteria of such vantage point from beyond brain which then will be explicated in a more concrete way in the subsequent parts.

A vantage point from beyond brain must allow us taking into view that what lies beyond the boundaries of the brain itself and, even more important, how that shapes the brain. More specifically, we need to consider how the world and its external dynamic shape and impact the brain as featured by its own internal dynamic (see below for the exact meaning of dynamic). Accordingly, we need to take into the relationship between world and brain, that is, how the world shapes the brain-this is what I recently described as "world-brain relation" (Northoff, 2016a,b,c,d, 2018; see Figure 2C).

The world-brain relation needs to be distinguished from the reverse relationship, that is, how the brain shapes and cognizes the world-this is described as "brainworld relation" (Northoff, 2016a,b,c,d, 2018; see also Figure 2B). The distinction between world-brain relation and brain-world relation is important in both aspects, empirically and methodologically.

Empirically, the brain-world relation entails that the brain imposes itself upon and shapes the world-this is the case in especially cognition and action. This is different in the case of the world-brain relation where the world primarily shapes the brain rather than the latter shaping and imposing itself upon the former. That is empirically supported by data showing how, for instance, early life events in the world shape the brain's temporospatial dynamic, e.g., its degree of entropy in ventromedial prefrontal cortex (Duncan et al., 2015), and internally-guided decision making (e.g., N200 in EEG; Nakao et al., 2013) later in adulthood.

Yet another empirical example of how the world shapes the brain is the phenomenon of entrainment where the neural activity of the brain actively adapts to the events in the environment like the rhythm of music or tone sequences (Lakatos et al., 2013; van Atteveldt et al., 2015). We all know such alignment of our brain to the world only too well as when we, for instance, unconsciously, tap our feet in the rhythm of the background music.

Together, these examples show that the brain's neural activity is strongly shaped by the world by either the latter imposing itself upon the former, e.g., as in the life events, or, alternatively, by the brain actively adapting to the world, e.g., as in entrainment. Common to both examples is that the world's external dynamics shapes the brain's internal dynamics-we, therefore, speak of "world-brain relation" as distinguished from brain-world relation where the brain's internal dynamics shapes and imposes itself upon the world's external dynamics. Note that the distinction of world-brain relation vs. brain-world relation is not an absolute and mutually exclusive. Instead, world-brain relation and brain-world relation stand in a dynamic balance with each other-their conceptual distinction is thus relative (rather than absolute).

Finally, one may be surprised why we almost completely neglect the body here. Recent data show that the brain and its internal dynamic align to the body's dynamics in more or less the same way as it aligns to the world's external dynamics. For instance, various studies by the group around Tallon-Baudry et al. (2018) demonstrated that the brain's internal dynamics aligns its phase onsets to the onsets of the heartbeat-one can thus speak of "body-brain relation" (Northoff, 2018). The brain thus recruits the same mechanisms for its alignment, e.g., relation to the body as it employs when synchronizing with its external environment, e.g., the world. Therefore, we assume that the body-brain relation can be subsumed (conceptually) under the more extended world-brain relation (given also that the body is part of the world; Northoff, 2018 for details).

\section{Vantage Point From Beyond Brain II-Post-Copernican Vantage Point in Neuroscience}

The distinction of world-brain relation and brain-world relation carries major methodological implications. Featuring how the 
brain shapes and imposes itself upon the world, the brainworld relation conceives the brain as center and the world as periphery. This presupposes a vantage point from within brain. One consequently comes to the assumption of the specialness of the brain (as distinguished from non-brains) and its dichotomy to the world-this resembles the pre-Copernican vantage points in astronomy and biology. Since mental features are supposed to be caused by or identical with the brain (see Solms, 2019 for the difference between causal theories and dual-aspect monistic accounts of mental features), the specialness of the brain and its dichotomy to the world do then also apply to mental features like consciousness, self, and affect (and other mental features).

This is different in the case of the world-brain relation. Unlike the brain-world relation, the world-brain relation is based on that what happens beyond the boundaries of the brain, e.g., the world's external dynamics, and how it shapes the brain's internal dynamics. That can be taking into view only when presupposing a post-Copernican vantage point from beyond brain-the brain is then no longer special (when compared to non-brains) nor dichotomous to the world (see Figure 2D).

Presupposing such vantage point from beyond brain, we can then take into view how mental features extend beyond the brain as they may be traced to and based on the world and how it shapes the brain, e.g., world-brain relation. Consciousness, self, affect and other mental features may consequently no longer be conceived as exclusively neuronal but neuro-ecological (see below for details). Most important, this implies that mental features are no longer special nor in dichotomous relation to the world. Presupposing a vantage point from beyond brain, we will now, in the next part, sketch (albeit very preliminary) such post-Copernican view of both brain, e.g., in terms of world-brain relation and free energy (third part), and mental features (fourth part; for more details, see Northoff, 2016a,b,c,d, 2018).

We here pursue a two-step procedure to explicate such post-Copernican approach. First, we explicate and detail what is meant by world-brain relation in more biological detail by characterizing it by the free-energy principle of Friston (2010). This amounts to a post-Copernican view of the brain (third part). Second, being characterized by free energy, we are then able to link world-brain relation in a necessary way to mental features; such necessary connection is provided by dynamic and more specifically temporo-spatial features as "common currency" of world, brain, and mental features (Northoff, 2018, 2019). This entails a post-Copernican view of mental features.

\section{PART III: POST-COPERNICAN VIEW OF THE BRAIN-FREE ENERGY}

\section{Free Energy I-Neuro-Ecological and Biological View}

The organism and its brain are not isolated from the world but deeply embedded within and dependent upon the world, e.g., its respective environmental context (which is the meaning in which understand the concept of "world" in the following). There is interaction between the world's external dynamics and the brain's internal dynamics. Both can interact in a bilateral or mutual way in that the brain's internal dynamics can conform to the world's external dynamics ("perception" as Friston says), e.g., world-brain relation, or, conversely, the world's external dynamics can conform to the brain's internal dynamics ("action" as Friston says), e.g., brain-world relation (Friston and Stephan, 2007; Friston, 2010; Bruineberg and Rietveld, 2014; Bruineberg et al., 2018a,b).

How is such bilateral interaction between world/environmental context and brain mediated? That is the moment where Friston's free energy principle comes in. Roughly, free energy provides the commonly shared reference of both world/environmental context and brain according to which they adjust and relate to each other. The interaction of organism/brain and world/environmental context is characterized by the attempt to minimize the amount of free energy that is discrepant between both systems (Friston and Stephan, 2007; Bruineberg and Rietveld, 2014). Friston thus speaks of "free energy minimization" as basic principle of the organism's life that specifically characterizes the brain (Friston and Stephan, 2007).

We need to be careful though. The concept of "free energy minimization" can be understood in different ways. One most commonly held assumption is that free energy minimization is a guiding principle within the brain itself; the different layers of neuronal activity and its hierarchy do then aim to minimize their amount of free energy against each other. Here, free energy minimization is taken to be closely linked to (if not almost identical with) predictive coding as central computational mechanism of the brain's neuronal activity (Hohwy, 2013; for an excellent discussion, see Bruineberg et al., 2018a). This amounts to a neuronal view of free energy which, conceptually, merges well with what I described as brainworld relation.

Such neuronal view of free energy stands in contrast to the here sketched more biological view. The biological view conceives free energy as basic principle and common reference for the interaction of world/environmental context and organism/brain. Free energy here is no longer restricted to the brain itself and confined within its boundaries. In contrast, free energy is supposed to operate at and beyond the boundaries of the brain by guiding its interaction with and attunement to the world/environmental context. That allows to view free energy as the central principle and reference for regulating the homeostasis of the organism/brain with the world/environmental context. I here follow such biological and neuro-ecological view of free energy (see Bruineberg and Rietveld, 2014; Bruineberg et al., $2018 \mathrm{a}, \mathrm{b}$ as it aligns well with what I describe as world-brain relation (see above).

\section{Free Energy II-Generative Model and Variational Density}

How can the organism and its brain access and modulate free energy relative to their respective environmental context? Friston assumes that the organism/brain's internal dynamics can be characterized by two features, that is, generative model and variational density. In a nutshell, the generative model describes the probability of co-occurrence between the brain's 
internal states and the environmental context's external state. Importantly, the generative model does not amount to a neuronal representation of the world within the brain itself. Instead, the generative model refers to the long-term stochastic regularities in the relationship between world/environmental context and organism/brain (see Bruineberg et al., 2018a who emphasize this point).

Since the generative model does not provide a neuronal representation of the world, it can not be thought of as a model of the world that the organism and its brain create within themselves. Instead, the organism and its brain are by themselves a model of the world and, more specifically, "being a model of their econiche" (Bruineberg and Rietveld, 2014). The concept of generative model describes the organism's eco-niche within the world by free energy - this entails a neuro-ecological rather than purely neuronal view of generative model. Based on such neuroecological understanding of generative model, free energy can be thought as basic biological principle that provides the coupling or attunement between the organism/brain's internal dynamics and the world/environments' external dynamics.

How can the organism and its brain actively modulate their free energy as to conform to their respective environmental context by minimizing free energy? Friston takes variational density as proxy for probability distribution within the organism itself including its body (like temperature) and brain [e.g., its "perception/action," as Friston says, which is interpreted in terms of "readiness states" by Bruineberg and Rietveld, 2014 as it does not really imply actual (or real) but only possible (not yet realized) perception/action]. Variational density is encoded in the organism/brain's internal dynamics (see below for details of this point) whose probability distribution can be changed to minimize free energy in its relationship to the world/environmental context.

Variational density, reflecting the brain's internal dynamics, is, for instance, changed by anticipation. If the organism can, through its brain, anticipate the state of the world/environmental context, the free energy between world/environmental context and organism/brain is minimized and thus low. In that case, there is strong coupling and high attunement of world and brain - that is, for instance, the case when dancing to the rhythm of the music. If, in contrast, anticipation remains impossible, free energy is rather high. That is manifest in low coupling with less attunement of world and brain - in that case, one cannot get into the rhythm of the music.

\section{Free Energy III-Vantage Point From Beyond Brain}

Free energy featured by generative model and variational density can be understood as the basic biological principle that guides the relationship of world/environmental context and organism/brain, e.g., world-brain relation as I coined it above. Specifically, free energy can be understood as the biological mechanism that establishes relationship between world and brain. Taken in such a way, free energy must be understood as intrinsically neuro-ecological and biological rather than as purely neuronal and neuroscientific.
Such neuro-ecological view of the brain as part of the world with their relation established by free energy is only possible by presupposing a vantage point that allows to take into view that what happens beyond the boundaries of the brain. The here suggested neuro-ecological and biological view of free energy (see Bruineberg et al., 2018a,b) thus presupposes a post-Copernican vantage point from beyond brain. Like in the cases of earth and human species in astronomy and biology (see above), such post-Copernican vantage point from beyond brain radically changes our view of the brain in neuroscience.

The brain as an organ that aims to minimize free energy is no longer special when compared to other organs, i.e., non-brains, which, being biological adhere to the same principle. Nor does the brain stand in a dichotomous relationship to the world anymore as it is intimately coupled to the latter through free energy minimization. This specifies and presupposes what I described as world-brain relation. If, in contrast, one shifts towards a neuronal and neuroscientific concept of free energy, one's view remains restricted to the brain itself without taking into view that what happens beyond the brain, that is, how it is related and coupled to the world. This entails a pre-Copernican vantage point from within brain where the brain remains special and stands in a dichotomous relation to the world thus presupposing brain-world relation (rather than world-brain relation).

\section{PART IV: POST-COPERNICAN VIEW OF MENTAL FEATURES-TEMPORO-SPATIAL DYNAMIC AS "MISSING INGREDIENT" AND "COMMON CURRENCY"}

\section{Mental Features I-Biological and Neuro-Ecological}

I characterized the world-brain relation by free energy that allows for relating and coupling world and brain. This raises the question of how such world-brain relation, as based on free energy, stands in relation to mental features like consciousness, self, and affect. Applied in this sense, free energy provides a biological and neuro-ecological characterization of mental features (see Figure 3A).

Such biological characterization in terms of free energy has indeed been suggested by various authors for different mental features. Without going into details of the various approaches, I here just mention some (which neglects various others). One model of consciousness that takes the computational mechanisms of free energy as a starting point is the "projective consciousness model" (Rudrauf et al., 2017). Yet others have applied the free energy principles to dreams (Hobson et al., 2014). Moreover, free energy has even been assumed to provide an answer to the hard problem of consciousness (Solms, 2019), that is, why is there consciousness rather than non-consciousness (Chalmers, 1996).

Yet another mental feature where free energy has been intensively applied is the self. Bodily approaches to the self, e.g., somatic self, extensively rely on free energy 

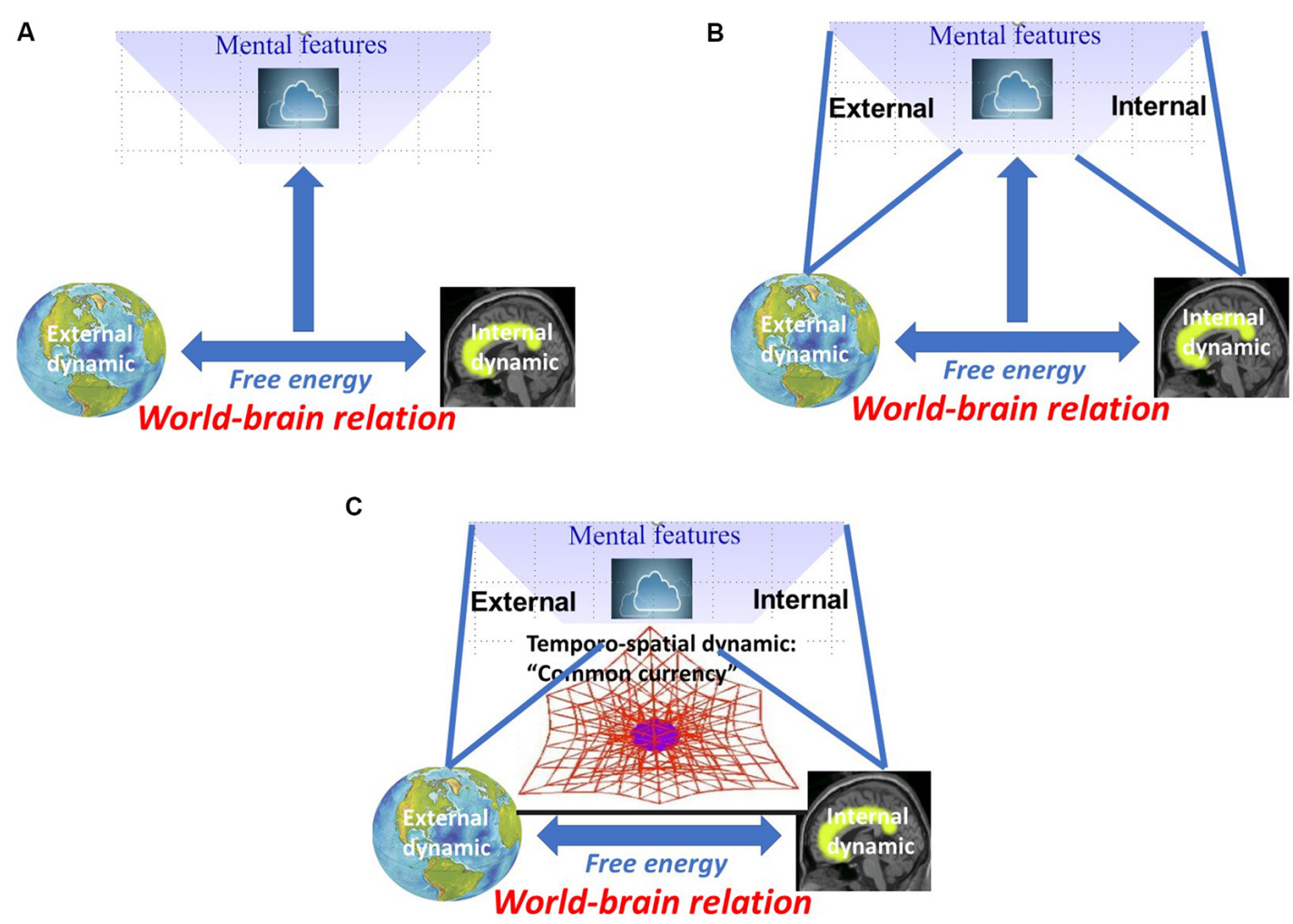

FIGURE 3 | (A) Neuro-biological characterization of mental features by free energy between world and brain. (B) Neuro-ecological characterization of mental features by internal-external relation. (C) Temporo-spatial dynamic as "common currency" of world-brain relation, free energy, and mental features.

(Seth and Tsakiris, 2018). More generally, the self as such, e.g., as distinguished from non-self, has been associated with free energy by Friston himself (Friston, 2018). Yet other recent approaches to the self like the dynamic pattern theory of self (Gallagher and Daly, 2018) and the subjective self ("I" vs. "me") strongly rely on free energy.

Finally, affect and emotion have also been related to free energy. One major proponent of such approach is Solms (2017, 2018, 2019). He associates the subcortical regions of the brain, as relying on Panksepp (1998a,b) and Damasio (2018), with affect and especially its subjective first-experiential features as paradigmatic and most basic manifestation of consciousness. Affective and its subjective feature are, in turn, assumed to be closely linked to free energy minimization with the environment (Solms, 2017, 2018, 2019). Hence, Solms links free energy to affect and consciousness in a unique way. Yet another proponent is Seth who develops an embodied theory of the free energy-based concept of active inference and how that relates to emotions (Seth and Friston, 2016).

Taken together, free energy is conceived central for mental features including consciousness, self, and affect (and others not mentioned here). If free energy characterizing the relation between world/environmental context and organism/brain is indeed central for mental features, one would expect the latter to be neither purely external, e.g., ecological, nor purely internal, e.g., neuronal, either. Instead, one would then assume mental features to be intrinsically neuro-ecological rather than merely neuronal.

\section{Mental Features II-Internal-External Relation}

Free energy regulating the relation of world/environmental context and organism/brain relates the former's external dynamics and the latter's external dynamics. If mental features do indeed depend upon free energy, one would expect them to signify different forms of internal-external relations (thus reflecting their neuro-ecological rather than neuronal characterization). This raises the question of how we can characterize the relationship between internal and external dynamics on both biological and mental levels.

On the biological level, the internal dynamics of the organism/brain is coupled to the external dynamics of the world/environmental context. Both share mutual information which is manifested in what Friston (2010) describes as "generalized synchrony" (as distinguished from representation). Generalized synchrony refers to the "coupled dynamics" between two systems who synchronize their different time scales with each other like the Huygens clocks where two clocks synchronize their time scales with each other over time-such synchronization can then be conceived as manifestation of free energy minimization (Bruineberg et al., 2018a). 
The same kind of synchronization now happens, analogously, in the relationship between world/environmental context and organism/brain when they couple with each other. For instance, when we tap our foot in the rhythm of the music, our brain and its internal dynamic entrain to the external dynamic of the latter. Neuronal investigation show, for instance, the brain synchronizes its phase onsets with those of continuous external stimuli (Lakatos et al., 2013) which seems to be disrupted in schizophrenia (Lakatos et al., 2013). One can thus see how, on the biological level, free energy allows for establishing synchronization between external and internal dynamics of world/environmental context and organism/brain.

We now assume that such synchronization between internal and external dynamics is also central for establishing relation between internal and external contents in mental features, i.e., internal-external relation. For instance, Honey et al. (2017) recently showed how perception, memory, and others can be characterized by different forms of internal-external relations. Yet another example is consciousness. Inner time consciousness, for instance, can be characterized by the relation between the subject's own "inner time speed" and how it perceives "outer time speed" (Fuchs, 2013). Usually, inner and outer time speed are somewhat in synchrony in our consciousness with both mutually adjusting and coupling to each other (Fuchs, 2013; see Figure 3B).

However, they can also differ and thus be non-adjusted. That is, for instance, the case in psychiatric conditions like depression and mania. In the case of depression, inner time speed in consciousness is too slow while the subjects perceive outer time speed, e.g., the time speed in the world, as too fast (Northoff, 2018). While the reverse happens in mania where subjects' inner time speed is fast (as manifested in fast action and psychomotor agitation) while they perceive outer time speed, relative to their abnormally fast inner time, as too slow-they thus become impatient (Northoff, 2018). Accordingly, as exemplified by our example of inner and outer time speed, consciousness can be characterized by specific relation of internal and external dynamic in our subjective experience, i.e., internal-external relation.

The same holds for other mental features like self and affect. The self is based on relating external stimuli and objects in the environment to the internal dynamics-this has been described as self-related processing (Northoff et al., 2007; Northoff, 2011, 2016a,b,c,d). For instance, even our own name is nothing but a collection of syllables which need to be put together by our brain to shape what we call our own name-that is possible only by processing the syllables more strongly related to the own brain and its internal dynamics than those of another person's name. Accordingly, what we describe as self-related (like our own name) reflects a certain constellation between the brain's internal dynamics, e.g., its spontaneous activity and the environment's external dynamics, e.g., the syllables. If that specific internalexternal relation is disrupted, as in schizophrenia, we may lose our sense of self (Northoff and Duncan, 2016). The self can thus be featured as relational and neuro-ecological (Northoff, 2016a,b,c,d).
Yet another example of internal-external relation shaping the self is transcultural differences. It has been well established that the self is constructed in a more inter-dependent, e.g., social way, in far eastern cultures (Markus and Kitayama, 1991; Han and Northoff, 2008). In contrast, the self is constructed in a more independent, e.g., isolated way in western culture. Most interestingly, such difference between inter- and in-dependent self is accompanied by neuronal differences (Han and Northoff, 2008; Han et al., 2013). Hence, the self in different cultures is neither purely internal nor exclusively external but is constituted by different degrees or balances of internal-external relation.

\section{Temporo-Spatial Dynamic I-World-Brain Relation and Free Energy}

One may now raise the question of why and how the coupling of world and brain, the world-brain relation, can give rise to mental features. This question focuses especially on the subjective experience and its phenomenal features like qualia, intentionality, transparency, unity, et cetera (for details Northoff, 2014a,b), that characterize all mental features like consciousness, self, and affect. In order for the world-brain relation and its free energy to yield mental features, both must share something that first and foremost makes possible the transformation of the former into the latter. This is the search for what we recently described as "common currency" (Northoff, 2019).

A "common currency" allows for exchange and mutual adaptation. Consider for instance the US dollar that provides the "common currency" between the different currencies in the global economy. By referring their own currency to the USdollar, different countries can exchange and trade goods. Goods can thus be transformed from one country to another country. We now assume that such "common currency" is also central in transforming the neuro-ecological activity of world-brain relation, e.g., free energy, into mental features like consciousness, self, and affect (for details, see Northoff, 2019).

What is the "common currency" of world-brain relation and its characterization by free energy on the one hand mental features on the other? To address this question, we first have to briefly address the notion of time and space and, secondly, how they characterize both worldbrain relation and free energy. Note that the conceptions of time and space are here not understood in the way we perceive or cognize time and space in terms of discrete points in time and space (see for instance, Buzsáki and Llinás, 2017; Drayton and Furman, 2018). Instead, we rather refer to time and space in a dynamic sense as in dynamical system theories where time and space are rather described in terms of attractors and trajectories (Cocchi et al., 2017) this amount to what we refer to as "temporospatial dynamic" (see also Northoff and Huang, 2017; Northoff, 2019).

Let us be more concrete. The brain constructs such dynamics in terms of different frequencies with oscillations and fluctuations (Buiszaki, 2006) that show a specific temporal structure with long range temporal correlations (LRTC) and scale-free activity (Linkenkaer-Hansen et al., 2001; He et al., 2010). As they connect different points in time by operating 
across different temporal scales, LRTC can be conceived as an example of temporal relation. Specifically, LRTCs and scale-free activity reflect the relationship between different frequencies and thus model different points in time relative to each other (see Northoff, 2019 as well as Northoff and Huang, 2017 for more details on the brain's temporo-spatial dynamic).

Importantly, the brain's construction is closely aligned to the way how it couples and relates to its respective environmental context, that is, in terms of free energy. This is reflected in the fact that the assumption of space and time takes also center stage in formulations in the free energy principle. That follows because the variational free energy is defined in terms of a generative model (see above) and the generative model includes information about the relation of world and brain, e.g., their degree of stochastic matching or convergence (Friston et al., 2006). This leads to the notion of deep temporal models that possess a necessary temporal thickness or depth (Seth and Friston, 2016). Together, albeit only hinted upon, both free energy and brain can be characterized by an elaborate temporospatial dynamic.

\section{Temporo-Spatial Dynamics II "Missing Ingredient" and "Common Currency" of Neuronal and Mental Features}

Mental features can also be characterized by time and space, that is, what phenomenological accounts describe as "spatiotemporality." Consciousness, for instance, can be characterized by a "stream" and the inclusion of presentation, prospection, and retrospection (James, 1890a,b; Husserl, 1921) this has been subsumed under the umbrella of "inner time consciousness" (Husserl, 1921; Fuchs, 2013; Northoff, 2014a,b). The same holds analogously for space (Ferri et al., 2015); for that reason, philosophers characterize consciousness by "spatiotemporality" (James, 1890a,b; Husserl, 1921; Zahavi, 2005; Fuchs, 2013).

Recent modeling further supports such view by showing that the "spatiotemporality" of mental features can be understood in dynamical terms, that is by temporo-spatial dynamics in terms of virtual trajectories in what has been described as "phenomenal space" (Prentner, 2019). Such dynamic temporo-spatial view of mental features like consciousness and self (for the latter, see Wolff et al., 2019) thus replaces the non-temporal view of mental features in traditional philosophy and the more recent static temporal approach to mental features in terms of perception and cognition.

We are now ready to address the quest for the "common currency." Albeit tentatively and laid out in more detail elsewhere (Northoff, 2014a,b, 2018; Northoff and Huang, 2017; Northoff, 2019), we assume that temporo-spatial dynamical features provide the link between neuro-ecological and mental levels-the former's temporo-spatial dynamic is thus supposed to be manifest in the latter's "spatiotemporality." We, therefore, suppose that temporo-spatial dynamic may be a good candidate to provide the "missing ingredient" (Lamme, 2018) and "common currency" (Northoff, 2019) of world-brain relation, free energy, and mental features (see Figure 3C).

How can we lend more concrete empirical support to the assumption of temporo-spatial dynamics providing the "common currency" of neuronal and mental features? This has recently been put into more specific terms when, for instance, assuming that the scale-free activity of the brain's spontaneous activity transforms into more or less analogous scale-freeness with the integration of different time scales on the psychological level of consciousness, e.g., its arousal or level/state (Tagliazucchi et al., 2013, 2016; Northoff, 2017; Cavanna et al., 2018). Analogously, recent studies demonstrated that the self is also mediated by temporo-spatial features of the spontaneous activity like scale-free activity, autocorrelation window and crossfrequency coupling which may be in analogous way manifest on the psychological level (Huang et al., 2016; Wolff et al., 2019). The different affects as described by Panksepp (1998a,b) and Solms $(2017,2018,2019)$ may then also be described by different forms of spatial and temporal coordinates in their subjective experience.

Yet another instance where temporo-spatial features transform from the neuronal to the mental level are psychiatric disorders like autism (Damiani et al., 2019) and bipolar disorder (Martino et al., 2016, 2018) where recently "Spatiotemporal Psychopathology" (Northoff, 2016a,b,c,d, 2017, 2018; Fingelkurts and Fingelkurts, 2019) has been proposed. This is supported by data on consciousness, self, and bipolar disorder (for details, see Northoff, 2019).

For instance, the above mentioned inner and outer time speed changes in the consciousness of depressed and manic patients are related to corresponding time speed changes in the neuronal activity in those networks mediating inner and outer time experience/perception (Northoff, 2018). Together, these examples support the view that temporo-spatial dynamics provides the "common currency" of neuronal and mental features in both healthy and pathological states.

\section{Temporo-Spatial Dynamics III-Post-Copernican Approach to Mental Features}

Why do we require a novel methodological strategy for postulating temporo-spatial dynamics as "common currency" of neuronal and mental features? I assume that this is only possible by presupposing a post-Copernican approach with a vantage point from beyond brain. Let me sketch that briefly.

The concept of "common currency" provides the necessary (rather than contingent) connection of neuronal and mental features that so far remained elusive to us. We simply do not know how neuronal and mental features are intrinsically linked to each other-we miss something, the "missing ingredient" (Lamme, 2018). I suggest in this article that the lack of insight into the necessary connection and thus the "missing ingredient" is due, at least in part, to our pre-Copernican methodological strategy.

Specifically, our currently pre-Copernican ego-centric vantage point from within brain (or within body, information, 
or cognition; see above) prevents us from taking into view that what happens beyond the brain in world (and body) and how that shapes the brain's neuronal activity in such that it is intrinsically and thus necessarily connected to mental features. We consequently assume mental features to be special as related to specific neuronal mechanisms (like the NCC) as distinguished from those underlying others, e.g., non-mental features. This renders impossible to take into view the necessary connection of neuronal and mental features. Moreover, that puts mental features in a dichotomous relation to the world and its non-mental features.

That changes once one shifts the pre-Copernican vantage point from within brain to a post-Copernican vantage point from beyond brain. The vantage point from beyond brain allows taking into view that what happens beyond the boundaries of the brain in the world, e.g., world-brain relation as featured by free energy, and how that, e.g., its temporo-spatial dynamics, shapes and yields mental features with their own spatiotemporality. Being a viable candidate to provide the "common currency," temporospatial dynamics establishes intrinsic and thus necessary connection of neuronal and mental features.

Most importantly, mental features are then no longer conceived as special (when compared to non-mental features) but rather non-special. Moreover, being temporo-spatial, mental features no longer stand in dichotomous relationship to the world but in a "temporo-spatial continuum" this specifies and explicates what, in more general terms, has been described as "embeddedness/enactivism" or "deep continuity of mind and life" (Thompson, 2007; Clark, 2013). Most important, such view of mental features resembles very much our current post-Copernican views of both earth and human species including their continuous relationship to universe and evolution that were established by Copernicus and Darwin.

\section{CONCLUSION}

I here propose a novel methodological strategy on how to approach brain and mental features. Relying on Copernicus and Darwin, I advocate changing our currently rather pre-Copernican vantage from within brain to a post-Copernican vantage point from beyond brain. This allows us taking into view that what happens beyond the boundaries of our brain, e.g., in world and body as described in the

\section{REFERENCES}

Aru, J., Bachmann, T., Singer, W., and Melloni, L. (2012). Distilling the neural correlates of consciousness. Neurosci. Biobehav. Rev. 36, 737-746. doi: 10.1016/j.neubiorev.2011.12.003

Blanke, O., Slater, M., and Serino, A. (2015). Behavioral, neural, and computational principles of bodily self-consciousness. Neuron 88, 145-166. doi: 10.1016/j. neuron.2015.09.029

Bruineberg, J., Kiverstein, J., and Rietveld, E. (2018a). The anticipating brain is not a scientist: the free-energy principle from an ecological-enactive perspective. Synthese 195, 2417-2444. doi: 10.1007/s11229-016-1239-1

Bruineberg, J., Rietveld, E., Parr, T., van Maanen, L., and Friston, K. J. (2018b). Free-energy minimization in joint agent-environment systems: a niche concept of world-brain relation, and how that shapes the brain in such way that it can yield mental features. Relying on Friston, I characterize such world-brain relation in a biological way by the free-energy principle. That renders the brain as intrinsically neuro-ecological rather than merely neuronal.

Such post-Copernican vantage point from beyond brain allows us taking into view that both free energy of world-brain relation and the brain itself are characterized by temporospatial dynamics. Based on empirical evidence, such temporospatial dynamic is also manifest in mental features, e.g., their spatiotemporality. I therefore conclude that a post-Copernican approach to the brain, e.g., a vantage point from beyond brain, allows us taking into view temporo-spatial dynamics as a viable candidate of the so far elusive "missing ingredient" and "common currency" of neuronal (or better neuro-ecological) and mental features (Northoff, 2019).

I conclude that neuroscience may benefit from a shift in its vantage point from within brain to beyond brain in its methodological approach to mental features. That amounts to nothing less than a Copernican turn or revolution in neuroscience akin to the ones in both astronomy and biology. Like in the latter disciplines, such methodological shift can, as I propose, provide a novel framework for neuroscience that will turn its search for the neural basis of mental features into a true and major success story at the beginning of the 21 st century.

\section{AUTHOR CONTRIBUTIONS}

GN being the sole author, contributed all parts of the article himself.

\section{FUNDING}

This project/research has received funding from the European Union's Horizon 2020 Framework Programme for Research and Innovation under the Specific Grant Agreement No. 785907 (Human Brain Project SGA2). GN is grateful for funding provided by UMRF (University Medical Research Funds), uOBMRI (University of Ottawa Brain and Mind Research Institute), CIHR (Canadian Institute of Health Research), and PSI (Physician Service Incorporated Foundation).

construction perspective. J. Theor. Biol. 455, 161-178. doi: 10.1016/j.jtbi.2018. 07.002

Bruineberg, J., and Rietveld, E. (2014). Self-organization, free energy minimization, and optimal grip on a field of affordances. Front. Hum. Neurosci. 8:599. doi: 10.3389/fnhum.2014.00599

Buiszaki, G. (2006). Rhythms in the Brain. Oxford, New York: Oxford University Press.

Buzsáki, G., and Llinás, R. (2017). Space and time in the brain. Science 358, 482-485. doi: 10.1126/science.aan8869

Cavanna, F., Vilas, M. G., Palmucci, M., and Tagliazucchi, E. (2018). Dynamic functional connectivity and brain metastability during altered states of consciousness. Neuroimage 180, 383-395. doi: 10.1016/j.neuroimage.2017. 09.065 
Chalmers, D. (1996). The Conscious Mind. Oxford, New York: Oxford University Press.

Chalmers, D. (Eds). (2001). The Neural Correlates of Consciousness. Oxford, New York: Oxford University Press.

Churchland, P. S. (2002). Brain-Wise. An Introduction to Neurophilosophy. Cambridge, MA: MIT Press.

Clark, A. (2013). Whatever next? Predictive brains, situated agents, and the future of cognitive science. Behav. Brain Sci. 36, 181-204. doi: 10.1017/s0140525x12 000477

Cocchi, L., Gollo, L. L., Zalesky, A., and Breakspear, M. (2017). Criticality in the brain: a synthesis of neurobiology, models and cognition. Prog. Neurobiol. 158, 132-152. doi: 10.1016/j.pneurobio.2017.07.002

Crick, F., and Koch, C. (1990). Some reflections on visual awareness. Cold Spring Harb. Symp. Quant. Biol. 55, 953-962. doi: 10.1101/sqb.1990.055. 01.089

Crick, F., and Koch, C. (2003). A framework for consciousness. Nat. Neurosci. 6, 119-126. doi: 10.1038/nn0203-119

Damiani, S., Scalabrini, A., Gomez-Pilar, J., Brondino, N., and Northoff, G. (2019). Increased scale-free dynamics in salience network in adult high-functioning autism. Neuroimage Clin. 21:101634. doi: 10.1016/j.nicl.2018.101634

Damasio, A. (2018). The Strange Order of Things: Life, Feeling, and the Making of Cultures. (New York: Deckle Edge).

Dehaene, S., Charles, L., King, J. R., and Marti, S. (2014). Toward a computational theory of conscious processing. Curr. Opin. Neurobiol. 25, 76-84. doi: 10.1016/j. conb.2013.12.005

Dehaene, S., Lau, H., and Kouider, S. (2017). What is consciousness and could machines have it? Science 358, 486-492. doi: 10.1126/science.aan8871

de Graaf, T. A., Hsieh, P. J., and Sack, A. T. (2012). The 'correlates' in neural correlates of consciousness. Neurosci. Biobehav. Rev. 36, 191-197. doi: 10.1016/j.neubiorev.2011.05.012

Drayton, L., and Furman, M. (2018). Thy mind, thy brain and time. Trends Neurosci. 41, 641-643. doi: 10.1016/j.tins.2018.09.001

Duncan, N. W., Hayes, D. J., Wiebking, C., Tiret, B., Pietruska, K., Chen, D. Q., et al. (2015). Negative childhood experiences alter a prefrontal-insular-motor cortical network in healthy adults: a preliminary multimodal rsfMRI-fMRIMRS-dMRI study. Hum. Brain Mapp. 36, 4622-4637. doi: 10.1002/hbm. 22941

Ferri, F., Costantini, M., Huang, Z., Perrucci, M. G., Ferretti, A., Romani, G. L., et al. (2015). Intertrial variability in the premotor cortex accounts for individual differences in peripersonal space. J. Neurosci. 35, 16328-16339. doi: 10.1523/JNEUROSCI.1696-15.2015

Fingelkurts, A. A., and Fingelkurts, A. A. (2019). Brain space and time in mental disorders: paradigm shift in biological psychiatry. Int. J. Psychiatry Med. 54, 53-63. doi: 10.1177/0091217418791438

Friston, K. (2010). The free-energy principle: a unified brain theory? Nat. Rev. Neurosci. 11, 127-138. doi: 10.1038/nrn2787

Friston, K. (2018). Am I self-conscious? (or does self-organization entail selfconsciousness?). Front. Psychol. 9:579. doi: 10.3389/fpsyg.2018.00579

Friston, K., Kilner, J., and Harrison, L. (2006). A free energy principle for the brain. J. Physiol. Paris 100, 70-87. doi: 10.1016/j.jphysparis.2006.10.001

Friston, K. J., and Stephan, K. E. (2007). Free-energy and the brain. Synthese 159, 417-458. doi: 10.1007/s11229-007-9237-y

Fuchs, T. (2013). Temporality and psychopathology. Phenomenol. Cogn. Sci. 12, 75-104. doi: 10.1007/s11097-010-9189-4

Gallagher, S. (2005). How the Body Shapes the Mind. Oxford, New York: Oxford University Press.

Gallagher, S., and Daly, A. (2018). Dynamical relations in the self-pattern. Front. Psychol. 9:664. doi: 10.3389/fpsyg.2018.00664

Han, S., Northoff, G., Vogeley, K., Wexler, B. E., Kitayama, S., and Varnum, M. E. (2013). A cultural neuroscience approach to the biosocial nature of the human brain. Annu. Rev. Psychol. 64, 335-359. doi: 10.1146/annurev-psych-071112054629

Han, S., and Northoff, G. (2008). Culture-sensitive neural substrates of human cognition: a transcultural neuroimaging approach. Nat. Rev. Neurosci. 9, 646-654. doi: 10.1038/nrn2456

He, B. J., Zempel, J. M., Snyder, A. Z., and Raichle, M. E. (2010). The temporal structures and functional significance of scale-free brain activity. Neuron 66, 353-369. doi: 10.1016/j.neuron.2010.04.020
Hobson, J. A., Hong, C. C., and Friston, K. J. (2014). Virtual reality and consciousness inference in dreaming. Front. Psychol. 5:1133. doi: 10.3389/fpsyg. 2014.01133

Hohwy, J. (2013). The Predictive Mind. New York, NY: Oxford University Press.

Honey, C. J., Newman, E. L., and Schapiro, A. C. (2017). Switching between internal and external modes: a multiscale learning principle. Netw. Neurosci. 1,339-356. doi: 10.1162/netn_a_00024

Hu, C., Di, X., Eickhoff, S. B., Zhang, M., Peng, K., Guo, H., et al. (2016). Distinct and common aspects of physical and psychological self-representation in the brain: a meta-analysis of self-bias in facial and self-referential judgements. Neurosci. Biobehav. Rev. 61, 197-207. doi: 10.1016/j.neubiorev.2015.12.003

Huang, Z., Zhang, J., Wu, J., Qin, P., Wu, X., Wang, Z., et al. (2016). Decoupled temporal variability and signal synchronization of spontaneous brain activity in loss of consciousness: An fMRI study in anesthesia. Neuroimage 124, 693-703. doi: 10.1016/j.neuroimage.2015.08.062

Husserl, E. (1921). Inner Time Consciousness. Amsterdam: Nijhaus Publisher.

James, W. (1890a). The Principles of Psychology: Vol. 1. London: MacMillan.

James, W. (1890b). The Principles of Psychology: Vol. 2. London: MacMillan.

Kant, I. (1781/1998). Critique of Pure Reason (trans. and eds by P. Guyer \& A.W. Wood). Cambridge, UK: Cambridge University Press.

Koch, C. (2004). The Quest for Consciousness. Oxford, New York: Oxford University Press.

Koch, C., Massimini, M., Boly, M., and Tononi, G. (2016). Neural correlates of consciousness: progress and problems. Nat. Rev. Neurosci. 17, 307-321. doi: $10.1038 / \mathrm{nrn} .2016 .22$

Lakatos, P., Schroeder, C. E., Leitman, D. I., and Javitt, D. C. (2013). Predictive suppression of cortical excitability and its deficit in schizophrenia. J. Neurosci. 33, 11692-11702. doi: 10.1523/JNEUROSCI.0010-13.2013

Lamme, V. A. F. (2018). Challenges for theories of consciousness: seeing or knowing, the missing ingredient and how to deal with panpsychism. Philos. Trans. R. Soc. Lond. B Biol. Sci. 373:20170344. doi: 10.1098/rstb.2017.0344

Lau, H., and Rosenthal, D. (2011). Empirical support for higher-order theories of conscious awareness. Trends Cogn. Sci. 15, 365-373. doi: 10.1016/j.tics.2011. 05.009

Levine, J. (1983). Materialism and qualia: The explanatory gap. Pacific Philosophical Quarterly 64, 354-361. doi: 10.1111/j.1468-0114.1983.tb00207.x

Linkenkaer-Hansen, K., Nikouline, V. V., Palva, J. M., and Ilmoniemi, R. J. (2001). Long-range temporal correlations and scaling behavior in human brain oscillations. J. Neurosci. 21, 1370-1377. doi: 10.1523/JNEUROSCI.21-0401370.2001

Markus, H. R., and Kitayama, S. (1991). Culture and the self: implications for cognition, emotion, and motivation. Psychol. Rev. 98, 224-253. doi: 10.1037/0033-295X.98.2.224

Martino, M., Magioncalda, P., Huang, Z., Conio, B., Piaggio, N., Duncan, N. W., et al. (2016). Contrasting variability patterns in the default mode and sensorimotor networks balance in bipolar depression and mania. Proc. Natl. Acad. Sci. U S A 113, 4824-4829. doi: 10.1073/pnas.1517558113

Martino, M., Magioncalda, P., Yu, H., Li, X., Wang, Q., Meng, Y., et al. (2018). Abnormal resting-state connectivity in a substantia nigra-related striato-thalamo-cortical network in a large sample of first-episode drug-naïve patients with schizophrenia. Schizophr. Bull. 44, 419-431. doi: 10.1093/schbul/ sbx067

McDowell, J. (1994). Mind and World. Cambridge, MA: Harvard University Press.

Nakao, T., Bai, Y., Nashiwa, H., and Northoff, G. (2013). Resting-state EEG power predicts conflict-related brain activity in internally guided but not in externally guided decision-making. Neuroimage 66, 9-21. doi: 10.1016/j.neuroimage. 2012.10.034

Noe, A. (2004). Action in Perception. Cambridge, MA: MIT Press.

Northoff, G. (2011). Self and brain: what is self-related processing? Trends Cogn. Sci. 15, 186-187; author reply 187-188. doi: 10.1016/j.tics.2011.03.001

Northoff, G. (2013). What the brain's intrinsic activity can tell us about consciousness? A tri-dimensional view. Neurosci. Biobehav. Rev. 37, 726-738. doi: 10.1016/j.neubiorev.2012.12.004

Northoff, G. (2014a). Unlocking the Brain. Volume I: Coding. New York, NY: Oxford University Press.

Northoff, G. (2014b). Unlocking the Brain. Volume II: Consciousness. Oxford: Oxford University. 
Northoff, G. (2016a). Neurophilosophy and the Healthy Mind. Learning from the Unwell Brain. New York, NY: Norton Publisher.

Northoff, G. (2016b). Neuroscience and Whitehead: Neuro-Ecological Model of Brain. New York, NY: Axiomathes.

Northoff, G. (2016c). Spatiotemporal psychopathology I: no rest for the brain's resting state activity in depression? Spatiotemporal psychopathology of depressive symptoms. J. Affect. Disord. 190, 854-866. doi: 10.1016/j.jad.2015. 05.007

Northoff, G. (2016d). Spatiotemporal Psychopathology II: how does a psychopathology of the brain's resting state look like? Spatiotemporal approach and the history of psychopathology. J. Affect. Disord. 190, 867-879. doi: 10.1016/j.jad.2015.05.008

Northoff, G. (2017). "Paradox of slow frequencies"-Are slow frequencies in upper cortical layers a neural predisposition of the level/state of consciousness (NPC)? Conscious. Cogn. 54, 20-35. doi: 10.1016/j.concog.2017.03.006

Northoff, G. (2018). The brain's spontaneous activity and its psychopathological symptoms-"Spatiotemporal binding and integration". Prog. Neuropsychopharmacol. Biol. Psychiatry 80, 81-90. doi: 10.1016/j.pnpbp. 2017.03.019

Northoff, G. (2019). The anxious brain and its heart-temporal brain-heart de-synchronization in anxiety disorders. J. Affect. Disord.

Northoff, G., and Duncan, N. W. (2016). How do abnormalities in the brain's spontaneous activity translate into symptoms in schizophrenia? From an overview of resting state activity findings to a proposed spatiotemporal psychopathology. Prog. Neurobiol. 145-146, 26-45. doi: 10.1016/j.pneurobio. 2016.08.003

Northoff, G., Bermpohl, F., Schoeneich, F., and Boeker, H. (2007). How does our brain constitute defense mechanisms? First-person neuroscience and psychoanalysis. Psychother. Psychosom. 76, 141-153. doi: 10.1159/0000 99841

Northoff, G., and Huang, Z. (2017). How do the brain's time and space mediate consciousness and its different dimensions? Temporo-spatial theory of consciousness (TTC). Neurosci. Biobehav. Rev. 80, 630-645.

Panksepp, J. (1998a). Affective Neuroscience. Oxford, NY: Oxford University Press.

Panksepp, J. (1998b). The preiconscious substrates of consciousness: affective states and the evolutionary origins of the SELF. J. Conscious. Stud. 5, 566-582.

Prentner, R. (2019). Consciousness and topologically structured phenomenal spaces. Conscious. Cogn. 70, 25-38. doi: 10.1016/j.concog.2019. 02.002

Rudrauf, D., Bennequin, D., Granic, I., Landini, G., Friston, K., and Williford, K. (2017). A mathematical model of embodied consciousness. J. Theor. Biol. 428, 106-131. doi: 10.1016/j.jtbi.2017.05.032

Ruse, M. (2009). The Darwinian revolution: rethinking its meaning and significance. Proc. Natl. Acad. Sci. U S A 106, 10040-10047. doi: 10.1073/pnas. 0901011106

Seth, A. K., and Friston, K. J. (2016). Active interoceptive inference and the emotional brain. Philos. Trans. R. Soc. Lond. B Biol. Sci. 371:20160007. doi: $10.1098 /$ rstb.2016.0007

Seth, A. K., and Tsakiris, M. (2018). Being a beast machine: the somatic basis of selfhood. Trends Cogn. Sci. 22, 969-981. doi: 10.1016/j.tics.2018.08.008

Sherburne, D. W. (1983). Whitehead, categories, and the completion of the copernican revolution. Monist 66, 367-386. doi: 10.5840/monist198366319
Solms, M. (2017). What is "the unconscious," and where is it located in the brain? A neuropsychoanalytic perspective. Ann. N Y Acad. Sci. 1406, 90-97. doi: $10.1111 /$ nyas. 13437

Solms, M. L. (2018). The neurobiological underpinnings of psychoanalytic theory and therapy. Front. Behav. Neurosci. 12:294. doi: 10.3389/fnbeh.2018. 00294

Solms, M. (2019). The hard problem of consciousness and the free energy principle. Front. Psychol. 9:2714. doi: 10.3389/fpsyg.2018.02714

Sui, J., and Humphreys, G. W. (2015). The integrative self: how self-reference integrates perception and memory. Trends Cogn. Sci. 19, 719-728. doi: 10.1016/j.tics.2015.08.015

Tagliazucchi, E., von Wegner, F., Morzelewski, A., Brodbeck, V., Jahnke, K., and Laufs, H. (2013). Breakdown of long-range temporal dependence in default mode and attention networks during deep sleep. Proc. Natl. Acad. Sci. U S A 110, 15419-15424. doi: 10.1073/pnas.1312848110

Tagliazucchi, E., Crossley, N., Bullmore, E. T., and Laufs, H. (2016). Deep sleep divides the cortex into opposite modes of anatomical-functional coupling. Brain Struct. Funct. 221, 4221-4234. doi: 10.1007/s00429-015-1162-0

Tallon-Baudry, C., Campana, F., Park, H.-D., and Babo-Rebelo, M. (2018). The neural monitoring of visceral inputs, rather than attention, accounts for first-person perspective in conscious vision. Cortex 102, 139-149. doi: 10.1016/j.cortex.2017.05.019

Thompson, E. (2007). Mind in Life. Cambridge, MA: MIT Press.

Tononi, G., and Koch, C. (2015). Consciousness: here, there and everywhere? Philos. Trans. R. Soc. Lond. B Biol. Sci. 370:20140167. doi: 10.1098/rstb.2014. 0167

Tononi, G., Boly, M., Massimini, M., and Koch, C. (2016). Integrated information theory: from consciousness to its physical substrate. Nat. Rev. Neurosci. 17, 450-461. doi: 10.1038/nrn.2016.44

van Atteveldt, N., Musacchia, G., Zion-Golumbic, E., Sehatpour, P., Javitt, D. C., and Schroeder, C. (2015). Complementary fMRI and EEG evidence for more efficient neural processing of rhythmic vs. unpredictably timed sounds. Front. Psychol. 6:1663. doi: 10.3389/fpsyg.2015.01663

Weinert, F. (2013). The March of Time. Heidelberg, New York, NY: Springer Publisher.

Whitehead, A. N. (1929/1978). Process and Reality: An Essay in Cosmology. D. R. Griffin and D. W. Sherburne (Eds). New York, NY: The Free Press.

Wolff, A., Di Giovanni, D. A., Gómez-Pilar, J., Nakao, T., Huang, Z., Longtin, A., et al. (2019). The temporal signature of self: temporal measures of restingstate EEG predict self-consciousness. Hum. Brain Mapp. 40, 789-803. doi: $10.1002 / \mathrm{hbm} .24412$

Zahavi, D. (2005). Subjectivity and Selfhood. Camrbidge, MA: MIT Press.

Conflict of Interest Statement: The author declares that the research was conducted in the absence of any commercial or financial relationships that could be construed as a potential conflict of interest.

Copyright (c) 2019 Northoff. This is an open-access article distributed under the terms of the Creative Commons Attribution License (CC BY). The use, distribution or reproduction in other forums is permitted, provided the original author(s) and the copyright owner(s) are credited and that the original publication in this journal is cited, in accordance with accepted academic practice. No use, distribution or reproduction is permitted which does not comply with these terms. 


\section{OPEN ACCESS}

Edited by:

Roberto Esposito,

Azienda Ospedaliera Ospedali Riuniti

Marche Nord, Italy

Reviewed by:

Wenbin Guo,

The Second Xiangya Hospital, Central

South University, China

Antonio Ferretti,

Università degli Studi "G. d'Annunzio"

Chieti-Pescara, Italy

*Correspondence:

Alessandra Splendiani

alessandra.splendiani@univaq.it

Specialty section:

This article was submitted to

Brain Imaging Methods,

a section of the journal

Frontiers in Neuroscience

Received: 03 April 2019

Accepted: 13 September 2019

Published: 27 September 2019

Citation:

Pistoia F, Conson M,

Quarantelli M, Panebianco L,

Carolei A, Curcio G, Sacco S,

Saporito G, Di Cesare E, Barile A, Masciocchi $C$ and Splendiani A (2019) Neural Correlates of Facial Expression Recognition in Earthquake Witnesses.

Front. Neurosci. 13:1038.

doi: 10.3389/fnins.2019.01038

\section{Neural Correlates of Facial Expression Recognition in Earthquake Witnesses}

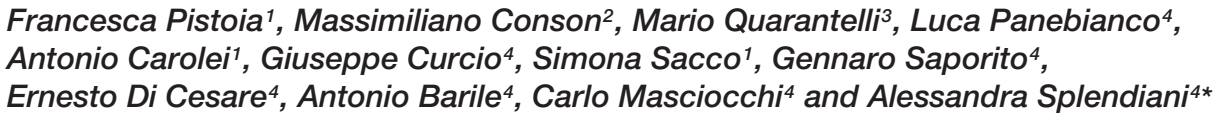

${ }^{1}$ Department of Biotechnological and Applied Clinical Sciences, Neurological Institute, University of L'Aquila, L'Aquila, Italy, ${ }^{2}$ Developmental Neuropsychology Laboratory, Department of Psychology, University of Campania Luigi Vanvitelli, Campania, Italy, ${ }^{3}$ Institute of Biostructure and Bioimaging, National Research Council, Naples, Italy, ${ }^{4}$ Department of Biotechnological and Applied Clinical Sciences, University of L'Aquila, L'Aquila, Italy

Major adverse events, like an earthquake, trigger different kinds of emotional dysfunctions or psychiatric disorders in the exposed subjects. Recent literature has also shown that exposure to natural disasters can increase threat detection. In particular, we previously found a selective enhancement in the ability to read emotional facial expressions in L'Aquila earthquake witnesses, suggesting hypervigilance to stimuli signaling a threat. In light of previous neuroimaging data showing that trauma exposure is related to derangement of resting-state brain activity, in the present study we investigated the neurofunctional changes related to the recognition of emotional faces in L'Aquila earthquake witnesses. Specifically, we tested the relationships between accuracy in recognizing facial expressions and activity of the visual network (VN) and of the default-mode network (DMN). Resting-state functional connectivity (FC) with the main hub of the VN (primary, ventral, right-dorsal, and left-dorsal visual cortices) and DMN (posterior cingulate/precuneus, medial prefrontal, and right and left inferior parietal cortices) was investigated through a seed-based functional magnetic resonance imaging (fMRI) analysis in both earthquake-exposed subjects and non-exposed persons who did not live in an earthquake-affected area. The results showed that, in earthquakeexposed subjects, there is a significant reduction in the correlation between accuracy in recognizing facial expressions and the $\mathrm{FC}$ of the dorsal seed of the $\mathrm{VN}$ with the right inferior occipito-temporal cortex and the left lateral temporal cortex, and of two parietal seeds of DMN, i.e., lower parietal and medial prefrontal cortex, with the precuneus bilaterally. These findings suggest that a functional modification of brain systems involved in detecting and interpreting emotional faces may represent the neurophysiological basis of the specific "emotional expertise" observed in the earthquake witnesses. 


\section{INTRODUCTION}

After a natural disaster, like an earthquake, people usually experience different kinds of emotional dysfunctions or disorders. Among post-earthquake psychiatric complications, the most frequently reported include post-traumatic stress disorder (PTSD), depression, anxiety, obsessive-compulsive disorders, and social phobia (Farooqui et al., 2017; Dube et al., 2018; Geng et al., 2018; Rafiey et al., 2019). The risk of sleep disorders and of prolonged grief symptoms also seems to be increased in earthquake-exposed subjects (Tang et al., 2018; Eisma et al., 2019). On the other hand, experiencing a natural disaster may trigger post-traumatic growth, which refers to positive personality changes following threatening life events and a higher level of functioning (Calhoun and Tedeschi, 2006). Therefore, the spectrum of trauma-related changes may encompass a wide range of manifestations, with subjects who have a poor coping ability being more likely to develop psychiatric disorders and subjects with greater adaptability being able to obtain advantages from adverse events. While the former experience a decreased quality of life after a natural disaster and often need specific psychological or pharmacological support, the latter may thrive (O'Leary and Ickovics, 1995). Independent of developing a clear psychopathological condition, such as PTSD, convergent evidence suggests that trauma exposure increases threat detection (Hayes et al., 2012; Zhang et al., 2014; Bell et al., 2017; Pistoia et al., 2018). In a relevant study on earthquakeexposed persons, Bell et al. (2017) demonstrated that both individuals who develop PTSD and individuals without PTSD are significantly more accurate than non-exposed controls in recognizing emotional facial expressions. The authors interpreted their results in terms of increased sensitivity to threat due to the prolonged exposure to aftershocks in the earthquake-exposed groups.

In this context, we recently reported on a specific emotional expertise developed by earthquake-exposed subjects without PTSD (Pistoia et al., 2018). In detail, we compared two groups of students, one with a permanent residence in the earthquake epicenter of L'Aquila (central Italy) on April 6, 2009, and one not living in an earthquake-affected area. Participants took part in two behavioral experiments aimed at evaluating their ability to recognize facial expressions and to evaluate emotionally evocative scenes. The results demonstrated that students living in the earthquake-affected areas were significantly more accurate than controls in recognizing facial expressions, whereas the two groups did not differ in the evaluation of emotionally evocative scenes. This enhanced recognition ability was not selective for specific emotions, at variance to what has previously been found in neurological patients (Mather and Carstensen, 2005; Pistoia et al., 2010). However, both positive (happiness, surprise) and negative (disgust, fear, anger, sadness) emotions were involved. These results were interpreted in terms of hypervigilance in respect of threats in earthquake witnesses, since trauma exposure, especially in an environment of ongoing threat, requires individuals to maintain their safety by systematically paying attention to potential signs of approaching threat, such as emotional facial expressions (Bell et al., 2017). This interpretation is even more convincing if we consider that the earthquake-exposed students living in L'Aquila experienced a long-lasting exposure to earthquakes with massive psychological distress, as the main event in 2009 was followed by hundreds of thousands of aftershocks in the months afterward and by additional earthquakes in 2016 and 2017.

Recently, Kleshchova et al. (2019) tested the hypothesis that since chronic hypervigilance is a persistent rather than a reactive state, brain correlates can be directly observable under restingstate conditions without the need for exposure to affectively charged stimuli. Results actually showed that, compared to no-trauma controls, trauma-exposed women showed greater connectivity between the amygdala and the cingulate cortex not only during affective processing but also at rest. Long et al. (2014) also demonstrated that testing functional brain changes using resting-state functional magnetic resonance imaging (rsfMRI) is a useful approach in classifying people who have survived an earthquake who develop psychological responses to trauma exposure. Furthermore, although earthquake witnesses, especially those without PTSD, may not have structural brain changes shortly after the trauma, functional brain changes have been demonstrated as occurring in as little as 1 month after an earthquake (Lui et al., 2009). Against this background, in the present study, we used rs-fMRI to investigate the neurofunctional basis of enhanced recognition of facial expressions in earthquake witnesses.

Data from different neurofunctional approaches demonstrate that the processing of facial expressions crucially involves central nodes of the visual network (VN), like the inferior occipitotemporal cortex, the fusiform and the lingual gyrus, and the lateral temporal cortex (Haxby et al., 2000; Gorno-Tempini et al., 2001; Ganel et al., 2005; Said et al., 2011). Several studies also support the role of the default-mode network (DMN) in interpreting emotional faces, with structures including the medial prefrontal and parietal cortices (Phillips et al., 1998; Harmer et al., 2001; Mattavelli et al., 2011, 2016); the DMN is especially relevant in conditions where explicit expression processing is required (Mattavelli et al., 2016; Zhao et al., 2017). Importantly, the involvement of the $\mathrm{VN}$ and the DMN has been demonstrated in trauma-exposed persons during processing of arousing stimuli (Fani et al., 2012; Todd et al., 2015).

By capitalizing on the above evidence, here we used a subsample of Pistoia et al.'s (2018) group of L'Aquila earthquake witnesses to test resting-state functional connectivity (FC) within the major hubs of the $\mathrm{VN}$ and the $\mathrm{DMN}$ and, crucially, to test FC as it relates to behavioral performance in facial expression recognition task (Pistoia et al., 2018). We hypothesized that enhanced recognition of facial expressions in earthquakeexposed persons could imply an altered pattern of FC between the seeds of the $\mathrm{VN}$ and $\mathrm{DMN}$ and brain regions related to detection and interpretation of emotional facial expressions, such as the inferior occipito-temporal cortex, the lateral temporal cortex, and the medial parietal cortex. 


\section{MATERIALS AND METHODS}

\section{Participants}

The original sample of the main neuropsychological study by Pistoia et al. (2018) included 107 students, 48 belonging to the experimental earthquake-exposed group with a permanent residence in L'Aquila at the time of the 2009 earthquake (20 males and 28 females, mean age $=22.6, S D=2.3$ years) and 59 belonging to the control group not living in an earthquake-affected area ( 30 males and 29 females, mean age $=23.1, S D=1.6$ years). The original inclusion criteria were: (i) no history of previous or coexistent neurological or psychiatric diseases including PTSD, as revealed by a psychiatric examination; (ii) no assumption of drugs or substances acting on the central nervous system; and (iii) signed informed consent to participate in the study.

Here, a subsample was selected from both the earthquakeexposed and the non-exposed group to perform the rs-fMRI study. MRI assessment was restricted to a subsample of subjects because neuroimaging assessment requires a longer time to organize and complete, and not all the subjects originally included were available at the time of the neurofunctional assessment. Following selection, 41 (38\%) subjects were included, 18 students belonging to the earthquake-exposed group (8 males and 10 females, mean age $=24.5, S D=1.8$ years) and 23 students belonging to the non-exposed control group (14 males and 9 females, mean age $=23.7, S D=2.0$ years); non-parametric between-group comparisons showed that the two groups did not differ with respect to both sex and age (both $p>0.05$ ).

The research protocol was approved by the Internal Review Board of the University of L'Aquila (January 2017). The study was conducted in accordance with the ethical standards of the Helsinki Declaration and signed informed consent was obtained from all the participants.

\section{Methods}

\section{Self-Report Measures}

All participants were assessed by means of a series of formalized self-report measures. The Beck Depression Inventory (BDI; Beck, 1967) is one of the most widely used self-report measures for the assessment of depression severity. The score can range from 0 to 63 , with higher scores indicating an increasing level of depressive symptoms. The score is usually taken as a dependent variable. The State-Trait Anxiety Inventory (STAI; Spielberger et al., 1983; Pedrabissi and Santiniello, 1989) is a commonly used measure of trait and state anxiety: here, only the 20 items for the assessment of trait anxiety were used. The score can range from 20 to 60; a high score reflects a high level of anxiety. This score was used as a dependent variable. The Insomnia Severity Index (ISI; Bastien et al., 2001; Castronovo et al., 2016) is a self-report questionnaire evaluating different dimensions of insomnia (sleep onset, sleep maintenance and early morning awakening problems, sleep dissatisfaction, interference with daytime functioning, noticeability of sleep problems by others, and distress caused by the sleep difficulties). The score ranges from 0 to 28 , with higher scores indicating higher severity of insomnia symptoms; the score was taken into consideration as a dependent variable. The Tolerance of Uncertainty Scale Short Form (IUS-12; Freeston et al., 1994) measures responses to uncertainty, ambiguous situations, and the future. It provides a measure of both prospective anxiety and inhibitory anxiety, as well as a total measure of uncertainty (by summing the scores to all the 12 items). We considered the total score as a dependent variable. The Uncertainty Response Scale (URS; Greco and Roger, 2001) is a scale for the evaluation of styles of coping with uncertainty and can provide a measure of three subscales (emotional uncertainty, desire for control, and cognitive uncertainty). We considered the three subscale scores and the total score as dependent variables. The Anxiety Sensitivity Index 3 (ASI-3; Taylor et al., 2007; Petrocchi et al., 2015) measures vulnerability to anxiety. Higher scores reflect higher levels of anxiety. We considered the physical concerns, social concerns, and cognitive concerns subscales as well as the total score (sum of all the three subscales) as dependent variables. Finally, the Eysenck Personality Questionnaire-Revised Short Form (EPQRS; Eysenck et al., 1985; Picconi et al., 2018) was used to assess the personality characteristics of participants. In particular, here we used the scores for neuroticism, extraversion/introversion, and psychoticism scales as dependent variables.

\section{Recognition of Facial Expressions Task}

In Pistoia et al.'s (2018) study, participants also took part in behavioral experiments aimed at evaluating their ability to recognize facial expressions (using the Ekman and Friesen Pictures of Facial Affect) and to evaluate emotionally evocative scenes (using the International Affective Picture System). In the present study, we specifically focused on the participants' accuracy in recognizing emotional facial expression, that is the ability to correctly identify actors from the Ekman and Friesen (1976) set of Pictures of Facial Affect (Ekman, 1993) displaying the six basic emotions: happiness, sadness, anger, fear, disgust, and surprise [see Pistoia et al. (2018) for a detailed description of the experimental procedure].

\section{rs-fMRI}

\section{Data Acquisition}

Magnetic resonance imaging studies were carried out at three Tesla (Discovery MR, General Electric Medical Systems, Erlangen, Germany), using a 32-channel head coil.

Structural T1w volumes were acquired using a threedimensional magnetization-prepared fast spoiled gradient echo sequence (144 sagittal partitions; TR $6.6 \mathrm{~ms}$; TE $2.3 \mathrm{~ms}$; TI $1100 \mathrm{~ms}$; flip angle $7^{\circ}$; voxel size $1 \times 1 \times 1 \mathrm{~mm}^{3}$ ).

Resting-state functional magnetic resonance imaging data were acquired using an EPI sequence (50 contiguous axial slices, $\mathrm{TR}=3000 \mathrm{~ms}, \mathrm{TE}=33 \mathrm{~ms}, \mathrm{FOV}=240 \mathrm{~mm}, 64 \times 64$ matrix, slice thickness $3.6 \mathrm{~mm}, 120$ time-points).

In addition, turbo-spin-echo FLAIR axial images were acquired (144 sagittal partitions; TR $8000 \mathrm{~ms}$; TE $119 \mathrm{~ms}$; TI $2032 \mathrm{~ms}$; flip angle $90^{\circ}$; voxel size $1 \times 1 \times 1 \mathrm{~mm}^{3}$ ) to help rule out the presence of chronic cerebrovascular disease or other CNS pathologies.

During the MRI study, the subjects were lying in a supine position with the head lightly fixed by straps and foam pads to 
minimize head movement. They were asked to relax with their eyes closed but not to fall asleep during the examination.

\section{Data Processing}

Magnetic resonance imaging data were pre-processed and analyzed using a toolbox for FC data analysis (CONN FC toolbox v18b, Gabrieli Lab., McGovern Institute for Brain Research, Massachusetts Institute of Technology ${ }^{1}$; Whitfield-Gabrieli and Nieto-Castanon, 2012) running in Matlab (MathWorks Inc.). CONN is a toolbox for fMRI analysis based on libraries from the Statistical Parametric Mapping package (SPM12, the Wellcome Department of Neurology, London, United Kingdom). Brain tissue probability maps were used to derive the white matter and Cerebro Spinal Fluid (CSF) mean signal time-courses for fMRI pre-processing (see the section "Data Processing") and to restrict the definition of seeds to each subject's GM voxels. To this end, for each subject, GM, WM, and CSF probability maps, normalized to the Montreal Neurological Institute (MNI) space, were obtained using the unified segmentation (Ashburner and Friston, 2005), implemented in SPM12. For all the segmentation preprocessing steps, the default SPM12 parameters were used. rs-fMRI preprocessing steps included the following: exclusion of the first five time-points to avoid the effects of the possible instability of the initial MRI signal; correction for differences in acquisition time across slices; motion correction by rigid-body co-registration of all the time-points to the first EPI volume (Friston et al., 1996); and band-pass filtering $(0.008-0.09 \mathrm{~Hz}$, to remove low-frequency signal drifts related to scanner instability and high frequency noise). rs-fMRI data were then normalized to the standard MNI space by first coregistering to the corresponding T1-weighted volumes (to avoid misregistration related to inter-sequence movements) and then applying the normalization parameters calculated for the $\mathrm{T} 1$ volumes in the segmentation step to the co-registered EPI volumes.

Normalized EPI volumes were then resampled to a voxel size of $3 \times 3 \times 3 \mathrm{~mm}^{3}$. A rigorous removal of signal contributions from head movements and from physiological variations unrelated to neuronal activity was implemented by regressing out the mean white matter and cerebro-spinal fluid signals (Whitfield-Gabrieli and Nieto-Castanon, 2012), along with six framewise motion parameters derived from the motion correction routine (i.e., rotations and shifts along the three orthogonal main axes).

In addition, a "scrubbing" procedure (Power et al., 2012) was applied, consisting of the introduction of dummy regressors to censor the effect of frames with excessive movements and/or signal changes. Accordingly, for each EPI sequence, volumes were identified that had, compared to the previous one, a mean signal difference exceeding three $Z$-values, and/or a mean framewise displacement exceeding $0.5 \mathrm{~mm}$.

On average, $10.1 \pm 9.7$ (mean $\pm \mathrm{SD}$ ) time-points were removed due to the scrubbing procedure $(10.3 \pm 10.2$ in the controls, $9.7 \pm 9.2$ in the exposed; $p=$ not significant). Analogously, neither mean framewise displacement $(0.15 \pm 0.11 \mathrm{~mm}$ vs. $14 \pm 0.07 \mathrm{~mm})$ nor mean global signal

${ }^{1}$ http://www.nitrc.org/projects/conn change $Z$-values $(1.1 \pm 0.5$ vs. $9 \pm 0.2)$ were significantly different between the two groups.

Functional magnetic resonance imaging volumes were finally smoothed with an isotropic Gaussian filter of $8 \mathrm{~mm}$ (FWHM). Voxel-wise maps of FC were then generated by calculating the Fisher-transformed Pearson correlation coefficients between the time course of each voxel and the time course averaged over each of eight seeds, sampling the major hubs of the VN (primary, ventral, right-dorsal, and left-dorsal visual cortices) and of the DMN (posterior cingulate/precuneus, medial prefrontal, right and left inferior parietal cortices). To this end, the seeds provided in CONN were used, which were obtained by independent component analysis of 497 normal subjects from the human connectome project dataset ${ }^{2}$, after masking by the GM map of each patient. The size and the MNI coordinates of the centers of mass of these eight seeds are reported in Table 1.

For all the pre-processing steps, an experienced operator, blind to participants' clinical conditions, visually assessed accuracy of the segmentation and spatial normalization.

\section{Statistical Analysis}

For each seed, FC maps were then entered in a second-level analysis. To identify differences between the two groups in the strength of the correlation with the $\mathrm{VN}$, or significant interactions between the group and the correlations of the FC of the seeds of both the VN and the DMN with the selected behavioral scores, FC maps were statistically analyzed using a multiple regression analysis within the general linear model framework. Both contrasts (exposed > non-exposed; non-exposed > exposed) were probed when comparing the two subject groups for both the between-group differences and the interaction analyses. Seedbased fMRI analysis was restricted to voxels falling in a GM mask, obtained thresholding at 0.2 the mean of the GM maps obtained in the segmentation step. For all the analyses, age and sex were included as nuisance covariates in the model, along with the mean framewise displacement derived from the motion correction procedure. Results, corrected for family-wise error (FWE) at cluster level, following a cluster-defining threshold of 0.001 , were considered significant when surviving an alpha level of 0.05 , corrected according to Bonferroni for the number of tests

${ }^{2}$ http://www.humanconnectomeproject.org

TABLE 1 |XYZ coordinates in the MNI space of the center of mass and size of the eight seeds used for the analysis.

\begin{tabular}{llcc}
\hline Network & Seed & $\begin{array}{c}\text { Center of mass } \\
\text { MNI coordinates } \\
\mathbf{( m m})\end{array}$ & $\begin{array}{c}\text { Size } \\
\mathbf{( m m}^{\mathbf{3}} \mathbf{)}\end{array}$ \\
\hline Visual & Primary & $2,-79,-12$ & 79,224 \\
& Ventral & $0,-93,-4$ & 48,712 \\
& Right dorsal & $38,-72,13$ & 33,968 \\
Default-mode & Left dorsal & $-37,-79,10$ & 24,832 \\
& Posterior cingulate/precuneus & $1,-61,38$ & 38,664 \\
& Medial prefrontal & $1,55,-3$ & 10,768 \\
& Right inferior parietal & $47,-67,29$ & 10,608 \\
& Left inferior parietal & $-39,-77,33$ & 8,328
\end{tabular}


performed ( $p=0.01$ for the comparison between the two groups and 0.006 for the imaging/clinical correlations).

\section{RESULTS}

Behavioral and rs-fMRI raw data are available upon request to the corresponding author.

\section{Self-Report Measures and Facial Expression Recognition Task}

Participants' scores on the self-report measures strongly overlapped with Pistoia et al.'s (2018) data (Table 2) in revealing higher scores in earthquake witnesses than in controls on several of the selected measures, although the multivariate results did not show significant effects for group [Pillai's Trace $=0.363$; Wilks' Lambda $=0.637 ; F(14,24)=0.978 ; p=0.501, \eta_{\mathrm{p}}^{2}=0.363$ ] and for sex [Pillai's Trace $=0.353$; Wilks' Lambda $=0.647$; $\left.F(14,24)=0.935 ; p=0.539, \eta_{\mathrm{p}}^{2}=0.353\right]$. The group by sex interaction was also not significant $(p>0.05)$.

TABLE 2 | Scores (mean and SD) of the two groups on the self-reported measures and on the facial expression recognition task.

\begin{tabular}{|c|c|c|c|c|}
\hline & \multicolumn{2}{|c|}{ Controls } & \multicolumn{2}{|c|}{$\begin{array}{l}\text { Earthquake } \\
\text { witnesses }\end{array}$} \\
\hline & Mean & $S D$ & Mean & $S D$ \\
\hline \multicolumn{5}{|l|}{ Self-report measures } \\
\hline$|S|$ & 4.52 & 3.1 & 5.06 & 3.15 \\
\hline IUS-12 & 32.52 & 10.4 & 44.22 & 11.7 \\
\hline URS-total score & 121.61 & 12.9 & 131.06 & 14.9 \\
\hline URS-emotional uncertainty & 27.43 & 6.5 & 34.17 & 8.8 \\
\hline URS-desire for control & 45.52 & 6.2 & 44.67 & 8.7 \\
\hline URS-cognitive uncertainty & 48.43 & 8.3 & 52.5 & 9.6 \\
\hline STAI-2 & 38.52 & 8.2 & 40.06 & 6.4 \\
\hline $\mathrm{BDI}$ & 5.78 & 4.5 & 6.89 & 4.9 \\
\hline ASI-total score & 9.35 & 6.2 & 16.56 & 15.9 \\
\hline ASI-physical concerns & 2.35 & 2.3 & 4.94 & 6.9 \\
\hline ASI-cognitive concerns & 4.13 & 3.7 & 5.33 & 5.8 \\
\hline ASI-social concerns & 2.87 & 3.3 & 6.28 & 5.8 \\
\hline EPQ-R-extraversion/introversion & 9.22 & 2.9 & 7.78 & 4.1 \\
\hline EPQ-R-neuroticism & 4.74 & 2.8 & 6.17 & 3.6 \\
\hline EPQ-R-psychoticism & 3.52 & 1.8 & 2.5 & 1.2 \\
\hline \multicolumn{5}{|l|}{ Recognition of facial expressions } \\
\hline Disgust & 0.76 & 0.1 & 0.91 & 0.1 \\
\hline Happiness & 0.98 & 0.1 & 1 & 0.2 \\
\hline Fear & 0.64 & 0.1 & 0.76 & 0.1 \\
\hline Anger & 0.77 & 0.2 & 0.97 & 0.2 \\
\hline Surprise & 0.91 & 0.2 & 0.99 & 0.2 \\
\hline Sadness & 0.71 & 0.1 & 0.77 & 0.2 \\
\hline
\end{tabular}

ISI, Insomnia Severity Index (Bastien et al., 2001; Castronovo et al., 2016); IUS12. Tolerance of Uncertainty Scale Short Form (Freeston et al., 1994); URS, Uncertainty Response Scale (Greco and Roger, 2001); STAl, State-Trait Anxiety Inventory (Spielberger et al., 1983; Pedrabissi and Santiniello, 1989); BDI, Beck Depression Inventory (Beck, 1967); ASI-3, Anxiety Sensitivity Index (Taylor et al., 2007; Petrocchi et al., 2015); EPQ-R, Eysenck Personality Questionnaire-Revised Short Form (Eysenck et al., 1985; Picconi et al., 2018).
The performance on the facial expression recognition task largely confirmed our previous data for the whole sample, demonstrating a higher accuracy by earthquake witnesses than non-exposed persons in recognizing all the six emotional categories (happiness, sadness, anger, fear, disgust, and surprise; percentages of correct responses are shown in Table 2). Indeed, the three-way mixed ANOVA on recognition accuracy, with emotion (disgust, happiness, fear, anger, surprise, and sadness) as a within-subject factor, and with group and sex as betweensubject factors, showed a significant main effect of emotion $\left[F(5,185)=11.547, p=0.0001, \eta_{\mathrm{p}}^{2}=0.238\right]$, with recognition of fear (0.70) being less accurate than all other emotions (disgust $=0.83$; happiness $=0.99$; anger $=0.87$; surprise $=0.95$; and sadness $=0.74)$. Importantly, results also showed significant main effects of group $\left[F(1,37)=8.844, p=0.005, \eta_{p}^{2}=0.193\right]$, with overall accuracy being higher in earthquake witnesses (mean $=0.90, S D=0.26)$ than in controls (mean $=0.79$, $S D=0.24)$, and of $\operatorname{sex}\left[F(1,37)=4.369, p=0.044, \eta_{\mathrm{p}}^{2}=0.106\right]$, with females (mean $=0.88, S D=0.26$ ) being more accurate than males $($ mean $=0.81, S D=0.25)$. No interaction was significant (all $p>0.05)$.

\section{rs-fMRI Data}

All participants were included in the analysis; when asked if they had fallen asleep even briefly, they all confirmed that they had remained awake the whole time.

No significant clusters of different FC with any of the tested seeds emerged when comparing the two groups independently of behavioral performance. Differences were found in the between-group correlation of the score for the facial expression recognition task and the $\mathrm{FC}$ with the $\mathrm{VN}$ and $\mathrm{DMN}$ seeds (interaction analysis) (Table 3). For the left dorsal visual seed of the $\mathrm{VN}$, differences emerged in the peripheral ventral occipital cortex on the right (Figure 1A, left) and in the middle temporal gyrus on the left (Figure 1A, right). For seeds of the DMN, differences were found in the precuneus for the medial prefrontal cortex (Figure 2A) and the left lower parietal (Figure 2B). These differences were due to a presence of an inverse correlation between FC and score in these regions in the exposed subjects, as opposed to the direct correlation detectable in the same regions in the non-exposed subjects (Figure 1B and right column of Figure 2).

\section{DISCUSSION}

The results of the study show significant between-group differences in the correlation of the score for the facial expression recognition task and the FC of the dorsal seed of the $\mathrm{VN}$ with the right occipito-temporal cortex and the left middle temporal cortex, and of the two parietal seeds of DMN, i.e., lower parietal and medial prefrontal cortex, with the precuneus bilaterally. These significant between-group differences are consistent with growing data demonstrating chronic and stable changes in functional brain connectivity at rest in persons exposed to trauma (Lui et al., 2009; Long et al., 2014; Chen et al., 2015; Kleshchova et al., 2019). In particular, Kleshchova et al. (2019) suggested that 
A

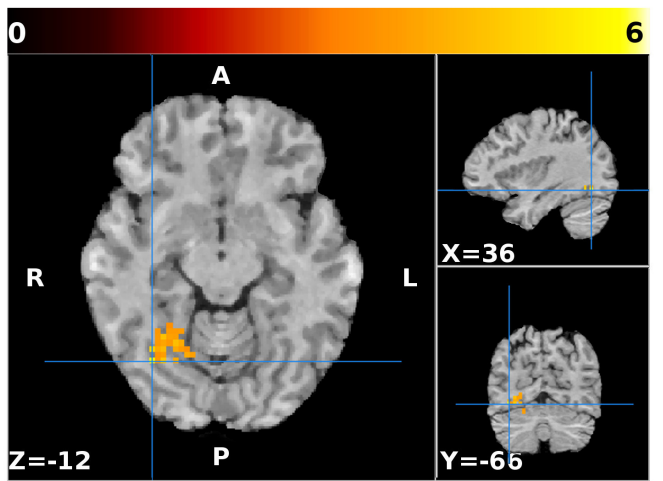

B
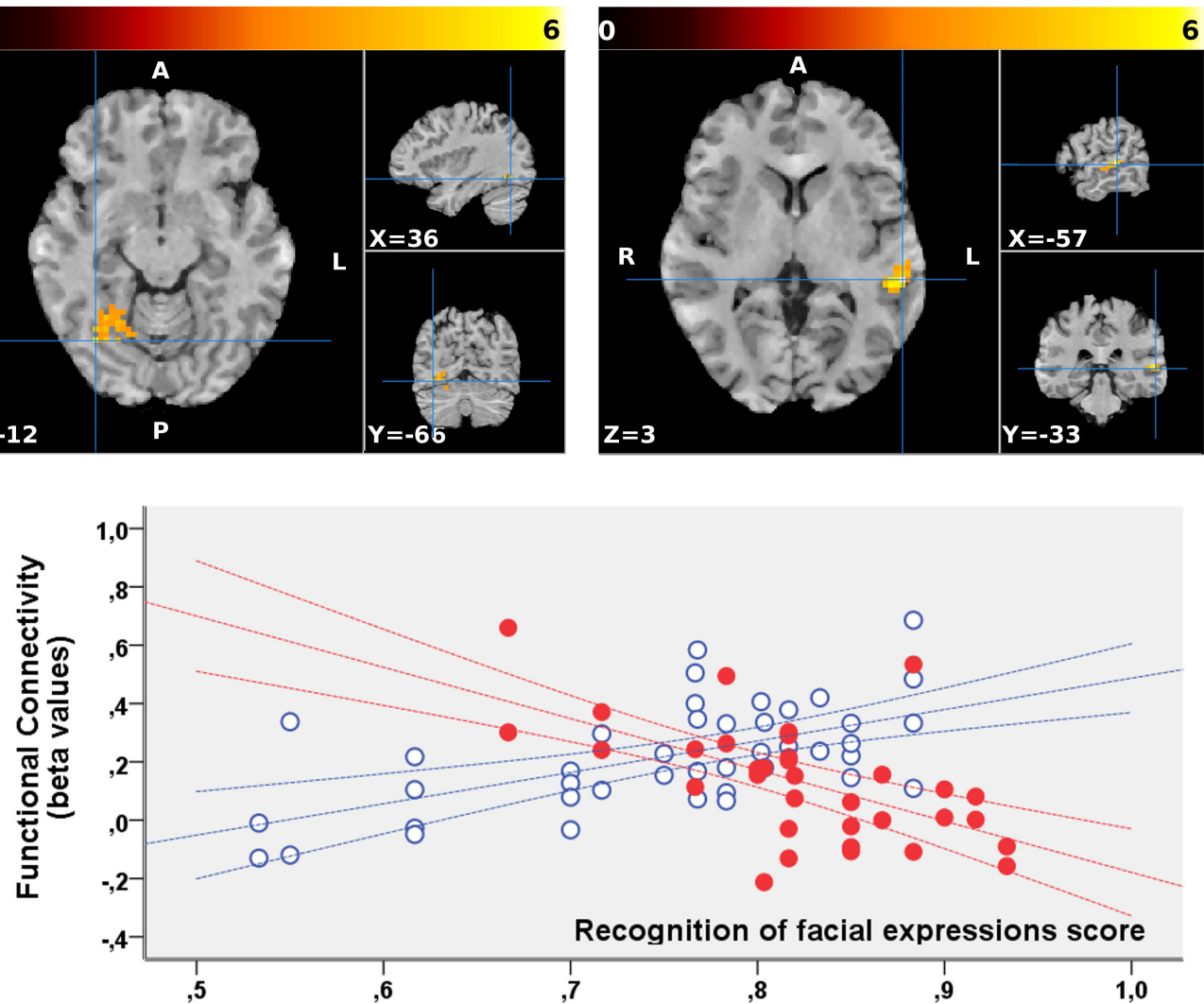

FIGURE 1 | (A) Regions showing a significant interaction between the groups (non-exposed > exposed) and correlation of the functional connectivity to the dorsal visual seed of the $\mathrm{VN}$ with the behavioral scores for the facial expression recognition task. The two clusters are superimposed to the T1-weighted volume from one of the participants to the study normalized to the MNI space. Color-scale maximum is set to a $T$-value of 6 . The three orthogonal planes are centered on the peak values of the two clusters [MNI coordinates, respectively, $(57,-33,3)$ and $(36,-66,-12)]$. No significant cluster emerged when probing the inverse (exposed > non-exposed) contrast. (B) Corresponding mean FC values are plotted against the scores obtained for the facial expression recognition task for exposed (red, filled marks) and non-exposed (blue, empty marks) subjects. The 95\% confidence intervals of the fit are also shown in the corresponding colors. In these regions, the exposed subjects display a significantly inverse correlation between the FC with the left dorsal visual seed and the score, whereas a direct correlation is present in the same regions in non-exposed subjects.

resting neurofunctional changes in trauma-exposed persons are part of an exaggerated neural alerting response to threat that can be observed even in the absence of physical threat, likely due to a chronic trauma-related condition of hypervigilance. It is worth remembering here that our sample of persons exposed to earthquake was actually in a chronic condition since, as noted above, the main L'Aquila earthquake in 2009 was followed by continuous aftershocks in the later months and by additional earthquakes in 2016 and 2017.

The difference we found between exposed and non-exposed participants in the relationship between facial expression recognition and the correlations of $\mathrm{FC}$ values in $\mathrm{VN}$ and DMN with occipito-temporal, lateral temporal, and medial parietal regions support the idea that the emotional expertise in earthquake witnesses involves neurofunctional changes in networks devoted to the processing of specific signals of potential threats, such as emotional faces. Indeed, although the occipitotemporal cortex represents a central node in the face identity recognition network (Haxby et al., 2000), a recent meta-analysis showed its involvement in the affective representation of a face
(Ganel et al., 2005; Said et al., 2011) when both implicit and explicit emotional processing are required (Gorno-Tempini et al., 2001; LeDoux, 2003; Litt et al., 2011; Brooks et al., 2012). Similarly, Mazza et al. (2012) investigated neural response to facial expressions implicitly presented during fMRI in a sample of L'Aquila earthquake witnesses affected by PTSD. Results showed that subliminal presentation of emotional faces (happy and sad) was related to activation of the occipito-temporal cortex, amygdala, and insula. Interestingly, the neurofunctional model of facial processing developed by Haxby et al. (2000) postulates that the core processing system not only involves the ventral occipito-temporal cortex but also the lateral (superior and middle) temporal cortex. Here, we actually found that the behavioral performance of earthquake witnesses was related to altered FC in the $\mathrm{VN}$ with the left middle temporal gyrus, consistent with Haxby et al.'s (2000) model, according to which this cortical region would be particularly involved in detecting the changeable aspects of faces, such as emotional expressions.

Facial expressions are actually among the most relevant signals conveying information on what is going on in other persons' 
A

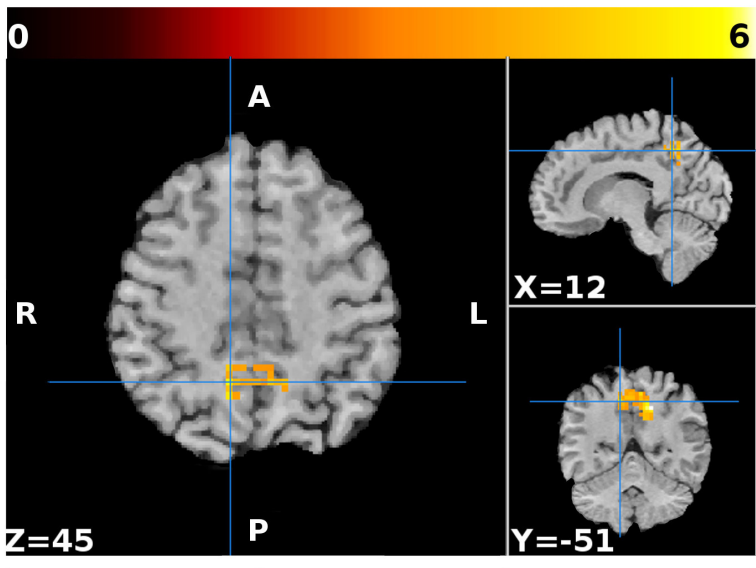

B

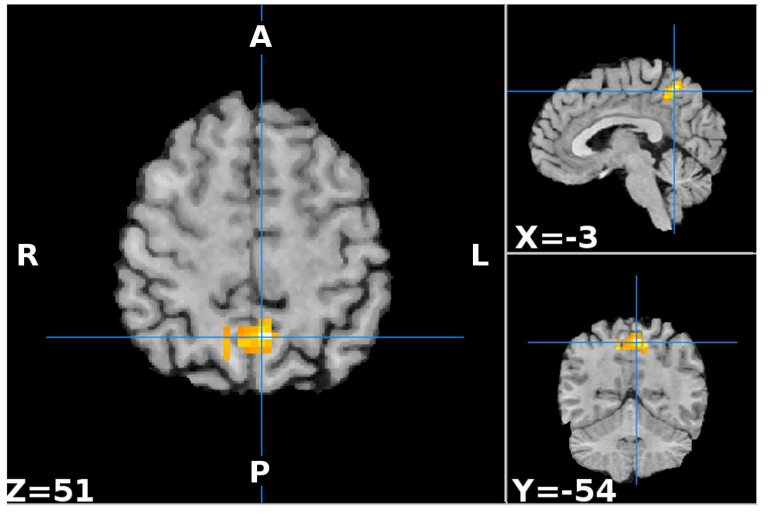

6
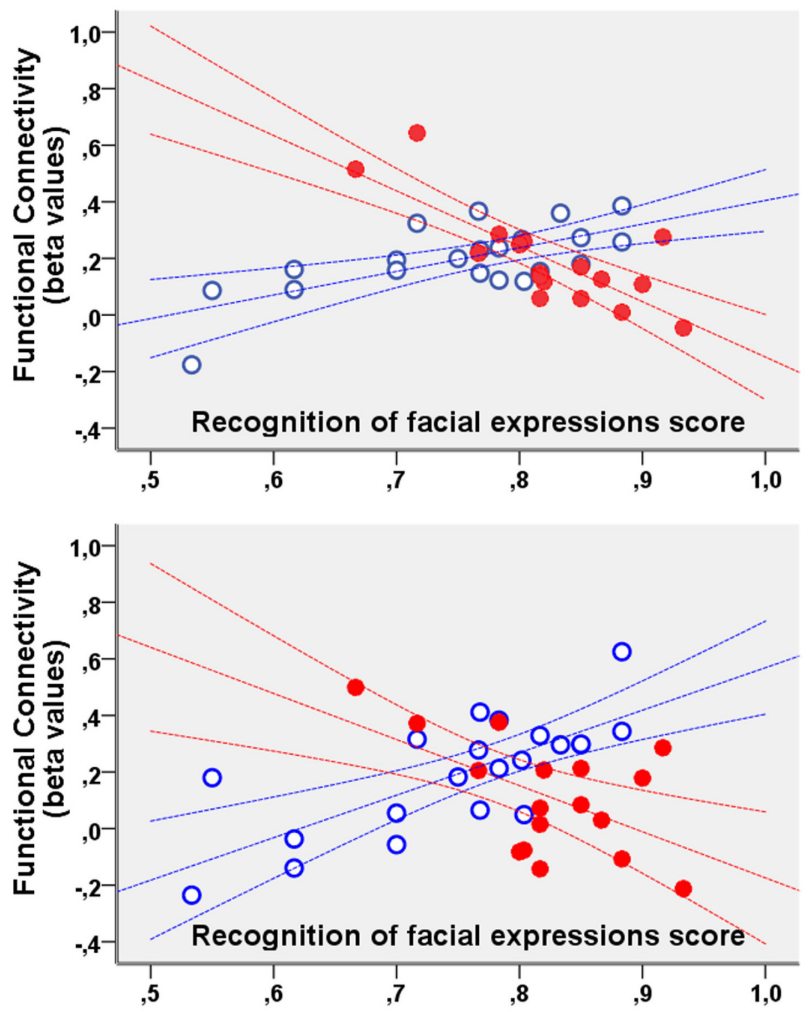

FIGURE 2 | Regions showing a significant interaction between the group and the correlation of the functional connectivity to the medial prefrontal cortex (A) and the left lower parietal (B) seeds of the DMN with the scores for the facial expression recognition task. For both seeds, the FC with the precuneus (part of the DMN) showed an altered correlation with the scores in the exposed subjects. The clusters are superimposed to the T1-weighted volume from one of the participants to the study normalized to the MNI space. The color-scale maximum is set to a $T$-value of 6 . For each cluster, the three orthogonal planes are centered on the peak value MNI coordinates. No significant cluster emerged when probing the inverse (exposed > non-exposed) contrast. On the right, the mean FC values of each cluster are plotted against the scores obtained for the facial expressions recognition task for exposed (red, filled marks) and non-exposed (blue, empty marks) subjects. The $95 \%$ confidence intervals of the fit are also shown in the corresponding colors. In the precuneus, the exposed subjects display a significantly inverse correlation between the FC with both these DMN seeds and the score, whereas non-exposed subjects show a direct correlation.

TABLE 3 | Clusters of altered correlation of the connectivity with the scores for the facial expression recognition task (Interaction). For each cluster, corresponding $p$-values (corrected at cluster level for family-wise error) and size (in cubic centimeters of gray matter) are reported, along with the local maxima $T$-values and coordinates.

\begin{tabular}{|c|c|c|c|c|c|c|c|c|}
\hline Network & Seed & $p$-value (FWE) & Size (cc) & $T$ & $x$ & $Y$ & $Z$ & Anatomical labels \\
\hline \multirow[t]{6}{*}{ Visual } & Left dorsal & $<10^{-5}$ & 1.4 & 6.33 & -57 & -33 & 3 & Left middle temporal gyrus \\
\hline & & & & 4.29 & -57 & -24 & 0 & Left middle temporal gyrus \\
\hline & & & & 3.82 & -51 & -18 & -6 & Left middle temporal gyrus \\
\hline & & $<10^{-6}$ & 1.8 & 5.61 & 36 & -66 & -12 & Right inferior occipital \\
\hline & & & & 5.16 & 24 & -57 & -6 & Right lingual \\
\hline & & & & 4.39 & 30 & -54 & -12 & Right fusiform \\
\hline \multirow[t]{5}{*}{ DMN } & Left lower parietal & $<10^{-13}$ & 4.1 & 6.68 & -3 & -54 & 51 & Left precuneus \\
\hline & & & & 6.14 & 12 & -57 & 45 & Right precuneus \\
\hline & & & & 5.72 & -6 & -48 & 45 & Left precuneus \\
\hline & Medial prefrontal cortex & $<10^{-11}$ & 3.7 & 5.54 & 12 & -51 & 45 & Right precuneus \\
\hline & & & & 5.32 & 9 & -57 & 39 & Right precuneus \\
\hline
\end{tabular}

Anatomical labeling is according to Tzourio-Mazoyer et al. (2002).

minds (Adolphs, 1999, 2003; LeDoux, 2003; Kanwisher and Yovel, 2006). Many imaging studies have examined the neural basis of understanding others' minds by different experimental tasks, such as judgments on facial expressions, stories, or moving shapes (Frith and Frith, 2006, 2007). Results always show the activation of a set of regions including medial prefrontal and 
parietal cortex, and posterior temporo-parietal areas around the temporo-parietal junction (Frith and Frith, 2006, 2007), a network of areas largely overlapping with the DMN (e.g., Mars et al., 2012). In particular, the left parietal and posterior midline nodes of the DMN are involved in processing emotional facial expressions, both in healthy individuals (Sreenivas et al., 2012) and in patients with different psychopathological conditions including social phobia (Gentili et al., 2009) and schizophrenia (Salgado-Pineda et al., 2011). Schilbach et al. (2008) explored the relationship between the neural basis for social cognition and the DMN, and found that the core nodes of the DMN overlap with those involved in social cognition (Vogeley and Fink, 2003; Schilbach et al., 2006). The authors suggested that the resting default state of the human brain is related to the predisposition of humans for social cognition as a default mental state. Consistently, here we demonstrated the involvement of key nodes of the DMN as the lateral parietal cortex, and the medial prefrontal and the parietal cortex. Therefore, we suggest that this default tendency to focus on the other person's mental state could be enhanced in persons exposed to traumatic experiences, as in the case of earthquake witnesses, in order to search relevant social signals, allowing rapid identification of possible threats in the environment (Zhang et al., 2014; Bell et al., 2017; Pistoia et al., 2018). This result fits with findings from a seminal rsfMRI study on witnesses of the Wenchun earthquake in China by Lui et al. (2009), who found a reduced temporal synchronization within the DMN in trauma victims, even immediately after trauma exposure. Moreover, a recent study investigating the correspondence between spontaneous neural activity in the DMN and the severity of PTSD symptoms showed that the atrest activity of the left inferior parietal lobule was positively correlated with symptom severity, thus suggesting that its activity is involved in the cognitive biases observed in persons with PTSD (Disner et al., 2018).

Future studies on earthquake witnesses are warranted to replicate the present results on a large sample; the size of the present group was small, although it was in line with the size of samples recruited in similar studies (e.g., Long et al., 2014; Kleshchova et al., 2019). Further, sensitivity of the study may have been limited by the relatively short scan duration which, however, was within the timeframe that has been shown to be required to stabilize the correlation strengths within and between the major networks (Van Dijk et al., 2010). While we preferred to keep acquisition short to reduce the risk of the subject falling asleep and/or moving, the increased $\mathrm{S} / \mathrm{N}$ ratio achievable with longer acquisitions may be considered in future studies, to increase sensitivity.

\section{REFERENCES}

Adolphs, R. (1999). Social cognition and the human brain. Trends Cogn. Sci. 3, 469-479.

Adolphs, R. (2003). Cognitive neuroscience of human social behaviour. Nat. Rev. Neurosci. 4, 165-178. doi: 10.1038/nrn1056

Ashburner, J., and Friston, K. J. (2005). Unified segmentation. Neuroimage 26, 839-851. doi: 10.1016/j.neuroimage.2005.02.018
Also, shorter TRs allowed by multiband acquisition (Feinberg and Setsompop, 2013), which was not available on our scanner, may in the future allow further boosting of the $\mathrm{S} / \mathrm{N}$ ratio, overcoming the limitations derived from the relatively long sampling interval (3s), which was used here to allow complete brain coverage while keeping a reasonable in-plane resolution with the available hardware.

Notwithstanding these limitations, our findings suggest that emotional expertise in earthquake witnesses goes through a functional modification of brain systems devoted to detection, identification, and interpretation of emotional faces, including the occipito-temporal cortex and the medial parietal cortex. Since we observed a general increase in anxiety and anticipation of threats, as well as emotional uncertainty, such emotional expertise, although first developing as a response of adaptive value, ends up being a maladaptive change to trauma, likely related to anxiety responses (see also Pistoia et al., 2018). It is possible to suggest that this emotional response is even more likely in young persons, in whom traumatic experiences tend to have a great impact on psychological functioning (Wang et al., 2015; Hong and Efferth, 2016). The earthquake witnesses who participated in the present study were young teenagers in 2009. Thus, in future studies, it could be interesting to investigate "emotional expertise" after a natural disaster in persons who were exposed to the traumatic experience at a later stage of their life.

\section{DATA AVAILABILITY STATEMENT}

The datasets generated for this study are available on request to the corresponding author.

\section{ETHICS STATEMENT}

The research protocol was approved by the Internal Review Board of the University of L'Aquila (01/2017). The study was conducted in accordance with the ethical standards of the Helsinki Declaration and signed informed consent was obtained from all the participants.

\section{AUTHOR CONTRIBUTIONS}

All authors equally contributed to the planning, development, and drafting of the manuscript.

Bastien, C. H., Vallières, A., and Morin, C. M. (2001). Validation of the Insomnia Severity Index as an outcome measure for insomnia research. Sleep Med. 2, 297-307. doi: 10.1016/s1389-9457(00)00065-4

Beck, A. T. (1967). Depression: Clinical, Experimental, and Theoretical Aspects. New York, NY: Harper \& Row.

Bell, C. J., Colhoun, H. C., Frampton, C. M., Douglas, K. M., McIntosh, V. V. W., Carter, F. A., et al. (2017). Earthquake brain: altered recognition and misclassification of facial expressions are related to trauma exposure but not 
posttraumatic stress disorder. Front. Psychiatry 8:278. doi: 10.3389/fpsyt.2017. 00278

Brooks, S. J., Savov, V., Allzén, E., Benedict, C., Fredriksson, R., and Schiöth, H. B. (2012). Exposure to subliminal arousing stimuli induces robust activation in the amygdala, hippocampus, anterior cingulate, insular cortex and primary visual cortex: a systematic meta-analysis of fMRI studies. Neuroimage 59, 2962-2973. doi: 10.1016/j.neuroimage.2011.09.077

Calhoun, L. G., and Tedeschi, R. G. (2006). "The foundations of posttraumatic growth: An expanded framework," in Handbook of Posttraumatic Growth, eds L. G. Calhoun, and R. G. Tedeschi, (Mahwah, NJ: Erlbaum), 1-23.

Castronovo, V., Galbiati, A., Marelli, S., Brombin, C., Cugnata, F., Giarolli, L., et al. (2016). Validation study of the Italian version of the Insomnia Severity Index (ISI). Neurol Sci. 37, 1517-1524. doi: 10.1007/s10072-016-2620-z

Chen, B., Xu, T., Zhou, C., Wang, L., Yang, N., Wang, Z., et al. (2015). Individual variability and test-retest reliability revealed by ten repeated resting-state brain scans over one month. PLoS One 10:e0144963. doi: 10.1371/journal.pone. 0144963

Disner, S. G., Marquardt, C. A., Mueller, B. A., Burton, P. C., and Sponheim, S. R. (2018). Spontaneous neural activity differences in posttraumatic stress disorder: a quantitative resting-state meta-analysis and fMRI validation. Hum. Brain Mapp. 39, 837-850. doi: 10.1002/hbm.23886

Dube, A., Moffatt, M., Davison, C., and Bartels, S. (2018). Health outcomes for children in haiti since the 2010 earthquake: a systematic review. Prehosp. Disaster Med. 33, 77-88. doi: 10.1017/S1049023X17007105

Eisma, M. C., Lenferink, L. I. M., Chow, A. Y. M., Chan, C. L. W., and Li, J. (2019). Complicated grief and post-traumatic stress symptom profiles in bereaved earthquake survivors: a latent class analysis. Eur. J. Psychotraumatol. 10:1558707. doi: 10.1080/20008198.2018.1558707

Ekman, P. (1993). Facial expression and emotion. Am. Psychol. 48, 384-392.

Ekman, P., and Friesen, W. V. (1976). Pictures of Facial Affect. Palo Alto, CA: Consulting Psychologists.

Eysenck, S. B. G., Eysenck, H. J., and Barrett, P. (1985). A revised version of the psychoticism scale. Pers. Individ. Dif. 6, 21-29. doi: 10.1016/0191-8869(85) 90026- 1

Fani, N., Jovanovic, T., Ely, T. D., Bradley, B., Gutman, D., Tone, E. B., et al. (2012). Neural correlates of attention bias to threat in post-traumatic stress disorder. Biol. Psychol. 90, 134-142. doi: 10.1016/j.biopsycho.2012.03.001

Farooqui, M., Quadri, S. A., Suriya, S. S., Khan, M. A., Ovais, M., Sohail, Z., et al. (2017). Posttraumatic stress disorder: a serious post-earthquake complication. Trends Psychiatry Psychother. 39, 135-143. doi: 10.1590/2237-6089-2016-0029

Feinberg, D. A., and Setsompop, S. K. (2013). Ultra-fast MRI of the human brain with simultaneous multi-slice imaging. J. Magn. Reson. 229, 90-100. doi: 10. 1016/j.jmr.2013.02.002

Freeston, M., Rhéaume, J., Letarte, H., Dugas, M. J., and Ladouceur, R. (1994). Why do people worry? Pers. Individ. Dif. 17, 791-802. doi: 10.1016/0191-8869(94) 90048-5

Friston, K. J., Williams, S., Howard, R., Frackowiak, R. S. J., and Turner, R. (1996). Movement-related effects in fMRI timeseries. Magn. Reson. Med 35, 346-355. doi: $10.1002 / \mathrm{mrm} .1910350312$

Frith, C. D., and Frith, U. (2006). The neural basis of mentalizing. Neuron 50, 531-534. doi: 10.1016/j.neuron.2006.05.001

Frith, C. D., and Frith, U. (2007). Social cognition in humans. Curr. Biol. 17, R724-R732.

Ganel, T., Valyear, K. F., Goshen-Gottstein, Y., and Goodale, M. A. (2005). The involvement of the "fusiform face area" in processing facial expression. Neuropsychologia 43, 1645-1654. doi: 10.1016/j.neuropsychologia.2005. 01.012

Geng, F., Liang, Y., Shi, X., and Fan, F. (2018). A prospective study of psychiatric symptoms among adolescents after the wenchuan earthquake. J. Trauma Stress 31, 499-508. doi: 10.1002/jts.22307

Gentili, C., Ricciardi, E., Gobbini, M. I., Santarelli, M. F., Haxby, J. V., Pietrini, P., et al. (2009). Beyond amygdala: default mode network activity differs between patients with social phobia and healthy controls. Brain Res. Bull. 79, 409-413. doi: 10.1016/j.brainresbull.2009.02.002

Gorno-Tempini, M. L., Pradelli, S., Serafini, M., Pagnoni, G., Baraldi, P., Porro, C., et al. (2001). Explicit and incidental facial expression processing: an fMRI study. Neuroimage 14, 465-473. doi: 10.1006/nimg.2001.0811
Greco, V., and Roger, D. (2001). Coping with uncertainty: the construction and validation of a new measure. Pers. Individ. Dif. 31, 519-534. doi: 10.1016/s01918869(00)00156-2

Harmer, C. J., Thilo, K. V., Rothwell, J. C., and Goodwin, G. M. (2001). Transcranial magnetic stimulation of medial prefrontal cortex impairs processing of angry facial expression. Nat. Neurosci. 4, 17-18. doi: 10.1038/82854

Haxby, J. V., Hoffman, E. A., and Gobbini, M. I. (2000). The distributed human neural system for face perception. Trends Cogn. Sci. 4, 223-233. doi: 10.1016/ s1364-6613(00)01482-0

Hayes, J. P., Vanelzakker, M. B., and Shin, L. M. (2012). Emotion and cognition interactions in PTSD: a review of neurocognitive and neuroimaging studies. Front. Integr. Neurosci. 6:89. doi: 10.3389/fnint.2012.00089

Hong, C., and Efferth, T. (2016). Systematic review on post-traumatic stress disorder among survivors of the wenchuan earthquake. Trauma Violence Abuse 17, 542-561. doi: 10.1177/1524838015585313

Kanwisher, N., and Yovel, G. (2006). The fusiform face area: a cortical region specialised for the perception of faces. Philos. Trans. R. Soc. Lond. B Biol. Sci. 361, 2109-2128. doi: 10.1098/rstb.2006.1934

Kleshchova, O., Rieder, J. K., Grinband, J., and Weierich, M. R. (2019). Resting amygdala connectivity and basal sympathetic tone as markers of chronic hypervigilance. Psychoneuroendocrinology 102, 68-78. doi: 10.1016/j.psyneuen. 2018.11.036

LeDoux, J. (2003). The emotional brain, fear, and the amygdala. Cell Mol. Neurobiol. 23, 727-738.

Litt, A., Plassmann, H., Shiv, B., and Rangel, A. (2011). Dissociating valuation and saliency signals during decision-making. Cereb. Cortex 21, 95-102. doi: 10.1093/cercor/bhq065

Long, J., Huang, X., Liao, Y., Hu, X., Hu, J., Lui, S., et al. (2014). Prediction of postearthquake depressive and anxiety symptoms: a longitudinal resting-state fMRI study. Sci. Rep. 4:6423. doi: 10.1038/srep06423

Lui, S., Huang, X., Chen, L., Tang, H., Zhang, T., Li, X., et al. (2009). High-field MRI reveals an acute impact on brain function in survivors of the magnitude 8.0 earthquake in China. Proc. Natl. Acad. Sci. U.S.A. 106, 15412-15417. doi: 10.1073/pnas.0812751106

Mars, R. B., Neubert, F. X., Noonan, M. P., Sallet, J., Toni, I., and Rushworth, M. F. (2012). On the relationship between the "default mode network" and the "social brain”. Front. Hum. Neurosci. 6:189. doi: 10.3389/fnhum.2012.00189

Mather, M., and Carstensen, L. L. (2005). Aging and motivated cognition: the positivity effect in attention and memory. Trends Cogn. Sci. 9, 496-502. doi: 10.1016/j.tics.2005.08.005

Mattavelli, G., Cattaneo, Z., and Papagno, C. (2011). Transcranial magnetic stimulation of medial prefrontal cortex modulates face expressions processing in a priming task. Neuropsychologia 49, 992-998. doi: 10.1016/j.neuropsychologia.2011.01.038

Mattavelli, G., Rosanova, M., Casali, A. G., Papagno, C., and Romero Lauro, L. J. (2016). Timing of emotion representation in right and left occipital region: evidence from combined TMS-EEG. Brain Cogn. 106, 13-22. doi: 10.1016/j. bandc.2016.04.009

Mazza, M., Catalucci, A., Mariano, M., Pino, M. C., Tripaldi, S., Roncone, R., et al. (2012). Neural correlates of automatic perceptual sensitivity to facial affect in posttraumatic stress disorder subjects who survived L'Aquila eartquake of April 6, 2009. Brain Imaging Behav. 6, 374-386. doi: 10.1007/s11682-0129151-x

O'Leary, V. E., and Ickovics, I. R. (1995). Resilience and thriving in response to challenge: an opportunity for a paradigm shift in women's health. Womens Health 1, 121-142.

Pedrabissi, L., and Santiniello, M. (1989). Manuale Dell'Adattamento Italiano Dello STAI Forma Y. Firenze: Organizzazioni Speciali.

Petrocchi, N., Tenore, K., Couyoumdjian, A., and Gragnani, A. (2015). The anxiety sensitivity index-3: factor structure and psychometric properties in Italian clinical and non-clinical samples. Boll. Psicol. Appl. 269, 53-64.

Phillips, M. L., Bullmore, E. T., Howard, R., Woodruff, P. W. R., Wright, I. W., Williams, S. C., et al. (1998). Investigation of facial recognition memory and happy and sad facial expression perception: an fMRI study. Psychiatry Res. 83, 127-138. doi: 10.1016/s0925-4927(98)00036-5

Picconi, L., Jackson, C. J., Balsamo, M., Tommasi, M., and Saggino, A. (2018). Factor structure and measurement invariance across groups of the Italian 
Eysenck Personality Questionnaire - Short form (EPP-S). Pers. Individ. Dif. 123, 76-80. doi: 10.1016/j.paid.2017.11.013

Pistoia, F., Conson, M., Carolei, A., Dema, M. G., Splendiani, A., Curcio, G., et al. (2018). Post-earthquake distress and development of emotional expertise in young adults. Front. Behav. Neurosci. 12:91. doi: 10.3389/fnbeh.2018.00091

Pistoia, F., Conson, M., Trojano, L., Grossi, D., Ponari, M., Colonnese, C., et al. (2010). Impaired conscious recognition of negative facial expressions in patients with locked-in syndrome. J. Neurosci. 30, 7838-7844. doi: 10.1523/ JNEUROSCI.6300-09.2010

Power, J. D., Barnes, K. A., Snyder, A. Z., Schlaggar, B. L., and Petersen, S. E. (2012). Spurious but systematic correlations in functional connectivity MRI networks arise from subject motion. Neuroimage 59, 2142-2154. doi: 10.1016/j. neuroimage.2011.10.018

Rafiey, H., Alipour, F., LeBeau, R., and Salimi, Y. (2019). Prevalence and determinants of PTSD 3 years after an earthquake in Iran. Commun. Ment. Health J. 55, 542-547. doi: 10.1007/s10597-019-00384-x

Said, C. P., Haxby, J. V., and Todorov, A. (2011). Brain systems for assessing the affective value of faces. Philos. Trans. R. Soc. Lond. B Biol. Sci. 366, 1660-1670. doi: 10.1098/rstb.2010.0351

Salgado-Pineda, P., Fakra, E., Delaveau, P., McKenna, P. J., Pomarol-Clotet, E., and Blin, O. (2011). Correlated structural and functional brain abnormalities in the default mode network in schizophrenia patients. Schizophrenia Res. 125, 101-109. doi: 10.1016/j.schres.2010.10.027

Schilbach, L., Eickhoff, S. B., Rotarska-Jagiela, A., Fink, G. R., and Vogeley, K. (2008). Minds at rest? Social cognition as the default mode of cognizing and its putative relationship to the default system of the brain. Consciousness Cogn. 17, 457-467. doi: 10.1016/j.concog.2008.03.013

Schilbach, L., Wohlschlaeger, A. M., Kraemer, N. C., Newen, A., Shah, N. J., Fink, G. R., et al. (2006). Being with virtual others: neural correlates of social interaction. Neuropsychologia 44, 718-730. doi: 10.1016/j.neuropsychologia. 2005.07.017

Spielberger, C. D., Gorsuch, R. L., Lushene, R., Vagg, P. R., and Jacobs, G. A. (1983). Manual for the State-Trait Anxiety Inventory. Palo Alto, CA: Consulting Psychologists Press.

Sreenivas, S., Boehm, S. G., and Linden, D. E. (2012). Emotional faces and the default mode network. Neurosci. Lett. 506, 229-234. doi: 10.1016/j.neulet.2011. 11.012

Tang, W., Lu, Y., Yang, Y., and Xu, J. J. (2018). An epidemiologic study of selfreported sleep problems in a large sample of adolescent earthquake survivors: the effects of age, gender, exposure, and psychopathology. Psychosom. Res. 113, 22-29. doi: 10.1016/j.jpsychores.2018.07.006

Taylor, S., Zvolensky, M. J., Cox, B. J., Deacon, B., Heimberg, R. G., Ledley, D. R., et al. (2007). Robust dimensions of anxiety sensitivity: development and initial validation of the anxiety sensitivity index-3. Psychol. Assess. 19, 176-188. doi: 10.1037/1040-3590.19.2.176

Todd, R. M., MacDonald, M. J., Sedge, P., Robertson, A., Jetly, R., Taylor, M. J., et al. (2015). Soldiers with Posttraumatic Stress Disorder see a world full of threat: magnetoencephalography reveals enhanced tuning to combat-related cues. Biol. Psychiatry 78, 821-829. doi: 10.1016/j.biopsych.2015.05.011

Tzourio-Mazoyer, N., Landeau, B., Papathanassiou, D., Crivello, F., Etard, O., Delcroix, N., et al. (2002). Automated anatomical labeling of activations in SPM using a macroscopic anatomical parcellation of the MNI MRI single-subject brain. Neuroimage 15, 273-289. doi: 10.1006/nimg.2001.0978

Van Dijk, K. R., Hedden, T., Venkataraman, A., Evans, K. C., Lazar, S. W., and Buckner, R. L. (2010). Intrinsic functional connectivity as a tool for human connectomics: theory, properties, and optimization. J. Neurophysiol. 103, 297321. doi: 10.1152/jn.00783.2009

Vogeley, K., and Fink, G. R. (2003). Neural correlates of the first-personperspective. Trends Cogn. Sci. 7, 38-42. doi: 10.1016/s1364-6613(02)00 003-7

Wang, L., Zhang, L., Armour, C., Cao, C., Qing, Y., Zhang, J., et al. (2015). Assessing the underlying dimensionality of DSM-5 PTSD symptoms in Chinese adolescents surviving the 2008 Wenchuan earthquake. J. Anxiety Disord. 31, 90-97. doi: 10.1016/j.janxdis.2015.02.006

Whitfield-Gabrieli, S., and Nieto-Castanon, A. (2012). Conn: a functional connectivity toolbox for correlated and anticorrelated brain networks. Brain Connect. 2, 125-141. doi: 10.1089/brain.2012.0073

Zhang, Y., Kong, F., Han, L., Najam, U., Hasan, A., and Chen, H. (2014). Attention bias in earthquake-exposed survivors: an event-related potential study. Int. J. Psychophysiol. 94, 358-364. doi: 10.1016/j.ijpsycho.2014. 09.004

Zhao, K., Zhao, J., Zhang, M., Cui, Q., and Fu, X. (2017). Neural responses to rapid facial expressions of fear and surprise. Front. Psychol. 8:761. doi: 10.3389/fpsyg. 2017.00761

Conflict of Interest: The authors declare that the research was conducted in the absence of any commercial or financial relationships that could be construed as a potential conflict of interest.

Copyright @ 2019 Pistoia, Conson, Quarantelli, Panebianco, Carolei, Curcio, Sacco, Saporito, Di Cesare, Barile, Masciocchi and Splendiani. This is an open-access article distributed under the terms of the Creative Commons Attribution License (CC BY). The use, distribution or reproduction in other forums is permitted, provided the original author(s) and the copyright owner(s) are credited and that the original publication in this journal is cited, in accordance with accepted academic practice. No use, distribution or reproduction is permitted which does not comply with these terms. 


\section{OPEN ACCESS}

Edited by:

Filippo Cieri,

Cleveland Clinic Lou Ruvo Center for Brain Health, United States

Reviewed by:

Wen Qin,

Tianjin Medical University General

Hospital, China

Dario J. Englot,

Vanderbilt University, United States Anbupalam Thalamuthu, University of New South Wales,

Australia

${ }^{*}$ Correspondence:

R. L. van den Brink r.van-den-brink@uke.de

Specialty section: This article was submitted to Brain Imaging and Stimulation, a section of the journa

Frontiers in Human Neuroscience

Received: 22 July 2019

Accepted: 17 September 2019

Published: 09 October 2019

Citation:

van den Brink RL, Pfeffer T and

Donner TH (2019) Brainstem Modulation of Large-Scale Intrinsic

Cortical Activity Correlations.

Front. Hum. Neurosci. 13:340. doi: 10.3389/fnhum.2019.00340
Brainstem Modulation of Large-Scale Intrinsic Cortical Activity Correlations

\author{
R. L. van den Brink ${ }^{1 *}$, T. Pfeffer ${ }^{1}$ and T. H. Donner ${ }^{1,2,3}$
}

1 Department of Neurophysiology and Pathophysiology, University Medical Center Hamburg-Eppendorf, Hamburg, Germany, ${ }^{2}$ Department of Psychology, University of Amsterdam, Amsterdam, Netherlands, ${ }^{3}$ Amsterdam Center for Brain and Cognition, Institute for Interdisciplinary Studies, Amsterdam, Netherlands

Brain activity fluctuates continuously, even in the absence of changes in sensory input or motor output. These intrinsic activity fluctuations are correlated across brain regions and are spatially organized in macroscale networks. Variations in the strength, topography, and topology of correlated activity occur over time, and unfold upon a backbone of longrange anatomical connections. Subcortical neuromodulatory systems send widespread ascending projections to the cortex, and are thus ideally situated to shape the temporal and spatial structure of intrinsic correlations. These systems are also the targets of the pharmacological treatment of major neurological and psychiatric disorders, such as Parkinson's disease, depression, and schizophrenia. Here, we review recent work that has investigated how neuromodulatory systems shape correlations of intrinsic fluctuations of large-scale cortical activity. We discuss studies in the human, monkey, and rodent brain, with a focus on non-invasive recordings of human brain activity. We provide a structured but selective overview of this work and distil a number of emerging principles. Future efforts to chart the effect of specific neuromodulators and, in particular, specific receptors, on intrinsic correlations may help identify shared or antagonistic principles between different neuromodulatory systems. Such principles can inform models of healthy brain function and may provide an important reference for understanding altered cortical dynamics that are evident in neurological and psychiatric disorders, potentially paving the way for mechanistically inspired biomarkers and individualized treatments of these disorders.

Keywords: functional connectivity, norepinepherine, dopamine, acetycholine, serotonin, brainstem, neuromodulation, resting-state

\section{INTRODUCTION}

Neural population activity in the cerebral cortex fluctuates continuously, even in the absence of changes in sensory input or motor output. These so-called intrinsic cortical activity fluctuations show remarkable structure across time and space: activity fluctuations correlate across sets of distributed brain areas, on the basis of which macroscale functional networks can be delineated (Biswal et al., 1995; Fox and Raichle, 2007). Such intrinsic activity correlations are commonly studied in a setting that is often referred to as the "resting state": the absence of motor output or structured sensory input (often with eyes-closed). However, intrinsic activity fluctuations that 
correlate across time and space also occur during active processing of sustained, unchanging, sensory input (Donner et al., 2013; Meindertsma et al., 2017; Pfeffer et al., 2018). We therefore use the term "intrinsic activity correlations," as it is agnostic about the behavioral context.

We focus on intrinsic correlations between cortical population signals that pool the activity across thousands of individual neurons. Intrinsic activity fluctuations have also been investigated at the level of single-neuron spiking, in this context commonly referred to as "noise correlations" (Zohary et al., 1994; Cohen and Kohn, 2011; Nienborg et al., 2012; Kohn et al., 2016). Similar to the intrinsic correlations between cortical population signals reviewed below, noise correlations between single neurons are state-dependent (e.g., Harris and Thiele, 2011; Reimer et al., 2014; Joshi and Gold, 2019). An important open question, beyond the scope of this review, is whether or not the same mechanistic principles account for the impact of state variations on neural correlations at these different (microscopic vs. macroscopic) scales. In this article, we use the term "intrinsic (cortical activity) correlations" to exclusively refer to correlations between cortical population signals.

Although predominantly studied with functional magnetic resonance imaging (fMRI), intrinsic correlations have also been shown to occur using electro-/magnetoencephalography (E/MEG), electrocorticography, and other imaging modalities (Friston et al., 1993; Mao et al., 2001; Nir et al., 2008; de Pasquale et al., 2010; Hipp et al., 2012; Lewis et al., 2016; Siems et al., 2016; Stitt et al., 2018; Hollensteiner et al., 2019) and show spatiotemporal correspondence across modalities (Hipp and Siegel, 2015; Siems et al., 2016). The temporal structure of intrinsic activity varies across cortical areas, which may reflect inter-areal variation in computational properties such as intrinsic timescales (Murray et al., 2014) that depend on inter-areal projections (Chaudhuri et al., 2015) and, possibly, functional interactions (Baria et al., 2013). Moreover, intrinsic activity correlations are largely predictive of taskrelated activation patterns (Cole et al., 2016; Tavor et al., 2016), and provide useful diagnostic and prognostic markers of neurological and psychiatric disorders (Fox and Greicius, 2010; van den Brink et al., 2018b). Thus, intrinsic activity correlations are a ubiquitous phenomenon and their quantitative features (Box 1) are potentially revealing indicators of the functional architecture of the brain.

A number of observations indicate that the features of intrinsic activity correlations are shaped by the architecture of anatomical connections of the cerebral cortex. First, intrinsic correlations within the visual system reflect established principles of cortico-cortical projections, such as retinotopic organization (Heinzle et al., 2011; Donner et al., 2013; Gravel et al., 2014; Bock et al., 2015). Second, computational models that are equipped with realistic anatomical connectivity can predict the topological features and temporal dynamics of empirical intrinsic correlations reasonably well (Honey et al., 2007, 2009; Cabral et al., 2012). Third, causal manipulation of anatomical connections alters the strength of functional interactions (O'Reilly et al., 2013). This line of inquiry thus suggests that the full repertoire
BOX 1 | Quantifying features of intrinsic activity correlations.

In this article, we discuss three characteristics of intrinsic activity correlations:

- The strength/magnitude of correlations in activity. Correlation strength can be used to examine the extent to which activity between any two brain regions is correlated (so-called seed-based correlation analysis), or to examine the overall "connectedness" of the brain by averaging the correlation coefficient across all brain region or deriving summary statistics such as "degree" (Rubinov and Sporns, 2010) or "functional connectivity density" (Tomasi and Volkow, 2010).

- Topography: A spatial representation of (some property of) a system. For example, the spatial distribution of a particular resting state network (RSN), defined as a set of brain regions or voxels that shows consistent spatio-temporal dynamics (usually above a particular threshold). The topography is often used to characterize the structure of individual RSNs, or experimental manipulation-related changes therein (Smith et al., 2009).

- Topology: The geometrical relationship between elements of a system. The human brain has been argued to approximate a small world topology, which forms a mixture of dense connections between neighboring brain regions and sparse long-range connections (Watts and Strogatz, 1998; Sporns and Zwi, 2004; Bassett and Bullmore, 2006; Bassett et al., 2006). Such a topology allows for both distributed and integrated processing, the balance between which relates to task-performance (Shine et al., 2016; Shine and Poldrack, 2018).

of functional interactions across the brain is shaped by the anatomical substrate upon which these interactions unfold (Deco et al., 2011).

However, other observations indicate that the anatomical connectome alone is not sufficient to account for the features of intrinsic activity correlations. The correspondence between the anatomical and functional connectome varies with attentional (Baria et al., 2013) and conscious (Barttfeld et al., 2015) state, and shows substantial temporal variability even within periods of rest (Chang and Glover, 2010; Sakoglu et al., 2010; Allen et al., 2014; Zalesky et al., 2014; Lurie et al., 2018). What are the sources of these variations of intrinsic activity correlations?

Here, we focus on one candidate source that has received surprisingly little attention in the resting-state literature, but, as we propose, is crucial for understanding the origin, dynamics, and diagnostic value of intrinsic activity correlation: the neuromodulatory systems of the brainstem. The term refers to a small set of brainstem nuclei with widespread projections to the forebrain, which synthesize and release specific modulatory neurotransmitters ("neuromodulators"; Figure 1 and Box 2). By virtue of their widespread projection profiles and effects on the state of cortical target networks, these systems can shape neural activity across the cortex in a coordinated fashion. Consequently, these systems are in an ideal position to shape intrinsic activity correlations. What is more, these brainstem systems are disturbed in several major psychiatric disorders, which also coincide with changes in intrinsic activity correlations (Calhoun et al., 2009; Rosazza and Minati, 2011; Wang et al., 2012; Vargas et al., 2013; Baggio et al., 2015; Dichter et al., 2015; Mulders et al., 2015; Giraldo-Chica and Woodward, 2017).

In what follows, we review the dependence of intrinsic activity correlations on neuromodulatory systems. An increasing number of studies over the past decade, conducted in humans, macaques, and rodents (rats and mice), have begun to provide 


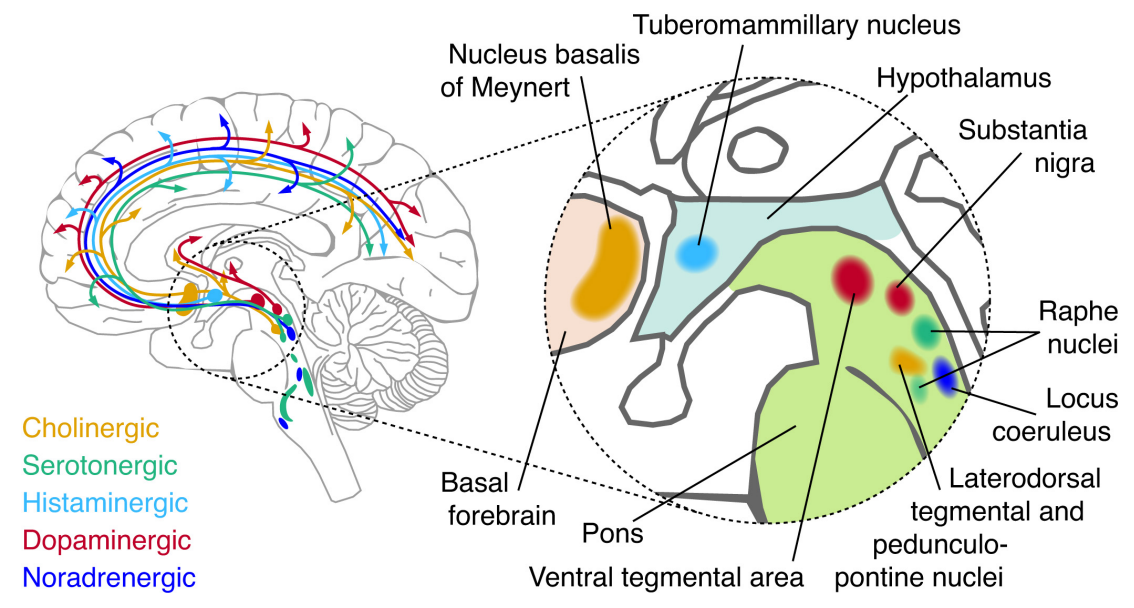

FIGURE 1 | Schematic of major neuromodulatory systems. Cerebellar, spinal, and temporal projections are omitted for brevity. The inset shows the approximate anatomical location of each nucleus that sends major afferents to the forebrain.

insight into this dependence. Our goal is to provide a structured overview of this nascent literature and distil from it a number of emerging principles. This article is a selective review, which focuses on emerging principles rather than a comprehensive coverage of the literature. Moreover, we focus on the neuromodulatory systems on which most work has been conducted. In particular, the catecholaminergic systems (norepinephrine, NE; and dopamine, DA) were among the first systems to be studied in relation to intrinsic activity correlations. This article also covers more recent work into the acetylcholine (ACh) and serotonin (5HT) systems. To date, comparatively little work has been conducted on the system-level effects of histamine, and it will therefore not be discussed in the current review.

\section{CANDIDATE MECHANISMS OF BRAINSTEM MODULATION OF INTRINSIC CORTICAL ACTIVITY CORRELATIONS}

The distinction between two modes of cortical state provides a useful heuristic for conceptualizing potential effects of brainstem neuromodulatory systems on cortical network dynamics: "activity state" and "dynamic state" (Curto et al., 2009; Safaai et al., 2015). This (likely oversimplified) dichotomy can be formalized by means of dynamical systems models. Curto et al. (2009) and Safaai et al. (2015) used the FitzHugh-Nagumo model [originally developed for describing action potential generation (FitzHugh, 1961; Nagumo et al., 1962)] for modeling cortical population dynamics (Curto et al., 2009; Safaai et al., 2015). In this framework, activity state refers to the set of parameters that vary on timescales from milliseconds to hundreds of milliseconds, and dynamic state refers to the set of parameters that vary more slowly (from seconds to tens of seconds) and interact multiplicatively with (i.e., modulate) the fast variations of activity states
(Curto et al., 2009; Luczak et al., 2009; Harris and Thiele, 2011; Safaai et al., 2015).

In physiological terms, activity state can be conceptualized as common measures of "neuronal activity": membrane potential or spiking activity. Changes in these measures of neuronal activity are caused by excitatory or inhibitory postsynaptic potentials, mediated by point-by-point synaptic transmission via ionotropic receptors (predominantly for glutamate and GABA). This form of synaptic transmission is the means of intracortical interactions and lies at the heart of current large-scale computational models of intrinsic activity correlations (Honey et al., 2007, 2009; Breakspear et al., 2009; Deco et al., 2013, 2014).

By contrast, variations in dynamic state can be conceptualized as the slower effects of neuromodulators, mediated by "volume transmission" (i.e., not point-by-point synapses) and by metabotropic receptors that do not alter the postsynaptic membrane potential directly. Activation of metabotropic receptors sets in motion intracellular signaling cascades that alter the way in which neurons respond to input over protracted periods of time, from changing the conductance of ionotropic receptors to altering the expression of genes. For example, catecholamines (in particular noradrenaline) change the balance between excitation and inhibition in the local microcircuit (Froemke, 2015; Martins and Froemke, 2015; Pfeffer et al., 2018). This circuit effects in turn increases the responsivity of cortical neurons to synaptic input (Moises et al., 1979; Rogawksi and Aghajanian, 1980; Seamans et al., 2001a,b; Wang and O'Donnell, 2001), an effect referred to as "neural gain": an increase in the slope of the input-output function (Berridge and Waterhouse, 2003; Murphy and Miller, 2003; Winterer and Weinberger, 2004; Disney et al., 2007; Polack et al., 2013). This mechanism of action corresponds to the common notion of "neuromodulation."

Importantly, while the majority of receptors for neuromodulators are metabotropic, some of them are ionotropic - in particular, the nicotinic class of ACh receptors 
BOX 2 | Major brainstem neuromodulatory systems.

Besides various peptides, five major neuromodulatory systems have been identified:

- Norepinephrine (NE) is released by the locus coeruleus (LC), and the A1/A2 regions of the brainstem (Sara, 2009). The LC projects to virtually all areas of the forebrain with the exception of the basal ganglia. Projection specificity of sub-populations of LC neurons has recently been shown (Chandler and Waterhouse, 2012; Chandler et al., 2014; Schwarz and Luo, 2015; Schwarz et al., 2015; Uematsu et al., 2015, 2017; Rho et al., 2018; Cerpa et al., 2019).

- Dopamine (DA) is predominantly released by two nuclei: the substantia nigra pars compacta (SNpc) and the ventral tegmental area (VTA) (Foote and Morrison, 1987). Four major dopaminergic branches exist of which three are ascending: the mesolimbic (VTA to ventral striatum), mesocortical (VTA to cortex) and nigrostriatal (SNpc to dorsal striatum) pathways. In addition, DA co-release by the LC (Takeuchi et al., 2016; Beas et al., 2018) and serotonergic dorsal raphe nuclei (Cho et al., 2017) have recently been shown. Other DA-producing neurons are found in the olfactory bulb (Pignatelli and Belluzzi, 2017) and pedunculopontine nucleus (French and Muthusamy, 2018). DA and NE have a similar chemical composition and are collectively known as catecholamines.

- Acetylcholine (ACh) is released by neurons in the basal forebrain (BF), which is comprised of several subdivisions, termed Ch1-Ch4, that contain cholinergic neurons. Ch4 corresponds to the nucleus basalis of Meynert, and is the major source of cortical ACh (Mesulam and van Hoesen, 1976; Mesulam et al., 1983; Mesulam and Changiz, 1988). This nucleus contains topographically organized clusters of neurons that preferentially innervate select portions of the cortex (Rho et al., 2018; Zaborszky et al., 2018; Ahmed et al., 2019). Other sources of ACh include the pedunculopontine nucleus and laterodorsal tegmental nucleus of the brainstem, which project to the thalamus, basal ganglia, hypothalums, and cortex (Statoh and Fibiger, 1986; Garcia-Rill, 1991; French and Muthusamy, 2018).

- Serotonin (5HT) originates from the raphe nuclei, which is a constellation of nuclei scattered throughout the brainstem (Törk, 1990). The raphe nuclei can be roughly subdivided in rostral and caudal portions, of which the rostral portion can be further subdivided in a dorsal (B6-B7) and median (B5/B8) portion. Both the dorsal rand median raphe project heavily to the cortex (Törk, 1990).

- Histamine is released by the tuberomammilary nucleus of the hypothalamus that projects to virtually the entire forebrain (Haas and Panula, 2003).

Most neuromodulatory brainstem nuclei do not consist of a single type of neuron releasing one neuromodulator, but contain a mixture of multiple types of neurons that can include GABAergic or glutamatergic types as well as other neuromodulators (Lin et al., 2015; Cho et al., 2017; Beas et al., 2018; Breton-Provencher and Sur, 2019). Furthermore, some of these nuclei are reciprocally connected and their activity tends to co-fluctuate in unison with changes in arousal and wakefulness (Foote and Morrison, 1987; Haas and Panula, 2003; Sara, 2009).

(Itier and Bertrand, 2001), and the $5 \mathrm{HT}_{3}$ subclass of serotonin receptors (Barnes et al., 2009). Activation of both receptor types leads to rapid activation of cortical networks (Puig et al., 2004; Fu et al., 2014; McCormick and Nusbaum, 2014; Wester and McBain, 2014; McGinley et al., 2015). Moreover, changes in the activity of neuromodulatory nuclei coincide with (Eschenko et al., 2012), and cause (Pinto et al., 2013) rapid fluctuations in activity state (e.g., the transition from the "down" to "up" state of synchronized cortical population activity). Serotonergic or cholinergic activation of ionotropic receptors thus constitutes a mechanism by which neuromodulatory brainstem system can rapidly change the cortical activity state.

In sum, neuromodulators may, in principle, alter intrinsic activity correlations in two ways. First, by rapidly changing the activity state of distributed sets of cortical regions through common (excitatory or inhibitory) drive (Drew et al., 2008). Second, neuromodulators may change the dynamic state (e.g., excitation-inhibition balance) of sets of cortical regions in a coordinated fashion but on slower timescales. Such coordinated changes in dynamic state, in turn, may directly produce correlations between population activity, but they can also modulate the correlations produced by cortical interactions through altering local dynamics (Deco et al., 2014; Pfeffer et al., 2018). The two principal mechanisms (modulation of activity state vs. dynamic state) reflect the distinct effects of cortical (ionotropic vs. metabotropic) receptors. Importantly, both mechanisms can produce intrinsic activity correlations (Leopold et al., 2003), even in the absence of any effect on cortico-cortical interactions.

While the projections of neuromodulatory nuclei to the cortex are commonly known as widespread or diffuse, there is substantial heterogeneity and specificity in these projections, part of which is only now being uncovered through novel anatomical tracing techniques (Foote and Morrison, 1987; Chandler and Waterhouse, 2012; Chandler et al., 2014; Schwarz and Luo, 2015; Schwarz et al., 2015; Uematsu et al., 2015, 2017; Kebschull et al., 2016; Breton-Provencher and Sur, 2019). What is more, the cortical distributions of the various different receptors for each neuromodulator are heterogeneous (Ramos and Arnsten, 2007; Zilles and Amunts, 2009; Nahimi et al., 2015; Salgado et al., 2016), which is evident for the human cortex in recent maps of receptor gene expression (Figure 2). Consequently, input from any neuromodulatory nucleus to the cortex, changing activity state, dynamic state, or both, might translate into spatially structured correlations of neural population signals in the cortex. For this reason, it is critical to consider the potential impact of neuromodulatory brainstem systems when making inferences about physiological cortico-cortical interactions (and "cortical networks") from the correlation of intrinsic cortical activity alone.

\section{CORRELATED CORTICAL ACTIVITY DRIVEN BY INTRINSIC FLUCTUATIONS OF BRAINSTEM ACTIVITY}

If neuromodulatory nuclei rapidly drive cortical activity in widespread target networks via ionotropic mechanisms then (i) removing neuromodulatory drive on the cortex should attenuate correlated activity within the cortex, and (ii) manipulating the time-varying activity of neuromodulatory nuclei should similarly affect the time-varying activity within the cortex, and (iii) activity within neuromodulatory nuclei should co-vary with intrinsic activity in large areas of the cortex. These predictions also hold if neuromodulatory nuclei potentiate drive from other sources such as the thalamus. 


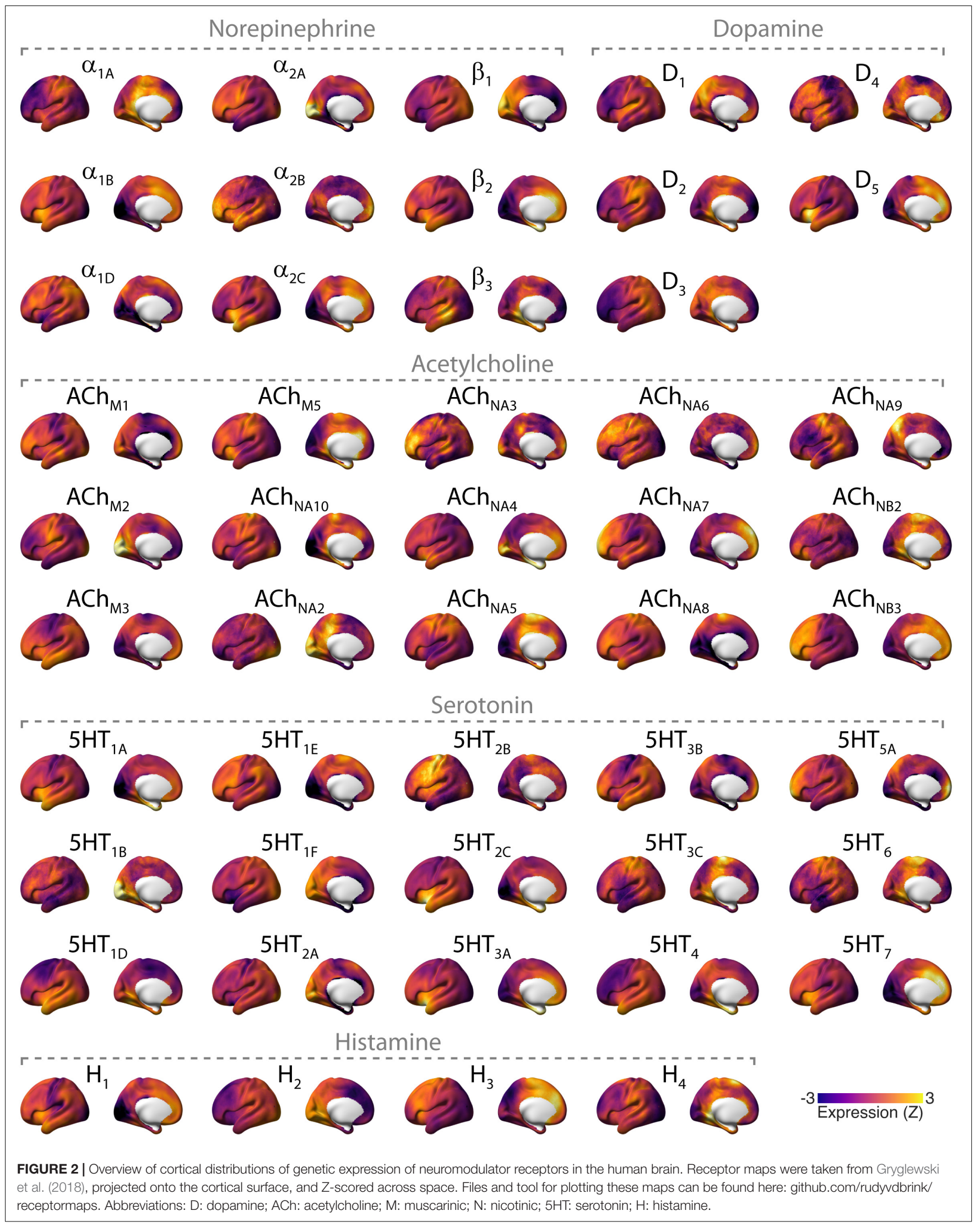




\section{Causal Manipulation of Time-Varying Neuromodulatory Activity}

Evidence for the first two predictions comes from studies in which the time varying activity of neuromodulatory nuclei is manipulated. Turchi et al. (2018) reversibly inactivated portions of the $\mathrm{BF}$ using the $\mathrm{GABA}_{\mathrm{A}}$ agonist muscimol in rhesus macaques, thus removing potential fluctuating common input to cortical areas. This manipulation reduced correlations between the "global" BOLD-fMRI signal (i.e., the average across all gray matter voxels) and voxel-wise activity topographically aligned with the afferents of the inactivated BF location. Because the $\mathrm{BF}$ sends GABAergic as well as cholinergic projections to the cortex, these findings do not necessarily reflect cholinergic effects. Grandjean et al. (2019) rhythmically stimulated serotonergic neurons in the DR with optogenetics in rodents, and measured the cerebral blood volume (CBV) response with fMRI (CBV was used because of putative signal-to-noise advantages over BOLDfMRI). Cortical CBV showed widespread, correlated troughs in amplitude in response to DR stimulation. Moreover, the cortical distribution of CBV responses correlated spatially with reductions in burst rate and delta-band power as measured electrophysiologically.

Combined, these two studies (Turchi et al., 2018; Grandjean et al., 2019) provide the strongest evidence to date of a mediation of (a part of) intrinsic activity correlations through rapid, common input from neuromodulatory brainstem nuclei that are consistent with correlated changes in activity state. A critical test of this notion would, however, use as a neural marker of interest correlations between spiking activity in different cortical regions, rather than between their BOLD or CBV signals. This is because changes in the latter signals may not necessarily reflect changes in cortical activity state (i.e., spiking activity; Maier et al., 2008) but may be produced by changes in dynamic state (Logothetis, 2008).

Analogous evidence for the LC-NE system comes from older positron emission tomography (PET) work by Coull et al. (1999) who reduced LC-activity via clonidine (Florin-Lechner et al., 1996) in healthy humans. During rest, clonidine caused broad reductions in directed coupling of PET activity between several cortical and sub-cortical regions. Due to the sluggish nature of the PET signals measured, however, it is difficult to attribute these results to changes in activity or dynamic state. In addition, DA depletion has similarly been reported to cause broad reductions in fMRI activity correlation strength (Shafiei et al., 2019).

\section{Temporal Co-variation Between the Brainstem and the Cortex}

The prediction that activity in neuromodulatory nuclei should co-vary with cortical activity can be tested using seed-based correlation analyses, where (fMRI) activity in neuromodulatory nuclei is correlated with cortical activity. A limited number of such studies have been conducted. Because of the small size and spatial proximity of these nuclei to the ventricles (Figure 1) and strong effects of physiological noise, these types of measurements require non-standard approaches to fMRI measurement and data analysis (Astafiev et al., 2010; Klein-Flugge et al., 2011; Brooks et al., 2013; Beissner, 2015; de Gee et al., 2017; Forstmann et al., 2017). Therefore, unless mentioned otherwise, we only considered studies where retrospective image correction for physiological noise was applied.

Zhang et al. (2015) assessed correlations between the cortex and LC, VTA, and SN, as defined by anatomical atlases (Ahsan et al., 2007; Keren et al., 2009). They reported widespread negative correlations between the cortex and all three brainstem nuclei and predominantly positive correlations between activity in these nuclei and other subcortical areas. Liu et al. (2018) found that peaks in the global (gray matter voxel averaged) signal coincided with troughs in activity of the $\mathrm{BF}$, suggesting an anti-correlation between $\mathrm{BF}$ activity and cortical activity. This is in line with earlier findings by Li et al. (2014), who reported widespread negative correlation between $\mathrm{BF}$ and cortical areas, although no correction for physiological noise was applied in this study. By contrast, Markello et al. (2018) found positive correlations between anatomically defined BF subdivisions (Zaborszky et al., 2008) and known cortical targets of BF projections, possibly due to the fact that in this study partial correlation was used to examine subdivision-specific correlations. Lastly, Beliveau et al. (2015) found relatively confined partial correlations between the cortex and anatomically and $\left[{ }^{11} \mathrm{C}\right] \mathrm{DASB}$ PET-binding constrained delineations of the dorsal and median raphe nuclei.

\section{Summary and Outstanding Issues}

The most direct evidence for intrinsic activity correlations through common drive of distributed cortical regions by neuromodulatory systems comes from direct manipulations of the activity of the corresponding brainstem nuclei. However, such studies are sparse and have not yet been conducted for all neuromodulatory nuclei. In addition, activity in most, but not all, neuromodulatory nuclei has been reported to covary with widespread areas of the cortex. However, to the best of our knowledge, no study to date has examined the individual contribution of the full set of neuromodulatory nuclei (Figure 1 and Box 2) to intrinsic activity fluctuations within the cortex. This leaves open the possibility that any correlation between an individual nucleus and the cortex is due to shared fluctuations across neuromodulatory nuclei (de Gee et al., 2017) rather than reflecting specific drive of one neuromodulatory nucleus on cortical activity. Moreover, removal of the global signal (as done by e.g., Beliveau et al., 2015) may obscure wide-spread correlations and reveal only those correlations that are stronger than global components of the cortical signal (i.e., the mean of all cortical regions). Another important limitation of all studies assessing intrinsic activity correlations by means of the fMRI signal, is that that the latter may reflect either changes in activity state (spike rate) or changes in dynamic state produced by neuromodulatory mechanisms (Logothetis, 2008).

In sum, the available studies are consistent with the notion of common drive of distributed cortical regions by the fluctuating activity of neuromodulatory nuclei. In this light, at least part of the spatial structure of intrinsic activity correlations within the cortex may reflect the spatial distribution of the projections of these brainstem nuclei, or their receptors, rather than the topography of cortico-cortical connections. Further experiments and direct comparisons of the contribution 
of individual neuromodulatory nuclei on intrinsic activity correlations are warranted, bearing in mind the above-mentioned interpretational caveats.

\section{CHANGES IN INTRINSIC CORTICAL CORRELATIONS UNDER PHARMACOLOGICAL INTERVENTION}

A major approach in the study of neuromodulatory systems is to manipulate neuromodulator levels via pharmacological intervention, and measure the resulting effects on cortical activity. Such pharmacological intervention will primarily exert its effects on cortical activity through sustained alterations of cortical circuit properties (i.e., shifts in cortical dynamic state), and less (or not at all) by altering the rapid drive of cortical regions, although it has been shown that the NEreuptake inhibitor atomoxetine alters the dynamics of LC activity (e.g., via auto-receptors) (Bari and Aston-Jones, 2013). For simplicity, we here heuristically treat pharmacological intervention as manipulations of changes in cortical dynamic state, and review the effects of these manipulations on intrinsic activity correlations.

We examine three key characteristics of these correlations (Box 1) in order to delineate commonalities or inconsistencies across findings. First, given the widespread projection profile of neuromodulatory systems (Figure 1 and Box 2), pharmacological manipulation of these systems may be expected to result in changes in correlation strength that encompass large areas of the cortex. We thus discuss literature that has examined such global changes of correlation strength (i.e., whole-brain increases or decreases). Second, the spatial heterogeneity of neuromodulatory projections and receptors (Figure 2) across the cortex suggests that neuromodulatory systems may not only change the global strength of intrinsic activity correlations, but also result in spatially inhomogeneous changes of correlation strength (van den Brink et al., 2018a). Third, computational modeling work indicates that modifications of circuit properties that are subject to neuromodulatory influence such as gain or excitation-inhibition balance (Servan-Schreiber et al., 1990; Polack et al., 2013; Froemke, 2015; Pfeffer et al., 2018) can alter the geometric properties of wholebrain intrinsic activity correlations (topology), even without any heterogeneity of neuromodulatory influences (Deco et al., 2014; Shine et al., 2018a). We therefore discuss literature that has examined the effect of pharmacological manipulation of neuromodulator systems on RSN topography and whole-brain functional topology.

\section{Changes of the Global Strength of Intrinsic Correlations}

Several studies have examined the effect of the NE reuptake inhibitor atomoxetine, which increases cortical catecholamine levels (Bymaster et al., 2002; Devoto et al., 2004; Swanson et al., 2006; Koda et al., 2010), on global correlation strength. Using atomoxetine, van den Brink et al. (2016) found reductions compared to placebo in graph-theoretic metrics of the global strength of intrinsic fMRI activity correlations in humans. Similarly, Guedj et al. (2017b) found reduced atomoxetineinduced fMRI correlation strength within and between various RSNs in rhesus macaques, with an overall net change of reduced correlation. This was also reflected in a reduction in the average brain-wide weighted correlation coefficient (Guedj et al., 2017a). By contrast, Pfeffer et al. (2019) found no significant atomoxetine-induced change in graph theoretic metrics of activity correlations measured with MEG during rest. However, during viewing of a perceptually ambiguous visual stimulus task, Pfeffer et al. (2019) found that atomoxetine increased the strength of correlations. A global increase in fMRI correlations following chemogenetic LC stimulation in anesthetized mice has also been reported (Zerbi et al., 2019).

The above mentioned MEG-study by Pfeffer et al. (2019) also investigated the effect of increased cortical ACh levels on correlated activity. This study used the acetylcholinesterase inhibitor donepezil and found reduced correlation strength during rest, and no effect during the presentation of an ambiguous visual stimulus. Three studies have examined the effect of pharmacological modulation of the 5HT system on the global strength of fMRI correlations. Schaefer et al. (2014) reported widespread reductions in correlation strength (quantified as the graph theoretic metric degree) following administration of the 5HT reuptake inhibitor escitalopram. Similarly, Preller et al. (2018) found a widespread shift of cortical intrinsic activity correlations toward zero due to the $5 \mathrm{HT}_{2} \mathrm{~A}$ receptor agonist LSD - an effect that did not occur when simultaneously administering the $5 \mathrm{HT}_{2 \mathrm{~A}}$ antagonist ketanserin. By contrast, Tagliazucchi et al. (2016) reported an LSD-induced increase in the overall strength of correlations within the cortex.

\section{Topographically Specific Changes of Intrinsic Correlation Strength}

Topographical effects of pharmacological manipulation may be informative about which sets of cortical regions ("networks") are particularly susceptible to neuromodulatory influence through a modulation of the dynamic state. Below, we provide a summary of consistent findings in the literature (see also Tables 1, 2). We review studies that either used whole-brain "dual regression," or correlation between a seed and the entire cortex. The term dual regression refers to sequential spatial and temporal regression of independent components with the purpose of identifying consistent spatiotemporal networks and manipulation-related changes therein (Beckmann, 2009).

Pharmacological elevations of the noradrenergic tone, while yielding a diversity of findings, have consistently produced effects that involve visual cortex. Coull et al. (1999) reported an $\alpha_{2}$ agonist clonidine-induced reduction in effective connectivity to- and from posterior extrastriate visual cortex. Reduced correlations between early visual cortex and frontoparietal cortical areas have also been reported following the $\alpha_{2}$ agonist dexmedetomidine (Akeju et al., 2016) and NET blocker atomoxetine (van den Brink et al., 2016). Similarly, Guedj et al. (2017b) reported atomoxetine-induced reductions in 
TABLE 1 | Summary of main findings of (pharmacological) manipulation of catecholamines (NE and DA).

\begin{tabular}{|c|c|c|c|c|}
\hline Study & Modality (species) & Analysis method & Manipulation & Main effects of manipulation on intrinsic correlations \\
\hline Coull et al., 1999 & PET (humans) & $\begin{array}{l}\text { Dynamic causal } \\
\text { modeling }\end{array}$ & Clonidine ( $\alpha 2$ agonist) & $\begin{array}{l}\text { Rest: reduced effective connectivity from PFC to thalamus, } \\
\text { and to and from visual cortex. Attentional task: general } \\
\text { increase in effective connectivity, with changes centered on } \\
\text { parietal cortex. }\end{array}$ \\
\hline $\begin{array}{l}\text { Achard and } \\
\text { Bullmore, } 2007\end{array}$ & fMRI (humans) & $\begin{array}{l}\text { Graph theoretic } \\
\text { analysis }\end{array}$ & Sulpride ( $\mathrm{D}_{2}$ antagonist) & Reduced metrics of global and local efficiency. \\
\hline Kelly et al., 2009 & fMRI (humans) & Seed-based correlation & L-DOPA (DA precursor) & $\begin{array}{l}\text { Increased correlation between putamen and cerebellum } \\
\text { and brainstem. Increased correlation between ventral } \\
\text { striatum and vIPFC. Reduced correlation between DMN, } \\
\text { and ventral striatum and caudate. }\end{array}$ \\
\hline $\begin{array}{l}\text { McCabe and } \\
\text { Mishor, } 2011\end{array}$ & fMRI (humans) & Seed-based correlation & Reboxetine (SNRI) & $\begin{array}{l}\text { Reboxetine: reduced amygdala-OFC correlation, and } \\
\text { reduced striatal-OFC correlation. }\end{array}$ \\
\hline Cole et al., 2013 & fMRI (humans) & Dual regression & $\begin{array}{l}\text { Haloperidol }\left(\mathrm{D}_{2}\right. \\
\text { antagonist) L-DOPA } \\
\text { (DA precursor) }\end{array}$ & $\begin{array}{l}\text { Linear increase (haloperidol < placebo < L-DOPA) between } \\
\text { BG network and sensorimotor cortex. Inerted-U between } \\
\text { BG network and dorsal anterior-mid cingulate } \\
\text { (placebo > haloperidol and L-DOPA). Linear decrease } \\
\text { (haloperidol > placebo > L-DOPA) between DMN and } \\
\text { sensorimotor cortex. Linear increase } \\
\text { (haloperidol < placebo < L-DOPA) between DMN and } \\
\text { SMG. }\end{array}$ \\
\hline Akeju et al., 2016 & fMRI (humans) & $\begin{array}{l}\text { Dual regression } \\
\text { Seed-based correlation }\end{array}$ & $\begin{array}{l}\text { Dexmedetomidine ( } \alpha 2 \\
\text { agonist) }\end{array}$ & $\begin{array}{l}\text { Reduced correlation between DMN and bilateral thalamus } \\
\text { and left cerebellum; increased correlation between DMN } \\
\text { and IFG, putamen, and insula. Reduced correlation } \\
\text { between rIFPN and cerebellum; increased correlation } \\
\text { between rIFPN and cerebellum, precuneus, parietal } \\
\text { operculum insula fusiform and angular gyri. Reduced } \\
\text { correlation between IIFPN and cerebellum, calcarine cortex, } \\
\text { MFG, and SFG. }\end{array}$ \\
\hline $\begin{array}{l}\text { Metzger et al., } \\
2015\end{array}$ & fMRI (humans) & Seed-based correlation & $\begin{array}{l}\text { Reboxetine (SNRI) } \\
\text { Amisulpride }\left(\mathrm{D}_{2 / 3}\right. \\
\text { antagonist) }\end{array}$ & $\begin{array}{l}\text { Reboxetine: increased correlation between brainstem, and } \\
\text { thalamus and PCC; and thalamus and accumbens; } \\
\text { reduced correlation between putamen-brainstem; } \\
\text { amygdala-ACC; and between the accumens and two } \\
\text { regions of the ACC; reduced correlation between } \\
\text { accumbens and two regions in ACC. Amisulpride: } \\
\text { increased correlation between: PCC-brainstem; amygdala, } \\
\text { brainstem and thalamus; putamen-brainstem. }\end{array}$ \\
\hline $\begin{array}{l}\text { van den Brink et al., } \\
2016\end{array}$ & fMRI (humans) & $\begin{array}{l}\text { Graph theoretic } \\
\text { analysis Seed-based } \\
\text { correlation }\end{array}$ & Atomoxetine (SNRI) & $\begin{array}{l}\text { Reduced metrics of global correlation strength and } \\
\text { clustering. Reduced correlation between FPN, and DMN } \\
\text { and visual network, and reduced correlation between the } \\
\text { visual and sensorimotor network. Reduced correlation } \\
\text { within a set of occipital regions. Reduced correlation } \\
\text { between early visual cortex and the rest of the brain. }\end{array}$ \\
\hline Guedj et al., 2017a & fMRI (rhesus macaque) & Dual regression & Atomoxetine (SNRI) & $\begin{array}{l}\text { Reduced correlation within FPN, somatosensory, } \\
\text { sensorimotor, visual, and superior temporal sulcus } \\
\text { networks. Reduced correlation between various networks } \\
\text { with an overall net change of reduced correlation. }\end{array}$ \\
\hline Guedj et al., 2017b & fMRI (rhesus macaque) & $\begin{array}{l}\text { Graph-theoretic } \\
\text { analysis }\end{array}$ & Atomoxetine (SNRI) & $\begin{array}{l}\text { Reduced global efficiency. Reduced global correlation } \\
\text { strength. Increased clustering. }\end{array}$ \\
\hline Ye et al., 2017 & fMRI (humans) & $\begin{array}{l}\text { Graph theoretic } \\
\text { analysis and } \\
\text { seed-based correlation }\end{array}$ & $\begin{array}{l}\text { Pramipexole }\left(\mathrm{D}_{2}\right. \\
\text { agonist) }\end{array}$ & $\begin{array}{l}\text { Reduced correlation between caudate and nodes of the } \\
\text { sensorimotor network. No change in topological metrics. }\end{array}$ \\
\hline Shine et al., 2018b & fMRI (humans) & $\begin{array}{l}\text { Graph theoretic } \\
\text { analysis }\end{array}$ & Atomoxetine (SNRI) & $\begin{array}{l}\text { Rest: reduced metrics of integration. N-back task: } \\
\text { increased metrics of integration. }\end{array}$ \\
\hline $\begin{array}{l}\text { van den Brink et al., } \\
2018 \mathrm{a}\end{array}$ & fMRI (humans) & $\begin{array}{l}\text { Generalized eigenvalue } \\
\text { decomposition }\end{array}$ & Atomoxetine (SNRI) & $\begin{array}{l}\text { Increased correlations in a network that loosely resembled a } \\
\text { rIFPN, and distribution of } \beta \text { (negatively) and } D_{2} \text { receptors. } \\
\text { Reduced correlations in a network that loosely resembled a } \\
\text { IIFPN, sensorimotor and DMN networks, and distribution of } \\
\beta \text { (positively) and } \alpha_{1} \text { receptors. }\end{array}$ \\
\hline Pfeffer et al., 2018 & MEG (humans) & $\begin{array}{l}\text { DFA + computational } \\
\text { model }\end{array}$ & Atomoxetine (SNRI) & $\begin{array}{l}\text { Rest: reduced } \alpha \text { scaling exponent due to atomoxetine. Task } \\
\text { (bistable perception): reduced } \alpha \text { scaling exponent due to } \\
\text { atomoxetine. Computational model accounted for the } \\
\text { findings as a change in excitation relative to inhibition. }\end{array}$ \\
\hline
\end{tabular}


TABLE 1 | Continued

\begin{tabular}{|c|c|c|c|c|}
\hline Study & Modality (species) & Analysis method & Manipulation & Main effects of manipulation on intrinsic correlations \\
\hline Shafiei et al., 2019 & fMRI (humans) & $\begin{array}{l}\text { Seed-based correlation } \\
\text { Graph theoretic } \\
\text { analysis }\end{array}$ & $\begin{array}{l}\text { Phenylalanine and } \\
\text { tyrosine depletion (DA } \\
\text { depletion) }\end{array}$ & $\begin{array}{l}\text { Reduced correlations strength in sensorimotor, salience, } \\
\text { and temporal networks. Reduced between-module } \\
\text { correlation of the sensorimotor and salience networks. }\end{array}$ \\
\hline Pfeffer et al., 2019 & MEG (humans) & $\begin{array}{l}\text { Graph theoretic } \\
\text { analysis + computational } \\
\text { model }\end{array}$ & Atomoxetine (SNRI) & $\begin{array}{l}\text { Rest: no effect of atomoxetine. Task (bistable perception): } \\
\text { Atomoxetine increased correlation strength. Computational } \\
\text { models indicate that effects can be accounted for by an } \\
\text { increase in gain. }\end{array}$ \\
\hline Zerbi et al., 2019 & fMRI (mice) & $\begin{array}{l}\text { Graph theoretic } \\
\text { analysis (FCD) Dual } \\
\text { regression }\end{array}$ & $\begin{array}{l}\text { Chemogenetic LC } \\
\text { stimulation }\end{array}$ & $\begin{array}{l}\text { Increase in global correlation strength. Increased correlation } \\
\text { in the Salience, amygdala, auditory, striato-motor, and DMN } \\
\text { networks. }\end{array}$ \\
\hline
\end{tabular}

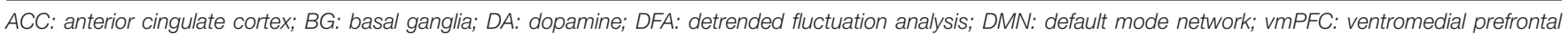

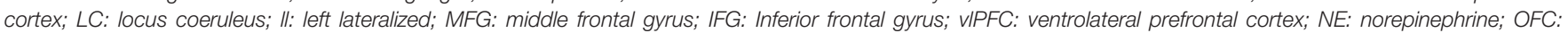

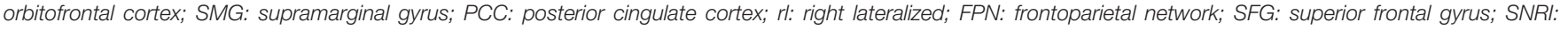
selective norepinephrine reuptake inhibitor.

correlation within a frontoparietal network and peripheral visual network. One study did not report changes in correlation of visual cortical areas following chemogenetic LC stimulation in rodents (Zerbi et al., 2019), potentially due to inter-species differences or differences between the effect of chemogenetic and pharmacological manipulation (Giorgi et al., 2017).

Manipulations of the cholinergic system have, likewise, yielded topographical effects that involve visual cortex. Tanabe et al. (2011) reported a nicotine-induced increase in correlation strength within an extrastriate network, and Klaassens et al. (2017) reported a galantamine (cholinesterase inhibitor) induced increase in correlation strength between a polar occipital network and widespread areas of the cortex. In addition, some, but not all (Guedj et al., 2017b), studies using noradrenergic or cholinergic agents have reported effects that involve the default mode network (DMN) (Tanabe et al., 2011; Akeju et al., 2016; van den Brink et al., 2016, 2018a; Klaassens et al., 2017; Zerbi et al., 2019). The observation that noradrenergic or cholinergic agents consistently produced effects on intrinsic correlations in visual cortical areas could be due to the fact that NE receptors $\alpha_{2 \mathrm{~A}}$ and $\beta_{1}$, and $\mathrm{ACh}$ receptors $\mathrm{NA}_{10}$ and $\mathrm{M}_{2}$ are prominently expressed these regions (Figure 2).

In contrast, primarily dopaminergic agents produced no effects on visual cortex, but instead on somatosensory and (pre-)motor cortex. For example, a positive relationship between DA levels and correlation strength between the basal ganglia and sensorimotor cortex (Cole et al., 2013), reduced correlation between the caudate and nodes of the sensorimotor network including pre- and postcentral gyri following $\mathrm{D}_{2}$ receptor agonism (Ye et al., 2017), and reduced correlation within the sensorimotor network following DA depletion (Shafiei et al., 2019). Dopaminergic effects in motor cortical regions may reflect direct modulations of dynamic state within the cortex, or downstream effects of modulations of the efficacy of dopaminergic projections from the brainstem to the basal ganglia (Figure 1).

Catecholaminergic manipulations have also been studied in the context of stress-related (re)activation patterns (Hermans et al., 2011; Gerlicher et al., 2018). These studies show prominent noradrenergic and dopaminergic effects on stress-induced changes in activation patterns or later reemergence thereof.
Studies using serotonergic agents have reported effects on intrinsic correlations resembling a combination of noradrenergic/cholinergic and dopaminergic effects: in other words, effects in both visual cortical and sensorimotor areas (Klaassens et al., 2015, 2017; Carhart-Harris et al., 2016; Tagliazucchi et al., 2016; Preller et al., 2018). In particular, studies that used the $5 \mathrm{HT}_{2} \mathrm{~A}$ receptor agonist LSD consistently reported effects on visual cortex. Indeed, the $5 \mathrm{HT}_{2} \mathrm{~A}$ receptor is prominently expressed in visual cortex (Figure 2).

\section{Topologically Specific Changes of Intrinsic Correlation Strength}

Various analytical tools exist to characterize the topology of functional brain organization (Rubinov and Sporns, 2010; Shine and Poldrack, 2018). For instance, the balance between topological segregation and integration of cortical ensembles is determined by the ratio of activity correlation strength within versus between separate modules (Mattar et al., 2015; Shine et al., 2016), and has been related to behavioral performance (Shine and Poldrack, 2018). Topological variations in the segregationintegration balance of fMRI activity during rest covary with pupil diameter (Shine et al., 2016), a non-invasive proxy for activity in neuromodulatory nuclei (Murphy et al., 2014; Varazzani et al., 2015; Joshi et al., 2016; Breton-Provencher and Sur, 2019). Thus, topological features of intrinsic activity correlations may be under neuromodulatory control.

Indeed, pharmacological upregulation of cortical NE levels using atomoxetine has been shown to result in a shift toward segregated processing during rest, and a converse shift toward integrated processing during the performance of a cognitively demanding (N-back) task (Shine et al., 2018b). Similarly, van den Brink et al. (2016) found that atomoxetine reduced betweenmodule correlation strength, and reduced metrics of integration (clustering coefficient and transitivity) during rest. Guedj et al. (2017a) reported reduced global efficiency, a metric of integration (Rubinov and Sporns, 2010), but reduced clustering, due to atomoxetine in rhesus macaques.

Studies using DA manipulations seem to indicate that DA facilitates integration. Achard and Bullmore (2007) reported 
TABLE 2 | Summary of main findings of (pharmacological) manipulation of 5HT and Ach.

\begin{tabular}{|c|c|c|c|c|}
\hline Study & Modality (species) & Analysis method & Manipulation & Main effects of manipulation on intrinsic correlations \\
\hline $\begin{array}{l}\text { Tanabe et al., } \\
2011\end{array}$ & fMRI (humans) & $\begin{array}{l}\text { ICA-based } \\
\text { back-reconstruction }\end{array}$ & $\begin{array}{l}\text { Nicotine (nACh } \\
\text { receptor agonist) }\end{array}$ & $\begin{array}{l}\text { Reduced correlations within the DMN. Increased } \\
\text { correlations within extrastriate cortex. }\end{array}$ \\
\hline $\begin{array}{l}\text { McCabe and } \\
\text { Mishor, } 2011\end{array}$ & fMRI (humans) & Seed-based correlation & Citalopram (SSRI) & Citalopram: reduced amygdala-vmPFC correlation. \\
\hline $\begin{array}{l}\text { McCabe et al., } \\
2011\end{array}$ & fMRI (humans) & Seed-based correlation & Citalopram (SSRI) & $\begin{array}{l}\text { Reduced correlation between left dmPFC and left } \\
\text { hippocampus. }\end{array}$ \\
\hline $\begin{array}{l}\text { van de Ven } \\
\text { et al., } 2013\end{array}$ & fMRI (humans) & Dual regression & Escitalopram (SSRI) & $\begin{array}{l}\text { Reduced DMN correlations with PCC, ACC, hippocampus, } \\
\text { and lateral parietal cortex. }\end{array}$ \\
\hline $\begin{array}{l}\text { Schaefer et al., } \\
2014\end{array}$ & fMRI (humans) & $\begin{array}{l}\text { Graph theoretic } \\
\text { analysis (FCD) }\end{array}$ & Escitalopram (SSRI) & $\begin{array}{l}\text { Reduced global strength of correlation. Local increases in } \\
\text { thalamus and cerebellum. }\end{array}$ \\
\hline $\begin{array}{l}\text { Klaassens } \\
\text { et al., } 2015\end{array}$ & fMRI (humans) & Dual regression & Sertraline (SSRI) & $\begin{array}{l}\text { Widespread decreases in correlation with DMN; executive } \\
\text { control; visual and sensorimotor networks. Increased } \\
\text { correlation between auditory network and PCC/precuneus. }\end{array}$ \\
\hline $\begin{array}{l}\text { Carhart-Harris } \\
\text { et al., } 2016\end{array}$ & fMRI (humans) & Seed-based correlation & LSD (5HT agonist) & $\begin{array}{l}\text { Increase in correlation between visual cortex and } \\
\text { widespread regions of the cortex and between the } \\
\text { parahippocampal cortex and the retrosplenial cortex and } \\
\text { PCC, and increased correlations between parahippocampal } \\
\text { cortex and dorsal mPFC and right dorsolateral PFC. }\end{array}$ \\
\hline $\begin{array}{l}\text { Tagliazucchi } \\
\text { et al., } 2016\end{array}$ & fMRI (humans) & $\begin{array}{l}\text { Graph theoretic } \\
\text { analysis. Seed-based } \\
\text { correlation }\end{array}$ & LSD (5HT agonist) & $\begin{array}{l}\text { Increased global correlation strength. Brain regions that } \\
\text { showed altered correlation strength overlapped with } \\
5 \mathrm{HT}_{2 \mathrm{~A}}-\mathrm{r} \text { distributions. Increased correlation between four } \\
\text { seeds (PFC, parietal cortex, precuneus, and thalamus) and } \\
\text { sensorimotor areas. Reduced modularity. Increased } \\
\text { participation coefficient of frontal and midline regions. } \\
\text { Reduced rich-club coefficient. }\end{array}$ \\
\hline $\begin{array}{l}\text { Klaassens } \\
\text { et al., } 2017\end{array}$ & fMRI (humans) & Dual regression & $\begin{array}{l}\text { Citalopram (SSRI) } \\
\text { Galantamine (nACh } \\
\text { receptor agonist) }\end{array}$ & $\begin{array}{l}\text { Citalopram: reduction of correlations within the } \\
\text { sensorimotor network, PFN, DMN, and executive control } \\
\text { network. Galantamine: increased correlations between } \\
\text { polar occipital network and distributed regions; between an } \\
\text { auditory network and regions of the DMN and } \\
\text { somatosensory cortex; reduced correlations within the } \\
\text { DMN, and between DMN and lateral and inferior occipital } \\
\text { cortices; between the FPN and DMN, inferior temporal and } \\
\text { cerebellar regions. }\end{array}$ \\
\hline $\begin{array}{l}\text { Deco et al., } \\
2018\end{array}$ & fMRI (humans) & $\begin{array}{l}\text { Dynamic } \\
\text { FC + computational } \\
\text { model }\end{array}$ & LSD (5HT agonist) & $\begin{array}{l}\text { The model captured the effect of } 5 \mathrm{HT}_{2 \mathrm{~A}}-\mathrm{r} \text { stimulation as an } \\
\text { increase in gain to explain the effect of LSD on the } \\
\text { distribution of FC dynamics. }\end{array}$ \\
\hline $\begin{array}{l}\text { Turchi et al., } \\
2018\end{array}$ & fMRI (rhesus macaque) & $\begin{array}{l}\text { Global signal correlation } \\
\text { and dual regression }\end{array}$ & $\begin{array}{l}\text { Pharmacological } \\
\text { inactivation of basal } \\
\text { forebrain }\end{array}$ & $\begin{array}{l}\text { Broad reductions in coupling of local activity with the global } \\
\text { signal, corresponding spatially to the inactivated location. } \\
\text { Topography of individual RSNs unaffected. }\end{array}$ \\
\hline $\begin{array}{l}\text { Lord et al., } \\
2018\end{array}$ & fMRI (humans) & Dynamic FC & $\begin{array}{l}\text { Psilocybin ( } 5 \mathrm{HT} \\
\text { agonist) }\end{array}$ & $\begin{array}{l}\text { Longer dwell times for a global FC component. Reduced } \\
\text { dwell times for FPN. }\end{array}$ \\
\hline $\begin{array}{l}\text { Preller et al., } \\
2018\end{array}$ & fMRI (humans) & $\begin{array}{l}\text { Graph theoretic } \\
\text { analysis (FCD) } \\
\text { Seed-based correlation }\end{array}$ & LSD (5HT agonist) & $\begin{array}{l}\text { Reduced correlation (from positive toward zero) in } \\
\text { associative networks. Increased correlation (from negative } \\
\text { toward zero) in sensorimotor and thalamic networks. LSD } \\
\text { effects correlated with } 5 \mathrm{HT}_{2 \mathrm{~A}}-\mathrm{r} \text { distributions. Reduced } \\
\text { correlation between sensorimotor areas and global signal. }\end{array}$ \\
\hline $\begin{array}{l}\text { Pfeffer et al., } \\
2018\end{array}$ & MEG (humans) & $\begin{array}{l}\text { DFA + computational } \\
\text { model }\end{array}$ & $\begin{array}{l}\text { Donepezil } \\
\text { (acetylcholinesterase } \\
\text { inhibitor) }\end{array}$ & No effect of donepezil. \\
\hline $\begin{array}{l}\text { Pfeffer et al., } \\
2019\end{array}$ & MEG (humans) & $\begin{array}{l}\text { Graph theoretic } \\
\text { analysis + computational } \\
\text { model }\end{array}$ & $\begin{array}{l}\text { Donepezil } \\
\text { (acetylcholinesterase } \\
\text { inhibitor) }\end{array}$ & $\begin{array}{l}\text { Rest: reduced correlation strength. Computational models } \\
\text { indicate that effect can be accounted for by an increase in } \\
\text { gain. }\end{array}$ \\
\hline $\begin{array}{l}\text { Grandjean } \\
\text { et al., } 2019\end{array}$ & fMRI, MUA, and LFP (mice) & GLM analysis & $\begin{array}{l}\text { Blockwise optogenetic } \\
\text { stimulation of DR }\end{array}$ & $\begin{array}{l}\text { Wide suppression of cortical CBV response. Suppression of } \\
\text { cortical MUA, and } \delta \text { LFP power, which spatially correlated } \\
\text { with the cortical CBV response. CBV response correlated } \\
\text { with distribution of } 5 \mathrm{HT} 1 \mathrm{~F}, 2 \mathrm{~A}, 2 \mathrm{C} \text { receptors, but not } 1 \mathrm{~A} \\
\text { and } 1 \mathrm{~B} \text { receptors. Correlations with receptor maps were } \\
\text { stronger than correlation with DR projection profile. }\end{array}$ \\
\hline
\end{tabular}

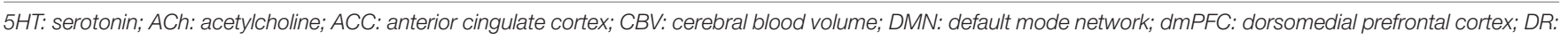

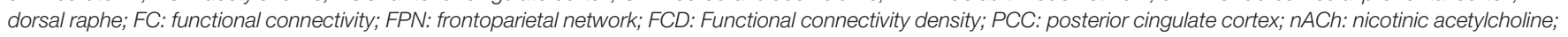
PCA: principle component analysis; SSRI: selective serotonin reuptake inhibitor. 
reduced metrics of global and local efficiency due to the $\mathrm{D}_{2}$ antagonist sulpride. Shafiei et al. (2019) reported that DA depletion reduced the participation coefficient (betweenmodule correlation) of the sensorimotor and salience networks. Thus, DA and NE may have dichotomous effects on network topology. However, null effects of DA agonism on various topological metrics, including metrics of integration, have also been reported (Ye et al., 2017). To the best of our knowledge, no studies to date have examined the effect of ACh manipulation on network topology, and one study has examined the effect of $5 \mathrm{HT}_{2 \mathrm{~A}}$ agonism (via LSD) on fMRI intrinsic correlation topology (Tagliazucchi et al., 2016). This study reported reduced modularity (increased integration), increased participation coefficient of frontal and midline regions (increased between-network correlation at the expense of within-network correlation), and reduced rich-club coefficient (less correlation with hub regions).

\section{Summary and Outstanding Issues}

Pharmacological manipulation of tonic neuromodulatory action, and the resulting, putative change in cortical dynamic state, consistently alters the global strength of intrinsic correlations. Less consistent, however, is the direction of these effects, even within classes of neuromodulators. Further studies are needed to corroborate or exclude the following possible reasons for these discrepancies: cross-study differences in preprocessing such as global signal regression (Preller et al., 2018); dosedependence of effects (Zahrt et al., 1997); or dependence of effects on cognitive/behavioral context (Coull et al., 1999; Shine et al., 2018b; Pfeffer et al., 2019) or baseline arousal (Warren et al., 2016).

Topographical effects following noradrenergic or cholinergic manipulation consistently involve visual cortex. Studies that used predominantly dopaminergic agents consistently report effects involving motor-related brain areas, but not visual brain areas. Studies that used a serotonergic agent report both visual and motor areas. This literature suggests that the regions that are most likely to be affected by pharmacological manipulation of neuromodulators are potentially distributed in accordance with the distribution of receptors across areas.

The predominant finding from studies on topological effects is that neuromodulators alter functional network topology, in particularly the catecholamines. These studies also suggest a possible distinction between the effects of $\mathrm{DA}$ and $\mathrm{NE}$ on network-level integration. Whereas NE reuptake during rest reduces topological metrics of integration, DA antagonism and depletion have the same effect, suggesting that DA facilitates integration. Similar to DA, 5HT seems to increase metrics of integration, but only one study has examined these effects. The influence of ACh on functional network topology remains to be studied.

A caveat with the findings on network topology is that some metrics of integration (in particular efficiency and clustering) are susceptible to changes in degree, even when artificially fixing degree of adjacency matrices by applying a fixed threshold (van Wijk et al., 2010). Since neuromodulators have been reported to alter degree as well (see section changes of the global strength of intrinsic correlations), future studies should carefully consider alterations in global degree when examining topological metrics. In addition, studies that have examined changes in the timevarying topology should take into account the influence of temporal fluctuations of the community structure on topological metrics (Thompson et al., 2019).

\section{CONCLUSION AND FUTURE DIRECTIONS}

Both (de)activation studies and seed-based correlation studies provide supporting evidence for ongoing fluctuations in the activity of neuromodulatory brainstem nuclei as a possible driving source of intrinsic activity correlations within the cortex. Temporary BF inactivation, NE release inhibition, DA synthesis inhibition, and rhythmic optogenetic serotonergic DR neuron stimulation all reduce intrinsic activity correlations. Furthermore, activity fluctuations in most neuromodulatory nuclei (BF; VTA; SN; LC) but not all (raphe nuclei) predict correlated activity fluctuations in broad areas of the cortex.

Pharmacological manipulation of cortical neuromodulator levels, which putatively alters cortical dynamic state, results in diverse changes of intrinsic activity correlations. First, pharmacological upregulation consistently changes the global strength of cortical correlations. Yet, in what direction the individual modulators exert their effects needs further study, since these effects are likely to be dependent on several factors, such as drug dose or behavioral context.

Second, several pharmacological studies have quantified neuromodulator-induced changes in the topography of cortical activity. Noradrenergic and cholinergic manipulation consistently alter activity correlations in visual cortical areas, dopaminergic manipulation affects motor cortical networks, and serotonergic manipulation affects both.

Finally, a number of studies have demonstrated that neuromodulators alter the topological properties of intrinsic activity correlations. These studies suggest a possible distinction between the effects of DA and NE on network-level functional integration. Similar to DA, 5HT seems to increase metrics of functional integration.

The studies discussed in this review may well have only scratched the surface the full spectrum of effects that neuromodulatory systems exert on intrinsic activity correlations. Even so, they open up exciting avenues for future work. An effort to map the contribution of each neuromodulatory system to the spatial and temporal features of intrinsic correlations may aid the identification of shared, independent, or antagonistic principles between the actions of different neuromodulatory systems and their diverse receptor classes. Such principles will inform models of healthy brain function and provide an important reference for the mechanistic understanding of neurological and psychiatric disorders. Ultimately, such principles may guide the way toward identification of specific molecular targets for mechanistically inspired and individualized pharmacological interventions in disorders of higher brain function. In what follows, we outline a number of important avenues for future research. Each of 
these could help advance our understanding of the principles that govern intrinsic brain dynamics and its dysfunctions in critical ways.

\section{Dissecting the Mechanisms of Brainstem Modulation of Cortical Correlations}

We have highlighted that neuromodulatory brainstem systems may alter intrinsic correlations of cortical population activity through diverse mechanistic pathways. The fluctuating activity of brainstem nuclei may provide common drive to large swathes of cortical regions, or drive other subcortical regions (such as the pulvinar nucleus of the thalamus) that can in turn cause widespread changes in cortical activity (Nakajima and Halassa, 2017; Arcaro et al., 2018). Second, neuromodulatory brainstem systems may also modulate the cortical dynamic state, thereby altering correlations in cortical population activity indirectly. These two mechanisms are non-mutually exclusive: the physiological effect of any neuromodulatory action likely results from a complex mixture of both. Nevertheless, careful experimental manipulations should tease these mechanisms apart and provide insight into the consequences of each mechanism for intrinsic activity fluctuations within the cortex.

For example, in order to test whether neuromodulatory systems induce temporal correlations in the cortex through common drive, one can manipulate the time varying activity of brainstem nuclei, and examine the time varying signature of this manipulation in cortical activity. Using optogenetics, specific neuron types can be targeted such that non-neuromodulatory (e.g., GABAergic) long-range projections that emanate from these nuclei are not directly affected by the experimental manipulation. Moreover, electrophysiological recordings within the cortex would circumvent interpretational caveats that are inherent to the transformation of neural activity into the fMRI signal (Logothetis, 2008). Experiments of this kind have shown promise (Grandjean et al., 2019). Combining such manipulations with the administration of pharmacological blockade of ionotropic receptors could ultimately provide decisive evidence in favor of or against the notion of drive of intrinsic activity correlations by neuromodulators.

Additionally, seed-based correlation studies can provide evidence of co-fluctuating activity in brainstem neuromodulatory nuclei and the cortex in humans. In order to elucidate the relationship between each neuromodulatory system and correlated activity within the cortex, studies are needed in which activity in all nuclei is measured simultaneously, and the covariation between the individual nuclei is taken into account. Such analyses have not yet been conducted, but can be readily implemented with existing techniques.

Another possible means to distinguishing multiple mechanisms of action is to examine the spatial correspondence between the effect of a manipulation of neuromodulators on cortical intrinsic activity correlations, and the distribution of specific receptors. These comparisons are now possible using open-access databases of genetic expression of receptor types (Hawrylycz et al., 2012), validated for use in neuroimaging (Gryglewski et al., 2018). Such studies may benefit from analytical tools that are tailored to distil manipulation-related effects on cortical correlations in a specific direction, without relying on a priori selection of correlated networks (e.g., van den Brink et al., 2018a). Examining the spatial relationship between the effect of a manipulation and the receptors may also be indicative of whether the cortical effect of a neuromodulator is determined primarily by the anatomical projection profile of the nucleus that releases it, or if the receptor distributions weigh more heavily (Grandjean et al., 2019). Moreover, such analyses should be used to contrast the impact of ionotropic and metabotropic receptors.

\section{Developing Mechanistic Models and Biomarkers for Neuropsychiatric Disorders}

A detailed understanding of how neuromodulators shape intrinsic activity correlations may aid the development of novel biomarkers for neurological and psychiatric disorders, as well as mechanistic models of these disorders. Several disorders are associated with dysfunctions in one or multiple neuromodulatory brainstem systems. For example, Parkinson's disease is caused by degeneration of the dopaminergic midbrain nuclei, along with the noradrenergic LC. Cognitive decline in aging, in particular Alzheimer's disease, coincides with degeneration of the cholinergic BF and possibly the LC. Major depression and schizophrenia are associated with disturbances in catecholaminergic and serotonergic systems.

These clear associations with neuromodulatory brainstem systems are currently not exploited for the early detection and classification of such disorders. In particular, the current classification and diagnosis of psychiatric disorders is solely based on subjective assessments of behavioral symptoms, irrespective of the underlying pathophysiological mechanisms (Insel et al., 2010; Krystal and State, 2014). One consequence of this coarse and phenomenological classification scheme is that patient populations in one diagnostic category are often heterogeneous in terms of the neural circuit deficits that give rise to the behavioral symptoms (Seaton et al., 2001; Insel et al., 2010). This hampers the development of individualized treatment plans that target the key circuit disorder that underlies the cognitive or behavioral deficits of a given patient.

The insight that neuromodulators profoundly shape intrinsic activity correlations that are evident with non-invasive neuroimaging techniques opens the door for overcoming these limitations in current clinical practice. The insight sets the stage for the development of neural markers of psychiatric disorders that are directly grounded in the underlying pathomechanisms and cortical signatures of neuromodulatory action. Specifically, the changes in correlation patterns associated with (manipulations of) a specific neuromodulatory system can provide a "reference template" to which alterations of correlation patterns associated with specific disorders can be compared. Such markers may prove to reflect an individual patient's precise deficit more reliably and help identify molecular targets for pharmacological intervention.

In this light, it is important to evaluate the influence of behavioral context on the effect that neuromodulators exert on 
intrinsic activity correlations. Manipulation of neuromodulator levels has been shown to result in opposing effects on intrinsic activity correlations under different behavioral contexts (Coull et al., 1999; Shine et al., 2018b; Pfeffer et al., 2019). Direct comparisons of various neuromodulators within the sameand between different cognitive contexts can thus provide interpretational constraints on alterations of correlation patterns that are associated with psychiatric disorders. Moreover, such an approach may help resolve standing discrepancies in the literature regarding the direction of pharmacological effects on intrinsic activity correlations.

Lastly, neuromodulators interact, through reciprocal connections between the brainstem nuclei, shared cortical afferents, and cortical receptor co-expression. Because of this, dysfunction in a single neuromodulatory system is unlikely to occur without affecting others. Moreover, any neuromodulatory dysfunction that is associated with a psychiatric disorder may not be observable in intrinsic activity correlations as a linear summation of the above described reference templates of the individual neuromodulatory systems that are dysfunctional. Thus, further study on how the joint actions of neuromodulators shape cortical interactions is needed. A starting

\section{REFERENCES}

Achard, S., and Bullmore, E. (2007). Efficiency and cost of economical brain functional networks. PLoS Comput. Biol. 3:e17. doi: 10.1371/journal.pcbi. 0030017

Ahmed, N. Y., Knowles, R., and Dehorter, N. (2019). New insights into cholinergic neuron diversity. Front. Mol. Neurosci. 12:204. doi: 10.3389/fnmol.2019.00204

Ahsan, R. L., Allom, R., Gousias, I. S., Habib, H., Turkheimer, F. E., Free, S., et al. (2007). Volumes, spatial extents and a probabilistic atlas of the human basal ganglia and thalamus. NeuroImage 38, 261-270. doi: 10.1016/j.neuroimage. 2007.06.004

Akeju, O., Loggia, M. L., Catana, C., Pavone, K. J., Vazquez, R., Rhee, J., et al. (2016). Disruption of thalamic functional connectivity is a neural correlate of dexmedetomidine-induced unconsciousness. eLife 3:e04499.

Allen, E. A., Damaraju, E., Plis, S. M., Erhardt, E. B., Eichele, T., and Calhoun, V. D. (2014). Tracking whole-brain connectivity dynamics in the resting state. Cereb. Cortex 24, 663-676. doi: 10.1093/cercor/bhs352

Arcaro, M. J., Pinsk, M. A., Chen, J., and Kastner, S. (2018). Organizing principles of pulvino-cortical functional coupling in humans. Nat. Commun. 9:5382. doi: 10.1038/s41467-018-07725-6

Astafiev, S. V., Snyder, A. Z., Shulman, G. L., and Corbetta, M. (2010). Comment on "modafinil shifts human locus coeruleus to low-tonic, high-phasic activity during functional MRI" and "homeostatic sleep pressure and responses to sustained attention in the suprachiasmatic area”. Science 328:309. doi: 10.1126/ science. 1177200

Baggio, H. C., Segura, B., and Junque, C. (2015). Resting-state functional brain networks in Parkinson's disease. CNS Neurosci. Ther. 21, 793-801. doi: 10.1111/ cns. 12417

Bari, A., and Aston-Jones, G. (2013). Atomoxetine modulates spontaneous and sensory-evoked discharge of locus coeruleus noradrenergic neurons. Neuropharmacology 64, 53-64. doi: 10.1016/j.neuropharm.2012.07.020

Baria, A. T., Mansour, A., Huang, L., Baliki, M. N., Cecchi, G. A., Mesulam, M. M., et al. (2013). Linking human brain local activity fluctuations to structural and functional network architectures. NeuroImage 73, 144-155. doi: 10.1016/j. neuroimage.2013.01.072

Barnes, N. M., Hales, T. G., Lummis, S. C., and Peters, J. A. (2009). The 5-HT3 receptor - the relationship between structure and function. Neuropharmacology 56, 273-284. doi: 10.1016/j.neuropharm.2008.08.003 point is to incorporate receptor co-expression into large-scale computational models of cortical function, combined with coupling terms that link activity of one neuromodulatory system to that of another. This may capture interactions between these systems more accurately, and yield mechanistic insight about how dysfunction in these systems manifests itself at the level of intra-cortical processes and behavior.

\section{AUTHOR CONTRIBUTIONS}

$\mathrm{RB}$ and TD conceived the idea for this article. RB wrote the manuscript. RB, TP, and TD commented on and edited the manuscript.

\section{FUNDING}

This work was supported by a fellowship for postdoctoral researchers funded by the Alexander von Humboldt Foundation (to $\mathrm{RB}$ ), and the following grants from the German Research Foundation (to TD): DO 1240/4-1, SFB 936/A7, and SFB 936/Z3.

Barttfeld, P., Uhrig, L., Sitt, J. D., Sigman, M., Jarraya, B., and Dehaene, S. (2015). Signature of consciousness in the dynamics of resting-state brain activity. Proc. Natl. Acad. Sci. U.S.A. 112, 887-892. doi: 10.1073/pnas.1418031112

Bassett, D. S., and Bullmore, E. (2006). Small-world brain networks. Neuroscientist $12,512-523$.

Bassett, D. S., Meyer-Lindenberg, A., Achard, S., Duke, T., and Bullmore, E. (2006). Adaptive reconfiguration of fractal small-world human brain functional networks. Proc. Natl. Acad. Sci. U.S.A. 103, 19518-19523. doi: 10.1073/pnas. 0606005103

Beas, B. S., Wright, B. J., Skirzewski, M., Leng, Y., Hyun, J. H., Koita, O., et al. (2018). The locus coeruleus drives disinhibition in the midline thalamus via a dopaminergic mechanism. Nat. Neurosci. 21, 963-973. doi: 10.1038/s41593018-0167-4

Beckmann, C. F. (2009). Group Comparison of Resting-State FMRI Data Using Multi-Subject ICA and Dual Regression. Minnesota: OHBM.

Beissner, F. (2015). Functional MRI of the brainstem: common problems and their solutions. Clin. Neuroradiol. 25(Suppl. 2), 251-257. doi: 10.1007/s00062-0150404-0

Beliveau, V., Svarer, C., Frokjaer, V. G., Knudsen, G. M., Greve, D. N., and Fisher, P. M. (2015). Functional connectivity of the dorsal and median raphe nuclei at rest. NeuroImage 116, 187-195. doi: 10.1016/j.neuroimage.2015.04.065

Berridge, C. W., and Waterhouse, B. D. (2003). The locus coeruleus-noradrenergic system: modulation of behavioral state and state-dependent cognitive processes. Brain Res. Rev. 42, 33-84. doi: 10.1016/s0165-0173(03)00143-7

Biswal, B., Yetkin, F. Z., Haughton, V. M., and Hyde, J. S. (1995). Functional connectivity in the motor cortex of resting human brain using echo-planar MRI. Magn. Reson. Med. 34, 537-541. doi: 10.1002/mrm.1910340409

Bock, A. S., Binda, P., Benson, N. C., Bridge, H., Watkins, K. E., and Fine, I. (2015). Resting-state retinotopic organization in the absence of retinal input and visual experience. J. Neurosci. 35, 12366-12382. doi: 10.1523/JNEUROSCI.4715-14. 2015

Breakspear, M., Terry, J. R., and Friston, K. J. (2009). Modulation of excitatory synaptic coupling facilitates synchronization and complex dynamics in a biophysical model of neuronal dynamics. Network 14, 703-732. doi: 10.1088/ 0954-898x_14_4_305

Breton-Provencher, V., and Sur, M. (2019). Active control of arousal by a locus coeruleus GABAergic circuit. Nat. Neurosci. 22, 218-228. doi: 10.1038/s41593018-0305-z 
Brooks, J. C., Faull, O. K., Pattinson, K. T., and Jenkinson, M. (2013). Physiological noise in brainstem FMRI. Front. Hum. Neurosci. 7:623. doi: 10.3389/fnhum. 2013.00623

Bymaster, F. P., Katner, J. S., Nelson, D. L., Hemrick-luecke, S. K., Threlkeld, P. G., Heiligenstein, J. H., et al. (2002). Atomoxetine increases extracellular levels of norepinephrine and dopamine in prefrontal cortex of rat: a potential mechanism for efficacy in attention deficit/hyperactivity disorder. Neuropsychopharmacology 27, 699-711. doi: 10.1016/s0893-133x(02)00346-9

Cabral, J., Hugues, E., Kringelbach, M. L., and Deco, G. (2012). Modeling the outcome of structural disconnection on resting-state functional connectivity. NeuroImage 62, 1342-1353. doi: 10.1016/j.neuroimage.2012.06.007

Calhoun, V. D., Eichele, T., and Pearlson, G. (2009). Functional brain networks in schizophrenia: a review. Front. Hum. Neurosci. 3:17. doi: 10.3389/neuro.09.017. 2009

Carhart-Harris, R. L., Muthukumaraswamy, S., Roseman, L., Kaelen, M., Droog, W., Murphy, K., et al. (2016). Neural correlates of the LSD experience revealed by multimodal neuroimaging. Proc. Natl. Acad. Sci. U.S.A. 113, 4853-4858. doi: $10.1073 /$ pnas. 1518377113

Cerpa, J. C., Marchand, A. R., and Coutureau, E. (2019). Distinct regional patterns in noradrenergic innervation of the rat prefrontal cortex. J. Chem. Neuroanat. 96, 102-109. doi: 10.1016/j.jchemneu.2019.01.002

Chandler, D., and Waterhouse, B. D. (2012). Evidence for broad versus segregated projections from cholinergic and noradrenergic nuclei to functionally and anatomically discrete subregions of prefrontal cortex. Front. Behav. Neurosci. 6:20. doi: $10.3389 /$ fnbeh. 2012.00020

Chandler, D. J., Gao, W.-J., and Waterhouse, B. D. (2014). Heterogeneous organization of the locus coeruleus projections to prefrontal and motor cortices. Proc. Natl. Acad. Sci. U.S.A. 111, 6816-6821. doi: 10.1073/pnas.1320827111

Chang, C., and Glover, G. H. (2010). Time-frequency dynamics of resting-state brain connectivity measured with fMRI. NeuroImage 50, 81-98. doi: 10.1016/ j.neuroimage.2009.12.011

Chaudhuri, R., Knoblauch, K., Gariel, M. A., Kennedy, H., and Wang, X. J. (2015). A large-scale circuit mechanism for hierarchical dynamical processing in the primate cortex. Neuron 88, 419-431. doi: 10.1016/j.neuron.2015. 09.008

Cho, J. R., Treweek, J. B., Robinson, J. E., Xiao, C., Bremner, L. R., Greenbaum, A., et al. (2017). Dorsal raphe dopamine neurons modulate arousal and promote wakefulness by salient stimuli. Neuron 94, 1205.e8-1219.e8.

Cohen, M. R., and Kohn, A. (2011). Measuring and interpreting neuronal correlations. Nat. Neurosci. 14, 811-819. doi: 10.1038/nn.2842

Cole, D. M., Beckmann, C. F., Oei, N. Y., Both, S., van Gerven, J. M., and Rombouts, S. A. (2013). Differential and distributed effects of dopamine neuromodulations on resting-state network connectivity. NeuroImage 78, 59-67. doi: 10.1016/j. neuroimage.2013.04.034

Cole, M. W., Ito, T., Bassett, D. S., and Schultz, D. H. (2016). Activity flow over resting-state networks shapes cognitive task activations. Nat. Neurosci. 19, 1718-1726. doi: 10.1038/nn.4406

Coull, J. T., Büchel, C., Friston, K. J., and Firth, C. D. (1999). Noradrenergically mediated plasticity in a human attentional neuronal network. NeuroImage 10, 705-715. doi: 10.1006/nimg.1999.0513

Curto, C., Sakata, S., Marguet, S., Itskov, V., and Harris, K. D. (2009). A simple model of cortical dynamics explains variability and state dependence of sensory responses in urethane-anesthetized auditory cortex. J. Neurosci. 29, 1060010612. doi: 10.1523/JNEUROSCI.2053-09.2009

de Gee, J. W., Colizoli, O., Kloosterman, N. A., Knapen, T., Nieuwenhuis, S., and Donner, T. (2017). Dynamic modulation of decision biases by brainstem arousal systems. eLife 6:e23232.

de Pasquale, F., Della Penna, S., Snyder, A. Z., Lewis, C., Mantini, D., Marzetti, L., et al. (2010). Temporal dynamics of spontaneous MEG activity in brain networks. Proc. Natl. Acad. Sci. U.S.A. 107, 6040-6045. doi: 10.1073/pnas. 0913863107

Deco, G., Jirsa, V. K., and McIntosh, A. R. (2011). Emerging concepts for the dynamical organization of resting-state activity in the brain. Nat. Rev. Neurosci. 12, 43-56. doi: 10.1038/nrn2961

Deco, G., Ponce-Alvarez, A., Mantini, D., Romani, G. L., Hagmann, P., and Corbetta, M. (2013). Resting-state functional connectivity emerges from structurally and dynamically shaped slow linear fluctuations. J. Neurosci. 33, 11239-11252. doi: 10.1523/JNEUROSCI.1091-13.2013
Deco, G., Ponce-Alvarez, A., Hagmann, P., Romani, G. L., Mantini, D., and Corbetta, M. (2014). How local excitation-inhibition ratio impacts the whole brain dynamics. J. Neurosci. 34, 7886-7898. doi: 10.1523/JNEUROSCI.5068-13. 2014

Deco, G., Cruzat, J., Cabral, J., Knudsen, G. M., Carhart-Harris, R. L., Whybrow, P. C., et al. (2018). Whole-brain multimodal neuroimaging model using serotonin receptor maps explains non-linear functional effects of LSD. Curr. Biol. 28, 3065.e6-3074.e6.

Devoto, P., Flore, G., Pira, L., Longu, G., and Gessa, G. L. (2004). Alpha2adrenoceptor mediated co-release of dopamine and noradrenaline from noradrenergic neurons in the cerebral cortex. J. Neurochem. 88, 1003-1009. doi: 10.1046/j.1471-4159.2003.02239.x

Dichter, G. S., Gibbs, D., and Smoski, M. J. (2015). A systematic review of relations between resting-state functional-MRI and treatment response in major depressive disorder. J. Affect. Disord. 172, 8-17. doi: 10.1016/j.jad.2014. 09.028

Disney, A. A., Aoki, C., and Hawken, M. J. (2007). Gain modulation by nicotine in macaque v1. Neuron 56, 701-713. doi: 10.1016/j.neuron.2007.09.034

Donner, T. H., Sagi, D., Bonneh, Y. S., and Heeger, D. J. (2013). Retinotopic patterns of correlated fluctuations in visual cortex reflect the dynamics of spontaneous perceptual suppression. J. Neurosci. 33, 2188-2198. doi: 10.1523/JNEUROSCI. 3388-12.2013

Drew, P. J., Duyn, J. H., Golanov, E., and Kleinfeld, D. (2008). Finding coherence in spontaneous oscillations. Nat. Neurosci. 11, 991-993. doi: 10.1038/nn0908-991

Eschenko, O., Magri, C., Panzeri, S., and Sara, S. J. (2012). Noradrenergic neurons of the locus coeruleus are phase locked to cortical up-down states during sleep. Cereb. Cortex 22, 426-435. doi: 10.1093/cercor/bhr121

FitzHugh, R. (1961). Impuses and physiological states in theoretical models of nerve membrane. Biophys. J. 1, 445-466. doi: 10.1016/s0006-3495(61)86902-6

Florin-Lechner, S. M., Druhan, J. P., Aston-Jones, G., and Valentino, R. J. (1996). Enhanced norepinephrine release in the prefrontal cortex with burst stimulation of the locus coeruleus. Brain Res. 742, 89-97. doi: 10.1016/s00068993(96)00967-5

Foote, S. L., and Morrison, J. H. (1987). Extrathalamic modulation of cortical function. Ann. Rev. Neurosci. 10, 67-95. doi: 10.1146/annurev.neuro.10.1.67

Forstmann, B. U., Hollander, G. D., Maanen, L. V., Alkemade, A., and Keuken, M. C. (2017). Towards a mechanistic understanding of the human subcortex. Nat. Rev. Neurosci. 18, 57-65. doi: 10.1038/nrn.2016.163

Fox, M. D., and Raichle, M. E. (2007). Spontaneous fluctuations in brain activity observed with functional magnetic resonance imaging. Nat. Rev. Neurosci. 8, 700-711. doi: 10.1038/nrn2201

Fox, M. D., and Greicius, M. (2010). Clinical applications of resting state functional connectivity. Front. Syst. Neurosci 4:19. doi: 10.3389/fnsys.2010.00019

French, I. T., and Muthusamy, K. A. (2018). A review of the pedunculopontine nucleus in Parkinson's disease. Front. Aging Neurosci. 10:99. doi: 10.3389/fnagi. 2018.00099

Friston, K. J., Firth, C. D., Liddle, P. F., and Frackowiak, R. S. J. (1993). Functional connectivity: the principal-component analysis of large (PET) data sets. J. Cereb. Blood Flow Metab. 13, 5-14. doi: 10.1038/jcbfm.1993.4

Froemke, R. C. (2015). Plasticity of cortical excitatory-inhibitory balance. Annu. Rev. Neurosci. 38, 195-219. doi: 10.1146/annurev-neuro-071714-034002

Fu, Y., Tucciarone, J. M., Espinosa, J. S., Sheng, N., Darcy, D. P., Nicoll, R. A., et al. (2014). A cortical circuit for gain control by behavioral state. Cell 156, 1139-1152. doi: 10.1016/j.cell.2014.01.050

Garcia-Rill, E. (1991). The pedunculopontine nucleus. Prog. Neurobiol. 36, 363-389. doi: 10.1016/0301-0082(91)90016-t

Gerlicher, A. M. V., Tuscher, O., and Kalisch, R. (2018). Dopamine-dependent prefrontal reactivations explain long-term benefit of fear extinction. Nat. Commun. 9:4294. doi: 10.1038/s41467-018-06785-y

Giorgi, A., Migliarini, S., Galbusera, A., Maddaloni, G., Mereu, M., Margiani, G., et al. (2017). Brain-wide mapping of endogenous serotonergic transmission via chemogenetic fMRI. Cell Rep. 21, 910-918. doi: 10.1016/j.celrep.2017.09.087

Giraldo-Chica, M., and Woodward, N. D. (2017). Review of thalamocortical resting-state fMRI studies in schizophrenia. Schizophr. Res. 180, 58-63. doi: 10.1016/j.schres.2016.08.005

Grandjean, J., Corcoba, A., Kahn, M. C., Upton, A. L., Deneris, E. S., Seifritz, E., et al. (2019). A brain-wide functional map of the serotonergic responses to acute stress and fluoxetine. Nat. Commun. 10:350. doi: 10.1038/s41467-018-08256-w 
Gravel, N., Harvey, B., Nordhjem, B., Haak, K. V., Dumoulin, S. O., Renken, R., et al. (2014). Cortical connective field estimates from resting state fMRI activity. Front. Neurosci. 8:339. doi: 10.3389/fnins.2014.00339

Gryglewski, G., Seiger, R., James, G. M., Godbersen, G. M., Komorowski, A., Unterholzner, J., et al. (2018). Spatial analysis and high resolution mapping of the human whole-brain transcriptome for integrative analysis in neuroimaging. NeuroImage 176, 259-267. doi: 10.1016/j.neuroimage.2018.04.068

Guedj, C., Meunier, D., Meunier, M., and Hadj-Bouziane, F. (2017a). Could LC-NE-dependent adjustment of neural gain drive functional brain network reorganization? Neural Plast. 2017:4328015. doi: 10.1155/2017/4328015

Guedj, C., Monfardini, E., Reynaud, A. J., Farne, A., Meunier, M., and HadjBouziane, F. (2017b). Boosting norepinephrine transmission triggers flexible reconfiguration of brain networks at rest. Cereb. Cortex 27, 4691-4700. doi: 10.1093/cercor/bhw262

Haas, H., and Panula, P. (2003). The role of histamine and the tuberomamillary nucleus in the nervous system. Nat. Rev. Neurosci. 4, 121-130. doi: 10.1038/ nrn 1034

Harris, K. D., and Thiele, A. (2011). Cortical state and attention. Nat. Rev. Neurosci. 12, 509-523. doi: 10.1038/nrn3084

Hawrylycz, M. J., Lein, S., Guillozet-Bongaarts, A. L., Shen, E. H., Ng, L., Miller, J. A., et al. (2012). An anatomically comprehensive atlas of the adult human brain transcriptome. Nature 489, 391-399. doi: 10.1038/nature11405

Heinzle, J., Kahnt, T., and Haynes, J. D. (2011). Topographically specific functional connectivity between visual field maps in the human brain. NeuroImage 56, 1426-1436. doi: 10.1016/j.neuroimage.2011.02.077

Hermans, E., Marle, H. V., Ossewaarde, L., Menckens, M., Qin, S., Kesteren, M. V., et al. (2011). Stress-related noradrenergic activity prompts large-scale neural network reconfiguration. Science 334, 1151-1153. doi: 10.1126/science. 1209603

Hipp, J. F., and Siegel, M. (2015). BOLD fMRI correlation reflects frequencyspecific neuronal correlation. Curr. Biol. 25, 1368-1374. doi: 10.1016/j.cub. 2015.03.049

Hipp, J. F., Hawellek, D. J., Corbetta, M., Siegel, M., and Engel, A. K. (2012). Large-scale cortical correlation structure of spontaneous oscillatory activity. Nat. Neurosci. 15, 884-890. doi: 10.1038/nn.3101

Hollensteiner, K. J., Galindo-Leon, E., Pieper, F., Engler, G., Nolte, G., and Engel, A. K. (2019). Large-scale functional connectivity in multisensory cortex predicts performance. BioRxiv

Honey, C. J., Kotter, R., Breakspear, M., and Sporns, O. (2007). Network structure of cerebral cortex shapes functional connectivity on multiple time scales. Proc. Natl. Acad. Sci. U.S.A. 104, 10240-10245. doi: 10.1073/pnas.070151 9104

Honey, C. J., Sporns, O., Cammoun, L., Gigandet, X., Thiran, J. P., Meuli, R., et al. (2009). Predicting human resting-state functional connectivity from structural connectivity. Proc. Natl. Acad. Sci. U.S.A. 106, 2035-2040. doi: 10.1073/pnas. 0811168106

Insel, T., Cuthbert, B., Garvey, M., Heinssen, R., Pine, D. S., Quinn, K., et al. (2010). Research domain criteria (RDoC): toward a new classification framework for research on mental disorders. Am. J. Psychiatry 167, 748-751. doi: 10.1176/ appi.ajp.2010.09091379

Itier, V., and Bertrand, D. (2001). Neuronal nicotinic receptors: from protein structure to function. FEBS Lett. 504, 118-125. doi: 10.1016/s0014-5793(01) 02702-8

Joshi, S., and Gold, J. I. (2019). Context-Dependent Relationships between Locus Coeruleus Activation and Coordinated Neural Activity Patterns in the Anterior Cingulate Cortex SSRN. Available at: https://ssrn.com/abstract= 3413098 (accessed July 1, 2019).

Joshi, S., Li, Y., Kalwani Rishi, M., and Gold Joshua, I. (2016). Relationships between pupil diameter and neuronal activity in the locus coeruleus, colliculi, and cingulate cortex. Neuron 89, 221-234. doi: 10.1016/j.neuron.2015. 11.028

Kebschull, J. M., Garcia da Silva, P., Reid, A. P., Peikon, I. D., Albeanu, D. F., and Zador, A. M. (2016). High-throughput mapping of single-neuron projections by sequencing of barcoded RNA. Neuron 91, 975-987. doi: 10.1016/j.neuron. 2016.07.036

Kelly, C., de Zubicaray, G., Di Martino, A., Copland, D. A., Reiss, P. T., Klein, D. F., et al. (2009). L-dopa modulates functional connectivity in striatal cognitive and motor networks: a double-blind placebo-controlled study. J. Neurosci. 29, 7364-7378. doi: 10.1523/JNEUROSCI.0810-09.2009
Keren, N. I., Lozar, C. T., Harris, K. C., Morgan, P. S., and Eckert, M. A. (2009). In vivo mapping of the human locus coeruleus. NeuroImage 47, 1261-1267. doi: 10.1016/j.neuroimage.2009.06.012

Klaassens, B. L., Rombouts, S. A., Winkler, A. M., van Gorsel, H. C., van der Grond, J., and van Gerven, J. M. (2017). Time related effects on functional brain connectivity after serotonergic and cholinergic neuromodulation. Hum. Brain Mapp. 38, 308-325. doi: 10.1002/hbm.23362

Klaassens, B. L., van Gorsel, H. C., Khalili-Mahani, N., van der Grond, J., Wyman, B. T., Whitcher, B., et al. (2015). Single-dose serotonergic stimulation shows widespread effects on functional brain connectivity. NeuroImage 122, 440-450. doi: 10.1016/j.neuroimage.2015.08.012

Klein-Flugge, M. C., Hunt, L. T., Bach, D. R., Dolan, R. J., and Behrens, T. E. (2011). Dissociable reward and timing signals in human midbrain and ventral striatum. Neuron 72, 654-664. doi: 10.1016/j.neuron.2011.08.024

Koda, K., Ago, Y., Cong, Y., Kita, Y., Takuma, K., and Matsuda, T. (2010). Effects of acute and chronic administration of atomoxetine and methylphenidate on extracellular levels of noradrenaline, dopamine and serotonin in the prefrontal cortex and striatum of mice. J. Neurochem. 114, 259-270. doi: 10.1111/j.14714159.2010.06750.x

Kohn, A., Coen-Cagli, R., Kanitscheider, I., and Pouget, A. (2016). Correlations and neuronal population information. Annu. Rev. Neurosci. 39, 237-256. doi: 10.1146/annurev-neuro-070815-013851

Krystal, J. H., and State, M. W. (2014). Psychiatric disorders: diagnosis to therapy. Cell 157, 201-214. doi: 10.1016/j.cell.2014.02.042

Leopold, D. A., Murayama, Y., and Logothetis, N. K. (2003). Very slow activity fluctuations in monkey visual cortex: implications for functional brain imaging. Cereb. Cortex 13, 422-433. doi: 10.1093/cercor/13.4.422

Lewis, C. M., Bosman, C. A., Womelsdorf, T., and Fries, P. (2016). Stimulusinduced visual cortical networks are recapitulated by spontaneous local and interareal synchronization. Proc. Natl. Acad. Sci. U.S.A. 113, E606-E615. doi: $10.1073 /$ pnas. 1513773113

Li, C. S., Ide, J. S., Zhang, S., Hu, S., Chao, H. H., and Zaborszky, L. (2014). Resting state functional connectivity of the basal nucleus of meynert in humans: in comparison to the ventral striatum and the effects of age. NeuroImage 97, 321-332. doi: 10.1016/j.neuroimage.2014.04.019

Lin, S. C., Brown, R. E., Hussain Shuler, M. G., Petersen, C. C., and Kepecs, A. (2015). Optogenetic dissection of the basal forebrain neuromodulatory control of cortical activation, plasticity, and cognition. J. Neurosci. 35, 13896-13903. doi: 10.1523/JNEUROSCI.2590-15.2015

Liu, X., de Zwart, J. A., Scholvinck, M. L., Chang, C., Ye, F. Q., Leopold, D. A., et al. (2018). Subcortical evidence for a contribution of arousal to fMRI studies of brain activity. Nat. Commun. 9:395. doi: 10.1038/s41467-017-02815-3

Logothetis, N. K. (2008). What we can do and what we cannot do with fMRI. Nature 453, 869-878. doi: 10.1038/nature06976

Lord, L.-D., Expert, P., Atasoy, S., Roseman, L., Rapuano, K., Lambiotte, R., et al. (2018). Altered trajectories in the dynamical repertoire of functional network states under psilocybin. BioRxiv

Luczak, A., Bartho, P., and Harris, K. D. (2009). Spontaneous events outline the realm of possible sensory responses in neocortical populations. Neuron 62 , 413-425. doi: 10.1016/j.neuron.2009.03.014

Lurie, D. J., Kessler, D., Bassett, D., Betzel, R., Breakspear, M., Keilholz, S., et al. (2018). On the nature of resting fMRI and time-varying functional connectivity. PsyRxiv

Maier, A., Wilke, M., Aura, C., Zhu, C., Ye, F. Q., and Leopold, D. A. (2008). Divergence of fMRI and neural signals in V1 during perceptual suppression in the awake monkey. Nat. Neurosci. 11, 1193-1200. doi: 10.1038/nn.2173

Mao, B.-W., Hamzei-Sichani, F., Arnov, D., Froemke, R. C., and Yuste, R. (2001). Dynamics of spontaneous activity in neocortical slices. Neuron 32, 883-898. doi: 10.1016/s0896-6273(01)00518-9

Markello, R. D., Spreng, R. N., Luh, W. M., Anderson, A. K., and De Rosa, E. (2018). Segregation of the human basal forebrain using resting state functional MRI. NeuroImage 173, 287-297. doi: 10.1016/j.neuroimage.2018.02.042

Martins, A. R., and Froemke, R. C. (2015). Coordinated forms of noradrenergic plasticity in the locus coeruleus and primary auditory cortex. Nat. Neurosci. 18, 1483-1492. doi: 10.1038/nn.4090

Mattar, M. G., Cole, M. W., Thompson-Schill, S. L., and Bassett, D. S. (2015). A Functional Cartography of Cognitive Systems. PLoS Comput. Biol. 11:e1004533. doi: 10.1371/journal.pcbi.1004533 
McCabe, C., and Mishor, Z. (2011). Antidepressant medications reduce subcortical-cortical resting-state functional connectivity in healthy volunteers. NeuroImage 57, 1317-1323. doi: 10.1016/j.neuroimage.2011.05.051

McCabe, C., Mishor, Z., Filippini, N., Cowen, P. J., Taylor, M. J., and Harmer, C. J. (2011). SSRI administration reduces resting state functional connectivity in dorso-medial prefrontal cortex. Mol. Psychiatry 16, 592-594. doi: 10.1038/ mp. 2010.138

McCormick, D. A., and Nusbaum, M. P. (2014). Editorial overview: neuromodulation: tuning the properties of neurons, networks and behavior. Curr. Opin. Neurobiol. 29, 4-7.

McGinley, M. J., Vinck, M., Reimer, J., Batista-Brito, R., Zagha, E., Cadwell, C. R., et al. (2015). Waking state: rapid variations modulate neural and behavioral responses. Neuron 87, 1143-1161. doi: 10.1016/j.neuron.2015. 09.012

Meindertsma, T., Kloosterman, N. A., Nolte, G., Engel, A. K., and Donner, T. H. (2017). Multiple transient signals in human visual cortex associated with an elementary decision. J. Neurosci. 37, 5744-5757. doi: 10.1523/JNEUROSCI. 3835-16.2017

Mesulam, M. M., and van Hoesen, G. W. (1976). Acetylcholinesterase-rich projections from the basal forebrain of the rhesus monkey to neocortex. Brain Res. 109, 152-157. doi: 10.1016/0006-8993(76)90385-1

Mesulam, M. M., and Changiz, G. (1988). Nucleus basalis (CH4) and cortial cholinergic innnervation in the human brain: observations based on the distribution of acetylcholinesterase and choline acetyltransferase. J. Comp. Neurol. 257, 216-240. doi: 10.1002/cne.902750205

Mesulam, M. M., Mufson, E. J., Levey, A. I., and Wainer, B. H. (1983). Cholinergic innervation of cortex by the basal forebrain: cytochemistry and cortical connections of the septal area, diagonal band nuclei, nucleus basalis (substantia Innominata), and hypothalamus in the rhesus monkey. J. Comp. Neurol. 214, 170-197. doi: 10.1002/cne.902140206

Metzger, C. D., Wiegers, M., Walter, M., Abler, B., and Graf, H. (2015). Local and global resting state activity in the noradrenergic and dopaminergic pathway modulated by reboxetine and amisulpride in healthy subjects. Int. J. Neuropsychopharmacol. 9:pyv080. doi: 10.1093/ijnp/pyv080

Moises, H. C., Woodward, B. J., Hoffer, B. J., and Freedman, R. (1979). Interactions of norepinephrine with Purkinje cell responses to putative amino acid neurotransmitters applied by microiontophoresis. Exp. Neurol. 64, 493-515. doi: 10.1016/0014-4886(79)90227-9

Mulders, P. C., van Eijndhoven, P. F., Schene, A. H., Beckmann, C. F., and Tendolkar, I. (2015). Resting-state functional connectivity in major depressive disorder: a review. Neurosc. Biobehav. Rev. 56, 330-344. doi: 10.1016/j. neubiorev.2015.07.014

Murphy, B. K., and Miller, K. D. (2003). Multiplicative gain changes are induced by excitation or inhibition alone. J. Neurosci. 23, 10040-10051. doi: 10.1523/ jneurosci.23-31-10040.2003

Murphy, P. R., O’Connell, R. G., O’Sullivan, M., Robertson, I. H., and Balsters, J. H. (2014). Pupil diameter covaries with BOLD activity in human locus coeruleus. Hum. Brain Mapp. 35, 4140-4154. doi: 10.1002/hbm.22466

Murray, J. D., Bernacchia, A., Freedman, D. J., Romo, R., Wallis, J. D., Cai, X., et al. (2014). A hierarchy of intrinsic timescales across primate cortex. Nat. Neurosci. 17, 1661-1663. doi: 10.1038/nn.3862

Nagumo, J., Arimoto, S., and Yoshizawa, S. (1962). An active pulse transmission line simulating nerve axon. Proc. Inst. Radio Eng. 50, 2061-2070. doi: 10.1109/ jrproc. 1962.288235

Nahimi, A., Jakobsen, S., Munk, O. L., Vang, K., Phan, J. A., Rodell, A., et al. (2015). Mapping alpha2 adrenoceptors of the human brain with $11 \mathrm{C}$-yohimbine. J. Nucl. Med. 56, 392-398. doi: 10.2967/jnumed.114.145565

Nakajima, M., and Halassa, M. M. (2017). Thalamic control of functional cortical connectivity. Curr. Opin. Neurobiol. 44, 127-131. doi: 10.1016/j.conb.2017.04. 001

Nienborg, H., Cohen, M. R., and Cumming, B. G. (2012). Decision-related activity in sensory neurons: correlations among neurons and with behavior. Annu. Rev. Neurosci. 35, 463-483. doi: 10.1146/annurev-neuro-062111-150403

Nir, Y., Mukamel, R., Dinstein, I., Privman, E., Harel, M., Fisch, L., et al. (2008). Interhemispheric correlations of slow spontaneous neuronal fluctuations revealed in human sensory cortex. Nat. Neurosci. 11, 1100-1108. doi: 10.1038/ nn. 2177
O’Reilly, J. X., Croxson, P. L., Jbabdi, S., Sallet, J., Noonan, M. P., Mars, R. B., et al. (2013). Causal effect of disconnection lesions on interhemispheric functional connectivity in rhesus monkeys. Proc. Natl. Acad. Sci. U.S.A. 10, 13982-13987. doi: 10.1073/pnas.1305062110

Pfeffer, T., Avramiea, A. E., Nolte, G., Engel, A. K., Linkenkaer-Hansen, K., and Donner, T. H. (2018). Catecholamines alter the intrinsic variability of cortical population activity and perception. PLoS Biol. 16:e2003453. doi: 10. 1371/journal.pbio.2003453

Pfeffer, T., Ponce-Alvarez, A., van den Brink, R. L., Engel, A. K., Nolte, G., Deco, G., et al. (2019). Double-Dissociation Between Catecholaminergic and Cholinergic Effects on Cortex-Wide Intrinsic Correlations of Neural Activity. Lisabon: Cosyne.

Pignatelli, A., and Belluzzi, O. (2017). Dopaminergic neurones in the main olfactory bulb: an overview from an electrophysiological perspective. Front. Neuroanat. 11:7. doi: 10.3389/fnana.2017.00007

Pinto, L., Goard, M. J., Estandian, D., Xu, M., Kwan, A. C., Lee, S. H., et al. (2013). Fast modulation of visual perception by basal forebrain cholinergic neurons. Nat. Neurosci. 16, 1857-1863. doi: 10.1038/nn.3552

Polack, P. O., Friedman, J., and Golshani, P. (2013). Cellular mechanisms of brain state-dependent gain modulation in visual cortex. Nat. Neurosci. 16, 1331-1339. doi: 10.1038/nn.3464

Preller, K. H., Bur, J. B., Ji, J. L., Charles, H., Schleifer, Adkinson, B. D., et al. (2018). Changes in global and thalamic brain connectivity in LSD-induced altered states of consciousness are attributable to the 5-HT2A receptor. eLife 7, e35082. doi: 10.7554/eLife.35082

Puig, M. V., Santana, N., Celada, P., Mengod, G., and Artigas, F. (2004). In vivo excitation of GABA interneurons in the medial prefrontal cortex through 5-HT3 receptors. Cereb. Cortex 14, 1365-1375. doi: 10.1093/cercor/bhh097

Ramos, B. P., and Arnsten, A. F. (2007). Adrenergic pharmacology and cognition: focus on the prefrontal cortex. Pharmacol. Ther. 113, 523-536. doi: 10.1016/j. pharmthera.2006.11.006

Reimer, J., Froudarakis, E., Cadwell, C. R., Yatsenko, D., Denfield, G. H., and Tolias, A. S. (2014). Pupil fluctuations track fast switching of cortical states during quiet wakefulness. Neuron 84, 355-362. doi: 10.1016/j.neuron.2014.09.033

Rho, H. J., Kim, J. H., and Lee, S. H. (2018). Function of selective neuromodulatory projections in the mammalian cerebral cortex: comparison between cholinergic and noradrenergic systems. Front. Neural Circuits 12:47. doi: 10.3389/fncir. 2018.00047

Rogawksi, M. A., and Aghajanian, G. K. (1980). Modulation of lateral geniculate neurone excitability by noradrenaline microiontophoresis or locus ceuruleus stimulation. Nature 287, 731-734. doi: 10.1038/287731a0

Rosazza, C., and Minati, L. (2011). Resting-state brain networks: literature review and clinical applications. Neurol. Sci. 32, 773-785. doi: 10.1007/s10072-0110636-y

Rubinov, M., and Sporns, O. (2010). Complex network measures of brain connectivity: uses and interpretations. NeuroImage 52, 1059-1069. doi: 10.1016/j.neuroimage.2009.10.003

Safaai, H., Neves, R., Eschenko, O., Logothetis, N. K., and Panzeri, S. (2015). Modeling the effect of locus coeruleus firing on cortical state dynamics and single-trial sensory processing. Proc. Natl. Acad. Sci. U.S.A. 112, 12834-12839. doi: 10.1073/pnas.1516539112

Sakoglu, U., Pearlson, G. D., Kiehl, K. A., Wang, Y. M., Michael, A. M., and Calhoun, V. D. (2010). A method for evaluating dynamic functional network connectivity and task-modulation: application to schizophrenia. MAGMA 23, 351-366. doi: 10.1007/s10334-010-0197-8

Salgado, H., Trevino, M., and Atzori, M. (2016). Layer- and area-specific actions of norepinephrine on cortical synaptic transmission. Brain Res. 1641, 163-176. doi: 10.1016/j.brainres.2016.01.033

Sara, S. J. (2009). The locus coeruleus and noradrenergic modulation of cognition. Nat. Rev. Neurosci. 10, 211-223. doi: 10.1038/nrn2573

Schaefer, A., Burmann, I., Regenthal, R., Arelin, K., Barth, C., Pampel, A., et al. (2014). Serotonergic modulation of intrinsic functional connectivity. Curr. Biol. 24, 2314-2318. doi: 10.1016/j.cub.2014.08.024

Schwarz, L. A., and Luo, L. (2015). Organization of the locus coeruleusnorepinephrine system. Curr. Biol. 25, R1051-R1056.

Schwarz, L. A., Miyamichi, K., Gao, X. J., Beier, K. T., Weissbourd, B., DeLoach, K. E., et al. (2015). Viral-genetic tracing of the input-output organization 
of a central noradrenaline circuit. Nature 524, 88-92. doi: 10.1038/nature 14600

Seamans, J. K., Gorelova, N., Durstewitz, D., and Yang, C. R. (2001b). Bidirectional dopamine modulation of GABAergic inhibition in prefrontal cortical pyramidal neurons. J. Neurosci. 21, 3628-3638. doi: 10.1523/jneurosci.21-10-03628. 2001

Seamans, J. K., Durstewitz, D., Christie, B. R., Stevens, C. F., and Sejnowski, T. J. (2001a). Dopamine D1/D5 receptor modulation of excitatory synaptic inputs to layer V prefrontal cortex neurons. Proc. Natl. Acad. Sci. U.S.A. 98, 301-306. doi: 10.1073/pnas.011518798

Seaton, B. E., Goldstein, G., and Allen, D. N. (2001). Sources of heterogeneity in schizophrenia: the role of neropsychological functioning. Neuropsychol. Rev. 11, 45-67.

Servan-Schreiber, D., Printz, H., and Cohen, J. D. (1990). A network model of catecholamine effects: gain, signal-to-noise ratio, and behavior. Science 249, 892-895. doi: 10.1126/science.2392679

Shafiei, G., Zeighami, Y., Clark, C. A., Coull, J. T., Nagano-Saito, A., Leyton, M., et al. (2019). Dopamine signaling modulates the stability and integration of intrinsic brain networks. Cereb. Cortex 29, 397-409. doi: 10.1093/cercor/ bhy264

Shine, J. M., and Poldrack, R. A. (2018). Principles of dynamic network reconfiguration across diverse brain states. NeuroImage 180, 396-405. doi: 10.1016/j.neuroimage.2017.08.010

Shine, J. M., Aburn, M. J., Breakspear, M., and Poldrack, R. A. (2018a). The modulation of neural gain facilitates a transition between functional segregation and integration in the brain. eLife 7, e31130. doi: 10.7554/eLife. 31130

Shine, J. M., van den Brink, R. L., Hernaus, D., Nieuwenhuis, S., and Poldrack, R. A. (2018b). Catecholaminergic manipulation alters dynamic network topology across cognitive states. Netw. Neurosci. 2, 381-396. doi: 10.1162/netn a_00042

Shine, J. M., Bissett, P. G., Bell, P. T., Koyejo, O., Balsters, J. H., Gorgolewski, K. J., et al. (2016). The dynamics of functional brain networks: integrated network states during cognitive task performance. Neuron 92, 544-554. doi: 10.1016/j. neuron.2016.09.018

Siems, M., Pape, A. A., Hipp, J. F., and Siegel, M. (2016). Measuring the cortical correlation structure of spontaneous oscillatory activity with EEG and MEG. NeuroImage 129, 345-355. doi: 10.1016/j.neuroimage.2016.01.055

Smith, S. M., Fox, P. T., Miller, K. L., Glahn, D. C., Fox, P. M., Mackay, C. E., et al. (2009). Correspondence of the brain's functional architecture during activation and rest. Proc. Natl. Acad. Sci. U.S.A. 106, 13040-13045. doi: 10.1073/pnas. 0905267106

Sporns, O., and Zwi, J. D. (2004). The small world of the cerebral cortex. Neuroinformatics 2, 145-162. doi: 10.1385/ni:2:2:145

Statoh, K., and Fibiger, H. C. (1986). Cholinergic neurons of the laterodorsal tegmental nucleus: efferent and afferent connections. J. Comp. Neurol. 353, 277-302. doi: 10.1002/cne.902530302

Stitt, I., Zhou, Z. C., Radtke-Schuller, S., and Frohlich, F. (2018). Arousal dependent modulation of thalamo-cortical functional interaction. Nat. Commun. 9:2455. doi: 10.1038/s41467-018-04785-6

Swanson, C. J., Perry, K. W., Koch-Krueger, S., Katner, J., Svensson, K. A., and Bymaster, F. P. (2006). Effect of the attention deficit/hyperactivity disorder drug atomoxetine on extracellular concentrations of norepinephrine and dopamine in several brain regions of the rat. Neuropharmacology 50, 755-760. doi: 10 . 1016/j.neuropharm.2005.11.022

Tagliazucchi, E., Roseman, L., Kaelen, M., Orban, C., Muthukumaraswamy, S. D., Murphy, K., et al. (2016). Increased global functional connectivity correlates with lsd-induced ego dissolution. Curr. Biol. 26, 1043-1050. doi: 10.1016/j.cub. 2016.02.010

Takeuchi, T., Duszkiewicz, A. J., Sonneborn, A., Spooner, P. A., Yamasaki, M., Watanabe, M., et al. (2016). Locus coeruleus and dopaminergic consolidation of everyday memory. Nature 537, 357-362. doi: 10.1038/nature19325

Tanabe, J., Nyberg, E., Martin, L. F., Martin, J., Cordes, D., Kronberg, E., et al. (2011). Nicotine effects on default mode network during resting state. Psychopharmacology 216, 287-295. doi: 10.1007/s00213-0112221-8

Tavor, I., Parker, Jones O, Mars, R. B., Smith, S. M., Behrens, T. E., and Jbabdi, S. (2016). Task-free MRI predicts individual differences in brain activity during task performance. Science 352, 216-220. doi: 10.1126/science. aad 8127

Thompson, W. H., Kastrati, G., Finc, K., Wright, J., Shine, J. M., and Poldrack, R. A. (2019). Time-varying nodal measures with temporal community structure: a cautionary note to avoid misquantification. BioRxiv

Tomasi, D., and Volkow, N. D. (2010). Functional connectivity density mapping. Proc. Natl. Acad. Sci. U.S.A. 107, 9885-9890. doi: 10.1073/pnas.1001414107

Törk, I. (1990). Anatomy of the serotonergic system. Ann. N. Y. Acad. Sci. 600, 9-34. doi: 10.1111/j.1749-6632.1990.tb16870.x

Turchi, J., Chang, C., Ye, F. Q., Russ, B. E., Yu, D. K., Cortes, C. R., et al. (2018). The basal forebrain regulates global resting-state fMRI fluctuations. Neuron 97, 940.e4-952.e4. doi: 10.1016/j.neuron.2018.01.032

Uematsu, A., Tan, B. Z., and Johansen, J. P. (2015). Projection specificity in heterogeneous locus coeruleus cell populations: implications for learning and memory. Learn. Mem. 22, 444-451. doi: 10.1101/lm.037283.114

Uematsu, A., Tan, B. Z., Ycu, E. A., Cuevas, J. S., Koivumaa, J., Junyent, F., et al. (2017). Modular organization of the brainstem noradrenaline system coordinates opposing learning states. Nat. Neurosci. 20, 1602-1611. doi: 10. $1038 / \mathrm{nn} .4642$

van de Ven, V., Wingen, M., Kuypers, K. P., Ramaekers, J. G., and Formisano, E. (2013). Escitalopram decreases cross-regional functional connectivity within the default-mode network. PLoS One 8:e68355. doi: 10.1371/journal.pone. 0068355

van den Brink, R. L., Nieuwenhuis, S., and Donner, T. H. (2018a). Amplification and suppression of distinct brainwide activity patterns by catecholamines. J. Neurosci. 38, 7476-7491. doi: 10.1523/JNEUROSCI.0514-18. 2018

van den Brink, R. L., Nieuwenhuis, S., van Boxtel, G. J. M., van Luijtelaar, G., Eilander, H. J., and Wijnen, V. J. M. (2018b). Task-free spectral EEG dynamics track and predict patient recovery from severe acquired brain injury. Neuroimage Clin. 17, 43-52. doi: 10.1016/j.nicl.2017.10.003

van den Brink, R. L., Pfeffer, T., Warren, C. M., Murphy, P. R., Tona, K. D., van der Wee, N. J., et al. (2016). Catecholaminergic neuromodulation shapes intrinsic MRI functional connectivity in the human brain. J. Neurosci. 36, 7865-7876. doi: 10.1523/JNEUROSCI.0744-16.2016

van Wijk, B. C. M., Stam, C. J., and Daffershofer, A. (2010). Comparing brain networks of different size and connectivity density using graph theory. PLoS One 5:e13701. doi: 10.1371/journal.pone.0013701

Varazzani, C., San-Galli, A., Gilardeau, S., and Bouret, S. (2015). Noradrenaline and dopamine neurons in the reward/effort trade-off: a direct electrophysiological comparison in behaving monkeys. J. Neurosci. 35, 7866-7877. doi: 10.1523/ JNEUROSCI.0454-15.2015

Vargas, C., López-Jaramillo, C., and Vieta, E. (2013). A systematic literature review of resting state network-functional MRI in bipolar disorder. J. Affect. Disord. 150, 727-735. doi: 10.1016/j.jad.2013.05.083

Wang, J., and O'Donnell, P. (2001). D1 dopamine receptors potentiate NMDAmediated excitability increase in layer $\mathrm{V}$ prefrontal cortical pyramidal neurons. Cereb. Cortex 11, 452-462. doi: 10.1093/cercor/11.5.452

Wang, L., Hermens, D. F., Hickie, I. B., and Lagopoulos, J. (2012). A systematic review of resting-state functional-MRI studies in major depression. J. Affect. Disord. 142, 6-12. doi: 10.1016/j.jad.2012.04.013

Warren, C. M., Eldar, E., van den, Brink RL, Tona, K. D., van der Wee, N. J., Giltay, E. J., et al. (2016). Catecholamine-mediated increases in gain enhance the precision of cortical representations. J. Neurosci. 36, 5699-5708. doi: 10.1523/JNEUROSCI.3475-15.2016

Watts, D. J., and Strogatz, S. H. (1998). Collective dynamics of 'small-workd' networks. Nature 393, 440-442. doi: 10.1038/30918

Wester, J. C., and McBain, C. J. (2014). Behavioral state-dependent modulation of distinct interneuron subtypes and consequences for circuit function. Curr. Opin. Neurobiol. 29, 118-125. doi: 10.1016/j.conb.2014.07.007

Winterer, G., and Weinberger, D. R. (2004). Genes, dopamine and cortical signalto-noise ratio in schizophrenia. Trends Neurosci. 27, 683-690. doi: 10.1016/j. tins.2004.08.002

Ye, Z., Hammer, A., and Munte, T. F. (2017). Pramipexole modulates interregional connectivity within the sensorimotor network. Brain Connect 7, 258-263. doi: 10.1089/brain.2017.0484

Zaborszky, L., Hoemke, L., Mohlberg, H., Schleicher, A., Amunts, K., and Zilles, K. (2008). Stereotaxic probabilistic maps of the magnocellular cell groups in 
human basal forebrain. NeuroImage 42, 1127-1141. doi: 10.1016/j.neuroimage. 2008.05.055

Zaborszky, L., Gombkoto, P., Varsanyi, P., Gielow, M. R., Poe, G., Role, L. W., et al. (2018). Specific basal forebrain-cortical cholinergic circuits coordinate cognitive operations. J. Neurosci. 38, 9446-9458. doi: 10.1523/JNEUROSCI. 1676-18.2018

Zahrt, J., Taylor, J., Mathew, R., and Arnsten, A. (1997). Supranormal stimulation of d1 dopamine receptors in the rodent prefrontal cortex impairs spatial working memory performance. J. Neurosci. 17, 8528-8535. doi: 10.1523/jneurosci.1721-08528.1997

Zalesky, A., Fornito, A., Cocchi, L., Gollo, L. L., and Breakspear, M. (2014). Time-resolved resting-state brain networks. Proc. Natl. Acad. Sci. U.S.A. 111, 10341-10346. doi: 10.1073/pnas.1400181111

Zerbi, V., Floriou-Servou, A., Markicevic, M., Vermeiren, Y., Sturman, O., Privitera, M., et al. (2019). Rapid reconfiguration of the functional connectome after chemogenetic locus coeruleus activation. Neuron 103, 1-17. doi: 10.1016/ j.neuron.2019.05.034

Zhang, S., Hu, S., Chao, H. H., and Li, C. R. (2015). Resting-state functional connectivity of the locus coeruleus in humans: in comparison with the ventral tegmental area/substantia nigra pars compacta and the effects of age. Cereb. Cortex 26, 3413-3427. doi: 10.1093/cercor/bhv172

Zilles, K., and Amunts, K. (2009). Receptor mapping: architecture of the human cerebral cortex. Curr. Opin. Neurol. 22, 331-339. doi: 10.1097/WCO. 0b013e32832d $95 \mathrm{db}$

Zohary, E., Shadlen, M. N., and Newsome, W. T. (1994). Correlated neuronal discharge rate and its implications for psychophysical performance. Nature 370, 140-143. doi: 10.1038/370140a0

Conflict of Interest: The authors declare that the research was conducted in the absence of any commercial or financial relationships that could be construed as a potential conflict of interest.

Copyright $\odot 2019$ van den Brink, Pfeffer and Donner. This is an open-access article distributed under the terms of the Creative Commons Attribution License (CC BY). The use, distribution or reproduction in other forums is permitted, provided the original author(s) and the copyright owner(s) are credited and that the original publication in this journal is cited, in accordance with accepted academic practice. No use, distribution or reproduction is permitted which does not comply with these terms. 


\section{OPEN ACCESS}

Edited by:

Roberto Esposito,

A.O. Ospedali Riuniti Marche Nord,

Italy

Reviewed by:

Yonggui Yuan,

Southeast University, China

Dong Zhou,

Sichuan University, China

*Correspondence:

Enrico Collanton

enrico.collantoni@gmail.com

Specialty section:

This article was submitted to

Brain Imaging Methods,

a section of the journal

Frontiers in Neuroscience

Received: 22 April 2019

Accepted: 19 September 2019

Published: 14 October 2019

Citation:

Collantoni E, Meneguzzo P.

Solmi M, Tenconi E, Manara $R$ and

Favaro A (2019) Functiona

Connectivity Patterns and the Role of 5-HTTLPR Polymorphism on Network Architecture in Female

Patients With Anorexia Nervosa.

Front. Neurosci. 13:1056.

doi: 10.3389/fnins.2019.01056

\section{Functional Connectivity Patterns and the Role of 5-HTTLPR Polymorphism on Network Architecture in Female Patients With Anorexia Nervosa}

\author{
Enrico Collantoni ${ }^{1 *}$, Paolo Meneguzzo ${ }^{1}$, Marco Solmi ${ }^{1,2}$, Elena Tenconi ${ }^{1,2}$, \\ Renzo Manara ${ }^{3}$ and Angela Favaro ${ }^{1,2}$ \\ ${ }^{1}$ Department of Neurosciences, University of Padua, Padua, Italy, ${ }^{2}$ Padova Neuroscience Center, University of Padua, \\ Padua, Italy, ${ }^{3}$ Radiology Unit, Department of Medicine and Surgery, Neuroscience Section, University of Salerno, Salerno, \\ Italy
}

Introduction: Recent neuroimaging studies suggest that anorexia nervosa (AN) symptoms emerge from failures in the relationships between spatially distributed networks that support different cognitive, emotional, and somatosensory functions. The 5-HTTLPR genotype has been shown to modulate all these abilities in AN, as well as the connectivity patterns between brain regions that support their functioning. This study aims at exploring the presence of any difference in functional connectome properties between AN patients and healthy controls $(\mathrm{HC})$ by means of graph theory tools. The effect of 5-HTTLPR genotype on regional and global network characteristics in AN and $\mathrm{HC}$ was also explored.

Methods: A sample of 74 subjects ( $38 \mathrm{HC}, 36 \mathrm{AN}$ ) underwent a resting state functional magnetic resonance imaging and was genotyped for 5-HTTLPR polymorphism. Comparisons of network properties were made between the AN and HC groups and, within each group, between 5-HTTLPR carriers of low-functioning alleles and carriers of the long-long genotype.

Results: Patients with AN displayed lower network clustering than $\mathrm{HC}(\mathrm{p}=0.04$ at Mann-Whitney $U$ test). Based on both degree and betweenness, a different distribution of network hubs emerged in the two groups. In particular, the anterior part of the anterior cingulate cortex was a hub only in the patient group. A correlation emerged between differences in brain volumes between patients and $\mathrm{HC}$ and differences in degree values of basal ganglia, nodes in the insula, and those in the parietal cortex. Carriers of the short allele of the 5-HTTLPR polymorphism were characterized by lower small-world properties $(p=0.027)$ and modularity $(p=0.031)$ in the patient group, and a trend toward higher modularity $(p=0.033)$ and small-world values $(p=0.123)$ in the HC group.

Discussion: Patients with AN showed differences in hubs distribution, providing evidence of the presence of a different functional architectural backbone in this group. Since some correlation emerged between different degree values of nodes and 
differences in volumes, further longitudinal studies are warranted to better understand the role of malnutrition on brain network architecture. The opposite effects of 5HTTLPR polymorphism on global network characteristics in the two groups suggest an interaction of the short allele and malnutrition in modulating brain network properties.

Keywords: eating disorders, anorexia nervosa, neuroimaging, graph theory, fMRI, resting state, 5-HTTLPR

\section{INTRODUCTION}

In recent years, studies that evaluate the neurobiological underpinnings of anorexia nervosa (AN) have greatly increased and revealed that this disorder is characterized by complex and multifaceted patterns of interaction between genetic, neuropsychological, and connective characteristics (Zipfel et al., 2015; Solmi et al., 2018; Frank et al., 2019).

Within neuroimaging research, most of the studies in AN are aimed to explore the neural processing of stimuli that activate specific processes (i.e., taste or reward processes), as well as the functional relationships between different areas during rest (Fuglset et al., 2016; Gaudio et al., 2016). Results of these studies are extremely heterogeneous, due to the complexity of the disorder and often different study protocols and designs. Nevertheless, a common point of all these observations is that brain functioning in $\mathrm{AN}$ is not characterized by isolated dysfunction in circumscribed brain areas but rather from disturbance in spatially distributed neural networks that support several functions and processes (Steinglass et al., 2018; Seidel et al., 2019).

The possibility to describe the interactions between anatomically distributed areas have been greatly supported, in recent years, by the application of graph theory tools to neuroimaging data (Bullmore and Sporns, 2009). Graph theory allows the mathematical representation of the brain as an architecture of nodes, which are represented by discrete brain areas, and edges, which represent the functional or structural correlations between the nodes. The advantage of this framework is its ability to describe the organizational principles that govern the interactions between different brain regions and to analyze the relevance that specific areas have in managing the connectivity within a network (Rubinov and Sporns, 2010).

To date, three studies evaluated brain connectivity in AN by means of graph theory tools but have considered very different samples. The first compared a sample of acutely ill patients with healthy controls (HC), and pointed out both global and regional anomalies. Regionally, patients with $\mathrm{AN}$ showed reduced connectivity strength in insula and a lower centrality of thalamus. Globally, they showed higher levels of separation between nodes in the overall network as well as a higher tendency of nodes with similar connectedness to link together (Geisler et al., 2016). The second one compared a sample of patients recovered from $\mathrm{AN}$ and $\mathrm{HC}$ and pointed out the presence, in the experimental group, of a higher tendency of the network to form clusters of densely interconnected nodes (increased clustering coefficient) and a higher tendency of nodes with similar connectedness to link together (increased assortativity). Furthermore, patients with AN reported a different balance between segregation and integration properties when compared to HC [reduced small-world index (SWI)] (Geisler et al., 2018). The third study was conducted on a sample of adolescent patients at first stages of AN and pointed out the presence of a decreased connectivity in a sub-network of connections that encompass the left and right rostral anterior cingulate cortex, the left paracentral lobule, the left cerebellum, the left posterior insula, the left medial fronto-orbital gyrus, and the right superior occipital gyrus in the experimental sample (Gaudio et al., 2018).

Alongside the evaluation of the rules that govern the relationships between different brain areas in the connectome, graph theory allows the identification of those regions of the brain that have a peculiar role in supporting network communication and integration and that are called "hubs" (van den Heuvel and Sporns, 2013; Oldham and Fornito, 2019). The ability of hub regions to mediate large proportion of communications in the brain, as well as their tendency to integrate distributed neural signals, makes them susceptible to different disease processes. Coherently with these observations, network hubs have been demonstrated to be points of vulnerability into a network, and alterations in their distribution have been evidenced in many neurologic and psychiatric disorders like depression, ADHD, schizophrenia, and Alzheimer disease (Rubinov and Bullmore, 2013; Dai et al., 2014; Hong et al., 2014; Korgaonkar et al., 2014).

No study to date investigated the presence of any alteration in hubs distribution in patients with AN. The evaluation of hubs distribution in AN could be particularly interesting both from a neurobiological and from a clinical point of view. In fact, an alteration or a disproportion in their distribution could help in identifying specific areas of vulnerability in the disorder as well as in better characterizing the functional correlates of specific cognitive dysfunctions and psychopathological dimensions.

Another point of interest, in the evaluation of network properties in $\mathrm{AN}$, seems to be the investigation of the role of genes in mediating the configuration of the connectome. In fact, the presence of specific polymorphisms, like the 5HTTLPR polymorphism for the serotonin transporter gene, have been evidenced to modulate the connectivity patterns in different functional networks in AN (Collantoni et al., 2016). Furthermore, in AN, the 5-HT modulation have been proposed to be involved in contributing to specific psychopathological and temperamental dimensions, in regulating satiety and food consumption, and in determining cognitive and executive functioning (Haleem, 2012; Tenconi et al., 2016). Thus, the evaluation of the role of serotoninergic circuits in modulating the architectural properties of the functional brain connectome in AN can help in better characterizing their role in the neurobiology of the disorder as well as their possible relevance as targets of serotoninergic drugs. 
Our purpose in this study is therefore to explore the presence of any difference in functional connectome properties between AN patients and HC by means of graph theory tools. The effect of 5-HTTLPR genotype on regional and global network characteristics in AN and $\mathrm{HC}$ was also examined. We hypothesized to find differences between patients and controls in assortativity values and hubs distribution. We also hypothesize the presence of a relationship between alterations in morphology of brain areas and their alterations in network properties, as observed in other psychiatric disorders (Crossley et al., 2014). Finally, we hypothesize to find effects of the 5-HTTLPR polymorphism that are similar in cases and controls.

\section{MATERIALS AND METHODS}

A total of 38 patients with acute $\mathrm{AN}$ and $38 \mathrm{HC}$ were included in this study.

Patients with AN were recruited from the Padova Hospital Eating Disorders Unit. All the subjects of the experimental group met DSM-5 criteria for AN. A sample of $\mathrm{HC}$ was recruited from the same geographical area. The HC group was similar to the patient group in age, ethnicity, educational level, and hand lateralization. Exclusion criteria for recruitment in both experimental and HC groups were male gender, history of head trauma or injury with loss of consciousness, history of any serious neurological or medical illness, active use of systemic steroids, pregnancy, active suicidality or major depression, history of substance/alcohol abuse or dependence, bipolar disorder or schizophrenia spectrum disorder, moderate mental impairment (IQ $<60$ ) or learning disabilities, use of medications other than antidepressants, and known contraindications to conventional MRI. In HC, history of any psychiatric disorder and any first-degree relatives with an eating disorder were considered additional exclusion criteria. After recruitment, we excluded 2 AN patients for technical reasons (see below).

Table 1 describes the main characteristics of the sample. Thirteen patients with AN were under drug treatment with antidepressants at the time of scanning (1 patient mirtazapine, 2 paroxetine, 2 escitalopram, 1 fluoxetine, and 7 sertraline).

Ethical permission was obtained from the Ethics Committee of the Hospital of Padova. After completely describing the study to the subjects, written informed consent was obtained.

\section{Clinical and Neuropsychological Assessment}

All subjects were investigated for AN diagnosis with a diagnostic interview according to the Eating Disorders Section of the Structured Clinical Interview for Diagnostic and Statistical Manual of Mental Disorder (DSM-5, American Psychiatric Association, 2013) and, also, a semi-structured interview was used in order to collect socio-demographic and clinical variables (Favaro et al., 2012, 2013). More information about subjects' psychopathology was achieved using the Hopkins Symptoms Checklist (Derogatis et al., 1974), the Eating Disorders Inventory (Garner et al., 1983), and the State-Trait Anxiety Inventory (Spielberger et al., 1999). Furthermore, the Edinburgh
TABLE 1 | Baseline characteristics of the two groups.

\begin{tabular}{|c|c|c|c|}
\hline & $\begin{array}{l}\text { Patients with AN } \\
\qquad(n=36)\end{array}$ & $\begin{array}{l}\text { Healthy women } \\
\qquad(n=38)\end{array}$ & $\begin{array}{c}\text { Mann-Whitney } U \\
\text { test }\end{array}$ \\
\hline & Mean (SD) & Mean (SD) & $p$ \\
\hline Age (years) & $26.0(7.0)$ & $25.3(6.3)$ & 0.729 \\
\hline Age at onset (years) & $18.6(5.0)$ & $=$ & $=$ \\
\hline $\begin{array}{l}\text { Duration of } \\
\text { illness (months) }\end{array}$ & $73.3(79.2)$ & $=$ & $=$ \\
\hline $\begin{array}{l}\text { Baseline } \\
\text { BMI }\left(\mathrm{kg} / \mathrm{m}^{2}\right)\end{array}$ & $15.8(1.8)$ & $21.7(2.9)$ & 0.000 \\
\hline $\begin{array}{l}\text { Lowest } \\
\text { BMI }\left(\mathrm{kg} / \mathrm{m}^{2}\right)\end{array}$ & $14.0(1.8)$ & $19.8(2.5)$ & 0.000 \\
\hline $\begin{array}{l}\text { Edinburgh laterality } \\
\text { index }\end{array}$ & $58.2(37.2)$ & $55.0(42.0)$ & 0.541 \\
\hline WCST global index & $48.0(38.6)$ & $42.2(29.6)$ & 0.995 \\
\hline $\begin{array}{l}\text { WCST number } \\
\text { persev. responses }\end{array}$ & $16.9(16.4)$ & $12.4(7.4)$ & 0.979 \\
\hline $\begin{array}{l}\text { Rey central } \\
\text { coherence index }\end{array}$ & $0.98(0.43)$ & $1.16(0.39)$ & 0.101 \\
\hline Rey visual memory & $17.5(5.4)$ & $20.6(4.6)$ & 0.026 \\
\hline \multirow{2}{*}{$\begin{array}{l}\text { lowa gambling task } \\
\text { net score }\end{array}$} & $3.6(27.1)$ & $21.5(29.5)$ & 0.009 \\
\hline & N/tot (\%) & & Pearson $\chi^{2}$ \\
\hline 5-httlpr short allele & $29 / 36(81 \%)$ & $26 / 38$ (69\%) & $1.43(0.23)$ \\
\hline Restricting subtype & 26/36 (72\%) & $=$ & $=$ \\
\hline
\end{tabular}

Handedness Inventory (Oldfield, 1971) was used to assess handedness (and left-handed individuals were excluded).

The neuropsychological assessment included the Wisconsin Card Sorting Task (WCST) and measures of intellectual abilities. WCST is one of the most widely used measures assessing abstract thinking and set-shifting abilities (Berg, 1948). The global score (a general measure of executive functioning) and number of perseverative responses (a measure of cognitive inflexibility) were used as main outcomes (Tenconi et al., 2010). Subjects were also assessed with the Rey-Osterrieth Complex Figure Test in order to assess participants' memory, visual-spatial abilities, and central coherence. Outcomes from this test is the Central Coherence Index (CCI), calculated from the style index and the copy order index, and the Visual Memory Index, calculated from the correct reproduction of the 18 items of the original figure (Tenconi et al., 2010). To exclude mental impairment, all participants over the age of 20 completed the Brief Intelligence Test (TIB), which measures premorbid intellectual ability (the test is very similar to the National Adult Reading Test for the Italian population) (Colombo et al., 2002). Participants younger than 21 completed the Information subtest of Wechsler Intelligence Scale (the children version if participants aged 16 or less, and the adult version if their age was between 17 and 20 years) as a measure of verbal intelligence (Wechsler, 1949).

\section{Data Acquisition}

Data were collected on a Philips Achieva 1.5-T scanner equipped for echo-planar imaging. A resting-state fMRI scan entailed 250 continuous functional volumes (repetition time $=2009 \mathrm{~ms}$, echo time $=50 \mathrm{~ms}$, flip angle $=90^{\circ}, 21$ slices, matrix $=128 \times 128$, 
acquisition voxel size $=1.8 \times 1.8 \times 6 \mathrm{~mm}$, acquisition time $=8 \mathrm{~min}$; field of view $=23 \mathrm{~cm}$ ). Participants were instructed to rest with their eyes closed during the scan. Highresolution 3D T1-weighted anatomical images were also acquired in a gradient-echo sequence (repetition time $=20 \mathrm{~s}$, echo time $=3.78 \mathrm{~ms}$, flip angle $=20^{\circ}, 160$ slices, acquisition voxel size $=1 \times 0.66 \times 0.66 \mathrm{~mm}$, field of view $=21-22 \mathrm{~cm})$.

\section{Data Processing and Statistics}

Structural images were preprocessed using the FreeSurfer package (Martinos Center for Biomedical Imaging, Massachusetts General Hospital, Boston) version 5.3.0. The cortex was then divided into 148 regions of interest (ROIs) (74 per hemisphere) with a specific sulco-gyral atlas (Destrieux atlas) (Destrieux et al., 2010). The 148 cortical regions and the subcortical nuclei volumes (caudate, putamen, accumbens, hippocampus, amygdala, palladum, and thalamus) obtained were then transformed and warped using a MNI_2 mm map, summed to build a probability map of every single brain areas, and then transformed in functional space using FLIRT. Seed ROIs were obtained by thresholding probability maps at $80 \%$.

Resting-state scans were preprocessed with both Analysis of Functional NeuroImages (version AFNI_2010_10_19_1028'; NIMH, Bethesda, Maryland) and FM-RIB Software Library (version FSL 4.1.6²; FMRIB, Oxford, United Kingdom).

Signal-to-noise ratio was computed as described by van Dijk et al. (2012) for each resting-state sequence and used to estimate data quality. Only resting-state scans with a mean slice-based temporal signal-to-noise ratio higher than 100 were included in the subsequent analyses. All but two scans (two patients with AN) passed this criterion.

Preprocessing was then performed as described in our previous reports (Favaro et al., 2013, 2014a,b). Details of preprocessing and processing are described in the Supplementary Information. A high-pass filter setting of $200 \mathrm{~s}(0.005 \mathrm{~Hz})$ was used to reduce very-low-frequency artifacts such as scanner drift and a low-pass filter to remove any components in the high-frequency spectrum $(40.1 \mathrm{~Hz})$.

A seed-based approach was used to explore the functional connectivity of brain areas obtained as described before. Nuisance signals were removed by multiple regression before functional connectivity analyses. Each individual's $4 \mathrm{D}$ time series were regressed on nine predictors, consisting of white matter, cerebrospinal fluid, the global signal, and six motion parameters (three cardinal directions and rotational movement around three axes). The time series of the nuisance signals were extracted by (a) averaging all voxels in the brain (global signal) across the time series; (b) segmenting each individual's high-resolution structural image (FAST, FSL) (Zhang et al., 2001), applying a threshold at $80 \%$ tissue type probability, and averaging all voxels within the thresholded mask (white matter and cerebrospinal fluid) across each time series; and (c) using the residuals obtained after motion correction by MCFLIRT (FMRIB, Oxford, United Kingdom). Each subject's residual $4 \mathrm{D}$ time series was transformed into

${ }^{1}$ http://afni.nimh.nih.gov/afni

${ }^{2}$ http://www.fmrib.ox.ac.uk
Montreal Neurological Institute space by means of a linear affine transformation implemented in FSL (FLIRT) and the time series extracted for each seed.

Analyses of network properties were performed with Graph Analysis Toolbox (GAT; Stanford University School of Medicine, Stanford, CA, United States) (Hosseini et al., 2012). Undirected weighted networks were constructed by averaging time series across all voxels in the seed ROI. A $148 \times 148$ association matrix was constructed for each individual by means of pairwise Pearson correlation coefficients. Each entry of the symmetric correlation matrices represents the strength of functional connectivity between two ROIs. By thresholding the correlation values between matrix entries, an adjacency matrix is derived for each association matrix. In particular, a range threshold of 0.1-0.5 with increments of 0.05 was applied in order to estimate the binary adjacency matrices. The statistical differences between groups in the network measures, which are quantified for each individual network, were determined by means of a nonparametric permutation test with 1000 repetitions. In addition to comparing global network measures at every density, AUC analyses were performed to make the between-group comparison less sensitive to the thresholding process.

\section{Graph-Based Metrics}

Segregation and integration are two major organizational principles of brain structure and function. Segregation properties of a network describe its tendency to be composed by specialized and functionally coherent areas. Parameters that describe the segregation of a network are the clustering coefficient (that indicate the density of connections between the neighbors of an individual node) and the modularity (that measures the correlation between the probability of having an edge that connect two nodes and the probability that nodes are part of the same community).

Integration properties of a network describe its ability to manage a globally distributed and efficient communication. Parameters that describe the integration of a graph are the global efficiency (which measures the efficiency of information transfer across the network, where maximal GE values indicate a fully connected network) and the characteristic path length (which indicates the number of edges that are present in the shortest path of two nodes, averaged over all pairs of nodes).

Assortativity is the correlation between the degrees of connected nodes and reflects the tendency of a brain region to connect with nodes of similar degree (Boccaletti et al., 2006; Rubinov and Sporns, 2010).

A network is small world if high clustering coexists with high efficiency. This means that small-world networks combine the ability to use a relatively small number of longdistance connections to synchronize the information flow and the advantage to use local connections to locally processing information. Therefore, the SWI is computed by comparing the $\mathrm{CPL}$ and the CF of a graph with the corresponding values of null random graphs with same number of nodes, edges, and degree distribution (Bassett and Bullmore, 2006).

Hubs are crucial regulators of information flow across the network and play a key role in network resilience to insult. Degree 
and betweenness centrality identify hub nodes by measuring the fraction of short paths between nodes of the network that pass through a given node. Nodes that are characterized by high degree and/or betweenness centrality values are likely to participate in many of the network's short paths and to have a great control over the flow of information within the network (van den Heuvel and Sporns, 2013).

\section{Genetic Analysis}

DNA samples were collected at the time of assessment in all participants. Participants were genotyped for the presence of the short variant of the 5-HTTLPR gene, and the A/G singlenucleotide polymorphism (SNP rs25531) of the 5-HTTLPR gene, according to previously described standard protocols (Favaro et al., 2014a). For the 5-HTTLPR gene, samples were split on the basis of the presence or absence of the short variant (S) of the 5-HT transporter, and polymorphism G were included in this short group variant.

\section{Statistical Analyses}

Global and local graph metrics is usually considered not normally distributed (Hosseini et al., 2012). For this reason, for between-group comparisons of graph metrics, non-parametric independent two-group Mann-Whitney $U$ tests with a critical $p$ value of 0.05 were performed. Given the explorative nature of the study, we decided to avoid correction for multiple comparisons for global graph metrics. However, to reduce the possibility of false-positive results, we chose to consider only six parameters of the available graph metrics. Local graph metrics were, on the contrary, corrected for multiple comparisons regarding the number of nodes with FDR.

\section{RESULTS}

\section{Comparison Between Patients and Healthy Women}

Patients with AN showed no differences when compared to HC in whole-brain segregation and integration measures, except for a slightly lower clustering score ( $p=0.04$ at Mann-Whitney $U$ test; Table 2). Measures of net integration/segregation did not show any significant correlation with age, body mass index (BMI), age of onset, duration of illness, eating psychopathology, and SCL depression or obsessive-compulsive scores. The lifetime lowest BMI showed a significant positive correlation with path length (rho $=0.42 ; p=0.011$ ) in the group of AN patients and a negative one (rho $=-0.42 ; p=0.011$ ) in the control group (Figure 1). In patients with $\mathrm{AN}$, the CCI (Rey Figure Task) correlated with clustering values (rho $=0.42 ; p=0.012$ ), whereas both state and trait STAI scores negatively correlated with global efficiency (rho $=-0.56 ; p=0.001 ;$ rho $=-0.39 ; p=0.019$ ). No differences in net measures emerged comparing diagnostic subtype groups, or comparing patients who were on antidepressant treatment and those who were not.

Based on both degree and betweenness values, a partially different distribution of network hubs emerged in patients with AN and HC (Table 2). In particular, analyzing betweenness, the left superior frontal gyrus was lacking in the AN group, while the anterior part of the cingulate gyrus represented a hub region only in patients with AN (Figure 2). For degree values, AN showed a higher betweenness value for the anterior part of the cingulate gyrus and right/left middle frontal gyri, whereas in healthy women, hubs were located in bilateral parahippocampal gyri, in the left transverse frontopolar gyri and in the posterior

TABLE 2 | Network properties and hubs distribution in AN patients and healthy women.

\begin{tabular}{|c|c|c|c|}
\hline & Patients with AN $(n=36)$ & Healthy women $(n=38)$ & \multirow{2}{*}{$\begin{array}{c}\text { Mann-Whitney } U \text { test } \\
p\end{array}$} \\
\hline & Mean (SD) & Mean (SD) & \\
\hline Assortativity & $0.29(0.11)$ & $0.33(0.14)$ & 0.187 \\
\hline Global efficiency & $0.49(0.06)$ & $0.48(0.05)$ & 0.443 \\
\hline Clustering & $0.61(0.04)$ & $0.62(0.04)$ & 0.034 \\
\hline Modularity & $0.28(0.09)$ & $0.31(0.09)$ & 0.217 \\
\hline Path length & $2.16(0.16)$ & $2.16(0.16)$ & 0.736 \\
\hline Sigma & $1.34(0.32)$ & $1.41(0.26)$ & 0.284 \\
\hline \multirow[t]{7}{*}{ Net hubs betweenness } & Anterior part of the left cingulate gyrus and sulcus & Left superior frontal gyrus & \\
\hline & Left precuneus & Left precuneus & \\
\hline & Lateral aspect of the left superior temporal gyrus & Lateral aspect of the left superior temporal gyrus & \\
\hline & Right superior frontal gyrus & Right superior frontal gyrus & \\
\hline & Right precuneus & Right precuneus & \\
\hline & Lateral aspect of the right superior temporal gyrus & Lateral aspect of the right superior temporal gyrus & \\
\hline & & Right middle temporal gyrus & \\
\hline \multirow[t]{6}{*}{ Net hubs degree } & Anterior part of the left cingulate gyrus and sulcus & Left transverse frontopolar gyri and sulci & \\
\hline & Left middle frontal gyrus & Left parahippocampal gyrus & \\
\hline & Left subcallosal area & Left subcallosal area & \\
\hline & Right middle frontal gyrus & Right parahippocampal gyrus & \\
\hline & Right subcallosal area & Right subcallosal area & \\
\hline & & Posterior segment right lateral sulcus & \\
\hline
\end{tabular}




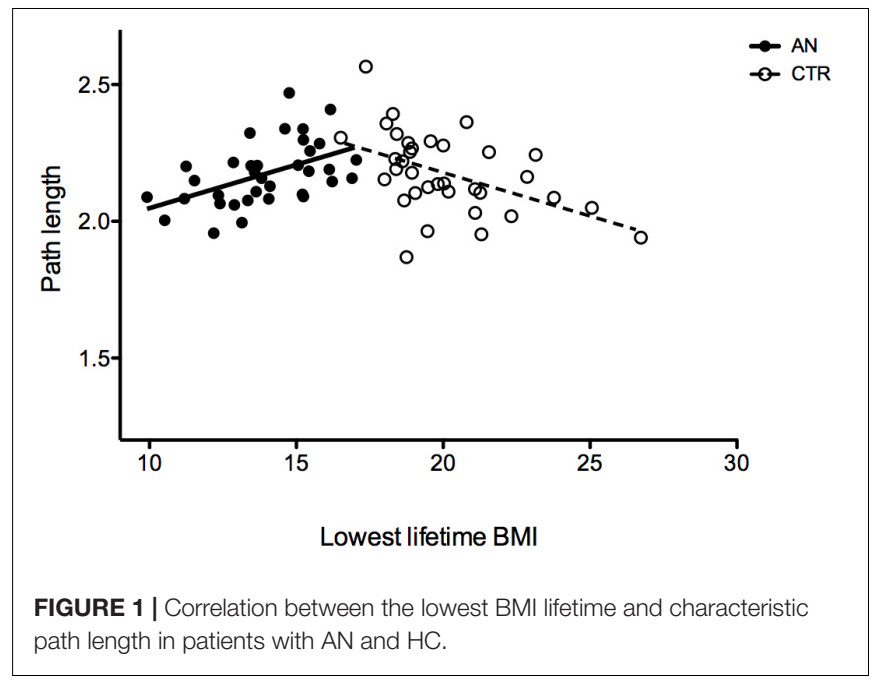

segment of the right lateral sulcus. The degree of the anterior part of the cingulate gyrus significantly negatively correlates $\left(p=0.014 ; R^{2}=0.19\right)$ with the number of perseveration errors at the WCST (Figure 3).

Testing the hypothesis of a relationship between brain volume decrease and changes in degree/betweenness values of brain nodes (Figure 4), we found trends for decreased values of degree along with increased differences in volumes in the comparison between patients and controls in nodes of the insula cortex $\left(p=0.033 ; R^{2}=0.38\right)$ and in basal ganglia $(p=0.05$; $\left.R^{2}=0.28\right)$. On the contrary, nodes of the parietal cortex showed an inverse relationship: increased degree values along with increased differences between patients and controls in both volume $\left(p=0.01 ; R^{2}=0.35\right)$ and thickness $(p=0.018$; $R^{2}=0.30$; Supplementary Figure 1) of the cortical areas. No significant correlation emerged for betweenness values. No significant differences emerged in regional network measures

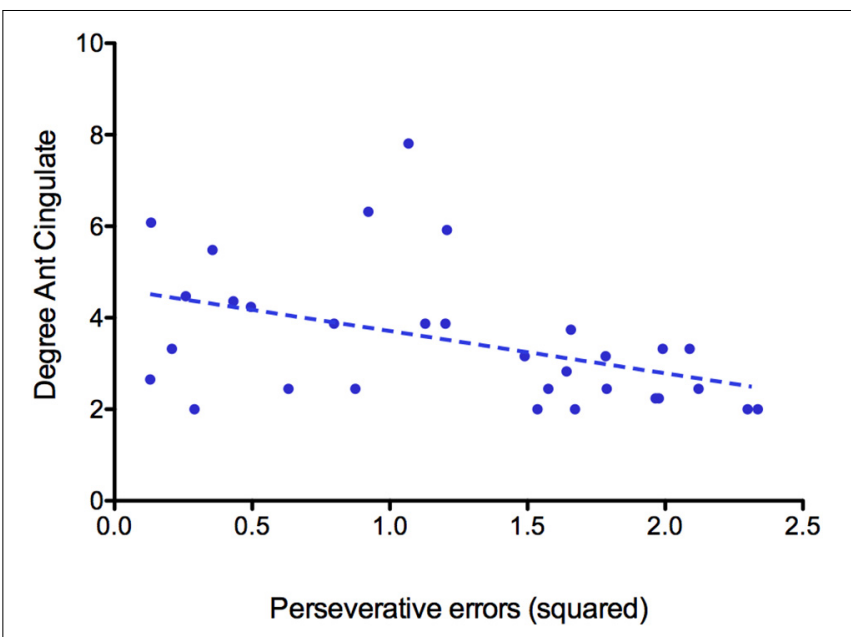

FIGURE 3 | Correlation between the degree of the anterior part of the anterior cingulate gyrus and the number of perseverative errors at WCST.

between patients with AN and HC. No differences emerged on graph metrics between patients who were taking antidepressants and those who did not (Mann-Whitney $U$ test: global efficiency: $p=0.43$, clustering coefficient: $p=0.54$, modularity: $p=0.54$, characteristic path length: $p=0.17$, SWI: $p=0.41$, assortativity: $p=0.90)$.

\section{HTTLPR Polymorphism Effects on Network Measures}

According to 5-HTTLPR polymorphism, in patients with AN, the low functioning genotype was associated with significantly lower small-world properties and lower modularity compared with the high functioning LL genotype (Table 3). On the contrary, in HC, the group who carried the $\mathrm{S}$ allele showed significantly higher
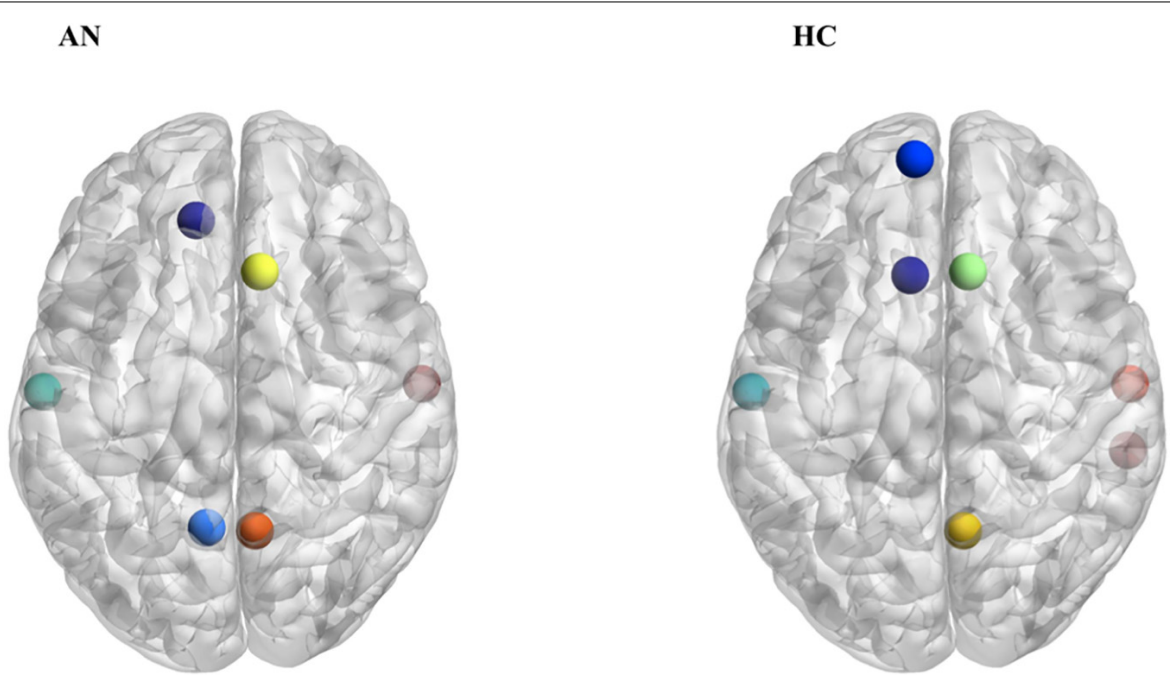

FIGURE 2 | Hubs distribution based on betweenness values in patients with AN and HC. 


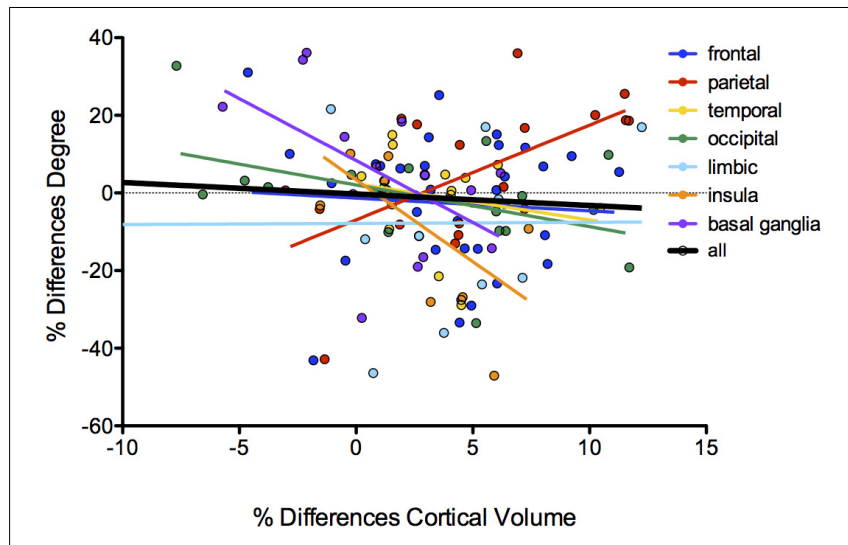

FIGURE 4 | Correlation between brain volumes changes and degree /betweenness values of brain nodes.

modularity and a trend toward higher values of small-worldness in comparison with the LL genotype (Table 4).

\section{DISCUSSION}

The present study evidences the presence of specific alterations in the configuration of functional connectivity architecture in AN. The experimental group showed, when compared to the HC group, a different distribution of hub regions based on both betweenness and degree values. The importance of evaluating hub regions in brain disorders is twofold, as these areas not only are crucial in supporting the network communication but also represent points of vulnerability to several pathogenic processes affecting the brain (Fornito et al., 2017). Therefore, the observation of a different distribution of hubs into the functional connectome in patients with AN does not have a univocal interpretation, being able to reflect their functional relevance in the overall connectome as well as the pathological consequence of disorder-related processes.

Our results pointed out that the hubs distribution between patients with AN and $\mathrm{HC}$ differs for some nodes, while others are identically distributed between the two groups. Interestingly, the main differences are identifiable mostly in a disproportion between frontal and subcortical areas, the former being more represented in AN patients, while the latter are more expressed in the control group. In particular, the left anterior cingulate gyrus is present only in patients with AN when hubs are computed on both betweenness and degree values. Hub regions are considered to offer an architectural backbone for brain processes that require high levels of functional integration, like cognitive and executive functions (Crossley et al., 2014). Therefore, the peculiar hubs distribution in patients with AN may represent a fundamental component of the functional connective architecture that supports the cognitive functioning in the disorder. The prevalence of frontal regions over subcortical ones in our experimental group may be coherent with this hypothesis, since it may sustain the presence of unbalanced top-down and bottom-up processes in AN (O'Hara et al., 2015). In $\mathrm{AN}$, the anterior cingulate gyrus has been shown to have an important role in performance monitoring and in the cognitive regulation of appetitive stimuli, and it has also been proposed to be functionally involved in set shifting abilities as well as in cognitive inflexibility (Kaye et al., 2013; Geisler et al., 2017). The observation that this node is highly

TABLE 3 | Network properties in patients with AN according to 5-HTTLPR genotype.

\begin{tabular}{|c|c|c|c|c|}
\hline & Patients with AN S allele $(n=29)$ & Patients with AN LL genotype $(n=7)$ & Mann-Whitney $U$ test & Cliff's delta \\
\hline & Mean (SD) & Mean (SD) & $p$ & Effect size \\
\hline Assortativity & $0.30(0.12)$ & $0.30(0.10)$ & 0.845 & 0.054 \\
\hline Global efficiency & $0.48(0.05)$ & $0.49(0.08)$ & 0.410 & 0.212 \\
\hline Clustering & $0.61(0.04)$ & $0.62(0.05)$ & 0.424 & 0.079 \\
\hline Modularity & $0.27(0.09)$ & $0.35(0.06)$ & 0.031 & 0.448 \\
\hline Path length & $2.18(0.12)$ & $2.10(0.27)$ & 0.838 & 0.034 \\
\hline Sigma & $1.29(0.29)$ & $1.64(0.32)$ & 0.027 & 0.517 \\
\hline
\end{tabular}

TABLE 4 | Network properties in HC according to 5-HTTLPR genotype.

\begin{tabular}{|c|c|c|c|c|}
\hline & Healthy women S allele $(n=26)$ & Healthy women LL genotype $(n=12)$ & Mann-Whitney $U$ test & Cliff's delta \\
\hline & Mean (SD) & Mean (SD) & $p$ & Effect size \\
\hline Assortativity & $0.35(0.15)$ & $0.29(0.12)$ & 0.207 & 0.263 \\
\hline Global efficiency & $0.48(0.06)$ & $0.49(0.05)$ & 0.958 & 0.006 \\
\hline Clustering & $0.63(0.03)$ & $0.61(0.03)$ & 0.198 & 0.263 \\
\hline Modularity & $0.33(0.09)$ & $0.28(0.09)$ & 0.033 & 0.359 \\
\hline Path length & $2.15(0.19)$ & $2.18(0.10)$ & 0.930 & 0.103 \\
\hline Sigma & $1.45(0.25)$ & $1.30(0.27)$ & 0.123 & 0.276 \\
\hline
\end{tabular}


connected in patients with AN but not in HC confirms the hypothesis, already supported by previous observations, that it plays a crucial role in AN neurobiology and psychopathology (Kuyck et al., 2009). The negative correlation between the degree of anterior cingulate gyrus and perseverative errors at WCST suggests that this area may be involved in the ability to modulate cognitive and behavioral flexibility in AN. Overall, these observations suggest a possible role of cognitive training protocols (i.e., cognitive remediation therapy) in the treatment of AN. Furthermore, brain-directed treatments (i.e., repetitive transcranial magnetic stimulation or transcranial electrical stimulation) that can modulate the functioning of prefrontal areas as well as the functional balance between cortical and subcortical functioning could have a good clinical potential (Dalton et al., 2017).

The importance of the network functional architecture in modulating specific cognitive and psychopathological traits of AN is confirmed by the correlations between clustering coefficient and CCI and between global efficiency and STAI scores. These correlations suggest that a higher network segregation is associated with a better global processing in $\mathrm{AN}$, and that a lower global network integration is associated with higher anxiety symptoms. The serious metabolic consequences of AN may in part explain these results. In fact, integration characteristics are more expensive, while segregation properties are less energetically costly and therefore more protective (Bullmore and Sporns, 2012). We can hypothesize that high segregation plays a protective role in AN, since it probably allows a more efficient cognitive processing, but we can also speculate that a lower integration may predispose to higher anxiety symptoms in the disorder. This finding supports the importance of therapeutically addressing anxiety symptoms when they occur in comorbidity with AN (Lloyd et al., 2019; Martín et al., 2019).

The opposite correlation between lowest lifetime BMI and characteristic path length in the experimental group and in the $\mathrm{HC}$ one suggests that starvation induced a reduced path length as a form of compensation to minimize the impact of malnutrition, while in healthy subjects, in the absence of malnutrition, a similar effect is observed with an increased BMI.

The relationships between brain structural differences in the two groups and the degree of cortical and subcortical nodes suggest that structural brain alterations in AN have a specific impact on the functional architecture of the brain network. In particular, the observation that these relationships have different directions in cortical and subcortical areas indicates that the functional centrality of these regions may be differently affected by brain atrophy in AN. The increase of degree along with the average decrease in volume was especially observed in nodes of the parietal lobe, including brain areas particularly involved in somatosensory functions and spatial reasoning (Favaro et al., 2012).

Our results also pointed out a role of the 5-HTTLPR polymorphism in modulating the configuration of the overall functional network in AN. Specifically, the low functioning genotype conferred lower small-world properties and a lower modularity in patients with AN, compared with high functioning genotype.

Small-world properties reflect the presence of a proper balance between integration and segregation characteristics of a network, and indicate how much a graph can use local connections to process local information and a small number of long-distance connections to synchronize the overall flow of information (Bassett and Bullmore, 2006). The presence of a peculiar impact of 5-HTTLPR polymorphism on the network architecture suggests a role of serotonin system in balancing segregation and integration properties of the functional connectome in AN. Several lines of evidence show that abnormal activity of the 5-HT system might be involved in AN neurobiology (Solmi et al., 2016). The involvement of the serotonin transporter gene polymorphism in determining the balance of the overall functional connectome in AN could contribute in explaining why the modulation of serotonin pathways is implicated in many processes that are involved in AN etiology, psychopathology, connectivity, and executive functioning (Baker et al., 2018; Teresa et al., 2019). In addition, the opposite role of the polymorphism in AN patients and healthy women might suggest an interaction between this polymorphism and malnutrition in determining the effects on brain network properties.

The present study has two main points of strength. First, it is one of the first studies that investigates the functional connectome in AN by means of graph theory tools and the first to report about hubs distribution. Second, it investigates the role of 5-HTTLPR polymorphism in determining the properties of the network, highlighting the role of serotonin pathways in modulating network architecture. However, some limitations should be mentioned as well. The main limitation is the small sample size, which is due to the pilot nature of the study. Secondly, the study has a cross-sectional design; thus, any casual or prognostic consideration is not allowed.

\section{CONCLUSION}

In conclusion, our results point out that hub regions are differently distributed in the functional connectomes of patients with AN and HC. The distribution of these regions, alongside the correlation between some of them and specific cognitive functions in AN, suggests that they may play an important role in AN neurobiology. The correlations that emerged between different degree values of nodes and differences in brain volumes suggest an impact of structural alterations on the functional network architecture, also highlighting the need of further longitudinal observations to better understand the role of malnutrition and weight loss on graph metrics. Furthermore, the opposite effects of 5-HTTLPR polymorphism on global network characteristics in the two groups suggest an interaction of the short allele with malnutrition in modulating brain network properties. 


\section{DATA AVAILABILITY STATEMENT}

The raw data supporting the conclusions of this manuscript will be made available by the authors, without undue reservation, to any qualified researcher.

\section{ETHICS STATEMENT}

Ethical permission was obtained from the Ethics Committee of the Hospital of Padova. After completely describing the study to the subjects, informed written informed consent was obtained.

\section{REFERENCES}

American Psychiatric Association (2013). Diagnostic and Statistical Manual of Mental Disorders, 5th Edn, Washington, DC: American Psychiatric Association.

Baker, J. H., Schaumberg, K., and Munn-chernoff, M. A. (2018). HHS public access. Curr. Psychiatry Rep. 19:84. doi: 10.1007/s11920-017-0842-2

Bassett, D. S., and Bullmore, E. (2006). Small-world brain networks. Neuroscientist 12, 512-523. doi: $10.1177 / 1073858406293182$

Berg, E. A. (1948). A simple objective technique for measuring flexibility in thinking. J. Gen. Psychol. 39, 15-22. doi: 10.1080/00221309.1948.9918159

Boccaletti, S., Latora, V., Moreno, Y., Chavez, M., and Hwang, D. U. (2006). Complex networks: structure and dynamics. Phys. Rep. 424, 175-308. doi: 10. 1016/j.physrep.2005.10.009

Bullmore, E., and Sporns, O. (2012). The economy of brain network organization. Nat. Rev. Neurosci. 13, 336-349. doi: 10.1038/nrn3214

Bullmore, E. T., and Sporns, O. (2009). Complex brain networks: graph theoretical analysis of structural and functional systems. Nat. Rev. Neurosci. 10, 186-198. doi: $10.1038 / \mathrm{nrn} 2575$

Collantoni, E., Michelon, S., Tenconi, E., Degortes, D., Titton, F., Manara, R., et al. (2016). Functional connectivity correlates of response inhibition impairment in anorexia nervosa. Psychiatry Res. Neuroimaging 247, 9-16. doi: 10.1016/j.pscychresns.2015.11.008

Colombo, L., Sartori, G., and Brivio, C. (2002). Stima del quoziente intellettivo tramite l'applicazione del TIB (Test di Intelligenza Breve). G. Ital. Psicol. 3, 613-637

Crossley, N. A., Mechelli, A., Scott, J., Carletti, F., Fox, P. T., McGuire, P., et al. (2014). The hubs of the human connectome are generally implicated in the anatomy of brain disorders. Brain 137, 2382-2395. doi: 10.1093/brain/awu132

Dai, Z., Yan, C., Li, K., Wang, Z., Wang, J., Cao, M., et al. (2014). Identifying and mapping connectivity patterns of brain network hubs in Alzheimer's disease. Cereb. Cortex 25, 3723-3742. doi: 10.1093/cercor/bhu246

Dalton, B., Campbell, I. C., and Schmidt, U. (2017). Neuromodulation and neurofeedback treatments in eating disorders and obesity. Curr. Opin. Psychiatry 30, 458-473. doi: 10.1097/YCO.0000000000000361

Derogatis, L. R., Lipman, R. S., Rickels, K., Uhlenhuth, E. H., and Covi, L. (1974), The hopkins symptom checklist (HSCL): a self report symptom inventory. Behav. Sci. 19, 1-15. doi: 10.1002/bs.3830190102

Destrieux, C., Fischl, B., Dale, A., and Halgren, E. (2010). Automatic parcellation of human cortical gyri and sulci using standard anatomical nomenclature. Neuroimage 53, 1-15. doi: 10.1016/j.neuroimage.2010.06.010

Favaro, A., Clementi, M., Manara, R., Bosello, R., Forzan, M., Bruson, A., et al. (2013). Catechol-O-methyltransferase genotype modifies executive functioning and prefrontal functional connectivity in women with anorexia nervosa. J. Psychiatry Neurosci. 38, 241-248. doi: 10.1503/jpn.120068

Favaro, A., Manara, R., Pievani, M., Clementi, M., Forzan, M., Bruson, A., et al. (2014a). Neural signatures of the interaction between the 5-HTTLPR genotype and stressful life events in healthy women. Psychiatry Res. Neuroimaging 223, 157-163. doi: 10.1016/j.pscychresns.2014.05.006

Favaro, A., Santonastaso, P., Manara, R., Bosello, R., Bommarito, G., Tenconi, E., et al. (2012). Disruption of visuospatial and somatosensory functional

\section{AUTHOR CONTRIBUTIONS}

EC and AF: conceptualization, formal analysis, and methodology. $\mathrm{EC}, \mathrm{AF}$, and RM: data curation. AF: supervision. EC and PM: writing original draft. AF, ET, and MS: writing, review, and editing.

\section{SUPPLEMENTARY MATERIAL}

The Supplementary Material for this article can be found online at: https://www.frontiersin.org/articles/10.3389/fnins. 2019.01056/full\#supplementary-material

connectivity in anorexia nervosa. Biol. Psychiatry 72, 864-870. doi: 10.1016/j. biopsych.2012.04.025

Favaro, A., Tenconi, E., Degortes, D., Manara, R., and Santonastaso, P. (2014b). Effects of obstetric complications on volume and functional connectivity of striatum in anorexia nervosa patients. Int. J. Eat. Disord. 47, 686-695. doi: $10.1002 /$ eat. 22320

Fornito, A., Bullmore, E. T., and Zalesky, A. (2017). Opportunities and challenges for psychiatry in the connectomic era. Biol. Psychiatry Cogn. Neurosci. Neuroimaging 2, 9-19. doi: 10.1016/j.bpsc.2016.08.003

Frank, G., Shott, M., and DeGuzman, M. C. (2019). Recent advances in understanding anorexia nervosa. F1000Res. 8:504. doi: 10.12688/f1000research. 17789.1

Fuglset, T. S., Landrø, N. I., Reas, D. L., and Rø, Ø. (2016). Functional brain alterations in anorexia nervosa: a scoping review. J. Eat. Disord. 4:32. doi: 10.1186/s40337-016-0118-y

Garner, D. M., Olmstead, M. P., and Polivy, J. (1983). Development and validation of a multidimensional eating disorder inventory for anorexia nervosa and bulimia. Int. J. Eat. Disord. 2, 15-34. doi: 10.1002/1098-108x(198321)2:2<15:: aid-eat2260020203>3.0.co;2-6

Gaudio, S., Olivo, G., Zobel, B. B., and Schioth, H. B. (2018). Altered cerebellarinsular-parietal-cingular subnetwork in adolescents in the earliest stages of anorexia nervosa: a network-based statistic analysis. Transl. Psychiatry 8:127. doi: 10.1038/s41398-018-0173-z

Gaudio, S., Wiemerslage, L., Brooks, S. J., and Schiöth, H. B. (2016). A systematic review of resting-state functional-MRI studies in anorexia nervosa: evidence for functional connectivity impairment in cognitive control and visuospatial and body-signal integration. Neurosci. Biobehav. Rev. 71, 578-589. doi: 10.1016/j. neubiorev.2016.09.032

Geisler, D., Borchardt, V., Boehm, I., King, J. A., Tam, F. I., Marxen, M., et al. (2018). Altered global brain network topology as a trait marker in patients with anorexia nervosa. Psychol. Med. 9, 1-9. doi: 10.1017/S0033291718004002

Geisler, D., Borchardt, V., Lord, A. R., Boehm, I., Ritschel, F., Zwipp, J., et al. (2016). Abnormal functional global and local brain connectivity in female patients with anorexia nervosa. J. Psychiatry Neurosci. 41, 6-15. doi: 10.1503/jpn.140310

Geisler, D., Ritschel, F., King, J. A., Bernardoni, F., Seidel, M., Boehm, I., et al. (2017). Increased anterior cingulate cortex response precedes behavioural adaptation in anorexia nervosa. Sci. Rep. 7, 1-10. doi: 10.1038/srep42066

Haleem, D. J. (2012). Serotonin neurotransmission in anorexia nervosa. Behav. Pharmacol. 23, 478-495. doi: 10.1097/FBP.0b013e328357440d

Hong, S. B., Zalesky, A., Fornito, A., Park, S., Yang, Y. H., Park, M. H., et al. (2014). Connectomic disturbances in attention-deficit/hyperactivity disorder: a whole-brain tractography analysis. Biol. Psychiatry 76, 656-663. doi: 10.1016/ j.biopsych.2013.12.013

Hosseini, S. M. H., Hoeft, F., and Kesler, S. R. (2012). Gat: a graph-theoretical analysis toolbox for analyzing between-group differences in large-scale structural and functional brain networks. PLoS One 7:e40709. doi: 10.1371/ journal.pone. 0040709

Kaye, W. H., Wierenga, C. E., Bailer, U. F., Simmons, A. N., and Bischoff-Grethe, A. (2013). Nothing tastes as good as skinny feels: the neurobiology of anorexia nervosa. Trends Neurosci. 36, 110-120. doi: 10.1016/j.tins.2013.01.003 
Korgaonkar, M. S., Fornito, A., Williams, L. M., and Grieve, S. M. (2014). Abnormal structural networks characterize major depressive disorder: a connectome analysis. Biol. Psychiatry 76, 567-574. doi: 10.1016/j.biopsych.2014.02.018

Kuyck, K., Van Gérard, N., Laere, K., Van Casteels, C., Pieters, G., Gabriëls, L. et al. (2009). Towards a neurocircuitry in anorexia nervosa: evidence from functional neuroimaging studies. J. Psychiatr. Res. 43, 1133-1145. doi: 10.1016/j.jpsychires. 2009.04.005

Lloyd, E. C., Haase, A. M., Foster, C. E., and Verplanken, B. (2019). A systematic review of studies probing longitudinal associations between anxiety and anorexia nervosa. Psychiatry Res. 276, 175-185. doi: 10.1016/j.psychres.2019.05. 010

Martín, J., Arostegui, I., Loroño, A., and Padierna, A. (2019). Anxiety and depressive symptoms are related to core symptoms, general health outcome, and medical comorbidities in eating disorders. Eur. Eat. Disord. Rev. doi: 10. 1002/erv.2677 [Epub ahead of print].

O'Hara, C. B., Campbell, I. C., and Schmidt, U. (2015). A reward-centred model of anorexia nervosa: a focussed narrative review of the neurological and psychophysiological literature. Neurosci. Biobehav. Rev. 52, 131-152. doi: 10. 1016/j.neubiorev.2015.02.012

Oldfield, R. C. (1971). The assessment and analysis of handedness: the edinburgh inventory. Neuropsychologia 9, 97-113. doi: 10.1016/0028-3932(71)90067-4

Oldham, S., and Fornito, A. (2019). Developmental cognitive neuroscience: the development of brain network hubs. Dev. Cogn. Neurosci. 36:100607. doi: 10 . 1016/j.dcn.2018.12.005

Rubinov, M., and Bullmore, E. (2013). Schizophrenia and abnormal brain network hubs. Dialogues Clin. Neurosci. 15, 339-349. doi: 10.1016/j.siny.2015.10.004

Rubinov, M., and Sporns, O. (2010). NeuroImage complex network measures of brain connectivity: uses and interpretations. Neuroimage 52, 1059-1069. doi: 10.1016/j.neuroimage.2009.10.003

Seidel, M., Borchardt, V., Geisler, D., King, J. A., Boehm, I., Pauligk, S., et al. (2019). Abnormal spontaneous regional brain activity in young patients with anorexia nervosa. J. Am. Acad. Child Adolesc. Psychiatry doi: 10.1016/j.jaac.2019.01.011 [Epub ahead of print].

Solmi, M., Collantoni, E., Meneguzzo, P., Tenconi, E., and Favaro, A. (2018). Network analysis of specific psychopathology and psychiatric symptoms in patients with anorexia nervosa. Eur. Eat. Disord. Rev. 51, 680-692. doi: 10.1002/ erv. 2633

Solmi, M., Gallicchio, D., Collantoni, E., Correll, C. U., Clementi, M., Pinato, C., et al. (2016). Serotonin transporter gene polymorphism in eating disorders: data from a new biobank and META-analysis of previous studies. World J. Biol. Psychiatry 17, 244-257. doi: 10.3109/15622975.2015.1126675

Spielberger, C. D., Sydeman, S. J., Owen, A. E., and Marsh, B. J. (1999). "Measuring anxiety and anger with the state-trait anxiety inventory (STAI) and the statetrait anger expression inventory (STAXI)," in The Use of Psychological Testing for Treatment Planning and Outcomes Assessment, 2nd Edn, ed. M. E. Maruish, (Mahwah, NJ: Lawrence Erlbaum Associates Publishers), 993-1021.

Steinglass, J. E., Berner, L. A., and Attia, E. (2018). Cognitive neuroscience of eating disorders. Psychiatr. Clin. N. Am. 42, 75-91. doi: 10.1016/j.psc.2018. 10.008

Tenconi, E., Degortes, D., Clementi, M., Collantoni, E., Pinato, C., Forzan, M., et al. (2016). Clinical and genetic correlates of decision making in anorexia nervosa. J. Clin. Exp. Neuropsychol. 38, 327-337. doi: 10.1080/13803395.2015.111 2878

Tenconi, E., Santonastaso, P., Degortes, D., Bosello, R., Titton, F., Mapelli, D., et al. (2010). Set-shifting abilities, central coherence, and handedness in anorexia nervosa patients, their unaffected siblings and healthy controls: exploring putative endophenotypes. World J. Biol. Psychiatry 11, 813-823. doi: 10.3109/ 15622975.2010 .483250

Teresa, M., Torres, T., Rodríguez, N., Boloc, D., Gassó, P., Moreno, E., et al. (2019). Genetic variability in the serotoninergic system and age of onset in anorexia nervosa and obsessive-compulsive disorder. Psychiatry Res. 271, 554-558. doi: 10.1016/j.psychres.2018.12.019

van den Heuvel, M. P., and Sporns, O. (2013). Network hubs in the human brain. Trends Cogn. Sci. 17, 683-696. doi: 10.1016/j.tics.2013.09.012

van Dijk, K. R. A., Sabuncu, M. R., and Buckner, R. L. (2012). The influence of head motion on intrinsic functional connectivity MRI. Neuroimage 59, 431-438. doi: 10.1016/j.neuroimage.2011.07.044

Wechsler, D. (1949). Wechsler Intelligence Scale for Children; Manual. Oxford: The Psychological Corp.

Zhang, Y., Brady, M., and Smith, S. (2001). Segmentation of brain MR images through a hidden markov random field model and the expectationmaximization algorithm. IEEE Trans. Med. Imaging 20, 45-57. doi: 10.1109/ 42.906424

Zipfel, S., Giel, K. E., Bulik, C. M., Hay, P., and Schmidt, U. (2015). Anorexia nervosa: aetiology, assessment, and treatment. Lancet Psychiatry 2, 1099-1111. doi: 10.1016/S2215-0366(15)00356-359

Conflict of Interest: The authors declare that the research was conducted in the absence of any commercial or financial relationships that could be construed as a potential conflict of interest.

Copyright (c) 2019 Collantoni, Meneguzzo, Solmi, Tenconi, Manara and Favaro. This is an open-access article distributed under the terms of the Creative Commons Attribution License (CC BY). The use, distribution or reproduction in other forums is permitted, provided the original author(s) and the copyright owner(s) are credited and that the original publication in this journal is cited, in accordance with accepted academic practice. No use, distribution or reproduction is permitted which does not comply with these terms. 


\section{OPEN ACCESS}

Edited by:

Roberto Esposito

A.O. Ospedali Riuniti Marche Nord,

Italy

Reviewed by:

Timo Ruusuvirta,

University of Turku, Finland

Gennady Knyazev,

State Scientific-Research Institute of

Physiology \& Basic Medicine,

Russia

Takefumi Ueno,

Hizen Psychiatric Center (NHO),

Japan

*Correspondence:

Annemarie Wolft

awolf037@uottawa.ca

Specialty section:

This article was submitted to

Neuroimaging and Stimulation,

a section of the journal

Frontiers in Psychiatry

Received: 10 June 2019 Accepted: 09 September 2019

Published: 15 October 2019

Citation:

Wolff A, de la Salle S, Sorgini A

Lynn E, Blier P, Knott $V$ and

Northoff G (2019) Atypical Temporal Dynamics of Resting State Shapes

Stimulus-Evoked Activity in

Depression-An EEG Study on Rest-

Stimulus Interaction.

Front. Psychiatry 10:719.

doi: 10.3389/fpsyt.2019.00719

\section{Atypical Temporal Dynamics of Resting State Shapes Stimulus- Evoked Activity in Depression- An EEG Study on Rest-Stimulus Interaction}

\author{
Annemnarie Wolff ${ }^{1,2,3 *}$, Sara de la Salle ${ }^{4}$, Alana Sorgini ${ }^{1}$, Emma Lynn ${ }^{2}$, Pierre Blier ${ }^{1,2,3}$, \\ Verner Knott ${ }^{1,2,4}$ and Georg Northoff ${ }^{1,2,3}$ \\ ${ }^{1}$ University of Ottawa Institute of Mental Health Research, Ottawa, ON, Canada, ${ }^{2}$ Department of Cellular and Molecular \\ Medicine and Neuroscience, Faculty of Medicine, University of Ottawa, Ottawa, ON, Canada, ${ }^{3}$ Brain and Mind Research \\ Institute, University of Ottawa, Ottawa, ON, Canada, ${ }^{4}$ Department of Psychology, Faculty of Social Sciences, University of \\ Ottawa, Ottawa, ON, Canada
}

Major depressive disorder (MDD) is a complex psychiatric disorder characterized by changes in both resting state and stimulus-evoked activity. Whether resting state changes are carried over to stimulus-evoked activity, however, is unclear. We conducted a combined rest ( $3 \mathrm{~min}$ ) and task (three-stimulus auditory oddball paradigm) EEG study in $n=28$ acute depressed MDD patients, comparing them with $n=25$ healthy participants. Our focus was on the temporal dynamics of both resting state and stimulus-evoked activity for which reason we measured peak frequency (PF), coefficient of variation (CV), LempelZiv complexity (LZC), and trial-to-trial variability (TTV). Our main findings are: i) atypical temporal dynamics in resting state, specifically in the alpha and theta bands as measured by peak frequency (PF), coefficient of variation (CV) and power; ii) decreased reactivity to external deviant stimuli as measured by decreased changes in stimulus-evoked variance and complexity - TTV, LZC, and power and frequency sliding (FS and PS); iii) correlation of stimulus related measures (TV, LZC, PS, and FS) with resting state measures. Together, our findings show that resting state dynamics alone are atypical in MDD and, even more important, strongly shapes the dynamics of subsequent stimulus-evoked activity. We thus conclude that MDD can be characterized by an atypical temporal dynamic of its rest-stimulus interaction; that, in turn, makes it difficult for depressed patients to react to relevant stimuli such as the deviant tone in our paradigm.

Keywords: EEG, Depression, resting state, Peak frequency, alpha oscillations, theta oscillations

\section{INTRODUCTION}

\section{Temporo-Spatial Dynamics Shape Rest and Task States}

Major depressive disorder (MDD) is a complex psychiatric disorder that includes affective, cognitive, vegetative, sensorimotor, social, and perceptual changes (1). Neuronally, changes in stimulusevoked activity in response to especially affective and cognitive stimuli have been reported in MDD, including event-related potential (ERP) changes during auditory target detection paradigms as 
well as atypical power in theta $(5-8 \mathrm{~Hz})$ and/or alpha $(7-13 \mathrm{~Hz})$ frequency ranges (2-6). The origin of these neuronal changes in stimulus-evoked activity in MDD, however, remains unclear.

Recent studies have demonstrated changes in the resting state in MDD (7). FMRI resting state studies show atypical functional connectivity, especially in anterior regions of the prefrontal cortex, as well as in other networks such as the default-mode network (8), while EEG resting state studies demonstrate atypical activity, especially in theta and alpha frequencies in $\operatorname{MDD}(7,9$, 10). Given the overlap of both rest and task findings in theta and alpha frequencies, one would suggest that resting state changes may also shape stimulus-evoked activity in depression. This link is yet to be shown.

The brain's resting state, or spontaneous activity, can be characterized by an intrinsic temporo-spatial dynamic (11-13). Such dynamics concern, among other features, various frequency bands, such as theta and alpha (14). Importantly, the strength of these frequencies can be characterized by peak frequencies (1517) which, for instance, has been shown to be atypically low in the resting state of first-episode psychosis patients (17). To our knowledge, no studies have examined peak frequency in MDD specifically; this temporo-spatial dynamic measure is yet to be probed in MDD. Other resting state studies, however, have found greater functional connectivity in the theta and alpha bands in MDD when compared with healthy controls (18) as well as a decrease in long-range temporal correlations (19). A recent review found that studies of alpha asymmetry in MDD patients had inconsistent findings (20), despite its early promise (21).

The brain's temporo-spatial dynamic extends beyond its spontaneous activity to its stimulus-evoked activity. Two studies have demonstrated the interaction of prestimulus activity levels with poststimulus activity (22-24) by dynamic measures which account for complexity and variance.

One recent EEG study in healthy participants showed the relationship of prestimulus complexity, as measured with Lempel-Ziv Complexity (LZC) (25), with poststimulus trialto-trial variability (TTV) (26-29). LZC, a measure from information theory (30), quantifies patterns, and their repetition, within a sequence, with higher values of complexity indicating less structure and predictability in the sequence (25). This study (29) showed that prestimulus complexity related to poststimulus variability reduction measured through TTV, and that the poststimulus decrease in LZC and variability may capture the increase in regularity of neural activity after stimulus onset, which has been shown in computational models (31). Whether LZC and TTV, or their relation, are atypical in MDD, however, is yet to be studied.

A measure that has been applied recently is the change in peak frequency $(\mathrm{PF})$ in specific bands, the alpha and theta bands in particular $(17,32,33)$. The PF is defined as the frequency within a specified band-alpha, theta, and beta-which has the maximal power (for example, the peak frequency in the alpha band could change from 9 to $10 \mathrm{~Hz}$ when doing a task (16)) (32-34).

The PF and its change can be measured during the resting state or in response to a stimulus-PF can increase in alpha when performing a task (16), termed frequency sliding (FS) (32). Changes in $\mathrm{PF}$ is based on the neuronal principle that the firing rate of a neuron is proportional to the strength of its input (35); it has been shown (32) in both computational models and human EEG rest and task data that if input to a neural network increases, the "speed" of oscillations of a specific frequency band, and thus its PF, will increase accordingly. As a result, FS has shown to be an index, which can be measured using scalp EEG, of input to a neural network (32).

\section{General and Specific Aims}

The general aim of our combined rest and task EEG study in MDD consisted in investigating how changes in the resting state's temporo-spatial dynamics shape stimulus-evoked activity. Our study can thus be conceived of as an example of rest-stimulus interaction $(23,24,36)$ in MDD. For that purpose, we investigated resting state and task activity (auditory oddball) in MDD.

We first applied the above-mentioned dynamic measures$\mathrm{PF}$ and its variance, and power-to the resting state in MDD and a control group. Based on previous findings $(16,17)$, we hypothesized lower PF in the theta $(4-8 \mathrm{~Hz})$ and alpha $(7-13 \mathrm{~Hz})$ bands in the MDD group in comparison to the control group.

Next, we applied the dynamic measures of LZC and TTV to stimulus-evoked activity in MDD and the control group. Given the previous findings of atypical ERP, we hypothesized decreases in both poststimulus TTV change and LZC poststimulus change in MDD. The employment of an auditory oddball paradigm also allowed us to distinguish between attention-demanding task-relevant deviant tones and task-irrelevant standard tones. Consistent with previous findings in $\operatorname{MDD}(2,4,6)$, we hypothesized atypical LZC and TTV results in the deviant tones in particular.

After the LZC and TTV analysis, we measured the dynamic FS - and corresponding power sliding (PS) - to the same deviant and standard tones in the theta and alpha frequency bands. We hypothesized that these measures in the MDD group would differ significantly from the healthy controls in the deviant tones, but not the standards.

Finally, we sought to relate rest and task measures. We hypothesized that the dynamical measures of the resting state (PF, power) correlate with poststimulus TTV and LZC change in both groups. The temporo-spatial dynamics of the resting state, including its changes, may thus be conveyed to the subsequent stimulus-evoked activity and its own dynamics.

\section{METHODS}

\section{Participants}

Twenty-eight participants with Major Depressive Disorder $(\mathrm{MDD})($ age: mean $=54 \pm 18$ years, range $=16-73$ years; 19 female) and twenty-five healthy controls (age: mean $=46 \pm 16$ years, range $=21-70$ years; 14 female) completed this study. MDD participants were recruited from an outpatient population with major depressive disorder (MDD) that were participating in a larger adjacent clinical trial. Clinical assessments and diagnosis of depression were conducted with a study psychiatrist. MDD participants were evaluated with the Montgomery-Åsberg Depression Rating Scale (MADRS) (37) and found to have scores 
between 14 and 37 (mean $=28 \pm 6$ ) (inclusive) at the time of the electroencephalography (EEG) session.

Exclusion criteria for the MDD participants were the following: DSM-IV disorder other than MDD; depression secondary to severe medical illness; positive urine drug screen for illicit substances or non-prescribed medicine; substance or alcohol abuse or dependence 6 months prior to enrollment; diagnosis of a neurological disorder; pregnancy or lactation; an unstable clinical finding that might be negatively affected by the treatment of the larger clinical trial; liver function tests three times the upper normal limit; inadequately corrected hypothyroidism or hyperthyroidism; clinically significant deviation from the reference range in clinical laboratory test results; imminent suicidal or homicidal risk; and participation in another clinical treatment study with a drug that had not yet received approval.

Age- and sex-matched healthy adults were recruited from the local community as a control group. Participants were assessed using the SCID non-patient version (SCID-NP; 38) and the Family Interview for Genetic Studies (FIGS; 39), following an initial telephone session and a subsequent faceto-face interview.

The Beck Depression Inventory (BDI) score was used to verify that the control participants did not have depression. We administered the BDI to the patient group as part of the larger adjacent clinical trial; BDI scores before and after treatment in the clinical study were compared. Only the BDI scores recorded before the treatment initiation were used here. MDD Participants had BDI scores greater than 13 (mean $=28 \pm 10$ ), while all healthy control participants had BDI scores below 13 $($ mean $=1 \pm 2)$.

The experimental protocols were approved by the research ethics committee of the University of Ottawa Institute of Mental Health Research, and the study was carried out with their permission. Written informed consent was obtained from each participant prior to study participation.

\section{EEG Session}

EEG data were recorded using a Brain Vision EasyCap with 32 $\mathrm{Ag} / \mathrm{AgCl}$ electrodes at a sampling rate of $500 \mathrm{~Hz}$. Electrode $\mathrm{AF}_{\mathrm{z}}$ served as the ground and an additional nose electrode served as the reference during recording.

Additional channels were added for independent component analysis (ICA) decomposition: vertical ocular (above and below the left eye), and horizontal ocular (the outer canthi of the right and left eyes). The impedance of all channels was maintained at less than $5 \mathrm{k} \Omega$ during recording.

Prior to beginning the task, participants completed threeminute resting state EEG recordings with their eyes closed.

The task was an auditory oddball paradigm (40) and was presented to participants using the Presentation software (Neurobehavioral Systems, Albany, CA, USA). Participants were presented with 800 tones in four blocks (200 tones per block). Eighty percent of the tones, standard tones, were $1,000 \mathrm{~Hz}$ and $70 \mathrm{~dB}$ pure tones $336 \mathrm{~ms}$ in length. Ten percent were deviant tones at $700 \mathrm{~Hz}(70 \mathrm{~dB}$ pure tones lasting for $336 \mathrm{~ms}$, identical to standards). Participants were instructed to respond to the deviant tones by a button press. The remaining $10 \%$ were novel non-target-participants did not respond by a button pressenvironmental sounds (ie., dog bark, horn, etc) at 65-75 dB for 169-399 ms. Between these stimuli, participants were presented with a fixation cross for $1 \mathrm{~s}$. Reaction times to target responses were recorded.

The standard and deviant stimuli were the focus of this study as they were alike; both were pure tones with the same length and power. The novel stimulus had varying lengths and was not a pure tone, so not comparable to the other two stimuli. The novel stimuli, however, were analyzed with TTV and LZC for differences between MDD and controls. This was done only to determine if any differences found in the deviant stimuli were due to the infrequent presentation of the stimulus.

\section{EEG Preprocessing}

All EEG data preprocessing was completed using EEGLAB (v14) (41), which required MATLAB (The MathWorks) v2018b, including the use of the Optimization, Statistics and Signal Processing Toolboxes. All statistical analyses were completed in MATLAB v2018b.

The raw continuous data was low- and high-pass FIR filtered from $1 \mathrm{~Hz}$ to $40 \mathrm{~Hz}$, and then visually inspected. If channels were flat longer than $5 \mathrm{~s}$, had less than 0.80 correlation with neighboring channels, or had line noise greater than four standard deviations difference compared to other channels, they were spherically interpolated.

The task data was then epoched-with no baseline correction-to stimulus onset. All files were re-referenced to the surface Laplacian reference, according to the methods of previous studies $(42,43)$, to spatially filter the data; activity recorded at the specified electrode had a higher correspondence with cortical activity just below it than had an alternative reference montage been used.

All stationary artifacts, specifically eye movements, were reduced using Independent Component Analysis (ICA) and the Multiple Artifact Rejection Algorithm (44, 45).

\section{Resting State Measures: Peak Frequency (PF), Coefficient of Variation (CV), Power}

We began our analysis by investigating the resting state data. All preprocessed resting state files were first cut to the same length (85,000 data points). According to previous studies (16, 17, 29, $32,34,46,47)$, our a priori interests were focused on activity in the alpha $(7-13 \mathrm{~Hz})$ and theta $(4-8 \mathrm{~Hz})$ bands. For this reason, and due to the findings of $\mathrm{dmPFC}$ in rest-task interactions (36) and its implication in MDD (48-50), all analyses were done with electrodes $\mathrm{Fz}$ for theta $(51,52)$ and broadband activity, and $\mathrm{Pz}$ for alpha related activity $(53,54)$. All data analysis was completed in MATLAB (v2018b).

The peak frequency (PF) was calculated according to the methods of MX Cohen (32). Specifically, the preprocessed broadband data was first FIR bandpass filtered-with 15\% transition zones added to each edge of the filtered range 
according to the published methods (32). The data were then Hilbert transformed to get the analytic signal, and the phaseangle timeseries was extracted using MATLAB's angle function. To compute the frequency of the peak within the filtered band, the first derivative of the phase-angle timeseries was taken. Finally, a median filter (filter order 10) was applied to the timeseries according to the published methods of this approach (32). The mean over the full three-minute resting state of the peak frequency was calculated at electrode Fz for the theta band $(4-8 \mathrm{~Hz})$ and $\mathrm{Pz}$ for the alpha band $(8-13 \mathrm{~Hz})$, as was the power in the same electrodes for the same bands.

The coefficient of variation $(\mathrm{CV})$ has previously been measured in neuroscientific data on single neuron spike trains, as well as data from larger arrays $(55,56)$. Recently, however, it has also been applied to EEG data $(28,57)$. The CV, also termed the Fano Factor (58), is a measure of relative variability as

$$
C V=\frac{\sigma}{\mu}
$$

with $\sigma$ being the standard deviation of the peak frequency in the resting state and $\mu$ being the mean. We measured the $\mathrm{CV}$ as it quantifies the stability of the peak and normalizes the standard deviation as it divides by the mean.

\section{Trial-To-Trial Variability}

Trial-to-Trial Variability (TTV) was calculated according to the methods of previous studies $(23,27-29)$. TTV is defined as the variability changes over trials with respect to variability at stimulus onset:

$$
\operatorname{TTV}(t)=\frac{\sigma_{o t}(t)-\sigma_{o t}(0)}{\sigma_{o t}(0)} x 100
$$

where $\sigma_{o t}(t)$ is the SD of the EEG signal over trials at timepoint $t$ and $\sigma_{o t}(0)$ is the SD over trials at stimulus onset. Therefore, each poststimulus timepoint is relative to stimulus onset.

In the task, eighty stimuli were deviants with many more standards. To compare the two stimuli, in this and subsequent analyses, eighty randomly chosen trials of standard stimuli were extracted. Therefore, all measures contained the same number of deviant and standard trials, with the standards randomly chosen.

To compare the TTV between stimuli and groups, the area under the curve (AUC) between stimulus onset $(0 \mathrm{~ms})$ and 500 ms was computed and compared statistically.

\section{Lempel-Ziv Complexity Analysis}

As computed in a previous study (29), we applied a measure of complexity from information theory (30) to the non-baseline corrected data. Lempel-Ziv Complexity (LZC) was calculated from previous studies $(25,29,59)$ in MATLAB v2018b using a custom script. In both the pre- and post-stimulus periods for which LZC was calculated, $300 \mathrm{~ms}$ of the signal was measured.

The EEG signal was first converted into a binary sequence with each data point in a timeseries $x(i)$ being converted to a symbol in the sequence $s(i)$ :

$$
s(i)=\left\{\begin{array}{l}
0 \text { if } x(i)<T_{d} \\
1 \text { if } x(i) \geq T_{d}
\end{array}\right.
$$

where $T_{d}$ is the threshold (25), the median of the timeseries here. The median was chosen according to previous studies $(25,29)$ as it is unaffected by extreme values in a sequence. This sequence $s(i)$ is then scanned from left to right. The complexity measure $c(n)$ is increased by one each time a new sequence of consecutive values occurs (25). Finally, the complexity $C(n)$ is normalized to control for signal length:

$$
C(n)=\frac{c(n)}{\frac{n}{\log _{2}(n)}}
$$

where $n$ is the length of the sequence. LZC has no units.

The difference between the pre- and post-stimulus was calculated (poststim LZC minus prestim LZC) and this was compared between MDD and controls $(\mathrm{CON})$.

\section{Peak Frequency Sliding (FS) and Power Sliding (PS) During Task}

Calculated in the same way as the PF in the resting state (see above) according to the methods of MX Cohen (32), the peak frequency sliding (FS) in theta $(4-8 \mathrm{~Hz})$ and alpha $(7-13 \mathrm{~Hz})$ was calculated. For each of the deviant and standard trials, the FS was computed, and the mean over all the trials was then calculated. To account for any changes in PF at stimulus onset between groups (see Supplementary Figure 1), the data were converted to percent change; the PF in the task is the percent change relative to stimulus onset.

Next, to calculate the power sliding (PS), the same analysis was done, with one difference: rather than extract the phase-angle timeseries, the modulus (absolute value) of the Hilbert transform was extracted. This was then squared-at each timepoint-to give a timeseries of the power in the specified band. As with the FS, the PS was converted to percent change relative to stimulus onset to correct for any differences in power between MDD and controls.

Finally, the AUC in $100 \mathrm{~ms}$ intervals was calculated for both measures and compared statistically. The time intervals in which the AUC was measured were as follows: $376-476 \mathrm{~ms}$ for the alpha FS; 150-250 ms for the alpha PS; 400-500 ms for the theta FS; $166-266 \mathrm{~ms}$ for the theta PS.

\section{Task-Task Correlation: Trial-To-Trial Variability (TTV) and Frequency/Power Sliding (FS/PS)}

To compare the results from the TTV analysis and the FS and PS analysis, two-tailed Spearman correlations were performed 
for both stimuli on all participants (MDD and CON) together. The significance level for all correlations was 0.05 . To correct for multiple statistical tests, the Benjamini-Hochberg False Discovery Rate (60) was applied to all $p$-values. The $p$-values stated in the results and figures are therefore corrected for multiple comparisons.

\section{Rest-Task Correlation: Resting State PF/ CV and Task (TTV, LZC, FS, and PS)}

Finally, to link the resting state findings (PF, CV, and Power) to those from the task (TTV, LZC, FS, and PS), two-tailed Spearman correlations were performed on all participants (MDD and $\mathrm{CON}$ ) together. Again, the significance level for all correlations was 0.05. As above, the Benjamini-Hochberg False Discovery Rate (60) was applied to all $p$-values, so all $p$-values reported in the results and figures have been corrected for multiple comparisons.

\section{Statistical Analyses}

All statistical analyses were done in MATLAB v2018b, using the Statistics toolbox, at a significance level of 0.05 .

It has been shown that large interindividual differences between participants exists and, in fact, is a particular focus of recent research $(29,44,61-65)$. Typical parametric tests, however, often fail to account for a factor that has an effect in many individuals since they focus on the average effect (66). With that in mind, and at the direction of a study on that exact idea (66), to compare the effects of stimulus (deviant, standard) and group (MDD, CON), Kolmogorov-Smirnov two-sample tests were used. The Kolmogorov-Smirnov test is a nonparametric statistical test which compares the distributions of data rather than any parameter (mean, median). In MATLAB, the function kstest 2 was used for this test.

For the correlations, the Spearman's two-tailed correlation was performed for all tests as most of the variable distributions were non-normal. The function corr, with type = "Spearman," was used in MATLAB for this correlation.

To correct for multiple statistical tests, the BenjaminiHochberg False Discovery Rate (60) was applied to all $p$-values.

\section{RESULTS}

\section{Modulation of Peak Frequency and Coefficient of Variation During the Resting State in Depression}

We began our analysis with the resting state data. Over the full 3 -min resting state, the mean peak frequency $(\mathrm{PF})$, coefficient of variation $(\mathrm{CV})$, and mean power in the theta $(4-8 \mathrm{~Hz})$ and alpha $(7-13 \mathrm{~Hz})$ bands was measured and statistically tested with Kolmogorov-Smirnov two-sample nonparametric tests.

In the theta band, we first found a difference between the MDD and control participants in the PF $\left(D_{28,25}=0.496, p=\right.$ $0.002)$. We then tested to see if there was an effect of group on the $\mathrm{CV}$ and power and found that this was also true in the theta band $\left(D_{28,25}=0.541, p=9.021 \times 10^{-4}\right.$ for $C V ; D_{28,25}=$ $0.670, p=1.042 \times 10^{-5}$ for power) (Figure 1A). In the MDD participants, the $\mathrm{PF}$ was higher, and $\mathrm{CV}$ and power lower than the healthy controls.

We then repeated the same statistical analysis for the alpha band. Again, we found a difference between MDD and controls in the PF $\left(D_{28,25}=0.546, p=3.947 \times 10^{-4}\right), \mathrm{CV}\left(D_{28,25}=0.554, p=\right.$ $\left.3.947 \times 10^{-4}\right)$, and power $\left(D_{28,25}=0.479, p=0.003\right)$ (Figure 1B).
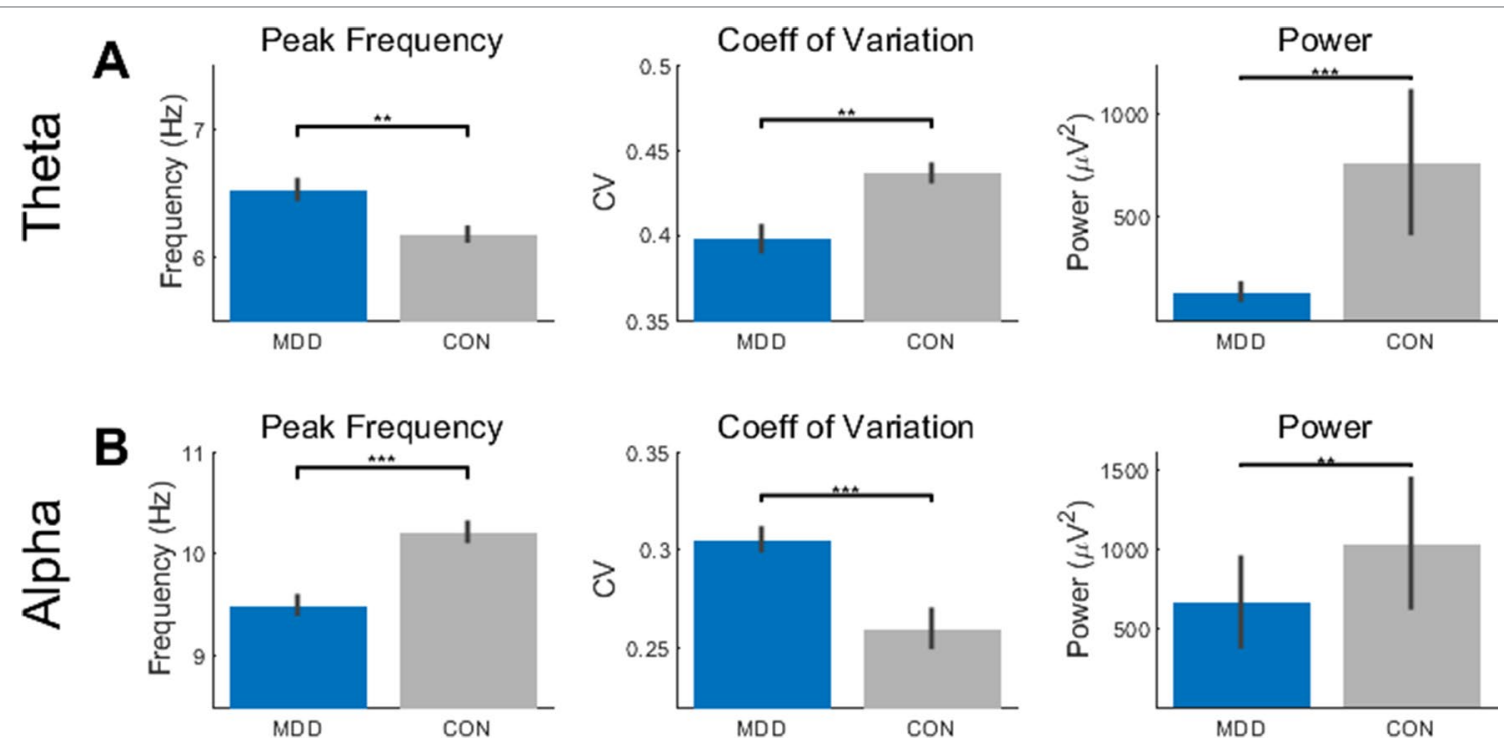

FIGURE 1 | Resting state activity showed differences in theta and alpha between the MDD group and healthy controls. (A) The peak frequency (PF), coefficient of variation (CV), and power measures showed higher PF, lower CV, and lower power in the MDD participants than the controls. (B) In the alpha band, the MDD group had lower PF, higher CV, and lower power. All $p$-values are FDR corrected for multiple comparisons. ${ }^{\star \star}:<0.01,{ }^{\star \star \star}:<0.001$. 
In contrast to the theta band, the PF and power were lower and $\mathrm{CV}$ higher in the MDD when compared to the healthy controls.

Taken together, these data suggest opposite, or reciprocal, modulation of PF and CV in theta and alpha in MDD. High PF and low $\mathrm{CV}$ were found in theta while the opposite pattern low PF and high CV - was observed in alpha. Interestingly, both alpha and theta show reduced power. Together, these findings suggest more unstable resting state activity pattern in theta and alpha in MDD.

\section{Trial-To-Trial Variability Reduced for Deviant Stimuli in Depression}

After completing the analysis of the resting state data, we then investigated the differences between the MDD and control group during the task. As TTV has been stated as measuring the effect of the stimulus to decrease/increase the ongoing spontaneous activity of the brain, we wanted to investigate whether there was an effect of group (MDD, control) or stimulus (deviant, standard) on TTV. To do so, the AUC of the TTV curve between stimulus onset and $500 \mathrm{~ms}$ at electrode $\mathrm{Fz}$ was calculated (Figure 2A).

A two-sample Kolmogorov-Smirnov test found a difference in AUC between the MDD and control participants when presented with the deviant stimuli $\left(D_{26,25}=0.489, p=0.005\right)$, with smaller AUC in the MDD participants. Therefore, there was less of a change in variability in the MDD participants when presented with the deviant stimuli than in the control participants. As the TTV was measured relative to stimulus onset, it can be inferred that the deviant stimulus made less of an impact on the ongoing spontaneous activity in the MDD group than in the control group. In contrast, no difference was found between groups in the standard stimuli $\left(D_{26,25}=0.326, p=0.105\right)$ (Figure 2B).

Since the deviant stimuli were only presented $10 \%$ of the time (see Methods), this difference may be due to the rarity of the stimulus; the control participants had more of a change in variability as they were presented with a rarer stimulus, not because they were presented with a rarer stimulus also relevant to the task. To determine if this was the case, we did the same analysis on the novel stimuli (baby cry, so environmental nontarget auditory stimulus) which were also presented for $10 \%$ of the trials.

We found no difference between MDD and controls for these novel stimuli $\left(D_{26,25}=0.169, p=0.823\right)$. This result supports the idea that the difference found in deviant stimuli is not simply because they are more infrequent than the standards, as were that true, a similar difference would also exist in the novel stimuli. This was not the case.

In sum, TTV change after stimulus onset was reduced in the task-relevant deviant tone in MDD, suggesting decreased impact of this tone on stimulus-evoked activity.

\section{Decreased Lempel-Ziv Complexity Change for Deviant Stimuli in Depression}

The next step in the task-related analysis was to measure LempelZiv Complexity (LZC). This was done to determine if there
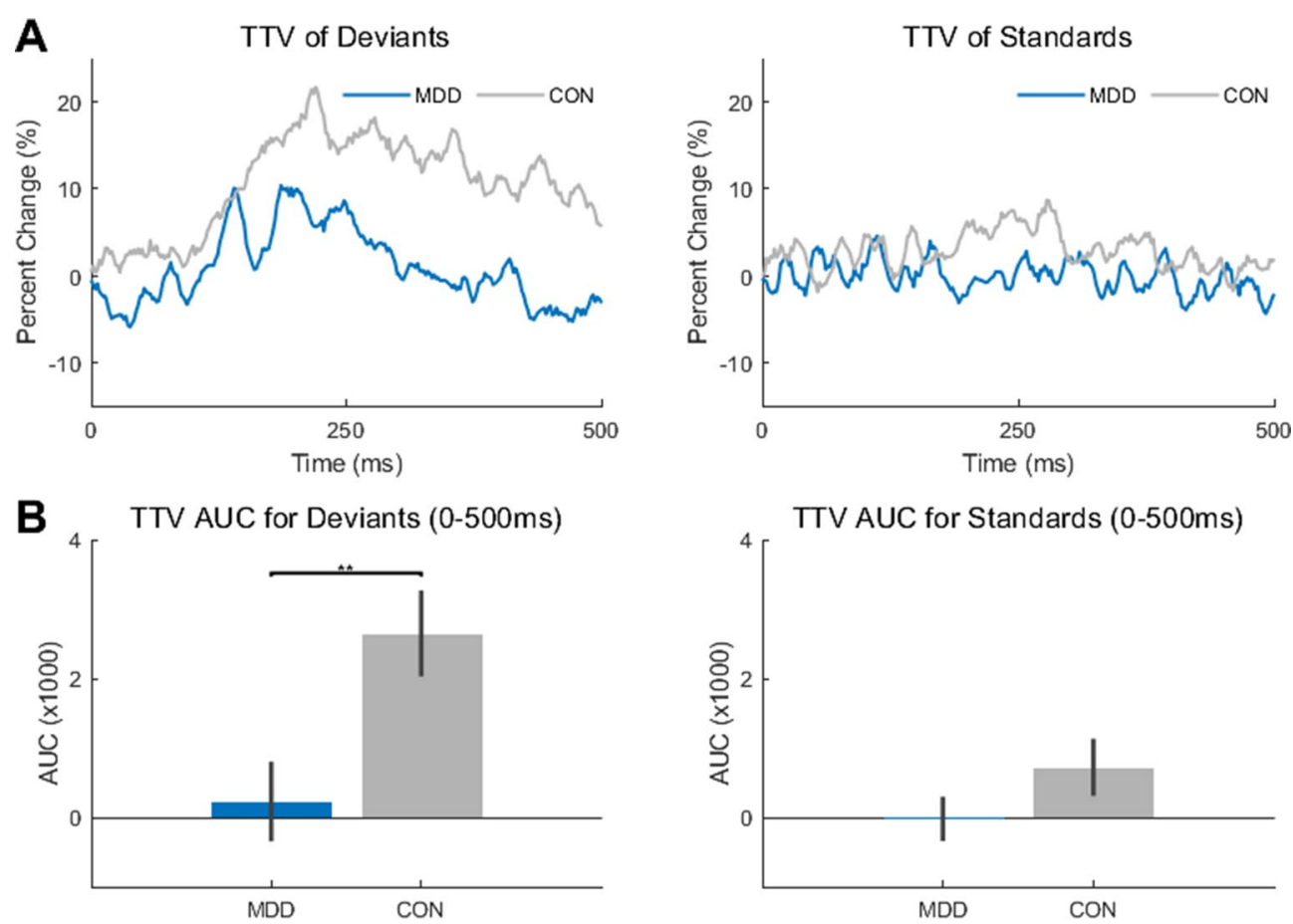

FIGURE 2 | Trial-to-trial variability (TTV) showed significantly less change in variability in the MDD group for deviant stimuli. (A) TTV curves (80 trials) in both groups for deviant and standard stimuli. (B) Area under the curve (AUC) was measured between 0-500ms for both groups and stimuli. The AUC in the MDD group was significantly lower in the deviant stimuli compared to the healthy controls. All $p$-values are FDR corrected for multiple comparisons. ${ }^{* *}:<0.01$. 
was an effect of group (MDD, control) or stimulus (deviant, standard) on the structure or pattern of the timeseries, which LZC measures. LZC (no units) was measured in $300 \mathrm{~ms}$ windows before and after stimulus onset. The difference was calculated by subtracting the prestim from the poststim as we wanted to see if the arrival of the stimulus coincided with a change in LZC and by what amount. It was tested, for each stimulus, for statistical difference between the two groups of participants with the twosample Kolmogorov-Smirnov test.

After calculating the difference-poststim minus prestimthere was found to be a difference between groups in the deviant stimuli $\left(D_{26,25}=0.402, p=0.049\right)$, but not in the standard stimuli $\left(D_{26,25}=0.308, p=0.133\right)$ (Figure 3). As we did in the TTV AUC above, we calculated the LZC difference in the novel stimuli to see if the difference found in the deviant stimuli was only because they are rarer than the standard stimuli. We found no difference between MDD and controls for the novel stimuli $\left(D_{26,25}=0.183\right.$, $p=0.742$ ). If there had been a difference between groups here, the difference found in the deviant stimuli would be due to the infrequent presentation of the stimulus, not due to the stimulus itself; we would conclude that the MDD group responded differently to rare stimuli, not task-related rare stimuli. As there was found to be no difference between the groups, we can infer that the finding in the deviant stimuli is not due to its frequency.

In the deviant stimuli then, the change in LZC was larger in the control participants than in the MDD group; MDD participants had less of a LZC decrease after hearing the deviant stimulus than healthy controls. One final statistical test determined that the prestim LZC for both stimuli did not differ between groups $\left(D_{26,25}=0.182, p=0.752\right.$ for deviants and $D_{26,25}=0.131, p=0.752$ for standards). This finding is important to consider as it is the degree of change that is relevant; this indicates that the MDD subjects show decreased propensity of change when presented the deviant stimulus.
In sum, MDD patients showed less changes in LZC from the prestim period to the poststim period in deviant stimuli. This again suggests decreased impact of the external stimulus on stimulus-evoked activity which, as the LZC findings suggest, is related to decreased propensity of prestimulus activity for change by the external stimulus.

\section{Frequency and Power Sliding in the Task Showed Differences in Depression}

Our final task-related analysis was to measure the effect of group (MDD, control) and stimulus (deviant, standard) on change in peak frequency (PF) and power (PS) after stimulus onset. To do so in the theta and alpha bands after stimulus onset, the AUC in a $100 \mathrm{~ms}$ interval was calculated.

In the alpha band, we found a difference between groups in FS when participants were presented with deviant stimuli $\left(D_{26,25}=\right.$ $0.430, p=0.032$ ) (Figure 4C). The MDD group had larger AUC than the control group. This difference was not found when the participants were presented with standard stimuli $\left(D_{26,25}=\right.$ 0.149, $p=0.917$ ) (Figure 4D). For the PS in the same band, the difference between the MDD group and the controls was significant for both stimuli $\left(D_{26,25}=0.460, p=0.006\right.$ for deviants and $D_{26,25}=0.539, p=0.001$ for standards), with larger AUC in the MDD participants than the controls.

Conversely, there was no difference in FS for both stimuli in the theta band $\left(D_{26,25}=0.332, p=0.188\right.$ for deviants and $D_{26,25}=$ $0.192, p=0.685$ for standards) (Figure 4A, B). As in the alpha band, however, there was a difference in PS in both the deviant stimuli $\left(D_{26,25}=0.922, p=2.453 \times 10^{-10}\right)$ and the standards $\left(D_{26,25}=\right.$ $0.462, p=0.006)$. The power for the MDD group was higher for the deviant stimuli and lower for the standard stimuli.

These same time intervals for AUC were analyzed again in the same way, however the absolute values of the FS- not the

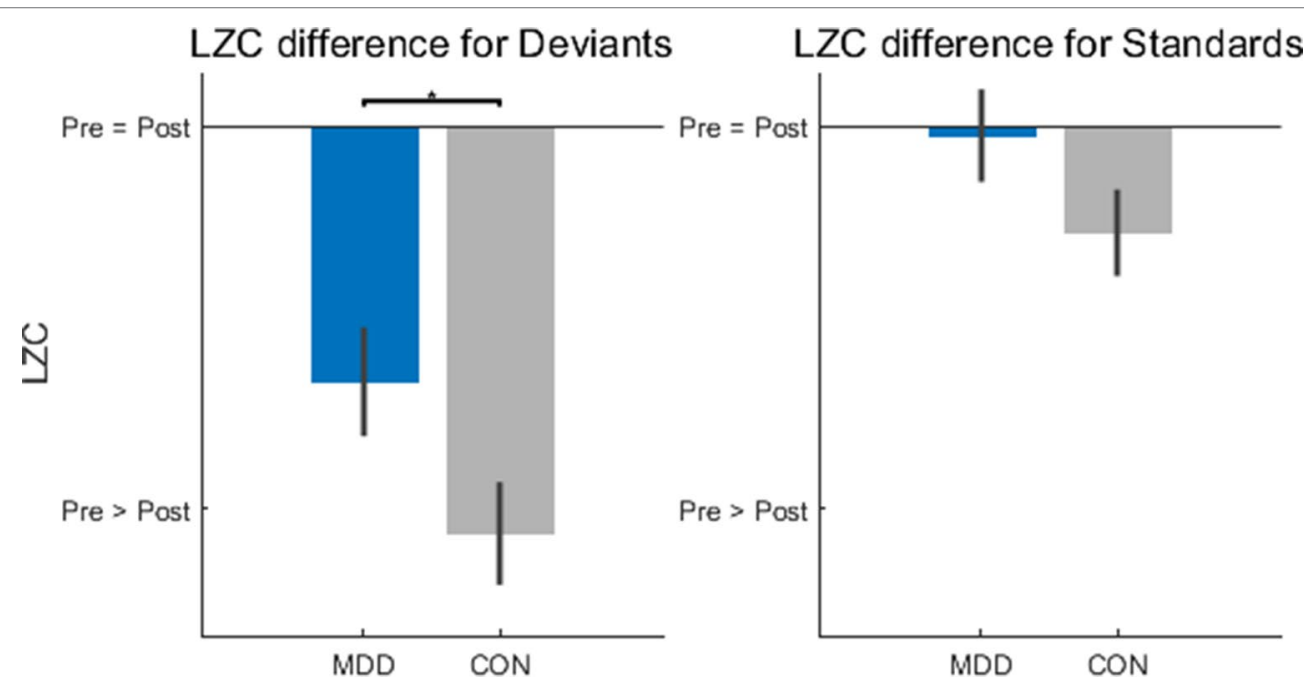

FIGURE 3 | Lempel-Ziv Complexity (LZC) changes after stimulus onset were significantly lower in the MDD group for deviant stimuli. LZC was measured in 300ms time windows before and after stimulus onset. When the difference was calculated (poststim minus prestim), for deviant stimuli the MDD group showed less of a decrease when compared to healthy controls. All $p$-values are FDR corrected for multiple comparisons. *: $<0.05$. 

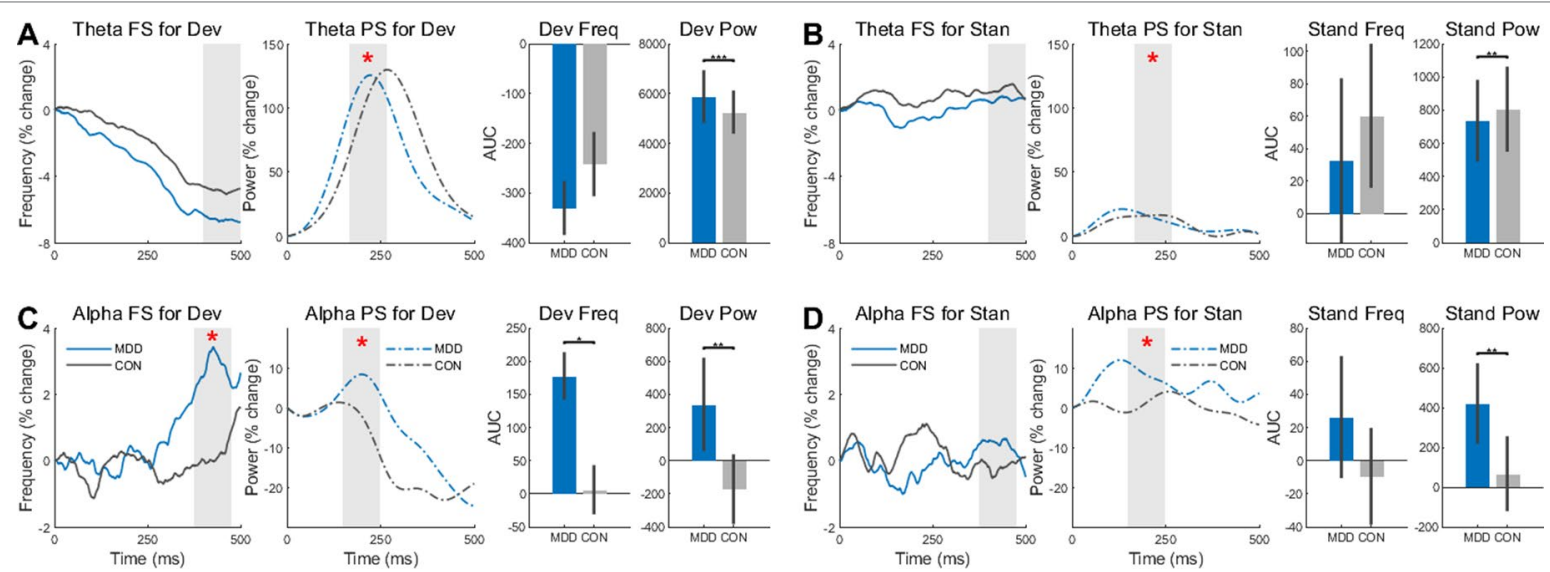

FIGURE 4 | Frequency Sliding (FS) and Power Sliding (PS) found greater changes to deviant stimuli in the MDD group for theta and alpha frequency bands. (A) In the deviant stimuli, Theta PS was higher in the MDD group and lower (B) for standard stimuli. (C) In the alpha band, both FS and PS were significantly higher for the deviant stimuli in the MDD group. (D) Only the PS was higher in the MDD group for standard stimuli in the alpha band. Area under the curve for 100ms time intervals were measured. Time intervals are shown with grey shading. All $p$-values are FDR corrected for multiple comparisons. ${ }^{\star}:<0.05,{ }^{\star \star}:<0.01,{ }^{\star \star \star}:<0.001$.

percent change-were measured to see if there was an absolute difference in these intervals, not just a relative difference. No difference in FS was found in the alpha band $\left(D_{26,25}=0.177, p=\right.$ 0.806 for deviants and $D_{26,25}=0.220, p=0.806$ for standards), however there was a difference in PS $\left(D_{26,25}=1.0, p=1.887 \times 10^{-12}\right.$ for deviants and $D_{26,25}=1.0, p=1.887 \times 10^{-12}$ for standards) (Supplementary Figures 1C, D). The same results were found in the FS $\left(D_{26,25}=0.145, p=0.934\right.$ for deviants and $D_{26,25}=0.188$, $p=0.934$ for standards $)$ and PS $\left(D_{26,25}=1.0, p=1.887 \times 10^{-12}\right.$ for deviants and $D_{26,25}=1.0, p=1.887 \times 10^{-12}$ for standards) in the theta band (Supplementary Figures 1A, B).

In both bands and both stimuli, there was a difference in PS between the MDD and control group, while FS showed a difference between groups in percent change only in the deviant stimuli of the alpha band. Together, the increased changes, especially in PS, suggest larger instability of poststimulus activity over time in MDD than in healthy subjects. Our data on the relative difference clearly point out a reduced propensity for change by the external task-related stimulus on the ongoing spontaneous neural activity.

\section{Task-Task Correlation Between Trial-To- Trial Variability and Frequency/Power Sliding}

After the results found above, we sought to determine if there was a relationship between the TTV and the FS/PS results. This was tested with two-tailed Spearman's correlations.

We first found significant correlations in the deviant stimuli between TTV AUC and both theta FS AUC $(\rho=0.334, p=0.023)$ and PS AUC $(\rho=0.377, p=0.023)$ (Figure 5A). This indicates that as the TTV variability increased after stimulus onset (larger AUC), two things happened: 1) theta peak frequency decreased; 2) theta power increased.

Next, we did the same analysis in the standard stimuli. We found a significant correlation between TTV AUC and PS AUC in the alpha $(\rho=-.324, p=0.023)$ and theta $(\rho=0.319$, $p=0.023$ ) band (Figure 5B). This indicates two points: 1 ) as the TTV variability increased in the alpha band, the power decreased; 2) as the TTV variability increased in the theta band, the power increased.

Finally, to link the neural activity to the behavioral data, we correlated the TTV AUC with the mean reaction times. We found a significant negative correlation in the deviant stimuli ( $\rho=-0.351, p=0.023$ ) (Figure 5C), but not in the standard stimuli. Therefore, participants with higher variability increase in the deviant stimuli had faster reaction times.

In sum, we had three findings: 1) participants had positive correlations between variability changes and theta power in both stimuli, with an increase in variability corresponding with an increase in theta power; 2 ) participants had a positive correlation between variability changes and PF in theta for the deviants, with an increase in variability corresponding with an increase in PF; 3) participants had a negative correlation between variability changes and PS in alpha for the standards, with an increase in variability corresponding with a decrease in PS. The TTV AUC also showed behavioral relevance with greater increases in variability showing faster reaction times.

\section{Rest-Task Correlations Between Resting State and Task-Related Measures}

The last group of analyses we did was to test whether there was a relationship between the resting state findings and the taskrelated findings. To do this, two-tailed Spearman's correlations, with the resting state measures as the independent variables, were done.

We first found a significant negative correlation between the resting state PF in the theta band and the prestimulus LZC for the standard stimuli ( $\rho=-0.345, p=0.018$ ) (Figure 6A); participants with higher PF had lower prestimulus complexity when presented with standard stimuli.

Next, we correlated the CV with the task-related measures. In the theta band we found a significant positive correlation between the CV and the TTV AUC in the deviant stimuli $(\rho=0.360, p=$ 

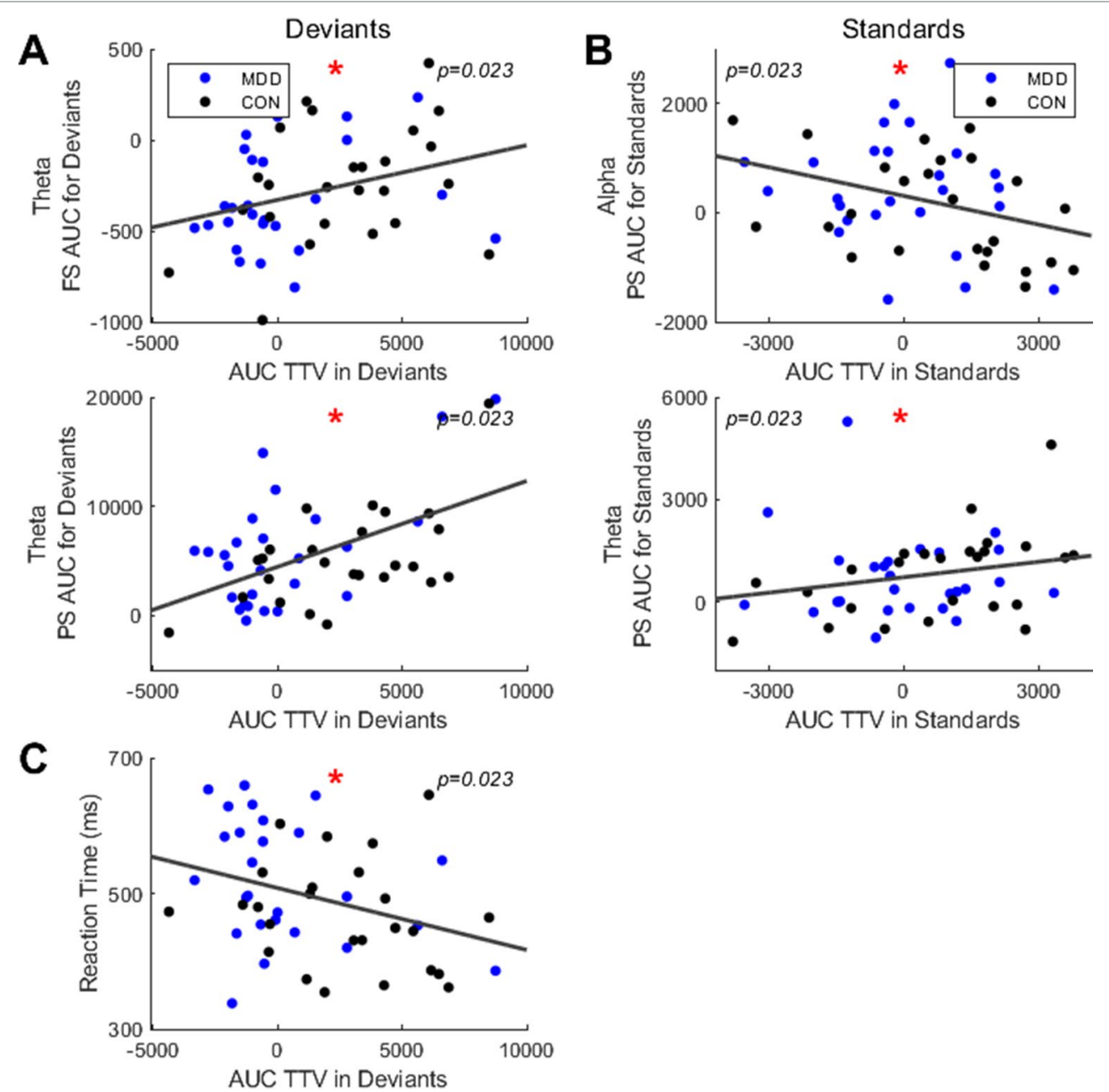

FIGURE 5 | Spearman correlations found significant relationships between area under the curve (AUC) in TTV and FS, PS and reaction times. (A) For deviant stimuli, AUC in TTV had significant correlations across all participants with FS and PS in the theta band. (B) For standard stimuli, AUC in TTV had significant correlations with PS in theta and alpha. (C) AUC in TTV for deviant stimuli correlated significantly with the mean reaction time. All $p$-values are FDR corrected for multiple comparisons. * : 0.05 .

0.016). A significant positive correlation between the CV and the PS for deviants was also found in the theta band ( $\rho=0.420, p=$ 0.009) (Figure 6B). Therefore, the greater the dispersion around the mean in the theta band, as measured by the $\mathrm{CV}$, the greater the a) variability increase and b) theta power increase for the deviant stimuli.

To finish, we correlated the resting state theta power with the task-related measures. We found significant negative correlations with both the pre- and poststimulus LZC in both stimuli: prestimulus LZC was $\rho=-0.411, p=0.009$ for deviants and $\rho=-0.311, p=0.029$ for standards; poststimulus LZC was $\rho=-0.397, p=0.009$ for deviants and $\rho=-0.308, p=0.029$ for standards (Figure 6C). Therefore, participants with higher resting state theta power had lower complexity both before and after the stimulus was presented in both stimuli.

In sum, only the measures in the resting state theta band correlated with the task measures; significant correlations were found between a) power and both pre- and poststimulus LZC in both stimuli; b) CV and both TTV AUC and PS in deviant stimuli only; c) PF and prestimulus LZC in standard stimuli only. This suggests that the increased instability of the resting state in MDD is carried over to the pre- and poststimulus activity, where it is manifest in decreased responsiveness to external stimuli as measured by decreased changes in LZC and TTV.

\section{DISCUSSION}

The main findings of our combined rest-task EEG study in MDD are as follows: i) opposite changes in peak frequency (PF) and coefficient of variation $(\mathrm{CV})$ in theta and alpha ranges in MDD; ii) decreased neural response to deviant stimuli in MDD as measured by the change in LZC and TTV, and an increased change in FS and PS in the alpha band; iii) significantly different PS change in both stimuli and bands with theta power in standard stimuli lower in MDD and the other stimulus and band showing a 

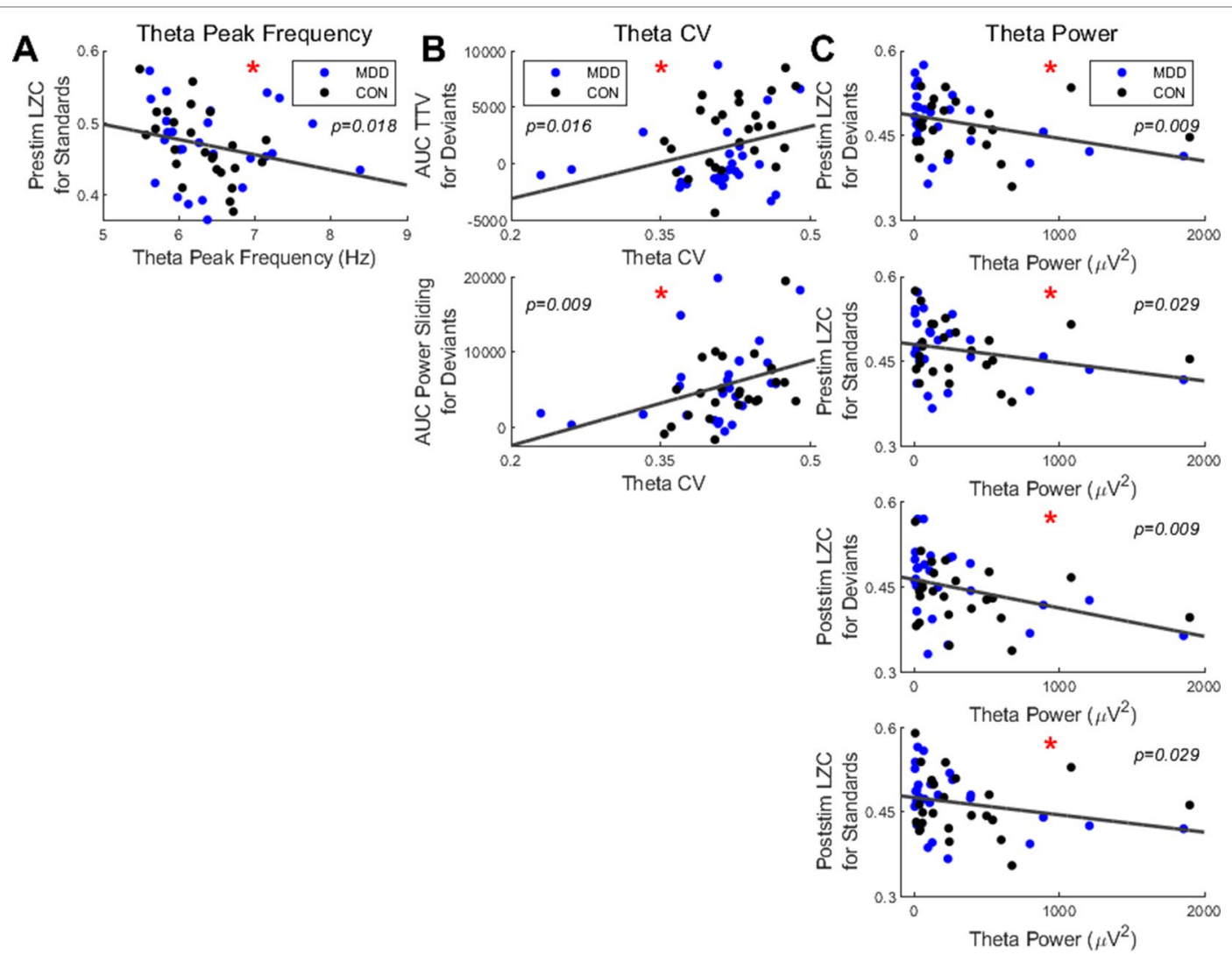

FIGURE 6 | Spearman correlations found significant relationships between resting state measures in the theta band and LZC in both stimuli and PS and TTV AUC in deviant stimuli. (A) Resting state theta PF had a negative correlation with prestim LZC in standard stimuli. (B) The CV in the theta band had significant correlations with PS and TTV AUC for deviant stimuli. (C) Resting state theta power had significant negative correlations with both pre- and poststim LZC in both bands. All $p$-values are FDR corrected for multiple comparisons. ${ }^{*}:<0.05$.

higher change in MDD; iv) significant correlation of the dynamic resting state measures with stimulus-evoked dynamics.

Together, our findings show how differences in the resting state's temporo-spatial dynamics, as seen here in participants with MDD, are carried over and thus shaping subsequent stimulus-evoked activity as has been previously shown in healthy participants $(23,24)$. MDD can thus be characterized by atypical temporal dynamics of rest-stimulus interaction-specifically in the theta and alpha frequency bands-during attentiondemanding stimuli such as the deviant tone in our auditory oddball paradigm.

\section{Resting State-Reciprocal Modulation of Theta-Alpha Dynamic}

EEG resting state studies have demonstrated differences between participants with MDD and healthy controls (5, 7, 18, 67-69), among them decreased selectivity of functional connectivity during the resting state in MDD, and some with a particular emphasis on the theta and alpha frequency bands (19-20, 21, 70).

Our findings in the theta band is consistent with previous studies and symptoms of MDD. Depression has been related to neuronal atrophy in the hippocampus (71) and almost the complete absence of long-range temporal correlations in the theta band (19). Theta oscillations have been closely related to activity of the hippocampus (72), and a common symptom of depression (1) is memory impairment (73), which has been localized to the hippocampus $(74,75)$. These studies link theta, the hippocampus and symptoms of depression with our results which may be due to issues in the hippocampus or in the wider limbic system (67).

To our knowledge, no studies have examined peak frequency changes in MDD specifically, however a recent study found participants with first episode psychosis to have lower alpha peak frequency during the resting state (17). Other alpha findings include a study that found decreased alpha activity in participants with MDD (76). Our study replicated these findings and extend them significantly.

Applying the measurement of $\mathrm{PF}$, in conjunction with a variance measure - coefficient of variation $(\mathrm{CV})$ - to resting state data, we could show opposing patterns of these two measures in theta and alpha. The CV is a measurement of the dispersion around the mean, with an increase in the $\mathrm{CV}$ corresponding to an increase in the dispersion of the data in the variable being measured. Also, since it divides the standard deviation of the data by the mean (Equation 1), it normalizes for individual mean values, thus negating any differences. As this measure was 
applied to the PF during the resting state, a lower CV indicates more stability and uniformity of the PF during the resting state.

We did not specifically examine the inter-dependence of theta and alpha dynamics to their respective psychological functions such as memory recall (theta) $(52,77)$ and attention/gating (alpha) (78-81), though a reciprocal link may exist. The shift towards slower PF and higher CV in theta may indicate that the memory recall $(52,77)$ is dominant over attention and gating (78-81) which may be diminished by higher PF and lower CV in alpha. This notion is speculative, though consistent with clinical symptoms long studied in MDD (1).

\section{Stimulus-Evoked Activity-Intrinsic and Extrinsic Components}

Though EEG studies have shown similar task-related changes in participants with MDD-decreased ERP amplitude in participants with MDD when presented with a novel task-related stimulus $(2,4,6)$ - and one similar resting state finding (76), our results extend these observations by showing altered dynamics in pre- (resting state activity) and poststimulus activity and apply methods new to psychiatric data.

We show first that the TTV has significantly lower change after the deviant stimulus is presented in participants with MDD. This finding implies a decreased response to the deviant stimulus of the prestimulus activity at stimulus onset as TTV change has been thought to show how a stimulus impacts the ongoing variability of activity $(23,24,26,29,82)$, and has shown to be different than controls in participants with autism (83). This is further supported by our second finding: decreased change in LZC after stimulus onset in MDD participants when presented with deviant stimuli, though there was no difference in the prestimulus period between groups. We therefore suggest that the prestimulus activity in MDD participants is less responsive to task-related stimuli when compared to healthy controls.

The specific role of the prestimulus' reactivity to this task-relevant stimulus is further supported by our PS and FS findings during stimulus-evoked activity. Both FS and PS show significantly larger relative changes [no difference in the absolute values (Supplemental Figure 1)], especially in the alpha band, in the patient group. This suggests the dynamic instability of poststimulus activity in MDD as the basis for these changes. The differences between the groups can be observed mainly in deviant tones, but differences in power were evident in standard tones.

We now assume that such decreased responsiveness to taskrelevant external stimuli, such as the deviant tone, is related to increased internal instability with higher variance across time in the poststimulus period. Our results on FS and PS support this; though we could not analyze single-trials, we could observe increased variance in both frequency and power in a time-resolved manner after stimulus onset. The decreased responsiveness to task-relevant external stimuli-seen in the decreased TTV and LZC changes-may thus be related to instability in the spontaneous activity during the stimulus period. This can be seen in the increased FS and PS; TTV changes are theorized to have the purpose of changing ongoing spontaneous activity as a way to increase the signal-to-noise ratio in favor of the stimulus $(26,27,29,82,84)$. Further support for this is the correlation of TTV with both FS and PS in alpha and theta. This thus connects more intrinsic measures (FS, PS as measured also during the resting state)_intrinsic as being changes of the ongoing spontaneous activity which is always present-and extrinsic-extrinsic as resulting from a stimulus from the external environment-(TTV) dynamic features of stimulus-evoked activity.

Taken together, all three findings-1) TTV and LZC, 2) FS and PS, and 3) their correlation-suggest the following: poststimulus activity in MDD may be characterized by increased neural activity instability. This in turn may decrease its ability to react to external task-relevant stimuli from the environment. Put more generally, MDD patients may suffer from an imbalance between ongoing spontaneous neural activity (internal) and components from the environment (external) in their stimulusevoked activity, with the internal component dominating the external one.

This imbalance seems to have a particularly strong impact on attention-demanding task-relevant stimuli (85-91). This is in accordance with the fact that we observed the main changes during the deviant tone that is more attention-demandingparticipants were told to respond to it-than the standard. We therefore hypothesize that the high degree of intrinsic noise from the ongoing spontaneous activity during stimulus-evoked activity (increased FS and PS differences) may make it impossible for the depressed participants to suppress this intrinsic noise when the stimulus is presented. They are required to devote attention to, and thus process, the task-relevant external stimulus (here the deviant tone) which we see in the decreased change in TTV and LZC in the healthy participants. Maybe, MDD participants are not capable of doing so, or doing so to the degree necessary; stimulus-evoked activity may be simply too noisy for attention-demanding stimuli to be properly processed. Our aim in this study was to determine if there were differences between groups, which we did, so a future study must be done specifically to examine this signal-to-noise ratio hypothesis in participants with MDD.

\section{Rest-Stimulus Interaction-Resting State Dynamics Shape Intrinsic and Extrinsic Components of Stimulus-Evoked Activity}

Our results, as measured by FS and PS, suggest differences in theta and alpha dynamic activity in the MDD participants during the poststimulus period. These dynamics may influence other stimulus-related activity which can be measured by TTV and LZC. The question for us, then, is what is the source of such dynamics in the ongoing spontaneous activity? One possibility is the resting state itself; its dynamics may transfer and shape that of the poststimulus period, as it has been shown that the state of this spontaneous activity when the stimulus is presented has a differential impact on the stimulus-evoked activity $(23,24)$.

We explored such a hypothesis by correlating resting state measures (PF, CV, and power) with those of stimulus-evoked activity (TTV, LZC, FS, and PS). As expected, this yielded 
significant correlations; these findings suggest a direct relationship between resting state dynamics and those of the stimulus-evoked activity as measured by LZC and TTV; the former is carried over to the latter, as was previously shown in healthy participants only $(23,24,29)$. Our aim in this study was to determine if there was a difference between groups, but the strength and boundaries of this transfer from ongoing spontaneous activity to stimulusevoked activity, as well as a possible role in psychiatric symptoms, however, must be examined specifically in a future study.

These findings suggest that the resting state dynamics in the MDD group, especially in the theta band, is related to the decreased reactivity to task-relevant external stimuli which was measured by TTV and LZC changes. This may be mediated by the resting state dynamics as measured by FS and PS. Together, these findings suggest that the differences in the resting state temporal dynamics of MDD participants are carried over to subsequent stimulus-evoked activity.

\section{Physiologic-Mechanistic Basis of Atypical Rest-Task Interaction in MDD}

It has been shown, through computational modelling (32) and human/animal studies $(16,92,93)$ that changes in input intensity at the neural network level can be measured by changes in PF, not power, measured on the scalp using EEG. It was also shown that changes in $\mathrm{PF}$ - as a result of changes in input to the corresponding neural network-can produce changes in the networks' subsequent response to inputs $(16,32,94)$. This leads to sensitization/desensitization of the neuron by lowering/raising the threshold to generate an action potential (32).

Together these findings suggested a hypothesis $(16,32)$ : changes in PF is a gain-control mechanism. Briefly, faster oscillations-which would have higher PF-allow for accurate but restrained responding as the neurons must meet a higher threshold before an action potential is generated. At the same time, slower oscillations-lower $\mathrm{PF}$-enable fast responding as the threshold to generate an action potential is lower. The drawback to this lower threshold is that these oscillations may be guided by noise as it permits a response to weaker inputs, so more inputs will meet this threshold.

In addition, input from a relatively slower oscillation will have a longer time window into which to integrate voltage; one cycle is longer in a slower frequency such as $9 \mathrm{~Hz}$ than a relatively faster frequency like $10 \mathrm{~Hz}$. Therefore, bands with decreased $\mathrm{PF}$ have longer time windows in which action potentials can occur [relevant for detecting temporally coincidental inputs at the synapse (14)]. This in turn leads to greater spike timing variability (32). This adaptive mechanism (16) responds to the demands presented by a task and may adjust the sampling rateincreasing the PF would allow for more cycles in the same period of time-of incoming information required by this task. This is supported by findings from several studies $(95,96)$.

How does this apply to our results? Our findings of opposing differences between groups in theta and alpha show that PF is directly related to uniformity; MDD participants had higher theta $\mathrm{PF}$ and a lower, more stable $\mathrm{CV}$, and lower alpha $\mathrm{PF}$ and a higher, less stable CV. The power in both bands was lower in the patient group, illustrating the nonlinear relationship between PF and power as shown previously $(32,97)$.

We found that the group with the faster PF also had the more stable $\mathrm{PF}$, as measured by the $\mathrm{CV}$. In the patients with depression, therefore, the resting state theta band was faster, more uniform-thus stable-and less powerful than the controls. In the resting state alpha band, the patients with depression were slower, less stable and less powerful, which is consistent with previous studies (76). This suggests an extreme instance of reciprocal inter-dependence in MDD between theta and alpha in opposite directions.

These theoretical studies that lead to the adaptive gaincontrol mechanism hypothesis are consistent with our results. As described above, a lower PF experiences more spike timing variability and is driven more by noise. Higher PF, in contrast, has more selective responses due to a higher threshold for generating action potentials. Our resting state results showed that the MDD group had lower PF and higher $\mathrm{CV}$, but they also had a greater relative response to the deviant stimuli in the alpha band. This could be an attempt to overcome the lower baseline level and to increase the sampling rate (increase the $\mathrm{PF}$ ), thereby increasing the information being taken in, as required by the task-relevant deviant tone. This PF increase response to the stimulus was also seen in the control group, however the baseline activity of the two groups differ (Figure 7), which may be the most important finding. The lower threshold in the alpha band that comes with a lower PF allows for more noise and variability generally in the signal. The signal-to-noise ratio (SNR) is then insufficient to respond to the task-relevant stimulus, despite this increase in alpha PF after stimulus onset.

This possibility may underlie the decreased changes in TTV and LZC after stimulus onset we found in the MDD group when compared with the healthy controls. It may be that the variability in the ongoing resting state alpha activity in the MDD group, specifically in this important band for sensory processing (33, 80, 98-101), due to a lower action potential threshold is too high for the PF increase seen in our results. The SNR is below what is required for changes in TTV and LZC after presentation of a task-relevant stimulus, as both these measures have been shown to relate to activity in the alpha band (29). If supported, this hypothesis would support symptoms of depression which include decreased response to sensory stimuli $(1,6)$, and present resting state alpha PF as a possible therapeutic target.

\section{Limitations}

There were several limitations to this study. To begin, the relatively small number of participants in each group $(<30)$, as well as their age (means of 54 and 46 years), made distilling out differences related to the MDD diagnosis-amid the large interindividual differences-more challenging. This was aided using the nonparametric Kolmogorov-Smirnov (KS) test which measures differences in distributions rather than the mean. This statistical test provided two advantages here: 1) it is robust to outlying participants while mean-based statistical tests are sensitive to them, and 2) it is effective with small sample sizes (less than 30 participants) (66). Therefore, though our study did have relatively 


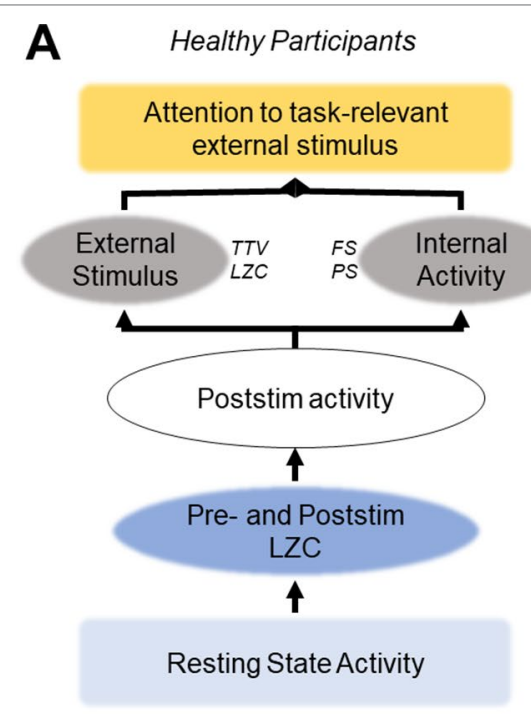

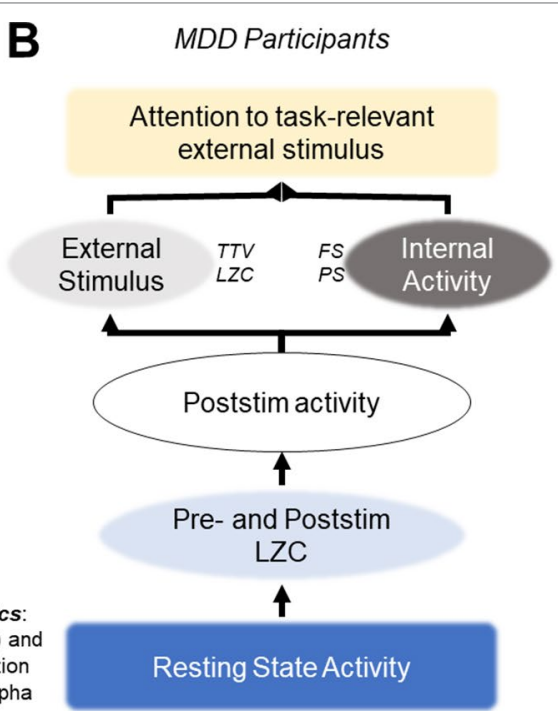

FIGURE 7 | Hypothesis: atypical resting state activity leads to increased internal activity and decreased response to external stimuli. (A) In healthy participants, normal resting state activity allows for the quenching of ongoing variability (TTV, LZC) and an optimal signal-to-noise ratio (SNR) to respond to task-relevant stimuli. (B) In MDD participants, atypical resting state activity leads to higher levels of noise in the SNR, overshadowing the task-relevant stimulus. Recent studies support this hypothesis; however, it must be tested directly in future studies.

small sample sizes, and there was large interindividual variability in some of our results, our use of the KS test lessened these factors.

Furthermore, a third possible factor may have accounted for the differences between groups found in our results. As noted above, the mean age difference between the MDD and control group was just under 10 years. This difference may have contributed, at least partially, to the differences we found (102-104). In fact, it may be the case that a third unknown factor contributed to our findings of differences between the MDD and control groups. Replication of this analysis would determine if this were to be the case.

Next, the recording of data would benefit in future studies from high density EEG or MEG rather than the 32 channels used here. High-density recordings would allow for source localization and analyses in source space, as well as greater topographical specificity of activity on the scalp.

Finally, though the task paradigm presented a simple sensory stimulus with behavioral response (reaction times), the short intertrial intervals may have prevented some of the measures in our analysis (FS, PS, LZC) from a return to baseline before the presentation of the subsequent stimulus. As the intertrial interval was only one second long, the stimulus-evoked activity may have carried over into the trial of the subsequent stimulus; the EEG activity measured just after onset of that next stimulus would have been the result of a) the ongoing spontaneous activity, b) activity evoked by the stimulus just presented, and c) activity carried over from the stimulus of the previous trial. For this reason, the analyses described here should be applied to other task paradigms and datasets to verify the results. Until then, these findings are preliminary.

\section{CONCLUSION}

We here investigated combined rest and task EEG in major depressive disorder (MDD). Our findings show differences in resting state dynamics between the patient group and healthy controls, and that this is carried over to subsequent stimulusevoked activity. We therefore tentatively postulate atypical temporal dynamics of rest-stimulus interaction in MDD. This difference in MDD patients may predispose them to respond in an atypical way to task-related external stimuli.

\section{DATA AVAILABILITY STATEMENT}

The datasets generated for this study are available on request to the corresponding author.

\section{ETHICS STATEMENT}

The studies involving human participants were reviewed and approved by University of Ottawa Institute of Mental Health Research Ethics Board. Written informed consent to participate in this study was provided by the participants themselves.

\section{AUTHOR CONTRIBUTIONS}

The data were acquired by SS, EL, and VK and the patients were referred to VK by PB. The data were analyzed by AW, AS, and GN and the manuscript was written by AW and GN. 


\section{FUNDING}

This work was supported by the EJLB-Michael Smith Foundation, the Canadian Institute of Health Research (CIHR), and the Hope of Depression Foundation (HDRF).

\section{ACKNOWLEDGEMENTS}

The first author (AW) would like to thank the computational neuroscience summer school of the Centre of Neural Dynamics at the University of Ottawa, and its organizers Andre Longtin and

\section{REFERENCES}

1. Beck AT, Alford BA. Depression: Causes and treatment. 2nd ed. University of Pennsylvania Press (2009). Vol. xxi. p. 405.

2. Pang X, Xu J, Chang Y, Tang D, Zheng Y, Liu Y, et al. Mismatch negativity of sad syllables is absent in patients with major depressive disorder. PLoS One (2014) 9:1-8. doi: 10.1371/journal.pone.0091995

3. Kalev K, Bachmann M, Orgo L, Lass J, Hinrikus H. Lempel-Ziv and multiscale Lempel-Ziv complexity in depression. Proc Annu Int Conf IEEE Eng Med Biol Soc EMBS (2015) 4158-61. doi: 10.1109/EMBC.2015.7319310

4. Mu Z, Chang Y, Xu J, Pang X, Zhang H, Liu X, et al. Pre-attentive dysfunction of musical processing in major depressive disorder: A mismatch negativity study. J Affect Disord (2016) 194:50-6. doi: 10.1016/j.jad.2016.01.028

5. Gärtner $M$, Irrmischer $M$, Winnebeck E, Fissler M, Huntenburg JM, Schroeter TA, et al. Aberrant long-range temporal correlations in depression are attenuated after psychological treatment. Front Hum Neurosci (2017) 11:1-10. doi: 10.3389/fnhum.2017.00340

6. Hirakawa N, Hirano Y, Nakamura I, Hirano S, Sato J, Oribe N, et al. Right hemisphere pitch-mismatch negativity reduction in patients with major depression: An MEG study. J Affect Disord (2017) 215:225-9. doi: 10.1016/j. jad.2017.03.046

7. Koo PC, Thome J, Berger C, Foley P, Hoeppner J. Current source density analysis of resting state EEG in depression: a review. J Neural Transm (2017) 124:S109-18. doi: 10.1007/s00702-015-1432-2

8. Kaiser RH, Andrews-Hanna JR, Wager TD, Pizzagalli DA. Large-scale network dysfunction in major depressive disorder: a meta-analysis of resting-state functional connectivity. JAMA Psychiatry (2015) 72:603-11. doi: 10.1001/jamapsychiatry.2015.0071

9. Knyazev GG, Savostyanov AN, Bocharov AV, Bazovkina DV, Proshina EA. Resting state networks mediate the effect of genotype by environment interaction on mental health. Neuroscience (2018) 369:139-51. doi: 10.1016/j. neuroscience.2017.11.010

10. Shim M, Im CH, Kim YW, Lee SH. Altered cortical functional network in major depressive disorder: A resting-state electroencephalogram study. NeuroImage Clin (2018) 19:1000-7. doi: 10.1016/j.nicl.2018.06.012

11. Raichle ME, MacLeod AM, Snyder AZ, Powers WJ, Gusnard DA, Shulman GL. A default mode of brain function. Proc Natl Acad Sci U S A (2001) 98:676-82. doi: 10.1073/pnas.98.2.676

12. Northoff G. How is our self altered in psychiatric disorders? A neurophenomenal approach to psychopathological symptoms. Psychopathology (2014) 47:365-76. doi: 10.1159/000363351

13. Northoff G. The brain's spontaneous activity and its psychopathological symptoms - "Spatiotemporal binding and integration." Prog NeuroPsychopharmacol Biol Psychiatry (2018) 80:81-90. doi: 10.1016/j. pnpbp.2017.03.019

14. Buzsáki G. Rhythms of the brain. Oxford University Press (2006). Vol. xv. p. 448 doi: 10.1093/acprof:oso/9780195301069.001.0001

15. Grandy TH, Werkle-Bergner M, Chicherio C, Schmiedek F, Lövdén M, Lindenberger U. Peak individual alpha frequency qualifies as a stable neurophysiological trait marker in healthy younger and older adults. Psychophysiology (2013) 50:570-82. doi: 10.1111/psyp.12043
Richard Naud, for their contribution towards the information theory/complexity aspect of this study.

\section{SUPPLEMENTARY MATERIAL}

The Supplementary Material for this article can be found online at: https://www.frontiersin.org/articles/10.3389/fpsyt.2019.00719/ full\#supplementary-material

SUPPLEMENTARY FIGURE 1 | Frequency Sliding (FS) and Power Sliding (PS) between groups for both stimuli in absolute values, not relative change. (A) Theta for deviant stimuli. (B) Theta for standard stimuli. (C) Alpha for deviant stimuli. (D) Alpha for standard stimuli. ${ }^{*}:<0.05,{ }^{* \star}:<0.01,{ }^{* \star *}:<0.001$.
16. Mierau A, Klimesch W, Lefebvre J. State-dependent alpha peak frequency shifts: Experimental evidence, potential mechanisms and functional implications. Neuroscience (2017) 360:146-54. doi: 10.1016/j. neuroscience.2017.07.037

17. Murphy M, Öngür D. Decreased peak alpha frequency and impaired visual evoked potentials in first episode psychosis. NeuroImage Clin (2019) 22. doi: 10.1016/j.nicl.2019.101693

18. Leuchter AF, Cook IA, Hunter AM, Cai C, Horvath S. Resting-state quantitative electroencephalography reveals increased neurophysiologic connectivity in depression. PLoS One (2012) 7:e32508. doi: 10.1371/journal. pone. 0032508

19. Linkenkaer-Hansen K, Monto S, Rytsälä H, Suominen K, Isometsä E, Kähkönen S. Breakdown of long-range temporal correlations in theta oscillations in patients with major depressive disorder. J Neurosci (2005) 25:10131-7. doi: 10.1523/JNEUROSCI.3244-05.2005

20. Kaiser AK, Gnjezda MT, Knasmüller S, Aichhorn W. Electroencephalogram alpha asymmetry in patients with depressive disorders: Current perspectives. Neuropsychiatr Dis Treat (2018) 14:1493-504. doi: 10.2147/NDT.S137776

21. Allen JJB, Cohen MX. Deconstructing the "resting" state: Exploring the temporal dynamics of frontal alpha asymmetry as an endophenotype for depression. Front Hum Neurosci (2010) 4:1-14. doi: 10.3389/ fnhum.2010.00232

22. Smith SM, Fox PT, Filippini N, Laird AR, Beckmann CF. Correspondence of the brain's functional architecture during activation and rest. PNAS (2009) 106:13040-5. doi: 10.1051/0004-6361:20052893

23. He BJ. Spontaneous and task-evoked brain activity negatively interact. J Neurosci (2013) 33:4672-82. doi: 10.1523/JNEUROSCI.2922-12.2013

24. Huang Z, Zhang J, Longtin A, Dumont G, Duncan NW, Pokorny J, et al. Is there a nonadditive interaction between spontaneous and evoked activity? Phase-dependence and its relation to the temporal structure of scale-free brain activity. Cereb Cortex (2017) 27:1-23. doi: 10.1093/ cercor/bhv288

25. Aboy M, Hornero R, Abásolo D, Álvarez D. Interpretation of the Lempel-Ziv complexity measure in the context of biomedical signal analysis. IEEE Trans Biomed Eng (2006) 53:2282-8. doi: 10.1109/TBME.2006.883696

26. Churchland MM, Yu BM, Cunningham JP, Sugrue LP, Cohen MR, Corrado GS, et al. Stimulus onset quenches neural variability: A widespread cortical phenomenon. Nat Neurosci (2010) 13:369-78. doi: 10.1038/nn.2501

27. Arazi A, Censor N, Dinstein I. Neural variability quenching predicts individual perceptual abilities. J Neurosci (2017a) 37:97-109. doi: 10.1523/ JNEUROSCI.1671-16.2017

28. Arazi A, Gonen-Yaacovi G, Dinstein I. The magnitude of trial-by-trial neural variability is reproducible over time and across tasks in humans. eNeuro (2017b) 4:0292-17.2017. doi: 10.1523/ENEURO.0292-17.2017

29. Wolff A, Yao L, Gomez-Pilar J, Shoaren M, Jiang N, Northoff G. Neural variability quenching during decision-making: neural individuality and its prestimulus complexity. Neuroimage (2019b) 192:1-14. doi: 10.1016/j. neuroimage.2019.02.070

30. Gershenson C, Fernandez N. Complexity and information: Measuring emergence, self-organization, and homeostasis at multiple scales. Complexity (2012) 2:3. doi: 10.1002/cplx.21424 
31. Deco G, Hugues E. Neural network mechanisms underlying stimulus driven variability reduction. PLoS Comput Biol (2012) 8:e1002395. doi: 10.1371/journal. pcbi.1002395

32. Cohen MX. Fluctuations in oscillation frequency control spike timing and coordinate neural networks. J Neurosci (2014) 34:8988-98. doi: 10.1523/ JNEUROSCI.0261-14.2014

33. Gulbinaite R, van Viegen T, Wieling M, Cohen MX, VanRullen R. Individual alpha peak frequency predicts $10 \mathrm{~Hz}$ flicker effects on selective attention. J Neurosci (2017) 37:10173-84. doi: 10.1523/JNEUROSCI.1163-17.2017

34. Furman AJ, Meeker TJ, Rietschel JC, Yoo S, Muthulingam J, Prokhorenko M, et al. Cerebral peak alpha frequency predicts individual differences in pain sensitivity. Neuroimage (2018) 167:203-10. doi: 10.1016/j. neuroimage.2017.11.042

35. Hong S, Lundstrom BN, Fairhall AL. Intrinsic gain modulation and adaptive neural coding. PLoS Comput Biol (2008) 4:e1000119.

36. Northoff G, Qin P, Nakao T. Rest-stimulus interaction in the brain: A review. Trends Neurosci (2010) 33:277-84. doi: 10.1016/j.tins.2010.02.006

37. Montgomery A, Asberg M. A new depression scale designed to be sensitive to change. Br J Psychiatry (1979) 134:382-9. doi: 10.1192/bjp.134.4.382

38. First M, Spitzer R, Gibbon M, Williams J. Structured Clinical Interview for DSM-IV-TR Axis I Disorders, Non-Patient Version. Washington, DC: American Psychiatric Association (2002).

39. Maxwell M. Interview for Genetic Studies (FIGS). Manual for FIGS. Clinical Neurogenetics Branch, Intramural Research Program, National Institute of Mental Health; Bethesda, MD: (1992).

40. Jaworska N, De Somma E, Blondeau C, Tessier P, Norris S, Fusee W, et al. Auditory P3 in antidepressant pharmacotherapy treatment responders, nonresponders and controls. Eur Neuropsychopharmacol (2013) 23:1561-9. doi: 10.1016/j.euroneuro.2013.03.003

41. Delorme A, Makeig S. EEGLAB: An open source toolbox for analysis of single-trial EEG dynamics. J Neurosci Methods (2004) 134:9-21. doi: 10.1016/j.jneumeth.2003.10.009

42. Cohen MX. Effects of time lag and frequency matching on phase-based connectivity. J Neurosci Methods (2015) 250:137-46. doi: 10.1016/j. jneumeth.2014.09.005

43. Carvalhaes C, De Barros JA. The surface Laplacian technique in EEG: Theory and methods. Int J Psychophysiol (2015) 97:174-88. doi: 10.1016/j. ijpsycho.2015.04.023

44. Winkler I, Haufe S, Tangermann M. Automatic classification of artifactual ica- components for artifact removal in EEG signals. Behav Brain Funct (2011) 7:30. doi: 10.1186/1744-9081-7-30

45. Winkler I, Brandl S, Horn F, Waldburger E, Allefeld C, Tangermann M. Robust artifactual independent component classification for BCI practitioners. J Neural Eng (2014) 11:035013. doi: 10.1088/1741-2560/11/3/035013

46. Harris AM, Dux PE, Jones CN, Mattingley JB. Distinct roles of theta and alpha oscillations in the involuntary capture of goal-directed attention. Neuroimage (2017) 152:171-83. doi: 10.1016/j.neuroimage.2017.03.008

47. Wolff A, Gomez-Pilar J, Nakao T, Northoff G. Interindividual neural differences in moral decision-making are mediated by alpha power and delta/theta phase coherence. Sci Rep (2019a) 9, 1-13. doi: 10.1038/ s41598-019-40743-y

48. Başar E, Güntekin B. Review of delta, theta, alpha, beta, and gamma response oscillations in neuropsychiatric disorders. Suppl Clin Neurophysiol (2013) 62:303-41. doi: 10.1016/B978-0-7020-5307-8.00019-3

49. Downar J, Geraci J, Salomons TV, Dunlop K, Wheeler S, McAndrews MP, et al. Anhedonia and reward-circuit connectivity distinguish nonresponders from responders to dorsomedial prefrontal repetitive transcranial magnetic stimulation in major depression. Biol Psychiatry (2014) 76:176-85. doi: 10.1016/j.biopsych.2013.10.026

50. Bakker N, Shahab S, Giacobbe P, Blumberger DM, Daskalakis ZJ, Kennedy $\mathrm{SH}$, et al. rTMS of the dorsomedial prefrontal cortex for major depression: Safety, tolerability, effectiveness, and outcome predictors for $10 \mathrm{~Hz}$ versus intermittent theta-burst stimulation. Brain Stimul (2015) 8:208-15. doi: 10.1016/j.brs.2014.11.002

51. Papenberg G, Hämmerer D, Müller V, Lindenberger U, Li SC. Lower theta inter-trial phase coherence during performance monitoring is related to higher reaction time variability: A lifespan study. Neuroimage (2013) 83:91220. doi: 10.1016/j.neuroimage.2013.07.032
52. Maurer U, Brem S, Liechti M, Maurizio S, Michels L, Brandeis D. Frontal midline theta reflects individual task performance in a working memory task. Brain Topogr (2015) 28:127-34. doi: 10.1007/s10548-014-0361-y

53. Babiloni $\mathrm{C}$, Marzano N, Iacoboni M, Infarinato F, Aschieri $\mathrm{P}$, Buffo $\mathrm{P}$, et al. Resting state cortical rhythms in athletes: A high-resolution EEG study. Brain Res Bull (2010) 81:149-56. doi: 10.1016/j.brainresbull.2009.10.014

54. Haegens S, Cousijn H, Wallis G, Harrison PJ, Nobre AC. Inter- and intraindividual variability in alpha peak frequency. Neuroimage (2014) 92:46-55. doi: 10.1016/j.neuroimage.2014.01.049

55. Holt GR, Softky WR, Koch C, Douglas RJ. Comparison of discharge variability in vitro and in vivo in cat visual cortex neurons. J Neurophysiol (1996) 75:1806-14. doi: 10.1152/jn.1996.75.5.1806

56. Riehle A, Brochier T, Nawrot M, Grün S. Behavioral context determines network state and variability dynamics in monkey motor cortex. Front Neural Circuits (2018) 12:1-17. doi: 10.3389/fncir.2018.00052

57. Haar S, Donchin O, Dinstein I. Individual movement variability magnitudes are explained by cortical neural variability. J Neurosci (2017) 37:9076-85. doi: 10.1523/JNEUROSCI.1650-17.2017

58. Rajdl K, Lansky P. Fano factor estimation. Math Biosci Eng (2014) 11:105-23. doi: 10.3934/mbe.2014.11.105

59. Casali AG, Gosseries O, Rosanova M, Boly M, Sarasso S, Casali KR, et al. A theoretically based index of consciousness independent of sensory processing and behavior. Sci Transl Med (2013) 5:198ra105. doi: 10.1126/scitranslmed.3006294

60. Benjamini $Y$, Hochberg Y. Controlling the false discovery rate : A practical and powerful approach to multiple testing. J R Stat Soc (1995) 57:289-300. doi: 10.1111/j.2517-6161.1995.tb02031.x

61. Thompson-Schill S, Braver TS, Jonides J. Individual differences. Cogn Affect Behav Neurosci (2002) 5:115-6. doi: 10.3758/CABN.5.2.115

62. Braver TS, Cole MW, Yarkoni T. Vive les differences! Individual variation in neural mechanisms of executive control. Curr Opin Neurobiol (2010) 20:242-50. doi: 10.1016/j.conb.2010.03.002

63. Reineberg AE, Andrews-hanna JR, Depue BE, Friedman NP, Banich MT. Resting-state networks predict individual differences in common and specific aspects of executive function. Neuroimage (2015) 104:69-78. doi: 10.1016/j.neuroimage.2014.09.045

64. Finn ES, Scheinost D, Finn DM, Shen X, Papademetris X, Constable RT. Can brain state be manipulated to emphasize individual differences in functional connectivity? Neuroimage (2017) 160:140-51. doi: 10.1016/j. neuroimage.2017.03.064

65. Seghier ML, Price CJ. Interpreting and utilising intersubject variability in brain function. Trends Cogn Sci (2018) 22:517-30. doi: 10.1016/j. tics.2018.03.003

66. Vindras P, Desmurget M, Baraduc P. When one size does not fit all: A simple statistical method to deal with across-individual variations of effects. PLoS One (2012) 7:e39059. doi: 10.1371/journal.pone.0039059

67. Goldapple K, Segal Z, Garson C, Lau M, Bieling P, Kennedy S, et al. Modulation of cortical-limbic pathways in major depression treatmentspecific effects of cognitive behavior therapy background: Functional imaging studies of major depressive disorder demonstrate response-specific regional changes following various modes. Arch. Gen. Psychiatry (2004) 61:34-41. doi: 10.1001/archpsyc.61.1.34

68. Fernández A, Al-Timemy AH, Ferre F, Rubio G, Escudero J. Complexity analysis of spontaneous brain activity in mood disorders: A magnetoencephalography study of bipolar disorder and major depression. Compr Psychiatry (2018) 84:112-7. doi: 10.1016/j.comppsych.2018.03.015

69. Gao W, Chen S, Biswal B, Lei X, Yuan J. Temporal dynamics of spontaneous default-mode network activity mediate the association between reappraisal and depression. Soc Cogn Affect Neurosci (2018) 13:1235-47. doi: 10.1093/ scan/nsy092

70. Northoff G. How do resting state changes in depression translate into psychopathological symptoms? from "Spatiotemporal correspondence" to "Spatiotemporal Psychopathology.". Curr Opin Psychiatry (2016) 29:18-24. doi: 10.1097/YCO.0000000000000222

71. Campbell S, Marriott M, Nahmias C, Macqueen GM. Lower hippocampal volume in patients suffering from depression: A meta-analysis. Am J Psychiatry (2004) 161:598-607. doi: 10.1176/appi.ajp.161.4.598

72. Buzsáki G. Theta oscillations in the hippocampus. Neuron (2002) 33:325-40. doi: 10.1016/S0896-6273(02)00586-X 
73. Rock PL, Roiser JP, Riedel WJ, Blackwell AD. Cognitive impairment in depression: A systematic review and meta-analysis. Psychol Med (2014) 44:2029-40. doi: 10.1017/S0033291713002535

74. Malykhin NV, Coupland NJ. Hippocampal neuroplasticity in major depressive disorder. Neuroscience (2015) 309:200-13. doi: 10.1016/j. neuroscience.2015.04.047

75. Travis S, Coupland NJ, Silversone PH, Huang Y, Fujiwara E, Carter R, et al. Dentate gyrus volume and memory performance in major depressive disorder. J Affect Disord (2015) 172:159-64. doi: 10.1016/j. jad.2014.09.048

76. Kan DPX, Lee PF. International Conference on BioSignal Analysis, Processing and Systems ICBAPS. 2015 Decrease alpha waves in depression: An electroencephalogram (EEG) study. IEEE (2015) pp. 156-61. doi: 10.1109/ ICBAPS.2015.7292237

77. Sauseng P, Griesmayr B, Freunberger R, Klimesch W. Control mechanisms in working memory: A possible function of EEG theta oscillations. Neurosci Biobehav Rev (2010) 34:1015-22. doi: 10.1016/j.neubiorev.2009.12.006

78. Klimesch W. Evoked alpha and early access to the knowledge system: The P1 inhibition timing hypothesis. Brain Res (2011) 1408:52-71. doi: 10.1016/j. brainres.2011.06.003

79. Klimesch W. Alpha-band oscillations, attention, and controlled access to stored information. Trends Cogn Sci (2012) 16:606-17. doi: 10.1016/j. tics.2012.10.007

80. Buchholz VN, Jensen O, Medendorp WP. Different roles of alpha and beta band oscillations in anticipatory sensorimotor gating. Front Hum Neurosci (2014) 8:1-9. doi: 10.3389/fnhum.2014.00446

81. Zumer J, Scheeringa R, Schoffelen JM, Norris D, Jensen O. Occipital alpha activity during stimulus processing gates the information flow to objectselective cortex. PLoS Biol (2014) 12:e1001965. doi: 10.1371/journal. pbio. 1001965

82. Schurger A, Sarigiannidis I, Naccache L, Sitt JD, Dehaene S. Cortical activity is more stable when sensory stimuli are consciously perceived. Proc Natl Acad Sci (2015) 112:E2083-92. doi: 10.1073/pnas.1418730112

83. Dinstein I, Heeger DJ, Lorenzi L, Minshew NJ, Malach R, Behrmann M. Unreliable Evoked Responses in Autism. Neuron (2012) 75:981-91. doi: 10.1016/j.neuron.2012.07.026

84. Huang Z, Zhang J, Wu J, Liu X, Xu J, Zhang J, et al. Disrupted neural variability during propofol-induced sedation and unconsciousness. Hum Brain Mapp (2018). 39, 1-12. doi: 10.1002/hbm.24304

85. Whitfield-Gabrieli S, Ford JM. Default mode network activity and connectivity in psychopathology. Annu Rev Clin Psychol (2012) 8:49-76. doi: 10.1146/annurev-clinpsy-032511-143049

86. Hamilton JP, Farmer M, Fogelman P, Gotlib IH. Depressive rumination, the default-mode network, and the dark matter of clinical neuroscience. Biol Psychiatry (2015) 78:224-30. doi: 10.1016/j.biopsych.2015.02.020

87. Coutinho JF, Fernandesl SV, Soares JM, Maia L, Goncalves OF, Sampaio A. Default mode network dissociation in depressive and anxiety states. Brain Imaging Behav (2016) 10:147-57. doi: 10.1007/s11682-015-9375-7

88. Kim SM, Park SY, Kim YI, Son YD, Chung U-S, Min KJ, et al. Affective network and default mode network in depressive adolescents with disruptive behaviors. Neuropsychiatr Dis Treat (2016) 12:49-56. doi: 10.2147/NDT. S95541

89. Rodriguez-Cano E, Alonso-Lana S, Sarro S, Fernandez-Corcuera P, Goikolea JM, Vieta E, et al. Differential failure to deactivate the default mode network in unipolar and bipolar depression. Bipolar Disord (2017) 19:386-95. doi: 10.1111/bdi.12517

90. Brakowski J, Spinelli S, Dörig N, Bosch OG, Manoliu A, Holtforth MG, et al. Resting state brain network function in major depression - Depression symptomatology, antidepressant treatment effects, future research. J Psychiatr Res (2017) 92:147-59. doi: 10.1016/j.jpsychires.2017.04.007
91. Shao J, Meng C, Tahmasian M, Brandl F, Yang Q, Luo G, et al. Common and distinct changes of default mode and salience network in schizophrenia and major depression. Brain Imaging Behav (2018) 12:1708-19. doi: 10.1007/ s11682-018-9838-8

92. Jadi MP, Sejnowski TJ. Cortical oscillations arise from contextual interactions that regulate sparse coding. Proc Natl Acad Sci (2015) 112:E341-1. doi: 10.1073/pnas. 1423665112

93. Lefebvre J, Hutt A, Knebel J-F, Whittingstall K, Murray MM. Stimulus statistics shape oscillations in nonlinear recurrent neural networks. J Neurosci (2015) 35:2895-903. doi: 10.1523/JNEUROSCI.3609-14.2015

94. Herrmann CS, Murray MM, Ionta S, Hutt A, Lefebvre J. Shaping intrinsic neural oscillations with periodic stimulation. J Neurosci (2016) 36:5328-37. doi: 10.1523/JNEUROSCI.0236-16.2016

95. Cecere R, Rees G, Romei V. Individual differences in alpha frequency drive crossmodal illusory perception. Curr Biol (2015) 25:231-5. doi: 10.1016/j. cub.2014.11.034

96. Haberbosch L, Schmidt S, Jooss A, Köhn A, Kozarzewski L, Rönnefarth $\mathrm{M}$, et al. Rebound or entrainment? The influence of alternating current stimulation on individual alpha. Front Hum Neurosci (2019) 13:1-10. doi: 10.3389/fnhum.2019.00043

97. Benwell CSY, London RE, Tagliabue CF, Veniero D, Gross J, Keitel C, et al. Frequency and power of human alpha oscillations drift systematically with time-on-task. Neuroimage (2019) 192:101-14. doi: 10.1016/j. neuroimage.2019.02.067

98. Foxe JJ, Snyder AC. The role of alpha-band brain oscillations as a sensory suppression mechanism during selective attention. Front Psychol (2011) 2:1-13. doi: 10.3389/fpsyg.2011.00154

99. Jensen O, Gips B, Bergmann TO, Bonnefond M. Temporal coding organized by coupled alpha and gamma oscillations prioritize visual processing. Trends Neurosci (2014) 37:357-69. doi: 10.1016/j.tins.2014.04.001

100. Bonnefond M, Jensen O. Gamma activity coupled to alpha phase as a mechanism for top-down controlled gating. PLoS One (2015) 10:e0128667. doi: 10.1371/journal.pone.0128667

101. Babu Henry Samuel I, Wang C, Hu Z, Ding M. The frequency of alpha oscillations: Task-dependent modulation and its functional significance. Neuroimage (2018) 183:897-906. doi: 10.1016/j.neuroimage.2018.08.063

102. Nikulin VV, Brismar T. Long-range temporal correlations in electroencephalographic oscillations: Relation to topography, frequency band, age and gender. Neuroscience (2005) 130:549-58. doi: 10.1016/j. neuroscience.2004.10.007

103. Berthouze L, James LM, Farmer SF. Human EEG shows long-range temporal correlations of oscillation amplitude in theta, alpha and beta bands across a wide age range. Clin Neurophysiol (2010) 121:1187-97. doi: 10.1016/j. clinph.2010.02.163

104. Ramanand P, Bruce MC, Bruce EN. Mutual information analysis of EEG signals indicates age-related changes in cortical interdependence during sleep in middle-aged vs. elderly women. J Clin Neurophysiol (2010) 27:27484. doi: 10.1097/WNP.0b013e3181eaa9f5

Conflict of Interest: The authors declare that the research was conducted in the absence of any commercial or financial relationships that could be construed as a potential conflict of interest.

Copyright () 2019 Wolff, de la Salle, Sorgini, Lynn, Blier, Knott and Northoff. This is an open-access article distributed under the terms of the Creative Commons Attribution License (CC BY). The use, distribution or reproduction in other forums is permitted, provided the original author(s) and the copyright owner(s) are credited and that the original publication in this journal is cited, in accordance with accepted academic practice. No use, distribution or reproduction is permitted which does not comply with these terms. 


\section{OPEN ACCESS}

Edited by:

Filippo Cieri,

Lou Ruvo Center for Brain Health,

Cleveland Clinic, United States

Reviewed by:

Maria Salsone,

Italian National Research Council

(CNR), Italy

Bo Gao,

Affiliated Hospital of Guizhou Medical

University, China

*Correspondence:

Silvia Minosse

silvia.minosse2@gmail.com

Specialty section:

This article was submitted to

Applied Neuroimaging,

a section of the journal

Frontiers in Neurology

Received: 29 April 2019 Accepted: 10 October 2019

Published: 25 October 2019

Citation:

Minosse S, Garaci F, Martucci A, Lanzafame S, Di Giuliano F, Picchi E, Cesareo M, Mancino R, Guerrisi M, Pistolese $C A$, Floris $R$, Nucci $C$ and Toschi N (2019) Primary Open Angle

Glaucoma Is Associated With

Functional Brain Network

Reorganization

Front. Neurol. 10:1134.

doi: 10.3389/fneur.2019.01134

\section{Primary Open Angle Glaucoma Is Associated With Functional Brain Network Reorganization}

\author{
Silvia Minosse ${ }^{1 *}$, Francesco Garaci ${ }^{2,3}$, Alessio Martucci $^{4}$, Simona Lanzafame ${ }^{1}$, \\ Francesca Di Giuliano ${ }^{5}$, Eliseo Picchi ${ }^{5}$, Massimo Cesareo ${ }^{4}$, Raffaele Mancino ${ }^{4}$, \\ Maria Guerrisi ${ }^{1}$, Chiara Adriana Pistolese ${ }^{5}$, Roberto Floris ${ }^{5}$, Carlo Nucci ${ }^{4}$ and \\ Nicola Toschi ${ }^{1,6}$
}

${ }^{1}$ Department of Biomedicine and Prevention, University of Rome "Tor Vergata", Rome, Italy, ${ }^{2}$ Neuroradiology Unit, Department of Biomedicine and Prevention, University of Rome "Tor Vergata", Rome, Italy, ${ }^{3}$ San Raffaele Cassino, Cassino, Italy, ${ }^{4}$ Ophthalmology Unit, Department of Experimental Medicine, University of Rome Tor Vergata, Rome, Italy, ${ }^{5}$ Diagnostic Imaging Unit, Department of Biomedicine and Prevention, University of Rome Tor Vergata, Rome, Italy, ${ }^{6}$ Athinoula A. Martinos Center for Biomedical Imaging, Harvard Medical School, Boston, MA, United States

Background: Resting-state functional magnetic resonance imaging ( $r s-f M R I)$ is commonly employed to study changes in functional brain connectivity. The recent hypothesis of a brain involvement in primary open angle Glaucoma has sprung interest for neuroimaging studies in this classically ophthalmological pathology.

Object: We explored a putative reorganization of functional brain networks in Glaucomatous patients, and evaluated the potential of functional network disruption indices as biomarkers of disease severity in terms of their relationship to clinical variables as well as select retinal layer thicknesses.

Methods: Nineteen Glaucoma patients and 16 healthy control subjects (age: 50-76, mean $61.0 \pm 8.2$ years) underwent rs-fMRI examination at 3T. After preprocessing, rs-fMRI time series were parcellated into 116 regions using the Automated Anatomical Labeling atlas and adjacency matrices were computed based on partial correlations. Graph-theoretical measures of integration, segregation and centrality as well as group-wise and subject-wise disruption index estimates (which use regression of graph-theoretical metrics across subjects to quantify overall network changes) were then generated for all subjects. All subjects also underwent Optical Coherence Tomography (OCT) and visual field index (VFI) quantification. We then examined associations between brain network measures and VFI, as well as thickness of retinal nerve fiber layer (RNFL) and macular ganglion cell layer (MaculaGCL).

Results: In Glaucoma, group-wise disruption indices were negative for all graph theoretical metrics. Also, we found statistically significant group-wise differences in subject-wise disruption indexes in all local metrics. Two brain regions serving as hubs in healthy controls were not present in the Glaucoma group. Instead, three hub regions were present in Glaucoma patients but not in controls. We found significant associations between all disruption indices and VFI, RNFL as well as MaculaGCL. The disruption index based on the clustering coefficient yielded the best discriminative power for 
differentiating Glaucoma patients from healthy controls [Area Under the ROC curve (AUC) 0.91, sensitivity, 100\%; specificity, 78.95\%].

Conclusions: Our findings support a possible relationship between functional brain changes and disease severity in Glaucoma, as well as alternative explanations for motor and cognitive symptoms in Glaucoma, possibly pointing toward an inclusion of this pathology in the heterogeneous group of disconnection syndromes.

Keywords: resting-state functional magnetic resonance imaging (rs-fMRI), open angle glaucoma, graph theoretical measures, functional brain networks, neurodegenerative diseases

\section{INTRODUCTION}

Glaucoma as one of the major causes of blindness in the world. Glaucoma is an optic neuropathy characterized by retinal ganglion cells death and degeneration of the optic nerve $(1,2)$. In this debilitating disease, any additional biomarker able to detect and quantify neuronal changes can aid in formulating better prognosis, monitor therapy outcomes and therefore influence quality of life (3).

Several diffusion tensor imaging (DTI) studies have demonstrated the involvement and degeneration of specific brain regions and white matter (WM) bundles in patients affected by Glaucoma $(2,4)$. Additionally, there is evidence of changes in the regional homogeneity and low frequency fluctuations in fMRI signals in Glaucoma patients compared to controls $(5,6)$. In this context, the recent hypothesis of brain involvement in pathologies of the visual system has sprung interest for neuroimaging studies in this realm, with a particular focus on primary open angle Glaucoma.

Overall, the mechanism underlying brain involvement in Glaucoma is hypothesized to be supported by a combination of both functional changes and structural damage. For example, a recent paper (7) demonstrated that connectivity between specific brain regions is associated with disease severity in as patients affected by Glaucoma, and that several structural brain abnormalities (as compared to healthy controls) can be detected in Glaucoma patients $(4,8,9)$. Also, a global reorganization of brain networks in Glaucoma has been shown in a study (10) focused on cortical region and excluding the cerebellum. Interestingly, the latter region may be of particular interest in the study of brain involvement in glaucoma in view of the motor difficulties faced by Glaucoma patients $(11,12)$. Finally, a few studies examined the correlation between visual function tests outcomes and structural MRI findings in the anterior visual pathway in Glaucoma patients $(13,14)$, and an association between structural, functional and metabolic brain changes and optical coherence tomography (OCT) measures was recently shown (15).

Resting-state functional magnetic resonance imaging (rsfMRI) is commonly employed to study changes in functional brain connectivity in a vast number of conditions, including neurodegenerative diseases such as Parkinson's or Alzheimer's disease. The interest in the so-called functional connectome (i.e., the complex network of cross-talks between brain areas) is ever increasing (16-18). To this end, recently several methods which stem from the realms of graph theory and network science have emerged as useful tools to study both local and global properties of complex brain networks. In detail, the brain is conceptualized as a graph, in which brain regions represent nodes and the relationships between the regions, defined through a variety of association measures fMRI time-series, represent edges which connect the nodes within the graph (19). Then, topological properties that highlight brain organization can be extracted (20). Recently, various studies have shown that graph-theoretical indices are sensitive to changes in brain network measures in both psychiatric and neurological diseases (16).

The purpose of this study was to evaluate a putative reorganization of functional brain networks in patients affected by primary open angle Glaucoma through graph- theoretical measures. To this end, we employ adjacency matrices based on partial correlation measures, in order to avoid the redundancies commonly introduced by the use of bivariate associations measures. Further, we exploit the recently introduced idea of a "disruption index" (21), which simultaneously takes global and local topological metric into account and allows to define the comparison between patients and controls in terms of how much the distribution of such measures is disrupted across the brain. Finally, in order to evaluate the potential of these disruption indices to serve as biomarkers to monitor disease severity, we explore their discriminative power between healthy and Glaucoma population as well as possible associations between functional brain reorganization indices, functional visual parameters, and thickness of select retinal layers measured through OCT.

\section{MATERIALS AND METHODS}

The overall workflow of our study is shown in Figure 1.

\section{Subjects}

Nineteen Glaucoma patients and 16 healthy control subjects were enrolled from the Glaucoma Clinic and the General Outpatients clinic at the University Hospital "Policlinico Tor Vergata" (Rome, Italy). Patient characteristics are detailed in Table 1. The study protocol was approved by the local Institutional Review Board and adhered to the tenets of the Declaration of Helsinki. All subjects provided written informed consent.

After Glaucoma diagnosis, Glaucoma patients were eligible for the current study if they fulfilled the following inclusion criteria: (i) best corrected visual acuity $>0.1 \log M A R$, (ii) 


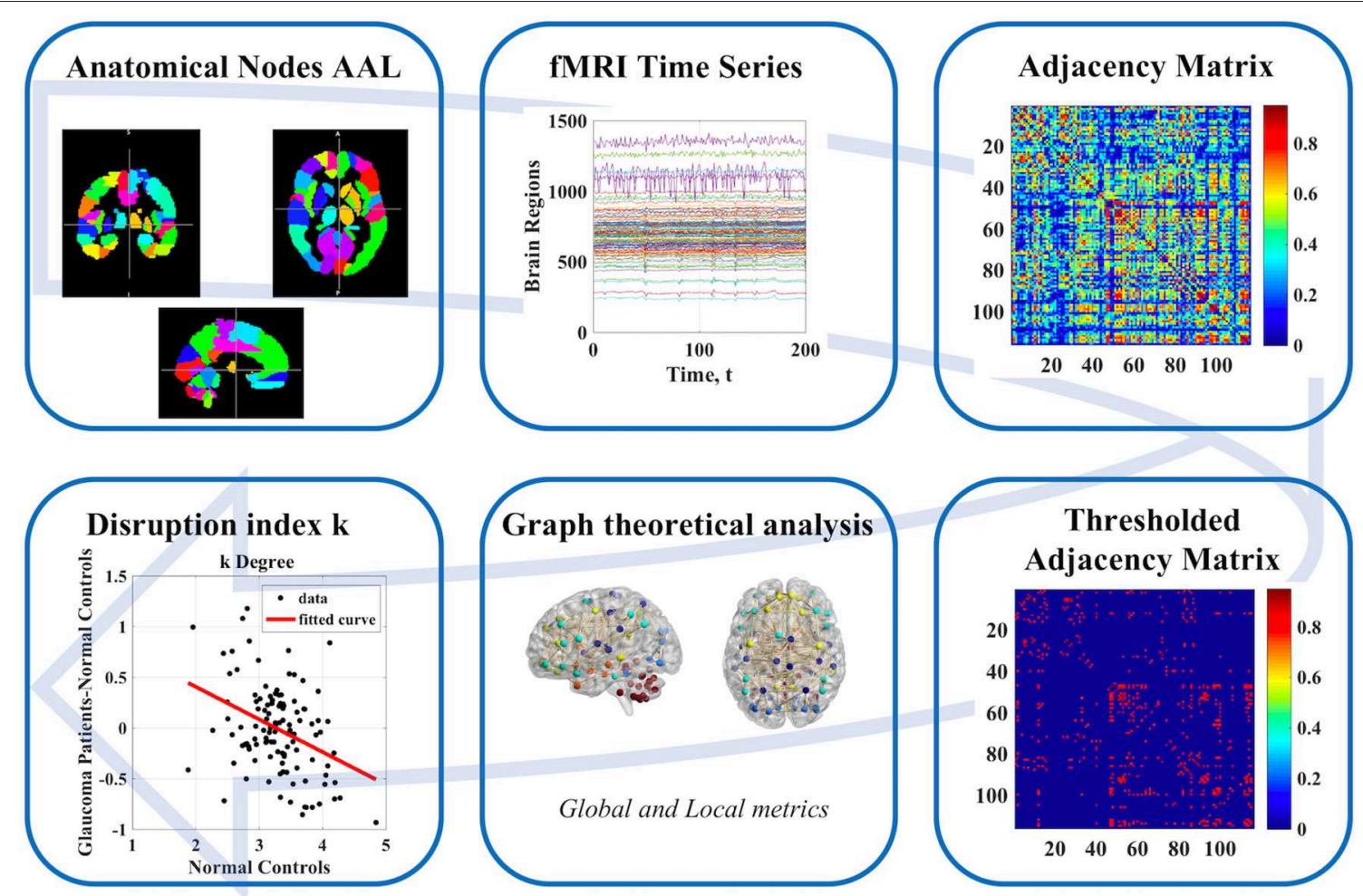

FIGURE 1 | Schematic illustration of workflow from data to association matrix and graph analysis.

TABLE 1 | Clinical characteristics of our study population.

\begin{tabular}{lcc}
\hline & Glaucoma & Controls \\
\hline Group size & 19 & 16 \\
Age (years) & $61.3(50-72)$ & $60.8(50-76)$ \\
Mean (range) & $8 / 11$ & $11 / 5$ \\
Sex (male/female) & & $15.44(12-18)$ \\
IOP in treatment & $15.89(12-19)$ & - \\
Mean (range) & & - \\
Disease stage & 9 & - \\
I & 2 & - \\
II & 4 & - \\
III & 1 & - \\
IV & 3 & \\
V & & \\
\hline
\end{tabular}

IOP, Intraocular pressure.

refractive error $< \pm 5$ spherical diopters or $< \pm 3$ cylindrical diopters, (iii) transparent ocular media, and (iv) open anterior chamber (Shaffer classification $>20^{\circ}$ ). Exclusion criteria for Glaucoma patients as well as healthy controls were: (i) previous or active optic neuropathies, (ii) retinal vascular diseases, (iii) preproliferative or proliferative diabetic retinopathy, (iv) macular degeneration, (v) hereditary retinal dystrophy, (vi) use of medication that could affect Visual Field, (vii) previous or active neurological, cerebrovascular, or neurodegenerative diseases. Normal tension Glaucoma patients were also excluded (22). A Glaucoma diagnosis was defined following the European Glaucoma Society criteria (23). Glaucoma patients were treated using topical beta-blockers, prostaglandin analogs, and carbonic anhydrase inhibitors, alone or in fixed or unfixed combination.

\section{Ophthalmological Data Collection}

After administering a medical history questionnaire, bestcorrected visual acuity, anterior segment examination, intraocular pressure (IOP) measurement, ultrasound pachymetry, gonioscopy, and standard automated perimetry tests were administered to all subjects. Visual Field (VF) examination was performed using Humphrey Swedish Interactive Threshold Algorithm (SITA) standard with 24-2 test point pattern (Carl Zeiss Meditec Inc., Dublin, CA). The visual filed index (VFI) is a recently introduced summary parameter which is automatically calculated from the pattern deviation map values, in such a way that the central points of the VF have a larger impact as compared to peripheral points. The VFI ranges from 100\% for a normal VF to $0 \%$ for a completely abolished VF (24). After pupillary dilation, slit-lamp fundus examination and spectral domain-optical coherence tomography (SD-OCT) using Glaucoma Module Premium Edition (GMPE) software (Heidelberg Retinal Engineering, Dossenheim, Germany) were assessed (22). The SD-OCT offers a tool for macular segmentation and thickness evaluation of individual retinal 
layers as well as Retinal Nerve Fiber Layer (RNFL) thickness. For each layer [macular total retina, Retinal Nerve Fiber Layer RNFL, Ganglion Cell Layer (GCL), Inner Plexiform Layer, Inner Nuclear Layer, Outer Plexiform Layer, Outer Nuclear Layer, Retinal Pigmented Epithelium, Inner Retinal Layers, and Outer Retinal Layers], thickness measurements of all sectors, as defined by the Early Treatment Diabetic Retinopathy Study scheme (temporal inner, superior inner, nasal inner, inferior inner, temporal outer, superior outer, nasal outer, and inferior outer), were employed.

\section{MR Imaging Protocol and Preprocessing}

The maximum time interval between MRI and Ophthalmological data collection examinations was 1 week. MRI examinations were performed on a 3T scanner system (Achieva, Philips Medical Systems, Best, The Netherlands) with dedicated 8-channel sensitivity encoding (SENSE) head coil. All MRI examinations included rs-fMRI and three-dimensional T1-weighted, magnetization prepared rapid gradient echo (MPRAGE) images. rs-fMRI was performed using single-shot echo planar imaging (EPI) with the following parameters: acquisition and reconstruction voxel size $3.31 \mathrm{~mm}^{3}$, repetition time $(\mathrm{TR})=3,000 \mathrm{~ms}$, echo time $(\mathrm{TE})=30 \mathrm{~ms}$, flip angle $=80^{\circ}$, field of view $(\mathrm{FOV})=212 \times 198 \mathrm{~mm}^{2}, 200$ volumes/subjects. The T1 weighted images (3D MPRAGE) were acquired with the following parameters: acquisition and reconstruction voxel size $1 \times 1 \times 1.2 \mathrm{~mm}^{3}, \mathrm{TR}=500(\mathrm{~ms}), \mathrm{TE}=50(\mathrm{~ms})$, flip angle $=8^{\circ}, \mathrm{FOV}=256 \times 240 \mathrm{~mm}^{2}$. rs-fMRI data was preprocessed in FLS v. 6.0 (25). The first three volumes were discarded to allow for scanner stabilization. Motion, distortion, and slice timing correction were performed in the FSL software suite. Finally, preprocessed functional scans were nonlinearly coregistered to standard space via the high-resolution T1 weighted MPRAGE image.

\section{Graph Theoretical Measures}

Network nodes were defined by parcellation of the whole brain into 116 regions defined through the automated anatomical labeling (AAL) atlas. After parcellation, node- and subjectspecific timeseries were extracted by voxel-wise averaging of the rs-fMRI signal in each region. Partial correlation between all 116 timeseries was used to generate subject-wise adjacency matrices. Subsequently range of density thresholds (from 5 to $40 \%$ in steps of $5 \%$ ) were applied to these matrices. Given that the main results were seen to be robust to the threshold, the results were reported based on a sparsity value of $10 \%$, as commonly adopted in brain network literature. We calculated the following graph-theoretical measures for each subject: two local nodal measures [degree and betweenness centrality $(\mathrm{BC})$ ], one functional integration measures (global efficiency), four measures of functional segregation (local efficiency, clustering coefficient, transitivity, and modularity), and one measure of resilience (assortativity). All metrics were calculated using the Brain Connectivity Toolbox (20).

\section{Disruption Indices}

Local measures were analyzed through the disruption index $k$ $(10,21)$, which measures the degree of overall reorganization of a specific property in the whole network. For network measure $N M_{i}$, where $\mathrm{i}=$ (degree, betweenness centrality, local efficiency, clustering coefficient, and spectral measure of centrality), the disruption index $k$ is defined [both for a group of subjects (Equation 2)] and for a single subject (Equation 1, see below for an example) through the following linear regressions across all nodes:

$$
\begin{aligned}
& N M_{i, l}-\frac{1}{C} \sum_{j=1}^{C} N M_{i, j}=k_{i, l}^{0}+k_{i, l} \cdot \frac{1}{C} \sum_{j=1}^{C} N M_{i, j}+\varepsilon_{l} \\
& \frac{1}{G} \sum_{j=1}^{G} N M_{i, j}-\frac{1}{C} \sum_{j=1}^{C} N M_{i, j}=k_{i}^{0}+k_{i} \cdot \frac{1}{C} \sum_{j=1}^{C} N M_{i, j}+\varepsilon
\end{aligned}
$$

where $N M_{i, l}, N M_{i, j=C}, N M_{i, j=P}$ are the $i$-th network measures for all subjects $(\mathrm{l}=\mathrm{C}+\mathrm{G})$, control group $(\mathrm{C})$ and Glaucoma patients (G), respectively. $N M_{i} \in \mathbb{R}^{N}$, where $N$ is the number of the node (1-116). $k_{i, l}$ and $k_{i}$ are the disruption indices relative to the $i$ th network measures for a single subject and for the Glaucoma patient group, respectively. $k_{i, l}^{0}$ and $k_{i}^{0}$ are constant terms, $\varepsilon_{l}$ and $\varepsilon$ are the linear regression residues.

The calculation of $k_{i, l}$ in the case of $\mathrm{i}=$ degree, subject $\mathrm{A}$ $=$ control and subject $\mathrm{b}=$ Glaucoma patient is exemplified in Figure 2. The $y$-axis represents the difference between the degree of the single node of the subject (A or B) and the mean value of the degree of each node obtained in the control group. This latter quantity is also reported in the $x$-axis (mean value of the degree of each node obtained in the control group). The slope of the linear regression is the disruption index $k$. A disruption index $k$ equal to zero implies that, on average, the degree of the node in a patient is close to the mean degree of the same node in a control group. If the disruption index $k$ is statistically different from zero, the degree of the patient's node does not reflect the average degree of the same node in the control group, i.e., the degree of nodes in the network is completely reorganized $(10,21)$. The same rationale and algorithm can be applied to all other local network metrics.

\section{Network Hubs}

In addition to calculating disruption indices, we identified subject-wise hub regions using all local network measures $N M_{i}$ separately. For each subject, each region was classified as a hub when the respective network measure $N M_{i}$ was at least 1.5 times higher than its whole-brain average.

\section{Measures of Brain Network and Clinical Parameters}

The clinical parameters we employed in conjunction with graphtheoretical metrics are: (i) Retinal Nerve Fiber Layer (RNFL), (ii) Macula Ganglion Cell Layer (GCL), and (iii) Visual Field Index (VFI). The first two quantities were obtained as averages from OCT measures as follows:

$$
\overline{R N F L}=\frac{1}{6} \sum_{i=1}^{6} R N F L_{i}
$$




\section{\begin{tabular}{l|l|l|l|l|} 
Only one subject & $\operatorname{Deg}(\mathrm{N}=1)$ & $\operatorname{Deg}(\mathrm{N}=2)$ & $\ldots$ & $\operatorname{Deg}(\mathrm{N}=116)$
\end{tabular}}
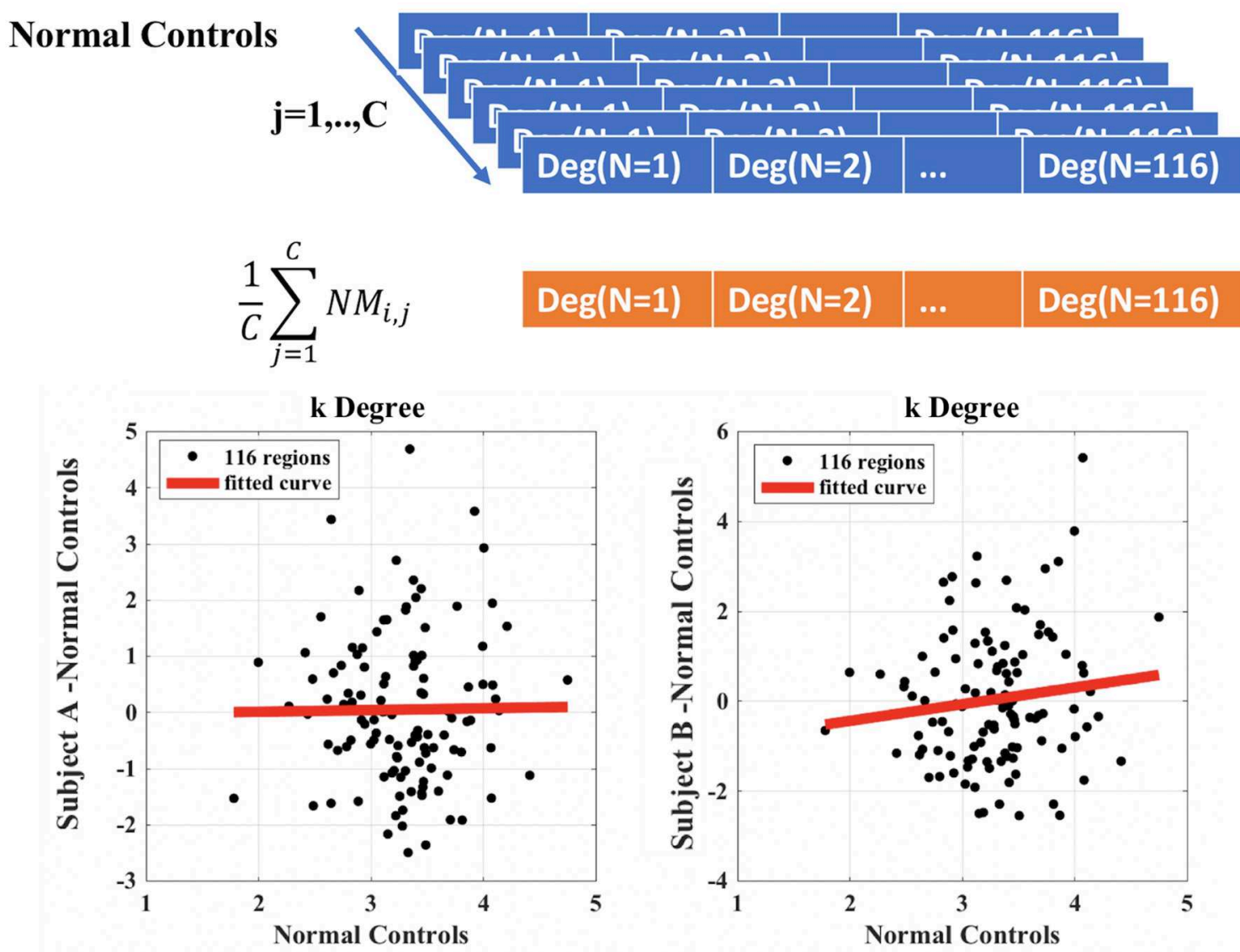

FIGURE 2 | Example of calculation of the disruption index $\mathrm{k}$ for a single patient compared to control group. Subject A represents a healthy control patient; subject B represents a Glaucoma patient.

$$
\overline{\text { MaculaGCL }}=\frac{1}{9} \sum_{j=1}^{9} \text { MaculaGCL } L_{j}
$$

where $i=$ (temporal superior, nasal superior, nasal, nasal inferior, temporal inferior, temporal) and $j=$ (Fovea, Temporal Inner, Superior Inner, Nasal Inner, Inferior Inner, Temporal Outer, Superior Outer, Nasal Outer, Inferior Outer). Both VFI and layer thicknesses were averaged across eyes for each patient. The leftright discrepancy between measures did not exceed 10\% (VFI: mean $5.9 \pm 8.8 \%$; RNFL: mean $7.6 \pm 6.7 \%$, MaculaGCL: mean $3.2 \pm 3.7 \%)$ in any of our patients.

\section{Statistical Analysis}

Subject-wise global as well as local graph-theoretical metrics and subject-wise disruption indexes were compared statistically across groups using the nonparametric Mann-Whitney $U$-Test. For group-wise disruption indexes, we report the statistical significance of the overall regression. The interaction between the presence/absence of a hub at any specific node and group membership was tested using a Fisher's exact test. The association between functional brain measures (global and local graphtheoretical measures as well as disruption indices) and clinical parameters (RNFL, MaculaGCL, VFI) was assessed though separate linear regression models which included age and gender as nuisance covariates; for regression which yielded statistically significant results, Cohen's $\mathrm{f}^{2}$ was employed as a standardized measure of effect size. In the case of local measures, to exclude false positive results under multiple testing, a false discovery rate $(\mathrm{FDR}$, alpha $=0.05)$ procedure was applied across all nodes (brain regions). $p<0.05$ (FDR corrected) was considered statistically significant. In addition, receiver operating characteristic (ROC) analysis through binary logistic regression was performed in order to quantify the discrimination potential (between Glaucoma patients and healthy controls) of each metric and disruption index. The optimal operating point of each ROC curve was determined using Youden's index (which maximizes 
TABLE 2 | Effect sizes (subject-wise disruption indices k) and regression slopes (group-wise disruption indices $\mathrm{k}$ ).

\begin{tabular}{lcccc}
\hline Network Measures & $\left(\hat{\boldsymbol{k}_{\boldsymbol{i}, \boldsymbol{C}}} \hat{\boldsymbol{k}_{\boldsymbol{i}, \boldsymbol{G}}}\right)$ & $\boldsymbol{p}$ & $\boldsymbol{k}$ & $\boldsymbol{p}$ \\
\hline Degree & 0.44 & 0.004 & -0.32 & $<0.001$ \\
Betweenness centrality & 0.46 & 0.004 & -0.38 & $<0.001$ \\
Local efficiency & 0.66 & $<0.001$ & -0.65 & $<0.001$ \\
Clustering coefficient & 0.69 & $<0.001$ & -0.72 & $<0.001$ \\
Spectral centrality measure & 0.52 & 0.006 & -0.37 & $<0.001$
\end{tabular}

Effect size (second column from left): difference between the median values of the subject wise disruption indices along with $p$-values resulting from Mann-Whitney-U-tests (third column). Group-wise disruption indices $k$ (fourth column from left) along with regression p-values (right column).

the sum of sensitivity and specificity), after which sensitivity, specificity, positive predicted value (PPV), and negative predicted value (NPV) were calculated. All data analysis was performed using in-house script written in MATLAB version 9.3.0, release 2017b (MathWorks, Natick, MA, USA).

\section{RESULTS}

We found no statistically significant differences in age $(p=0.4$, Mann-Whitney- $U$-Test) and gender $(p=0.5$, Chi-Square160 Test) between the two groups. Table 2 summarizes the results obtained with group-wise disruption indices along with effect sizes (regression lines obtained while calculating the disruption indices are shown on the left of Figure 3). Figure 3 (right) also shows the results of group comparisons in subject-wise disruption indexes.

We found no statistically significant differences in global or local graph-theoretical metrics between Glaucoma and control patients. However, we found that all group-wise disruption indices were negative, and statistically different from 0 for all graph theoretical metrics (Figure 3 and Table 2). Additionally, statistically significant group-wise differences in subject-wise disruption indexes were found in all local metrics (Figure 3). For all statistically significant comparisons (i.e., in all disruption indices), the disruption index was lower in the Glaucoma group as compared to the healthy control group, highlighting a complex functional brain network reorganization pattern in Glaucoma patients.

Figure 4 summarizes differences in regions which were classified as hubs between Glaucoma and control subjects. The left lobule VIIB of the cerebellar hemisphere $(p=0.035)$ was classified as a betweenness centrality hub in healthy controls but not in Glaucoma patients, and the right inferior occipital cortex $(p=0.010)$ behaved in the opposite manner. Also, we found that the right angular gyrus $(p=0.035)$ was classified as a spectral measure of centrality hub in healthy controls but not in Glaucoma patients, and that the right inferior temporal gyrus ( $p$ $=0.047$ ) behaved in an opposite manner. Finally, the left lobule IX of cerebellar hemisphere $(p=0.047)$ was classified as a local efficiency hub in Glaucoma patients but not in healthy controls.

We found no statistically significant associations between global graph-theoretical metrics and clinical parameters.
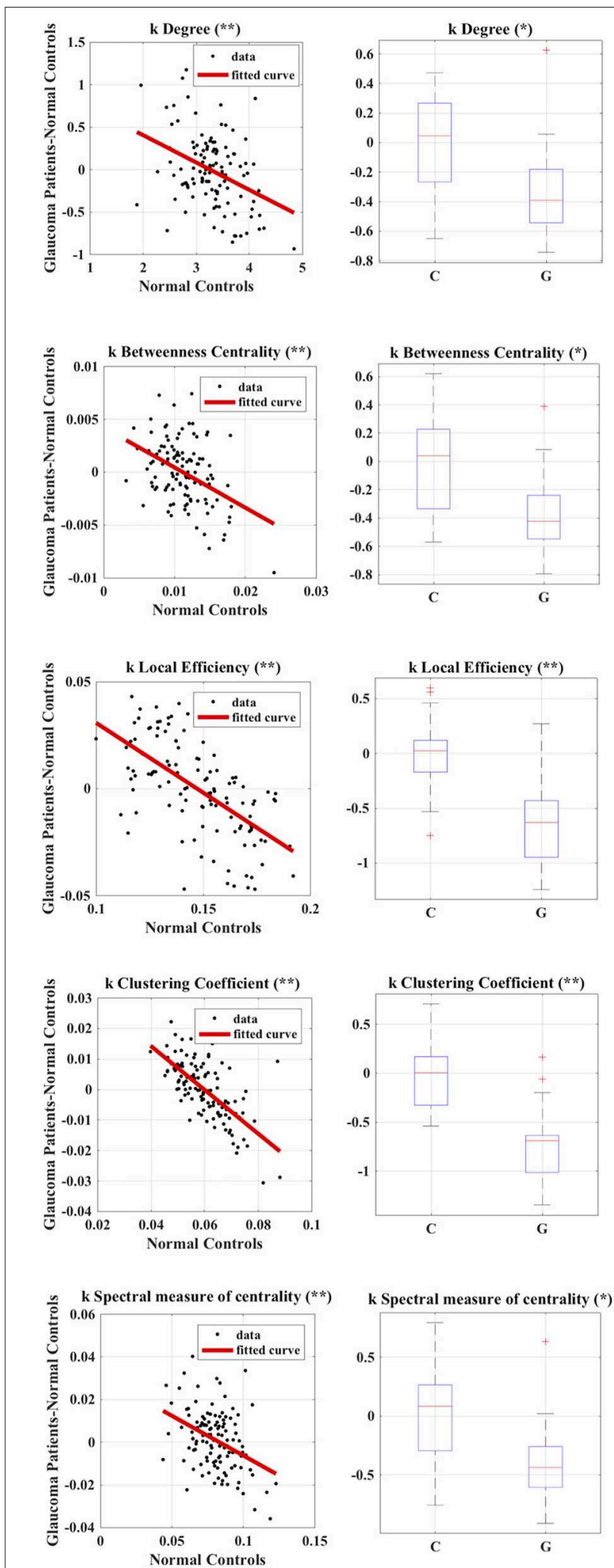

FIGURE 3 | Calculation of group-wise disruption index (left) and group-wise differences in subject-wise disruption index $k$ when comparing Glaucoma patients to healthy controls (right) in all local graph measures. ${ }^{\star} p<0.05$, ${ }^{\star *} p<0.001$. 

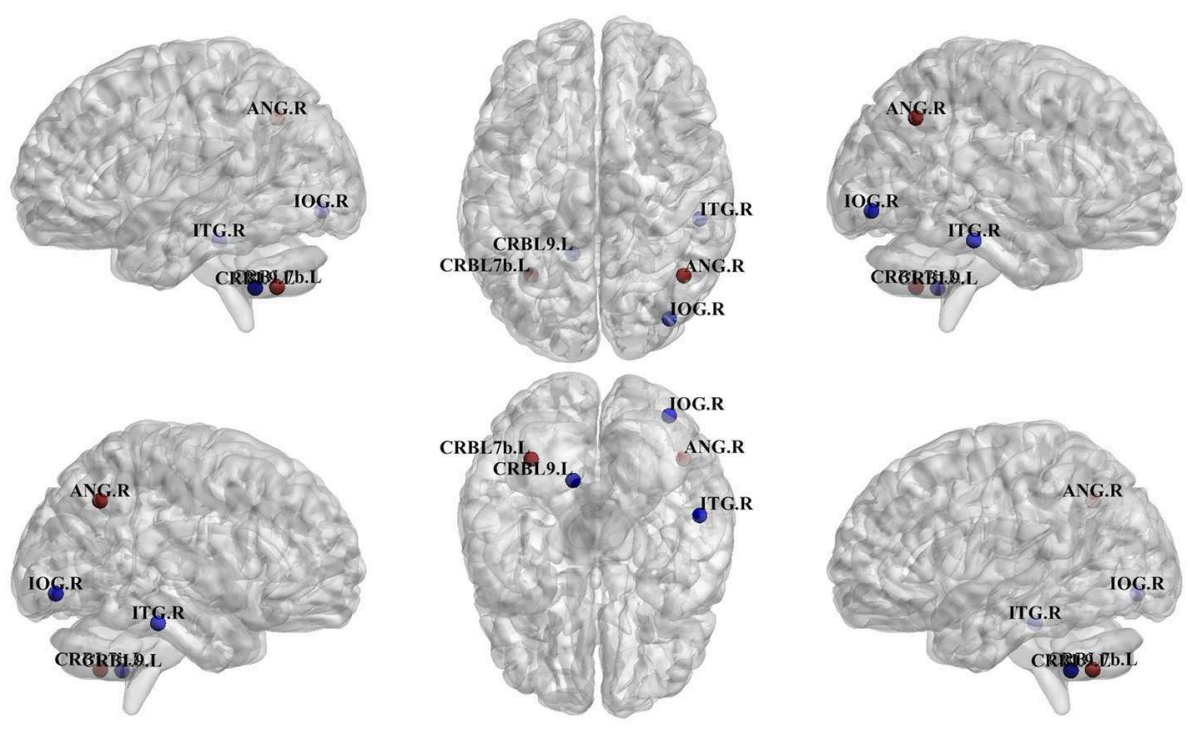

FIGURE 4 | Differences in regions classified as hub in control vs. Glaucoma patients. The blue hubs "appear" and the red hubs "disappear" in Glaucoma patient as compared to controls. IOG.R, right inferior occipital; ANG.R, right angular gyrus; ITG.R, right inferior temporal gyrus; CRBL7b.L, left lobule VIIB of cerebellar hemisphere; CRBL9.L, left lobule IX of cerebellar hemisphere.

However, we found significant positive associations between disruption indices and VFI, and (Table 3). When looking at associations between local graph-theoretical metrics and clinical parameters, significant positive associations were found in a number of regions, including the right parahippocampal gyrus, right transverse temporal gyrus and lobule X of vermis (Table 4).

The results of ROC analysis for disruption indices and global network measures are shown in Table 5. Overall, all disruption index yielded good to excellent (AUC $=0.773-$ 0.911 ) discriminative power. The disruption index based on the clustering coefficient metrics yielded the best performance $($ AUC $=0.911$, sensitivity $=100 \%$; specificity $=78.95 \%)$ (Figure 5). Instead, global graph-theoretical metrics yielded fair to poor discriminative power. Finally, Table 6 shows the top 25 AUCs obtained when employed all single local graph-theoretical metrics as independent variables, which only yielded moderate discrimination performance (top AUC value $=0.72$ ).

\section{DISCUSSION}

Advanced neuroimaging techniques have very recently begun to be employed to study the structural, functional, and metabolic changes in Glaucoma patients, including damage of gray matter atrophy and loss of structural connectivity (4), functional connectivity changes (10), and metabolite concentration (26). In this context, the involvement in Glaucoma of brain areas not directly responsible for the processing of visual information is beginning to emerge $(4,7,9,27)$.

In this study, we used advanced graph theoretical methods, including the recently defined idea of subject-wise and groupwise disruption index, to analyze the topological properties of brain connectivity in patients affected by Glaucoma.
Our results provide novel insights into subtle functional alterations in the brain of Glaucoma patients, also extending recent findings on functional brain network reorganization in Glaucoma (10). As opposed to Wang et al. (10), in the present study we included cerebellar regions, an extensive array of graph-theoretical measures and used a non-redundant, fully multivariate associations measure to construct adjacency matrices, and used three different graph metrics (betweenness centrality, local efficiency and a spectral measure of centrality) to identify hubs, and analyzed correlations with the Visual Field Index, as opposed to the VFI MD. These multiple methodological differences may explain minor discrepancies between our findings and the results reported in Wang et al. (10). We found a profound whole-brain functional reorganization in Glaucomatous patients (all disruption indices were significantly lower in the Glaucoma group as compared to healthy controls) which was also reflected in network disruption and appearancedisappearance of specific hubs as compared to healthy controls. This in in keeping with a recently highlighted extensive brain dysfunction with and showed different spatial distribution in short- and long-range functional connectivity density in Glaucoma (28). ROC analysis confirmed that disruption indices yield remarkably high sensitivity and specificity and are therefore particularly useful in discriminating Glaucoma patients from healthy controls, hence candidating such indices as biomarkers for monitoring brain involvement and reorganization in Glaucoma. Their robust positive association with VFI and retinal thickness values further corroborates this possibility.

Two hub regions present in healthy controls "disappeared" in Glaucoma patients as compared to controls: (A) the right angular gyrus (which was classified as a spectral measure of centrality hub in healthy controls only, but not in Glaucoma patients). 
TABLE 3 | Results of linear regressions of disruption indices index $k$ against clinical parameters.

\begin{tabular}{|c|c|c|c|c|c|c|c|c|c|}
\hline \multirow[t]{2}{*}{ Network measures } & \multicolumn{3}{|c|}{ VFI } & \multicolumn{3}{|c|}{$\overline{\text { RNFL }}$} & \multicolumn{3}{|c|}{$\overline{\text { MaculaGCL }}$} \\
\hline & $\mathbf{s}$ & $f^{2}$ & $p$ & $\mathbf{s}$ & $\mathbf{f}^{2}$ & $p$ & $\mathbf{s}$ & $f^{2}$ & $p$ \\
\hline k Degree & + & 0.059 & 0.129 & + & 0.207 & $0.023^{\star}$ & + & 0.104 & 0.073 \\
\hline k Betweenness centrality & + & 0.040 & 0.129 & + & 0.221 & $0.018^{\star}$ & + & 0.122 & $0.047^{*}$ \\
\hline k Local efficiency & + & 0.718 & $0.002^{\star}$ & + & 1.140 & $<0.001^{\star}$ & + & 0.993 & $0.001^{*}$ \\
\hline k Clustering coefficient & + & 0.136 & $0.038^{*}$ & + & 0.657 & $<0.001^{*}$ & + & 0.454 & $0.002^{*}$ \\
\hline k Spectral centrality measure & + & 0.060 & 0.108 & + & 0.192 & $0.023^{\star}$ & + & 0.099 & 0.065 \\
\hline
\end{tabular}

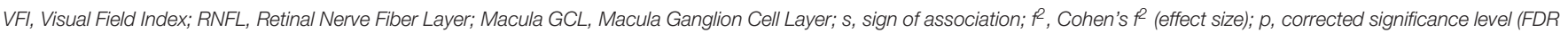
across 15 comparisons, alpha $=0.05)$; the asterisk $\left(^{*}\right)$ indicates statistically significant correlation $(p<0.05)$.

TABLE 4 | Results of linear regressions of local graph-theoretical measures against clinical parameters.

\begin{tabular}{|c|c|c|c|c|c|c|c|c|c|c|}
\hline \multirow[t]{2}{*}{ Regions } & \multirow[t]{2}{*}{ Network measures } & \multicolumn{3}{|c|}{ VFI } & \multicolumn{3}{|c|}{$\overline{\text { RNFL }}$} & \multicolumn{3}{|c|}{$\overline{\text { MaculaGCL }}$} \\
\hline & & $\mathbf{s}$ & $f^{2}$ & $p$ & $\mathbf{s}$ & $\mathbf{f}^{2}$ & $p$ & $\mathbf{s}$ & $\mathbf{f}^{2}$ & $p$ \\
\hline R parahippocampal gyrus & $\mathrm{C}$ & + & 0.193 & $0.022^{\star}$ & + & 0.171 & $0.023^{\star}$ & + & 0.239 & $0.019^{*}$ \\
\hline \multirow[t]{3}{*}{$R$ transverse temporal gyrus } & Deg & + & 0.142 & $0.048^{\star}$ & & & ns & + & 0.206 & $0.022^{*}$ \\
\hline & El & + & 0.118 & $0.046^{\star}$ & & & ns & & & ns \\
\hline & v & + & 0.158 & $0.046^{\star}$ & + & 0.139 & $0.048^{\star}$ & + & 0.277 & $0.014^{*}$ \\
\hline Lobule $X$ of Vermis & $\mathrm{C}$ & + & 0.208 & $0.049^{\star}$ & & & ns & & & $\mathrm{ns}$ \\
\hline
\end{tabular}

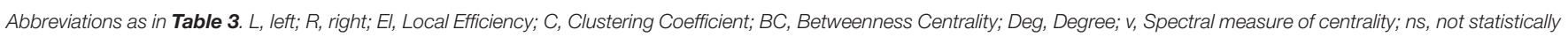
significant, s: sign of association, $f^{2}$, Cohen's $f^{2}$ (effect size); the asterisk $\left(^{*}\right)$ indicates statistically significant correlation $(p<0.05)$.

TABLE 5 | Discrimination performance for global graph-theoretical metrics and disruption index for differentiating Glaucoma patients from healthy controls.

\begin{tabular}{lccccc}
\hline Network measures & AUC & Sens (\%) & Spec (\%) & PPV (\%) & NPV (\%) \\
\hline k Clustering coefficient & 0.911 & 100 & 78.95 & 80.00 & 100 \\
k Local efficiency & 0.885 & 87.50 & 78.95 & 77.78 & 88.24 \\
k Degree & 0.786 & 81.25 & 68.42 & 68.42 & 81.25 \\
k Betweenness centrality & 0.786 & 68.75 & 89.47 & 84.62 & 77.27 \\
k Spectral measure of centrality & 0.773 & 81.25 & 68.42 & 68.42 & 81.25 \\
Betweenness centrality & 0.582 & 87.50 & 42.11 & 56.00 & 80.00 \\
Transitivity & 0.549 & 62.50 & 52.63 & 52.63 & 62.50 \\
Modularity & 0.530 & 62.50 & 57.89 & 55.56 & 64.71 \\
Clustering coefficient & 0.520 & 87.50 & 36.84 & 53.85 & 77.78 \\
Global efficiency & 0.500 & 56.25 & 57.89 & 52.94 & 61.11 \\
Degree & 0.490 & 56.25 & 47.37 & 47.37 & 56.25 \\
Assortativity & 0.470 & 56.25 & 47.37 & 47.37 & 56.25 \\
Eigenvector centrality & 0.352 & 56.25 & 36.84 & 42.86 & 50.00
\end{tabular}

k, disruption index; AUC, area under the receiver operating characteristic curve; Sens, sensitivity; Spec, specificity; PPV, positive predicted value; NPV, negative predicted value. AUC are ordered from high to low, top-down.

This region, located in the anterolateral region of parietal lobe, plays an important role in processing concepts rather than percepts when interfacing perception-to-recognition-to-action (29), possibly offering an alternative, non-mutually exclusive explanation (in addition to impaired vision) for the difficulty in distinguishing faces documented in Glaucoma patients (12); (B) The left lobule VIIB of cerebellar hemisphere (which was

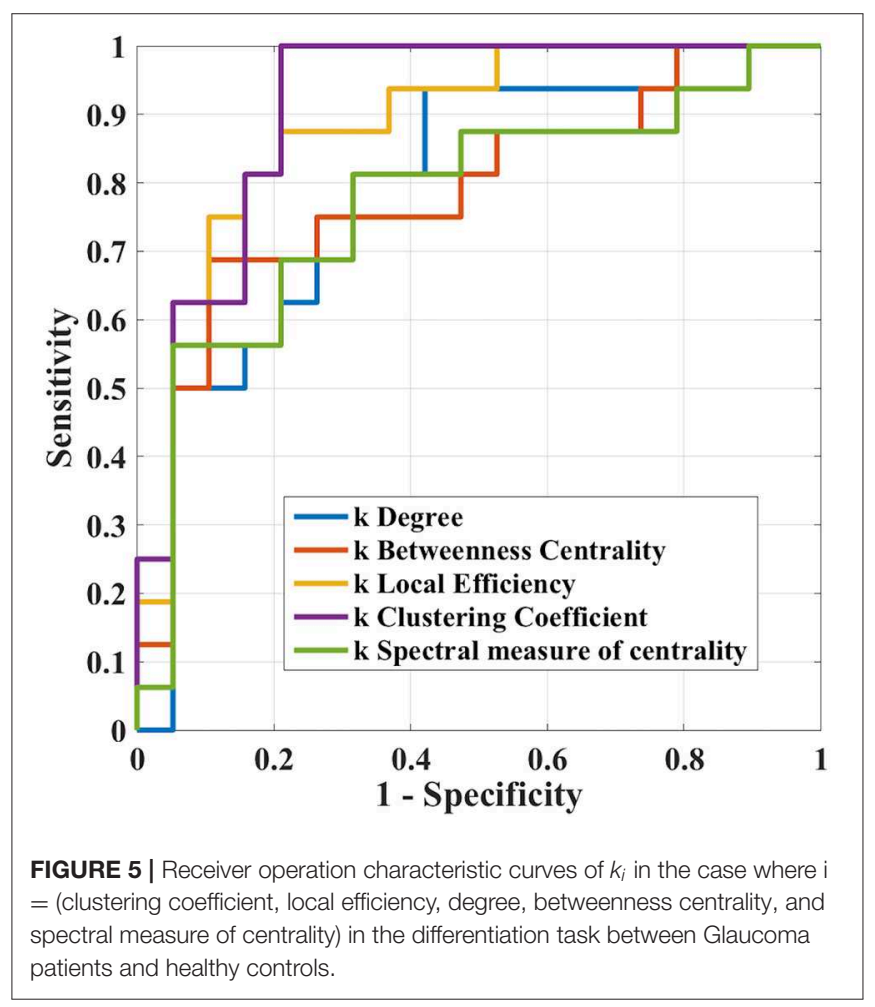

classified as a spectral measure of centrality hub in healthy controls only, but not in Glaucoma patients) plays an important 
TABLE 6 | Discrimination performance for local graph-theoretical metrics for differentiating Glaucoma patients from healthy controls (top 25).

\begin{tabular}{|c|c|c|c|c|c|c|}
\hline Regions & Network measures & AUC & Sens (\%) & Spec (\%) & PPV (\%) & NPV (\%) \\
\hline L globus pallidus & $\mathrm{BC}$ & 0.719 & 81.25 & 63.16 & 65.00 & 80.00 \\
\hline R parahippocampal gyrus & $\mathrm{BC}$ & 0.712 & 68.75 & 73.68 & 68.75 & 73.68 \\
\hline L paracentral lobule & El & 0.704 & 68.75 & 73.68 & 68.75 & 73.68 \\
\hline L supplementary motor area & $\mathrm{SmC}$ & 0.701 & 81.25 & 63.16 & 65.00 & 80.00 \\
\hline L precuneus & $\mathrm{BC}$ & 0.699 & 68.75 & 63.16 & 61.11 & 70.59 \\
\hline R middle frontal gyrus & $\mathrm{SmC}$ & 0.697 & 75.00 & 78.95 & 75.00 & 78.95 \\
\hline$R$ cuneus & El & 0.697 & 62.50 & 89.47 & 83.33 & 73.91 \\
\hline L superior occipital gyrus & $\mathrm{C}$ & 0.697 & 68.75 & 63.16 & 61.11 & 70.59 \\
\hline Rsupramarginal gyrus & El & 0.694 & 62.50 & 73.68 & 66.67 & 70.00 \\
\hline L globus pallidus & $\mathrm{SmC}$ & 0.691 & 68.75 & 68.42 & 64.71 & 72.22 \\
\hline L supplementary motor area & Deg & 0.688 & 62.50 & 78.95 & 71.43 & 71.43 \\
\hline R parahippocampal gyrus & Deg & 0.688 & 68.75 & 63.16 & 61.11 & 70.59 \\
\hline R supramarginal gyrus & Deg & 0.688 & 56.25 & 68.42 & 60.00 & 65.00 \\
\hline R supplementary motor area & C & 0.686 & 81.25 & 68.42 & 68.42 & 81.25 \\
\hline L lobule VI of cerebellar hemisphere & $\mathrm{BC}$ & 0.684 & 68.75 & 63.16 & 61.11 & 70.59 \\
\hline$R$ caudate nucleus & $\mathrm{BC}$ & 0.679 & 75.00 & 68.42 & 66.67 & 76.47 \\
\hline L olfactory cortex & C & 0.676 & 68.75 & 73.68 & 68.75 & 73.68 \\
\hline R lobule IV, V of cerebellar hemisphere & $\mathrm{BC}$ & 0.676 & 56.25 & 84.21 & 75.00 & 69.57 \\
\hline R superior occipital gyrus & $\mathrm{C}$ & 0.674 & 68.75 & 68.42 & 64.71 & 72.22 \\
\hline L inferior occipital & Deg & 0.674 & 62.50 & 78.95 & 71.43 & 71.43 \\
\hline L inferior occipital & El & 0.674 & 81.25 & 57.89 & 61.90 & 78.57 \\
\hline R supramarginal gyrus & $\mathrm{BC}$ & 0.674 & 68.75 & 63.16 & 61.11 & 70.59 \\
\hline $\mathrm{L}$ transverse temporal gyrus & El & 0.674 & 56.25 & 73.68 & 64.29 & 66.67 \\
\hline R superior parietal lobule & $\mathrm{SmC}$ & 0.671 & 68.75 & 73.68 & 68.75 & 73.68 \\
\hline L globus pallidus & Deg & 0.671 & 81.25 & 63.16 & 65.00 & 80.00 \\
\hline
\end{tabular}

Abbreviations as in Table 5. L, left; R, right; BC, Betweenness Centrality; El, Local Efficiency; SmC, Spectral measure of centrality; C, Clustering Coefficient; Deg, Degree.

role in fine motor coordination, in particular in the inhibition of involuntary movement via inhibitory neurotransmitters (30). Importantly, this could provide an alternative explanation (other than impaired vision) for the motor disturbances experienced by Glaucoma patients (12).

In contrast, three hubs were present in Glaucoma patients only (but not in healthy controls): (A) the right inferior occipital cortex (betweenness centrality hub): this region is located in the occipital lobe, which contains the primary visual pathway (15); (B) the right inferior temporal gyrus (spectral measure of centrality hub), this regions is located in the temporal lobe and has been found to be a key area in terms of simple processing of the visual field (31); (C) the left lobule IX of the cerebellar hemisphere (local efficiency hub); this area is considered essential for the visual guidance of movement (32). In this context, the first cortical transmission and processing station of the visual pathway is the primary visual cortex, from which information is transmitted to the parietal lobe and temporal lobe. There, information is processed and feedback is provided to the primary visual cortex. Given that the hubs not present in Glaucoma patients (i.e., in the parietal lobe and cerebellum) belong to secondary visual pathways, and that the hubs present only in Glaucoma patients are located in the occipital lobe, this reorganization could be hypothesized to reflect a complex interplay between neurodegeneration and functional compensatory mechanisms. In addition, our findings are not limited to the primary visual pathway. This is in agreement with previous structural $(2,4,33)$ and functional imaging studies, which also highlight changes in brain areas related to working memory and attention in Glaucoma patients $(4,7,9)$. Also, the fact that out of there three hubs, two were localized in the right hemisphere, may lend itself to a lateralization hypothesis, which should however be tested statistically in a larger patient sample.

Finally, while several studies have investigated associations between structural, functional, and metabolic brain measures and clinical parameters such as RNFL thickness and VFI (14, 15), such associations have not yet been studies through local and global disruption indices. Indeed, indices of brain network reorganization were significantly and positively related to VFI as well as structural retinal layer thicknesses. In addition, select local (as opposed to global) graph measures were positively related to VFI as well as structural retinal layer thicknesses. This points toward a direct link between the extent in functional rearrangement in both visual and extra-visual areas and both functional vision parameters (e.g., VFI) as well as structural indicators of disease severity (retinal thickness values), further corroborating the role of such disruption indices as possible biomarkers in Glaucoma. It should be noted that, since this is an associational and cross-sectional study, no definite inference is possible about the causality of the interactions we observed 
between visual impairments and functional brain reorganization. Indeed, altered functional connectivity of the primary visual cortex has been demonstrated in early and late blindness $(34,35)$. However, the fact that our findings involve not only primary, but also secondary visual regions could lead to speculate about a putative role of the latter secondary regions as contributors to the pathogenesis of Glaucoma. Taken together, our data highlight cerebral reorganization of brain networks in Glaucoma patients (36-39) supporting the interpretation of Glaucoma as central nervous system disease, likely part of the heterogeneous group of recently described disconnection syndromes (40). However, it should be noted that the number of patients assessed qualifies this work as an exploratory study, and that the optimal disruption index cut-off values estimated in this study may vary between centers due to e.g., differences in rs-fMRI acquisition protocols. Also, our experimental protocol did not include neurocognitive testing-we therefore cannot examine the putative associations between neurocognitive status and MRI parameters. Also, given that our study was performed in a relatively small sample size, future multicentric investigations in a larger number of patients and with longitudinal observations are warranted to precisely evaluate the true direction of the putative causal relationships between visual and brain manifestations of Glaucoma, and to quantify the potential of brain disruption indices as sensitive biomarkers of disease progression and brain involvement in this disease. Also, our patients were treated using topical beta-blockers, prostaglandin analogs and carbonic anhydrase inhibitors, alone or in fixed or unfixed combination. A recent study assessing glaucoma patients using resting state f-MRI reports that the possible subtle impact of these medications on intrinsic brain dynamics are not yet determined (41). Also, another study on patients treated with a beta-blocker or a prostaglandin analog reported that macular thickness, measured using OCT, did not to vary significantly both between the two

\section{REFERENCES}

1. Whitmore A V., Libby RT, John SWM. Glaucoma: thinking in new ways - A rôle for autonomous axonal self-destruction and other compartmentalised processes? Prog Retin Eye Res. (2005) 24:639-62. doi: 10.1016/j.preteyeres.2005.04.004

2. Garaci FG, Bolacchi F, Cerulli A, Melis M, Spanò A, Cedrone C, et al. Optic nerve and optic radiation neurodegeneration in patients with glaucoma: in vivo analysis with 3-T diffusion-tensor MR imaging. Radiology. (2009) 252:496-501. doi: 10.1148/radiol.2522081240

3. Cesareo M, Ciuffoletti E, Ricci F, Missiroli F, Giuliano MA, Mancino R, et al. Visual disability and quality of life in glaucoma patients. Prog Brain Res. (2015) 221:359-74. doi: 10.1016/bs.pbr.2015.07.003

4. Giorgio A, Zhang J, Costantino F, De Stefano N, Frezzotti P. Diffuse brain damage in normal tension glaucoma. Hum Brain Mapp. (2018) 39:532-41. doi: $10.1002 / \mathrm{hbm} .23862$

5. Liu Z, Tian J. Amplitude of low frequency fluctuation in primary open angle glaucoma: a resting state fMRI study. In: 2014 36th Annual International Conference of the IEEE Engineering in Medicine and Biology Society, EMBC. Chicago, IL (2014).

6. Song $\mathrm{Y}, \mathrm{Mu} \mathrm{K}$, Wang J, Lin F, Chen Z, Yan X, et al. Altered spontaneous brain activity in primary open angle glaucoma: a resting-state functional magnetic resonance imaging study. PLoS ONE. (2014) 9:e89493. doi: 10.1371/journal.pone.0089493 groups and within each group during the 6-month evaluation (42). It is therefore likely that drug treatment ahs significantly interfered with our findings.

In summary, our data lend further support to the involvement of the central nervous system in Glaucoma supporting the hypothesis that glaucoma is a neurodegenerative disease. From the clinical point of view, this supports the usefulness of neuroprotective strategies in the treatment of glaucoma in association to the standard hypotensive treatments $(36,43-47)$.

\section{DATA AVAILABILITY STATEMENT}

The datasets for this manuscript are not publicly available because the data was acquired in our institution and is not available online. Requests to access the datasets should be directed to SM (silvia.minosse2@gmail.com).

\section{ETHICS STATEMENT}

The study protocol was approved by the local Institutional Review Board and adhered to the tenets of the Declaration of Helsinki. All subjects provided written informed consent.

\section{AUTHOR CONTRIBUTIONS}

FG, CN, and NT managed the overall project (conceptualization, methodology, interpretation). SM and NT performed data preprocessing, statistical analysis, results interpretation, and prepared the original manuscript draft. FG, SL, FD, EP, MG, $\mathrm{CP}$, and RF performed MRI data acquisition and database maintenance. $\mathrm{AM}, \mathrm{MC}, \mathrm{RM}$, and $\mathrm{CN}$ were responsible for recruitment and ophthalmological examinations and results interpretation. All authors critically reviewed, read, and approved the submitted version of the manuscript.

7. Frezzotti P, Giorgio A, Toto F, De Leucio A, De Stefano N. Early changes of brain connectivity in primary open angle glaucoma. Hum Brain Mapp. (2016) 37:4581-96. doi: 10.1002/hbm.23330

8. Chen WW, Wang N, Cai S, Fang Z, Yu M, Wu Q, et al. Structural brain abnormalities in patients with primary open-angle glaucoma: a study with 3T MR imaging. Investig Ophthalmol Vis Sci. (2013) 54:545-54. doi: $10.1167 /$ iovs.12-9893

9. Frezzotti P, Giorgio A, Motolese I, De Leucio A, Iester M, Motolese E, et al. Structural and functional brain changes beyond visual system in patients with advanced glaucoma. PLoS ONE. (2014) 9:e105931. doi: 10.1371/journal.pone.0105931

10. Wang J, Li T, Wang N, Xian J, He H. Graph theoretical analysis reveals the reorganization of the brain network pattern in primary open angle glaucoma patients. Eur Radiol. (2016) 26:3957-67. doi: 10.1007/s00330-01 6-4221-x

11. Wu J, Coffey M, Reidy A, Wormald R. Impaired motion sensitivity as a predictor of subsequent field loss in glaucoma suspects: the Roscommon Glaucoma Study. Br J Ophthalmol. (1998) 82:534-7. doi: 10.1136/bjo.82.5.534

12. Sotimehin AE, Ramulu PY. Measuring disability in glaucoma. J Glaucoma. (2018) 27:939-49. doi: 10.1097/IJG.0000000000001068

13. Furlanetto RL, Teixeira SH, Gracitelli CPB, Lottenberg CL, Emori F, Michelan M, et al. Structural and functional analyses of the optic nerve and lateral geniculate nucleus in glaucoma. PLoS ONE. (2018) 13:e0194038. doi: 10.1371/journal.pone.0194038 
14. Nucci C, Mancino R, Martucci A, Bolacchi F, Manenti G, Cedrone C, et al. 3-T Diffusion tensor imaging of the optic nerve in subjects with glaucoma: correlation with GDx-VCC, HRT-III and Stratus optical coherence tomography findings. Br J Ophthalmol. (2012) 96:976-8. doi: 10.1136/bjophthalmol-2011-301280

15. Nuzzi R, Dallorto L, Rolle T. Changes of visual pathway and brain connectivity in glaucoma: a systematic review. Front Neurosci. (2018) 12:363. doi: 10.3389/fnins.2018.00363

16. Bullmore E, Sporns O. Complex brain networks: graph theoretical analysis of structural and functional systems. Nat Rev Neurosci. (2009) 10:186-98. doi: $10.1038 / \mathrm{nrn} 2618$

17. Sporns O, Betzel RF. Modular brain networks. Annu Rev Psychol. (2016) 67:613-40. doi: 10.1146/annurev-psych-122414-033634

18. Sporns O. The human connectome: origins and challenges. Neuroimage. (2013) 80:53-61. doi: 10.1016/j.neuroimage.2013.03.023

19. Bullmore ET, Bassett DS. Brain graphs: graphical models of the human brain connectome. Annu Rev Clin Psychol. (2011) 7:113-40. doi: 10.1146/annurev-clinpsy-040510-143934

20. Rubinov M, Sporns O. Complex network measures of brain connectivity: uses and interpretations. Neuroimage. (2010) 52:1059-69. doi: 10.1016/j.neuroimage.2009.10.003

21. Achard S, Delon-Martin C, Vertes PE, Renard F, Schenck M, Schneider F, et al. Hubs of brain functional networks are radically reorganized in comatose patients. Proc Natl Acad Sci USA. (2012) 109:20608-13. doi: 10.1073/pnas.1208933109

22. Martucci A, Toschi N, Cesareo M, Giannini C, Pocobelli G, Garaci F, et al. Spectral domain optical coherence tomography assessment of macular and optic nerve alterations in patients with glaucoma and correlation with visual field index. J Ophthalmol. (2018) 2018:6581846. doi: 10.1155/2018/6581846

23. Guidelines E. European Glaucoma Society Terminology and Guidelines for Glaucoma, 4th Edition - Chapter 2: Classification and terminologySupported by the EGS Foundation. Br J Ophthalmol. (2017) 101:73-127. doi: 10.1136/bjophthalmol-2016-EGSguideline.002

24. Gros-Otero J, Castejón M, Paz-Moreno J, Mikropoulos D, Teus M. Perimetric progression using the visual field index and the advanced glaucoma intervention study score and its clinical correlations. J Optom. (2015) 8:232-8. doi: 10.1016/j.optom.2014.07.009

25. Jenkinson M, Beckmann CF, Behrens TEJ, Woolrich MW, Smith SM. FSL - review. Neuroimage. (2012) 62:782-90. doi: 10.1016/j.neuroimage.2011.09.015

26. Zhang Y, Chen X, Wen G, Wu G, Zhang X. Proton magnetic resonance spectroscopy (1H-MRS) reveals geniculocalcarine and striate area degeneration in primary glaucoma. PLoS ONE. (2013) 8:e73197. doi: 10.1371/journal.pone.0073197

27. Chen W, Zhang L, Xu Y, Zhu K, Luo M. Primary angle-closure glaucomas disturb regional spontaneous brain activity in the visual pathway: an fMRI study. Neuropsychiatr Dis Treat. (2017) 13:1409-17. doi: $10.2147 /$ NDT.S134258

28. Chen L, Li S, Cai F, Wu L, Gong H, Pei C, et al. Altered functional connectivity density in primary angle-closure glaucoma patients at resting-state. Quant Imaging Med Surg. (2019) 9:603-14. doi: 10.21037/qims.2019.04.13

29. Seghier ML. The angular gyrus: multiple functions and multiple subdivisions. Neuroscientist. (2013) 19:43-61. doi: 10.1177/1073858412440596

30. Manto M, Bower JM, Conforto AB, Delgado-García JM, Da Guarda SNF, Gerwig M, et al. Consensus paper: roles of the cerebellum in motor control-the diversity of ideas on cerebellar involvement in movement. Cerebellum. (2012) 11:457-87. doi: 10.1007/s12311-011-0331-9

31. Bonilha L, Hillis AE, Hickok G, Den Ouden DB, Rorden C, Fridriksson J. Temporal lobe networks supporting the comprehension of spoken words. Brain. (2017) 140:2370-80. doi: 10.1093/brain/awx169

32. Glickstein M, Gerrits N, Kralj-Hans I, Mercier B, Stein J, Voogd J. Visual pontocerebellar projections in the macaque. J Comp Neurol. (1994) 349:51-72. doi: $10.1002 / \mathrm{cne} .903490105$

33. Boucard CC, Hanekamp S, Curčić-Blake B, Ida M, Yoshida M, Cornelissen FW. Neurodegeneration beyond the primary visual pathways in a population with a high incidence of normal-pressure glaucoma. Ophthalmic Physiol Opt. (2016) 36:344-53. doi: 10.1111/opo.12297

34. Yu C, Liu Y, Li J, Zhou Y, Wang K, Tian L, et al. Altered functional connectivity of primary visual cortex in early blindness. Hum Brain Mapp. (2008) 29:53343. doi: $10.1002 / \mathrm{hbm} .20420$

35. Wen Z, Zhou FQ, Huang X, Dan HD, Xie BJ, Shen Y. Altered functional connectivity of primary visual cortex in late blindness. Neuropsychiatr Dis Treat. (2018) 2018:3317-27. doi: 10.2147/NDT.S183751

36. Nucci C, Russo R, Martucci A, Giannini C, Garaci F, Floris R, et al. New strategies for neuroprotection in glaucoma, a disease that affects the central nervous system. Eur J Pharmacol. (2016) 787:119-26. doi: 10.1016/j.ejphar.2016.04.030

37. Garaci F, Altobelli S, Toschi N, Mancino R, Nucci C, Schillaci O, et al. Brain imaging in glaucoma from clinical studies to clinical practice. Prog Brain Res. (2015) 221:159-75. doi: 10.1016/bs.pbr.2015.06.004

38. Nucci C, Martucci A, Cesareo M, Mancino R, Russo R, Bagetta G, et al. Brain involvement in glaucoma: advanced neuroimaging for understanding and monitoring a new target for therapy. Curr Opin Pharmacol. (2013) 13:128-33. doi: 10.1016/j.coph.2012.08.004

39. Teipel SJ, Grothe M, Lista S, Toschi N, Garaci FG, Hampel H. Relevance of magnetic resonance imaging for early detection and diagnosis of alzheimer disease. Med Clin North Am. (2013) 97:399-424. doi: 10.1016/j.mcna.2012.12.013

40. Jenkinson M, Beckmann CF, Behrens TEJ, Woolrich MW, Smith SM, Thiebaut De Schotten M, et al. From phineas gage and monsieur leborgne to H.M.: revisiting disconnection syndromes. Cereb Cortex. (2015) 25:4812-27. doi: 10.1093/cercor/bhv173

41. Cai F, Gao L, Gong H, Jiang F, Pei C, Zhang X, et al. Network centrality of resting-state $\mathrm{fMRI}$ in primary angle-closure glaucoma before and after surgery. PLoS ONE. (2015) 10:e0141389. doi: 10.1371/journal.pone.0141389

42. Martin E, Martinez-de-la-Casa JM, Garcia-Feijoo J, Troyano J, Larrosa JM, Garcia-Sanchez J. A 6-month assessment of bimatoprost $0.03 \%$ vs timolol maleate 0.5\%: hypotensive efficacy, macular thickness and flare in ocular-hypertensive and glaucoma patients. Eye. (2007) 21:164-8. doi: 10.1038/sj.eye.6702149

43. Martucci A, Nucci C. Evidence on neuroprotective properties of coenzyme Q10 in the treatment of glaucoma. Neural Regen Res. (2019) 14:197-200. doi: 10.4103/1673-5374.244781

44. Raffaele M, Cesareo M, Martucci A, Di Carlo E, Ciuffoletti E, Giannini C, et al. Neurodegenerative process linking the eye and the brain. Curr Med Chem. (2018) 26:3754-63. doi: 10.2174/0929867325666180307114332

45. Mancino R, Martucci A, Cesareo M, Giannini C, Corasaniti MT, Bagetta G, et al. Glaucoma and Alzheimer disease: one age-related neurodegenerative disease of the brain. Curr Neuropharmacol. (2017) 16:9717. doi: 10.2174/1570159X16666171206144045

46. Nucci C, Martucci A, Cesareo M, Garaci F, Morrone LA, Russo R, et al. Links among glaucoma, neurodegenerative, and vascular diseases of the central nervous systemb. Prog Brain Res. (2015) 221:49-65. doi: 10.1016/bs.pbr.2015.04.010

47. Nucci C, Martucci A, Giannini C, Morrone LA, Bagetta G, Mancino R. Neuroprotective agents in the management of glaucoma. Eye. (2018) 32:93845. doi: 10.1038/s41433-018-0050-2

Conflict of Interest: The authors declare that the research was conducted in the absence of any commercial or financial relationships that could be construed as a potential conflict of interest.

Copyright (c) 2019 Minosse, Garaci, Martucci, Lanzafame, Di Giuliano, Picchi, Cesareo, Mancino, Guerrisi, Pistolese, Floris, Nucci and Toschi. This is an openaccess article distributed under the terms of the Creative Commons Attribution License (CC BY). The use, distribution or reproduction in other forums is permitted, provided the original author(s) and the copyright owner(s) are credited and that the original publication in this journal is cited, in accordance with accepted academic practice. No use, distribution or reproduction is permitted which does not comply with these terms. 


\section{OPEN ACCESS}

Edited by:

Alessandra Griffa,

Vrije Universiteit Amsterdam,

Netherlands

Reviewed by:

Gareth Barnes,

University College London,

United Kingdom

Camillo Porcaro,

Institute of Cognitive Sciences and

Technologies (CNR), Italy

George O'Neill,

University College London,

United Kingdom

*Correspondence:

Stefania Della Penna

stefania.dellapenna@itab.unich.it

Specialty section:

This article was submitted to

Brain Imaging Methods,

a section of the journal

Frontiers in Neuroscience

Received: 30 April 2019

Accepted: 02 October 2019

Published: 25 October 2019

Citation:

Della Penna S, Corbetta M,

Wens V and de Pasquale F (2019)

The Impact of the Geometric Correction Scheme on MEG

Functional Topology at Rest.

Front. Neurosci. 13:1114.

doi: 10.3389/fnins.2019.01114

\section{The Impact of the Geometric Correction Scheme on MEG Functional Topology at Rest}

\author{
Stefania Della Penna1*, Maurizio Corbetta ${ }^{2,3}$, Vincent Wens ${ }^{4,5}$ and \\ Francesco de Pasquale ${ }^{6}$
}

\begin{abstract}
'Department of Neuroscience, Imaging and Clinical Sciences, and Institute for Advanced Biomedical Technologies, "G. d'Annunzio" University of Chieti-Pescara, Chieti, Italy, ${ }^{2}$ Department of Neuroscience and Padova Neuroscience Center, University of Padua, Padua, Italy, ${ }^{3}$ Department of Neurology, Radiology, and Anatomy and Neurobiology, Washington University, St. Louis, MO, United States, ${ }^{4}$ Laboratoire de Cartographie Fonctionnelle du Cerveau, UNI-ULB Neuroscience Institute, Université Libre de Bruxelles, Brussels, Belgium, ${ }^{5}$ Magnetoencephalography Unit, Department of Functional Neuroimaging, Service of Nuclear Medicine, CUB-Hôpital Erasme, Brussels, Belgium, ${ }^{6}$ Faculty of Veterinary Medicine, University of Teramo, Teramo, Italy
\end{abstract}

Spontaneous activity is correlated across brain regions in large scale networks (RSN) closely resembling those recruited during several behavioral tasks and characterized by functional specialization and dynamic integration. Specifically, MEG studies revealed a set of central regions (dynamic core) possibly facilitating communication among differently specialized brain systems. However, source projected MEG signals, due to the fundamentally ill-posed inverse problem, are affected by spatial leakage, leading to the estimation of spurious, blurred connections that may affect the topological properties of brain networks and their integration. To reduce leakage effects, several correction schemes have been proposed including the Geometric Correction Scheme (GCS) whose theory, simulations and empirical results on topography of a few RSNs were already presented. However, its impact on the estimation of fundamental graph measures used to describe the architecture of interactions among brain regions has not been investigated yet. Here, we estimated dense, MEG band-limited power connectomes in theta, alpha, beta, and gamma bands from 13 healthy subjects (all young adults). We compared the connectivity and topology of MEG uncorrected and GCS-corrected connectomes. The use of GCS considerably reorganized the topology of connectivity, reducing the local, within-hemisphere interactions mainly in the beta and gamma bands and increasing across-hemisphere interactions mainly in the alpha and beta bands. Moreover, the number of hubs decreased in the alpha and beta bands, but the centrality of some fundamental regions such as the Posterior Cingulate Cortex (PCC), Supplementary Motor Area (SMA) and Middle Prefrontal Cortex (MPFC) remained strong in all bands, associated to an increase of the Global Efficiency and a decrease of Modularity. As a comparison, we applied orthogonalization on connectomes and ran the same topological analyses. The correlation values were considerably reduced, and orthogonalization mainly decreased local within-hemisphere interactions in all bands, similarly to GCS. Notably, the centrality of the PCC, SMA and MPFC was preserved in 
all bands, as for GCS, together with other hubs in the posterior parietal regions. Overall, leakage correction removes spurious local connections, but confirms the role of dynamic hub regions, specifically the anterior and posterior cingulate, in integrating information in the brain at rest.

Keywords: functional connectivity, band-limited power correlation, MEG connectome, leakage correction, functional hubs

\section{INTRODUCTION}

The spontaneous activity of the brain at rest is spatially and temporally organized in large-scale networks of cortical and subcortical regions, denoted Resting State Networks (RSNs). The topography of RSNs is similar to that of brain networks recruited by different cognitive tasks (Biswal et al., 1995; Attwell and Laughlin, 2001; Fox et al., 2005), and for this reason RSNs have been named according to their putative function and pattern of task activation: dorsal and ventral attention, visual, somatomotor, auditory, language, executive control, and default systems (Doucet et al., 2011; Hacker et al., 2013; Glasser et al., 2016).

As behavior unfolds, these functionally specific systems must integrate to ensure efficient processing and transfer of information in the brain. This seems to be achieved through a specialized architecture of brain regions, as shown by fMRI, DTI, EEG and MEG studies (van den Heuvel and Sporns, 2013; de Pasquale et al., 2018). A possible mechanism allowing for the dynamic integration of activity in different brain regions is the existence of structural and functional 'hub' regions that, through the architecture of their interactions, structural, as shown by DTI studies (van den Heuvel et al., 2012), and functional, as shown by fMRI studies (Zuo et al., 2012; Power et al., 2013), act as waystations of integration thus facilitating the communication within/across RSNs. Studies at higher temporal resolution, using for instance MEG, revealed a more complex scenario where nodes of the Default Mode, Dorsal Attention and Somato-Motor Networks act as dynamic cortical cores of integration in the beta and alpha bands (de Pasquale et al., 2010, 2012, 2018). Furthermore, these areas are not independent but their centrality tend to cofluctuate, hence forming a so called "dynamic core network of interaction" (de Pasquale et al., 2016). These findings nicely link with other MEG studies, showing a temporally varying organization of brain subnetworks - MEG states- (Baker et al., 2014), and with the DTI-supported notion of a structural Rich Club organization within the brain connectome. Many of these functional and structural models strongly depend on which measures of connectivity are adopted, e.g., correlationbased measures of interaction, and on which measures are used to analyze graph properties. In the case of MEG, even though neuromagnetic signals have broader frequency content and higher temporal resolution than fMRI, and are not influenced by neurovascular coupling, a serious drawback is the inherent "spatial leakage" which generates a spurious codependence among the reconstructed activity of distinct sources. In fact, in order to solve the ill-posed inverse problem, linear source projection schemes such as MNE (Hamalainen and Ilmoniemi, 1994) or Beamformers (Van Veen et al., 1997; Hillebrand et al., 2005) will inevitably yield a spatially blurred representation of the underlying source distribution, with signals reconstructed at different locations affected by activity from neighboring brain areas. These effects will largely affect the connectivity estimation. To overcome this problem, several measures insensitive to spatial leakage have been introduced: the imaginary coherence (Nolte et al., 2004), the multivariate interaction measure (Marzetti et al., 2013) or the phase lag index (Stam and Reijneveld, 2007; Hillebrand et al., 2012) for phase coupling on the fast signal (activity), the orthogonalized correlation (Brookes et al., 2012; Hipp et al., 2012; O’Neill et al., 2015) and the symmetrical multivariate leakage corrections (Colclough et al., 2015) for amplitude coupling on the slow signal (activity envelope). The common idea behind all these correction schemes is that spatial leakage can only induce zerolag linear spurious coupling, which can be in turn eliminated by an appropriate regression model (Wens, 2015), and that it does not affect non-zero-lag connectivity (see Palva et al., 2018 for a critical overview on this assumption). However, a potential issue with these approaches is that physiological interactions involving zero-lag linear coupling may be suppressed as well. This is particularly important since, with synaptic delays in the range of 5-25 $\mathrm{ms}$ from neighboring to remote regions, zerolag interactions are expected to be physiologically dominant. This has been widely documented in empirical data as well as modeling studies (Gollo et al., 2014). In fact, these mechanisms have been ascribed to a range of crucial neuronal functions, from perceptual integration to the execution of coordinated motor behaviors (Roelfsema et al., 1997; Singer, 1999; Varela et al., 2001; Uhlhaas et al., 2009). A recent work also suggests the existence of zero-lag correlations at rest, specifically within the Default Mode Network (Sjogard et al., 2019).

A possible alternative method for preserving zero-phase lag correlation is the Geometric Correction Scheme (GCS) proposed by Wens et al. (2015), which models spatial leakage from a seed location based on the forward and inverse models. The fundamental theoretical aspects of the GCS as well as simulation- and data-based proof-of-concept were developed in Wens (2015) and Wens et al. (2015), but they were limited to the study of RSN topographies. Here, we investigate the effect of this leakage correction on a dense connectome (155 nodes, involving 9 separate RSNs) as a function of the oscillatory band and the eventual impact on the estimated topological features. Specifically, we estimated the dense connectome based on band-limited power (BLP) computed in the theta, alpha, beta and gamma bands without leakage correction and with the GCS. We first compared the overall topology through 
Network Based Statistics (NBS). Furthermore, we considered topological features such as the Betweenness Centrality and Global Efficiency, to understand the impact of the GCS on the identification of hub regions and the efficiency of integration. Our hypothesis is that GCS will affect local connections, producing a decrease of local links, and a partial reorganization of the brain hubs. We expect that leakage reorganization will occur mainly in the alpha and beta bands, which are the main correlates of fMRI RSNs (Mantini et al., 2008). Finally, to assess the impact on the topology of removing 0-lag correlations as compared to maintaining them, we ran the same analyses also on connectomes where leakage was corrected through orthogonalization similarly to Brookes et al. (2012). Based on the overall similarity of RSN topography shown in Wens et al. (2015), we expect to find some agreement between results obtained from the two approaches.

\section{MATERIALS AND METHODS}

\section{Subjects and Recordings}

MEG signals were recorded from 13 healthy adult subjects (mean age $29 \pm 6$ years, 5 females), already used in de Pasquale et al. (2016). Subjects were asked to remain still in the MEG system while fixating a cross on a screen. We recorded 2 or 3 scans lasting $5 \mathrm{~min}$ from each subject. MEG signals were recorded using the 153-channel MEG system developed and installed at the University of Chieti (Della Penna et al., 2000). The system is placed within a four-layer magnetically shielded room allowing magnetometric recordings. Two EOG and two ECG channels were recorded simultaneously with the MEG signals, to be used for physiological artifact rejection. Neuromagnetic and electrical signals were filtered in the band 0.16$250 \mathrm{~Hz}$ and were sampled at $1 \mathrm{kHz}$. Before and after each resting state run, the signal generated by five positioning coils placed on the subject's head were recorded and used to coregister functional and anatomical data. Anatomical images were acquired using a 1.5 T Siemens Vision scanner, through a sagittal magnetization-prepared rapid acquisition gradient echo $\mathrm{T} 1$ weighted sequence (MP-RAGE) with repetition time $(\mathrm{TR})=9.7 \mathrm{~s}$; echo time $(\mathrm{TE})=4 \mathrm{~ms} ; \alpha=12^{\circ}$; inversion time $=1,200 \mathrm{~ms}$; voxel size $=1 \times 1 \times 1.25 \mathrm{~mm}^{3}$.

\section{MEG Data Preprocessing}

An extensive description of the approach used to preprocess our MEG data can be found in Mantini et al. (2011). In brief, we applied a pipeline based on ICA (similar to the one described in Larson-Prior et al., 2013) to separate and identify environmental and physiological (e.g., cardiac, ocular) artifacts, which were removed from sensor-space MEG data. Runs affected by excessive noise (e.g., movement of the subject's head in the helmet) were discarded from further analysis. This reduced our final sample to a total of 27 runs. The sensor maps of non-artifactual ICs (brain ICs) were projected into the individual source space through a weighted minimum norm estimator using Curry 6 (Neuroscan, Hamburg, Germany). The source space was mapped into a $3 \mathrm{D}$ grid of cubic voxels with a $4 \mathrm{~mm}$ side, coregistered to the MNI atlas. The vector activity $\boldsymbol{\Psi}(t)=\sum_{\text {ic }} A_{\text {ic }} s_{\text {ic }}(t)$ at each voxel in the brain was obtained as the linear combination of the brain IC timecourses weighted by the related source-projected IC maps.

\section{Connectivity Estimation and Geometric Leakage Correction}

We estimated a dense connectome based on BLP correlations among a set of 155 nodes obtained in a previous meta-analysis on fMRI data (Hacker et al., 2013; Baldassarre et al., 2014), which yielded 9 RSNs (Dorsal Attention Network -DAN, Ventral Attention Network - VAN, Somato-Motor Network - SMN, Visual Network - VIS, Auditory Network - AUD, Language Network - LAN, Default Mode Network - DMN, Fronto-Parietal Network - FPN, Control Network - CON). Subcortical ROIs were removed from the original set. Source activity $\boldsymbol{\Psi}(t)$ was filtered into 4 frequency bands: theta $(4-7 \mathrm{~Hz})$, alpha $(7-14 \mathrm{~Hz})$, beta $(14-25 \mathrm{~Hz})$, gamma $(25-70 \mathrm{~Hz})$ and their BLP time series were obtained by integrating the square amplitude of the signal over $150 \mathrm{~ms}$ windows sliding by $20 \mathrm{~ms}$. Then, for each pair of nodes, we computed the Pearson correlation coefficient between their respective BLP time series over non-overlapping epochs lasting $40 \mathrm{~s}$ and then averaged them across the whole run. This procedure yielded the original, uncorrected connectivity matrices. To obtain their leakage-corrected version, each node was successively taken as a seed and spatial leakage emanating from it was modeled using the GCS (Wens, 2015; Wens et al., 2015), which we review in the following.

The GCS was applied on the source space maps of each brain IC before estimating the leakage-corrected activity as follows. Given a seed $\boldsymbol{r}_{0}$, and the linear inverse operator $\boldsymbol{W}=\sum_{i c} \boldsymbol{A}_{i c} \boldsymbol{u}_{i c}$ associated with our source reconstruction pipeline (Mantini et al., 2011; Betti et al., 2018), where $\boldsymbol{A}_{i c}$ is the source-space map for a generic component IC with dimensions $\left(\mathrm{N}_{\text {voxels }} \mathrm{x} 3\right)$, coregistered to the MNI atlas, $\boldsymbol{u}_{i c}$ is the row of the unmixing matrix with dimensions ( $1 \mathrm{x} \mathrm{N}_{\text {channels }}$ ), and the sum is over the brain ICs, the corrected source map was computed as:

$$
\boldsymbol{A}_{i c}^{G C S}=\boldsymbol{A}_{i c}-\left(\sum_{i c} \boldsymbol{A}_{i c} \boldsymbol{u}_{i c} \boldsymbol{L}\left(\boldsymbol{r}_{0}\right)\right)\left(\sum_{i c} \boldsymbol{A}_{i c}\left(\boldsymbol{r}_{0}\right) \boldsymbol{u}_{i c} \boldsymbol{L}\left(\boldsymbol{r}_{0}\right)\right)^{+} \boldsymbol{A}_{i c}\left(\boldsymbol{r}_{0}\right)
$$

where $\boldsymbol{L}\left(\boldsymbol{r}_{0}\right)$ is the leadfield associated to the seed, with dimensions $\left(\mathrm{N}_{\text {channels }} \times 3\right)$ also coregistered to the MNI atlas, and + denotes pseudoinversion. Given the seed, the leakagecorrected vector activity of all the other nodes was then rebuilt as:

$$
\boldsymbol{\Psi}^{G C S}(t)=\sum_{\text {ic }} \boldsymbol{A}_{i c}^{G C S} s_{i c}(t)
$$

where $s_{\mathrm{ic}}(t)$ are the brain IC timecourses. The row of the connectivity matrix corresponding to this seed was obtained by correlation of the BLP obtained from the uncorrected activity $\boldsymbol{\Psi}(t)=\sum_{\mathrm{ic}} \boldsymbol{A}_{\mathrm{ic}} s_{\mathrm{ic}}(t)$ at the seed and the BLP of all the corrected activities $\boldsymbol{\Psi}^{G C S}(t)$ at the other nodes.

GCS connectomes were then symmetrized by averaging the upper and lower triangles to avoid the slight asymmetries induced by leakage correction. Of note, spatial leakage is symmetrical in 
minimum norm estimates (Hauk and Stenroos, 2014), so this step discarded only slight asymmetries.

Finally, as suggested in Wens et al. (2015), to remove the contribution of possible local under-correction effects due to seed mis-localization in the GCS connectomes, we masked out node pairs closer than $35 \mathrm{~mm}$. To avoid biasing comparisons between connectomes, this distance mask was applied to both uncorrected and the corrected connectomes. Thus, all the correlation values between node pairs closer than $35 \mathrm{~mm}$ were not considered in all the analyses applied to the two types of connectomes and described in the following subsections. The number of node pairs masked in our analysis was 1155, corresponding to less than 5\% of the total number of node pairs (equal to 23870).

\section{Analysis of Topological Effects of Leakage Correction}

First, we analyzed possible decreases of correlation strength by estimating the $\mathrm{z}$-Fisher transformed correlation averaged over the whole connectome, for each subject and band, and performing paired $t$-tests assessing the effect of the GCS over each frequency band. Then, topological changes induced by the GCS were investigated through the NBS Toolbox (Zalesky et al., 2010) at each frequency band. NBS directly operates on the connectivity values and searches for graph components (subgraphs made of connected nodes) comprising suprathreshold links obtained from a t-statistics testing connectivity differences. We looked for components representing decreases and increases separately, using a large primary threshold for the t-statistic, namely 6 . This value represents a good compromise between the size of the significant components produced by NBS, which was already large for all bands at this threshold, and the sake of clarity in showing and interpreting the obtained components. However, it must be noted that the adoption of a smaller threshold for the t-statistics, e.g., $t=3$ as in Betti et al. (2018), produced very similar results. We are aware that the choice of this threshold certainly influences the size of the obtained components and thus to draw any conclusions on its absolute value within a condition can be misleading, also due to the contribution of false positive links in a component (Zalesky et al., 2012). Nevertheless, in this work we adopted the same threshold in all bands when comparing GCS-corrected vs. non-corrected data. For this reason, since all the data will be affected in the same way by the choice of the threshold, we limited our observations on the relative increase or decrease of the component size observed across frequency bands.

The significance level of each component was set to $a<0.01$ assessed through permutation testing (number of permutations $n=200)$. Then, we adopted the MATLAB toolbox BrainNet Viewer for the graph visualization (Xia et al., 2013).

\section{Graph Analyses}

In this work, the graph measures were computed on the BLP connectomes averaged across subjects. Thus, from the z-score connectivity matrices estimated for each run, obtained after Fisher transformation of the original correlation values, we first averaged across runs and then across subjects to obtain mean connectivity matrices. This applies both for the GCS corrected and the uncorrected data. Then, we thresholded the computed matrices to obtain fully connected binarized connectomes (Bordier et al., 2017). To this aim, the graph components were estimated at several thresholds. Then, the maximum threshold ensuring a single graph component, and thus full connectedness, was selected. This procedure allowed us to compare graphs in the same condition (a path between any pair of nodes exists), across uncorrected and leakage corrected scenarios and across bands. Now, to characterize the topology of the graph, measures such as centrality, connection density, modularity and efficiency were computed on these binarized connectomes. To quantify the centrality of the graph nodes, we adopted the binary Betweenness Centrality $(\mathrm{BC})$ that is related to the number of times a node acts as a bridge between the strongest connections of any two nodes. Thus, nodes with high BC participate in many shortest paths. The $\mathrm{BC}$ is computed according to the following formula:

$$
B C(v)=\frac{2}{(N-1)(N-2)} \sum_{i \neq j \neq v} \frac{\sigma_{i j}(v)}{\sigma_{i j}}
$$

where $\sigma_{i j}$ is the total number of shortest paths from node $i$ to node $j, \sigma_{i j}(v)$ is the fraction of those paths passing through the node $v$ and $N$ is the graph order (Rubinov and Sporns, 2010; Sporns, 2011). To compare the pattern of BC values across different conditions (GCS corrected and non-corrected graphs) and bands, this was normalized to the sum of BC across nodes.

Notably, to assess the significance of the BC, we compared the obtained values with those from a population of random graphs. These were generated by the approach described in Rubinov and Sporns (2011) where a randomization function preserving the degree and strength distributions was employed (each graph edge was randomly rewired five times and 1000 iterations were run). From the obtained population of random graphs, we computed the 95th percentile of the obtained distribution and we considered as significant only those BC values exceeding such value.

To characterize the global integration properties of the graph we adopted the Global Efficiency (GE), a measure related to the information exchange across the whole graph (Rubinov and Sporns, 2010; Sporns, 2011). GE is defined as the average inverse shortest path length in the network, which is inversely related to the characteristic path length:

$$
G E=\frac{1}{N[N-1]} \sum_{i, j \in N, i \neq j} \frac{1}{d_{i j}}
$$

where $d_{i j}$ is the shortest path length between the nodes $i$ and $j$. In order to evaluate significant effects of the GCS on GE, we performed a paired $t$-test comparing GE obtained with leakage correction and without it, across resting state sessions, within each frequency band. Probability values were Bonferroni corrected for multiple comparisons.

We further investigated how eventual changes in GE related to the graph Modularity and Density of connections. Modularity is a measure that identifies the community structure of a network as a subdivision of non-overlapping groups of nodes in a way that maximizes the number of within-group edges and minimizes the 
number of between-group edges. Thus, this represents a statistic related to the degree to which the network may be subdivided into clearly delineated modules. Different approaches can be adopted to this aim, here we used the Louvain algorithm which provides a fast and accurate community detection (Blondel et al., 2008). We also checked that any modulation of the GE was not a mere effect of a different density of connections. The effect of the GCS on these two measures was also assessed with paired $t$-tests, with significance Bonferroni-corrected for multiple comparisons.

All the above quantities have been estimated by means of the MATLAB toolbox, the Brain Connectivity Toolbox ${ }^{1}$ (Rubinov and Sporns, 2010) and for graph visualization the BrainNet Viewer $^{2}$ (Xia et al., 2013) was adopted.

\section{Control Analyses Using Orthogonalization}

In order to compare the GCS approach with another popular leakage correction approach, we performed the same topological analyses described in the previous subsections on orthogonalized connectomes. Signal orthogonalization is another correction scheme based on linear regression to remove all zerolag correlations (Brookes et al., 2012; Hipp et al., 2012). A multivariate version designed for beamformer inverse solution, ensuring symmetrical connectomes has also become widespread (Colclough et al., 2015), but we did not apply it since spatial leakage is symmetrical in minimum norm estimates. Here, we used the basic pairwise linear regression of Brookes et al. (2012). Specifically, given a seed $\boldsymbol{r}_{0}$, the orthogonalized vector activity was obtained by regressing out the seed signal $\boldsymbol{\Psi}_{r 0}(t)$ :

$$
\boldsymbol{\Psi}^{O R T}(t)=\boldsymbol{\Psi}(t)-\boldsymbol{\beta} \boldsymbol{\Psi}_{r_{0}}(t)
$$

where the $3 \mathrm{~N}_{\text {voxels }} \times 3$ matrix $\boldsymbol{\beta}=\boldsymbol{\Psi} \boldsymbol{\Psi}_{r 0}^{+}$encodes the regression coefficients. In this last equation, $\boldsymbol{\Psi}$ gathers all samples $\boldsymbol{\Psi}(t)$ of the activity vector in a $3 \mathrm{~N}_{\text {voxels }} \times \mathrm{N}_{\text {time }}$ matrix, and correspondingly the seed activity $\boldsymbol{\Psi}_{r 0}(t)$ is a $3 \times \mathrm{N}_{\text {time }}$ matrix.

As in the GCS case, the row of the connectivity matrix corresponding to each seed was obtained by correlation of the BLP obtained from the uncorrected activity $\boldsymbol{\Psi}_{\boldsymbol{r}_{0}}(t)$ at the seed and the BLP of all the corrected activities $\boldsymbol{\Psi}^{\text {ORT }}(t)$ at the other nodes.

Orthogonalized connectomes were then symmetrized, to account for numerical errors. The distance mask over node pairs closer than $35 \mathrm{~mm}$ was applied also in this case, given that orthogonalization also leads to similar under-correction effects (Palva et al., 2018), albeit less extensively than the GCS (Wens et al., 2015).

As in the GCS vs. uncorrected case, we ran the following analyses: (i) we compared the mean z-Fisher correlation values between uncorrected and orthogonalized connectomes; (ii) we applied NBS at each frequency band, using a threshold for the t-statistics equal to 6, as for the GCS vs. uncorrected comparison; (iii) given the disruptive effect of orthogonalization on the $z$-score correlation values, we also used a higher t-threshold, namely 10,

${ }^{1}$ https://sites.google.com/site/bctnet/

${ }^{2}$ http://www.nitrc.org/projects/bnv/ to display and discuss the component size; (iv) we binarized the band-specific, group-averaged, orthogonalized connectomes imposing the full connectedness, as for the uncorrected and GCS connectomes; (v) we estimated BC and its significance for each node in the graph; (vi) we estimated GE, graph modularity and density and we compared these values with the ones obtained from the uncorrected connectomes through a $t$-test, with significance Bonferroni-corrected for multiple comparisons.

\section{RESULTS}

\section{Global Topology of GCS vs. Uncorrected Connectomes}

To investigate the effect of signal leakage correction on the architecture of within and across RSN interactions, we computed RSN-based connectomes, obtained by averaging the dense connectomes first across the RSN nodes (see Table 1 for a complete list of nodes, RSNs and abbreviations), discarding the node pairs closer than $35 \mathrm{~mm}$, and then across subjects. The results for the uncorrected and GCS-corrected data are shown in Figure 1 (left column: UNCORRECTED, right column: GCS) at the different frequency bands. Although a spatial modulation of the RSN-based connectome is apparent across all bands, local reductions and increases of connectivity seem to be balanced. In fact, the connectivity averaged over the whole connectome does not differ significantly with and without GCS ( $t$-tests over subjects, all $p s>0.35$ for all bands). This suggests that GCS might preserve the global strength of connectivity. In addition, these RSN-based connectomes reveal a common modulation of connectivity patterns: within-VIS correlation seems to decrease for all bands after GCS, while within-AUD correlation seems to increase.

In order to assess the statistical significance of the topological changes underlying these modulations, we applied NBS to the dense connectomes to identify graph components containing significantly different connections. As it can be seen in Figure 2A, when comparing uncorrected with GCS-corrected connectomes, we obtained components representing significant GCS-induced decrements of BLP correlation in all the physiological bands. These results are overlaid onto an MNI reference brain (BrainNetViewer, Xia et al., 2013) and nodes are color-coded based on the membership to fMRI RSNs (see reported colormap in Figure 2). Notably, at the same t-statistics threshold for all bands, the number of links in the decreased component assumed a different size as a function of the frequency band. Specifically, the decreased components in the beta, gamma and alpha bands involved a larger number of nodes than in the theta band. The pie chart reported in Figure 2B shows the percentage of links significantly decreased after GCS in each frequency band with respect to the total number of links decreased across all bands, which was 1022 at the $t$-threshold $=6$. It can be noted a similar proportion of links removed in the beta/gamma bands (around $30 \%$ ) followed by the theta (21\%) and alpha band (only 16\%). Interestingly, Figure $\mathbf{2 C}$ shows that the majority of links removed by GCS were intra-hemispheric (WITHIN HEM. - red bars), as compared to the inter-hemispheric ones (ACROSS HEM. - black 
TABLE 1 | List of abbreviations of the node and network labels adopted in this work.

\begin{tabular}{|c|c|c|c|c|c|c|c|c|c|c|c|}
\hline & \multicolumn{3}{|c|}{ MNI Coordinates } & \multirow[t]{2}{*}{ Label } & \multirow[t]{2}{*}{ RSN } & & \multicolumn{3}{|c|}{ MNI Coordinates } & \multirow[t]{2}{*}{ Label } & \multirow[t]{2}{*}{ RSN } \\
\hline & $x$ & $y$ & z & & & & $x$ & y & z & & \\
\hline 2 & -26.3 & -11.8 & 52.7 & IFEF & DAN & 53 & -24 & -32 & 63 & LdPoCe & SMN \\
\hline 3 & 23.2 & -69.4 & 48.6 & rpips & DAN & 54 & -54 & -23 & 37 & LvPoCe & SMN \\
\hline 4 & 46 & -32 & 50 & RalPs & DAN & 55 & 54 & -18 & 37 & RvPoCe & SMN \\
\hline 7 & 26 & -69 & 30 & RvIPS2 & DAN & 58 & 22 & -18 & 58 & RdPrCe & SMN \\
\hline 8 & -25.3 & -67.3 & 47.6 & Ipips & DAN & 59 & 11 & -46 & 59 & RSPL-preCun & SMN \\
\hline 9 & -32 & -42 & 45 & LalPS & DAN & 60 & -18 & -36 & 55 & LdCS & SMN \\
\hline 10 & -22 & -53 & 52 & LSPL & DAN & 61 & -7 & -44 & 56 & LdmSPL & SMN \\
\hline 11 & -31 & -80 & 18 & LVIPS1 & DAN & 62 & 9 & -43 & 53 & RmdSPL & SMN \\
\hline 16 & 46 & -51 & -14 & RvitG1 & DAN & 67 & -7.26 & -98.79 & 6.79 & IV2 & VIS \\
\hline 17 & 39 & 30 & 12 & RIFG & DAN & 68 & 27.5 & -88.5 & 14.5 & rV3 & VIS \\
\hline 18 & -49 & -5 & 32 & LvPrCe & DAN & 69 & -16.11 & -92.7 & 17.83 & IV3 & VIS \\
\hline 19 & -40 & -42 & -19 & LVITG1 & DAN & 70 & 26.67 & -71.44 & -13.94 & rV4 & VIS \\
\hline 20 & -45 & -34 & 45 & LPoCe & DAN & 71 & -30.98 & -76.54 & -17.13 & IV4 & VIS \\
\hline 21 & -53 & -29 & 37 & LvPoCe & DAN & 72 & 32.23 & -78.38 & 25.12 & rV7 & VIS \\
\hline 22 & 41 & 17 & 31 & $\mathrm{RMFG}$ & VAN & 73 & -23.1 & -78.13 & 26.12 & IV7 & VIS \\
\hline 23 & 12 & 5 & 61 & RMFG2 & VAN & 74 & -7 & -86 & 36 & LV7-POSd & VIS \\
\hline 24 & 41 & 2 & 50 & RMFG3 & VAN & 75 & -22 & -71 & 6 & LV7 & VIS \\
\hline 25 & 52 & -48 & 28 & $\mathrm{RSMG}$ & VAN & 76 & 34 & -88 & -4 & RLO & VIS \\
\hline 33 & -44 & 10 & 8 & LVIFG & VAN & 84 & -11 & -74 & -6 & LVP & VIS \\
\hline 34 & 42 & 28 & 1 & RIFG-Al & VAN & 85 & 17 & -64 & -5 & RVP & VIS \\
\hline 35 & -33 & 17 & -5 & LAl & VAN & 86 & 18 & -76 & 26 & RPOSd & VIS \\
\hline 36 & -10 & -32 & 43 & LPC & VAN & 87 & 60 & -22 & 6 & rMSTG & AUD \\
\hline 37 & 39 & 11 & 21 & RvPrCe & VAN & 88 & -41 & -28 & 6 & IMSTG & AUD \\
\hline 38 & -60 & -28 & 24 & $|S| \mid$ & SMN & 89 & -51 & -22 & 5 & LmSTG & AUD \\
\hline 39 & 35 & -26 & 55 & rCs & SMN & 90 & -56 & -33 & 16 & LSTG1 & AUD \\
\hline 40 & 56 & -2 & 23 & RPrCe & SMN & 91 & -43 & -34 & 11 & LSTG2 & AUD \\
\hline 41 & -37 & -19 & 53 & ICS & SMN & 92 & 54 & -43 & 12 & rIST & AUD \\
\hline 42 & -57 & -8 & 21 & LPoCe & SMN & 93 & -54 & -40 & 10 & $\| S T$ & AUD \\
\hline 43 & 57 & -28 & 23 & rSII & SMN & 94 & 38 & -19 & 12 & Rpl & AUD \\
\hline 44 & -1 & -17 & 55 & ISMA & SMN & 95 & -35 & -20 & 14 & Lpl & AUD \\
\hline 45 & -10 & -12 & 60 & ISMA2 & SMN & 96 & 34 & -24 & 17 & Rpl & AUD \\
\hline 46 & -12 & -20 & 40 & ISMA3 & SMN & 97 & 36 & 0 & 12 & $\mathrm{Rml}$ & AUD \\
\hline 47 & 4 & -15 & 53 & rSMA & SMN & 98 & -38 & -4 & 11 & Lml & AUD \\
\hline 48 & 10 & -33 & 52 & RParaCe & SMN & 99 & 50 & -12 & 17 & $\mathrm{Rml}$ & AUD \\
\hline 49 & -30 & -18 & 10 & IPUT & SMN & 100 & 38 & -6 & 4 & $\mathrm{Rml}$ & AUD \\
\hline 50 & 30 & -17 & 9 & rPUT & SMN & 101 & -37 & -8 & 3 & Lml & AUD \\
\hline 51 & -42 & -17 & 46 & LcPrCe & SMN & 102 & -30 & 0 & 15 & Lml3 & AUD \\
\hline
\end{tabular}


TABLE 1 | Continued

\begin{tabular}{|c|c|c|c|c|c|c|c|c|c|c|c|}
\hline & \multicolumn{3}{|c|}{ MNI Coordinates } & \multirow[t]{2}{*}{ Label } & \multirow[t]{2}{*}{ RSN } & & \multicolumn{3}{|c|}{ MNI Coordinates } & \multirow[t]{2}{*}{ Label } & \multirow[t]{2}{*}{ RSN } \\
\hline & $\mathbf{x}$ & $\mathbf{y}$ & $\mathbf{z}$ & & & & $\mathbf{x}$ & $\mathbf{y}$ & $\mathbf{z}$ & & \\
\hline 103 & -48 & 31 & -1 & IFG & LAN & 130 & -19 & 22 & 52 & LSFG & DMN \\
\hline 104 & -50 & 19 & 9 & lifg2 & AUD & 131 & 17 & 24 & 51 & RSFG & DMN \\
\hline 105 & -45 & 13 & 24 & MFG & LAN & 132 & 13 & 40 & 40 & RSFG2 & DMN \\
\hline 106 & -7 & 9 & 60 & $\operatorname{Imfc} 4$ & AUD & 133 & 59 & -25 & -12 & RSTS & DMN \\
\hline 107 & -50 & -54 & 22 & STS & LAN & 134 & -5 & 31 & -4 & LAC3 & DMN \\
\hline 108 & -56 & -12 & -3 & aSTG & LAN & 135 & -9 & 36 & 8 & LAC2 & DMN \\
\hline 109 & -55 & -48 & 15 & pSTG & LAN & 136 & -3 & 28 & 55 & LmSFG2 & DMN \\
\hline 110 & -48 & -44 & 3 & Istg3 & AUD & 137 & 52 & -1 & -25 & RMTG1 & DMN \\
\hline 111 & -56 & -33 & 3 & lstg2 & AUD & 138 & -6 & 16 & 63 & LmSFG1 & DMN \\
\hline 112 & -52 & -54 & 12 & Istg4 & AUD & 139 & 42 & 10 & -29 & RMTG2 & DMN \\
\hline 113 & -54 & -23 & -3 & $\operatorname{lstg} 1$ & AUD & 140 & -41 & 7 & -31 & LITG & DMN \\
\hline 114 & 47 & 25 & -4 & rifg1 & AUD & 141 & -31 & -59 & 42 & LIPS & FPN \\
\hline 115 & 44 & -36 & 6 & rstg2 & AUD & 142 & 30 & -61 & 39 & RIPS & FPN \\
\hline 116 & 53 & 23 & 7 & rifg2 & AUD & 143 & 51 & -47 & 42 & $\mathrm{RIPL}$ & FPN \\
\hline 117 & 61 & -43 & 8 & rstg1 & AUD & 144 & 10 & -69 & 39 & RprCu & FPN \\
\hline 118 & 2 & 52.6 & 23.5 & rMPFC & $\mathrm{DMN}$ & 145 & -43 & 22 & 34 & LdIPFC & FPN \\
\hline 119 & -2 & 50.5 & 1.7 & IMPFC & $\mathrm{DMN}$ & 146 & -9 & -72 & 37 & LprCu & FPN \\
\hline 120 & -13.1 & 51.5 & 23.4 & IMPFC2 & DMN & 147 & 43 & 22 & 34 & RdIPFC & FPN \\
\hline 121 & -2 & 40 & 27 & LmPFC2 & $\mathrm{DMN}$ & 148 & -41 & 3 & 36 & LFC & FPN \\
\hline 122 & -3 & -54 & 31 & IPCC & $\mathrm{DMN}$ & 149 & -51 & -51 & 36 & LIPL & FPN \\
\hline 123 & 51 & -64 & 32 & rAG & $\mathrm{DMN}$ & 150 & -28 & 51 & 15 & LaPFC & FPN \\
\hline 124 & -43 & -76 & 35 & $\mid A G$ & $\mathrm{DMN}$ & 151 & 39 & 1 & 42 & $\mathrm{RdPrCe}$ & FPN \\
\hline 125 & -56.6 & -25.1 & -16.9 & ॥TG & $\mathrm{DMN}$ & 152 & -33 & 13 & 9 & Lal_CO & CON \\
\hline 126 & 8 & -51 & 29 & RPCPreCun & $\mathrm{DMN}$ & 153 & 36 & 16 & 4 & RalfO & CON \\
\hline 127 & 0 & -65 & 31 & RPreCun & $\mathrm{DMN}$ & 154 & -1 & 10 & 46 & dACCmsFC & CON \\
\hline 128 & -1 & 44 & -2 & LAC1 & $\mathrm{DMN}$ & 155 & 8 & 3 & 51 & RpreSMA & CON \\
\hline 129 & -4 & 42 & 45 & LmSFG3 & $\mathrm{DMN}$ & & & & & & \\
\hline
\end{tabular}

bars). We acknowledge that care should be taken in interpreting the number of components' nodes and edges for each band, since NBS controls the family wise error rate in the weak sense. Thus, the significance is associated to the whole component and not to single links, some of which might represent false positives (Zalesky et al., 2012). However, since we used the same t-test threshold across bands, we expect approximately the same number of false positives in all bands and thus the comparison of the number of edges in the decreased components in the beta and gamma bands versus the other bands is reasonable. In addition to components representing significant decreases, we also obtained components representing significant increases in all bands (see Figure 3A). In this case, the majority of increments in the number of links occurred in the alpha and beta bands and to a lesser extent in the theta band, while the gamma band involved fewer links. The percentage of links (with respect to the total number of increased connections across all bands, which was 1022) increasing after leakage correction was approximately $40 \%$ for alpha and beta bands and it decreased to about 20 and $6 \%$ in theta and gamma bands, respectively (see Figure 3B). Again, because of false positives, the absolute number of supra-threshold links should be interpreted cautiously. However, the main result is that the size of the increased component in the alpha and beta bands is considerably larger than for the theta and gamma bands.
Furthermore, as it can be seen in Figure 3C, most involved links were between hemispheres (black - ACROSS HEM.) and only a few links were within hemispheres (red - WITHIN HEM.). To summarize, the GCS considerably changed the topology of the interactions, producing local, within-hemisphere decreases, and long-range, between-hemisphere increases. These effects were stronger in the beta and gamma bands for the decreases, and in the alpha and beta band for the increases.

\section{Integration/Segregation in GCS vs. Uncorrected Connectomes}

In order to investigate how the modulation of topology affected the integration and segregation in the whole brain network, we computed the Betweenness Centrality of the considered nodes, over the binary graphs obtained from uncorrected and GCScorrected connectomes, in each frequency band (see Figure 4). First, we note that leakage correction led to a difference in the number of significant hubs: we obtained a slightly larger number of hubs in the theta band $(+9 \%)$ as compared to the uncorrected data, while in the gamma band the number of hubs almost doubled $(+93 \%)$. On the contrary, in the alpha $(-20 \%)$ and beta $(-42 \%)$ bands the GCS reduced the number of central regions. This presumably indicates that in these 


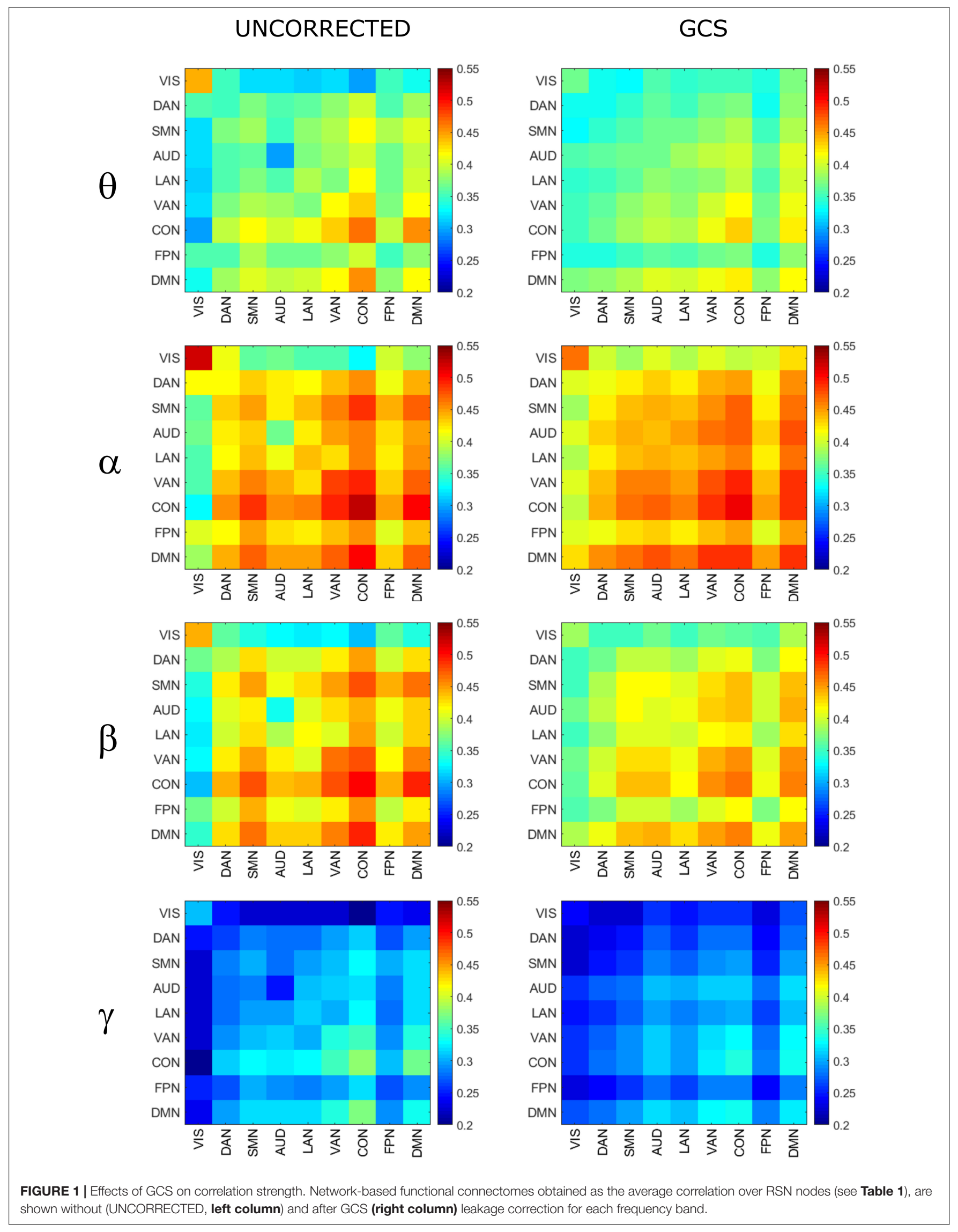


A

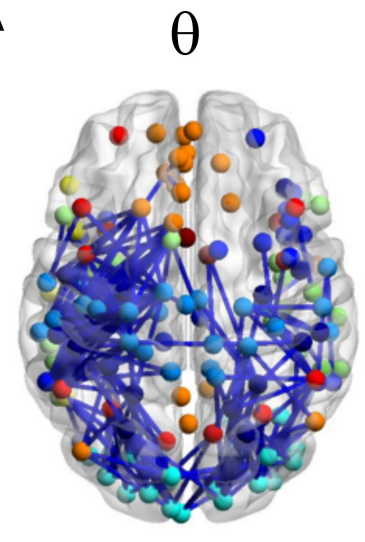

$\alpha$

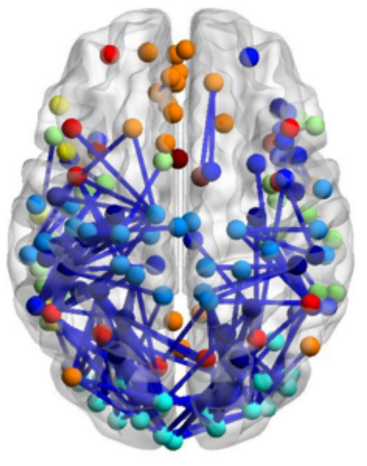

$\beta$

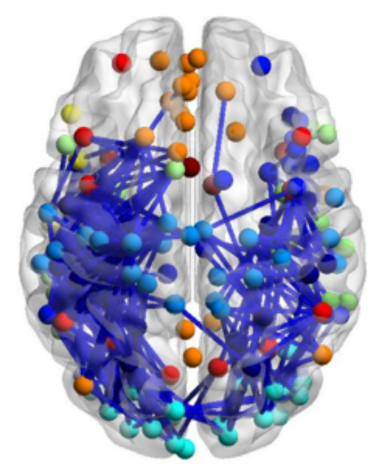

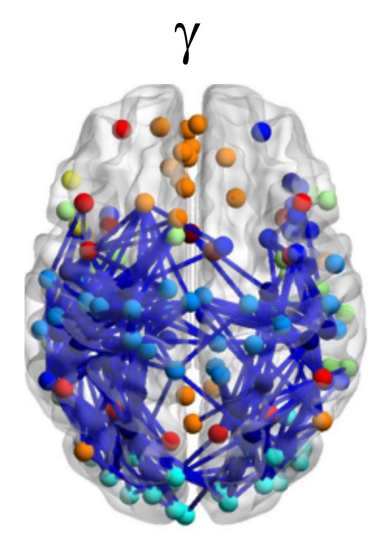

DAN VAN SMN VIS AUD LAN DMN FPN CON

B

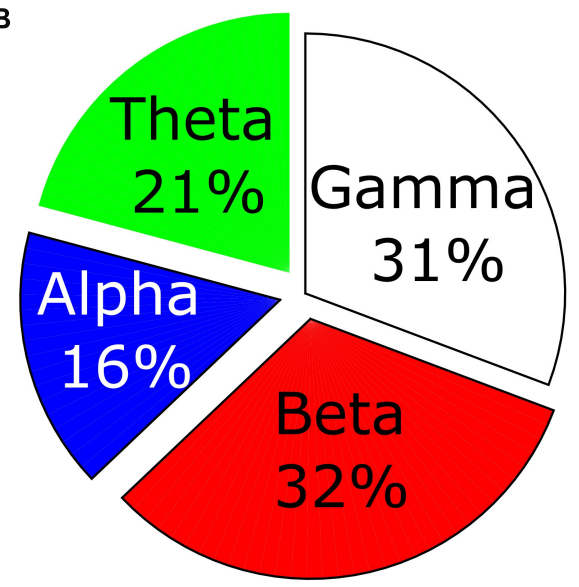

C

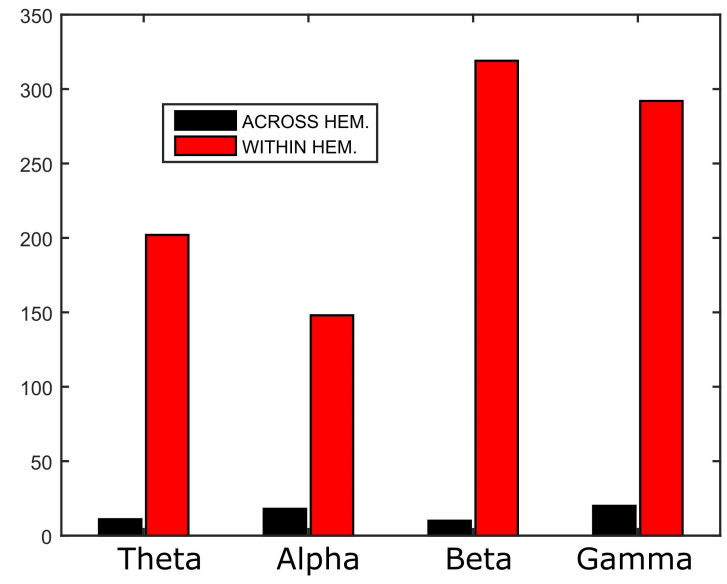

FIGURE 2 | Analysis of global topology: decreased components after GCS. Results of the NBS on uncorrected and corrected connectomes are shown for each frequency band. (A) Decreased components (blue) displayed over an MNI brain. (B) Relative sizes of the decreased components (normalized to the total number of decreased links across bands). (C) Number of links, in the decreased components, changing within (red bars - WITHIN HEM.) and across hemispheres (black bars - ACROSS HEM.).

bands the centrality of uncorrected graphs was inflated by local spurious connections due to spatial leakage. Interestingly, apart from the observed changes, it must also be noted that some hub regions remained central both with and without GCS (see dashed contours in Figure 4). In all bands, medial prefrontal and posterior cingulate/precuneus nodes (except for the theta band) of the DMN remained central after leakage correction. Similarly, we found consistent hubs near/at the Supplementary Motor Area part of the SMN. In contrast, the IPS region, part of the DAN, did not result central after GCS. In summary, the topology of central nodes is partially maintained after the GCS.

Eventually, to understand the effect of the GCS on the global integration, we estimated the GE of communication, which measures the overall efficiency of integration of the considered graph. As it can be seen in Figure 5A, we obtained a statistically significant ( $p<0.01$, Bonferroni corrected) increase of GE in GCS (white bars) vs. uncorrected (black bars) connectomes across all frequency bands. This suggests that the GCS might lead, in general, to a more efficiently integrated connectome as compared to the uncorrected data. Then, since we observed a loss of local links and an increase of long-range ones, to interpret the GE modulation we addressed some further graph measures that might influence the GE. First, we computed the Modularity (Figure 5B) and the number of modules (Figure 5C) that can be identified in the average connectome. As it can be seen in Figure 5B, a general decrease of modularity due to GCS was obtained in all bands ( $p<0.01$, Bonferrroni corrected), while the number of modules did not significantly change in all bands (Figure 5C). These results, taken together, are fundamental since they show that while the number of functional communities remain the same (same number of modules), they are less segregated once leakage corrected (smaller modularity). As a fundamental control on the GE modulation, we considered the density of connections in every band (Figure 5D). This is necessary because GE and density are known to be dependent, i.e., higher density leads to higher GE, and at least in a certain 

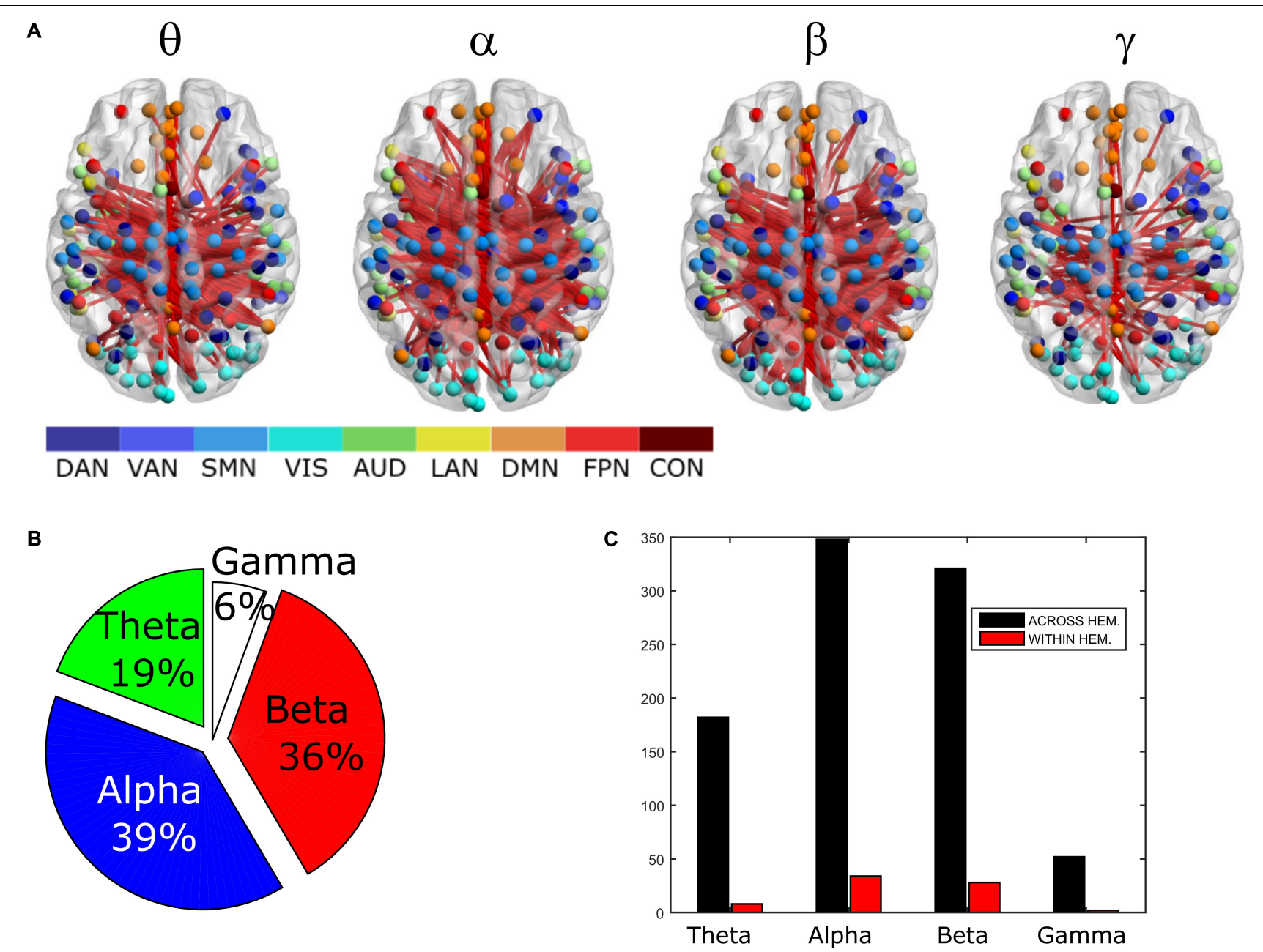

FIGURE 3 | Analysis of global topology: increased components after GCS. Results of the NBS on uncorrected and corrected connectomes at each frequency band. (A) Increased components (red) displayed over an MNI brain. (B) Relative sizes of the increased components (normalized to the total number of increased links across bands). (C) Number of links, in the increased components, changing within (red bars - WITHIN HEM.) and across hemispheres (black bars - ACROSS HEM.).

regime such dependence is linear (Fornito et al., 2016; Strang et al., 2018). Thus, one may suspect that the higher values of GE obtained with GCS are simply driven by higher density. To address this issue, we first statistically tested the densities obtained at the different frequency bands, see Figure 5D. As it can be noted, although on average the density values in GCS are larger than in uncorrected connectomes, a paired $t$-test showed that these are not significantly different (apart from the theta band, $p<0.01$, Bonferroni corrected). This provides a first indication that the GE differences might not be ascribed to the density variations. However, since the obtained density values lie in the range where a linear relationship is expected between GE and density (see Strang et al., 2018), we performed a regression analysis to check whether the density may predict the GE differences. We obtained no significant effects in the alpha and beta bands ( $p<0.01$, Bonferroni corrected). These results suggest that in these bands, the influence of density changes have a minor impact on the observed increase of integration for the GCS.

\section{Global Topology and Integration/Segregation in Orthogonalized vs. Uncorrected Connectomes}

To compare the impact of the GCS approach with current and popular leakage correction schemes, we performed the same analyses on data corrected through the orthogonalization approach described in the Methods section. The orthogonalized RSN-based connectomes are shown in Figure 6, left column. Please note that a scale different from Figure 1 was adopted to appreciate the connectome patterns (connectomes with the same scale of the uncorrected ones are reported in Supplementary Figure S1, left column). Further, to be consistent with the previous comparisons, correlations of node pairs closer than $35 \mathrm{~mm}$ were not considered in these analyses. It can be noted that, differently from the GCS, in this case, the correlation averaged over the whole connectomes considerably decreased in all bands ( $p<0.0002$, Bonferroni corrected). However, when 


\section{UNCORRECTED}

\section{GCS}

$\theta$
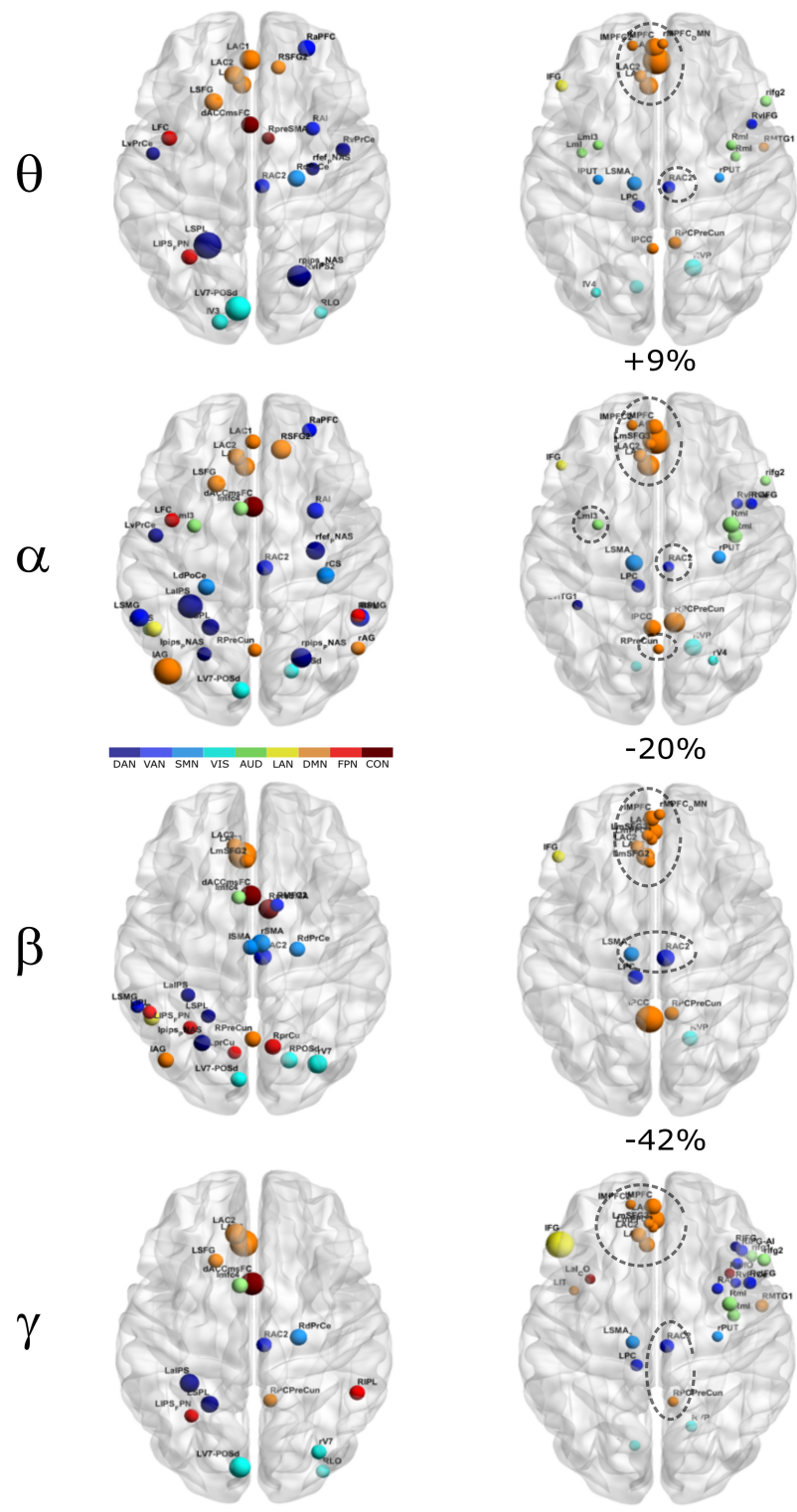
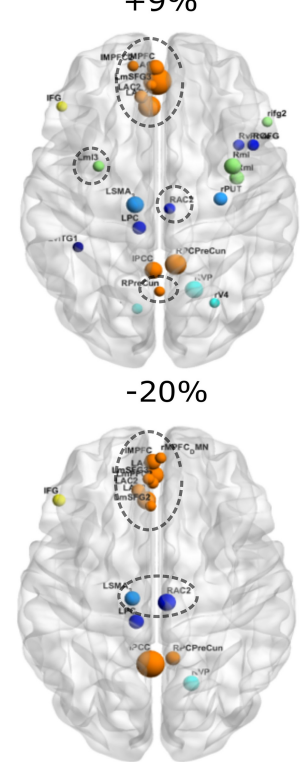

$-42 \%$

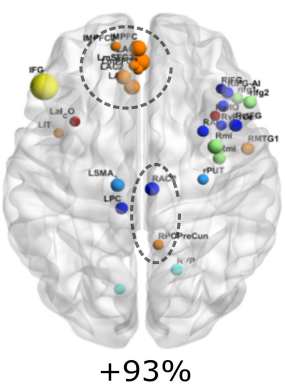

FIGURE 4 | Functional hubs before and after GCS. Functional hubs identified through the $\mathrm{BC}$ are reported at the considered frequency bands without (UNCORRECTED) and with (GCS) leakage correction. A set of hubs belonging to the DMN (orange) and SMN (light blue) are consistently observed (dotted circle) after the leakage correction. For each frequency band, the percentage increase of the number of hubs due to the GCS is reported.

inspecting Figure 6, some patterns in the uncorrected and orthogonalized RSN-based connectomes seem to be preserved: within-VIS correlation seems to be stronger than VIS-other RSNs in all bands, and the relative difference between within-AUD correlation and the averaged correlation seems to be positive, as with the GCS. Accordingly, when comparing orthogonalized and uncorrected dense connectomes, NBS produced only

components representing significant decreases (see Figure 6, middle column and Supplementary Figure S1, right column). If the same t-statistics threshold as for the uncorrected vs. GCS comparison is applied (Supplementary Figure S1), the components sizes are large for all bands and involve all the nodes of the connectomes. Specifically, the percentage of edges (with respect to the total number of decreased edges across all bands) in the decreased components is similarly shared across bands (about 25\% in theta, 28\% in alpha, 26\% in beta, and 21\% in gamma). For display and interpretation purposes, we show the results of NBS statistics using a higher threshold ( $t=10$, middle column in Figure 6) to appreciate possible differences across bands. In this case, the decreased components in alpha and beta bands involved a larger number of nodes (about 130) than in theta and gamma bands (about 100). The percentage of links with respect to the total number of decreased links across all bands reduced by orthogonalization is larger in alpha/beta bands $(28 \%)$ followed by the theta $(22 \%)$ and gamma band (only 16\%). Analogously to the GCS, the majority of links removed by orthogonalization were intra-hemispheric (209 in theta, 243 in alpha, 265 in beta, 101 in gamma), as compared to the inter-hemispheric ones (21 in theta, 33 in alpha, 22 in beta, 58 in gamma).

Moreover, the $\mathrm{BC}$ analysis on orthogonalized connectomes (see Figure 6, right column) revealed an increase in the number of hubs with respect to the uncorrected connectomes in the theta and gamma bands, and a decrease in the alpha band, as occurred for GCS-based connectomes (+32\%, $+67 \%$, and $-33 \%$ respectively). However, differently from GCS, the number of hubs slightly increased in the beta band $(+12 \%)$. Hubs in the medial prefrontal (in all bands) and posterior cingulate/precuneus nodes (in the beta and gamma bands) of the DMN, together with hubs near/at the Supplementary Motor Area part of the SMN (in the beta band) remained central, as with the GCS (Figure 6, contoured nodes, left column and Figure 4, left column). In addition, hubs in the IPS regions of the DAN and FPN (in all bands), and in the AG (DMN, alpha and beta bands), were found in both the uncorrected and orthogonalized connectomes. These results suggest that for orthogonalized matrices, a partial overlap with uncorrected data on the hub topography can be revealed. Interestingly, some of these hubs overlap also with those obtained after applying the GCS.

As far as it regards the integration analyses, the effects of orthogonalization were slightly different from the ones obtained for GCS (see Figure 5). Interestingly, in the orthogonalized data (ORT) no statistically significant increases in the GE were observed (Figure 5A, gray bars). Nevertheless, a significant decrease of modularity was observed across all bands $(p<0.01$ Bonferroni corrected, Figure 5B), while the number of modules did not change, as with GCS (Figure 5C). The density of connections, although on average larger than in the uncorrected connectomes, reached statistical significance only in the theta and gamma bands $(p<0.01$, Bonferroni corrected). In summary, the orthogonalization seems not to affect the global integration as measured via GE. 
A

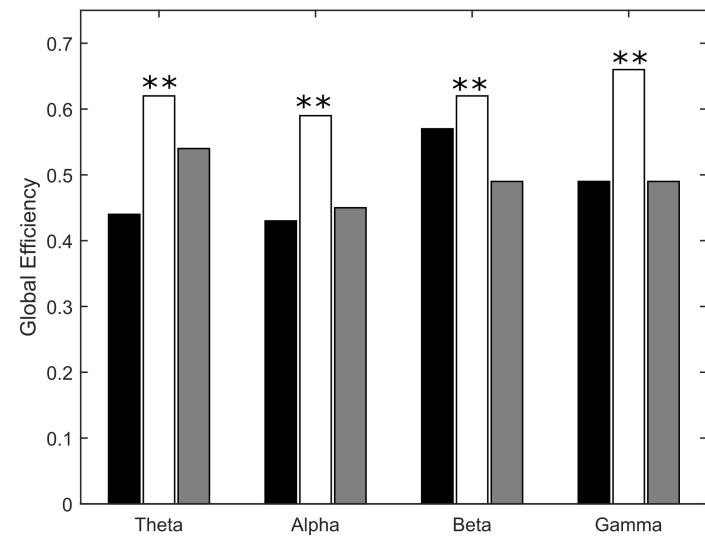

C

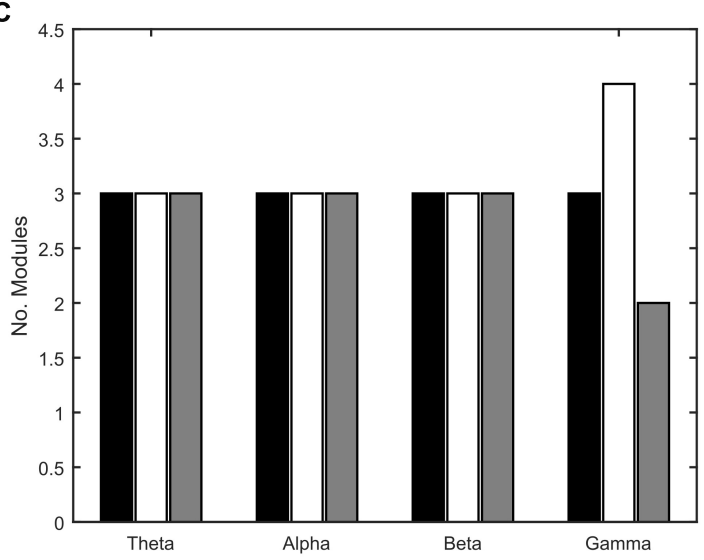

B

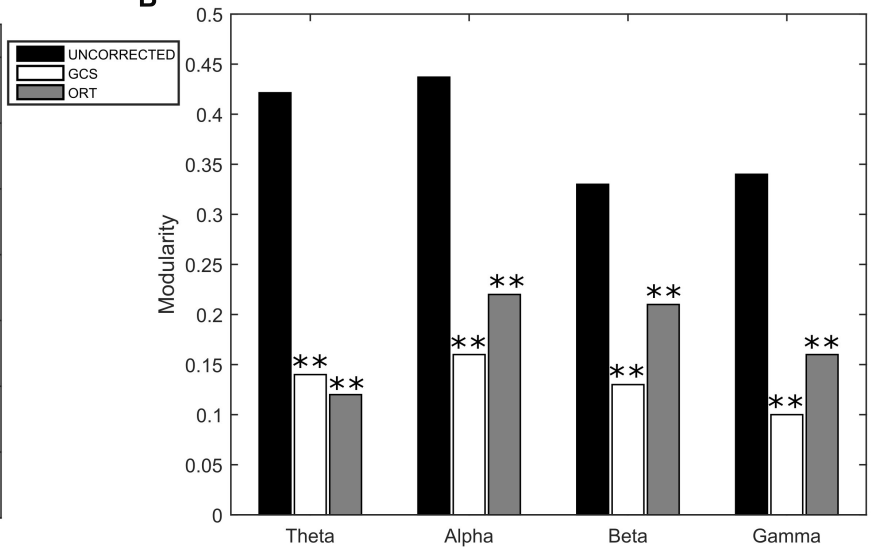

D

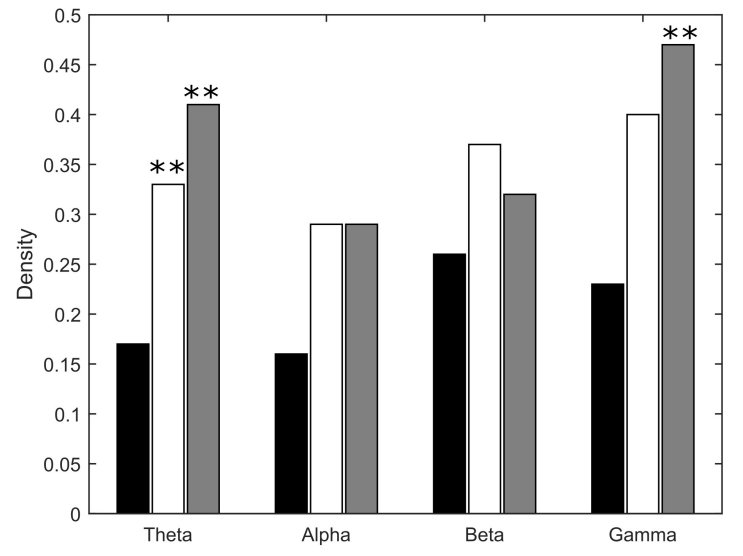

FIGURE 5 | Effect of GCS and ORT on the global integration. (A) Mean Global Efficiency (GE) values computed before (black bars - UNCORRECTED) and after leakage correction (GCS- white bars, ORT - gray bars). A statistically significant difference was found in each frequency band (** $p<0.01$, Bonferroni corrected) for the GCS corrected data. The GCS seems to increase the efficiency of communication in the brain. This does not apply to the ORT correction where no statistically significant difference was found. (B) Mean modularity as a function of the frequency band. All the differences were statistically significant both for GCS and ORT $\left({ }^{* *} p<0.01\right)$. The decrease of modularity shows that, after GCS and ORT, the segregation of the functional communities is lower. (C) The average number of modules is constant in all frequency bands except for gamma, despite the t-statistics is not significant. (D) No significant increase of the average density of connections is observed in general, apart from the theta band for GCS and theta and gamma for ORT (** $p<0.01$ ).

\section{DISCUSSION}

In this work, we analyzed the impact of the GCS in minimizing the effect of MEG spatial leakage on the topology of connectivity at rest. We compared the effect of GCS with a popular orthogonalization approach (Brookes et al., 2012). We focused on integration/segregation measures observing several interesting spatially- and frequency-specific aspects. First, we observed that the GCS significantly modified the overall topology: the connectivity decreased within each hemisphere, mainly in the gamma and beta bands, and increased across hemispheres, especially in the alpha and beta bands. On the other hand, orthogonalization only produced a decreased connectivity in every band, mainly involving intra-hemispheric links, as for GCS.

Second, in terms of BC, a set of hubs in the medial frontal and parietal areas, especially in the DMN and SMN, survived both leakage corrections, while more lateral regions (IPS) were preserved by orthogonalization but not by the GCS.
Third, after GCS, we observed an increase of GE across all bands. This change occurred with a significant decrease in the Modularity corresponding to a constant number of modules, hence supporting a stronger integration among functional communities. The modulations of GE and Modularity, after GCS, could not be explained by a significant decrease in connection density (apart from the theta band).

\section{Impact of the Geometric Correction Scheme on the Overall Connectivity}

The analysis performed by means of NBS on the overall connectivity structure showed that, despite the mean strength of GCS-corrected correlation was not significantly modified by leakage correction, a profound alteration of the topology was observed in all bands (Figures 2, 3), suggesting that uncorrected connectomes should be interpreted with care. In general, the first topological change consisted of a massive 


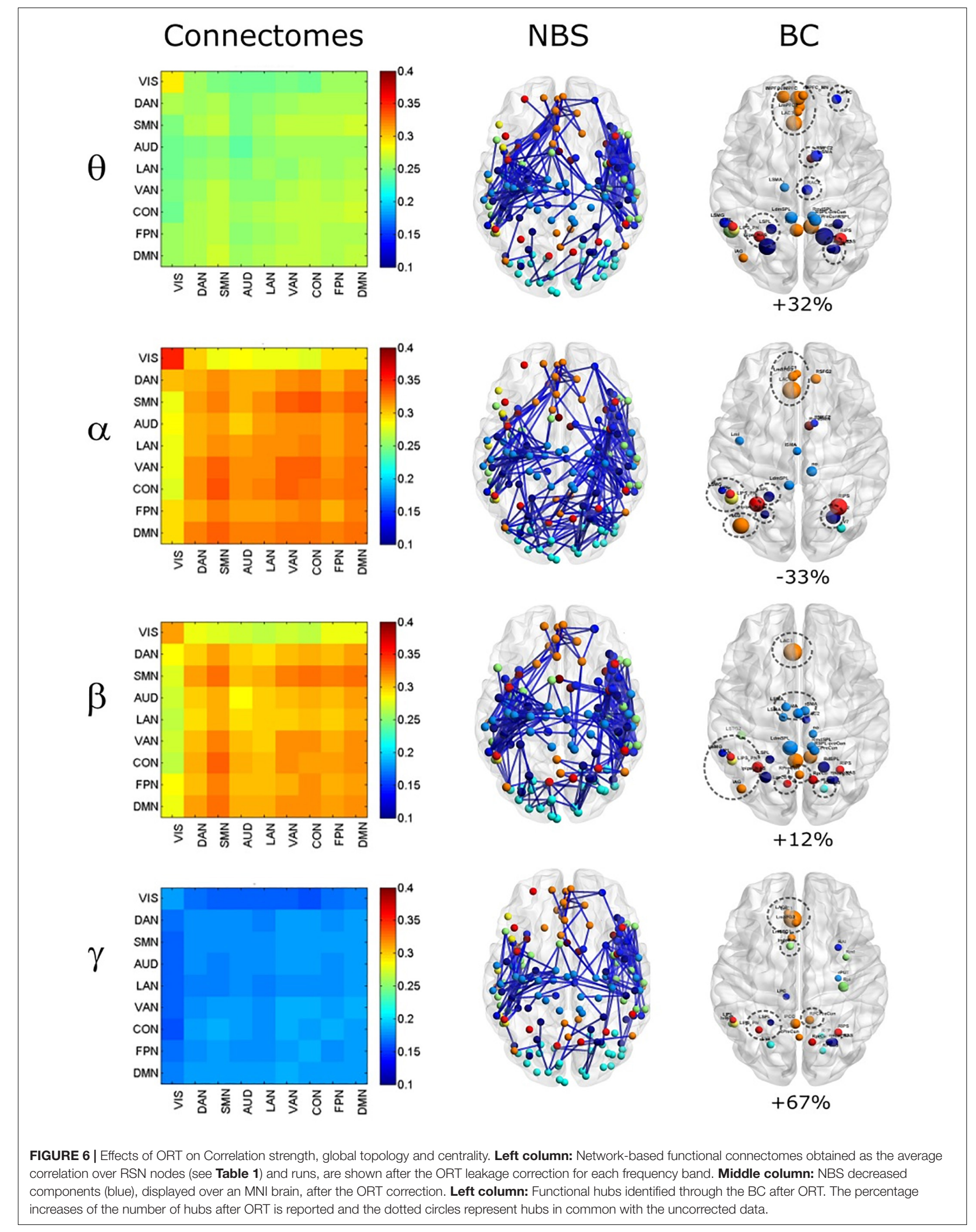


decrease of within-hemisphere connections among neighboring nodes (see Figure 2). This local effect is not surprising, since the leakage effect mainly consists of the influence of one source on its neighbors, due to the spatial spread of the reconstructed sources (Hauk et al., 2011; Wens, 2015; Wens et al., 2015) and their mis-localization. The edges involved in the decreased component were spatially located mainly in the parieto-occipital regions. Although leakage effects have different spatial distribution according to the location of the seed in the brain, the larger involvement of the parieto-occipital regions might simply reflect the higher density of the parcellation scheme in those areas. We also found that the size of the decreased components was spectrally specific, with larger components in the beta and gamma bands (Figure 2). It must be noted that although the spatial leakage itself is not frequency-dependent (Brookes et al., 2014; Wens et al., 2015) - a property that is built in the GCS - the induced effect on connectivity does depend on the frequency-specific signal-to-noise ratio (SNR) (O’Neill et al., 2015). Lower SNRs such as those expected in the theta and gamma bands tend to induce sharper local spurious connections. This may explain the larger component sizes found in beta, gamma, and theta as compared to alpha (which exhibits the largest SNR in MEG). Another possible explanation for the stronger post-GCS decreases in the beta and gamma bands could be a mitigation of the seed mislocation which affects the GCS (Wens et al., 2015). Specifically, seed mis-location, i.e., the position of the actual seed does not overlap with a true seed, generates spurious connectivity corresponding to a smaller loss of edges after GCS. Given that local synchronization at high frequencies is stronger, as reported in the influential work of Buzsaki and Draguhn (2004), the effect of mis-location is mitigated as there are many interacting seeds very close to each other.

Correspondingly, orthogonalization also led to massive connectivity decreases mainly involving intra-hemispheric links (Figure 6). However, this correction affected more links in the alpha/beta band, which exhibit high SNR, then in the theta and gamma bands, where SNR is lower. This might reflect an effect of SNR in the estimation of the regression coefficients, which could be biased by physiological bands with the larger power (i.e., alpha and beta). As such, orthogonalization tends to produce milder effects when the SNR is low. Alternatively, this difference could be ascribed to the existence of 0-lag interactions, which are preserved by the GCS but cancelled by orthogonalization. This is in line with the dominance of alpha/beta bands regarding connectivity increases post-GCS, that we discuss in the following.

The GCS yielded an increased component involving mainly edges connecting parietal nodes between hemispheres and edges connecting occipital and frontal nodes (Figure 3). An increase of connectivity was rather unexpected as leakage correction generically leads to lower connectivity due to the elimination of spurious couplings. However, some increased interhemispheric connectivity after leakage correction has already been reported when using orthogonalization or closely-related regressions (Brookes et al., 2012, 2014; Hipp et al., 2012; Maldjian et al., 2014; Wens et al., 2015), but they are milder and may be explained by an over-correction effect (Wens, 2015). Accordingly, in our data no such increases were detected with orthogonalization. Here, we report a global, not network-specific, increase of interhemispheric connectivity after GCS, suggesting that spatial leakage might screen some genuine, sufficiently long-range connectivity. Since a major distinction between the GCS and orthogonalization is the preservation of 0-lag interactions, this seems to suggest that this rather counter-intuitive effect relates to inter-hemispheric, short-lag correlations. In fact, we demonstrate mathematically in the Appendix that such screening emerges in the presence of linear correlation, which enables a non-linear contribution of spatial leakage to BLP correlations (despite its linearity in source activity). When the spatial leakage is strong, i.e., for local connections, it induces a spurious increase of short-range connectivity. This is in line with the results showing that both leakage corrections clean local connections mainly (Figures 2, 6). When spatial leakage is weaker, i.e., for longer-range connectivity, it may lead, through non-linear effects induced by 0-lag correlation, to a spurious reduction of connectivity. This fits with our results in Figure 3. As a matter of fact, in the uncorrected connectomes, the local connections composing the decreased components shown in Figure 2 were always significantly stronger than the long-range connections comprised in the increased components in Figure 3 (separate $t$-tests for each band, $p<10^{-5}$ for all bands).

Spectrally, as mentioned above, we noted a larger size of the increased component mainly in the alpha and beta bands which have been reported as the most consistent spectral signatures of fMRI RSNs (however, see Liu et al. (2017) showing that only whole band EEG RSNs match the spatial patterns of fMRI RSNs). These systems in fact cover mainly occipital, parietal and temporal regions and involve long-range interhemispheric or antero-posterior connections (Mantini et al., 2007; de Pasquale et al., 2010, 2012; Brookes et al., 2011; Hipp et al., 2012). Specifically, the alpha band was associated to the VIS, DAN, DMN, SMN, AUD in an EEG-fMRI study (Mantini et al., 2007); to SMN and DAN in an ECoG study (Hacker et al., 2017); and to VIS, VAN, LAN, SMN, DMN in MEG studies (Brookes et al., 2011, 2014; de Pasquale et al., 2012). Analogously, the beta characterized the CON in Mantini et al. (2007); the SMN, DMN, DAN, VIS, VAN, LAN, FPN in MEG studies (Brookes et al., 2011, 2014; de Pasquale et al., 2012). Eventually, a spatial concordance of these RSNs over a whole larger band was found also in Hipp and Siegel (2015). Interestingly, an increase of interhemispheric interactions in the alpha band in the VIS and in the beta band in the SMN was also observed after symmetrical orthogonalization in Colclough et al. (2015). In this work, we used a quite different pipeline (different inverse solver, keeping all components of source activity, denser connectome but based on pointwise source estimates, and the GCS for leakage correction) and extended Colclough's findings identifying more extensive increases. This is possibly also in line with our mathematical description of the connectivity screening effect: symmetrical orthogonalization does not enforce strict pairwise orthogonalization but is rather based on a multivariate optimization, so some zero-lag correlations may survive and lead to connectivity increases post-correction as per our Appendix. 


\section{Functional Hubs}

In this work, the comparison between hubs identified with and without the leakage correction was based on the BC measure and the constraint of full connectedness of the investigated connectomes. We obtained that a set of regions survived the correction while others were 'canceled', suggesting that they probably were spurious byproducts of spatial leakage. On the other hand, other regions resulted central after the correction (see Figures 4, 6). In fact, if the number of hubs slightly increased in the theta band and almost doubled in the gamma band, they decreased in the alpha. In the beta band, the number of hubs decreased with GCS and slightly increased with orthogonalization. Hubs that did not replicate with GCS were those in the IPS region, part of the DAN; while new hub regions were identified in right prefrontal cortex part of the Auditory and Ventral Attention Networks in the theta and alpha bands. As it can be seen in Figure 4, a set of interesting nodes survived the GCS and these areas comprised nodes from the DMN (orange) and SMN (blue). Apart from the specific nodes involved, the frontal areas of the DMN were consistent across frequency bands while the parietal nodes were mainly observed in the alpha and beta bands with some involvement of the gamma band. The preservation of hubs in the DMN and SMN in all bands was confirmed also after orthogonalization (Figure 6), which also maintained AG in the DMN and parietal nodes in the DAN and FPN, and introduced new hubs in the SPL-PreCun region.

The consistency of hubs observed across GCS and orthogonalization approaches nicely fit with previous MEG findings observed in de Pasquale et al. (2010, 2016, 2017, 2018) as well as fMRI findings (de Pasquale et al., 2013, 2017). Notably, at least for the parietal nodes of the DMN and SMN, our results extend the findings of Marino et al. (2019), in that the DMN hubs are mainly found from theta to beta bands, but at a lesser extent in the gamma bands, while the SMN hubs are also found in the gamma band, reflecting a balance between low- and high-frequency oscillations in the Cognitive (as the DMN) and the Perceptual (as the SMN) networks. The parietal regions of the DMN and, specifically, the Posterior Cingulate cortex/Precuneus have been shown to play a fundamental role of integration across several RSNs in the alpha and beta bands. Notably, these observations are in line with the work of Maldjian et al. (2014) where, in the leakage corrected MEG data, the DMN topography closely resembled the fMRI one and functional hubs were consistently observed in the posterior cingulate and bilateral parietal areas of this network. These results are interesting since they suggest that the centrality of these regions was not dominated by the leakage effects.

Analogously, SMN nodes like RAC2 (all bands for GCS, theta and beta for orthogonalization) and SMA (mainly in beta band for GCS and theta, alpha and beta for orthogonalization) were consistently observed both with and without leakage correction. These nodes have been previously reported as functional hubs of the SomatoMotor Network but more importantly these nodes together with DMN nodes seem to form a fundamental axis of integration related to the interplay between the internal vs external cognition (see for example de Pasquale et al., 2013) and lately part of a dynamic core network of integration (de Pasquale et al., 2016).

Finally, it is not unexpected that some differences between hub topographies was found when comparing the two leakage correction approaches. These differences could be partially ascribed to the contribution of the interhemispheric edges that increase with GCS but are not modified by orthogonalization. However, since the ground truth is unknown, fully proving this claim would require turning to simulated data and inspect the impact of the leakage correction methods on the estimated connectomes. This would require developing largescale simulations of the brain connectome wherein topological features such as BC (and GE, as discussed below) are controlled, to compare estimated connectomes with known ground truth regarding network topology. Instead, we used here orthogonalization as a control to contrast with the GCS. In fact, Wens et al. (2015) presented small-scale but controlled network simulations that revealed two major differences: (i) orthogonalization suppresses linear, 0-lag correlations while GCS preserves it, and (ii) orthogonalization is more resilient than the GCS to local correction errors due to seed mislocation. Further, the simulations in Wens et al. (2015) showed that these local correction errors are confined to node pairs closer than $3 \mathrm{~cm}$. Our use of a $35 \mathrm{~mm}$ mask thus mitigated this difference. In this setup, it is thus reasonable to expect that the preservation/cancelation of 0-lag correlations represent the only distinction between the two correction methods. As suggested by our mathematical model of the leakage "screening" effect (see Appendix), we expect our detection of post-GCS connectivity increases to be a consequence of that distinction. That said, full proof of this statement would require the abovementioned large scale simulations of connectome topology, which thus represents an interesting avenue for future work. Until then, we suggest that both leakage corrections should be used to provide a more comprehensive overview on MEG functional architecture.

\section{Global Efficiency}

The strong interaction realized by the functional axis DMNSMN through the involvement of the hubs in the cingulate cortex has been shown to relate to a global optimization criterion (de Pasquale et al., 2016). Such topology seems to optimize the efficiency of information transfer as measured via the global efficiency. Now, since we confirmed the presence of these hub regions with leakage correction, it would be interesting to see their impact on these global measures. In fact, the effect of reducing connections (Figures 2, 6) might induce a decrease of the global integration. As it can be seen in Figure 5A this does not seem to be case: we observed a significant increase of GE in the alpha and beta bands after GCS (but not after orthogonalization). These results suggest that the spurious connections removed by GCS and orthogonalization from the connectomes were not serving the mentioned optimal criterion. On the other 
hand, the increase of interhemispheric links following GCS seems to promote the integration across the brain components. This result must be interpreted also considering the decrease of Modularity (Figure 5B) and the fact that the number of modules remained constant (see Figure 5C). Thus, in the leakage-corrected connectomes, it is more difficult to identify segregated communities (higher modularity) but not because the number of modules increased leading to more fragmented communities. In fact, the number of modules is constant, thus the same communities are, without the spurious contributions of spatial leakage, less segregated and more integrated, in the case of GCS. More generally, the topological effect of spatial leakage to spuriously increase of local connectivity explains its negative impact on optimal integration and modularity of the brain. Interestingly, we show in the Appendix that using orthogonalization, instead of the GCS, might tend to mitigate the detection of higher integration. This is confirmed by our results reported in Figure $\mathbf{5 A}$ where no significant changes in the GE were observed after orthogonalization.

\section{CONCLUSION}

We showed that a proper leakage correction is necessary to study MEG functional topology and reinforces the findings that the brain functioning at rest relies on a topologically optimal local and global integration principle.

\section{DATA AVAILABILITY STATEMENT}

The datasets generated for this study are available on request to the corresponding author.

\section{REFERENCES}

Attwell, D., and Laughlin, S. B. (2001). An energy budget for signaling in the grey matter of the brain. J. Cereb. Blood Flow Metab. 21, 1133-1145. doi: 10.1097/ 00004647-200110000-1

Baker, A. P., Brookes, M. J., Rezek, I. A., Smith, S. M., Behrens, T., Probert Smith, P. J., et al. (2014). Fast transient networks in spontaneous human brain activity. eLife 3:e01867. doi: 10.7554/eLife.01867

Baldassarre, A., Ramsey, L., Hacker, C. L., Callejas, A., Astafiev, S. V., Metcalf, N. V., et al. (2014). Large-scale changes in network interactions as a physiological signature of spatial neglect. Brain 137(Pt 12), 3267-3283. doi: 10.1093/brain/ awu297

Betti, V., Corbetta, M., de Pasquale, F., Wens, V., and Della Penna, S. (2018). Topology of functional connectivity and hub dynamics in the beta band as temporal prior for natural vision in the human brain. J. Neurosci. 38, 3858-3871. doi: 10.1523/JNEUROSCI.1089-17.2018

Biswal, B., Yetkin, F. Z., Haughton, V. M., and Hyde, J. S. (1995). Functional connectivity in the motor cortex of resting human brain using echo-planar MRI. Magn. Reson. Med. 34, 537-541. doi: 10.1002/mrm.1910340409

Blondel, V. D., Guillaume, J. L., Lambiotte, R., and Lefebvre, E. (2008). Fast unfolding of communities in large networks. J. Stat. Mech. 10, 1008-1020.

Bordier, C., Nicolini, C., and Bifone, A. (2017). Graph analysis and modularity of brain functional connectivity networks: searching for the optimal threshold. Front. Neurosci. 11:441. doi: 10.3389/fnins.2017.00441

\section{ETHICS STATEMENT}

This study was carried out in accordance with the recommendations of "Code of Ethics of the World Medical Association, and the Institutional Review Board and Ethics Committee at the University of Chieti" with written informed consent from all subjects. All subjects gave written informed consent in accordance with the Declaration of Helsinki. The protocol was approved by the Ethics Committee at the University of Chieti.

\section{AUTHOR CONTRIBUTIONS}

$\mathrm{SD}, \mathrm{MC}, \mathrm{VW}$, and FP designed the research, interpreted the results, and wrote the manuscript. SD and FP performed the research and analyzed the data. SD, VW, and FP contributed analytic tools.

\section{FUNDING}

VW acknowledges financial support from the Fonds Erasme for MEG research at CUB Hôpital Erasme (research convention "Les Voies du Savoir," Fonds Erasme, Brussels, Belgium).

\section{SUPPLEMENTARY MATERIAL}

The Supplementary Material for this article can be found online at: https://www.frontiersin.org/articles/10.3389/fnins. 2019.01114/full\#supplementary-material

FIGURE S1 | Effects of ORT on correlation strength (left column) with the same scale as Figure $\mathbf{1}$ and on global topology (right column) with the same t-threshold as Figure 2A.

Brookes, M., Woolrich, M., Luckhoo, H., Price, D., Hale, J. R., Stephenson, M. C., et al. (2011). Investigating the electrophysiological basis of resting state networks using magnetoencephalography. Proc. Natl. Acad. Sci. U.S.A. 108, 16783-16788. doi: 10.1073/pnas.1112685108

Brookes, M. J., O’Neill, G. C., Hall, E. L., Woolrich, M. W., Baker, A., Palazzo Corner, S., et al. (2014). Measuring temporal, spectral and spatial changes in electrophysiological brain network connectivity. Neuroimage 91, 282-299. doi: 10.1016/j.neuroimage.2013.12.066

Brookes, M. J., Woolrich, M. W., and Barnes, G. R. (2012). Measuring functional connectivity in MEG: a multivariate approach insensitive to linear source leakage. Neuroimage 63, 910-920. doi: 10.1016/j.neuroimage.2012. 03.048

Buzsaki, G., and Draguhn, A. (2004). Neuronal oscillations in cortical networks. Science 304, 1926-1929. doi: 10.1126/science.1099745304/5679/ 1926

Colclough, G. L., Brookes, M. J., Smith, S. M., and Woolrich, M. W. (2015). A symmetric multivariate leakage correction for MEG connectomes. Neuroimage 117, 439-448. doi: 10.1016/j.neuroimage.2015.03.071

de Pasquale, F., Corbetta, M., Betti, V., and Della Penna, S. (2018). Cortical cores in network dynamics. Neuroimage 180(Pt B), 370-382. doi: 10.1016/j.neuroimage. 2017.09.063

de Pasquale, F., Della Penna, S., Sabatini, U., Caravasso Falletta, C., and Peran, P. (2017). The anatomical scaffold underlying the functional centrality of known cortical hubs. Hum. Brain Mapp. 38, 5141-5160. doi: 10.1002/hbm.23721 
de Pasquale, F., Della Penna, S., Snyder, A. Z., Lewis, C., Mantini, D., Marzetti, L., et al. (2010). Temporal dynamics of spontaneous MEG activity in brain networks. Proc. Natl. Acad. Sci. U.S.A. 107, 6040-6045. doi: 10.1073/pnas. 0913863107

de Pasquale, F., Della Penna, S., Snyder, A. Z., Marzetti, L., Pizzella, V., Romani, G. L., et al. (2012). A cortical core for dynamic integration of functional networks in the resting human brain. Neuron 74, 753-764. doi: 10.1016/j. neuron.2012.03.031

de Pasquale, F., Della Penna, S., Sporns, O., Romani, G. L., and Corbetta, M. (2016). A dynamic core network and global efficiency in the resting human brain. Cereb. Cortex 26, 4015-4033. doi: 10.1093/cercor/bhv185

de Pasquale, F., Sabatini, U., Della Penna, S., Sestieri, C., Caravasso, C., Formisano, R., et al. (2013). The connectivity of functional cores reveals different degrees of segregation and integration in the brain at rest. Neuroimage 69, 51-61. doi: 10.1016/j.neuroimage.2012.11.051

Della Penna, S., Del Gratta, C., Granata, C., Pasquarelli, A., Pizzella, V., Rossi, R., et al. (2000). Biomagnetic systems for clinical use. Philos. Mag. B 80, 937-948. doi: 10.1080/014186300254899

Doucet, G., Naveau, M., Petit, L., Delcroix, N., Zago, L., Crivello, F., et al. (2011). Brain activity at rest: a multiscale hierarchical functional organization. J. Neurophysiol. 105, 2753-2763. doi: 10.1152/jn.00895.2010

Fornito, A., Zalesky, A., and Bullmore, E. (2016). Fundamentals of Brain Network Analysis. Cambridge, MA: Academic Press.

Fox, M. D., Snyder, A. Z., Vincent, J. L., Corbetta, M., Van Essen, D. C., and Raichle, M. E. (2005). The human brain is intrinsically organized into dynamic, anticorrelated functional networks. Proc. Natl. Acad. Sci. U.S.A. 102, 9673-9678. doi: $10.1073 /$ pnas.0504136102

Glasser, M. F., Coalson, T. S., Robinson, E. C., Hacker, C. D., Harwell, J., Yacoub, E., et al. (2016). A multi-modal parcellation of human cerebral cortex. Nature 536, 171-178. doi: 10.1038/nature18933

Gollo, L. L., Mirasso, C., Sporns, O., and Breakspear, M. (2014). Mechanisms of zero-lag synchronization in cortical motifs. PLoS Comput. Biol. 10:e1003548. doi: 10.1371/journal.pcbi.1003548

Hacker, C. D., Laumann, T. O., Szrama, N. P., Baldassarre, A., Snyder, A. Z., Leuthardt, E. C., et al. (2013). Resting state network estimation in individual subjects. Neuroimage 82, 616-633. doi: 10.1016/j.neuroimage.2013. 05.108

Hacker, C. D., Snyder, A. Z., Pahwa, M., Corbetta, M., and Leuthardt, E. C. (2017). Frequency-specific electrophysiologic correlates of resting state fMRI networks. Neuroimage 149, 446-457. doi: 10.1016/j.neuroimage.2017. 01.054

Hamalainen, M. S., and Ilmoniemi, R. J. (1994). Interpreting magnetic fields of the brain: minimum norm estimates. Med. Biol. Eng. Comput. 32, 35-42. doi: $10.1007 / \mathrm{bf} 02512476$

Hauk, O., and Stenroos, M. (2014). A framework for the design of flexible cross-talk functions for spatial filtering of EEG/MEG data: DeFleCT. Hum. Brain Mapp. 35, 1642-1653. doi: 10.1002/hbm.22279

Hauk, O., Wakeman, D. G., and Henson, R. (2011). Comparison of noisenormalized minimum norm estimates for MEG analysis using multiple resolution metrics. Neuroimage 54, 1966-1974. doi: 10.1016/j.neuroimage.2010. 09.053

Hillebrand, A., Barnes, G. R., Bosboom, J. L., Berendse, H. W., and Stam, C. J. (2012). Frequency-dependent functional connectivity within restingstate networks: an atlas-based MEG beamformer solution. Neuroimage 59, 3909-3921. doi: 10.1016/j.neuroimage.2011.11.005

Hillebrand, A., Singh, K. D., Holliday, I. E., Furlong, P. L., and Barnes, G. R. (2005). A new approach to neuroimaging with magnetoencephalography. Hum. Brain Mapp. 25, 199-211. doi: 10.1002/hbm.20102

Hipp, J. F., Hawellek, D. J., Corbetta, M., Siegel, M., and Engel, A. K. (2012). Large-scale cortical correlation structure of spontaneous oscillatory activity. Nat. Neurosci. 15, 884-890. doi: 10.1038/nn.3101

Hipp, J. F., and Siegel, M. (2015). BOLD fMRI correlation reflects frequencyspecific neuronal correlation. Curr. Biol. 25, 1368-1374. doi: 10.1016/j.cub. 2015.03.049

Larson-Prior, L. J., Oostenveld, R., Della Penna, S., Michalareas, G., Prior, F., Babajani-Feremi, A., et al. (2013). Adding dynamics to the human connectome project with MEG. Neuroimage 80, 190-201. doi: 10.1016/j.neuroimage.2013. 05.056
Liu, Q., Farahibozorg, S., Porcaro, C., Wenderoth, N., and Mantini, D. (2017). Detecting large-scale networks in the human brain using high-density electroencephalography. Hum. Brain Mapp. 38, 4631-4643. doi: 10.1002/hbm. 23688

Maldjian, J. A., Davenport, E. M., and Whitlow, C. T. (2014). Graph theoretical analysis of resting-state MEG data: identifying interhemispheric connectivity and the default mode. Neuroimage 96, 88-94. doi: 10.1016/j.neuroimage.2014. 03.065

Mantini, D., Della Penna, S., Marzetti, L., de Pasquale, F., Pizzella, V., Corbetta, M., et al. (2011). A signal-processing pipeline for magnetoencephalography resting-state networks. Brain Connect. 1, 49-59. doi: 10.1089/brain.2011.0001

Mantini, D., Franciotti, R., Romani, G. L., and Pizzella, V. (2008). Improving MEG source localizations: an automated method for complete artifact removal based on independent component analysis. Neuroimage 40, 160-173. doi: 10.1016/j. neuroimage.2007.11.022

Mantini, D., Perrucci, M. G., Del Gratta, C., Romani, G. L., and Corbetta, M. (2007). Electrophysiological signatures of resting state networks in the human brain. Proc. Natl. Acad. Sci. U.S.A. 104, 13170-13175. doi: 10.1073/pnas.070066 8104

Marino, M., Liu, Q., Samogin, J., Tecchio, F., Cottone, C., Mantini, D., et al. (2019). Neuronal dynamics enable the functional differentiation of resting state networks in the human brain. Hum. Brain Mapp. 40, 1445-1457. doi: 10.1002/ hbm. 24458

Marzetti, L., Della Penna, S., Snyder, A. Z., Pizzella, V., Nolte, G., de Pasquale, F., et al. (2013). Frequency specific interactions of MEG resting state activity within and across brain networks as revealed by the multivariate interaction measure. Neuroimage 79, 172-183. doi: 10.1016/j.neuroimage.2013. 04.062

Nolte, G., Bai, O., Wheaton, L., Mari, Z., Vorbach, S., and Hallett, M. (2004). Identifying true brain interaction from EEG data using the imaginary part of coherency. Clin. Neurophysiol. 115, 2292-2307. doi: 10.1016/j.clinph.2004. 04.029

O’Neill, G. C., Bauer, M., Woolrich, M. W., Morris, P. G., Barnes, G. R., and Brookes, M. J. (2015). Dynamic recruitment of resting state sub-networks. Neuroimage 115, 85-95. doi: 10.1016/j.neuroimage.2015.04.030

Palva, J. M., Wang, S. H., Palva, S., Zhigalov, A., Monto, S., Brookes, M. J., et al. (2018). Ghost interactions in MEG/EEG source space: a note of caution on inter-areal coupling measures. Neuroimage 173, 632-643. doi: 10.1016/j. neuroimage.2018.02.032

Power, J. D., Schlaggar, B. L., Lessov-Schlaggar, C. N., and Petersen, S. E. (2013). Evidence for hubs in human functional brain networks. Neuron 79, 798-813. doi: 10.1016/j.neuron.2013.07.035

Roelfsema, P. R., Engel, A. K., Konig, P., and Singer, W. (1997). Visuomotor integration is associated with zero time-lag synchronization among cortical areas. Nature 385, 157-161. doi: 10.1038/385157a0

Rubinov, M., and Sporns, O. (2010). Complex network measures of brain connectivity: uses and interpretations. NeuroImage 52, 1059-1069. doi: 10.1016/j.neuroimage.2009.10.003

Rubinov, M., and Sporns, O. (2011). Weight-conserving characterization of complex functional brain networks. Neuroimage 56, 2068-2079. doi: 10.1016/ j.neuroimage.2011.03.069

Singer, W. (1999). Neuronal synchrony: a versatile code for the definition of relations? Neuron 24, 49-65, 111-125.

Sjogard, M., De Tiege, X., Mary, A., Peigneux, P., Goldman, S., Nagels, G., et al. (2019). Do the posterior midline cortices belong to the electrophysiological default-mode network? Neuroimage 200, 221-230. doi: 10.1016/j.neuroimage. 2019.06.052

Sporns, O. (2011). Networks of the Brain. Cambridge: The MIT Press.

Stam, C. J., and Reijneveld, J. C. (2007). Graph theoretical analysis of complex networks in the brain. Nonlinear Biomed. Phys. 1:3. doi: 10.1186/1753-4631$1-3$

Strang, A., Haynes, O., Cahill, N. D., and Narayan, D. A. (2018). Generalized relationships between characteristic path length, efficiency, clustering coefficients, and density. Soc. Netw. Anal. Min. 8:14. doi: 10.1007/s13278-018-0492-3

Uhlhaas, P. J., Pipa, G., Lima, B., Melloni, L., Neuenschwander, S., Nikolic, D., et al. (2009). Neural synchrony in cortical networks: history, concept and current status. Front. Integr. Neurosci. 3:17. doi: 10.3389/neuro.07.017.2009 
van den Heuvel, M. P., Kahn, R. S., Goni, J., and Sporns, O. (2012). High-cost, highcapacity backbone for global brain communication. Proc. Natl. Acad. Sci. U.S.A. 109, 11372-11377. doi: 10.1073/pnas.1203593109

van den Heuvel, M. P., and Sporns, O. (2013). Network hubs in the human brain. Trends Cogn. Sci. 17, 683-696. doi: 10.1016/j.tics.2013.09.012

Van Veen, B. D., van Drongelen, W., Yuchtman, M., and Suzuki, A. (1997). Localization of brain electrical activity via linearly constrained minimum variance spatial filtering. IEEE Trans. Biomed. Eng. 44, 867-880. doi: 10.1109/ 10.623056

Varela, F., Lachaux, J. P., Rodriguez, E., and Martinerie, J. (2001). The brainweb: phase synchronization and large-scale integration. Nat. Rev. Neurosci. 2, 229-239. doi: 10.1038/35067550

Wens, V. (2015). Investigating complex networks with inverse models: analytical aspects of spatial leakage and connectivity estimation. Phys. Rev. E Stat. Nonlin. Soft. Matter. Phys. 91:012823.

Wens, V., Marty, B., Mary, A., Bourguignon, M., Op de Beeck, M., Goldman, S., et al. (2015). A geometric correction scheme for spatial leakage effects in MEG/EEG seed-based functional connectivity mapping. Hum. Brain Mapp. 36, 4604-4621. doi: 10.1002/hbm.22943

Xia, M., Wang, J., and He, Y. (2013). BrainNet viewer: a network visualization tool for human brain connectomics. PLoS One 8:e68910. doi: 10.1371/journal.pone. 0068910
Zalesky, A., Cocchi, L., Fornito, A., Murray, M. M., and Bullmore, E. (2012). Connectivity differences in brain networks. Neuroimage 60, 1055-1062. doi: 10.1016/j.neuroimage.2012.01.068

Zalesky, A., Fornito, A., and Bullmore, E. T. (2010). Network-based statistic: identifying differences in brain networks. NeuroImage 53, 1197-1207. doi: 10.1016/j.neuroimage.2010.06.041

Zuo, X. N., Ehmke, R., Mennes, M., Imperati, D., Castellanos, F. X., Sporns, O., et al. (2012). Network centrality in the human functional connectome. Cereb. Cortex 22, 1862-1875. doi: 10.1093/cercor/bh r269

Conflict of Interest: The authors declare that the research was conducted in the absence of any commercial or financial relationships that could be construed as a potential conflict of interest.

Copyright (c) 2019 Della Penna, Corbetta, Wens and de Pasquale. This is an openaccess article distributed under the terms of the Creative Commons Attribution License (CC BY). The use, distribution or reproduction in other forums is permitted, provided the original author(s) and the copyright owner(s) are credited and that the original publication in this journal is cited, in accordance with accepted academic practice. No use, distribution or reproduction is permitted which does not comply with these terms. 


\section{APPENDIX}

\section{Analytical Demonstration of Connectivity Increases After GCS}

To better understand the a priori counter-intuitive detection of higher connectivity values after GCS, we analyze here mathematically the effect of spatial leakage and characterize the changes brought by its correction. We demonstrate that: (i) the GCS decreases connectivity when leakage is strong enough (as expected for nearby sources), (ii) the GCS may increase connectivity for sufficiently weak leakage (as expected for well separated sources), and (iii) this increase occurs only in the presence of linear correlations. The last claim explains why no such connectivity increase was obtained after orthogonalization.

We consider source activity $\boldsymbol{\Psi}_{0}$ at a seed location and $\boldsymbol{\Psi}_{1}$ at a target location, which for simplicity will be assumed one-dimensional time courses. The linear mixing expected from linear, zero-lag spatial leakage can be written as

$$
\boldsymbol{\Psi}_{1}=\boldsymbol{\Psi}_{1}^{G C S}+k \boldsymbol{\Psi}_{0}
$$

where $\boldsymbol{\Psi}_{1}^{\mathrm{GCS}}$ is the corrected target source activity and $k$ denotes the coupling constant encoding the spatial leakage effect (Wens, 2015). As described in the Methods, the corresponding BLP time series are obtained by averaging the squared signal over windows $w$, so $B L P_{0,1}(w)=\left\langle\left(\boldsymbol{\Psi}_{0,1}\right)^{2}\right\rangle_{w}$ and $B L P_{1}^{G C S}(w)=\left\langle\left(\boldsymbol{\Psi}_{1}^{G C S}\right)^{2}\right\rangle_{w}$. The uncorrected BLP time course at the target source now depends quadratically on the leakage constant $k$ :

$$
B L P_{1}(w)=B L P_{1}^{G C S}(w)+k^{2} B L P_{0}(w)+2 k L C(w),
$$

where $L C(w)=\left\langle\boldsymbol{\Psi}_{1}^{G C S} \boldsymbol{\Psi}_{0}\right\rangle_{w}$ is a time-dependent measure of linear coupling between the seed and corrected target sources. Finally, connectivity is computed using BLP correlation over the windows $w$. However, the root of the argument can be explained more easily by considering the BLP covariance:

$$
\operatorname{cov}\left(B L P_{1}, B L P_{0}\right)=\operatorname{cov}\left(B L P_{1}^{G C S}, B L P_{0}\right)+k^{2} \operatorname{var}\left(B L P_{0}\right)+2 k \operatorname{cov}\left(L C, B L P_{0}\right) .
$$

Here the left-hand side represents uncorrected connectivity, and the first term in the right-hand side the corrected estimate. The second term is the coupling between the seed's $B L P_{0}$ and its linear contribution $k^{2} B L P_{0}$ to the target's $B L P_{1}$. Due to its positivity, it inflates the uncorrected connectivity, as may be intuitively expected. The third and key term in our argument depends on the covariation of the seed's $B L P_{0}$ and the time-dependent seed-target linear coupling $L C$. It can either increase or decrease the uncorrected connectivity, depending on the relative sign of $k$ and $\operatorname{cov}\left(L C, B L P_{0}\right)$. When this term is negative, it may or may not overcome the second, positive term depending on the leakage coupling strength $|k|$. The critical value $k_{\mathrm{c}}$ determined by the equality of these two contributions is

$$
k_{c}=-2 \frac{\operatorname{cov}\left(L C, B L P_{0}\right)}{\operatorname{var}\left(B L P_{0}\right)} .
$$

We conclude that: (i) When the leakage coupling is strong enough, i.e., $|k|>\left|k_{c}\right|$, the second, positive term dominates, so $\operatorname{cov}\left(B L P_{1}, B L P_{0}\right)>\operatorname{cov}\left(B L P_{1}^{G C S}, B L P_{0}\right)$ and the GCS thus decreases connectivity. (ii) When the leakage coupling is weak, i.e., $|k|<\left|k_{c}\right|$, the third term dominates, and if its sign is appropriate we have $\operatorname{cov}\left(B L P_{1}, B L P_{0}\right)<\operatorname{cov}\left(B L P_{1}^{G C S}, B L P_{0}\right)$ so the GCS may increase connectivity. (iii) Finally, this increase in connectivity requires the existence of a linear coupling $L C$, since otherwise $k_{\mathrm{c}}=0$ and the possibility (ii) cannot occur. Interestingly, orthogonalization strives to eliminate any linear, zero-lag correlation (in the most drastic case, non-stationary versions of orthogonalization set $L C(w) \approx 0$ within each window, see O'Neill et al., 2015). So, using signal orthogonalization instead of the GCS would by design tend to mitigate this effect and thus may miss the increased optimization in brain integration reported in the main text. 
OPEN ACCESS

Edited by:

Filippo Cieri,

Cleveland Clinic Lou Ruvo Center for Brain Health, United States

Reviewed by:

Lili Jiang,

Institute of Psychology (CAS), China Jinhui Wang,

South China Normal University, China

*Correspondence:

Gopikrishna Deshpande gopi@auburn.edu

Specialty section:

This article was submitted to Brain Imaging Methods,

a section of the journal

Frontiers in Neuroscience

Received: 01 June 2019 Accepted: 27 December 2019 Published: 06 February 2020

Citation:

Deshpande G and Jia H (2020) Multi-Level Clustering of Dynamic Directional Brain Network Patterns and Their Behavioral Relevance.

Front. Neurosci. 13:1448. doi: 10.3389/fnins.2019.01448

\section{Multi-Level Clustering of Dynamic Directional Brain Network Patterns and Their Behavioral Relevance}

\author{
Gopikrishna Deshpande ${ }^{1,2,3,4,5,6,7,8 *}$ and Hao $\mathrm{Jia}^{9}$ \\ ${ }^{1}$ Department of Electrical and Computer Engineering, AU MRI Research Center, Auburn University, Auburn, AL, \\ United States, ${ }^{2}$ Department of Psychology, Auburn University, Auburn, AL, United States, ${ }^{3}$ Center for Neuroscience, Auburn \\ University, Auburn, AL, United States, ${ }^{4}$ Center for Health Ecology and Equity Research, Auburn, AL, United States, \\ ${ }^{5}$ Alabama Advanced Imaging Consortium, Birmingham, AL, United States, ${ }^{6}$ Department of Psychiatry, National Institute \\ of Mental Health and Neurosciences, Bengaluru, India, ' School of Psychology, Capital Normal University, Beijing, China, \\ ${ }^{8}$ Key Laboratory for Learning and Cognition, Capital Normal University, Beijing, China, ${ }^{9}$ Department of Automation, College \\ of Information Engineering, Taiyuan University of Technology, Taiyuan, China
}

Dynamic functional connectivity (DFC) obtained from resting state functional magnetic resonance imaging (fMRI) data has been shown to provide novel insights into brain function which may be obscured by static functional connectivity (SFC). Further, DFC, and by implication how different brain regions may engage or disengage with each other over time, has been shown to be behaviorally relevant and more predictive than SFC of behavioral performance and/or diagnostic status. DFC is not a directional entity and may capture neural synchronization. However, directional interactions between different brain regions is another putative mechanism by which neural populations communicate. Accordingly, static effective connectivity (SEC) has been explored as a means of characterizing such directional interactions. But investigation of its dynamic counterpart, i.e., dynamic effective connectivity (DEC), is still in its infancy. Of particular note are methodological insufficiencies in identifying DEC configurations that are reproducible across time and subjects as well as a lack of understanding of the behavioral relevance of DEC obtained from resting state fMRI. In order to address these issues, we employed a dynamic multivariate autoregressive (MVAR) model to estimate DEC. The method was first validated using simulations and then applied to resting state $\mathrm{fMRI}$ data obtained in-house $(N=21)$, wherein we performed dynamic clustering of DEC matrices across multiple levels [using adaptive evolutionary clustering (AEC)] - spatial location, time, and subjects. We observed a small number of directional brain network configurations alternating between each other over time in a quasi-stable manner akin to brain microstates. The dominant and consistent DEC network patterns involved several regions including inferior and mid temporal cortex, motor and parietal cortex, occipital cortex, as well as part of frontal cortex. The functional relevance of these DEC states were determined using meta-analyses and pertained mainly to memory and emotion, but also involved execution and language. Finally, a larger cohort of resting-state fMRI 
and behavioral data from the Human Connectome Project (HCP) (N = 232, Q1-Q3 release) was used to demonstrate that metrics derived from DEC can explain larger variance in 70 behaviors across different domains (alertness, cognition, emotion, and personality traits) compared to SEC in healthy individuals.

Keywords: dynamic brain connectivity, resting state fMRI, effective connectivity, clustering, behavioral relevance, human connectome

\section{INTRODUCTION}

The view that human brain functions as a coordinated system with functional segregation and integration between different regions has been corroborated and widely accepted (Friston et al., 1993; Greicius et al., 2009; Guye et al., 2010; Rogers et al., 2010). A bulk of this evidence at the macro-level comes from connectivity studies based on non-invasive resting state functional magnetic resonance imaging (fMRI). Functional connectivity (FC) is a term used to describe measures of synchronous, non-directional, correlation of inter-regional brain activity in time. Effective connectivity (EC), on the other hand, is a term used to describe measures of directional relationships between brain activity in different brain regions (Friston, 1994; Deshpande et al., 2011b; Valdes-Sosa et al., 2011; Deshpande and $\mathrm{Hu}, 2012)$. Previous studies mainly investigated static FC (van de Ven et al., 2004) and EC (Roebroeck et al., 2005; Stilla et al., 2007, 2008; Deshpande et al., 2009b, 2013; Hampstead et al., 2011; Sathian et al., 2011, 2013; Liang et al., 2014) characteristics, assuming that connectivity is stationary in time, and the relevance of FC (Greicius et al., 2007; Kelly et al., 2008; Han et al., 2013) and EC (Liao et al., 2010; Inman et al., 2012) to behavior and brain disorders. Further, some studies have reported that static EC relationships at rest represent a mode of communication between brain regions whose activities are not synchronized (Deshpande et al., 2011b), and hence, both FC and EC taken together, provide complementary characterizations of brain connectivity at rest. However, recent evidence points to the fact that resting state FC is not stationary in time and consequently an array of methods have been proposed to capture dynamic variations in FC (Deshpande et al., 2006; Sato et al., 2006; Britz et al., 2010; Chang and Glover, 2010; Sakoðlu et al., 2010; Chang et al., 2013a,b; Majeed et al., 2011; Cribben et al., 2012; Dimitriadis et al., 2012; Fornito et al., 2012; Handwerker et al., 2012; Hutchison et al., 2012, 2013; Rack-Gomer and Liu, 2012; Tagliazucchi et al., 2012; Keilholz et al., 2013; Lee et al., 2013; Leonardi et al., 2013). This raises the possibility that dynamic alterations in resting state EC cannot be ignored and needs to be investigated. However, to the best of our knowledge, there has been scant literature on dynamic EC of resting state fMRI (but see Jin et al., 2017; Zhao et al., 2017; Rangaprakash et al., 2018), and most investigations of dynamic EC have focused on taskbased fMRI (Sato et al., 2006; Havlicek et al., 2010; Grant et al., 2014, 2015; Lacey et al., 2014; Wheelock et al., 2014; Hutcheson et al., 2015; Feng et al., 2016, 2018; Hampstead et al., 2016; Wang et al., 2017; Ramaihgari et al., 2018; Rao et al., 2018).
In order to holistically characterize connectivity in resting state brain networks, it is necessary to employ regions across the whole brain to conduct connectivity analysis. In this regard, there exists many studies exploring whole-brain static FC (Shirer et al., 2012; Zeng et al., 2012), and several others exploring whole-brain dynamic FC (Allen et al., 2013; Leonardi et al., 2013; Syed et al., 2017, 2019; Zhao et al., 2018) using principle component analysis (PCA) or independent component analysis (ICA)-based methods as well as using full pairwise connectomes instead of seed-based analysis. However, wholebrain EC analyses are less numerous due to associated challenges such as computational complexity and model discovery (Stephan and Roebroeck, 2012). For example, methods such as dynamic causal modeling (Friston et al., 2003) and structural equation modeling (McIntosh and Gozales-Lima, 1994; Zhuang et al., 2005) impose restrictions on the number of regions (but see whole brain DCM based on sparsity constraints: Frassle et al., 2018) that can be included in the model. In addition, it becomes difficult to formulate a priori hypotheses regarding connections between all brain regions (Lohmann et al., 2012), which are required by these methods. Therefore, data-driven approaches have become popular while investigating EC between large numbers of brain regions. One such model is the multivariate autoregressive (MVAR) model, which is used to capture time-lagged Granger causal influences between brain regions (Granger, 1969; Geweke, 1982; Deshpande et al., 2008, 2009a,b, 2010a,b, 2011a,b; Krueger et al., 2011; Wen et al., 2013). It has been previously demonstrated that the precision of the MVAR model increases when more variables containing information regarding the underlying system are included in the model (Kus et al., 2004). Yet, to estimate the parameters of an MVAR model fit using all voxel time series in the brain would require more data (in terms of the length of the time series and number of subjects) as well as require very large computational power (in terms of time and memory requirements) that may make it practically impossible. Besides, an MVAR model with too many voxel time series as regressors is ill-conditioned and highly sensitive to noise. To address this issue, dimensionality reduction is often employed and an exemplary work employing wholebrain regions/voxels for static EC can be found in Wu et al. (2013). These challenges become even more acute while computing whole-brain dynamic EC. In this present paper, we address these challenges by adopting a dynamic MVAR for characterizing dynamic EC in combination with a dimensionality reduction strategy based on multi-level clustering of dynamic EC patterns across spatial location, time, and subjects. 
Clustering of dynamic EC patterns over time is motivated by evidence from dynamic FC analysis with fMRI and EEG data which show that the synchronized blood oxygenation level dependent (BOLD) signal fluctuations over the brain organize into a finite number of configurations alternating with each other in time (Britz et al., 2010; Chang and Glover, 2010; Musso et al., 2010; Li et al., 2014). One principled approach to find dynamic FC configurations which are quasi-stable for a certain period of time can be found in $\mathrm{Li}$ et al. (2014). This follows from similar quasi-stable scalp voltage configurations, called microstates, obtained from agglomerative clustering of EEG data (Britz et al., 2010; Musso et al., 2010). Such approaches assume that a single FC configuration exists across the entire brain at any given time instant. Additionally, they also assume that the dynamics of connectivity is essentially due to the brain changing from one across-the-brain connectivity configuration to another. In this work, we investigated this issue with regard to dynamic EC. To find the dynamic EC configurations over time, we employed clustering of EC patterns over time using the adaptive evolutionary clustering (AEC) algorithm (Jia et al., 2014). Specifically, we performed simulations in order to demonstrate the efficacy of the temporal clustering for capturing dynamic EC regimes and subsequently, we applied it to resting state fMRI data.

The amount of information obtained from the assessment of dynamic EC over the whole brain can be quite large. Hence, it has been often difficult to interpret the underlying neuroscientific meaning (Chang and Glover, 2010). Some previous studies showed snapshots of dynamic FC at various points during the experiment obtained by using different window lengths (Handwerker et al., 2012; Lee et al., 2013) or template pattern matching (Majeed et al., 2011). Although a very good exploratory technique, the results, and interpretation from such an approach can be subjective, depending on the window length and frames chosen. Time-frequency analysis can overcome this difficulty by projecting dynamics of connectivity onto time-frequency plane (Chang and Glover, 2010) using wavelet-based methods. But the information obtained this way is difficult to interpret. Besides, the approaches based on agglomerative clustering (Britz et al., 2010; Musso et al., 2010) (used in microstate analysis) and principal component analysis (Leonardi et al., 2013) make an assumption that the connectivity networks may have different weights in spatial or temporal domains, but the spatial configuration of connectivity networks themselves do not change with time. This assumption may suffer from loss of generality. Therefore, in this paper, we propose an approach which does not make such an assumption. The AEC algorithm yields time-varying clustering configurations wherein the clusters (networks) themselves could change over time, as well as the ECs in each network. For instance, there are two networks shown in Figure 1A. Each network has the same nodes over time, so the network itself does not change, only the connections between nodes change with time. By contrast, the case shown in Figure 1B is more general wherein both connections between nodes and the networks themselves change with time, i.e., the networks have different nodes at different time instants. The clustering algorithm used

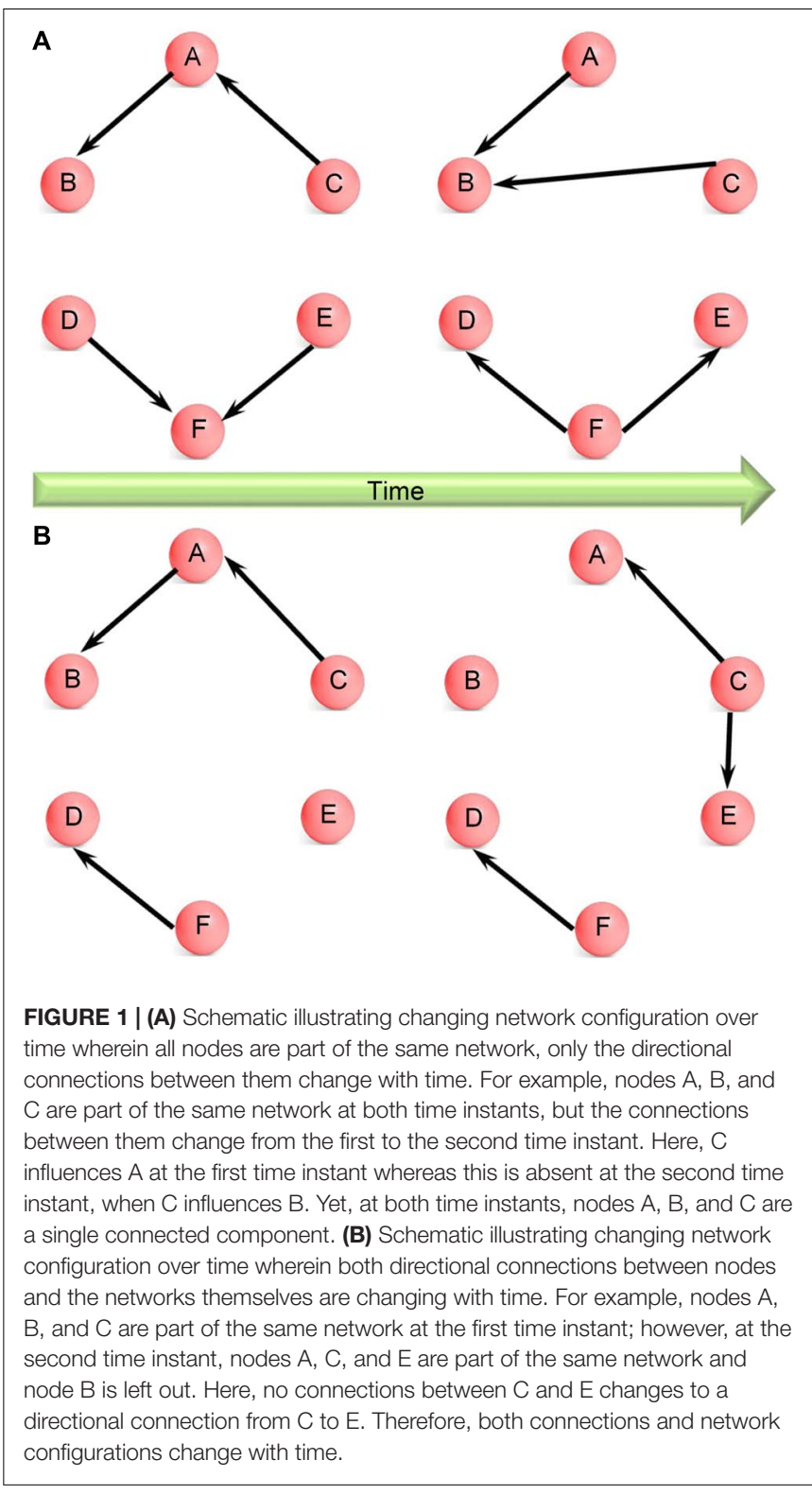

in this work is capable of modeling the more general case. With this merit, the proposed method is likely to hold promise in a variety of situations.

In order to determine EC configurations between brain regions which may be reproducible across different time instants within a given run, as well as across different subjects, two additional levels of clustering were employed across all fMRI runs and subjects, one level for determining most reproducible spatial configurations across time instants and another level for determining such consistent patterns across subjects. As we have shown in the case of static connectivity (Deshpande et al., 2011b), absence of significant synchronous connectivity does not imply the absence of brain connectivity, rather, such regions could be communicating via non-synchronous relationships (such as causality) that may be captured via EC. Our work can complement existing FC studies, since both synchronization and 
causality are established mechanisms of brain connectivity and one needs to assess both measures in order to gain a complete understanding of brain connectivity (Deshpande and Hu, 2012).

Once group-level dynamic EC patterns have been found using the proposed multilevel clustering approach, we tested the hypothesis that such dynamic EC patterns may be behaviorally salient. Specifically, we tested the hypotheses that (i) greater temporal variability of EC increases the adaptability and efficiency of brain networks, leading to better behavioral performance, and (ii) dynamic EC may better predict behavior than their static counterparts. These hypotheses were motivated by evidence in their favor in the context of dynamic FC as in our previous study (Jia et al., 2014). In order to test these hypotheses, we used resting-state fMRI and behavioral data from the Human Connectome Project (HCP) to correlate dynamic and static ECbased metrics with behavioral data in various domains, such as alertness, emotion, cognition, and personality traits.

\section{MATERIALS AND METHODS}

\section{Data}

\section{Data Acquisition and Pre-processing (Cohort-1)}

Resting-state fMRI data were acquired from a 3T Siemens Verio scanner at the Auburn University MRI Research Center from 21 healthy adults (aged $29.68 \pm 11.06$ years, nine females). Informed consent was obtained from all subjects after explaining and reviewing detailed written information about the study protocol, which was approved by the IRB of Auburn University. In all experiments, subjects were lying at rest during the scan with eyes open, and they were instructed to be awake and let their mind wander and not think about anything in particular. After the scan, all subjects confirmed adherence to these guidelines. $\mathrm{T} 2 *$-weighted echo planar imaging with the following parameters were used for fMRI data acquisition: 1000 volumes (time points) per run, in-plane matrix of $64 \times 64$, 16 axial slices covering the entire cerebral cortex, field of view $(\mathrm{FOV})=225 \mathrm{~mm} \times 225 \mathrm{~mm}$, flip angle $(\mathrm{FA})=90^{\circ}, \mathrm{TR}$ (repetition time)/TE (echo time) $=1000 \mathrm{~ms} / 29 \mathrm{~ms}$, in-plane voxel size $=3.5 \mathrm{~mm} \times 3.5 \mathrm{~mm}$, slice thickness of $5 \mathrm{~mm}$ with $1.25 \mathrm{~mm}$ gap. Standard anatomical MPRAGE data were also acquired from each subject for spatial normalization. Functional MRI preprocessing was performed using Data Processing Assistant for Resting-State fMRI software (DPARSF) (Yan and Zang, 2010) and included slice timing correction, rigid body registration, normalization to MNI template with resampling to $2 \mathrm{~mm} \times 2 \mathrm{~mm} \times 2 \mathrm{~mm}$ resolution, spatial smoothing with $4 \mathrm{~mm} \times 4 \mathrm{~mm} \times 4 \mathrm{~mm}$ full width at half magnitude (FWHM) Gaussian kernel, 0.01-0.1 Hz band pass filtering, detrending of mean and linear trend, and regressing out of nuisance covariates such as physiological artifacts and residual motion using aCompCor (Muschelli et al., 2014). Then, the 190-region version of the CC200 brain atlas (Craddock et al., 2012) was used as the reference brain parcellation template. We extracted the mean time series from 164 cerebral regions for subsequent use (26 regions of the CC200 template belonging to the cerebellum were not considered because our field of view covered only the cerebrum; this was done in order to reduce the TR which would be beneficial for EC analysis).

\section{Behavioral and Individual Difference Measures (Cohort-2)}

The subjects from the first cohort described above were used for demonstration of the proposed method in experimental data. However, we did not have detailed behavioral phenotyping of these subjects in order to demonstrate the behavioral relevance of the dynamic EC patterns. Therefore, we also used a second cohort consisting of resting-state fMRI data obtained from the $\mathrm{HCP}\left(N=232, \mathrm{Q} 1-\mathrm{Q} 3^{1}\right)$. These data underwent the same preprocessing pipeline as the first cohort. Behavioral measurements from same subjects have also been used in order to test the relative ability of static and dynamic EC for explaining behavior. HCP mainly measures behavioral data developed for NIH Toolbox Assessment of Neurological and Behavioral function $^{2}$ and some other measurements that are not covered by the NIH toolbox. The behavioral measures employed in this work belonged to the following domains: alertness, cognition, personality, and emotion. Notably, motor and sensory functions were not included in our analysis since they may be more relevant to while examining task data.

\section{Analysis Methodology Dynamic Effective Connectivity Model}

The traditional static formulation of the multivariate vector autoregressive (MVAR) model is shown below

$$
Y(t)=B+\sum_{m=1}^{p} K(m) \cdot Y(t-m)+\mathrm{N}(t)
$$

where $\boldsymbol{Y}(t)=\left[\begin{array}{llll}y_{1}(t) & y_{2}(t) & \cdots & y_{l}(t)\end{array}\right]$ is a vector autoregressive process including $l$ individual univariate processes [in our scenario, $l$ is 164 , the number of regions spanning the cerebrum in the CC200 brain parcellation atlas (Craddock et al., 2012)], $\boldsymbol{B}$ is the intercept vector representing the non-zero mean component, $m$ denotes the time lag (in terms of TRs), $\boldsymbol{K}(m)$ corresponds to the model coefficient matrix, $p$ is the model order, and $N(t)$ is the vector noise process. Since during preprocessing, the data were detrended and mean centered, $\boldsymbol{B}$ vanished. Then, the Granger causality (static version) representing direct causal influences from region $i$ to region $j$ is formulated as below.

$$
G_{i j}=\sum_{m=1}^{p}\left[k_{i j}(m)\right]^{2}
$$

where each $k_{i j}, i, j=1: l$, is one entry of matrix $K$ with row number being $i$ and column number being $j$. $K$ is determined in the least square sense. The order $p$ of this MVAR model can be determined according to Bayesian Information Criterion (BIC) (Schwartz, 1978; Roebroeck et al., 2005) (in our data, $p=1$ since we are interested in relationships with lags equal to or

\footnotetext{
${ }^{1}$ https://www.humanconnectome.org/storage/app/media/documentation/q3/Q3 Release_Reference_Manual.pdf

${ }^{2}$ www.nihtoolbox.org
} 
less than a TR). Then, estimation of EC dynamics was obtained through dynamic Granger causality (DGC) which differs from static Granger causality in terms of coefficient matrix $\boldsymbol{K}$, which is allowed to vary over time. Then the MVAR model in Eq. 1 changes to Eq. 3 accordingly.

$$
Y(t)=B(t)+\sum_{m=1}^{p} K(m, t) \cdot Y(t-m)+\mathrm{N}(t) .
$$

Note here that both coefficient matrix $\boldsymbol{K}(m, t)$ and $\boldsymbol{B}(t)$ are a function of both lag $m$ and time $t$. The DGC metric is then formulated as below.

$$
\operatorname{DGC}_{i j}(t)=\sum_{m=1}^{p}\left[k_{i j}(m, t)\right]^{2}
$$

Model coefficients $\boldsymbol{K}(m, t)$ were estimated based on a previously used procedure which utilizes Kalman filtering (Arnold et al., 1998). For Kalman updating of coefficient matrix $\boldsymbol{K}(m, t)$, we imported a parameter called forgetting factor, $F$, to control the way of updating. $1-F$ is actually the weighting of recent past Kalman estimate of $\boldsymbol{K}$ in the current estimate of $\boldsymbol{K}$. The weighting for most recent past $K$ is $1-F$, and exponentially decreases when moving backward. This is due to the consideration of boosting estimation stability and $F$ was optimized by minimizing the variance of estimated error energy as follows (Schlogl et al., 2000; Havlicek et al., 2010).

$$
F=\arg \left\{\min \left(\operatorname{var}\left(\tilde{N}(t)^{2}\right)\right\}\right.
$$

where $\tilde{N}(t)$ is the estimate of $N(t)$ and "var" is the variance operation over time.

In order to estimate a reasonable initial condition for the Kalman filter, we used the following procedure. The Kalman filter coefficients were randomly initialized and updated coefficients were obtained from the first run of the first subject which were in turn used as initial conditions for the following run/subject. Using this procedure iteratively, Kalman coefficients which were updated using the entire subject sample were obtained. This represented Kalman coefficients of the entire group as a whole. This group value was used as the initial condition for all runs/subjects and DGC values were re-estimated at the individual subject level. This procedure ensured that for each subject, the Kalman filter coefficients were initialized to the same value which was representative of the group average, which helped in relatively quick convergence. However, the DGCs obtained from the first 50 time points were discarded before being input into the clustering algorithm for the following reasons. First, initial time points in fMRI time series are routinely discarded to allow the MR signal to achieve T1 equilibration. Second, even with a group-averaged Kalman coefficients as the starting condition, the Kalman filter needed time to converge to ground truth connectivity as shown by our simulations (see simulation results for illustration).

\section{Clustering}

The DGC matrix calculated via the above procedures was of size $X \times X \times Y \times Z$ where $X=164$, the number of cerebral regions,
$Y=950$, the number of TRs in DGC calculation (each run had a total of 1000 TRs, first 50 TRs discarded), and $Z=21$, the number of runs/subjects. So, for each run, at each time instant, the EC between all pairs of regions had a dimension of $X \times X$. This DGC matrix was then fed into the AEC algorithm (Xu et al., 2013). This algorithm dynamically clustered all 164 regions according to their distances (the distances were transformed from the EC metric, for details see the section "First Level Clustering") at every time instant. Likewise, a forgetting factor was introduced to control the impact of the recent past of clustering results on the current calculation, with the purpose of enhancing stability of the clustering operation. This clustering is termed first level clustering and is described in detail in the following section. As mentioned in the section "Introduction," this clustering strategy can accommodate not only the changes of EC between nodes in a given network, but also the network configuration itself in terms of the nodes that may make up the network (Figure 1B). The first level clustering result revealed time-varying brain network configurations which were then fed to second level clustering as members in order to determine the distribution and consistency of the first level configurations across different time instants across a given run. Last but not the least, the dominating second level patterns from all runs were identified and used as members for the third level clustering. Resultantly, by the third level clustering, the dominant and consistent brain EC network patterns across all runs and subjects were identified. A graphical presentation of the hierarchy of three levels of clustering is given in Figure 2.

\section{First Level Clustering}

The first level clustering was implemented using the AEC algorithm employing a distance measure computed from the DGC matrix. A reasonable assumption is that the higher the absolute value of DGC, the closer the two regions are in feature space. Also noteworthy is that the DGC value between regions cannot be utilized directly as distances between regions for clustering. We know that the distance is inversely proportional to the closeness between regions, but the DGC metric is proportional to the closeness. Next, distance measure is greater or equal to zero, being zero only when it is measured from one region to itself. However, DGC metrics have both positive and negative values, and the diagonal entries of DGC matrix measuring auto-DGC are not zero. Moreover, DGC matrices are not symmetric, i.e., the distance from one region to another is not equal to the other way around, violating the condition of reciprocity required of any distance measure.

In order to convert DGC values into a distance measure, we devised a transformation algorithm as described below. We represent the $\mathrm{DGC}$ from region $i$ to region $j$ by $\mathrm{DGC}_{i j}$, and the other way around is $\mathrm{DGC}_{j i}$. DGC was transformed as shown below to meet the non-negative and reciprocity requirements.

$$
C=\left(\left|D G C_{i j}\right|^{m}+\left|D G C_{j i}\right|^{m}\right)^{1 / n}
$$

where $m$ and $n$ determine the characteristics of this transformation. According to Eq. 6, C will increase along with the increase of either $\mathrm{DGC}_{i j}$ or $\mathrm{DGC}_{j i}$. We chose $m=2$ and 


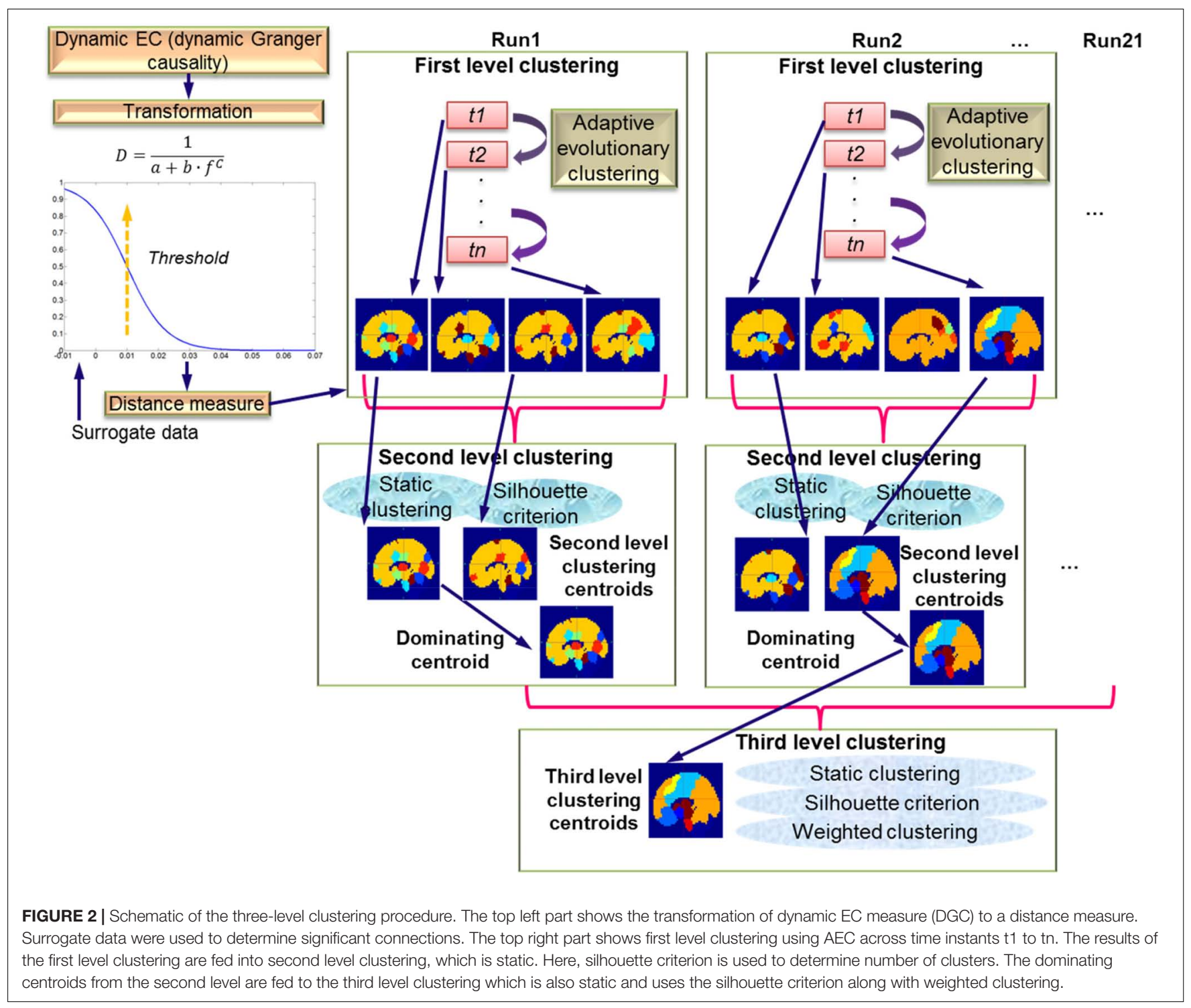

$n=1$ in this work, as such is usually employed for a second order matrix norm. If $n$ is relatively large compared to $m$, then $C$ would not be sensitive to the change of DGC, thus cannot distinguish significant connectivity from trivial ones. If $m$ is relatively large compared to $n$, the result will be sensitive to noise. Therefore, $m=2$ and $n=1$ seemed to be an optimal choice. Next, we used a reversed "S" shaped function applied to $C$ to meet the requirement of a monotonically decreasing transformation:

$$
D=\frac{1}{a+b \cdot f^{C}}
$$

where $a, b$, and $f$ are control parameters determining the behavior of this function. After this transformation, the significant connectivity between two regions are highlighted while non-significant ones are not. In order to find the boundary between significant and insignificant connectivities, we employed the method of surrogate data (Theiler et al., 1992; Deshpande et al., 2009b). Specifically, we transformed the time series to their frequency domain representation, randomized the phase of time series from all 164 regions in the frequency domain with magnitude unchanged, reconverted the phase-randomized data into time domain signals, and then the DGC was recalculated. Since the temporal structure of time series relative to each other was destroyed due to phase randomization, the DGCs obtained belonged to a null distribution of no influence between regions. After this procedure was repeated in a Monte Carlo manner (1000 times), a statistical null distribution of insignificant DGCs was obtained for each connection. Then we applied Eq. 6 to get the null distribution of $C$ and found the threshold at 95th percentile, denoted by $t h_{0}$. It is obvious that parameter $a$ just controls the scaling of $D$, thus is trivial, and hence we set it to 1 for normalization. In this way, $D$ can attain its maximum at $1 /(1+b)$ and an asymptotic minimum at 0 . Assume $b$ is sufficiently small such that the maximal value that $D$ can reach is asymptotically equal to 1 . Then it is reasonable to let $D$ be 
0.5 when $C$ is equal to $t h_{0}$, and when $C$ approaches $0, D$ is asymptotically equal to 1 . With surrogate data, we found tho to be equal to 0.01 . To find $b$, we followed previous work (Kent et al., 1972; McDowall and Dampney, 2006). Accordingly, we restricted $D$ to be no $<0.8$ and steepness to be $<0.02$ when $C$ is 0 . So, when $D=0.8$, steepness is 0.02 when $C$ is 0 , we calculated $b$ to be 0.2 , and $f$ as $5^{100}$ which were used in the following. Above sigmoid curve design aims to balance the separation of null and significant connectivity, and sensitivity to noise. The sigmoid curve design and parameters determination has been widely applied in many fields (Kent et al., 1972; McDowall and Dampney, 2006). After the transformation of Eq. 7, we let the Ds which were from one region to itself to be zero, resulting in the final distance measure $D_{\text {final }}$.

The estimated distance matrix $D_{\text {final }}$ at each time point was fed into the AEC algorithm (Xu et al., 2013). With this algorithm, we did clustering over all 164 regions at one time instant, taking the clustering result at recent previous time instants into account with adaptive weighting. The weighting was calculated through a forgetting factor, which was determined by BIC (Roebroeck et al., 2005). The clustering method was chosen to be hierarchical (Joe and Ward, 1963).

The number of clusters can be chosen either based on prior information about the neurophysiological system being investigated (which is preferable when that information is available) or based on mathematical criteria such as the silhouette index (Rousseeuw, 1987) (which is preferable when no a priori heuristics are available). Many previous studies have reported on the appropriate number of clusters, or in other words, the number of resting state networks (RSNs). To derive an eloquent result, various methods have been tried. The most representative one is ICA-based methods. In particular, probabilistic ICA (PICA) (De Luca et al., 2006) and tensor PICA (Damoiseaux et al., 2006) are variants of ICA which has attracted a lot of attention recently. Fully exploratory network ICA (FENICA) (Schöpf et al., 2010, 2011; Kalcher et al., 2012; Wang et al., 2012) has also been shown to find consistent networks among a group which may include thousands of subjects. Besides, fuzzy clustering (Lee et al., 2012) and graph theory (Moussa et al., 2012) are also two prevailing methods to find the number of RSNs with their own merits. Except a few of the above studies which employed task-related data (Schöpf et al., 2011), most studies focus on resting-state fMRI data. However, these findings are in terms of FC, and corresponding EC results are very sparse. A data-driven pilot study conducted by $\mathrm{Wu}$ et al. (2013), reported six communities from resting-state EC networks. Therefore, we used six as the number of clusters in first level clustering. Also, we assume that EC networks are hierarchically organized, similar to FC networks (Kalcher et al., 2012; Lee et al., 2012). Accordingly, if the specified number of networks or clusters increases, some networks will split into sub-networks, such as into left and right lateral parts or peripheral and foveal parts, rather than reshape into a new set of networks which has no relation with the previous one. Based on this assumption, if the number of networks/clusters is heuristically specified to be 6, it may not lead to loss of generality.

\section{Second Level Clustering}

We performed second level clustering for characterizing consistently recurring first-level patterns over time. This aided us to answer the question about whether there exists finite number of directional brain network patterns which consistently recur in time at an individual subject level. The difference from first level clustering is that this is a static clustering along the time axis. Hierarchical method was employed as the clustering method, and the number of clusters was determined by silhouette criterion in the absence of prior heuristics.

At this stage, we devised the distance measures between first level clustering configurations at different time instants using the following strategy. For any given two first level clustering configurations, we assume the first one has $M$ clusters denoted by $a_{m}, m=1,2, \ldots, M$, and the second one has $N$ clusters denoted by $b_{n}, n=1,2, \ldots, N$. For each pair of clusters with one picked out from the first configuration and the other picked out from the second configuration, the number of common regions is computed and among them, the maximal one is found. For the very pair having the maximal number of common regions, all regions in them are given a uniform label. For example, suppose the 5th cluster comprised of region $\# 1$, region $\# 2$, region $\# 3$ in the first configuration and 4 th cluster comprised of region $\# 2$, region $\# 3$, region $\# 5$ in the second configuration have the most common regions (regions \#2 and \#3), then regions $\# 1, \# 2$, and \#3 in the first configuration and regions $\# 2, \# 3$, and \#5 in the second configurations are given the same label (1). Next, we delete this pair and for all remaining clusters, redo the above operation, i.e., find the pair of clusters which have most common regions and give all clusters inside them the same label (2). Then we delete the second pair and redo the above operation. When the maximal number of common regions becomes zero over many iterations, we give the clusters in the first configuration a label, for example, (3) and those in the second configuration a different label, for example, (4). Then we delete this pair and redo the above operation. We iterate this procedure until no pair is left. After that, we set the initial distance measure to be zero, then we transverse all regions, if one region has different labels in the first and second configurations, the distance measure adds by 1 , otherwise it does not change. So the final distance measure is actually the number of regions having different labels in the two configurations. It is self-evident that this distance measure meets the requirement of reciprocity, nonnegativeness, triangular inequality, and the distance from one region to itself is zero.

After second level clustering, similar first level clustering configurations are grouped together, forming one pattern. Since a cluster can be represented by its centroid, we discuss how to represent each second level cluster centroid. The theoretical centroid is the weighted sum of first level configurations indicating that each region in the theoretical centroid owns fuzzy memberships to all first level clusters inside a given second level cluster. This centroid is very awkward to use, especially for third level clustering. Therefore, we represent the theoretical centroid by an agent which is described below. For every second level cluster, we average out all distance matrices $D_{i}$ s of all its 
members, resulting in the mean matrix $\boldsymbol{D}_{\text {mean }}$. Then the first level configuration inside this second level cluster with smallest Euclidean distance to $\boldsymbol{D}_{\text {mean }}$ is set as the agent (denoted by $i_{\text {agent }}$ ) for theoretical centroid, i.e.,

$$
i_{\text {agent }}=\arg \left(\min \left(\sum_{r=1}^{R} \sum_{c=1}^{C}\left(D_{i}(r, c)-D_{\text {mean }}(r, c)\right)^{2}\right), i\right)
$$

where $C$ and $R$ represent the number of columns and rows in the distance matrices.

\section{Third Level Clustering}

The second level clustering gives clustered patterns over time for each run and for each subject. The dominating second level patterns/clusters were predicated based on the histogram of second level clusters' occurrence times. Here the occurrence times is formulated as follows. For each run, each second level cluster covered a number of first level clustering configurations, the number of which was the times this second level cluster "occurred," so was defined as occurrence times for the given second level cluster. The histogram mentioned above was calculated over second level clusters from all runs. The threshold separating dominating, and non-dominating clusters was determined as follows. The point where the histogram value first hits zero, and the corresponding first-order derivative is also zero, is set as the threshold. In our data, this threshold was found to be at 95 (occurrence times). There was clearly a gap encompassing 95, and the majority of second level clusters had occurrence times $<95$, while beyond 95, second level clusters were relatively scarce and mainly distributed over the range: $100-200,270-360$, and 450-850, clearly indicating that they are dominant clusters. In order to assess the consistency of these dominating patterns across subjects, we performed third level clustering.

To calculate the distance measure between dominating second level centroid agents, it is inappropriate to adopt the strategy adopted in second level clustering since each centroid has a weight that we need to account for, i.e., the occurrence times of the pattern it represents. Therefore, a weighted clustering was adopted and is described as follows. To represent each dominating second level centroid agent in feature space, we vectorized its distance matrix (as illustrated in Eqs. 7 and 8) without recruiting diagonal entries (diagonal entries are all zeros and hence useless), such that the resulting vector is the representative in feature space. Next, the weighted $K$-means clustering was performed over these vector representatives, resulting in several patterns at the third level. As before, the number of clusters were optimized using the silhouette criterion. Each third level cluster's theoretical centroid is represented by its agent since the regions in the theoretical centroid have fuzzy memberships to dominating second level clusters. The agent has smallest Euclidean distance to the theoretical centroid in terms of distance. The three-level clustering procedure for dynamic EC described above is illustrated in Figure 2.

\section{Potential Correlates With Real World Functionalities}

Through the third level clustering, the brain's EC network patterns which were dominant and consistent across all subjects were obtained. To interpret the neural connotations of these patterns, we related them to real world cognitive functionalities using the Brainmap Sleuth search engine (Brainmap.Org, 2019). Specifically, for a given third level centroid agent, the 164 regions inside the cerebrum were divided into six clusters. It should be noted that one of the six clusters (networks) was trivial because it included all other brain regions which were not present in the five other clusters. This is because the clustering algorithm partitions all members, and if the five networks are definitive networks, the 6th one will include every other member not inside the five networks. The trivial cluster can be separated from five other networks by visual inspection since it embodies the most regions. As such, for each of the five definitive networks, we used the list of regions it included as input to Brainmap Sleuth search engine to find functional that those regions/networks may be engaged in based on previous literature. The Brainmap Sleuth search engine allows this kind of reverse inference to me made in a mathematically principled way.

\section{Simulations}

Simulations were performed in order to validate the proposed method for calculating DGC and the efficacy of subsequent clustering using the AEC algorithm. We simulated time series with a total length of 1000 time points from 12 regions using an autoregressive model as given below:

$$
Z(t)=\sum_{m=1}^{p} K_{m} \cdot Z(t-m)+N(t)
$$

where $Z(t)$ denotes the vector of signals from multiple regions, $\boldsymbol{K}_{m}$ is the regression coefficient matrix, and $\boldsymbol{N}(t)$ represents noise term with covariance matrix Cov, which has autocorrelation coefficients normalized to 1 . The order $p$ is chosen to be 1 . Then, three scenarios are used:

1. Cov was identity matrix so as to remove the effect of instantaneous correlation. The 12 time series were divided into four clusters each having three members (regions 1, 2, and 3 were in one cluster, regions 4,5 , and 6 were in one cluster, regions 7,8 , and 9 were in one cluster, and regions 10,11 , and 12 were in one cluster). Thus, $\boldsymbol{K}_{1}$ had block structure with $3 \times 3$ blocks on the diagonal, but the causality coefficients were constant over time. Each non-zero element in $\boldsymbol{K}_{1}$ was selected such that $K_{1}$ had all eigenvalues within the unit circle with all diagonal terms being negative. This ensured that the simulated time series were stable and its power spectral energy was concentrated in the low frequency band, in accordance with the fact that the signal of interest in experimental fMRI data lies in the low frequency band.

2. Cov was still an identity matrix, and 12 time series were divided into the same four clusters as in (i). As before, $K_{1}$ had block structure with $3 \times 3$ blocks on the diagonal, all eigenvalues within the unit circle, and all diagonal terms being negative. But each non-zero entry in $\boldsymbol{K}_{1}$ was oscillating 
over time sinusoidally with period equal to $200 \pi$ and randomized phases. The extent of this sinusoidal oscillation was bounded under the consideration of maintaining the stability of time series.

3. Cov was still an identity matrix, and $\boldsymbol{K}_{1}$ had all eigenvalues within the unit circle and all diagonal terms being negative. Initially, we set $K_{1}$ to have a block structure with $3 \times 3$ blocks on the diagonal and the same four clusters as in (i) and (ii). But after every 200 time points, $\boldsymbol{K}_{1}$ was circularly shifted by one column and one row aiming to change the cluster belongingness of each region. For example, from time point 1 to 200, regions 1, 2, and 3 belong to the same cluster (indicating they are inter-connected), regions 4,5 , and 6 belong to the same cluster, regions 7, 8, and 9 belong to the same cluster, and regions 10,11 , and 12 belong to the same cluster. But from time point 201 to 400 , regions 2,3 , and 4 belong to the same cluster, regions 5 , 6 , and 7 belong to the same cluster, ..., and regions 11 , 12 , and 1 belong to the same cluster. Then from time point 401 to 600 , region 3,4 , and 5 belong to the same cluster, and so on.

For each scenario listed above, the simulation was conducted 1000 times to get a group of simulated MVAR processes, and then the statistics of the DGCs were obtained.

\section{Behavioral Relevance of Dynamic EC and Static EC}

While the previous section described analyses of Cohort1 , we now describe the analysis procedure employed for Cohort-2. We used variance of dynamic EC as the metric of EC dynamics, and the absolute value of static EC as the metric of EC strength across the run. We input these two metrics into a GLM as explanatory variables and behavioral scores as dependent variables, as is given below:

$$
B_{i, j}=\alpha_{i, j} \cdot \mathrm{DEC}_{i, j}+\beta_{i, j} \cdot \mathrm{SEC}_{i, j}+\varepsilon_{i, j}
$$

where $i$ indexes different behavioral tests, $j$ indexes the ECs between different pairs of regions, $B_{i, j}$ is a vector of behavioral scores for all subjects, $\mathrm{DEC}_{i, j}$, and $\mathrm{SEC}_{i, j}$ are vectors of corresponding dynamic/static EC metrics for all subjects. $\alpha_{i, j}, \beta_{i, j}$ are their coefficients, respectively, and $\varepsilon_{i, j}$ are residuals. It should be noted that we had two runs and one behavior score for each of the 44 subjects, so each subject's behavioral score was used twice in this GLM. The coefficients obtained from this GLM were tested for statistical significance using a $z$-test. A Bonferronicorrected $p$-value threshold of $p=0.05 / 70=0.00071(70$ is the number of behavioral tests) was used in this test. Then, the variance explained in this GLM by each metric was calculated. For example $\overline{\left(\alpha_{i, j} \cdot \mathrm{DEC}_{i, j}\right)^{2}}$ is the variance explained by dynamic effective connectivity (DEC). The overline denotes mean operation over all is and $j$. And $\overline{\left(\alpha_{i, j} \cdot \mathrm{DEC}_{i, j}\right)^{2}} /\left(\overline{\left(\alpha_{i, j} \cdot \mathrm{DEC}_{i, j}\right)^{2}}+\overline{\left(\beta_{i, j} \cdot \mathrm{SEC}_{i, j}\right)^{2}}\right)$ represents the relative percentage of variance explained by DEC.

\section{RESULTS}

\section{Simulations}

The simulation results are shown in Figure 3. In each partfigure in Figure 3, the simulated ground truth of DGCs are shown on the left and the estimated DGCs using the dynamic MVAR model employed in this work are shown on the right. The estimated DGCs converged to the ground truth quickly, and suitably responded to dynamic variations in ground truth DGC as shown in Figures 3B,C. Also, the regions belonging to different clusters had nearly zero causality, such as region $1 \rightarrow$ region 12 in Figure $\mathbf{3 A}$ and region $4 \rightarrow$ region 8 in Figure $3 \mathrm{~B}$, indicating no false positives. The standard deviation of estimated DGCs over all instantiations of the AR process was modest, indicating good fidelity. Figure 3D presents a representative realization of first level clustering using AEC algorithm for simulated DGCs in scenario (iii). Along the time axis, regions rendered the same color belong to the same cluster. It can be seen that in Figure 3D, the AEC first level clustering clearly separates the 12 regions into clusters with correct memberships in a time-varying manner. In summary, the simulations demonstrate that the proposed DGC model qualifies for tracking true dynamic ECs, and the true timevarying clustering patterns can be reliably reproduced by the AEC algorithm (first level clustering).

\section{Experimental Data (Cohort-1)}

We estimated the DGC metric for each subject of pre-processed resting-state fMRI data and fed it into the three-level clustering algorithm. The results are shown in Figures 4-6 and relevant statistics are summarized in Tables 1, 2. Figure 4 presents exemplary second level clustering patterns along the time axis. Different colors represent different clusters. The number of second level clusters from the top bar to the bottom bar in Figure 4 is $10,6,6,10,11$, and 11 . These numbers are representative numbers of second level clusters, as reflected in Table 1. Please note that the same colors in different subjects do not mean they represent the same pattern. Table 1 presents corresponding second level clustering statistics for all subjects. By the histogram method described in the previous section, we identified one to three dominant clusters at the second level (varies from subject to subject). Critically, we can observe features similar to quasi-stability in Figure 4, i.e., each dominant pattern lasts for a period of time, during which it may swiftly switch to a few non-dominant pattern $s$ and switch back, and then switches to another dominant pattern. Certainly, dominant patterns last longer than non-dominant patterns, as expected.

Table 1 also conveys information about the number of second level clusters for each subject and the time spent before state (pattern) transition. Normally, the larger the number of clusters, the smaller the mean time (and its standard deviation) spent before a state transition. Their relationship is illustrated in Figure 5 using linear regression. The regression of mean time spent before state transition with respect to the number of clusters is shown in Figure $\mathbf{5 A}$, and corresponding results for 

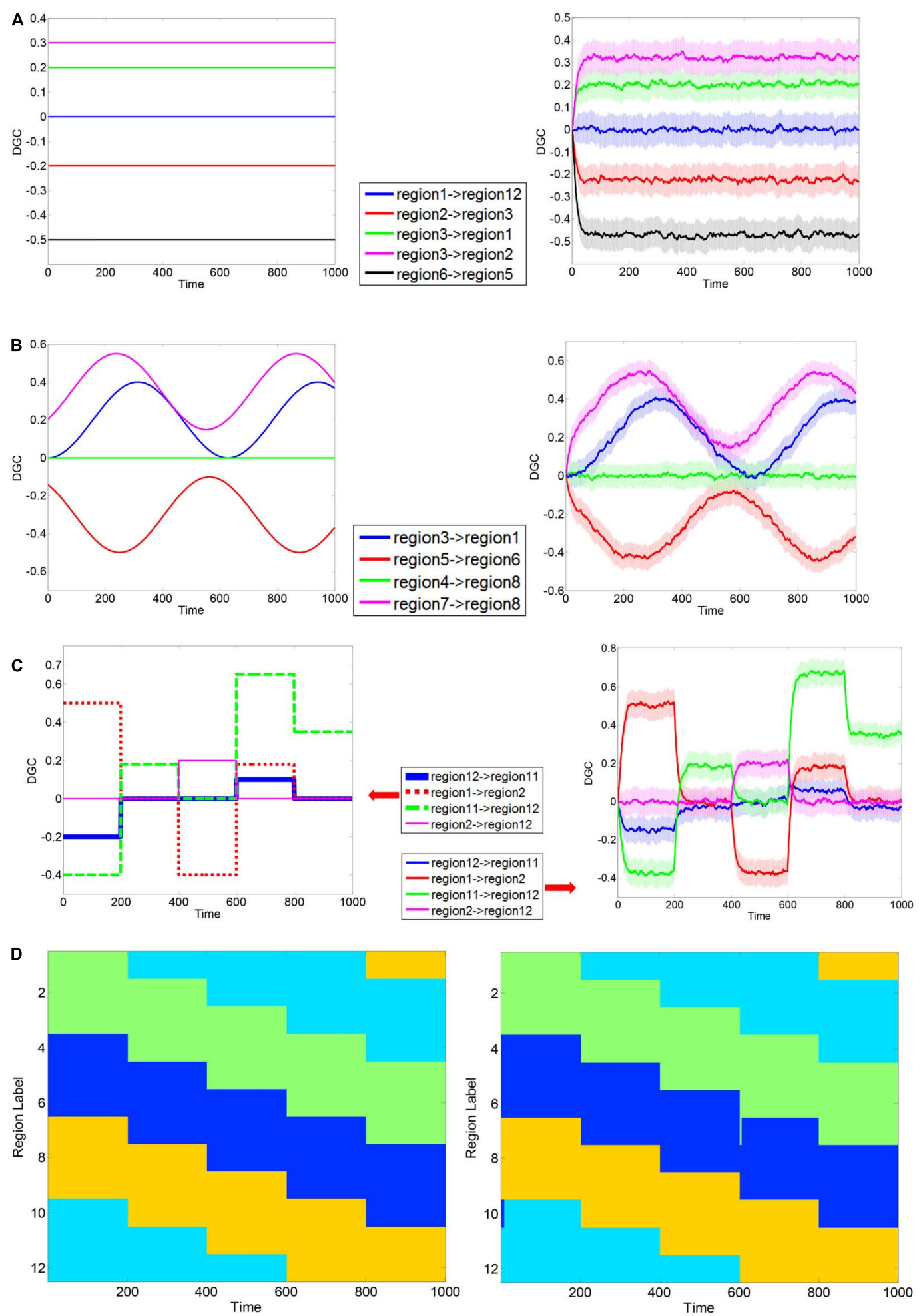

FIGURE 3 | Exemplary simulation result for dynamic Granger causality and first level AEC clustering. MVAR processes of 12 regions were simulated, with a length of 1000 time points. Three scenarios were used to corroborate the validity of formulated DGC. Exemplary ground truth causality of scenario (i) is shown in panel

(A) Left, and corresponding mean \pm standard deviation (std) of calculated DGCs is shown at Right. Color bands extend from mean-std to mean + std with mean values at the center. Below is same. Exemplary ground truth causality of scenario (ii) is shown in panel (B) Left, and corresponding mean \pm standard deviation (std) of calculated DGCs is shown at Right. Exemplary ground truth causality of scenario (iii) is shown in panel (C) Left, and corresponding mean \pm standard deviation (std) of calculated DGCs is shown at Right. Exemplary ground truth clustering pattern corresponding to scenario (iii) is shown in panel (D) Left and corresponding clustering result estimated using AEC algorithm is shown at Right. Regions rendered the same color belong to the same cluster. 

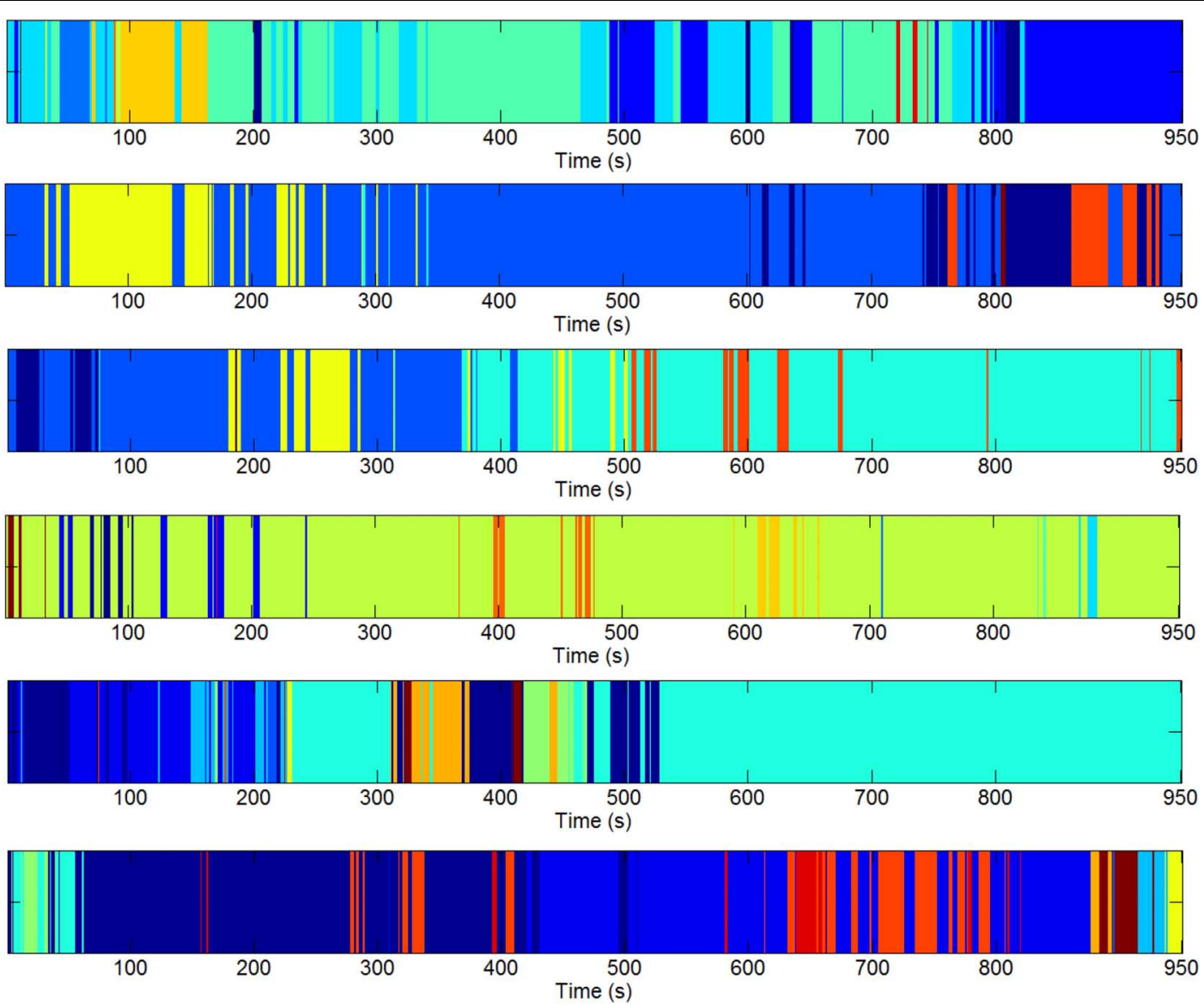

FIGURE 4 | Exemplary second level clustering patterns over time axis from six runs. Along each bar, each color represents one second level cluster and the time instants it occupies indicate the first-level configurations at these time instants belong to it. Different colors represent different second-level clusters. The number of second-level clusters for each bar is 10, 6, 6, 10, 11, 11 (from top to bottom). Please note the same colors in different runs do not mean they are of the same pattern.
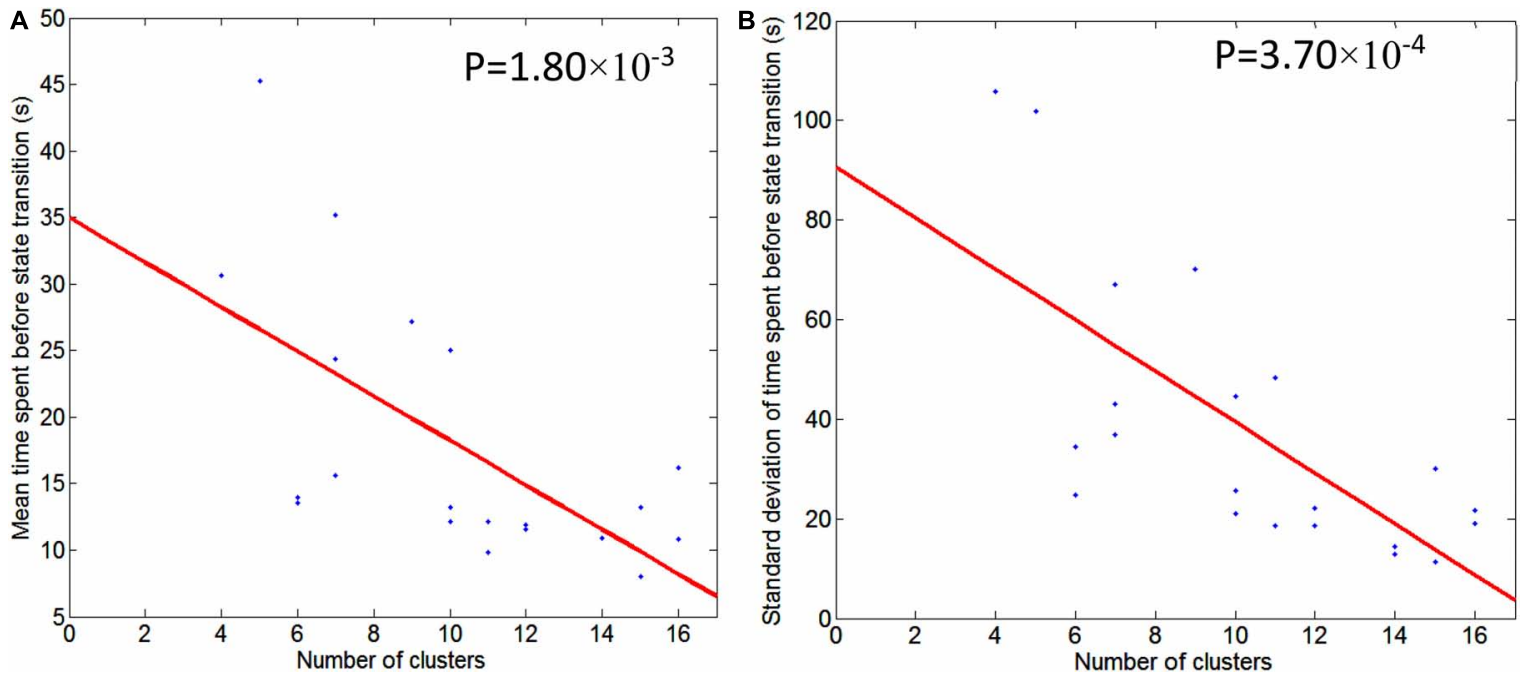

FIGURE 5 | Illustration of regression of mean/std of time spent before state transition with respect to the number of second level clusters. Graph (A) is for mean time spent before state transition and Graph $(\mathbf{B})$ is for standard deviation of time spent before state transition. Regression line is shown in red, and scattered dots represent data points from 21 subjects. The $p$-value for the significance of the fit using the regression line is also indicated. 


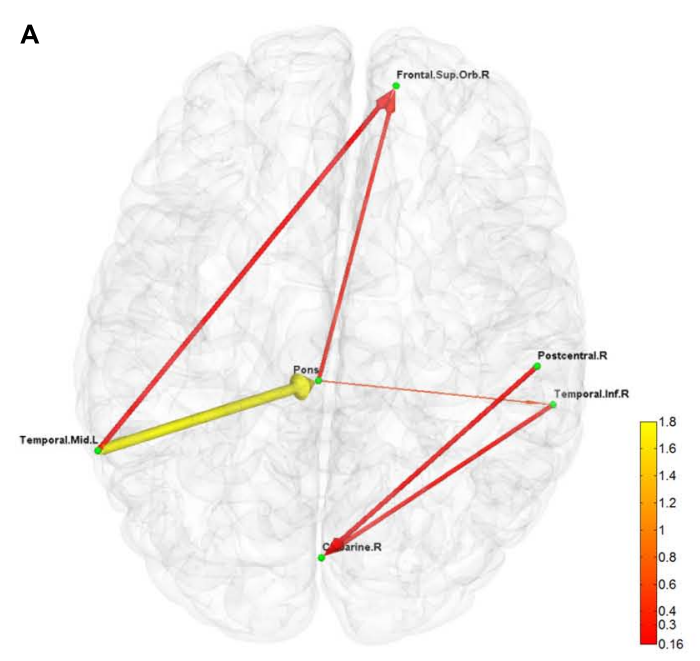

C

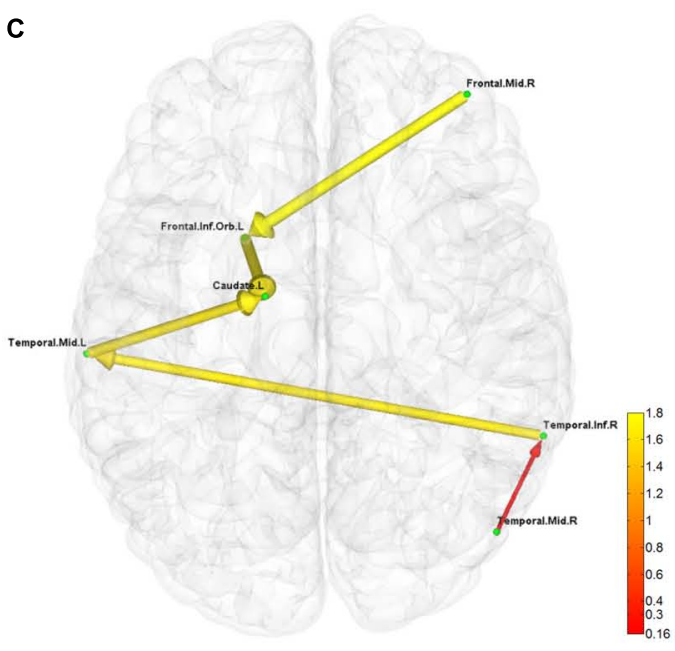

E

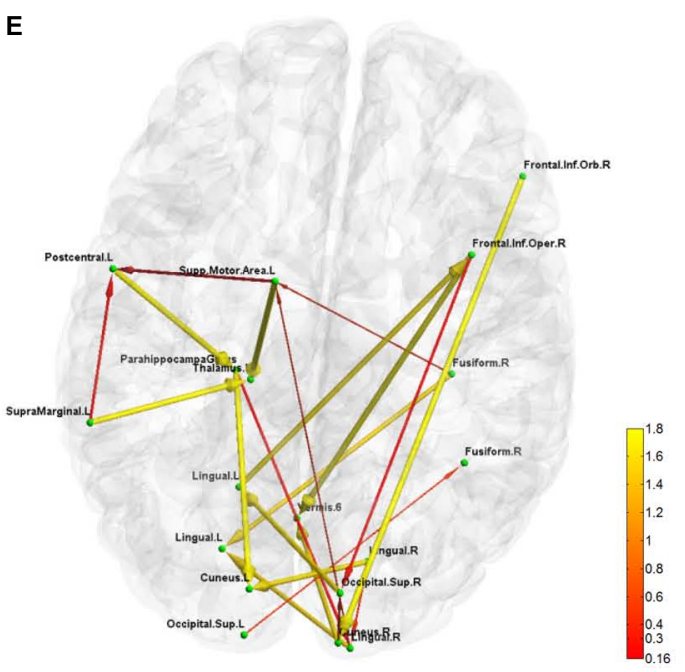

B

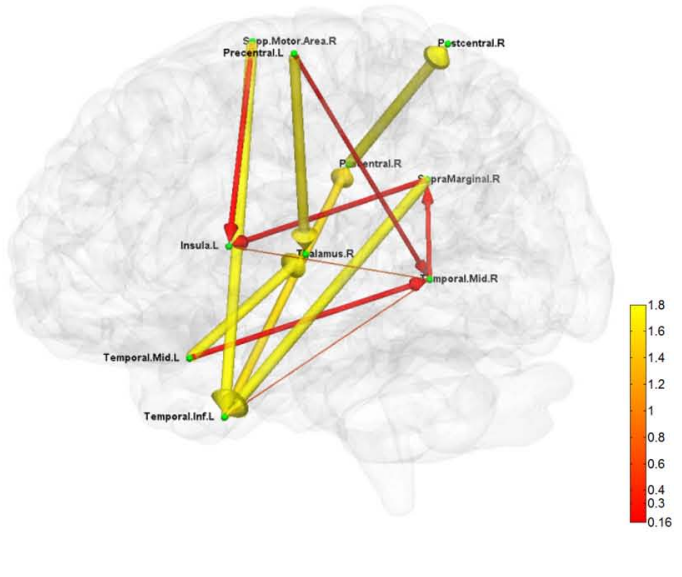

D

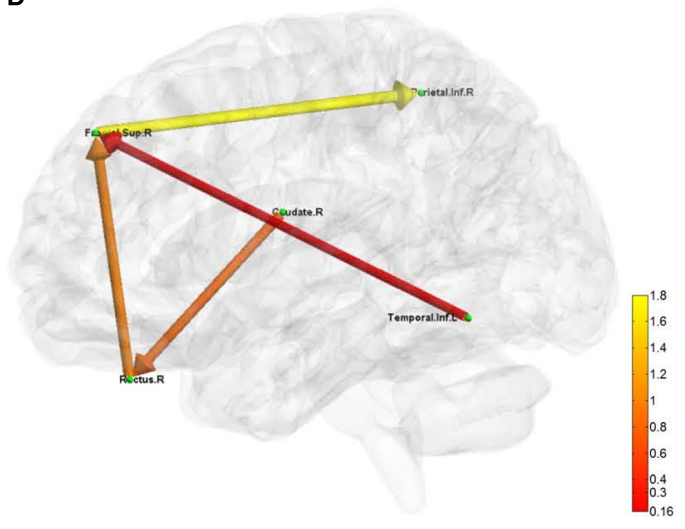

FIGURE 6 | Five directional connectivity networks of the most reproducible third level clustering centroid. In each part figures (A-E), green dots represent the centers of corresponding functionally homogeneous CC200 regions and arrowed paths represent directional connectivity between regions with thickness and color representing the absolute connectivity value. Autumn color map is used with red indicating small value and yellow indicating big value. 
TABLE 1 | Summary of statistical characteristics of second level clusters.

\begin{tabular}{|c|c|c|c|}
\hline Subject & $\begin{array}{c}\text { Number of } \\
\text { clusters }\end{array}$ & $\begin{array}{c}\text { Time spent (s) before any state } \\
\text { transition (mean } \pm \text { standard } \\
\text { deviation) }\end{array}$ & Time spent (s) in each cluster (descending) \\
\hline \#1 & 11 & $9.79 \pm 18.65$ & $350,329,116,33,31,28,22,17,13,10,1$ \\
\hline \#2 & 7 & $35.19 \pm 67.08$ & $777,69,60,26,13,4,1$ \\
\hline \#3 & 16 & $16.18 \pm 21.62$ & $456,172,79,69,62,27,22,19,11,10,6,4,4,3,3,3$ \\
\hline \#4 & 4 & $30.65 \pm 105.84$ & $732,179,24,15$ \\
\hline \#5 & 12 & $11.59 \pm 18.61$ & $348,307,112,70,68,16,8,8,5,4,2,2$ \\
\hline \#6 & 7 & $15.57 \pm 36.98$ & $775,135,24,9,3,2,2$ \\
\hline$\# 7$ & 6 & $13.97 \pm 34.38$ & $636,143,105,57,5,4$ \\
\hline \#8 & 15 & $13.19 \pm 30.04$ & $662,78,59,56,23,19,18,11,9,7,4,1,1,1,1$ \\
\hline \#9 & 14 & $10.92 \pm 14.45$ & $294,149,132,85,81,65,53,49,14,12,9,5,1,1$ \\
\hline$\# 10$ & 10 & $12.18 \pm 20.94$ & $369,226,220,68,26,25,8,6,1,1$ \\
\hline$\# 11$ & 7 & $24.36 \pm 43.06$ & $591,196,112,26,20,4,1$ \\
\hline \#12 & 6 & $13.57 \pm 24.68$ & $506,277,77,51,38,1$ \\
\hline$\# 13$ & 15 & $7.98 \pm 11.36$ & $574,147,47,39,36,34,15,15,15,12,5,5,3,2,1$ \\
\hline$\# 14$ & 11 & $12.18 \pm 48.36$ & $534,140,123,53,38,36,12,7,4,2,1$ \\
\hline \#15 & 16 & $10.78 \pm 19.03$ & $346,296,147,65,42,11,11,5,5,5,4,3,3,3,2,2$ \\
\hline \#16 & 10 & $13.19 \pm 25.68$ & $839,29,23,21,16,9,8,3,1,1$ \\
\hline$\# 17$ & 5 & $45.24 \pm 101.86$ & $851,74,15,9,1$ \\
\hline \#18 & 14 & $10.88 \pm 12.97$ & $476,116,108,96,52,34,26,11,7,6,5,5,4,4$ \\
\hline \#19 & 12 & $11.88 \pm 22.20$ & $659,169,25,22,15,13,13,12,8,7,6,1$ \\
\hline \#20 & 9 & $27.14 \pm 70.18$ & $813,84,20,17,7,4,2,2,1$ \\
\hline \#21 & 10 & $25.00 \pm 44.62$ & $582,326,13,11,6,4,3,2,2,1$ \\
\hline Summary (mean \pm standard deviation) & $10.52 \pm 4.05$ & $13.47 \pm 35.95$ & $90.27 \pm 177.96$ \\
\hline
\end{tabular}

standard deviation of time spent before state transition are shown in Figure 5B.

The result for third level clustering is illustrated in Figure 6 and Table 2, and potential neural correlates of those connectivity patterns to real world functionalities are depicted in Figure 7. At the third level, seven clusters were found from all dominating second level clusters obtained from all subjects, but only the 4th cluster was dominant and consistent across all subjects (Table 2). Figure 6 visualizes the five first level clusters which make up the centroid of the 4 th third level cluster. It is noted that at the first level, the number of clusters was set to 6 , and one of those clusters (networks) was trivial because it included all other brain regions which were not present in the five other

TABLE 2 | Summary of number of members and total occurrence times for third level clusters.

\begin{tabular}{lcc}
\hline Clusters & $\begin{array}{c}\text { Number of members (dominant } \\
\text { second level clustering } \\
\text { patterns) }\end{array}$ & $\begin{array}{c}\text { Total occurrence } \\
\text { times (TRs) }\end{array}$ \\
\hline$\# 1$ & 3 & 789 \\
$\# 2$ & 3 & 899 \\
$\# 3$ & 2 & 910 \\
$\# 4$ & 31 & 11,850 \\
$\# 5$ & 4 & 796 \\
$\# 6$ & 2 & 783 \\
$\# 7$ & 2 & 721 \\
Sum & 47 & 16,748
\end{tabular}

clusters. This happens because clustering partitions the input space, and if there are five definitive clusters, the 6th cluster will include everything that was excluded in the five clusters. In Figure 6, green dots represent the centers of the functionally homogeneous CC200 regions under consideration, and arrowed paths represent directional connectivities between regions with thickness and color indicating the absolute connectivity value. Network \#1 in Figure 6A illustrates directional causal influences among right mid temporal area, left calcarine, left postcentral, and left inferior temporal area, as well as pons and left superior orbital frontal area. According to activation likelihood estimation (ALE)-based meta-analyses using the BrainMap database these regions are co-activated by emotional stimuli (Shapira et al., 2003), language processing (Fu et al., 2002), working memory (Sailer et al., 2007), and spatial information processing (Ricciardi et al., 2006). Network \#2 in Figure 6B involves supplementary motor area (SMA), postcentral area, supramarginal area, mid temporal area, and thalamus in the right hemisphere, and insula, mid, and inferior temporal area, and precentral area in the left hemisphere. These regions are mostly distributed in parietal lobe, temporal lobe, as well as limbic regions. According to ALE-based meta-analyses using BrainMap, the co-activation of most of these regions is due to interoception (Karnath et al., 2005; Hu et al., 2008), working memory (Medaglia et al., 2012), language (Bookheimer et al., 2000), observation (Hu et al., 2008), execution (Bookheimer et al., 2000), as well as emotion regulation (Garrett and Maddock, 2006; Bokde et al., 2009). Besides the functionalities for areas above mentioned in Network \#1, the SMA and precentral area are involved in movement control and 
A

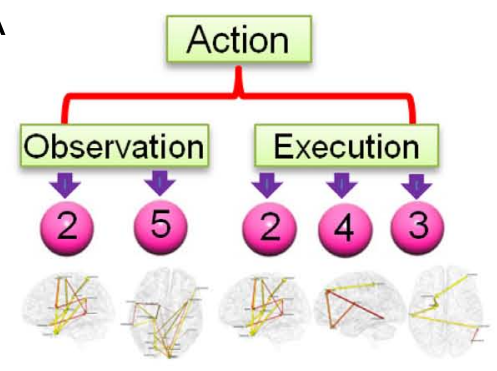

B

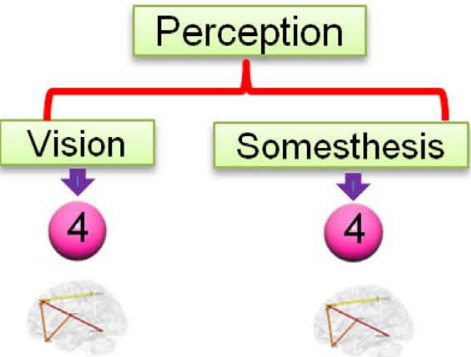

C

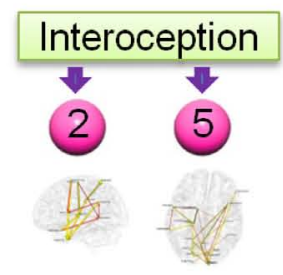

D

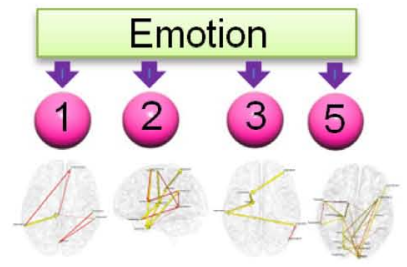

E

Cognition

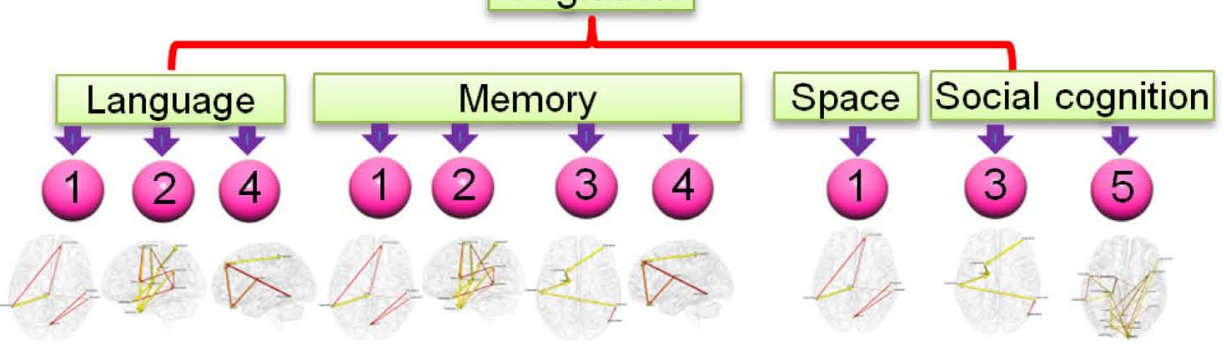

FIGURE 7 | Functional relevance of the five networks obtained from the most consistent third level clustering centroid. The digits in pink balls mark the corresponding directional networks in Figure 6. The head nodes of networks indicate the functionality that co-activates the regions of corresponding networks as ascertained through activation likelihood estimation (ALE)-based meta-analyses using the BrainMap database. Flowchart (A) is for functionality of action, (B) is for perception, (C) is for interoception, (D) is for emotion, and (E) is for cognition.

execution (Ellermann et al., 1998); insula involved in emotion, perception, motor control, self-awareness, and interoception; postcentral area is involved in tactile sense. Network \#3 shown in Figure 6C involves middle frontal area, inferior and mid temporal areas in the right hemisphere, and mid temporal area, caudate, and inferior orbital frontal area in the left hemisphere. 


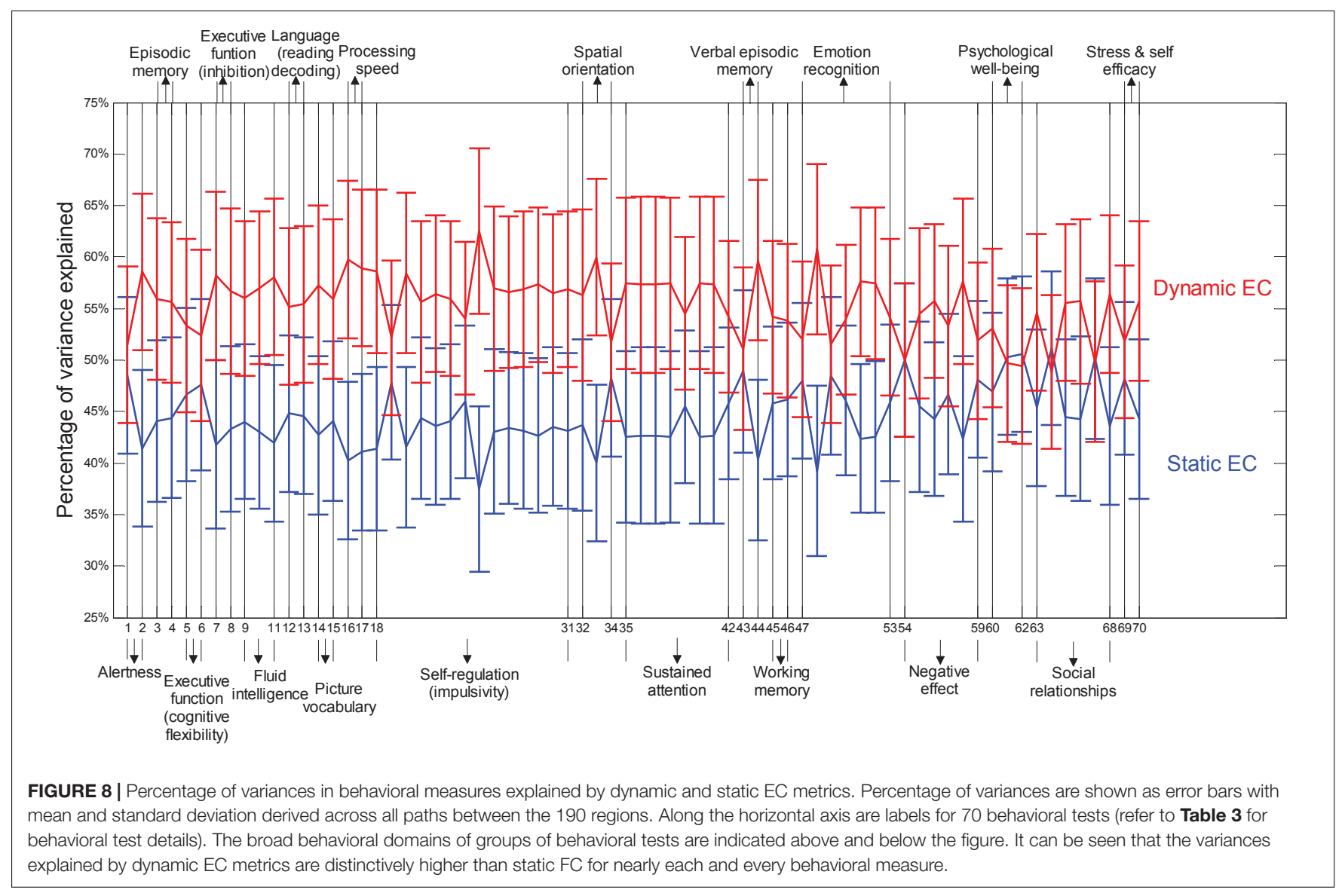

We can clearly see the temporal to caudate causal pathway and frontal to caudate causal pathway. Caudate nucleus has been demonstrated to be involved in learning and memory (Graybiel, 2005), particularly regarding feedback processing (reward and motivation) (Kinnison et al., 2012), as well as emotion (Aron et al., 2005; Ishizu and Zeki, 2011). Together with temporal area and frontal area, these two pass ways indicate several functionalities using Brainmap Sleuth: memory (Malhi et al., 2007), execution, emotion (Lee et al., 2006), and social cognition (Strathearn et al., 2008). Network \#4 shown in Figure 6D includes inferior parietal area, caudate, rectus, and superior frontal area in the right hemisphere, as well as left inferior temporal area. Rectal gyrus has been linked to attention and memory processing (Morecraft et al., 1992). Inferior parietal lobule is involved in interpretation of sensory input and perception of emotions. By using Brainmap Sleuth, the co-activation of most these areas was found to involve the functionalities of execution (Lissek et al., 2007), memory (Achim and Lepage, 2005), visual and somesthesis perception (Bingel et al., 2006), and language processing ( $\mathrm{Fu}$ et al., 2002; Liljeström et al., 2008). Lastly, Network \#5 in Figure 6E involves left SMA, left postcentral area, left supramarginal area, bilateral lingual gyrus, bilateral cuneus, superior bilateral occipital area, right fusiform, right inferior orbital and opercular frontal area, left parahippocampus gyrus, part of vermis, and left thalamus. Using Brainmap Sleuth, most of these areas are co-activated by emotion and social cognition
(Strathearn et al., 2008), interoception and observation (Hu et al., 2008). Fusiform, superior occipital area, cuneus relate to visual perception and processing, lingual gyrus participates in visual processing and visual memory encoding, supramaginal relates to language processing, and postcentral area and parahippocampal gyrus relate to memory encoding and retrieval.

\section{Experimental Data (Cohort-2)}

Figure 8 presents relative percentage of variance explained by static EC and dynamic EC for 70 behavioral scores such as alertness, cognition, emotion, and personality traits (refer to Table 3 for behavioral test details) obtained from Cohort-2. Noticeably, it is clear that the variances explained by dynamic EC metrics are distinctively higher than Static FC for nearly each and every behavioral measure. This implies that dynamic EC can be a better predictor of human behavior than conventional static EC. Scatter plots of behavioral measures against two metrics indicate that the correlations were not caused by outliers.

\section{DISCUSSION}

We propose a multi-level clustering algorithm for characterizing and understanding directional network patterns across spatial locations, time, and subjects. We validate the proposed method using simulations and demonstrate its utility using a dynamic 
TABLE 3 | Description of categorized behavioral measures employed in this work.

\begin{tabular}{llll}
\hline Variable name & Description & Values
\end{tabular}

\begin{tabular}{lc}
\hline Category: Alertness \\
\hline 1. MMSE score & Instrument: Cognitive status (Mini Mental Status Exam/MMSE) \\
& MiniMental Status Exam Total Score \\
2. PSQI score & Instrument: Sleep (Pittsburgh Sleep Questionnaire/PSQI) \\
& Sleep (Pittsburgh Sleep Questionnaire) Total Score \\
Category: Cognition
\end{tabular}

Instrument: Episodic memory (Picture Sequence Memory)

3. Picture sequence unadjusted $\quad \mathrm{NIH}$ Toolbox Picture Sequence Memory Test Unadjusted Scale Score

4. Picture sequence adjusted

$\mathrm{NIH}$ Toolbox Picture Sequence Memory Test Age-Adjusted Scale Score

Instrument: Executive function/cognitive flexibility (Dimensional Change Card Sort)

\section{Card sort unadjusted \\ 6. Card sort adjusted}

\section{Flanker task unadjusted}

8. Flanker task adjusted

9. PMAT24_A_CR

10. PMAT24_A_SI

11. PMAT24_A_RTCR

12. Reading test unadjusted

14. Picture vocabulary test unadjusted

15. Picture vocabulary test adjusted

16. Processing speed unadjusted

16. Processing speed unadjusted
17. Processing speed adjusted

18. SV_1mo_200

19. SV_6mo_200

20. SV_1yr_200

21. SV_3yr_200

22. SV_5yr_200

23. SV_10yr_200

24. SV_1mo_40K

25. SV_6mo_40K

26. SV_1yr_40K

27. SV_3yr_40K

28. SV_5yr_40K

29. SV_10yr_40K
$\mathrm{NIH}$ Toolbox Dimensional Change Card Sort Test

Unadjusted Scale Score

$\mathrm{NIH}$ Toolbox Dimensional Change Card Sort Test

Age-Adjusted Scale Scre

Instrument: Executive function/inhibition (Flanker Task)

$\mathrm{NIH}$ Toolbox Flanker Inhibitory Control and Attentio Test

Unadjusted Scale Score

NIH Toolbox Flanker Inhibitory Control and Attention Test Adjusted Scale Score

Instrument: Fluid intelligence (Penn Progressive Matrices)

Penn Matrix Test: Number of Corect Resonses

Penn Matrix Test: Total Skipped Items (items not presented

because maximum errors allowed reached)

Penn Matrix Test: Median Reaction Time for Correct

Responses

Instrument: Language/reading decoding (Oral Reading Recognition)

$\mathrm{NIH}$ Toolbox Oral Reading Recognition Test Unadjusted

Scale Score

$\mathrm{NIH}$ Toolbox Oral Reading Recognition Test Adjusted Scale

Score

Instrument: Language/receptive vocabulary

$\mathrm{NIH}$ Toolbox Picture Vocabulary Test Unadjusted Scale

Score

$\mathrm{NIH}$ Toolbox Picture Vocabulary Test Adjusted Scale Score

Instrument: processing speed (Pattern Completion Processing Speed)

$\mathrm{NIH}$ Toolbox Pattern Comparison Processing Speed Test

Unadjusted Scale Score

NIH Toolbox Pattern Comparison Processing Speed Test Adjusted Scale Score

Instrument: Self-regulation/impulsivity (Delay Discounting)

Subjective value for $\$ 200$ at 1 month

Subjective value for $\$ 200$ at 6 months

Subjective value for $\$ 200$ at 1 year

Subjective value for $\$ 200$ at 3 years

Subjective value for $\$ 200$ at 5 years

Subjective value for $\$ 200$ at 10 years

Subjective value for $\$ 40,000$ at 1 month

Subjective value for $\$ 40,000$ at 6 months

Subjective value for $\$ 40,000$ at 1 year

Subjective value for $\$ 40,000$ at 3 years

Subjective value for $\$ 40,000$ at 5 years

Subjective value for $\$ 40,000$ at 10 years

\section{$0-24$}

0-19

Typical range: $2730-51,730$ 
TABLE 3 | Continued

\begin{tabular}{|c|c|c|}
\hline Variable name & Description & Values \\
\hline 30. AUC_200 & Area under the curve for discounting of $\$ 200$ & $0.0-1.0$ \\
\hline 31. AUC_40K & Area under the curve for discounting of $\$ 40,000$ & $0.0-.0$ \\
\hline \multicolumn{3}{|c|}{ Instrument: Spatial orientation (Variable Short Penn Line Orientation Test) } \\
\hline 32. VSPLOT_TC & Penn line orientation: total number correct & $0-24$ \\
\hline 33. VSPLOT_CRTE & Penn line orientation: median reaction time divided by expected number of clicks for correct trials & Typical range: 640-2800 \\
\hline 34. VSPLOT_OFF & Penn line orientation: total positions off for all trials & $0-165$ \\
\hline \multicolumn{3}{|c|}{ Instrument: Sustained attention (Short Penn Continuous Performance Test) } \\
\hline 35. SCPT_TP & Short Penn CPT true positives $=$ sum of CPN_TP and CPL_TP & $0-60$ \\
\hline 36. SCPT_TN & Short Penn CPT true negatives $=$ sum of CPN_TN and CPL_TPN & $0-120$ \\
\hline 37. SCPT_FP & Short Penn CPT false positives $=$ sum of CPN_FN and CPL_FN & $0-120$ \\
\hline 38. SCPT_FN & Short Penn CPT False Negatives = Sum of CPN_FN and CPL_FN & $0-60$ \\
\hline 39. SCPT_TPRT & Short Penn CPT median response time for true positive responses & $0-1000 \mathrm{~ms}$ \\
\hline 40. SCPT_SEN & Short Penn CPT Sensitivity = SCPT_TP/(SCPT_TP + SCPT_FN) & Typical range: 0.8-1.0 \\
\hline 41. SCPT_SPEC & Short Penn CPT specificity= SCPT_TN/(SCPT_TN+SCPT_FP) & Typical range: 0.8-1.0 \\
\hline 42. SCPT_LRNR & Short Penn CPT longest run of non-responses) & $5-16$ \\
\hline \multicolumn{3}{|c|}{ Instrument: Verbal episodic memory (Penn Word Memory Test) } \\
\hline 43. IWRD_TOT & Penn word memory: total number of correct responses & $0-40$ \\
\hline 44. IWRD_RTC & Penn word memory: median reaction time for correct responses & Typical range: 1100-3200 \\
\hline \multicolumn{3}{|c|}{ Instrument: Working memory (List Sorting) } \\
\hline 45. List sorting unadjusted & NIH Toolbox List Sorting Working Memory Test Unadjusted Scale Score & \\
\hline 46. List sorting adjusted & NIH Toolbox List Sorting Working Memory Test Adjusted Scale Score & \\
\hline \multicolumn{3}{|c|}{ Category: Emotion } \\
\hline \multicolumn{3}{|c|}{ Instrument: Emotion recognition (Penn Emotion Recognition Test) } \\
\hline 47. ER40_CR & Penn emotion recognition: number of correct responses & $0-40$ \\
\hline 48. ER40_CRT & Penn emotion recognition: correct responses median response time (ms) & Typical range: $1435-2746$ \\
\hline 49. ER4OANG & Penn emotion recognition: correct anger identifications & $0-8$ \\
\hline 50. ER40FEAR & Penn emotion recognition: correct fear identificatins & $0-8$ \\
\hline 51. ER4OHAP & Penn emotion recognition: correct happy identifications & $0-8$ \\
\hline 52. ER4ONOE & Penn emotion recognition: correct neutral identifications & $0-8$ \\
\hline 53. ER40SAD & Penn emotion recognition: correct sad identifications & $0-8$ \\
\hline \multicolumn{3}{|c|}{ Instrument: Negative affect } \\
\hline 54. Anger-affect & NIH Toolbox Anger-Affect Survey & \\
\hline 55. Anger-hostility & NIH Toolbox Anger-Hostility Survey & \\
\hline 56. Anger-aggression & NIH Toolbox Anger-Physical Aggression Survey & \\
\hline 57. Fear-affect & NIH Toolbo Fear-Affect Survey & \\
\hline 58. Fear-somatic & NIH Toolbox Fear-Somatic Arousal Survey & \\
\hline 59. Sadness & NIH Toolbox Sadness Survey & \\
\hline \multicolumn{3}{|c|}{ Instrument: Psychological well-being } \\
\hline 60. Life satisfaction & NIH Toolbox General Life Satisfaction Survey & \\
\hline 61. Meaning and purpose & NIH Toolbox Meaning and Purpose Survey & \\
\hline 62. Positive affect & NIH Toolbox Positive Affect Survey & \\
\hline \multicolumn{3}{|c|}{ Instrument: Social relationships } \\
\hline 63. Friendship & NIH Toolbox Friendship Survey & \\
\hline 64. Loneliness & NIH Toolbox Loneliness Survey & \\
\hline 65. Perceived hostility & NIH Toolbox Perceived Hostility Survey & \\
\hline 66. Perceived rejection & NIH Toolbox Perceived Rejection Survey & \\
\hline 67. Emotional support & NIH Toolbox Emotional Support Survey & \\
\hline 68. Instrumental support & NIH Toolbox Instrumental upport Survey & \\
\hline \multicolumn{3}{|c|}{ Instrument: Stress and self-efficacy } \\
\hline 69. Perceived stress & NIH Toolbox Perceived Stess Survey & \\
\hline 70. Self-efficacy & NIH Toolbox Self-Efficacy Survey & \\
\hline
\end{tabular}

For more details, refer to HCP Q3 release manual http://humanconnectome.org/documentation/Q3/Q3_Release_Reference_Manual.pdf. 
EC measure. Further, we relate such clustering patterns to realword functionalities using meta-analysis and demonstrate that dynamic EC explains more variance in behavioral measures across 70 different behavioral domains as compared conventional static EC. Below, we elaborate on major contributions of this work.

The alternating first level brain EC clusters or network patterns were subjected to second level clustering, and from this, we were able to identify one to three dominating patterns in individual subjects, with quasi-stable property. A dominating pattern will dominate for a period of time and during this period, no other dominating patterns exist. After this period, another dominating pattern takes over. During the period when one dominating pattern dominates, there may appear several sub-patterns, and like the dominating patterns, each sub-pattern will appear and alternate with the dominating one for only a section of time, and after this time, another sub-pattern takes over. Then, under the sub-patterns, there may appear even smaller sub-patterns which behave similarly to sub-patterns, but at a finer scale. Previous reports have found this fractal-like quasistable phenomenon using EEG/fMRI analysis (Britz et al., 2010; Musso et al., 2010; Van de Ville et al., 2010). Both functional connectivity networks and the topography of the spontaneous EEG show stable global brain states remaining quasi-stationary for a period of time, called microstates. Our results suggest that quasi-stable EC network configurations may support or be related to brain microstates. Further study is necessary to investigate this phenomenon and is one of our future research directions.

At the third level, we obtained seven clusters and among them, we found one dominating and consistent EC network pattern across all subjects. Detailed analysis of all networks in this pattern demonstrates that there are five EC networks in this pattern, and these networks contain several regions: mid and inferior temporal cortex, frontal cortex, SMA, pre and postcentral area, parietal cortex, and occipital cortex. These areas are frequently recruited when the brain is engaged in memory retrieval, observation, execution, and emotion regulation. These cognitive functions are most frequently encountered for restingstate human brain when lying in scanner. Other regions such as caudate, hippocampus, fusiform, and lingual cortex are also implicated in above functions (Bogousslavsky et al., 1987; Bliss and Collingridge, 1993; Grahn et al., 2008). The language function and execution function involve three networks which is less than memory, and emotion. This may be due to the reason that language and execution processes are not as frequently recruited as the other three at rest. Functionalities including observation, somesthesis, vision, interoception, spatial cognition, and social cognition involve even less networks maybe due to the reason these functions are less frequently employed when lying rest during $\mathrm{fMRI}$ scan.

It must be noted that three levels of clustering is required to find patterns consistent across three varying factors: time, spatial location, and subjects. However, it is not necessary in all cases. For example, if only patterns across time are of interest, a single level AEC across time will suffice. There are multiple studies adopting $K$-means or hierarchical clustering across spatial locations to find brain networks. Our point is that single level clustering has always existed and we are proposing multi-level clustering for estimating consistent patterns across different varying factors (such as time, spatial location, and subjects). The framework could also be extended to have more than three levels of clustering if someone wants to investigate consistent patterns across more than three varying factors.

Previously we investigated behavioral relevance of static and dynamic functional connectivity (DFC) (Jia et al., 2014). Since EC has been shown to be a complimentary mode of communication between brain regions in resting state, we used a similar framework for dynamic and static EC metrics here. Akin to our previous study, the results from the current study demonstrate that dynamic EC is able to explain more variance in behavioral performance tasks compared to SEC metric for a total of 70 behavioral tests included, which contains domains such as alertness, cognition, emotion, and personality. This supports our hypothesis that greater temporal variability increases the adaptability of brain networks, leading to better behavioral performance.

Admittedly, this work suffers from several drawbacks. First, the data did not cover cerebellum such that this brain region was not considered in the analysis. For future work, using restingstate data covering the whole brain for analysis of this kind is necessary. On the other hand, the switching pattern of brain network configurations in this work is similar to that of EEG microstates that have been shown to have fractal property (Van de Ville et al., 2010). In the future, a similar analysis using simultaneous EEG-fMRI data may allow for a comparison of quasi-stable network patterns obtained from EEG and fMRI.

\section{DATA AVAILABILITY STATEMENT}

The datasets generated for this study are available on request to the corresponding author.

\section{ETHICS STATEMENT}

The studies involving human participants were reviewed and approved by the IRB at University of Minnesota (for HCP data) and the IRB at Auburn University (for in-house MRI data). The patients/participants provided their written informed consent to participate in this study.

\section{AUTHOR CONTRIBUTIONS}

GD conceived the idea in consultation with HJ. HJ implemented the idea under guidance from GD. HJ wrote the first draft of the manuscript and GD substantially added and edited it.

\section{FUNDING}

Human Connectome Project (HCP) data used in the study was acquired using funding from the National Institutes of Health (NIH). The rest of the study was funded by the Auburn University MRI Research Center. 


\section{REFERENCES}

Achim, A. M., and Lepage, M. (2005). Neural correlates of memory for items and for associations: an event-related functional magnetic resonance imaging study. J. Cogn. Neurosci. 17, 652-667. doi: 10.1162/0898929053467578

Allen, E., Damaraju, E., Plis, S., Erhardt, E., Eichele, T., and Calhoun, V. (2013). Tracking whole-brain connectivity dynamics in the resting state. Cereb. Cortex 24, 663-676. doi: 10.1093/cercor/bhs352

Arnold, M., Miltner, W., Witte, H., Bauer, R., and Braun, C. (1998). Adaptive AR modeling of nonstationary time series by means of kalman filtering. IEEE Trans. Biomed. Eng. 45, 553-562. doi: 10.1109/10.668741

Aron, A., Fisher, H., Mashek, D., Strong, G., Li, H., and Brown, L. (2005). Reward, motivation, and emotion systems associated with early-stage intense romantic love. J. Neurophysiol. 94, 327-337. doi: 10.1152/jn.00838.2004

Bingel, U., Lorenz, J., Schoell, E., Weiller, C., and Büchel, C. (2006). Mechanisms of placebo analgesia: rACC recruitment of a subcortical antinociceptive network. Pain 120, 8-15. doi: 10.1016/j.pain.2005.08.027

Bliss, T., and Collingridge, G. (1993). A synaptic model of memory: long-term potentiation in the hippocampus. Nature 361, 31-39. doi: 10.1038/361031a0

Bogousslavsky, J., Miklossy, J., Deruaz, J., Assal, G., and Regli, F. (1987). Lingual and fusiform gyri in visual processing: a clinico-pathologic study of superior altitudinal hemianopia. J. Neurol. Neurosurg. Psychiatry 50, 607-614. doi: 10. 1136/jnnp.50.5.607

Bokde, L., Karmann, M., Teipel, S. J., Born, C., Lieb, M., Reiser, M. F., et al. (2009). Decreased activation along the dorsal visual pathway after a 3-month treatment with galantamine in mild Alzheimer disease: a functional magnetic resonance imaging study. J. Clin. Psychopharmacol. 29, 147-156. doi: 10.1097/ JCP.0b013e31819a8f2e

Bookheimer, S., Zeffiro, T., Blaxton, T., Gaillard, P., and Theodore, W. (2000). Activation of language cortex with automatic speech tasks. Neurology 8, 1151-1157. doi: 10.1212/wnl.55.8.1151

Brainmap.Org (2019). Available at: http://www.brainmap.org/sleuth/. (accessed August 31, 2015).

Britz, J., Van De Ville, D., and Michel, C. (2010). BOLD correlates of EEG topography reveal rapid resting-state network dynamics. Neuroimage 52, $1162-$ 1170. doi: 10.1016/j.neuroimage.2010.02.052

Chang, C., and Glover, G. H. (2010). Time-frequency dynamics of resting-state brain connectivity measured with fMRI. Neuroimage 50, 81-98. doi: 10.1016/j. neuroimage.2009.12.011

Chang, C., Liu, Z., Chen, M., Liu, X., and Duyn, J. (2013a). EEG correlates of timevarying BOLD functional connectivity. Neuroimage 72, 227-236. doi: 10.1016/ j.neuroimage.2013.01.049

Chang, C., Metzger, C., Glover, G., Duyn, J., Heinze, H., and Walter, M. (2013b). Association between heart rate variability and fluctuations in resting-state functional connectivity. Neuroimage 68, 93-104. doi: 10.1016/j.neuroimage. 2012.11.038

Craddock, R., James, G., Holtzheimer, P., Hu, X., and Mayberg, H. (2012). A whole brain fMRI atlas generated via spatially constrained spectral clustering. Hum. Brain Mapp. 33, 1914-1928. doi: 10.1002/hbm.21333

Cribben, I., Haraldsdottir, R., Atlas, L., Wager, T., and Lindquist, M. (2012). Dynamic connectivity regression: determining state-related changes in brain connectivity. Neuroimage 61, 907-920. doi: 10.1016/j.neuroimage.2012.03.070

Damoiseaux, J., Rombouts, S., Barkhof, F., Scheltens, P., Stam, C., Smith, S., et al. (2006). Consistent resting-state networks across healthy subjects. Proc. Natl. Acad. Sci. U.S.A. 103, 13848-13853. doi: 10.1073/pnas.0601417103

De Luca, M., Beckmann, C., De Stefano, N., Matthews, P., and Smith, S. (2006). fMRI resting state networks define distinct modes of long-distance interactions in the human brain. Neuroimage 29, 1359-1367. doi: 10.1016/j.neuroimage. 2005.08.035

Deshpande, G., and Hu, X. (2012). Investigating effective brain connectivity from fMRI data: past findings and current issues with reference to granger causality analysis. Brain Connectivity 2, 235-245. doi: 10.1089/brain.2012.0091

Deshpande, G., Hu, X., Lacey, S., Stilla, R., and Sathian, K. (2010a). Object familiarity modulates effective connectivity during haptic shape perception. Neuroimage 49, 1991-2000. doi: 10.1016/j.neuroimage.2009.08.052

Deshpande, G., Hu, X., Stilla, R., and Sathian, K. (2008). Effective connectivity during haptic perception: a study using granger causality analysis of functional magnetic resonance imaging data. Neuroimage 40, 1807-1814. doi: 10.1016/j. neuroimage.2008.01.044
Deshpande, G., LaConte, S., James, G., Peltier, S., and Hu, X. (2009a). Multivariate Granger causality analysis of brain networks. Hum. Brain Mapp. 30, 1361-1373. doi: 10.1002/hbm.20606

Deshpande, G., LaConte, S., James, G., Peltier, S., and Hu, X. (2009b). Multivariate Granger causality analysis of fMRI data. Hum. Brain Mapp. 30, 1361-1373. doi: 10.1002/hbm.20606

Deshpande, G., LaConte, S., Peltier, S., and Hu, X. (2006). Connectivity analysis of human functional MRI data:from linear to nonlinear and static to dynamic. Lecture Notes Comput. Sci. 4091, 17-24. doi: 10.1007/11812715_3

Deshpande, G., Libero, L., Sreenivasan, K., Deshpande, H., and Kana, R. (2013). Identification of neural connectivity signatures of autism using machine learning. Front. Hum. Neurosci. 7:670. doi: 10.3389/fnhum.2013.00670

Deshpande, G., Priya, S., and Hu, X. (2011a). Instantaneous and causal connectivity in resting state brain networks derived from functional MRI data. Neuroimage 54, 1043-1052. doi: 10.1016/j.neuroimage.2010.09.024

Deshpande, G., Santhanam, P., and Hu, X. (2011b). Instantaneous and causal connectivity in resting state brain networks derived from functional MRI data. Neuroimage 54, 1043-1052. doi: 10.1016/j.neuroimage.2010. 09.024

Deshpande, G., Sathian, K., and Hu, Z. (2010b). Assessing and compensating for zero-lag correlation effects in time-lagged granger causality analysis of fMRI. IEEE Trans. Biomed. Eng. 57, 1446-1456. doi: 10.1109/TBME.2009.203 7808

Dimitriadis, S., Laskaris, N., Tsirka, V., Vourkas, M., and Micheloyannis, S. (2012). An EEG study of brain connectivity dynamics at the resting state. Nonlinear Dyn. Psychol. Life Sci. 16, 5-22.

Ellermann, J., Siegal, J., Strupp, J., Ebner, T., and Ugurbil, K. (1998). Activation of visuomotor systems during visually guided movements: a functional MRI study. J. Magn. Reson. 131, 272-285. doi: 10.1006/jmre.1998.1379

Feng, C., Deshpande, G., Liu, C., Gu, R., Luo, Y., and Krueger, F. (2016). Diffusion of responsibility attenuates altruistic punishment: a functional magnetic resonance imaging effective connectivity study. Hum. Brain Mapp. 37, 663-677. doi: 10.1002/hbm.23057

Feng, C., Feng, X., Wang, L., Gu, R., Ni, A., Deshpande, G., et al. (2018). The neural signatures of egocentric bias in normative decision making. Brain Imaging Behav. 13, 685-698. doi: 10.1007/s11682-018-9893-1

Fornito, A., Harrison, B., Zalesky, A., and Simons, J. (2012). Competitive and cooperative dynamics of large-scale brain functional networks supporting recollection. Proc. Natl. Acad. Sci. U.S.A. 109, 12788-12793. doi: 10.1073/pnas. 1204185109

Frassle, S., Lomakina, E., Kasper, L., Manjaly, Z., Leff, A., Pruessmann, K., et al. (2018). A generative model of whole-brain effective connectivity. Neuroimage 179, 505-529. doi: 10.1016/j.neuroimage.2018.05.058

Friston, K. (1994). Functional and effective connectivity in neuroimaging: a synthesis. Hum. Brain Mapp. 2, 56-78. doi: 10.1002/hbm.46002 0107

Friston, K., Frith, C., Liddle, P., and Frackowiak, R. (1993). Functional connectivity: the principal-component analysis of large (PET) data sets. J. Cereb. Blood Flow Metab. 13, 5-14. doi: 10.1038/jcbfm.1993.4

Friston, K., Harrison, L., and Penny, W. (2003). Dynamic causal modeling. Neuroimage 19, 1273-1302.

Fu, S., Chen, Y., Smith, S. M., Iversen, S. D., and Matthews, P. M. (2002). Effects of word form on brain processing of written Chinese. Neuroimage 17, 1538-1548. doi: 10.1006/nimg.2002.1155

Garrett, A. S., and Maddock, R. J. (2006). Separating subjective emotion from the perception of emotion-inducing stimuli: an fMRI study. Neuroimage 33, 263-274. doi: 10.1016/j.neuroimage.2006.05.024

Geweke, J. (1982). Measurement of linear dependence and feedback between multiple time series. J. Am. Stat. Assoc. 77, 304-313. doi: 10.1080/01621459. 1982.10477803

Grahn, J., Parkinson, J., and Owen, A. (2008). The cognitive functions of the caudate nucleus. Prog. Neurobiol. 86, 141-155. doi: 10.1016/j.pneurobio.2008. 09.004

Granger, C. (1969). Investigating causal relations by econometric models and cross-spectral methods. Econometrica 37, 424-438.

Grant, M., White, D., Hadley, J., Hutcheson, N., Shelton, R., Sreenivasan, K., et al. (2014). Early life trauma and directional brain connectivity within major depression. Hum. Brain Mapp. 35, 4815-4826. doi: 10.1002/hbm. 22514 
Grant, M., Wood, K., Sreenivasan, K., Wheelock, M., White, D., Thomas, J., et al. (2015). Influence of early life stress on intra- and extra-amygdaloid causal connectivity. Neuropsychopharmacology 40, 1782-1793. doi: 10.1038/npp. 2015.28

Graybiel, A. M. (2005). The basal ganglia: learning new tricks and loving it. Curr. Opin. Neurobiol. 15, 638-644. doi: 10.1016/j.conb.2005.10.006

Greicius, M., Supekar, K., Menon, V., and Dougherty, R. (2009). Resting-state functional connectivity reflects structural connectivity in the default mode network. Cereb. Cortex 1, 72-78. doi: 10.1093/cercor/bhn059

Greicius, M. D., Flores, B., Menon, V., Glover, G., Solvason, H., Kenna, H., et al. (2007). Resting-state functional connectivity in major depression: abnormally increased contributions from subgenual cingulate cortex and thalamus. Biol. Psychiatry 5, 429-437. doi: 10.1016/j.biopsych.2006.09.020

Guye, M., Bettus, G., Bartolomei, F., and Cozzone, P. (2010). Graph theoretical analysis of structural and functional connectivity MRI in normal and pathological brain networks. MAGMA 5-6, 409-421. doi: 10.1007/s10334-0100205-z

Hampstead, B., Khoshnoodi, M., Yan, W., Deshpande, G., and Sathian, K. (2016). Patterns of effective connectivity between memory encoding and retrieval differ between patients with mild cognitive impairment and healthy older adults. Neuroimage 124, 997-1008. doi: 10.1016/j.neuroimage.2015.10.002

Hampstead, B. M., Stringer, A., Stilla, R., Deshpande, G., Hu, X., Moore, A., et al. (2011). Activation and effective connectivity changes following explicit-memory training for face-name pairs in patients with mild cognitive impairment: a pilot study. Neurorehabil. Neural Repair 25, 210-222. doi: 10. $1177 / 1545968310382424$

Han, C. E., Yoo, S., Seo, S., Na, D., and Seong, J. (2013). Cluster-based statistics for brain connectivity in correlation with behavioral measures. PLoS One 8:e72332. doi: 10.1371/journal.pone.0072332

Handwerker, D., Roopchansingh, V., Gonzalez-Castillo, J., and Bandettini, P. (2012). Periodic changes in fMRI connectivity. Neuroimage 63, 1712-1719. doi: 10.1016/j.neuroimage.2012.06.078

Havlicek, M., Jan, J., Milan, B., and Calhoun, V. D. (2010). Dynamic Granger causality based on Kalman filter for evaluation of functional network connectivity in fMRI data. Neuroimage 53, 65-77. doi: 10.1016/j.neuroimage. 2010.05.063

Hu, S., Wei, N., Wang, Q., Yan, L., Wei, E., Zhang, M., et al. (2008). Patterns of brain activation during visually evoked sexual arousal differ between homosexual and heterosexual men. Am. J. Neuroradiol. 29, 1890-1896. doi: 10.3174/ajnr.A1260

Hutcheson, N., Sreenivasan, K., Deshpande, G., Reid, M., Hadley, J., White, D., et al. (2015). Effective connectivity during episodic memory retrieval in Schizophrenia participants before and after antipsychotic medication. Hum. Brain Mapp. 36, 1442-1457. doi: 10.1002/hbm.22714

Hutchison, R., Womelsdorf, T., Allen, E., Bandettini, P., Calhoun, V., Corbetta, M., et al. (2013). Dynamic functional connectivity: promise, issues, and interpretations. Neuroimage 80, 360-378. doi: 10.1016/j.neuroimage.2013. 05.079

Hutchison, R., Womelsdorf, T., Gati, J., Everling, S., and Menon, R. (2012). Restingstate networks show dynamic functional connectivity in awake humans and anesthetized macaques. Hum. Brain Mapp. 34, 2154-2177. doi: 10.1002/hbm. 22058

Inman, C. S., James, G., Hamann, S., Rajendra, J., Pagnoni, G., and Butler, A. (2012). Altered resting-state effective connectivity of fronto-parietal motor control systems on the primary motor network following stroke. Neuroimage 1, 227-237. doi: 10.1016/j.neuroimage.2011.07.083

Ishizu, T., and Zeki, S. (2011). Toward a brain-based theory of beauty. PLoS One 6:e21852. doi: 10.1371/journal.pone.0021852

Jia, H., Hu, X., and Deshpande, G. (2014). Behavioral relevance of the dynamics of the functional brain connectcome. Brain Connect. 4, 741-759. doi: 10.1089/ brain. 2014.0300

Jin, C., Jia, H., Lanka, P., Rangaprakash, D., Li, L., Liu, T., et al. (2017). Dynamic brain connectivity is a better predictor of PTSD than static connectivity. Hum. Brain Mapp. 38, 4479-4496. doi: 10.1002/hbm.23676

Joe, H., and Ward, J. (1963). Hierarchical Grouping to Optimize an Objective Function. J. Am. Stat. Assoc. 58, 236-244. doi: 10.1080/01621459.1963. 10500845

Kalcher, K., Huf, W., Boubela, R., Filzmoser, P., Pezawas, L., Biswal, B., et al. (2012). Fully exploratory network independent component analysis of the 1000 functional connectomes database. Front. Hum. Neurosci. 6:301. doi: 10.3389/ fnhum.2012.00301

Karnath, H., Baier, B., and Nägele, T. (2005). Awareness of the functioning of one's own limbs mediated by the insular cortex? J. Neurosci. 25, 7134-7138. doi: 10.1523/jneurosci.1590-05.2005

Keilholz, S., Magnuson, M., Pan, W., Willis, M., and Thompson, G. (2013). Dynamic properties of functional connectivity in the rodent. Brain Connect. 3, 31-40. doi: 10.1089/brain.2012.0115

Kelly, A. M., Uddin, L., Biswal, B., Castellanos, F., and Milham, M. (2008). Competition between functional brain networks mediates behavioral variability. Neuroimage 39, 527-537. doi: 10.1016/j.neuroimage.2007.08.008

Kent, B., Drane, J., Blumenstein, B., and Manning, J. (1972). A mathematical model to assess changes in the baroreceptor reflex. Cardiology 57, 295-310. doi: $10.1159 / 000169528$

Kinnison, J., Padmala, S., Choi, J., and Pessoa, L. (2012). Network analysis reveals increased integration during emotional and motivational processing. J. Neurosci. 32, 8361-8372. doi: 10.1523/JNEUROSCI.0821-12.2012

Krueger, F., Landgraf, S., van der Meer, E., Deshpande, G., and Hu, X. (2011). Effective connectivity of the multiplication network: a functional MRI and multivariate granger causality mapping study. Hum. Brain Mapp. 32, 14191431. doi: 10.1002/hbm.21119

Kus, R., Kaminski, M., and Blinowska, K. (2004). Determination of EEG activity propagation: pair-wise versus multichannel estimate. IEEE Trans. Biomed. Eng. 51, 1501-1510. doi: 10.1109/tbme.2004.827929

Lacey, S., Stilla, R., Sreenivasan, K., Deshpande, G., and Sathian, K. (2014). Spatial imagery in haptic shape perception. Neuropsychologia 60, 144-158. doi: 10. 1016/j.neuropsychologia.2014.05.008

Lee, H., Zahneisen, B., Hugger, T., LeVan, P., and Hennig, J. (2013). Tracking dynamic resting-state networks at higher frequencies using MRencephalography. Neuroimage 65, 216-222. doi: 10.1016/j.neuroimage.2012. 10.015

Lee, M., Hacker, C., Snyder, A., Corbetta, M., Zhang, D., Leuthardt, E., et al. (2012). Clustering of resting state networks. PLoS One 7:e40370. doi: 10.1371/journal. pone. 0040370

Lee, T., Josephs, O., Dolan, R., and Critchley, H. (2006). Imitating expressions: emotion-specific neural substrates in facial mimicry. Soc. Cogn. Affect. Neurosci. 1, 122-135. doi: 10.1093/scan/ns1012

Leonardi, N., Richiardi, J., Gschwind, M., Simioni, S., Annoni, J., Schluep, M., et al. (2013). Principal components of functional connectivity: a new approach to study dynamic brain connectivity during rest. Neuroimage 83, 937-950. doi: 10.1016/j.neuroimage.2013.07.019

Li, X., Zhu, D., Jiang, X., Jin, C., Zhang, X., Guo, L., et al. (2014). Dynamic functional connectomics signatures for characterization and differentiation of PTSD patients. Hum. Brain Mapp. 35, 1761-1778. doi: 10.1002/hbm.22290

Liang, P., Li, Z., Deshpande, G., Wang, Z., Hu, X., and Li, K. (2014). Altered causal connectivity of resting state brain networks in amnesic MCI. PLoS One 9:e88476. doi: 10.1371/journal.pone.0088476

Liao, W., Qiu, C., Gentili, C., Walter, M., Pan, Z., Ding, J., et al. (2010). Altered effective connectivity network of the amygdala in social anxiety disorder: a resting-state FMRI study. PLoS One 12:e15238. doi: 10.1371/journal.pone. 0015238

Liljeström, M., Tarkiainen, A., Parviainen, T., Kujala, J., Numminen, J., Hiltunen, J., et al. (2008). Perceiving and naming actions and objects. Neuroimage 41, 1132-1141. doi: 10.1016/j.neuroimage.2008.03.016

Lissek, S., Hausmann, M., Knossalla, F., Peters, S., Nicolas, V., Güntürkün, O., et al. (2007). Sex differences in cortical and subcortical recruitment during simple and complex motor control: an fMRI study. Neuroimage 37, 912-926. doi: 10.1016/j.neuroimage.2007.05.037

Lohmann, G., Erfurth, K., Muller, K., and Turner, R. (2012). Critical comments on dynamic causal modelling. NeuroImage 59, 2322-2329. doi: 10.1016/j. neuroimage.2011.09.025

Majeed, W., Magnuson, M., Hasenkamp, W., Schwar, H., Schumacher, E., Barsalou, L., et al. (2011). Spatiotemporal dynamics of low frequency BOLD fluctuations in rats and humans. Neuroimage 54, 1140-1150. doi: 10.1016/j.neuroimage. 2010.08.030

Malhi, G., Owen, L. J. A., Ivanovski, B., Shnier, R., and Sachdev, P. (2007). Reduced activation to implicit affect induction in euthymic bipolar patients: an fMRI study. J. Affect. Disord. 97, 109-122. doi: 10.1016/j.jad.2006.06.005 
McDowall, L., and Dampney, R. (2006). Calculation of threshold and saturation points of sigmoidal baroreflex function curves. Am. J. Physiol. Heart Circ. Physiol. 291, H2003-H2007.

McIntosh, A., and Gozales-Lima, F. (1994). Structural equation modelling and its application to network analysis in functional brain imaging. Hum. Brain Mapp. $2,2-22$.

Medaglia, J., Chiou, K., Slocomb, J., Fitzpatrick, N., Wardecker, B., Ramanathan, D., et al. (2012). The less BOLD, the wiser: support for the latent resource hypothesis after traumatic brain injury. Hum. Brain Mapp. 33, 979-993. doi: $10.1002 / \mathrm{hbm} .21264$

Morecraft, R., Geula, C., and Mesulam, M. (1992). Cytoarchitecture and neural afferents of orbitofrontal cortex in the brain of the monkey. J. Comp. Neurol. 323, 341-358. doi: 10.1002/cne.903230304

Moussa, M., Steen, M., Laurienti, P., and Hayasaka, S. (2012). Consistency of network modules in resting-state FMRI connectome data. PLoS One 7:e44428. doi: 10.1371/journal.pone.0044428

Muschelli, J., Nebel, M., Caffo, B., Barber, A., Pekar, J., and Mostofsky, S. (2014). Reduction of motion-related artifacts in resting state fMRI using aCompCor. Neuroimage 96, 22-35. doi: 10.1016/j.neuroimage.2014.03.028

Musso, F., Brinkmeyer, J., Mobascher, A., Warbrick, T., and Winterer, G. (2010). Spontaneous brain activity and EEG microstates. A novel EEG/fMRI analysis approach to explore resting-state networks. Neuroimage 52, 1149-1161. doi: 10.1016/j.neuroimage.2010.01.093

Rack-Gomer, A. L., and Liu, T. (2012). Caffeine increases the temporal variability of resting-state BOLD connectivity in the motor cortex. Neuroimage 59, 2994 3002. doi: 10.1016/j.neuroimage.2011.10.001

Ramaihgari, B., Pustovyy, O., Waggoner, P., Beyers, R., Wildey, C., Morrison, E., et al. (2018). Zinc nanoparticles enhance brain connectivity in the canine olfactory network: evidence from an fMRI study in unrestrained awake dogs. Front. Vet. Sci. 5:127. doi: 10.3389/fvets.2018.00127

Rangaprakash, D., Dretsch, M., Venkataraman, A., Katz, J., Denney, T., and Deshpande, G. (2018). Identifying disease foci from static and dynamic effective connectivity networks: Illustration in Soldiers with trauma. Hum. Brain Mapp. 39, 264-287. doi: 10.1002/hbm.23841

Rao, N., Deshpande, G., Gangadhar, K., Arasappa, R., Varambally, S., Venkatasubramaian, G., et al. (2018). Directional brain networks underlying OM chanting. Asian J. Psychiatry 37, 20-25. doi: 10.1016/j.ajp.2018.08.001

Ricciardi, E., Bonino, D., Gentili, C., Sani, L., Pietrini, P., and Vecchi, T. (2006). Neural correlates of spatial working memory in humans: a functional magnetic resonance imaging study comparing visual and tactile processes. Neuroscience 139, 339-349. doi: 10.1016/j.neuroscience.2005.08.045

Roebroeck, A., Formisano, E., and Goebel, R. (2005). Mapping directed influence over the brain using Granger causality and fMRI. Neuroimage 25, 230-242. doi: 10.1016/j.neuroimage.2004.11.017

Rogers, B. P., Katwald, S. B., Morgana, V. L., and Asplunde, C. L. C. (2010). Functional MRI and multivariate autoregressive models. Magn. Reson. Imaging 28, 1058-1065. doi: 10.1016/j.mri.2010.03.002

Rousseeuw, P. J. (1987). Silhouettes: a graphical aid to the interpretation and validation of cluster analysis. J. Comput. Appl. Math. 20, 53-65. doi: 10.1016/ 0377-0427(87)90125-7

Sailer, U., Robinson, S., Fischmeister, F. P., Moser, E., Krypsin, I.-E., and Bauer, H. (2007). Imaging the changing role of feedback during learning in decisionmaking. Neuroimage 37, 1474-1486. doi: 10.1016/j.neuroimage.2007.07.012

Sakoð̋lu, U., Pearlson, G., Kiehl, K., Wang, Y., Michael, A., and Calhoun, V. (2010). A method for evaluating dynamic functional network connectivity and taskmodulation: application to schizophrenia. MAGMA 23, 351-366. doi: 10.1007/ s10334-010-0197-8

Sathian, K., Deshpande, G., and Stilla, R. (2013). Neural changes with tactile learning reflect decision level reweighting of perceptual readout. J. Neurosci. 33, 5387-5398. doi: 10.1523/JNEUROSCI.3482-12.2013

Sathian, K., Lacey, S., Stilla, R., Gibson, G., Deshpande, G., Hu, X., et al. (2011). Dual pathways for haptic and visual perception of spatial and texture information. Neuroimage 57, 462-475. doi: 10.1016/j.neuroimage.2011.05.001

Sato, J., Junior, E., Takahashi, D., de Maria, F. M., Brammer, M., and Morettin, P. (2006). A method to produce evolving functional connectivity maps during the course of an fMRI experiment using wavelet-based time-varying Granger causality. Neuroimage 31, 187-196. doi: 10.1016/j.neuroimage.2005.11.039
Schlogl, A., Roberts, S., and Pfurtscheller, G. (2000). "A criterion for adaptive autoregressive models," in Proceedings of the 22nd IEEE International Conference on Engineering in Medicine and Biology (Piscataway, NJ: IEEE).

Schöpf, V., Kasess, C., Lanzenberger, R., Fischmeister, F., Windischberger, C., and Moser, E. (2010). Fully exploratory network ICA (FENICA) on resting-state fMRI data. J. Neurosci. Methods 192, 207-213. doi: 10.1016/j.jneumeth.2010. 07.028

Schöpf, V., Windischberger, C., Robinson, S., Kasess, C., Fischmeister, F., Lanzenberger, R., et al. (2011). Model-free fMRI group analysis using FENICA. Neuroimage 55, 185-193. doi: 10.1016/j.neuroimage.2010.11.010

Schwartz, G. (1978). Estimating the dimension of a model. Ann. Stat. 5, 461-464. doi: $10.1214 / \mathrm{aos} / 1176344136$

Shapira, N. A., Liu, Y., He, A. G., Bradley, M. M., Lessig, M. C., James, G. A., et al. (2003). Brain activation by disgust-inducing pictures in obsessive-compulsive disorder. Biol. Psychiatry 54, 751-756. doi: 10.1016/s0006-3223(03)00003-9

Shirer, W., Ryali, S., Rykhlevskaia, E., Menon, V., and Greicius, M. (2012). Decoding subject-driven cognitive states with whole-brain connectivity patterns. Cereb. Cortex 22, 158-165. doi: 10.1093/cercor/bhr099

Stephan, K., and Roebroeck, A. (2012). A short history of causal modeling of fMRI data. Neuroimage 62, 856-863. doi: 10.1016/j.neuroimage.2012.01.034

Stilla, R., Deshpande, G., Laconte, S., Hu, X., and Sathian, K. (2007). Posteromedial parietal cortical activity and inputs predict tactile spatial acuity. J. Neurosci. 27, 11091-11102. doi: 10.1523/jneurosci.1808-07.2007

Stilla, R., Hanna, R., Mariola, E., Deshpande, G., Hu, X., and Sathian, K. (2008). Neural processing underlying tactile microspatial discrimination in the blind: a functional magnetic resonance imaging study. J. Vis. 8, 13.1-19. doi: 10.1167/8. 10.13

Strathearn, L., Li, J., Fonagy, P., and Montague, P. (2008). What's in a smile? maternal brain responses to infant facial cues. Pediatrics 122, 40-51. doi: 10. 1542/peds.2007-1566

Syed, M., Yang, Z., Hu, X., and Deshpande, G. (2017). Investigating brain connectomic alterations in autism using the reproducibility of independent components derived from resting state functional MRI data. Front. Neuroinform. 11:459. doi: 10.3389/fnins.2017.00459

Syed, M., Yang, Z., Rangaprakash, D., Hu, X., Dretsch, M., Katz, J., et al. (2019). DisConICA: a software package for assessing reproducibility of brain networks and their discriminability across disorders. Neuroinformatics doi: 10.1007/ s12021-019-09422-1 [Epub ahead of print],

Tagliazucchi, E., von Wegner, F., Morzelewski, A., Brodbeck, V., and Laufs, H. (2012). Dynamic BOLD functional connectivity in humans and its electrophysiological correlates. Front. Hum. Neurosci. 6:339. doi: 10.3389/ fnhum.2012.00339

Theiler, J., Eubank, S., Longtin, A., Galdrikian, B., and Farmer, D. (1992). Testing for nonlinearity in time series:The method of surrogate data. Physica D 58, 77-94. doi: 10.1016/0167-2789(92)90102-s

Valdes-Sosa, P., Roebroeck, A., and Daunizeau, J. F. K. (2011). Effective connectivity: influence, causality and biophysical modeling. Neuroimage 58, 339-361. doi: 10.1016/j.neuroimage.2011.03.058

van de Ven, V., Formisano, E., Prvulovic, D., Roeder, C., and Linden, D. (2004). Functional connectivity as revealed by spatial independent component analysis of fMRI measurements during rest. Hum. Brain Mapp. 22, 165-178. doi: 10. $1002 / \mathrm{hbm} .20022$

Van de Ville, D., Britz, J., and Michel, C. (2010). EEG microstate sequences in healthy humans at rest reveal scale-free dynamics. Proc. Natl. Acad. Sci. U.S.A. 42, 18179-18184. doi: 10.1073/pnas.1007841107

Wang, N., Zeng, W., and Chen, L. (2012). A fast-FENICA method on resting state fMRI data. J. Neurosci. Methods 209, 1-12. doi: 10.1016/j.jneumeth.2012. 05.007

Wang, Y., Katwal, S., Rogers, B., Gore, J., and Deshpande, G. (2017). Experimental validation of dynamic Granger causality for inferring stimulus-evoked sub-100 ms timing differences from fMRI. IEEE Trans. Neural Syst. Rehabil. Eng. 25, 539-546. doi: 10.1109/TNSRE.2016.2593655

Wen, X., Rangarajan, G., and Ding, M. (2013). Is granger causality a viable technique for analyzing fMRI data? PLoS One 8:e67428. doi: 10.1371/journal. pone.0067428

Wheelock, M., Sreenivasan, K., Wood, K., Ver Hoeff, L., Deshpande, G., and Knight, D. (2014). Threat-related learning relies on distinct dorsal 
prefrontal cortex network connectivity. Neuroimage 102, 904-912. doi: 10.1016/ j.neuroimage.2014.08.005

Wu, G., Stramaglia, S., Chen, H., Liao, W., and Marinazzo, D. (2013). Mapping the voxel-wise effective connectome in resting state fMRI. PLoS One 8:e73670. doi: 10.1371/journal.pone.0073670

Xu, K. S., Kliger, M., and Hero, A. O. (2013). Adaptive evolutionary clustering. Data Min. Knowl. Discov. 28, 304-336.

Yan, C.-G., and Zang, Y.-F. (2010). Available: http://www.restfmri.net (accessed August 31, 2015).

Zeng, L., Shen, H., Liu, L., Wang, L., Li, B., Fang, P., et al. (2012). Identifying major depression using whole-brain functional connectivity: a multivariate pattern analysis. Brain 135, 1498-1507. doi: 10.1093/brain/aws059

Zhao, S., Rangaprakash, D., Venkataraman, A., Liang, P., and Deshpande, G. (2017). Investigating focal connectivity deficits in Alzheimer's disease using directional brain networks derived from resting-state fMRI. Front. Aging Neurosci. 9:211.

Zhao, X., Rangaprakash, D., Yuan, B., Denney, T., Katz, J., Dretsch, M., et al. (2018). Investigating the correspondence of clinical diagnostic grouping with underlying neurobiological and phenotypic clusters using unsupervised machine learning. Front. Appl. Math. Stat. 4:25. doi: 10.3389/fams.2018. 00025

Zhuang, J., LaConte, S., Peltier, S., Zhang, K., and Hu, H. (2005). Connectivity exploration with structural equation modeling: an fMRI study of bimanual motor coordination. Neuroimage 25, 462-470. doi: 10.1016/j.neuroimage.2004. 11.007

Conflict of Interest: The authors declare that the research was conducted in the absence of any commercial or financial relationships that could be construed as a potential conflict of interest.

Copyright (c) 2020 Deshpande and Jia. This is an open-access article distributed under the terms of the Creative Commons Attribution License (CC BY). The use, distribution or reproduction in other forums is permitted, provided the original author(s) and the copyright owner(s) are credited and that the original publication in this journal is cited, in accordance with accepted academic practice. No use, distribution or reproduction is permitted which does not comply with these terms. 


\section{OPEN ACCESS}

Edited by:

Nicoletta Cera,

University of Porto, Portugal

Reviewed by:

Yann Quidé,

School of Psychiatry, UNSW, Australia

Silvia Tommasin,

Sapienza University of Rome, Italy

${ }^{*}$ Correspondence:

Jennifer Kornelsen

jennifer.kornelsen@umanitoba.ca

tThese authors have contributed equally to this work

Specialty section:

This article was submitted to Applied Neuroimaging,

a section of the journal

Frontiers in Neurology

Received: 06 November 2019 Accepted: 14 January 2020

Published: 18 February 2020

Citation:

Kornelsen J, Wilson A, Labus JS,

Witges K, Mayer EA and Bernstein CN (2020) Brain Resting-State Network

Alterations Associated With Crohn's

Disease. Front. Neurol. 11:48.

doi: 10.3389/fneur.2020.00048

\section{Brain Resting-State Network Alterations Associated With Crohn's Disease}

\author{
Jennifer Kornelsen ${ }^{1,2 \times t}$, Alyssia Wilson ${ }^{3 \dagger}$, Jennifer S. Labus ${ }^{4}$, Kelcie Witges ${ }^{2}$, \\ Emeran A. Mayer ${ }^{4}$ and Charles N. Bernstein ${ }^{2,3}$ \\ ${ }^{1}$ Department of Radiology, University of Manitoba, Winnipeg, MB, Canada, ${ }^{2}$ IBD Clinical and Research Centre, University of \\ Manitoba, Winnipeg, MB, Canada, ${ }^{3}$ Department of Internal Medicine, University of Manitoba, Winnipeg, MB, Canada, \\ ${ }^{4}$ G. Oppenheimer Family Center for Neurobiology of Stress and Resilience, David Geffen School of Medicine at UCLA, \\ Los Angeles, CA, United States
}

Inflammatory bowel disease (IBD) is a chronic disease that is associated with aspects of brain anatomy and activity. In this preliminary MRI study, we investigated differences in brain structure and in functional connectivity $(\mathrm{FC})$ of brain regions in 35 participants with Crohn's disease $(\mathrm{CD})$ and 21 healthy controls $(\mathrm{HC})$. Voxel-based morphometry (VBM) analysis was performed to contrast $\mathrm{CD}$ and $\mathrm{HC}$ structural images. Region of interest (ROI) analyses were run to assess FC for resting-state network nodes. Independent component analysis (ICA) identified whole brain differences in FC associated with resting-state networks. Though no structural differences were found, ROI analyses showed increased FC between the frontoparietal (FP) network and salience network $(\mathrm{SN})$, and decreased FC between nodes of the default mode network (DMN). ICA results revealed changes involving cerebellar (CER), visual (VIS), and SN components. Differences in FC associated with sex were observed for both ROI analysis and ICA. Taken together, these changes are consistent with an influence of CD on the brain and serve to direct future research hypotheses.

Keywords: Crohn's disease (CD), resting-state networks (RSNs), functional connectivity (FC), functional magnetic resonance imaging (fMRI), inflammatory bowel disease (IBD)

\section{INTRODUCTION}

Inflammatory bowel disease (IBD) consists of two main conditions, Crohn's disease (CD) and ulcerative colitis (UC) (1), which are rising in prevalence around the world (2). The main working etiologic hypothesis is that in persons genetically predisposed to develop $\mathrm{CD}$, the gut microbiome triggers a maladaptive immune response causing chronic gut inflammation $(1,3)$. Patient symptoms may vary in severity and activity, but normally include abdominal pain, diarrhea, and weight loss, which can greatly affect quality of life and mental health (4). Chronic disorders, such as CD not only affect physical health, but also mental health, thereby increasing the chances of acquiring comorbid mood disorders including depression or anxiety (5-7). In fact, it has also been shown that persons with $\mathrm{CD}$ are more likely to have depression or anxiety antedating $\mathrm{CD}$ diagnosis by years, suggesting possible shared risk factors for both chronic mental health disorders and CD $(8,9)$. Physiologically, as a threat to homeostasis, there are a number of mechanisms by which stress may impact the gastrointestinal tract (10). Mood and CD demonstrate a reciprocal effect on stress and flare-ups through the brain-gut axis (11). Stress (12-14), pain (15-18), and mood disorders $(15,19-21)$ are all factors that influence structure and function of the brain. 
Investigations into altered brain structure and function in $\mathrm{CD}$ have increased; however, the existing body of literature is limited and there is a lack of consistency among the techniques used and the results reported that prohibits any key brain alterations to be identified. Studies of brain structure in $\mathrm{CD}$ may be in agreement on one result, altered gray matter (GM) of the superior frontal gyrus, although laterality and direction of alteration are inconsistent (22-24). Studies of brain function in $\mathrm{CD}$ have reported numerous FC alterations with very little overlap (25-32) aside from some agreement on changes in brain networks or regions involved in executive function and/or default mode $(26,30,32)$. Our study aimed to identify brain structural and functional changes in $\mathrm{CD}$ as compared to HC. In an attempt to isolate the relationship of $\mathrm{CD}$ and the brain in the absence of psychiatric comorbidity, the study exclusion criteria include depression and anxiety. Differences between groups were expected for both GM volume and functional connectivity (FC). Sex differences in FC were also expected. Voxel-based morphometry was used to assess structural difference in GM volume, and both region of interest (ROI) analysis and independent component analysis (ICA) were used to detect differences in the FC of resting-state networks (RSNs). By employing three methods of assessing change in brain structure or function in the same study participants, this preliminary approach will identify changes in the brain that are associated with $\mathrm{CD}$ and provide hypothesis-formation guidance for future work.

\section{METHODS}

\section{Participants}

This study was approved by the University of Manitoba Research Ethics Board and UCLA Institutional Review Board and all participants signed an informed consent form. For this study, 35 individuals with $\mathrm{CD}$ [18 males and 17 females, mean age $32.6 \pm 11.4$ years, body mass index (BMI) $25.5 \pm 4.4 ; 17$ at UCLA and 18 at UM] were recruited. Patients had been previously diagnosed with $\mathrm{CD}$ and were recruited through their previous involvement in a local longitudinal IBD cohort study, a provincial-based research registry, regional gastroenterology clinics, and via website (ibdmanitoba.org). We reviewed medical records to confirm IBD diagnoses and contacted physicians for additional information as needed. Twenty-one healthy controls (HC) (14 males, 7 females, mean age $26.9 \pm 10.0$ years, $\mathrm{BMI}=25.5 \pm 4.9 ; 16$ at UCLA and 5 at UM) with no history of gastrointestinal diagnosis or symptoms were enlisted.

The inclusion criteria for $\mathrm{CD}$ patients included righthanded, ambulatory English-speaking adults aged between 18 and 55 years. Exclusion criteria included non-CDrelated structural abnormalities of the gastrointestinal tract, additional gastrointestinal disease or non-CD-related surgery, or involvement in an ongoing clinical trial. Inclusion criteria for $\mathrm{HC}$ were age between 18 and 55 years, and no gastrointestinal history or a diagnosis or symptoms of irritable bowel syndrome (IBS) meeting the ROME III diagnostic criteria. Exclusion criteria for all subjects included left-handedness, illicit drug use, any neurological or psychiatric conditions (including depression and anxiety), morbid obesity (BMI > 35), post-menopausal status, or MRI incompatibility.

\section{Study Design}

Before imaging, all patients and controls provided written informed consent. Age, sex, and BMI were recorded for all participants. Patients completed the Harvey-Bradshaw questionnaire to determine symptomatic disease activity (33). Patients were considered to have active disease with a score of 5 or higher. Bowel habit was classified as abnormal for constipation, diarrhea, alternating, mixed, or unspecified, and normal otherwise. Patients were phenotyped using the Montreal classification system (34) to determine location [L1 (ileum), L2 (colonic), L3 (ileocolonic), and L4 (only upper disease)] and disease behavior [B1 (only inflammatory disease), B2 (fibrostenosing disease), B3 (fistula), P (perianal disease)]. Patient use of biologic therapy (use of adalimumab or infliximab) and of non-biologic therapy was documented. Disease duration in years was recorded. All study participants were screened for MRI safety before entering the scanner.

\section{MRI Acquisition}

Using a Siemens Magnetom Verio 3T at UM and a Siemens Magnetom Trio 3T at UCLA and 12-channel head coil [Erlangen, Germany], we acquired T1-weighted three-dimensional magnetization-prepared rapid gradient echo (MPRAGE) scans. The imaging protocol file was identical at each site and quality assurance checks were conducted during separate pilot testing prior to study commencement. Data were acquired with the following parameters: $\mathrm{TR}=2,300 \mathrm{~ms}, \mathrm{TE}=3.02 \mathrm{~ms}$, field of view $=256 \times 240 \mathrm{~mm}$, matrix $=256 \times 256$, voxel size was $1.0 \times 1.0$ $\times 1.0 \mathrm{~mm}^{3}$, flip angle $9^{\circ}$, and slices $=240$. Functional data were acquired using $\mathrm{T} 2 *$-weighted whole brain echo planar imaging (EPI) sequence with $\mathrm{TR}=2,000 \mathrm{~ms}, \mathrm{TE}=28 \mathrm{~ms}$, field of view $=$ $220 \times 220 \mathrm{~mm}$, matrix $=64 \times 64$, flip angle $=77^{\circ}$, and slices $=37$ with $4 \mathrm{~mm}$ slice thickness and $12 \%$ distance factor, with participants' eyes closed.

\section{Voxel-Based Morphometry Analysis}

To assess GM volume, structural MRI data were preprocessed and analyzed using SPM12 software (http://www.fil.ion.ucl.ac. $\mathrm{uk} / \mathrm{spm}$ ) and CAT12 toolbox, which was run on MATLAB (version 2018a; Mathworks Inc., Natick, MA USA). T1-weighted images were segmented into GM and white matter (WM). Default settings for the CAT12 segmentation pipeline were used, including affine regularization with the ICBM space templateEuropean brains and spatial registration used Diffeomorphic Anatomical Registration Through Exponentiated Lie Algebra (DARTEL) (35) IXI555_MNI152 template (http://www.braindevelopment.org) with an isotropic voxel size of $1.5 \mathrm{~mm}$. Modulated normalized GM and WM images were saved, with scaling by the Jacobian determinants. GM segments were checked for homogeneity and images were smoothed in SPM12 with an 8-mm full width at the half maximum (FWHM) Gaussian kernel. Total intracranial volume (TIV) was estimated and saved. 
Using SPM12 to examine GM volume differences between CD patients and HCs, a two-sample $t$-test with an absolute masking threshold of 0.2 controlled for TIV, age, sex, BMI, and acquisition site was run. The statistical threshold was set at $p<0.05$, FDRcorrected at the cluster level, with a minimum cluster size of 5 voxels. In the case that significant differences between groups were detected, additional analyses were planned for further investigation of significant structural differences within the CD group by covarying disease duration with volumetric measures, and performing two-sample $t$-tests of Montreal classification L1 vs. L2 and L3 combined, and Montreal classification B1 vs. B2 and $\mathrm{B} 3$ combined.

\section{Functional Pre-processing}

Resting-state FC was assessed using the CONN toolbox Version 17.f (36) to preprocess and analyze the functional imaging data. The functional data were functionally realigned and unwarped; translated by centering to $(0,0,0)$ coordinates; slice time corrected; scrubbed with ART-based identification for outlier scans; segmented into GM, WM, and CSF and normalized to the Montreal Neurological Institute (MNI) template; and smoothed using an 8-mm Gaussian kernel. Structural data were translated by centering to $(0,0,0)$ coordinates, segmented into GM, WM, and CSF, and normalized to the MNI template. Artifact detection was run using the ART toolbox. During the denoising processes, WM, CSF, and outliers detected by the ART toolbox were entered into the linear regression as confounding effects. Subject motion correction was performed via realignment parameters entered in the linear regression of confounding effects (first-order derivatives, no polynomial expansion). No significant differences between groups were found for realignment average raw scores (1-tailed $p=0.176)$ or average framewise (scan-toscan) differences (1-tailed $p=0.170)$. Linear de-trending was performed, and the default band-pass filter of [0.008 0.09] $\mathrm{Hz}$ was applied.

\section{ROI Analysis}

Functional analysis was performed using the ROI-to-ROI function in the CONN toolbox using the General Linear Model (GLM, correlation analysis settings, and no weighting applied). The ROI-to-ROI analysis was run using CONN RSN nodes (36), which include 32 seeds/targets [Default Mode Network (DMN): medial pre-frontal cortex (MPFC), precuneus cortex (PCC), bilateral lateral parietal (LP); Sensorimotor Network (SMN): Superior, bilateral Lateral; Visual Network (VIS): Medial, Occipital, bilateral Lateral; Salience Network (SN): anterior cingulate cortex (ACC), bilateral anterior insula (AI), rostral pre-frontal cortex (RPFC), and supramarginal gyrus (SMG); Dorsal Attention Network (DA): bilateral frontal eye field (FEF) and intraparietal sulcus (IPS); Fronto-parietal Network (FP): bilateral lateral pre-frontal cortex (LPFC) and posterior parietal cortex (PPC); Language Network (LAN): bilateral inferior frontal gyrus (IFG) and posterior superior temporal gyrus (pSTG); and Cerebellar Network (CER): Anterior, Posterior].

\section{Independent Component Analysis}

Using default settings for CONN 17.f, the ICA was run with a FastICA for estimation of independent spatial components and GICA1 back-projection for individual subject level spatial map estimation. Dimensionality reduction was set to 64 and number of components was set to 31 . The number of components was estimated using GIFT 4.0b software, using minimum description length (MDL) criteria, to estimate components for each individual separately, and then compute the estimated number of components for the entire data set using the mean, median, and maximum standard deviation of individual results. The correlational spatial match-to-template approach was used in CONN to identify within each component the brain RSNs for the cerebellar (CER), default mode (DMN), sensorimotor $(\mathrm{SMN})$, frontoparietal (FP), dorsal attention (DA), salience (SN), language (LAN), and visual (VIS) networks.

\section{Statistics}

Between-subjects [CD (1) HC (-1)] contrasts were run for both ROI and ICA analyses, controlling for age, sex, BMI, and acquisition site. For the ROI analysis, all network nodes were used as both sources and targets, and ROI-to-ROI connections were set to a threshold by intensity of two-sided FDR-corrected $p$ $<0.05$. ICA results were displayed at $p<0.001$ uncorrected and a cluster-wise threshold of $p<0.05$, FDR-corrected. Additional analyses were run to explore differences in $\mathrm{FC}$ in $\mathrm{CD}$ patients. The relationship between FC and disease duration in years was assessed with linear regression, and Montreal classification L1 vs. L2 and L3 combined as well as Montreal classification B1 vs. B2 and B3 combined, while controlling for age, sex, BMI, and acquisition site were assessed with two-tailed two-sample $t$-tests. The $t$-test of $\mathrm{L} 1$ vs. L2/L3 combined compares isolated small bowel disease to small bowel plus colon or colon alone, to see if colon involvement is associated with different outcomes, whereas B1 vs. B2/B3 combined compares pure inflammatory vs. complicated (stricture and/or fistula) disease. Sex differences were investigated by contrasting [CD females (1) CD males $(-1)$ ], [CD females (1) HC females (-1)], and [CD males (1) HC males $(-1)]$, while controlling for age, BMI, and acquisition site.

\section{Post-hoc Multimodal Analysis}

Following the structural and functional data analyses, a multimodal analysis was performed using MRIcroGL version 1.2.20190902. The areas of significantly different FC identified by the ICA were summed such that the five components formed a single layer representing the ICA results. Similarly, the significant differences between groups identified in the ROI-to-ROI analysis were summed into a single layer. The ICA, ROI-to-ROI, and spm152 atlas layers were overlaid. The Additive Overlay Blending option was selected to identify areas of spatial overlap. Although the VBM analysis did not produce significant results when corrected for multiple comparisons, the spmT map of the contrast between groups before correction for multiple comparisons was used as a layer to compare with the ICA and ROI-to-ROI layers independently. Finally, all three layers were overlaid with the spm152 template and assessed for areas of spatial overlap. 
TABLE 1 | Demographics and relevant clinical data for all subjects.

\begin{tabular}{|c|c|c|c|}
\hline & $\mathrm{CD}(n=35)$ & $\mathrm{HC}(n=21)$ & $p$-value \\
\hline Sex (male/female) & $18 / 17$ & $14 / 7$ & 0.273 \\
\hline Age (years) & $32.6 \pm 11.4$ & $26.9 \pm 10.0$ & 0.066 \\
\hline BMI & $27.1 \pm 4.4$ & $25.5 \pm 4.9$ & 0.801 \\
\hline Harvey-Bradshaw (active/inactive) & $7 / 28$ & - & - \\
\hline Bowel habits (normal/abnormal) & $14 / 21$ & $21 / 0$ & $<0.001$ \\
\hline Disease duration (years) & $11.3 \pm 7.1$ & - & - \\
\hline \multicolumn{4}{|l|}{ Montreal classification } \\
\hline \multicolumn{4}{|l|}{ Location } \\
\hline L1 & 18 & & \\
\hline L2 & 3 & - & - \\
\hline L3 & 13 & - & - \\
\hline$\left\llcorner 4^{\star}\right.$ & 3 & - & - \\
\hline \multicolumn{4}{|l|}{ Behavior } \\
\hline B1 & 20 & - & - \\
\hline B2 & 7 & - & - \\
\hline B3 & 10 & - & - \\
\hline$P$ & 5 & - & - \\
\hline IBD medications (biologics/no biologics) & $13 / 22$ & - & - \\
\hline \multicolumn{4}{|c|}{$\begin{array}{l}\text { Values for age, } B M I \text {, and disease duration are presented as mean values and standard } \\
\text { deviations. Values for sex, bowel habits, Harvey-Bradshaw, Montreal classification, and } \\
\text { IBD medications are presented as number of subjects in that category. BMI, body mass } \\
\text { index; } L 1 \text {, ileum; } L 2 \text {, colonic; } L 3 \text {, ileocolonic; } L 44^{*} \text {, upper gastrointestinal tract disease } \\
\text { (participants could have } L 4 \text { disease and any of } L 1, L 2 \text {, and } L 3 \text { as well); } B 1 \text {, only } \\
\text { inflammatory; } B 2, \text { stricture in bowel; } B 3 \text {, fistula; } P \text {, perianal disease; IBD, inflammatory } \\
\text { bowel disease. }\end{array}$} \\
\hline
\end{tabular}

\section{RESULTS}

\section{Demographic Results}

As seen in Table 1, no significant differences were found between CD patients and HC for sex, age, or BMI. However, bowel habits between the two groups were significantly different.

\section{Voxel-Based Morphometry Results}

No GM volume differences were found between $C D$ patients and HCs when using FDR correction for multiple comparisons. As no differences were found between the groups, no further analyses were run.

\section{ROI Results}

The ROI analysis shows that $\mathrm{CD}$ patients have reciprocally increased FC between the right lateral pre-frontal cortex (LPFC) of the FP network and bilateral supramarginal gyrus nodes of the $\mathrm{SN}$, and decreased FC between the medial pre-frontal cortex (MPFC) and the left lateral parietal (LP) node of the DMN (Table 2, Figure 1A). Bivariate correlations between right LPFC and bilateral SMG indicate that $\mathrm{HC}$ had negative FC whereas $\mathrm{CD}$ had positive FC between nodes. FC was less positive for $\mathrm{CD}$ than HC for the MPFC and left LP nodes.

When comparing $\mathrm{CD}$ and $\mathrm{HC}$ females (Table 2, Figure 1B), we found increased FC of the SN left anterior insula (AI) with all four FP network nodes, with a reciprocal FC of the right LPFC and PPC nodes with the left AI. In all four cases, negative FC between nodes for the $\mathrm{HC}$ was increased to near or above zero
TABLE 2 | ROI-to-ROI results showing functional connectivity differences between Crohn's disease patients and healthy controls $(\mathrm{CD}>\mathrm{HC})$ controlling for age, sex, $\mathrm{BMI}$, and acquisition site followed by results of contrast between patient females and healthy females (CDF > HCF) controlling for age, BMl, and acquisition site (threshold ROI-to-ROI connections by intensity, FDR $p<0.05$, two-sided).

\begin{tabular}{|c|c|c|c|c|c|}
\hline Seed & Target & $t$ & $p$ & $r \mathrm{CD}$ & $r \mathrm{HC}$ \\
\hline \multicolumn{6}{|l|}{$\mathrm{CD}>\mathrm{HC}$} \\
\hline \multirow{2}{*}{$\begin{array}{l}\text { Lateral pre-frontal } \\
\text { cortex R }\end{array}$} & Supramarginal gyrus $L$ & 3.53 & 0.019 & 0.07 & -0.10 \\
\hline & Supramarginal gyrus $R$ & 3.43 & 0.019 & 0.10 & -0.11 \\
\hline Supramarginal gyrus $L$ & Lateral pre-frontal cortex $\mathrm{R}$ & 3.53 & 0.028 & 0.07 & -0.10 \\
\hline Supramarginal gyrus $\mathrm{R}$ & Lateral pre-frontal cortex $\mathrm{R}$ & 3.43 & 0.038 & 0.10 & -0.11 \\
\hline $\begin{array}{l}\text { Medial pre-frontal } \\
\text { cortex }\end{array}$ & Lateral parietal L & -3.93 & 0.008 & 0.19 & 0.39 \\
\hline Lateral parietal $L$ & Medial pre-frontal cortex & -3.93 & 0.008 & 0.19 & 0.39 \\
\hline \multicolumn{6}{|l|}{ CDF $>$ HCF } \\
\hline \multirow[t]{5}{*}{ Anterior insula L } & Lateral pre-frontal cortex $\mathrm{L}$ & 3.01 & 0.004 & 0.07 & -0.03 \\
\hline & Lateral pre-frontal cortex $\mathrm{R}$ & 3.03 & 0.004 & 0.00 & -0.13 \\
\hline & Posterior parietal cortex $L$ & 3.23 & 0.017 & 0.00 & -0.07 \\
\hline & Posterior parietal cortex $\mathrm{R}$ & 3.52 & 0.001 & 0.06 & -0.05 \\
\hline & Visual medial & -3.81 & 0.001 & -0.14 & -0.05 \\
\hline \multirow[t]{2}{*}{ Visual occipital } & Supramarginal gyrus $R$ & -4.69 & 0.001 & -0.11 & -0.07 \\
\hline & Superior temporal R & -3.51 & 0.015 & -0.06 & 0.05 \\
\hline \multirow[t]{2}{*}{ Supramarginal gyrus $\mathrm{R}$} & Visual occipital & -4.69 & 0.001 & -0.11 & -0.07 \\
\hline & Visual medial & -3.20 & 0.036 & -0.12 & -0.04 \\
\hline \multirow[t]{3}{*}{ Visual medial } & Anterior insula $L$ & -3.81 & 0.012 & -0.14 & -0.05 \\
\hline & Anterior insula $\mathrm{R}$ & -3.57 & 0.012 & -0.10 & -0.05 \\
\hline & Supramarginal gyrus $R$ & -3.20 & 0.024 & -0.12 & -0.04 \\
\hline Anterior insula $\mathrm{R}$ & Visual medial & -3.57 & 0.025 & -0.10 & -0.05 \\
\hline Superior temporal $\mathrm{R}$ & Visual occipital & -3.51 & 0.029 & -0.06 & 0.05 \\
\hline
\end{tabular}

$\overline{C D}$, Crohn's disease; $H C$, healthy control; $C D F$, Crohn's disease female; $H C F$, healthy control female; $t, t$-value; $p, p$-value; $r C D$, mean bivariate correlation for $C D$ group; $r$ HC, mean bivariate correlation for HC group; R, right; L, left; FP, frontoparietal network; SN, salience network; DMN, default mode network; LAN, language network, VIS, visual network.

for CD. Negative FC between the medial VIS network node and bilateral SN AI were decreased for CD. No differences were found between $\mathrm{CD}$ females and $\mathrm{CD}$ males, or $\mathrm{CD}$ males and $\mathrm{HC}$ males. For the $\mathrm{CD}$ group, neither Montreal classifications nor disease duration correlated significantly with the FC findings.

\section{ICA Results}

Results from the ICA analysis (Table 3, Figure 2) show FC decrease from positive to negative between the CER network with superior lateral occipital cortex and SN with left planum temporale in $\mathrm{CD}$ patients compared to HCs. CD patients also showed an increase in FC from negative to positive between the VIS network and the left putamen and right occipital pole compared to HCs. Sex differences were observed for the ICA. $\mathrm{CD}$ females, as compared with $\mathrm{HC}$ females, showed multiple differences, including a decrease from positive to negative FC of the DMN with the left insula and of the VIS with the right fusiform gyrus, and an increase in FC from negative to positive correlation of the DMN with the right temporal pole and of the SMN with the left orbitofrontal cortex. CD males showed a decrease in positive FC of the DMN with the right 

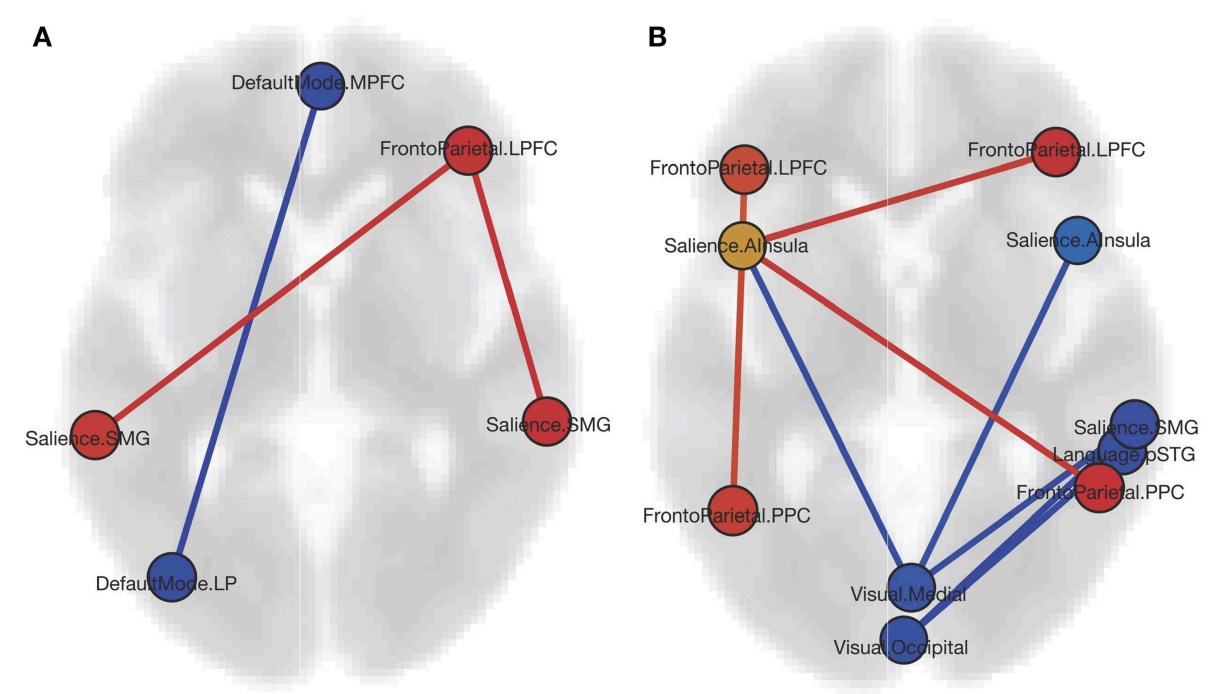

FIGURE 1 | (A) Graphic of ROI-to-ROI contrast showing nodes with increased FC (red) and decreased FC (blue) for the CD group as compared to HCs. (B) Graphic of ROI-to-ROI contrast showing nodes with increased FC (red), decreased FC (blue), and both increased and decreased FC (yellow) for the CD females as compared to the $\mathrm{HC}$ females. Both graphics are displayed in axial orientation with the anterior aspect toward the top and right on the right, with $\mathrm{ROI}$-to-ROI connection threshold set by intensity at FDR $p<0.05$, two-sided (MPFC, medial pre-frontal cortex; LPFC, lateral pre-frontal cortex; SMG, supramarginal gyrus; LP, lateral parietal; Al, anterior insula; PPC, posterior parietal cortex; STG, superior temporal gyrus).

angular gyrus, as compared to the $\mathrm{HC}$ males. There was no significant difference between the sexes within the CD group. No significant correlations were found for Montreal classifications or disease duration.

\section{Post-hoc Multimodal Analysis}

Areas of spatial overlap were identified for the combinations of the ICA and ROI-to-ROI, the ICA and VBM, and the ROI-to-ROI and VBM layers, as well as for the combination of all three layers. The overlay of the two functional results showed spatial overlap in bilateral supplementary motor area, right parahippocampal gyrus, and left insula and thalamus. The overlay of the ICA and VBM layers showed spatial overlap in bilateral supplementary motor area, right cerebellum, and left insula, thalamus, and hippocampus. Overlay of the ROI-to-ROI and VBM layers showed spatial overlap in bilateral cerebellum and supplementary motor area, and left paracentral lobule, fusiform gyrus, insula, and rolandic operculum. Spatial overlap of all three layers was observed for bilateral supplementary motor area and a region spanning the left insula and rolandic operculum.

\section{DISCUSSION}

This study compared the structural and functional brain images of patients with CD and HC. An ROI-to-ROI analysis of network nodes displayed increased FC between nodes of cognitive control (frontoparietal network) and salience networks, and decreased FC within nodes of the DMN for the CD group as compared to the HC. Using ICA, we identified altered network FC in the cerebellar, visual, and salience networks. A preliminary look at the effect of CD that differs based on sex suggests that the alterations in the DMN are driven by the males in the study while the frontoparietal, salience, and visual network alterations appear driven by the females. A multimodal assessment of the spatial overlap of the structural and two functional results indicated that bilateral supplementary motor area and left insula were common to all three analyses.

The ROI analysis revealed that pre-frontal cortex FC is altered in $\mathrm{CD}$. The FC of the frontoparietal node, right LPFC, is increased reciprocally with bilateral supramarginal gyrus salience network nodes. The frontoparietal network is a cognitive control network involved in modulating attention and expectancy-induced pain modulation. Within the DMN, MPFC is decreased reciprocally with the left LP cortex. Altered pre-frontal cortex FC is consistent with previous studies of $\mathrm{CD}$ including differences in amplitude of low-frequency fluctuations (29), in regional homogeneity for CD patients without pain (28), and in relation to effective treatment (30). The altered FC between anterior (MPFC) and posterior (LP) nodes within the DMN is also consistent with previous work (29). Although reduced GM volume of MPFC for CD as compared to HC has also been reported (23), this finding was not reproduced in the current study. The involvement of prefrontal cortex changes in CD may be explained by its role in mediating antinociceptive effects and modulation of pain (37). MPFC-nucleus accumbens connectivity has been linked to the chronification of pain (37). An investigation into pre-frontal cortex alterations and its associations with pain, psychiatric comorbidity, and cognitive deficits within CD is warranted. As participants in the current study were screened for depression 
TABLE 3 | Differences in FC between Crohn's disease patients and healthy controls (CD > HC) controlling for age, sex, BMI, and acquisition site, and between CD and $\mathrm{HC}$ females (CDF $>\mathrm{HCF})$, and $\mathrm{CD}$ and $\mathrm{HC}$ males $(\mathrm{CDM}>\mathrm{HCM})$ controlling for age, $\mathrm{BMl}$, and acquisition site $(T$ threshold $=3.5$, uncorrected height threshold $p<0.001$, FDR-corrected cluster threshold $p<0.05)$.

\begin{tabular}{|c|c|c|c|c|c|c|c|c|c|}
\hline \multirow[t]{2}{*}{ Network } & \multirow[t]{2}{*}{ Region } & \multirow[t]{2}{*}{ Voxels } & \multicolumn{3}{|c|}{ Peak MNI coordinates } & \multirow[t]{2}{*}{$t$} & \multirow[t]{2}{*}{$p$} & \multirow[t]{2}{*}{ CD } & \multirow[t]{2}{*}{ HC } \\
\hline & & & $x$ & $y$ & $z$ & & & & \\
\hline \multicolumn{10}{|l|}{$\mathrm{CD}>\mathrm{HC}$} \\
\hline CER & Superior lateral occipital L & 137 & -46 & -68 & 22 & -5.75 & 0.005 & -0.53 & 0.81 \\
\hline \multirow[t]{2}{*}{ VIS } & Putamen L & 97 & -30 & 00 & -8 & 5.43 & 0.029 & 0.77 & -0.70 \\
\hline & Occipital pole R & 126 & 18 & -82 & 14 & 4.91 & 0.007 & 1.40 & -0.57 \\
\hline SN & Planum temporale L & 113 & -46 & -30 & 12 & -4.96 & 0.020 & -0.34 & 1.36 \\
\hline \multicolumn{10}{|c|}{ CDF > HCF } \\
\hline \multirow[t]{2}{*}{ DMN } & Temporal pole R & 89 & 32 & 8 & -24 & 4.64 & 0.038 & 0.55 & -2.76 \\
\hline & Insula L & 103 & -28 & -24 & 26 & -7.25 & 0.038 & -0.80 & 2.70 \\
\hline SMN & Orbital frontal cortex $L$ & 135 & -8 & 14 & -26 & 5.42 & 0.012 & 0.92 & -2.94 \\
\hline VIS & Fusiform cortex R & 124 & 36 & -54 & -22 & -5.38 & 0.011 & -0.48 & 1.03 \\
\hline \multicolumn{10}{|c|}{$\mathrm{CDM}>\mathrm{HCM}$} \\
\hline DMN & Angular gyrus R & 106 & 56 & -56 & 16 & -5.72 & 0.025 & 1.18 & 3.40 \\
\hline
\end{tabular}

The number of voxels per cluster, MNI coordinates of the peak intensity voxel per cluster, $t, p$, and mean values are given.

$C D$, Crohn's disease; HC, healthy control; CDF, Crohn's disease female; HCF, healthy control female; CDM, Crohn's disease male; HCM, healthy control male; R, right; L, left; CER, cerebellar network; VIS, visual network; SN, salience network; DMN, default mode network; SMN, sensorimotor network.

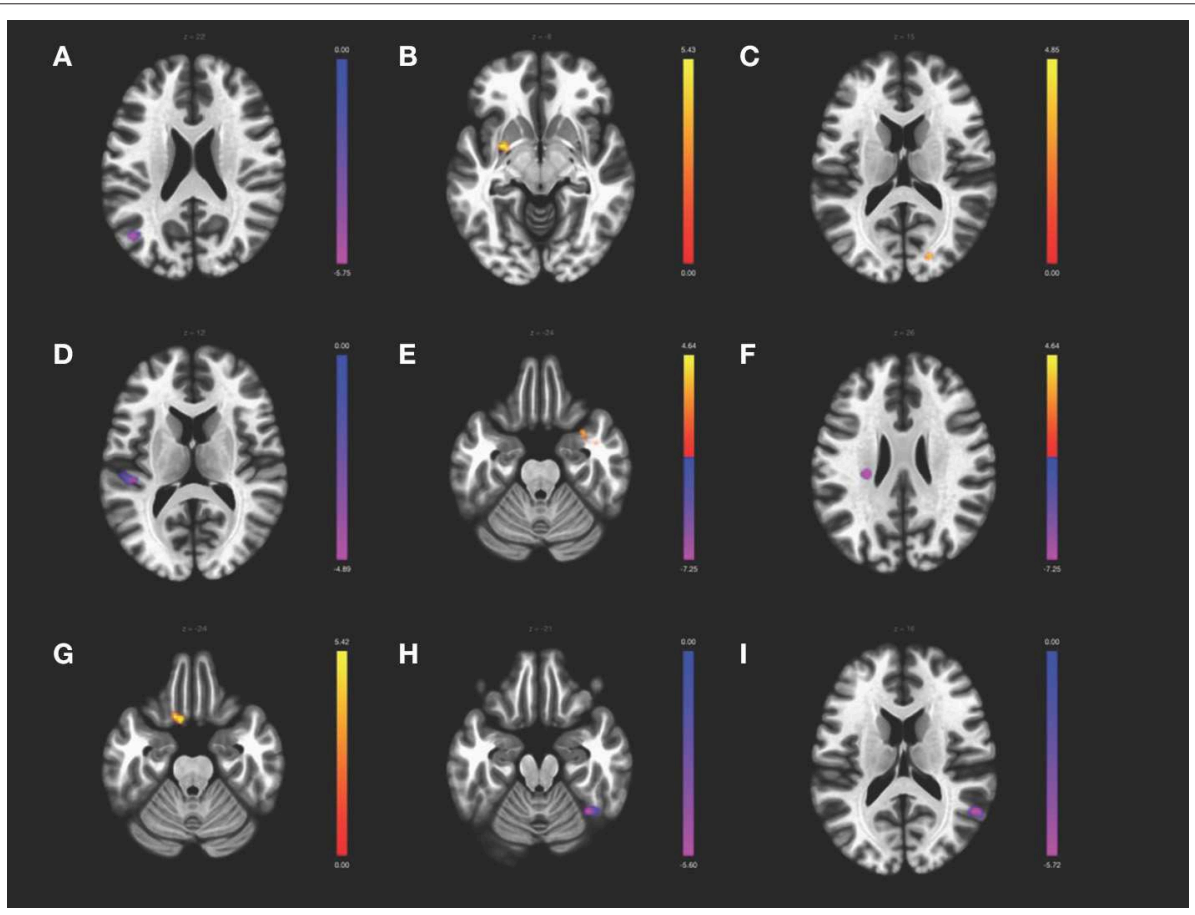

FIGURE 2 | ICA contrast between CD > HC for (A) the cerebellar network FC with left superior lateral occipital, (B) the visual network FC with left putamen and (C) the right occipital pole, and (D) the salience network FC with the left planum temporale. The female CD $>\mathrm{HC}$ contrast revealed altered FC in the (E) DMN with the right temporal pole and $(\mathbf{F})$ left insula, $(\mathbf{G})$ the SMN with left orbitofrontal cortex, and $(\mathbf{H})$ the visual network with the fusiform gyrus, whereas the male CD $>$ HC contrast showed altered FC of (I) the DMN with the right angular gyrus. All slices correspond to the peak activation coordinates with the color bar representing positive $t$-values in orange and negative $t$-values in blue. Slices are shown in neurological orientation (left hemisphere on the left of each slice, anterior toward the top of each slice) and are displayed at $T$ threshold $=3.5$, uncorrected height threshold $p<0.001$, and FDR-corrected cluster threshold $p<0.05$.

or anxiety as exclusion criteria and no cognitive measures were obtained, the associations between brain alterations and psychiatric comorbidity or cognition cannot be assessed. The exclusion of CD patients with psychiatric comorbidity facilitated the interpretation of the effects of $\mathrm{CD}$ on the brain. Future work contrasting $\mathrm{CD}$ patients with and without comorbidities will prove informative regarding brain changes associated with the disease. 
The increased FC between the frontoparietal and salience network nodes is suggestive of stronger ties between cognitive control and meaningful external/internal stimulus detection. It has been proposed that the salience network acts as a "switch" between task-positive networks, such as the frontoparietal network involved in cognitive control (38) and the tasknegative network involved in internal mentation, the DMN (39). It is possible that the stronger coupling of awareness and cognitive process is a reflection of the physical discomfort and the adverse effect that $\mathrm{CD}$ has on cognition (40). The decreased within-network FC of the DMN may indicate that the CD patients experience disrupted mind-wandering or selfreferential thought. The enhanced functional coupling of the salience network "switch" and frontoparietal network cognitive control, combined with the reduced DMN integrity, underlies this interpretation.

Whereas the ROI-to-ROI analysis calculates only the FC between each of the 32 network nodes, the ICA performs at a voxel-to-voxel level assessing the FC of the entire brain. ICA has the advantage of identifying functional connections of brain regions not restricted to the boundaries of the network nodes, within each of the components associated with RSNs. The ICA results revealed differences between $\mathrm{CD}$ and $\mathrm{HC}$ in three RSNs that, taken together, suggest alterations in sensorimotor processes. The cerebellar network showed decreased FC with the superior lateral occipital cortex, a brain region involved in visual processing. Increased FC was observed for the visual network and the occipital pole, a region involved in vision. FC of the salience network with the left planum temporale, a region involved in locating sound in space, was decreased. This may indicate an alteration of the salience network, which detects salient stimuli from the environment, with an auditory network; however, this cannot be known from the current study as no auditory network was investigated. Increased FC was observed within the visual network component with the putamen, a region involved in movement, decision-making, and learning, which may correspond to an alteration of sensorimotor and behavioral processes related to $\mathrm{CD}$ symptoms. It is possible that for persons with $\mathrm{CD}$, sensitivity to visceral sensory information, and modulation of a physical response to that information, is more acute and the need to learn and adapt behavior accordingly may be greater than for HC. As examples, the necessity of strategically planned outings or an urgent need to locate a bathroom while out may be stressors for persons living with a disease that include abdominal pain and diarrhea as common symptoms. The increased co-activity of brain regions involved in sensorimotor and executive functions may make sense in this context.

In an analysis of whether the group level results were related to sex, it became apparent that the females in the study largely drove the frontoparietal, salience and visual network differences whereas the males drove the DMN alteration. Although no differences were detected in the ROI analysis between $\mathrm{CD}$ females and males, or $\mathrm{CD}$ males and $\mathrm{HC}$ males, numerous differences between $\mathrm{CD}$ and $\mathrm{HC}$ females were observed. Notably, the $\mathrm{CD}$ females had increased FC between frontoparietal and salience network nodes. FC was increased in the right frontoparietal nodes, LPFC and PPC, with the salience node left anterior insula that, in return, had increased FC with all four frontoparietal nodes, bilateral LPFC and PPC. The CD females had decreased FC of both the medial and occipital visual network nodes with numerous other salience network nodes. As no differences in FC were observed for the frontoparietal, salience, or visual network nodes for the males, a trend emerges that suggests the females in this study may possibly drive these network changes.

Curiously, there were no group differences in the ICA for the frontoparietal or DMN components, as there were for the ROI analysis. However, when investigating sex differences for each of the RSNs, there did appear to be an explanation for this: $\mathrm{CD}$ males, as compared to $\mathrm{HC}$ males, showed a decrease in FC for the DMN with the angular gyrus, whereas CD females as compared to $\mathrm{HC}$ females showed decreased FC for the left insula and increased FC for the right temporal pole for the DMN component. Whereas, the males showed decreased FC within the DMN, the females showed altered FC of the DMN with brain regions outside of the network. Given that the DMN was functionally connected to disparate brain regions for each sex may account for the lack of significant difference in the ICA at the group level. It is worth noting that the decreased FC of the DMN with the angular gyrus is consistent with the ROI analysis, which showed a decrease in FC between the DMN nodes MFPC and LP-in fact, the angular gyrus and LP cortex share some of the same coordinates but are labeled differently depending on the atlas used. Therefore, the ICA results support the ROI results and, further, suggest that the males in the study are behind this observed alteration. The multimodal analysis showed spatial overlap of the ICA and ROI layers in a medial frontal region, the bilateral supplementary motor areas, although not in MPFC. The left insula was also shown to have spatial overlap for the ICA and ROI layers (in fact, both supplementary motor area and insula have spatial overlap for the overlay of the ICA, ROI, and VBM layers, although the VBM layer represents an uncorrected t-map and therefore should be interpreted with caution). According to the sex differences observed in the ICA and ROI analyses, it would appear that the males might contribute to the medial frontal region overlap whereas the females contribute to the insula overlap, in the multimodal analysis of group-level results. However, the subgroups of males and females are small and a larger dataset is required to test this idea.

ICA results show CD females had decreased FC of the visual network with the right temporal occipital fusiform gyrus, which supports the multiple ROI findings of altered visual network FC for CD females. Although perhaps not intuitive that visual network changes would be involved in $\mathrm{CD}$, differences in visual processing regions have been reported in previous CD studies, including cortical thickness differences in left lateral occipital cortex (24), hypergyrification of left lingual gyrus (41) for CD, and increased FC within visual medial and frontoparietal networks in IBD (42), and similar reports are seen for other chronic conditions, such as knee osteoarthritis (43), persistent somatoform pain disorder (44), postherpetic neuralgia (45), migraine (46), and fibromyalgia (47). Having recognized the plethora of studies reporting visual system FC alterations in chronic pain populations, Shen et al. (48) 
investigated the FC of visual network nodes in a chronic low back pain population using an ROI analysis and a support vector machine classifier. Significant FC alterations were found for primary and bilateral dorsal visual network seeds with somatosensory and motor brain regions and a classification accuracy of $79.3 \%$ was reported for distinguishing chronic low back pain from HC. The authors proposed an adaptation or self-adjustment mechanism and cross-modal interaction between visual, somatosensory, motor, attention, and saliency networks to account for their findings (48). The results presented in the current study are supportive of cross-modal interactions between these networks.

It is important to note the study limitations. This is a preliminary study with a modest sample size necessitating replication in future studies. Greater sample size may be required for detecting GM volume differences in particular. Regarding the ROI results, the FC decrease in the DMN with the insula is consistent with reports of increased anticorrelations between salience network AI and DMN in osteoarthritis (49) and decreased FC between MPFC and AI in IBS (50); however, the finding in the current study must be interpreted with caution as the peak coordinate was in insula but the majority of the cluster voxels were in non-labeled atlas coordinates. Similarly, the results of the multimodal analyses revealed the insula to have spatial overlap among layers, but this should be interpreted with caution given that the VBM layer displayed results uncorrected for multiple comparisons.

In summary, this study reports on a ROI analysis that shows increased FC between the cognitive control and salience networks and decreased within the DMN for persons with CD. Both results have altered pre-frontal cortex FC for CD. The ICA results show differences in cerebellar connectivity and visual and auditory processing regions that require further investigation.

\section{REFERENCES}

1. Torres J, Mehandru S, Colombel J-F, Peyrin-Biroulet L. Crohn's disease. Lancet. (2017) 389:1741-55. doi: 10.1016/S0140-6736(16)31711-1

2. Ng SC, Shi HY, Hamidi N, Underwood FE, Tang W, Benchimol EI, et al. Worldwide incidence and prevalence of inflammatory bowel disease in the 21st century: a systematic review of population-based studies. Lancet. (2017) 390:2769-78. doi: 10.1016/S0140-6736(17)32448-0

3. Lerebours E, Gower-Rousseau C, Merle V, Brazier F, Debeugny S, Marti R, et al. Stressful life events as a risk factor for inflammatory bowel disease onset: a population-based case-control study. Am J Gastroenterol. (2007) 102:122-31. doi: 10.1111/j.1572-0241.2006.00931.x

4. Long MD, Drossman DA. Inflammatory bowel disease, irritable bowel syndrome, or what: a challenge to the functional-organic dichotomy. Am J Gastroenterol. (2010) 105:1796-8. doi: 10.1038/ajg.2010.162

5. Cámara RJA, Schoepfer AM, Pittet V, Begré S, von Känel R. Mood and nonmood components of perceived stress and exacerbation of crohn's disease. Inflamm Bowel Dis. (2011) 17:2358-65. doi: 10.1002/ibd.21623

6. Farrokhyar F, Marshall JK, Easterbrook B, Irvine EJ. Functional gastrointestinal disorders and mood disorders in patients with inactive inflammatory bowel disease: prevalence and impact on health. Inflamm Bowel Dis. (2006) 12:38-46. doi: 10.1097/01.MIB.0000195391.49762.89

7. Rampton D. Does stress influence inflammatory bowel disease? Dig Dis. (2009) 27(Suppl. 1):76-9. doi: 10.1159/000268124
Contrasts based on sex revealed that $\mathrm{CD}$ males and females might differ in how their disease affects their RSN FC. This preliminary analysis will be instrumental in guiding hypotheses for future work.

\section{DATA AVAILABILITY STATEMENT}

The datasets for this article are not publicly available due to restrictions of institutional research ethics regarding confidential/identifiable human data. Requests to access the datasets should be directed to Jennifer Kornelsen: jennifer.kornelsen@umanitoba.ca.

\section{ETHICS STATEMENT}

The studies involving human participants were reviewed and approved by University of Manitoba Research Ethics Board and UCLA Institutional Review Board. The patients/participants provided their written informed consent to participate in this study.

\section{AUTHOR CONTRIBUTIONS}

$\mathrm{JK}, \mathrm{JL}, \mathrm{EM}$, and $\mathrm{CB}$ contributed to the conception and design of the study. KW organized the database. AW and JK performed the statistical analysis. AW wrote the first draft of the manuscript. JK wrote the sections of the manuscript. All authors contributed to the manuscript revision, read, and approved the submitted version.

\section{FUNDING}

This work was funded in part by NIH grant RO1-081913.
8. Marrie RA, Walld R, Bolton JM, Sareen J, Walker JR, Patten SB, et al. Increased incidence of psychiatric disorders in immune-mediated inflammatory disease. J Psychosom Res. (2017) 101:17-23. doi: 10.1016/j.jpsychores.2017.07.015

9. Walker JR, Ediger JP, Graff LA, Greenfeld JM, Clara I, Lix L, et al. The manitoba IBD cohort study: a population-based study of the prevalence of lifetime and 12-month anxiety and mood disorders. Am J Gastroenterol. (2008) 103:1989-97. doi: 10.1111/j.1572-0241.2008.01980.x

10. Bonaz BL, Bernstein CN. Brain-gut interactions in inflammatory bowel disease. Gastroenterology. (2013) 144:36-49. doi: 10.1053/j.gastro.2012.10.003

11. Mayer, Emeran A, Tillisch K. The brain-gut axis in abdominal pain syndromes. Annu Rev Med. (2011) 62:381-96. doi: 10.1146/annurev-med-012309-103958

12. Soares JM, Sampaio A, Ferreira LM, Santos NC, Marques P, Marques F, et al. Stress impact on resting state brain networks. PLoS ONE. (2013) 8:e66500. doi: 10.1371/journal.pone.0066500

13. Kim P, Evans GW, Angstadt M, Ho SS, Sripada CS, Swain JE, et al. Effects of childhood poverty and chronic stress on emotion regulatory brain function in adulthood. Proc Natl Acad Sci USA. (2013) 110:18442-7. doi: $10.1073 /$ pnas. 1308240110

14. Thomason ME, Hamilton JP, Gotlib IH. Stress-induced activation of the HPA axis predicts connectivity between subgenual cingulate and salience network during rest in adolescents. J Child Psychol Psychiatr. (2011) 52:1026-34. doi: 10.1111/j.1469-7610.2011. 02422.x 
15. Kim ES, Cho KB, Park KS, Jang BI, Kim KO, Jeon SW, et al. Predictive factors of impaired quality of life in Korean and mood disorders. J Clin Gastroenterol. (2013) 47:38-44. doi: 10.1097/MCG.0b013e318266fff5

16. Tagliazucchi E, Balenzuela P, Fraiman D, Chialvo DR. Brain resting state is disrupted in chronic back pain patients. Neurosci Lett. (2010) 485:26-31. doi: 10.1016/j.neulet.2010.08.053

17. Tian T, Guo L, Xu J, Zhang S, Shi J, Liu C, et al. Brain white matter plasticity and functional reorganization underlying the central pathogenesis of trigeminal neuralgia. Sci Rep. (2016) 6:36030. doi: 10.1038/srep36030

18. Yoshino A, Okamoto Y, Okada G, Takamura M, Ichikawa N, Shibasaki $\mathrm{C}$, et al. Changes in resting-state brain networks after cognitivebehavioral therapy for chronic pain. Psychol Med. (2018) 48:1148-56. doi: 10.1017/S0033291717002598

19. Femenía T, Gómez-Galán M, Lindskog M, Magara S. Dysfunctional hippocampal activity affects emotion and cognition in mood disorders. Brain Res. (2012) 1476:58-70. doi: 10.1016/j.brainres.2012.03.053

20. Wiebking C, Bauer A, De Greck M, Duncan NW, Tempelmann C, Northoff G. Abnormal body perception and neural activity in the insula in depression: an fMRI study of the depressed material me. World J Biol Psychiatr. (2010) 11:538-49. doi: 10.3109/15622970903563794

21. Bär K-J, Wagnera G, Koschke M, Boettger S, Boettger MK, Schlösser R, et al. Increased prefrontal activation during pain perception in major depression. Biol Psychiatr. (2007) 62:1281-7. doi: 10.1016/j.biopsych.2007.02.011

22. Agostini A, Benuzzi F, Filippini N, Bertani A, Scarcelli A, Farinelli V, et al. New insights into the brain involvement in patients with crohn's disease: a voxel-based morphometry study. Neurogastroenterol Motil. (2013) 25:147-82. doi: $10.1111 / \mathrm{nmo} .12017$

23. Bao CH, Liu P, Liu HR, Wu LY, Shi Y, Chen WF, et al. Alterations in brain grey matter structures in patients with crohn's disease and their correlation with psychological distress. J Crohn's Colitis. (2015) 9:532-40. doi: 10.1093/ecco-jcc/jjv057

24. Nair VA, Beniwal-Patel P, Mbah I, Young BM, Prabhakaran V, Saha S. Structural imaging changes and behavioral correlates in patients with crohn's disease in remission. Front Hum Neurosci. (2016) 10:460. doi: $10.3389 /$ fnhum.2016.00460

25. Thomann AK, Griebe M, Thomann PA, Hirjak D, Ebert MP, Szabo K, et al. Intrinsic neural network dysfunction in quiescent crohn's disease. Sci Rep. (2017) 7:978-80. doi: 10.1038/s41598-017-11792-y

26. Thomann AK, Griebe M, Thomann PA, Reindl W, Wolf C. Altered intrinsic brain function in crohn's disease. J Crohn's Colitis. (2017) 11(Suppl. 1):S126-7. doi: 10.1093/ecco-jcc/jjx002.225

27. Agostini A, Ballotta D, Righi S, Moretti M, Bertani A, Scarcelli A, et al. Stress and brain functional changes in patients with crohn's disease: a functional magnetic resonance imaging study. Neurogastroenterol Motil. (2017) 29:1-10. doi: $10.1111 / \mathrm{nmo} .13108$

28. Bao CH, Liu P, Liu HR, Wu LY, Jin XM, Wang SY, et al. Differences in regional homogeneity between patients with crohn's disease with and without abdominal pain revealed by resting-state functional magnetic resonance imaging. Pain. (2016) 157:1037-44. doi: 10.1097/j.pain.0000000000000479

29. Bao C, Liu P, Liu H, Jin X, Shi Y, Wu L, et al. Difference in regional neural fluctuations and functional connectivity in crohn's disease: a restingstate functional MRI study. Brain Imaging Behav. (2018) 12:1795-803. doi: 10.1007/s11682-018-9850-Z

30. Bao C, Liu P, Liu H, Jin X, Calhoun VD, Wu L, et al. Different brain responses to electro-acupuncture and moxibustion treatment in patients with crohn's disease. Sci Rep. (2016) 6:36636. doi: 10.1038/srep36636

31. Liu P, Li R, Bao C, Wei Y, Fan Y, Liu Y, et al. Altered topological patterns of brain functional networks in crohn's disease. Brain Imaging Behav. (2018) 12:1466-78. doi: 10.1007/s11682-017-9814-8

32. Hou J, Mohanty R, Nair VA, Dodd K, Beniwal-Patel P, Saha S, et al. Alterations in resting-state functional connectivity in patients with crohn's disease in remission. Sci Rep. (2019) 9:7412. doi: 10.1038/s41598-019-43878-0

33. Evertsz' FB, Hoeks CCMQ, Nieuwkerk PT, Stokkers PCF, Ponsioen CY, Bockting CLH, et al. Development of the patient harvey bradshaw index and a comparison with a clinician-based harvey bradshaw index assessment of crohn's disease activity. J Clin Gastroenterol. (2013) 47:850-6. doi: 10.1097/MCG.0b013e31828b2196

34. Satsangi J, Silverberg MS, Vermeire S, Colombel JF. The montreal classification of inflammatory bowel disease: controversies, consensus, and implications. Gut. (2006) 55:749-53. doi: 10.1136/gut.2005.082909
35. Ashburner J. A fast diffeomorphic image registration algorithm. Neuroimage. (2007) 38:95-113. doi: 10.1016/j.neuroimage.2007.07.007

36. Whitfield-Gabrieli S, Nieto-Castanon A. Conn: a functional connectivity toolbox for correlated and anticorrelated brain networks. Brain Connect. (2012) 2:125-41. doi: 10.1089/brain.2012.0073

37. Ong WY, Stohler CS, Herr DR. Role of the prefrontal cortex in pain processing. Mol Neurobiol. (2019) 56:1137-66. doi: 10.1007/s12035-018-1130-9

38. Marek S, Dosenbach NUF. The frontoparietal network: function, electrophysiology, and importance of individual precision mapping. Dial Clin Neurosci. (2018) 20:133-40.

39. Sridharan D, Levitin DJ, Menon V. A critical role for the right fronto-insular cortex in switching between central-executive and default-mode networks. Proc Natl Acad Sci USA. (2008) 105:12569-74. doi: 10.1073/pnas.0800005105

40. Whitehouse CE, Fisk JD, Bernstein CN, Berrigan LI, Bolton JM, Graff LA, et al. Comorbid anxiety, depression, and cognition in MS and other immune-mediated disorders. Neurology. (2019) 92:E406-17. doi: 10.1212/WNL.0000000000006854

41. Thomann AK, Thomann PA, Wolf RC, Hirjak D, Schmahl C, Ebert MP, et al. Altered markers of brain development in crohn's disease with extraintestinal manifestations-a pilot study. PLoS ONE. (2016) 11:e0163202. doi: 10.1371/journal.pone.0163202

42. Neeb L, Bayer A, Bayer KE, Farmer A, Fiebach JB, Siegmund B, et al. Transcranial direct current stimulation in inflammatory bowel disease patients modifies resting-state functional connectivity: a RCT. Brain Stimul. (2019) 12:978-80. doi: 10.1016/j.brs.2019.03.001

43. Pujol J, Martínez-Vilavella G, Llorente-Onaindia J, Harrison BJ, LópezSolà $\mathrm{M}$, López-Ruiz $\mathrm{M}$, et al. Brain imaging of pain sensitization in patients with knee osteoarthritis. Pain. (2017) 158:1831-8. doi: $10.1097 /$ j.pain.0000000000000985

44. Zhao Z, Huang T, Tang C, Ni K, Pan X, Yan C, et al. Altered restingstate intra- and inter- network functional connectivity in patients with persistent somatoform pain disorder. PLoS ONE. (2017) 12:e176494. doi: 10.1371/journal.pone.0176494

45. Cao S, Qin B, Zhang Y, Yuan J, Fu B, Xie P, et al. Herpes zoster chronification to postherpetic neuralgia induces brain activity and grey matter volume change. Am J Transl Res. (2018) 10:184-99.

46. Liu J, Zhao L, Li G, Xiong S, Nan J, Li J, et al. Hierarchical alteration of brain structural and functional networks in female migraine sufferers. PLOS ONE. (2012) 7:e51250. doi: 10.1371/journal.pone.0051250

47. Pujol J, Macià D, Garcia-Fontanals A, Blanco-Hinojo L, LópezSolà M, Garcia-Blanco S, et al. The contribution of sensory system functional connectivity reduction to clinical pain in fibromyalgia. Pain. (2014) 155:1492-503. doi: 10.1016/j.pain.2014. 04.028

48. Shen W, Tu Y, Gollub RL, Ortiz A, Napadow V, Yu S, et al. Visual network alterations in brain functional connectivity in chronic low back pain: a resting state functional connectivity and machine learning study. NeuroImage Clin. (2019) 22:101775. doi: 10.1016/j.nicl.2019. 101775

49. Cottam WJ, Iwabuchi SJ, Drabek MM, Reckziegel D, Auer DP. Altered connectivity of the right anterior insula drives the pain connectome changes in chronic knee osteoarthritis. Pain. (2018) 159:929-38. doi: 10.1097/j.pain.0000000000001209

50. Qi R, Liu C, Ke J, Xu Q, Zhong J, Wang F, et al. Intrinsic brain abnormalities in irritable bowel syndrome and effect of anxiety and depression. Brain Imaging Behav. (2016) 10:1127-34. doi: 10.1007/s11682-0159478-1

Conflict of Interest: The authors declare that the research was conducted in the absence of any commercial or financial relationships that could be construed as a potential conflict of interest.

Copyright (c) 2020 Kornelsen, Wilson, Labus, Witges, Mayer and Bernstein. This is an open-access article distributed under the terms of the Creative Commons Attribution License (CC BY). The use, distribution or reproduction in other forums is permitted, provided the original author(s) and the copyright owner(s) are credited and that the original publication in this journal is cited, in accordance with accepted academic practice. No use, distribution or reproduction is permitted which does not comply with these terms. 


\title{
Decreased Functional Connectivity Between the Left Amygdala and Frontal Regions Interferes With Reading, Emotional, and Executive Functions in Children With Reading Difficulties
}

\author{
Ohad Nachshon ${ }^{1}$, Rola Farah ${ }^{1}$ and Tzipi Horowitz-Kraus 1,2* \\ ${ }^{1}$ Educational Neuroimaging Center, Faculty of Education in Science and Technology, Faculty of Biomedical Engineering, \\ Technion - Israel Institute of Technology, Haifa, Israel, ${ }^{2}$ Reading and Literacy Discovery Center, Cincinnati Children's Hospital \\ Medical Center, Cincinnati, OH, United States
}

OPEN ACCESS

Edited by:

Chella Kamarajan,

SUNY Downstate Health Sciences

University, United States

Reviewed by:

Panagiotis G. Simos,

University of Crete, Greece

Zhen Yuan,

University of Macau, China

${ }^{*}$ Correspondence:

Tzipi Horowitz-Kraus

Tzipi.kraus@technion.ac.il

Specialty section:

This article was submitted to

Speech and Language,

a section of the journal

Frontiers in Human Neuroscience

Received: 08 May 2019 Accepted: 09 March 2020

Published: 28 April 2020

Citation:

Nachshon O, Farah $R$ and Horowitz-Kraus $T$ (2020) Decreased Functional Connectivity Between the Left Amygdala and Frontal Regions Interferes With Reading, Emotional, and Executive Functions in Children With Reading Difficulties.

Front. Hum. Neurosci. 14:104. doi: 10.3389/fnhum.2020.00104
Introduction: Dyslexia is a reading disorder characterized by significant difficulty in reading, as well as reports of altered executive functions (EF). Children with reading difficulties (RD) experience a broad range of social and emotional problems. Recently it was suggested that children with RD have altered functional connections within the amygdala, which is related to emotional processing. Altered brain laterality related to reading was previously reported in children with $\mathrm{RD}$. Hence, we sought to determine the differences in functional connectivity between the right and left emotional network as related to emotional challenges and the other reported difficulties in reading and EF in children with RD compared to typical readers.

Methods: Sixty-four 8 to 12 year old children, 27 children with RD and 37 age-matched typical readers, participated in the study. Reading, emotional, and EF abilities were assessed. Global efficiency of the emotional network was calculated and compared between the groups, and left vs. right functional connectivity of the amygdala was tested using the CONN toolbox. Functional connectivity measures were then associated with measures of reading, emotional, and EF abilities.

Results: Children with RD showed significantly decreased emotional and EF abilities compared to typical readers. A negative correlation between reading, emotional, and EF abilities was determined in both groups. Neuroimaging results showed decreased global efficiency measures within the emotional network in children with RD, who also showed lower functional connectivity between the amygdala and the left and right frontal pole regions. Results also indicated increased functional connectivity of the right vs. left amygdala with left and right pre-central and post-central gyri regions, which were related to decreased reading, emotional, and EF abilities in both typical readers and children with RD. 


\begin{abstract}
Conclusion: The positive relationship between EF and emotional abilities in children with $\mathrm{RD}$ strengthens the relationship between EF difficulties and emotional stress, which in turn may lower EF abilities (monitoring, inhibition, and attention) as well as decreased reading abilities. The emotional challenges in children with $\mathrm{RD}$ were associated with decreased functional connectivity of the left amygdala with pre/post central gyrus and cognitive-control regions. These findings suggest that although the right hemisphere is thought to be related to emotional stress, it was the decreased control of the left hemisphere that was related to emotional disturbance in children with RD.
\end{abstract}

Keywords: anxiety, emotional state, executive functions, functional connectivity, reading, reading task, reading difficulties, resting state

\section{HIGHLIGHTS}

- Decreased emotional and cognitive control abilities and the relationship between these abilities in children with $\mathrm{RD}$ vs. typical readers.

- Decreased global efficiency of the emotional network during rest in children with $\mathrm{RD}$ vs. typical readers, related to lower emotional abilities.

- Increased functional connectivity between the left vs. right amygdala and the right frontal pole, and between the left amygdala and the left frontal pole in typical readers compared to children with RD.

- Increased functional connections between the left vs. right amygdala in typical readers compared to children with $\mathrm{RD}$, related to better cognitive control, and emotional and reading abilities.

\section{INTRODUCTION}

\section{Emotional Difficulties in Children With Reading Difficulties}

Dyslexia is a reading disorder characterized by significant difficulty in reading that is not explained by any other intelligence, motivational, or environmental deficit (International Dyslexia Association [IDA], 2011). Recent studies suggest additional alterations in cognitive control, also called executive functions (EF), may indicate a common malfunction in $\mathrm{EF}$ in individuals with reading difficulties (RD) (Habib, 2000), with some suggesting the EF challenge as the source for the reading-based difficulties shared by children with RD (Pennington, 2006). Interestingly, it was also found that children with $\mathrm{RD}$ experience emotional problems including low self-esteem, anxiety, and depression (Grills-Taquechel et al., 2012). We recently demonstrated that in addition to their impaired reading, children with $\mathrm{RD}$ demonstrated decreased emotional and EF abilities compared to typical readers, which were also positively correlated with each other (Nachshon and Horowitz-Kraus, 2018). Cleary, children with $\mathrm{RD}$ face several challenges and it is challenging to objectively identify each one. With the development of imaging technologies, a better understanding of the neural circuits involved in these RD challenges is emerging. Therefore, the goal of the current research study was to determine the neurobiological correlates for the emotional difficulties reported in children with RD.

\section{Neural Circuitry Involved in Reading Difficulties}

Evidence of the neurobiological, neuropsychological, and neurophysiological basis for RD has existed since the end of the 19th century (Habib, 2000). Traditional studies suggested that a decreased activation in "classical" reading-related neural circuits, such as the left fusiform gyrus, is related to orthographical processing (see, for example, Pugh et al., 2000). Children with $\mathrm{RD}$ showed greater activation in frontal lobes, i.e., in regions responsible for $\mathrm{EF}$, during a narrative comprehension task compared to typical readers (Horowitz-kraus et al., 2016). Decreased functional connectivity between reading and EF regions (i.e., anterior cingulate cortex) during reading in children with $\mathrm{RD}$ compared to typical readers was also observed (Horowitz-Kraus and Holland, 2015). Interestingly, several studies have indicated a right-lateralization during reading in these children, specifically in reading-related regions such as the right fusiform gyrus, suggesting a heavier reliance on the right hemisphere for reading as opposed to the left fusiform gyrus in children with $\mathrm{RD}$ compared to typical readers (HorowitzKraus et al., 2014). Recent studies also reported differences in functional connectivity related to cognitive control networks in children with $\mathrm{RD}$. Levinson et al. reported greater functional connectivity in children with $\mathrm{RD}$ between the $\mathrm{EF}$ network and visual, language, and cognitive control regions during the Stroop task (Levinson et al., 2018). They suggested that children with RD might exploit neural circuits supporting EF when performing a cognitive task more than typical readers. Nevertheless, since emotional and cognitive abilities were found to be linked (Ochsner and Gross, 2005), a question arises as to the neurobiological signatures for the emotional difficulties in children with $\mathrm{RD}$. This study set out to find these signatures, if they exist.

\section{Neural Circuitry Related to Emotional Difficulties}

Emotional difficulties such as anxiety, stress, and depression are currently defined using the Diagnostic and Statistical Manual of Mental Disorders (American Psychiatric Association [APA], 2013). Anxiety is characterized by continuous excitation due 
to expectations of bad outcomes or constant threat (Kim et al., 2011). Previous neuroimaging studies reported that increased amygdala activation was correlated with higher anxiety levels. For example, Bishop et al. (2004) reported that increased amygdala activity when faced with unattended fearful faces was correlated with higher levels of self-reported anxiety. Etkin et al. (2004) revealed a positive association between the unconscious processing of fearful faces by the basolateral amygdala and subjects' trait anxiety levels. Finally, anxiety was associated with elevated amygdala activity when faced with threat-related stimuli and non-threat-related stimuli, suggesting that elevated amygdala activity may reflect greater anxiety levels even in the absence of a clear threat (Somerville et al., 2004).

Recent work indicated the contribution of subregions of the amygdala to emotional and reading abilities in children with $\mathrm{RD}$. Increased functional connectivity between the amygdala and the medial prefrontal cortex (mPFC) in children with $\mathrm{RD}$ compared to typical readers was demonstrated (Davis et al., 2018). Children with RD exhibited a positive functional connectivity between the left basolateral amygdala and the mPFC compared to a negative functional connectivity between these regions in typical readers. Children with $\mathrm{RD}$ and typical readers showed similar functional connectivity between the bilateral centro-medial amygdala and the mPFC. This connectivity was negatively correlated with reading ability, whereas functional connectivity between the right centro-medial amygdala and the left $\mathrm{mPFC}$ were positively correlated with anxiety symptoms in the entire sample. The authors theorized that a similar pattern of unequal functional connectivity exists in individuals with anxiety disorder and this reinforces the co-occurrence of emotional and reading difficulties in children with RD. The authors, however, did not examine the relationship between the amygdala laterality, i.e., right vs. left functional connections with other regions in the brain related to reading or cognitive control, as related to reading or emotional abilities in children with $\mathrm{RD}$. Due to the overall role of the right hemisphere in processing emotional information (Borod et al., 1988), and based on previously observed laterality in children with RD, specifically as related to reading words (i.e., decreased activation of the left occipital regions in children with $\mathrm{RD}$ during reading) (Horowitz-Kraus and Breznitz, 2014), an explicit examination of the amygdala's laterality (i.e., the relationship between the right vs. left amygdala functional connections with other regions in the brain), and reading, emotional, and EF abilities in children with $\mathrm{RD}$ is warranted. Such an examination may elucidate the overall different lateralization in children with $\mathrm{RD}$ related to their reading, emotional, and $\mathrm{EF}$ abilities.

The overarching goal of this study was to close the gap in knowledge regarding the neurobiological evidence for the involvement of emotional difficulties in children with RD, specifically focusing on the functional connections between the left vs. right amygdala and the entire brain related to reading and emotional abilities. We hypothesized that children with RD would show decreased functional connectivity patterns within the emotional network compared to typical readers during rest. We also anticipated that children with RD would show different functional connectivity in the left vs. right amygdala compared to typical readers, which would be related to decreased reading, emotional, and EF abilities in children with RD.

\section{MATERIALS AND METHODS}

\section{Participants}

Sixty-four 8 to12 year old children [27 children with RD (mean age $=10.16, \mathrm{SD}=1.11,15$ females) and 37 age-matched typical readers (mean age $=9.93, \mathrm{SD}=1.05,15$ females) $]$ participated in the study (no significant age difference between the group, $t=0.829$, ns). All participants were in the normal range of nonverbal IQ tests, as measured by the test of non-verbal intelligence (TONI) (Brown et al., 1997). No differences were found in general verbal ability, as tested by the Peabody picture vocabulary test (PPVT) (Dunn and Dunn, 2007), between children with RD and typical readers.

All participants were native English speakers, Caucasian, with average socioeconomic status. All were right-handed, displayed normal or corrected-to normal vision in both eyes and had normal hearing. All children were without a history of neurological or emotional disorders, and no differences were found between the two reading groups in attention ability [as measured using the Conners questionnaire (Conners, 1989)]. Participants were recruited from posted online ads and through commercial advertisements. All participants signed informed written assent and their parents provided informed written consent prior to inclusion in the study; all were compensated for their participation. The Cincinnati Children's Hospital Medical Center (CCHMC) Institutional Review Board approved the study. All participants underwent thorough baseline behavioral (reading, EF) and emotional (anxiety questionnaire) testing. The data were collected at the Pediatric Imaging Research Consortium (PNRC) at CCHMC in Cincinnati, Ohio. Behavioral data acquisition (reading, EF, and emotional questionnaires) lasted approximately $2 \mathrm{~h}$.

\section{Reading Measures}

Children with $\mathrm{RD}$ were assigned to the $\mathrm{RD}$ group based on a previous diagnosis. Their reading ability was verified using a set of reading tests (Nachshon and Horowitz-Kraus, 2018), on which they had to score in less than the 25th percentile for at least two of the subtests. Children assigned to the typical readers group were age-matched students without previous reports of impaired reading and who scored in greater than the 25 th percentile for all of the reading tests performed.

To evaluate their reading ability, the children's reading was tested using the following: Word level reading (1) Automatic word reading accuracy/orthography, the Test of Words Reading Efficiency [TOWRE (Torgesen et al., 1999)]; (2) Automatic decoding, the pseudowords reading efficiency subtest from the TOWRE; (3) Non-timed word reading accuracy/orthography (Letter-Word subtest) (Woodcock et al., 1989); and (4) Nontimed decoding of pseudoword reading (Word-Attack subtest) (Woodcock et al., 1989), phonemic awareness, using the Elision subtest from the Comprehensive Test of Phonological Processing 
(Wagner et al., 1999), and contextual reading using the passage comprehension subtest (Woodcock et al., 1989).

\section{Executive Functions and Emotional Measures}

Using several subtests, the EF were mapped to their sub-domains: (1) Attention using speed and accuracy subtests from the TEA-Ch battery [Sky Search, Score! and Sky-search DT subtests] (Manly et al., 1999); (2) Verbal fluency using the Delis-Kaplan Executive Functions System [D-KEF battery (Dellis et al., 2001)]; (3) Speed of processing using the "object naming" subtest from the CTOPP (Wagner et al., 1999); (4) Switching using the Wisconsin Card Sorting Task (Nyhus and Barceló, 2009); (5) Inhibition using the Stroop subtests from the D-KEFS (Dellis et al., 2001); and (6) Overall EF measured by the Behavior Rating Inventory of Executive Functions (BRIEF) (Gioia et al., 2000). Emotional abilities were assessed using the Emotional Control scale from the BRIEF (Nyhus and Barceló, 2009). Associations between reading, EF and emotional abilities were determined using Pearson correlations. Data was corrected using a Bonferroni correction.

\section{Neuroimaging Measures}

\section{Data Acquisition and Preprocessing}

All images were acquired using a Philips Achieva 3T MRI scanner (Philips Medical Systems, Best, Netherlands). A T2*-weighted, gradient-echo, echo planar imaging (EPI) sequence was used with fMRI parameters: $\mathrm{TR} / \mathrm{TE}=2000 / 38 \mathrm{msec}$, matrix size $=64 \times 64$, slice thickness $=5 \mathrm{~mm}$, resulting in a voxel size $=4 \times 4 \times 5 \mathrm{~mm}^{3}$.

\section{Resting-state task}

Two five-minute resting-state scans were acquired, resulting in a total of 300 whole-brain volumes acquired in a total imaging time of $10 \mathrm{~min}$. The initially acquired 10 time points were discarded to allow for $\mathrm{T} 1$ relaxation equilibrium. In addition, a high-resolution T1-weighted 3D anatomical scan was acquired using an inversion recovery (IR)-prepared turbo gradient-echo acquisition protocol with a spatial resolution of $1 \times 1 \times 1 \mathrm{~mm}^{3}$. Participants were acclimated and desensitized to the scanner to condition them for comfort during imaging (Vannest et al., 2014) and were instructed to keep their eyes open and to look at a cross on the screen during the resting state condition.

\section{Functional Connectivity Analysis}

Regions for the emotional network were based on the amygdala seeds and were defined based on the literature (see Table 1). Functional-connectivity analysis during resting state was carried out using the CONN toolbox (Whitfield-Gabrieli and NietoCastanon, 2012). Normalized bias-corrected $T_{1}$ images were generated in $\mathrm{SPM}^{1}$ and segmented into gray matter, white matter, and cerebral spinal fluid (CSF). The principle eigenvariate of the BOLD time-courses from the white matter and CSF, as well as the six motion-correction parameters, were included as regressors of no interest and removed from the fMRI time-series data. The data was band-pass filtered between 0.008 and $0.2 \mathrm{~Hz}$ [as recommended (Baria et al., 2011)], and only data within

${ }^{1}$ http://www.fil.ion.ucl.ac.uk/spm/
TABLE 1 | Regions of interest in the emotional network for the current analyses [based on Roy et al. (2009)].

\begin{tabular}{lccc}
\hline Specific site & X & Y & Z \\
\hline Amygdala BLA (R) & 22 & -4 & -22 \\
Amygdala CAN (R) & 24 & -8 & -18 \\
Amygdala BLA (L) & -26 & -9 & -14 \\
Amygdala CAN (L) & -24 & -8 & -18 \\
\hline
\end{tabular}

BLA, basolateral amygdaloidal complex; CAN, central amygdala nucleus; $L$, left; $R$, right; $X Y / Z$, centroid coordinates.

this range was included in the analysis. Networks were defined according to the regions of interest (ROIs) listed in Table $\mathbf{1}$ and shown in Figure 1, by equally weighting each of the ROIs within each network. Then, several functional connectivity analyses were performed: functional-connectivity within the amygdala and between the amygdala and other regions in the brain during rest in children with $\mathrm{RD}$ and in typical readers.

Functional connectivity on the network level (i.e., global efficiency) was calculated in CONN using a formula based on Latora and Marchiori (2001):

$$
E=\frac{1}{n} \sum_{i \in N} E_{i}=\frac{1}{n} \sum_{i \in N} \frac{\sum_{j \in N, j \neq 1} d_{i j}^{-1}}{n-1}
$$

where $E_{i}$ is the efficiency of node $i, n$ is the number of network nodes, $N$ is the set of all network nodes, and $d_{i j}^{-1}$ is the inverse shortest path length between nodes $j$ and $I$.

\section{Seed-to-Voxel Analysis}

To address the lateralization question, additional seed-to-voxel analyses were conducted. ROIs related to the amygdala (as implemented in the FSL Harvard-Oxford atlas in CONN) were

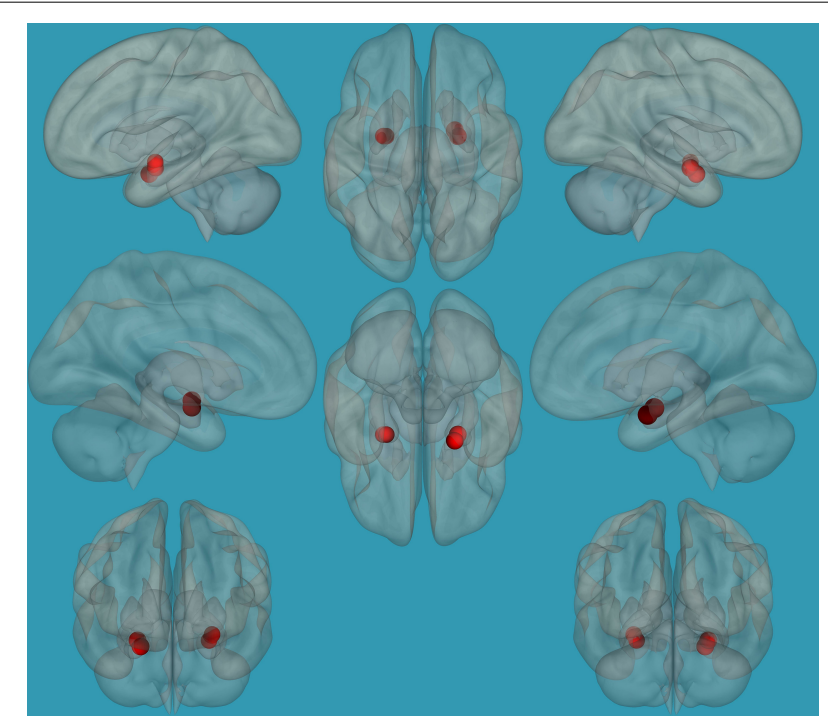

FIGURE 1 | Seeds for the amygdala network. The seeds for the amygdala network: sagittal, axial, and coronal axis. The red color represents the seeds. Neurological orientation ( $L$, left; $R$, right). 
divided into left and right ROIs, and each was defined as a seed in our analysis. We related to the left and right amygdala separately as seeds and correlated their BOLD response with the residual BOLD signal of each voxel in the brain. We then set a threshold for the resulting map of $p=0.05$ voxel-height, false-discoveryrate (FDR) corrected for multiple comparisons and $p=0.05$ cluster-size, FDR-corrected. To address the study questions related to laterality differences in the amygdala network, a seedto-voxel analysis (functional-connectivity) between (1) the right amygdala, (2) the left amygdala, (3) the left vs. the right amygdala, and (4) the right vs. the left amygdala and all voxels in the brain for each group separately, as well as comparing children with RD and typical readers, was performed.

\section{Correlation Analyses of Functional Connectivity of the Left and Right Amygdala and Reading, Emotional, and EF Abilities}

To assess the relationship between EF abilities as measured by the BRIEF (Gioia et al., 2000), a seed-to-voxel analysis was performed over a range of voxels that spans a greater extent of functionally relevant areas. After determining the left and right amygdala seed-to-voxel functional connectivity matrix, we performed a seed-to-voxel correlation analysis between the left and right amygdala with reading, emotional and EF abilities, with a voxel-height threshold of $p=0.05$, FDR corrected.

\section{Prediction of Reading, Emotional and EF Abilities Based on Functional Connections of the Left vs. Right Amygdala in Typical Readers and Children With RD}

To determine if the difference between functional connectivity of the left and right amygdala in children with $\mathrm{RD}$ and typical readers predicts reading, emotional, and EF abilities, separate regression analyses were conducted using reading, the BRIEF emotional subtest, and the general EF score (from the BRIEF) tasks.

\section{RESULTS}

\section{Behavioral Results}

Children with $\mathrm{RD}$ demonstrated significantly decreased reading (in all domains of reading), emotional, and EF abilities compared to typical readers (see Table 2 for details). In the current study, significant positive correlations were found between reading measures, EF and emotional measures across groups; i.e., decreased reading ability was related to decreased emotional and EF abilities. Emotional and EF abilities also showed positive correlations, whereby better emotional abilities were related to better EF in both groups (see Supplementary Material Table 1 for details).

\section{Neuroimaging Results \\ Differences in Global Efficiency of the Amygdala Between Children With RD and Typical Readers}

Global efficiency values of the amygdala network were defined ( $p<0.05$, FDR corrected for multiple comparisons), following which a two-sample $t$-test analysis was conducted for global efficiency within the amygdala network. The analysis revealed that children with $\mathrm{RD}$ showed significantly lower global efficiency values within the amygdala network compared to typical readers (children with $\mathrm{RD}$ : mean $=0.08$, $\mathrm{SD}=0.001$, typical readers: mean $=1, \mathrm{SD}=0.02$, $t=290.442, p<0.01)$.

\section{Correlation Between the Global Efficiency of the Amygdala and EF and Emotional Measures}

Pearson correlations between the global efficiency of the amygdala (see Table 1 for the regions comprising the amygdala) and emotional and EF behavioral measures revealed significant negative correlations between the global efficiency of the amygdala network and EF abilities (with the emotional BRIEF sub-test: $[r=-0.248, p<0.05]$; the EF BRIEF sub-test: working memory $[r=-0.451, p<0.01]$; planning and organizing $[r=-0.571, p<0.01]$, and monitoring $[r=-0.506, p<0.01])$. Increased functional connectivity within this network was associated with increased emotional ability, and lower BRIEF scores across groups. Increased functional connectivity within this network was associated with increased EF abilities (lower BRIEF scores) in both groups.

\section{Differences in Functional Connectivity of the Amygdala Within Each Group Separately: Seed-to-Voxel Analysis}

To establish functional connectivity of the right and left amygdala with the whole brain in each group separately, seed-to-voxel analysis was conducted (a voxel-height threshold of $p=0.05$, FDR corrected). Voxel clusters that showed significant functional connectivity with the right and left amygdala in each group are presented in Table 2 in the Supplementary Material. Based on the results demonstrating the differences in functional connectivity within the amygdala between children with $\mathrm{RD}$ and typical readers, a two-sample $t$-test analysis comparing the functional connectivity between the two amygdala seeds (left vs. right amygdala) was conducted within each group separately $(p<0.05$, FDR corrected). Typical readers demonstrated greater functional connectivity between the right vs. left amygdala and the right temporal pole. When the opposite contrast was compared (left vs. right amygdala), these readers demonstrated greater functional connectivity with the left insular cortex and left cingulate gyrus. Children with RD demonstrated greater functional connectivity of the right vs. left amygdala with the right temporal pole and the right middle frontal gyrus. These children also demonstrated a greater functional connectivity between the left vs. right amygdala and the left hippocampus; see Table 3 and Figures 2-5.

\section{Differences in Functional Connectivity of the Amygdala Between the Groups: Seed-to-Voxel Analysis}

Based on the results demonstrating the differences in functional connectivity within the amygdala network between children with $\mathrm{RD}$ and typical readers, a two-sample $t$-test analysis comparing 
TABLE 2 | Baseline behavioral, reading measure, cognitive control and emotional test scores for children with RD and typical readers.

\begin{tabular}{|c|c|c|c|c|c|}
\hline Measure & Description (test) & Children with RD (A) & Typical Readers (B) & t-test P-value & Contrast \\
\hline \multirow[t]{2}{*}{ General Ability } & General non-verbal intelligence (Toni, Standard Score) & $97.57(11.15)$ & $101.57(14.09)$ & $1.22(\mathrm{~ns})$ & $A<B$ \\
\hline & General verbal ability (PPVT, Standard Score) & $102.48(12.52)$ & $110.89(21.65)$ & $1.81(\mathrm{~ns})$ & $\mathrm{A}<\mathrm{B}$ \\
\hline Attention & $\begin{array}{l}\text { Visual attention accuracy (Number of Correct items, } \\
\text { TEA-Ch, Sky Search, Scaled Score) }\end{array}$ & $9.67(3.42)$ & $11.03(2.52)$ & $1.83(\mathrm{~ns})$ & $A<B$ \\
\hline \multirow[t]{6}{*}{ Reading } & $\begin{array}{l}\text { Phonemic awareness (Ellison subset, CTOPP, Scaled } \\
\text { Score) }\end{array}$ & $7.7(2.32)$ & $11.3(2.43)$ & $5.96(* \star)$ & $A<B$ \\
\hline & $\begin{array}{l}\text { Words reading efficiency (SWE subset, TOWRE, } \\
\text { Percentile) }\end{array}$ & $23.52(25.25)$ & $65.65(23.09)$ & $6.93\left({ }^{\star \star \star}\right)$ & $A<B$ \\
\hline & $\begin{array}{l}\text { Pseudo-words reading efficiency (PDE subset, TOWRE, } \\
\text { Scaled Score) }\end{array}$ & $83.52(13.12)$ & $108.84(9.75)$ & $8.86\left({ }^{\star \star \star}\right)$ & $A<B$ \\
\hline & $\begin{array}{l}\text { Non-timed decoding of word reading (Letter-Word, } \\
\text { Woodcock-Johnson, Standard Score) }\end{array}$ & $89.93(14.85)$ & $112.65(9.61)$ & $7.43(* \star *)$ & $A<B$ \\
\hline & $\begin{array}{l}\text { Non-timed reading comprehension (Passage } \\
\text { comprehension, Woodcock-Johnson, Scaled Score) }\end{array}$ & $84.41(14.49)$ & $103.57(6.76)$ & $7.07(* \star *)$ & $A<B$ \\
\hline & $\begin{array}{l}\text { Non-timed decoding of pseudo-words reading } \\
\text { (Word-Attack subtest, Woodcock-Johnson, Standard } \\
\text { Score) }\end{array}$ & $93.67(9.54)$ & $108.86(8.6)$ & $\left.6.677^{\star \star \star}\right)$ & $A<B$ \\
\hline \multirow[t]{20}{*}{ Cognitive Control } & $\begin{array}{l}\text { Speed of processing: Numbers (Number Naming, } \\
\text { CTOPP, Scaled Score) }\end{array}$ & $8.63(2.9)$ & $10.93(2.14)$ & $3.65\left({ }^{\star \star \star}\right)$ & $A<B$ \\
\hline & $\begin{array}{l}\text { Speed of processing: Words (Letter Naming, CTOPP, } \\
\text { Scaled Score) }\end{array}$ & $8.04(2.78)$ & $9.98(2.36)$ & $3.02(* \star)$ & $A<B$ \\
\hline & $\begin{array}{l}\text { Inhibition abilities: STROOP (Color Word Condition, } \\
\text { Time, D-KEFS, Standard Score) }\end{array}$ & $9.7(2.37)$ & $11.62(2.23)$ & $3.31\left(^{\star \star}\right)$ & $A<B$ \\
\hline & $\begin{array}{l}\text { Inhibition abilities: Stroop (Color Word Condition, } \\
\text { Corrected Errors, D-KEFS, Standard Score) }\end{array}$ & $37.93(31.93)$ & $54.05(26.97)$ & $2.19\left(^{*}\right)$ & $A<B$ \\
\hline & Working memory (Digit Span, WISC, Standard Score) & $8.41(2.21)$ & $10.12(2.04)$ & $3.2\left({ }^{\star \star}\right)$ & $\mathrm{A}<\mathrm{B}$ \\
\hline & $\begin{array}{l}\text { Working memory (Digit Span, WISC, } \\
\text { Forward-last-attempted) }\end{array}$ & $5.37(1.28)$ & $6.54(1.41)$ & $3.42\left(^{\star \star}\right)$ & $A<B$ \\
\hline & $\begin{array}{l}\text { Working memory (Digit Span, WISC, } \\
\text { Backward-last-attempted) }\end{array}$ & $3.67(1.04)$ & $4.51(1.02)$ & $3.26\left(^{\star \star}\right)$ & $A<B$ \\
\hline & $\begin{array}{l}\text { Information speed of processing (Coding, WISC, } \\
\text { Standard Score) }\end{array}$ & $9.11(2.78)$ & $10.97(4.21)$ & 2 (ns) & $A<B$ \\
\hline & $\begin{array}{l}\text { Information speed of processing (Symbol Search, } \\
\text { WISC, Standard Score) }\end{array}$ & $11.19(1.96)$ & $11.81(2.04)$ & 1.23 (ns) & $A<B$ \\
\hline & $\begin{array}{l}\text { Inhibition abilities: Stroop (Color naming Time, D-KEFS, } \\
\text { Standard Score) }\end{array}$ & $8.81(3.43)$ & $11.95(2.74)$ & $4.06\left(^{\star \star \star}\right)$ & $A<B$ \\
\hline & $\begin{array}{l}\text { Inhibition abilities: Stroop (Color Word naming Time, } \\
\text { D-KEFS, Standard Score) }\end{array}$ & $9.22(2.98)$ & 11.84 (2.39) & $3.9\left(^{\star \star \star}\right)$ & $A<B$ \\
\hline & $\begin{array}{l}\text { Visual attention speed (Time Per Target, TEA-Ch Sky } \\
\text { Search, Scaled Score) }\end{array}$ & $11.89(17.93)$ & $14.92(17.03)$ & 0.69 (ns) & $A<B$ \\
\hline & $\begin{array}{l}\text { Visual attention accuracy (Attention, TEA-Ch Sky } \\
\text { Search, Scaled Score) }\end{array}$ & 7.67 (2.96) & $9.05(2.61)$ & $1.98(\mathrm{~ns})$ & $A<B$ \\
\hline & $\begin{array}{l}\text { Switching abilities (Perseverative Error Percent, } \\
\text { Wisconsin, T score) }\end{array}$ & 7.67 (2.9) & 9.35 (2.86) & $2.32\left(^{\star}\right)$ & $A<B$ \\
\hline & $\begin{array}{l}\text { Switching abilities (Non-perseverative Error Percent, } \\
\text { Wisconsin, T score) }\end{array}$ & $51.45(11)$ & $57.29(9.37)$ & $2.29\left(^{\star}\right)$ & $A<B$ \\
\hline & $\begin{array}{l}\text { Switching abilities (Categories Completed, Wisconsin, } \\
\text { Percentile) }\end{array}$ & $50.77(12.54)$ & $56.33(11.06)$ & $1.88(\mathrm{~ns})$ & $A<B$ \\
\hline & $\begin{array}{l}\text { Switching abilities (Learning to Learn, Wisconsin, } \\
\text { Percentile) }\end{array}$ & 14.47 (6.3) & 15.96 (5.99) & $0.96(\mathrm{~ns})$ & $A<B$ \\
\hline & $\begin{array}{l}\text { Switching abilities (Failure to maintain set, Wisconsin, } \\
\text { Percentile) }\end{array}$ & 13.06 (4.87) & $16(0)$ & $3.68\left(^{\star \star \star}\right)$ & $A<B$ \\
\hline & $\begin{array}{l}\text { BRIEF Questionnaire: Executive Functions (Inhibit, } \\
\text { BRIEF parent report, T Score) }\end{array}$ & $14.92(2.47)$ & $13.82(4.6)$ & $-1.13(\mathrm{~ns})$ & $B<A$ \\
\hline & $\begin{array}{l}\text { BRIEF Questionnaire: Executive Functions (Shift, BRIEF } \\
\text { parent report, T Score) }\end{array}$ & $53.41(12.44)$ & $46.97(9.11)$ & $-2.39\left(^{\star}\right)$ & $\mathrm{B}<\mathrm{A}$ \\
\hline
\end{tabular}


TABLE 2 | Continued

\begin{tabular}{|c|c|c|c|c|c|}
\hline Measure & Description (test) & Children with RD (A) & Typical Readers (B) & t-test P-value & Contrast \\
\hline & $\begin{array}{l}\text { BRIEF Questionnaire: Executive Functions (Initiate, } \\
\text { BRIEF parent report, T Score) }\end{array}$ & $53.48(12.97)$ & $47.73(10.24)$ & $-1.98(n s)$ & $\mathrm{B}<\mathrm{A}$ \\
\hline & $\begin{array}{l}\text { BRIEF Questionnaire: Executive Functions (Working } \\
\text { Memory, BRIEF parent report, T score) }\end{array}$ & 53.26 (11.38) & $46.92(8.48)$ & $-2.55\left(^{\star}\right)$ & $B<A$ \\
\hline & $\begin{array}{l}\text { BRIEF Questionnaire: Executive Functions (Plan } \\
\text { Organize, BRIEF parent report, T Score) }\end{array}$ & $55.97(11.71)$ & $45.51(9.47)$ & $-3.95\left(^{(\star \star}\right)$ & $B<A$ \\
\hline & $\begin{array}{l}\text { BRIEF Questionnaire: Executive Functions (Monitor, } \\
\text { BRIEF parent report, T score) }\end{array}$ & $57.66(10.86)$ & $44.84(8.13)$ & $\left.-5.41^{(\star \star}\right)$ & $B<A$ \\
\hline & $\begin{array}{l}\text { BRIEF Questionnaire: Executive Functions (GEC, BRIEF } \\
\text { parent report, T score) }\end{array}$ & $55.35(10.61)$ & $45.46(6.68)$ & $\left.-4.57^{(\star \star \star}\right)$ & $B<A$ \\
\hline Emotion-al ability & $\begin{array}{l}\text { BRIEF Questionnaire: Emotional Score (Emotional, } \\
\text { BRIEF parent report, T score) }\end{array}$ & $56.29(11.43)$ & $45.22(9.47)$ & $\left.-4.23^{(\star \star \star}\right)$ & $\mathrm{B}<\mathrm{A}$ \\
\hline
\end{tabular}

$R D$, reading difficulties; TR, typical readers. Results are presented as mean (standard deviation). * $p<0.05 ;{ }^{* *} p<0.01 ;{ }^{* * *} p<0.001$.

TABLE 3 | Analyses ( $t$-test) for the seed-voxel functional connectivity analysis for typical readers and children with reading difficulties; contrast: Left amygdala vs. right amygdala $(p<0.05$, FDR corrected).

\begin{tabular}{|c|c|c|c|c|c|c|c|c|}
\hline Group & Amygdala seed (contrast) & Clusters (anatomical regions) & Number of voxels in the cluster & $\mathbf{x}$ & $\mathbf{Y}$ & $\mathbf{Z}$ & Effect size (beta) & $\mathbf{T}$ \\
\hline TR & $R>L$ & Right temporal pole & 5027 & 24 & 0 & -16 & 0.15 & 14.9 \\
\hline \multirow[t]{2}{*}{ TR } & $L>R$ & Left insular cortex & 2671 & -24 & 0 & -16 & 0.16 & 15.6 \\
\hline & & Left cingulate gyrus & 317 & -22 & -16 & 44 & 0.07 & 4.97 \\
\hline \multirow[t]{2}{*}{$\mathrm{RD}$} & $R>L$ & Right temporal pole & 2201 & 26 & 0 & -16 & 0.24 & 9.03 \\
\hline & & Right middle frontal gyrus & 267 & 28 & 32 & 26 & 0.11 & 4.64 \\
\hline $\mathrm{RD}$ & $L>R$ & Left hippocampus & 2937 & -26 & 0 & -18 & 0.21 & 7.88 \\
\hline
\end{tabular}

FDR, false discovery rate; TR, typical readers; $R D$, children with reading difficulties; $L$, left; $R$, right; $X Y / Z$, centroid coordinates.

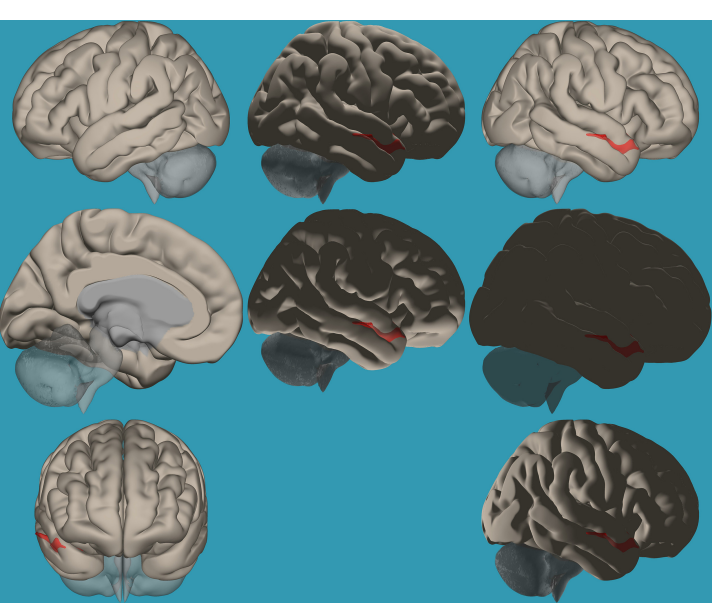

FIGURE 2 | Seed-voxel functional connectivity between the right vs. left amygdala and all voxels in the brain in typical readers. Seed-to-voxel analysis for typical readers, right amygdala $>$ left amygdala. Hot colors represent voxels with higher connectivity values ( $p<0.05$, FDR corrected). Neurological orientation ( $L$, left; $R$, right). The colors in the figures do not represent the level of functional connections (a specific scale) but the existence of functional connections (all or none). This applies to all figures.

the functional connectivity between the two amygdala seeds (left vs. right) was conducted. Results revealed increased functional connectivity between the left vs. right amygdala and the right frontal pole in typical readers vs. children with $\mathrm{RD}(p<0.05$, FDR corrected); see Table 4 and Figure 6.

To pinpoint the functional connections between the left amygdala, as the seed showing significant differences, and the whole brain in typical readers versus children with $\mathrm{RD}$, two-way $t$-tests examining the functional connectivity between the left amygdala and the whole brain in both groups were conducted. Results revealed a significantly greater functional connectivity between the left amygdala and the left frontal pole in typical readers vs. children with $\mathrm{RD}(p<0.05$, FDR corrected) (Figure 7). Choosing the right amygdala as a seed and contrasting typical readers with children with $\mathrm{RD}$ did not reveal significant results within a threshold of 0.05 corrected for multiple comparisons. Lowering the threshold to $p<0.01$ uncorrected for multiple comparisons revealed that typical readers demonstrated an increased functional connectivity between the right amygdala and the left thalamus compared to children with RD; see Table 4 and Figures 7, 8.

\section{Pearson Correlation Between Functional Connectivity of the Amygdala and the Entire Brain (Seed-to-Voxel Analysis) and Behavioral Measures (Reading, Emotional and EF Abilities)}

Due to the observed differences in functional connectivity of the right vs. left amygdala and the entire brain between typical readers and children with $\mathrm{RD}$, Pearson correlation analyses between functional connectivity of the left and right 


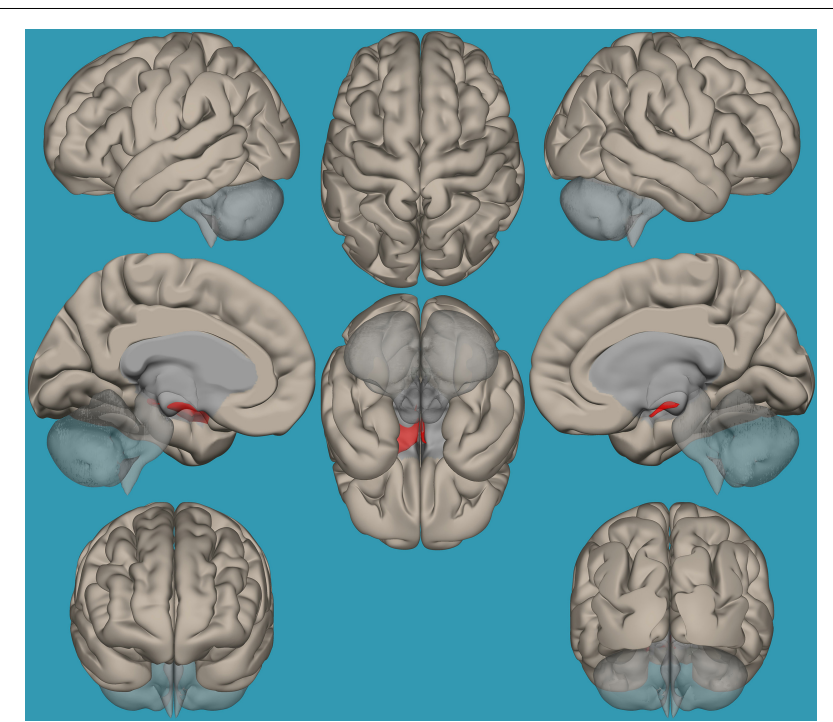

FIGURE 3 | Seed-voxel functional connectivity between the left vs. right amygdala and all the voxels in the brain in typical readers. Seed-to-voxel analysis for typical readers, left amygdala > right amygdala. Hot colors represent voxels with higher connectivity values ( $\rho<0.05$, FDR corrected). Neurological orientation ( $L$, left; $R$, right).

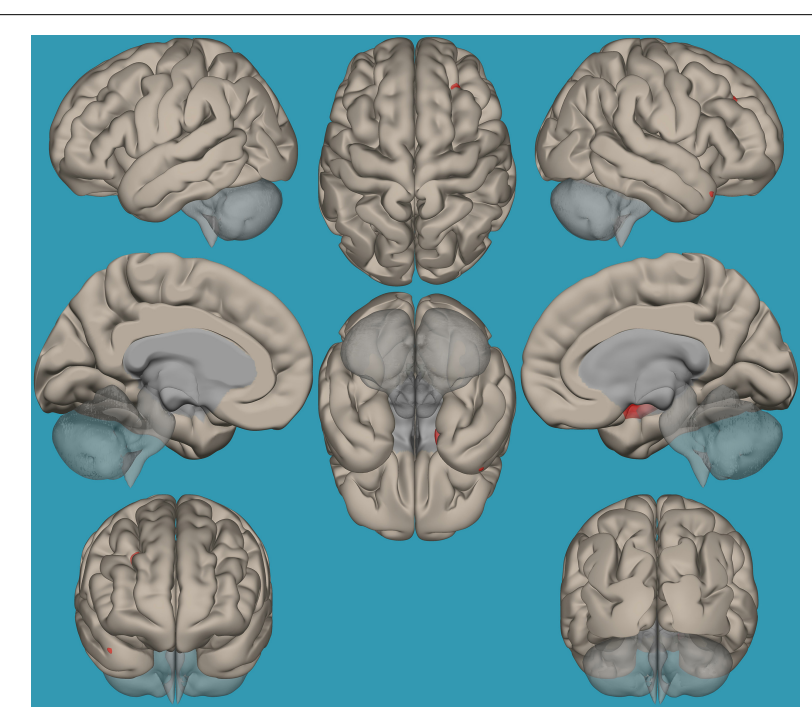

FIGURE 4 | Seed-voxel functional connectivity between the right vs. left amygdala and all the voxels in the brain in children with reading difficulties (RD). Seed-to-voxel analysis for children with $\mathrm{RD}$, right amygdala > left amygdala. Hot colors represent voxels with higher connectivity values ( $p<0.05$, FDR corrected). Neurological orientation ( $L$, left; $R$, right).

amygdala separately and the entire brain, with several behavioral measures for reading, emotional, and EF abilities in the entire study population (children with $\mathrm{RD}$ and typical readers), were conducted. Positive correlation was found between reading measures (phonological processing abilities [CTOPP Ellison subset $(r=0.348, p<0.01)]$, timed-word reading ability [TOWRE $(r=0.248, p<0.05)$ ], and untimed reading ability

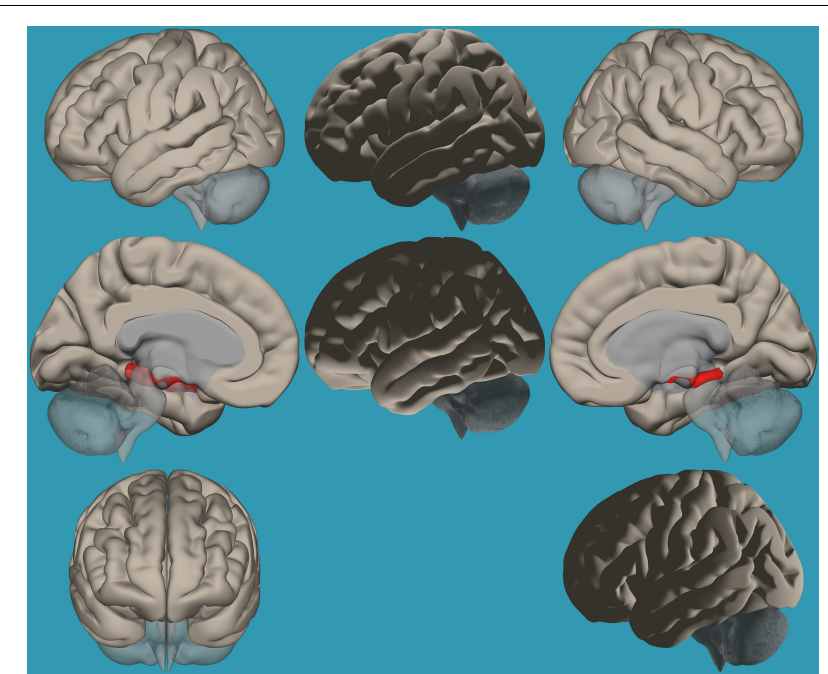

FIGURE 5 | Seed-voxel functional connectivity between the left vs. right amygdala and all voxels in the brain in children with reading difficulties (RD). Seed-to-voxel analysis for children with $\mathrm{RD}$, left amygdala > right amygdala. Hot colors represent voxels with higher connectivity values $(p<0.05$, FDR corrected). Neurological orientation (L, left; R, right).

[Letter-Word $(r=0.446, p<0.01)])$ and functional connectivity of the left amygdala with both the left and right frontal poles. Negative correlation was found between emotional abilities and functional connectivity of the left amygdala with the right frontal pole [BRIEF emotional subtest $(r=-0.314, p<0.01)]$. Positive correlation was found between EF measures and functional connectivity of the left amygdala with both the left and right frontal poles for switching abilities [Stroop Color Word Condition, Corrected Errors $(r=0.247, p<0.05)]$, working memory [digit span test $(r=0.247, p<0.05)$ ], and learning from errors [Wisconsin Non-perseverative Error $(r=0.356, p<0.01)$ ]. Negative correlation was found between the BRIEF cognitive subtests and functional connectivity of the left amygdala with the right frontal pole using the BRIEF overall general score $(r=-0.317, p<0.01)$. Increased functional connectivity of the left amygdala and the whole brain was related to an increased reading ability, as well as emotional and EF performance, across all participants.

\section{Regression Analyses Between Functional Connectivity Results and Reading, Emotional, and EF Abilities}

To determine whether the functional connectivity in the left vs. right amygdala in typical readers vs. children with RD explains reading, emotional, and EF abilities in these groups, three separate regression analysis models were conducted.

\section{Reading measures}

A linear regression established that functional connectivity of the left vs. right amygdala to the right frontal pole and left amygdala to the left frontal pole significantly predicted passage comprehension subtest scores in both groups $[F(1,62)=7.73$, $p<0.05]$ and accounted for $52 \%$ of the explained variability in 
TABLE 4 | Analyses ( $t$-test) for the seed-voxel functional connectivity analysis for typical readers compared to children with reading difficulties ( $p<0.05$, FDR corrected).

\begin{tabular}{|c|c|c|c|c|c|c|c|c|}
\hline Group contrast & Seed & Clusters (anatomical regions) & Number of voxels in the cluster & $\mathbf{x}$ & $\mathbf{Y}$ & $\mathbf{Z}$ & Effect size (beta) & $\mathbf{T}$ \\
\hline $\mathrm{TR}>\mathrm{RD}$ & $L>R$ & Right frontal pole & 336 & 26 & 38 & 28 & 0.12 & 4.49 \\
\hline $\mathrm{TR}>\mathrm{RD}$ & $L$ & Left frontal pole & 324 & -38 & 62 & 6 & 0.17 & 4.45 \\
\hline $\mathrm{TR}>\mathrm{RD}$ & $\mathrm{R}$ & Left thalamus & 1388 & -12 & 0 & 10 & 0.12 & $5.59^{\star}$ \\
\hline
\end{tabular}

${ }^{*} P$ threshold of 0.01 uncorrected for multiple comparisons. FDR, false discovery rate; XY/Z, centroid coordinates; TR, typical readers; RD, children with reading difficulties.
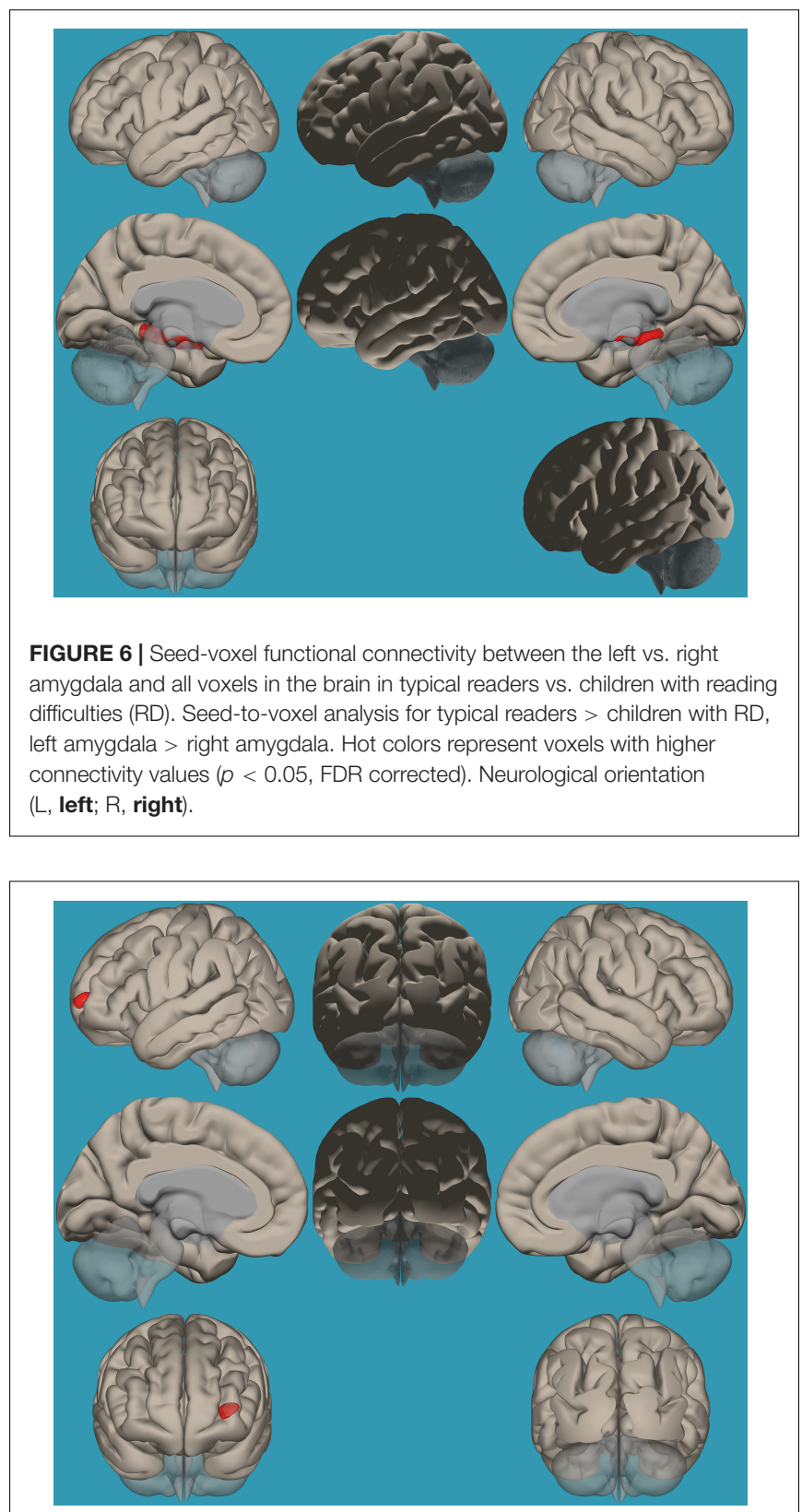

FIGURE 7 | Seed-voxel functional connectivity between the left amygdala and all voxels in the brain in typical readers vs. children with reading difficulties (RD). Seed-to-voxel analysis for typical readers > children with $R D$, with the seed as the left amygdala. Hot colors represent voxels with higher connectivity values. Contrast: $(p<0.05$, FDR corrected). Neurological orientation $(L$, left; $R$, right).

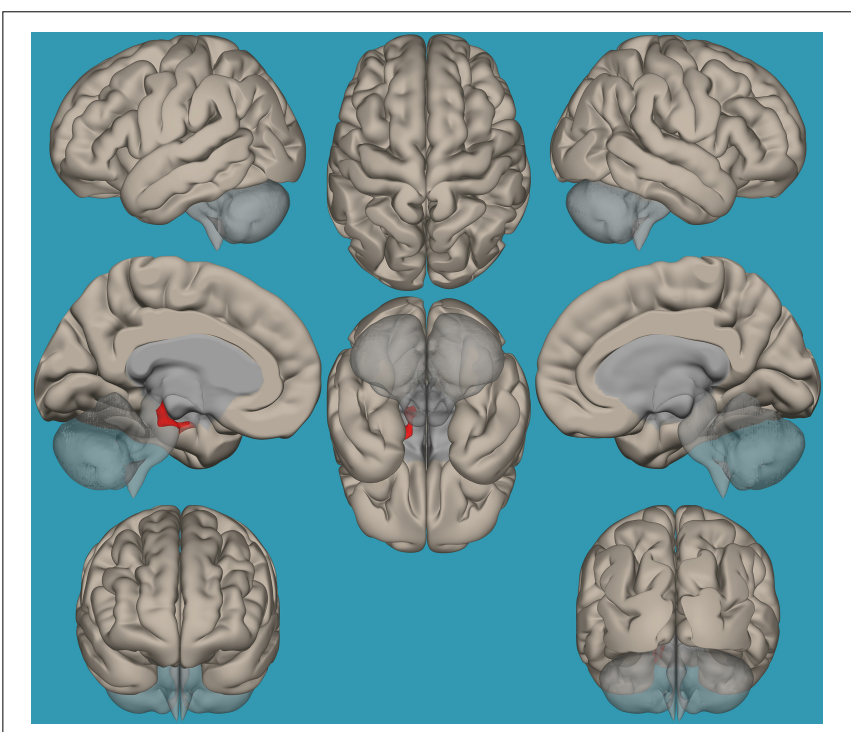

FIGURE 8 | Seed-voxel functional connectivity between the right amygdala and all voxels in the brain in typical readers vs. children with reading difficulties (RD). Seed-to-voxel analysis for typical readers > children with RD, with the seed as the right amygdala. Hot colors represent voxels with higher connectivity values. Contrast ( $p<0.01$, FDR uncorrected). Neurological orientation ( $L$, left; $R$, right).

passage comprehension scores. Increased functional connectivity between these regions was associated with higher reading scores.

\section{Emotional abilities}

A linear regression established that functional connectivity of the left vs. right amygdala to the right frontal pole significantly predicted emotional abilities in both groups $[F(1,62)=6.785$, $p<0.05$ ] and accounted for $31.4 \%$ of the explained variability in the BRIEF emotional scores. Higher functional connectivity between the left vs. right amygdala and the right frontal pole predicted lower scores in the BRIEF emotional subsets, reflecting less emotional difficulties within the entire population.

\section{EF measures}

A linear regression established that functional connectivity of the left vs. right amygdala to the right frontal pole and the left amygdala to the left frontal pole significantly predicted switching abilities (Wisconsin test switching abilities (Non-perseverative Error Percent) $[F(1,62)=13.02, p<0.05]$ and accounted for $42 \%$ of the explained variability in switching abilities. Increased functional connectivity between these regions was associated with higher cognitive scores. 


\section{DISCUSSION}

The current study was designed to reveal neurobiological evidence of the involvement of emotional difficulties in children with $\mathrm{RD}$, focusing on amygdala laterality. We determined the existence of such neurobiological evidence by assessing the differences in functional connectivity within the amygdala, its left and right components, and the entire brain between children with $\mathrm{RD}$ and typical readers.

In addition to the decreased EF and emotional abilities previously found in children with $\mathrm{RD}$ and typical readers (Nachshon and Horowitz-Kraus, 2018), our results suggest that the global efficiency of the amygdala is decreased in those with $\mathrm{RD}$ compared to typical readers, and that the left and right amygdala contribute to reading, emotional, speed of processing and EF abilities in an unequal manner; i.e., greater functional connections between the left vs. right amygdala and frontal cortices were related to increased reading, emotional, speed of processing and EF abilities. Therefore, for the first time, we have demonstrated a linkage between the laterality of the amygdala functional connectivity and frontal regions and reading, emotional and $\mathrm{EF}$ abilities.

\section{Decreased Emotional Skills Are Associated With Decreased EF Abilities}

We previously reported emotional difficulties in children with RD (Nachshon and Horowitz-Kraus, 2018), which supported other earlier findings (Grills-Taquechel et al., 2012). These difficulties are also found among children facing challenges in EF, and there exist correlations between these challenges and reading. This connection exemplifies the reading-EF-emotional triangle in children with RD. The behavioral reports of impaired emotional abilities in children with $\mathrm{RD}$ are supported by the current neuroimaging study. Our results indicate that children with RD showed a significantly lower global efficiency of the amygdala network, which included the left and right amygdala seeds, compared to typical readers. The global efficiency measure is related to the average inverse shortest path length to all other nodes in the network, which is related to better synergy between the seeds in the network (Rubinov, 2010). These results enhance previously reported results of decreased global efficiency in EF networks (cingulo-opercular) that was related to decreased reading abilities in children with $\mathrm{RD}$ (Horowitz-Kraus et al., 2015). Decreased global efficiency is related to the inefficiency of the network nodes to activate simultaneously, which may be a factor contributing to the emotional difficulties in children with $\mathrm{RD}$. The fact that the neuroimaging condition used in the current study is a resting state condition, without any written stimuli, strengthens the assumption that the impairment in this emotional network among children with $\mathrm{RD}$ is not specific to their interaction with written stimuli, but can be generalized to non-reading conditions and everyday life experiences.

\section{Separate Roles for the Left and Right Amygdala}

Our results confirm our hypothesis that children with RD would show different functional connectivity in the left vs. right amygdala compared to typical readers, which will be related to their behavioral performance. We found that children with RD demonstrated decreased functional connectivity between the left vs. right amygdala and the left and right frontal poles compared to typical readers, which was related to decreased reading, emotional, and EF abilities in this group. Since the difference between functional connectivity of the left and right amygdala and the entire brain was reduced in children with $\mathrm{RD}$, we suggest that either overactivation of the right amygdala or decreased activity of the left amygdala in children with RD is driving these differences, compared to typical readers. The role of the right hemisphere in processing emotional stimuli was previously described by others. Morris et al. (1999) showed a significant role of the right amygdala seeds in processing of "unseen" fear stimuli, while left amygdala seeds did not show any change in connectivity. Davis et al. (2018) also showed an increased functional connectivity between the left basolateral amygdala and left mPFC, and between the bilateral centro-medial amygdala and left mPFC in children with RD, while decreased functional connectivity was observed in typical readers. In addition, they showed that functional connectivity of the right centro-medial amygdala and the left mPFC positively predicted anxiety symptoms, as was suggested in the current study. The role of the right amygdala, and specifically connectivity with prefrontal regions, was previously related to processing of subconscious fear stimuli (Morris et al., 1999).

The fact that no significant differences were found when comparing typical readers and children with $\mathrm{RD}$ on functional connectivity of the right hemisphere with all the voxels in the brain (i.e., results did not survive the correction for multiple comparisons and only showed results at $p<0.01$, uncorrected for multiple comparisons), however, may rule out the assumption that the differences in lateralization between the left and right amygdala result from decreased right-lateralized connectivity. It does, nevertheless, strengthen the assumption that the differences stem from decreased functional connections of the left hemisphere with all the voxels in the brain in children with RD. Our results not only support a relationship between decreased EF abilities and functional connections of the right amygdala and frontal cortices, but also suggest that the left amygdala demonstrates greater functional connections with frontal regions compared to the right amygdala in children with RD. Moreover, the results suggest that these greater connections between the left vs. right amygdala in children with $\mathrm{RD}$ are related to their reading, emotional and EF performance. This may indicate that not only is the right amygdala processing emotional stimuli in an imprecise way in children with $\mathrm{RD}$, but that the left amygdala, which would have been expected to have demonstrated greater functional connections than the right amygdala, does not demonstrate this pattern in children with RD.

An alternative explanation may be derived from reports showing overall increased activation in the right hemisphere in children with RD (Horowitz-Kraus and Breznitz, 2014). Increased right-lateralized activation in reading-related regions was reported in children with $\mathrm{RD}$, with the authors of that study theorizing that this ineffective processing pattern may be one cause of the RD (Horowitz-Kraus and Breznitz, 2014). Based 
on our results, we suggest that children with $\mathrm{RD}$ may show a generally greater reliance on the right hemisphere, which was suggested previously as one reason for the increased artistic and creative abilities in this population (Wolff and Lundberg, 2002). The increased reliance on the right hemisphere may also be related to increased functional connections of the right amygdala in these readers compared to typical readers. Future research should explore this further and create a lateralization index for the right vs. left hemispheres in children with $\mathrm{RD}$.

\section{Study Limitations}

When reviewing the results of this study, the following limitations should be weighed. First, although the BRIEF questionnaire utilized 10 questions to cover the emotional abilities domain, only one emotional subtest was used in this study, compared to a variety of subtests used for EF and reading. Nevertheless, it is important to note that the test used covers a variety of domains, such as emotional control. A future study should collect additional emotional measures, e.g., for anxiety, selfefficacy, self-esteem, and social behavior. A second limitation is related to not yet determining whether emotional difficulty is the source of reading impairment or if the reverse is the case. A longitudinal research study may reveal the origins of the variety of difficulties in children with RD. Third, the current study used resting state data to examine the association between the amygdala functional connectivity and reading and EF. Future research should include a specific emotional-based paradigm to examine the emotional network and its association with reading and $\mathrm{EF}$.

\section{CONCLUSION}

The results of this study demonstrated that children with $\mathrm{RD}$ also suffer from lower emotional skills that conjoin with impairments in both reading and EF, and strengthen the role of the amygdala in controlling aspects of their EF and reading abilities. Both global efficiency of the amygdala and functional connectivity of the left vs. right amygdala to frontal regions predicted and explained differences in EF and reading abilities, which highlight the amygdala as a critical region of the reading network to be considered in future studies (Stanislas, 2009).

\section{REFERENCES}

American Psychiatric Association [APA] (2013). Diagnostic and Statistical Manual of Mental Disorders. Arlington, VA: APA.

Baria, A. T., Baliki, M. N., Parrish, T., and Apkarian, A. V. (2011). Anatomical and functional assemblies of brain BOLD oscillations. J. Neurosci. 31, 7910-7919.

Bishop, S. J., Duncan, J., and Lawrence, A. D. (2004). State anxiety modulation of the amygdala response to unattended threat-related stimuli. J. Neurosci. 24, 10364-10368.

Borod, J., Kent, J., Koff, E., Martin, C., and Alpert, M. (1988). Facial asymmetry while posing positive and negative emotions: Support for the right hemisphere hypothesis. Neuropsychologia 26, 759-764.

Brown, L., Sherbenou, R., and Johnsen, S. (1997). Test of Nonverbal Intelligence (TONI-3): A Language-Free Measure of Cognitive Ability. Austin, TX: PRO-ED. Conners, K. (1989). Manual for Conners Rating Scales: Instruments for Use with Children and Adolescents. Tonawanda, NY: Multi-Health Systems.

\section{DATA AVAILABILITY STATEMENT}

The raw data supporting the conclusions of this article will be made available by the authors, without undue reservation.

\section{ETHICS STATEMENT}

This study was carried out in accordance with the recommendations of Cincinnati Children's Hospital review board with written informed assent signed by participants and informed consent signed by parents in accordance with the Declaration of Helsinki. The protocol was approved by the CCHMC IRB Committee.

\section{AUTHOR CONTRIBUTIONS}

ON: data analysis, interpretation, manuscript writing, and approving final version. TH-K: study design, data acquisition, analysis, interpretation, manuscript writing and revising, and approving final version for publication. RF: data analysis and interpretation, manuscript writing and revising, and approving final version for publication.

\section{FUNDING}

This study was funded by a grant from The Eunice Kennedy Shriver National Institute of Child Health (grant number R01HD 086011) and Human Development, National Institutes of Health (PI: TH-K).

\section{SUPPLEMENTARY MATERIAL}

The Supplementary Material for this article can be found online at: https://www.frontiersin.org/articles/10.3389/fnhum. 2020.00104/full\#supplementary-material

TABLE S1 | Pearson correlations between emotional, reading and EF abilities in the typical readers group and in the reading difficulties group.

TABLE S2 | Voxel clusters showing significant functional connectivity with the right and left amygdala in each group.

Davis, K., Margolis, A. E., Thomas, L., Huo, Z., and Marsh, R. (2018). Amygdala sub-regional functional connectivity predicts anxiety in children with reading disorder. Dev. Sci. 21, 1-9.

International Dyslexia Association [IDA] (2011). Definition of Dyslexia International Dyslexia Association. Baltimore, MD: International Dyslexia Association.

Dellis, D. C., Kaplan, E., and Kramer, J. H. (2001). Delis-Kaplan Executive Function System. San Antonio: Psychological Corporation.

Dunn, D., and Dunn, L. (2007). Peabody picture vocabulary test: Manual.

Etkin, A., Klemenhagen, K. C., Dudman, J. T., Rogan, M. T., Hen, R., Kandel, E. R., et al. (2004). Individual differences in trait anxiety predict basolateral amygdala response only to unconsciously processed fearful faces. Neuron 44, 1043-1055.

Gioia, G. A., Isquith, K., Guy, S. C., and Kenworthy, L. (2000). TEST review behavior rating inventory of executive function behavior rating inventory of executive function. Child Neuropsychol. 6, 235-238. 
Grills-Taquechel, A. E., Fletcher, J. M., Vaughn, S. R., and Stuebing, K. K. (2012). Anxiety and reading difficulties in early elementary school: evidence for unidirectional- or bi-directional relations? Child Psychiatry Hum. Dev. 43, 35-47.

Habib, M. (2000). The neurological basis of developmental dyslexia: an overview and working hypothesis. Brain 123(Pt 12), 2373-2399.

Horowitz-Kraus, T., and Breznitz, Z. (2014). Can reading rate acceleration improve error monitoring and cognitive abilities underlying reading in adolescents with reading difficulties and in typical readers? Brain Res. 1544, 1-14.

Horowitz-kraus, T., Buck, C., and Dorrmann, D. (2016). Altered neural circuits accompany lower performance during narrative comprehension in children with reading difficulties : an fMRI study. Ann. Dyslexia 66, 301-318.

Horowitz-Kraus, T., DiFrancesco, M., Kay, B., Wang, Y., and Holland, S. K. (2015). Increased resting-state functional connectivity of visual- and cognitive-control brain networks after training in children with reading difficulties. Neuroimage Clin. 8, 619-630.

Horowitz-Kraus, T., and Holland, S. K. (2015). Greater functional connectivity between reading and error-detection regions following training with the reading acceleration program in children with reading difficulties. Ann. Dyslexia 65, 1-23. doi: 10.1007/s11881-015-0096-9

Horowitz-Kraus, T., Vannest, J. J., Kadis, D., Cicchino, N., Wang, Y. Y., and Holland, S. K. (2014). Reading acceleration training changes brain circuitry in children with reading difficulties. Brain Behav. 4, 886-902.

Kim, M. J., Loucks, R. A., Palmer, A. L., Brown, A. C., Solomon, K. M., Marchante, A. N., et al. (2011). The structural and functional connectivity of the amygdala: from normal emotion to pathological anxiety. Behav. Brain Res. 223, 403-410.

Latora, V., and Marchiori, M. (2001). Efficient behavior of small-world networks. Phys. Rev. Lett. 87:198701. doi: 10.1103/PhysRevLett.87.198701

Levinson, O., Hershey, A., Farah, R., and Horowitz-Kraus, T. (2018). Altered functional connectivity of the executive functions network during a stroop task in children with reading difficulties. Brain Connect. 8, 516-525.

Manly, T., Reader, S. K., Preston, A. S., Fennell, E. B., Puyana, O. E., Gill, N., et al. (1999). The Test of Everyday Attention for Children (TEA-Ch). Suffolk: Thames Valley Test Company.

Morris, J. S., Ohman, A., and Dolan, R. J. (1999). A subcortical pathway to the right amygdala mediating "unseen” fear. Proc. Natl. Acad. Sci. U.S.A. 96, 1680-1685.

Nachshon, O., and Horowitz-Kraus, T. J. A. (2018). Cognitive and emotional challenges in children with reading difficulties. Acta Paediatr. 108, 1110-1114.

Nyhus, E., and Barceló, F. (2009). Brain and cognition the wisconsin card sorting test and the cognitive assessment of prefrontal executive functions : a critical update. Brain Cogn. 71, 437-451.

Ochsner, K. N., and Gross, J. J. (2005). The cognitive control of emotion. Trends Cogn. Sci. 9, 242-249.
Pennington, B. F. (2006). From single to multiple de W cit models of developmental disorders. Cognition 101, 385-413.

Pugh, K. R., Mencl, W. E., Jenner, A. R., Katz, L., Frost, S. J., Lee, J. R., et al. (2000). Functional neuroimaging studies of reading and reading disability (developmental dyslexia). Ment. Retard. Dev. Disabil. Res. Rev. 6, 207-213.

Roy, A. K., Shehzad, Z., Margulies, D. S., Kelly, A. M., Uddin, L. Q., Gotimer, K., et al. (2009). Functional connectivity of the human amygdala using resting state fMRI. Neuroimage 45, 614-626.

Rubinov, M. (2010). Complex network measures of brain connectivity: uses and interpretations. Neuroimage 52, 1059-1069.

Somerville, L. H., Kim, H., Johnstone, T., Alexander, A. L., and Whalen, P. J. (2004). Human amygdala responses during presentation of happy and neutral faces: correlations with state anxiety. Biol. Psychiatry 55, 897-903.

Stanislas, D. (2009). Reading in the Brain: The New Science of How We Read? London: Penguin.

Torgesen, J., Wagner, R., and Rashotte, C. (1999). TOWRE: Test of Word Reading Efficiency. Austin, TX: Pro-Ed.

Vannest, J., Rajagopal, A., Cicchino, N. D., Franks-Henry, J., Simpson, S. M., Lee, G., et al. (2014). Factors determining success of awake and asleep magnetic resonance imaging scans in nonsedated children. Neuropediatrics 46, 370-377.

Wagner, R., Torgesen, J., and Rashotte, C. (1999). CTOPP: Comprehensive Test of Phonological Processing. London: Pearson.

Whitfield-Gabrieli, S., and Nieto-Castanon, A. (2012). Conn: a functional connectivity toolbox for correlated and anticorrelated brain networks. Brain Connect. 2, 125-141.

Wolff, U., and Lundberg, I. (2002). The prevalence of Dyslexia among art students. Dyslexia 8, 34-42.

Woodcock, R., Johnson, M., and Mather, N. (1989). Woodcock-Johnson PsychoEducational Battery-Revised. Hoboken, NJ: John Wiley \& Sons.

Conflict of Interest: The authors declare that the research was conducted in the absence of any commercial or financial relationships that could be construed as a potential conflict of interest.

Copyright (c) 2020 Nachshon, Farah and Horowitz-Kraus. This is an open-access article distributed under the terms of the Creative Commons Attribution License (CC BY). The use, distribution or reproduction in other forums is permitted, provided the original author(s) and the copyright owner(s) are credited and that the original publication in this journal is cited, in accordance with accepted academic practice. No use, distribution or reproduction is permitted which does not comply with these terms. 


\section{OPEN ACCESS}

Edited by:

Roberto Esposito,

ASUR Marche, Italy

Reviewed by:

Jen-Yin Chen,

Chi Mei Medical Center, Taiwan

Fuqing Zhou,

First Affiliated Hospital of Nanchang

University, China

${ }^{*}$ Correspondence:

Lizu Xiao

1417343432@qq.com

Yongxin $\mathrm{Li}$

yxin-li@163.com

${ }^{\dagger}$ These authors have contributed equally to this work

Specialty section:

This article was submitted to Applied Neuroimaging,

a section of the journal

Frontiers in Neurology

Received: 08 February 2020 Accepted: 01 September 2020

Published: 22 October 2020

Citation:

Huang J, Li Y, Xie H, Yang S, Jiang C, Sun W, Li D, Liao Y, Ba X and Xiao L (2020) Abnormal Intrinsic Brain Activity and Neuroimaging-Based fMRI Classification in Patients With Herpes Zoster and Postherpetic Neuralgia. Front. Neurol. 11:532110. doi: 10.3389/fneur.2020.532110

\section{Abnormal Intrinsic Brain Activity and Neuroimaging-Based fMRI Classification in Patients With Herpes Zoster and Postherpetic Neuralgia}

\author{
Jiabin Huang ${ }^{1 \dagger}$, Yongxin $\mathrm{Li}^{2 * \dagger}$, Huijun Xie ${ }^{2}$, Shaomin Yang ${ }^{1}$, Changyu Jiang ${ }^{1}$, Wuping Sun ${ }^{1}$, \\ Disen $\mathrm{Li}^{1}$, Yuliang Liao ${ }^{1}$, Xiyuan $\mathrm{Ba}^{1}$ and Lizu Xiao ${ }^{1 *}$ \\ 1 Department of Pain Medicine and Shenzhen Municipal Key Laboratory for Pain Medicine, Huazhong University of Science \\ and Technology Union Shenzhen Hospital, Shenzhen, China, ${ }^{2}$ Formula-Pattern Research Center, School of Traditional \\ Chinese Medicine, Jinan University, Guangzhou, China
}

Objective: Neuroimaging studies on neuropathic pain have discovered abnormalities in brain structure and function. However, the brain pattern changes from herpes zoster $(\mathrm{HZ})$ to postherpetic neuralgia $(\mathrm{PHN})$ remain unclear. The present study aimed to compare the brain activity between $\mathrm{HZ}$ and $\mathrm{PHN}$ patients and explore the potential neural mechanisms underlying cognitive impairment in neuropathic pain patients.

Methods: Resting-state functional magnetic resonance imaging (MRI) was carried out among 28 right-handed $\mathrm{HZ}$ patients, 24 right-handed $\mathrm{PHN}$ patients, and 20 healthy controls (HC), using a $3 T \mathrm{MRI}$ system. The amplitude of low-frequency fluctuation (ALFF) was analyzed to detect the brain activity of the patients. Correlations between ALFF and clinical pain scales were assessed in two groups of patients. Differences in brain activity between groups were examined and used in a support vector machine (SVM) algorithm for the subjects' classification.

Results: Spontaneous brain activity was reduced in both patient groups. Compared with $\mathrm{HC}$, patients from both groups had decreased ALFF in the precuneus, posterior cingulate cortex, and middle temporal gyrus. Meanwhile, the neural activities of angular gyrus and middle frontal gyrus were lowered in $\mathrm{HZ}$ and $\mathrm{PHN}$ patients, respectively. Reduced ALFF in these regions was associated with clinical pain scales in PHN patients only. Using SVM algorithm, the decreased brain activity in these regions allowed for the classification of neuropathic pain patients $(\mathrm{HZ}$ and $\mathrm{PHN})$ and $\mathrm{HC}$. Moreover, $\mathrm{HZ}$ and $\mathrm{PHN}$ patients are also roughly classified by the same model.

Conclusion: Our study indicated that mean ALFF values in these pain-related regions can be used as a functional MRI-based biomarker for the classification of subjects with different pain conditions. Altered brain activity might contribute to $\mathrm{PHN}$-induced pain.

Keywords: herpes zoster, postherpetic neuralgia, neuropathic pain, resting-state fMRI, aptitude of low-frequency fluctuation (ALFF), support vector machine 


\section{INTRODUCTION}

Herpes zoster (HZ) is one of the leading causes of severe pain in China, with a prevalence estimated to be $7.7 \%$ of the population who seek medical care. Moreover, 29.8\% of $\mathrm{HZ}$ patients may develop postherpetic neuralgia (PHN) that causes pain for months or even years (1). Neuropathic pain is well-understood to have a negative impact on the quality of life and a significant impact on cognitive function, including attention, memory, and executive functions (2). Previous studies have shown that peripheral neuropathic pain arises from injury of the peripheral and the central nervous systems (3-5). HZ is caused by the reactivation of varicella zoster virus and produces typical neuropathic pain. It can be classified as $\mathrm{HZ}$ and PHN. HZ is characterized by a painful erythematous rash in the affected dermatome (6). PHN is a prototypical human chronic neuropathic condition exhibiting multiple signs of peripheral and central neuropathy (7). The clinical manifestations include burning, tingling, hyperesthesia, and allodynia in the affected dermatome.

Although previous studies have shown that neuropathic pain can change brain plasticity (8), the basis of the brain structural and functional changes in patients with neuropathic pain is not clear. Previous neuroimaging studies have reported that patients with PHN showed anatomical changes in the bilateral insula, superior temporal gyrus, left middle frontal gyrus, and right thalamus (9). PHN's effects on brain activity have been studied by resting-state functional magnetic resonance imaging (MRI) and task-functional MRI. Abnormal functional activation and intrinsic activity were detected in regions including the thalamus, insula, somatosensory, putamen, amygdala, brainstem, prefrontal lobe, and cerebellum (10-12). Quantitative cerebral blood flow (CBF) mapping in PHN patients showed significantly increased CBF values in the striatum, thalamus, insula, amygdala, and primary somatosensory cortex and decreased CBF values in the frontal cortex (5). The functional connectivity between these pain-related regions and the notable connections between the putamen and other brain regions were altered in PHN patients (13). A negative correlation was found between PHN patients' pain scores and intrinsic activity in the prefrontal cortex (14). These works indicated that the functional connectivity between the prefrontal regions and other cortical regions was modulated by pain intensity. Additionally, a graph-theoretic approach was used to calculate the small-world network alterations in PHN patients. The PHN patients exhibited decreased local efficiency and significant changes of regional nodal efficiency in the postcentral gyrus, inferior parietal gyrus, thalamus, parahippocampus, and putamen (15). All the above studies indicate that chronic pain in PHN patients would modulate the activity and the connectivity of these pain-related regions.

The vast majority of these previous neuroimaging studies were restricted to PHN patients. Only a few studies have used neuroimaging methods to explore the differences in brain activity between HZ and PHN $(11,16)$. A previous study identified that $\mathrm{HZ}$ patients showed significant functional changes in the cerebellum, occipital lobe, temporal lobe, parietal lobe, and limbic lobe in contrast to PHN patients (11). Compared with the healthy controls (HC), both $\mathrm{HZ}$ and PHN patients demonstrated significantly decreased functional connectivity density (FCD) in the precuneus. However, there is no significant difference in the FCD values between HZ and PHN patients (16). One recent longitudinal neuroimaging study also assessed the brain imaging changes from HZ to PHN and found that the activity of the cerebellum and frontal lobes increased and the activity of the occipital lobe and limbic lobe decreased significantly during this transition (17).

Although previous literature confirmed that the brain function of patients with $\mathrm{HZ}$ and PHN was changed compared with the $\mathrm{HC}$, the differences in brain activity between $\mathrm{HZ}$ and PHN were inconsistent across these studies, and the neural mechanism is still unclear. Based on previous literature, we hypothesized that patients with $\mathrm{HZ}$ and PHN exhibit significant changes in spontaneous brain activity, which can be used to classify them from healthy controls. Thus, the present study aimed to explore the effects of $\mathrm{HZ}$ and PHN on brain activity and detect the neural mechanism underlying cognitive impairment in neuropathic pain patients. Resting-state functional MRI data were collected from all participants, and the amplitude of lowfrequency fluctuation (ALFF) was calculated. The ALFF is the most common and widely used method for characterizing the dynamic properties of the neuronal processing unit (18). The correlations between clinical pain scales and spontaneous brain activity were also assessed. Additionally, to evaluate the stability of the group comparison results, imaging features with significant differences between groups were applied for classification using support vector machine (SVM).

\section{METHODS}

\section{Subjects}

Fifty-two patients (right-handed; $28 \mathrm{HZ}$ and $24 \mathrm{PHN}$ ) and 20 HCs (right-handed) were recruited from the Pain Medicine Department of Huazhong University of Science and Technology Union Shenzhen Hospital. Before the imaging data were collected, we explained the purpose of the study, the study procedures, and the possible risks and discomfort to all participants. Then, written confirmed consent was obtained from each participant or their companion. This study was carried out following the Declaration of Helsinki and was approved by the Ethics Committee of Huazhong University of Science and Technology Union Shenzhen Hospital. The inclusion criteria were $\mathrm{HZ}$ within 30 days after onset of the painful rash, dermatome below $\mathrm{C} 2$, age older than 50 years old, and with persistent pain [visual analog scale (VAS) $\geq 5$ ]. All patients were diagnosed as PHN based on persistent pain (VAS $\geq 5$ ) for more than 30 days following the initial rash caused by $\mathrm{HZ}$ (19). Patients who had other pain disorders were excluded. All patients underwent MRI scanning within $24 \mathrm{~h}$ of enrollment. For ethical consideration, all patients had taken medicines to reach tolerable pain level before imaging. The baseline treatment is $8 \mathrm{mg}$ amitriptyline (Shanghai Fosun Pharmaceutical group) and $75 \mathrm{mg}$ pregabalin (Pfizer).

Before MRI scanning, spontaneous pain intensity was assessed using the VAS, which measures pain intensity on a scale of 0 
TABLE 1 | Demographic and clinical information data of the subjects.

\begin{tabular}{lccc}
\hline Characteristics & $\begin{array}{c}\text { HZ patients } \\
(\boldsymbol{n}=\mathbf{2 8})\end{array}$ & $\begin{array}{c}\text { PHN patients } \\
(\boldsymbol{n}=\mathbf{2 4})\end{array}$ & HC ( $\mathbf{n}=\mathbf{2 0})$ \\
\hline Gender (male/female) & $12 / 16$ & $15 / 9$ & $7 / 13$ \\
Age (years, mean \pm & $58.2 \pm 13.1$ & $67.0 \pm 14.1$ & $63.1 \pm 12.2$ \\
SD) & & & \\
Handedness (right/left) & $28 / 0$ & $24 / 0$ & $20 / 0$ \\
Duration (days, mean \pm & $15.7 \pm 5.6$ & $227.0 \pm 72.1$ & $/$ \\
SD) & & & \\
VAS score (mean \pm SD) & $7.6 \pm 1.3$ & $6.7 \pm 1.6$ & $/$ \\
PRI score (mean \pm SD) & $12.4 \pm 6.0$ & $16.4 \pm 9.2$ & $/$ \\
PPI score (mean \pm SD) & $2.9 \pm 1.0$ & $3.0 \pm 1.1$ & $/$
\end{tabular}

HC, healthy controls; $H Z$, herpes zoster; PHN, postherpetic neuralgia; VAS, visual analog scale; PRI, pain rating index; PPI, present pain intensity; SD, standard deviation.

to 10 , with 0 indicating no pain and 10 indicating the highest tolerable pain. Additionally, to identify the qualities of pain, the McGill pain questionnaire was used, including the Pain Rating Index (PRI) and the Present Pain Index (PPI). The PRI contains four subscales to evaluate the sensory, affective and evaluative, and miscellaneous aspects of pain. The PPI is a six-level pain intensity scale, including none (0), mild (1), discomforting (2), distressing (3), horrible (4), and excruciating (5) (20, 21). The exclusion criteria included any active major psychiatric illnesses, neurological illnesses, head injuries, or alcohol or drug abuse. Twenty healthy controls (all right-handed, 13 females, mean age: $63.05 \pm 26.20$ months) were also recruited. Detailed information can be found in Table 1 .

\section{MRI Data Acquisition}

All participants underwent MRI scans using a 3T Siemens scanner (MAGNETOM Skyra, Siemens, Germany) at the Huazhong University of Science and Technology Union Shenzhen Hospital, Shenzhen, China. Foam cushions were used in the scan process to reduce head translation and rotation. The resting-state functional MRI scans were obtained using an echo-planar imaging sequence with the parameters as follows: repetition time/echo time $=2,430 / 30 \mathrm{~ms}$, field-of-view $=240 \times$ $240 \mathrm{~mm}^{2}$, matrix $=64 \times 64$, flip angle $=90^{\circ}$, slice thickness $=3.6 \mathrm{~mm}, 40$ interleaved axial slices, and 180 volume. Highresolution T1-weighted 3D MPRAGE images were acquired for all subjects: repetition time/echo time $=1,900 / 2.12 \mathrm{~ms}$, field-ofview $=256 \times 256 \mathrm{~mm}^{2}, 320$ sagittal slices, $0.6 \mathrm{~mm}$ slice thickness, flip angle $=9^{\circ}$. All the participants were instructed to lie still with their eyes closed while remaining awake. After the scan, the subjects were asked whether they remained awake during the entire scan. All acquisitions were visually inspected for the presence of imaging artifacts. None of the participants were excluded on this basis.

\section{Resting-State Data Preprocessing and Statistical Analysis}

The resting-state functional MRI data were processed using Data Processing Assistant for Resting-State Functional MRI
(DPARSF) software (http://www.restfmri.net) (22). The first 10 functional images per subject were excluded from the analysis to ensure magnetization equilibrium. The functional MRI data were then slice-timed with a reference point at the median image and realigned to the first image. A mean functional image was obtained for each participant. No translation or rotation parameters in any given data set exceeded $\pm 2 \mathrm{~mm}$ or $\pm 2^{\circ}$. Afterward, each participant's T1-weighted structural image was co-registered to their mean functional image and then segmented. Nine nuisance covariates (six head motion parameters, global signal, white matter signal, and cerebrospinal fluid signal) were removed. The functional images were then normalized into the standard Montreal Neurological Institute space using the $\mathrm{T} 1$ image unified segmentation, resampled to $3 \mathrm{~mm}$, and smoothed using a 6-mm full-width at half-maximum Gaussian smoothing kernel. Linear trends were removed before the time course data from each voxel. Finally, an approach of the ALFF method was used for detecting regional signal change of spontaneous activity (23).

The ALFF for each voxel was normalized, and one-way ANOVA was used to determine whether there were any statistically significant differences in the ALFF between these groups $(p<0.001$, cluster size $=5)$. Post hoc analyses using two-sample $t$-tests were also conducted among the three groups. The significant results of ANOVA were selected as an output mask in the following $t$-tests. A $p<0.05$ (a minimum cluster size of five voxels) corrected by false discovery rate (FDR) correction was considered a difference between groups. Age and gender were controlled as covariates in all the above-mentioned statistical analyses.

All the significantly different clusters from the above ALFF analyses were extracted and considered as one region-of-interest (ROI). The mean ALFF of this ROI was extracted and normalized with Fisher's z-transformed function. The associations between the transformed ALFF value and the scores of clinical pain scales were tested using Pearson's correlation. A $p<0.05$ was considered a statistically significant correlation.

\section{Subjects' Classification With Support Vector Machine}

A multivariate pattern analysis was used in neuroimaging data to extract patterns and to categorize individual observations into different categories $(24,25)$. We used a specific multivariate pattern analysis approach known as SVM to classify the patients in the present study. SVM was implemented using the Pattern Recognition for Neuroimaging Toolbox software, version 2.0 (http://www.mlnl.cs.ucl.ac.uk/pronto/). Individual resting-state functional MRI was treated as points located in a high-dimensional space defined by the ALFF values in the preprocessed images. Significant clusters of the above ALFF analyses results were extracted and considered as a mask. This mask was applied to each preprocessed functional MRI to select the normalized ALFF values as a feature in the modeling. The classifier created in the present work (i.e., HZ vs. HC, PHN vs. HC, HZ vs. PHN) is based on binary SVMs. During the cross-validation step, a "leave-one-subject-out" method was used 
$(24,26)$. The data were split into a training set consisting of the samples from all but one subject and a validation set consisting of the samples from the left-out subject. To assess the SVM's overall accuracy, this procedure was repeated for each subject pair. The classification procedure was repeated 1,000 times.

\section{RESULTS}

\section{Demographic and Clinical Features}

The clinical characteristics of all the participants are shown in Table 1. All of the recruited patients in both groups suffered pain with VAS scores $\geq 5$, indicating moderate-to-severe pain. PRI and PPI were also assessed immediately before MRI scanning in most patients (24 HZ and $20 \mathrm{PHN}$ ). Eight patients were not assessed for PRI and PPI because of old age and being dialect-speaking. The doctor-patient communication is not smooth, which had caused a challenge in evaluating the scale accurately.

\section{Between-Group ALFF Differences}

One-way ANOVA test demonstrated that several regions showed significant differences in ALFF values among the three groups (Supplementary Figure 1). These regions included the bilateral precuneus, bilateral superior occipital gyrus, left posterior cingulate cortex (PCC), right middle frontal gyrus (MFG), right calcarine, right middle temporal gyrus (MTG), and right angular gyrus (AG). Compared with $\mathrm{HC}$, patients with $\mathrm{HZ}$ showed decreased ALFF in the bilateral precuneus, left PCC, right MTG, and right AG (FDR corrected; Table 2, Figure 1). No region showed a significant increase of ALFF in the HZ group. In the PHN group, compared with the control subjects, a significant decrease of ALFF values was found in the bilateral precuneus, right MFG, and left PCC (FDR corrected; Table 2, Figure 1). However, the ALFF was not significantly different between the $\mathrm{HZ}$ and the PHN groups.

\section{Correlation Between ALFF and Clinical Scale}

We selected the regions showing significant changes in the abovementioned between-group ALFF comparisons as our ROI (see Figure 2). The mean ALFF value of this ROI was extracted, and the correlations between the mean ALFF and the clinical scale were tested. There was a significant correlation between the mean ALFF and PRI in the PHN group, but not in the HZ group (PHN: $r=-0.535, p=0.015$; HZ: $r=-0.343, p=0.101$; see Figure 3). No significant correlation was observed between the mean ALFF and the other clinical scales.

\section{SVM Classification Results}

ALFF in the ROI demonstrated a significant difference in patients. The SVM classification between the $\mathrm{HZ}$ and the $\mathrm{HC}$ achieved a classification accuracy of $81.25 \%$ (sensitivity $75 \%$, specificity $85.71 \%, P<0.001$; Figure 4, left panel). Similarly, using the ALFF values of the same ROI allowed for the classification of $\mathrm{PHN}$ and $\mathrm{HC}$ subjects and achieved a classification accuracy of $86.36 \%$ (sensitivity $85 \%$, specificity $87.5 \%, P<0.001$; Figure 4, middle panel). Finally, for $\mathrm{HZ}$ and PHN classification, the use of ALFF in the same ROI achieved a classification accuracy of $61.54 \%$ (sensitivity $60.71 \%$, specificity $62.5 \%, P<0.081$; Figure 4, right panel).

\section{DISCUSSION}

In the present study, resting-state functional MRI and a machine learning method were combined to explore the abnormalities of spontaneous neuronal activity in patients with $\mathrm{HZ}$ and PHN. We demonstrated that, compared with HC, spontaneous brain activity was reduced in both patient groups. For $\mathrm{HZ}$ patients, reduction in neural activity was observed in the bilateral

TABLE 2 | Significant differences in amplitude of low-frequency fluctuation among three groups.

\begin{tabular}{|c|c|c|c|c|c|c|c|}
\hline Comparisons & \multicolumn{3}{|c|}{ Statistical values } & \multicolumn{4}{|c|}{ Coordinates' anatomical location } \\
\hline & 8 & 4.89 & 0.000 & -3 & -63 & 27 & $\mathrm{~L}$ precuneus \\
\hline & 15 & 5.46 & 0.000 & -6 & -48 & 27 & LPCC \\
\hline & 9 & 4.62 & 0.000 & 54 & -51 & 36 & $\mathrm{R}$ angular \\
\hline \multirow[t]{4}{*}{$\mathrm{HC}>\mathrm{PHN}$} & 72 & 5.80 & 0.000 & 9 & -48 & 24 & R precuneus \\
\hline & & 5.76 & 0.000 & -6 & -48 & 27 & LPCC \\
\hline & & 4.83 & 0.000 & 0 & -63 & 27 & $\mathrm{~L}$ precuneus \\
\hline & 6 & 4.66 & 0.000 & 42 & 48 & 27 & R MFG \\
\hline
\end{tabular}

The Montreal Neurological Institute coordinates and $t$-values for the local maxima of the centers of the voxel clusters. The threshold for significant clusters reported here was set at $p<$ 0.05 (false discovery rate corrected, cluster size of 5).

R, right hemisphere; L, left hemisphere; HC, healthy controls; HZ, herpes zoster; PHN, postherpetic neuralgia; PCC, posterior cingulate cortex; MTG, middle temporal gyrus; MFG, middle frontal gyrus. 

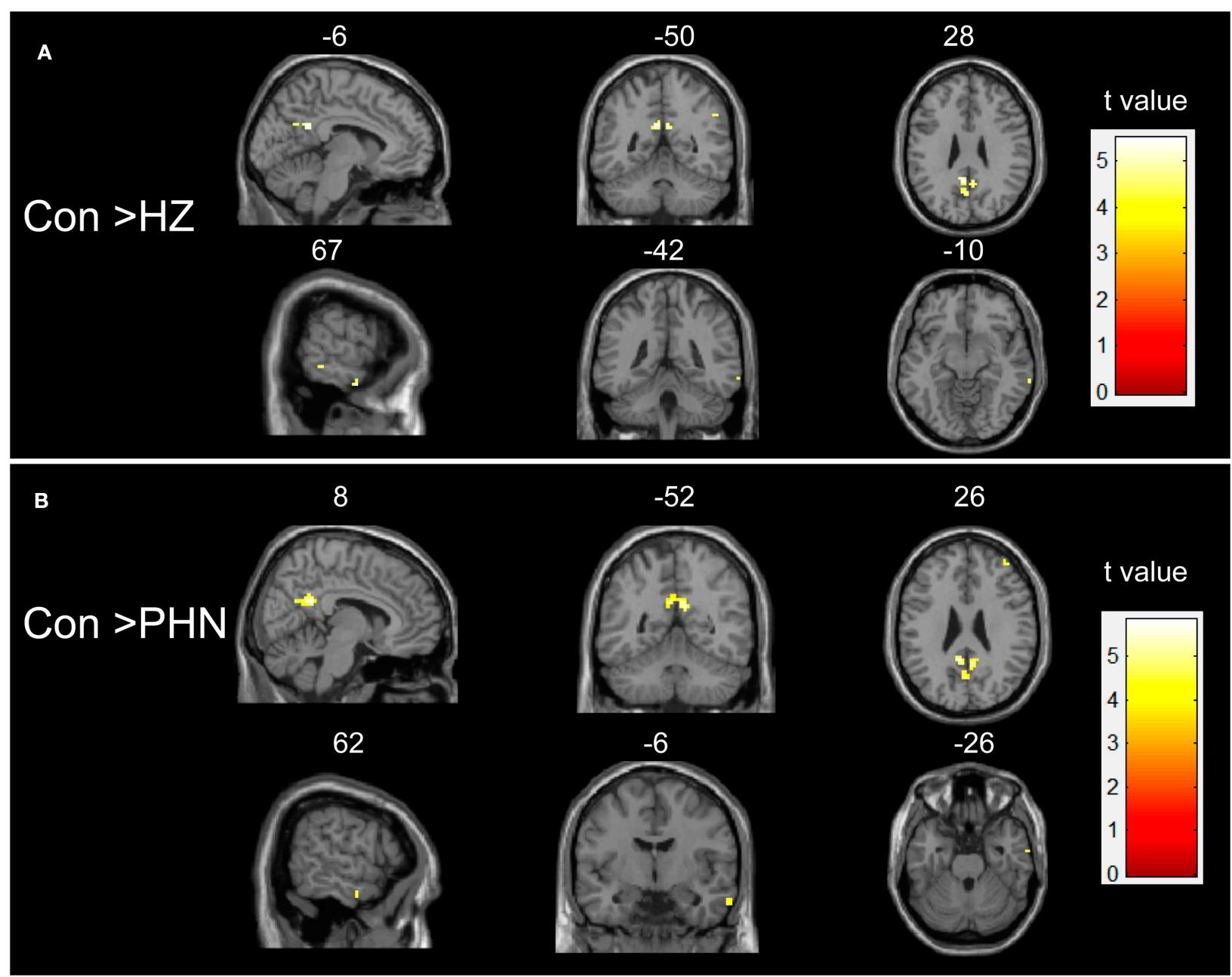

FIGURE 1 | Intergroup difference in amplitude of low-frequency fluctuation (ALFF). Both comparisons of HZ vs. healthy controls (HC) (A) and PHN vs. HC (B) groups showed a significant decrease in ALFF in some brain areas. Threshold of the whole brain: $p<0.05$, false discovery rate corrected, cluster size $>5$. HZ, herpes zoster; $\mathrm{PHN}$, postherpetic neuralgia; Con, normal controls.

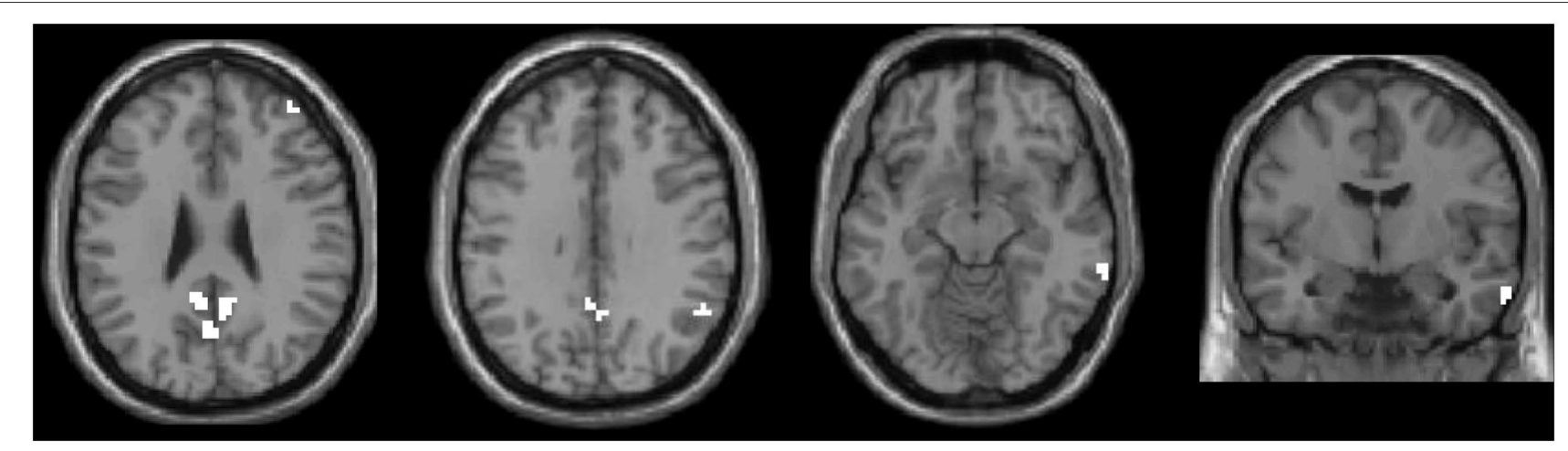

FIGURE 2 | All significant clusters of the intergroup amplitude of low-frequency fluctuation analyses were extracted as one region of interest (ROI). This ROI was used in the following correlation and classification analyses. 

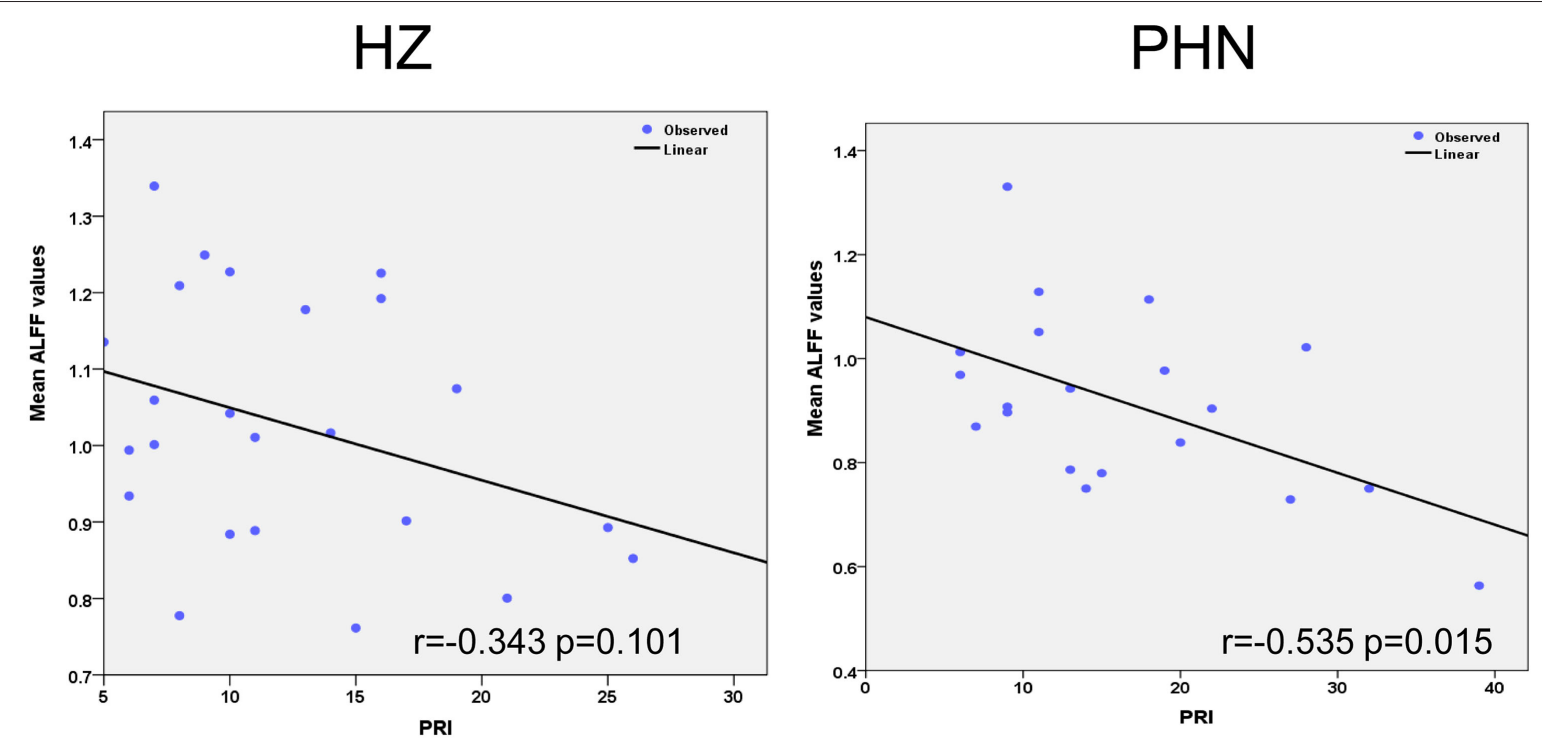

FIGURE 3 | Significant correlation between the amplitude of low-frequency fluctuation and clinical variables. The left panel shows the correlation results in the herpes zoster group. The right panel showed the correlation results in the postherpetic neuralgia group.

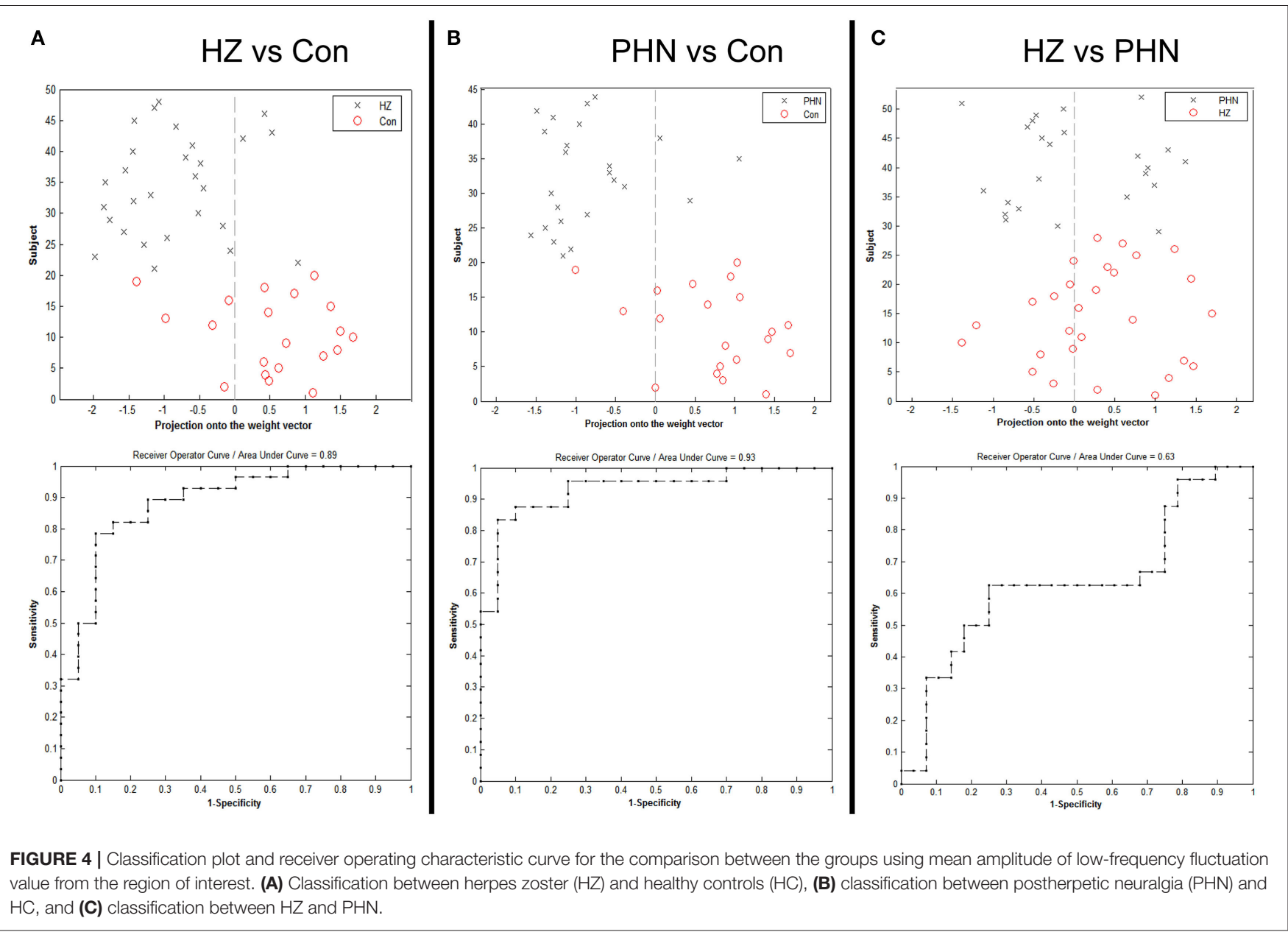


precuneus, left PCC, right MTG, and right AG. For PHN patients, the decreased activity was noted in the bilateral precuneus, left PCC, right MTG, and MFG. The mean ALFF values extracted from all these regions were significantly correlated with the clinical pain scales in the PHN group, but not in the HZ group. This result suggested that the spontaneous activity of these regions, in some respects, reflects the pain severity in PHN. In particular, machine learning classification using SVM showed that neuropathic pain patients ( $\mathrm{HZ}$ and $\mathrm{PHN}$ ) and healthy subjects could be classified by the decreased activity in these regions. The classifier also partially categorized patients into $\mathrm{HZ}$ and PHN groups $(p<0.081)$. These results may have important implications for understanding the brain abnormalities and the clinical characteristics of this disorder. Mean ALFF values in these pain-related regions may be used for the classification of neuropathic pain patients and healthy subjects.

\section{Significant Decrease in Spontaneous Neuronal Activity in Patients}

One notable finding of this study is the significantly decreased ALFF values of the bilateral precuneus and the left PCC between groups of patients. The precuneus and the PCC are a core component of the default mode network (27). The default mode network is a network in which activity is higher than in other parts of the brain at baseline. The hub regions of this network include the bilateral PCC/precuneus, inferior parietal cortex/angular gyrus, and medial prefrontal/anterior cingulate cortex (28). The brain regions in this network are deactivated during typical cognitive load tasks, while these regions show the highest activity at baseline. Previous studies have found that PCC and the adjacent precuneus are involved in monitoring sensory information $(29,30)$. Information from memory and perception was combined with bottom-up attention by the precuneus/PCC. Research on brain functional connectivity of precuneus/PCC has shown disrupted connectivity in patients with PHN $(16,31)$. Furthermore, the precuneus has various connections with other cortical and subcortical areas, which facilitates the integration between external and internal information (32). Previous studies have also demonstrated that the precuneus plays an essential role in the implementation of a wide range of higher-order cognitive functions (33).

In the present study, we found that the ALFF values were decreased in the precuneus/PCC in the two patient groups. It has been reported that the impairment of the bilateral precuneus was associated with cognitive and behavioral impairments observed in patients with pain (34). Our finding shed light on the neural basis of increased risk of dementia in $\mathrm{HZ}$ and $\mathrm{PHN}$ patients (PMID: 29244265 and PMID: 23900759). Generally, cognitive and emotional factors have substantial impacts on pain (35). In 1979, the International Association for the Study of Pain approved a definition of pain: an unpleasant sensory and emotional experience associated with actual or potential tissue damage or described in terms of such damage (36). When actual damage is present, although the unpleasantness could rapidly disappear, the fear memory would last long (37). This result implied that the impairment of the precuneus might contribute to the emotional experience associated with pain.

In contrast to the commonly affected brain regions, decreased ALFF values in the right AG were observed only in $\mathrm{HZ}$ patients. The AG is an important part of the brain, which is responsible for processing language, numbers, and various logic-oriented processes. Decreased ALFF in the right AG might account for changes in the cognitive functions of $\mathrm{HZ}$ patients. However, no significantly decreased ALFF was found in the AG of patients with PHN. This phenomenon warrants further study in the future.

Another pain-condition-dependent brain region is MFG. In this study, a significant decrease of ALFF in the right MFG was found in patients with PHN, but not in patients with HZ. This result is consistent with previous studies that decreased ALFF value and cerebral blood flow in the MFG were detected in the patients with $\mathrm{PHN}(5,12)$. The patients with $\mathrm{PHN}$ also showed notably abnormal tissue microstructure in the MFG (9). The right MFG has been proposed to be a site of convergence of the dorsal and the ventral attention network (38). Previous studies have identified that this region is implicated in emotion modulation and executive function (39). Therefore, our finding indicated that repressed neural activity in the MFG might be responsible for the emotional or attention aspect of $\mathrm{PHN}$ pain.

In addition to the precuneus/PCC, decreased ALFF values were also detected in the right MTG of both patient groups. This finding was consistent with previous studies which reported functional impairment of the right MTG in patients with PHN and $\operatorname{HZ}(11,12,17)$. However, there was no significant difference in ALFF between HZ and PHN. This result did not agree with a previous study in which PHN patients showed a decreased activity in the limbic system, right MTG, and parietal lobe compared with the $\mathrm{HZ}$ patients (11). We speculate that this inconsistency may arise due to the different definitions of PHN. Cao (11) defined PHN as persistent pain for more than 3 months after the zoster rash, whereas 1 month was adopted to identify PHN patients in our study (40). It is possible that this transition from $\mathrm{HZ}$ to $\mathrm{PHN}$ is gradually developed in a time-dependent fashion. A recent neuroimaging study using a similar PHN definition demonstrated that functional connectivity density was not significantly different between the PHN and the $\mathrm{HZ}$ patients (16). Interestingly, the results of a previous study by Hong and Cao were mildly consistent with our present study. We recently reported that temporary spinal cord stimulation could effectively prevent the development of PHN for the HZ patients (duration $<3$ months), compared with the $\mathrm{PHN}$ patients (duration $>3$ months) (41). Moreover, it may indicate that early neuromodulation of herpetic neuralgia could prevent the PHN effectively due to the fact that central sensitization has not yet been developed.

\section{Correlation Between Neuroimaging Index and the Clinical Pain Scales}

More importantly, our results showed a significant correlation between the mean ALFF in the selected ROI and the clinical pain scales in the PHN group. The negative correlation suggested that 
the abnormal spontaneous brain activity may be due to the pain. Chronic pain condition can lead to anatomical and functional alterations in the brain regions associated with psychological modulation, resulting in not only pain but also altered cognition and affection. Cognitive behavioral therapy could reduce stress and control pain (35). Brain activity and connectivity would, in some aspects, reflect patients' cognitive ability and the pain intensity $(14,42)$. Chronic pain may affect the cognitive functions that depend on functional connectivity between the pain-related regions. Previous studies in brain anatomy also showed a negative correlation between brain microstructure abnormalities and PHN pain (9). Our results were consistent with these previous neuroimaging studies, which indicated that the activity of painrelated regions has potential value in objectively estimating the severity of PHN pain.

The selected ROI included regions such as the bilateral precuneus, left PCC, right MTG, right MFG, and right AG. All these regions are pain-related areas. Previous neuroimaging studies have found that the brain activity and the gray matter volume in the right MTG have a significant correlation with pain duration and intensity of patients with PHN $(12,17)$. The functional connectivity density of bilateral precuneus in PHN patients also showed a significant correlation with VAS scores (16). In the present study, the negative correlation results in our study indicated that the patients with more pain would show greater reduction in spontaneous brain activity in these regions. This negative correlation was not significant in the $\mathrm{HZ}$ group. This may be explained by the short duration of pain condition in these patients. Future studies are needed to verify this explanation.

\section{Identification of Patients With PHN From HC and Patients With HZ}

In this study, the patients with neuropathic pain and the healthy controls were classified by the mean ALFF values of these altered regions using the linear SVM classifier. There is a high classification accuracy of $81.25 \%$ between the $\mathrm{HZ}$ and $\mathrm{HC}$ and of $86.36 \%$ between the PHN and HC. In recent years, multivariate pattern analysis methods have been applied to distinguish patients from healthy controls $(43,44)$ and predict surgery outcomes $(25,45)$. For patients with neuropathic pain, one previous neuroimaging study has found that the voxelmirrored homotopic connectivity in the dorsolateral prefrontal cortex, precuneus, and PCC discriminated between patients with PHN and healthy subjects (31). Our recent study measuring gray matter volume found that alterations in several brain regions, including the middle frontal cortex, ACC, precuneus, and cuneus, had a significant predictive power to classify $\mathrm{HZ}$ patients with different responses to medications (46). Along with these findings, our observations suggested that the frontal gyrus and the precuneus have a significant predictive power to distinguish neuropathic pain patients from the healthy controls.

In the present study, we used the mean ALFF of ROI as a feature for classifying the subtypes of patients with neuropathic pain (i.e., PHN and HZ). The SVM classifier performed between $\mathrm{HZ}$ and PHN patients had a classification accuracy of $61.54 \%$. We did not achieve as high a classification accuracy as in previous studies $(31,46)$. It should be noted that, in our study, all patients have taken pregabalin and amitriptyline to alleviate the pain intensity before the MRI scan. The previous findings revealed that amitriptyline may reduce pain-related central activations in the ACC in patients with irritable bowel syndrome (47). Similarly, pregabalin suppressed evoked neural activity in ACC, when compared with tramadol, in neuropathic pain patients (48). The current results indicated that these medicines strongly inhibited the ACC, but not the PCC, in HZ and PHN patients compared to healthy controls. Because of long-term analgesia in PHN patients clinically, it is difficult to quantify the central changes due to drug therapy or the refractory pain condition in human research.

\section{Limitations}

There are several limitations in our study. Firstly, our present research used a cross-sectional design in which the MRI data of the subjects were obtained at just one time-point. This method may lead to questions about whether the results are the consequence of preexisting differences between groups. Future studies should consider a longitudinal design, which can directly monitor activity changes from acute pain to chronic pain. Secondly, all patients are allowed to take the drug therapy of the pregabalin and amitriptyline which may certainly inhibit or reduce the brain function. A well-controlled group should be established in further studies.

\section{CONCLUSION}

In the present study, we discovered reduced spontaneous brain activity in the precuneus/PCC, MTG, AG, and MFG of patients with neuropathic pain. The reduced ALFF in these regions was associated with the clinical pain scales of patients with PHN. The SVM classification indicated that these abnormalities might accurately discriminate between neuropathic pain patients and healthy controls at the level of the individual. A similar analysis also demonstrated that the abnormalities in ALFF values showed potentials to distinguish patients with PHN from those with HZ. These results are likely to be valuable in explaining the underlying neural mechanisms and understanding the interaction between neuropathic pain and brain abnormalities. The present results provided new insights into functional MRI as a pain biomarker to help diagnose and classify patients with neuropathic pain. Future work should combine functional information with structural imaging data to examine whether this leads to a higher diagnostic accuracy.

\section{DATA AVAILABILITY STATEMENT}

All datasets generated for this study are included in the article/Supplementary Material.

\section{ETHICS STATEMENT}

The studies involving human participants were reviewed and approved by the Ethics Committee of 
the Huazhong University of Science and Technology Union Shenzhen Hospital. The patients/participants provided their written informed consent to participate in this study.

\section{AUTHOR CONTRIBUTIONS}

WS, SY, and LX designed this work. JH, XB, DL, and YL collected the data. YL and HX analyzed the data. YL, JH, and LX discussed the results. YL wrote the initial draft. YL, JH, LX, and CJ substantially revised the manuscript. LX acquired the funding. All authors agreed to be accountable for all aspects of this work.

\section{FUNDING}

This work was supported by the Shenzhen Municipal Scheme for Basic Research (No. JCYJ20160429181451546) and the National Natural Science Foundation of China (Nos. 81601483 and

\section{REFERENCES}

1. Yang F, Yu S, Fan B, Liu Y, Chen YX, Kudel I, et al. The epidemiology of herpes zoster and postherpetic neuralgia in China: results from a cross-sectional study. Pain Ther. (2019) 8:249-59. doi: 10.1007/s40122-01 9-0127-z

2. Moriarty O, Mcguire BE, Finn DP. The effect of pain on cognitive function: a review of clinical and preclinical research. Prog Neurobiol. (2011) 93:385-404. doi: 10.1016/j.pneurobio.2011.01.002

3. Vartiainen N, Kirveskari E, Kallio-Laine K, Kalso E, Forss N. Cortical reorganization in primary somatosensory cortex in patients with unilateral chronic pain. J Pain. (2009) 10:854-9. doi: 10.1016/j.jpain.2009.02.006

4. Gondo M, Moriguchi Y, Kodama N, Sato N, Sudo N, Kubo C, et al. Daily physical complaints and hippocampal function: an fMRI study of pain modulation by anxiety. Neuroimage. (2012) 63:1011-9. doi: 10.1016/j.neuroimage.2012.07.025

5. Liu J, Hao Y, Du M, Wang X, Zhang J, Manor B, et al. Quantitative cerebral blood flow mapping and functional connectivity of postherpetic neuralgia pain: a perfusion fMRI study. Pain. (2013) 154:110-8. doi: 10.1016/j.pain.2012.09.016

6. Jeon YH. Herpes zoster and postherpetic neuralgia: practical consideration for prevention and treatment. Korean J Pain. (2015) 28:177-84. doi: $10.3344 / \mathrm{kjp} .2015 .28 .3 .177$

7. Oaklander AL. The density of remaining nerve endings in human skin with and without postherpetic neuralgia after shingles. Pain. (2001) 92:139-45. doi: 10.1016/S0304-3959(00)00481-4

8. Apkarian AV, Hashmi JA, Baliki MN. Pain and the brain: specificity and plasticity of the brain in clinical chronic pain. Pain. (2011) 152:S49-64. doi: 10.1016/j.pain.2010.11.010

9. Zhang Y, Yu T, Qin B, Li Y, Song G, Yu B. Microstructural abnormalities in gray matter of patients with postherpetic neuralgia: a diffusional kurtosis imaging study. Pain Phys. (2016) 19:E601-11. Available online at: http:// europepmc.org/article/MED/27228

10. Geha PY, Baliki MN, Chialvo DR, Harden RN, Paice JA, Apkarian AV. Brain activity for spontaneous pain of postherpetic neuralgia and its modulation by lidocaine patch therapy. Pain. (2007) 128:88-100. doi: 10.1016/j.pain.2006.09.014

11. Cao S, Li Y, Deng WW, Qin BY, Zhang Y, Xie P, et al. Local brain activity differences between herpes zoster and postherpetic neuralgia patients: a resting-state functional MRI study. Pain Physician. (2017) 20:E687-99.

12. Cao S, Song GJ, Zhang Y, Xie P, Tu Y, Li Y, et al. Abnormal local brain activity beyond the pain matrix in postherpetic neuralgia patients:
81630104). This work was also supported by the Administration of Traditional Chinese Medicine of Guangdong Province, China (Nos. 20182023 and 20191083).

\section{ACKNOWLEDGMENTS}

We would like to thank all the subjects who participated in this study for their cooperation. We are also grateful to the radiographers at the Department of Radiology, Huazhong University of Science and Technology Union Shenzhen Hospital, who scanned the imaging data of the subjects.

\section{SUPPLEMENTARY MATERIAL}

The Supplementary Material for this article can be found online at: https://www.frontiersin.org/articles/10.3389/fneur. 2020.532110/full\#supplementary-material

Supplementary Figure 1 | One-way ANOVA comparison on amplitude of low-frequency fluctuation maps among the three groups.

a resting-state functional MRI study. Pain Phys. (2017) 20:E303-14. doi: 10.36076/ppj.2017.E314

13. Geha PY, Baliki MN, Wang X, Harden RN, Paice JA, Apkarian AV. Brain dynamics for perception of tactile allodynia. (touchinduced pain) in postherpetic neuralgia. Pain. (2008) 138:641-56. doi: 10.1016/j.pain.2008.02.021

14. Li J, Huang X, Sang K, Bodner M, Ma K, Dong XW. Modulation of prefrontal connectivity in postherpetic neuralgia patients with chronic pain: a resting-state functional magnetic resonance-imaging study. J Pain Res. (2018) 11:2131-44. doi: 10.2147/JPR.S166571

15. Zhang Y, Liu J, Li L, Du M, Fang W, Wang D, et al. A study on small-world brain functional networks altered by postherpetic neuralgia. Magn Reson Imaging. (2014) 32:359-65. doi: 10.1016/j.mri.2013.12.016

16. Hong S, Gu L, Zhou F, Liu J, Huang M, Jiang J, et al. Altered functional connectivity density in patients with herpes zoster and postherpetic neuralgia. J Pain Res. (2018) 11:881-8. doi: 10.2147/JPR.S154314

17. Cao S, Qin BY, Zhang Y, Yuan J, Fu B, Xie P, et al. Herpes zoster chronification to postherpetic neuralgia induces brain activity and grey matter volume change. Am J Transl Res. (2018) 10:184-99.

18. Song XW, Dong ZY, Long XY, Li SF, Zuo XN, Zhu CZ, et al. REST: a toolkit for resting-state functional magnetic resonance imaging data processing. PLoS ONE. (2011) 6:e25031. doi: 10.1371/journal.pone.0025031

19. Klompas M, Kulldorff M, Vilk Y, Bialek SR, Harpaz R. Herpes zoster and postherpetic neuralgia surveillance using structured electronic data. Mayo Clin Proc. (2011) 86:1146-53. doi: 10.4065/mcp.2011.0305

20. Melzack R, Wall PD. Pain mechanisms: a new theory. Science. (1965) 150:9719. doi: 10.1126/science.150.3699.971

21. Melzack R. The McGill pain questionnaire: major properties and scoring methods. Pain. (1975) 1:277-99. doi: 10.1016/0304-3959(75)90044-5

22. Yan C, Zang Y. DPARSF: a MATLAB toolbox for "pipeline" data analysis of resting-state fMRI. Front Syst Neurosci. (2010) 4:13. doi: 10.3389/fnsys.2010.00013

23. Zou Q-H, Zhu C-Z, Yang Y, Zuo X-N, Long X-Y, Cao Q-J, et al. An improved approach to detection of amplitude of low-frequency fluctuation. (ALFF) for resting-state fMRI: fractional ALFF. J Neurosci Methods. (2008) 172:137-41. doi: 10.1016/j.jneumeth.2008.04.012

24. Li F, Huang X, Tang W, Yang Y, Li B, Kemp GJ, et al. Multivariate pattern analysis of DTI reveals differential white matter in individuals with obsessive-compulsive disorder. Hum Brain Mapp. (2014) 35:2643-51. doi: $10.1002 / \mathrm{hbm} .22357$

25. Wu MJ, Mwangi B, Bauer IE, Passos IC, Sanches M, Zunta-Soares $\mathrm{GB}$, et al. Identification and individualized prediction of clinical 
phenotypes in bipolar disorders using neurocognitive data, neuroimaging scans and machine learning. Neuroimage. (2017) 145(Pt. B):254-64. doi: 10.1016/j.neuroimage.2016.02.016

26. Saeb S, Lonini L, Jayaraman A, Mohr DC, Kording KP. The need to approximate the use-case in clinical machine learning. Gigascience. (2017) 6:1-9. doi: 10.1093/gigascience/gix019

27. Utevsky AV, Smith DV, Huettel SA. Precuneus is a functional core of the default-mode network. J Neurosci. (2014) 34:932-40. doi: 10.1523/JNEUROSCI.4227-13.2014

28. van Oort ESB, van Cappellen Van Walsum AM, Norris DG. An investigation into the functional and structural connectivity of the default mode network. Neuroimage. (2014) 90:381-9. doi: 10.1016/j.neuroimage.2013.12.051

29. Raichle ME, Macleod AM, Snyder AZ, Powers WJ, Gusnard DA, Shulman GL. A default mode of brain function. Proc Natl Acad Sci USA. (2001) 98:676-82. doi: 10.1073/pnas.98.2.676

30. Andrews-Hanna JR, Reidler JS, Sepulcre J, Poulin R, Buckner RL. Functionalanatomic fractionation of the brain's default network. Neuron. (2010) 65:55062. doi: 10.1016/j.neuron.2010.02.005

31. Jiang J, Gu LL, Bao D, Hong SD, He W, Tan YM, et al. Altered homotopic connectivity in postherpetic neuralgia: a resting state fMRI study. J Pain Res. (2016) 9:877-86. doi: 10.2147/JPR.S117787

32. Scheuerecker J, Meisenzahl EM, Koutsouleris N, Roesner M, Schopf V, Linn J, et al. Orbitofrontal volume reductions during emotion recognition in patients with major depression. J Psychiatr Neurosci. (2010) 35:311-20. doi: 10.1503/jpn.090076

33. Cavanna AE, Trimble MR. The precuneus: a review of its functional anatomy and behavioural correlates. Brain. (2006) 129:564-83. doi: 10.1093/brain/awl004

34. Baliki MN, Geha PY, Apkarian AV, Chialvo DR. Beyond feeling: chronic pain hurts the brain, disrupting the default-mode network dynamics. J Neurosci. (2008) 28:1398-403. doi: 10.1523/JNEUROSCI.4123-07.2008

35. Bushnell MC, Ceko M, Low LA. Cognitive and emotional control of pain and its disruption in chronic pain. Nat Rev Neurosci. (2013) 14:502-11. doi: $10.1038 / \mathrm{nrn} 3516$

36. Pain terms: a list with definitions and notes on usage. recommended by the IASP subcommittee on taxonomy. Pain. (1979) 6:249.

37. Nielsen FA, Balslev D, Hansen LK. Mining the posterior cingulate: segregation between memory and pain components. Neuroimage. (2005) 27:520-32. doi: 10.1016/j.neuroimage.2005.04.034

38. Su Q, Liang M, Iannetti GD, Mouraux A. Spatial patterns of brain activity preferentially reflecting transient pain and stimulus intensity. Cereb Cortex. (2019) 29:2211-27. doi: 10.1093/cercor/bhz026

39. Schoenbaum G, Roesch M. Orbitofrontal cortex, associative learning, and expectancies. Neuron. (2005) 47:633-6. doi: 10.1016/j.neuron.2005.07.018
40. Gauthier A, Breuer J, Carrington D, Martin M, Rémy V. Epidemiology and cost of herpes zoster and post-herpetic neuralgia in the United Kingdom. Epidemiol Infect. (2009) 137:38-47. doi: 10.1017/S0950268808000678

41. Huang J, Yang S, Yang J, Sun W, Jiang C, Zhou J, et al. Early treatment with temporary spinal cord stimulation effectively prevents development of postherpetic neuralgia. Pain Phys. (2020) 23:E219-30.

42. Cardoso-Cruz H, Lima D, Galhardo V. Impaired spatial memory performance in a rat model of neuropathic pain is associated with reduced hippocampus-prefrontal cortex connectivity. J Neurosci. (2013) 33:2465-80. doi: 10.1523/JNEUROSCI.5197-12.2013

43. De Vos F, Koini M, Schouten TM, Seiler S, Van Der Grond J, Lechner A et al. A comprehensive analysis of resting state fMRI measures to classify individual patients with Alzheimer's disease. Neuroimage. (2018) 167:62-72. doi: 10.1016/j.neuroimage.2017.11.025

44. Tripp B. A deeper understanding of the brain. Neuroimage. (2018) 180:114-6. doi: 10.1016/j.neuroimage.2017.12.079

45. Memarian N, Kim S, Dewar S, Engel J, Jr., Staba RJ. Multimodal data and machine learning for surgery outcome prediction in complicated cases of mesial temporal lobe epilepsy. Comput Biol Med. (2015) 64:67-78 doi: 10.1016/j.compbiomed.2015.06.008

46. Zeng P, Huang J, Wu S, Qian C, Chen F, Sun W, et al. Characterizing the structural pattern predicting medication response in herpes zoster patients using multivoxel pattern analysis. Front Neurosci. (2019) 13:534. doi: 10.3389/fnins.2019.00534

47. Morgan V, Pickens D, Gautam S, Kessler R, Mertz H. Amitriptyline reduces rectal pain related activation of the anterior cingulate cortex in patients with irritable bowel syndrome. Gut. (2005) 54:601-7. doi: 10.1136/gut.2004.0 47423

48. Wanigasekera V, Wartolowska K, Huggins JP, Duff EP, Vennart W, Whitlock $\mathrm{M}$, et al. Disambiguating pharmacological mechanisms from placebo in neuropathic pain using functional neuroimaging. Br J Anaesth. (2018) 120:299-307. doi: 10.1016/j.bja.2017.11.064

Conflict of Interest: The authors declare that the research was conducted in the absence of any commercial or financial relationships that could be construed as a potential conflict of interest.

Copyright (C) 2020 Huang, Li, Xie, Yang, Jiang, Sun, Li, Liao, Ba and Xiao. This is an open-access article distributed under the terms of the Creative Commons Attribution License (CC BY). The use, distribution or reproduction in other forums is permitted, provided the original author(s) and the copyright owner(s) are credited and that the original publication in this journal is cited, in accordance with accepted academic practice. No use, distribution or reproduction is permitted which does not comply with these terms. 


\section{OPEN ACCESS}

Edited by:

Nicoletta Cera

University of Porto, Portugal

Reviewed by: Yi Zhang,

Xidian University, China

Heng Chen,

Guizhou University, China

Xujun Duan,

University of Electronic Science and

Technology of China, China

*Correspondence:

Peihai Zhang

zhangpeihai@126.com

Degui Chang

624440310@qq.com

tThese authors have contributed equally to this work and share first

authorship

Specialty section:

This article was submitted to Neuroimaging and Stimulation, a section of the journal

Frontiers in Psychiatry

Received: 16 July 2020 Accepted: 09 September 2020

Published: 27 October 2020

Citation:

Yin T, Liu Q, Ma Z, Li Z, Sun R, Ren F Li G, Huang $X$, Chang $D$ and Zhang $P$

(2020) Associations Between Altered

Cerebral Activity Patterns and

Psychosocial Disorders in Patients

With Psychogenic Erectile

Dysfunction: A Mediation Analysis of

fMRI. Front. Psychiatry 11:583619.

doi: 10.3389/fpsyt.2020.583619
Associations Between Altered Cerebral Activity Patterns and Psychosocial Disorders in Patients With Psychogenic Erectile

\section{Dysfunction: A Mediation Analysis of fMRI}

Tao Yin ${ }^{1,2 \dagger}$, Qi Liu ${ }^{3+}$, Ziyang Ma ${ }^{4,5 \dagger}$, Zhengjie Li $^{1,2}$, Ruirui Sun ${ }^{1,2}$, Feiqiang Ren ${ }^{4}$, Guangsen $\mathrm{Li}^{4}$, Xiaopeng Huang ${ }^{4}$, Degui Chang ${ }^{4 *}$ and Peihai Zhang ${ }^{4 *}$

'Acupuncture and Tuina School/The 3rd Teaching Hospital, Chengdu University of Traditional Chinese Medicine, Chengdu, China, ${ }^{2}$ Acupuncture-Brain Science Research Center, Chengdu University of Traditional Chinese Medicine, Chengdu, China, ${ }^{3}$ Acupuncture and Tuina School, Shaanxi University of Chinese Medicine, Xian, China, ${ }^{4}$ Hospital of Chengdu University of Traditional Chinese Medicine, Chengdu, China, ${ }^{5}$ Clinical Medical School, Chengdu University of Traditional Chinese Medicine, Chengdu, China

Previous studies had illustrated the significant neural pathological changes in patients with psychogenic erectile dysfunction (pED), while few works focused on the neural underpinning of the psychosocial status in patients with pED. This study aimed to investigate the associations among the altered cerebral activity patterns, impaired erectile function, and the disrupted psychosocial status in patients with pED. Thirty-two patients with pED and 28 healthy controls (HCs) were included. The amplitude of low-frequency fluctuations (ALFF), region-of-interest-based functional connectivity (FC), as well as Pearson correlation analyses and mediation analyses between neuroimaging outcomes and clinical outcomes were performed. Compared to HCs, patients with pED manifested lower erectile function, disrupted psychosocial status, as well as decreased ALFF in the left dorsolateral prefrontal cortex (dIPFC) and reduced FC between the left dIPFC and left angular gyrus, and left posterior cingulate cortex (PCC) and precuneus, which belonged to the default mode network (DMN). Moreover, both the ALFF of the left dIPFC and FC between the left dIPFC and left PCC and precuneus were significantly correlated with the sexual function and psychosocial status in patients with pED. The disrupted psychosocial status mediated the influence of atypical FC between dIPFC and DMN on decreased erectile function. This study widened our understanding of the important role of psychosocial disorders in pathological neural changes in patients with pED.

Keywords: psychogenic erectile dysfunction, psychosocial status, amplitude of low-frequency fluctuations, functional connectivity, fMRI, mediation analysis 


\section{INTRODUCTION}

Erectile dysfunction (ED), the most common male sexual disorder, is defined as the persistent inability to attain or maintain sufficient penile erection for successful sexual performance (1). Population-based cross-sectional studies estimated that $11 \%$ of men in their 30 s and $24 \%$ of men in their 40 s suffered from this sexual dysfunction $(2,3)$. A recent review on the epidemiological surveys of ED similarly indicated that the prevalence of ED among young people was as high as 30\% (4). ED is now recognized as a major public health issue, not only for its high prevalence but also for its severe impacts on sexual satisfaction and quality of life (QOL) of patients and their partners (5-8). Among the different subtypes of ED, psychogenic $\mathrm{ED}(\mathrm{pED})$ has always been a focus of researchers for the high incidence and complex pathogenesis $(7,9,10)$. Different from organic ED, which has physical causes and evident pathological characteristics, pED is generally caused by psychosocial factors and lacks specific biomarkers $(11,12)$.

Male sexual arousal is described as a complicated biopsychosocial process that involves the coordination of psychological, neurological, endocrine, and vascular systems (13). With the application of neuroimaging technologies, the close correlation between sexuality and the central nervous system (CNS) is becoming clearer (14-16). Many male sexual dysfunctions, such as $\operatorname{ED}(17-22)$, premature ejaculation $(23,24)$, and anejaculation (25) have been detected to be associated with alterations in the structure and function of the brain. For example, patients with pED demonstrated the atrophied gray matter volume $(17,19)$, altered white matter microstructure (26), aberrant activity patterns (20), and disrupted topological properties (22) in multiple brain regions, such as prefrontal cortex, cingulate cortex, and insular cortex. These studies indicated that the structural and functional aberrancies of the brain might be the critical pathogenesis properties of pED. However, most of these previous studies paid primary attention to the neural mechanism of the low erectile function, and few works focused on the associations between the altered cerebral activity and the psychosocial status of pED patients. As a typical psychosocial disorder, patients with pED demonstrated not only decreased erectile function but also severe symptomrelated psychosocial disorders, such as poor sexual relations, diminished sexual satisfaction, and low self-esteem. Therefore, in addition to investigating the correlations between the altered cerebral activity properties and the physiological function, it is necessary to explore the neural underpinning of the disrupted psychosocial status in patients with $\mathrm{pED}$, so that to more fully understand the neuropathological features underlying pED. The amplitude of low-frequency fluctuations (ALFF) and region-of-interest (ROI)-based functional connectivity (FC) are two commonly used approaches to investigate human brain activity patterns. The ALFF is a data-driven algorithm to measure the spontaneous activity of the brain (27), and the ROI-based FC is a purpose-driven algorithm that reflects the synchronization of different brain regions and indicates the tendency of cortical networks to be co-activated (28). The combination of ALFF and ROI-based FC provides valuable perspectives for detecting the neural underpinning of diseases $(29,30)$.

In this study, we aimed to investigate the alterations of spontaneous cerebral activity and synchronous cerebral activity of patients with pED using ALFF and ROI-based FC analysis methods, and to examine the potential associations among the altered cerebral activity patterns and the decreased erectile function as well as the disrupted psychosocial status in patients with pED. To deepen the understanding of the impacts of psychosocial status on clinical symptoms and cerebral activity patterns, the mediation analysis with psychological status as a mediator was also conducted. We hypothesized that the cerebral activity patterns were altered in patients with pED compared to healthy controls (HCs), and that the disordered psychosocial status mediated the relationship between the aberrant cerebral activity patterns and the low erectile function.

\section{MATERIALS AND METHODS}

\section{Participants Selection}

Thirty-two patients with pED and 28 HCs were enrolled in this study. The patients were recruited at the outpatient department of Andrology in the Hospital of Chengdu University of Traditional Chinese Medicine and Sichuan Integrative Medicine Hospital from November 2018 to April 2019. All the potential patients were diagnosed with comprehensive history taking, physical examinations, laboratory tests, and specific examinations. The details of the preceding items could be found in our early study (26). Patients were included if they fulfilled the following inclusion criteria: (1) matched the diagnosis criteria of the guidelines for the diagnosis and treatment of Chinese male diseases (2013 edition) (31); (2) were right-handed and 20 to 45 years old; (3) had impotence symptoms for at least 6 months; (4) had a stable heterosexual partner for more than 1 year; (5) avoided taking medications affecting sexual function over 30 days before enrollment; and (6) signed the informed consent. Patients were excluded if they: (1) were diagnosed with organic ED or mixed ED by history taking or examinations; (2) suffered from any organic or metabolic disease of urological, cardiovascular, respiratory, gastrointestinal system or had other severe primary diseases; (3) were alcohol or drug addicts or had neuropsychiatric disorders; (4) had a history of head trauma or urological surgery; (5) were participating in other current clinical trials; or (6) had any contraindication of MRI scans, such as implanted ferromagnetic metal and claustrophobia. The 28 righthanded HCs (ranged from 20 to 45 years old) were recruited by advertisement. These volunteers had never been diagnosed with $\mathrm{ED}$ or other sexual dysfunction and undergone the same clinical examinations as patients before inclusion. HCs with a history of neurological or psychiatric disorders, or urinary tract surgery were excluded.

\section{Symptom Assessments}

In addition to a brief assessment of sexual function using the International Index of Erectile Function 5 (IIEF-5) routinely, we selected the Self-Esteem and Relationship Questionnaire 
(SEARQ) and Quality of Erection Questionnaire (QEQ) to assess each participant's psychosocial status (self-esteem, sexual relationships, and erectile satisfaction). Moreover, the Self-Rating Anxiety Scale (SAS) (32) and the Self-Rating Depression Scale (SDS) (33) were also exploited to measure the mental states. The IIEF-5 is a multidimensional self-reported instrument for the assessment of male sexual function (34). It is recommended as the standard screening and diagnostic tool for ED in clinical (35). The 14-item SEARQ is a commonly used patient-administered and disease-specific psychosocial questionnaire to evaluate the psychosocial status of males. It contains three dimensions: selfesteem, sexual confidence, and sexual relationship (36-38). The QEQ is a self-reported questionnaire that specifically evaluates male satisfaction with erectile quality (39). The symptoms assessments were performed prior to the MRI scans, and the lower scores of these questionnaires indicated more severe symptoms, lower self-esteem, poorer sexual relationships, and lower erectile satisfaction.

\section{MRI Data Acquisition}

The MRI data were acquired with a 3.0T GE scanner at the Department of Radiology, Hospital of Chengdu University of Traditional Chinese Medicine, Chengdu, China. Each participant underwent a high-resolution three-dimensional T1-weighted imaging scan and a resting-state blood oxygenation level-dependent fMRI (BOLD-fMRI) sequence scan with eyes blindfolded and ears plugged. The structural scanning parameters were as follows: repetition time /echo time= $1,900 / 2.26 \mathrm{~ms}$, slices $=176$, slice thickness $=1 \mathrm{~mm}$, matrix size $=256 \times 256$, field of view $=256 \times 256 \mathrm{~mm}^{2}$. The BOLD-fMRI data were acquired with the following parameters: repetition time/echo time $=2,000 / 30 \mathrm{~ms}$, slices $=30$, slice thickness $=$ $5 \mathrm{~mm}$, flip angle $=90^{\circ}$, matrix size $=64 \times 64$, field of view $=$ $240 \times 240 \mathrm{~mm}^{2}$, total volume $=180$.

\section{Data Analysis \\ Clinical Data Analysis}

The demographic characteristics and clinical measurements were analyzed via SPSS 20.0 (SPSS Inc. USA). Age, Body Mass Index (BMI), IIEF-5 score, SEARQ score, and QEQ score were described as mean \pm standard deviation and compared by the two-sample $t$-test. The significance threshold was set at 0.05 (two-tailed).

\section{MRI Data Analysis}

The MRI data were preprocessed and analyzed by Statistical Parametric Mapping 12.0 (SPM12, http://www.fil.ion.ucl.ac. uk/spm), Data Processing Assistant for Resting-State fMRI (DPARSF) (40) (http://rfmri.org/DPARSF), and FC toolbox (CONN) (http://www.nitrc.org/projects/conn) working on MATLAB 2013b (Mathworks Inc. USA).

\section{MRI Data Preprocessing}

The data preprocessing were carried out with DPARSF, including (1) discarded the first 10 timepoints; (2) perfomed slice-timing correction; (3) performed realignment and discarded the subjects with a mean framewise displacement (FD) value exceeding
$0.2 \mathrm{~mm}$ or a maximum displacement greater than one voxel size $(41,42)$; (4) reoriented the functional images and T1 images with six rigid-body parameters; (5) coregistered the $\mathrm{T} 1$ images to functional space for each subject, respectively, and performed new segment and DARTEL (diffeomorphic anatomical registration through exponentiated lie algebra); (6) regressed out nuisance covariates including linear trend, white matter and cerebrospinal fluid signals, and head motion parameters [Friston 24 parameter model $(43,44)$ ]; $(7)$ normalized the functional images to MNI space and then resampled the functional images to $3 \mathrm{~mm}$ cubic voxels; (8) smoothed images with a $6 \mathrm{~mm}$ Gaussian kernel of full-width at half maximum; and (9) performed temporal filtering $(0.01-0.08 \mathrm{~Hz})$ (45). Following Yan et al.'s recommendation (44), the scrubbing procedure was conducted to remove the bad time points when performing FC analysis. The bad time points were defined as those whose FD was larger than $0.5 \mathrm{~mm}$.

\section{ALFF Analysis}

The zALFF maps, which normalized with the normal ztransformation, were applied to examine the differences of the spontaneous cerebral activity between patients with $\mathrm{pED}$ and HCs. The ALFF analysis was conducted with a twosample $t$-test via SPM12. The multiple-comparisons corrections were performed based on Gaussian random field theory (46, $47)$ and the threshold was set to voxel-level $p<0.001$ uncorrected and cluster-level $p<0.05$ familywise error (FWE) corrected (48).

\section{ROI-Based FC Analysis}

The voxels exceeding the established statistical threshold in the ALFF analysis were selected as the ROI, and the ROI-based FC analysis was performed with CONN. We first extracted the average BOLD time-series of the ROI and calculated the temporal synchronization between ROI and other voxels across the whole brain. And then, transferred the correlation coefficients into $\mathrm{z}$-scores using Fisher's transformation to allow for the normal distribution. Again, the two-sample $t$-test with the same statistical threshold as ALFF analysis was performed to investigate the ROI-based FC performance of patients with $\mathrm{pED}$ and HCs. Both age and BMI were controlled at ALFF and ROIbased FC analysis.

\section{Correlation Analysis}

To investigate the associations between symptom severity, psychosocial status, and brain functional alterations in pED, the clusterwise correlation analyses were performed between the ALFF/FC value and duration/ IIEF-5 score/ SEARQ score/ QEQ score in pED group, with age, BMI, and mean FD controlled. The significance threshold was set to $p<0.05$ with Bonferroni corrected.

\section{Mediation Analysis}

Mediation analyses were conducted within SPSS 20.0 using the PROCESS macro (49). A full mediation model (model 4) was applied to examine the potential mediating role of the psychosocial status (lower SEARQ score) on the relationship between cerebral activity patterns and decreased erectile function 
TABLE 1 | The comparisons of demographic and clinical characteristics between patients with pED and HCs.

\begin{tabular}{|c|c|c|c|c|c|c|c|c|c|}
\hline & Age (Year) & BMI & Duration (Months) & IIEF-5 & SEARQ & QEQ & SAS & SDS & Mean FD \\
\hline $\mathrm{pED}(n=31)$ & $33.16 \pm 5.89$ & $22.16 \pm 1.87$ & $33.06 \pm 28.67$ & $13.97 \pm 3.6$ & $37.58 \pm 7.96$ & $35.48 \pm 15.72$ & $35.24 \pm 7.92$ & $35.97 \pm 8.02$ & $0.122 \pm 0.037$ \\
\hline $\mathrm{HCs}(n=24)$ & $31.17 \pm 6.57$ & $22.7 \pm 3.54$ & / & $22.21 \pm 0.98$ & $61.92 \pm 3.73$ & $81.36 \pm 6.8$ & $34.01 \pm 5.33$ & $33.49 \pm 5.26$ & $0.113 \pm 0.451$ \\
\hline$p$-value & 0.241 & 0.467 & / & $<0.001$ & $<0.001$ & $<0.001$ & 0.515 & 0.196 & 0.385 \\
\hline
\end{tabular}

No significant difference was obtained in age, BMI, SAS, SDS, and mean FD ( $p>0.05)$. Patients with pED had lower IIEF-5 score, SEARQ score, and QEQ score than HCs ( $p<$

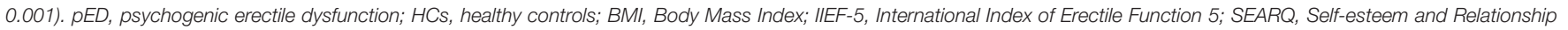
Questionnaire; QEQ, Quality of Erection Questionnaire; SAS, Self-rating Anxiety Scale; SDS, Self-rating Depression Scale; FD, framewise displacement.

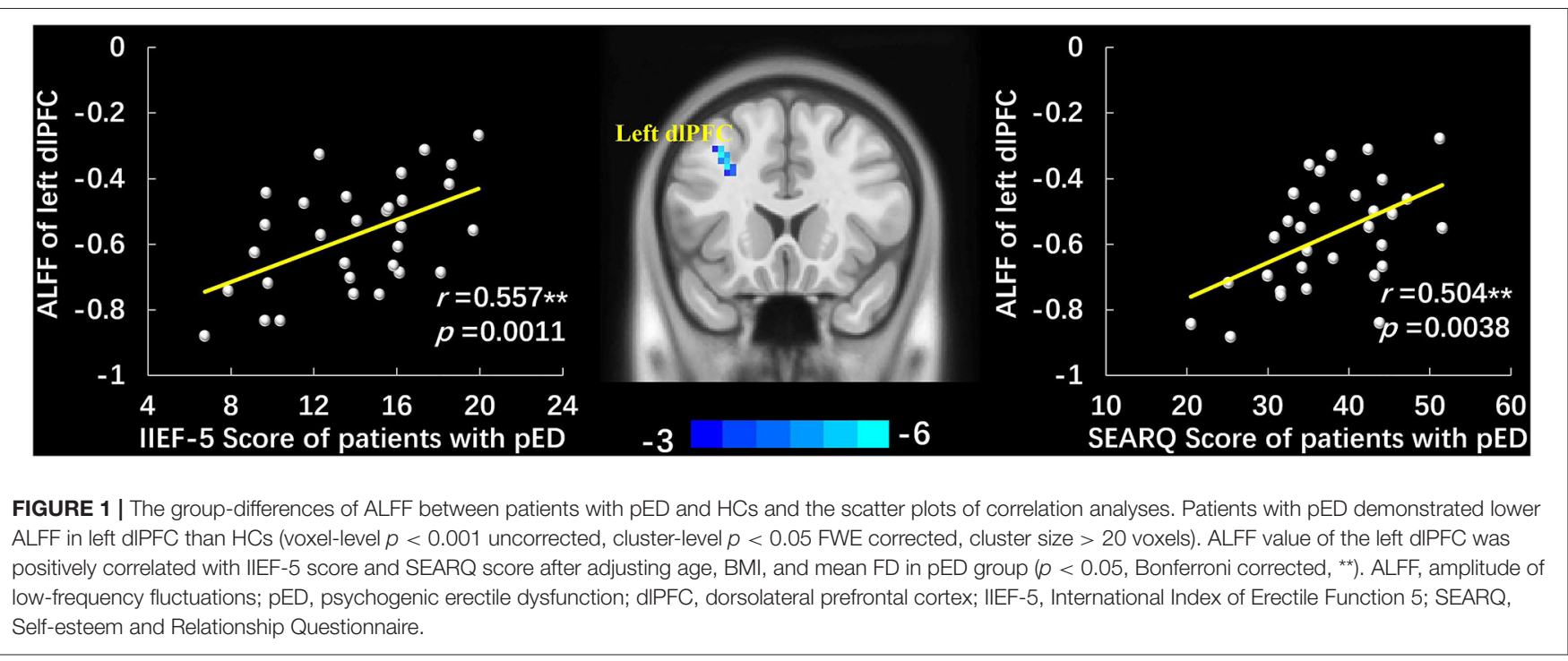

(lower IIEF-5 score), with age, BMI, and mean FD included as covariates. Similar to the previous studies $(50,51)$, the non-parametric bootstrapping analysis was utilized to calculate the desired statistic in each resample. Bias-corrected bootstrap confidence intervals (CI) were estimated from 10,000 bootstrap samples, and the mediation effects were considered statistically significant if the bootstrapped 95\% CI did not include zero.

\section{RESULTS}

All the participants completed the MRI scan. Two HCs were excluded due to incomplete functional images. One patient and two HCs were excluded due to excessive head motion. Therefore, 31 patients with pED and $24 \mathrm{HCs}$ were included in the final data analysis. There was no significant difference in mean FD between patients and HCs $(p>0.05)$.

\section{Demographic and Clinical Characteristics}

There was no significant difference in age, BMI, SAS, and SDS between patients with $\mathrm{pED}$ and HCs $(p>0.05)$. While patients with $\mathrm{pED}$ had lower IIEF-5 score, SEARQ score, and QEQ score than HCs $(p<0.001)$, which indicated the impaired erectile function, damaged sexual relationships, decreased self-esteem, and diminished erectile satisfaction in pED group (Table 1). In addition, The IIEF- 5 was found to be positively correlated with the SEAR score $(r=0.688, p<0.0001)$ and QEQ score $(r=0.754$, $p<0.0001)$, but not with the duration, SAS, or SDS in patients with $\mathrm{pED}$.

\section{Between-Group ALFF Comparisons}

Compared to HCs, patients with pED demonstrated significant ALLF decrease at the left dlPFC (voxel-level $p<0.001$ uncorrected, cluster-level $p<0.05$ FWE corrected, cluster size $>$ 20 voxels) (Figure 1, Table 2). No region with higher ALFF was observed in pED group. The correlation analysis demonstrated that the ALFF value of the left dlPFC was positively correlated with the IIEF-5 score $(r=0.557, p=0.0011)$ and SEARQ score $(r=0.504, p=0.0038)$, while not with duration and QEQ scores in patients with $\mathrm{pED}$ after controlling age, BMI, and mean FD (Bonferroni correction, $p<0.05 / 4=0.0125$ ) (Figure 1).

\section{Between-Group FC Comparisons}

The left dlPFC, which manifested significantly lower ALFF in pED group, was selected as the ROI. Compared to HCs, the decreased FC were observed between the ROI and the left dlPFC, left angular gyrus (AG), and left PCC and precuneus in patients with pED (voxel-level $p<0.001$ uncorrected, cluster-level $p$ $<0.05$ FWE corrected, cluster size $>20$ voxels) (Figure 2, Table 3). No significantly increased FC between ROI and voxels was found in $\mathrm{pED}$ group. The correlation analysis manifested that the FC between ROI and left PCC and precuneus was positively correlated with IIEF-5 score $(r=0.578, p=0.0007)$ and SEARQ 
score $(r=0.607, p=0.0003)$ in patients with pED after adjusting the impacts of age, BMI, and mean FD (Bonferroni correction, $p$ $<0.05 / 12=0.0042)$. Furthermore, FC between ROI and left AG was found to be associated with the IIEF-5 score $(r=0.459, p=$ $0.0094)$ and SEARQ score $(r=0.404, p=0.0242)$ in patients with $\mathrm{pED}$ at the threshold of uncorrected $p<0.05$. However, the above correlation results were no longer significant after Bonferroni correction (Figure 2).

Given the significant correlation between the duration and the SEARQ score $(r=-0.513, p=0.003)$, we conducted the correlation analyses between neuroimaging data and clinical

TABLE 2 | Differences in ALFF between patients with pED and HCs.

\begin{tabular}{|c|c|c|c|c|c|c|c|}
\hline \multirow{2}{*}{$\begin{array}{l}\text { Contrast } \\
\text { pED < HCs }\end{array}$} & \multirow{2}{*}{$\begin{array}{l}\text { Foci } \\
\text { L dIPFC }\end{array}$} & \multirow{2}{*}{$\begin{array}{c}\text { Voxels } \\
22\end{array}$} & \multirow{2}{*}{$\begin{array}{r}\text { BA } \\
46\end{array}$} & \multicolumn{3}{|c|}{ MNI coordinate $(x, y, z)$} & \multirow{2}{*}{$\begin{array}{l}\boldsymbol{t} \text {-value } \\
-5.24\end{array}$} \\
\hline & & & & $\begin{array}{l}-33 \\
-33\end{array}$ & $\begin{array}{l}18 \\
24\end{array}$ & 4251 & \\
\hline
\end{tabular}

Patients with pED manifested lower ALFF in left dIPFC than HCs (voxel-level $p<$ 0.001 uncorrected, cluster-level $p<0.05$ FWE corrected, cluster size $>20$ voxels). $p E D$, psychogenic erectile dysfunction; HCs, healthy controls; L, left; dIPFC, dorsolateral prefrontal cortex; BA, Brodmann area; MNI, Montreal Neurological Institute. symptoms with duration as an additional covariate to exclude its impact. These results are displayed at Supplementary Material.

\section{Mediation Analysis}

The mediation analyses demonstrated that the associations between the altered brain activity synchronization and ED symptoms were mediated through the psychosocial status after controlling the impacts of age, BMI, and mean FD. Namely, the SEARQ score mediated the influences of the dlPFC-left PCC and precuneus connectivity on the IIEF-5 score (indirect effect = 0.3284; 95\% CI: 0.0593-0.6694) (Figure 3A). Interestingly, the total effect of left dlPFC-left PCC and precuneus connectivity on IIEF-5 score was significant $(\beta c=0.5937, p=0.0013)$, but after taking the significant mediation effect of SEARQ score into consideration, the remaining direct effect of cerebral activity on erectile function was reduced and no longer significant $\left(\beta c^{\prime}=0.2652, p=0.1559\right)$. Similarly, we also found the fully mediating effect of SEARQ score on the relationships between left dlPFC-left AG connectivity and IIEF-5 score (indirect effect $=0.24$; 95\% CI: 0.0024-0.5117) (Figure 3B). No significant mediation effect was found between ALFF value and clinical measurements.

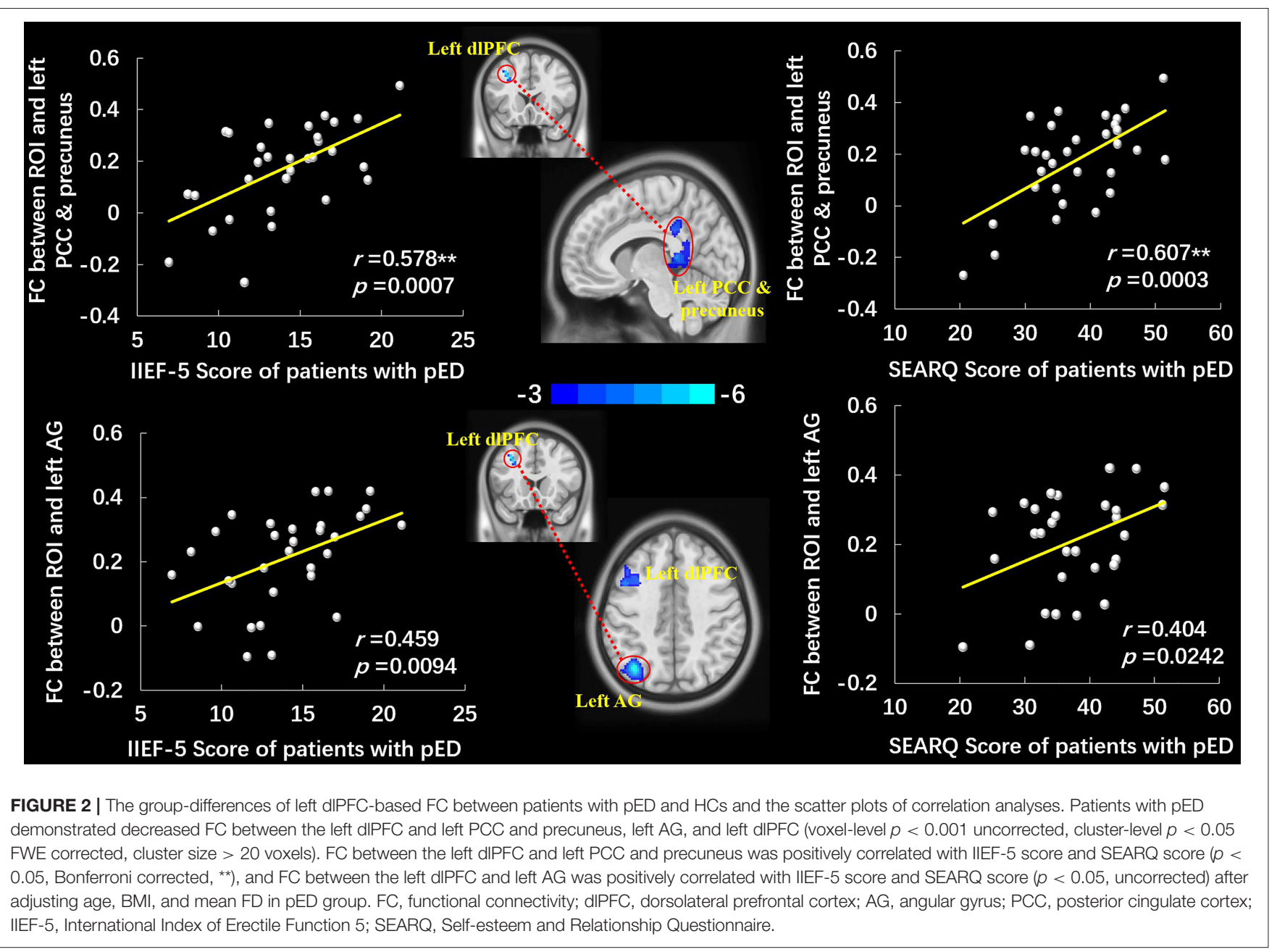


TABLE 3 | Differences of ROI-based FC between patients with pED and HCs.

\begin{tabular}{|c|c|c|c|c|c|c|c|}
\hline \multirow[t]{2}{*}{ Contrast } & \multirow{2}{*}{$\begin{array}{l}\text { Foci } \\
\text { L dIPFC }\end{array}$} & \multirow{2}{*}{$\begin{array}{l}\text { Voxels } \\
581\end{array}$} & \multirow{2}{*}{$\frac{\text { BA }}{46}$} & \multicolumn{3}{|c|}{ MNI coordinate $(x, y, z)$} & \multirow{2}{*}{$\begin{array}{c}\boldsymbol{t} \text {-value } \\
-4.15\end{array}$} \\
\hline & & & & -34 & 18 & 40 & \\
\hline & LAG & 552 & 39 & -38 & -68 & 42 & -5.55 \\
\hline \multirow[t]{2}{*}{$\mathrm{pED}<\mathrm{HCs}$} & LPCC & 539 & 23/29/31 & -8 & -40 & 36 & -3.82 \\
\hline & L precuneus & & 31 & -2 & -64 & 28 & -3.77 \\
\hline
\end{tabular}

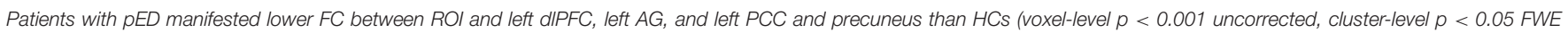

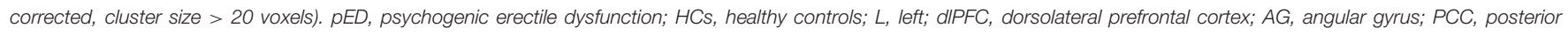
cingulate cortex; BA, Brodmann area; MNI, Montreal Neurological Institute.

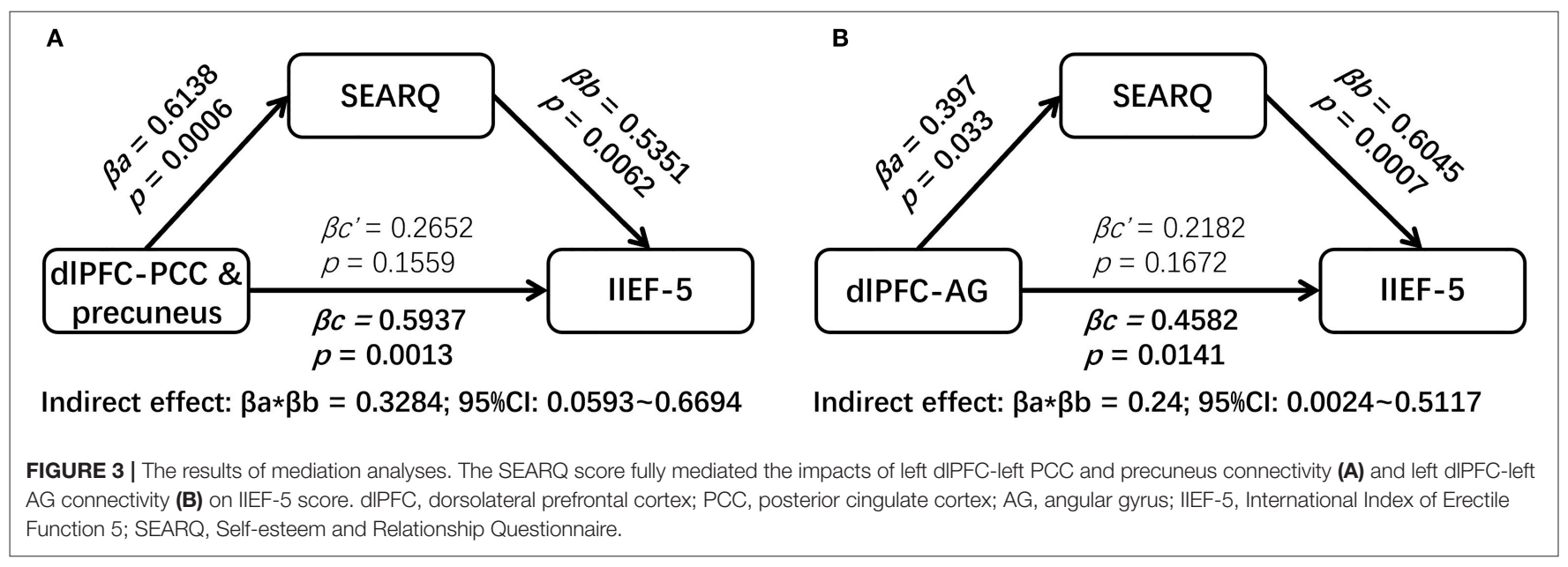

\section{DISCUSSION}

This study investigated the associations among the cerebral activity patterns, erectile function, and psychosocial status in patients with $\mathrm{pED}$. The current findings indicated that patients with pED manifested not only the impaired erectile function and disrupted psychosocial status, but also the lower spontaneous activity in the left dlPFC and lower synchronous activity between the left dlPFC and the left AG, left PCC and precuneus, and left dlPFC compared to HCs. Moreover, we also found that both the ALFF of left dlPFC and FC between left dlPFC and left PCC and precuneus were positively correlated with the sexual function and psychosocial status of patients with $\mathrm{pED}$, and that the disrupted psychosocial status fully mediated the influence of altered brain activity synchronization on impaired erectile function.

A great number of studies have indicated that the abnormal psychosocial status was the important cause of $\mathrm{pED}$, and that patients with $\mathrm{pED}$ manifested significantly decreased self-esteem, damaged sexual relationships, and diminished sexual confidence than healthy men (52-54). The current findings illustrated that patients with pED displayed symptom-related SEARQ scores and QEQ scores decrease, which was consistent with previous studies (54-56). They all indicated that patients with pED had significant psychosocial disorders that were closely related to the severity of symptoms. Another interesting finding of the present study was that the psychological status fully mediated the influences of atypical FC on the clinical symptoms. Namely, the altered left dlPFC-left PCC and precuneus connectivity as well as left dlPFC-left AG connectivity could indirectly affect the clinical symptoms of pED patients by influencing their psychological status. This contributed to the understanding of the mechanisms behind the correlations between abnormal brain functional synchronization and impaired erectile function, and further enhanced our knowledge of the critical roles played by psychosocial disorders in the pathogenesis of pED. To some extent, this finding implied the rationale and feasibility of modulating abnormal brain activity and regulating abnormal psychosocial status to improve clinical symptoms of $\mathrm{pED}$, which has been tentatively demonstrated in several clinical studies (57-59).

This result showed that patients with $\mathrm{pED}$ had reduced spontaneous activity at the left dlPFC, and another study also observed the aberrant activity patterns at dlPFC in patients with pED (20). These two studies illustrated that the abnormal functional activity in the dlPFC might be an important neural pathological feature of $\mathrm{pED}$. That dlPFC playing a critical role in male sexual arousal and sexual behavior has been extensively investigated in both healthy subjects and patients (60-62). For example, researchers demonstrated that visual sexual stimulus could significantly produce dlPFC activation and that healthy males manifested higher and more rapid dlPFC activation than females (63). Compared to HCs, patients with problematic hypersexual behavior experienced stronger sexual desire and higher dlPFC activation when exposed to the visual sexual stimulus (64). In contrast, patients with 
hypoactive sexual desire disorder showed lower BOLD signal activity in dlPFC during a similar stimulus (65). As the core region of central executive network (CEN) (66), dlPFC primarily implicates in response inhibition, cognitive control, and attention (62). It was reported that patients with minor dlPFC damage demonstrated disinterest in their surroundings and inhibition in behavior (67). Sexual inhibition, as one of the most critical characteristics of $\mathrm{pED}$, has been found to be associated with the dlPFC activity in the previous researches $(62,68)$. In the current study, we found the abnormally decreased activity at left dlPFC in patients with $\mathrm{pED}$, and the activity intensity of left dlPFC was positively correlated with the sexual ability and sexual satisfaction. This result identified that patients with pED had dlPFC-involved aberrant sexual inhibition and insufficient cognition and attention to sexual targets.

In addition to the altered spontaneous activity of the brain, we also detected the decreased FC between the left dlPFC and multiple key regions of default mode network (DMN) in pED group, and the FC values of ROI to foci in DMN were positively associated with the erectile function and psychosocial status. These results suggested that the abnormal synchronization of functional activity between dlPFC and regions of DMN might contribute to the pathogenesis of pED. DMN is a highinterconnected anatomical brain network that contains four functional hubs: the PCC, the precuneus, the AG, and the medial PFC $(69,70)$. DMN participates in regulating the male erection $(62,71-73)$. The aberrances of some $\mathrm{DMN}$ regions contribute to the abnormal sexual arousal and behavior patterns, manifesting as pED $(17,19)$ and other sexual dysfunctions $(74,75)$. DMN involves in many functions, such as selfreferential introspection, self-esteem, thinking about others, remembering the past, and looking forward to the future (7678). As the core regions of DMN, the PCC and precuneus mainly participate in autobiographical and emotional memories, as well as the integration of self-evaluation, perception, memories, and attention $(69,79,80)$; while the AG engages in the manipulation of mental representations and conceptual knowledge, and helps with the recall of episodic memories $(81,82)$. The erection is a complex physiological process involving perception, introspection, cognitive, and affective components $(83,84)$. The occurrence of pED is primarily related to traumatic memories, as well as self-cognitive, affective, and interpersonal factors (9, 85). Therefore, in addition to the dlPFC-involved abnormal sexual inhibition, it seemed that patients with $\mathrm{pED}$ might also have DMN-related improper introspection, damaged selfesteem, overthinking about nervous sexual relationships, and undue consolidation and extraction of traumatic memories about failed intercourses. In addition, the aberrant function of DMN has been confirmed to be relevant to psychosocial stress (86), major depression (87), posttraumatic stress disorder (PTSD) (88), and many other psychosocial disorders $(89,90)$. As a typical biopsychosocial illness, the incidence of $\mathrm{pED}$ was inseparable from the influence of psychosocial factors, such as performance anxiety, nervous sexual relationships, and failed intercourse memories. Our present study found that the disordered psychosocial status mediated the impacts of dlPFCDMN FC on impotence symptoms, which further illustrated the close associations among the aberrant psychosocial status, impaired erectile function, and altered cerebral activity patterns in patients with $\mathrm{pED}$.

To some degree, the current results appeared to be able to deduce the viewpoint that patients with pED had aberrant connectivity patterns between CEN and DMN. There are complex connectivity and mutual interactions between CEN and DMN (91). The DMN is responsible for the processing of endogenous or self-referential mental activity, and the CEN participates in the processing of exogenous or cognitively demanding mental activity (92). CEN and DMN together involve in cognitive control, self-emotion regulation, and attention to the external and internal worlds. In our study, patients with pED not only demonstrated the decreased spontaneous activity of the CEN hub (dlPFC), but also manifested the reduced synchronous activity between the core regions of $\mathrm{CEN}$ and $\mathrm{DMN}$. It indicated that patients with pED had different CEN-DMN connectivity models from healthy subjects. Similarly, the weak CEN-DMN connectivity was also detected at many other psychosocial disorders, such as major depression (93), PTSD (94), and acute stress (95). Therefore, based on the previous findings and our current results, we put forward the deduction that patients with $\mathrm{pED}$ had decreased synchronous activity between the CEN and the DMN.

Some limitations should be concerned in this study. First, the sample size was relatively small; studies with larger samples are encouraged to repeat the current findings. Second, because no significant difference was found between patients with $\mathrm{pED}$ and HCs in SAS and SDS scores, this study did not further investigate the correlation or mediation effects of imaging data and emotional condition. Third, patients enrolled in this study were aged from 20 to 45 years old, so the findings reflected the disease characteristics of younger patients only. As we know, the incidence of ED increases significantly with age, and the majority of cases are in the middle-aged and elderly population. Therefore, future studies should pay more attention to older pED patients.

\section{CONCLUSION}

In conclusion, this study demonstrated that patients with $\mathrm{pED}$ had symptom-related abnormal psychosocial status, decreased spontaneous activity in dlPFC, and damaged FC between dlPFC and $\mathrm{DMN}$, and that the disrupted psychosocial status fully mediated the influence of aberrant dlPFC-DMN connectivity on ED symptoms. This study widened our understanding of the important role of psychosocial disorders in pathological neural changes in patients with $\mathrm{pED}$. The future intervention for $\mathrm{pED}$ should fully consider the impacts of psychosocial disorders on this disease.

\section{DATA AVAILABILITY STATEMENT}

All datasets generated for this study are included in the article/Supplementary Material. 


\section{ETHICS STATEMENT}

The studies involving human participants were reviewed and approved by Institutional Review Boards and Ethics Committees of Hospital of Chengdu University of Traditional Chinese Medicine (Approved number: 2018KL-064). The patients/participants provided their written informed consent to participate in this study.

\section{AUTHOR CONTRIBUTIONS}

PZ and DC were responsible for this study. PZ, TY, QL, and $\mathrm{ZM}$ contributed to the study conception and design. QL and ZL participated in material preparation and patient recruitment. $\mathrm{ZM}, \mathrm{FR}, \mathrm{GL}$, and $\mathrm{XH}$ contributed to patient recruitment. TY,

\section{REFERENCES}

1. Najari BB, Kashanian JA. Erectile dysfunction. JAMA. (2016) 316:1838. doi: 10.1001/jama.2016.12284

2. Rosen RC, Fisher WA, Eardley I, Niederberger C, Nadel A, Sand M. The multinational Men's Attitudes to Life Events and Sexuality. (MALES) study: I. Prevalence of erectile dysfunction and related health concerns in the general population. Curr Med Res Opin. (2004) 20:607-17. doi: 10.1185/030079904125003467

3. Quilter M, Hodges L, von Hurst P, Borman B, Coad J. Male sexual function in New Zealand: a population-based cross-sectional survey of the prevalence of erectile dysfunction in men aged 40-70 years. J Sex Med. (2017) 14:928-36. doi: 10.1016/j.jsxm.2017.05.011

4. Nguyen HMT, Gabrielson AT, Hellstrom WJG. Erectile dysfunction in young men-a review of the prevalence and risk factors. Sex Med Rev. (2017) 5:508-20. doi: 10.1016/j.sxmr.2017.05.004

5. Boddi V, Corona G, Fisher AD, Mannucci E, Ricca V, Sforza A, et al. "It takes two to tango": the relational domain in a cohort of subjects with erectile dysfunction. (ED). J Sex Med. (2012) 9:3126-36. doi: 10.1111/j.1743-6109.2012.02948.x

6. Li HJ, Bai WJ, Dai YT, Xu WP, Wang CN, Li HZ. An analysis of treatment preferences and sexual quality of life outcomes in female partners of Chinese men with erectile dysfunction. Asian J Androl. (2016) 18:773-9. doi: 10.4103/1008-682X.159719

7. Yafi FA, Jenkins L, Albersen M, Corona G, Isidori AM, Goldfarb S, et al. Erectile dysfunction. Nat Rev Dis Primers. (2016) 2:16003. doi: $10.1038 / \mathrm{nrdp} .2016 .3$

8. Coward RM, Stetter C, Kunselman A, Trussell JC, Lindgren MC, Alvero RR, et al. Fertility related quality of life, gonadal function and erectile dysfunction in male partners of couples with unexplained infertility. J Urol. (2019) 202:379-84. doi: 10.1097/JU.00000000000 00205

9. Shamloul R, Ghanem H. Erectile dysfunction. Lancet. (2013) 381:153-65. doi: 10.1016/S0140-6736(12)60520-0

10. McMahon CG. Current diagnosis and management of erectile dysfunction. Med J Aust. (2019) 210:469-76. doi: 10.5694/mja2.50167

11. Rastrelli G, Maggi M. Erectile dysfunction in fit and healthy young men: psychological or pathological? Transl Androl Urol. (2017) 6:79-90. doi: 10.21037/tau.2016.09.06

12. Chen L, Shi GR, Huang DD, Li Y, Ma CC, Shi M, et al. Male sexual dysfunction: a review of literature on its pathological mechanisms, potential risk factors, and herbal drug intervention. Biomed Pharmacother. (2019) 112:108585. doi: 10.1016/j.biopha.2019.01.046

13. Prieto D. Physiological regulation of penile arteries and veins. Int J Impot Res. (2008) 20:17-29. doi: 10.1038/sj.ijir.3901581

14. Brunetti M, Babiloni C, Ferretti A, Del Gratta C, Merla A, Olivetti Belardinelli $\mathrm{M}$, et al. Hypothalamus, sexual arousal and psychosexual identity in human
RS, and QL performed the data collection and data analysis. TY wrote the first draft of the manuscript. All authors commented on previous versions of the manuscript, read, and approved the final manuscript.

\section{FUNDING}

This study was financially supported by the National Natural Science Foundation of China (No. 81774137).

\section{SUPPLEMENTARY MATERIAL}

The Supplementary Material for this article can be found online at: https://www.frontiersin.org/articles/10.3389/fpsyt. 2020.583619/full\#supplementary-material males: a functional magnetic resonance imaging study. Eur J Neurosci. (2008) 27:2922-7. doi: 10.1111/j.1460-9568.2008.06241.x

15. Courtois F, Carrier S, Charvier K, Guertin PA, Journel NM. The control of male sexual responses. Curr Pharm Des. (2013) 19:4341-56. doi: 10.2174/13816128113199990333

16. Wu SL, Chow MSM, L JY, Yang J, Zhou H, Yew DT. Visual sexual stimulation and erection, a brief review with new fMRI data. Curr Med Chem. (2017) 24:1139-46. doi: 10.2174/0929867323666161213102528

17. Cera N, Delli Pizzi S, Di Pierro ED, Gambi F, Tartaro A, Vicentini C, et al. Macrostructural alterations of subcortical grey matter in psychogenic erectile dysfunction. PLoS ONE. (2012) 7:e39118. doi: 10.1371/journal.pone.0039118

18. Cera N, Di Pierro ED, Ferretti A, Tartaro A, Romani GL, Perrucci MG. Brain networks during free viewing of complex erotic movie: new insights on psychogenic erectile dysfunction. PLoS ONE. (2014) 9:e105336. doi: 10.1371/journal.pone.0105336

19. Zhao L, Guan M, Zhang X, Karama S, Khundrakpam B, Wang M, et al. Structural insights into aberrant cortical morphometry and network organization in psychogenic erectile dysfunction. Hum Brain Mapp. (2015) 36:4469-82. doi: 10.1002/hbm.22925

20. Wang $\mathrm{Y}$, Dong $\mathrm{M}$, Guan $\mathrm{M}$, Wu J, He Z, Zou Z, et al. Aberrant insula-centered functional connectivity in psychogenic erectile dysfunction patients: a resting-state fMRI study. Front Hum Neurosci. (2017) 11:221. doi: 10.3389/fnhum.2017.00221

21. Chen J, Chen Y, Gao Q, Chen G, Dai Y, Yao Z, et al. Impaired prefrontalamygdala pathway, self-reported emotion, and erection in psychogenic erectile dysfunction patients with normal nocturnal erection. Front Hum Neurosci. (2018) 12:157. doi: 10.3389/fnhum.2018.00157

22. Chen J, Huang X, Liu S, Lu C, Dai Y, Yao Z, et al. Disrupted topological properties of brain networks in erectile dysfunction patients owing predominantly to psychological factors: a structural and functional neuroimaging study. Andrology. (2020) 8:381-91. doi: 10.1111/andr.12684

23. Xu Z, Yang X, Gao M, Liu L, Sun J, Liu P, et al. Abnormal resting-state functional connectivity in the whole brain in lifelong premature ejaculation patients based on machine learning approach. Front Neurosci. (2019) 13:448. doi: 10.3389/fnins.2019.00448

24. Chen J, Huang X, Lu C, Liu T, Dai Y, Yao Z, et al. Graph analysis of DTI-based connectome: decreased local efficiency of subcortical regions in PE patients with high sympathetic activity. Andrology. (2020) 8:400-6. doi: 10.1111/andr.12701

25. Chen J, Yang J, Huang X, Ni L, Fan Q, Liu T, et al. Reduced segregation and integration of structural brain network associated with sympathetic and dorsal penile nerve activity in anejaculation patients: a graphbased connectome study. Andrology. (2020) 8:392-9. doi: 10.1111/andr. 12715

26. Zhang P, Liu J, Li G, Pan J, Li Z, Liu Q, et al. White matter microstructural changes in psychogenic erectile dysfunction patients. Andrology. (2014) 2:379-85. doi: 10.1111/j.2047-2927.2014.00191.x 
27. Zang YF, He Y, Zhu CZ, Cao QJ, Sui MQ, Liang M, et al. Altered baseline brain activity in children with ADHD revealed by resting-state functional MRI. Brain Dev. (2007) 29:83-91. doi: 10.1016/j.braindev.2006.07.002

28. Smitha KA, Akhil Raja K, Arun KM, Rajesh PG, Thomas B, Kapilamoorthy TR, et al. Resting state fMRI: a review on methods in resting state connectivity analysis and resting state networks. Neuroradiol J. (2017) 30:30517. doi: 10.1177/1971400917697342

29. Cheng R, Qi H, Liu Y, Zhao S, Li C, Liu C, et al. Abnormal amplitude of lowfrequency fluctuations and functional connectivity of resting-state functional magnetic resonance imaging in patients with leukoaraiosis. Brain Behav. (2017) 7:e00714. doi: 10.1002/brb3.714

30. Tang Y, Zhou Q, Chang M, Chekroud A, Gueorguieva R, Jiang X, et al. Altered functional connectivity and low-frequency signal fluctuations in early psychosis and genetic high risk. Schizophr Res. (2019) 210:172-9. doi: 10.1016/j.schres.2018.12.041

31. Wang XF, Zhu JC, Deng CH. Guidelines for the Diagnosis and Treatment of Chinese Male Diseases. 2013 ed. Beijing: People’s Medical Publishing House (2013).

32. Zung WWK. A rating instrument for anxiety disorders. Psychosomatics. (1971) 12:371-9. doi: 10.1016/S0033-3182(71)71479-0

33. Zung WW. A self-rating depression scale. Arch Gen Psychiatry. (1965) 12:6370. doi: 10.1001/archpsyc.1965.01720310065008

34. Rosen RC, Cappelleri JC, Smith MD, Lipsky J, Pena BM. Development and evaluation of an abridged, 5-item version of the International Index of Erectile Function. (IIEF-5) as a diagnostic tool for erectile dysfunction. Int J Impot Res. (1999) 11:319-26. doi: 10.1038/sj.ijir.3900472

35. Cappelleri JC, Rosen RC. The Sexual Health Inventory for Men. (SHIM): a 5-year review of research and clinical experience. Int J Impot Res. (2005) 17:307-19. doi: 10.1038/sj.ijir.3901327

36. Althof SE, Cappelleri JC, Shpilsky A, Stecher V, Diuguid C, Sweeney $\mathrm{M}$, et al. Treatment responsiveness of the Self-Esteem and Relationship questionnaire in erectile dysfunction. Urology. (2003) 61:888-92. doi: 10.1016/S0090-4295(03)00041-4

37. Cappelleri JC, Althof SE, Siegel RL, Shpilsky A, Bell SS, Duttagupta S. Development and validation of the Self-Esteem And Relationship. (SEAR) questionnaire in erectile dysfunction. Int J Impot Res. (2004) 16:30-8. doi: 10.1038/sj.ijir.3901095

38. McCabe MP, Althof SE. A systematic review of the psychosocial outcomes associated with erectile dysfunction: does the impact of erectile dysfunction extend beyond a man's inability to have sex? J Sex Med. (2014) 11:347-63. doi: 10.1111/jsm.12374

39. Porst H, Gilbert C, Collins S, Huang X, Symonds T, Stecher V, et al. Development and validation of the quality of erection questionnaire. $J$ Sex Med. (2007) 4:372-81. doi: 10.1111/j.1743-6109.2006.00422.x

40. Yan CG, Wang XD, Zuo XN, Zang YF. DPABI: Data Processing and Analysis for (Resting-State) brain imaging. Neuroinformatics. (2016) 14:33951. doi: 10.1007/s12021-016-9299-4

41. Power JD, Barnes KA, Snyder AZ, Schlaggar BL, Petersen SE. Spurious but systematic correlations in functional connectivity MRI networks arise from subject motion. Neuroimage. (2012) 59:2142-54. doi: 10.1016/j.neuroimage.2011.10.018

42. Fiorenzato E, Strafella AP, Kim J, Schifano R, Weis L, Antonini A, et al. Dynamic functional connectivity changes associated with dementia in Parkinson's disease. Brain. (2019) 142:2860-72. doi: 10.1093/brain/awz192

43. Friston KJ, Williams S, Howard R, Frackowiak RS, Turner R. Movementrelated effects in fMRI time-series. Magn Reson Med. (1996) 35:346-55. doi: 10.1002/mrm.1910350312

44. Yan CG, Cheung B, Kelly C, Colcombe S, Craddock RC, Di Martino A, et al. A comprehensive assessment of regional variation in the impact of head micromovements on functional connectomics. Neuroimage. (2013) 76:183201. doi: 10.1016/j.neuroimage.2013.03.004

45. Biswal B, Yetkin FZ, Haughton VM, Hyde JS. Functional connectivity in the motor cortex of resting human brain using echo-planar MRI. Magn Reson Med. (1995) 34:537-41. doi: 10.1002/mrm.1910340409

46. Chumbley JR, Friston KJ. False discovery rate revisited: FDR and topological inference using Gaussian random fields. Neuroimage. (2009) 44:62-70. doi: 10.1016/j.neuroimage.2008.05.021
47. Chumbley J, Worsley K, Flandin G, Friston K. Topological FDR for neuroimaging. Neuroimage. (2010) 49:3057-64. doi: 10.1016/j.neuroimage.2009.10.090

48. Zeng F, Sun R, He Z, Chen Y, Lei D, Yin T, et al. Altered functional connectivity of the amygdala and sex differences in functional dyspepsia. Clin Transl Gastroenterol. (2019) 10:e00046. doi: 10.14309/ctg.0000000000000046

49. Preacher KJ, Hayes AF. Asymptotic and resampling strategies for assessing and comparing indirect effects in multiple mediator models. Behav Res Methods. (2008) 40:879-91. doi: 10.3758/BRM.40.3.879

50. Gu Y, Vorburger RS, Gazes Y, Habeck CG, Stern Y, Luchsinger JA, et al. White matter integrity as a mediator in the relationship between dietary nutrients and cognition in the elderly. Ann Neurol. (2016) 79:1014-25. doi: 10.1002/ana.24674

51. Callaghan BL, Dandash O, Simmons JG, Schwartz O, Byrne ML, Sheeber $\mathrm{L}$, et al. Amygdala resting connectivity mediates association between maternal aggression and adolescent major depression: a 7-year longitudinal study. J Am Acad Child Adolesc Psychiatry. (2017) 56:983-91.e983. doi: 10.1016/j.jaac.2017.09.415

52. Tiefer L, Schuetz-Mueller D. Psychological issues in diagnosis and treatment of erectile disorders. Urol Clin North Am. (1995) 22:767-73.

53. Cappelleri JC, Bell SS, Althof SE, Siegel RL, Stecher VJ. Comparison between sildenafil-treated subjects with erectile dysfunction and control subjects on the Self-Esteem And Relationship questionnaire. J Sex Med. (2006) 3:274-82. doi: 10.1111/j.1743-6109.2005.00205.x

54. Moncada I, Martinez-Jabaloyas JM, Rodriguez-Vela L, Gutierrez PR, Giuliano F, Koskimaki J, et al. Emotional changes in men treated with sildenafil citrate for erectile dysfunction: a double-blind, placebo-controlled clinical trial. J Sex Med. (2009) 6:3469-77. doi: 10.1111/j.1743-6109.2009.01514.x

55. Patel HR, Ilo D, Shah N, Cuzin B, Chadwick D, Andrianne R, et al. Effects of tadalafil treatment after bilateral nerve-sparing radical prostatectomy: quality of life, psychosocial outcomes, and treatment satisfaction results from a randomized, placebo-controlled phase IV study. BMC Urol. (2015) 15:31. doi: 10.1186/s12894-015-0022-9

56. Tang WH, Zhuang XJ, Ma LL, Hong K, Zhao LM, Liu DF, et al. Effect of sildenafil on erectile dysfunction and improvement in the quality of sexual life in China: a multi-center study. Int J Clin Exp Med. (2015) 8:11539-43.

57. Ponomarenko GN, Bin'iash TG, Raigorodskii Iu M, Guliaev AS, Shul'diakov VA, Kiriliuk AM, et al. Transcranial magneto- and electrostimulation in patients with obesity and erectile dysfunction [Article in Russian]. Vopr Kurortol Fizioter Lech Fiz Kult. (2009) 5:30-3.

58. Andersson E, Walén C, Hallberg J, Paxling B, Dahlin M, Almlöv J, et al. A randomized controlled trial of guided Internet-delivered cognitive behavioral therapy for erectile dysfunction. J Sex Med. (2011) 8:2800-9. doi: 10.1111/j.1743-6109.2011.02391.x

59. Wassersug R, Wibowo E. Non-pharmacological and non-surgical strategies to promote sexual recovery for men with erectile dysfunction. Transl Androl Urol. (2017) 6(Suppl. 5):S776-94. doi: 10.21037/tau.2017.04.09

60. Spinella M. The role of prefrontal systems in sexual behavior. Int J Neurosci. (2007) 117:369-85. doi: 10.1080/00207450600588980

61. Walter M, Witzel J, Wiebking C, Gubka U, Rotte M, Schiltz K, et al. Pedophilia is linked to reduced activation in hypothalamus and lateral prefrontal cortex during visual erotic stimulation. Biol Psychiatry. (2007) 62:698-701. doi: 10.1016/j.biopsych.2006.10.018

62. Cheng JC, Secondary J, Burke WH, Fedoroff JP, Dwyer RG. Neuroimaging and sexual behavior: identification of regional and functional differences. Curr Psychiatry Rep. (2015) 17:55. doi: 10.1007/s11920-015-0593-x

63. Leon-Carrion J, Martin-Rodriguez JF, Damas-Lopez J, Pourrezai K, Izzetoglu K, Barroso YMJM, et al. Does dorsolateral prefrontal cortex. (DLPFC) activation return to baseline when sexual stimuli cease? The role of DLPFC in visual sexual stimulation. Neurosci Lett. (2007) 416:55-60. doi: 10.1016/j.neulet.2007.01.058

64. Seok JW, Sohn JH. Neural substrates of sexual desire in individuals with problematic hypersexual behavior. Front Behav Neurosci. (2015) 9:321. doi: 10.3389/fnbeh.2015.00321

65. Cacioppo S. Neuroimaging of female sexual desire and hypoactive sexual desire disorder. Sex Med Rev. (2017) 5:434-44. doi: 10.1016/j.sxmr.2017.07.006 
66. Seeley WW, Menon V, Schatzberg AF, Keller J, Glover GH, Kenna $\mathrm{H}$, et al. Dissociable intrinsic connectivity networks for salience processing and executive control. J Neurosci. (2007) 27:2349-56. doi: 10.1523/JNEUROSCI.5587-06.2007

67. Hoffmann M. The human frontal lobes and frontal network systems: an evolutionary, clinical, and treatment perspective. ISRN Neurol. (2013) 2013:892459. doi: 10.1155/2013/892459

68. Beauregard M, Levesque J, Bourgouin P. Neural correlates of conscious self-regulation of emotion. J Neurosci. (2001) 21:Rc165. doi: 10.1523/JNEUROSCI.21-18-j0001.2001

69. Laird AR, Eickhoff SB, Li K, Robin DA, Glahn DC, Fox PT. Investigating the functional heterogeneity of the default mode network using coordinate-based meta-analytic modeling. J Neurosci. (2009) 29:14496-505. doi: 10.1523/JNEUROSCI.4004-09.2009

70. Andrews-Hanna JR, Smallwood J, Spreng RN. The default network and self-generated thought: component processes, dynamic control, and clinical relevance. Ann N Y Acad Sci. (2014) 1316:29-52. doi: 10.1111/nyas.12360

71. Hu SH, Wei N, Wang QD, Yan LQ, Wei EQ, Zhang MM, et al. Patterns of brain activation during visually evoked sexual arousal differ between homosexual and heterosexual men. Am J Neuroradiol. (2008) 29:1890-6. doi: 10.3174/ajnr.A1260

72. Huh J, Park K, Hwang IS, Jung SI, Kim HJ, Chung TW, et al. Brain activation areas of sexual arousal with olfactory stimulation in men: a preliminary study using functional MRI. J Sex Med. (2008) 5:619-25. doi: 10.1111/j.1743-6109.2007.00717.x

73. Stoleru S, Fonteille V, Cornelis C, Joyal C, Moulier V. Functional neuroimaging studies of sexual arousal and orgasm in healthy men and women: a review and meta-analysis. Neurosci Biobehav Rev. (2012) 36:1481509. doi: 10.1016/j.neubiorev.2012.03.006

74. Sumich AL, Kumari V, Sharma T. Neuroimaging of sexual arousal: research and clinical utility. Hosp Med. (2003) 64:28-33. doi: 10.12968/hosp.2003.64.1.2378

75. Poeppl TB, Langguth B, Laird AR, Eickhoff SB. Meta-analytic evidence for neural dysactivity underlying sexual dysfunction. J Sex Med. (2019) 16:614-17. doi: 10.1016/j.jsxm.2019.02.012

76. Buckner RL, Andrews-Hanna JR, Schacter DL. The brain's default network: anatomy, function, and relevance to disease. Ann N Y Acad Sci. (2008) 1124:1-38. doi: 10.1196/annals.1440.011

77. Raichle ME. The brain's default mode network. Annu Rev Neurosci. (2015) 38:433-47. doi: 10.1146/annurev-neuro-071013-014030

78. Pan W, Liu C, Yang Q, Gu Y, Yin S, Chen A. The neural basis of trait selfesteem revealed by the amplitude of low-frequency fluctuations and resting state functional connectivity. Soc Cogn Affect Neurosci. (2016) 11:367-76. doi: $10.1093 /$ scan/nsv119

79. Fransson P, Marrelec G. The precuneus/posterior cingulate cortex plays a pivotal role in the default mode network: evidence from a partial correlation network analysis. Neuroimage. (2008) 42:1178-84. doi: 10.1016/j.neuroimage.2008.05.059

80. Hoefler A, Athenstaedt U, Corcoran K, Ebner F, Ischebeck A. Coping with self-threat and the evaluation of self-related traits: an fMRI study. PLOS ONE. (2015) 10:e0136027. doi: 10.1371/journal.pone.0136027

81. Seghier ML. The angular gyrus: multiple functions and multiple subdivisions. Neuroscientist. (2013) 19:43-61. doi: 10.1177/1073858412440596

82. Thakral PP, Madore KP, Schacter DL. A role for the left angular gyrus in episodic simulation and memory. J Neurosci. (2017) 37:8142-9. doi: 10.1523/JNEUROSCI.1319-17.2017

83. Ferretti A, Caulo M, Del Gratta C, Di Matteo R, Merla A, Montorsi F, et al. Dynamics of male sexual arousal: distinct components of brain activation revealed by fMRI. Neuroimage. (2005) 26:1086-96. doi: 10.1016/j.neuroimage.2005.03.025
84. Seok JW, Park MS, Sohn JH. Neural pathways in processing of sexual arousal: a dynamic causal modeling study. Int J Impot Res. (2016) 28:184-8. doi: 10.1038/ijir.2016.27

85. Carson CC, Dean JD. Erectile dysfunction - etiology and risk factors. Management of Erectile Dysfunction in Clinical Practice. London: Springer London (2007). p. 19-39.

86. Grandjean J, Azzinnari D, Seuwen A, Sigrist H, Seifritz E, Pryce CR, et al. Chronic psychosocial stress in mice leads to changes in brain functional connectivity and metabolite levels comparable to human depression. Neuroimage. (2016) 142:544-52. doi: 10.1016/j.neuroimage.2016. 08.013

87. Wise T, Marwood L, Perkins AM, Herane-Vives A, Joules R, Lythgoe DJ, et al. Instability of default mode network connectivity in major depression: a two-sample confirmation study. Transl Psychiatry. (2017) 7:e1105. doi: 10.1038/tp.2017.40

88. Akiki TJ, Averill CL, Wrocklage KM, Scott JC, Averill LA, Schweinsburg B, et al. Default mode network abnormalities in posttraumatic stress disorder: a novel network-restricted topology approach. Neuroimage. (2018) 176:489-98. doi: 10.1016/j.neuroimage.2018.05.005

89. Lee D, Lee J, Lee JE, Jung YC. Altered functional connectivity in default mode network in Internet gaming disorder: influence of childhood ADHD. Prog Neuropsychopharmacol Biol Psychiatry. (2017) 75:135-41. doi: 10.1016/j.pnpbp.2017.02.005

90. Uytun MC, Karakaya E, Oztop DB, Gengec S, Gumus K, Ozmen S, et al. Default mode network activity and neuropsychological profile in male children and adolescents with attention deficit hyperactivity disorder and conduct disorder. Brain Imaging Behav. (2017) 11:1561-70. doi: 10.1007/s11682-016-9614-6

91. Li R, Zhang S, Yin S, Ren W, He R, Li J. The fronto-insular cortex causally mediates the default-mode and central-executive networks to contribute to individual cognitive performance in healthy elderly. Hum Brain Mapp. (2018) 39:4302-11. doi: 10.1002/hbm.24247

92. Bressler SL, Menon V. Large-scale brain networks in cognition: emerging methods and principles. Trends Cogn Sci. (2010) 14:277-90. doi: $10.1016 /$ j.tics.2010.04.004

93. Mulders PC, van Eijndhoven PF, Schene AH, Beckmann CF, Tendolkar I. Resting-state functional connectivity in major depressive disorder: a review. Neurosci Biobehav Rev. (2015) 56:330-44. doi: 10.1016/j.neubiorev.2015.07.014

94. Akiki TJ, Averill CL, Abdallah CG. A network-based neurobiological model of PTSD: evidence from structural and functional neuroimaging studies. Curr Psychiatry Rep. (2017) 19:81. doi: 10.1007/s11920-0170840-4

95. van Oort J, Tendolkar I, Hermans EJ, Mulders PC, Beckmann $\mathrm{CF}$, Schene $\mathrm{AH}$, et al. How the brain connects in response to acute stress: a review at the human brain systems level. Neurosci Biobehav Rev. (2017) 83:281-97. doi: 10.1016/j.neubiorev.2017. 10.015

Conflict of Interest: The authors declare that the research was conducted in the absence of any commercial or financial relationships that could be construed as a potential conflict of interest.

Copyright (C) 2020 Yin, Liu, Ma, Li, Sun, Ren, Li, Huang, Chang and Zhang. This is an open-access article distributed under the terms of the Creative Commons Attribution License (CC BY). The use, distribution or reproduction in other forums is permitted, provided the original author(s) and the copyright owner(s) are credited and that the original publication in this journal is cited, in accordance with accepted academic practice. No use, distribution or reproduction is permitted which does not comply with these terms. 


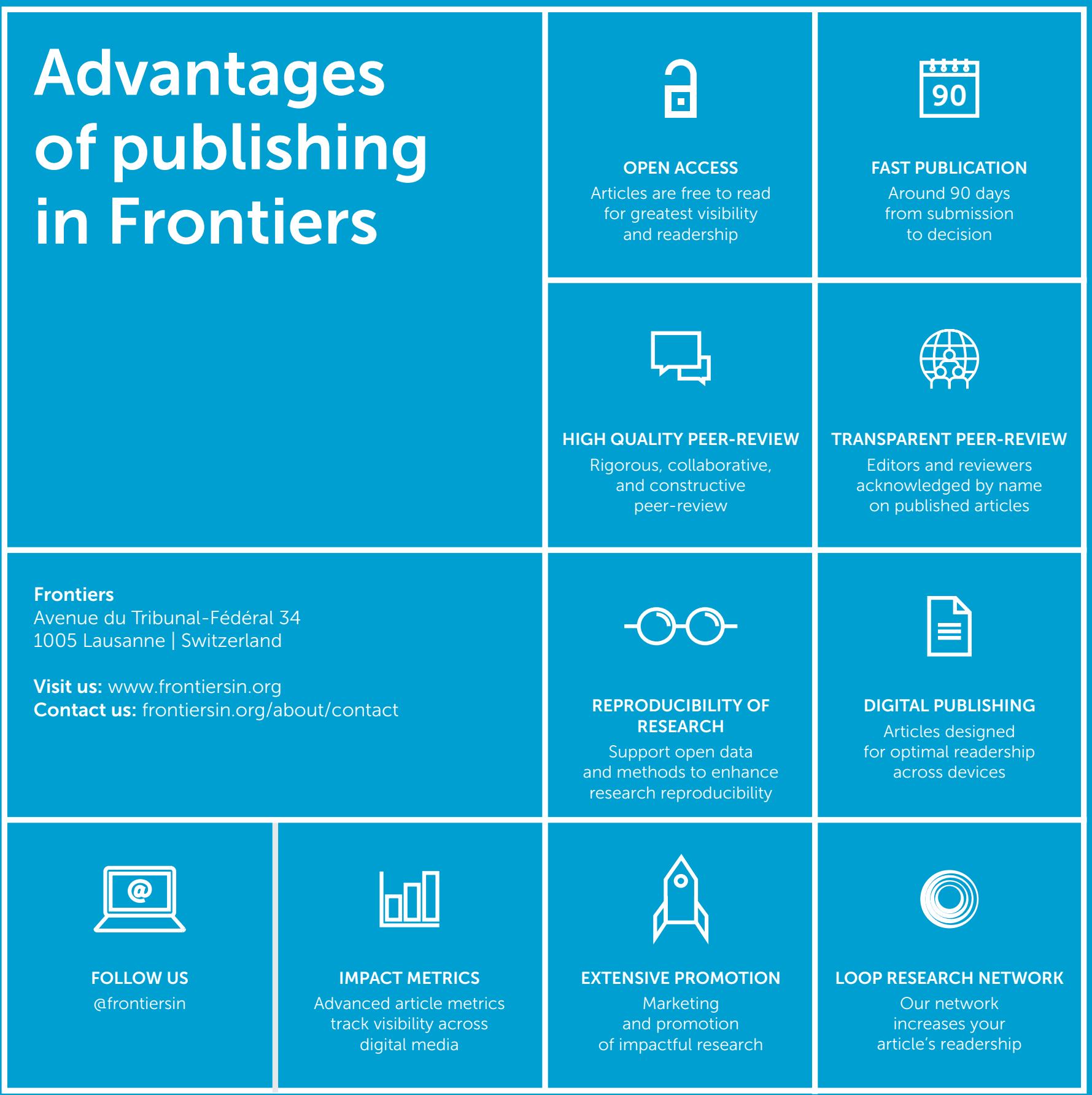

